\title{
UPDATE TO ASSESSMENT OF DIRECT DISPOSAL IN UNSATURATED TUFF OF SPENT NUCLEAR FUEL AND HIGH-LEVEL WASTE OWNED BY U.S. DEPARTMENT OF ENERGY
}

\author{
Idaho National Engineering and Environmental Laboratory \\ Lockheed Martin Idaho Technologies Company \\ Idaho Falls, Idaho 83415
}

September 1998

This report was not produced under a quality assurance program that satisfies the requirements of the National Spent Nuclear Fuel Program and DOE/RW-0333P, Office of Civilian Radioactive Waste Management Quality Assurance Requirements Description. Therefore, the data in the report are not considered qualified and are not to be relied upon to address safety and waste isolation issues until an accepted qualification process has been completed.

Prepared for the

U.S. Department of Energy

Assistant Secretary for Environmental Management Under DOE Idaho Operations Office Contract No. DE-AC07-94ID13223 


\section{DISCLAIMER}

This report was prepared as an account of work sponsored by an agency of the United States Government. Neither the United States Government nor any agency thereof, nor any of their employees, make any warranty, express or implied, or assumes any legal liability or responsibility for the accuracy, completeness, or usefulness of any information, apparatus, product, or process disclosed, or represents that its use would not infringe privately owned rights. Reference herein to any specific commercial product, process, or service by trade name, trademark, manufacturer, or otherwise does not necessarily constitute or imply its endorsement, recommendation, or favoring by the United States Government or any agency thereof. The views and opinions of authors expressed herein do not necessarily state or reflect those of the United States Government or any agency thereof. 


\section{DISCLAIMER}

Portions of this document may be illegible in electronic image products. Images are produced from the best available original document. 
DOE/SNF/REP-015

INEEL/EXT-98-00185

SAND98-0795

Rev. 0

\title{
Update to Assessment of Direct Disposal in Unsaturated Tuff of Spent Nuclear Fuel and High-Level Waste Owned by U.S. Department of Energy
}

\author{
Rob P. Rechard, Editor \\ Nuclear Waste Management Center \\ Sandia National Laboratories \\ P.O. Box 5800 \\ Albuquerque, New Mexico 87185
}

\begin{abstract}
The overall purpose of this study is to provide information and guidance to the Office of Environmental Management of the U.S. Department of Energy (DOE) about the level of characterization necessary to dispose of DOE-owned spent nuclear fuel (SNF). The disposal option modeled was codisposal of DOE SNF with defense high-level waste (DHLW). A specific goal was to demonstrate the influence of DOE SNF, expected to be minor, in a predominately commercial repository using modeling conditions similar to those currently assumed by the Yucca Mountain Project (YMP). A performance assessment (PA) was chosen as the method of analysis. The performance metric for this analysis (referred to as the $1997 \mathrm{PA}$ ) was dose to an individual; the time period of interest was $100,000 \mathrm{yr}$. Results indicated that cumulative releases of ${ }^{99} \mathrm{Tc}$ and ${ }^{237} \mathrm{~Np}$ (primary contributors to human dose) from commercial SNF exceed those of DOE SNF both on a per MTHM and per package basis. Thus, if commercial SNF can meet regulatory performance criteria for dose to an individual, then the DOE SNF can also meet the criteria. This result is due in large part to lower burnup of the DOE SNF (less time for irradiation) and to the DOE SNF's small percentage of the total activity (1.5\%) and mass $(3.8 \%)$ of waste in the potential repository. Consistent with the analyses performed for the YMP, the $1997 \mathrm{PA}$ assumed all cladding as failed, which also contributed to the relatively poor performance of commercial SNF compared to DOE SNE.
\end{abstract}




\section{Preface}

This study, the 1997 performance assessment (1997 PA), evaluates whether spent nuclear fuel (SNF) owned by the Office of Environmental Management of the U.S. Department of Energy (DOE) performs noticeably better (or worse) than commercial SNF, and identifies important parameters that influence this performance after disposal. The latter results, in particular, are intended to help define appropriate requirements for waste characterization with regard to DOE-owned SNF being accepted for disposal. The study is part of a broader DOE program, the National Spent Nuclear Fuel Program, for developing a safe, cost-effective technical strategy for the interim management and ultimate disposition of the foreign and domestic spent nuclear fuel under the DOE's jurisdiction. The DOE-owned SNF is currently stored at three primary sites (Idaho National Engineering and Environmental Laboratory [INEEL], the Hanford reservation, and the Savannah River plant) and several secondary DOE sites. The SNF originated in experimental, defense, and foreign reactors.

\section{Organization of Report}

This report is a detailed account of the analysis design and results. The introductory chapter describes the analysis goals and approach, highlights some of the analysis decisions made during the assessment, and sets the stage for the data and concepts that are presented in later chapters. Chapter 2 provides information on the performance measures and assumptions made with regard to modeling style.

The remaining chapters cover the tasks of performance assessment as they were pursued in this study, i.e., disposal system characterization (Chapters 3, 4, and 5); analysis design (Chapter 6); consequence modeling (Chapters 7 through 10), results (Chapter 11), and guidance based on the results (Chapter 12). The consequence modeling task is grouped in chapters by topic to help the reader locate material of particular interest, specifically, radionuclide source term modeling (Chapter 7), unsaturated zone flow and transport (Chapter 8), saturated zone flow and transport (Chapter 9), and biosphere modeling (Chapter 10). Two appendices containing data provided by INEEL on the content of the fuel categories are provided; a third appendix provides background information for this study.

Although the report documents both the method of analysis and the data used, the organization of the report emphasizes the method of analysis. Therefore, the data are not isolated, but instead are found in related chapters. Most model parameters for characterization of the disposal system are included in Chapters 3,4,5, and 7 .

\section{Acknowledgments}

The authors appreciate the reviews by P. Wheatley, H. Loo, L. Taylor, D. Fillmore, and J. R. Wilson of INEEL; J. Boyd of DOE-ID; P. Gottlieb and J. Duguid of the YMP; and R.F. Weiner of Sandia National Laboratories. The technical assistance of James E. Bean of Sandia with BRAGFLO_T modifications was appreciated. Also, the editorial help on the text and illustrations provided by J.M. Chapman, L.C. Tartaglia, H.N. Olmstead, and S.K. Best of Tech Reps, Inc., Albuquerque, NM, greatly improved the report.

A performance assessment of a complex system using stochastic simulation requires a tremendous volume of long running calculations; thus, the reliability of the computer system is very important. The help of C.M. Williamson, J. Geilow, and $\mathrm{K}$. Aragon of the Computational Support Department is acknowledged. 


\section{Contents}

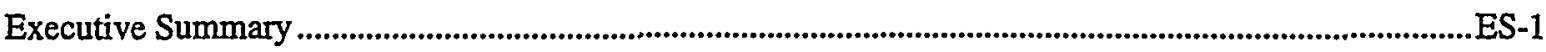

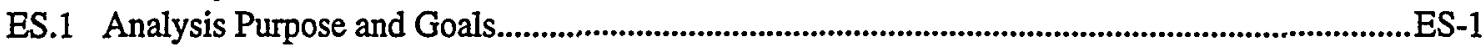

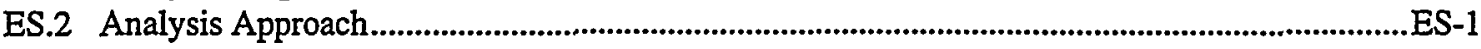

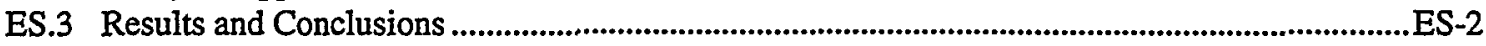

ES.3.1 Performance of DOE SNF Compared to Commercial SNF...........................................ES-2

ES.3.2 Performance of Individual DOE SNF Categories ..........................................................ES-2

ES.3.3 Performance of Disposal Container......................................................................................ES-3

ES.3.4 Behavior of Metallic Uranium..............................................................................................ES-3

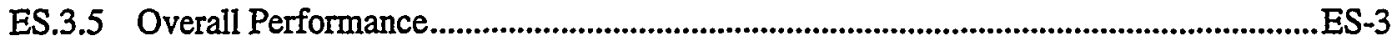

ES.4 Characterization Needs for DOE SNF ............................................................................................ES-4

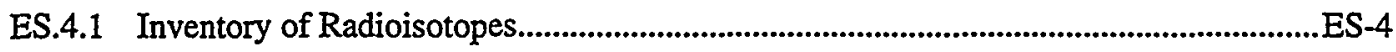

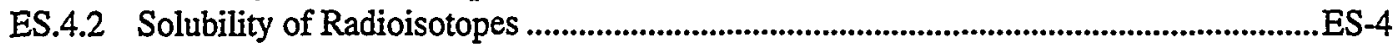

ES.4.3 Corrosion Parameters ............................................................................................................ES-4

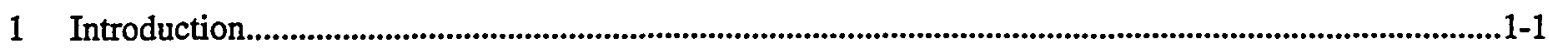

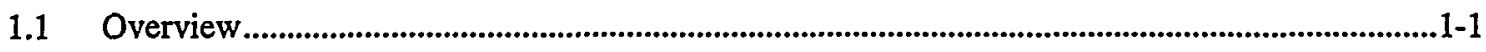

1.2 General Purpose of the Analysis ....................................................................................................... $1-2$

1.3 Organization and Mechanics of the Performance Assessment .........................................................1-3

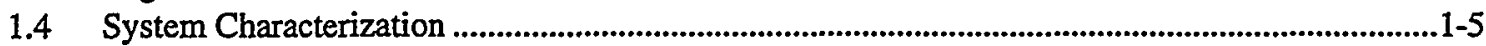

1.4.1 Waste Containment System Terminology ................................................................................... 1-5

1.4.2 Characterization of Waste Package ..............................................................................................1-7

1.4.3 Characterization of Geologic Barrier ...................................................................................1-12

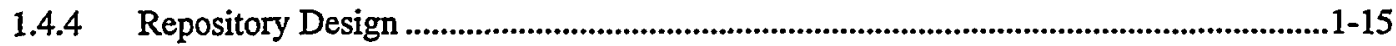

1.5 Scenario Development .............................................................................................................1-19

1.5.1 Selected Features, Events, and Processes.................................................................1-19

1.5.2 Calculational Design ........................................................................................................1-20

1.5.3 Conceptual Models ..................................................................................................................1-21

1.6 Probability Modeling ...............................................................................................................1-21

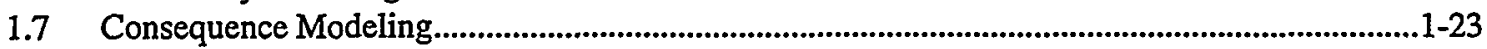

1.7.1 Corrosion and Source Term Modeling .................................................................1-24

1.7.2 Unsaturated Zone, Flow and Transport Modeling ............................................................1-25

1.7.3 Saturated Zone, Flow and Transport Modeling.................................................................1-26

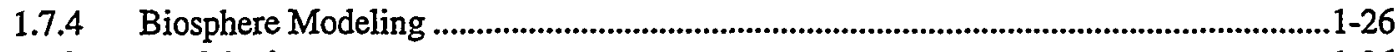

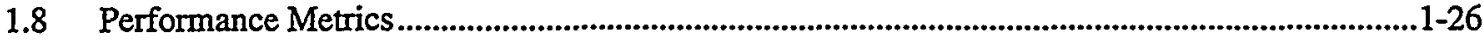

1.9 Sensitivity Analysis ......................................................................................................................... 1-27

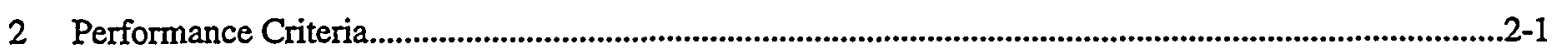

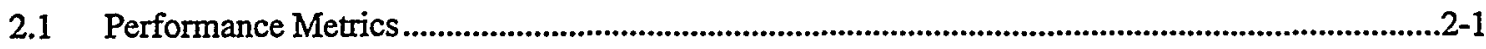

2.1.1 Relative versus Absolute Metrics ..............................................................................................2-1

2.1.2 Overall System Requirements ..........................................................................................

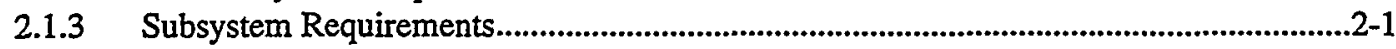

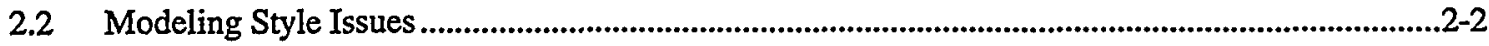

2.2.1 Location of Accessible Environment ...............................................................................2-2

2.2.2 Exposure Pathways to Critical Subgroups of Population....................................................2-2

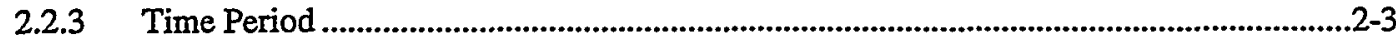

3 System Characterization: Waste Packages ...................................................................................................3-1

3.1 Description of Modeled Spent Fuel Categories ..........................................................................................

3.1.1 Uranium Metal Fuel, Category 1 ...............................................................................................

3.1.2 Uranium-Zirconium Alloy Fuel, Category 2 ......................................................................3-6 
3.1.3 Uranium-Molybdenum Alloy Fuel, Category 3 .....................................................................3-8

3.1.4 Uranium Oxide Fuel-Intact Clad, Category 4 …...............................................................3-10

3.1.5 Uranium Oxide Fuel-Failed Clad, Category 5 ................................................................3-12

3.1.6 Uranium Aluminum Alloy Fuel, Category 6 .......................................................................3-14

3.1.7 Uranium Silicide Fuel, Category 7......................................................................................3-16

3.1.8 Uranium/Thorium Carbide Fuel-Intact Clad, Category 8................................................3-18

3.1.9 Uranium/Thorium Carbide Fuel-Failed Clad, Category 9..............................................3-20

3.1.10 Uranium/Plutonium Carbide Fuel, Category 10....................................................................3-22

3.1.11 Mixed Oxide Fuel, Category 11 .............................................................................3-24

3.1.12 Uranium-Thorium Oxide Fuel, Category 12 ......................................................................3-26

3.1.13 Uranium/Zirconium Hydride Fuel, Category 13 .................................................................3-28

3.1.14 21-PWR Commercial - Uranium Oxide Fuel, Category 14 …............................................3-30

3.1.15 44-BWR Commercial - Uranium Oxide Fuel, Category 15 ............................................3-32

3.1.16 Defense High-Level Waste....................................................................................................3-34

3.2 Handling and Disposal Containers..............................................................................................3-36

3.2.1 Handling Containers..............................................................................................3-36

3.2.2 Disposal Containers...........................................................................................................3-37

3.3 Codisposal Configurations...........................................................................................................3-38

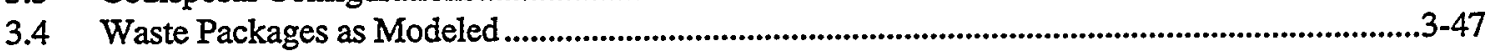

3.4.1 Layers and Matrices per Waste Package ...............................................................................3-48

3.4.2 Radioisotope Inventory per Waste Package ........................................................................3-48

3.5 Summary of Assumptions for Waste Package Conceptual Model ...................................................3-70

3.6 Modeling Parameters for Waste Package .................................................................................................3-71

3.6.1 Layers and Matrices Modeled per Waste Package.............................................................3-71

3.6.2 Activity per Category and Radionuclide Half-Life ...........................................................3-71

4 System Characterization: Geologic Barrier ................................................................................................4-1

4.1 Data Sources and Previous Studies..............................................................................................

4.2 Regional Setting and Climate ........................................................................................................4-1

4.2.1 Regional Geologic Setting.................................................................................................4-1

4.2.2 Regional Hydrology ...........................................................................................................4-8

4.2.3 Regional Geochemistry ....................................................................................................4-12

4.2.4 Regional Climate ...........................................................................................................12

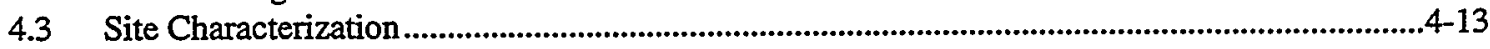

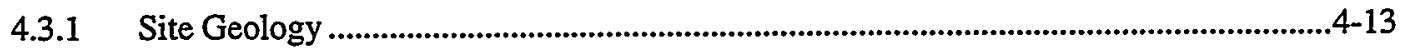

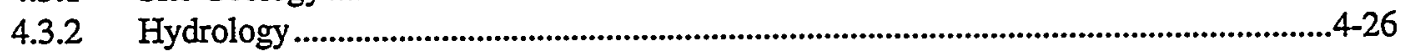

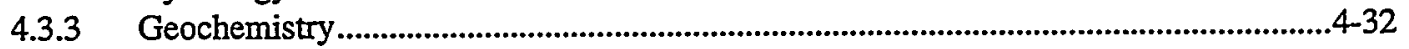

4.3.4 Climate ...................................................................................................................4-41

4.4 Summary of Assumptions for Geologic Barrier Conceptual Model ............................................4-46

4.5 Modeling Parameters for Geologic Barrier.....................................................................................4-48

5 System Characterization: Repository Design .............................................................................................5-1

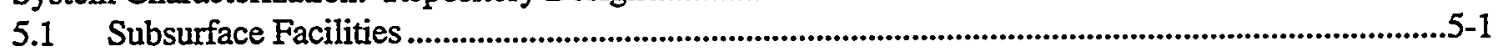

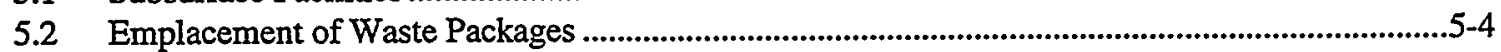

5.2.1 Waste Package Spacing ......................................................................................................5-4

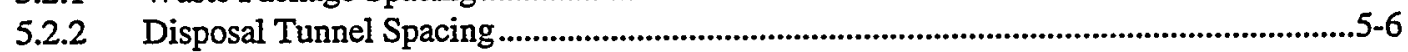

5.2.3 Enclosed Area...............................................................................................................5-8

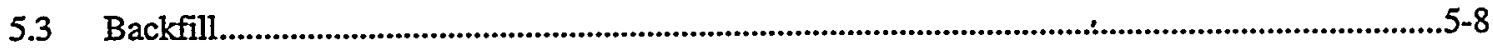

5.4 Summary of Assumptions for Repository Design Conceptual Model..............................................5-9

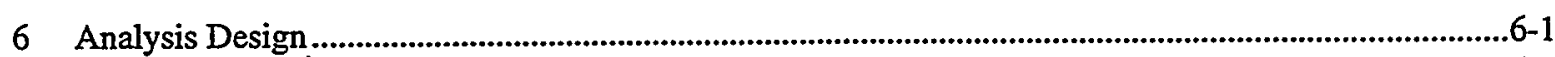

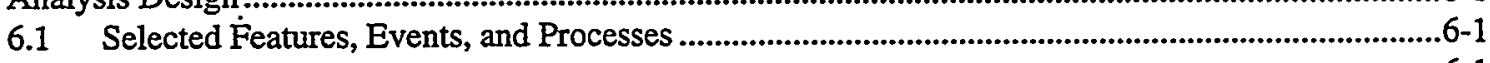

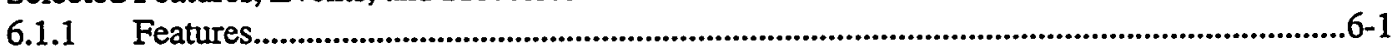




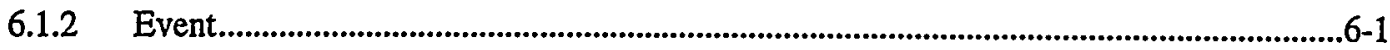

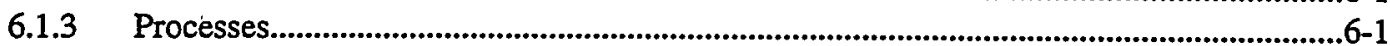

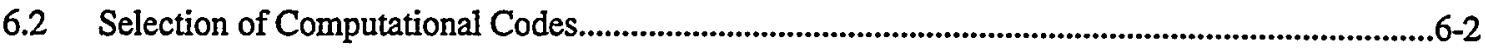

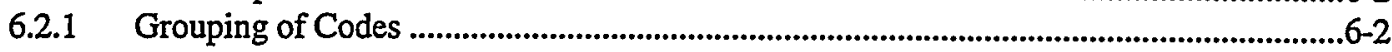

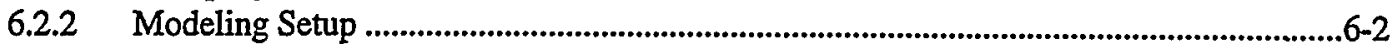

6.2.3 Corrosion and Source Term Consequence Modeling.........................................................6-6

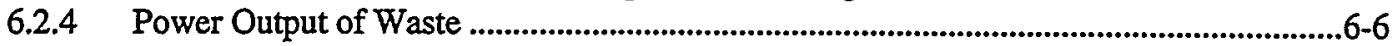

6.2.5 Unsaturated Zone, Flow Consequence Modeling …......................................................6-7

6.2.6 Unsaturated Zone, Transport Modeling ........................................................................6-8

6.2.7 Saturated Zone, Flow and Transport Consequence Modeling ........................................6-8

6.2.8 Biosphere Consequence Modeling ..................................................................................6-9

6.2.9 Probability Modeling and CCDF Construction .................................................................6-9

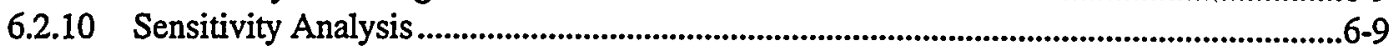

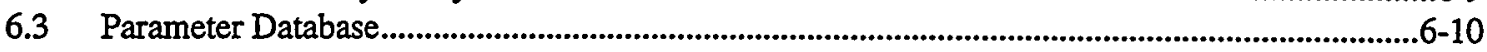

6.4 Sampled Parameters........................................................................................................................6-10

6.4.1 Parameters Selected for Sampling .......................................................................6-10

6.4.2 Parameters Held Constant .............................................................................................6-11

7 Consequence Modeling: Corrosion and Source-Term Model .......................................................................7-1

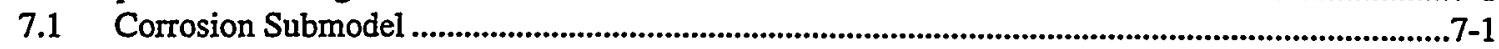

7.1.1 Overview of Waste Packages, Corrosion, and Corrosion Submodel ................................... $7-1$

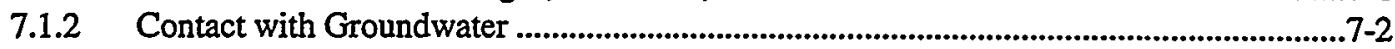

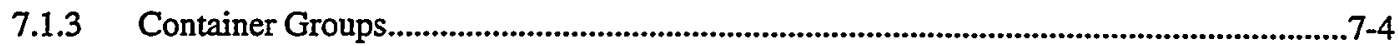

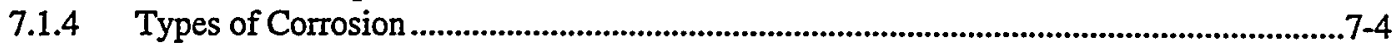

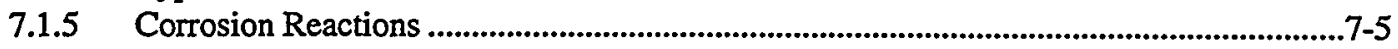

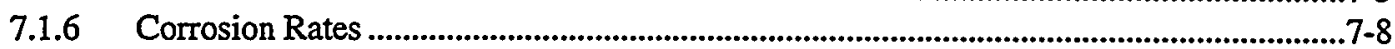

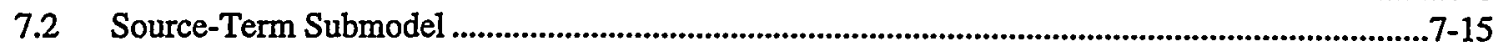

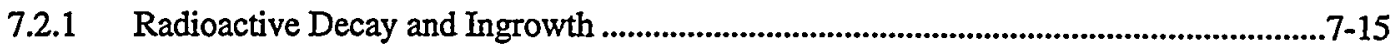

7.2.2 Distribution of Radioisotopes within the Waste Packages...............................................7-16

7.2.3 Transport of Radioisotopes from the Waste Packages to the Intact Host Rock..............7-19

7.3 Kinetic Parameters Used for the 1997 PA...........................................................................7-21

7.3.1 Disposal Container, Handling Container, Basket, Cladding, and Binder Materials .......7-21

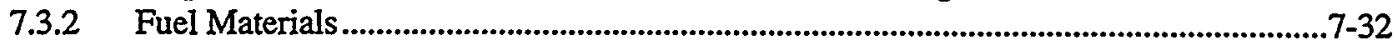

7.4 Summary of Assumptions for Corrosion and Source-Term Conceptual Models ...........................7-42

7.5 Modeling Parameters for Corrosion and Source-Term Submodels ................................................ 7-43

8 Consequence Modeling: Unsaturated Zone Flow and Transport ...........................................................8-1

8.1 Repository Fluid Flow and Temperature Modeling...........................................................................

8.1.1 Mathematical Model Description ..............................................................................8

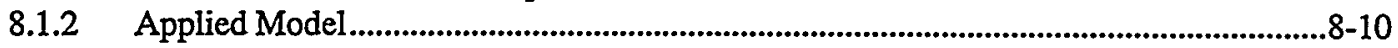

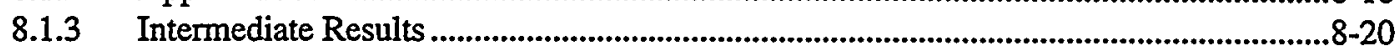

8.2 Thermal Calculations for Waste Package in Near Field ............................................................8-28

8.2.1 Code Descriptions for Thermal Conduction Calculations...............................................8-28

8.2.2 Computed Thermal Histories .........................................................................................8

8.3 Radionuclide Transport Modeling in Unsaturated Zone ...........................................................8-33

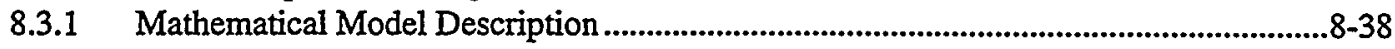

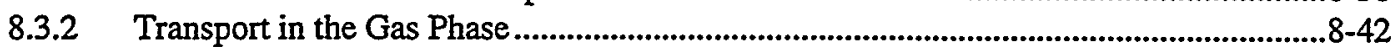

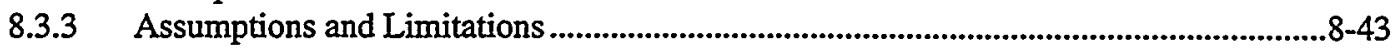

8.3.4 Implementation of Mathematical Model (Computational Model) .................................8-44

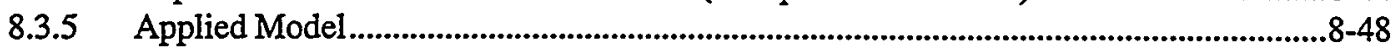


9 Consequence Modeling: Saturated Zone Flow and Transport ...................................................................9-1

9.1 Fluid Flow Model ......................................................................................................................................9-1

9.1.1 Mathematical Model Description ............................................................................................9-1

9.1.2 Applied Model........................................................................................................................9-5

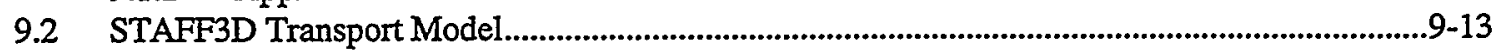

9.2.1 Mathematical Model Description ......................................................................................9-13

9.2.2 Applied Model......................................................................................................................9-16

10 Consequence Modeling: Biosphere Transport and Dose......................................................................10-1

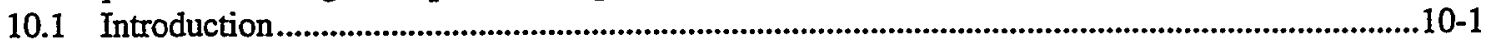

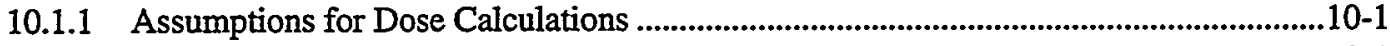

10.1.2 Methodology ................................................................................................................10-1

10.1.3 Criteria ............................................................................................................................10-3

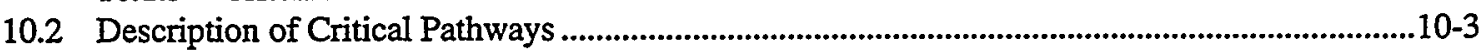

10.2.1 Critical Pathway for Ranch Case .........................................................................................10-3

10.2.2 Critical Pathways for Farm Case ...............................................................................10-5

10.2.3 Critical Pathways for Small Community .................................................................10-5

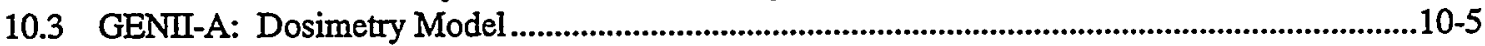

10.3.1 Types of Doses Calculated ...........................................................................................10-5

10.3.2 Data Input ............................................................................................................................10-6

10.3.3 Calculating Dose .......................................................................................................10-7

10.3.4 Types of Exposure......................................................................................................10-10

10.3.5 Link to 1997 PA Data (PREGENII) ...............................................................................10-10

10.4 Conceptual Models for Exposure Cases ........................................................................................10-11

10.4.1 Model for the Ranch Case ...........................................................................................10-11

10.4.2 Model for Farm Case.........................................................................................................10-11

10.4.3 Model for Small Community...........................................................................................10-14

10.5 Biosphere Conversion Factors ......................................................................................................10-15

11 Selected Overall Results .....................................................................................................................11-1

11.1 Findings for Waste Form ......................................................................................................11-1

11.1.1 Description of Spent Nuclear Fuel ......................................................................................11-1

11.1.2 Parameters Sampled ...............................................................................................................11-1

11.1.3 Releases of ${ }^{99} \mathrm{Tc}$ and ${ }^{237} \mathrm{~Np}$ from Waste Packages ..................................................11-5

11.1.4 Fraction of Waste Exposed, Altered, and Released ............................................................11-9

11.1.5 Changes in Modeling of Waste Form................................................................................11-12

11.2 Findings for Waste Container ............................................................................................................11-18

11.2.1 Description of Containers.......................................................................................11-18

11.2.2 Parameters Sampled .........................................................................................................11-18

11.2.3 Results for Waste Container ....................................................................................11-20

11.2.4 Potential Changes in Container .........................................................................11-24

11.3 Findings for Repository Design ..................................................................................................11-28

11.3.1 Description ..........................................................................................................11-28

11.3.2 Results for Repository Design.....................................................................................11-28

11.4 Findings for Geologic Barrier and Climate Change ...................................................................11-31

11.4.1 Description ..........................................................................................................................11-31

11.4.2 Parameters Sampled .......................................................................................................11-31

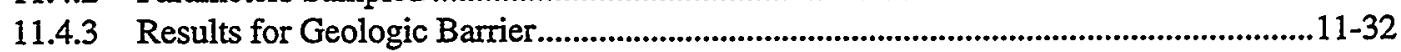

11.5 Findings on Behavior of Overall Disposal System...................................................................11-39

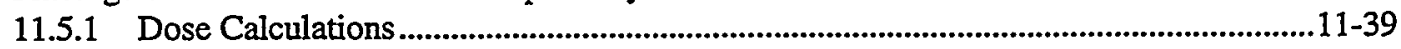

11.5.2 Change of Dose With Time ................................................................................11-39 


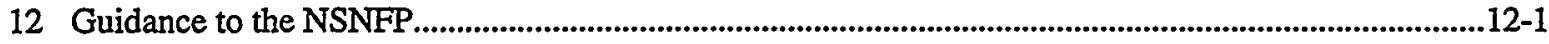

12.1 Guidance for Repository Data Needs Based on Performance Assessment ..................................12-1

12.1.1 Inventory of Radioisotopes..................................................................................................12-1

12.1.2 Handling Containers for DOE SNF............................................................................12-2

12.1.3 Solubility of Radioisotopes .........................................................................................12-2

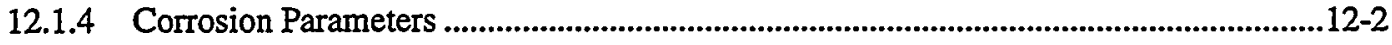

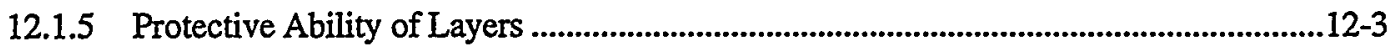

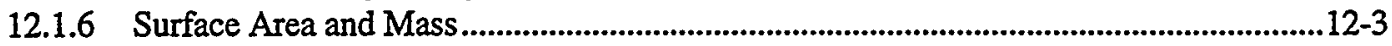

12.2 Recommended Future Analysis Tasks................................................................................................12-3

12.2.1 Screen Features, Events, and Processes ..................................................................12-3

12.2.2 Continued Assessment of Unique Aspects of DOE SNF..................................................12-4

12.2.3 Additional Evaluations ...............................................................................................12-4

12.2.4 Produce Abstractions from Detailed Analyses...............................................................12-5

12.2.5 Upgrade Quality Assurance...............................................................................12-5

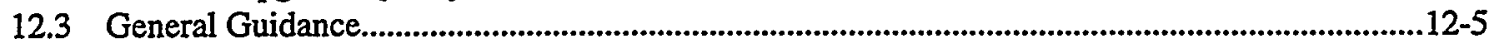

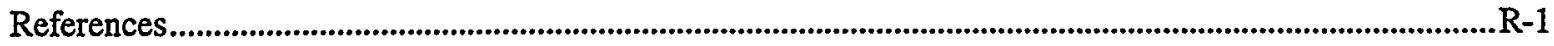

Appendix A: DOE Spent Nuclear Fuel Information

Appendix B: SNF Grouping for TSPA, 2035 Data

Appendix C: Background on 1997 Performance Assessment

Appendix D: Parameters for Waste Package Materials for Final 1997 PA 


\section{Figures}

1-1 Sandia's method for conducting DOE SNF/DHLW Performance Assessment calculations involves six general tasks (after Rechard, 1989, Figure 3.1) ............................................................. 1-4

1-2 Subsystems and components of a radioactive waste containment system ........................................ 1-6

1-3 Examples of packaging of spent nuclear fuel and high level waste ..................................................... 1-10

1-4 Location of potential repository at Yucca Mountain, Nevada. ............................................................ 1-13

1-5 Simplified stratigraphic column of Yucca Mountain tuffs and 16 hydrologic modeling units with similar porosity and/or welding that were used in the simulations ..................................... 1-14

1-6 Layout of disposal tunnels and access drifts for potential repository in tuff...................................... 1-16

1-7 Orientation of waste packages...................................................................................................... 1-17

1-8 Spacing pattern of waste packages to obtain fairly uniform distribution of temperature in host rock. ............................................................................................................................................... 1-18

1-9 Schematic of water movement and eventual degradation of container(s) and waste in potential unsaturated tuff repository............................................................................................................ 1-22

1-10 Four categories of codes used for consequence modeling .................................................................. 1-23

2-1 Dose comparisons from various sources ...................................................................................... $2-2$

3-1 Thirty-four original groupings of DOE spent nuclear fuel and 15 categories of spent

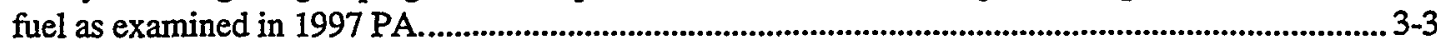

3-2 Category 1 spent fuel, represented by N-Reactor fuel assembly........................................................... 3-5

3-3 Category 3 spent fuel, represented by fuel pins only (center portion of figure)

for Enrico Fermi Reactor................................................................................................................................... 3-9

3-4 Category 4 spent fuel, represented by Shippingport PWR fuel assembly........................................... 3-11

3-5 Category 5 spent fuel, represented by Three Mile Island failed fuel assemblies............................... 3-13

3-6 Category 6 spent fuel, represented by Advanced Test Reactor (ATR) fuel assembly..................... 3-15

3-7 Category 7 spent fuel, represented by fuel from the Materials Testing Reactor (MTR) and Foreign Research Reactor (FRR)....................................................................................... 3-17

3-8 Category 8 spent fuel, represented by Fort St. Vrain fuel assembly.................................................. 3-19

3-9 Category 9 spent fuel, represented by Peach Bottom fuel assembly................................................. 3-21

3-10 Category 11 spent fuel, represented by Fast Flux Text Facility standard driver fuel assembly........ 3-25

3-11 Category 12 spent fuel, represented by Shippingport Light Water Breeder Reactor (LWBR)

fuel assembly............................................................................................................................................. 3-27

3-12 Category 13 spent fuel, represented by Training, Research, and Isotope production-

General Atomic (TRIGA) standard fuel assembly...............................................................................3-29

3-13 Category 14 spent fuel, represented by 21-PWR fuel assembly. ......................................................... 3-31

3-14 Category 15 spent fuel, represented by 44-BWR fuel assembly......................................................... 3-33

3-15 High-level waste DOE standard handling container. ........................................................................... 3-35

3-16 Codisposal configuration for Category 1, Uranium Metal, including four DOE SNF

handling containers and no DHLW handling containers. .................................................................. 3-39

3-17 Codisposal configuration for Category 2, Uranium-Zirconium Alloy, including one

DOE SNF handling container and five DHLW handling containers ................................................. 3-39

3-18 Codisposal configuration for Category 3, Uranium-Molybdenum Alloy, including one

DOE SNF handling container and four DHLW handling containers................................................... 3-40

3-19 Codisposal configuration for Category 4, Uranium Oxide-Intact Clad, including one

3-20 Codisposal configuration for Category 5, Uranium Oxide-Failed Clad, including one

3-21 Codisposal configuration for Category 6, Uranium Aluminum Alloy, including one

3-22 Codisposal configuration for Category 7, Uranium Silicide, including one DOE SNF handling container and five DHLW handling containers 


\section{Figures (continued)}

3-23 Codisposal configuration for Category 8, Uranium-Thorium Carbide-Intact Clad, including one DOE SNF handling container and five DHLW handling containers. ......................... 3-42

3-24 Codisposal configuration for Category 9, Uranium-Thorium Carbide-Failed Clad, including one DOE SNF handling container and five DHLW handling containers .......................... 3-43

3-25 Codisposal configuration for Category 10, Uranium-Plutonium Carbide, including one DOE SNF handling container and four DHLW handling containers.

3-26 Codisposal configuration for Category 11, Mixed Oxide Fuel, including one DOE SNF handling container and four DHLW handling containers.

3-27 Codisposal configuration for Category 12, Uranium-Thorium Oxide, including one DOE SNF handling container and three DHLW handling containers $3-44$

3-28 Codisposal configuration for Category 13, Uranium/Zirconium Hydride, including one DOE SNF handling container and five DHLW handling containers ..........................................3-45

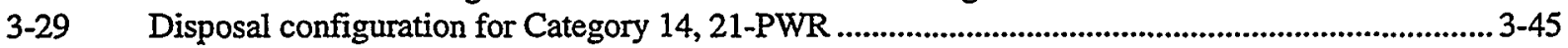

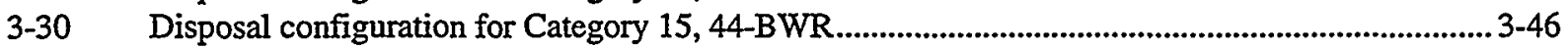

3-31 Curie content by waste package. ................................................................................................................3-51

3-32 Thermal power by waste package, based on total of 43 radioisotopes. ............................................ 3-52

3-33 Initial (year 2030) activity of 49 radioisotopes considered in the performance assessment for waste packages with Category 1 fuel (uranium metal) ............................................... 3-54

3-34 Initial (year 2030) activity of 49 radioisotopes considered in the performance assessment for waste packages with Category 2 fuel (uranium-zirconium alloy codisposed with DHLW).

3-35 Initial (year 2030) activity of 49 radioisotopes considered in the performance assessment for waste packages with Category 3 fuel (uranium-molybdenum alloy codisposed with DHLW).

3-36 Initial (year 2030) activity of 49 radioisotopes considered in the performance assessment for waste packages with Category 4 fuel (uranium oxide - intact clad, codisposed with DHLW)......... 3-57

3-37 Initial (year 2030) activity of 49 radioisotopes considered in the performance assessment for waste packages with Category 5 fuel (uranium oxide - failed clad, codisposed with DHLW). ....... 3-58

3-38 Initial (year 2030) activity of 49 radioisotopes considered in the performance assessment for waste packages with Category 6 fuel (uranium aluminum alloy, codisposed with DHLW)............ 3-59

3-39 Initial (year 2030) activity of 49 radioisotopes considered in the performance assessment for waste packages with Category 7 fuel (uranium silicide codisposed with DHLW).

3-40 Initial (year 2030) activity of 49 radioisotopes considered in the performance assessment for waste packages with Category 8 fuel (uranium-thorium carbide - intact clad, codisposed with DHLW).

3-41 Initial (year 2030) activity of 49 radioisotopes considered in the performance assessment for waste packages with Category 9 fuel (uranium-thorium carbide - failed clad, codisposed with DHLW).....

3-42 Initial (year 2030) activity of 49 radioisotopes considered in the performance assessment for waste packages with Category 10 fuel (uranium-plutonium carbide, codisposed with DHLW)...... 3-63

3-43 Initial (year 2030) activity of 49 radioisotopes considered in the performance assessment for waste packages with Category 11 fuel (mixed oxide fuel, codisposed with DHLW)................. 3-64

3-44 Initial (year 2030) activity of 49 radioisotopes considered in the performance assessment for waste packages with Category 12 fuel (uranium-thorium oxide, codisposed with DHLW)....... 3-65

3-45 Initial (year 2030) activity of 49 radioisotopes considered in the performance assessment for waste packages with Category 13 fuel (uranium/zirconium hydride, codisposed with DHLW)...... 3-66

3-46 Initial (year 2030) activity of 49 radioisotopes considered in the performance assessment for waste packages with Category 14 fuel (PWR commercial SNF).

3-47 Initial (year 2030) activity of 49 radioisotopes considered in the performance assessment for waste packages with Category 15 fuel (BWR commercial SNF)...

3-48 Initial (year 2030) activity of 49 radioisotopes considered in the performance assessment for total for all 15 waste package types. 


\section{Figures (continued)}

4-1 Potential repository at Yucca Mountain, located northwest of Las Vegas on and adjacent to southwest portions of Nevada Test Site.

4-2 Boundaries of the Basin and Range physiographic province including three of the larger subprovinces (after DOE, 1988b, Figure 1-2).

4-3 Walker Lane and major associated faults (from DOE, 1988b, after Stewart, 1985).......................... 4-5

4-4 Generalized geologic map of Yucca Mountain showing major faults and stratigraphic units and location of generalized structure cross section E-E'.

4-5 Schematic geologic cross section E-E' at Yucca Mountain

4-6 Hydrogeologic flow patterns of the three groundwater subbasins (of the Death

Valley System) in the region of Yucca Mountain

4-7 Stratigraphy in the potential repository area at Yucca Mountain with designations of aquifers and aquitards, or confining units, based on Winograd and Thordarson (1975) .............. 4-11

4-8 Selected stratigraphic units in the vicinity of Yucca Mountain that are important to the hydrology...

4-9 Comparison of (a) formal/informal stratigraphic layering with (b) modeling unit layering for Yucca Mountain from various studies

4-10 Idealized lateral and vertical configuration of welding zones in a simple cooling unit ....................... 4-20

4-11 1997 PA modeling unit column (far right) showing wellbores used for composite

and a plot of core porosity values from those wellbores (middle) ..................................................4-27

4-12 Preliminary potentiometric surface, Yucca Mountain...................................................................... 4-30

4-13 Revised poteniometric surface of an area of small hydraulic gradient, Yucca Mountain.................. 4-31

4-14 Assumed solubility of uranium and plutonium under oxic and reduced conditions in J-13 and UE-25P\#1 well water at Yucca Mountain, Nevada.

4-15 Estimates of distribution coefficients for uranium and plutonium adsorption on rust (i.e., goethite) and volcanic tuff at Yucca Mountain, Nevada, in J-13 well water............................4-42

4-16 Location of weather stations in the vicinity of Yucca Mountain, including Yucca Flat, which is cited as having weather representative of that at Yucca Mountain.

5-1 Location of potential repository at Yucca Mountain, Nevada.

5-2 Layout of disposal tunnels and access drifts for potential repository in tuff with

in-drift emplacement.

5-3 Disposal tunnel and emplacement of waste package for horizontal, in-drift emplacement......................5-5

5-4 Spacing pattern of waste packages to obtain fairly uniform distribution of temperature

in host rock.

Probability distributions of alteration rate of waste and cladding.

6-4 Early clad failure fractions for N-Reactor (Category 1 ) and commercial PWR

(Category 14) fuel.

6-5 Probability distributions for solubility of radioisotopes in expected Yucca Mountain groundwater.

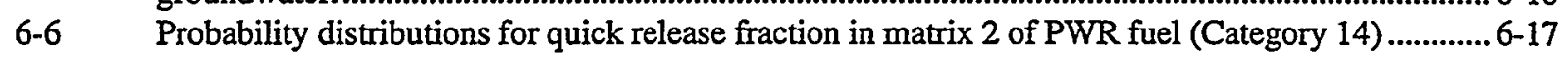

6-7 Activity inventory of neptunium and technetium in PWR (Category 14) and BWR (Category 15) fuels.

6-8 Probability distributions of alteration rate of waste package layer.

Probability distributions of radioisotopes (uranium, plutonium, neptunium, and zirconium) on rust of outer carbon steel container.

6-10 Probability distributions of diffusion of radionuclides through the breached containers, area above the container that contributes water (e.g., drips), that in turn contacts the container surface, fraction of containers with failed inner layer, and fraction of containers with rubble. 


\section{Figures (continued)}

6-11 Probability distributions for biosphere parameters, including irrigation rate, and consumption of drinking water, vegetables, milk, and beef.

6-12 Probability distributions of bulk, saturated hydraulic conductivity of modeling layers

TSw, CHnv, CHnz, and PPw.

Probability distributions of hydraulic conductivity of layers.

Probability distributions of spacing of fractures assigned to modeling layers TSw, CHnv, CHnz, and PPw.

Probability distributions for distribution coefficients for neptunium for vitrified tuff, uranium and plutonium for devitrified tuff, uranium for zeolitic tuff, and for dispersivity fraction.

7-1 Emplaced waste package with air gap (left) and host rock rubble (right)

8-1 Comparison of van Genuchten and piecewise models. ........................................................... 8-6

8-2 Median value capillary pressure functions for both matrix and fracture in TSw................................ 8-7

Repository temperature, matrix water saturation, and fracture water saturation

8-12 Repository oxygen mass fraction and water mass fraction at the center and down-dip edge of the repository.

8-13 Vertical water flow rate at the center of the repository, and water pressure and gas pressure at the center and down-dip edge of the repository.

8-14 Infiltration rate and cumulative volume of water passing through the top and bottom surfaces of the repository and the ground surface.

8-15 Infiltration rate and cumulative volume of water passing through the upper TSv and lower TSv layers and into the water table.

8-16 Oxygen availability as (a) oxygen density at center of repository, (b) water, nitrogen, and oxygen mass fraction at center of repository, and (c) cumulative reaction fluids over time. ........... 8-27

9-1 $X-Y$ "surface" view of STAFF3D grid used for aqueous flow and transport ......................................9-7

9-2 Calculated hydraulic head field for units intersecting water table near repository ............................. 9-12

9-3 Conceptual hydrologic model of the aquifer............................................................................. 9-14

9-4 ${ }_{99}^{99} \mathrm{Tc}$ concentrations in the $\mathrm{PPw}$ unit for various times....................................................................9-26

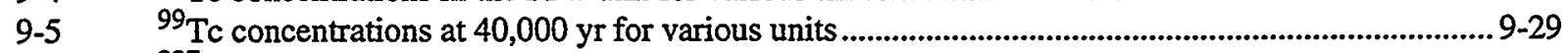

9-6 ${ }^{237} \mathrm{~Np}$ concentrations in the TSwe unit for various times. ................................................................9-31 


\section{Figures (continued)}

9-7 $\quad{ }^{237} \mathrm{~Np}$ concentrations at 10,000 yr for various units $9-34$

10-1 Critical pathways used in the 1997 PA: (a) Ranch case, and (b) Farm case $10-4$

10-2 Situation showing an internal effective dose equivalent (EDE) as a committed EDE (area under curve) from 1-yr internal intake dose.

10-3 Schematic of biosphere transport modeling, including data input by means of PREGENII. 10-12

11-1 A large portion (84\%) of the total radioisotope inventory is commercial spent nuclear fuel (SNF), with only 13\% defense high level waste (DHLW), and 3\% DOE SNF11-2

11-2 Comparison of neptunium solubilities used in various performance assessments.

11-3 Alteration ("corrosion") rates for matrices of spent nuclear fuel (SNF) as depth altered per time, based on parameter values reported in Chapter 7 for the Arrhenius equation.

11-4 Fraction of releases from the waste package in the deterministic run using mean model parameter values

11-5 Comparison of inventory of ${ }^{99} \mathrm{Tc}$ and ${ }^{237} \mathrm{~Np}$ in each SNF category shows that the inventory of DHLW in the codisposal option dominates the categories, making them appear to be similar ... 11-7

11-6 Content of ${ }^{99} \mathrm{Tc}$ and ${ }^{237} \mathrm{~Np}$ per MTHM for SNF categories, with and without codisposal with DHLW.

11-7 Performance of waste package in 1997 and 1994 PAs............................................................. 11-10

11-8 Location of radioisotopes in various compartments of the source-term model for (a) ${ }^{99} \mathrm{Tc}$ and (b) ${ }^{237} \mathrm{~Np}$.

11-9 Advective and diffusive repository releases of technetium and neptunium using median properties for (a) 1997 PA, 75,336-MTHM repository, and (b) 1994 PA, 12,060-MTHM repository.

11-10 Releases from the waste package when cladding is included for the deterministic run using mean model parameters.

11-11 Location of radioisotopes when cladding is included. $11-14$

11-12 Location of radioisotopes in various components of the source term model when doubling surface area of fuel matrix on commercial SNF and N-Reactor fuel in the deterministic run, using mean model parameter values for all other parameters for (a) ${ }^{99} \mathrm{Tc}$ and (b) ${ }^{237} \mathrm{~Np}$.

11-13 Location of radioisotopes when solubility is set at maximum values (and sorption, $\mathrm{K}_{\mathrm{D}}$, of $\mathrm{Np}$ on rust of $0.025 \mathrm{~kg} / \mathrm{m}^{3}$ )

11-14 Fraction of releases from the waste package when the inventory of DOE SNF is tripled for the deterministic run using mean model parameter values.

11-15 Corrosion rates for layers of waste containers and cladding on waste............................................. 11-19

11-16 Time of first breach for each container group across the repository for Waste Package 1

(N-Reactor).

11-17 Neptunium release rates from (a) deterministic run, using median properties, for 1997 PA hot repository compared to (b) overall repository release rate of ${ }^{237} \mathrm{~Np}$ in $1994 \mathrm{PA}$

11-18 Production of reaction material within repository, using mean parameter values ........................... 11-23

11-19 Fraction of release from the waste package when using Hastaloy C-22 in place of Inconel 625 in the deterministic run using mean model parameter values

11-20 Location of radioisotopes when the size of the container is halved through waste consolidation for the deterministic run using mean model parameter values

11-21 When amount of adsorptive rust is decreased by one-half using the maximum solubility of $10^{-4} \mathrm{M}$, the time of ${ }^{237} \mathrm{~Np}$ release occurs earlier even at small $\mathrm{K}_{\mathrm{D}}$ values $\left(0.025 \mathrm{~m}^{3} / \mathrm{kg}\right) \ldots \ldots \ldots . .11-26$

11-22 Mass fraction of $\mathrm{O}_{2}$ available for oxic alteration of fuel matrix at (a) center and (b) edge of potential repository over $10^{5} \mathrm{yr}$.

11-23 Mass fraction of $\mathrm{O}_{2}$ available for oxic alteration of fuel matrix at (a) center and

(b) edge of potential repository over $100,000 \mathrm{y}$

11-24 Cumulative volume of water passing vertically through various strata over the repository area in the unsaturated zone over $50,000 \mathrm{yr}$. 


\section{Figures (continued)}

11-25 Advective gas flow field in the repository model using mean parameter values at (a) $10 \mathrm{yr}$, (b) $100 \mathrm{yr}$, (c) $500 \mathrm{yr}$, and (d) $2000 \mathrm{yr}$

11-26 Releases and concentrations $\left(\mathrm{kg} / \mathrm{m}^{3}\right)$ for ${ }^{99} \mathrm{Tc}$ in deterministic run using mean parameter values.

11-27 Releases and concentrations $\left(\mathrm{kg} / \mathrm{m}^{3}\right)$ for ${ }^{237} \mathrm{~Np}$ in deterministic run using mean parameter values

11-28 Release of radioisotopes are significantly delayed by just an order of magnitude reduction in water passing through the container.

11-29 The distribution of radioisotopes is significantly affected by the reduction in water passing through the container for (a) ${ }^{99} \mathrm{Tc}$ and (b) ${ }^{237} \mathrm{~Np}$.

11-30 Dose as a function of time for (a) mean and median calculations for Farm case, water and vegetable consumption; (b) mean and median calculations for Ranch case, beef consumption only, and (c) mean totals for three cases (Farm case, drinking water only case, and Ranch case)..

11-31 Dose at 5-km boundary from drinking water as a function of time for (a) deterministic run using mean parameter values, (b) 87 realizations in $1997 \mathrm{PA}$, and (c) quantiles on 87 realizations. 


\section{Tables}

1-1 Categories of Spent Nuclear Fuel and High Level Waste Used in 1997 PA ....................................1-8

1-2 Codisposal Configuration Options for Categories 1 through 13 ...................................................1-11

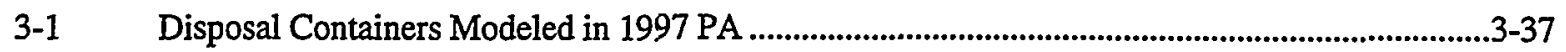

3-2 Codisposal Configuration Options for Categories 1 through 13 ...................................................3-38

3-3 Waste Package Types Modeled in the 1997 Performance Assessment ..........................................3-47

3-4 Radioisotopes Considered in 1997 Performance Assessment.......................................................3-53

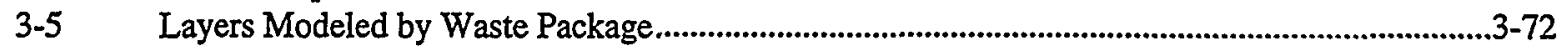

3-7 Total Curies (DSNF and DHLW) by Category .................................................................................3-88

3-8 Radioisotope Halflives (idpram: HALFLIFE) ................................................................................

4-1 Comparison of Luckey et al. (1996) Volcanic Aquifer and Confining Units

to Formal Geologic Units, Ortiz et al. (1985), and 1997 PA Modeling Units ..................................4-9

4-2 Treatment of Fractures in the 1997 PA Conceptual Models .............................................................4-15

4-3 1997 PA Thermal/Hydrologic Modeling Units modified from Ortiz et al. (1985) ..........................4-23

4-4 Designation of Rock Type Grouping for the 1997 PA Modeling Units

for Purposes of Association with $K_{D}$ Value Ranges from Wilson et al. (1994) ..............................4-25

4-5 Comparison of Modeling Approach for Hydrologic Systems and Processes for 1994

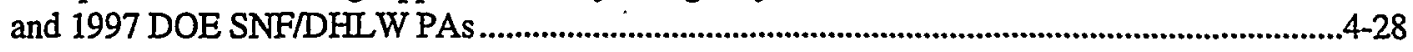

4-6 Composition of Representative Subunit of the Topopah Spring Formation from

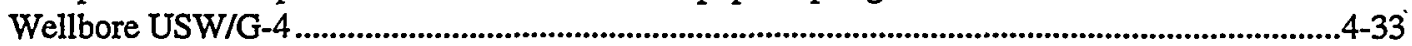

4-7 Composition of Water Samples from Four Wellbores in Vicinity of Yucca Mountain..................4-36

4-8 Climate and Meteorological Conditions Typical for Yucca Mountain ............................................4-43

4-9 Summary of Geologic Barrier Conceptual Models including Geology, Hydrology,

Geochemistry and Climate ...................................................................................................................4-46

4-10 Tiva Canyon Welded Tuff (idmtrl: TCW) Thermal/Mechanical Hydrologic Parameters .............4-23

4-11 Paintbrush Non-Welded Tuff (idmtrl: PTN) Thermal/Mechanical Hydrologic Parameters..........4-23

4-12 Topopah Springs Vitrophyre (idmtrl: TSV) Thermal/Mechanical Hydrologic Parameters...........4-23

4-13 Topopah Springs Upper Non-Lithophysal (idmtrl: TSUL) Thermal/Mechanical

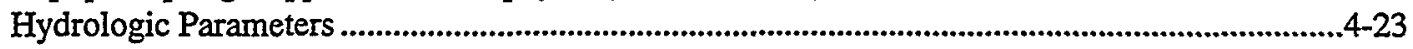

4-14 Topopah Springs Middle Non-Lithophysal (idmtrl: TSMNL) Thermal/Mechanical

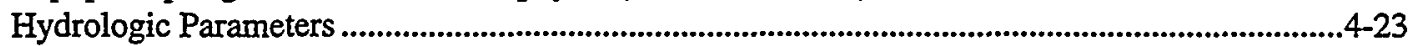

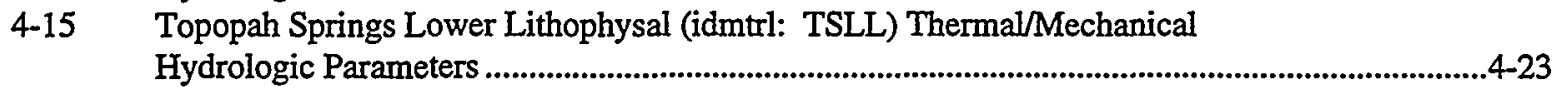

4-16 Topopah Springs Lower Non-Lithophysal (idmtrl: TSLNL) Thermal/Mechanical

Hydrologic Parameters ...........................................................................................................................4-23

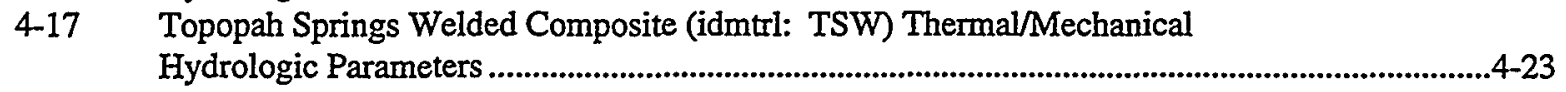

4-18 Calico Hills Non-Welded Vitric Tuff (idmtrl: CHNV) Thermal/Mechanical

4-19 Calico Hills Non-Welded Zeolitic Tuff (idmtrl: CHNZ) Thermal/Mechanical

4-20 Prow Pass Welded, Divitrified Tuff (idmtrl: PPW) Thermal/Mechanical Hydrologic

4-21 Bullfrog/Prow Pass Member of Crater Flat Tuff, Nonwelded, Zeolitic (idmtrl: CFUN)

4-22 Agents Acting on the Tuff Disposal System (idmtrl: GLOBAL) Parameters...............................4-24

4-23 STAFF3D Modeling Parameters for Topopah Springs Welded Tuff (idmtrl: TSWC).................4-24

4-24 STAFF3D Modeling Parameters for Topopah Springs Welded, Vitrophyre (idmtrl: TSLV) .......4-25

4-25 STAFF3D Modeling Parameters for Calico Hills Non-Welded Vitric Tuff (idmtrl: CHNV) .......4-25

4-26 STAFF3D Modeling Parameters for Calico Hills Non-Welded Zeolitic Tuff (idmtrl: CHNZ) ...4-25

4-27 STAFF3D Modeling Parameters for Prow Pass Welded, Divitrified Tuff (idmtrl: PPW) ............4-25 


\section{Tables (continued)}

4-28 STAFF3D Modeling Parameters for Bullfrog/Prow Pass Member of Crater Flat Tuff, Non-Welded, Zeolitic (idmtrl: CFUN). STAFF3D Modeling Parameters for Bullfrog Welded Tuff (idmtrl: BFW) ................................4-26 STAFF3D Modeling Parameters for Middle Member of Crater Flat Tuff (idmtrl: CFMN).........4-26

4-31

7-3 Wet Anoxic Corrosion Reactions and Stoichiometric Factors Used in CST for the 1997 PA Calculations

Kinetic Parameters Used for Aluminum in the 1997 PA .................................................................7-27 


\section{Tables (continued)}

7-27 Modeling Parameters for Thorium-Uranium-Carbide Spent Fuel (idmtrl: MC2).........................7-70

7-28 Modeling Parameters for TRISO (idmtrl: TRISO) .....................................................................7-73

7-29 Modeling Parameters for Uranium-Dioxide Spent Fuel (idmtrl: UO2) ........................................7-76

7-30 Modeling Parameters for Uranium, Metallic, Spent Fuel (idmtrl: URANIUM)............................7-80

7-31 Modeling Parameters for Uranium-Molybdenum-Alloy Spent Fuel (idmtrl: U_MO) ..................7-84

7-32 Modeling Parameters for Uranium Silicide Alloy Spent Fuel (idmtrl: U_SI) ..............................7-87

7-33 Modeling Parameters for Uranium-Zirconium-Hydride Spent Fuel (idmtrl: U_ZR_H)...............7-91

7-34 Modeling Parameters for Zircaloy_2 Cladding (idmtrl: ZIRCALOY) ..........................................7-94

8-1 TSw Median Capillary Pressure and Relative Permeability Parameters..............................................8-7

8-2 Thermal Conduction and Radiation Properties ....................................................................................8-29

8-3 Repository Design Summary for 1997 PA........................................................................................8-31

9-1 Summary of Previous Conceptual Models for Flow and Transport in the Saturated

Zone at Yucca Mountain .........................................................................................................................9-2

9-2 Well Data Used for STAFF3D Conceptual Model Calibration .......................................................9-8

9-3 Technetium-99 - Maximum Concentration and Location ...........................................................9-20

9-4 Iodine-129 - Maximum Concentration and Location ................................................................9-21

9-5 Neptunium-237-Maximum Concentration and Location .......................................................9-22

9-6 Technetium-99 - Maximum Concentration at 5-km Boundary ..........................................................9-23

9-7 Iodine-129 - Maximum Concentration at 5-km Boundary ............................................................9-24

9-8 Neptunium-237 - Maximum Concentration at 5-km Boundary .......................................................9-25

10-1 Various Human Organ Weights ........................................................................................................10-7

10-2 Types of Exposure Included in the 1997 PA................................................................................10-10

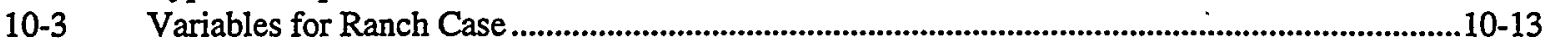

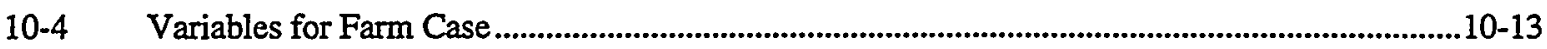

10-5 Growing Cycle and Consumption Data for GENII-A ........................................................10-14

10-6 Variables for Small Community Case ..................................................................................................10-14

10-7 Biosphere Dose Conversion Factors for Unit Concentrations of $1 \mathrm{pCi}$ in Groundwater for Significant Radioisotopes Transported in 1997 PA..............................................................10-15

11-1 Summary of Model Results for Waste Form..................................................................................11-17

11-2 Summary of Model Results for Waste Container.................................................................11-27

11-3 Major Contributors to Initial Power ........................................................................................11-28

11-4 Summary of Model Results for Repository..........................................................................................11-30

11-5 Summary of Model Results for Geologic Barrier and Climate Change........................................11-38

11-6 Summary of Model Results for Total System Performance............................................................11-42

September 30, $1998 \quad$ xvii 


\section{Executive Summary}

This summary describes the performance assessment conducted by the Performance Assessment Department at Sandia National Laboratories. The study was done for the Nati nal Spent Nuclear Fuel Program (NSNFP), coordinated by the Idaho National Engineering and Environmental Laboratory (INEEL) for the Office of Environmental Management of the U. S. Department of Energy (DOE/EM).

\section{ES.1 Analysis Purpose and Goals}

The purpose of the 1997 performance assessment (1997 PA) was to identify the behavior, after permanent disposal, of the spent nuclear fuel (SNF) now under the jurisdiction of DOE/EM. It evaluated whether this DOE SNF performs better or worse than commercial SNF, which can be used as a benchmark in the absence of explicit acceptance criteria. The disposal system modeled is the potential repository at Yucca Mountain, Nevada, containing commercial SNF, defense high level waste (DHLW), and DOE SNF. The performance assessment assumed the codisposal of DOE SNF, without treatment, with vitrified DHLW, in which DOE SNF is packaged with DHLW in the same disposal container.

A second goal, closely related to the first, was to identify the most sensitive parameters through analysis of the results to determine which DOE SNF characteristics should be carefully estimated or measured and which could be neglected, after demonstrating their minor influence. Such information is useful for developing performance-based requirements for repository acceptance criteria, that is, defining characterization requirements only for those spent fuel types and parameters that demand them, thus substantially reducing data gathering and preparation costs for DOE SNF.

The total inventory was 75,336 metric tons of heavy metal (MTHM). The inventory exceeded the 70,000 MTHM limit currently imposed by law in order to include all DOE SNF and sufficient DHLW for codisposal. Most of this inventory was commercial SNF (84\%). High-level waste derived from the reprocessing of SNF was about $13 \%$ of the waste. The DOE SNF categories were primarily based on the chemical characteristics of the fuel matrix and the cladding material. The DOE SNF was about $3 \%$ of the MTHM inventory but only $1.5 \%$ of the radionuclide activity. This analysis did not include fuel from Navy propulsion reactors.

Although the inventory of DOE SNF is relatively small when compared with commercial SNF, it does present unique challenges for characterization because of the large number of different fuel types, materials of construction, and enrichments. Because of the fuels development mission of the DOE, cladding on DOE SNF includes aluminum, zirconium, and carbide, and there are over 250 different fuel types. It is was necessary to group several types together to ease the computational burden.

Thirteen categories of DOE SNF were included in the 1997 PA. Twelve of the DOE SNF categories were codisposed with DHLW in borosilicate glass (Categories 2 through 13). Category 1 (N-Reactor SNF), which was 88\% of the DOE SNF, was modeled in four multi-purpose canister overpacks (MCOs) in a single disposal container.

\section{ES.2 Analysis Approach}

Extensive performance assessment calculations were performed to estimate the behavior of DOE SNF. The same approach was used in two previous performance assessments in 1993 and 1995. Sensitivity analysis was conducted to gain insight into the relative importance of specific DOE SNF properties or parameters. Sensitivity analysis was also performed to understand the influence of (a) including cladding on commercial SNF, (b) substituting Hastaloy C-22 for Inconel 625 as the inner layer of the disposal container, and (c) varying the surface area of container layers and waste matrices. 
The model for degradation of DOE SNF was enhanced in the 1997 PA. The upgraded model included transport of $\mathrm{O}_{2}$ in order to determine whether $\mathrm{O}_{2}$ was limited enough to reduce the rate of degradation of containers or waste. To facilitate the comparison with total system performance assessments (TSPAs) by the Office of Civilian Radioactive Waste Management (OCRWM), the 1997 PA used assumptions that matched those used by OCRWM in an abbreviated TSPA conducted in 1997. The 1997 PA neglected credit for cladding of SNF, assumed a similar radioisotope inventory, and used updated geologic data.

To improve traceability and repeatability, the 1997 PA used a centralized database for all parameters in its models. The database included about 3024 total parameters: 2755 assigned constants and 269 assigned distributions, 63 of which were varied.

\section{ES.3 Results and Conclusions}

The mass and the activity of the DOE SNF in the repository are modest in relation to those of the commercial SNF. The 1997 PA demonstrated that the unique characteristics of DOE SNF do not outweigh this relationship: they do not adversely influence the behavior of the disposal system. Moreover, the effects of the unique characteristics are further diminished because the radionuclides in the codisposal waste package are completely dominated by the DHLW radionuclides. Therefore, DOE SNF is expected to meet repository acceptance criteria if commercial SNF can meet them. Direct disposal of DOE SNF can remain the primary option considered by the DOE/EM.

\section{ES.3.1 Performance of DOE SNF Compared to Commercial SNF}

If commercial SNF can comply with regulatory dose criteria, then DOE SNF can also comply. The inventory of DOE SNF is, therefore, probably within the error band of the commercial SNF inventory.

- Relative to commercial SNF, DOE SNF is a small contributor to doses from the total repository. It contains less than $2 \%$ of the ${ }^{237} \mathrm{~Np}$ and $7 \%$ of the ${ }^{99} \mathrm{Tc}$, which are the most important contributors to estimated doses.

- Because DHLW contains more ${ }^{99} \mathrm{Tc}$ and ${ }^{237} \mathrm{~Np}$ than the DOE SNF, releases from a breached waste package with the codisposal option are dominated by radioisotopes in DHLW containers.

- Releases of ${ }^{237} \mathrm{~Np}$ were limited by its solubility; hence the solubility of neptunium is an important model parameter.

\section{ES.3.2 Performance of Individual DOE SNF Categories}

When no credit for cladding is taken, the different categories of DOE SNF behaved similarly: the protective container layers are the same for each category and the alteration rates of fuel matrices differ only slightly.

- Based on the corrosion behavior assumed in the $1997 \mathrm{PA}$, intact cladding does not fail within $10^{5} \mathrm{yr}$. Only SNF with already failed cladding when emplaced in a container releases radioisotopes.

- If credit is taken for cladding on commercial SNF, releases from commercial SNF are reduced by about two orders of magnitude (the amount of intact cladding) to about one order of magnitude below releases from codisposed DOE SNF. (Most of the release is from DHLW.) Thus DOE SNF categories behave similarly.

- For performance assessment calculations, treating the 13 categories of DOE SNF in a similar manner would appear to be warranted. 


\section{ES.3.3 Performance of Disposal Container}

If regulators do not allow performance assessments to take credit for cladding, it may be desirable to improve the disposal containers (ASTM Grade 60 carbon steel and Inconel 625).

- Carbon steel essentially provides no protection after disposal.

- Inconel 625 also rapidly fails because temperatures remain near $100^{\circ} \mathrm{C}$ for some time within the repository.

- Halving the corrosion rate for Inconel 625 , by decreasing the surface area, does not substantially improve behavior.

- The upgraded modeling of corrosion and $\mathrm{O}_{2}$ reduction did not significantly improve these potential difficulties.

- If Hastaloy C-22 is substituted for Inconel 625 in the disposal container, Hastaloy C-22 remains intact for $10^{5} \mathrm{yr}$, based on the excellent corrosion behavior assumed.

- The Hastaloy C-22 remains intact even when its corrosion rate is doubled.

\section{ES.3.4 Behavior of Metallic Uranium}

The 1997 PA and past performance assessments conducted for NSNFP have found no reason to negate any potential pyrophoric and potentially combustible characteristics of metallic uranium in N-Reactor fuel after disposal in the potential Yucca Mountain repository.

- The heat released from hydrating all $\mathrm{N}$-Reactor fuel, which contains the metallic uranium, is much lower than the heat from other sources in the repository. It is only $1 / 100$ th of the heat from radioactive decay of just the DOE SNF and DHLW in the first year after disposal.

- Most container failures occurred between 100 and $1000 \mathrm{yr}$, when $\mathrm{O}_{2}$ was depleted in the potential repository.

Other calculations are warranted, however, to evaluate the local effects that heat from a DOE SNF container may exert on nearby commercial containers or during transportation.

\section{ES.3.5 Overall Performance}

Although the intention of the 1997 PA was to study only the relative performance of commercial and DOE SNF, the study's estimates of doses allow for comparison with other studies. In addition, the study's sensitivity and uncertainty analyses suggest that improved data may lower the estimates significantly.

The 1997 PA showed that the potential dose up to about 50,000 yr depends on releases of ${ }^{99} \mathrm{Tc}$, while at later times it is due to ${ }^{237} \mathrm{~Np}$. The uncertainties in the study's source term affect the estimates of peak releases of ${ }^{99} \mathrm{Tc}$ more strongly than the estimates for ${ }^{237} \mathrm{~Np}$. Improving the accuracy of the alteration rate of the SNF matrix or fraction of ${ }^{99} \mathrm{Tc}$ in gaps of the fuel would reduce this uncertainty in the estimated dose from ${ }^{99} \mathrm{Tc}$. (The other two parameters influencing ${ }^{99} \mathrm{Tc}$ release rates are solubility of $\mathrm{Tc}$ and amount of water flowing through the container.)

The estimates of the doses from ${ }^{237} \mathrm{~Np}$ may decrease if more accurate values for several parameters are provided. If, by comparison with the assumptions in the $1997 \mathrm{PA}$, the actual mean solubility of ${ }^{237} \mathrm{~Np}$ is significantly lower, the longer-term doses will be lower. (Additional accuracy in the amount of ${ }^{237} \mathrm{~Np}$ present is not necessary, especially for the $2 \%$ represented by the DOE SNF fuel.) The rate of fluid flow through the mountain and the number of containers contacted by water also contribute heavily to the ${ }^{237} \mathrm{~Np}$ doses. Reduced values for any of these three parameterssolubility, fluid flow, and number of containers in contact with water-would improve the system's compliance with a future dose standard. 


\section{ES.4 Characterization Needs for DOE SNF}

An improvement in the modeling of post-disposal behavior will require additional characterization data for the DOE SNF. According to the 1997 PA, only some characteristics of DOE SNF significantly affect behavior and thus may warrant a concerted effort to obtain more precise data.

\section{ES.4.1 Inventory of Radioisotopes}

The list of radioisotopes important to repository performance is fairly short, which means that characterization resources can be focused on the few significant radioisotopes. The radioisotopes that are most important in both the DOE and commercial SNF are those long-lived isotopes dependent upon burnup: ${ }^{99} \mathrm{Tc},{ }^{237} \mathrm{~Np}$, and ${ }^{129} \mathrm{I}$. The 1997 PA confirmed that ${ }^{99} \mathrm{Tc}$ was the most important isotope in determining dose in the first $2 \times 10^{4} \mathrm{yr}$ and ${ }^{237} \mathrm{~Np}$ was the most important in the first $10^{5} \mathrm{yr}$, as seen in an earlier analysis. Other radioisotopes contributing to the dose for these times were ${ }^{231} \mathrm{~Pa},{ }^{79} \mathrm{Se}$, and the uranium isotopes $\left({ }^{233} \mathrm{U},{ }^{234} \mathrm{U},{ }^{235} \mathrm{U}\right.$, and ${ }^{236} \mathrm{U}$, but not $\left.{ }^{238} \mathrm{U}\right)$. Between $10^{5}$ and $10^{6} \mathrm{yr},{ }^{226} \mathrm{Ra}$ and ${ }^{210} \mathrm{~Pb}$ contribute to dose although they are not important in the initial inventory.

Radioisotopes important to evaluating the power production from the containers, and thereby the temperature of the repository, are ${ }^{137 \mathrm{~m}} \mathrm{Ba},{ }^{90} \mathrm{Y},{ }^{238} \mathrm{Pu},{ }^{241} \mathrm{Am},{ }^{137} \mathrm{Cs},{ }^{244} \mathrm{Cm},{ }^{90} \mathrm{Sr}$, and ${ }^{240} \mathrm{Pu}$, which contribute $98.6 \%$ of the initial power.

Because the activity of the DOE SNF, which comes largely from N-Reactor fuel (Category 1), is a small percentage of the total initial activity of the repository, its activity will probably remain within the error band of the commercial SNF. Therefore, when an error band of activity for each important radionuclide in the commercial SNF is known or estimated, then an allowable error for the DOE SNF can be calculated. An error band of $200 \%$ for the activity of ${ }^{99} \mathrm{Tc}$ and ${ }^{237} \mathrm{~Np}$ in the DOE SNF is probably appropriate, assuming a $\pm 1 \%$ error band for the commercial SNF inventory until a more accurate error band is known:

\section{ES.4.2 Solubility of Radioisotopes}

In general, the solubility of radioisotopes in the repository environment will be identical for both commercial and DOE SNF; hence, this information will be developed and supplied by the OCRWM. The 1997 PA confirmed some issues with regard to solubility that were indicated in previous studies. The study, which included higher water flow rates through the repository than previous analyses, suggested that among the radioisotopes the most important parameter by far is the solubility of ${ }^{237} \mathrm{~Np}$. The release of ${ }^{237} \mathrm{~Np}$ is controlled directly by its solubility (at least in commercial SNF). For highly soluble radioisotopes like ${ }^{99} \mathrm{Tc}$ or ${ }^{129} \mathrm{I}$, release is determined by the amount of matrix material exposed at the maximum solubility. Solubility becomes a limiting factor only for the middle and lower values for ${ }^{99} \mathrm{Tc}$ and ${ }^{129} \mathrm{I}$.

\section{ES.4.3 Corrosion Parameters}

The corrosion (or alteration) of layers and matrices can potentially affect (a) the time of penetration of the layers, (b) the consumption and production of gas and liquid, (c) the generation of rust adsorbent, and (d) the release of encapsulated radioisotopes. The corrosion rate of each material is potentially a function of the oxygen content (oxic or anoxic) and the moisture available (wet, humid, or dry). The corrosion rates of the materials in the disposal container and the zircaloy cladding, as well as the alteration rate of the uranium dioxide matrix, will be identical for both commercial and DOE SNF. Only the stoichiometry and corrosion (or alteration) rates of materials unique to DOE SNF must be evaluated by NSNFP. This material includes metallic uranium and other uranium matrices, and the aluminum, stainless steel, graphite, and TRISO cladding. Alteration rates for these materials should be pursued, but the analysis showed that evaluating these rates as a function of oxygen content was not particularly important. The alteration rate of the matrix is important only in determining the release rate of ${ }^{99} \mathrm{Tc}$ at its highest solubility values. At lower solubility values for ${ }^{99} \mathrm{Tc}$ and all solubility values for ${ }^{237} \mathrm{~Np}$, the accuracy of the matrix alteration rate is unimportant. 


\title{
1. Introduction
}

\author{
R. P. Rechard
}

\subsection{Overview}

The purpose of this 1997 performance assessment is to identify for the U.S. Department of Energy (DOE) performance characteristics of DOE-owned* spent nuclear fuel (DOE SNF) after disposal. The disposal site under study is the potential repository in unsaturated tuff at Yucca Mountain, Nevada. The study is part of a broader DOE program for developing a safe, cost-effective technical strategy for the interim management and ultimate disposition of the foreign and domestic spent nuclear fuel already under the DOE's jurisdiction. In this study, the codisposal option is modeled in which the DOE SNF is packaged with defense high-level waste (DHLW). The DOE SNF and DHLW are currently stored at the Idaho National Engineering and Environmental Laboratory (INEEL), the Hanford Reservation, the Savannah River Plant, and other DOE sites. The spent nuclear fuel originated in defense and experimental reactors; the high-level waste was generated during reprocessing of the spent nuclear fuel.

The performance assessment (PA) described here is a continuation of two earlier DOE SNF/DHLW studies conducted by Sandia National Laboratories in support of the DOE on the disposal of its spent nuclear fuel and high-level waste. The earlier analyses examined specific treatment options and disposal in two hypothetical repositories (1993 PA) and the direct disposal option in a relatively small but Yucca Mountain-like repository (1994 PA). The current performance assessment (1997 PA) assembles data and then evaluates the performance after disposal of 13 separate DOE SNF categories in containers with DHLW (i.e., the codisposal option) and 2 commercial spent fuel categories. A major focus of the current study is to improve the understanding of spent fuel performance in an unsaturated tuff repository by including the most current description of the potential Yucca Mountain disposal system. As modeled, the repository in the 1997 PA includes 75,336 metric tons of heavy metal (MTHM ${ }^{\dot{1}, \dot{*}}$ ) of waste (including 2415 MTHM of DOE SNF, 9842 MTHM of DHLW, and 63,080 MTHM of commercial spent fuel). Data for site characterization in the 1997 PA are taken primarily from Sandia's 1993 Total-System Performance Assessment (TSPA1993) of the Yucca Mountain Project (YMP) (Wilson et al., 1994), with updated data in several areas such as an orderof-magnitude increase in the average precipitation infiltration and a two order-of-magnitude decrease in neptunium solubility. In general, the data used in the 1997 PA are similar to those used in a-TSPA-1997, which is an abbreviated Total System Performance Assessment (TSPA) conducted by TRW for the YMP. The repository and container designs represent the most current conceptual designs. The fuel characterization data for the 1997 PA were collected and interpreted by the National Spent Nuclear Fuel Program (NSNFP), which is responsible for coordinating storage and disposal of DOE SNF.

The 1997 PA compares the performance of DOE SNF to that of commercial spent fuel, using criteria that represent a reasonable means of measuring performance in a potential repository at this time. The 1997 PA is not intended to evaluate compliance with standards; in fact, at present, no final standards exist. However, DOE SNF's behavior as compared to commercial spent fuel's can be used to inform decisions about future actions with regard to DOE SNF; in turn, the performance of commercial spent fuel measured against specific performance criteria provides information about broader issues that relate to both spent fuel types. Thus, performance criteria were selected based primarily on two sources: the Environmental Protection Agency (EPA) Standard, 40 CFR 191 (EPA, 1993), and guidance provided by the National Academy of Sciences (NAS) in 1995 regarding a proposed future standard for repository licensing (NAS/NRC, 1995). The criteria examined in the 1997 PA are the probabilistic maximum doses received by an individual; the time period under consideration is $100,000 \mathrm{yr}$.

The process for the $1997 \mathrm{PA}$ is the same as that used in the earlier performance assessments, which incorporated complex models directly into the probabilistic analysis to capture spent fuel behavior as accurately as possible. For example, the $1997 \mathrm{PA}$ considers transport of $\mathrm{O}_{2}$ in its hydrologic model, along with corrosion dependence in the radi-

\footnotetext{
* The DOE SNF is spent fuel owned by the U.S. Department of Energy, Office of Environmental Management. This study does not evaluate Navy SNF, which the DOENaval Nuclear Propulsion Program (NNPP) owns and is evaluating.

$\dagger$ MTHM is the mass of all isotopes of uranium, plutonium, and thorium before irradiation in a reactor, expressed in metric tons (1000 kg).

† The current legal limit, as specified in the Nuclear Waste Policy Act of 1982, is 70,000 MTHM.
} 
onuclide source term. In this way, the 1997 PA complements performance assessments of the YMP by providing additional information for their analysis, which uses a different methodology that includes simplified models that have been abstracted from detailed simulations. To provide a complete picture of DOE SNF performance in the repository, the 1997 PA results can be compared with the results from the YMP performance assessment, a-TSPA1997. Contrasting the results of the two methodologies provides a benchmark-type comparison, which creates confdence with regard to modeling.

The remainder of this chapter expands upon the information just presented, outlines important concepts in the performance assessment, and highlights decisions that affect how the disposal system was modeled. Chapters 2 through 10 provide additional details on the concepts and assumptions presented here; Chapter 11 contains the results of the assessment; Chapter 12 provides guidance to the NSNFP, based on the results.

\subsection{General Purpose of the Analysis}

The overall purpose of this study is to provide information and guidance to the Office of Environmental Management of the DOE (designated herein as DOE/EM) about the level of characterization of DOE SNF necessary for disposal. The currently planned disposal location for the DOE SNF is the potential Yucca Mountain repository. Although intended primarily for disposal of commercial SNF, the potential repository has a portion reserved for DOE SNF and DHLW in accordance with the Nuclear Waste Policy Act and a Presidential decision on disposal of DOE SNF with commercial SNF. Yucca Mountain is the first site to be characterized by the Office of Civilian Radioactive Waste Management (OCRWM) of the DOE as a potential repository for high-level nuclear waste and spent nuclear fuel. The OCRWM is ultimately responsible for setting the acceptance requirements for the potential repository. The value of this study, then, is that the DOE/EM can use the results to estimate (in a timely and cost-effective fashion) the appropriate level and type of characterization and packaging necessary for disposal of DOE SNF to comply with overall regulatory criteria for the repository. The results can also be used by the OCRWM to (a) develop appropriate abstractions $^{* *}$ and/or benchmark its results for DOE/EM fuel and (b) develop performance-based requirements for repository acceptance criteria (RAC). To this purpose, Sandia's performance assessments in 1993 and 1994 provided relevant information to the DOE/EM. The 1997 PA reported here is a continuation of these earlier studies.

The first goal of this analysis was to demonstrate the influence of the DOE SNF, expected to be minor, in a predominately commercial repository using modeling conditions similar to those currently assumed by the YMP when it conducts the TSPA. The assumptions are based on YMP's abbreviated TSPA in 1997 (a-TSPA-1997; M\&O, 1997), an analysis in 1996 (M\&O, 1996), and the TSPA in 1995 (TSPA-1995; M\&O, 1995). Toward this goal, the 1997 PA evaluates whether DOE SNF performs noticeably better (or worse) than commercial spent fuel, and identifies important parameters that influence this performance after disposal. The latter results, in particular, are intended to help define appropriate requirements for spent fuel characterization with regard to DOE SNF being accepted for storage and disposal. Major changes to assumptions that will be incorporated into the TSPA-VA (Verification Assessment) are not reflected in this report. However, in anticipation of these changes, this study examined how such changes might influence DOE SNF performance.

A second goal, closely related to the first, was to identify the most sensitive parameters through a preliminary analysis of the results to determine which DOE SNF characteristics should be quantitatively estimated or measured and which could be neglected, after demonstrating their minor influence. Such information is useful for developing performance-based requirements for repository acceptance criteria, which offers a significant advantage over establishing general repository acceptance criteria. The primary advantage is in defining characterization requirements only for those spent fuel types that demand them, thus potentially reducing preparation costs.

Because the source term was already very influential and then made even more important by the use of dose performance criteria, a third goal was to continue development and implementation of detailed process models for the

\footnotetext{
** Results of previous performance assessments have already been used in this way. For example, the current a-TSPA-1997, performed by the YMP Managing and Operating Contractor, TRW, under the OCRWM, uses formulations that were developed in the 1994 PA, conducted by the DOE/EM, for the alteration of metallic and ceramic DOE SNF.
} 
degradation of the containers and fuel matrices within the potential repository. An important phenomenon with regard to degradation of containers, alteration of SNF, and solubility of radioisotopes is the availability of oxygen. Hence, the primary model modifications for this year's analysis were (a) adding oxygen transport within the twophase flow code, BRAGFLO_T, and (b) using oxygen-dependent alteration rates of materials within the corrosion and source-term model, CST.

This report was commissioned to provide information to the DOE concerning the level of characterization of DOE SNF necessary for disposal in the potential Yucca Mountain repository. Hence, the general results are presented in this summary with the understanding that the reader appreciates that the findings are conditional on the models and data used to generate them. Data used in the models are based on the best information currently available, but should still be considered preliminary.

Although the environmental regulations for SNF disposal in the United States are currently undergoing revision, this assessment primarily used the performance criteria in 40 CFR Part 191, in particular the 5-km exclusion zone and the Individual Protection Requirements. The Containment Requirements were also used in some cases for comparison with the 1994 PA, thus offering the reader a historical perspective. Dissolved radionuclide releases from the engineered barrier and releases reaching the water table below the repository were also evaluated and presented. The time period chosen for calculating dose in the 1997 PA was 100,000 yr. In the next few years, the YMP plans to evaluate numerous design options and various natural phenomena for the license application to the NRC. In anticipation of these studies, this report also presents the sensitivity of the 1997 PA results to changes in container material, cladding use, and inventory of the DOE SNF.

\subsection{Organization and Mechanics of the Performance Assessment}

One means of determining whether spent fuel is acceptable for inclusion as part of a geologic disposal system is a performance assessment. The term performance assessment is generally defined as the process of assessing whether a system meets a set of performance criteria (Rechard, 1995, p. 1-1). Hi For a geologic disposal system, the performance criteria are various long-term $(10,000 \mathrm{yr}$ and longer) environmental metrics specified in government regulations. For assessment of the long-term consequences of a geologic disposal system, the technique used is computer simulation; thus, the system for the performance assessment is a computational model that represents the geologic disposal system, not the system itself. Consequently, a performance assessment for evaluating geologic disposal of nuclear waste is intimately tied to the general scientific processes of modeling.

For discussion in this report, the process for assessing the performance of the disposal system can be conveniently categorized into six general steps (Figure 1-1). Together, these tasks draw upon varied scientific disciplines. The six tasks ${ }^{\ddagger \ddagger}$ are as follows (Rechard, 1995):

1. Disposal System and Regional Characterization. The first step is the collection of data on (a) spent fuel and high-level waste (Chapter 3), (b) geology and hydrology near the site (Chapter 4), and (c) facility design that characterizes the disposal system and regional area (Chapter 5). System characterization is the first phase in conceptual model development. The results of the first step are used to determine the parameter space for the conceptual model. The parameters are subdivided into three disjoint subsets: uncertain parameters typically classified as aleatoric variables and thus often studied through scenarios (e.g., event and undetected feature parameters; none in this study), uncertain parameters typically expressed as random or epistemic variables

it This more general definition of a performance assessment is used herein; however, the 40 CFR 191 regulation defines a performance assessment as "an analysis that (1) identifies the processes and events that might affect the disposal system, (2) examines the effects of these processes and events on the performance of the disposal system, and (3) estimates the cumulative releases of radionuclides, considering the associated uncertainties, caused by all significant processes and events." In essence, this definition of performance assessment is tied specifically to one particular performance metric, the evaluation of cumulative releases of radionuclides. Also, the phrase "considering the associated uncertainties" implies the use of stochastic simulation as the evaluation technique.

¥ These six steps can also describe a probabilistic risk assessment (PRA) which, like a performance assessment, is a stochastic simulation. The main difference between the two assessments is that the term "PRA" is, in general, associated with only engineered facilities over short geologic time scales, while the term "PA" is associated with a combination of natural and engineered systems over long geologic time scales. 
1. Introduction
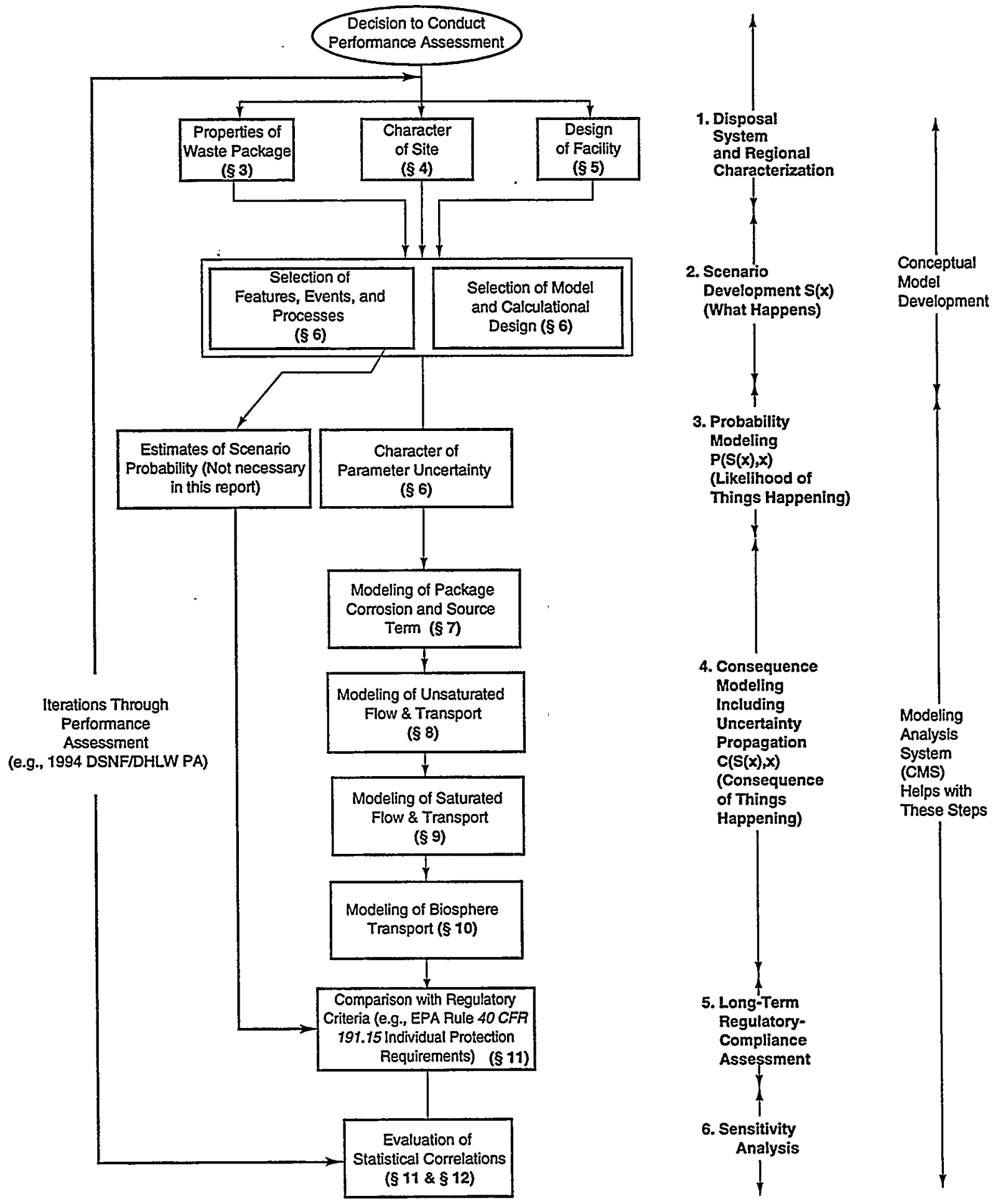

TRI-6342-5259-1

Figure 1-1. Sandia's method for conducting DOE SNF/DHLW Performance Assessment calculations involves six general tasks (after Rechard, 1989, Figure 3.1). This report is organized around those tasks; the section numbers in parentheses indicate the chapter in which the task is discussed. 
(e.g., material property parameters; 63 parameters in this study), and parameters selected to be fixed or varied one at a time (e.g., model domain parameters, physical constants; 3024 parameters in this study).

2. Scenario Development. The second step includes (a) the identification of features and agents (i.e., continuous phenomena such as corrosion, sometimes called processes, and short-term phenomena such as intrusion, called events) of the disposal system whereby radionuclides might be released to the accessible environment, and (b) selection of a set of mutually exclusive scenarios from known features, events, and processes (FEPs) (selecting scenarios is not necessary for this study). Scenario development is the second phase in conceptual model development (i.e., selecting what to model). At this step, the parameter space for the performance assessment model can be identified, which usually contains only minor modifications to the original parameter space (see 1, above) (Chapter 6).

3. Probability Modeling. The development and execution of probability models, which quantify the uncertainty in model parameters and predict the likelihood of the scenarios, is considered the third step. This step creates two types of results: models that evaluate the probability of the scenario occurring (not necessary for this study) and distributions that express the uncertainty of parameters (Chapter 6).

4. Consequence Modeling. The fourth step involves the development and execution of consequence models to predict the amount of radionuclide release to the accessible environment, including evaluating the uncertainties associated with the predictions. The results of this step are the consequences of the simulations. The models for the 1997 PA are discussed in the following chapters: waste and waste package, Chapter 7; unsaturated zone, Chapter 8; saturated zone, Chapter 9, and biosphere, Chapter 10.

5. Long-Term Regulatory Compliance Assessment. In assessments performed in support of licensing, comparison of the predicted doses with government regulations is considered the fifth step. Herein, plausible criteria are selected with which to compare relative performance, because current regulations are uncertain. Some possible comparisons are shown in Chapter 11.

6. Sensitivity Analysis. The final step is an identification of the important model parameters that most influence the calculated results. Sensitivity analysis is shown in Chapters 11 and 12.

Normally, a performance assessment is conducted several times because each iteration provides enhanced information in precisely those subject areas where it is required. The NSNFP last conducted a DOE SNF performance assessment in 1994.

\subsection{System Characterization}

System characterization is the first phase in conceptual model development. In the following text, the physical systems such as the spent fuel, the geologic setting in which the repository is located, and the potential repository design are defined.

\subsubsection{Waste Containment System Terminology}

As used in 40 CFR 191, a radioactive waste containment system includes three major subsystems (institutional controls, geologic barriers, and engineered barriers) and their corresponding major components (Figure 1-2). The first subsystem, institutional controls, consists of components such as legal ownership of the land and resources by the U.S. Government, fencing and signs around the property, permanent markers, public records and archives, and other methods of preserving knowledge about the disposal system (Trauth et al., 1993; Rechard et al., 1993). Effects of this subsystem are not included in the 1997 PA. 
1. Introduction

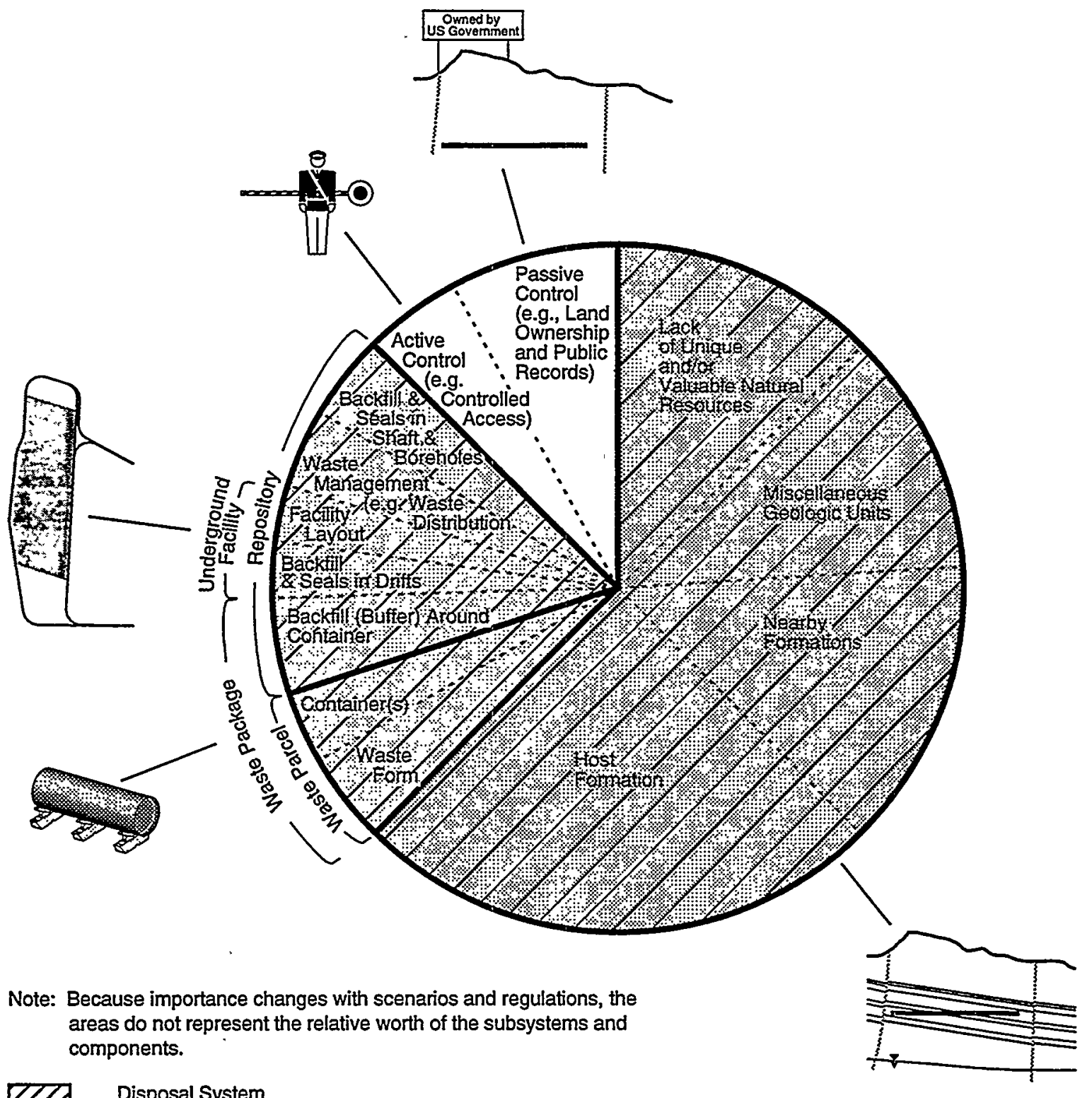

Q72 Disposal System

Engineered Barrier Subsystem

10 CFR 60 omits shaft and borehole backfill and waste management from engineered barrier system.

Institutional Control Subsystem

40 CFR 191 does not allow the DOE to depend upon the enforcement of active institutional controls after $100 \mathrm{yr}$, for performance assessments.

Geologic Barrier Subsystem

(Maximum $5 \mathrm{~km}$ distance around waste disposal area)

[ Various groupings of the engineered barrier -- "waste package" and "repository" are primarily used in this report.

The term waste parcel was used in the 1994 PA.

TRI-6342-5536-0

Figure 1-2. Subsystems and components of a radioactive waste containment system. The disposal system, as defined in 40 CFR 191, comprises the geologic and engineered subsystems of the waste containment system (after Rechard et al., 1990, Figure 1-10). Herein, the engineered barrier subsystem is discussed as a waste package and a repository. 
The second subsystem, the geologic barrier, is limited to the lithosphere up to the ground surface and a possible exclusion zone. As explained in Chapter 2, the exclusion zone used in the $1997 \mathrm{PA}$ is $5 \mathrm{~km}(-3 \mathrm{mi})$ from the outer boundary of the disposal tunnels.

The physical features of the repository (e.g., design of the repository, waste form, handling and disposal containers, and backfill) are components of the third subsystem, engineered barriers. For purposes of discussion, the components of the engineered barrier system are grouped into two subsystems: the waste package ${ }^{* * *}$ and the repository.

The disposal system, as defined by 40 CFR 191, includes only the geologic and engineered barrier subsystems. For the performance assessment, different parts of the geologic and engineered barrier subsystems are assembled into a disposal system that can be modeled. The information used to model the waste package, geologic barrier, and potential repository is described below. The modeling systems themselves are described in Section 1.7, and Chapters 7 through 10.

\subsubsection{Characterization of Waste Package}

The most inclusive term, waste package, is used in this report to describe the spent fuel or high-level waste as it would reside in the repository. The waste package includes the spent fuel and/or high-level waste, disposal and/or handling containers, and any backfill material placed between the disposal container and the host rock (none in this study). Spent fuel refers here to the packaged components including fuel rods, brackets, and hardware.

The 1997 PA emphasized the performance of 13 categories of DOE SNF after disposal compared to that of two commercial spent fuel categories. The DOE SNF categories are packaged with DHLW to reflect the DOE's proposed packaging plan as of September 1997; packaging DOE SNF with DHLW is called the codisposal option. All information about the spent fuel and packaging was provided by NSNFP (Appendix A). Chapter 3 provides more information about the spent fuel categories and containers as they were modeled in the 1997 PA. The conceptual model for the waste package focused on package corrosion, fuel oxidation, and resulting release rates; more information about these processes can be found in Chapter 7. This section provides a brief overview of the spent fuel categories, handling and disposal containers, codisposal configurations, and waste packages as modeled in the 1997 PA.

Spent Fuel Categories. Fifteen categories of spent nuclear fuel are included in the study: 13 categories of DOE SNF and 2 categories of commercial spent nuclear fuel (Pressurized Water Reactor [PWR] and Boiling Water Reactor [BWR]). Table 1-1 lists the spent fuel categories; Categories 1 to 13 represent over 250 types of spent nuclear fuel owned by the DOE, with Category $1, \mathrm{~N}$-Reactor fuel, accounting for about $88 \%$ of the DOE SNF inventory. ${ }^{\text {it }}$ The DOE/EM grouped its spent fuel into categories based on the chemical composition of the fuel matrix; for modeling purposes, a representative fuel was selected for each category (Table 1-1). Cladding condition is described, but was not a primary consideration for the grouping. The categories include a range of enrichments: low enriched uranium (LEU, $\leq 5 \%$ enrichment); medium enriched uranium (MEU, 5\% < enrichment $<20 \%$ ); and high enriched uranium (HEU, $\geq 20 \%$ enrichment).

The inventory from the DOE sites also includes high-level waste immobilized in borosilicate glass from the Savannah River Plant near Aiken, SC; the Hanford reservation, Hanford, WA; INEEL; and the West Valley Demonstration Project, West Valley, NY. For the 1997 PA, the DHLW is modeled as being packaged for disposal with the DOE SNF (Categories 2 to 13).

A total of 75,336 MTHM is modeled in the 1997 PA, including DOE SNF (2415 MTHM), DHLW (9842 MTHM), and commercial spent nuclear fuel (63,080 MTHM). The commercial spent fuel represents the majority of activity and mass in the potential repository; 41,440 MTHM of the total is PWR spent fuel and 21,640 MTHM is BWR spent fuel. The inventory used in the analysis was sanctioned by the YMP. Because the DOE SNF itself repre-

\footnotetext{
*** Another term used in the 1993 and 1994 PAs is waste parcel, which included all components of the waste package except backfill between the container and the host rock. In the 1997 PA, the waste package and the waste parcel are the same because only an air gap surrounds the waste package.

tff U.S. Navy fuel was not included in the 1997 PA; it will be evaluated by the Navy.
} 
Table 1-1. Categories of Spent Nuclear Fuel and High Level Waste Used in 1997 PA

\begin{tabular}{|c|c|c|c|c|c|c|c|}
\hline No. & $\begin{array}{l}\text { Spent Nuclear Fuel or } \\
\text { High-Level Waste }\end{array}$ & Represented by & $\begin{array}{l}\text { Represen- } \\
\text { tative } \\
\text { Cladding }\end{array}$ & $\begin{array}{l}\text { Activity } \\
\text { (2030 yr) } \\
\text { (Ci/ } \\
\text { Category) }\end{array}$ & $\begin{array}{c}\text { Mass } \\
\text { (MTHM/ } \\
\text { Category) }\end{array}$ & $\begin{array}{l}\text { Mass } \\
\text { (Total } \\
\text { MTHM/ } \\
\text { SNF \& } \\
\text { HLW) }\end{array}$ & $\begin{array}{l}\text { Number } \\
\text { of Waste } \\
\text { Packages }\end{array}$ \\
\hline 1 & Uranium metal & $\begin{array}{l}N-\text { Reactor ( } 4 \text { handling } \\
\text { containers/pkg) }\end{array}$ & $\begin{array}{l}\text { Zircaloy, } \\
\text { Failed }\end{array}$ & $2.45 \times 10^{7}$ & 2133 & 2133 & 118 \\
\hline 2 & Uranium-zirconium alloy & Chicago Pile 5 (CP-5) & Zircaloy & $2.32 \times 10^{5}$ & 0.041 & 35.4 & 9 \\
\hline 3 & $\begin{array}{l}\text { Uranium-molybdenum } \\
\text { alloy }\end{array}$ & Fermi Reactor & Zircaloy & $4.76 \times 10^{4}$ & 3.91 & 119 & 55 \\
\hline 4 & Uranium oxide-intact clad & $\begin{array}{l}\text { Shippingport } \\
\text { Pressurized Water Reac- } \\
\text { tor (PWR) }\end{array}$ & Zircaloy & $2.69 \times 10^{7}$ & 79.8 & 610 & 203 \\
\hline 5 & Uranium oxide-failed clad & $\begin{array}{l}\text { Three Mile Island } \\
\text { (TMI-2) }\end{array}$ & $\begin{array}{l}\text { Zircaloy, } \\
\text { Failed }\end{array}$ & $3.04 \times 10^{7}$ & 88.2 & 1334 & 595 \\
\hline 6 & Uranium aluminum alloy & $\begin{array}{l}\text { Advanced Test } \\
\text { Reactor (ATR) }\end{array}$ & Aluminum & $3.53 \times 10^{7}$ & 9.38 & 2951 & 750 \\
\hline 7 & Uranium silicide & $\begin{array}{l}\text { Materials Testing } \\
\text { Reactors (MTR) }\end{array}$ & Aluminum & $5.99 \times 10^{6}$ & 12.7 & 895 & 225 \\
\hline 8 & $\begin{array}{l}\text { Uranium-thorium carbide- } \\
\text { intact clad }\end{array}$ & Fort St. Vrain & Graphite & $4.75 \times 10^{6}$ & 24.7 & 2162 & 545 \\
\hline 9 & $\begin{array}{l}\text { Uranium-thorium carbide- } \\
\text { failed clad }\end{array}$ & Peach Bottom & Graphite & $4.40 \times 10^{5}$ & 1.61 & 405 & 103 \\
\hline 10 & $\begin{array}{l}\text { Uranium/plutonium car- } \\
\text { bide }\end{array}$ & $\begin{array}{l}\text { Sodium Reactor } \\
\text { Experiment (SRE) }\end{array}$ & $\begin{array}{l}\text { Stainless } \\
\text { steel }\end{array}$ & $1.14 \times 10^{5}$ & 0.057 & 15.7 & 5 \\
\hline 11 & Mixed oxide fuel & $\begin{array}{l}\text { Fast Flux Test } \\
\text { Facility (FFTF) }\end{array}$ & $\begin{array}{l}\text { Stainless } \\
\text { steel }\end{array}$ & $5.78 \times 10^{6}$ & 3.96 & 1110 & 352 \\
\hline 12 & Uranium-thorium oxide & $\begin{array}{l}\text { Shippingport Light Water } \\
\text { Breeder Reactor (LWBR) }\end{array}$ & Zircaloy & $7.33 \times 10^{5}$ & 55.5 & 218 & 69 \\
\hline 13 & $\begin{array}{l}\text { Uranium/zirconium } \\
\text { hydride }\end{array}$ & $\begin{array}{l}\text { Training, Research, and } \\
\text { Isotope production-Gen- } \\
\text { eral Atomic (TRIGA) }\end{array}$ & $\begin{array}{l}\text { Stainless } \\
\text { steel }\end{array}$ & $1.21 \times 10^{6}$ & 2.22 & 269 & 102 \\
\hline 14 & Commercial fuel, PWR & $\begin{array}{l}\text { PWR (21 assemblies/ } \\
\text { pkg) }\end{array}$ & Zircaloy & $1.43 \times 10^{10}$ & 41,440 & 41,440 & 4820 \\
\hline \multirow[t]{3}{*}{15} & Commercial fuel, BWR & $\begin{array}{l}\text { BWR (Boiling Water } \\
\text { Reactor) ( } 44 \text { assemblies/ } \\
\text { pkg) }\end{array}$ & Zircaloy & $6.01 \times 10^{9}$ & 21,640 & 21,640 & 2859 \\
\hline & $\begin{array}{l}\text { Defense High Level } \\
\text { Waste }\end{array}$ & $\begin{array}{l}\text { Borosilicate Glass } \\
\text { (9795 4.6-m and } 4125 \\
\text { 3-m handling containers } \\
\text { in Categories 2-13) }\end{array}$ & - & $3.29 \times 10^{9}$ & $9842^{b}$ & -- & - \\
\hline & TOTAL & & & & 75,336 & 75,336 & 10,810 \\
\hline b & \multicolumn{7}{|c|}{$\begin{array}{l}\text { This column shows the approximate mass of heavy metal (uranium, plutonium, and thorium) based on the total radioisotope inven- } \\
\text { tory that was used in the calculations. In most cases, these values provide a lower bound on the MTHM reported in Appendices A } \\
\text { and B; however, errors are present in the inventories. Because the inventory deternines the heat load and source term for the cal- } \\
\text { culation, the } 1997 \text { PA's estimate of SNF MTHM was used in the calculations to be consistent with the radioisotope inventory. A } \\
\text { comparison of reported and calculated values is presented in Section } 3.5 .2 \text {. } \\
\text { The MTHM equivalents for all DHLW when note } 1 \text { (d) of Appendix A of } 40 \text { CFR } 191 \text { is applied, assuming a radioisotope inventory } \\
\text { in } 2030 \text {, are } 9842 \text {. The MTHM equivalents assuming } 0.5 \text { MTHM per 3-m handling container and } 0.75 \text { MTHM per } 4.6-m \text { handling } \\
\text { container are } 9409 \text {. }\end{array}$} \\
\hline
\end{tabular}


sents only $3 \%$ of the regulatory mass of heavy metal modeled, its influence on total repository performance can be expected to be small.

Handling and Disposal Containers. A handling container is the container in which the fuel matrix and cladding, if any, is placed. The outermost container is referred to as the disposal container, which is sometimes called a repository overpack in other studies. The handling and disposal containers are important in determining performance of the fuels after disposal, because they represent layers of material that protect and confine the fuel. Degradation of the protective layers and alteration and dissolution of the fuel matrix are modeled in the 1997 PA by means of the Corrosion and Source Term (CST) submodel, which is described thoroughly in Chapter 7. This section describes the protective layers, represented by the handling and disposal containers, for the different fuels under study based on information provided by NSNFP (see Appendix A). In the 1997 PA, the commercial spent fuel, PWR and BWR assemblies, are packaged in a disposal container only (Figure 1-3a). Handling containers of DOE SNF and DHILW are placed together in a disposal container; disposing of DOE SNF/DHLW in one container is referred to as the codisposal option (Figure 1-3b). Illustrations depicting the containers as modeled are provided in Chapter 3.

Handling Containers. The handling container for Category $1, N$-Reactor fuel, is the Multi-Canister Overpack (MCO). The MCO has a $61-\mathrm{cm}$ outer diameter stainless steel shell that is $416.6 \mathrm{~cm}$ long and $0.95 \mathrm{~cm}$ thick; it is similar in size to the high-level waste standard DOE handling container.

All handling containers for spent fuel Categories 2 through 13 are constructed of 6.35-mm 304L stainless steel. Because lengths and diameters of the individual spent fuels differ, their varying measurements are accommodated by

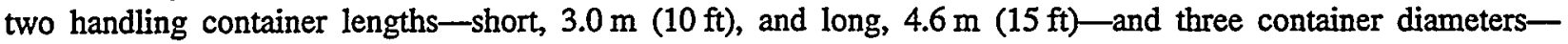
$25.4 \mathrm{~cm}, 43.2 \mathrm{~cm}$, or $61.0 \mathrm{~cm}$ (10 in., 17 in., or 24 in.).

The DHLW handling container is modeled as the 3.0-m-long, 61-cm diameter (10-ft-long, 24-in.-diameter) stainless steel standard DOE handling container or a $4.6-\mathrm{m}-$ long container.

Disposal Containers. The disposal container for all categories is a large disposal package (LDP), with a 10-cmthick outer carbon steel ${ }^{\ddagger}+\frac{1}{7}$ layer and a 2 -cm-thick inner Inconel 625 layer. The lengths and diameters vary based on respective fuel lengths, cross-sectional areas, and fissile content. In the $1997 \mathrm{PA}$, the disposal containers are described as either short $(3.79 \mathrm{~m})$ or long $(5.3 \mathrm{~m})$, and either standard $(1.725 \mathrm{~m}$ diameter) or super $(2.0 \mathrm{~m}$ diameter) (see Appendix A and Chapter 3).

Codisposal Configurations. Current packaging plans propose that DOE SNF be codisposed with DHLW whenever possible, primarily because of criticality concerns. Although criticality is not investigated in the $1997 \mathrm{PA}$, it is important that the analysis reflect the most current packaging plan. All proposed DOE SNF/DHLW codisposal configurations are listed in Appendix A; in the $1997 \mathrm{PA}$, the most frequently used configuration per spent fuel category was generally modeled. ${ }^{* * * *}$ Note that the commercial spent fuel (Categories 14 and 15) is packaged without the codisposal option, i.e., no DHLW is included. Table 1-2 lists the codisposal configurations selected for spent fuel Categories 2 through 13 . itti $^{\text {iti }}$

Waste Packages as Modeled. The waste package is the modeling unit in a performance assessment that represents the radioactive waste and its protective layers. In the 1997 PA, 15 types of waste packages are modeled, which represent the 13 DOE SNF categories, codisposed with DHLW, and the 2 commercial spent fuel categories, in their handling and/or disposal containers.

Mass of Heavy Metal per Waste Package. Table 1-1 lists the mass of heavy metal per spent fuel category in the 1997 PA inventory. The regulatory mass for DOE SNF, as provided by NSNFP in Appendix A, reported only the uranium mass, omitting the inventory of plutonium and thorium. Consequently, for consistency, the mass of heavy metal

和 The carbon steel layer is ASTM A516 Grade 60 steel.

****As of November 1997, the DOE had determined that only the $1 \times 5$ configuration will be considered in the future; however, that information is not reflected in the 1997 PA, which was based on information available as of September 1997.

Htti In the 1997 PA, Category 1 is not modeled with DHLW in the waste package. However, the proposed packaging plan as of September 1997 did anticipate a small number of codisposal packages for this category; see Appendix A, "Category 1." 
1. Introduction

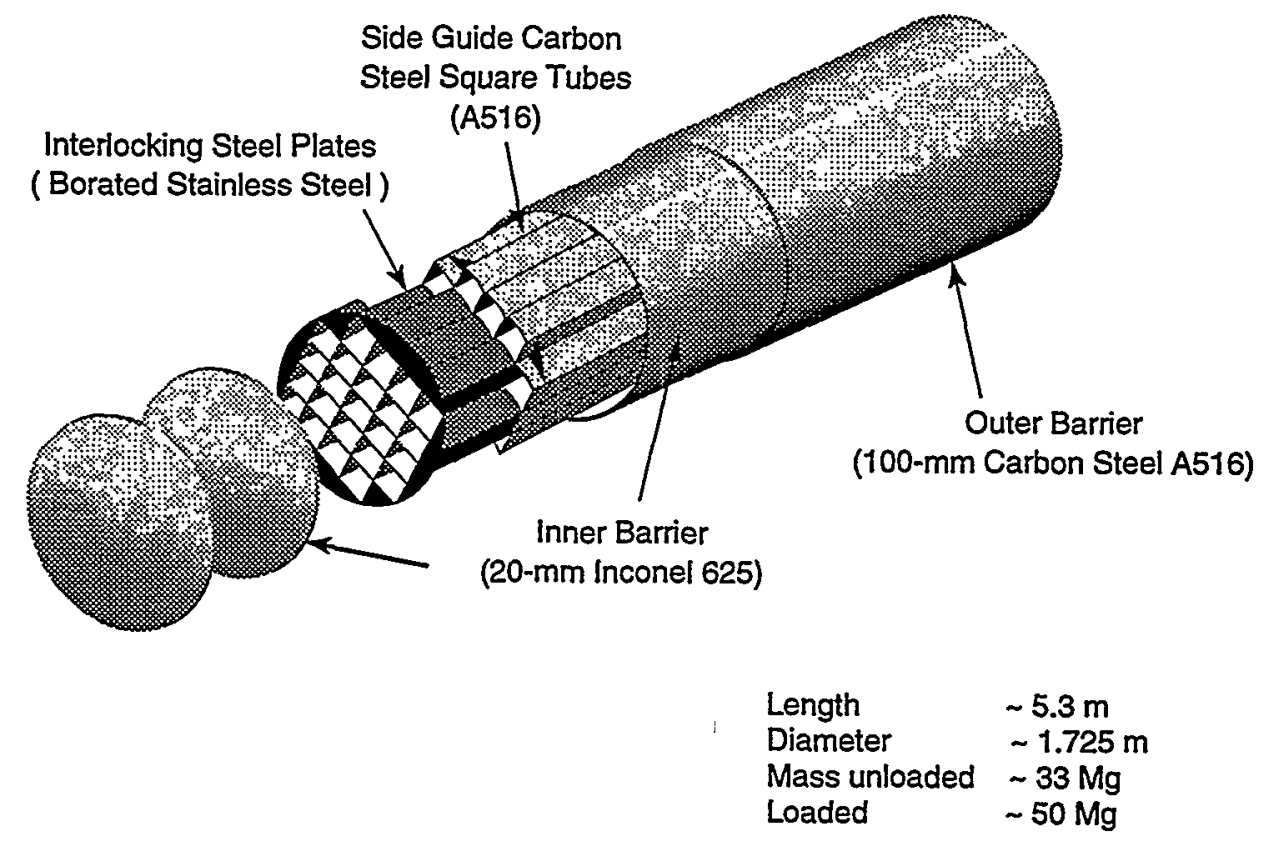

(a) 21 PWR Disposal Container

TRI-6342-5391-0

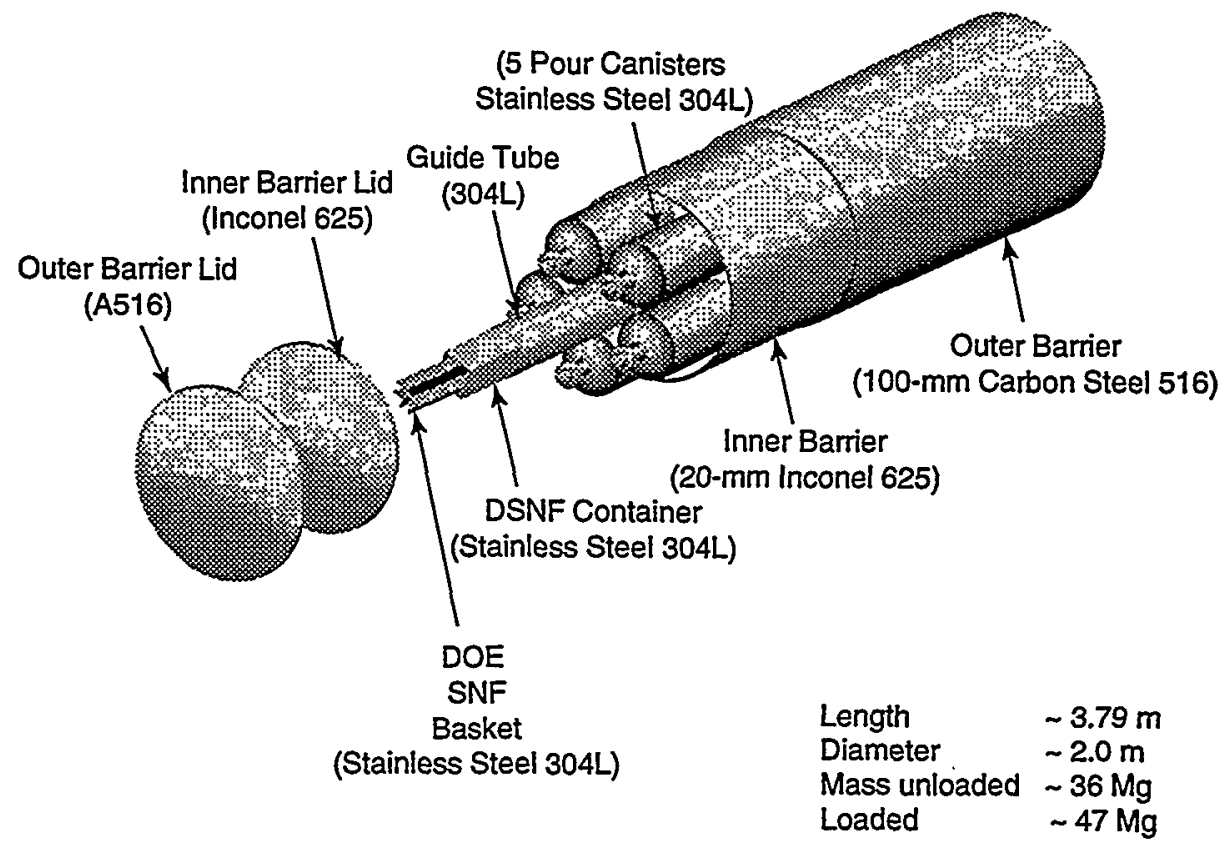

(b) DSNFDHLW Handling and Disposal Containers

TRI-6342-5504-0

Figure 1-3. Examples of packaging of spent nuclear fuel and high level waste. (a) Packaging for 21 assemblies of pressurized water reactor (PWR) commercial fuel and (b) one of the codisposal configurations for DOE-owned spent nuclear fuel and defense high level waste (DOE SNFIDHLW). 
Table 1-2. Codisposal Configuration Options for Categories 1 through 13

\begin{tabular}{|c|c|c|c|c|c|c|c|c|}
\hline \multirow[b]{3}{*}{$\begin{array}{c}\text { Spent } \\
\text { Fuel } \\
\text { Categories }\end{array}$} & \multicolumn{4}{|c|}{ Handling Container } & \multirow{2}{*}{\multicolumn{3}{|c|}{ Disposal Container }} & \multirow{3}{*}{$\begin{array}{c}\text { Total } \\
\text { No. of } \\
\text { Waste } \\
\text { Packages }\end{array}$} \\
\hline & \multicolumn{2}{|c|}{ DOE SNF } & \multicolumn{2}{|c|}{ DHLW } & & & & \\
\hline & $\begin{array}{l}\text { Number } \\
\text { per Waste } \\
\text { Package }\end{array}$ & $\begin{array}{l}\text { Diameter } \\
\text { (cm) }\end{array}$ & $\begin{array}{l}\text { Number } \\
\text { per Waste } \\
\text { Package }\end{array}$ & $\begin{array}{l}\text { Diameter } \\
\text { (cm) }\end{array}$ & $\begin{array}{l}\text { Length } \\
\text { (m) }\end{array}$ & $\begin{array}{l}\text { Diameter } \\
\text { (m) }\end{array}$ & $\begin{array}{c}\text { Mass } \\
(\mathrm{Mg})\end{array}$ & \\
\hline 1 & 4 & 61 & 0 & - & 5.30 & 1.725 (std) & 16.73 & 118 \\
\hline 12 & 1 & 61 & 3 & 61 & 5.30 & $1.725(\mathrm{std})$ & 15.12 & 69 \\
\hline 3,5 & 1 & 25.4 & 4 & 61 & 3.79 & 1.725 (std) & 15.12 & 650 \\
\hline 10,11 & 1 & 25.4 & 4 & 61 & 5.30 & $1.725(\mathrm{std})$ & 21.20 & 357 \\
\hline 4,13 & 1 & 43.2 & 5 & 61 & 3.79 & $2 . Q$ (super) & 19.02 & 305 \\
\hline $2,6,7,8,9$ & 1 & 43.2 & 5 & 61 & 5.30 & 2.0 (super) & 26.38 & 1632 \\
\hline (TOTALS) & $3480^{*}$ & & $13,920^{*}$ & & & & & 3131 \\
\hline
\end{tabular}

was adjusted in the 1997 PA to match the inventory of radioisotopes (provided in Appendix A) and the matrix masses were adjusted to include plutonium and thorium. The MTHM equivalents of DHLW were calculated using note 1 (d) of Appendix A of 40 CFR 191. This value is similar to that calculated if one assumed that the DHLW mass of heavy metal is 0.5 MTHM per DHLW handling container per short $(3.79 \mathrm{~m}$ ) waste package and 0.75 MTHM per DHLW handling container per long ( $5.3 \mathrm{~m}$ ) waste package, as described in Appendix A of this report.

Activity per Waste Package. Table 1-1 lists the initial activity per waste package. For the 1997 PA, the analysts calculated the activity per codisposal package by adding the DHLW inventory to the DOE SNF inventory per package by category. The inventories were provided by NSNFP (see Chapter 3 and Appendix A).

Thermal Power per Waste Package. The heat generated by the spent fuel within the waste packages is significant because a repository design consideration is the spacing of waste packages to ensure a hot repository (see Chapter 5). Values for the heat generated by the spent fuels after disposal are also essential to computations of near-field hydrologic response, which affects corrosion rates of the packages. For the 1997 PA, the activity inventories and decay parameters (e.g., half-lives) were used to obtain an initial thermal power for each waste package type modeled.

Radioisotopes Considered in the 1997 PA. For the heat generation model, a total of 43 radioisotopes were considered in the 1997 PA, which included the 41 radioisotopes identified by NSNFP (see Appendix A and Chapter 3), plus two additional radioisotopes-barium and yttrium, daughters of ${ }^{135} \mathrm{Cs}$ and ${ }^{90} \mathrm{Sr}$ - which were added because they contribute to heat in the first $100 \mathrm{yr}$. The 41 radioisotopes identified by NSNFP were modeled by CST in the source term submodel to predict their dissolution and solubility when exposed to groundwater and subsequent effects on releases. Only three of the 41 radioisotopes- ${ }^{129} \mathrm{I},{ }^{237} \mathrm{~Np}$, and ${ }^{99} \mathrm{Tc}$-were considered in the transport calculations because earlier studies indicated that these radioisotopes were the most significant with regard to release (Rechard, ed., 1995). For the DHLW, a total of 49 radioisotopes were considered. Like the 1994 PA, the 1997 PA used the inventory provided in the 1992 DOE report on Characteristics of Potential Repository Waste (DOE, 1992; Rechard, ed., 1995). 


\subsubsection{Characterization of Geologic Barrier}

The potential repository site is an unsaturated zone above a water table aquifer at Yucca Mountain, Nevada, located in a high desert climate (Figure 1-4). The complete characterization at Yucca Mountain as well as the natural setting, including climate, hydrology, and geochemistry, requires a significant amount of data. The brief discussion in this section centers on geologic, geochemical, hydrologic, and climatic factors with the most direct bearing on development of parameters affecting containment, flow, and transport. Other potentially disruptive geologic factors that might impact containment, including volcanism and seismicity, have been eliminated from study or shown to have small infiuence in previous iterations of the performance assessment by the YMP (M\&O, 1995a; Wilson et al., 1994).

The stratigraphy of Yucca Mountain consists of several thousand meters of widespread sheetlike volcanic ejecta ("tuff') deposited between $14.1 \times 10^{6} \mathrm{yr}$ and $11.3 \times 10^{6} \mathrm{yr}$ (Tertiary Period). The siliceous tuff formed as the hot volcanic ash and fragments were quickly deposited near a volcanic eruption and welded together as they compacted. The welding is more complete near the center of the deposit and is typically sandwiched between nonwelded upper and lower portions. Usually recrystallization of the welded vitric (glassy) volcanic ejecta (devitrification) occurs and occasionally lithophysae (stone bubbles) are formed when gases are trapped in the cooling welded tuff. The major welded ash-flow tuffs are typically 100 to 300 meters thick, separated by thinner intervals (a few meters to tens of meters thick) of nonwelded ash-falls and reworked tuffaceous rocks. The zones of reworked and/or bedded tuff indicate a hiatus after deposition when the volcanic material is eroded by wind or water. The nonwelded and reworked tuffaceous rocks are more susceptible to alteration and occasionally form zeolites through dissolution, hydration, and ion exchange. Yucca Mountain, a normal fault block mountain, formed over the last $7 \times 10^{6} \mathrm{yr}$ along with other features in the Basin and Range topographical province. The tuff layers are tilted slightly to the east near the potential repository.

Because of the manner of formation, a detailed description of the tuff stratigraphy is difficult. The character of the tuff in any one deposit changes vertically because of cooling history and horizontally because the ash flows generally become thinner away from the source caldera. Herein, the stratigraphy is idealized as a series of constant thickness modeling units ("pancakes") with a dip of $4.6^{\circ}$. The modeling units consist of consecutive layers of tuff with similar porosity. These porosity data are based on three exploratory wells, USW GU/G-3, USW G-4, and UZ-16 (Figure 1-4), and are combined using an approach similar to that of Rautman (1995) to define the modeling units (Figure 1-5). The GU/G-3 well is located a few miles south of the potential repository. Because the well penetrates all of the geologic strata found at Yucca Mountain and layer transitions are fairly easy to identify, the thicknesses of the modeling units were primarily based on these porosity data. However, because of differences in thickness data between the GU/G-3 and G-4 wells for the host rock, data from the G-4 well, which was drilled near the potential repository, were used for the TS modeling units (a major portion of the Topopah Spring Member of the Paintbrush Formation).

For the most part, interpreted data from the past performance assessment of the potential repository at Yucca Mountain by Sandia (Wilson et al., 1994; Schenker et al., 1995; M\&O, 1995a) were used for all geologic components of the disposal system without re-evaluation. Exceptions are parameters for which new data were available, specifically, saturation curves (as defined by the van Genuchten model [van Genuchten, 1980]) and the porosity of the modeling units. The bulk of the geologic barrier characterization is similar to that described by Rechard, ed. (1995) with some incorporation of observations from other studies such as Rautman (1995), Ho et al ${ }^{\ddagger \ddagger}$, and Altman et al. (1996). Updated sorption coefficients $\left(\mathrm{K}_{\mathrm{D}}\right)$, which impact the retardation effects of some geologic layers are used in the 1997 PA. As compared to the 1994 PA, the Topopah Spring modeling units have been refined using Rautman (1995), inclusion of new permeability data from LeCain (1997), and updated infiltration data from Flint et al. (in draft "Conceptual and Numerical Model of Infiltration for the Yucca Mountain Area, Nevada"). Relatively little new information has come from the YMP regarding characterization of the saturated zone; thus, differences between the 1994 and 1997 PAs largely reflect refinements of the modeling approach and are explained in Section 1.7. Because the emphasis of the 1997 PA is on the waste form, many geologic parameters are not varied. The geologic barrier discussion in Chapter 4 summarizes data and conceptual model attributes to illuminate the choices made in the 1997 PA.

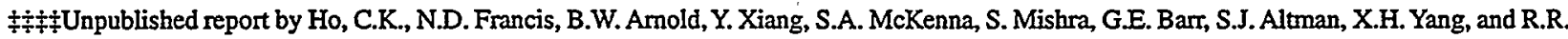
Eaton. "Thermo-Hydrologic Modeling of the Potential Repository at Yucca Mountain Including the Effects of Heterogeneities and Aiternative Conceptual Models of Fractured Porous Media." Level 3 Yucca Mountain Milestone Report T6536, M\&O Milestone Report. Records Information System (RIS) accession number MOL.19961219.0269. 


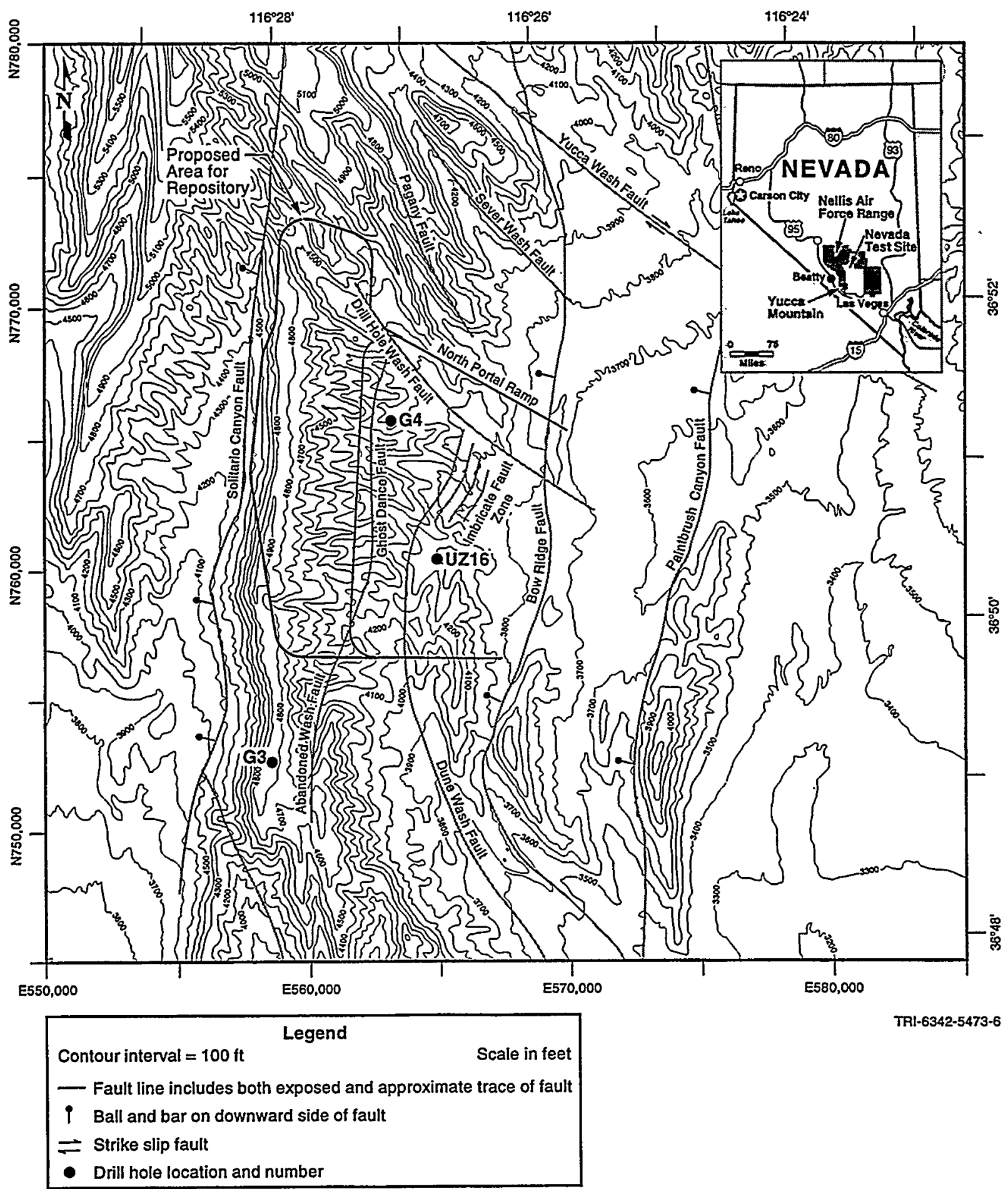

Figure 1-4. Location of potential repository at Yucca Mountain, Nevada. 
1. Introduction

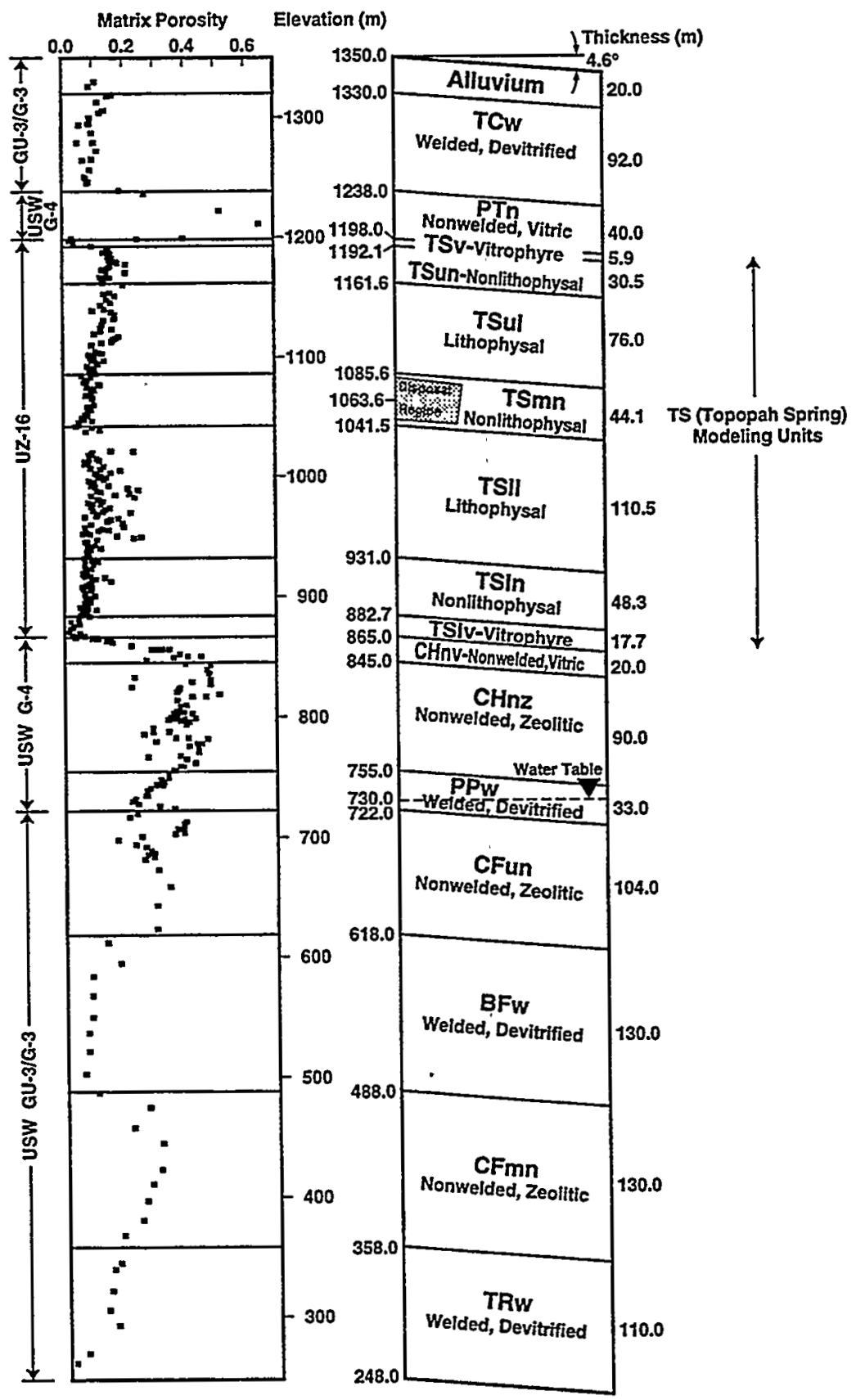

TAI- $5342-4535-3$

Figure 1-5. Simplified stratigraphic column of Yucca Mountain tuffs and 16 hydrologic modeling units with similar porosity and/or welding that were used in the simulations (after Rautman, 1995, Figure 8). 
The 1997 PA approach to geologic barrier characterization and modeling and the ways in which it differs from the 1994 PA (Rechard, ed., 1995) are discussed in a later section (Section 1.7 and Appendix C). A more detailed comparison of the present study with other studies (M\&O, 1995a; Ho et al. ${ }^{* * * *}$; Altman et al., 1996) is presented in Chapter 4.

\subsubsection{Repository Design}

Components of the engineered barriers that are specific to the repository design include (1) the subsurface facility layout, (2) the design for waste emplacement, and (3) specially placed backfill (a subset of which is often called a "seal") in the drifts and ramps. These components are briefly discussed below and more thoroughly described in Chapter 5, Repository Design. The waste package, another component of the engineered barrier, was discussed in Section 1.4.2. Because many of the precise details of the repository-such as the need for ramps or ventilation, and the size of the operational area-depend more on the operational phase of the potential repository than behavior after closure, the discussion below and in Chapter 5 highlights only the dimensions considered pertinent to postclosure behavior, such as the minimum spacing of waste packages and disposal tunnels.

Subsurface Facilities. For the 1997 PA, the repository modeled is located west of the Ghost Dance Fault and contains both DOE-owned and commercial spent fuel. The mine design for center in-drift emplacement is a tunnel and pillar configuration, with the entire repository consisting of $1236-\mathrm{m}$-long tunnels bored by machine. The tunnels are surrounded and connected by access drifts, $7.62 \mathrm{~m}$ in diameter, which lead to ramps that connect to the surface (Figure 1-6).

Waste Placement in Repository. In addition to size, design features of the repository that can affect its longterm performance are the orientation of the waste package and the spacing of waste packages and disposal tunnels.

Orientation of Waste Packages. For the 1997 PA, the orientation for placement of waste packages is center indrift on pedestal (CDP) emplacement (Figure 1-7), which is the most recent design choice by the YMP (M\&O, 1996). With the CIDP option, waste packages are placed by means of a remote controlled rail-mounted gantry crane on permanent pedestals, composed of carbon steel, that are prepositioned in the drift (M\&O, 1995a, p. 3-7). In the 1997 PA, no gravel or other material is placed beneath the pedestal.

Spacing of Waste Packages and Disposal Tunnels. The design goal in the 1997 PA was to use the "hot" repository concept, which is defined according to density of heavy-metal mass, which, for commercial spent nuclear fuel, is roughly equivalent to calculating areal power density. The design-basis MTHM density used by the YMP is 2.1 $\mathrm{MTHM} / \mathrm{m}^{2}$ (85 MTHM/acre). This goal is achieved by controlling the spacing among nearest-neighbor waste packages. The purpose of designing a hot repository is to dry out the host rock for hundreds of years. The amount of heat produced varies with time and is a function of the time that the radioisotopes have decayed (fuel "age") and the original irradiation of the fuel in the nuclear reactors (fuel "burnup"). (The heat rates and MTHM totals for the spent fuel modeled in the 1997 PA are shown in Chapter 5.)

In this performance assessment, the waste is uniformly distributed throughout the repository, with DOE-owned waste comingled with commercial waste. The DOE-owned waste, which is cooler than the commercial waste, is placed relatively close to the commercial waste (see Figure 1-8). The intention is to produce a temperature profile throughout the repository that is fairly uniform. Figure 1-8 illustrates the spacing of the DOE-owned and commercial waste assumed per 77-m section of the repository; this spacing pattern is repeated throughout each 1236-m disposal tunnel. (The 1236-m length includes two 2-m access tunnels.)

The maximum density of $2.1 \mathrm{MTHM} / \mathrm{m}^{2}$ was evaluated by the YMP (M\&O, 1995a) and depends on (1) a maximum centerline temperature in a waste package to prevent excessive failures of fuel cladding, (2) a host rock/waste

*****Unpublished report by Ho, C.K., N.D. Francis, B.W. Amold, Y. Xiang, S.A. McKenna, S. Mishra, G.E. Barr, S.J. Altman, X.H. Yang, and R.R. Eaton. "Thermo-Hydrologic Modeling of the Potential Repository at Yucca Mountain Including the Effects of Heterogeneities and Alternative Conceptual Models of Fractured Porous Media." Level 3 Yucca Mountain Milestone Report T6536, M\&O Milestone Report. Records Information System (RIS) accession number MOL.19961219.0269. 
1. Introduction

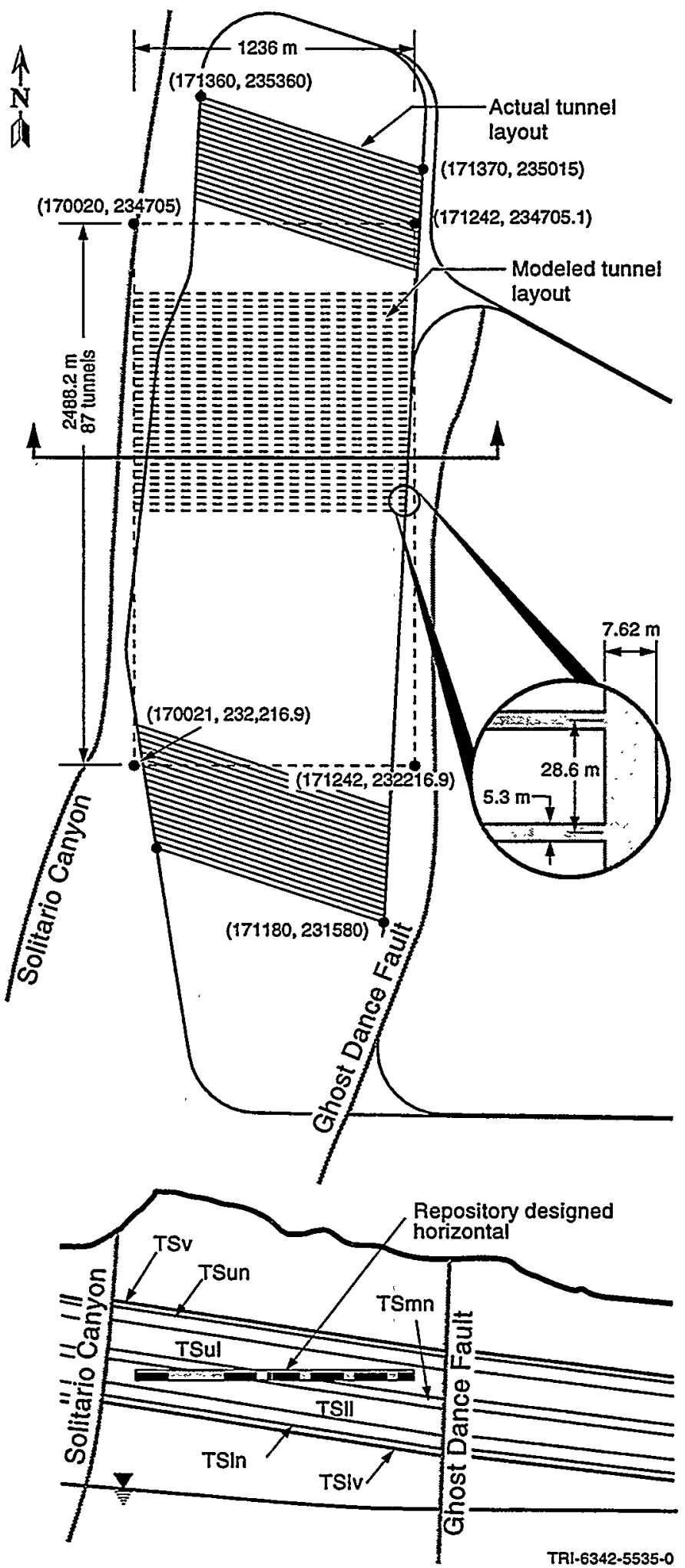

Figure 1-6. Layout of disposal tunnels and access drifts for potential repository in tuff. 

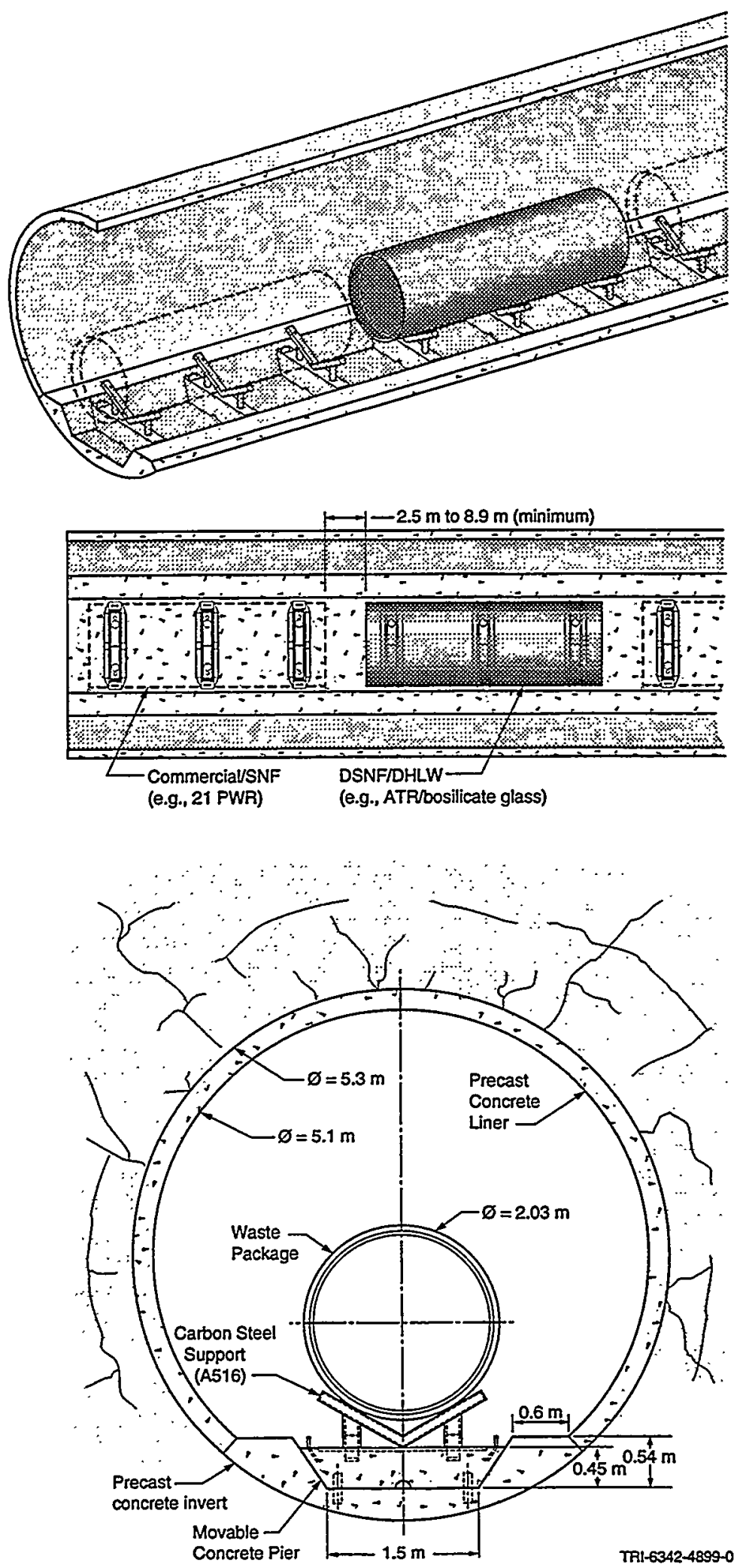

Figure I-?. Orientation of waste packages. 


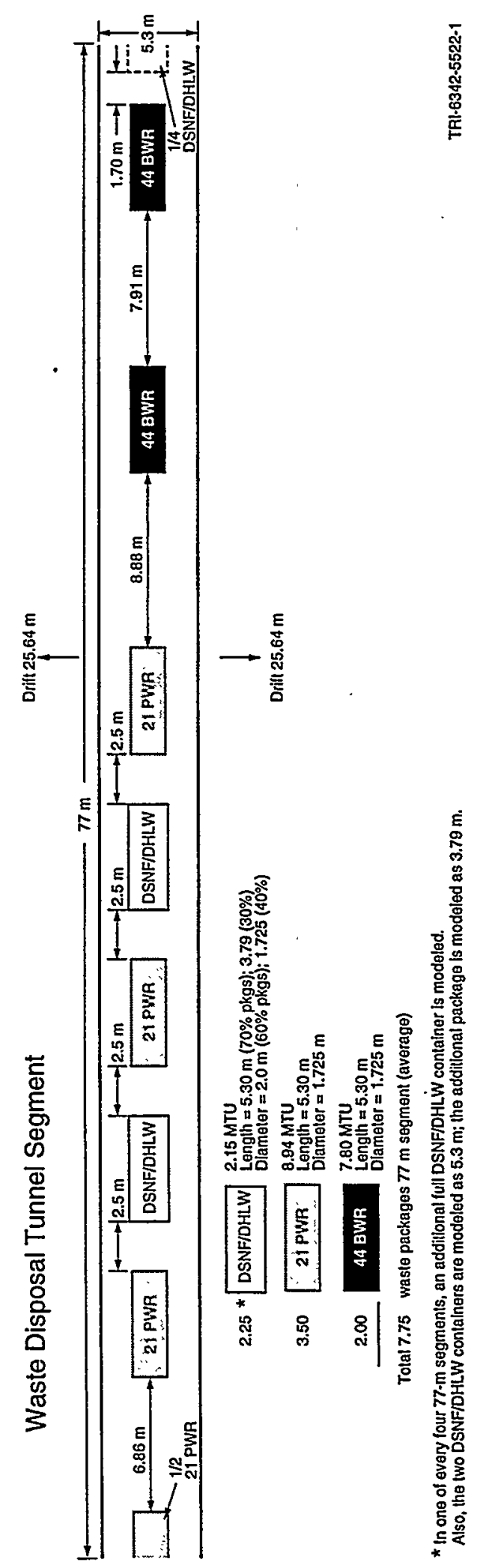

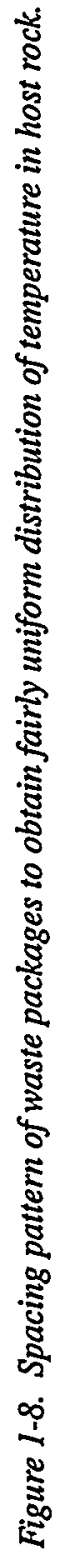


package interface temperature to prevent undesirable thermal stresses around the room, and (3) a maximum temperature in the zeolitic layer below the repository to prevent excessive alteration.

Disposal Tunnels. In the potential repository layout described in TSPA-1995 (M\&O, 1995a), the disposal tunnel spacing is $28 \mathrm{~m}$. Based on heat loads of the waste used in the $1997 \mathrm{PA}$, a spacing of $28.6 \mathrm{~m}$ between the 5.3-m-wide tunnels was used to maintain a heat load equivalent of $2.1 \mathrm{MTHM} / \mathrm{m}^{2}$ for commercial fuel. The disposal tunnels are assumed to be lined with a 0.2 -m-thick precast concrete liner. A thick segment on the base of the tunnel forms an invert into which a minimum of 3 piers can be set for container support (Figure 1-7).

Backfill. As used herein, backfill is the material that fills the excavated openings of the repository. Special categories of backfill include (a) seais for providing immediate sealing of access drifts and ramps and (b) backfill that is placed directly around the waste package. In the 1997 PA, only the ramps to the disposal area were assumed to be backfilled and effectively sealed.

Backfill was not used in the access drifts, disposal tunnels, or directly around or below the waste package in the invert. An air gap was selected in place of backfill because the trapped air in crushed-tuff backfill, which has a low thermal conductivity, would act as an insulator, thus increasing centerline temperatures. Also, the gap provides a capillary break, which does not eliminate dripping but does limit contact of water percolating through the matrix, which is important to the corrosion calculations.

\subsection{Scenario Development}

Herein, scenario development is considered the second phase of conceptual model development (the first phase being system characterization, Section 1.4). Generally speaking, scenario development is the process of deciding what may happen to the disposal system in the future and how to model it.

\subsubsection{Selected Features, Events, and Processes}

The selected features, events, and processes were drawn largely from previous studies, e.g., TSPA-1993 (Wilson et al., 1994). Because the focus of the 1997 PA is the performance of DOE SNF, some combinations of events and features were not evaluated, e.g., volcanism was not considered based on results from a previous performance assessment, which showed a probability of occurrence to be just above $10^{-4}$ in $10,000 \mathrm{yr}$ in conjunction with insignificant releases (Wilson et al., 1994). The basic features, events, and processes selected for modeling in this performance assessment are briefly summarized below.

Features. The basic features explored in the 1997 PA are (1) areas of concentrated infiltration above the repository; (2) a highly fractured, tilted pancake stratigraphy; (3) a large unsaturated zone that holds a repository; (4) horizontal emplacement of waste packages; and (5) an underlying aquifer. These features are described in Section 1.4 and Chapters 3,4 , and 5 .

Event. No events are considered in the 1997 PA; neither human intrusion nor criticality is specifically modeled. (However, related criticality calculations are performed as part of the broader DOE program for developing a safe, cost-effective technical strategy for the interim management and ultimate disposition of DOE-owned spent fuel and high-level waste.)

Processes. Basic processes are explored for the geologic barrier, waste form, waste package, and repository.

Geologic Barrier. For the geologic barrier, processes considered are two-phase flow with multicomponent gases coupled with heat conduction, allowing possible phase change in a fractured, porous matrix, and infiltration variations from climate change. The transport process of radionuclides in the liquid phase with large degrees of sorption is also included, as is transport of $\mathrm{O}_{2}$ from the surface to the repository horizon, which is an important focus of this analysis. 
Waste Form. For the waste form, the process considered is control of radionuclide release by solubility; matrix alteration or immediate release of some radionuclides in gaps and grain boundaries of spent fuel; and degradation of the cladding and the matrix containing radionuclides. Note that the 1997 PA code, BRAGFLO_T, is capable of modeling the retarding effects of cladding on fuel matrix exposure, but this effect was not modeled in the 1997 PA because of a requirement for consistency with a-TSPA-1997. Therefore, all cladding was modeled as $100 \%$ failed with regard to its protective features, as required by NSNFP. The effect of cladding degradation on oxygen consumption, however, was modeled.

Waste Package and Repository. For the waste package and repository, processes considered are $\mathrm{O}_{2}$ consumption from several layers because of general oxic corrosion, radionuclide release through the container(s) controlled by localized corrosion, diffusion from concentration gradient, and advective flow through container breach into the host rock.

The incorporation of the features, events, and processes is discussed in the chapters on consequence modeling, Chapters 7 through 10.

\subsubsection{Calculational Design}

In general, the approach for this analysis was the same as in the 1994 PA (Rechard, ed., 1995), with some aspects updated. Specifically, the analysis (a) used the methodology established by Sandia National Laboratories for assessing the long-term performance of the Waste Isolation Pilot Plant (WIPP) geologic disposal system, (b) coupled the model of the source term with the model of two-phase flow in the unsaturated tuff, (c) used updated data collected for the potential repository at Yucca Mountain, Nevada, and (d) improved the traceability and repeatability of the analysis. These aspects are summarized below and also are discussed in more detail in Chapter 6, Analysis Design. Model descriptions and assumptions can be found in Chapters 4 through 10.

Selection of the appropriate level of model sophistication is an important aspect of a performance assessment. Past experience has shown that use of simple analytic expressions of complicated phenomena, even though considered to be conservative, is not always acceptable. For example, the U.S. Environmental Protection Agency (EPA) questioned the one conservative simple model in the performance assessment analysis of the WIPP because the model could not be certified as reasonable. In response, the WIPP Project had to adopt a more complicated model that could be defended scientifically as reasonable (Rechard, 1998).

For the 1997 PA (which is essentially an update and improvement to the 1994 PA), Sandia

- simulated the complex details of contaminant transport in heated, unsaturated tuff directly in its analysis to complement the development of abstractions used in a-TSPA- 1997.

- included transport of $\mathrm{O}_{2}$ in order to evaluate whether natural processes significantly limit concentrations of reactants such as $\mathrm{O}_{2}$ and $\mathrm{H}_{2} \mathrm{O}$, reducing the oxidation rate of container or waste.

- neglected credit for cladding of SNF and used the same inventory to match a-TSPA-1997.

To update the 1997 PA and coordinate a better comparison of its results with a-TSPA-1997, Sandia

- attended the workshops conducted by the YMP performance assessment team to learn of the most recent designs, modeling issues, and data available on the potential Yucca Mountain repository.

To improve traceability and repeatability, the 1997 PA for the DOE/EM has

- used a centralized database for all parameters in the performance assessment models in the current analysis (3024 parameters: 2755 assigned constants, 269 with assigned distributions, 63 of which were varied), including references.

- provided extensive documentation on the conceptual and applied models used in the current analysis.

- incorporated configuration management software and detailed batch scripting of the simulations in the current analysis. 
The YMP's PA (a-TSPA-1997) was deterministic in that model parameters were set at their "best estimates." However, several parameters were varied to determine the sensitivity of the results. The 1997 PA was probabilistic in that 63 parameters were treated as uncertain, with 90 simulations run to develop a distribution of results. In addition, two deterministic runs, with parameters set at their mean and median values, were conducted.

\subsubsection{Conceptual Models}

In a performance assessment of radioactive waste after disposal in an underground repository, the conceptual model describes the processes by which radionuclides might be released and the ways in which these processes are modeled. Given the requirement that the repository be located in the unsaturated zone and that packages of spent fuel would be placed in a dry portion of the repository and in good condition, several models and disciplines are necessary in the 1997 PA for simulating plausible alteration of the handling and disposal containers. Flow modeling must account for percolation flux through the unsaturated repository horizon, which arises from the infiltration of precipitation at the ground surface minus the amount that is returned to the atmosphere through evapotranspiration. ${ }^{f+t}{ }^{t} t$ The portion of the infiltration that reaches deeper into the tuff percolates downward to the water table (top of the saturated zone) under the control of capillary and gravity forces. The heat from the radioactive decay of the waste influences this downward percolation and must be considered in performance assessment. Heat generated as the waste decays can disrupt any normal water movement through the tuff very near the repository, potentially drying out areas of the repository; however, this dry-out zone eventually disappears as the waste cools (Figure 1-9). The amount of dry-out zone present depends on the design of the repository (i.e., the spacing of disposal tunnels, the spacing and thermal loading of the waste packages, the type of waste, and the age of the waste) and the movement of water in the microand macro-fractures in the tuff from water dripping on the packages and water flow through the failed packages.

Degradation of the protective layers and alteration and dissolution of the waste form requires a detailed source term model incorporating corrosion chemistry and geochemistry with coupling to a fluid flow code. Once radioisotopes are leached from the wastes by means of either diffusion or advection, they can migrate through the unsaturated zone to the water table and then be transported to the accessible environment through the saturated groundwater flow. In the unsaturated zone, the migration of radioisotopes is a function of the percolation flux, dispersion, and retardation of radioisotopes by sorption to mineral surfaces along the flow path or by diffusion into slower percolation zones (diffusion from the fractures into the rock matrix where flow is slower). Sorption is a function of the chemistry of the specific radioisotope, the chemistry of the fiuid, and the chemistry of the minerals along the flow path. Dispersion is the spreading of the plume caused by the tortuous paths that the radioisotopes follow through the openings (pores and fractures) in the rock.

At the water table, the concentration of radioisotopes is diluted by the flow in the saturated zone. The radioisotopes are then transported through the saturated zone to the accessible environment where their concentration can be compared to a performance metric, in this case, dose to an individual. Along the transport paths to the accessible environment, the concentration of radioisotopes is reduced by dispersion and delayed by retardation (lagging behind the flow because of sorption and matrix diffusion). The delay caused by retardation may provide time for reduction of concentration through radioactive decay, depending on the delay time in relationship to the half-life of the specific radioisotope.

Further information about conceptual models can also be found in the discussions of their related computational models (Chapters 7 through 10).

\subsection{Probability Modeling}

In general, for a performance assessment, two types of probability models are used. The first estimates the likelihood of uncertain parameter values by constructing distributions for imprecisely known model parameters. The second estimates the likelihood of the broad classes of the parameter space, called summary scenarios; however, the

$+\dagger+\uparrow \nmid$ Much of this description was originally summarized in Bodvarsson et al., eds., 1997. 
1. Introduction

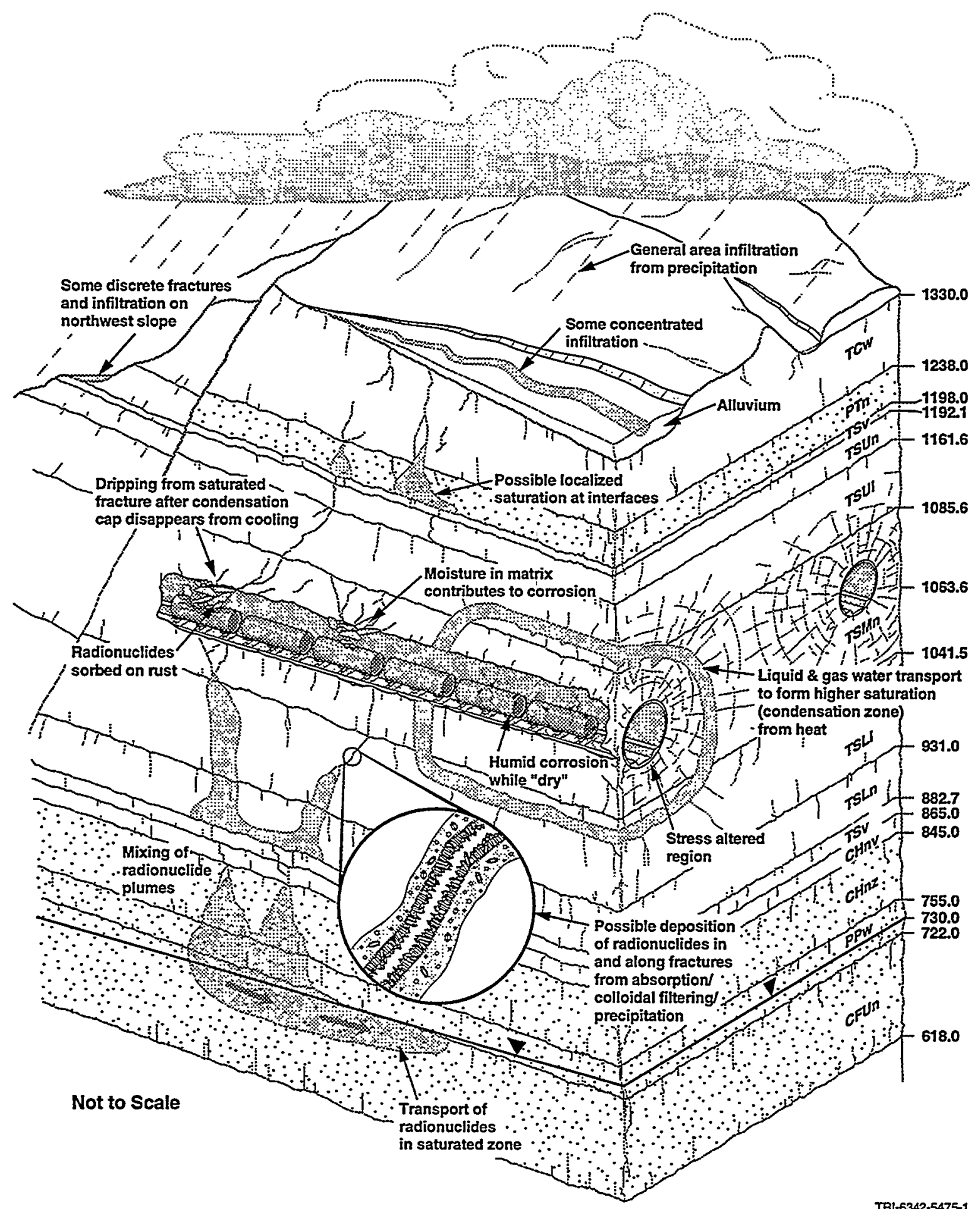

Figure 1-9. Schematic of water movement and eventual degradation of container(s) and waste in potential unsaturated tuff repository. 
latter are not used for this analysis. The model parameters themselves are discussed primarily in the chapters that characterize the system (Chapters 3, 4, and 5).

Identification of model parameters considered uncertain and the assignment of distributions are described in Chapter 6, Analysis Design. In this performance assessment, this parameter uncertainty is propagated through a Monte Carlo technique. Integration of mathematical equations through Monte Carlo techniques is discussed in more detail in Rechard (1995). Latin hypercube sampling is used to minimize the number of random samples needed in the Monte Carlo technique to adequately capture variability in the parameters and, thus, the number of simulations run in the consequence models (McKay et al., 1979; Iman and Conover, 1980).

\subsection{Consequence Modeling}

A major portion of the performance assessment methodology consists of simulating the selected features, events, and processes to estimate the amount of contaminants released to the accessible environment. The simulation also includes propagating the uncertainties of model parameters to evaluate the uncertainties associated with the predictions. In this section, the most significant aspects of the simulation (or "consequence modeling") are highlighted.

The 1997 PA examined the acceptability of minimally treated waste forms, which relies on the characteristics of the spent fuel as is and the engineered design of the waste package. Thus, an effort of prime importance was the modeling of phenomena related to the release of radionuclides near the package. The model used for consequence modeling is actually a composite function of several models (Figure 1-10), which are discussed briefly below and in more detail in later chapters of this report:

- Corrosion and source term submodel (Chapter 7)

- Unsaturated zone, flow and transport submodels (Chapter 8)

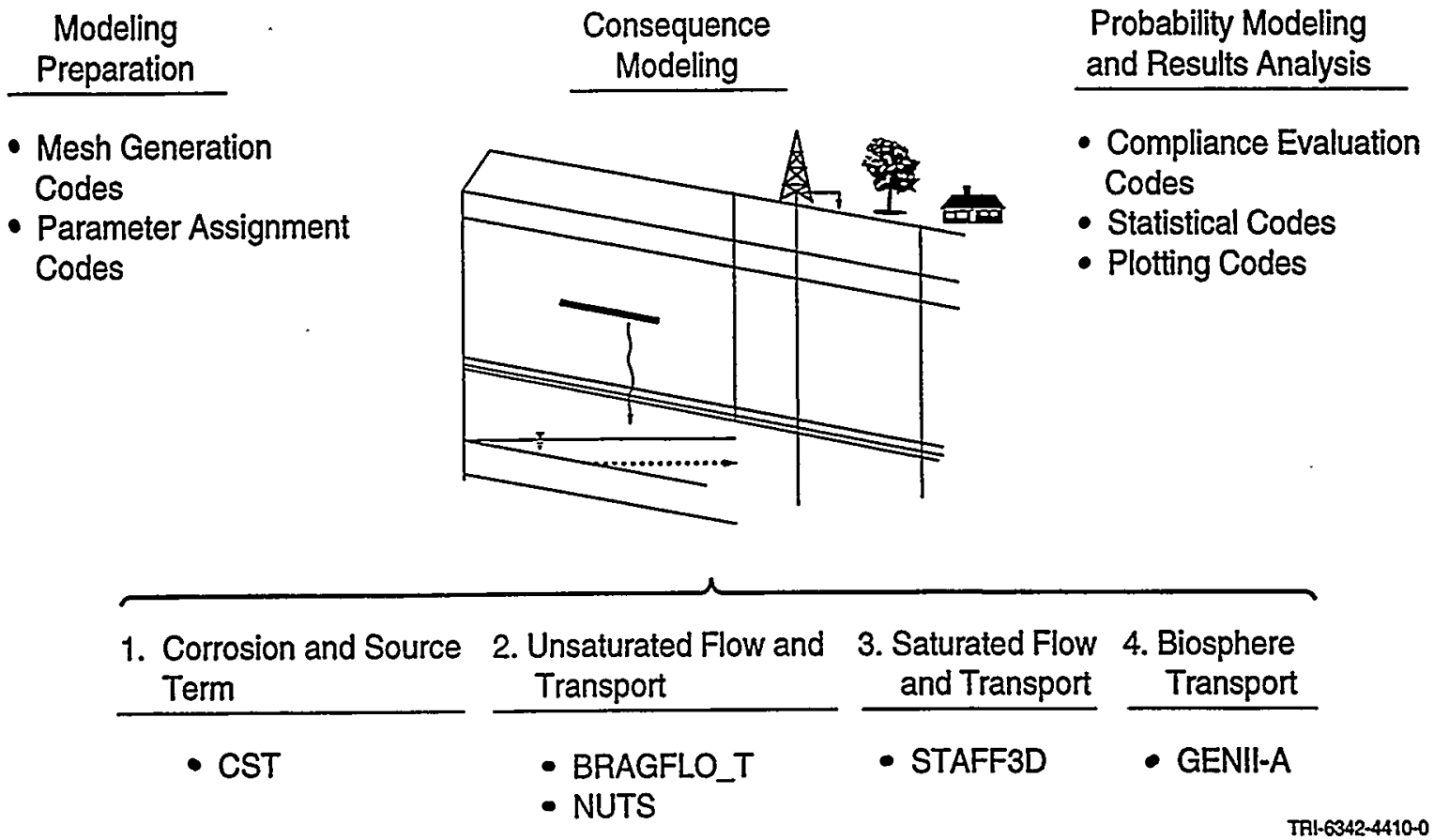

Figure 1-10. Four categories of codes used for consequence modeling. The primary focus of the model was on phenomena related to the release of radioisotopes near the waste package. Linkage of these codes is depicted in Chapter 6. 
- Saturated zone, flow and transport submodels (Chapter 9)

- Biosphere transport submodel (Chapter 10).

\subsubsection{Corrosion and Source Term Modeling}

Most phenomena associated with the waste package are included in the source term code, CST, which is a submodel to the flow and transport codes. CST is a modified source term model that replaces the code used in the 1994 PA, USATCONC. The primary improvement in the source term model in 1997 is the ability to account for $\mathrm{O}_{2}$ consumption. Chapter 7 provides additional details.

Modeling the corrosion of the container and fuel is a complex process because of (a) the wide variety of waste forms, which include radionuclides residing in up to 10 different matrices and up to 8 nested layers of material, (b) the need to track breach of a protective layer from localized corrosion, (c) the necessity of monitoring consumption/production of gases from all layers, (d) the need to evaluate the availability of oxygen, and (e) the possibilities of water contact, i.e., through either direct contact with the tuff matrix or dripping from fractures (see Section 1.5.3). For example, a container of ATR fuel (Category 6) has five layers (two of stainless steel) that must be breached before water can liberate the radioisotopes in the uranium-aluminide fuel matrix.

Capabilities of CST. CST tracks radioisotopes in four locations within the container to properly account for their ultimate release. CST's capabilities include (1) decay of any number of radioisotopes, (2) defining many different waste packages, each with different protective and nonprotective layers and different matrices containing the radioisotopes, (3) basing the amount of water for corrosion on saturation in fractures at each time step as calculated in the unsaturated flow model (Section 1.7.2), (4) defining wet and dry anoxic and oxic corrosion, (5) temperature dependence of corrosion rates, (6) diffusion through rubble around the waste packages, and (7) radioisotope sorption on corroded containers (e.g., iron in 1997 PA).

The corrosion section of the source term submodel estimates the mass of material corroded in each protective layer of the container or fuel over a time step, determines when a layer is breached and the underlying layer is exposed, and estimates the amount of gas and liquid corrosion products that are produced or consumed and the total summed mass of material that readily absorbs radioisotopes (e.g., rust). In the unsaturated zone, sufficient oxygen available for oxic corrosion might be assumed. However, it is entirely possible that corrosion of the large quantities of steel can deplete the oxygen such that corrosion is anoxic, a situation that may apply especially deep inside the waste package.

Once the waste is exposed, CST determines the release of radioisotopes by means of the rate of release from the matrix containing the material, the solubility of the material in the groundwater, and advection and diffusion from the package. For example, once exposed, some uranium metal matrices alter quickly relative to uranium dioxide (e.g., $\mathrm{UAl}_{\mathrm{x}}$ in ATR fuel and $\mathrm{UC}_{2}$ from graphite fuel), but because neptunium in the matrix is not very soluble, additional water is necessary to advect it from the package. Also, both intact air gaps and gaps filled with rubble from degradation of the disposal tunnel over time were considered because (1) the presence of the rubble meant that, in addition to dripping, the matrix could have contact with water percolation and (2) a diffusion pathway through the rubble was a potential release mechanism for radioisotopes.

Radioisotope Solubilities. In conjunction with performance assessments for the YMP in 1993, radioisotope solubilities were estimated based on experiments and geochemical calculations to account for differences in values for parameters that influence solubility. These results, combined with expert opinion, were then used to develop values for radioisotope solubilities. Although a formal elicitation process, such as that performed by Trauth et al. (1992), was not pursued, opinions from several project scientists were solicited in TSPA-1993 to define distributions for the solubility of radioisotopes in an oxic environment, assuming water conditions bound by J-13 and UE-25P\#1 well water in the underlying aquifer (Wilson et al., 1994, p. 9-6; also see Chapter 4). The 1997 PA decreased the solubility of ${ }^{237} \mathrm{~Np}$ in a Yucca Mountain environment by two orders of magnitude from that used in the 1994 PA and TSPA1995 because of more recent interpretation of experimental results, as explained in Chapter 7. 


\subsubsection{Unsaturated Zone, Flow and Transport Modeling}

In the performance assessment, flow and transport codes are used to model phenomena related to migration of radioisotopes away from the container (see Figure 1-9). The gas consumed during corrosion and the amount of radioisotopes released from the container are calculated in the corrosion and source term submodel (CST, see Section 1.7.1) and coupled to the flow and transport modeling codes. The 1997 PA unsaturated zone model is two-dimensional using BRAGFLO_T, which handles two phase flow (water in both liquid and vapor phases) and heat transfer. The modeling code NUTS is used for transport modeling. NUTS uses flow field results from BRAGFLO_T to evaluate transport of contaminants, as explained in Chapter 8 (see also Figure 1-10).

Capabilities of BRAGFLO_T. BRAGFLO_T (Version 3.10) was used to compute the time-dependent flow of three components $\left(\mathrm{O}_{2}, \mathrm{~N}_{2}\right.$, and water in both the liquid and vapor phases) near a waste package. These results were then used to evaluate consumption of $\mathrm{O}_{2}$ or $\mathrm{H}_{2} \mathrm{O}$ during alteration of the container and spent fuel and determine failure times of containers and release of radioisotopes. BRAGFLO_T (Rechard, ed., 1995) is an enhanced version of the isothermal multiphase flow code, BRAGFLO (WIPP Performance Assessment Dept., 1997; Bean et al., 1996). One of several enhancements of BRAGFLO_T was the addition of an energy balance equation, so that thermal effects on both fluid and rock properties are incorporated. Heat transport is by conduction and convection, the latter including both specific heat that results in temperature change and latent heat. BRAGFLO_T uses a finite-difference formulation to solve four partial differential equations that describe the mass and energy balance of the three-component, two-phase system. Fick's Law and a multiphase extension of Darcy's Law are used to describe fluid flow. Features such as vapor pressure lowering due to capillary pressure and diffusive mass fiux in the gas phase, similar to TOUGH2 (Pruess, 1991), are included.

Because volcanic tuff is highly fractured, the mathematical models that underlie these codes for both the unsaturated and saturated fiow regimes are special cases of a dual-permeability model. A dual-permeability model consists of a fracture system and a matrix system that are fully coupled in properties such as pressures of each phase, temperatures, and velocities. In BRAGFLO_T, the composite porosity simplification (Peters and Klaveter, 1988) (also called equivalent continuum [Pruess et al., 1990]) of the dual permeability formulation is used for fluid flow, that is, the fractures and matrix are assumed to have thermodynamic equilibrium (phase pressures and temperatures) between matrix and fractures. Consequently, one set of thermodynamic variables (e.g., a single saturation and permeability function) is assumed to represent the state of the fracture and matrix system.

Computational Grid. In the 1997 PA, the modeling unit layers, which represent stratigraphy, exhibit surface topography and stratigraphic dip by assigned rock properties that vary from unit to unit, and lateral homogeneity within each unit. The modeled cross section extends from west of the Solitario Canyon Fault near well H-6 to just east of the Ghost Dance Fault (Figures 1-4 and 1-6) passing near the location of well SD-12. Faults are represented by assigning different hydrologic properties from surrounding units, and the units were offset in accordance with the geologic framework data. Note that, in contrast, the 1994 PA did not model faults in the unsaturated zone and the modeling units were flat lying (because the cross section was north-south where stratigraphic dip is about one to two degrees).

Infiltration Model. Climate change was included in the analysis because it can enhance infiltration and thus percolation of water through the unsaturated zone and repository. The average injection rate over the model was described as a cosinusoidal function to simulate potential variation. The resulting average infiltration over the model grid was then varied according to a representative infiltration profile obtained from Flint et al. (in draft "Conceptual and Numerical Model of Infiltration for the Yucca Mountain Area, Nevada") (see also, Altman et al., 1996). In addition to this regional variation, at one cell over the repository near the crest of Yucca Mountain, a sample factor focused infiltration between 1 and 10 times that over the rest of the repository. Additional details are provided in Chapter 8. 


\subsubsection{Saturated Zone, Flow and Transport Modeling}

The saturated zone (see Figure 1-9) was modeled in three dimensions with STAFF3D (Huyakorn et al., 1992) (Figure 1-10), assuming a pancake layering as previously described (Section 1.4.3), except that the two top units, TCw and PTn, were omitted from the model and the TS units were grouped as one unit (TSwc), unlike the treatment in the unsaturated zone model (see Chapter 4, Figure 4-9). For the simulation, the dual-porosity simplification of the dual-permeability conceptual model is used. In the dual-porosity formulation implemented in STAFF3D, radioisotopes move through the fracture continuum by convection and dispersion and between the fracture continuum and the porous matrix by diffusion orthogonal to the fractures. The injected water from infiltration has only a small influence on fluid flow in the saturated zone; hence, a steady state simulation was used for each sample vector with an average infiltration value calculated over the 100,000 -yr simulation period from the sampled cosinusoidal function. The twodimensional unsaturated NUTS transport model was coupled to the three-dimensional saturated zone STAFF3D model by taking the radionuclide flux $(\mathrm{kg} / \mathrm{s})$ across the grid block elements just above the water table from NUTS and using it as the source radionuclide flux input $(\mathrm{kg} / \mathrm{s})$ just below the water table in STAFF3D.

The cursory description of the mathematical model and a detailed description of the applied model is provided in Chapter 9.

\subsubsection{Biosphere Modeling}

Dose to humans was evaluated in the 1997 PA by means of the software code, GENII-A. In addition, calculations similar to the method used in a-TSPA-1997 were conducted for purposes of comparison. The performance metric was maximum annual effective dose equivalent (EDE) to an individual from release of radioactivity by way of the potential repository.

The software code, GENII-A (Napier et al., 1988a,b,c) was used to evaluate individual dose from the migration of radioisotopes through the biosphere that reach the accessible environment by means of a water well. GENII-A uses the concentrations for the transported radioisotopes from STAFF3D (for the 5-km boundary location). The 1997 PA considers three cases: (1) a Ranch case, in which a rancher is exposed to radioisotopes by means of beef consumption only, (2) a Farm case, in which a member of a farming family is exposed to radioisotopes by means of food consumption, water consumption, and inhalation, and (3) a Small Community case, in which an average resident of a small community uses the contaminated water for drinking and consumes some foods grown locally from the Farm activities, including vegetables, fruits, dairy, and meat products.

For each case, dose is evaluated from peak concentrations of the transported radioisotopes- ${ }^{129} \mathrm{I},{ }^{237} \mathrm{~Np}$, and ${ }^{99} \mathrm{Tc}$ - at the 5-km boundary, i.e., as if the Rancher, Farmer, or Small Community had drilled a water well into the saturated zone at the point on the $5-\mathrm{km}$ boundary that showed the highest radioisotope concentrations. A more detailed description of biosphere modeling in the $1997 \mathrm{PA}$ is found in Chapter 10.

\subsection{Performance Metrics}

The results of this report are intended to serve as a tool for making rational decisions regarding resource allocation for characterizing spent fuel and for evaluating the current disposal option. Thus, the results of most importance are (1) the relative position of the performance measures with respect to the reference waste form, commercial spent fuel, and (2) the relative importance of model parameters in determining the value of the performance measure. The latter information is discovered through sensitivity analysis (Section 1.9). The results of the performance assessment are discussed further in Chapter 11. It is also useful to measure the performance of DOE SNF and commercial spent fuel against specific performance criteria, because the results provide information about broader issues that relate to both spent fuel types. 
Currently final standards do not exist for the potential repository at Yucca Mountain; 40 CFR 191 (EPA, 1985; 1993) does not apply. Furthermore, implementing regulations such as 10 CFR 60 (NRC, 1993) must be modified. In selecting performance criteria, the 1997 PA based its decisions on current information with regard to probable future standards. The 1997 PA included dose to an individual, which was used in a-TSPA-1997 and also has been recommended by the NAS (NAS/NRC, 1995). The time period selected was $100,000 \mathrm{yr}$, which was longer than the suggested period of $10^{4} \mathrm{yr}$ by the EPA in 40 CFR 191, but less than the $10^{6}$-yr period suggested by the NAS. The 1997 PA also relied on the requirements of the EPA's standard, 40 CFR 191, including the 5-km exclusion zone and Individual Protection Requirements. The results at several intermediate points were also evaluated and presented.

\subsection{Sensitivity Analysis}

The final step of the performance assessment is sensitivity analysis. Sensitivity analysis is used to determine the effect of varying model parameters on the consequences. The sensitivity analysis reported herein basically includes creating scatterplots and developing regression models between the parameters and the various results (see, for example, Helton et al., 1992) and using the absolute values of standardized regression coefficients (or the mathematically related partial correlation coefficients) from regression models.

Because uncertainty and sensitivity analyses are inherently conditional on the models, data distributions, and techniques used to generate them, they cannot provide insight about parameters not sampled, conceptual and computational models not used in the analysis in question, or processes that have been oversimplified in the analysis. Hence, qualitative judgment about the modeling system must be used in combination with the results of sensitivity analyses to set priorities for additional data acquisition and model development. The sensitivity analyses are discussed along with other results in Chapters 11 and 12. 


\title{
2. Performance Criteria
}

\author{
R. P. Rechard
}

The 1997 PA compares the performance of DOE SNF to that of commercial spent fuel, using criteria that represent a reasonable means of measuring performance in a potential repository at this time. The 1997 PA is not intended to evaluate compliance with standards; in fact, at present, no final standards exist. However, DOE SNF's behavior as compared to commercial spent fuel's can be used to inform decisions about future actions with regard to DOE SNF; in turn the performance of commercial spent fuel measured against specific performance criteria provides information about broader issues that relate to both spent fuel types. Thus, performance criteria were selected based primarily on two sources: the Environmental Protection Agency (EPA) Standard, 40 CFR 191 (EPA, 1993), and guidance provided by the National Academy of Sciences (NAS) in 1995 regarding a proposed future standard for repository licensing (NAS/NRC, 1995).

\subsection{Performance Metrics}

Previously, two general types of performance metrics existed for the proposed commercial repository at Yucca Mountain: (1) requirements for the entire disposal system, developed by the EPA in 40 CFR 191, and (2) requirements for several components of the disposal system, developed by the NRC in 10 CFR 60 . Current choices with regard to these requirements for disposal system behavior are discussed below.

\subsubsection{Relative versus Absolute Metrics}

At present, final standards for disposal of radioactive waste in the potential repository at Yucca Mountain do not exist; 40 CFR 191 does not currently apply. Furthermore, implementing regulations such as 10 CFR 60 must be modified. Thus, the performance criteria selected by the 1997 PA do not represent final, absolute criteria. However, performance is evaluated against these criteria so that results of this study can be compared to previous or future studies. More important in this analysis, however, is the performance of DOE SNF relative to the performance of commercial spent fuel. Thus a relative comparison of spent fuel forms is the primary focus of the 1997 PA.

\subsubsection{Overall System Requirements}

The Energy Policy Act of 1992 expresses the strong desire of Congress to use a dose or health risk as an overall system requirement for the repository. In addition, the NAS and currently proposed legislation adopt a risk limit, while 40 CFR 191 as repromulgated in 1993 has a dose limit of $150 \mu \mathrm{Sv}$ (15 mrem) (Figure 2-1). The latter value is used in this analysis and roughly corresponds to the limit, proposed in current legislation, of an increase of 1 in 1000 risk of latent cancer.

\subsubsection{Subsystem Requirements}

The NAS twice has recommended against subsystem requirements as they currently exist in 10 CFR 60 (NAS/ NRC, 1995; NAS/NRC, 1992a). Consequently, no subsystem comparisons were made in this analysis. 


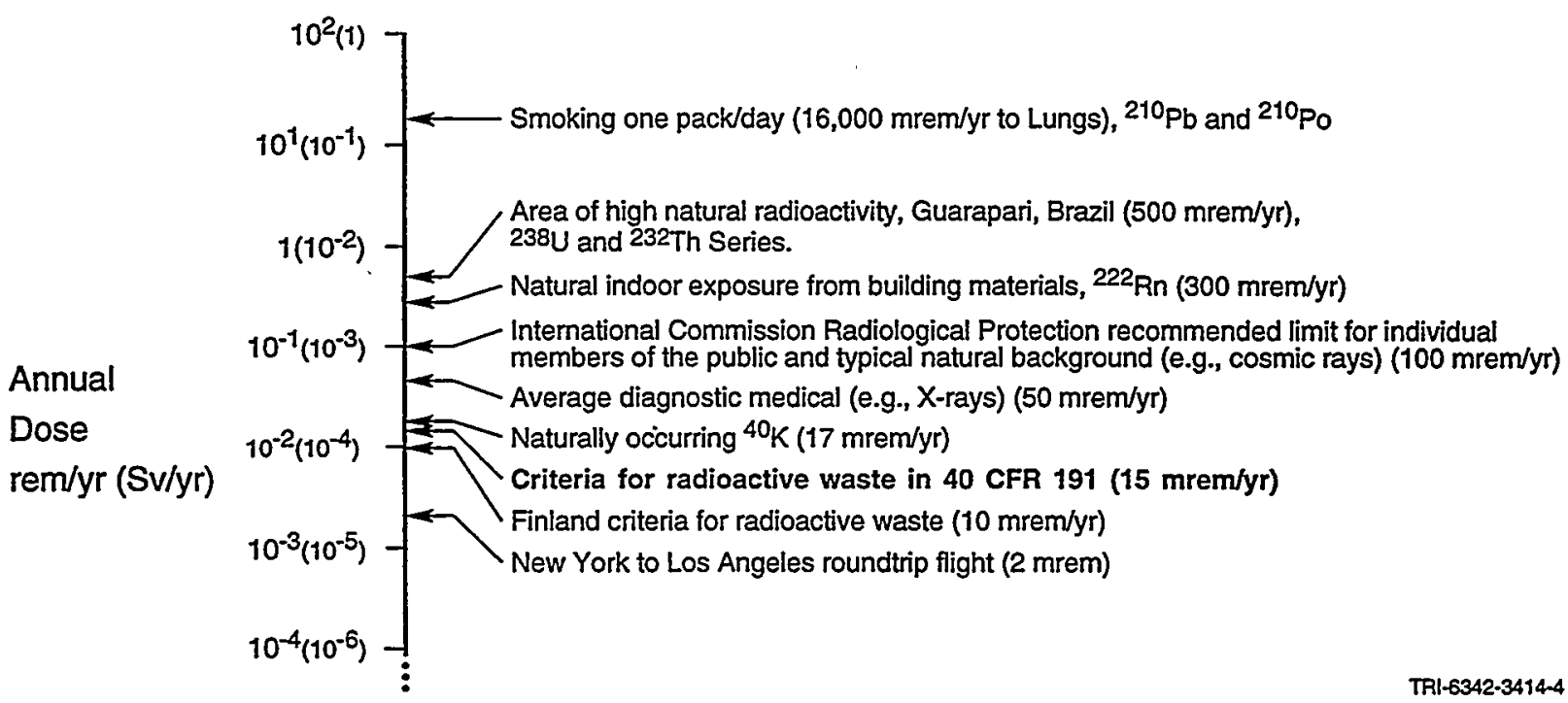

Figure 2-1. Dose comparisons from various sources. The 1997 PA uses the 40 CFR 191 dose limit of 15 mrem, which roughly corresponds to the limit proposed in current legislation of an increase of 1 in 1000 risk of latent cancer.

\subsection{Modeling Style Issues}

Modeling style refers to the guiding philosophy used to construct models. The current laws and regulations have provided three important influences with regard to modeling style: location of the accessible environment, exposure pathways, and the time period examined.

\subsubsection{Location of Accessible Environment}

In 40 CFR 191, the EPA defined the exclusion zone to be an area out to $5 \mathrm{~km}$ from the disposal region; the area beyond was defined as the accessible environment. The NAS did not recommend an exclusion zone, but did advise looking at current living patterns to determine populations at risk. Current proposed legislation mandates a similar approach but restricts the search for current living patterns to a 50-mile radius about the repository. At Yucca Mountain, locations where populations currently use water from the aquifer under the potential repository are quite far from the potential repository itself. Although the locations of potentially exposed populations or an exclusion zone is important in determining the absolute risk or dose to individuals, the location is not particularly significant when making relative comparisons among different types of fuel. Furthermore, the long distances to current population locations in the region would require an excessively large numerical mesh for simulating transport of radioisotopes. Consequently, this report uses one arbitrary boundary $-5 \mathrm{~km}$ - to evaluate doses to exposed individuals. Potential doses are also calculated at the boundary of the waste package and at the water table below the repository in specific instances.

\subsubsection{Exposure Pathways to Critical Subgroups of Population}

Besides the location of the exposed individuals, as discussed above, the calculation of a dose or health risk to a critical subgroup of the population depends upon other assumptions about the habits of individuals in the future. Consistent with most analyses, the behavior and knowledge of future potentially exposed populations were assumed 
to be the same as those today; current proposed legislation contains a definition of a critical group. A critical subgroup of today's population was assumed to be ranchers, who consumed meat from cattle that drank contaminated water, and subsistence farmers who draw well water from the contaminated aquifer for drinking and irrigation of all their food. Further discussion of this topic, along with the method of calculating the dose, is found in Chapter 10.

\subsubsection{Time Period}

For its regulatory time period, the EPA chose maximum dose over $10^{4} \mathrm{yr}$; current pending legislation mandates a similar requirement. The NAS recommended that peak maximum doses be calculated ovet a $10^{6}$-yr simulation. Based on previous work (e.g., Rechard, ed., 1995), doses out to $10^{4} \mathrm{yr}$ were thought to be negligible even at a well located $2.4 \mathrm{~km}$ from the repository. Hence, the $1997 \mathrm{PA}$ chose to calculate the peak maximum dose over a 100,000-yr simulation $^{*}$ (i.e., between the time periods suggested by the EPA and current regulations $\left[10^{4} \mathrm{yr}\right]$ and the NAS $\left.\left[10^{6} \mathrm{yr}\right]\right)$.

Mean and median runs for all models were run to 100,000 yr. For BRAGFLO_T and NUTS, the 50 realizations were run to 50,000 yr only. 


\title{
3. System Characterization: Waste Packages
}

\author{
L. J. Storz, R. P. Rechard, C. T. Stockman, and L. Taylor
}

This chapter discusses spent fuel characteristics and packaging, and related modeling parameters necessary to predict DOE SNF performance in the potential repository; the spent fuel and packaging are characterized as part of the engineered barrier system. (Repository design is another component of the engineered barrier system; see Chapter 5.) In the present PA, modeling the rates of package corrosion, fuel oxidation, and resulting release rates was emphasized.

The most inclusive term, waste package ${ }^{*}$, is used in this report to describe the spent fuel or high-level waste as it would reside in the repository. The waste package includes the spent fuel and high-level waste, handling and/or disposal containers, and any material placed between the disposal container and the host rock (none in this study). Spent fuel refers here to the packaged components including fuel rods, brackets and hardware. The high-level waste in this study is defense waste immobilized in borosilicate glass.

In the $1997 \mathrm{PA}$, thirteen categories of DOE SNF are under study and their performance is compared to that of two commercial spent fuel categories. The DOE SNF categories are packaged with DHLW to reflect DOE's proposed packaging plan as of September 1997; packaging DOE SNF with DHLW is called the codisposal option. In this chapter, we identify the DOE SNF and commercial fuel categories under study; discuss the types of handling and disposal containers for all spent fuel categories; describe the codisposal configurations; and then provide data about the 15 waste packages modeled in the $1997 \mathrm{PA}$, including related parameters. All information about the spent fuel and packaging was provided by NSNFP, as shown in Appendix $A$.

\subsection{Description of Modeled Spent Fuel Categories}

The INEEL manages approximately 38\% (by volume) of the DOE SNF and is projected to receive more in the future for an inventory that will eventually include about $60 \%$ (by volume) of the total. While awaiting licensing of a permanent repository, the DOE SNE, which is presently packaged and stored, may be characterized and treated; therefore, information about the need for additional fuel characterization and treatment is of importance to the DOE/EM.

Cladding and fuel matrix were selected as the two most important variables related to the fuel. Cladding is the outer coating of the fuel matrix such as a tube or a plate and is intended to isolate the fuel matrix from the external environment. The main classes of cladding in the DOE SNF inventory are zircaloy, stainless steel, aluminum, graphite, and no clad, all of which were modeled in the 1997 PA. Though there are several types of zircaloy, only Zircaloy2 was modeled in the current PA. The condition of the cladding can be described as intact, unclad/declad, or severely breached. Cladding integrity can be expressed by giving a percentage of elements with penetrations through the cladding and a description of the size of the penetrations, such as pinhole. BRAGFLO_T is capable of modeling the retarding effects of cladding on fuel matrix exposure, but this effect was not modeled in the 1997 PA because of an NSNFP requirement for consistency with a-TSPA-97 (M\&O, 1997). Therefore, all cladding was modeled as if $100 \%$ failed, so the retarding effect of the cladding was neglected. The effect of cladding on oxygen consumption, however, was modeled for the cladding, with the exception of TRISO and glass, for which no stoichiometry is presently available.

Fuel matrix includes the physical form and composition of the substance that holds the fissile material such as $\mathrm{UO}_{2}$ pellets, uranium metal, or $\mathrm{UO}_{2}$ beads embedded in aluminum. (In other studies, fuel meat is a term sometimes used in place of fuel matrix.) In its common usage it includes the fissile material as well. The fuel matrix enrichment

- Another term used in the 1993 and 1994 PAs is waste parcel, which included all components of the waste package except backfill between the container and the host rock. In the 1997 PA, the waste package and the waste parcel are the same because only an air gap surrounds the waste package. 
is the percentage of the fuel matrix that is fissile. The ${ }^{235} \mathrm{U}$ equivalent is $<5 \%$ for low enriched uranium (LEU); $5 \%<$ enrichment $<20 \%$ for medium enriched uranium (MEU); and $>20 \%$ for high enriched uranium (HEU). Because the current regulations do not allow credit for cladding as a protective layer, the chemical composition of the fuel matrix presently determines the rate of release of various radioisotopes based on leachability and solubility (Appendix A).

Within the NSNFP DOE SNF inventory are more than 250 individual types of spent nuclear fuel in various matrices and configurations with fissile material enrichments that range from depleted to $97 \%$. The spent fuel and its cladding occur in various conditions from declad to minor breaches; some fuel elements are crushed or melted. Because of the varied conditions of the spent fuel, it would be time consuming and expensive to characterize them in detail, especially because they represent only a small portion of the spent fuel for disposal. For example, the 250 spent fuel types represent less than 20\% of the entire DOE SNF inventory (N-Reactor fuel represents about $80 \%$ of the inventory) and, given the 70,000 MTHM capacity of the potential repository, the 250 types of spent fuel represent less than $1 \%$ of the full repository. To focus time and resources, the DOE/EM grouped the spent fuel types into categories based on the chemical composition of the fuel matrix (Figure 3-1 and Appendices A and B). Cladding condition is described, but was not a primary consideration for the grouping. It should be noted that over years of study DOE SNF has been grouped into categories using differing criteria depending on the analysis or disposal configuration being analyzed, and the current category names and numbers will differ from those selected for other purposes.

The 15 categories of spent fuel under study in the 1997 PA include 13 DOE SNF categories and two categories of commercial spent fuel (21-PWR and 44-BWR) (see Figure 3-1 and Table 1-1 in Chapter 1). Data about the spent fuels under study was provided by NSNFP and can be found in Appendix A. The spent fuel categories and their general characteristics are presented in Sections 3.1.1 through 3.1.16. Currently, the spent fuels are distributed among INEEL (Categories 1-5, 8-13), Savannah River (Categories 5-7), and Hanford (Categories 1, 4-5, 10-11, 13). Details regarding handling and disposal containers, codisposal configurations, and the 15 modeled waste packages are provided in Sections 3.2, 3.3, and 3.4, respectively. 


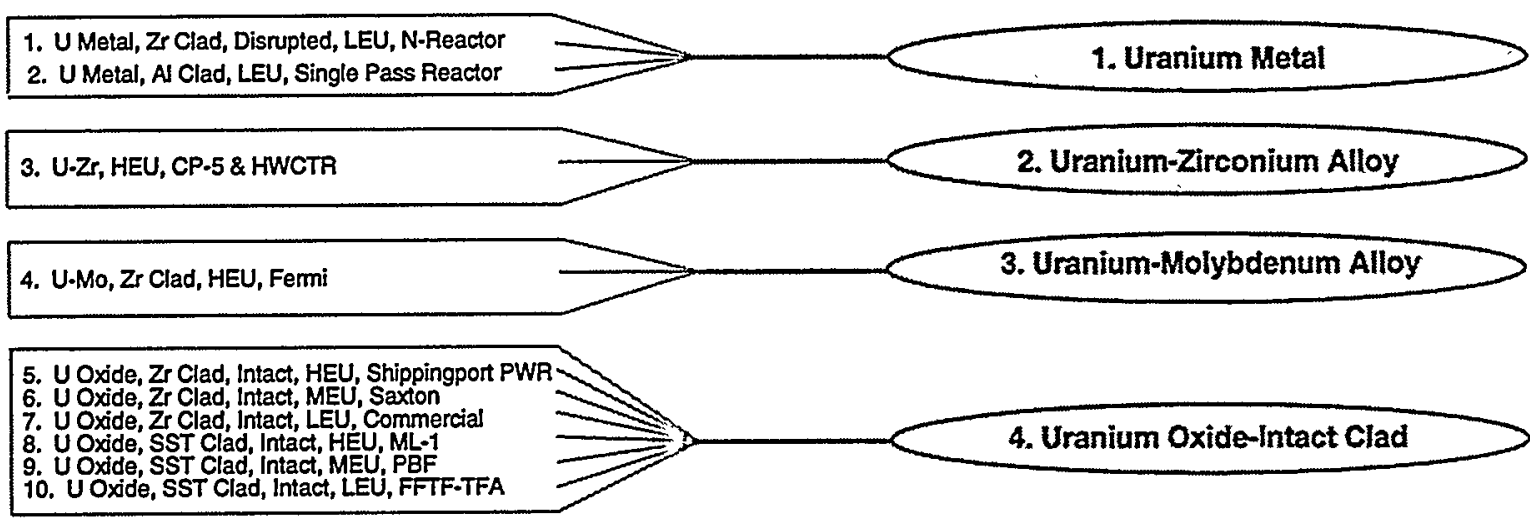

11. $U$ Oxide Failed, or Declad, HEU, SM-1A

11. U Oxide, Fail or Declad, MEU, ORNL SST \& Zr

13. U Oxide, Fail or Declad, LEU, TMI-2

14. U Oxide, Al Clad, HEU, HFIR

15. U Oxide, Al Clad, MEU, FRR, MTR

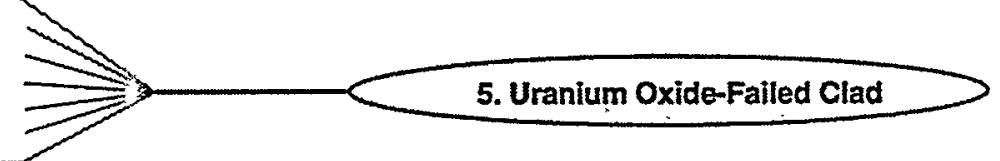

16. U-AI or U-AIX, AI Clad, HEU, ATR

17. U-Al or U-AIX, AI Clad, MEU, FRR, MTR

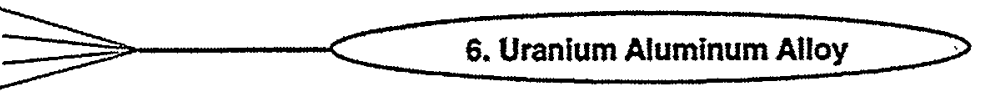

18. U.Si, A Clad, HEU, MEU, FRR, MTR

19. U/Th Carbide, Graphite, Hi-Integrity, HEU,
Ft. St. Vrain

20. U/Th Carbide, Graphite, Low-Integrity, HEU,
Peachbottom

21. U or U/PU Carbide, Nongraphite, MEU FGE,
SRE, FFTF Carbide

22. MOX, Zr Clad, HEU FGE, GE Test

23. MOX, SST, HEU FGE, FFTF-DFA

24. MOX, Misc Clad, MEU \& LEU FGE, FFTF-TFA-ACO
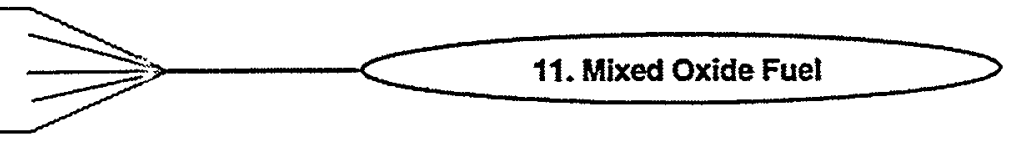

25. U/Th Oxide, Zr Clad, HEU FGE, LWBR

26. U/Th Oxide, SST Clad, HEU \& FGE, Dresden

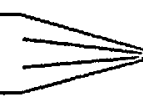

12. Uranium-Thorium Oxide

27. U-Zr-Hx, SST/nncoloy Clad, HEU, TRIGA Flip

28. U-Zr-Hx, SST/nncoloy Clad, MEU, TRIGA Std

29. U-Zr-Hx, AI Clad, MEU, TRIGA Alum

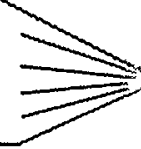

13. Uranium/Zirconium Hydride

30. U-Zr.Hx, DeClad, HEU, SNAP

31. Na-Bonded, SSTMisc, HEU, MEU, \& LEU,
Fermi I Blanket
32. Classified, HEU, Navy
33. Canyon Stab., HEU \& LEU, SRS Target
34. Misc. SNF, HEU, MEU, \& LEU, Misc. Unknown

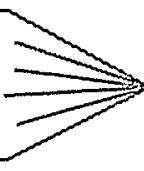

Not Evaluated

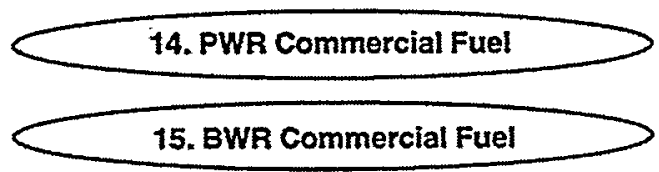

TRI-6342-5289-0

Figure 3-1. Thirty-four original groupings of DOE spent nuclear fuel and 15 categories of spent fuel as examined in 1997 PA. 


\subsubsection{Uranium Metal Fuel, Category 1}

Fuel Description. The uranium metal fuel, which is represented by N-Reactor fuel ( $98.4 \%$ of category), is currently stored at the Hanford site in Washington state. Category 1 also contains metallic fuel with aluminum cladding from Foreign Research Reactors (FRR) and EBR II (1.5\% of category). The fuel elements consist of two concentric tubes made of uranium metal (601 alloy) co-extruded with zirconium alloy (Zircaloy-2) cladding (Figure 3-2). Zircaloy- 2 is primarily zirconium with about $1 \%$ tin and trace quantities of other elements, such as iron, chromium, nickel, and carbon. There are two basic types of fuel elements-Mark IV and IA-differentiated by uranium enrichment: Mark IV fuel elements have a preirradiation enrichment of $0.947 \%$ in both tubes, and Mark IA fuel elements have a preirradiation enrichment of $1.25 \%$ in the outer tube and $0.947 \%$ in the inner tube. The average uranium weight is $22.7 \mathrm{~kg}$ for Mark IV fuels and $16.3 \mathrm{~kg}$ for Mark IA fuels. Both fuels have an outside diameter of $6.1 \mathrm{~cm}$; the lengths are 44,59,62, or $66 \mathrm{~cm}$ for Mark IV fuels, and 38, 50, or $53 \mathrm{~cm}$ for Mark IA fuels.

$\mathrm{N}$-Reactor fuel is categorized as either weapon grade or fuel grade, depending on plutonium composition. The ${ }^{240} \mathrm{Pu}$ isotopic content of weapon fuel is less than $6 \%$; the content of fuel grade is equal to or greater than $6 \%$.

Because of the degraded condition of the N-Reactor fuels, remediation currently includes drying for interim storage in a container labeled as a Multi-Canister Overpack (MCO) (see Section 3.2). No DHLW is packaged with DOE SNF in this category in the 1997 PA, although there is a small number of codisposal packages in the proposed plan (see Appendix A, Section 6.1).

Quantity Summary. Listed below are the amounts of reported MTHM and the percentage this category represents with regard to all DOE SNF and all disposed fuel (commercial, DOE SNF, and DHLW) modeled in the 1997 PA.

- Regulatory Mass 2137 MTHM

- Percentage of Total DOE SNF (2415 MTHM) $88.3 \%$

- Percentage of Total Disposed Fuel (75,336 MTHM) $2.8 \%$

Physical Characteristics. See Figure 3-2.

- Fuel Matrix Uranium metal

- Cladding 100\% failed Zircaloy

- Representative Fuel N-Reactor ( $98.4 \%$ of category)

- Other Fuels in Category Single Pass Reactor (aluminum clad)

- Enrichment (compare to CSNF, $1.2 \times 10^{-5}{ }^{235} \mathrm{U}$ and $9.4 \times 10^{5} \mathrm{Ci}^{239} \mathrm{Pu}$ ) Natural; $34.5 \mathrm{Ci}^{235} \mathrm{U}, 2.3 \times 10^{5} \mathrm{Ci}^{239} \mathrm{Pu}$

- Burnup (yr 2035) Low (per MTHM, $72 \mathrm{Ci}^{237} \mathrm{~Np} ; 2800 \mathrm{Ci}^{99} \mathrm{Tc}$ )

- Pyrophoricity

Finely divided uranium metal pyrophoric in atmosphere; limited $\mathrm{O}_{2}$ in repository would oxidize fuel too slowly to catch fire; even if fire occurred, amount of heat released would be insignificant in comparison to heat of radioisotope decay. 
Repository Disposal Considerations. Listed below are considerations specific to this fuel category with regard to potential fuel performance after disposal in an underground geologic repository.

- Because of its low burnup, this waste stream can dilute ${ }^{237} \mathrm{~Np}$ and ${ }^{99} \mathrm{Tc}$ in other waste streams when vitrified.

- Failed cladding; if protection equivalent to CSNF is required, an additional protective container inside disposal container can be used.

- Uranium metal alters rapidly (possibly 2 orders of magnitude faster than $\mathrm{UO}_{2}$ ); borosilicate glass treatment would cause alteration at a similar or slower rate than $\mathrm{UO}_{2}$, so treatment might reduce release rates of uranium.

- Reprocessing can remove uranium and plutonium; alternatively, can dissolve and dilute with ${ }^{238} \mathrm{U}$ or other waste streams before vitrification.

- Vitrification would oxidize uranium metal, which presents a difficulty with regard to current $10 \mathrm{CFR} 60$ regulations, but because heat of reaction is less than radioisotope decay, this will likely not pose a problem in the repository environment. A performance assessment can help to determine whether amount will compromise repository.

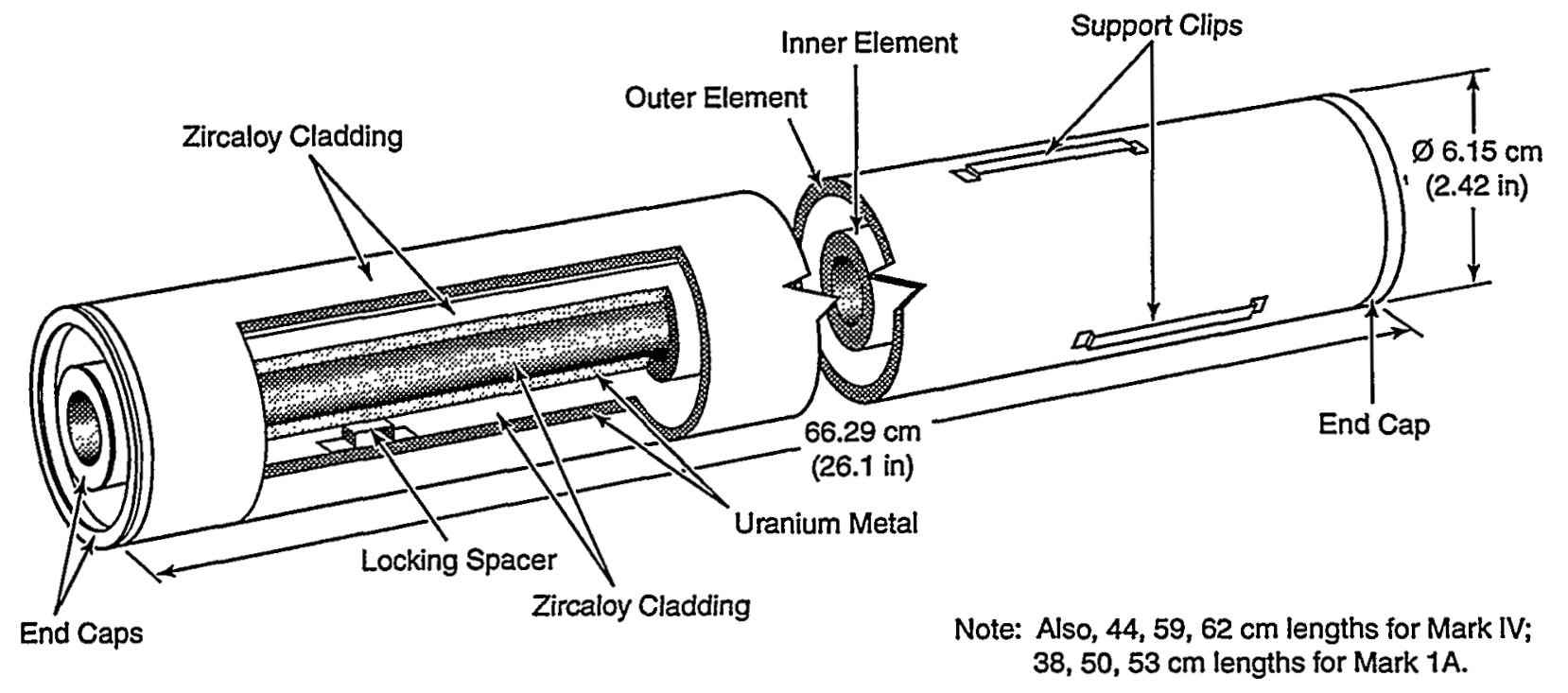

(Not to scale)

TRI-6342-5380-0

Figure 3-2. Category 1 spent fuel, represented by N-Reactor fuel assembly. 


\subsubsection{Uranium-Zirconium Alloy Fuel, Category 2}

Fuel Description. Category 2 is represented by the Chicago Pile 5 (CP-5) reactor fuel assembly. Other fuels in the category include those from the Heavy Water Component Test Reactor (HWCTR), which is a tank type, fully enriched (93\%) uranium, heavy water moderated and cooled reactor. The purpose of the reactor was to test fuel elements, materials, and components for heavy water reactors at power reactor conditions. The reactor had a nominal thermal power of $61 \mathrm{MW}$. The driver fuel elements were located around the outside portion of the reactor with up to 12 of the test fuel elements placed in the reactor center.

The HWCTR driver fuels are tube type design with $5.84 \mathrm{~cm}$ (2.3 in) outside diameter, $4.98 \mathrm{~cm}$ (1.96 in) inside diameter, and $287 \mathrm{~cm}$ (113 in) long. The fuel meat is $0.348 \mathrm{~cm}(0.137$ in) thick, consisting of $93 \%$ enriched uranium alloyed with $90.7 \mathrm{wt} \%$ zirconium. The test elements are made of natural or slightly enriched uranium metal or uranium oxide; thus, they are not included in this category.

Quantity Summary. Listed below are the amounts of reported MTHM and the percentage this category represents with regard to all DOE SNF and all disposed fuel (commercial, DOE SNF, and DHLW) modeled in the 1997 PA.

- Regulatory Mass

0.04 MTHM

- Percentage of Total DOE SNF (2415 MTHM) $0.0017 \%$

- Percentage of Total Disposed Fuel (75,336 MTHM) $0.00005 \%$

Physical Characteristics. Ilustration not available.

- Fuel Matrix Uranium-Zirconium Alloy

- Cladding Zircaloy

- Representative Fuel Chicago Pile 5 (CP-5) (28\% of category)

- Other Fuels in Category Heavy Water Component Test Reactor (HWCTR) fuel

- Enrichment (compare to CSNF, $1.2 \times 10^{-5}{ }^{235} \mathrm{U}$ and $9.4 \times 10^{5} \mathrm{Ci}^{239} \mathrm{Pu}$ ) Highly enriched: $0.064 \mathrm{Ci}^{235} \mathrm{U}$ and $8.6 \mathrm{Ci}^{239} \mathrm{Pu}$

- Burnup (yr 2035) Very low (per MTHM, $0.61 \mathrm{Ci}^{237} \mathrm{~Np} ; 7.9 \mathrm{Ci}^{99} \mathrm{Tc}$ )

- Pyrophoricity

Finely divided uranium metal pyrophoric in atmosphere; limited $\mathrm{O}_{2}$ in repository would oxidize fuel too slowly to catch fire; even if fire occurred, amount of heat released would be insignificant in comparison to heat of radioisotope decay. 


\subsection{Description of Modeled Spent Fuel Categories}

Repository Disposal Considerations. Listed below are considerations specific to this fuel category with regard to potential fuel performance after disposal in an underground geologic repository.

- Because of its low burnup, this waste stream can dilute ${ }^{237} \mathrm{~Np}$ and ${ }^{99} \mathrm{Tc}$ in other waste streams when vitrified.

- Zircaloy cladding provides excellent protection, but possibly $10 \%$ failed.

- Uranium metal alters rapidly (possibly 2 orders of magnitude faster than $\mathrm{UO}_{2}$ ); borosilicate glass treatment would cause alteration at a similar or slower rate than $\mathrm{UO}_{2}$, so treatment might reduce release rates of uranium.

- Reprocessing can remove uranium and plutonium; alternatively, can dissolve and dilute with ${ }^{238} U$ or other waste streams before vitrification.

- Vitrification would oxidize uranium metal. 


\subsubsection{Uranium-Molybdenum Alloy Fuel, Category 3}

Fuel Description. Category 3 spent fuel is represented by the Fermi driver fuel pins for the Enrico Fermi Reactor. Fermi was a sodium-cooled fast breeder reactor with intermediate sodium loops, sodium-to-water steam generators, and an associated steam-driven turbine generator. The lower reactor section of the reactor vessel has a $289.56 \mathrm{~cm}$ ( 9.5 feet) outside diameter and is $245.11 \mathrm{~cm}$ (96.5 in) in height. Core and blanket subassemblies are housed within the lower reactor vessel and are cooled by sodium that flows from the bottom of the lower reactor through the subassemblies and up into the upper reactor vessel. Each subassembly has a nozzle attached to the bottom end for insertion into the two 2-in. support plates spaced $14 \mathrm{in.}$ apart. The core and blanket of Fermi was made up of $6.72-\mathrm{cm}(2.646 \mathrm{in}$.) square driver core and blanket subassemblies positioned to approximate a right circular cylinder approximately 80 in. in diameter and 70 in. tall. Figure 3-3 shows the configuration of the core subassembly. The reactor core region was $30.5 \mathrm{in}$. in diameter and $31.2 \mathrm{in}$. tall and was completely enclosed by a thick breeder blanket designed to give a high breeding ratio and provide shielding.

The radial blanket fuel subassembly is made up of an inlet nozzle, a lower axial blanket, a fuel section, and an upper axial blanket. The radial blanket fuel subassemblies were made up of 25 cylindrical rods fabricated from depleted U-Mo alloy, encased in stainless steel tubes and bonded with sodium. The radial blanket subassemblies are currently stored dry in ICPP-749. The radial blanket subassembly rods contain depleted uranium and sodium and thus will be treated prior to final disposition. These are not part of the Category 3 inventory.

The Fermi driver fuel subassembly was designed with three active regions-a lower axial blanket, a fuel section, and an upper axial blanket. The lower and upper axial blanket subassemblies have been cropped off from the central core fuel section and are currently stored with the radial blanket subassemblies in ICPP-749 and will be treated prior to final disposal. A type 347 stainless steel square tube measuring 2.646 in. square with a 0.096 in. wall thickness was used as the outside structure to hold the three regions together. The fuel section contained 144 fuel pins, made up of $25.69 \%$ enriched uranium-molybdenum alloy. Four stainless steel structural support pins were inserted into the comer positions of the $12 \times 12$ array to add structural support to the fuel section and the fuel subassembly. The fuel pins were closely packed into the $2.646 \mathrm{in}$. square tube. The fuel pins were maintained on a square pitch of $0.200 \mathrm{in}$. in a cartridge made of stainless steel wires and plates.

Quantity Summary. Listed below are the amounts of reported MTHM and the percentage this category represents with regard to all DOE SNF and all disposed fuel (commercial, DOE SNF, and DHLW) modeled in the 1997 PA.

- Regulatory Mass 3.93 MTHM

- Percentage of Total DOE SNF (2415 MTHM) $0.2 \%$

- Percentage of Total Disposed Fuel (75,336 MTHM) $0.005 \%$

Physical Characteristics. See Figure 3-3.

- Fuel Matrix Uranium-Molybdenum Alloy

- Cladding Zircaloy

- Representative Fuel Fermi Reactor (100\% of category)

- Other Fuels in Category None

- Enrichment (compare to CSNF, $1.2 \times 10^{-5}{ }^{235} \mathrm{U}$ and $9.4 \times 10^{5} \mathrm{Ci}^{239} \mathrm{Pu}$ ) Highly enriched: $230 \mathrm{Ci}^{235} \mathrm{U}$ and $1.4 \times 10^{4} \mathrm{Ci}^{239} \mathrm{Pu}$

- Burnup (yr 2035)

$$
\text { High (per MTHM, } 2.4 \mathrm{Ci}^{237} \mathrm{~Np} ; 325 \mathrm{Ci}{ }^{99} \mathrm{Tc} \text { ) }
$$


- Pyrophoricity

Finely divided uranium metal pyrophoric in atmosphere; limited $\mathrm{O}_{2}$ in repository would oxidize fuel too slowly to catch fire; even if fire occurred, amount of heat released would be insignificant in comparison to heat of radioisotope decay

Repository Disposal Considerations. Listed below are considerations specific to this fuel category with regard to potential fuel performance after disposal in an underground geologic repository.

- Because of its high burnup, ${ }^{237} \mathrm{~Np}$ and ${ }^{99} \mathrm{Tc}$ in this waste stream could be diluted by other waste streams when vitrified.

- Zircaloy cladding provides excellent protection, but possibly $10 \%$ failed.

- Uranium-molybdenum metal alters rapidly (possibly 3 orders of magnitude faster than $\mathrm{UO}_{2}$ ); borosilicate glass treatment would cause alteration at a similar or slower rate than $\mathrm{UO}_{2}$, so treatment might reduce release rates of uranium.

- Reprocessing can remove uranium and plutonium; alternatively, can dissolve and dilute with ${ }^{238} \mathrm{U}$ or other waste streams before vitrification.

- Vitrification would oxidize uranium metal.

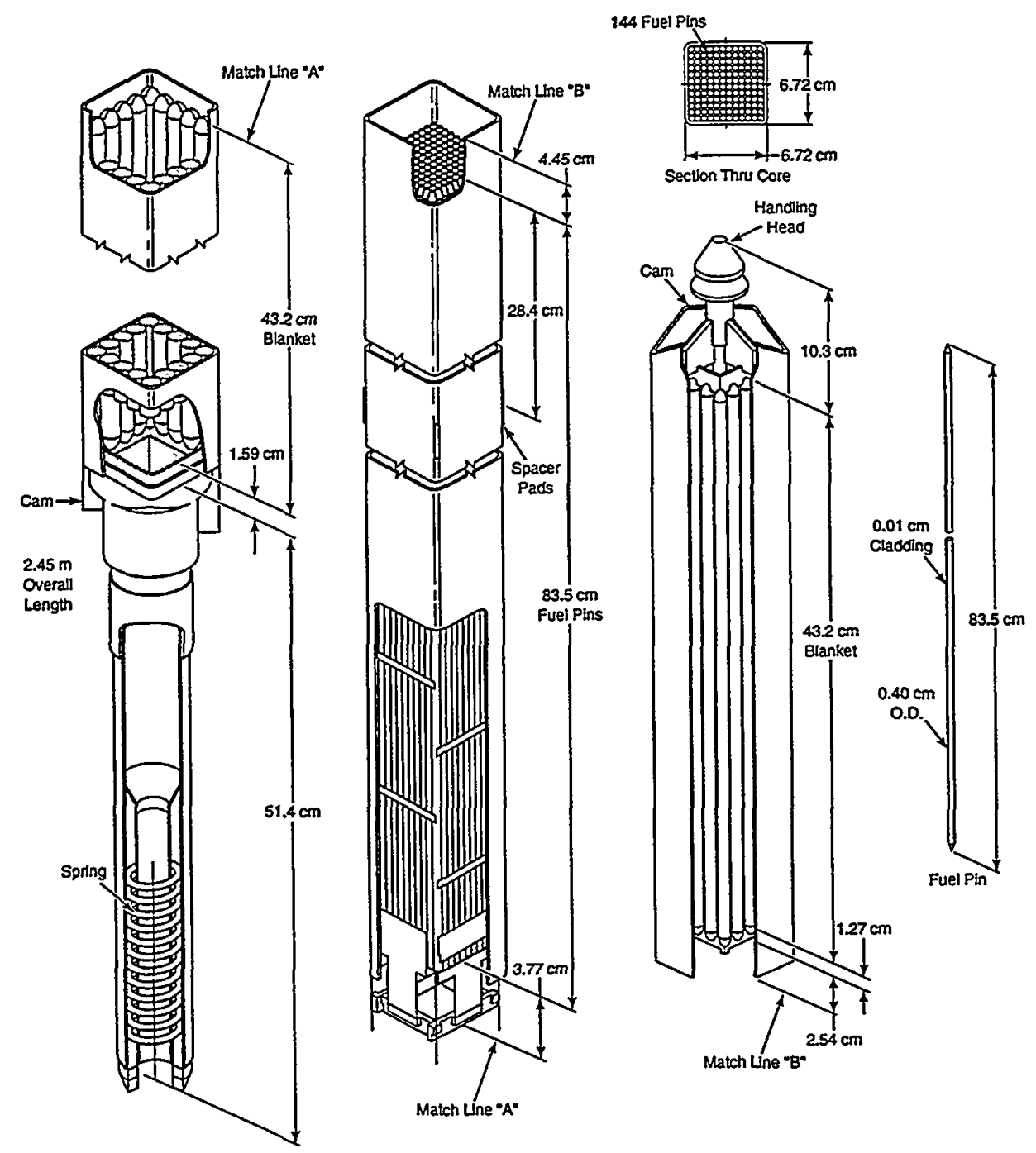

TF1-6342-5351-0

Figure 3-3. Category 3 spent fuel, represented by fuel pins only (center portion of figure) for Enrico Fermi Reactor. 


\subsubsection{Uranium Oxide Fuel-Intact Clad, Category 4}

Fuel Description. Category 4 is represented by Shippingport PWR fuel, which was a developmental fuel in the DOE PWR research program. It was the second of three cores irradiated in the Shippingport Atomic Power Station at Shippingport, Pennsylvania. The high power density, high total energy core required the development of a fuel element of high loading that would withstand long irradiation exposure at high heat fluxes. The fuel cluster consists of two oxide fuel plate subassemblies welded together to form a square structure and two Zircaloy-4 cluster extensions welded to the ends of the subassemblies (Figure 3-4). Category 4 also includes the radioisotope inventory for fuel from commercial (LEU), the Saxton reactor in Pennsylvania (MEU), the Mobile Low Power Reactor (ML-1) (HEU), the Power Burst Facility (PBF) (MEU), and the Fast Flux Test Reactor-Test Fuel Assembly (FFTR-TFA) (LEU).

Quantity Summary. Listed below are the amounts of reported MTHM and the percentage this category represents with regard to all DOE SNF and all disposed fuel (commercial, DOE SNF, and DHLW) modeled in the 1997 PA.

- Regulatory Mass 98 MTHM

- Percentage of Total DOE SNF (2415 MTHM) $4.1 \%$

- Percentage of Total Disposed Fuel (75,336 MTHM) $0.13 \%$

Physical Characteristics. See Figure 3-4.

- Fuel Matrix Uranium-Oxide

- Cladding Zircaloy

- Representative Fuel Shippingport PWR (18\% of category)

- Other Fuels in Category Saxton, Commercial, Mobile Low Power Reactor (ML-1), Power Burst Facility (PBF), Fast Flux Test Reactor Test Fuel Assembly (FFTF-TFA)

- Enrichment (compare to CSNF, $1.2 \times 10^{-5235} \mathrm{U}$ and $9.4 \times 10^{5} \mathrm{Ci}^{239} \mathrm{Pu}$ ) Similar to CSNF

- Burnup (yr 2035) Similar to commercial (per MTHM, $35.2 \mathrm{Ci}^{237} \mathrm{~Np} ; 1330 \mathrm{Ci}^{99} \mathrm{Tc}$ )

- Pyrophoricity Not pyrophoric

Repository Disposal Considerations. Listed below are considerations specific to this fuel category with regard to potential fuel performance after disposal in an underground geologic repository.

- Similar to commercial spent nuclear fuel. 


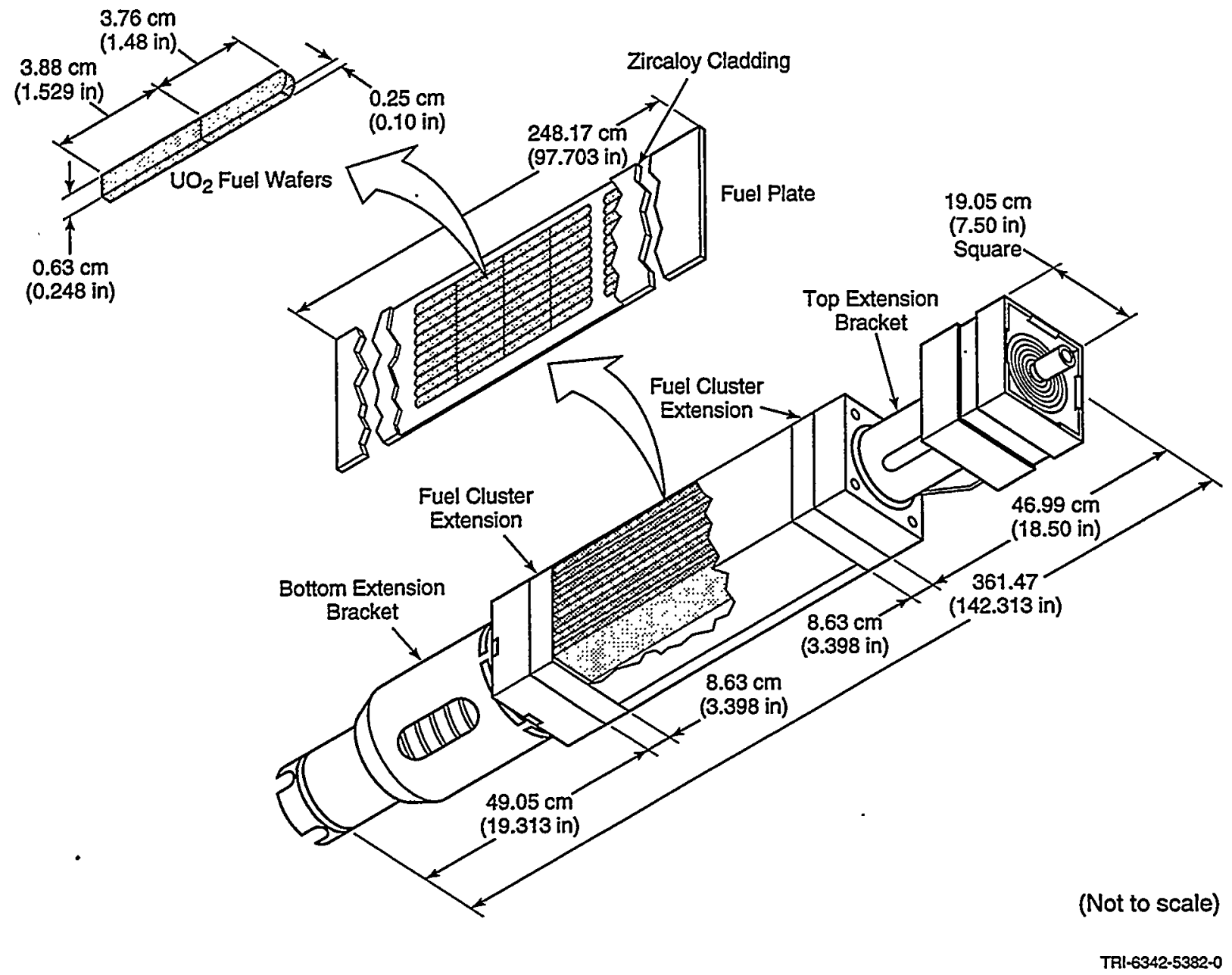

Figure 3-4. Category 4 spent fuel, represented by Shippingport $P W R$ fuel assembly. 


\subsubsection{Uranium Oxide Fuel-Failed Clad, Category 5}

Fuel Description. The fuels in this category represent materials that are already damaged, disrupted, or fragile in terms of immediate fissile and fission product movement upon package breach. The representative fuel type is the TMI-2 fuel type from Three Mile Island, which was a typical commercial pressurized water nuclear reactor fuel until it melted in a reactor accident. It now consists of material with sizes ranging from fine-sized pieces to nearly intact assemblies, some of which have been melted. The fuel debris was placed into three types of stainless steel handling containers: filter canisters that contain the fines, knockout canisters that contain material of gravel consistency, and fuel canisters that contain large pieces of melted or unaffected assemblies (Figure 3-5). Category 5 also includes the radioisotope inventory from type 1A fuel from the Stationary Medium Power Plant (SM-1A) (MEU), and fuel from the High Flux Isotope Reactor (HFIR), Foreign Research Reactor (FRR), and Materials Testing Reactor (MTR).

Quantity Summary. Listed below are the amounts of reported MTHM and the percentage this category represents with regard to all DOE SNF and all disposed fuel (commercial, DOE SNF, and DHLW) modeled in the 1997 PA.

- Regulatory Mass 87.0 MTHM

- Percentage of Total DOE SNF (2415 MTHM) $3.6 \%$

- Percentage of Total Disposed Fuel (75,336 MTHM) $0.12 \%$

Physical Characteristics. See Figure 3-5.

- Fuel Matrix Uranium Oxide

- Cladding $100 \%$ Failed Zircaloy

- Representative Fuel Three Mile Island (TMI-2) (93.8\% of category)

- Other Fuels in Category Stationary Medium Power Plant (SM-1A), High Flux Isotope Reactor (HFIR), Foreign Research Reactors (FRR), Materials Testing Reactor (MTR)

- Enrichment (compare to CSNF, $1.2 \times 10^{-5}{ }^{235} \mathrm{U}$ and $9.4 \times 10^{5} \mathrm{Ci}^{239} \mathrm{Pu}$ ) Similar to CSNF

- Burnup (yr 2035) Slightly less than CSNF (per MTHM, $16.8 \mathrm{Ci}^{237} \mathrm{~Np} ; 893 \mathrm{Ci}^{99} \mathrm{Tc}$ )

- Pyrophoricity Not pyrophoric

Repository Disposal Considerations. Listed below are considerations specific to this fuel category with regard to potential fuel performance after disposal in an underground geologic repository.

- Failed cladding; if protection equivalent to CSNF is required, an additional protective container inside disposal container can be used. 


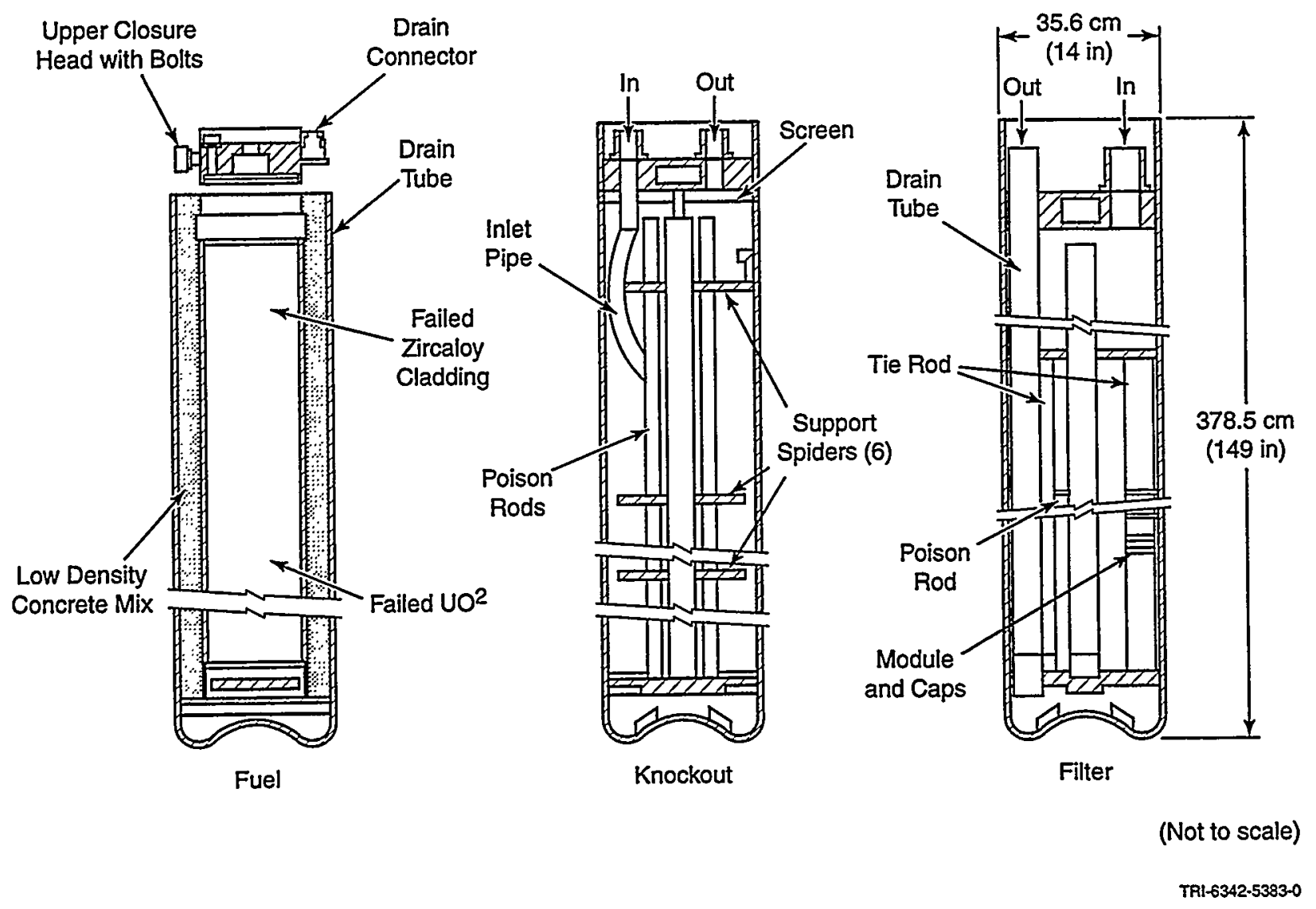

Figure 3-5. Category 5 spent fuel, represented by Three Mile Island failed fuel assemblies. 


\subsubsection{Uranium Aluminum Alloy Fuel, Category 6}

Fuel Description. The ATR fuel, which represents Category 6, originated in the Advanced Test Reactor (ATR), an experimental test reactor that provided for the insertion of numerous experiments into the core. Both the fuel and the core were designed to produce a more heterogeneous flux than a PWR. The ATR has four mechanical systems for reactivity control instead of the usual single system.

The ATR fuel is contained in plates of an aluminum-uranium matrix, which is cladded with pure aluminum (Figure 3-6). The fuel assemblies contain no control rods or other control components found in most PWR fuel assemblies. Each element forms a 45-degree sector of a right circular cylinder (wedge-shaped) and consists of 19 fuel plates with coolant channels between each plate. The fuel plates are $1.26 \mathrm{~m}$ (49.5 in.) long, with an active fuel length of $11.22 \mathrm{~m}$ (48 in.) and are loaded with a highly enriched uranium matrix consisting of uranium-aluminide (UAl ${ }_{3}$ ) and clad in ASTM B 209 aluminum alloy 6061-TG. The total fuel content of an ATR fuel assembly is nominally $1075 \mathrm{~g}$ (maximum $1100 \mathrm{~g}$ ) of uranium enriched to $93 \%{ }^{235} \mathrm{U}$. Certain plates contain boron carbide $\left(\mathrm{B}_{4} \mathrm{C}\right.$ ) mixed with the fuel as a burnable poison to minimize radial power peaking and extend the life cycle of the fuel element. Category 6 also includes the radioisotope inventory for fuel from the Materials Testing Reactor (MTR) and the Foreign Research Reactor (FRR) (MEU).

Quantity Summary. Listed below are the amounts of reported MTHM and the percentage this category represents with regard to all DOE SNF and all disposed fuel (commercial, DOE SNF, and DHLW) modeled in the 1997 PA.

- Reguiatory Mass

8.97 MTHM

- Percentage of Total DOE SNF (2415 MTHM)

$0.37 \%$

- Percentage of Total Disposed Fuel (75,336 MTHM) $0.01 \%$

Physical Characteristics. See Figure 3-6.

- Fuel Matrix

Uranium Aluminum Alloy

- Cladding

Aluminum

- Representative Fuel Advanced Test Reactor (ATR) (28\% of category)

- Other Fuels in Category Materials Testing Reactor (MTR), Foreign Research Reactors (FRR)

- Enrichment (compare to CSNF, $1.2 \times 10^{-5235} \mathrm{U}$ and $9.4 \times 10^{5} \mathrm{Ci}^{239} \mathrm{Pu}$ ) Highly enriched (some fuels initially $>90 \%$ )

- Burnup (yr 2035) Varies, but most fuels have high burnup (per MTHM, $23 \mathrm{Ci}^{237} \mathrm{~Np} ; 1210 \mathrm{Ci}^{99} \mathrm{Tc}$ )

- Pyrophoricity

Finely divided uranium metal pyrophoric in atmosphere; limited $\mathrm{O}_{2}$ in repository would oxidize fuel too slowly to catch fire; even if fire occurred, amount of heat released would be insignificant in comparison to heat of radioisotope decay. 
Repository Disposal Considerations. Listed below are considerations specific to this fuel category with regard to potential fuel performance after disposal in an underground geologic repository.

- Because of its high burnup, ${ }^{237} \mathrm{~Np}$ and ${ }^{99} \mathrm{Tc}$ in this waste stream could be diluted by other waste streams when vitrified.

- Aluminum cladding offers little long-term protective value; if protection equivalent to CSNF is required, an additional protective container inside disposal container can be used.

- Current codisposal option places only $14.4 \mathrm{~kg}^{235} \mathrm{U}$ in a waste package to avoid criticality; also, since total of 9 MTHM is only $0.01 \%$ of repository total and only $68 \%$ fissile, the added fission products are within the error band of calculated fission products in CSNF, even if all went critical.

- Reprocessing can remove uranium; alternatively, can dissolve and dilute with ${ }^{238} \mathrm{U}$ or other waste streams before vitrification.

- Vitrification would oxidize uranium metal.
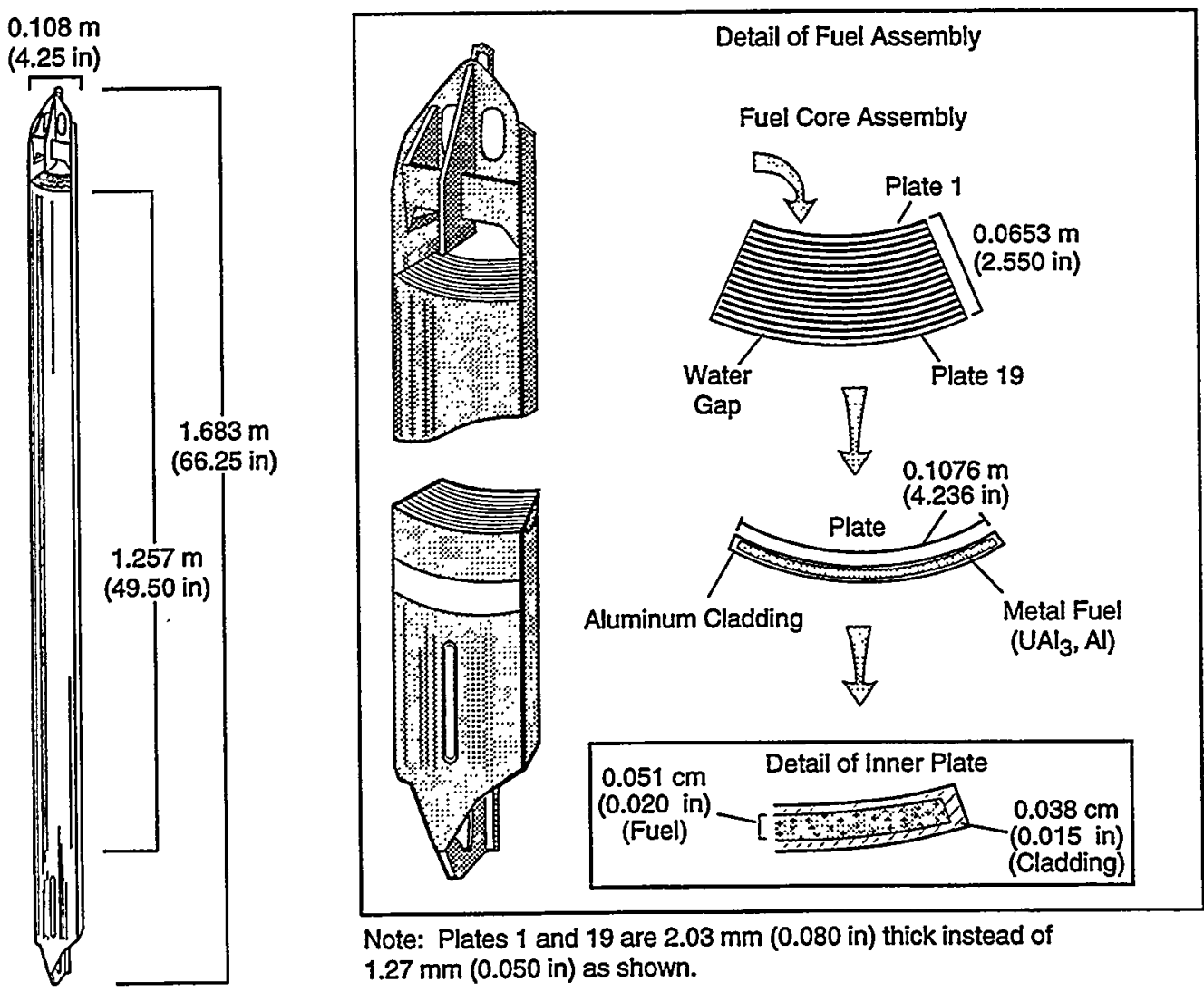

Note: Plates 1 and 19 are $2.03 \mathrm{~mm}(0.080$ in) thick instead of $1.27 \mathrm{~mm}(0.050 \mathrm{in})$ as shown.

(Not to scale)

TAI-6342-5384-0

Figure 3-6. Category 6 spent fuel, represented by Advanced Test Reactor (ATR) fuel assembly. 


\subsubsection{Uranium Silicide Fuel, Category 7}

Fuel Description. Category 7 is represented by fuel from the Materials Testing Reactor (MTR) and Foreign Research Reactor (FRR) (HEU, MEU). Most foreign research reactors will continue to operate during the next several years. Foreign reactors use a number of different fuel designs. These designs can be placed into five groups: (1) plate-type design, (2) concentric tube-type design, (3) pin-type design, (4) special-type design, and (5) rod-type design.

The plate type design is described here because it is used in the majority of the FRR (Figure 3-7). The thermal power of these reactors ranges from $1 \mathrm{MW}$ to $50 \mathrm{MW}$. Each fuel assembly contains between 6 to 23 plates and an initial ${ }^{235} \mathrm{U}$ content between 37 and 420 grams. The fuel matrix consists of $U$-Si dispersed in aluminum.

Quantity Summary. Listed below are the amounts of reported MTHM and the percentage this category represents with regard to all DOE SNF and all disposed fuel (commercial, DOE SNF, and DHLW) modeled in the 1997 PA.

- Regulatory Mass

11.4 MTHM

- Percentage of Total DOE SNF (2415 MTHM)

$0.47 \%$

- Percentage of Total Disposed Fuel (75,336 MTHM)

$0.02 \%$

Physical Characteristics. See Figure 3-7.

- Fuel Matrix

Uranium silicide

- Cladding

Aluminum

- Representative Fuel Materials Testing Reactor (MTR) (85.2\% of category)

- Other Fuels in Category Foreign Research Reactor (FRR)

- Enrichment (compare to CSNF, $1.2 \times 10^{-5}{ }^{235} \mathrm{U}$ and $9.4 \times 10^{5} \mathrm{Ci}^{239} \mathrm{Pu}$ ) Highly enriched $\left(4.61 \mathrm{Ci}^{235} \mathrm{U}\right.$ and $\left.2.5 \times 10^{3} \mathrm{Ci}^{239} \mathrm{Pu}\right)$

- Burnup (yr 2035) Moderately low (per MTHM, $0.92 \mathrm{Ci}^{237} \mathrm{~Np} ; 214 \mathrm{Ci}^{99} \mathrm{Tc}$ )

- Pyrophoricity

Not assumed pyrophoric in atmosphere

Repository Disposal Considerations. Listed below are considerations specific to this fuel category with regard to potential fuel performance after disposal in an underground geologic repository.

- Aluminum cladding offers little long-term protective value; if protection equivalent to CSNF is required, an additional protective container inside disposal container can be used.

- Uranium silicide matrix modeled at 0.1 of uranium metal; borosilicate glass treatment would cause alteration at a similar or slower rate than $\mathrm{UO}_{2}$, so treatment might reduce release rates of uranium.

- Reprocessing can remove uranium and plutonium; alternatively, can dissolve and dilute with ${ }^{238} \mathrm{U}$ or other waste streams before vitrification. 


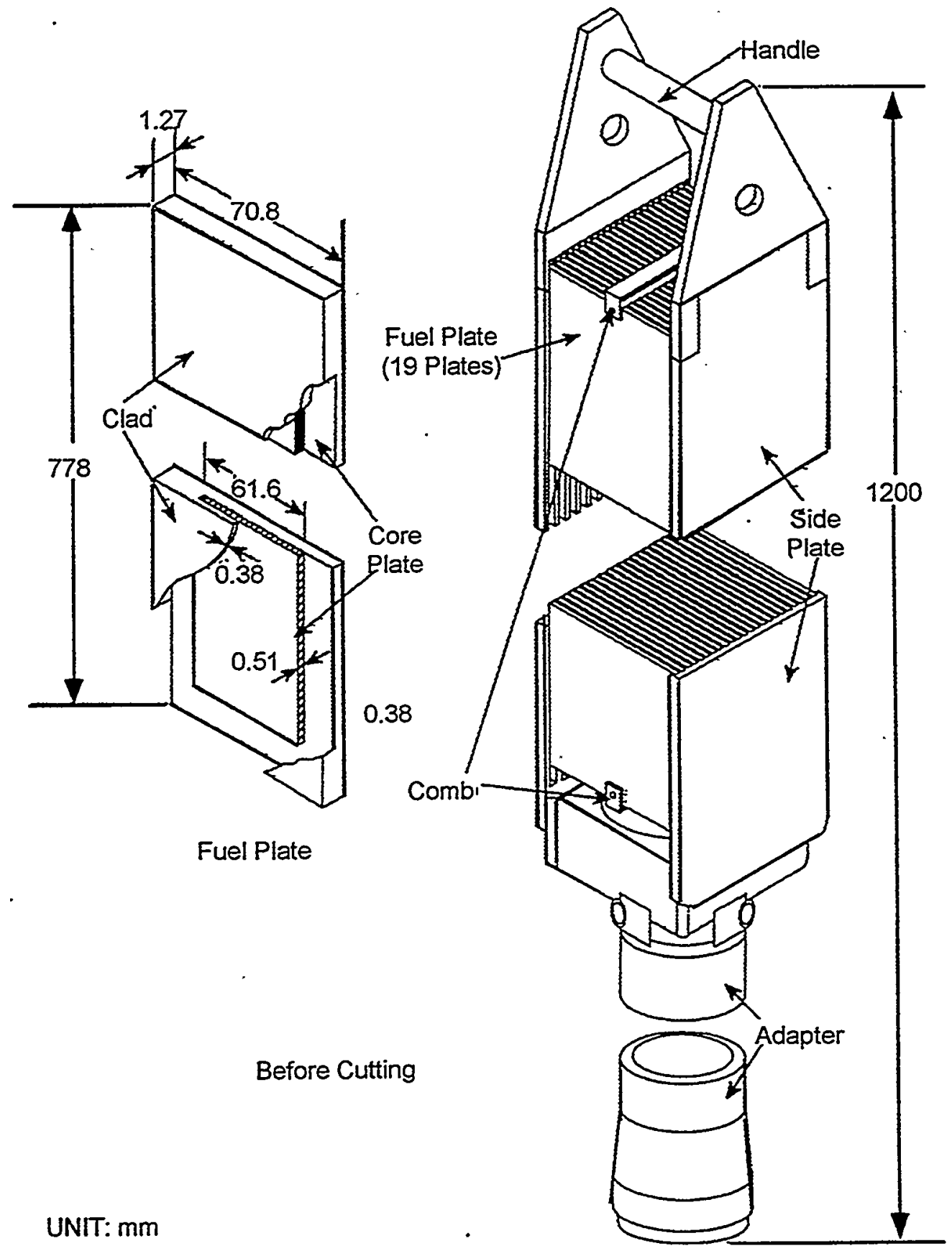

Figure 3-7. Category 7 spent fuel, represented by fuel from the Materials Testing Reactor (MTR) and Foreign Research Reactor (FRR). Illustration shows typical boxed-typelflat plate FRR fuel element (from NSNFP, Appendix A). 


\subsubsection{Uranium/Thorium Carbide Fuel-Intact Clad, Category 8}

Fuel Description. Graphite spent fuel from Fort St. Vrain, which represents Category 8, contains a homogeneous mixture of two types of particles, fissile and fertile. As manufactured and prior to irradiation, fissile particles contain thorium and $93.5 \%$ enriched uranium; fertile particles contain only thorium. The fuel kernels are coated with three fission-product retaining layers by means of a fluidized-bed vapor-phase deposition process. The inner and outer layers are of isotropic carbon, and the middle layer is of silicon carbide (SiC) (hence the name, TRISO-coated) (considered as cladding). A fourth layer of porous carbon, called the "buffer," is next to the kernel of fissile or fertile material and provides a volume for accumulation of fission-product gases without excessive pressure buildup. The $\mathrm{SiC}$ layer is highly resistant to both oxidation and moisture, even at extremely high temperatures. The fissile and fertile particles are blended and then molded into $1.27-\mathrm{cm}$ diameter $\times 5.08-\mathrm{cm}$ long fuel rods (compacts), that are in turn inserted in large hexagonal blocks of graphite (Figure 3-8).

Quantity Summary. Listed below are the amounts of reported MTHM and the percentage this category represents with regard to all DOE SNF and all disposed fuel (commercial, DOE SNF, and DHLW) modeled in the 1997 PA.

- Regulatory Mass

24.7 MTHM

- Percentage of Total DOE SNF (2415 MTHM) $1.0 \%$

- Percentage of Total Disposed Fuel (75,336 MTHM) $0.03 \%$

Physical Characteristics. See Figure 3-8.

- Fuel Matrix Uranium/Thorium Carbide

- Cladding Graphite and TRISO

- Representative Fuel Fort St. Vrain (94.3\% of category)

- Other Fuels in Category Peach Bottom, Core 2

- Enrichment (compare to CSNF, $1.2 \times 10^{-5}{ }^{235} \mathrm{U}$ and $9.4 \times 10^{5} \mathrm{Ci}^{239} \mathrm{Pu}$ ) Highly enriched $\left(1.08 \mathrm{Ci}^{235} \mathrm{U}\right.$ and $\left.113 \mathrm{Ci}^{239} \mathrm{Pu}\right)$

- Burnup (yr 2035) Moderately low (per MTHM, 8.16 $\mathrm{Ci}^{237} \mathrm{~Np} ; 372 \mathrm{Ci}^{99} \mathrm{Tc}$ )

- Pyrophoricity Uranium thorium would rapidly hydrate when exposed to water, but amount of heat released is insignificant in comparison to heat of radioisotope decay

Repository Disposal Considerations. Listed below are considerations specific to this fuel category with regard to potential fuel performance after disposal in an underground geologic repository.

- Graphite and TRISO (silicon carbide) coating offers better protective value than the Zircaloy cladding in CSNF; only $1 \%$ failed.

- Uranium thorium carbide matrix alters rapidly (possibly 10 orders of magnitude faster than $\mathrm{UO}_{2}$ ); borosilicate glass treatment would cause alteration at a similar or slower rate than $\mathrm{UO}_{2}$, so treatment might reduce release rates of uranium. However, it would destroy excellent protection from TRISO (silicon carbide) coating.

- Reprocessing can remove uranium and plutonium; alternatively, can dissolve and dilute with ${ }^{238} \mathrm{U}$ or other waste streams before vitrification.

- Vitrification would hydrate uranium thorium but would lose excellent protection of silicon carbide coating. 

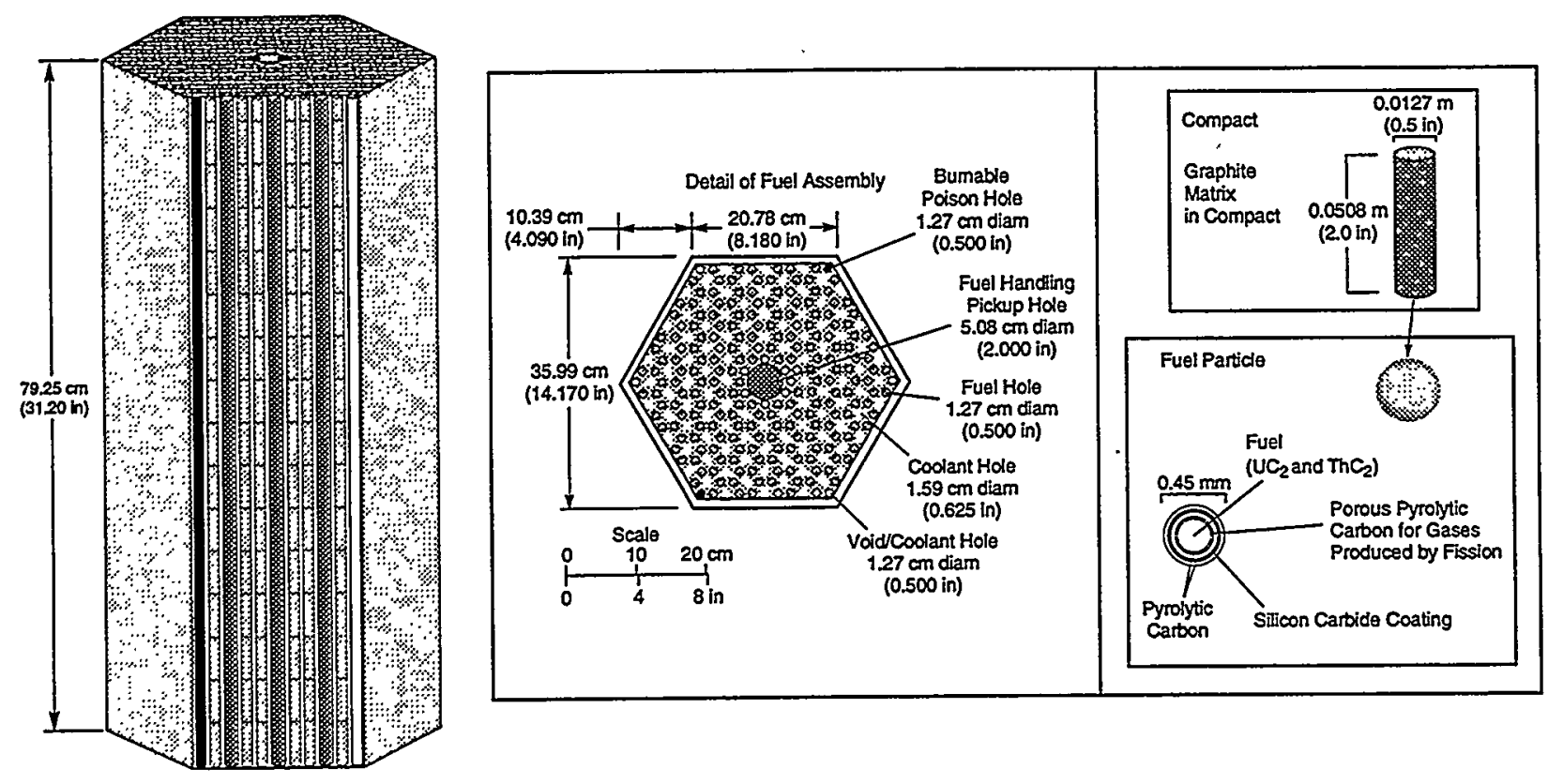

TRI-6342-5385-0

Figure 3-8. Category 8 spent fuel, represented by Fort St. Vrain fuel assembly. 


\subsubsection{Uranium/Thorium Carbide Fuel-Failed Clad, Category 9}

Fuel Description. The Peach Bottom Cores I and II spent fuel, which represents Category 9, is similar in characteristic to the Fort St. Vrain graphite fuel (Section 3.1.8) except that the Peach Bottom Core I particles do not contain the protective $\mathrm{SiC}$ layer. The Peach Bottom compacts are $6.86-\mathrm{cm}$ outside diameter, $3.25-\mathrm{cm}$ inside diameter $X$ 7.57-cm-long hollow cylinders (Figure 3-9).

Quantity Summary. Listed below are the amounts of reported MTHM and the percentage this category represents with regard to all DOE SNF and all disposed fuel (commercial, DOE SNF, and DHLW) modeled in the 1997 PA.

- Regulatory Mass 1.66 MTHM

- Percentage of Total DOE SNF (2415 MTHM) $0.07 \%$

- Percentage of Total Disposed Fuel (75,336 MTHM) . $0.002 \%$

Physical Characteristics. See Figure 3-9.

- Fuel Matrix Uranium/Thorium Carbide

- Cladding 100\% Failed Graphite

- Representative Fuel Peach Bottom, Core 1 (100\% of category)

- Other Fuels in Category None

- Enrichment (compare to CSNF, $1.2 \times 10^{-5}{ }^{235} \mathrm{U}$ and $9.4 \times 10^{5} \mathrm{Ci}{ }^{239} \mathrm{Pu}$ ) Highly enriched $\left(0.4 \mathrm{Ci}^{235} \mathrm{U}\right.$ and $\left.24.6 \mathrm{Ci}^{239} \mathrm{Pu}\right)$

- Burnup (yr 2035) Moderate (per MTHM, $0.44 \mathrm{Ci}^{237} \mathrm{~Np} ; 24.3 \mathrm{Ci}^{99} \mathrm{Tc}$ )

- Pyrophoricity Uranium-thorium would rapidly hydrate when exposed to water, but amount of heat released is insignificant in comparison to heat of radioisotope decay

Repository Disposal Considerations. Listed below are considerations specific to this fuel category with regard to potential fuel performance after disposal in an underground geologic repository.

- Uranium thorium carbide alters rapidly (possibly 10 orders of magnitude faster than $\mathrm{UO}_{2}$ ); borosilicate glass treatment would cause alteration at a similar or slower rate than $\mathrm{UO}_{2}$, so treatment might reduce release rates of uranium.

- Reprocessing can remove uranium and plutonium; alternatively, can dissolve and dilute with ${ }^{238} \mathrm{U}$ or other waste streams before vitrification.

- Vitrification would hydrate uranium. 

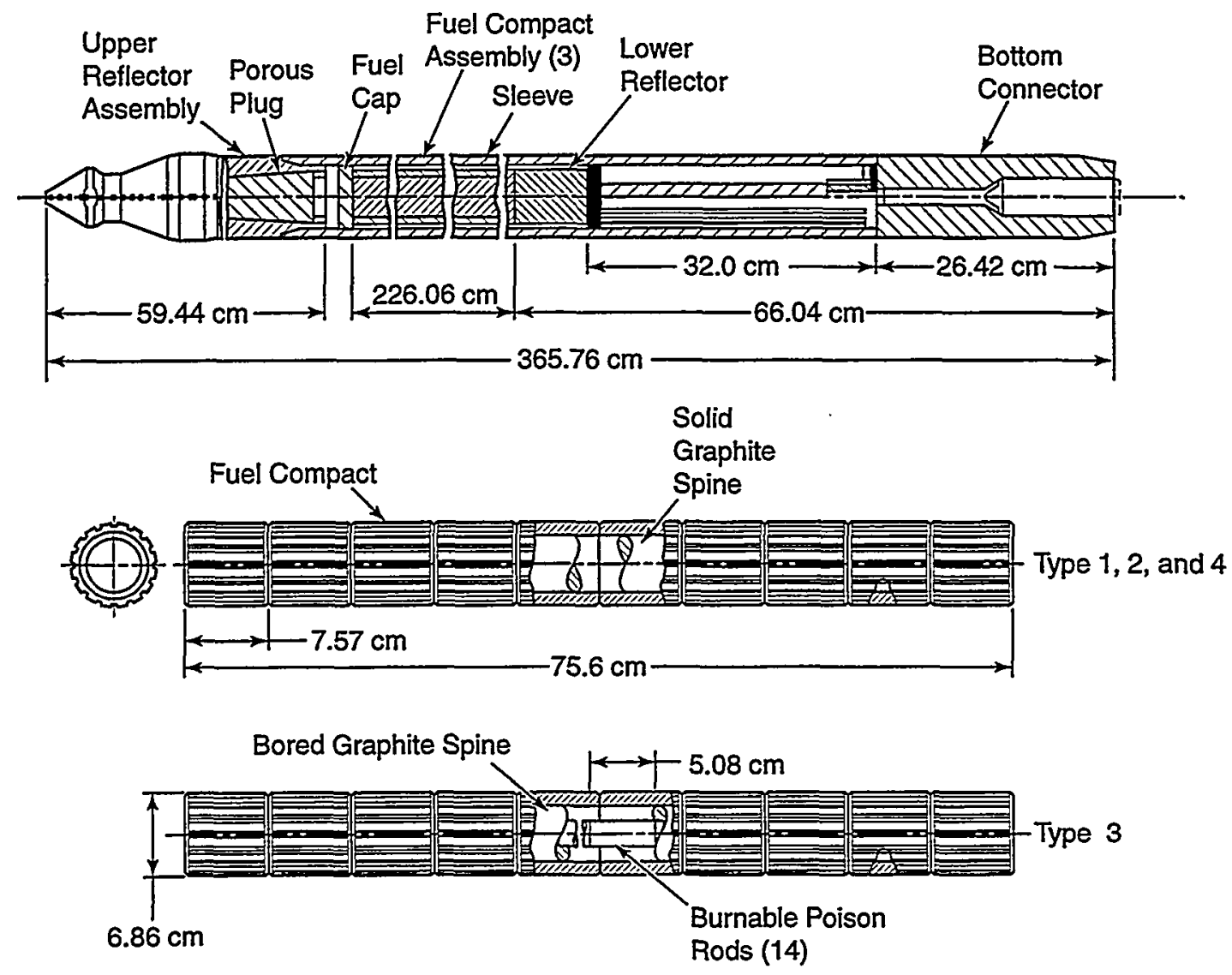

(Not to scale)

TRl-6342-5386-0

Figure 3-9. Category 9 spent fuel, represented by Peach Bottom fuel assembly. 


\subsubsection{Uranium/Plutonium Carbide Fuel, Category 10}

Fuel Description. Category 10 is represented by fuel from the Sodium Reactor Experiment (SRE). Category 10 also includes the radioisotope inventory for fuel from the Fast Flux Test Facility (FFTF) carbide (MEU fissile gram equivalent [FGE]). FFTF was designed to provide testing capability for a wide range of development needs of the United States advanced reactor program. The mission of the FFTF included irradiation and evaluation of different types of fuel assemblies and different materials for fuel assembly construction. The purposes of the test fuel assemblies vary; however, in general, the assemblies support the fuel or material requirements for large scale breeder reactors.

As an example, the FFTF-ACN-1 fuel in this category was tested to develop information on helium-and sodiumbonded mixed-carbide fuel pins with full length fuel columns at prototypic fluence and exposure conditions. Additionally, it tests the relative effects of $20 \%$ cold-worked 316 SS and $25 \%$ cold-worked D9 cladding on the carbide fuel pins. The assembly contains 18 sodium-bonded and 19 helium-bonded carbide fuel pins, enclosed in a 316 SS inner duct similar to the SRF-3. The outer region contains 90 standard driver fuel pins and is enclosed by a D9 duct. The test fuel assemblies configuration is similar to the FFTF driver fuels shown for Category 11 . More examples of Category 10 assemblies are found in Appendix A.

Quantity Summary. Listed below are the amounts of reported MTHM and the percentage this category represents with regard to all DOE SNF and all disposed fuel (commercial, DOE SNF, and DHLW) modeled in the 1997 PA.

- Regulatory Mass

0.163 MTHM

- Percentage of Total DOE SNF (2415 MTHM) $0.007 \%$

- Percentage of Total Disposed Fuel (75,336 MTHM) $0.0002 \%$

Physical Characteristics. Mllustration not available.

- Fuel Matrix Uranium/Plutonium Carbide

- Cladding Stainless steel

- Representative Fuel Sodium Reactor Experiment (SRE) (27\% of category)

- Other Fuels in Category Fast Flux Test Reactor (FFTR)

- Enrichment (compare to CSNF, $1.2 \times 10^{-5}{ }^{235} \mathrm{U}$ and $9.4 \times 10^{5} \mathrm{Ci}^{239} \mathrm{Pu}$ ) Moderate $\left(8.4 \times 10^{-5} \mathrm{Ci}^{235} \mathrm{U}\right.$ and $\left.2.8 \times 10^{3} \mathrm{Ci}^{239} \mathrm{Pu}\right)$

- Burnup (yr 2035) Low (per MTHM, $0.027 \mathrm{Ci}^{237} \mathrm{~Np} ; 0 \mathrm{Ci}^{99} \mathrm{Tc}$ )

- Pyrophoricity

Uranium plutonium carbide would rapidly hydrate when exposed to water, but amount of heat released is insignificant in comparison to heat of radioisotope decay. 
Repository Disposal Considerations. Listed below are considerations specific to this fuel category with regard to potential fuel performance after disposal in an underground geologic repository.

- Stainless steel cladding offers little long-term protective value; if protection equivalent to CSNF is required, an additional protective container inside disposal container can be used.

- Uranium-plutonium carbide matrix alters rapidly (possibly 10 orders of magnitude faster than $\mathrm{UO}_{2}$ ); borosilicate glass treatment would cause alteration at a similar or slower rate than $\mathrm{UO}_{2}$, so treatment might reduce release rates of uranium.

- Reprocessing can remove uranium and plutonium; alternatively, can dissolve and dilute with ${ }^{238} \mathrm{U}$ or other waste streams before vitrification.

- Vitrification would hydrate uranium-plutonium carbide. 


\subsubsection{Mixed Oxide Fuel, Category 11}

Fuel Description. Category 11 is represented by the Fast Flux Test Facility (FFTF) standard driver fuel assembly. Category 11 also includes the radioisotope inventory for GE Test fuel from the SE experimental fast oxide reactor (HEU fissile gram equivalent [FGE]), and advanced oxide (ACO) fuel assemblies from the FFTR-Test Fuel Assembly (TFA) (LEU and MEU FGE). Additional information about Category 11 was not available as of September 1997. Figure 3-10 illustrates the representative fuel for Category 11.

Quantity Summary. Listed below are the amounts of reported MTHM and the percentage this category represents with regard to all DOE SNF and all disposed fuel (commercial, DOE SNF, and DHLW) modeled in the 1997 PA.

- Regulatory Mass

12.0 MTHM

- Percentage of Total DOE SNF (2415 MTHM)

$0.5 \%$

- Percentage of Total Disposed Fuel (75,336 MTHM)

$0.016 \%$

Physical Characteristics. See Figure 3-10.

- Fuel Matrix Mixed Oxide

- Cladding Stainless steel

- Representative Fuel Fast Flux Test Facility (FFTF) (75\% of category)

- Other Fuels in Category GE Test Fuel, advanced oxide (ACO) fuel

- Enrichment (compare to CSNF, $1.2 \times 10^{-5235} \mathrm{U}$ and $9.4 \times 10^{5} \mathrm{Ci}^{239} \mathrm{Pu}$ ) Highly enriched $\left(0.044 \mathrm{Ci}^{235} \mathrm{U}\right.$ and $\left.1.34 \times 10^{5} \mathrm{Ci}^{239} \mathrm{Pu}\right)$

- Burnup (yr 2035) Low (per MTHM, $1.78 \mathrm{Ci}^{237} \mathrm{~Np} ; 18.4 \mathrm{Ci}^{99} \mathrm{Tc}$ )

- Pyrophoricity Not pyrophoric

Repository Disposal Considerations. Listed below are considerations specific to this fuel category with regard to potential fuel performance after disposal in an underground geologic repository.

- Because of its low burnup, this waste stream can dilute ${ }^{237} \mathrm{~Np}$ and ${ }^{99} \mathrm{Tc}$ in other waste streams when vitrified.

- Stainless steel cladding offers little long-term protective value; if protection equivalent to CSNF is required, an additional protective container inside disposal container can be used.

- Mixed oxide matrix alters rapidly (possibly 2 orders of magnitude faster than $\mathrm{UO}_{2}$ ); borosilicate glass treatment would cause alteration at a similar or slower rate than $\mathrm{UO}_{2}$ so treatment might reduce release rates.

- Reprocessing can remove uranium and plutonium; alternatively, can dissolve and dilute with ${ }^{238} \mathrm{U}$ or other waste streams before vitrification. 


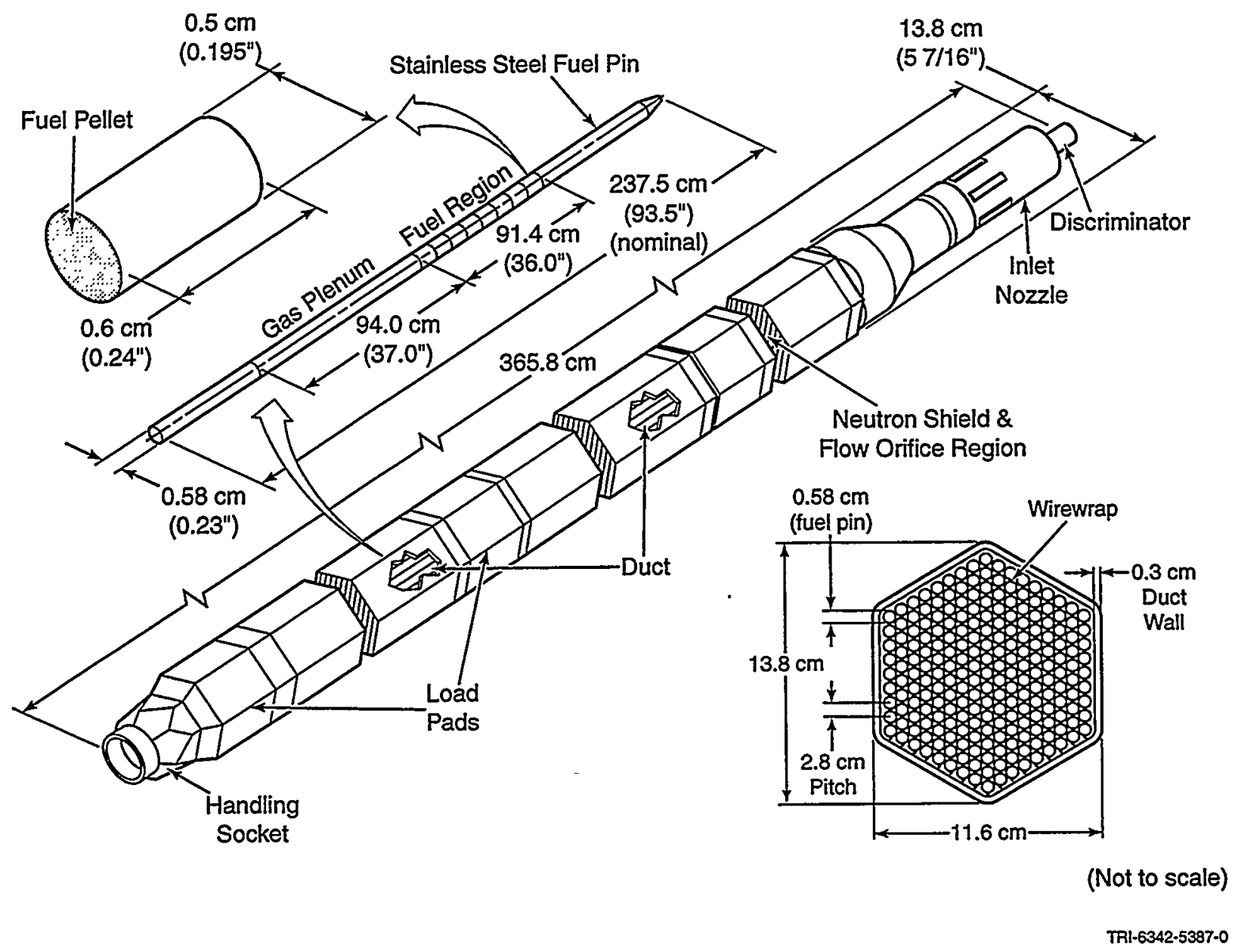

Figure 3-10. Category 11 spent fuel, represented by Fast Flux Text Facility standard driver fuel assembly. 


\subsubsection{Uranium-Thorium Oxide Fuel, Category 12}

Fuel Description. The representative fuel type for Category 12 is the Shippingport LWBR (Light Water Breeder Reactor). The $L W B R$ was used to demonstrate the production of ${ }^{233} U$ from thorium in a water-cooled operating reactor (Figure 3-11). The fuel was uranium oxide, enriched up to $98 \%$ in ${ }^{233} \mathrm{U}$ mixed with thorium oxide, and made into cylindrically shaped ceramic pellets. The fuel pellets were loaded in $0.762-\mathrm{cm}(0.3-\mathrm{in}$.) diameter Zircaloy-4 tubes whose ends are capped and seal welded. These tubes were made into assemblies. The LWBR has four different types of assemblies: 12 seed assemblies used the highly enriched uranium (HEU) to produce power; 12 blanket assemblies were used to capture neutrons and convert the thorium to ${ }^{233} \mathrm{U}$, and 9 type-IV reflector assemblies and 6 type- $\mathrm{V}$ reflector assemblies were used to reflect neutrons back into the reactor. (Note that his group does not include the nine canisters of disrupted LWBR fuel; they are in Category 5.) Category 12 also includes the radioisotope inventory for fuel from the Dresden Reactor located at Morris, Mlinois.

Quantity Summary. Listed below are the amounts of reported MTHM and the percentage this category represents with regard to all DOE SNF and all disposed fuel (commercial, DOE SNF, and DHLW) modeled in the 1997 PA.

- Regulatory Mass

49.7 MTHM

- Percentage of Total DOE SNF (2415 MTHM) $2 \%$

- Percentage of Total Disposed Fuel (75,336 MTHM) $0.07 \%$

Physical Characteristics. See Figure 3-11.

- Fuel Matrix Uranium-Thorium Oxide

- Cladding Zircaloy

- Representative Fuel Shippingport IWBR ( $86 \%$ of category)

- Other Fuels in Category Dresden Reactor fuel

- Enrichment (compare to CSNF, $1.2 \times 10^{-5} 235 \mathrm{U}$ and $9.4 \times 10^{5} \mathrm{Ci}{ }^{239} \mathrm{Pu}$ ) Highly enriched $\left(0.28 \mathrm{Ci}^{235} \mathrm{U}\right.$ and $\left.12.7 \mathrm{Ci}^{239} \mathrm{Pu}\right)$

- Burnup (yr 2035) Low (per MTHM, $0.05 \mathrm{Ci}^{237} \mathrm{~Np} ; 162 \mathrm{Ci}^{99} \mathrm{Tc}$ )

- Pyrophoricity Not pyrophoric

Repository Disposal Considerations. Listed below are considerations specific to this fuel category with regard to potential fuel performance after disposal in an underground geologic repository.

- Because of its low burnup, this waste stream can dilute ${ }^{237} \mathrm{~Np}$ and ${ }^{99} \mathrm{Tc}$ in other waste streams when vitrified.

- Possibly $30 \%$ of Zircaloy cladding has failed; if protection equivalent to CSNF is required, an additional protective container inside disposal container can be used.

- Reprocessing can remove uranium and plutonium; alternatively, can dissolve and dilute with ${ }^{238} \mathrm{U}$ or other waste streams before vitrification. 


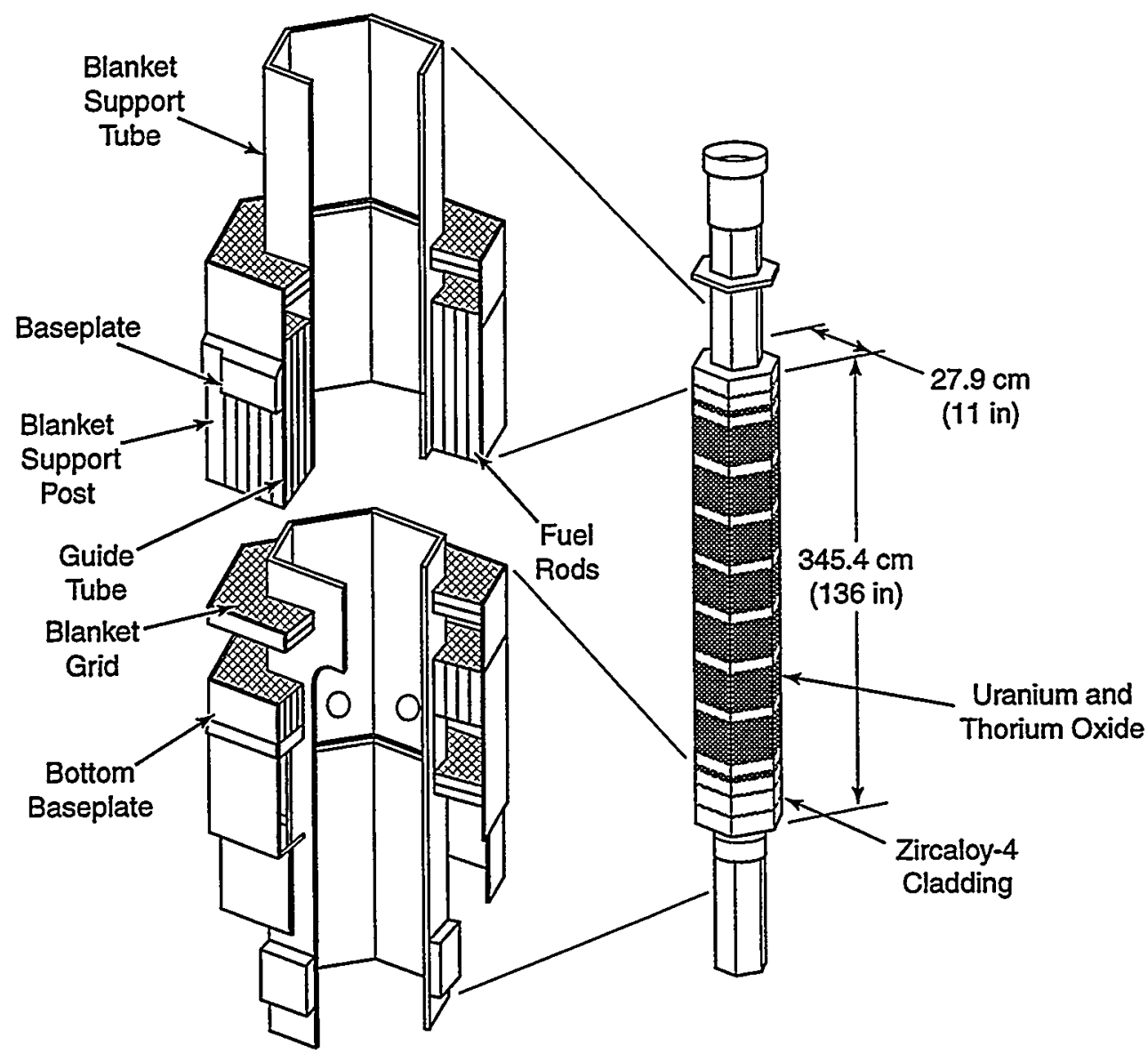

(Not to scale)

TRI-6342-5388-0

Figure 3-11. Category 12 spent fuel, represented by Shippingport Light Water Breeder Reactor (LWBR) fuel assembly. 


\subsubsection{Uranium/Zirconium Hydride Fuel, Category 13}

Fuel Description. The representative fuel for Category 13 is the Training, Research, and Isotope production General Atomic (TRIGA) fuel, made from uranium/zirconium hydride that was formed into either solid or hollow rods, 3.94-cm (1.4-in.) diameter by about 35.5 to $38 \mathrm{~cm}$ (14 or $15 \mathrm{in}$ ) long. Graphite plugs and samarium discs were placed on the end of the fuel element. Including the cladding and end pieces, the rods are about $72 \mathrm{~cm}(28.37 \mathrm{in}$.) long. The rods are not placed into assemblies, but each is handled separately (see Figure 3-12). Category 13 also includes the radioisotope inventory for fuel from the TRIGA Fuel Life Improvement Program (FLIP) (HEU), TRIGA aluminum-cladded fuel (MEU), and fuel from the Systems for Nuclear Auxiliary Power (SNAP) (HEU).

Quantity Summary. Listed below are the amounts of reported MTHM and the percentage this category represents with regard to all DOE SNF and all disposed fuel (commercial, DOE SNF, and DHLW) modeled in the 1997 PA.

- Regulatory Mass 2.00 MTHM

- Percentage of Total DOE SNF (2415 MTHM) $0.08 \%$

- Percentage of Total Disposed Fuel (75,336 MTHM) $0.003 \%$

Physical Characteristics. See Figure 3-12.

- Fuel Matrix Uranium/Zirconium Hydride

- Cladding Stainless steel

- Representative Fuel Training, Research, and Isotope production-General Atomic (TRIGA)

- Other Fuels in Category TRIGA Fuel Life Improvement Program, TRIGA aluminum-clad, Systems for Nuclear Auxiliary Power fuel

- Enrichment (compare to CSNF, $1.2 \times 10^{-5}{ }^{235} \mathrm{U}$ and $9.4 \times 10^{5} \mathrm{Ci}^{239} \mathrm{Pu}$ ) Moderate $\left(0.98 \mathrm{Ci}^{235} \mathrm{U}\right.$ and $\left.454 \mathrm{Ci}^{239} \mathrm{Pu}\right)$

- Burnup (yr 2035) Low (per MTHM, $0.19 \mathrm{Ci}^{237} \mathrm{~Np} ; 43.3 \mathrm{Ci}^{99} \mathrm{Tc}$ )

- Pyrophoricity Not pyrophoric

Repository Disposal Considerations. Listed below are considerations specific to this fuel category with regard to potential fuel performance after disposal in an underground geologic repository.

- Because of its low burnup, this waste stream can dilute ${ }^{237} \mathrm{~Np}$ and ${ }^{99} \mathrm{Tc}$ in other waste streams when vitrified.

- Stainless steel cladding offers little long-term protection; if protection equivalent to CSNF is required, an additional protective container inside disposal container can be used.

- Uranium/zirconium hydride matrix alters rapidly (possibly 1 order of magnitude faster than $\mathrm{UO}_{2}$ ); borosilicate glass treatment would cause alteration at a similar or slower rate than $\mathrm{UO}_{2}$, so treatment might reduce release rates of uranium.

- Reprocessing can remove uranium and plutonium; alternatively, can dissolve and dilute with ${ }^{238} \mathrm{U}$ or other waste streams before vitrification. 


\subsection{Description of Modeled Spent Fuel Categories}

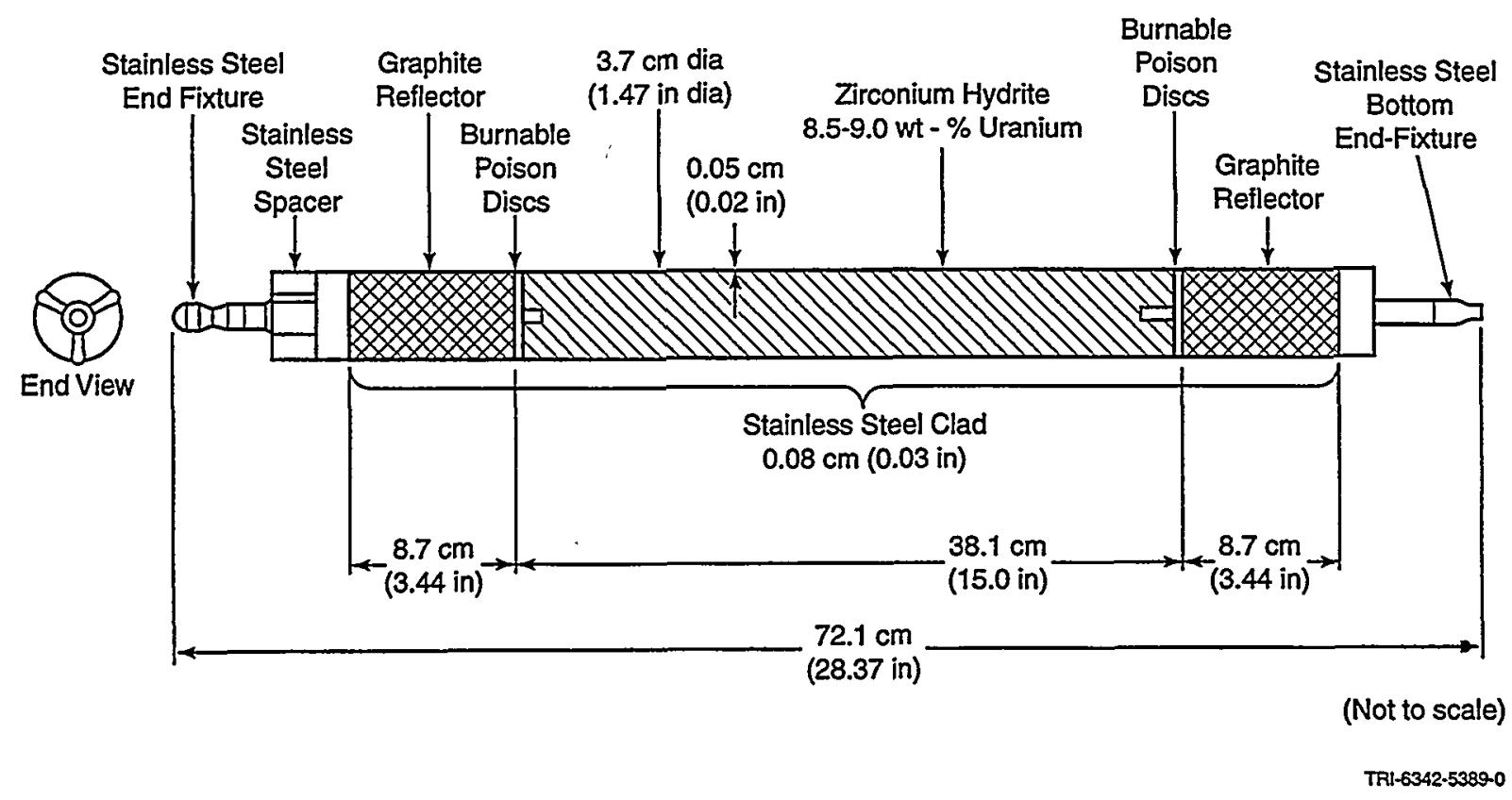

Figure 3-12. Category 13 spent fuel, represented by Training, Research, and Isotope production-General Atomic (TRIGA) standard fuel assembly. 


\subsubsection{1-PWR Commercial - Uranium Oxide Fuel, Category 14}

Fuel Description. Category 14 is represented by a single characteristic set of data that reflects the various configurations currently under consideration for both 21-PWR and 12-PWR commercial spent fuel packages. Currently, there are two configurations for both 21-PWR and 12-PWR. Figure 3-13 illustrates the 21-PWR package, which is modeled in the $1997 \mathrm{PA}$.

Quantity Summary. Listed below are the amounts of reported MTHM and the percentage this category represents with regard to all DOE SNF and all disposed fuel (commercial, DOE SNF, and DHLW) modeled in the 1997 PA.

- Regulatory Mass

$$
\text { 41,440 MTHM }
$$

- Percentage of Total DOE SNF (2415 MTHM)

N/A

- Percentage of Total Disposed Fuel (75,336 MTHM)

$55 \%$

Physical Characteristics. See Figure 3-13.

- Fuel Matrix

$$
\text { Uranium Oxide (PWR) }
$$

- Cladding

Zircaloy

- Representative Fuel 21-PWR

- Other Fuels in Category 12-PWR

- Enrichment

Low (uranium inventory $98 \%{ }^{238} \mathrm{U}$ ) $1.2 \times 10^{-5}{ }^{235} \mathrm{U}$ and $9.4 \times 10^{5} \mathrm{Ci}{ }^{239} \mathrm{Pu}$

- Burnup (yr 2035) $40 \mathrm{GWd} / \mathrm{MTU}\left(40,540 \mathrm{Ci}^{237} \mathrm{~Np} ; 23,910 \mathrm{Ci}^{99} \mathrm{Tc}\right.$

- Pyrophoricity Not pyrophoric

Repository Disposal Considerations. Listed below are considerations specific to this fuel category with regard to potential fuel performance after disposal in an underground geologic repository.

- Modeling of cladding would improve modeled performance of CSNF.

- Uranium oxide alters slowly; pitchblende mineral form in nature; borosilicate glass treatment would cause alteration at a similar or slower rate than $\mathrm{UO}_{2}$ so treatment might reduce release rates of uranium.

- Reprocessing can remove uranium. 


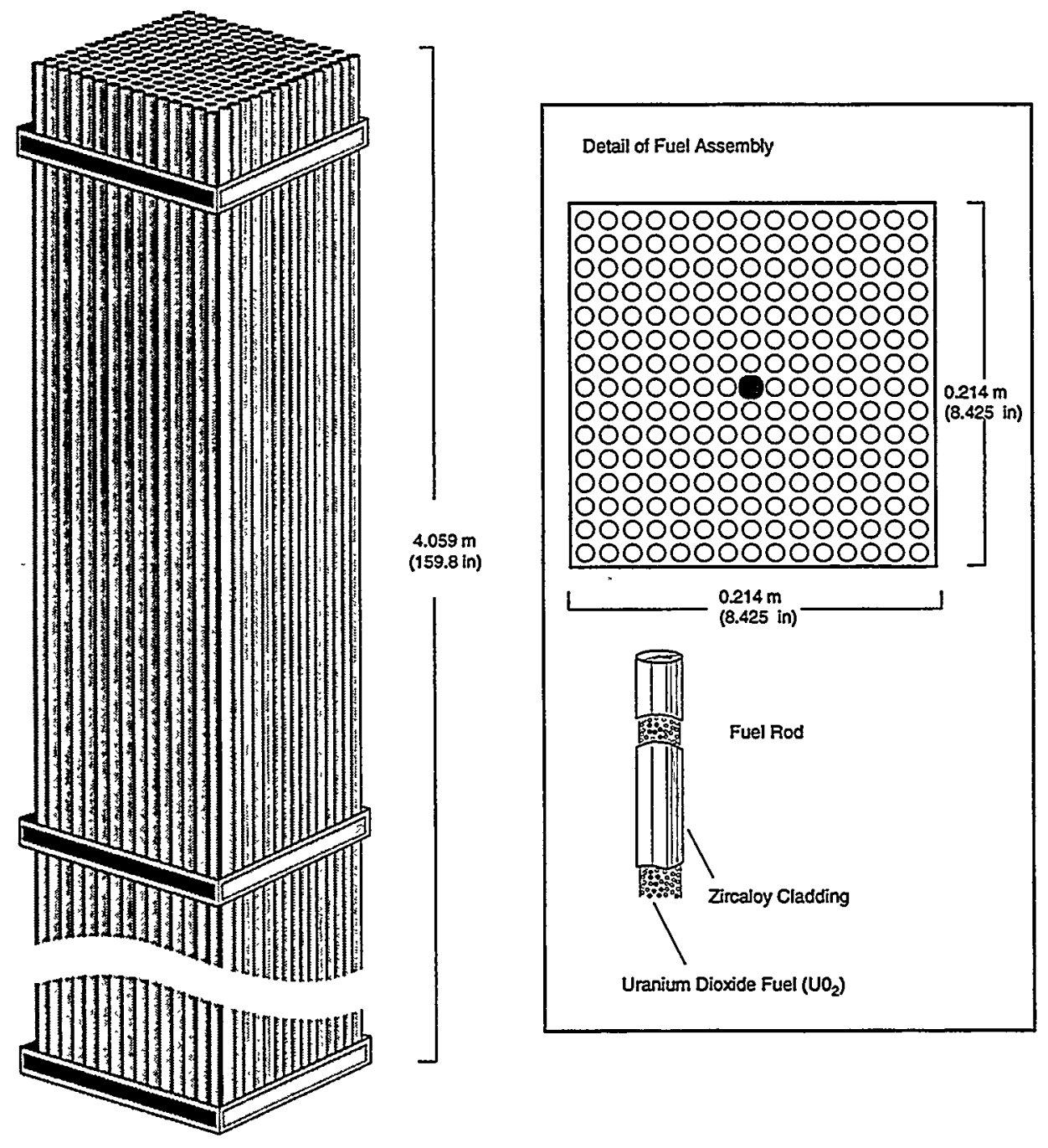

TA1-6342-3753-1

Figure 3-13. Category 14 spent fuel, represented by 21-PWR fuel assembly. 


\subsubsection{4-BWR Commercial - Uranium Oxide Fuel, Category 15}

Fuel Description. Category 15 is represented by a single characteristic set of data that reflects the various configurations currently under consideration for both 44-BWR and 24-BWR commercial spent nuclear fuel packages. Currently, there are three 44-BWR and one 24-BWR configurations. Figure 3-14 illustrates the 44-BWR package.

Quantity Summary. Listed below are the amounts of reported MTHM and the percentage this category represents with regard to all DOE SNF and all disposed fuel (commercial, DOE SNF, and DHLW) modeled in the 1997 PA.

- Regulatory Mass 21,640 MTHM

- Percentage of Total DOE SNF (2415 MTHM) N/A

- Percentage of Total Disposed Fuel (75,336 MTHM) $29 \%$

Physical Characteristics. See Figure 3-14.

- Fuel Matrix Uranium Oxide (BWR)

- Cladding Zircaloy

- Representative Fuel 44-BWR

- Other Fuels in Category 24-BWR

- Enrichment Low (uranium inventory $98 \%{ }^{238} \mathrm{U}$ ) $1.2 \times 10^{-5}{ }^{235} \mathrm{U}$ and $9.4 \times 10^{5} \mathrm{Ci}^{239} \mathrm{Pu}$

- Burnup (yr 2035) $40 \mathrm{GWd} / \mathrm{MTU}\left(16,870 \mathrm{Ci}^{237} \mathrm{~Np} ; 10,920 \mathrm{Ci}^{99} \mathrm{Tc}\right)$

- Pyrophoricity Not pyrophoric

Repository Disposal Considerations. Listed below are considerations specific to this fuel category with regard to potential fuel performance after disposal in an underground geologic repository.

- Modeling of cladding would improve modeled performance of CSNF.

- Uranium oxide alters slowly; pitchblende mineral form in nature; borosilicate glass treatment would cause alteration at a similar or slower rate than $\mathrm{UO}_{2}$ so treatment might reduce release rates of uranium

- Reprocessing can remove uranium. 

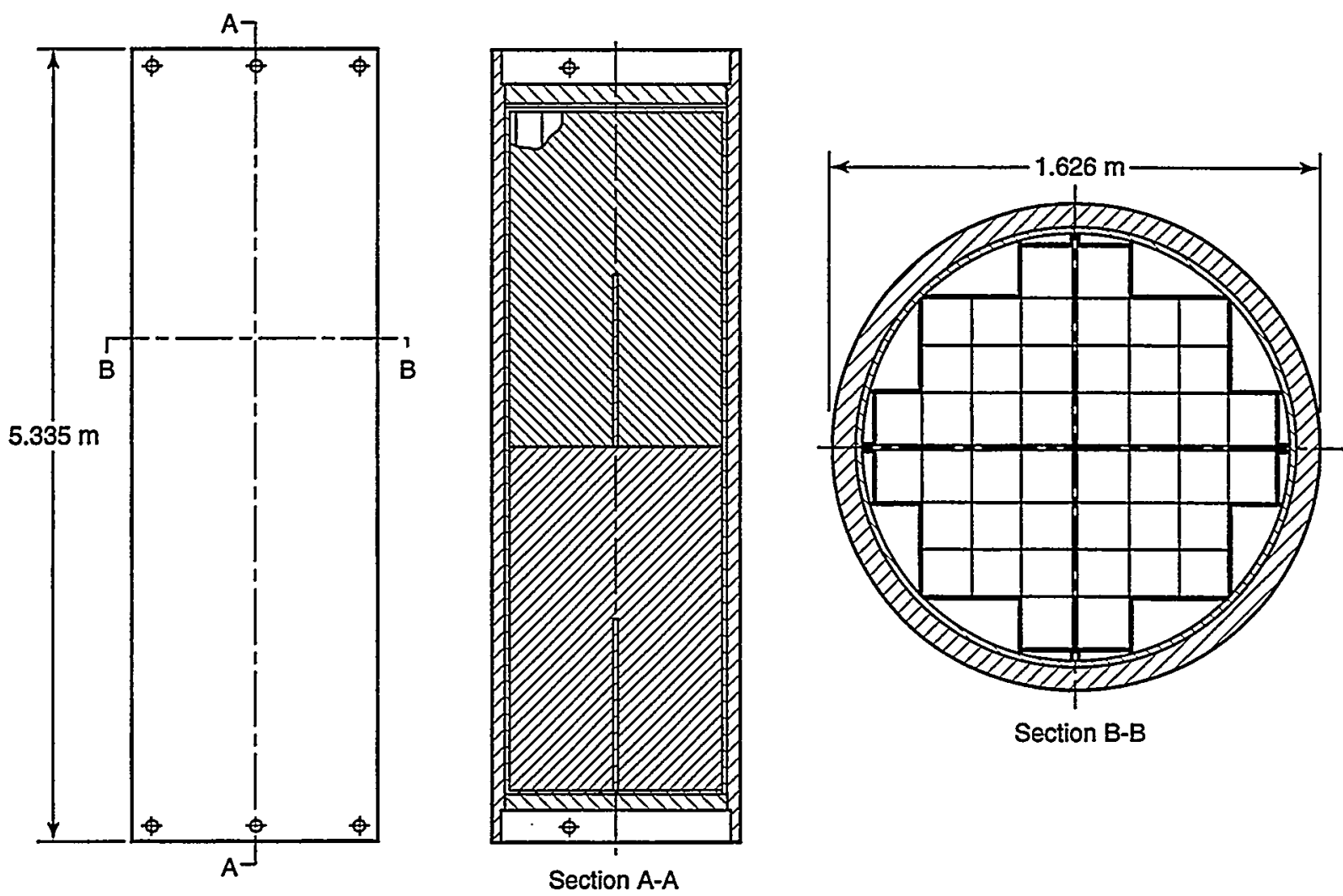

TRI-6342-5390-0

Figure 3-14. Category 15 spent fuel, represented by 44-BWR fuel assembly. 


\subsubsection{Defense High-Level Waste}

Fuel Description. Defense high-level waste from Savannah River, Hanford, INEEL, and West Valley will be packaged with Categories 1 through $13 .^{\dagger}$ Plans call for the borosilicate glass to be produced from neutralized highlevel waste. The DHLW has a much lower transuranic loading than does DOE SNF; Appendix A (pp. 1-2) contains a more detailed description of the source of DHLW.

For the 1997 PA only one type of DHLW, borosilicate glass, which represents the "average" characteristics of the several types, is modeled for consistency with a-TSPA-97 (M\&O, 1997). Figure 3-15 illustrates the standard DHLW DOE handling container. The MTHM of the high-level waste was calculated using 0.5 MTHM per standard DHLW container, which is a method of estimating that had been developed in the 1980s for DOE planning and was also used for MTHM estimates in a-TSPA-1997 (M\&O, 1997). For DHLW packaged in the long (5.3 m) containers, 0.75 MTHM per package was estimated, as outlined in Appendix A. The result is a total of 9842 MTHM for DHLW based on 13,920 containers ${ }^{\ddagger}$ required for the codisposal option. The difference between this value and the value of 9590 MTHM used in the 1994 PA is the result of differences in estimating MTHM; in the 1994 PA, MTHM was evaluated based on 40 CFR 191, Appendix A, note 1. Note also that the 1994 PA value of 9590 MTHM was calculated assuming that note 1(d) in 40 CFR 191 applied to all sites; instead, note 1(c) should apply to Savannah River's waste, which would have reduced the value to 6400 .

Quantity Summary. Listed below are the amounts of reported MTHM and the percentage this category represents with regard to all disposed fuel (commercial, DOE SNF, and DHLW) modeled in the 1997 PA.

- Regulatory Mass 9842 MTHM

- Percentage of Total DOE SNF (2415 MTHM) N/A

- Percentage of Total Disposed Fuel (75,336 MTHM) $13 \%$

Physical Characteristics. See Figure 3-15.

- Fuel Matrix Borosilicate Glass High-Level Waste

- Cladding N/A

- Enrichment N/A

- Burnup (yr 2035) Low (0.5 MTHM per container: $\left.0.007 \mathrm{Ci}^{237} \mathrm{~Np} ; 0.825 \mathrm{Ci}^{99} \mathrm{Tc}\right)$

- Pyrophoricity Not pyrophoric

Repository Disposal Considerations. Listed below are considerations specific to this waste type with regard to potential performance after disposal in an underground geologic repository.

- Borosilicate glass treatment means that particulates (e.g., in calcine high-level waste) do not pose a problem with regard to 10 CFR 60 .

$\dagger$ The proposed packaging plan (Appendix A) inciudes a small number of codisposal packages for Category 1; however, Category 1 is modeled in the 1997 PA without codisposal (see Section 3.3).

₹ The 1992 DOE report, Characteristics of Potential Repository Waste, (DOE, 1992), predicts 15,317 containers, but the codisposal option incorporates only 13,920 . Thus -1400 high-level waste containers of the predicted amount are not included in the 1997 PA. 


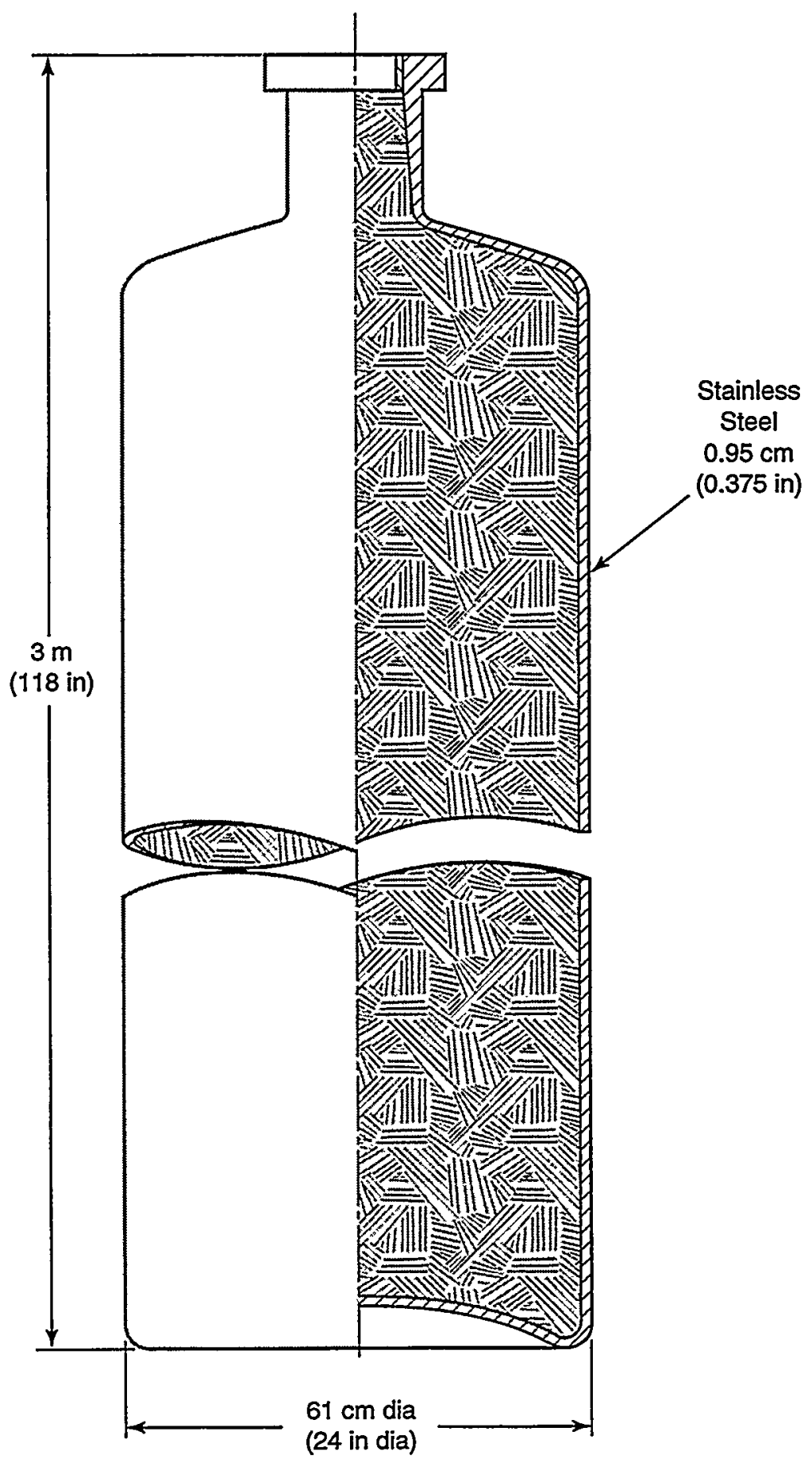

TRL-6342-5520-0

Figure 3-15. High-level waste DOE standard handling container. 


\subsection{Handling and Disposal Containers}

All spent fuel and high-level waste is placed in handling and/or disposal containers before emplacement in the potential Yucca Mountain repository. In the 1997 PA, DOE SNF categories are placed in both handling and disposal containers, as is the DHLW; commercial spent fuel is placed in a disposal container only. A handling container is the container in which the fuel matrix and cladding, if any, are placed. If the waste form is solidified, it is called the pour container; also the handling container is often called a canister. In some cases a handling container is the sole container for storage, handling, and transportation prior to disposal preparation or disposal itself (Wilson et al., 1994). In the present study the term handling container is used to designate its protective layer, which is stainless steel.

The outermost container is referred to as the disposal container, which is also referred to as a repository overpack in other studies. The disposal container consists of a secondary container, or containers, around the handling container that serves some specialized function such as corrosion resistance or structural support. In some literature about the potential Yucca Mountain repository, the disposal container is called a container barrier assembly.

The handling and disposal containers are important in determining performance of the spent fuels after disposal, because they represent layers of material that protect and confine the spent fuel. Degradation of the protective layers and alteration and dissolution of the fuel matrix are modeled in the 1997 PA by means of the Corrosion and Source Term (CST) submodel, which is described thoroughly in Chapter 7. With regard to the handling and disposal containers, CST predicts (a) the rates at which corrosion will penetrate the container layers in a potential Yucca Mountain repository, thereby exposing radionuclides to possible transport to the accessible environment and (b) the effects of corrosion on the gas, especially oxygen, and water contents of the near field, i.e., the portion of the repository that is significantly affected by the disposed spent fuel.

In the $1997 \mathrm{PA}$, the handling and disposal containers are considered layer(s) that consume or produce water or gas. (Note that the fuel matrix and cladding are also considered layers in this context.) Handling and disposal containers can be composed of corrosion-allowance materials, e.g., carbon steel, which oxidize rapidly in corrosive environments and require thick layers, or corrosion-resistant materials, e.g., Inconel, which oxidize slowly and can be thinner. Depending on the material type, CST considers one or two types of corrosion - general and/or localized-as it determines the sequential failure of the layers. General corrosion can be likened to peeling off layers of an onion, and localized corrosion can be thought of as cutting through layers of skin.

CST considers corrosion reactions and rates in its predictions of layer failure. Corrosion occurs when oxidants react with the layers to form corrosion products that no longer effectively isolate the fuel matrix from the near field. Potential oxidants include oxygen, water, peroxide, and others. Waste packages would come in contact with oxidants through processes such as dripping groundwater or, at later times, capillarity, when rubble has fallen into the air gap around the waste package.

Determination of the final packaging systems for DOE SNF and DHLW is still ongoing (INEEL SNFTT, 1997). Packaging systems must be suitable for interim storage, transportation to the repository, and repository storage. Safety requirements for transportation have been established by the DOT and NRC, along with very demanding requirements for long-term performance in the repository including low cost and resistance to degradation. Final engineering decisions and controlling regulations that impact these conditions are not known at this time, so packaging information used in this study may change in future iterations.

\subsubsection{Handling Containers}

Handling Container for DOE SNF Category 1. As a means of fuel remediation by drying, the Multi-Canister Overpack (MCO) is used as interim storage for Category 1 only, the $\mathrm{N}$-Reactor fuels. The basic unit will contain a close packed arrangement of either Mark IV or Mark IA fuels. While the original concept of the MCO is not intended as a repository-approved handling container, no alternative or proposed package exists at this time. The physical size of the MCO is similar to the standard DHLW handling container and is therefore modeled as a 4-pack within the dis- 
posal container. Each MCO consists of a $61-\mathrm{cm}$ outer diameter stainless steel shell that is $416.6 \mathrm{~cm}$ long and $0.95 \mathrm{~cm}$ thick. The approximate mass of the empty MCO is $1673.5 \mathrm{~kg}$.

Handling Containers for DOE SNF Categories 2 through 13. All handling containers for DOE SNF Categories 2 through 13 are constructed of $6.35-\mathrm{mm} 304 \mathrm{~L}$ stainless steel. Because lengths and diameters of the individual spent fuels differ, their varying measurements are accommodated by two handling container lengths: short, $3.0 \mathrm{~m}$

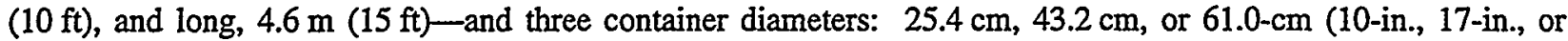
24-in.). The intent of combining the individual fuels into containers is to facilitate the handling of the great variety of fuels currently stored and to accommodate their small parts and pieces. In the 1997 PA, most spent fuels (70\% of total) are loaded within the long handling containers.

Handling Container for DHLW. The DHLW handling container is modeled as the 3-m-long, 61-cm-diameter (10-ft-long, 24-in.-diameter) stainless steel standard DOE container or the 4.6-m-long handling container.

Commercial Spent Nuclear Fuel. The commercial spent fuel is placed directly into a disposal container (see Section 3.2.2).

\subsubsection{Disposal Containers}

The disposal container for all categories is a large disposal package (LDP), with a 10-cm-thick outer carbon steel layer and a 2-cm-thick inner Inconel 625 layer. The lengths and diameters of the dispośal containers vary based on respective fuel lengths, cross-sectional areas, and fissile content. In the 1997 PA, four sizes are modeled (Table 3-1).

Table 3-1. Disposal Containers Modeled in 1997 PA

\begin{tabular}{lccc}
\hline \multicolumn{1}{c}{ Classification } & $\begin{array}{c}\text { Length (external) } \\
(\mathrm{m})\end{array}$ & $\begin{array}{c}\text { Diameter (external) } \\
(\mathrm{m})\end{array}$ & $\begin{array}{c}\text { Weight (empty pkg) } \\
(\mathbf{M g})\end{array}$ \\
\hline Standard short & 3.79 & 1.725 & 15.12 \\
Standard long & 5.30 & 1.725 & 21.20 \\
Super short & 3.79 & 2.0 & 19.02 \\
Super long & 5.30 & 2.0 & 26.35 \\
\hline
\end{tabular}




\subsection{Codisposal Configurations}

Current packaging plans propose that DOE SNF be codisposed with DHLW, i.e., that one DOE SNF handling container, together with three or more handling containers of DHLW, be placed in one disposal container. The primary reason for codisposal of DOE SNF with DHLW is related to criticality concerns; codisposal reduces criticality concerns by combining DHLW, which has a lower fissile content, in the package with DOE SNF. Although fissile load limits on a per disposal container basis have not been established for the potential repository, Appendix A (p. 5) provides the limit used for the current study, which is the same as for a-TSPA-97 (M\&O, 1997). Codisposal also takes advantage of otherwise unused space within disposal containers and is more cost effective than other methods to limit the reactivity of individual waste packages.

Although criticality is not investigated in the $1997 \mathrm{PA}$, it is important that the 1997 PA reflect the most current packaging plan. To model a codisposal package, the analysts must factor in attributes of both DOE SNF and DHLW. Appendix A lists all proposed DOE SNF/DHLW configurations by DOE SNF category. In some cases, more than one configuration has been proposed. $^{* *}$ For example, plans for DOE SNF Category 2 included a configuration that packages four DHLW handling containers with one DOE SNF handling container in a standard long disposal container (1 $\times 4 \times$ standard long), and another configuration that packages five DHLW containers with one DOE SNF container in a super long disposal container ( $1 \times 5 \times$ super long). In the $1997 \mathrm{PA}$, the most frequently used configuration per DOE SNF category is generally modeled. Note that the commercial spent fuel (Categories 14 and 15) is packaged without the codisposal option, i.e., no DHLW is included.

Table 3-2 lists the codisposal configurations selected for DOE SNF Categories 1 through $13^{\text {it }}$. The listing shows the number of DOE SNF and DHLW handling containers included in each waste package, and the size of the handling and disposal containers modeled. The waste package size, its containers, and the number of packages per category are significant with regard to physical arrangement within the repository (e.g., repository design; see also Chapter 5) and the type of protection provided. Figures 3-16 through 3-30 show the codisposal configuration per spent fuel category (including the disposal configurations for the commercial fuel, Categories 14 and 15).

Table 3-2. Codisposal Configuration Options for Categories 1 through 13

\begin{tabular}{|c|c|c|c|c|c|c|c|c|}
\hline \multirow[b]{3}{*}{$\begin{array}{c}\text { Spent } \\
\text { Fuel } \\
\text { Categories }\end{array}$} & \multicolumn{4}{|c|}{ Handling Container } & \multirow{2}{*}{\multicolumn{3}{|c|}{ Disposal Container }} & \multirow[b]{3}{*}{$\begin{array}{c}\text { Total } \\
\text { No. of } \\
\text { Waste } \\
\text { Packages }\end{array}$} \\
\hline & \multicolumn{2}{|c|}{ DOE SNF } & \multicolumn{2}{|c|}{ DHLW } & & & & \\
\hline & $\begin{array}{l}\text { Number } \\
\text { per Waste } \\
\text { Package }\end{array}$ & $\begin{array}{l}\text { Diameter } \\
\text { (cm) }\end{array}$ & $\begin{array}{l}\text { Number } \\
\text { per Waste } \\
\text { Package }\end{array}$ & $\begin{array}{l}\text { Diameter } \\
\text { (cm) }\end{array}$ & $\begin{array}{l}\text { Length } \\
\text { (m) }\end{array}$ & $\begin{array}{l}\text { Diameter } \\
\text { (m) }\end{array}$ & $\begin{array}{l}\text { Weight } \\
\text { (Mg) }\end{array}$ & \\
\hline 1 & 4 & 61 & 0 & - & 5.30 & 1.725 (std) & 16.73 & 118 \\
\hline 12 & 1 & 61 & 3 & 61 & 5.30 & 1.725 (std) & 15.12 & 69 \\
\hline 3,5 & 1 & 25.4 & 4 & 61 & 3.79 & 1.725 (std) & 15.12 & 650 \\
\hline 10,11 & 1 & 25.4 & 4 & 61 & 5.30 & 1.725 (std) & 21.20 & 357 \\
\hline 4,13 & 1 & 43.2 & 5 & 61 & 3.79 & 2.0 (super) & 19.02 & 305 \\
\hline $2,6,7,8,9$ & 1 & 43.2 & 5 & 61 & 5.30 & 2.0 (super) & 26.38 & 1632 \\
\hline (TOTALS) & $3485^{*}$ & & $13,920^{*}$ & & & & & 3131 \\
\hline
\end{tabular}

- The number of modeled and actual containers differs because more than one codisposal configuration per DOE SNF category was anticipated in the proposed disposal plan, but only one codisposal configuration was modeled per DOE SNF category in the 1997 PA.

\footnotetext{
** As of November 1997, the DOE/EM has proposed that only the $1 \times 5$ codisposal configuration be considered; however, that decision was made after calculations were performed for the 1997 PA and so is not reflected in this report.

It In the 1997 PA, DOE SNF Waste Category 1 is not modeled with DHLW in the waste package. However, the proposed packaging plan does anticipate a small number of codisposal packages for this category; see Appendix A, "Section 6.1, Category 1."
} 


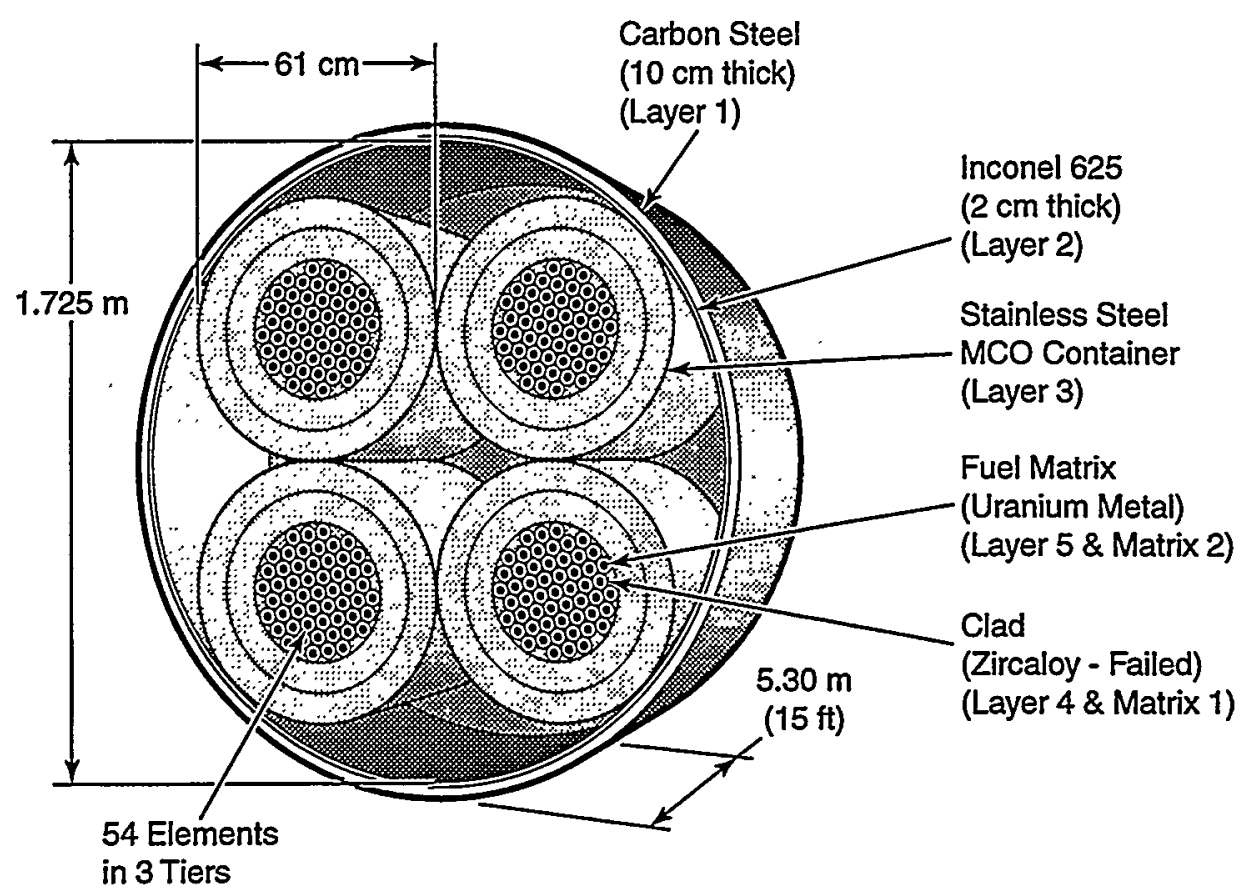

(Not to scale)

TFI-6342-5503-0

Figure 3-16. Codisposal configuration for Category 1, Uranium Metal, including four DOE SNF handling containers and no DHLW handling containers. Layers and matrices modeled in the 1997 PA are noted (see Section 3.4.1).

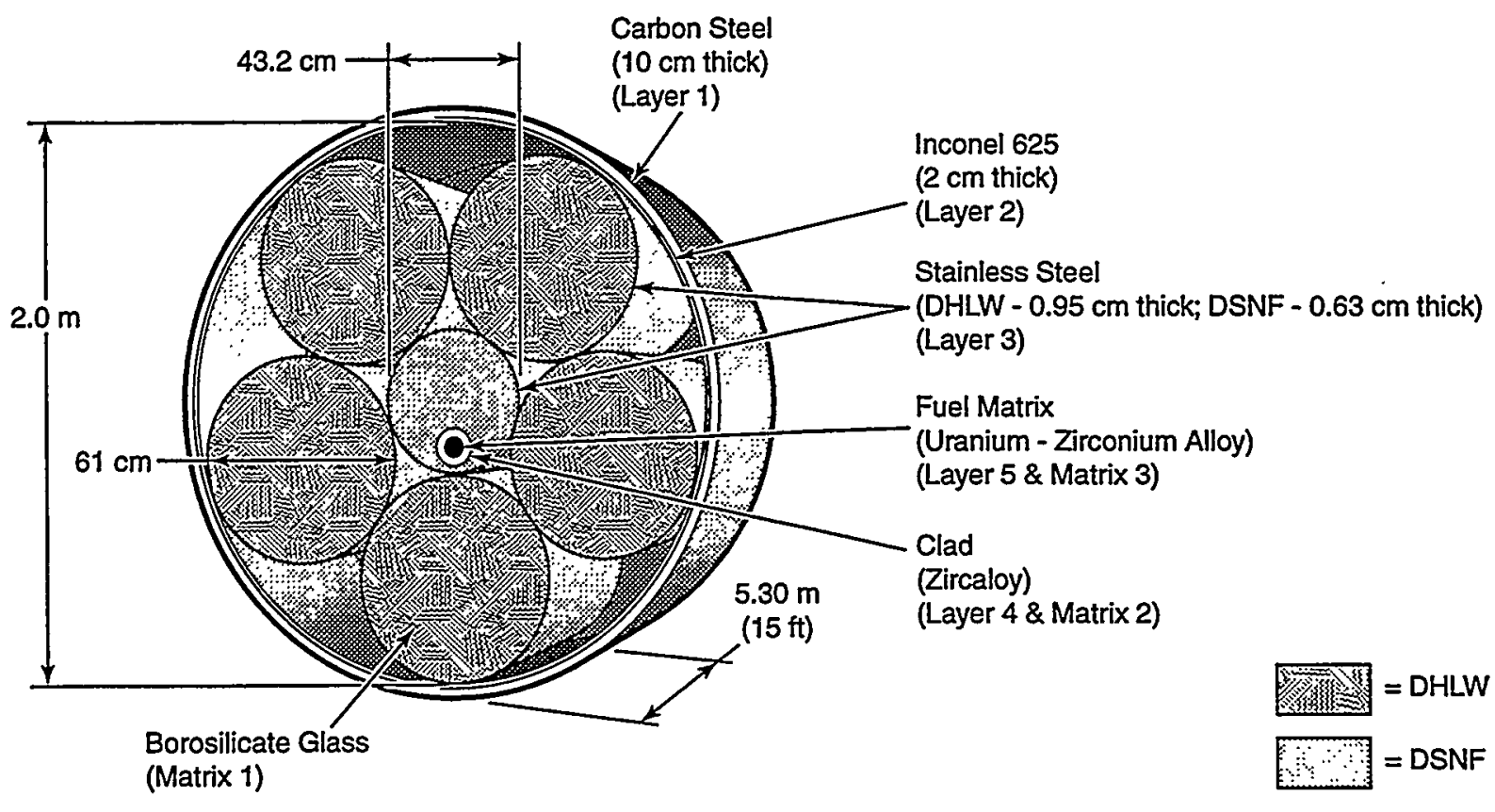

(Not to scale)

TR1-6342-5506-0

Figure 3-17. Codisposal configuration for Category 2, Uranium-Zirconium Alloy, including one DOE SNF handling container and five DHLW handling containers. Layers and matrices modeled in the 1997 PA are noted (see Section 3.4.1). 


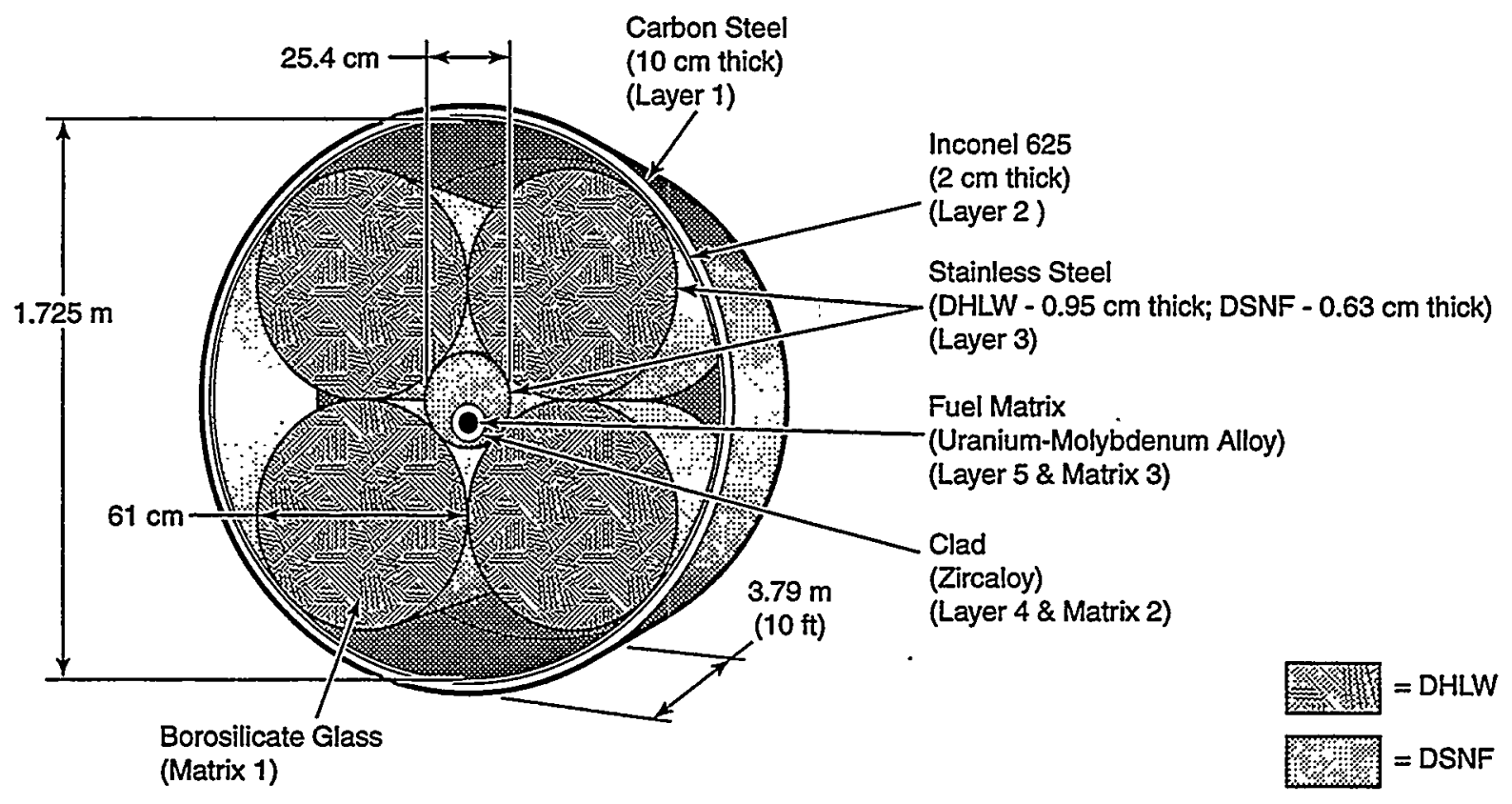

(Not to scale)

TRI-6342-5507-0

Figure 3-18. Codisposal configuration for Category 3, Uranium-Molybdenum Alloy, including one DOE SNF handling container and four DHLW handling containers. Layers and matrices modeled in the 1997 PA are noted (see Section 3.4.1).

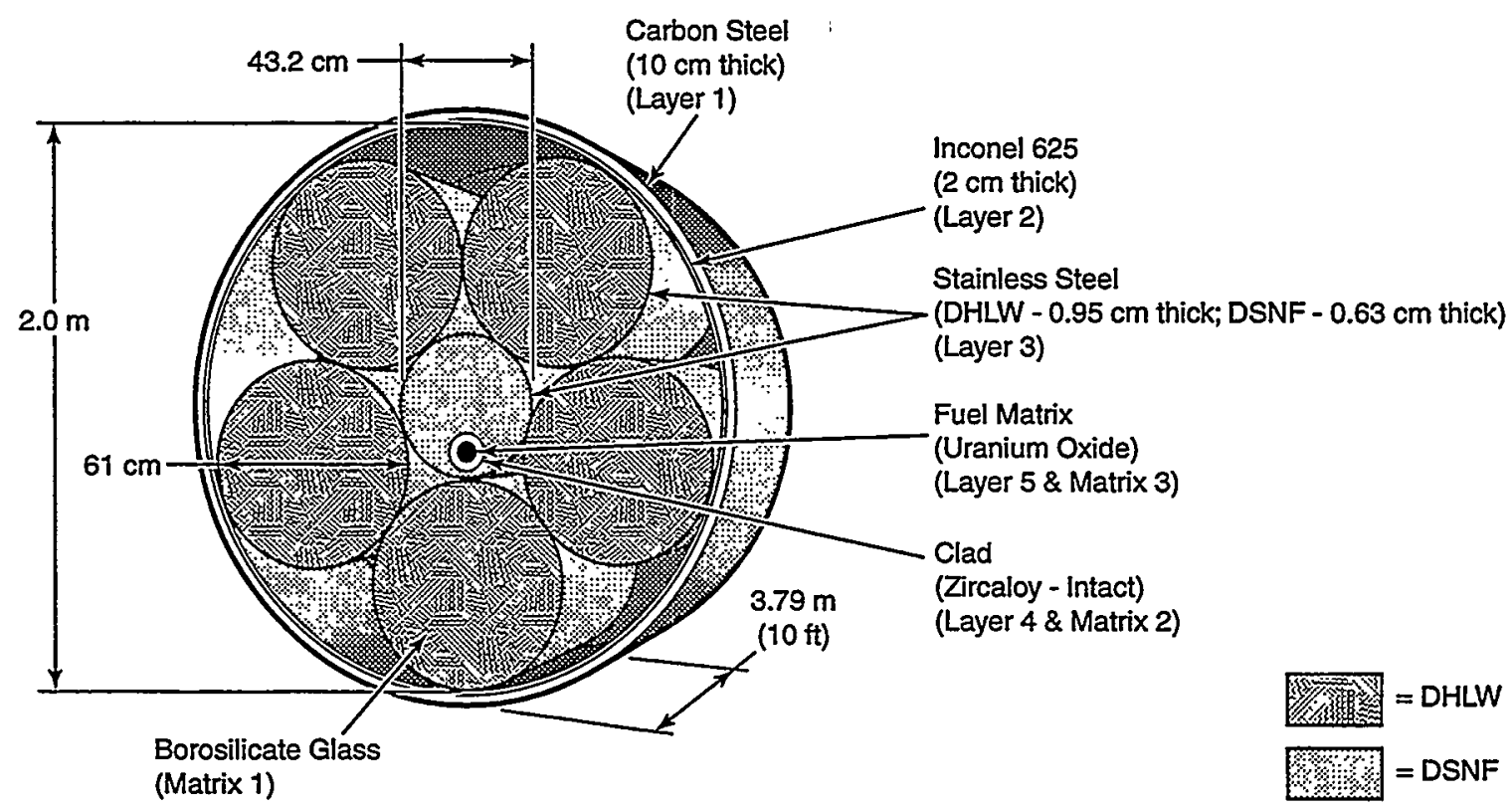

(Not to scale)

TA1-6342-5508-0

Figure 3-19. Codisposal configuration for Category 4, Uranium Oxide-Intact Clad, including one DOE SNF handling container and five DHLW handling containers. Layers and matrices modeled in the 1997 PA are noted (see Section 3.4.1). 


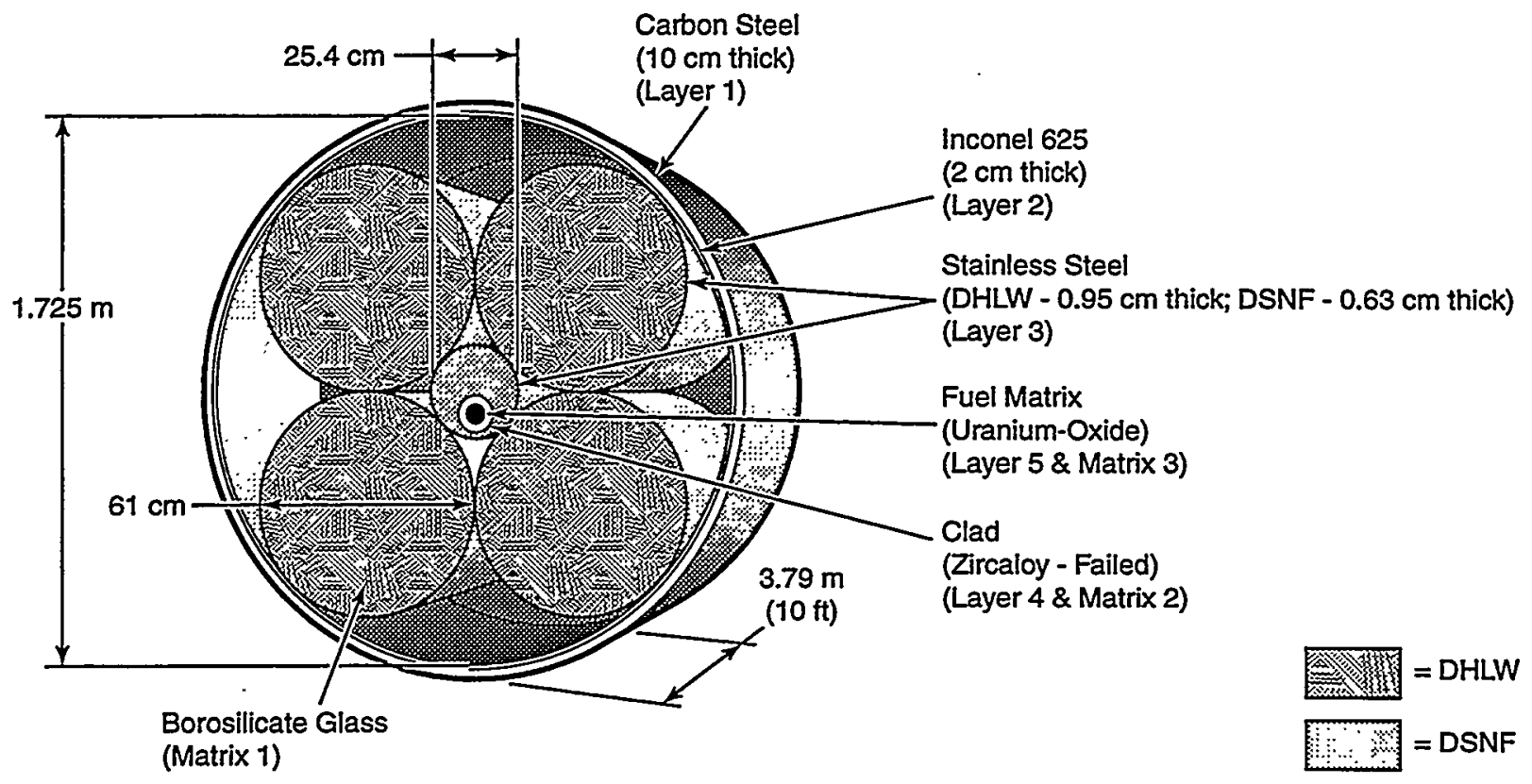

(Not to scale)

TA1-6342-5509-0

Figure 3-20. Codisposal configuration for Category 5, Uranium Oxide-Failed Clad, including one DOE SNF handling container and four DHLW handling containers. Layers and matrices modeled in the 1997 PA are noted (see Section 3.4.1).

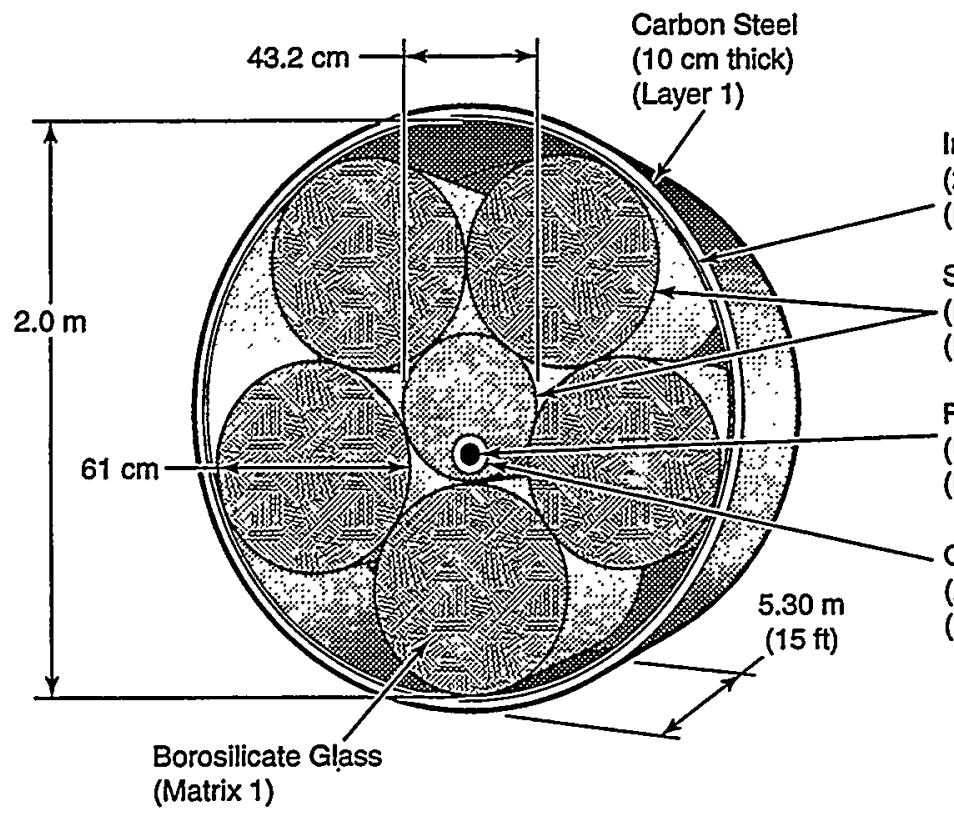

Inconel 625

( $2 \mathrm{~cm}$ thick)

(Layer 2)

Stainless Steel

(DHLW - $0.95 \mathrm{~cm}$ thick; DSNF - $0.63 \mathrm{~cm}$ thick)

(Layer 3)

Fuel Matrix

(Uranium Aluminum Alloy)

(Layer 5 \& Matrix 3)

Clad

(Aluminum)

(Layer 4 \& Matrix 2)

(Matrix 1)

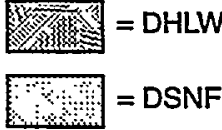

(Not to scale)

TRI-6342.5510-0

Figure 3-21. Codisposal configuration for Category 6, Uranium Aluminum Alloy, including one DOE SNF handling container and five DHLW handling containers. Layers and matrices modeled in the 1997 PA are noted (see Section 3.4.1).

September 30, 1998 


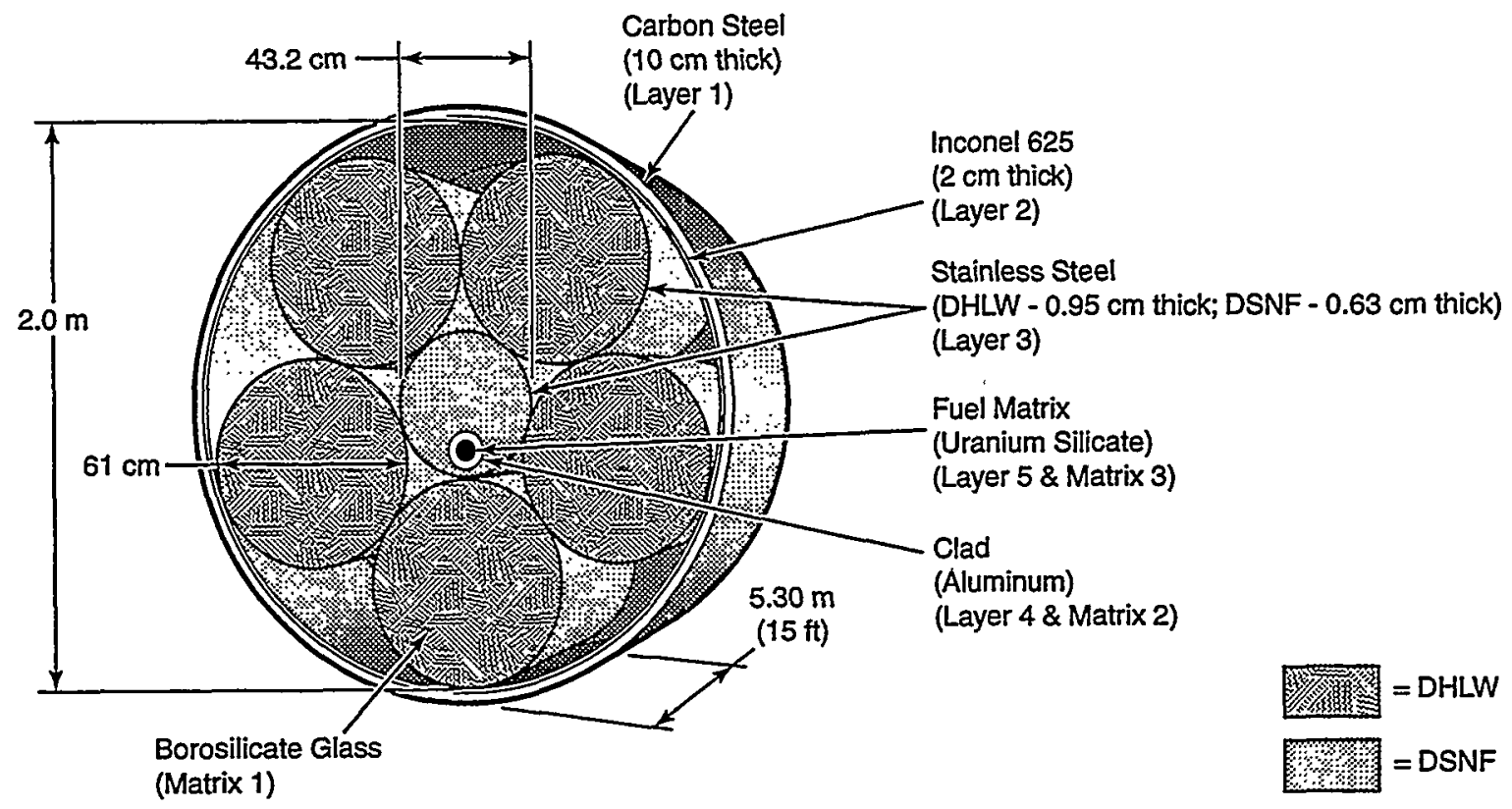

- (Not to scale)

TRI-6342-5511-0

Figure 3-22. Codisposal configuration for Category 7, Uranium Silicide, including one DOE SNF handling container and five DHLW handling containers. Layers and matrices modeled in the 1997 PA are noted (see Section 3.4.1).

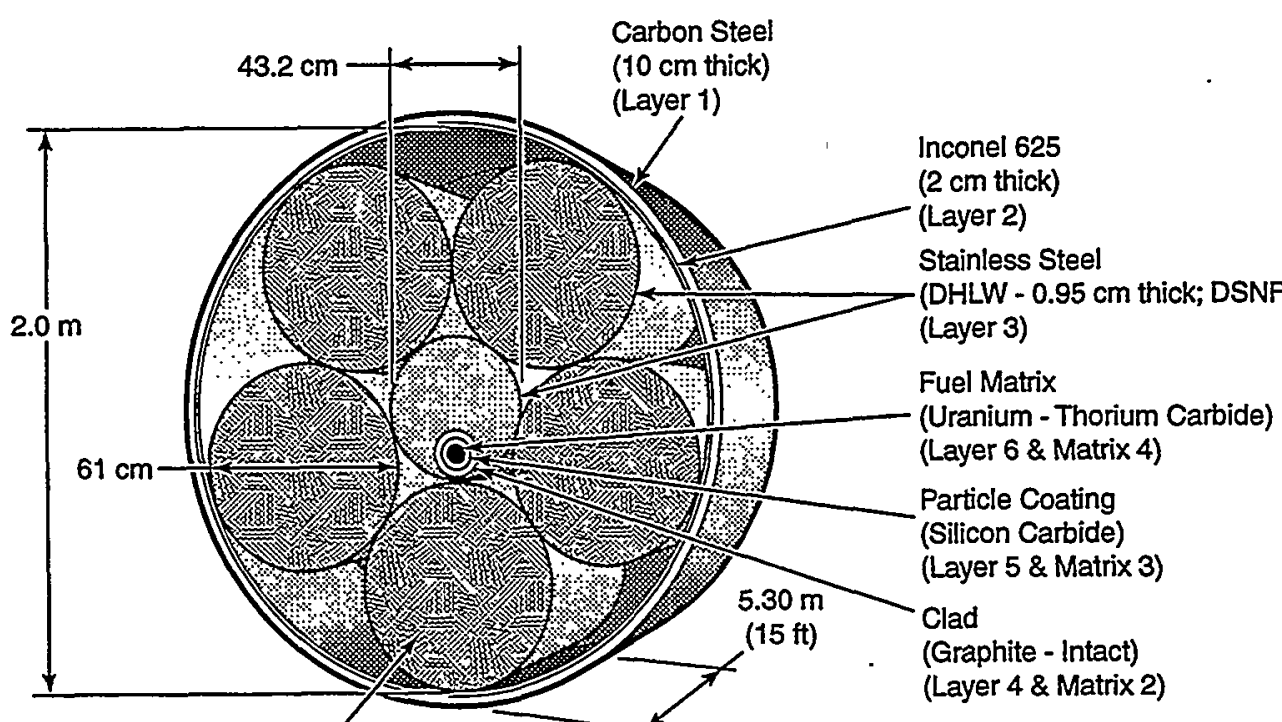

Borosilicate Glass

(Matrix 1)

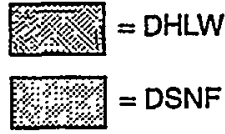

(Not to scale)

TR1-6342-5512-0

Figure 3-23. Codisposal configuration for Category 8, Uranium-Thorium Carbide-Intact Clad, including one DOE SNF handling container and five DHLW handling containers. Layers and matrices modeled in the 1997 $P A$ are noted (see Section 3.4.1). 


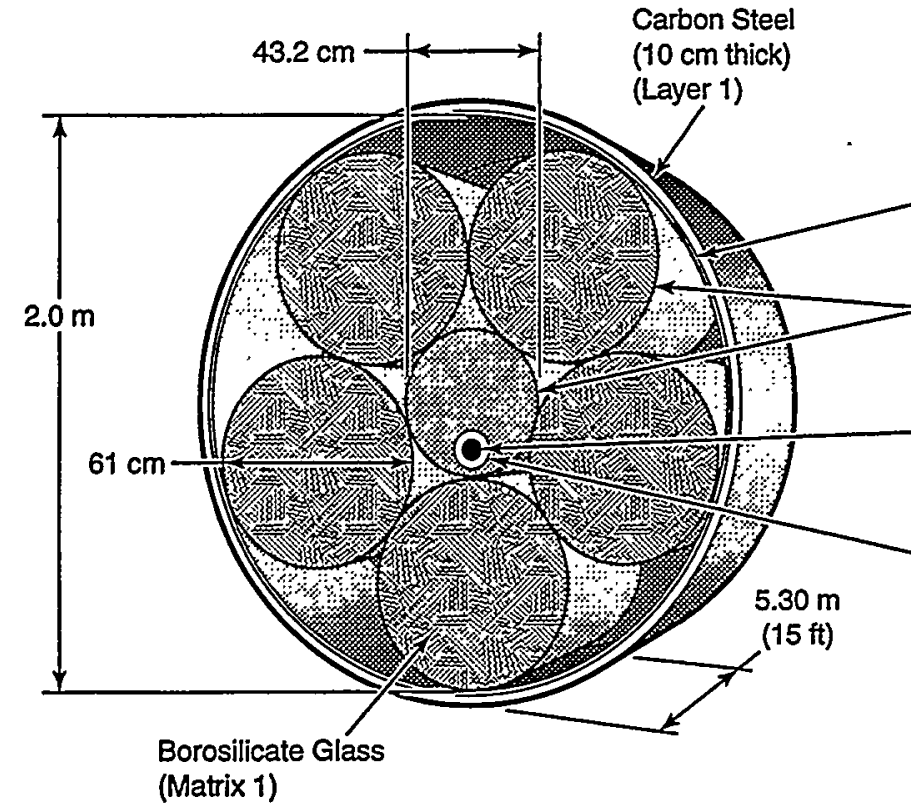

- No protective SiC layer (Layer 5)
Inconel 625

(2 cm thick)

(Layer 2)

Stainless Steel

(DHLW - $0.95 \mathrm{~cm}$ thick; DSNF - $0.63 \mathrm{~cm}$ thick) (Layer 3)

Fuel Matrix

(Uranium - Thorium Carbide)

(Layer 6* \& Matrix 3)

(Graphite - Failed)

(Layer 4 \& Matrix 2)

Figure 3-24. Codisposal configuration for Category 9, Uranium-Thorium Carbide-Failed Clad, including one DOE SNF handling container and five DHLW handling containers. Layers and matrices modeled in the 1997 PA are noted (see Section 3.4.1).

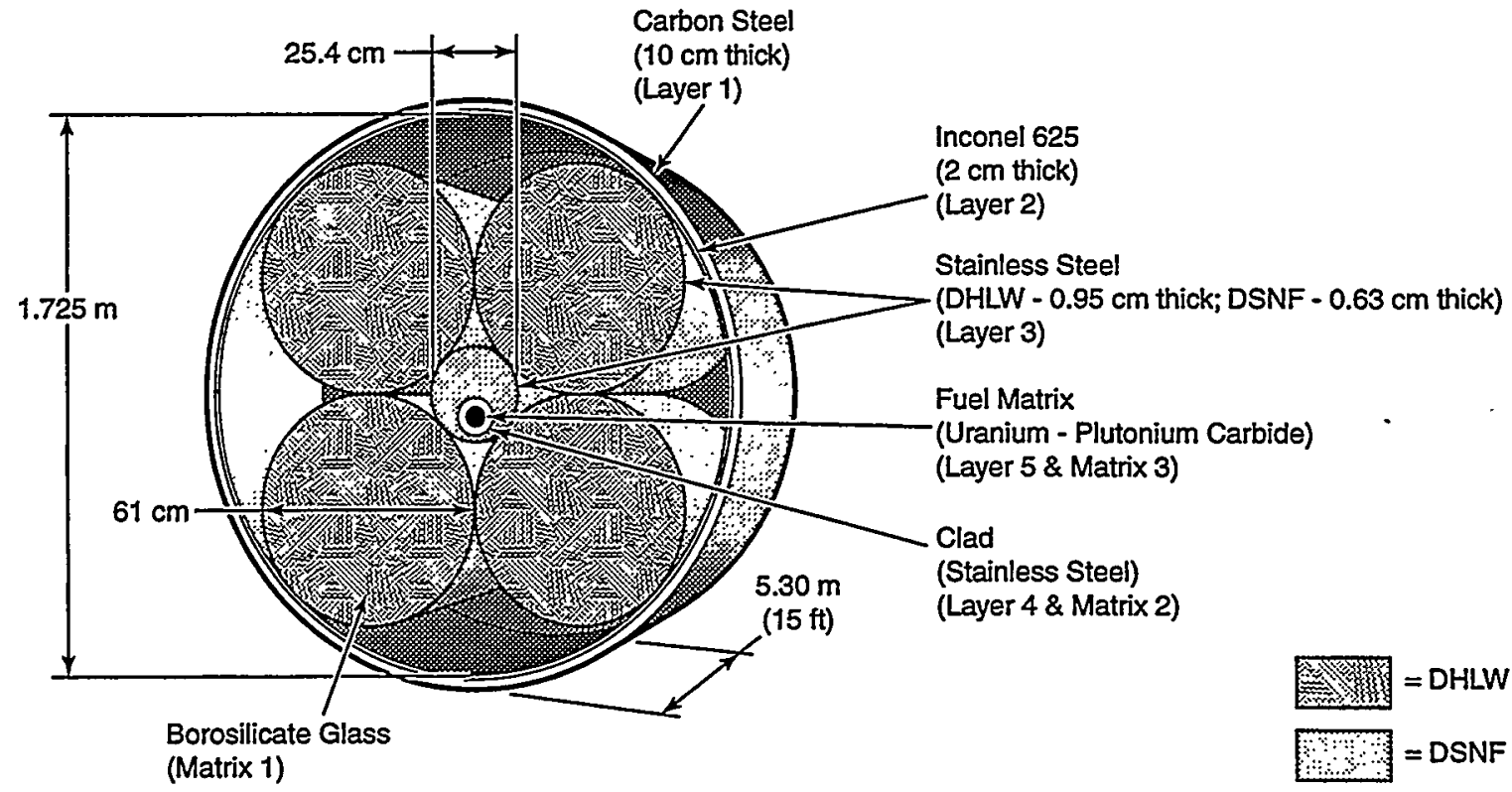

(Not to scale)

TRI-6342-5514-0

Figure 3-25. Codisposal configuration for Category 10, Uranium-Plutonium Carbide, including one DOE SNF handling container and four DHLW handling containers. Layers and matrices modeled in the 1997 PA are noted (see Section 3.4.1). 


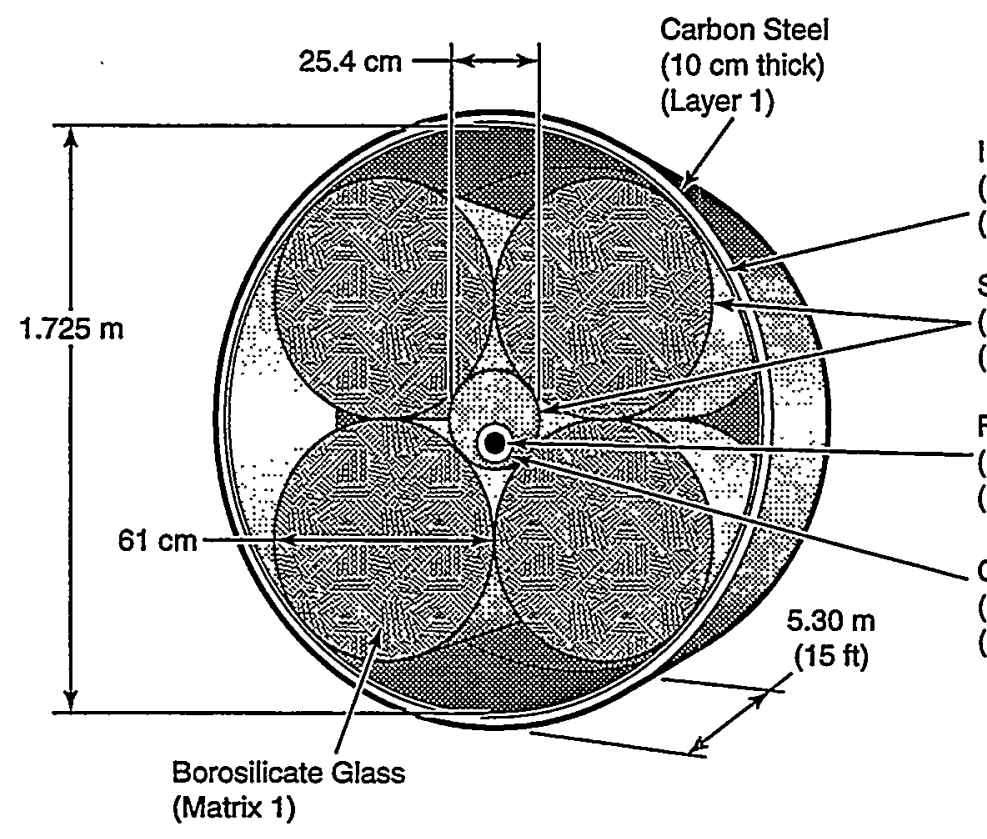

Inconel 625

(2 cm thick)

(Layer 2)

Stainless Steel

(DHLW - $0.95 \mathrm{~cm}$ thick; DSNF - $0.63 \mathrm{~cm}$ thick)

(Layer 3)

Fuel Matrix

(Mixed Oxide Fuel)

(Layer 5 \& Matrix 3)

\section{Clad}

(Stainless Steel)

(Layer 4 \& Matrix 2)

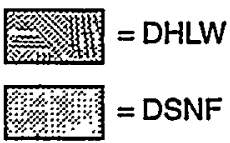

(Not to scale)

TRI.6342.5515-0

Figure 3-26. Codisposal configuration for Category 11, Mixed Oxide Fuel, including one DOE SNF handling container and four DHLW handling containers. Layers and matrices modeled in the 1997 PA are noted (see Section 3.4.1).

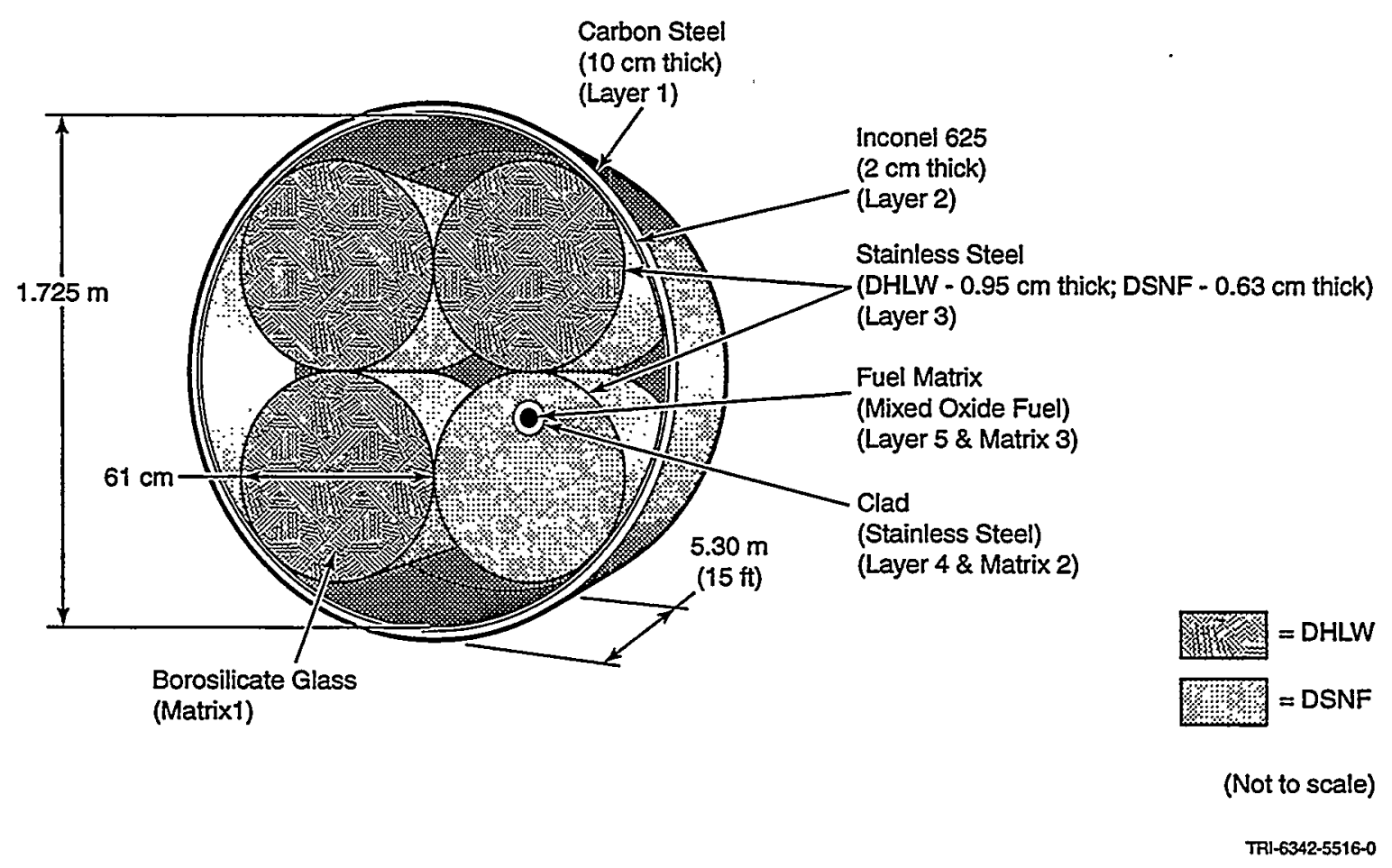

Figure 3-27. Codisposal configuration for Category 12, Uranium-Thorium Oxide, including one DOE SNF handling container and three DHLW handling containers. Layers and matrices modeled in the 1997 PA are noted (see Section 3.4.1). 


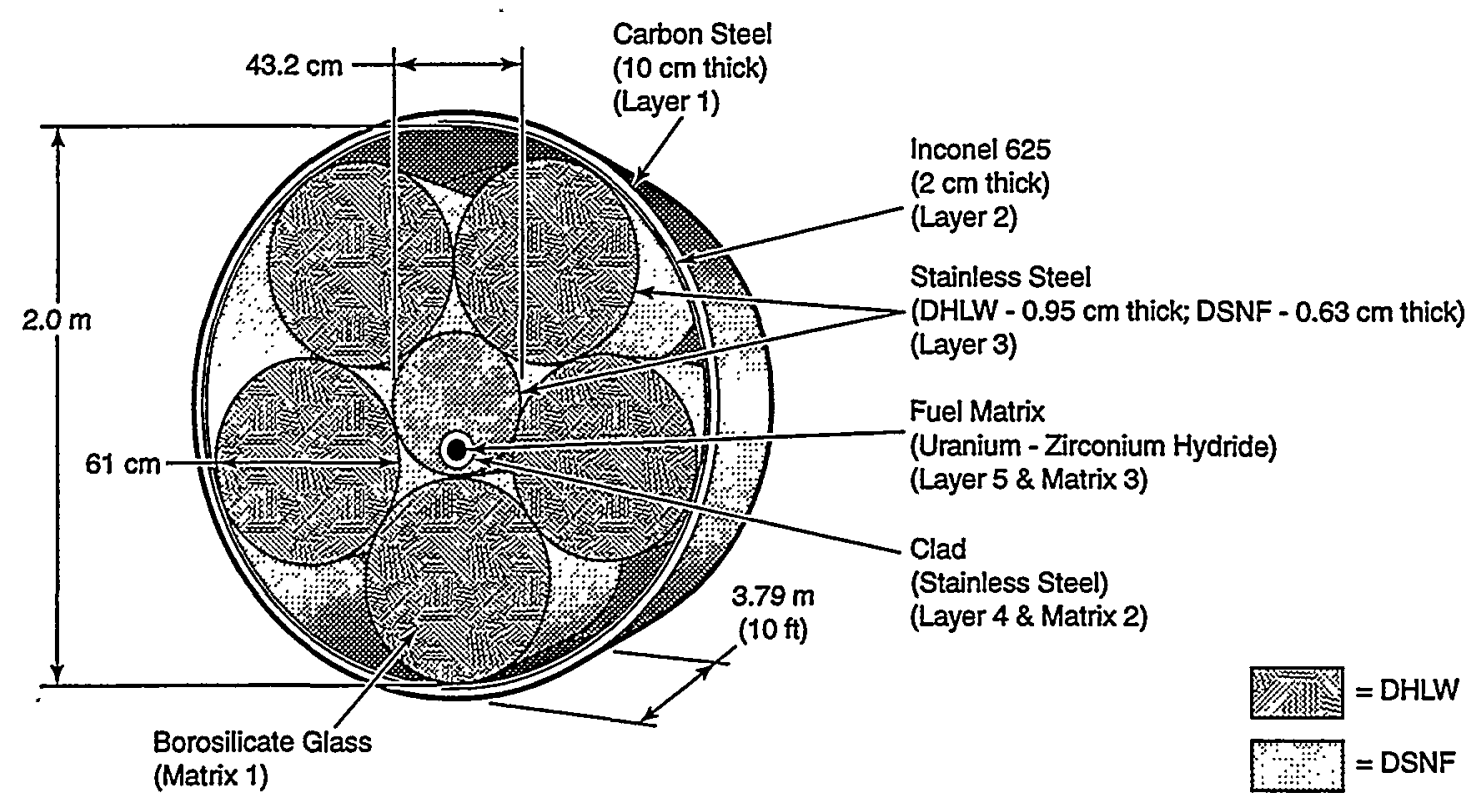

(Not to scale)

TRI- $\$ 342-5517-0$

Figure 3-28. Codisposal configuration for Category 13, Uranium/Zirconium Hydride, including one DOE SNF handling container and five DHLW handling containers. Layers and matrices modeled in the 1997 PA are noted (see Section 3.4.1).

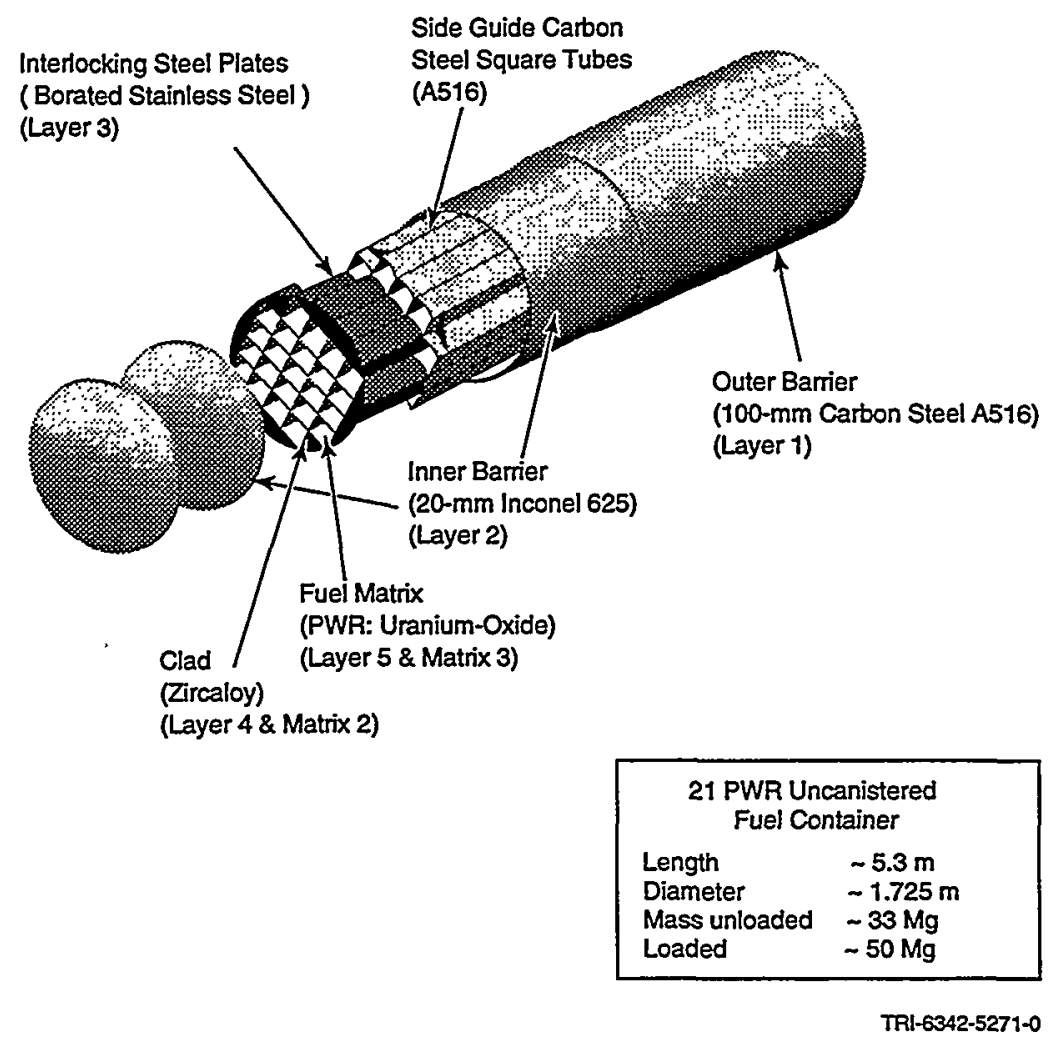

Figure 3-29. Disposal configuration for Category 14, 21-PWR. Layers and matrices modeled in the 1997 PA are noted (see Section 3.4.1). 
3. System Characterization: Waste Packages

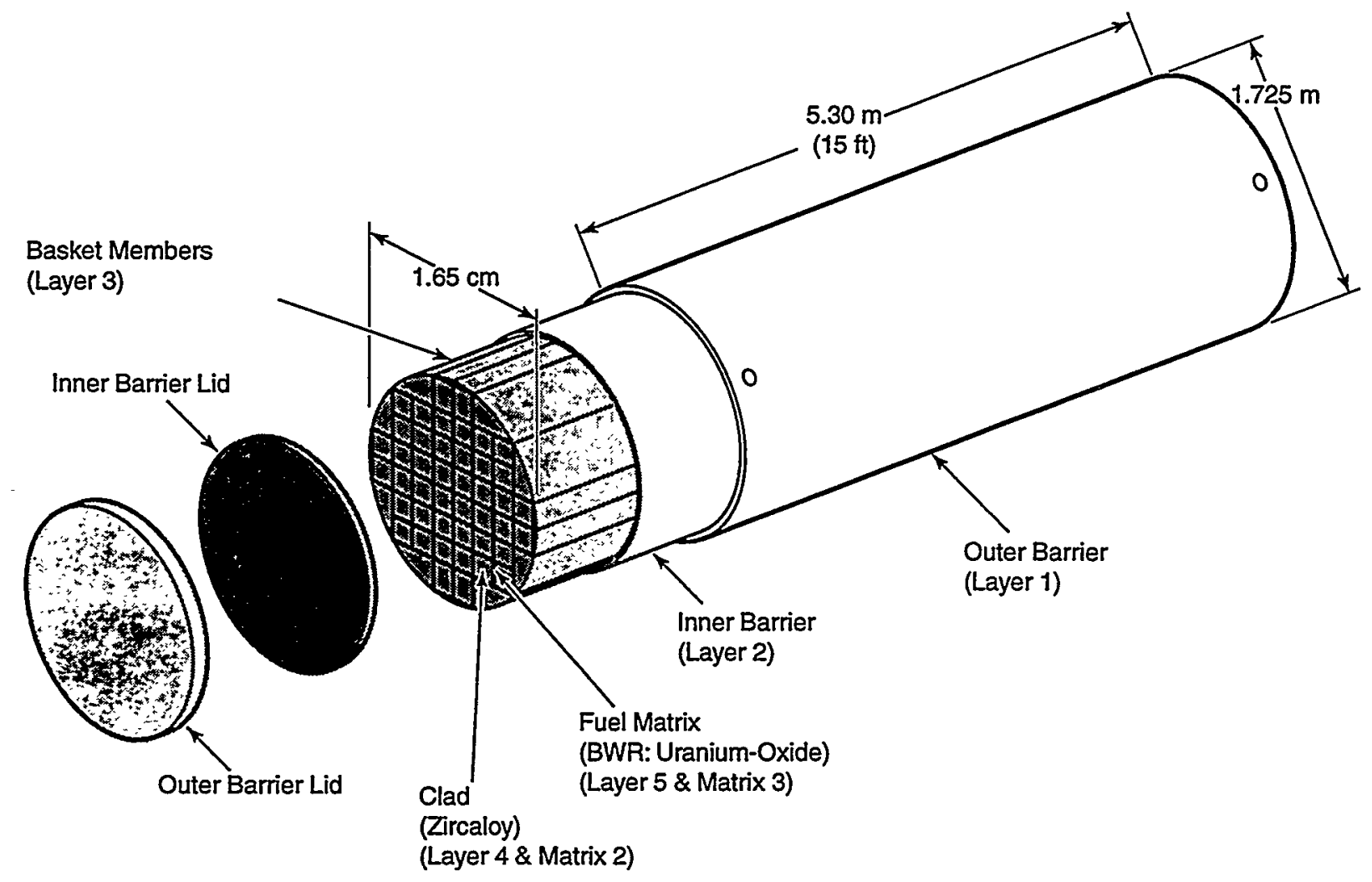

TAI-6342-5519-0

Figure 3-30. Disposal configuration for Category 15, 44-BWR. Layers and matrices modeled in the 1997 PA are noted (see Section 3.4.1). 


\subsection{Waste Packages as Modeled}

The waste package is the modeling unit in a performance assessment that represents the radioactive fuel and its protective layers. In the 1997 PA, 15 types of waste packages are modeled, which represent the 13 DOE SNF categories, codisposed with $\mathrm{DHLW}$, and the 2 commercial fuel categories in their handling and/or disposal containers. The intent of the 1997 PA is to compare the performance of each waste package type containing DOE SNF/DHLW with the commercial waste packages.

To properly model the waste packages, analysts must describe the type and amount of radioactive fuel in the package, including its activity and thermal power, and the degree of protection from corrosion. Table 3-3 summarizes information about the waste package types as modeled, including the number of packages per spent fuel category modeled in the 1997 PA; the DOE SNF/DHLW configuration, if applicable; and the mass, radioactivity, and thermal power per waste package.

Table 3-3. Waste Package Types Modeled in the 1997 Performance Assessment

\begin{tabular}{|c|c|c|c|c|c|c|c|c|c|}
\hline \multirow[b]{2}{*}{$\begin{array}{c}\text { Waste } \\
\text { Package } \\
\text { ID }\end{array}$} & \multirow[b]{2}{*}{$\begin{array}{l}\text { Number of } \\
\text { Waste } \\
\text { Packages in } \\
\text { Repository }\end{array}$} & \multirow[b]{2}{*}{$\begin{array}{l}\text { Configur- } \\
\text { ation } \\
\text { (DOE } \\
\text { SNF X } \\
\text { DHLW) }\end{array}$} & \multicolumn{5}{|c|}{ Mass per Waste Package } & \multicolumn{2}{|c|}{ Inventory at 2030} \\
\hline & & & $\begin{array}{c}\text { Average } \\
\text { Gross } \\
\text { (Mg) }\end{array}$ & $\begin{array}{c}\text { DOE SNF } \\
\text { Regulatory } \\
\text { (reported) } \\
\text { (MTHM) }\end{array}$ & $\begin{array}{c}\text { DOE SNF } \\
\text { Heavy } \\
\text { Metal } \\
\text { (inventory) } \\
\text { (MTHM) }\end{array}$ & $\begin{array}{l}\text { DHLW } \\
\text { Regula- } \\
\text { tory } \\
\text { (repor- } \\
\text { ted) } \\
\text { (MTHM) }\end{array}$ & $\begin{array}{c}\text { Fissile } \\
\text { (kg) }\end{array}$ & $\begin{array}{c}\text { Initial } \\
\text { Activity } \\
\text { (Ci) }\end{array}$ & $\begin{array}{c}\text { Initial } \\
\text { Thermal } \\
\text { Power } \\
\left(\mathrm{W} / \mathrm{m}^{3}\right)\end{array}$ \\
\hline 1 & 118 & $4 \times 0 \times s t-1^{*}$ & 66.41 & 18.10 & 18.07 & 0 & 60.7 & $1.15 \times 10^{5}$ & 64.02 \\
\hline 2 & 9 & $1 \times 5 \times s u-1$ & 47.6 & $4.01 \times 10^{-3}$ & $4.51 \times 10^{-3}$ & 3.75 & - & $5.61 \times 10^{4}$ & 20.60 \\
\hline 3 & 55 & $1 \times 4 \times s t-s$ & 33.7 & 0.0714 & 7.652 & 2.00 & 18.2 & $2.46 \times 10^{4}$ & 17.39 \\
\hline 4 & 203 & $1 \times 5 \times s u-s$ & 34 & 0.471 & 0.393 & 2.50 & -- & $1.06 \times 10^{5}$ & 53.50 \\
\hline 5 & 595 & $1 \times 4 \times s t-s$ & 26.3 & 0.193 & 0.148 & 3.00 & 0.65 & $4.89 \times 10^{4}$ & 32.97 \\
\hline 6 & 750 & $1 \times 5 \times s u-J$ & 33.7 & 0.012 & 0.012 & 3.75 & 7.09 & $6.68 \times 10^{4}$ & 24.32 \\
\hline 7 & 225 & $1 \times 5 \times s u-1$ & 47.67 & 0.050 & 0.057 & 3.75 & 4.39 & $5.66 \times 10^{4}$ & 20.66 \\
\hline 8 & 545 & $1 \times 5 \times s u-1$ & 48.18 & 0.0023 & 0.045 & 3.75 & 1.82 & $4.75 \times 10^{4}$ & 17.72 \\
\hline 9 & 103 & $1 \times 5 \times s u-1$ & 48.14 & 0.0020 & 0.016 & 3.75 & 1.72 & $4.52 \times 10^{4}$ & 16.82 \\
\hline 10 & 5 & $1 \times 4 \times s t-1$ & 36.65 & 0.029 & 0.011 & 3.00 & 5.641 & $4.89 \times 10^{4}$ & 25.59 \\
\hline 11 & 352 & $1 \times 4 \times s t-1$ & 36.64 & 0.027 & 0.011 & 3.00 & 7.18 & $4.49 \times 10^{4}$ & 23.17 \\
\hline 12 & 69 & $1 \times 3 \times s t-1$ & 33.97 & 0.0120 & 0.80 & 2.25 & 10.85 & $3.13 \times 10^{4}$ & 15.74 \\
\hline 13 & 102 & $1 \times 5 \times s u-s$ & 33.77 & 0.020 & 0.022 & 2.50 & 5.06 & $2.90 \times 10^{4}$ & 14.87 \\
\hline 14 & 4820 & $21 \mathrm{PWR}$ & - & 8.64 & 8.62 & 0 & - & $1.74 \times 10^{6}$ & 772.62 \\
\hline 15 & 2859 & 44 BWR & - & 7.87 & 7.57 & 0 & - & $1.29 \times 10^{6}$ & 536.88 \\
\hline
\end{tabular}




\subsubsection{Layers and Matrices per Waste Package}

The waste package layers and matrices modeled in the 1997 PA are identified in Figures 3-16 through 3-30. The total area and mass considered for the entire spent fuel category are labeled, per layer/matrix. In the 1997 PA, a layer represents a material that consumes or produces water or gas, i.e., protects or contributes to corrosion. A matrix represents a material that contains radioisotopes. For example, five or more protective layers must be penetrated in a waste package before release of radioisotopes, such as (1) outer layer of disposal container, (2) inner layer of disposal container, (3) handling container layer, (4) cladding, and (5) fuel. In the 1997 PA, CST monitors these layers for water and gas content and whether the layer has been breached. Examples of matrices are the fuel matrix, the cladding, and the borosilicate glass. Note that some materials, such as fuel matrix, are considered both a layer and a matrix, because they provide protection but also contain radionuclides. Matrices are used by CST, which predicts (1) radioactive decay and ingrowth of radionuclides, (2) distribution of radionuclides among aqueous and solid phases within the waste packages; and (3) the transport of radionuclides from the waste packages to the intact host rock. A listing of the layers and matrices modeled per waste package is provided in Modeling Parameters, Section 3.6.

\subsubsection{Radioisotope Inventory per Waste Package}

A total of 41 radioisotopes were considered in the inventory provided by NSNFP, as listed in Appendix A. This inventory is the projected inventory of the spent fuel or high-level waste for disposal in a repository by the year 2030 . INEEL determined the inventory for each spent fuel category with the computer code ORIGEN2 (Oak Ridge Isotope Generation), which is widely accepted for calculating spent fuel radioisotope inventories (ORNL, 1996).

The specific radioisotopes included in the inventory were selected based on screening that was conducted for TSPA-1993 (Andrews et al., 1994). Radioisotopes were screened by means of two methods. First, they were screened on the basis of the ratio of their inventory to the release limits specified in Table I of 40 CFR 191 (EPA, 1995). The fractional contribution to release was calculated assuming a delay caused by a combination of waste package lifetime and retarded transport over time. Radioisotopes that contributed to at least a fraction of the EPA's release limit at any of four selected times $(1000,10,000,100,000$, and $1,000,000 \mathrm{yr})$ passed this screening. The entire decay chain for daughters that contributed greater than $10^{-5}$ of the EPA release limit at any time was also included (Andrews et al., 1994). The second method was to make an additional selection of radioisotopes based on their contribution to dose for the four selected time periods (see M\&O, 1997, for the manner in which dose was calculated). All radioisotopes contributing less than $10^{-5}$ of total dose at any of the four selected times were eliminated from the inventory, unless they were in the decay chain for daughters that contributed $10^{-5}$ of total dose at any time. The results of these screening methods yielded the 41 radioisotopes listed in Appendix A.

To estimate the inventory of the 41 radioisotopes per spent fuel category, INEEL ran ORIGEN2 to model the spent fuel types (based on information in the National Spent Fuel Data Base [NSFDB]) (DOE, 1996). Required input for ORIGEN2 calculations includes fuel core composition, power history of the reactor, operating conditions of the reaction, and the nuclear cross section libraries for each fuel type. If actual data for a spent fuel type were not available, then those input data were estimated. For each fuel entry selected in the category, a representative ORIGEN2 run was made. The inventory was then calculated as a scaled amount based on the uranium content of each spent fuel type in the category. Totals for the category were then calculated for each of the 41 radioisotopes.

Spent fueis by category for which ORIGEN2 runs were used directly are as follows:

- Category 1 (N-Reactor fuel)

- Category 2 (none-represented by ATR ORIGEN2 run)

- Category 3 (Enrico Fermi Reactor fuel)

- Category 4 (Commercial Pressurized Water Reactor [PWR], Pathfinder, Power Burst Facility [PBF], Transient Reactor Test fuel)

- Category 5 (Pulstar Buffalo, Three Mile Island)

- Category 6 (Advanced Test Reactor [ATR]) 
- Category 7 (none-represented by ATR ORIGEN2 run)

- Category 8 (Fort St. Vrain, General Atomics-High Temperature Gas Cooled Reactor [GA-HTGR])

- Category 9 (Peach Bottom)

- Category 10 (Fast Flux Test Facility [FFTF] Carbide)

- Category 11 (FFTF Oxide)

- Category 12 (Shippingport LWBR)

- Category 13 (Training Research Isotopes production-General Atomics [TRIGA])

Results from these spent fuels were also used as templates for fuels for which no direct runs were available (e.g., Categories 2 and 7).

The inventory calculated in this manner by INEEL was a total per DOE SNF category.

Mass of Heavy Metal per Waste Package. Table 3-3 lists the mass of heavy metal per fuel category for both regulatory reporting and as modeled in the 1997 PA. The regulatory mass for DOE SNF, as provided by INEEL in Appendix A, reported only the uranium mass, omitting the inventory of plutonium and thorium. Consequently, for consistency, the mass of heavy metal was adjusted in the 1997 PA to match the inventory of radioisotopes and the matrix masses were adjusted to include plutonium and thorium. Future work can more thoroughly examine these discrepancies and determine the best approach for achieving consistency between the total inventory and the reported mass of heavy metal used for regulatory accounting and comparison.

The MTHM equivalents for all DHLW were estimated as 9842 based on note 1(d) of Appendix of 40 CFR 191 . When the DHLW mass of heavy metal is calculated as 0.5 MTHM per DHLW handling container per short (3.79 m) waste package, and 0.75 MTHM per DHLW handling container per long $(5.3 \mathrm{~m})$ waste package, as described in Appendix A of this report, the value is 9409.

Activity per Waste Package. Radionuclide inventory is the amount of radioactive elements in a fuel and is usually given in curies per metric ton of heavy metal (MTHM) initially in the fuel. For the 1997 PA, the analysts calculated the activity per DOE SNF/DHLW package by adding the DHLW inventory to the DOE SNF inventory per package by category. Table 3-3 and Figure 3-31 shows the initial activity per waste package by category used in the 1997 PA.

The activity per handling container of spent fuel (see Appendix A) was calculated by NSNFP for the DOE SNF categories by dividing the cumulative curie count for each fuel category by the total number of packages in the category. The number of handling containers was determined by adding the values for the "repository pkg count" row for each spent fuel category listed in Appendix A. Composite spreadsheet totals of the curies in a particular category were apportioned equally across the total number of packages in that category. This method resulted in a mean value per handling container that assumed containers within a category were the same size. Thus, no distinction in curie inventory was made among the differing diameters or container lengths. The 1997 PA used the inventory by DOE SNF handling container as provided by NSNFP.

In the $1997 \mathrm{PA}$, the DHLW inventory per waste package was calculated using the data in Appendix A, Table A-9, which is the same as that used in TSPA-1995. Note that the inventory as listed represents four standard handling containers. For each DOE SNF category, the 1997 PA calculated the DHLW inventory based on the codisposal configuration selected. For example, if the configuration was $1 \times 4 \times$ short, then the inventory in Table A-9 was used as is per waste package. If the selected configuration was $1 \times 5 \times$ long, then the inventory in Table A-9 was multiplied by $5 / 4$ (to account for $5 \mathrm{DHLW}$ packages instead of 4 ) and then by 1.5 (to account for a long waste package, rather than the short).

Thermal Power per Waste Package. The heat generated by the fuel within the waste packages is significant to the repository environment. A repository design consideration is. the spacing of waste packages to ensure a hot repos-

Repository package in Appendix $A$ is equivalent to waste package. 


\section{System Characterization: Waste Packages}

itory (see Chapter 5). Values for the heat generated by the spent fuels after disposal are also essential to computations of near-field hydrologic response, which affects corrosion rates of the packages.

For the 1997 PA, the activity inventories and decay parameters (e.g., half-lives) were used to obtain an initial thermal power and a thermal power time history for each waste package type modeled and for the entire inventory (see Table 3-3 and Figure 3-32). In the initial thermal power calculations, two additional radioisotopes were added to the 41 radioisotopes listed in Appendix A, because the heat generated by barium and yttrium, daughters of ${ }^{135} \mathrm{Cs}$ and ${ }^{90} \mathrm{Sr}$, influence short-term heat calculations although their half-lives are not long enough to be significant with regard to potential radioactive release. In the $1997 \mathrm{PA}$, further thermal conduction and radiative transfer calculations were performed to generate temperatures per waste package after disposal (see Chapter 8, Section 8.2) to predict the effect of heat on fuel performance.

Radioisotopes Considered in the 1997 PA. A total of 43 radioisotopes were considered in the performance assessment for the heat generation model (Table 3-4). This total includes the 41 radioisotopes identified by NSNFP (Appendix A), plus two additional radioisotopes-barium and yttrium, daughters of ${ }^{135} \mathrm{Cs}$ and ${ }^{90} \mathrm{Sr}$ - which were added because they contribute to heat in the short term, although they did not pass the radioisotope screening performed by INEEL, as described above. The 41 radioisotopes identified by NSNFP were modeled by CST in the source term submodel to predict their dissolution and solubility when exposed to groundwater and subsequent effects on releases. Only three of the 41 radioisotopes $-{ }^{129} \mathrm{I},{ }^{237} \mathrm{~Np},{ }^{99} \mathrm{Tc}$-were considered in the transport calculations because earlier studies had indicated that these radioisotopes were the most significant with regard to release.

For the DHLW, a total of 49 radioisotopes were considered. Like the 1994 PA, the 1997 PA used the inventory provided in the 1992 DOE report on Characteristics of Potential Repository Waste (DOE, 1992; Rechard, ed., 1995). The initial activities (in curies) of the 49 radioisotopes considered for the 1997 PA are shown in Figures 3-33 through $3-48$. 


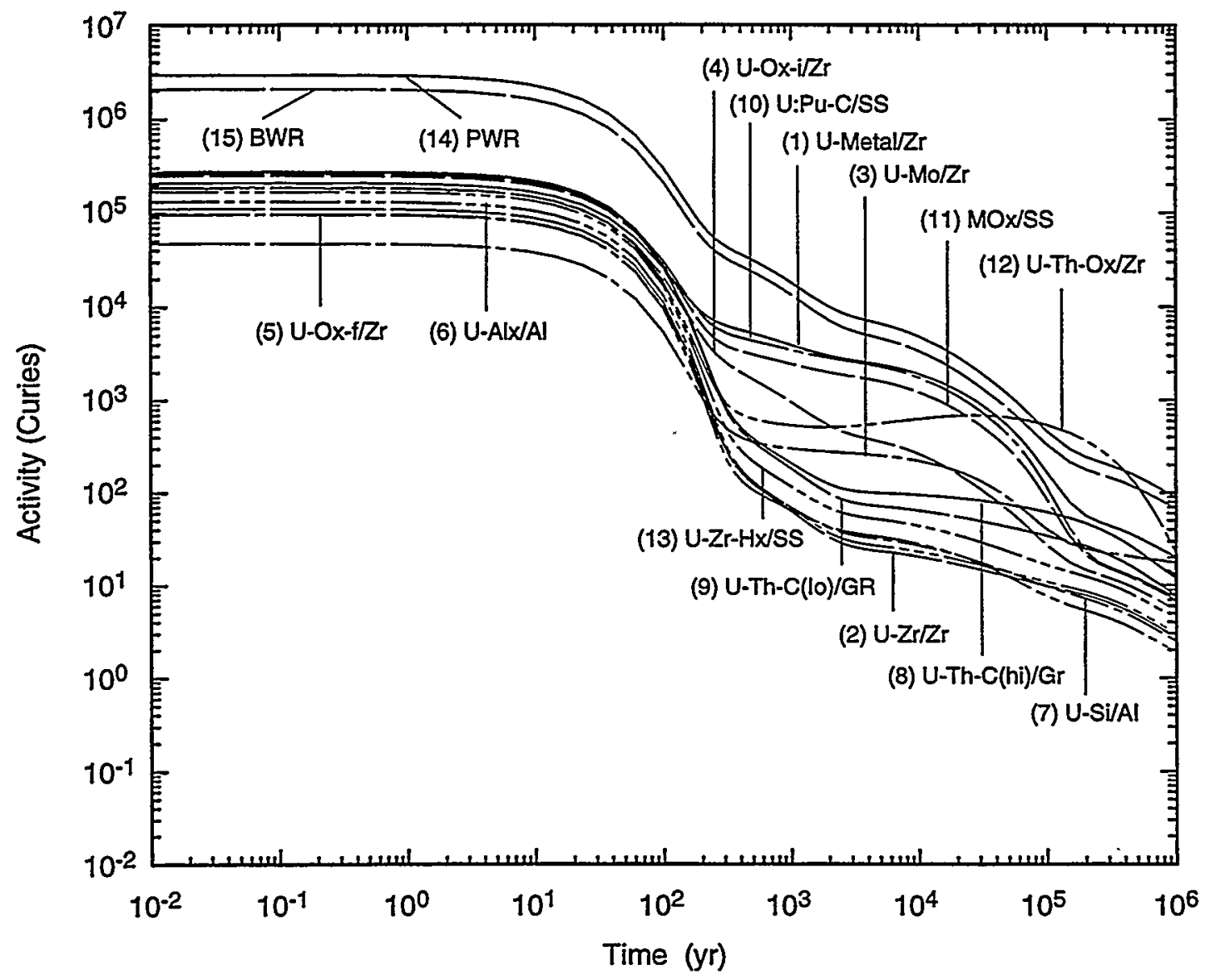

TRI-6342-5268-0

Figure 3-31. Curie content by waste package. 


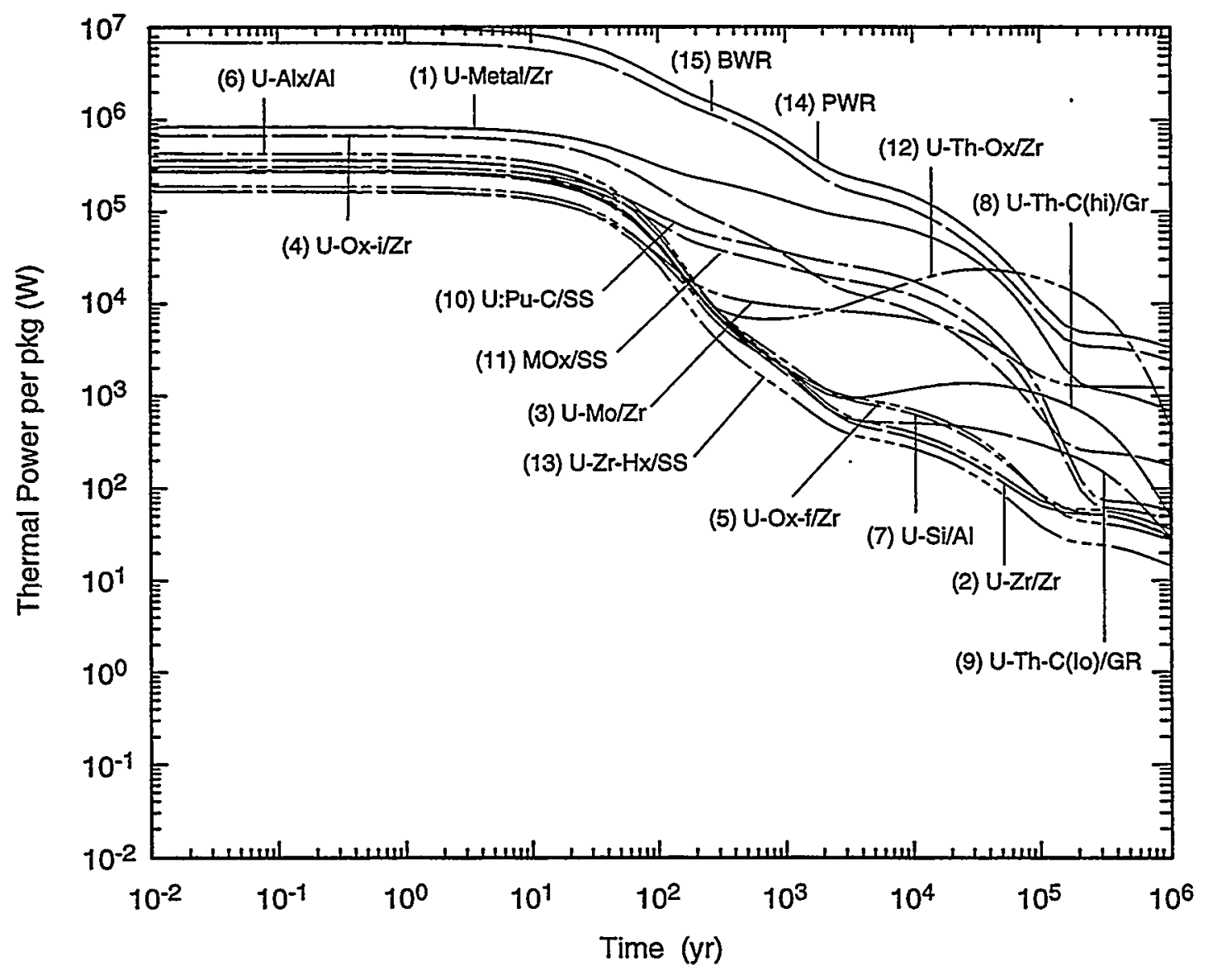

TRDI-6342-5269-0

Figure 3-32. Thermal power by waste package, based on total of 43 radioisotopes. 
Table 3-4. Radioisotopes Considered in 1997 Performance Assessment

\begin{tabular}{|c|c|c|c|}
\hline $\begin{array}{l}\text { Element } \\
\text { Name }\end{array}$ & $\begin{array}{l}\text { Radioisotopes Included } \\
\text { in Heat Calculations }\end{array}$ & $\begin{array}{l}\text { Radioisotopes Included in } \\
\text { Source-Term Model (CST) }\end{array}$ & $\begin{array}{l}\text { Radioisotopes Included in } \\
\text { Transport Model (NUTS) }\end{array}$ \\
\hline Actinium & ${ }^{227} \mathrm{AC}$ & ${ }^{227} \mathrm{AC}$ & \\
\hline \multirow[t]{3}{*}{ Americium } & ${ }^{241} \mathrm{Am}$ & ${ }^{241} \mathrm{Am}$ & \\
\hline & ${ }^{242 m} \mathrm{Am}$ & ${ }^{242 m} \mathrm{Am}$ & \\
\hline & ${ }^{243} \mathrm{Am}$ & ${ }^{243} \mathrm{Am}$ & \\
\hline Barium & $137 \mathrm{~m}_{\mathrm{Ba}}$ & & \\
\hline Carbon & ${ }^{14} \mathrm{C}$ & ${ }^{14} \mathrm{C}$ & • \\
\hline Chlorine & ${ }^{36} \mathrm{Cl}$ & ${ }^{36} \mathrm{Cl}$ & \\
\hline \multirow[t]{3}{*}{ Curium } & ${ }^{244} \mathrm{Cm}$ & ${ }^{244} \mathrm{Cm}$ & \\
\hline & ${ }^{245} \mathrm{Cm}$ & ${ }^{245} \mathrm{Cm}$ & \\
\hline & ${ }^{246} \mathrm{Cm}$ & ${ }^{246} \mathrm{Cm}$ & \\
\hline \multirow[t]{2}{*}{ Cesium } & ${ }^{135} \mathrm{Cs}$ & ${ }^{135} \mathrm{Cs}$ & \\
\hline & ${ }^{137} \mathrm{Cs}$ & ${ }^{137} \mathrm{Cs}$ & \\
\hline lodine & 129 & 129 & 129 \\
\hline \multirow[t]{2}{*}{ Niobium } & ${ }^{93} \mathrm{~m} \mathrm{Nb}$ & $93 \mathrm{~m} \mathrm{Nb}$ & \\
\hline & ${ }^{94} \mathrm{Nb}$ & ${ }^{94} \mathrm{Nb}$ & \\
\hline \multirow[t]{2}{*}{ Nickel } & ${ }^{59} \mathrm{Ni}$ & ${ }^{59} \mathrm{Ni}$ & \\
\hline & $63 \mathrm{Ni}$ & ${ }^{63} \mathrm{Ni}$ & \\
\hline Neptunium & ${ }^{237} \mathrm{~Np}$ & ${ }^{237} \mathrm{~Np}$ & ${ }^{237} \mathrm{~Np}$ \\
\hline Protactinium & ${ }^{231} \mathrm{~Pa}$ & ${ }^{231} \mathrm{~Pa}$ & \\
\hline Lead & ${ }^{210} \mathrm{~Pb}$ & ${ }^{210} \mathrm{~Pb}$ & \\
\hline Palladium & ${ }^{107} \mathrm{Pd}$ & ${ }^{107} \mathrm{Pd}$ & \\
\hline \multirow[t]{5}{*}{ Plutonium } & ${ }^{238} \mathrm{Pu}$ & ${ }^{238} \mathrm{Pu}$ & \\
\hline & ${ }^{239} \mathrm{Pu}$ & ${ }^{239} \mathrm{Pu}$ & \\
\hline & ${ }^{240} \mathrm{Pu}$ & ${ }^{240} \mathrm{Pu}$ & \\
\hline & ${ }^{241} \mathrm{Pu}$ & ${ }^{241} \mathrm{Pu}$ & \\
\hline & ${ }^{242} \mathrm{Pu}$ & $242 \mathrm{pu}$ & \\
\hline \multirow[t]{2}{*}{ Radium } & ${ }^{226} \mathrm{Ra}$ & ${ }^{226} \mathrm{Ra}$ & \\
\hline & ${ }^{228} \mathrm{Ra}$ & ${ }^{228} \mathrm{Ra}$ & \\
\hline Selenium & ${ }^{79} \mathrm{Se}$ & ${ }^{79} \mathrm{Se}$ & \\
\hline Samarium & ${ }^{151} \mathrm{Sm}$ & ${ }^{151} \mathrm{Sm}$ & \\
\hline Tin & ${ }^{126} \mathrm{Sn}$ & ${ }^{126} \mathrm{Sn}$ & \\
\hline Strontium & ${ }^{90} \mathrm{Sr}$ & ${ }^{90} \mathrm{Sr}$ & \\
\hline Technetium & ${ }^{99} \mathrm{TC}$ & ${ }^{99} \mathrm{TC}$ & ${ }^{99} \mathrm{TC}$ \\
\hline \multirow[t]{3}{*}{ Thorium } & ${ }^{229} \mathrm{Th}$ & ${ }^{229} \mathrm{Th}$ & \\
\hline & ${ }^{230} \mathrm{Th}$ & ${ }^{230} \mathrm{Th}$ & \\
\hline & ${ }^{232} \mathrm{Th}$ & ${ }^{232} \mathrm{Th}$ & \\
\hline \multirow[t]{5}{*}{ Uranium } & ${ }^{233} \mathrm{U}$ & ${ }^{233} \mathrm{U}$ & \\
\hline & ${ }^{234} U$ & ${ }^{234} \mathrm{U}$ & \\
\hline & $235 \mathrm{U}$ & ${ }^{235} \mathrm{U}$ & \\
\hline & ${ }^{236} \mathrm{U}$ & ${ }^{236} \mathrm{U}$ & \\
\hline & ${ }^{238} U$ & ${ }^{238} \mathrm{U}$ & \\
\hline Yttrium & $90 Y$ & & \\
\hline Zirconium & ${ }^{93} \mathrm{Zr}$ & ${ }^{93} \mathrm{Zr}$ & \\
\hline
\end{tabular}


Total Curies of each Isotope used in Performance Assessment (The Sum of Category 1)

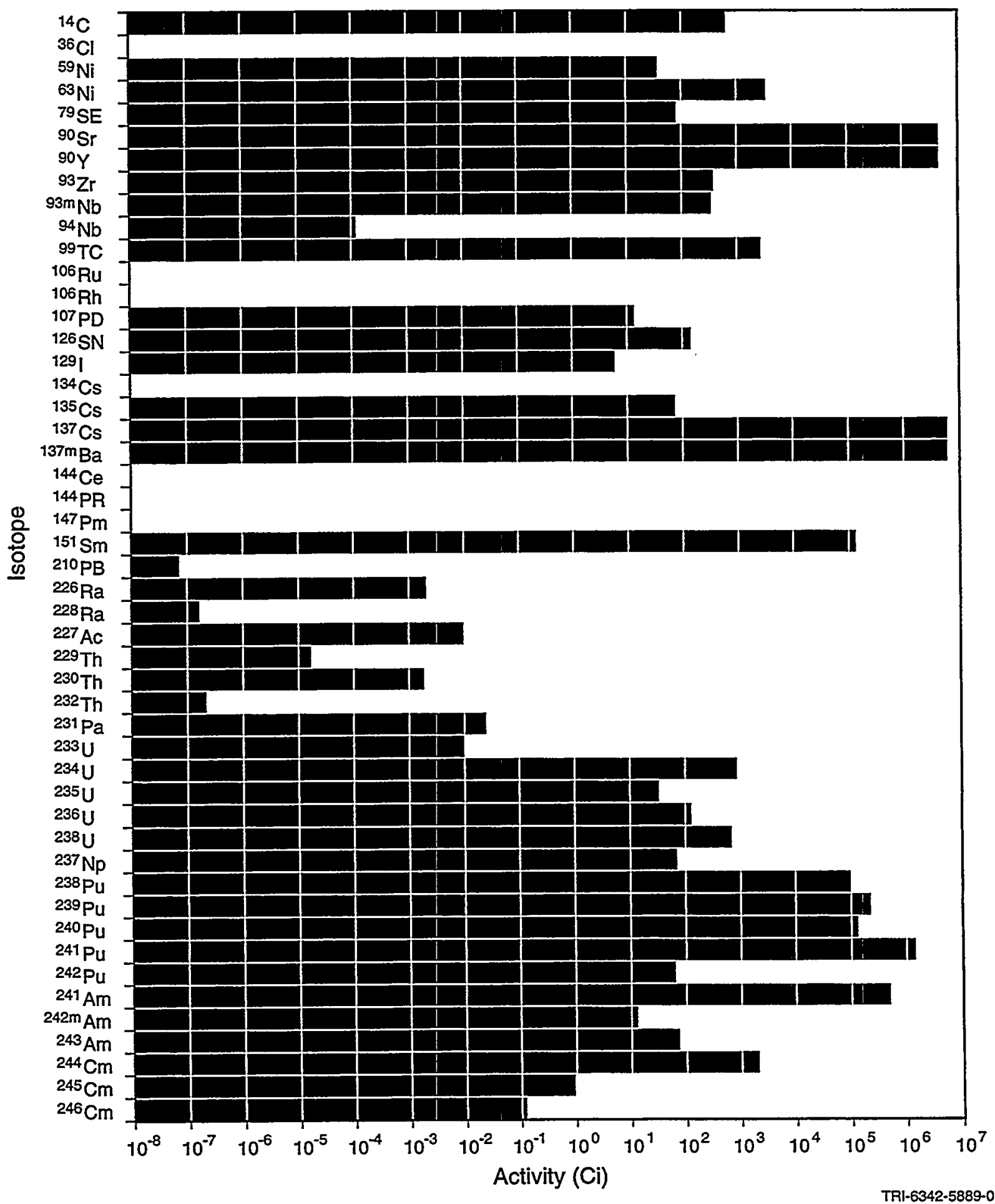

Figure 3-33. Initial (year 2030) activity of 49 radioisotopes considered in the performance assessment for waste packages with Category 1 fuel (uranium metal). 
Total Curies of each Isotope used in Performance Assessment (The Sum of Category 2)

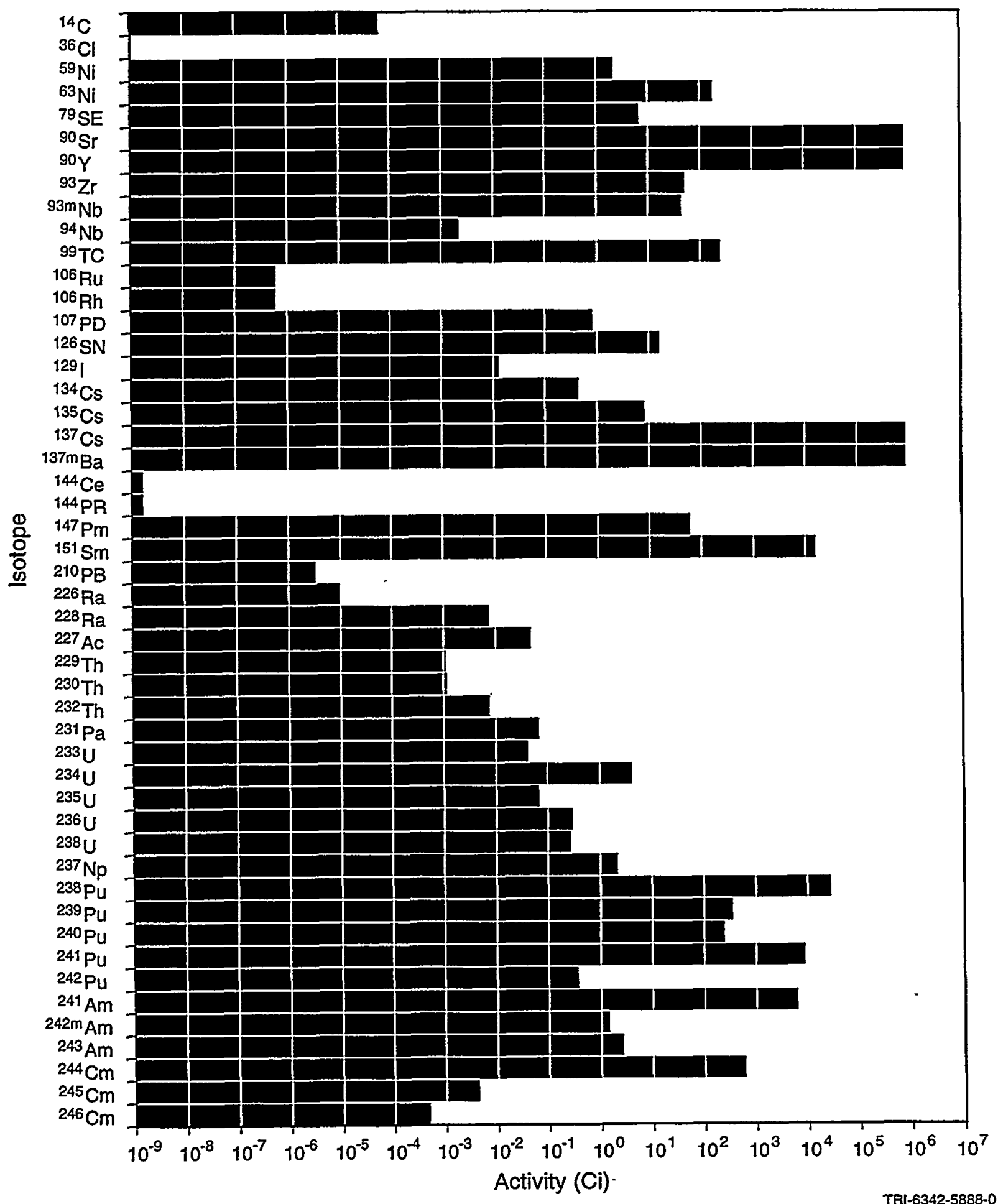

Figure 3-34. Initial (year 2030) activity of 49 radioisotopes considered in the performance assessment for waste packages with Category 2 fuel (uranium-zirconium alloy codisposed with DHLW). 
Total Curies of each Isotope used in Performance Assessment

(The Sum of Category 3)

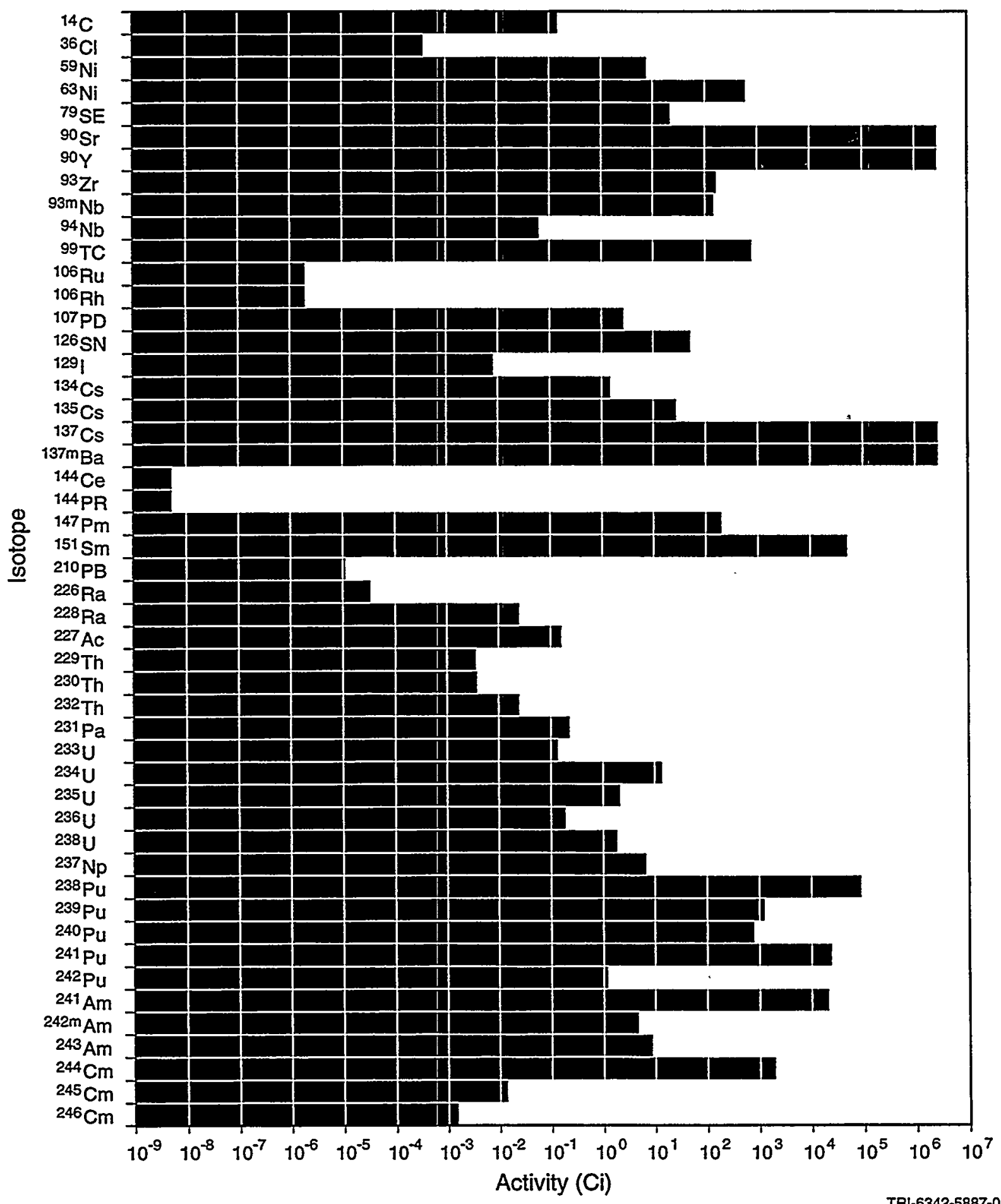

Figure 3-35. Initial (year 2030) activity of 49 radioisotopes considered in the performance assessment for waste packages with Category 3 fuel (uranium-molybdenum alloy codisposed with DHLW). 
Total Curies of each Isotope used in Performance Assessment (The Sum of Category 4)

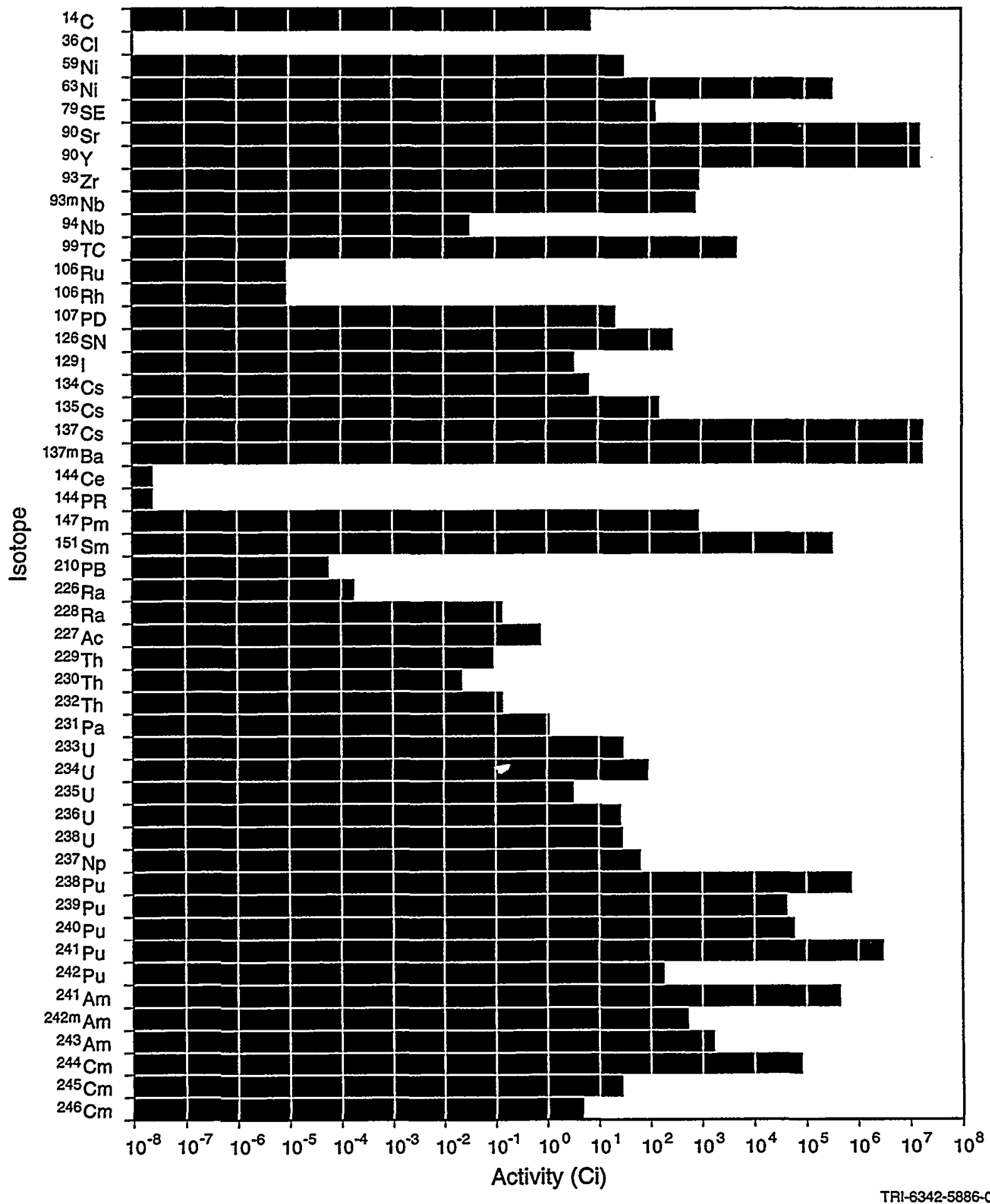

Figure 3-36. Initial (year 2030) activity of 49 radioisotopes considered in the performance assessment for waste packages with Category 4 fuel (uranium oxide - intact clad, codisposed with DHLW). 
Total Curies of each Isotope used in Performance Assessment (The Sum of Category 5)

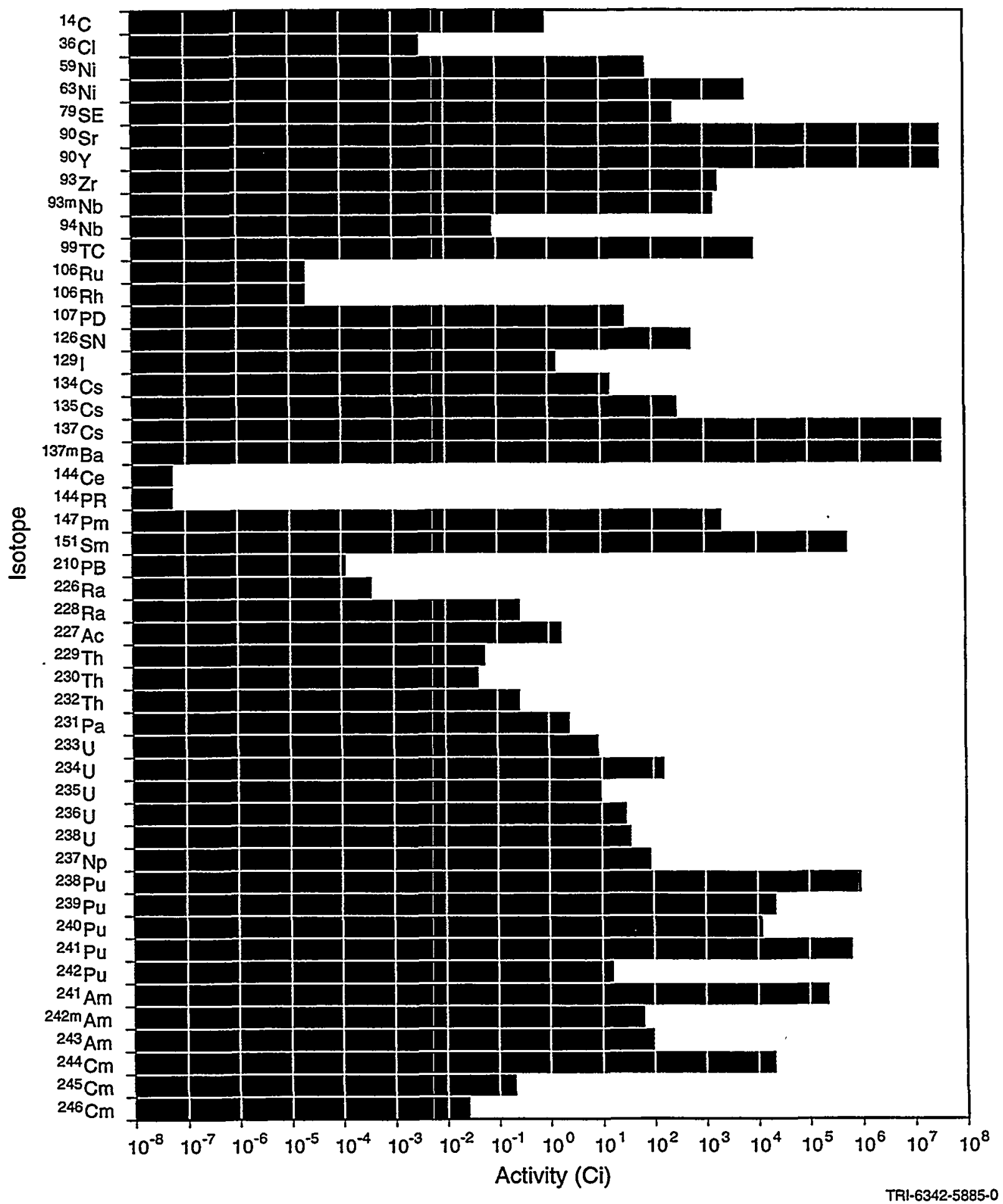

Figure 3-37. Initial (year 2030) activity of 49 radioisotopes considered in the performance assessment for waste packages with Category 5 fuel (uranium oxide - failed clad, codisposed with DHLW). 
Total Curies of each Isotope used in Performance Assessment (The Sum of Category 6)

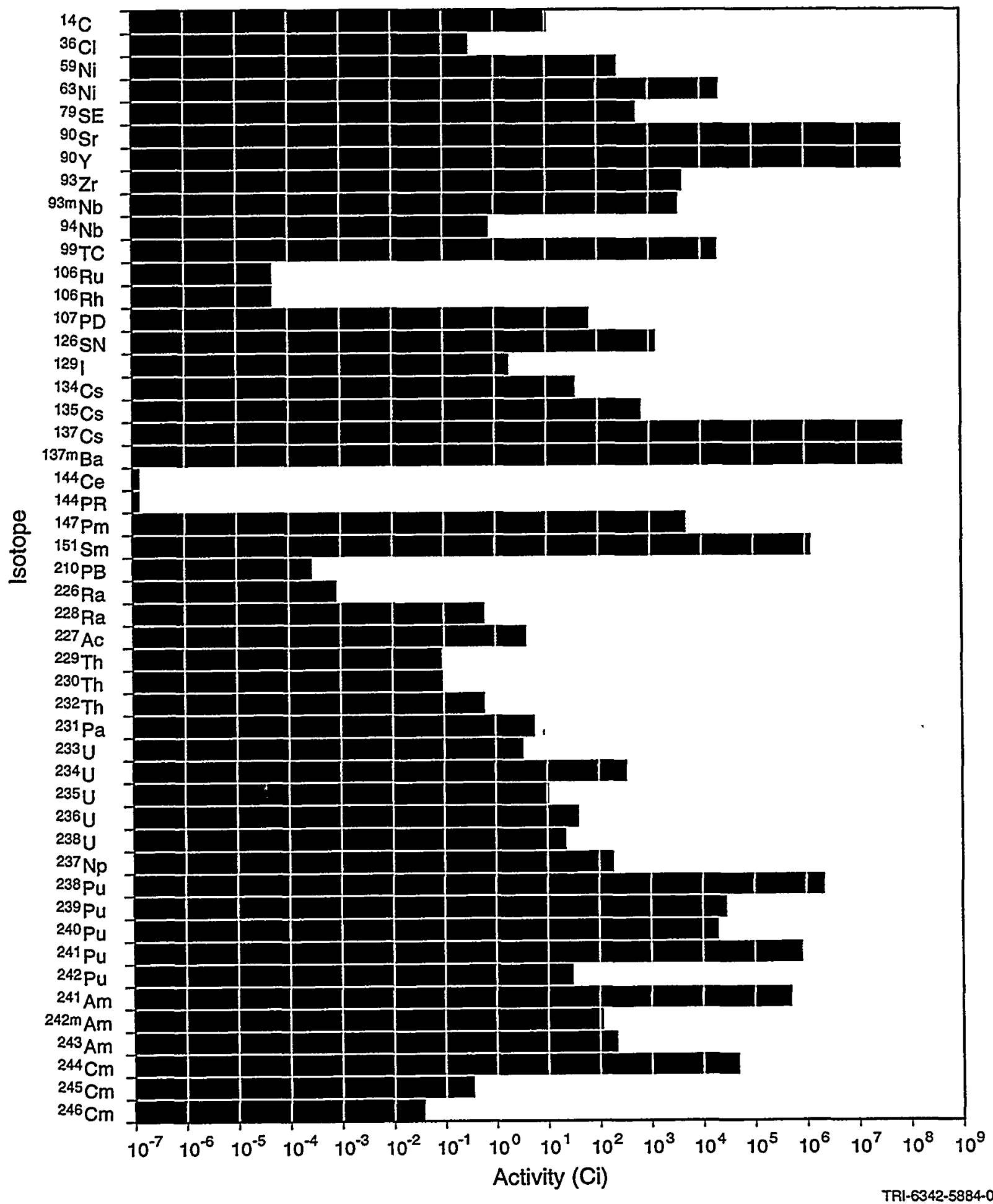

Figure 3-38. Initial (year 2030) activity of 49 radioisotopes considered in the performance assessment for waste packages with Category 6 fuel (uranium aluminum alloy, codisposed with DHLW). 
Total Curies of each Isotope used in Performance Assessment

(The Sum of Category 7)

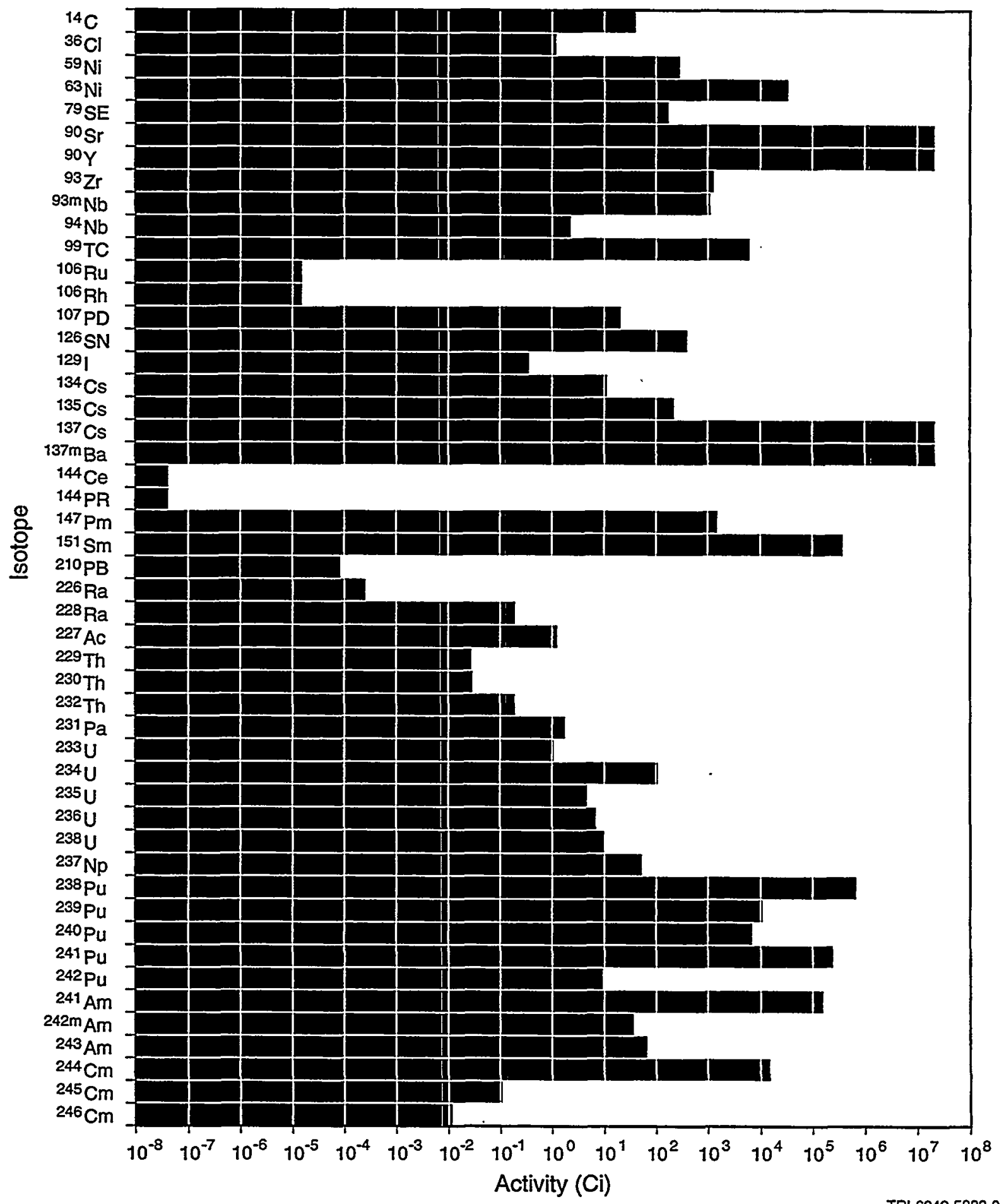

Figure 3-39. Initial (year 2030) activity of 49 radioisotopes considered in the performance assessment for waste packages with Category 7 fuel (uranium silicide codisposed with DHLW). 
Total Curies of each Isotope used in Performance Assessment (The Sum of Category 8)

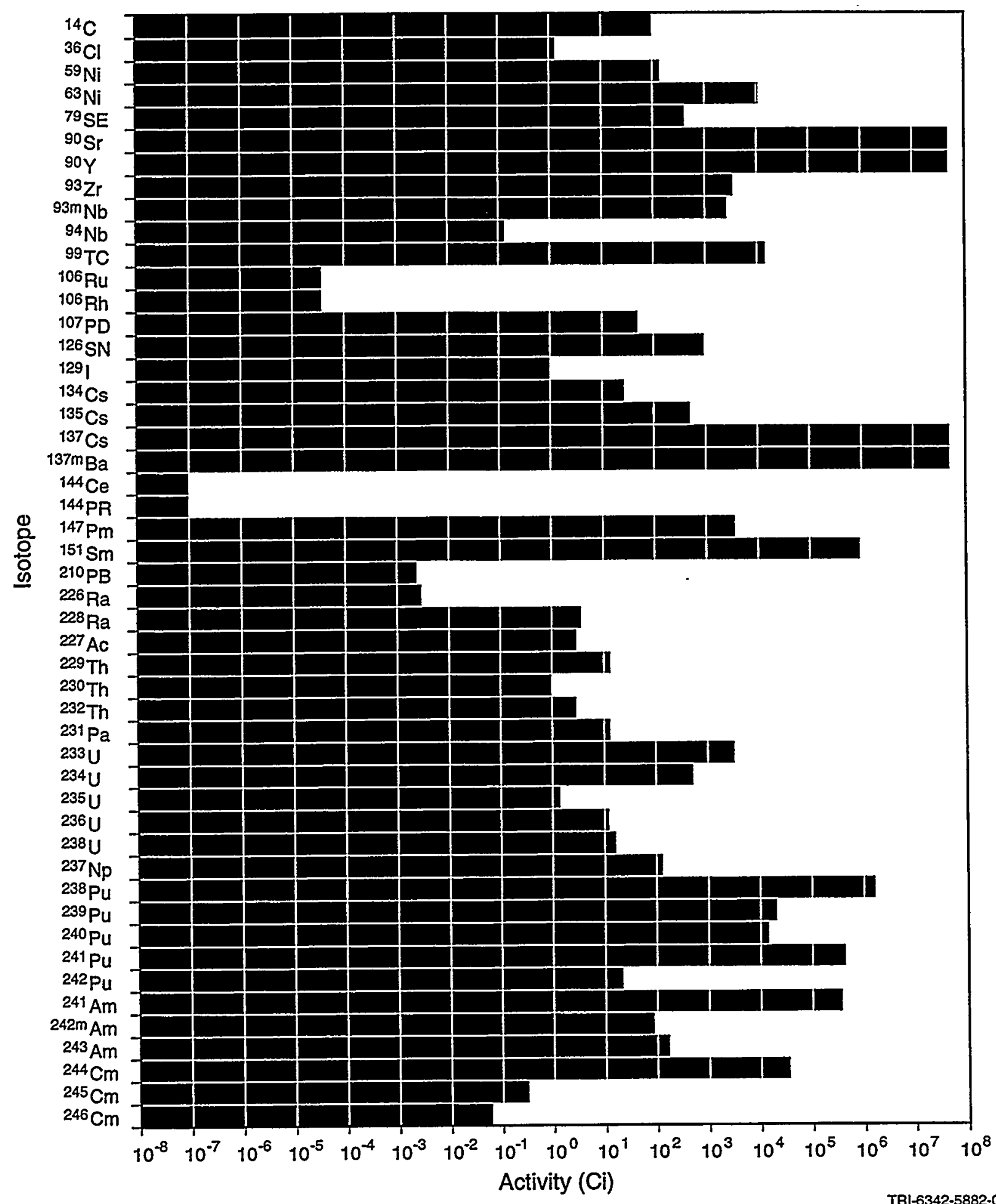

Figure 3-40. Initial (year 2030) activity of 49 radioisotopes considered in the performance assessment for waste packages with Category 8 fuel (uranium-thorium carbide - intact clad, codisposed with DHLW). 
Total Curies of each Isotope used in Performance Assessment (The Sum of Category 9)

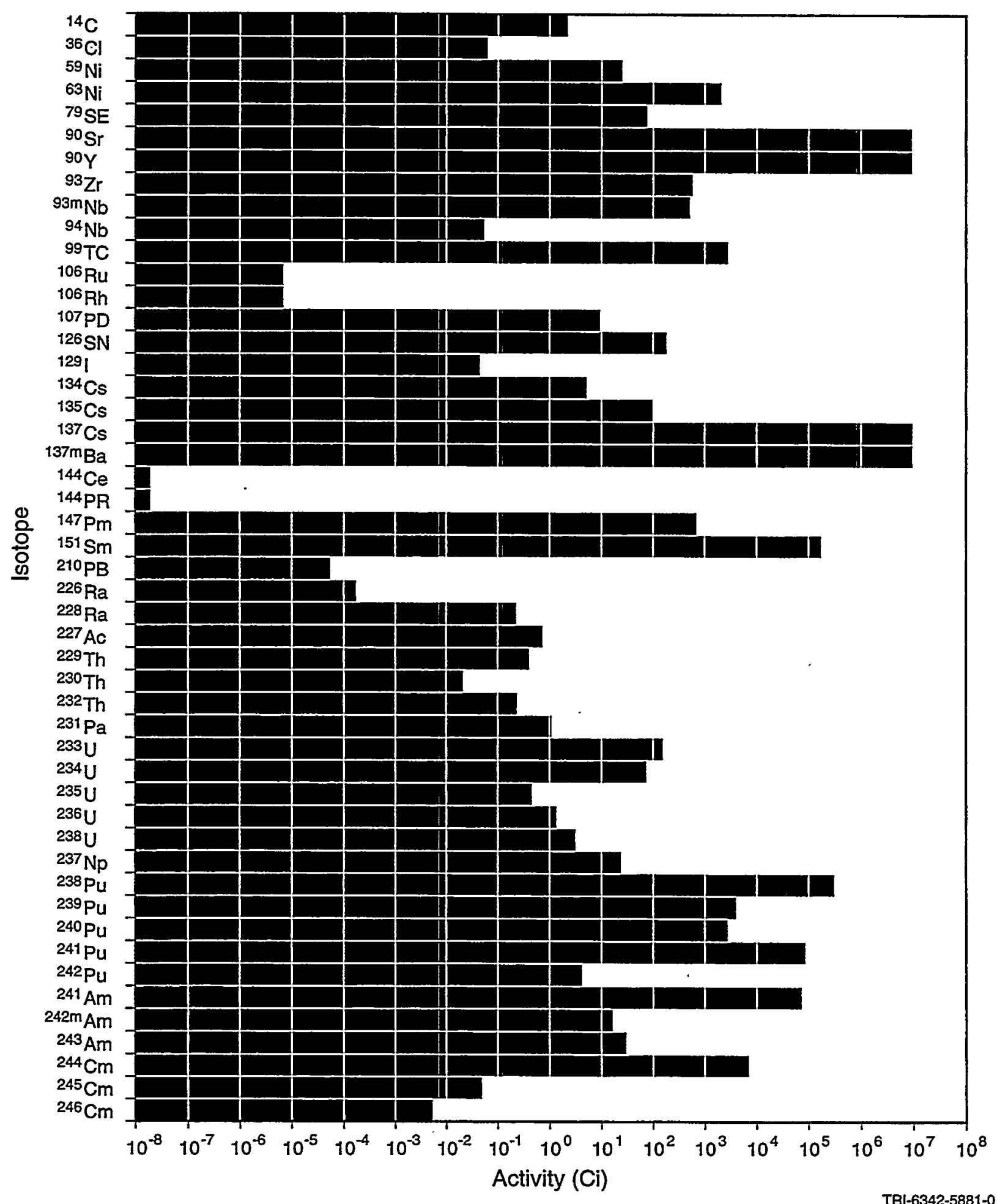

Figure 3-41. Initial (year 2030) activity of 49 radioisotopes considered in the performance assessment for waste packages with Category 9 fuel (uranium-thorium carbide - failed clad, codisposed with DHLW). 
Total Curies of each Isotope used in Performance Assessment (The Sum of Category 10)

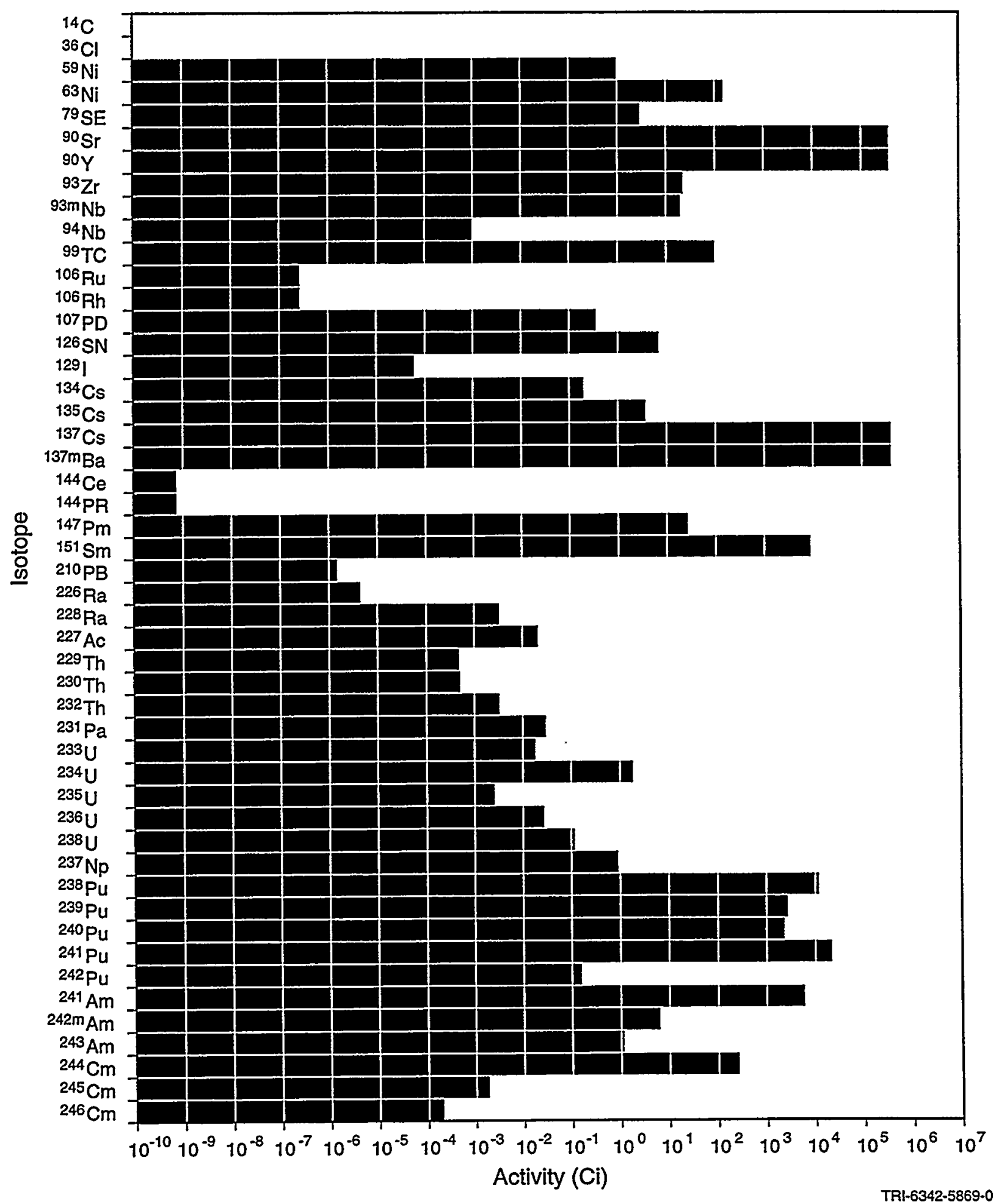

Figure 3-42. Initial (year 2030) activity of 49 radioisotopes considered in the performance assessment for waste packages with Category 10 fuel (uranium-plutonium carbide, codisposed with DHLW). 
Total Curies of each Isotope used in Performance Assessment (The Sum of Category 11)

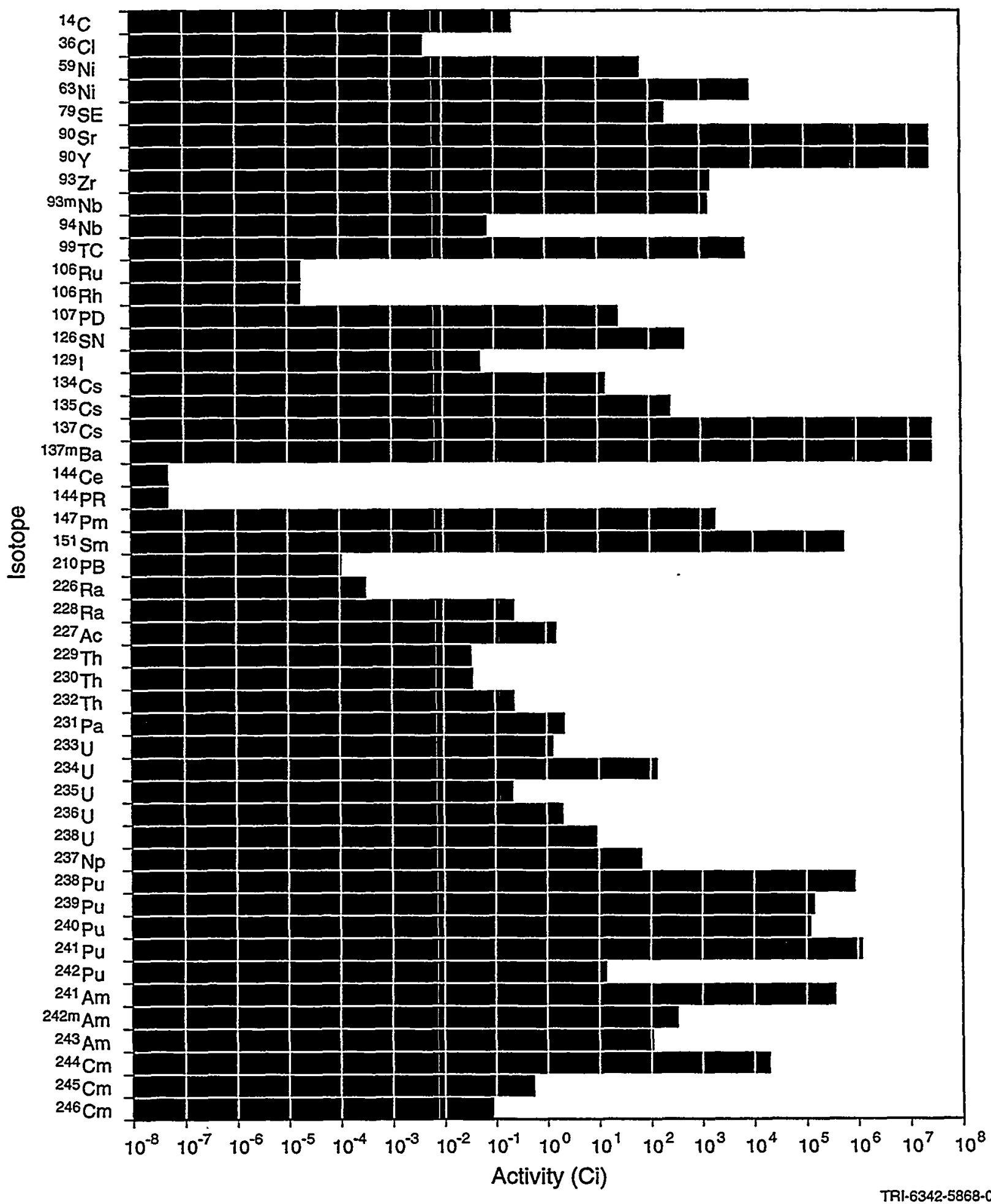

Figure 3-43. Initial (year 2030) activity of 49 radioisotopes considered in the performance assessment for waste packages with Category 11 fuel (mixed oxide fuel, codisposed with DHLW). 
Total Curies of each Isotope used in Performance Assessment (The Sum of Category 12)

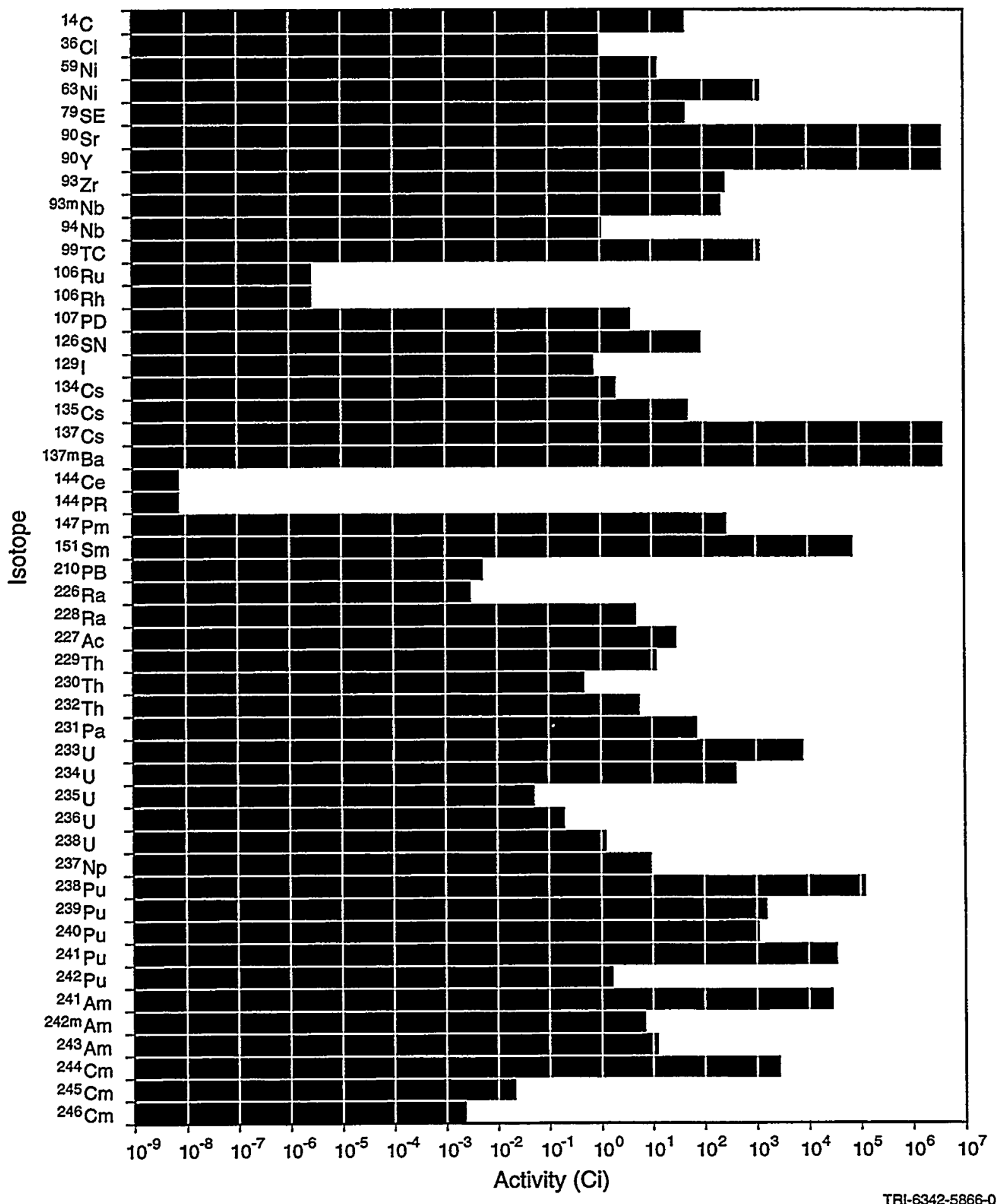

Figure 3-44. Initial (year 2030) activity of 49 radioisotopes considered in the performance assessment for waste packages with Category 12 fuel (uranium-thorium oxide, codisposed with DHLW). 


\section{Total Curies of each Isotope used in Performance Assessment}

(The Sum of Category 13)

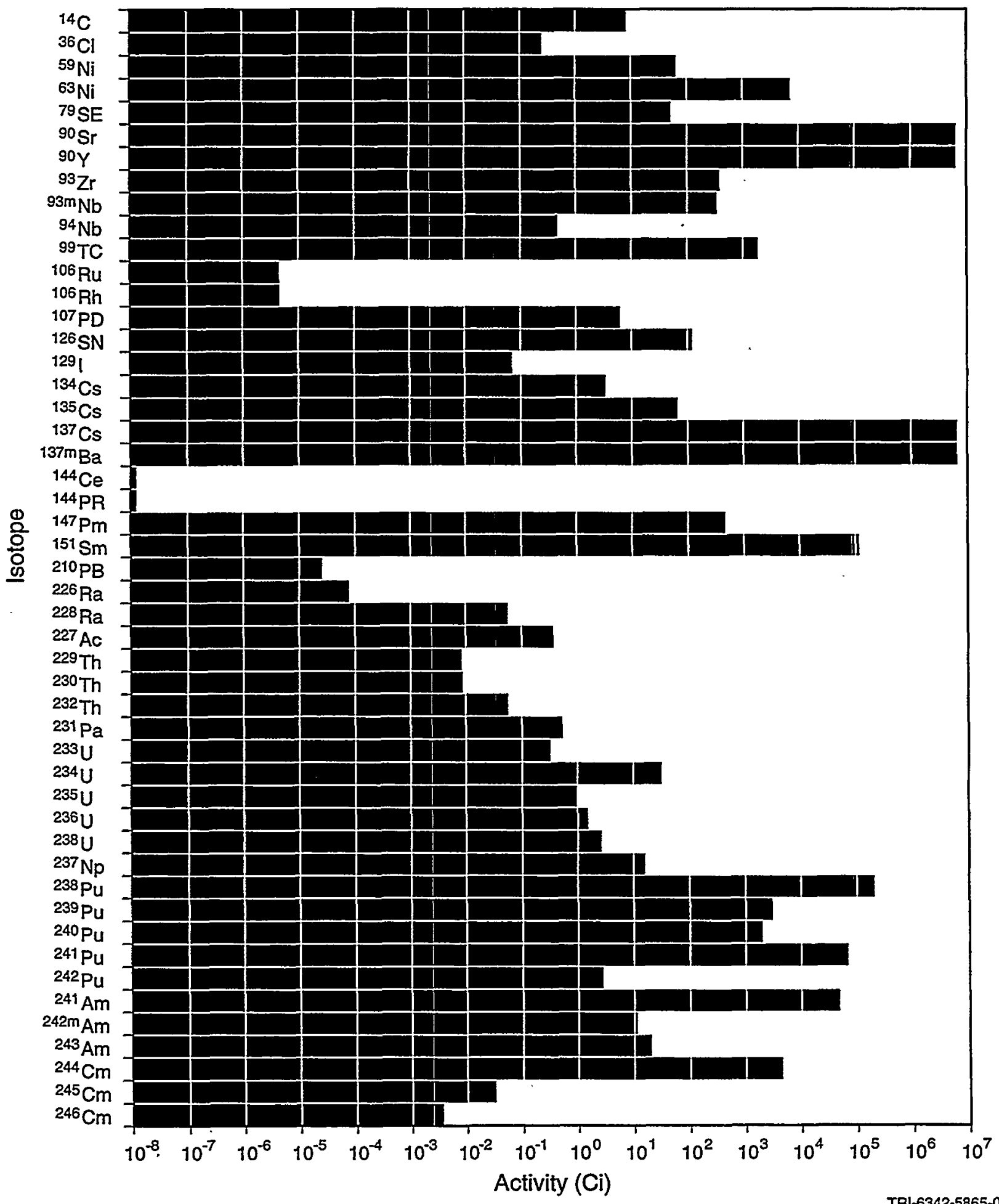

Figure 3-45. Initial (year 2030) activity of 49 radioisotopes considered in the performance assessment for waste packages with Category 13 fuel (uranium/zirconium hydride, codisposed with DHLW). 
Total Curies of each Isotope used in Performance Assessment (The Sum of Category 14)

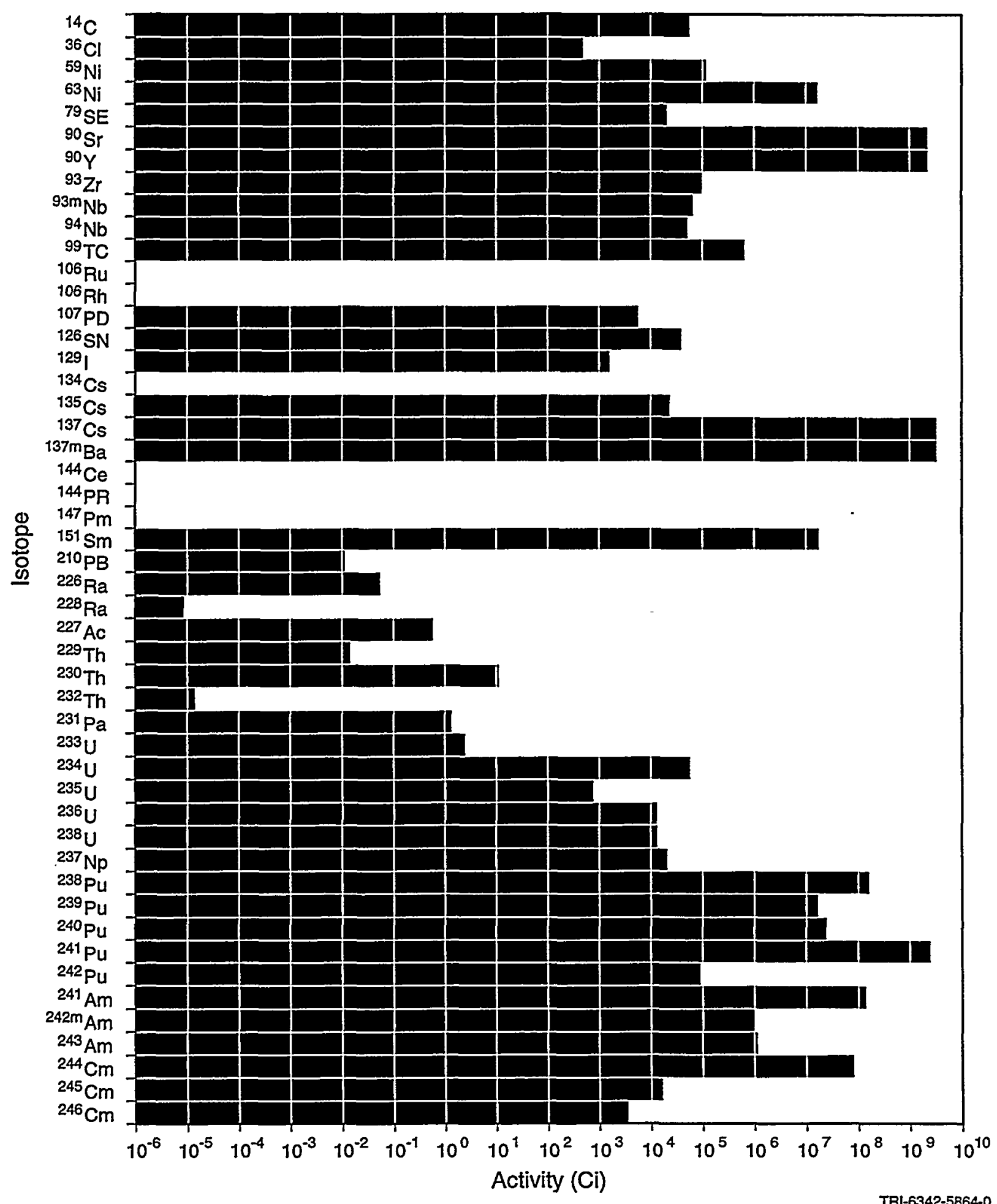

Figure 3-46. Initial (year 2030) activity of 49 radioisotopes considered in the performance assessment for waste packages with Category 14 fuel (PWR commercial SNF). 
Total Curies of each Isotope used in Performance Assessment (The Sum of Category 15)

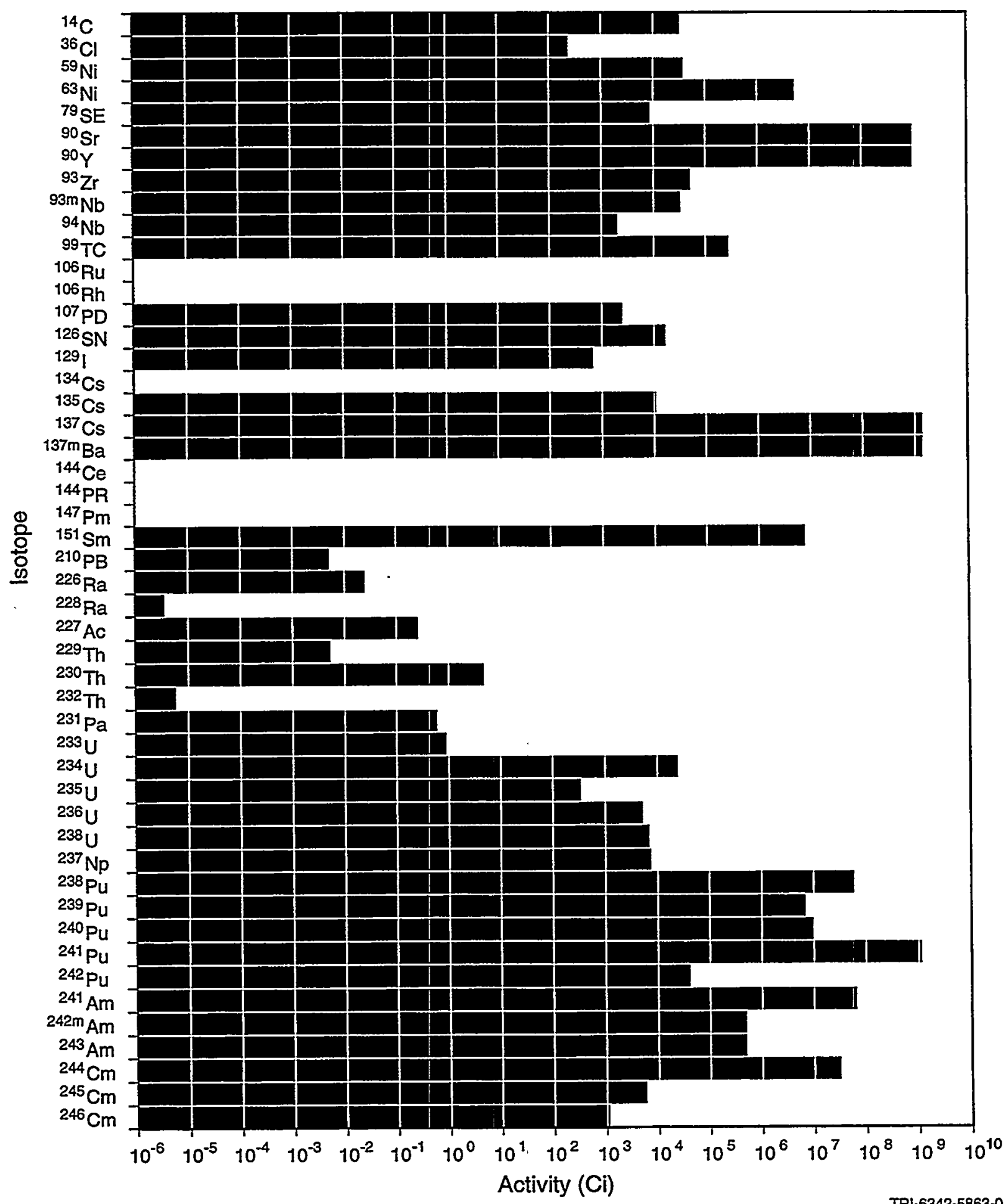

Figure 3-47. Initial (year 2030) activity of 49 radioisotopes considered in the performance assessment for waste packages with Category 15 fuel (BWR commercial SNF). 
Total Curies of each Isotope used in Performance Assessment (The Sum of All 15 Categories)

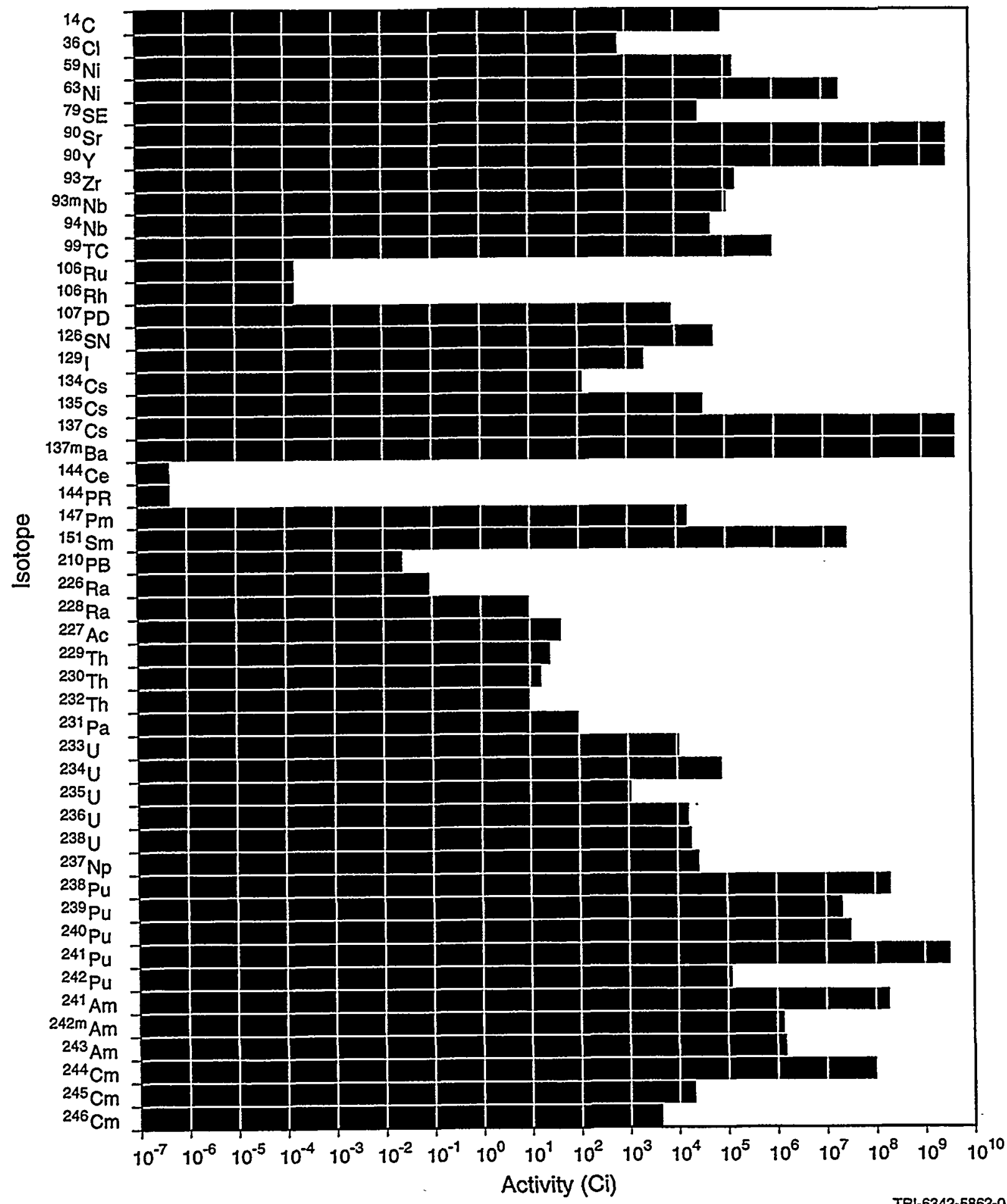

Figure 3-48. Initial (year 2030) activity of 49 radioisotopes considered in the performance assessment for total for all 15 waste package types. 


\subsection{Summary of Assumptions for Waste Package Conceptual Model}

- Disposal of the waste inventory into an unsaturated tuff geologic repository is assumed to occur in the year 2030.

- An initial inventory of 41 radioisotopes was taken as is from NSNFP (see Appendix A); 2 additional radionuclides-barium and yttrium, daughters of ${ }^{135} \mathrm{Cs}$ and ${ }^{90} \mathrm{Sr}$-were included in the heat generation calculations; 41 radioisotopes were considered in the source term model for releases from groundwater; 3 radioisotopes were considered in the transport calculations.

- Thirteen DOE SNF spent fuel categories are modeled, based on a grouping by DOE; two commercial spent fuel categories are modeled; DHLW in the form of borosilicate glass is modeled.

- One type of spent fuel per category represents the DOE SNF grouping for Categories 1 through 13.

- Fifteen types of waste packages are modeled. Waste Package 1 includes N-Reactor fuel only; Waste Packages 2 through 13 include DOE SNF categories 2 through 13 codisposed with DHLW; Waste Packages 14 and 15 include 21-PWR and 44-BWR, respectively.

- The codisposal configuration selected for Categories 1 through 13 was based on information provided by NSNFP for the 1997 PA and was generally the most frequently used configuration (see Appendix A).

- Four types of disposal containers are modeled: standard short, standard long, super short, and super long. The short containers are $3.79 \mathrm{~m}$ (nominal $10 \mathrm{ft}$ ); the long containers are $5.30 \mathrm{~m}$ (nominal $15 \mathrm{ft}$ ). The standard diameter is $1.725 \mathrm{~m}$; the super diameter is $2.0 \mathrm{~m}$.

- Handling containers for DOE SNF are constructed of $6.35-\mathrm{mm} 304 \mathrm{~L}$ stainless steel. Lengths are $3.0 \mathrm{~m}$ (10 ft) and $4.6 \mathrm{~m}(15 \mathrm{ft})$. Diameters are $25.4 \mathrm{~cm}, 43.2 \mathrm{~cm}$, or $61.0-\mathrm{cm}$ (10-in., 17-in., or 24-in.). Length and diameter were modeled by category based on information provided by NSNFP for the 1997 PA.

- Handling container for DHLW is the standard DOE high-level waste container, with 0.95-cm-thick stainless steel.

- The disposal.containers for all categories include a 10-cm-thick outer carbon steel layer and a 2-cm-thick inner Inconel 625 layer.

See also Chapter 7 for assumptions regarding corrosion of the waste package and transport of the source term. 


\subsection{Modeling Parameters for Waste Package}

Modeling parameters are listed for waste packages and radioisotopes.

\subsubsection{Layers and Matrices Modeled per Waste Package}

Table 3-5 provides a listing of the layers and matrices modeled per waste package. Below is a key to the parameter names.

$\begin{array}{ll}\text { WDG } & \text { Label for waste package } \\ \text { COUNT } & \text { Number of waste packages modeled } \\ \text { NLAYERS } & \text { Number of layers in waste package } \\ \text { NMATRIX } & \text { Number of matrices in waste package } \\ \text { AREALn } & \text { Area of Layer } \mathrm{n} \\ \text { COMPLn } & \text { Composition of Layer } \mathrm{n} \\ \text { DIAMLn } & \text { Diameter of Layer } \mathrm{n} \\ \text { LENGTHLn } & \text { Length of Layer } \mathrm{n} \\ \text { MASSLn } & \text { Mass of Layer } \mathrm{n} \\ \text { AREAMn } & \text { Area of Matrix } \mathrm{n} \\ \text { COMPMn } & \text { Composition of Matrix } \mathrm{n} \\ \text { COVLAYMn } & \text { Cover layer of Matrix } \mathrm{n} \\ \text { INFRALmn } & \text { Inventory fraction for all isotopes, Matrix m, Inventory } \mathrm{n} \\ \text { INFRCmn } & \text { Inventory fraction of carbon, Matrix m, Inventory } \mathrm{n} \\ \text { INVIDmn } & \text { Inventory identification of Matrix } \mathrm{m} \text {, Inventory } \mathrm{n} \\ \text { MASSMn } & \text { Mass of Matrix } \mathrm{m}\end{array}$

\subsubsection{Activity per Category and Radionuclide Half-Life}

Tables 3-6 and 3-7 list the total curies (DOE SNF and DHLW) per category and the half-lives of the radioisotopes considered in the inventory, respectively. 
3. System Characterization: Waste Package

Table 3-5. Layers Modeled by Waste Package

\begin{tabular}{|c|c|c|c|}
\hline Waste Package 1 & $0 \times 4 \times 15^{\prime}$ & 24" & (N-Reactor) \\
\hline $\begin{array}{l}\text { WDG } \\
\text { Count } \\
\text { Nlayers } \\
\text { Nmatrix }\end{array}$ & $\begin{array}{r}\text { WP01 } \\
118 \\
5 \\
1\end{array}$ & & \\
\hline $\begin{array}{l}\text { Layer 1 } \\
\text { AreaL1 } \\
\text { Compl1 } \\
\text { DiamL1 } \\
\text { Lengtil1 } \\
\text { MassL1 }\end{array}$ & $\begin{array}{r}\text { Outer Package } \\
33.40 \\
1 \\
1.725 \\
5.30 \\
24140.00\end{array}$ & $\begin{array}{l}\mathrm{m}^{2} \\
\text { carbon steel } \\
\mathrm{m} \\
\mathrm{m} \\
\mathrm{kg}\end{array}$ & \\
\hline $\begin{array}{l}\text { Layer 2 } \\
\text { Areal2 } \\
\text { Compl2 } \\
\text { DiamL2 } \\
\text { LengthL2 } \\
\text { MassL2 }\end{array}$ & $\begin{array}{r}\text { Inner Package } \\
27.13 \\
17 \\
1.525 \\
4.9 \\
4629.00\end{array}$ & $\begin{array}{l}m^{2} \\
\text { Alloy } \mathrm{C}-22 \\
\mathrm{~m} \\
\mathrm{~m} \\
\mathrm{~kg}\end{array}$ & \\
\hline $\begin{array}{l}\text { Layer3 } \\
\text { Areal3 } \\
\text { CompL3 } \\
\text { DiamL3 } \\
\text { Lengthl3 } \\
\text { MassL3 }\end{array}$ & $\begin{array}{r}\text { SNF Canister } \\
36.80 \\
6 \\
0.61 \\
4.572 \\
2773.60\end{array}$ & $\begin{array}{l}\mathrm{m}^{2} \\
304 \mathrm{LSS} \\
\mathrm{m} \\
\mathrm{m} \\
\mathrm{kg}\end{array}$ & \\
\hline $\begin{array}{l}\text { Layer } 4 \\
\text { AreaL4 } \\
\text { CompL4 } \\
\text { MassL4 }\end{array}$ & $\begin{array}{r}286.00 \\
3 \\
1650.00\end{array}$ & $\begin{array}{l}\mathrm{m}^{2} \\
\text { Zircaloy } \\
\mathrm{kg}\end{array}$ & ( $40 \%$ of U Mass) \\
\hline $\begin{array}{l}\text { Layer 5 } \\
\text { AreaL5 } \\
\text { CompL5 } \\
\text { MassL5 }\end{array}$ & $\begin{array}{rr}\text { Fuel Meat } & \\
286.00 \\
11 \\
18073.00\end{array}$ & $\begin{array}{l}\mathrm{m}^{2} \\
\mathrm{U} \text { Metal } \\
\mathrm{kg}\end{array}$ & (metal model) \\
\hline $\begin{array}{l}\text { Matrix } 1 \\
\text { AreaM1 } \\
\text { CompM1 } \\
\text { CovLayM1 } \\
\text { InFrA11 } \\
\text { invld11 } \\
\text { MassM1 }\end{array}$ & $\begin{array}{lr}\text { U metal } & \\
& 286.00 \\
& 11 \\
& 4 \\
& 1 \\
& 1 \\
& 18073.00\end{array}$ & $\begin{array}{l}\mathrm{m}^{2} \\
\mathrm{U} \text { Metal } \\
\mathrm{kg}\end{array}$ & (metal model) \\
\hline
\end{tabular}


Table 3-5. Layers Modeled by Waste Package (Continued)

\begin{tabular}{|c|c|c|}
\hline Waste Package 2 & $1 \times 5 \times 15^{\prime} 17^{\prime \prime}$ & (CP-5, HWCTR) \\
\hline $\begin{array}{l}\text { WDG } \\
\text { Count } \\
\text { Nlayers } \\
\text { Nmatrix }\end{array}$ & $\begin{array}{r}\text { WP02 } \\
9 \\
5 \\
2\end{array}$ & \\
\hline $\begin{array}{l}\text { Layer 1 } \\
\text { AreaL1 } \\
\text { CompL1 } \\
\text { DiamL1 } \\
\text { LengthL1 } \\
\text { MassL1 }\end{array}$ & $\begin{array}{l}\text { Outer Package } \\
\qquad \begin{array}{c}40.27 \mathrm{~m}^{2} \\
1 \text { carbon steel } \\
2.03 \mathrm{~m} \\
5.30 \mathrm{~m} \\
29390.00 \mathrm{~kg}\end{array}\end{array}$ & \\
\hline $\begin{array}{l}\text { Layer 2 } \\
\text { Areal2 } \\
\text { Compl2 } \\
\text { DiamL2 } \\
\text { Length12 } \\
\text { MassL2 }\end{array}$ & $\begin{array}{l}\text { Inner Package } \\
\qquad 33.43 \mathrm{~m}^{2} \\
17 \text { Alloy C-22 } \\
1.83 \mathrm{~m} \\
4.9 \mathrm{~m} \\
5717.00 \mathrm{~kg}\end{array}$ & \\
\hline $\begin{array}{l}\text { Layer3 } \\
\text { AreaL3 } \\
\text { CompL3 } \\
\text { DiamL3 } \\
\text { LengthL3 } \\
\text { MassL3 }\end{array}$ & $\begin{array}{rl}H L W & + \text { SNF Canisters } \\
52.90 & \mathrm{~m}^{2} \\
6 & 304 L \mathrm{SS} \\
0.61 & \mathrm{~m} \\
4.572 & \mathrm{~m} \\
3975.80 \mathrm{~kg}\end{array}$ & \\
\hline $\begin{array}{l}\text { Layer } 4 \\
\text { AreaL4 } \\
\text { CompL4 } \\
\text { Massl.4 }\end{array}$ & $\begin{array}{l}0.223 \mathrm{~m}^{2} \\
3 \text { Zircaloy } \\
29 \mathrm{~kg}\end{array}$ & (65\% of U Mass) \\
\hline $\begin{array}{l}\text { Layer 5 } \\
\text { AreaL5 } \\
\text { CompL5 } \\
\text { MassL5 }\end{array}$ & $\begin{array}{l}0.223 \mathrm{~m}^{2} \\
10 \mathrm{U}-\mathrm{Zr} \\
4.46 \mathrm{~kg}\end{array}$ & (metal model; treat as U Metal) \\
\hline $\begin{array}{l}\text { Matrix 1 } \\
\text { AreaM1 } \\
\text { CompM1 } \\
\text { CovLayM1 } \\
\text { InFrAl11 } \\
\text { Invid11 } \\
\text { MassM1 }\end{array}$ & $\begin{array}{c}42.30 \mathrm{~m}^{2} \\
15 \text { Glass } \\
3 \\
3.60 \mathrm{E}-03 \\
16 \\
14250.00 \mathrm{~kg}\end{array}$ & \\
\hline $\begin{array}{l}\text { Matrix } 2 \\
\text { AreaM2 } \\
\text { CompM2 } \\
\text { CovLayM2 } \\
\text { InFrAl21 } \\
\text { Invid21 } \\
\text { MassM2 }\end{array}$ & $\begin{array}{c}0.223 \mathrm{~m}^{2} \\
10 \mathrm{U}-\mathrm{Zr} \\
4 \\
1 \\
2 \\
4.46 \mathrm{~kg}\end{array}$ & (metal model; treat as U Meta) \\
\hline
\end{tabular}


3. System Characterization: Waste Package

Table 3-5. Layers Modeled by Waste Package (Continued)

\begin{tabular}{|c|c|c|c|}
\hline Waste Package 3 & $1 \times 4 \times 10^{\prime}$ & $10^{\prime \prime}$ & (Fermi) \\
\hline $\begin{array}{l}\text { WDG } \\
\text { Count } \\
\text { Nlayers } \\
\text { Nmatrix }\end{array}$ & $\begin{array}{r}\text { WP03 } \\
55 \\
5 \\
2\end{array}$ & & \\
\hline $\begin{array}{l}\text { Layer 1 } \\
\text { Areal1 } \\
\text { CompL1 } \\
\text { DiamL1 } \\
\text { LengthL1 } \\
\text { MassL1 }\end{array}$ & $\begin{array}{r}\text { Outer Package } \\
25.21 \\
1 \\
1.725 \\
3.79 \\
18080.00\end{array}$ & $\begin{array}{l}\mathrm{m}^{2} \\
\text { carbon steel } \\
\mathrm{m} \\
\mathrm{mg}\end{array}$ & \\
\hline $\begin{array}{l}\text { Layer 2 } \\
\text { AreaL2 } \\
\text { Compl2 } \\
\text { DiamL2 } \\
\text { LengthL2 } \\
\text { MassL2 }\end{array}$ & $\begin{array}{r}\text { Inner Package } \\
8.80 \\
17 \\
1.645 \\
3.4 \\
1608.00\end{array}$ & $\begin{array}{l}\mathrm{m}^{2} \\
\text { Alloy } \mathrm{C}-22 \\
\mathrm{~m} \\
\mathrm{mg}\end{array}$ & . \\
\hline $\begin{array}{l}\text { Layer } 3 \\
\text { AreaL3 } \\
\text { CompL3 } \\
\text { DiamL3 } \\
\text { Lengthl3 } \\
\text { MassL3 }\end{array}$ & $\begin{array}{r}\text { HLW + SNF Caniste } \\
27.82 \\
6 \\
0.61 \\
3.048 \\
2028.60\end{array}$ & $\begin{array}{l}\text { ers } \\
\mathrm{m}^{2} \\
304 L S S \\
\mathrm{~m} \\
\mathrm{~m} \\
\mathrm{~kg}\end{array}$ & . \\
\hline $\begin{array}{l}\text { Layer 4 } \\
\text { Areal4 } \\
\text { CompL4 } \\
\text { MassL4 }\end{array}$ & $\begin{array}{r}4.86 \\
3 \\
19.58\end{array}$ & $\begin{array}{l}\mathrm{m}^{2} \\
\text { Zircaloy } \\
\mathrm{kg}\end{array}$ & (27.4\% of U Mass) \\
\hline $\begin{array}{l}\text { Layer } 5 \\
\text { AreaL5 } \\
\text { CompL5 } \\
\text { MassL5 }\end{array}$ & $\begin{array}{r}4.86 \\
5 \\
71.45\end{array}$ & $\begin{array}{l}\mathrm{m}^{2} \\
\mathrm{U}-\mathrm{Mo}_{0}\end{array}$ & (Treat as U metal $\times 10$ ) \\
\hline $\begin{array}{l}\text { Matrix 1 } \\
\text { AreaM1 } \\
\text { CompM1 } \\
\text { CovLayM1 } \\
\text { InFrAl11 } \\
\text { Invid11 } \\
\text { MassM1 }\end{array}$ & $\begin{array}{r}22.56 \\
15 \\
3 \\
1.18 E-02 \\
16 \\
7600.00\end{array}$ & $\begin{array}{l}\mathrm{m}^{2} \\
\text { Glass } \\
\mathrm{kg}\end{array}$ & \\
\hline $\begin{array}{l}\text { Matrix } 2 \\
\text { AreaM2 } \\
\text { CompM2 } \\
\text { CovLayM2 } \\
\text { InFrAl21 } \\
\text { Invld21 } \\
\text { MassM2 }\end{array}$ & $\begin{array}{r}4.86 \\
5 \\
4 \\
1 \\
3 \\
71.45\end{array}$ & $\begin{array}{l}\mathrm{m}^{2} \\
\mathrm{U}-\mathrm{Mo}_{0} \\
\mathrm{~kg}\end{array}$ & (Treat as U metal $\times 10$ ) \\
\hline
\end{tabular}


Table 3-5. Layers Modeled by Waste Package (Continued)

\begin{tabular}{|c|c|c|c|}
\hline Waste Package 4 & $1 \times 5 \times 10^{\prime}$ & 17" (Shipp & ngport(HEU), Commercial (LEU), Saxton (MEU)) \\
\hline $\begin{array}{l}\text { WDG } \\
\text { Count } \\
\text { Nlayers } \\
\text { Nmatrix }\end{array}$ & $\begin{array}{r}\text { WPO4 } \\
203 \\
5 \\
2\end{array}$ & & \\
\hline $\begin{array}{l}\text { Layer 1 } \\
\text { AreaL1 } \\
\text { Compl1 } \\
\text { DiamL1 } \\
\text { LengthL1 } \\
\text { MassL1 }\end{array}$ & $\begin{array}{r}\text { Outer Package } \\
30.10 \\
1 \\
203 \\
3.79 \\
22200.00\end{array}$ & $\begin{array}{l}\mathrm{m}^{2} \\
\text { carbon steel } \\
\mathrm{m} \\
\mathrm{m} \\
\mathrm{kg}\end{array}$ & . \\
\hline $\begin{array}{l}\text { Layer } 2 \\
\text { Areal2 } \\
\text { CompL2 } \\
\text { DiamL2 } \\
\text { LengthL2 } \\
\text { MassL2 }\end{array}$ & $\begin{array}{r}\text { Inner Package } \\
24.81 \\
17 \\
1.83 \\
3.4 \\
4235.00\end{array}$ & $\begin{array}{l}\mathrm{m}^{2} \\
\text { Alloy } \mathrm{C}-22 \\
\mathrm{~m} \\
\mathrm{~m} \\
\mathrm{~kg}\end{array}$ & . \\
\hline $\begin{array}{l}\text { Layer } 3 \\
\text { AreaL3 } \\
\text { CompL3 } \\
\text { DiamL3 } \\
\text { LengthL3 } \\
\text { MassL3 }\end{array}$ & $\begin{array}{r}\text { HLW + SNF Caniste } \\
36.32 \\
6 \\
0.61 \\
3.048 \\
2727.30\end{array}$ & $\begin{array}{l}\mathrm{m}^{2} \\
304 \mathrm{LSS} \\
\mathrm{m} \\
\mathrm{mg}\end{array}$ & . \\
\hline $\begin{array}{l}\text { Layer } 4 \\
\text { AreaL4 } \\
\text { CompL4 } \\
\text { MassL4 }\end{array}$ & $\begin{array}{r}24.39 \\
3 \\
178.98\end{array}$ & $\begin{array}{l}\mathrm{m}^{2} \\
\text { Zircaloy } \\
\mathrm{kg}\end{array}$ & ( $38 \%$ of U Mass) \\
\hline $\begin{array}{l}\text { Layer } 5 \\
\text { AreaL5 } \\
\text { CompL5 } \\
\text { MassL5 }\end{array}$ & $\begin{array}{r}24.39 \\
14 \\
471.00\end{array}$ & $\begin{array}{l}\mathrm{m}^{2} \\
\mathrm{UO} 2 \\
\mathrm{~kg}\end{array}$ & (commercial model) \\
\hline $\begin{array}{l}\text { Matrix } 1 \\
\text { AreaM1 } \\
\text { CompM1 } \\
\text { CovLayM1 } \\
\text { InFrAl11 } \\
\text { Invld11 } \\
\text { MassM1 }\end{array}$ & $\begin{array}{r}28.2 \\
15 \\
3 \\
5.42 E-02 \\
16 \\
9500.00\end{array}$ & $\begin{array}{l}\mathrm{m}^{2} \\
\text { Glass } \\
\mathrm{kg}\end{array}$ & \\
\hline $\begin{array}{l}\text { Matrix } 2 \\
\text { AreaM2 } \\
\text { CompM2 } \\
\text { CovLayM2 } \\
\text { InFrAl21 } \\
\text { Invid21 } \\
\text { MassM2 }\end{array}$ & $\begin{array}{r}24.39 \\
14 \\
4 \\
1 \\
4 \\
471.00\end{array}$ & $\begin{array}{l}\mathrm{m}^{2} \\
\text { UO2 } \\
\mathrm{kg}\end{array}$ & (commercial model) \\
\hline
\end{tabular}


3. System Characterization: Waste Package

Table 3-5. Layers Modeled by Waste Package (Continued)

\begin{tabular}{|c|c|c|}
\hline Waste Package 5 & $1 \times 4 \times 10^{\prime} 10^{\prime \prime}(\mathrm{SM}-1)$ & LSST and $Z r$ (MEU), TMI-2 (LEU)) \\
\hline $\begin{array}{l}\text { WDG } \\
\text { Count } \\
\text { Nlayers } \\
\text { Nmatrix }\end{array}$ & $\begin{array}{r}\text { WP05 } \\
595 \\
5 \\
2\end{array}$ & \\
\hline $\begin{array}{l}\text { Layer 1 } \\
\text { Areal1 } \\
\text { CompL1 } \\
\text { DiamL1 } \\
\text { LengthL1 } \\
\text { MassL1 }\end{array}$ & $\begin{array}{l}\text { Outer Package } \\
\qquad \begin{array}{c}25.21 \mathrm{~m}^{2} \\
11 \mathrm{carbon}^{2} \text { steel } \\
1.725 \mathrm{~m} \\
3.79 \mathrm{~m} \\
18080.00 \mathrm{~kg}\end{array}\end{array}$ & \\
\hline $\begin{array}{l}\text { Layer 2 } \\
\text { Areal2 } \\
\text { Compl2 } \\
\text { DiamL2 } \\
\text { LengthL2 } \\
\text { MassL2 }\end{array}$ & $\begin{array}{l}\text { Inner Package } \\
\qquad .8 \mathrm{~m}^{2} \\
17 \mathrm{Alloy}^{\mathrm{C}-22} \\
1.645 \mathrm{~m} \\
3.4 \mathrm{~m} \\
1608.00 \mathrm{~kg}\end{array}$ & \\
\hline $\begin{array}{l}\text { Layer3 } \\
\text { AreaL3 } \\
\text { CompL3 } \\
\text { DiamL3 } \\
\text { Lengthl3 } \\
\text { MassL3 }\end{array}$ & $\begin{array}{c}\text { HLW + SNF Canisters } \\
27.82 \mathrm{~m}^{2} \\
6 \\
304 L S S \\
0.61 \mathrm{~m} \\
3.048 \mathrm{~m} \\
2028.60 \mathrm{~kg}\end{array}$ & \\
\hline $\begin{array}{l}\text { Layer } 4 \\
\text { AreaL4 } \\
\text { CompL4 } \\
\text { MassL4 }\end{array}$ & $\begin{array}{l}739.50 \mathrm{~m}^{2} \\
3 \text { Zircaloy } \\
116.00 \mathrm{~kg}\end{array}$ & (80\% of U Mass) \\
\hline $\begin{array}{l}\text { Layer } 5 \\
\text { AreaL5 } \\
\text { CompL5 } \\
\text { MassL5 }\end{array}$ & $\begin{array}{rl}739.50 & \mathrm{~m}^{2} \\
14 & \mathrm{UO} 2 \\
145 \mathrm{~kg}\end{array}$ & (commercial model) \\
\hline $\begin{array}{l}\text { Matrix } 1 \\
\text { AreaM1 } \\
\text { CompM1 } \\
\text { CovLayM1 } \\
\text { InFrA11 } \\
\text { Invld11 } \\
\text { MassM1 }\end{array}$ & 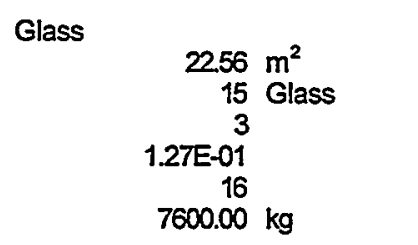 & \\
\hline $\begin{array}{l}\text { Matrix } 2 \\
\text { AreaM2 } \\
\text { CompM2 } \\
\text { CovLayM2 } \\
\text { InFrA121 } \\
\text { Invld21 } \\
\text { MassM2 }\end{array}$ & $\begin{array}{rl}739.50 & \mathrm{~m}^{2} \\
14 & \mathrm{UO} 2 \\
4 \\
1 \\
5 \\
145 \mathrm{~kg}\end{array}$ & (commercial model) \\
\hline
\end{tabular}


Table 3-5. Layers Modeled by Waste Package (Continued)

\begin{tabular}{|c|c|c|c|}
\hline Waste Package 6 & $1 \times 5 \times 15^{\prime}$ & $17 "$ & (ATR (HEU), MTR, FRR (MEU)) \\
\hline $\begin{array}{l}\text { WDG } \\
\text { Count } \\
\text { Nlayers } \\
\text { Nmatrix }\end{array}$ & $\begin{array}{r}\text { WP06 } \\
750 \\
5 \\
2\end{array}$ & & \\
\hline $\begin{array}{l}\text { Layer 1 } \\
\text { Areal1 } \\
\text { CompL1 } \\
\text { DiamL1 } \\
\text { LengthL1 } \\
\text { MassL1 }\end{array}$ & $\begin{array}{r}\text { Outer Package } \\
4027 \\
1.00 \\
203 \\
5.30 \\
29390.00\end{array}$ & $\begin{array}{l}\mathrm{m}^{2} \\
\text { carbon steel } \\
\mathrm{m} \\
\mathrm{m} \\
\mathrm{kg}\end{array}$ & \\
\hline $\begin{array}{l}\text { Layer2 } \\
\text { AreaL2 } \\
\text { Compl2 } \\
\text { DiamL2 } \\
\text { Lengthl2 } \\
\text { MassL2 }\end{array}$ & $\begin{array}{r}\text { Inner Package } \\
33.43 \\
17 \\
1.83 \\
4.9 \\
5717.00\end{array}$ & $\begin{array}{l}\mathrm{m}^{2} \\
\text { Alloy } \mathrm{C}-22 \\
\mathrm{~m} \\
\mathrm{~m} \\
\mathrm{~kg}\end{array}$ & \\
\hline $\begin{array}{l}\text { Layer3 } \\
\text { AreaL3 } \\
\text { CompL3 } \\
\text { DiamL3 } \\
\text { LengthL3 } \\
\text { MassL3 }\end{array}$ & $\begin{array}{r}\text { HLW + SNF Caniste } \\
52.90 \\
6 \\
0.61 \\
4.572 \\
3975.80\end{array}$ & $\begin{array}{l}\text { ters } \\
\mathrm{m}^{2} \\
304 L S S \\
\mathrm{~m} \\
\mathrm{~m} \\
\mathrm{~kg}\end{array}$ & \\
\hline $\begin{array}{l}\text { Layer 4 } \\
\text { Areal44 } \\
\text { CompL4 } \\
\text { MassL4 }\end{array}$ & $\begin{array}{r}15.46 \\
4 \\
10.71\end{array}$ & $\begin{array}{l}\mathrm{m}^{2} \\
\text { Aluminum } \\
\mathrm{kg}\end{array}$ & (90\% of U Mass) \\
\hline $\begin{array}{l}\text { Layer } 5 \\
\text { AreaL5 } \\
\text { CompL5 } \\
\text { MassL5 }\end{array}$ & $\begin{array}{r}15.46 \\
11 \\
11.90\end{array}$ & $\begin{array}{l}\mathrm{m}^{2} \\
\mathrm{U}-\mathrm{Al} \\
\mathrm{kg}\end{array}$ & (metal model; treat as U metal) \\
\hline $\begin{array}{l}\text { Matrix 1 } \\
\text { AreaM1 } \\
\text { CompM1 } \\
\text { CovLayM1 } \\
\text { InFrAl11 } \\
\text { Invld11 } \\
\text { MassM1 }\end{array}$ & $\begin{array}{r}42.30 \\
15 \\
3 \\
3.01 \mathrm{E}-01 \\
16 \\
14250.00\end{array}$ & $\begin{array}{l}0 \mathrm{~m}^{2} \\
5 \text { Glass } \\
3 \\
1 \\
5 \\
\mathrm{~kg}\end{array}$ & \\
\hline $\begin{array}{l}\text { Matrix } 2 \\
\text { AreaM2 } \\
\text { CompM2 } \\
\text { CovLayM2 } \\
\text { InFrAl21 } \\
\text { Invid21 } \\
\text { MassM2 }\end{array}$ & $\begin{array}{r}15.46 \\
11 \\
4 \\
1 \\
6 \\
11.90\end{array}$ & $\begin{array}{l}6 \mathrm{~m}^{2} \\
1 \mathrm{U}-\mathrm{Al} \\
4 \\
6 \\
6 \mathrm{~kg}\end{array}$ & (metal model; treat as U metal) \\
\hline
\end{tabular}


3. System Characterization: Waste Package

Table 3-5. Layers Modeled by Waste Package (Continued)

\begin{tabular}{|c|c|c|c|}
\hline Waste Package 7 & $1 \times 5 \times 15^{\prime}$ & $17^{\prime \prime}$ & (MTR, FRR (HEU, MEU)) \\
\hline $\begin{array}{l}\text { WDG } \\
\text { Count } \\
\text { Nlayers } \\
\text { Nmatrix }\end{array}$ & $\begin{array}{r}\text { WP07 } \\
225 \\
5 \\
2\end{array}$ & & \\
\hline $\begin{array}{l}\text { Layer 1 } \\
\text { AreaL1 } \\
\text { CompL1 } \\
\text { DiamL1 } \\
\text { Lengthl1 } \\
\text { MassL1 }\end{array}$ & $\begin{array}{r}\text { Outer Package } \\
40.27 \\
1.00 \\
2.03 \\
5.30 \\
29390.00\end{array}$ & $\begin{array}{l}\mathrm{m}^{2} \\
\text { carbon steel } \\
\mathrm{m} \\
\mathrm{m} \\
\mathrm{kg}\end{array}$ & \\
\hline $\begin{array}{l}\text { Layer } 2 \\
\text { AreaL2 } \\
\text { CompL2 } \\
\text { DiamL2 } \\
\text { LengthL2 } \\
\text { MassL2 }\end{array}$ & $\begin{array}{r}\text { Inner Package } \\
33.43 \\
17 \\
1.83 \\
4.9 \\
5717.00\end{array}$ & $\begin{array}{l}\mathrm{m}^{2} \\
\text { Alloy } \mathrm{C}-22 \\
\mathrm{~m} \\
\mathrm{~m} \\
\mathrm{~kg}\end{array}$ & \\
\hline $\begin{array}{l}\text { Layer } 3 \\
\text { AreaL3 } \\
\text { Compl3 } \\
\text { DiamL3 } \\
\text { LengthL3 } \\
\text { Massl33 }\end{array}$ & $\begin{array}{r}\text { HLW + SNF Caniste } \\
52.90 \\
6 \\
0.61 \\
4.572 \\
3975.80\end{array}$ & $\begin{array}{l}\mathrm{m}^{2} \\
304 L S S \\
\mathrm{~m} \\
\mathrm{~m}\end{array}$ & \\
\hline $\begin{array}{l}\text { Layer } 4 \\
\text { Areal4 } \\
\text { CompL44 } \\
\text { MassL4 }\end{array}$ & $\begin{array}{r}65.68 \\
4 \\
35.36\end{array}$ & $\begin{array}{l}\mathrm{m}^{2} \\
\text { Aluminum } \\
\mathrm{kg}\end{array}$ & (70\% of U Mass) \\
\hline $\begin{array}{l}\text { Layer } 5 \\
\text { AreaL5 } \\
\text { CompL5 } \\
\text { MassL5 }\end{array}$ & $\begin{array}{r}65.68 \\
7 \\
50.52\end{array}$ & $\mathrm{~m}_{\mathrm{kg}}^{\mathrm{U}^{2}}$ & (Treat as $0.1 \times$ U Metal) \\
\hline $\begin{array}{l}\text { Matrix } 1 \\
\text { AreaM1 } \\
\text { CompM1 } \\
\text { CovLayM1 } \\
\text { InFrAl11 } \\
\text { Invld11 } \\
\text { MassM1 }\end{array}$ & Glass $\begin{array}{r}42.30 \\
15 \\
3 \\
9.02 E-02 \\
16 \\
14250.00\end{array}$ & $\begin{array}{l}\mathrm{m}^{2} \\
\text { Giass } \\
\mathrm{kg}\end{array}$ & $\cdot$ \\
\hline $\begin{array}{l}\text { Matrix } 2 \\
\text { AreaM2 } \\
\text { CompM2 } \\
\text { CovLayM2 } \\
\text { InFrAl21 } \\
\text { Invld21 } \\
\text { MassM2 }\end{array}$ & $\begin{array}{r}65.68 \\
7 \\
4 \\
1 \\
7 \\
50.52\end{array}$ & $\begin{array}{l}\mathrm{m}^{2} \\
\mathrm{U}-\mathrm{Si} \\
\mathrm{kg}\end{array}$ & (Treat as $0.1 \times$ U Metal) \\
\hline
\end{tabular}


Table 3-5. Layers Modeled by Waste Package (Continued)

\begin{tabular}{|c|c|c|}
\hline Waste Package 8 & $1 \times 5 \times 15^{\prime} 17^{\prime \prime}$ & (Ft. St. Vrain (HEU)) \\
\hline $\begin{array}{l}\text { WDG } \\
\text { Count } \\
\text { Nlayers } \\
\text { Nmatrix }\end{array}$ & $\begin{array}{r}\text { WPO8 } \\
545 \\
6 \\
3\end{array}$ & \\
\hline $\begin{array}{l}\text { Layer 1 } \\
\text { AreaL1 } \\
\text { CompL1 } \\
\text { DiamL1 } \\
\text { LengthL1 } \\
\text { MassL1 }\end{array}$ & $\begin{array}{l}\text { Outer Package } \\
\qquad \begin{array}{l}40.27 \mathrm{~m}^{2} \\
1.00 \mathrm{carbon}^{2} \text { steel } \\
2.03 \mathrm{~m} \\
5.30 \mathrm{~m} \\
29390.00 \mathrm{~kg}\end{array}\end{array}$ & \\
\hline $\begin{array}{l}\text { Layer 2 } \\
\text { AreaL2 } \\
\text { Compl2 } \\
\text { DiamL2 } \\
\text { LengthL2 } \\
\text { MassL2 }\end{array}$ & $\begin{array}{l}\text { Inner Package } \\
\qquad 33.43 \mathrm{~m}^{2} \\
17 \text { Alloy C-22 } \\
1.83 \mathrm{~m} \\
4.9 \mathrm{~m} \\
5717.00 \mathrm{~kg}\end{array}$ & \\
\hline $\begin{array}{l}\text { Layer3 } \\
\text { Areal3 } \\
\text { CompL3 } \\
\text { DiamL3 } \\
\text { LengthL3 } \\
\text { MassL3 }\end{array}$ & $\begin{array}{c}\text { HLW + SNF Canisters } \\
52.90 \mathrm{~m}^{2} \\
6104 \mathrm{LSS} \\
0.61 \mathrm{~m} \\
4.572 \mathrm{~m} \\
3875.80 \mathrm{~kg}\end{array}$ & \\
\hline $\begin{array}{l}\text { Layer } 4 \\
\text { AreaL4 } \\
\text { CompL4 } \\
\text { MassL4 }\end{array}$ & $\begin{aligned} & 7.85 \mathrm{~m}^{2} \\
& 12 \text { Graphite } \\
& 2.2638 \mathrm{~kg}\end{aligned}$ & ( $98 \%$ of U Mass) \\
\hline $\begin{array}{l}\text { Layer 5 } \\
\text { AreaL5 } \\
\text { CompL5 } \\
\text { MassL5 }\end{array}$ & $\begin{array}{l}\text { Particle Coating } \\
\qquad \begin{aligned} 1 \mathrm{~m}^{2} \\
13 \mathrm{TRISO} \\
0.65 \mathrm{~kg}\end{aligned}\end{array}$ & \\
\hline $\begin{array}{l}\text { Layer 6 } \\
\text { AreaL6 } \\
\text { CompL6 } \\
\text { MassL6 }\end{array}$ & $\begin{array}{rl} & 1 \mathrm{~m}^{2} \\
8 & \mathrm{U}^{-T h}-\mathrm{C} \\
231 & \mathrm{~kg}\end{array}$ & (carbide model) \\
\hline $\begin{array}{l}\text { Matrix 1 } \\
\text { AreaM1 } \\
\text { CompM1 } \\
\text { CovLayM1 } \\
\text { InFrAl11 } \\
\text { Invld11 } \\
\text { MassM1 }\end{array}$ & 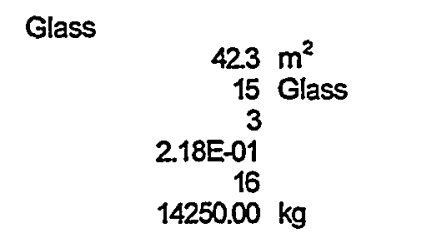 & \\
\hline $\begin{array}{l}\text { Matrix } 2 \\
\text { AreaM2 } \\
\text { CompM2 } \\
\text { CovLayM2 } \\
\text { InfrAL1 } \\
\text { InFrC_21 } \\
\text { Invid21 } \\
\text { MassM2 }\end{array}$ & $\begin{array}{c}7.85 \mathrm{~m}^{2} \\
12 \text { Graphite } \\
3 \\
0 \\
0.65 \\
8 \\
2.638 \mathrm{~kg}\end{array}$ & (98\% of U Mass) \\
\hline $\begin{array}{l}\text { Matrix } 3 \\
\text { AreaM3 } \\
\text { CompM3 } \\
\text { CovLayM3 } \\
\text { InFrAl31 } \\
\text { InFrC } 31 \\
\text { Invid31 } \\
\text { MassM3 }\end{array}$ & $\begin{array}{l}1 \mathrm{~m}^{2} \\
8 \mathrm{U}^{-T h-C} \\
5 \\
1 \\
0.35 \\
8 \\
231 \mathrm{~kg}\end{array}$ & (carbide model) \\
\hline
\end{tabular}


3. System Characterization: Waste Package

Table 3-5. Layers Modeled by Waste Package (Continued)

\begin{tabular}{|c|c|c|c|c|}
\hline Waste Package9 & $1 \times 5 \times 15^{1}$ & $17^{\prime \prime}$ & (Peachbottom (HEU)) & \\
\hline $\begin{array}{l}\text { WDG } \\
\text { Count } \\
\text { Nlayers } \\
\text { Nmatrix }\end{array}$ & $\begin{array}{r}\text { WP09 } \\
103 \\
5 \\
3\end{array}$ & & & \\
\hline $\begin{array}{l}\text { Layer 1 } \\
\text { AreaL1 } \\
\text { CompL1 } \\
\text { DiamL1 } \\
\text { LengthL1 } \\
\text { MassL1 }\end{array}$ & $\begin{array}{r}\text { Outer Package } \\
40.27 \\
1.00 \\
2.03 \\
5.30 \\
29390.00\end{array}$ & $\begin{array}{l}\mathrm{m}^{2} \\
\text { carbon steel } \\
\mathrm{m} \\
\mathrm{m} \\
\mathrm{kg}\end{array}$ & & - \\
\hline $\begin{array}{l}\text { Layer 2 } \\
\text { AreaL2 } \\
\text { CompL2 } \\
\text { DiamL2 } \\
\text { LengthL2 } \\
\text { MassL2 }\end{array}$ & $\begin{array}{r}\text { Inner Package } \\
33.43 \\
17 \\
1.83 \\
4.9 \\
5717.00\end{array}$ & $\begin{array}{l}\mathrm{m}^{2} \\
\text { Alloy } \mathrm{C}-22 \\
\mathrm{~m} \\
\mathrm{mg}\end{array}$ & & \\
\hline $\begin{array}{l}\text { Layer3 } \\
\text { AreaL3 } \\
\text { CompL3 } \\
\text { DiamL3 } \\
\text { Lengthl3 } \\
\text { MassL3 }\end{array}$ & $\begin{array}{r}\text { HLW + SNF Caniste } \\
5290 \\
6 \\
0.61 \\
4.572 \\
3975.80\end{array}$ & $\begin{array}{l}\mathrm{ers} \\
\mathrm{m}^{2} \\
304 L S S \\
\mathrm{~m} \\
\mathrm{~m} \\
\mathrm{~kg}\end{array}$ & & \\
\hline $\begin{array}{l}\text { Layer } 4 \\
\text { Areal 4 } \\
\text { CompL4 } \\
\text { MassL4 }\end{array}$ & $\begin{array}{r}0.10 \\
12 \\
1.997\end{array}$ & $\begin{array}{l}\mathrm{m}^{2} \\
\text { Graphite } \\
\mathrm{kg}\end{array}$ & ( $99.6 \%$ of U Mass) & \\
\hline $\begin{array}{l}\text { Layer } 5 \\
\text { AreaL5 } \\
\text { CompL5 } \\
\text { MassL5 }\end{array}$ & $\begin{array}{r}1 \\
8 \\
2.005\end{array}$ & $\begin{array}{l}\mathrm{m}^{2} \\
\mathrm{U}-\mathrm{Th}-\mathrm{C} \\
\mathrm{kg}\end{array}$ & (carbide model) & \\
\hline $\begin{array}{l}\text { Matrix 1 } \\
\text { AreaM1 } \\
\text { CompM1 } \\
\text { CovLayM1 } \\
\text { InFrAl11 } \\
\text { Invld11 } \\
\text { MassM1 }\end{array}$ & $\begin{array}{r}42.30 \\
15 \\
3 \\
4.13 E-02 \\
16 \\
14250.00\end{array}$ & $\begin{array}{l}\mathrm{m}^{2} \\
\text { Glass } \\
\mathrm{kg}\end{array}$ & & \\
\hline $\begin{array}{l}\text { Matrix 2 } \\
\text { AreaM22 } \\
\text { CompM2 } \\
\text { CovLayM2 } \\
\text { InFrAl21 } \\
\text { InFrC_21 } \\
\text { Invld21 } \\
\text { MassM2 }\end{array}$ & $\begin{array}{r}0.10 \\
12 \\
3 \\
0 \\
0 \\
0.65 \\
9 \\
1.997\end{array}$ & $\begin{array}{l}\mathrm{m}^{2} \\
\text { Graphite } \\
\mathrm{kg}\end{array}$ & ( $99.6 \%$ of $\cup$ Mass) & \\
\hline
\end{tabular}


Table 3-5. Layers Modeled by Waste Package (Continued)

\begin{tabular}{|c|c|c|c|}
\hline Waste Package 9 & $1 \times 5 \times 15^{\prime} 17^{\prime \prime}$ & (Peachbottom (HEU)) & \\
\hline $\begin{array}{l}\text { Matrix } 3 \\
\text { AreaM3 } \\
\text { CompM3 } \\
\text { CovLayM3 } \\
\text { InFrAl31 } \\
\text { InFrC 31 } \\
\text { Invid31 } \\
\text { MassM3 }\end{array}$ & $\begin{array}{c}1 \mathrm{~m}^{2} \\
8 \mathrm{U}-\mathrm{Th}-\mathrm{C} \\
4 \\
1 \\
0.35 \\
9 \\
2.005 \mathrm{~kg}\end{array}$ & (carbide model) & $\cdot$ \\
\hline
\end{tabular}


3. System Characterization: Waste Package

Table 3-5. Layers Modeled by Waste Package (Continued)

\begin{tabular}{|c|c|c|}
\hline Waste Package 10 & $1 \times 4 \times 15$ & 10" (SRE (MEU FGE), FFTF Carbide (MEU FGE)) \\
\hline $\begin{array}{l}\text { WDG } \\
\text { Count } \\
\text { Nlayers } \\
\text { Nmatrix }\end{array}$ & $\begin{array}{r}\text { WP10 } \\
5 \\
5 \\
2\end{array}$ & \\
\hline $\begin{array}{l}\text { Layer 1 } \\
\text { Areal1 } \\
\text { CompL1 } \\
\text { DiamL1 } \\
\text { Lengthl1 } \\
\text { MassL1 }\end{array}$ & $\begin{array}{r}\text { OuterPackage } \\
33.40 \\
1 \\
1.725 \\
5.30 \\
24140.00\end{array}$ & $\begin{array}{l}\mathrm{m}^{2} \\
\text { carbon steel } \\
\mathrm{m} \\
\mathrm{m} \\
\mathrm{kg}\end{array}$ \\
\hline $\begin{array}{l}\text { Layer 2 } \\
\text { AreaL2 } \\
\text { CompL2 } \\
\text { DiamL2 } \\
\text { LengthL2 } \\
\text { MassL2 }\end{array}$ & $\begin{array}{r}\text { Inner Package } \\
27.13 \\
17 \\
1.525 \\
4.9 \\
4629.00\end{array}$ & $\begin{array}{l}\mathrm{m}^{2} \\
\text { Alloy } \mathrm{C}-22 \\
\mathrm{~m} \\
\mathrm{mg}\end{array}$ \\
\hline $\begin{array}{l}\text { Layer } 3 \\
\text { Areal3 } \\
\text { CompL3 } \\
\text { DiamL3 } \\
\text { Lengthl33 } \\
\text { MassL3 }\end{array}$ & $\begin{array}{r}\text { HLW + SNF Canist } \\
40.55 \\
6 \\
0.61 \\
4.572 \\
2957.60\end{array}$ & $\begin{array}{l}\mathrm{m}^{2} \\
304 \mathrm{LSS} \\
\mathrm{m} \\
\mathrm{m} \\
\mathrm{kg}\end{array}$ \\
\hline $\begin{array}{l}\text { Layer } 4 \\
\text { AreaL4 } \\
\text { CompL4 } \\
\text { MassL4 }\end{array}$ & $\begin{array}{r}\text { Clad } \\
\quad 1.51 \\
6 \\
21.9\end{array}$ & $\begin{array}{l}\mathrm{m}^{2} \\
\text { Stainless Steel } \\
\mathrm{kg}\end{array}$ \\
\hline $\begin{array}{l}\text { Layer } 5 \\
\text { AreaL5 } \\
\text { CompL5 } \\
\text { MassL5 }\end{array}$ & $\begin{array}{r}1 \\
8 \\
29.2\end{array}$ & $\begin{array}{l}\mathrm{m}^{2} \\
\mathrm{Ug} / \mathrm{Pu}-\mathrm{C}\end{array}$ \\
\hline $\begin{array}{l}\text { Matrix } 1 \\
\text { AreaM1 } \\
\text { CompM1 } \\
\text { CovLayM1 } \\
\text { InFrAl11 } \\
\text { invid11 } \\
\text { MassM1 }\end{array}$ & Glass $\begin{array}{r}33.84 \\
15 \\
3 \\
1.60 \mathrm{E}-03 \\
16 \\
11400.00\end{array}$ & $\begin{array}{l}\mathrm{m}^{2} \\
\text { Glass } \\
\mathrm{kg}\end{array}$ \\
\hline $\begin{array}{l}\text { Matrix } 2 \\
\text { AreaM2 } \\
\text { CompM2 } \\
\text { CovLayM2 } \\
\text { InFrAl21 } \\
\text { InFrC_21 } \\
\text { Invid21 } \\
\text { MassM2 }\end{array}$ & $\begin{array}{lr}\text { U/Pu-C } & 1 \\
& 8 \\
& 4 \\
& 1 \\
& 0.35 \\
& 10 \\
29.2\end{array}$ & $\begin{array}{l}\mathrm{m}^{2} \\
\mathrm{U} / \mathrm{Pu}-\mathrm{C} \\
\mathrm{kg}\end{array}$ \\
\hline
\end{tabular}


Table 3-5. Layers Modeled by Waste Package (Continued)

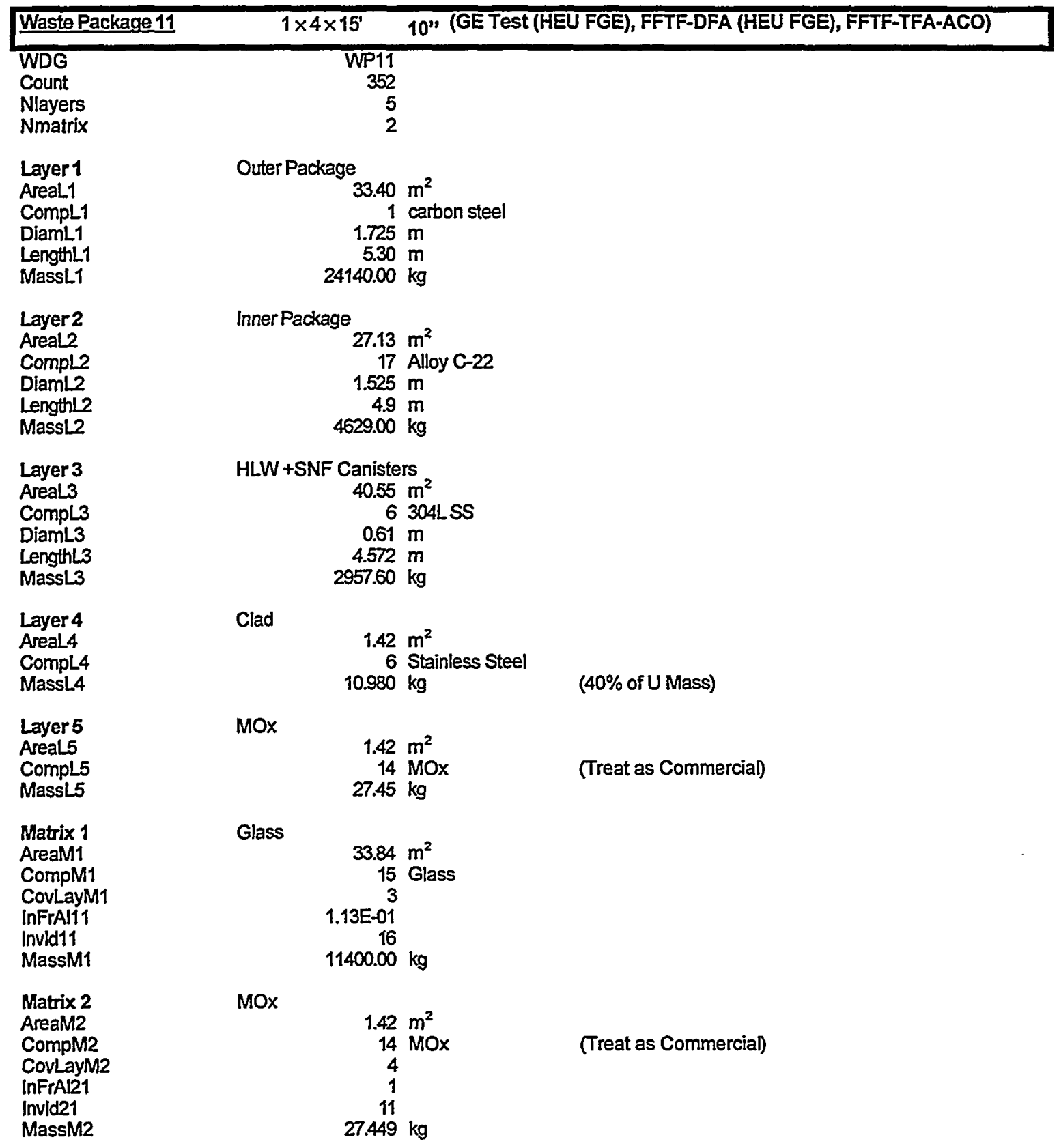


3. System Characterization: Waste Package

Table 3-5. Layers Modeled by Waste Package (Continued)

\begin{tabular}{|c|c|c|}
\hline Waste Package 12 & $1 \times 3 \times 15^{\prime}$ & 17" (LWBR (HEU FGE), Dresdes (HEU FGE) \\
\hline $\begin{array}{l}\text { WDG } \\
\text { Count } \\
\text { Nlayers } \\
\text { Nmatrix }\end{array}$ & $\begin{array}{r}\text { WP12 } \\
69 \\
4 \\
2\end{array}$ & \\
\hline $\begin{array}{l}\text { Layer 1 } \\
\text { AreaL1 } \\
\text { Compl1 } \\
\text { DiamL1 } \\
\text { Lengthl1 } \\
\text { MassL1 }\end{array}$ & $\begin{array}{r}\text { Outer Package } \\
33.40 \\
1 \\
1.725 \\
5.30 \\
24140.00\end{array}$ & $\begin{array}{l}\mathrm{m}^{2} \\
\text { carbon steel } \\
\mathrm{m} \\
\mathrm{m} \\
\mathrm{kg}\end{array}$ \\
\hline $\begin{array}{l}\text { Layer 2 } \\
\text { Areal2 } \\
\text { Compl2 } \\
\text { DiamL2 } \\
\text { Lengthl2 } \\
\text { MassL2 }\end{array}$ & $\begin{array}{r}\text { Inner Package } \\
27.13 \\
17 \\
1.525 \\
4.9 \\
4629.00\end{array}$ & $\begin{array}{l}\mathrm{m}^{2} \\
\text { Alloy } \mathrm{C}-22 \\
\mathrm{~m} \\
\mathrm{~m} \\
\mathrm{~kg}\end{array}$ \\
\hline $\begin{array}{l}\text { Layer } 3 \\
\text { AreaL3 } \\
\text { Compl3 } \\
\text { DiamL3 } \\
\text { Lengthl3 } \\
\text { MassL3 }\end{array}$ & $\begin{array}{r}\text { HLW + SNF Canist } \\
34.50 \\
6 \\
0.61 \\
4.572 \\
2589.00\end{array}$ & $\begin{array}{l}\mathrm{m}^{2} \\
304 \mathrm{LSS} \\
\mathrm{m} \\
\mathrm{mg}\end{array}$ \\
\hline $\begin{array}{l}\text { Layer } 4 \\
\text { AreaL4 } \\
\text { CompL4 } \\
\text { MassL4 }\end{array}$ & $\begin{array}{rr}\text { Clad } & 1.32 \\
& 3 \\
& 11.626\end{array}$ & $\begin{array}{l}\mathrm{m}^{2} \\
\text { Zircaloy } \\
\mathrm{kg}\end{array}$ \\
\hline $\begin{array}{l}\text { Matrix } 1 \\
\text { AreaM1 } \\
\text { CompM1 } \\
\text { CovLayM1 } \\
\text { InFrAl11 } \\
\text { Invid11 } \\
\text { MassM1 }\end{array}$ & Glass $\begin{array}{r}25.38 \\
15 \\
3 \\
1.66 E-02 \\
16 \\
8550.00\end{array}$ & $\begin{array}{l}\mathrm{m}^{2} \\
\text { Glass } \\
\mathrm{kg}\end{array}$ \\
\hline $\begin{array}{l}\text { Matrix } 2 \\
\text { AreaM2 } \\
\text { CompM2 } \\
\text { CovLayM2 } \\
\text { InFrAl21 } \\
\text { Invld21 } \\
\text { MassM2 }\end{array}$ & $\begin{array}{r}1.32 \\
9 \\
4 \\
1 \\
12 \\
11.986\end{array}$ & $\begin{array}{l}\mathrm{m}^{2} \\
\mathrm{THO} 2 \\
\mathrm{~kg}\end{array}$ \\
\hline
\end{tabular}


Table 3-5. Layers Modeled by Waste Package (Continued)

\begin{tabular}{|c|c|c|}
\hline Waste Package 13 & $1 \times 5 \times 10^{\prime}$ & 17" (TRIGA Flip (HEU), TRIGA Std. \& alum. (MEU), SNAP (HEU)) \\
\hline $\begin{array}{l}\text { WDG } \\
\text { Count } \\
\text { Nlayers } \\
\text { Nmatrix }\end{array}$ & $\begin{array}{r}\text { WP13 } \\
102 \\
5 \\
2\end{array}$ & - \\
\hline $\begin{array}{l}\text { Layer 1 } \\
\text { AreaL1 } \\
\text { CompL1 } \\
\text { DiamL1 } \\
\text { LengthL1 } \\
\text { MassL1 }\end{array}$ & $\begin{array}{r}\text { Outer Package } \\
30.64 \\
1 \\
2.03 \\
3.79 \\
22200.00\end{array}$ & $\begin{array}{l}\mathrm{m}^{2} \\
\text { carbon steel } \\
\mathrm{m} \\
\mathrm{m}\end{array}$ \\
\hline $\begin{array}{l}\text { Layer 2 } \\
\text { AreaL2 } \\
\text { Compl2 } \\
\text { DiamL2 } \\
\text { LengthL2 } \\
\text { MassL2 }\end{array}$ & $\begin{array}{r}\text { Inner Package } \\
24.81 \\
17 \\
1.83 \\
3.4 \\
4235.00\end{array}$ & $\begin{array}{l}\mathrm{m}^{2} \\
\text { Alloy } \mathrm{C}-22 \\
\mathrm{~m} \\
\mathrm{~m} \\
\mathrm{~kg}\end{array}$ \\
\hline $\begin{array}{l}\text { Layer } 3 \\
\text { AreaL3 } \\
\text { CompL3 } \\
\text { DiamL3 } \\
\text { LengthL3 } \\
\text { MassL3 }\end{array}$ & $\begin{array}{r}\text { HLW + SNF Canist } \\
36.32 \\
6 \\
0.61 \\
3.048 \\
2727.30\end{array}$ & $\begin{array}{l}\text { ers } \\
\mathrm{m}^{2} \\
304 L S S \\
\mathrm{~m} \\
\mathrm{~m} \\
\mathrm{~kg}\end{array}$ \\
\hline $\begin{array}{l}\text { Layer } 4 \\
\text { AreaL4 } \\
\text { Compl44 } \\
\text { MassL4 }\end{array}$ & $\begin{array}{r}37.22 \\
6 \\
18.61\end{array}$ & $\begin{array}{l}\mathrm{m}^{2} \\
\text { Stainless Steel } \\
\mathrm{kg}\end{array}$ \\
\hline $\begin{array}{l}\text { Layer } 5 \\
\text { AreaL5 } \\
\text { CompL5 } \\
\text { MassL5 }\end{array}$ & $\begin{array}{r}37.22 \\
10 \\
19.59\end{array}$ & $\mathrm{~m}_{\mathrm{Ug}}^{2} \mathrm{Zr}-\mathrm{Hx}$ \\
\hline $\begin{array}{l}\text { Matrix } 1 \\
\text { AreaM1 } \\
\text { CompM1 } \\
\text { CovLayM1 } \\
\text { InFrAl11 } \\
\text { Invld11 } \\
\text { MassM1 }\end{array}$ & Glass $\begin{array}{r}28.20 \\
15 \\
3 \\
2.18 E-02 \\
16 \\
9500.00\end{array}$ & $\begin{array}{l}\mathrm{m}^{2} \\
\text { Glass } \\
\mathrm{kg}\end{array}$ \\
\hline $\begin{array}{l}\text { Matrix } 2 \\
\text { AreaM2 } \\
\text { CompM2 } \\
\text { CovLayM2 } \\
\text { InFrAl21 } \\
\text { Invld21 } \\
\text { MassM2 }\end{array}$ & $\begin{array}{rr}\text { U-Zr-Hx } & 37 \frac{22}{10} \\
4 \\
1 \\
13 \\
19.588\end{array}$ & $\begin{array}{l}\mathrm{m}^{2} \\
\mathrm{U}-\mathrm{Zr}-\mathrm{Hx} \\
\mathrm{kg}\end{array}$ \\
\hline
\end{tabular}


3. System Characterization: Waste Package

Table 3-5. Layers Modeled by Waste Package (Continued)

\begin{tabular}{|c|c|}
\hline Waste Package 14 & Commercial \\
\hline $\begin{array}{l}\text { WDG } \\
\text { Count } \\
\text { Nlayers } \\
\text { Nmatrix }\end{array}$ & $\begin{array}{r}\text { WP14 } \\
4820 \\
5 \\
2\end{array}$ \\
\hline $\begin{array}{l}\text { Layer 1 } \\
\text { Areal1 } \\
\text { CompL1 } \\
\text { DiamL1 } \\
\text { Lengthl1 } \\
\text { MassL1 }\end{array}$ & 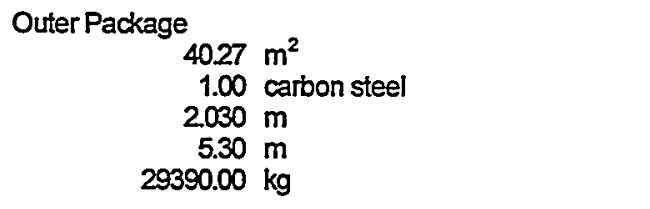 \\
\hline $\begin{array}{l}\text { Layer 2 } \\
\text { Areal2 } \\
\text { CompL2 } \\
\text { DiamL2 } \\
\text { LengthL2 } \\
\text { MassL2 }\end{array}$ & $\begin{array}{l}\text { Inner Package } \\
\qquad \begin{aligned} & 33.43 \mathrm{~m}^{2} \\
& 17 \text { Alloy C-22 } \\
& 1.83 \mathrm{~m} \\
& 4.9 \mathrm{~m} \\
& 5717.00 \mathrm{~kg}\end{aligned}\end{array}$ \\
\hline $\begin{array}{l}\text { Layer } 3 \\
\text { Areal3 } \\
\text { Compl3 } \\
\text { MassL3 }\end{array}$ & $\begin{array}{l}\text { 304L Stainless Steel } \\
\begin{array}{rl}180.00 & \mathrm{~m}^{2} \\
6 & 304 \mathrm{~L} \text { Stainless Steel } \\
3609.00 \mathrm{~kg}\end{array}\end{array}$ \\
\hline $\begin{array}{l}\text { Layer } 4 \\
\text { AreaL4 } \\
\text { CompL4 } \\
\text { MassL4 }\end{array}$ & $\begin{aligned} & 530.00 \mathrm{~m}^{2} \\
& 3 \text { Zircaloy } \\
& 2700.00 \mathrm{~kg}\end{aligned}$ \\
\hline $\begin{array}{l}\text { Layer } 5 \\
\text { AreaL5 } \\
\text { Compl5 } \\
\text { MassL5 }\end{array}$ & $\begin{array}{l}\text { UO2 } \\
\begin{array}{l}560.00 \mathrm{~m}^{2} \\
14 \\
8644.00 \mathrm{~kg} 2\end{array}\end{array}$ \\
\hline $\begin{array}{l}\text { Matrix } 1 \\
\text { AreaM1 } \\
\text { CompM1 } \\
\text { CovLayM1 } \\
\text { InFrAl11 } \\
\text { Invid11 } \\
\text { MassM1 }\end{array}$ & $\begin{array}{l}\text { Borated Stainless Steel } 316 \\
\qquad \begin{array}{c}180.00 \mathrm{~m}^{2} \\
16 \text { Borated Stainless Steel } 316 \\
2 \\
1 \\
14 \\
3609.00 \mathrm{~kg}\end{array}\end{array}$ \\
\hline $\begin{array}{l}\text { Matrix } 2 \\
\text { AreaM2 } \\
\text { CompM2 } \\
\text { CovLayM2 } \\
\text { InFrAl21 } \\
\text { Invld21 } \\
\text { MassM2 }\end{array}$ & $\begin{array}{rl}560.00 \mathrm{~m}^{2} \\
14 & \mathrm{UO} 2 \\
4 \\
1 \\
14 \\
8644.00 \mathrm{~kg}\end{array}$ \\
\hline
\end{tabular}


Table 3-5. Layers Modeled by Waste Package (Continued)

\begin{tabular}{|c|c|}
\hline Waste Package 15 & Commercial \\
\hline $\begin{array}{l}\text { WDG } \\
\text { Count } \\
\text { Nlayers } \\
\text { Nmatrix }\end{array}$ & $\begin{array}{r}\text { WP15 } \\
2859 \\
5 \\
2\end{array}$ \\
\hline $\begin{array}{l}\text { Layer 1 } \\
\text { AreaL1 } \\
\text { Compl1 } \\
\text { DiamL1 } \\
\text { LengthL1 } \\
\text { MassL1 }\end{array}$ & $\begin{array}{l}\text { Outer Package } \\
\qquad \begin{array}{c}40.27 \mathrm{~m}^{2} \\
1.00 \mathrm{carbon}^{2} \text { steel } \\
2.030 \mathrm{~m} \\
5.30 \mathrm{~m} \\
29390.00 \mathrm{~kg}\end{array}\end{array}$ \\
\hline $\begin{array}{l}\text { Layer } 2 \\
\text { AreaL2 } \\
\text { Compl2 } \\
\text { DiamL2 } \\
\text { LengthL2 } \\
\text { MassL2 }\end{array}$ & $\begin{array}{l}\text { Inner Package } \\
\qquad \begin{aligned} & 33.43 \mathrm{~m}^{2} \\
& 17 \text { Alloy C-22 } \\
& 1.83 \mathrm{~m} \\
& 4.9 \mathrm{~m} \\
& 5717.00 \mathrm{~kg}\end{aligned}\end{array}$ \\
\hline $\begin{array}{l}\text { Layer } 3 \\
\text { AreaL3 } \\
\text { CompL3 } \\
\text { MassL3 }\end{array}$ & $\begin{array}{l}304 \mathrm{~L} \text { Stainless Steel } \\
258.00 \mathrm{~m}^{2} \\
6 \mathrm{304L} \text { Stainless Steel } \\
3627.00 \mathrm{~kg}\end{array}$ \\
\hline $\begin{array}{l}\text { Layer } 4 \\
\text { AreaL4 } \\
\text { CompL4 } \\
\text { MassL4 }\end{array}$ & $\begin{array}{rl}420.00 & \mathrm{~m}^{2} \\
3 & \text { Zircaloy } \\
2500.00 & \mathrm{~kg}\end{array}$ \\
\hline $\begin{array}{l}\text { Layer } 5 \\
\text { AreaL5 } \\
\text { CompL5 } \\
\text { MassL5 }\end{array}$ & $\begin{array}{rl}440.00 & \mathrm{~m}^{2} \\
14 & \mathrm{UO2} \\
7872.00 \mathrm{~kg}\end{array}$ \\
\hline $\begin{array}{l}\text { Matrix } 1 \\
\text { AreaM1 } \\
\text { CompM1 } \\
\text { CovLayM1 } \\
\text { InFrAl11 } \\
\text { Invld11 } \\
\text { MassM1 }\end{array}$ & $\begin{array}{l}\text { Borated Stainless Steel } 316 \\
440.00 \mathrm{~m}^{2} \\
16 \text { Borated Stainless Steel } 316 \\
2 \\
1 \\
15 \\
3627.00 \mathrm{~kg}\end{array}$ \\
\hline $\begin{array}{l}\text { Matrix } 2 \\
\text { AreaM2 } \\
\text { CompM2 } \\
\text { CovLayM2 } \\
\text { InFrAl21 } \\
\text { Invid21 } \\
\text { MassM2 }\end{array}$ & $\begin{array}{rl}31173.12 & \mathrm{~m}^{2} \\
14 & \mathrm{UO} 2 \\
4 \\
1 \\
15 \\
7872.00 \mathrm{~kg}\end{array}$ \\
\hline
\end{tabular}


3. System Characterization: Waste Package

Table 3-6. Total Curies (DOE SNF and DHLW) by Category

COMPLEX CUM TSPA Category

SNF, not HLW

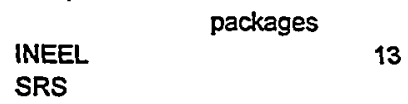

Hanford 105

Total 118

SNF (Ci/pkg)
(based upon 4 MCO's / package)

Isotopes

C 14

CL 36

NI 59

NI 63

SE 79

SR9O

Y90

ZR93

NB $93 M$

NB 94

TC 99

RU106

RH105

PD107

SN126

1129

CS134

CS135

CS137

BA137M

CE144

PR144

PM147

SM151

PB210

RA226

RA228

AC227

TH229

TH230

TH232

PA231

U233

U234

U235

U236

U238

NP237

PU238

PU239

PU240

PU241

PU242

AM241

AM242M

AM243

CM244

CM245

CM246
1

$4 \times 0 \times 15^{1}$

8

$0.00 E+00$

3.21E-01

3.03E $\div 01$

7.06E-01

$3.96 E+04$

$3.96 \mathrm{E}+04$

3.31E+00

$3.03 E+00$

$1.06 \mathrm{E}-06$

2.37E+01

1.18E-01

1.23E+00

$5.18 \mathrm{E}-02$

6.30E-01

5.32E+04

$5.32 E+04$

1.16E+03

$6.35 \mathrm{E}-10$

1.80E-05

1.46E-09

8.59E-05

1.46E-07

1.61E-05

1.90E-09

2.16E-04

9.01E-05

$7.64 E+00$

2.92E-01

$1.11 E+00$

$6.01 E+00$

6.10E-01

$8.56 \mathrm{E}+02$

$1.95 E+03$

1.13E+03

1.24E+04

5.46E-01

4.27E+03

1.14E-01

6.44E-01

$1.78 E+01$

8.06E-03

1.07E-03
Total Curies

Per Category

$6.72 E+02$

$0.00 E+00$

$3.79 E+01$

3.57E+03

$8.33 E+01$

4.67E+06

4. $67 E+06$

3.91E+02

$3.57 \mathrm{E}+02$

1.25E-04

$2.80 E+03$

1.39E+01

$1.46 \mathrm{E}+02$

6. $12 \mathrm{E}+00$

7.43E+01

$6.28 \mathrm{E}+06$

$6.28 \mathrm{E}+06$

1.36E+05

7.49E-08

2.13E-03

$1.72 E-07$

1.01E-02

1.73E-05

1.90E-03

2.25E-07

2.55E-02

1.06E-02

$9.02 E+02$

$3.45 \mathrm{E}+01$

$1.31 E+02$

7.09E+02

$7.20 \mathrm{E}+01$

$1.01 E+05$

2.30E+05

1.33E+05

1.46E+06

$6.44 E+01$

$5.04 E+05$

1.34E+01

7.60E +01

2.10E+03

9.52E-01

1.26E-01

2.45E+07

Total Cilpkg.

Total Ci/category
2.07E +05

$2.45 E+07$ 
Table 3-6. Total Curies (DOE SNF and DHLW) by Category (continued)

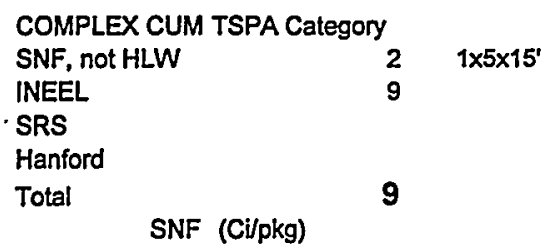

$1 \times 5 \times 15^{\prime}$

(1)

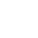

7.27E-06

$0.00 E+00$

$0.00 E+00$

$0.00 E+00$

2.61E-02

6.26E+03

$6.26 E+03$

1.34E-01

7.71E-03

1.45E-06

8.81E-01

1.01E-03

2.34E-02

1.45E-03

6.83E-03

$6.52 E+03$

$6.52 \mathrm{E}+03$

2.36E+01

7.65E-13

1.95E-12

1.10E-13

4.23E-09

9.41E-11

7.60E-09

1.80E-12

2.03E-07

8.09E-07

7.60E-04

7.27E-03

2.98E-02

1.33E-04

1.78E-02

3.83E+01

9.52E-01

5.44E-01

1.87E+02

8.15E-04

3.42E-01

1.03E-03

3.37E-03

1.39E-01

7.10E-06

4.89E-07
SNF (Ci/cat)

\subsection{E-05}

$0.00 E+00$

$0.00 E+00$

$0.00 E+00$

2.35E-01

$5.63 E+04$

5.63E+04

$1.21 E+00$

6.94E-02

1.31E-05

$7.93 E+00$

$0.00 E+00$

$0.00 E+00$

9.12E-03.

2.11E-01

1.30E-02

$0.00 E+00$

6.14E-02

5.87E+04

$5.87 E+04$

$0.00 E+00$

$0.00 E+00$

$0.00 E+00$

2.12E+02

6.89E-12

1.75E-11

9.91E-13

3.80E-08

8.47E-10

6.84E-08

1.62E-11

1.83E-06

7.28E-06

$6.84 \mathrm{E}-03$

6.54E-02

2.69E-01

1.20E-03

1.60E-01

$3.45 E+02$

$8.57 E+00$

$4.90 E+00$

$1.68 E+03$

7.33E-03

$3.08 E+00$

9.31E-03

3.04E-02

$1.25 E+00$

6.39E-05

4.40E-06
DHLW (Ci/can)

$0.00 E+00$ per package

$0.00 E+00$
$0.00 E+00$

3.31E-02 2.49E-01

$3.58 E+00 \quad 2.68 E+01$

1.01E-01 7.57E-01

$3.04 E+04 \quad 2.28 E+05$

$3.08 E+04 \quad 2.31 E+05$

7.71E-01 $5.78 E+00$

1.35E-01 $1.02 E+00$

3.33E-05 2.49E-04

$3.63 E+00 \quad 2.72 E+01$

$2.05 E+03$

2.05E+03 $1.54 E+04$

1.24E-02 $9.27 \mathrm{E}-02$

2.39E-01 1.79E+00

2.09E-06 1.56E-05

$2.42 E+03 \quad 1.81 E+04$

1.27E-01 9.49E-01

3.05E+04 2.28E+05

$2.88 \mathrm{E}+04 \quad 2.16 \mathrm{E}+05$

1.25E+04 $9.41 E+04$

$1.25 E+04 \quad 9.41 E+04$

$2.12 E+04$

$3.09 E+02 \quad 2.32 E+03$

$0.00 E+00 \quad 0.00 E+00$

$0.00 E+00 \quad 0.00 E+00$

1.07E-04 8.04E-04

5.80E-06 4.35E-05

1.48E-05 1.11E-04

4.24E-06 3.18E-05

1.16E-04 8.69E-04

1.07E-03 8.04E-03

6.38E-04 4.78E-03

1.27E-02 9.55E-02

8.70E-05 6.52E-04

4.76E-04 3.57E-03

4.15E-03 3.11E-02

3.02E-02 2.27E-01

$5.58 \mathrm{E}+02 \quad 4.18 \mathrm{E}+03$

$5.20 \mathrm{E}+00 \quad 3.90 \mathrm{E}+01$

$3.57 \mathrm{E}+00 \quad 2.68 \mathrm{E}+01$

$6.89 \mathrm{E}+02 \quad 5.17 \mathrm{E}+03$

5.52E-03 4.14E-02

8.20E+01 6.15E+02

2.60E-02 1.95E-01

4.05E-02 3.03E-01

$3.92 \mathrm{E}+01 \quad 2.94 \mathrm{E}+02$

6.44E-05 $\quad 4.83 \mathrm{E}-04$

7.24E-06

5.43E-05

$1.31 E+06$

Total Ci/pkg. =

Total Cilcategory $=$
Total Ci (SNF Total Ci

+ DHLW Per Category

7.27E-06 6.54E-05

$0.00 E+00 \quad 0.00 E+00$

2.49E-01 2.24E+00

$2.68 E+01 \quad 2.41 E+02$

7.83E-01 7.05E+00

$2.34 E+05 \quad 2.11 E+06$

2.37E+05 2.13E+06

$5.92 E+00 \quad 5.33 E+01$

$1.02 E+00 \quad 9.21 E+00$

2.51E-04 2.26E-03

$2.81 E+01 \quad 2.53 E+02$

$1.53 E+04 \quad 1.38 E+05$

$1.54 E+04 \quad 1.38 E+05$

9.38E-02 8.44E-01

$1.82 E+00 \quad 1.64 E+01$

$1.46 \mathrm{E}-03 \quad 1.32 \mathrm{E}-02$

$1.81 E+04 \quad 1.63 E+05$

9.56E-01 8.60E+00

2.35E+05 2.11E+06

2.22E+05 2.00E+06

9.41E+04 8.47E+05

9.41E+04 8.47E+05

$1.59 E+05 \quad 1.43 E+06$

$2.34 \mathrm{E}+03 \quad 2.11 \mathrm{E}+04$

7.65E-13 6.89E-12

$1.95 \mathrm{E}-12 \quad 1.75 \mathrm{E}-11$

8.04E-04 7.23E-03

4.35E-05 3.91E-04

1.11E-04 1.00E-03

3.18E-05 2.86E-04

8.69E-04 7.82E-03

8.04E-03 7.24E-02

4.79E-03 4.31E-02

9.63E-02 8.66E-01

7.92E-03 7.13E-02

3.34E-02 3.01E-01

3.13E-02 2.82E-01

2.44E-01 2.20E+00

4.22E+03 3.80E+04

4.00E+01 3.60E+02

$2.73 E+01$ - 2.46E+02

$5.35 E+03 \quad 4.82 E+04$

4.22E-02 3.80E-01

$6.15 E+02 \quad 5.54 E+03$

$1.96 \mathrm{E}-01 \quad 1.76 \mathrm{E}+00$

3.07E-01 2.76E+00

$2.94 E+02 \quad 2.65 E+03$

4.90E-04 4.41E-03

$5.48 E-05 \quad 4.93 E-04$

1.34E+06

$1.20 E+07$ 
Table 3-6. Total Curies (DOE SNF and DHLW) by Category (continued)

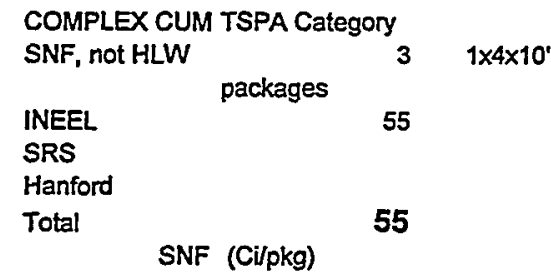

\section{Isotopes}

C 14

CL 36

NI 59

NI 63

SE 79

SR90

Y90

ZR93

NB 93M

NB 94

TC 99

RU106

RH106

PD107

SN126

1129

CS134

CS135

CS137

BA137M

CE144

PR144

PM147

SM151

PB210

RA226

RA228

AC227

TH229

TH230

TH232

PA231

U233

U234

U235

U236

U238

NP237

PU238

PU239

PU240

PU241

PU242

AM241

AM242M

AM243

CM244

CM245

CM246

SNF (Cipkg)

$$
\text { SNF (Ci/Cat) }
$$

2.73E-03

6.93E-06

8.53E-03

1.78E-01

2.07E-03

1.95E+02

$1.95 \mathrm{E}+02$

8.27E-03

5.75E-03

1.04E-03

5.62E-02

1.60E-04

4.72E-03

$1.44 \mathrm{E}-04$

5.63E-03

$2.32 E+02$

$2.32 E+02$

8. $64 \mathrm{E}+00$

1.79E-10

8.00E-10

5.01E-09

8.63E-06

2.93E-09

1.43E-07

5.29E-09

2.44E-05

1.23E-06

6.15E-04

3.92E-02

1.59E-03

$1.78 \mathrm{E}-02$

4.14E-04

2.07E-02

2. $45 E+00$

7.58E-03

7.53E-04

4.77E-11

5.66E-05

$0.00 E+00$

9.13E-13

8.50E-14

2.32E-19

7.59E-23

\begin{tabular}{|c|c|c|}
\hline Cat) & DHLW (CiJcan) & $\begin{array}{l}\text { Total Ci DHLW } \\
\text { per package }\end{array}$ \\
\hline 1.50E-01 & $0.00 E+00$ & $0.00 E+00$ \\
\hline 3.81E-04 & $0.00 E+00$ & $0.00 E+00$ \\
\hline 4.69E-01. & 3.31E-02 & 1.33E-01 \\
\hline $9.79 E+00$ & $3.58 \mathrm{E}+00$ & $1.43 E+01$ \\
\hline $1.14 \mathrm{E}-01$ & $1.01 E-01$ & 4.04E-01 \\
\hline $1.07 E+04$ & $3.04 E+04$ & $1.22 \mathrm{E}+05$ \\
\hline $1.07 E+04$ & $3.08 E+04$ & $1.23 E+05$ \\
\hline 4.55E-01 & 7.71E-01 & $3.08 E+00$ \\
\hline $3.16 \mathrm{E}-01$ & $1.35 \mathrm{E}-01$ & 5.42E-01 \\
\hline $5.72 E-02$ & $3.33 E-05$ & 1.33E-04 \\
\hline $3.09 E+00$ & $3.63 E+00$ & $1.45 E+01$ \\
\hline $0.00 \mathrm{E}+00$ & 2.05E+03 & $8.18 E+03$ \\
\hline $0.00 E+00$ & 2.05E+03 & 8.19E+03 \\
\hline 8.80E-03 & $1.24 E-02$ & 4.95E-02 \\
\hline $2.60 E-01$ & 2.39E-01 & 9.57E-01 \\
\hline 7.92E-03 & 2.09E-06 & 8.34E-06 \\
\hline $0.00 E+00$ & $2.42 E+03$ & $9.66 E+03$ \\
\hline $3.10 E-01$ & $1.27 E-01$ & 5.06E-01 \\
\hline $1.28 \mathrm{E}+04$ & $3.05 E+04$ & $1.22 E+05$ \\
\hline $1.28 \mathrm{E}+04$ & $2.88 E+04$ & $1.15 E+05$ \\
\hline $0.00 E+00$ & $1.25 E+04$ & $5.02 E+04$ \\
\hline $0.00 \mathrm{E}+00$ & 1.25E+04 & $5.02 E+04$ \\
\hline $0.00 E+00$ & 2.12E+04 & $8.49 E+04$ \\
\hline 4.75E+02 & $3.09 E+02$ & $1.24 E+03$ \\
\hline $9.85 E-09$ & $0.00 E+00$ & $0.00 E \div 00$ \\
\hline 4.40E-08 & $0.00 E+00$ & $0.00 E+00$ \\
\hline 2.76E-07 & $1.07 \mathrm{E}-04$ & $4.29 E-04$ \\
\hline 4.75E-04 & $5.80 \mathrm{E}-06$ & $2.32 E-05$ \\
\hline 1.61E-07 & $1.48 \mathrm{E}-05$ & 5.92E-05 \\
\hline 7.87E-06 & $4.24 \mathrm{E}-06$ & 1.69E-05 \\
\hline 2.91E-07. & $1.16 E-04$ & 4.63E-04 \\
\hline $1.34 \mathrm{E}-03^{\circ}$ & $1.07 E-03$ & 4.29E-03 \\
\hline $6.77 E-05$ & $6.38 \mathrm{E}-04$ & $2.55 E-03$ \\
\hline $3.38 E-02$ & 1.27E-02 & 5.09E-02 \\
\hline $2.16 \mathrm{E}+00$ & $8.70 \mathrm{E}-05$ & $3.48 \mathrm{E}-04$ \\
\hline $8.75 E-02$ & $4.76 E-04$ & $1.90 \mathrm{E}-03$ \\
\hline $9.79 E-01$ & $4.15 E-03$ & $1.66 \mathrm{E}-02$ \\
\hline $2.28 E-02$ & $3.02 E-02$ & 1.21E-01 \\
\hline $1.14 E+00$ & $5.58 E+02$ & $2.23 E+03$ \\
\hline 1.35E+02 & $5.20 \mathrm{E}+00$ & $2.08 E+01$ \\
\hline 4.17E-01 & $3.57 E+00$ & $1.43 E+01$ \\
\hline 4.14E-02 & $6.89 E+02$ & $2.76 E+03$ \\
\hline 2.62E-09 & $5.52 \mathrm{E}-03$ & 2.21E-02 \\
\hline 3.11E-03 & $8.20 E+01$ & $3.28 \mathrm{E}+02$ \\
\hline $0.00 \mathrm{E} \div 00$ & 2.60E-02 & $1.04 \mathrm{E}-01$ \\
\hline $5.02 E-11$ & $4.05 E-02$ & $1.62 E-01$ \\
\hline $4.68 \mathrm{E}-12$ & $3.92 E+01$ & $1.57 E+02$ \\
\hline 1.28E-17 & $6.44 E-05$ & $2.58 E-04$ \\
\hline \multirow[t]{3}{*}{ 4.17E-21 } & 7.24E-06 & $2.90 E-05$ \\
\hline & & $7.00 E+05$ \\
\hline & & tegory = \\
\hline
\end{tabular}

Prepared by Lenny Stor 9/4/98
Total Ci (SNF Total Ci + DHLW) Per Category 2.73E-03 1.50E-01 6.93E-06 3.81E-04 $1.41 E-01 \quad 7.76 E+00$ $1.45 \mathrm{E}+01 \quad 7.97 \mathrm{E}+02$ 4.06E-01 2.23E+01 $1.22 E+05 \quad 6.70 E+06$ $1.23 \mathrm{E}+05 \quad 6.78 \mathrm{E}+06$ $3.09 E+00 \quad 1.70 E+02$ 5.48E-01 3.01E+01 1.17E-03 6.45E-02 $1.46 E+01 \quad 8.02 E+02$ $8.18 \mathrm{E}+03 \quad 4.50 \mathrm{E}+05$ 8.19E+03 4.51E+05 4.96E-02 2.73E+00 9.62E-01 5.29E+01 1.52E-04 8.38E-03 $9.66 E+03 \quad 5.32 E+05$ 5.12E-01 2.82E+01 $1.22 E+05 \quad 6.71 E+06$ $1.15 \mathrm{E}+05 \quad 6.34 \mathrm{E}+06$ $5.02 E+04 \quad 2.76 E+06$ $5.02 E+04 \quad 2.76 E+06$ 8.49E+04 4.67E+06 $1.24 E+03 \quad 6.84 E+04$ $1.79 \mathrm{E}-10 \quad 9.85 \mathrm{E}-09$ 8.00E-10 4.40E-08 4.29E-04 2.36E-02 3.18E-05 1.75E-03 5.93E-05 3.26E-03 1.71E-05 9.40E-04 4.63E-04 2.55E-02 4.31E-03 2.37E-01 2.55E-03 1.40E-01 5.16E-02 2.84E+00 3.95E-02 2.18E+00 3.49E-03 1.92E-01 3.44E-02 1.89E+00 1.21E-01 6.67E+00 $2.23 E+03 \quad 1.23 E+05$ 2.33E+01 1.28E+03 1.43E+01 7.86E+02 $2.76 E+03 \quad 1.52 E+05$ 2.21E-02 1.22E +00 $3.28 E+02 \quad 1.80 E+04$ 1.04E-01 5.72E+00 1.62E-01 8.90E+00 $1.57 E+02 \quad 8.63 E+03$ 2.58E-04 1.42E-02 2.90E-05 1.59E-03

$7.01 E+05$ $3.85 E+07$ 
Table 3-6. Total Curies (DOE SNF and DHLW) by Category (continued)

\begin{tabular}{|c|c|c|c|c|c|c|}
\hline \multicolumn{7}{|c|}{ COMPLEX CUM TSPA Category } \\
\hline SNF, not $\mathrm{H}$ & 4 & $1 \times 5 \times 10^{\prime}$ & & & & \\
\hline & ges & & & & & \\
\hline INEEL & 182 & & & & . & \\
\hline SRS & & & & & & \\
\hline Hanford & 21 & & & & & \\
\hline Total & 203 & & & & & \\
\hline & & & & & & \\
\hline & & & W (Ci/can) & Total Ci DHLW & Total Ci (SNF & Total Ci \\
\hline Isotopes & & & & per package & + DHLW) & Per Category \\
\hline C 14 & 3.75E-02 & $7.61 E+00$ & $0.00 E+00$ & $0.00 E+00$ & 3.75E-02 & $7.61 E+00$ \\
\hline CL 36 & $0.00 E+00$ & $0.00 E+00$ & $0.00 E+00$ & $0.00 E+00$ & $0.00 E+00$ & $0.00 E+00$ \\
\hline N1 59 & $0.00 E+00$ & $0.00 E+00$ & $3.31 E-02$ & $1.66 \mathrm{E}-01$ & $1.66 \mathrm{E}-01$ & $3.36 E+01$ \\
\hline NI 63 & $1.72 E+03$ & $3.48 E+05$ & $3.58 E+00$ & $1.79 E+01$ & $1.73 E+03$ & $3.52 E+05$ \\
\hline SE 79 & $1.88 E-01$ & $3.82 E+01$ & $1.01 E-01$ & $5.05 E-01$ & 6.93E-01 & $1.41 E+02$ \\
\hline SR9O & $2.37 E+04$ & $4.81 E+06$ & $3.04 E+04$ & $1.52 E+05$ & 1.76E+05 & 3.57E+07 \\
\hline Y90 & 2.37E +04 & $4.81 E+06$ & $3.08 E+04$ & 1.54E+05 & $1.78 \mathrm{E}+05$ & $3.60 \mathrm{E}+07$ \\
\hline ZR93 & $8.83 E-01$ & $1.79 E+02$ & 7.71E-01 & $3.86 E+00$ & $4.74 E+00$ & $9.62 E+02$ \\
\hline NB 93M & 5.93E-01 & $1.20 E+02$ & 1.35E-01 & 6.77E-01 & $1.27 \mathrm{E}+00$ & $2.58 E+02$ \\
\hline NB 94 & $0.00 E+00$ & $0.00 E+00$ & 3.33E-05 & $1.66 E-04$ & $1.66 \mathrm{E}-04$ & 3.38E-02 \\
\hline TC 99 & $6.53 E+00$ & 1.33E+03 & $3.63 E+00$ & $1.82 E+01$ & 2.47E+01 & $5.01 E+03$ \\
\hline RU106 & & $0.00 E+00$ & $2.05 E+03$ & $1.02 E+04$ & $1.02 E+04$ & $2.08 E+06$ \\
\hline RH106 & & $0.00 E+00$ & 2.05E +03 & $1.02 E+04$ & $1.02 E+04$ & $2.08 E+06$ \\
\hline PD107 & $4.79 E-02$ & $9.72 E+00$ & $1.24 E-02$ & $6.18 E-02$ & 1.10E-01 & $2.23 E+01$ \\
\hline SN126 & 2.38E-01 & $4.83 E+01$ & 2.39E-01 & $1.20 E+00$ & $1.43 E+00$ & 2.91E+02 \\
\hline 1129 & $1.77 E-02$ & $3.58 E+00$ & 2.09E-06 & $1.04 E-05$ & 1.77E-02 & $3.59 E+00$ \\
\hline CS134 & & $0.00 E+00$ & $2.42 E+03$ & $1.21 E+04$ & $1.21 E+04$ & $2.45 E+06$ \\
\hline CS135 & 1.52E-01 & $3.08 E+01$ & 1.27E-01 & $6.33 E-01$ & 7.85E-01 & $1.59 E+02$ \\
\hline CS137 & $3,19 E+04$ & $6.47 E+06$ & $3.05 E+0.4$ & $1.52 E+05$ & $1.84 E+05$ & $3.74 E+07$ \\
\hline BA137M & $3.19 E+04$ & $6.47 E+06$ & $2.88 E+04$ & $1.44 E+05$ & $1.76 E+05$ & 3.57E+07 \\
\hline CE144 & & $0.00 E+00$ & $1.25 E+04$ & $6.27 E+04$ & $6.27 E+04$ & $1.27 E+07$ \\
\hline PR144 & & $0.00 E+00$ & $1.25 E+04$ & $6.27 E+04$ & $6.27 E+04$ & $1.27 E+07$ \\
\hline PM147 & & $0.00 E+00$ & $2.12 E+04$ & 1.06E+05 & $1.06 E+05$ & $2.15 E+07$ \\
\hline SM151 & $5.42 E+02$ & $1.10 E+05$ & $3.09 E+02$ & 1.54E+03 & $2.09 E+03$ & $4.24 E+05$ \\
\hline PB210 & $3.57 E-08$ & $7.25 E-06$ & $0.00 E+00$ & $0.00 E+00$ & $3.57 E-08$ & $7.25 E-06$ \\
\hline RA226 & $1.49 E-07$ & $3.02 E-05$ & $0.00 E+00$ & $0.00 E+00$ & $1.49 E-07$ & $3.02 E-05$ \\
\hline RA228 & $1.29 E-04$ & 2.62E-02 & $1.07 E-04$ & 5.36E-04 & 6.65E-04 & 1.35E-01 \\
\hline$A C 227$ & 1.60E-04 & $3.26 E-02$ & $5.80 E-05$ & 2.90E-05 & $1.89 E-04$ & $3.84 E-02$ \\
\hline TH229 & 3.84E-04 & 7.79E-02 & $1.48 E-05$ & 7.41E-05 & $4.58 E-04$ & 9.30E-02 \\
\hline TH230 & $2.65 E-05$ & 5.39E-03 & 4.24E-06 & 2.12E-05 & 4.77E-05 & $9.69 E-03$ \\
\hline TH232 & $1.36 E-04$ & $2.76 E-02$ & $1.16 E-04$ & 5.79E-04 & 7.15E-04 & 1.45E-01 \\
\hline PA231 & 2.81E-04 & 5.71E-02 & 1.07E-03 & 5.36E-03 & $5.64 E-03$ & $1.15 E+00$ \\
\hline U233 & 1.49E-01 & $3.02 E+01$ & 6.38E-0. & 3.19E-03 & $1.52 E-01$ & $3.08 E+01$ \\
\hline U234 & $1.43 E-01$ & $2.90 E+01$ & 1.27E-02 & 6.37E-02 & 2.06E-01 & 4.19E+01 \\
\hline U235 & $1.61 E-02$ & $3.27 \mathrm{E} \div 00$ & $8.70 E-05$ & 4.35E-0.4 & $1.65 E-02$ & $3.36 E+00$ \\
\hline U236 & $1.35 E-01$ & $2.73 E+01$ & $4.76 \mathrm{E}-04$ & 2.38E-03 & $1.37 E-01$ & $2.78 E+01$ \\
\hline U238 & $1.26 \mathrm{E}-01$ & $2.56 \mathrm{E}+01$ & 4.15E-03 & $2.08 E-02$ & 1.47E-01 & $2.98 E+01$ \\
\hline NP237 & 1.73E-01 & $3.52 E+01$ & $3.02 E-02$ & $1.51 E-01$ & $3.24 E-01$ & $6.58 E+01$ \\
\hline PU238 & $1.68 E+03$ & $3.41 E+05$ & $5.58 \mathrm{E}+02$ & $2.79 E+03$ & $4.47 E+03$ & $9.07 E+05$ \\
\hline PU239 & $1.85 E+02$ & $3.76 \mathrm{E}+04$ & $5.20 E+00$ & $2.60 E+01$ & $2.11 E+02$ & $4.28 E+04$ \\
\hline PU240 & $2.76 E+02$ & $5.61 E+04$ & 3.57E+00 & $1.78 \mathrm{E} \div 01$ & $2.94 E+02$ & 5.97E+04 \\
\hline PU241 & $1.47 E+04$ & $2.98 E+06$ & $6.89 E+02$ & 3.44E $\div 03$ & $1.81 E+04$ & $3.68 E+06$ \\
\hline PU242 & $9.12 E-01$ & $1.85 E+02$ & $5.52 E-03$ & 2.76E-02 & $9.40 \mathrm{E}-01$ & $1.91 E+02$ \\
\hline AM241 & 1.81E+03 & $3.67 E+05$ & $8.20 E+01$ & 4.10E+02 & $2.22 E+03$ & $4.50 E+05$ \\
\hline AM242M & $2.62 E+00$ & $5.31 E+02$ & 2.60E-02 & $1.30 E-01$ & $2.75 E+00$ & $5.57 E+02$ \\
\hline AM243 & $8.29 E+00$ & $1.68 E+03$ & $4.05 E-02$ & 2.02E-01 & $8.49 E+00$ & $1.72 E+03$ \\
\hline CM244 & $3.60 E+02$ & $7.31 E+04$ & $3.92 E+01$ & 1.96E+02 & $5.56 E+02$ & 1.13E $\div 05$ \\
\hline CM245 & $1.42 E-01$ & $2.89 E+01$ & $6.44 E-05$ & $3.22 E-04$ & 1.43E-01 & $2.90 E+01$ \\
\hline CM246 & $2.42 E-02$ & $4.91 E+00$ & 7.24E-06 & 3.62E-05 & 2.42E-02 & $4.92 E+00$ \\
\hline & & & & $8.75 E+05$ & & \\
\hline & $1.32 E+05$ & & & $\begin{array}{l}\text { lpkg. = } \\
\text { /category = }\end{array}$ & $\begin{array}{l}1.01 E+06 \\
2.04 E+08\end{array}$ & \\
\hline
\end{tabular}


Table 3-6. Total Curies (DOE SNF and DHLW) by Category (continued)

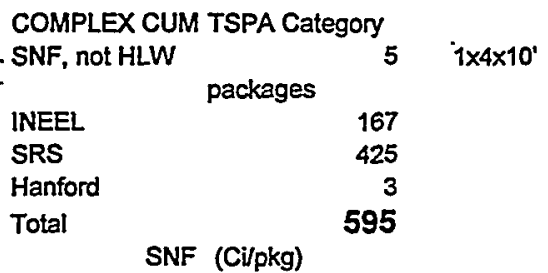

$\begin{array}{lc}\text { Isotopes } & \\ \text { C 14 } & 1.65 E-03 \\ \text { CL 36 } & 6.08 E-06 \\ \text { NI 59 } & 1.19 E-03 \\ \text { NI 63 } & 1.46 E-01 \\ \text { SE 79 } & 4.45 E-02 \\ \text { SR90 } & 1.22 E+04 \\ \text { Y90 } & 1.22 E+04 \\ \text { ZR93 } & 2.27 E-01 \\ \text { NB 93M } & 2.11 E-02 \\ \text { NB 94 } & 1.30 E-05 \\ \text { TC 99 } & 1.50 E+00 \\ \text { RU106 } & \\ \text { RH106 } & \\ \text { PD107 } & 2.32 E-03 \\ \text { SN126 } & 4.40 E-02 \\ \text { I129 } & 2.55 E-03 \\ \text { CS134 } & \\ \text { CS135 } & 1.80 E-02 \\ \text { CS137 } & 1.30 E+04 \\ \text { BA137M } & 1.30 E+04 \\ \text { CE144 } & \\ \text { PR144 } & \\ \text { PM147 } & \\ \text { SM151 } & \\ \text { PB210 } & 5.42 E+01 \\ \text { RA226 } & 6.82 E-09 \\ \text { RA228 } & 2.60 E-08 \\ \text { AC227 } & 1.15 E-05 \\ \text { TH229 } & 1.40 E-05 \\ \text { TH230 } & 3.31 E-05 \\ \text { TH232 } & 3.82 E-06 \\ \text { PA231 } & 1.21 E-05 \\ \text { U233 } & 2.49 E-05 \\ \text { U234 } & 1.26 E-02 \\ \text { U235 } & 1.48 E-02 \\ \text { U236 } & 1.74 E-02 \\ \text { U238 } & 4.95 E-05 \\ \text { NP237 } & \\ \text { PU238 } & \end{array}$

\begin{tabular}{|c|c|c|c|}
\hline DHLW (Ci/can) & $\begin{array}{l}\text { Total Ci DHLW } \\
\text { per package }\end{array}$ & $\begin{array}{l}\text { Total Ci (SNF } \\
\text { + DHLW) }\end{array}$ & $\begin{array}{l}\text { Total } \mathrm{Ci} \\
\text { Per Category }\end{array}$ \\
\hline $0.00 \mathrm{E}+00$ & $0.00 E+00$ & 1.65E-03 & $3 \quad 9.80 \mathrm{E}-01$ \\
\hline $0.00 E+00$ & $0.00 E \div 00$ & $6.08 \mathrm{E}-06$ & $3.62 E-03$ \\
\hline 3.31E-02 & 1.33E-01 & $1.34 \mathrm{E}-01$ & $7.96 E+01$ \\
\hline $3.58 E+00$ & $1.43 E+01$ & $1.45 E+01$ & $8.60 E+03$ \\
\hline $1.01 E-01$ & 4.04E-01 & $4.48 \mathrm{E}-01$ & 2.67E+02 \\
\hline $3.04 E+04$ & $1.22 E+05$ & $1.34 E+05$ & $7.96 E+07$ \\
\hline $3.08 E+04$ & $1.23 E+05$ & 1.35E+05 & $8.05 E+07$ \\
\hline 7.71E-01 & $3.08 E+00$ & $3.31 E+00$ & $1.97 E+03$ \\
\hline $1.35 \mathrm{E}-01$ & $5.42 E-01$ & 5.63E-01 & $3.35 E+02$ \\
\hline 3.33E-05 & 1.33E-04 & 1.46E-04 & 8.69E-02 \\
\hline $3.63 \mathrm{E}+00$ & $1.45 E+01$ & $1.60 E+01$ & $9.54 E+03$ \\
\hline $2.05 E+03$ & $8.18 E+03$ & $8.18 E+03$ & $4.87 E+06$ \\
\hline $2.05 E+03$ & $8.19 E+03$ & $8.19 E+03$ & $4.88 E+06$ \\
\hline $1.24 \mathrm{E}-02$ & 4.95E-02 & $5.18 \mathrm{E}-02$ & $3.08 \mathrm{E}+01$ \\
\hline $2.39 \mathrm{E}-01$ & $9.57 \mathrm{E}-01$ & $1.00 E+00$ & $5.96 E+02$ \\
\hline $2.09 E-06$ & 8.34E-06 & 2.56E-03 & $1.52 E+00$ \\
\hline $2.42 E+03$ & $9.66 E+03$ & $9.66 E+03$ & $5.75 E+06$ \\
\hline 1.27E-01 & $5.06 \mathrm{E}-01$ & $5.24 E-01$ & $3.12 E+02$ \\
\hline $3.05 E+04$ & $1.22 E+05$ & 1.35E+05 & $8.02 E+07$ \\
\hline $2.88 \mathrm{E}+0.4$ & $1.15 E+05$ & $1.28 E+05$ & $7.62 E+07$ \\
\hline $1.25 E+04$ & $5.02 E+04$ & $5.02 E+04$ & 2.99E+07 \\
\hline $1.25 E+04$ & $5.02 E+04$ & $5.02 E+04$ & $2.99 E+07$ \\
\hline $2.12 E+04$ & $8.49 E+04$ & $8.49 E+04$ & $5.05 E+07$ \\
\hline $3.09 \mathrm{E}+02$ & $1.24 E+03$ & $1.29 E+03$ & $7.67 \mathrm{E}+05$ \\
\hline $0.00 E+00$ & $0.00 E+00$ & $6.82 E-09$ & 4.06E-06 \\
\hline $0.00 E+00$ & $0.00 E+00$ & 2.60E-08 & $1.54 E-05$ \\
\hline $1.07 E-04$ & 4.29E-04 & $4.40 \mathrm{E}-04$ & 2.62E-01 \\
\hline $5.80 E-06$ & 2.32E-05 & $3.72 E-05$ & 2.21E-02 \\
\hline $1.48 \mathrm{E}-05$ & $5.92 E-05$ & $9.24 E-05$ & $5.50 \mathrm{E}-02$ \\
\hline 4.24E-08 & 1.69E-05 & 2.08E-05 & $1.24 \mathrm{E}-02$ \\
\hline 1.16E-04 & $4.63 E-04$ & $4.75 \mathrm{E}-04$ & 2.83E-01 \\
\hline 1.07E-03 & 4.29E-03 & 4.31E-03 & $2.57 E+00$ \\
\hline $6.38 E-04$ & 2.55E-03 & $1.52 \mathrm{E}-02$ & $9.04 E+00$ \\
\hline $1.27 \mathrm{E}-02$ & $5.09 \mathrm{E}-02$ & $6.57 \mathrm{E}-02$ & $3.91 E+01$ \\
\hline 8.70E-05 & $3.48 \mathrm{E}-04$ & 1.77E-02 & $1.05 E+01$ \\
\hline $4.76 E-04$ & $1.90 E-03$ & 5.11E-02 & $3.04 E+01$ \\
\hline 4.15E-03 & $1.66 \mathrm{E}-02$ & $6.32 \mathrm{E}-02$ & $3.76 \mathrm{E}+01$ \\
\hline $3.02 E-02$ & $1.21 E-01$ & $1.49 E-01$ & $8.87 E+01$ \\
\hline $5.58 E+02$ & $2.23 E+03$ & $2.29 E+03$ & $1.36 E+06$ \\
\hline $5.20 E+\infty 0$ & $2.08 \mathrm{E}+01$ & $3.91 E+01$ & $2.33 E+04$ \\
\hline $3.57 E+00$ & $1.43 E+01$ & $2.10 E+01$ & $1.25 E+04$ \\
\hline $6.89 E+02$ & $2.76 E+03$ & $3.42 E+03$ & $2.03 E+06$ \\
\hline $5.52 E-03$ & $2.21 E-02$ & $2.75 E-02$ & $1.64 E+01$ \\
\hline $8.20 \mathrm{E}+01$ & $3.28 E+02$ & $3.39 E+02$ & $2.01 E+05$ \\
\hline $2.60 \mathrm{E}-02$ & $1.04 \mathrm{E}-01$ & 1.26E-01 & $7.47 E+01$ \\
\hline 4.05E-02 & $1.62 \mathrm{E}-01$ & $1.72 E-01$ & $1.02 E+02$ \\
\hline $3.92 \mathrm{E}+01$ & $1.57 E+02$ & $1.57 E+02$ & $9.36 E+04$ \\
\hline $6.44 E-05$ & 2.58E-04 & $3.60 E-04$ & $2.14 E-01$ \\
\hline \multirow[t]{3}{*}{$7.24 E-06$} & $2.90 E-05$ & 4.54E-05 & $2.70 E-02$ \\
\hline & & $7.51 E+05$ & \\
\hline & ego & 4.47E+08 & \\
\hline
\end{tabular}

Prepared by Lenny Storz 9/4/98 
Table 3-6. Total Curies (DOE SNF and DHLW) by Category (continued)

COMPLEX CUM TSPA Category SNF, not HLW packages

INEEL

SRS

Hanford

Total SNF (Ci/pkg)

Isotopes

C 14

CL 36

NI 59

NI 63

SE 79

SR90

Y90

ZR93

NB 93M

NB 94

TC 99

RU106

RH106

PD107

SN126

1129

CS134

CS135

CS137

BA137M

CE144

PR144

PM147

SM151

PB210

RA226

RA228

AC227

TH229

TH230

TH232

PA231

U233

U234

U235

U236

U238

NP237

PU238

PU239

PU240

PU241

PU242

AM241

AM242M

AM243

CM244

CM245

CM246
750

$1.52 E-02$

4.58E-04

8.93E-02

1.10E+01

4.78E-02

$1.14 E+04$

1.14E+04

2.47E-01

1.46E-02

8.34E-04

$1.61 E+00$

1.87E-03

4.28E-02

2.65E-03

1.72E-02

1.19E+04

1.19E+04

4.49E+01

1.42E-12

$3.64 \mathrm{E}-12$

7.33E-10

1.71E-08

3.63E-10

1.37E-08

2.16E-09

5.09E-07

2.44E-06

1.35E-03

1.43E-02

5.41E-02

1.52E-03

3.12E-02

$6.70 \mathrm{E}+01$

2.57E+00

$1.30 \mathrm{E}+00$

$3.47 E+02$

$1.46 E-03$

$6.29 E-01$

2.04E-03

5.91E-03

2.43E-01

1.24E-05

8.53E-07
$1 \times 5 \times 15^{\prime}$

\begin{tabular}{|c|c|c|c|}
\hline DHLW (Ci/can) & $\begin{array}{l}\text { Total Ci DHLW } \\
\text { per package }\end{array}$ & $\begin{array}{l}\text { Total Ci (SNF } \\
+ \text { DHLW) }\end{array}$ & $\begin{array}{l}\text { Total Ci } \\
\text { Per Category }\end{array}$ \\
\hline $0.00 E+00$ & $0.00 E+00$ & $1.52 E-02$ & $1.14 E+01$ \\
\hline $0.00 E+00$ & $0.00 E+00$ & $4.58 \mathrm{E}-04$ & $3.43 E-01$ \\
\hline $3.31 \mathrm{E}-02$ & $2.49 E-01$ & 3.38E-01 & $2.53 E+02$ \\
\hline $3.58 \mathrm{E}+00$ & $2.68 E+01$ & $3.78 E+01$ & $2.84 E+04$ \\
\hline $1.01 E-01$ & 7.57E-01 & 8.05E-01 & $6.04 E+02$ \\
\hline $3.04 E+04$ & $2.28 \mathrm{E}+05$ & $2.39 E+05$ & $1.80 E+08$ \\
\hline $3.08 E+04$ & $2.31 E+05$ & $2.42 E+05$ & $1.82 E+08$ \\
\hline 7.71E-01 & $5.78 \mathrm{E}+00$ & $6.03 E+00$ & $4.52 E+03$ \\
\hline $1.35 \mathrm{E}-01$ & $1.02 E+00$ & $1.03 E+00$ & $7.73 E+02$ \\
\hline 3.33E-05 & 2.49E-04 & $1.08 E-03$ & 8.13E-01 \\
\hline $3.63 E+00$ & $2.72 E+01$ & $2.88 E+01$ & 2.16E+04 \\
\hline $2.05 E+03$ & $1.53 E+04$ & $1.53 E+04$ & $1.15 E+07$ \\
\hline $2.05 E+03$ & $1.54 \mathrm{E}+04$ & $1.54 E+0.4$ & $1.15 E+07$ \\
\hline $1.24 E-02$ & $9.27 E-02$ & $9.46 E-02$ & $7.10 E+01$ \\
\hline 2.39E-01 & $1.79 E+00$ & $1.84 E+00$ & $1.38 E+03$ \\
\hline 2.09E-06 & 1.56E-05 & $2.66 E-03$ & $2.00 E+00$ \\
\hline $2.42 E+03$ & $1.81 E+04$ & $1.81 E+04$ & 1.36E $\div 07$ \\
\hline 1.27E-01 & $9.49 E-01$ & $9.66 E-01$ & $7.25 E+02$ \\
\hline $3.05 E+04$ & $2.28 \mathrm{E}+05$ & $2.40 E+05$ & $1.80 E+08$ \\
\hline $2.88 E+04$ & $2.16 \mathrm{E}+05$ & $2.28 E+05$ & $1.71 E+08$ \\
\hline $1.25 E+04$ & $9.41 E+04$ & $9.41 E+04$ & $7.06 E+07$ \\
\hline $1.25 E+04$ & $9.41 E+04$ & $9.41 E+04$ & $7.06 E+07$ \\
\hline $2.12 E+04$ & $1.59 E+05$ & 1.59E+05 & $1.19 E+08$ \\
\hline $3.09 E+02$ & $2.32 E+03$ & $2.36 E+03$ & 1.77E+06 \\
\hline $0.00 E+00$ & $0.00 E+00$ & $1.42 \mathrm{E}-12$ & $1.07 E-09$ \\
\hline $0.00 \mathrm{E}+00$ & $0.00 E+00$ & $3.64 \mathrm{E}-12$ & 2.73E-09 \\
\hline $1.07 E-0.4$ & $8.04 E-04$ & $8.04 E-04$ & 6.03E-01 \\
\hline 5.80E-06 & 4.35E-05 & 4.35E-05 & 3.26E-02 \\
\hline $1.48 \mathrm{E}-05$ & 1.11E-04 & $1.11 E-04$ & 8.33E-02 \\
\hline 4.24E-06 & 3.18E-05 & $3.18 E-05$ & $2.38 E-02$ \\
\hline $1.16 E-04$ & 8.69E-04 & 8.69E-04 & $6.51 E-01$ \\
\hline 1.07E-03 & 8.04E-03 & 8.04E-03 & $6.03 E \div 00$ \\
\hline $6.38 \mathrm{E}-0.4$ & 4.78E-03 & $4.79 E-03$ & $3.59 E+00$ \\
\hline $1.27 E-02$ & 9.55E-02 & 9.69E-02 & $7.26 E+01$ \\
\hline 8.70E-05 & $6.52 E-04$ & $1.50 \mathrm{E}-02$ & $1.12 E+01$ \\
\hline $4.76 \mathrm{E}-04$ & $3.57 E-03$ & $5.77 \mathrm{E}-02$ & 4.33E+01 \\
\hline 4.15E-03 & 3.11E-02 & $3.27 \mathrm{E}-02$ & $2.45 E+01$ \\
\hline $3.02 E-02$ & 2.27E-01 & $2.58 E-01$ & $1.93 E+02$ \\
\hline $5.58 E+02$ & $4.18 E+03$ & $4.25 E+03$ & $3.19 E+06$ \\
\hline $5.20 E+00$ & 3.90E+01 & 4.16E+01 & $3.12 E+04$ \\
\hline $3.57 E+00$ & $2.68 E+01$ & $2.81 E+01$ & $2.11 E+04$ \\
\hline $6.89 E+02$ & $5.17 E+03$ & $5.51 E+03$ & $4.14 E+06$ \\
\hline 5.52E-03 & 4.14E-02 & $4.29 E-02$ & $3.22 E+01$ \\
\hline $8.20 E+01$ & $6.15 E+02$ & $6.16 E+02$ & $4.62 E+05$ \\
\hline $2.60 E-02$ & 1.95E-01 & 1.97E-01 & $1.48 E+02$ \\
\hline 4.05E-02 & 3.03E-01 & $3.09 \mathrm{E}-01$ & $2.32 E+02$ \\
\hline $3.92 E+01$ & $2.94 E+02$ & $2.95 \mathrm{E}+02$ & $2.21 E+05$ \\
\hline $6.44 E-05$ & 4.83E-0.4 & $4.96 \mathrm{E}-04$ & 3.72E-01 \\
\hline \multirow[t]{3}{*}{$7.24 \mathrm{E}-06$} & 5.43E-05 & 5.52E-05 & 4.14E-02 \\
\hline & & 1365106 & \\
\hline & Total Ci/category = & $1.02 E+09$ & \\
\hline
\end{tabular}

Prepared by Lenny Storz 9/4/98 
3. System Characterization: Waste Package

Table 3-6. Total Curies (DOE SNF and DHLW) by Category (continued)

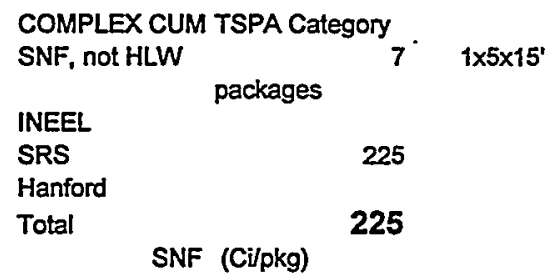

\begin{tabular}{|c|c|}
\hline Isotopes & \\
\hline C 14 & $1.82 \mathrm{E}-01$ \\
\hline $\mathrm{CL} 36$ & $5.48 E-03$ \\
\hline NI 59 & $1.07 E+00$ \\
\hline NI 63 & $1.32 E+02$ \\
\hline SE 79 & $2.81 E-02$ \\
\hline SR90 & $6.37 E+03$ \\
\hline Y90 & $6.37 E+03$ \\
\hline ZR93 & $1.69 E-01$ \\
\hline NB 93M & 1.49E-02 \\
\hline NB 94 & $9.96 \mathrm{E}-03$ \\
\hline TC 99 & 9.51E-01 \\
\hline RU106 & \\
\hline RH106 & \\
\hline PD107 & $1.33 E-03$ \\
\hline SN126 & 2.62E-02 \\
\hline 1129 & $1.58 \mathrm{E}-03$ \\
\hline CS134 & \\
\hline CS135 & 6.41E-02 \\
\hline CS137 & $6.72 E+03$ \\
\hline BA137M & $6.72 E+03$ \\
\hline CE144 & \\
\hline PR144 & \\
\hline PM147 & \\
\hline SM151 & $4.81 E+01$ \\
\hline PB210 & 1.13E-12 \\
\hline RA226 & $3.04 E-12$ \\
\hline RA228 & 8.77E-09 \\
\hline AC227 & 1.17E-07 \\
\hline TH229 & 2.39E-09 \\
\hline TH230 & 5.51E-09 \\
\hline TH232 & 2.59E-08 \\
\hline PA231 & $1.88 \mathrm{E}-06$ \\
\hline U233 & $1.24 E-05$ \\
\hline U234 & 3.24E-04 \\
\hline U235 & 2.05E-02 \\
\hline U236 & $2.78 \mathrm{E}-02$ \\
\hline U238 & $1.55 \mathrm{E}-02$ \\
\hline NP237 & $4.08 E-03$ \\
\hline PU238 & $5.65 E+00$ \\
\hline PU239 & $1.10 E+01$ \\
\hline PU240 & $4.26 E+00$ \\
\hline PU241 & $2.73 E+02$ \\
\hline PU242 & 5.33E-04 \\
\hline AM241 & 4.26E-01 \\
\hline AM242M & 2.95E-03 \\
\hline AM243 & 6.07E-04 \\
\hline CM244 & 1.71E-02 \\
\hline CM245 & $6.98 \mathrm{E}-07$ \\
\hline CM246 & 4.67E-08 \\
\hline
\end{tabular}

\begin{tabular}{|c|c|c|c|}
\hline DHLW (Ci/can) & $\begin{array}{l}\text { Total Ci DHLW } \\
\text { per package }\end{array}$ & $\begin{array}{l}\text { Total Ci (SNF } \\
+ \text { DHLW) }\end{array}$ & $\begin{array}{l}\text { Total Ci } \\
\text { Per Category }\end{array}$ \\
\hline $0.00 \mathrm{E}+00$ & $0.00 E+00$ & 1.82E-01 & 4.09E +01 \\
\hline $0.00 E+00$ & $0.00 E+00$ & $5.48 \mathrm{E}-03$ & $1.23 E+00$ \\
\hline 3.31E-02 & 2.49E-01 & $1.32 E+00$ & $2.97 E \div 02$ \\
\hline $3.58 \mathrm{E}+00$ & $2.68 E+01$ & $1.58 E+02$ & $3.56 \mathrm{E}+04$ \\
\hline $1.01 E-01$ & 7.57E-01 & 7.85E-01 & $1.77 \mathrm{E}+02$ \\
\hline $3.04 \mathrm{E}+04$ & $2.28 E+05$ & $2.34 E+05$ & 5.27E+07 \\
\hline $3.08 E+04$ & 2.31E+05 & 2.37E+05 & 5.34E $\div 07$ \\
\hline 7.71E-01 & $5.78 \mathrm{E}+00$ & $5.95 \mathrm{E} \div 00$. & $1.34 E+03$ \\
\hline 1.35E-01 & $1.02 E+00$ & $1.03 E+00$ & $2.32 E+02$ \\
\hline 3.33E-05 & 2.49E-04 & $1.02 E-02$ & $2.30 E+00$ \\
\hline $3.63 E+00$ & $2.72 E+01$ & $2.82 E+01$ & $6.34 \mathrm{E}+03$ \\
\hline 2.05E+03 & 1.53E+04 & $1.53 E+04$ & $3.45 E+06$ \\
\hline $2.05 E+03$ & $1.54 \mathrm{E}+04$ & $1.54 E+04$ & $3.46 E+06$ \\
\hline $1.24 \mathrm{E}-02$ & $9.27 E-02$ & 9.41E-02 & $2.12 E+01$ \\
\hline 2.39E-01 & $1.79 E+00$ & $1.82 E+00$ & 4.10E+02 \\
\hline $2.09 \mathrm{E}-06$ & 1.56E-05 & 1.60E-03 & $3.59 E-01$ \\
\hline $2.42 E+03$ & $1.81 \mathrm{E}+04$ & $1.81 E+04$ & $4.08 E+06$ \\
\hline $1.27 \mathrm{E}-01$ & 9.49E-01 & $1.01 E+00$ & $2.28 E+02$ \\
\hline $3.05 E+04$ & $2.28 \mathrm{E}+05$ & $2.35 E+05$ & $5.29 E+07$ \\
\hline $2.88 E+04$ & 2.16E+05 & $2.23 E+05$ & 5.01E+07 \\
\hline $1.25 E+04$ & 9.41E+04 & $9.41 E+04$ & 2.12E+07 \\
\hline $1.25 E+04$ & $9.41 E+04$ & $9.41 E \div 04$ & 2.12E $\div 07$ \\
\hline $2.12 E+04$ & 1.59E+05 & $1.59 E \div 05$ & $3.58 \mathrm{E} \div 07$ \\
\hline $3.09 E+02$ & $2.32 E+03$ & 2.37E +03 & $5.32 E+05$ \\
\hline $0.00 E+00$ & $0.00 E+00$ & 1.13E-12 & 2.54E-10 \\
\hline $0.00 E+00$ & $0.00 E+00$ & $3.04 E-12$ & $6.84 E-10$ \\
\hline 1.07E-04 & 8.04E-04 & 8.04E-04 & 1.81E-01 \\
\hline $5.80 \mathrm{E}-06$ & 4.35E-05 & 4.36E-05 & $9.81 E-03$ \\
\hline $1.48 \mathrm{E}-05$ & 1.11E-04 & 1.11E-04 & $2.50 E-02$ \\
\hline $4.24 E-06$ & 3.18E-05 & 3.18E-05 & $7.15 E-03$ \\
\hline $1.16 \mathrm{E}-04$ & 8.69E-04 & 8.69E-04 & 1.95E-01 \\
\hline $1.07 E-03$ & 8.04E-03 & 8.04E-03 & $1.81 E+00$ \\
\hline $6.38 \mathrm{E}-04$ & 4.78E-03 & $4.80 E-03$ & $1.08 E+00$ \\
\hline 1.27E-02 & 9.55E-02 & $9.58 E-02$ & $2.16 E+01$ \\
\hline $8.70 E-05$ & $6.52 \mathrm{E}-04$ & 2.12E-02 & 4.76E +00 \\
\hline $4.76 E-04$ & 3.57E-03 & $3.14 \mathrm{E}-02$ & $7.06 \mathrm{E} \div 00$ \\
\hline 4.15E-03 & $3.11 E-02$ & 4.66E-02 & $1.05 E+01$ \\
\hline $3.02 E-02$ & 2.27E-01 & 2.31E-01 & $5.19 E+01$ \\
\hline $5.58 \mathrm{E}+02$ & $4.18 E+03$ & $4.19 E+03$ & $9.43 E+05$ \\
\hline $5.20 \mathrm{E}+00$ & $3.90 E+01$ & $5.00 E \div 01$ & $1.12 E \div 04$ \\
\hline 3.57E+00 & $2.68 \mathrm{E}+01$ & 3.10E+01 & $6.98 E+03$ \\
\hline $6.89 E+02$ & 5.17E+03 & $5.44 E+03$ & $1.22 E+06$ \\
\hline $5.52 E-03$ & 4.14E-02 & 4.20E-02 & $9.44 E+00$ \\
\hline $8.20 E+01$ & $6.15 E+02$ & $6.15 E+02$ & $1.38 E+05$ \\
\hline $2.60 \mathrm{E}-02$ & 1.95E-01 & 1.98E-01 & 4.45E+01 \\
\hline 4.05E-02 & 3.03E-01 & 3.04E-01 & $6.84 E+01$ \\
\hline $3.92 E+01$ & $2.94 E+02$ & $2.94 E+02$ & $6.62 E+04$ \\
\hline $6.44 E-05$ & 4.83E-04 & 4.84E-04 & 1.09E-01 \\
\hline $7.24 \mathrm{E}-06$ & 5.43E-05 & 5.43E-05 & $1.22 \mathrm{E}-02$ \\
\hline & Total Ci/pkg. = & 1.34E+06 & \\
\hline
\end{tabular}

Prepared by Lenny Storz 9/4/98
Page 7 
Table 3-6. Total Curies (DOE SNF and DHLW) by Category (continued)

COMPLEX CUM TSPA Category

$\begin{array}{lrrr}\text { SNF, not HLW } & \text { packages } & & 1 \times 5 \times 15^{\prime} \\ & & 545 & \\ \text { INEEL } & & & \\ \text { SRS } & & & \\ \text { Hanford } & & 545 \\ \text { Total } & & \end{array}$

\begin{tabular}{|c|c|}
\hline & \\
\hline Isotopes & \\
\hline C 14 & 1.99E-01 \\
\hline CL 36 & 2.49E-03 \\
\hline NI 59 & $1.59 \mathrm{E}-02$ \\
\hline NI 63 & $3.82 \mathrm{E}-01$ \\
\hline SE 79 & 2.71E-02 \\
\hline SR90 & $2.08 E+03$ \\
\hline Y90 & $2.08 E+03$ \\
\hline ZR93 & $9.90 E-01$ \\
\hline NB 93M & 8.52E-03 \\
\hline NB 94 & $0.00 E+00$ \\
\hline TC 99 & 6.83E-01 \\
\hline RU106 & \\
\hline RH106 & \\
\hline PD107 & $8.03 E-04$ \\
\hline SN126 & $1.28 E-02$ \\
\hline 1129 & $1.91 E-03$ \\
\hline CS134 & \\
\hline CS135 & 1.59E-02 \\
\hline CS137 & $2.21 E+03$ \\
\hline BA137M & $2.21 \mathrm{E}+03$ \\
\hline CE144 & \\
\hline PR144 & \\
\hline PM147 & \\
\hline SM151 & $4.15 E+01$ \\
\hline PB210 & 4.22E-06 \\
\hline RA226 & $4.48 E-06$ \\
\hline RA228 & $6.04 E-03$ \\
\hline AC227 & $0.00 E+00$ \\
\hline TH229 & 2.56E-02 \\
\hline TH230 & 1.75E-03 \\
\hline TH232 & $4.68 \mathrm{E}-03$ \\
\hline PA231 & $1.71 E-02$ \\
\hline U233 & $6.35 E+00$ \\
\hline U234 & 5.16E-01 \\
\hline U235 & $1.99 \mathrm{E}-03$ \\
\hline U236 & $1.96 \mathrm{E}-02$ \\
\hline U238 & $4.94 \mathrm{E}-05$ \\
\hline NP237 & $1.50 \mathrm{E}-02$ \\
\hline PU238 & $7.49 E+01$ \\
\hline PU239 & 2.07E-01 \\
\hline PU240 & $3.49 E-01$ \\
\hline PU241 & $0.00 E+00$ \\
\hline PU242 & $0.00 E+00$ \\
\hline AM241 & $3.42 E+00$ \\
\hline AM242M & $9.27 E-04$ \\
\hline AM243 & 2.45E-02 \\
\hline CM244 & $7.68 \mathrm{E}-01$ \\
\hline CM245 & $1.28 \mathrm{E}-0.4$ \\
\hline CM246 & $6.40 E-05$ \\
\hline
\end{tabular}

\begin{tabular}{|c|c|c|c|}
\hline DHLW (Ci/can) & $\begin{array}{l}\text { Total Ci DHLW } \\
\text { per package }\end{array}$ & $\begin{array}{l}\text { Total Ci (SNF } \\
\text { + DHLW) }\end{array}$ & $\begin{array}{l}\text { Total Ci } \\
\text { Per Category }\end{array}$ \\
\hline $0.00 E+00$ & $0.00 E+00$ & $1.99 \mathrm{E}-01$ & $1.08 E+02$ \\
\hline $0.00 E+00$ & $0.00 E+00$ & 2.49E-03 & $1.36 \mathrm{E}+00$ \\
\hline $3.31 E-02$ & 2.49E-01 & 2.64E-01 & $1.44 E+02$ \\
\hline $3.58 \mathrm{E}+00$ & $2.68 E+01$ & $2.72 E+01$ & $1.48 E+04$ \\
\hline $1.01 E-01$ & 7.57E-01 & 7.84E-01 & 4.27E+02 \\
\hline $3.04 E+04$ & $2.28 \mathrm{E}+05$ & $2.30 E+05$ & $1.25 E+08$ \\
\hline $3.08 E+04$ & $2.31 E+05$ & $2.33 E \div 05$ & $1.27 E+08$ \\
\hline 7.71E-01 & $5.78 E+00$ & $6.77 \mathrm{E}+00$ & $3.69 E+03$ \\
\hline $1.35 E-01$ & $1.02 E+00$ & $1.02 E \div 00$ & $5.58 E+02$ \\
\hline 3.33E-05 & 2.49E-04 & 2.49E-04 & 1.36E-01 \\
\hline $3.63 E+00$ & $2.72 E+01$ & $2.79 E+01$ & $1.52 E+04$ \\
\hline $2.05 E+03$ & $1.53 E+04$ & $1.53 E \div 04$ & $8.36 E+06$ \\
\hline $2.05 E+03$ & $1.54 E+04$ & $1.54 E+04$ & 8.37E+06 \\
\hline $1.24 E-02$ & $9.27 E-02$ & $9.35 E-02$ & $5.10 E+01$ \\
\hline 2.39E-01 & $1.79 E+00$ & $1.81 E+00$ & $9.85 E+02$ \\
\hline $2.09 E-06$ & $1.56 \mathrm{E}-05$ & $1.92 \mathrm{E}-03$ & $1.05 E+00$ \\
\hline $2.42 E+03$ & $1.81 E+04$ & $1.81 E+04$ & $9.88 E+05$ \\
\hline $1.27 \mathrm{E}-01$ & 9.49E-01 & $9.65 E-01$ & $5.26 E+02$ \\
\hline $3.05 E+04$ & $2.28 E+05$ & $2.31 E+05$ & $1.26 E+08$ \\
\hline $2.88 E+04$ & $2.16 E+05$ & $2.18 E+05$ & $1.19 E+08$ \\
\hline $1.25 E+04$ & $9.41 E+04$ & $9.41 E+04$ & $5.13 E+07$ \\
\hline $1.25 E \div 04$ & $9.41 E+04$ & $9.41 E+04$ & $5.13 E+07$ \\
\hline 2.12E+04 & $1.59 E+05$ & $1.59 E+05$ & $8.68 E+07$ \\
\hline $3.09 E+02$ & $2.32 E+03$ & 2.36E+03 & $1.29 E+06$ \\
\hline $0.00 E+00$ & $0.00 E+00$ & $4.22 E-06$ & 2.30E-03 \\
\hline $0.00 E+00$ & $0.00 E+\infty 0$ & 4.48E-06 & 2.44E-03 \\
\hline $1.07 E-04$ & $8.04 E-04$ & $6.84 E-03$ & $3.73 E+00$ \\
\hline $5.80 E-06$ & 4.35E-05 & 4.35E-05 & $2.37 E-02$ \\
\hline $1.48 E-05$ & 1.11E-04 & 2.57E-02 & $1.40 E+01$ \\
\hline $4.24 E-06$ & $3.18 E-05$ & $1.78 \mathrm{E}-03$ & $9.72 E-01$ \\
\hline $1.16 E-04$ & 8.69E-04 & $5.54 \mathrm{E}-03$ & $3.02 E+00$ \\
\hline $1.07 E-03$ & $8.04 E-03$ & 2.52E-02 & $1.37 E+01$ \\
\hline $6.38 E-04$ & $4.78 \mathrm{E}-03$ & $6.35 \mathrm{E}+00$ & $3.46 E+03$ \\
\hline $1.27 E-02$ & $9.55 E-02$ & 6.12E-01 & $3.33 E+02$ \\
\hline $8.70 E-05$ & $6.52 E-04$ & $2.64 \mathrm{E}-03$ & $1.44 E+00$ \\
\hline 4.76E-04 & 3.57E-03 & 2.32E-02 & $1.26 E+01$ \\
\hline 4.15E-03 & 3.11E-02 & $3.12 E-02$ & $1.70 E+01$ \\
\hline $3.02 E-02$ & 2.27E-01 & 2.42E-01 & $1.32 E+02$ \\
\hline $5.58 E \div 02$ & 4.18E+03 & $4.26 E+03$ & $2.32 E+06$ \\
\hline $5.20 E+00$ & $3.90 E+01$ & $3.92 E+01$ & $2.14 E+04$ \\
\hline $3.57 E+00$ & $2.68 E+01$ & $2.71 E+01$ & $1.48 E+04$ \\
\hline $6.89 E+02$ & $5.17 \mathrm{E}+03$ & $5.17 E+03$ & $2.82 E+06$ \\
\hline $5.52 E-03$ & $4.14 E-02$ & 4.14E-02 & $2.26 E+01$ \\
\hline $8.20 E+01$ & $6.15 E+02$ & $6.18 E+02$ & $3.37 E+05$ \\
\hline $2.60 E-02$ & 1.95E-01 & $1.96 \mathrm{E}-01$ & $1.07 E+02$ \\
\hline 4.05E-02 & 3.03E-01 & $3.28 E-01$ & $1.79 E+02$ \\
\hline $3.92 E+01$ & $2.94 E+02$ & $2.95 E+02$ & 1.61E+05 \\
\hline $6.44 E-05$ & 4.83E-0.4 & 6.12E-04 & 3.33E-01 \\
\hline $7.24 \mathrm{E}-06$ & $5.43 E-05$ & $1.18 \mathrm{E}-04$ & $6.45 E-02$ \\
\hline & & 1.32E+06 & \\
\hline & ego & $7.20 E+08$ & \\
\hline
\end{tabular}

Prepared by Lenny Storz 9/4/98 
Table 3-6. Total Curies (DOE SNF and DHLW) by Category (continued)

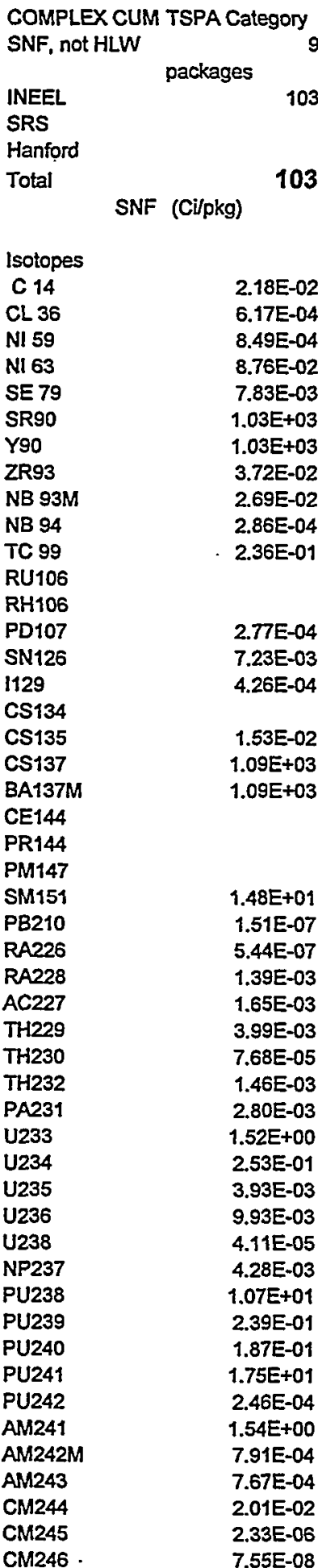

$91 \times 5 \times 15^{\prime}$

103

03

DHLW (Ci/can) Tot

$0.00 E+00$
$0.00 E+00$
$3.31 E-02$
$3.58 E+00$
$1.01 E-01$
$3.04 E+04$
$3.08 E+04$
$7.71 E-01$
$1.35 E-01$
$3.33 E-05$
$3.63 E+00$
$2.05 E+03$
$2.05 E+03$
$1.24 E-02$
$2.39 E-01$
$2.09 E-06$
$2.42 E+03$
$1.27 E-01$
$3.05 E+04$
$2.88 E+04$
$1.25 E+04$
$1.25 E+04$
$2.12 E+04$
$3.09 E+02$
$0.00 E+00$
$0.00 E+00$
$1.07 E-04$
$5.80 E-06$
$1.48 E-05$
$4.24 E-06$
$1.16 E-04$
$1.07 E-03$
$6.38 E-04$
$1.27 E-02$
$8.70 E-05$
$4.76 E-04$
$4.15 E-03$
$3.02 E-02$
$5.58 E+02$
$5.20 E+00$
$3.57 E+00$
$6.89 E+02$
$5.52 E-03$
$8.20 E+01$
$2.60 E-02$
$4.05 E-02$
$3.92 E+01$
$6.44 E-05$
$7.24 E-06$

Total Ci DHLW

per package

$0.00 \mathrm{E}+00$

6.17E-04 6.35E-02

$2.49 \mathrm{E}-01 \quad 2.49 \mathrm{E}-01 \quad 2.57 \mathrm{E}+01$

$2.68 E+01 \quad 2.69 E+01 \quad 2.77 E+03$

7.57E-01 7.65E-01 7.88E+01

$2.28 E+05 \quad 2.29 E+05 \quad 2.36 E+07$

$2.31 E+05 \quad 2.32 E+05 \quad 2.39 E+07$

$5.78 E+00 \quad 5.82 E+00 \quad 5.99 E+02$

$1.02 E+00 \quad 1.04 E+00 \quad 1.07 E+02$

2.49E-04 5.35E-04 5.51E-02

$2.72 E+01 \quad 2.75 E+01 \quad 2.83 E+03$

$1.53 E+04 \quad 1.53 E+04 \quad 1.58 E+06$

$1.54 E+04 \quad 1.54 E+04 \quad 1.58 E+06$

$\begin{array}{lll}9.27 E-02 & 9.30 E-02 & 9.58 E+00\end{array}$

$1.79 E+00 \quad 1.80 E+00 \quad 1.86 E+02$

$\begin{array}{lll}1.56 E-05 & 4.42 \mathrm{E}-04 & 4.55 \mathrm{E}-02\end{array}$

$1.81 E+04 \quad 1.81 E+04 \quad 1.87 E+06$

9.49E-01 9.65E-01 9.93E+01

$2.28 E+05 \quad 2.30 E+05 \quad 2.36 E+07$

$2.16 E+05 \quad 2.17 E+05 \quad 2.23 E+07$

$9.41 E+04 \quad 9.41 E+04 \quad 9.69 E+06$

$9.41 E+04 \quad 9.41 E+04 \quad 9.69 E+06$

$1.59 E+05 \quad 1.59 E+05 \quad 1.64 E+07$

$2.32 E+03 \quad 2.33 E+03 \quad 2.40 E+05$

$0.00 E+00 \quad 1.51 E-07 \quad 1.56 E-05$

$0.00 E+00 \quad 5.44 E-07 \quad 5.60 E-05$

8.04E-04 2.19E-03 2.26E-01

4.35E-05 1.69E-03 1.74E-01

1.11E-04 4.10E-03 4.22E-01

$3.18 E-05 \quad 1.09 E-04 \quad 1.12 \mathrm{E}-02$

8.69E-04 2.33E-03 2.40E-01

$\begin{array}{lll}8.04 E-03 & 1.08 E-02 & 1.12 E+00\end{array}$

4.78E-03 1.53E+00 $1.57 E+02$

9.55E-02 3.48E-01 3.59E +01

6.52E-04 4.59E-03 4.72E-01

$3.57 \mathrm{E}-03 \quad 1.35 \mathrm{E}-02 \quad 1.39 \mathrm{E}+00$

3.11E-02 3.12E-02 3.21E+00

$2.27 E-01 \quad 2.31 E-01 \quad 2.38 E+01$

4.18E+03 4.20E+03 4.32E+05

$3.90 E+01 \quad 3.93 E+01 \quad 4.04 E+03$

$2.68 E+01 \quad 2.70 E+01 \quad 2.78 E+03$

$5.17 E+03 \quad 5.18 E+03 \quad 5.34 E+05$

4.14E-02 4.17E-02 4.29E+00

$6.15 \mathrm{E}+02 \quad 6.17 \mathrm{E}+02 \quad 6.35 \mathrm{E}+04$

1.95E-01 1.96E-01 2.02E+01

$3.03 E-01 \quad 3.04 E-01 \quad 3.13 E+01$

$2.94 E+02 \quad 2.94 E+02 \quad 3.03 E+04$

4.83E-04 4.86E-04 5.00E-02

$\begin{array}{lll}5.43 E-05 & 5.44 E-05 & 5.60 E-03\end{array}$

Total Ci/pkg. =

Total Ci/category =

$1.32 E+06$

$1.36 E+08$ 
Table 3-6. Total Curies (DOE SNF and DHLW) by Category (continued)

COMPLEX CUM TSPA Category

$\begin{array}{llcc}\text { SNF, not HLW } & & 10 & 1 \times 4 \times 15^{\prime} \\ & \text { packages } & & \\ \text { INEEL } & & 4 & \\ \text { SRS } & & & \\ \text { Hanford } & & 1 & \\ \text { Total } & & 5 & \\ & \text { SNF (Cilpkg) } & & \end{array}$

\begin{tabular}{|c|c|}
\hline Isotopes & \\
\hline C 14 & $0.00 E+00$ \\
\hline CL. 36 & $0.00 E+00$ \\
\hline NI 59 & $0.00 E+00$ \\
\hline NI 63 & 1.47E+01 \\
\hline SE 79 & $0.00 E+00$ \\
\hline SR90 & $2.13 E+03$ \\
\hline Y9O & 2.13E+03 \\
\hline ZR93 & $0.00 E+00$ \\
\hline N8 93M & $0.00 E+00$ \\
\hline NB 94 & $0.00 E+00$ \\
\hline TC 99 & $0.00 E+00$ \\
\hline RU106 & \\
\hline RH106 & \\
\hline PD107 & $0.00 E+00$ \\
\hline SN126 & $0.00 \mathrm{E}+00$ \\
\hline 1129 & $0.00 E+00$ \\
\hline CS134 & \\
\hline CS135 & $0.00 E+00$ \\
\hline CS137 & $6.11 E+03$ \\
\hline BA137M & $6.11 E+03$ \\
\hline CE144 & \\
\hline PR144 & \\
\hline PM147 & \\
\hline SM151 & $4.04 E+02$ \\
\hline PB210 & $0.00 E+00$ \\
\hline RA226 & $6.69 \mathrm{E}-09$ \\
\hline RA228 & $1.39 \mathrm{E}-12$ \\
\hline AC227 & $1.45 \mathrm{E}-09$ \\
\hline TH229 & $1.70 E-10$ \\
\hline TH230 & - $1.47 E-06$ \\
\hline TH232 & 2.31E-12 \\
\hline PA231 & 5.53E-09 \\
\hline U233 & 3.14E-07 \\
\hline U234 & 1.01E-02 \\
\hline U235 & $1.69 \mathrm{E}-05$ \\
\hline U236 & 3.01E-03 \\
\hline U238 & $3.14 \varepsilon-14$ \\
\hline NP237 & 5.39E-03 \\
\hline PU238 & $1.02 E+02$ \\
\hline PU239 & $5.53 E+02$ \\
\hline PU240 & $4.78 E+02$ \\
\hline PU241 & $4.00 E+03$ \\
\hline PU242 & $1.28 E-05$ \\
\hline$A M 241$ & $6.97 E+02$ \\
\hline AM242M & $1.21 E+00$ \\
\hline AM243 & $0.00 E+00$ \\
\hline CM244 & $0.00 E+00$ \\
\hline CM245 & $0.00 E+00$ \\
\hline CM246 & $0.00 E+00$ \\
\hline
\end{tabular}

\begin{tabular}{|c|c|c|c|}
\hline DHLW (Ci/can) & $\begin{array}{l}\text { Total Ci DHLW } \\
\text { per package }\end{array}$ & $\begin{array}{l}\text { Total Ci (SNF } \\
\text { + DHLW) }\end{array}$ & $\begin{array}{l}\text { Total Ci } \\
\text { Per Category }\end{array}$ \\
\hline $0.00 E+00$ & $0.00 E+00$ & $0.00 E+00$ & $0.00 E+00$ \\
\hline $0.00 E+00$ & $0.00 \mathrm{E}+00$ & $0.00 E+00$ & $0.00 E+00$ \\
\hline 3.31E-02 & 1.99E-01 & 1.99E-01 & $9.94 E-01$ \\
\hline $3.58 \mathrm{E}+00$ & 2.15E+01 & $3.62 E+01$ & $1.81 E+02$ \\
\hline 1.01E-01 & 6.06E-01 & $6.06 \mathrm{E}-01$ & $3.03 E+00$ \\
\hline $3.04 E+04$ & $1.82 E+05$ & 1.85E+05 & $9.23 E+05$ \\
\hline $3.08 E+04$ & 1.85E+05 & $1.87 E+05$ & $9.34 E+05$ \\
\hline 7.71E-01 & $4.63 E+00$ & $4.63 E+00$ & $2.31 E+01$ \\
\hline 1.35E-01 & 8.13E-01 & 8.13E-01 & $4.06 E \div 00$ \\
\hline 3.33E-05 & $2.00 E-04$ & $2.00 E-04$ & $9.98 E-04$ \\
\hline $3.63 E+00$ & $2.18 \mathrm{E}+01$ & $2.18 E+01$ & $1.09 E+02$ \\
\hline $2.05 E+03$ & $1.23 E+04$ & $1.23 E+04$ & $6.14 E+04$ \\
\hline $2.05 E+03$ & $1.23 E+04$ & 1.23E+04 & $6.15 E+04$ \\
\hline $1.24 \mathrm{E}-02$ & 7.42E-02 & 7.42E-02 & 3.71E-01 \\
\hline $2.39 \mathrm{E}-01$ & $1.44 E+00$ & $1.44 E+00$ & $7.18 E+00$ \\
\hline 2.09E-06 & 1.25E-05 & $1.25 E-05$ & $6.26 E-05$ \\
\hline $2.42 E+03$ & 1.45E+04 & $1.45 E+04$ & $7.25 E+04$ \\
\hline $1.27 \mathrm{E}-01$ & 7.59E-01 & 7.59E-01 & $3.80 E+00$ \\
\hline $3.05 E+04$ & $1.83 E+05$ & $1.89 E+05$ & $9.44 E+05$ \\
\hline $2.88 E+04$ & 1.73E+05 & 1.79Eะ05 & $8.94 E+05$ \\
\hline $1.25 E+04$ & $7.53 E+04$ & $7.53 E+04$ & $3.76 E+05$ \\
\hline $1.25 E+04$ & $7.53 E+04$ & $7.53 E+04$ & $3.76 E+05$ \\
\hline $2.12 E+04$ & $1.27 E+05$ & 1.27E+05 & $6.37 E+05$ \\
\hline $3.09 E+02$ & $1.85 E+03$ & $2.26 E+03$ & 1.13E+04 \\
\hline $0.00 E+00$ & $0.00 E+00$ & $0.00 E+00$ & $0.00 E+00$ \\
\hline $0.00 E+00$ & $0.00 E+00$ & $6.69 \mathrm{E}-09$ & $3.34 E-08$ \\
\hline $1.07 \mathrm{E}-04$ & 6.43E-04 & $6.43 E-04$ & $3.22 E-03$ \\
\hline $5.80 \mathrm{E}-06$ & $3.48 \mathrm{E}-05$ & $3.48 E-05$ & $1.74 \mathrm{E}-04$ \\
\hline $1.48 \mathrm{E}-05$ & $8.89 E-05$ & 8.89E-05 & $4.44 E-04$ \\
\hline 4.24E-06 & $2.54 E-05$ & 2.69E-05 & $1.34 E-04$ \\
\hline $1.16 \mathrm{E}-04$ & 6.95E-04 & $6.95 E-04$ & $3.47 E-03$ \\
\hline 1.07E-03 & $6.43 E-03$ & $6.43 E-03$ & 3.22E-02 \\
\hline $6.38 \mathrm{E}-04$ & $3.83 E-03$ & $3.83 E-03$ & $1.91 E-02$ \\
\hline $1.27 \mathrm{E}-02$ & $7.64 E-02$ & $8.65 E-02$ & 4.33E-01 \\
\hline $8.70 \mathrm{E}-05$ & $5.22 E-04$ & 5.39E-04 & $2.69 E-03$ \\
\hline $4.76 E-04$ & 2.86E-03 & $5.86 E-03$ & 2.93E-02 \\
\hline $4.15 E-03$ & 2.49E-02 & $2.49 E-02$ & $1.25 E-01$ \\
\hline $3.02 E-02$ & 1.81E-01 & $1.87 E-01$ & 9.33E-01 \\
\hline $5.58 E+02$ & $3.35 \mathrm{E}+03$ & $3.45 E+03$ & $1.72 E+04$ \\
\hline $5.20 E+00$ & $3.12 E+01$ & $5.84 E+02$ & $2.92 E+03$ \\
\hline $3.57 E+00$ & 2.14E $\div 01$ & $4.99 E+02$ & $2.50 E+03$ \\
\hline $6.89 E+02$ & 4.13E+03 & $8.13 E+03$ & 4.07E+04 \\
\hline 5.52E-03 & 3.31E-02 & 3.31E-02 & $1.66 \mathrm{E}-09$ \\
\hline $8.20 E+01$ & $4.92 E+02$ & $1.19 E+03$ & $5.94 E+03$ \\
\hline $2.60 E-02$ & 1.56E-01 & $1.37 E+00$ & $6.83 E+00$ \\
\hline 4.05E-02 & 2.43E-01 & $2.43 E-01$ & $1.21 E+00$ \\
\hline $3.92 E+01$ & $2.35 E+02$ & $2.35 E+02$ & $1.18 E+03$ \\
\hline $6.44 \mathrm{E}-05$ & $3.87 E-04$ & 3.87E-04 & 1.93E-03 \\
\hline \multirow[t]{3}{*}{$7.24 E-06$} & 4.34E-05 & 4.34E-05 & 2.17E-04 \\
\hline & Total C & 1.07E+06 & \\
\hline & Total C & $5.36 E+06$ & \\
\hline
\end{tabular}

Prepared by Lenny Storz 9/4/98
Page 10 
Table 3-6. Total Curies (DOE SNF and DHLW) by Category (continued)

COMPLEX CUM TSPA Category

$\begin{array}{lccc}\text { SNF, not HLW } & & 11 & 1 \times 4 \times 15^{\prime} \\ & \text { packages } & & \\ \text { INEEL } & & 25 . \\ \text { SRS } & & 327 \\ \text { Hanford } & & 352 \\ \text { Total } & & \\ & \text { SNF (Ci/pkg) } & \end{array}$

Isotopes

C 14

CL 36

6.75E-04

1.26E-05

2.45E-03

1.04E+01

$1.50 E-03$

$1.61 E+03$

$1.61 E+03$

7.03E-03

$5.09 E-03$

2.28E-05

$5.22 \mathrm{E}-02$

4.12E-04

1.95E-03

$1.46 \mathrm{E}-0.4$

1.38E-03

4.39E $\div 03$

$4.39 E+03$

2.81E+02

1.24E-10

5.18E-09

2.21E-11

1.44E-09

$7.58 \mathrm{E}-10$

1.14E-06

$6.23 E-11$

5.51E-08

5.33E-07

7.80E-03

1.24E-04

3.06E-03

1.11E-03

5.05E-03

7.82E+01

$3.80 \mathrm{E}+02$

$3.29 E+02$

$2.86 \mathrm{E}+03$

7.95E-03

4.90E+02

8.51E-01

7.22E-02

$3.03 E+00$

1.24E-03

2.11E-04
DHLW (Ci/can)

$\begin{array}{rr}0.00 E+00] & \text { per package } \\ 0.00 \mathrm{E}+00 & 0.00 \mathrm{E}+00 \\ 3.31 \mathrm{E}-02 & 0.00 \mathrm{E}+00 \\ 3.58 \mathrm{E}+00 & 1.99 \mathrm{E}-01 \\ 1.01 \mathrm{E}-01 & 2.15 \mathrm{E}+01 \\ 3.04 \mathrm{E}+04 & 6.06 \mathrm{E}-01 \\ 3.08 \mathrm{E}+04 & 1.82 \mathrm{E}+05 \\ 7.71 \mathrm{E}-01 & 1.85 \mathrm{E}+05 \\ 1.35 \mathrm{E}-01 & 4.63 \mathrm{E}+00 \\ 3.33 \mathrm{E}-05 & 8.13 \mathrm{E}-01 \\ 3.63 \mathrm{E}+00 & 2.00 \mathrm{E}-04\end{array}$

2.18E+01

1.23E+04

$1.23 \mathrm{E}+04$

7.42E-02

1. $44 \mathrm{E}+00$

1.25E-05

1.45E+04

7.59E-01

1.83E+05

1.73E+05

$7.53 \mathrm{E}+04$

$7.53 E \div 04$

$1.27 E+05$

1.85E+03

$0.00 E+00$

$0.00 E+00$

6.43E-04

$3.48 \mathrm{E}-05$

8.89E-05

2.54E-05

6.95E-04

6.43E-03

3.83E-03

7.64E-02

5.22E-04

2.86E-03

2.49E-02

1.81E-01

$3.35 E+03$

3.12E+01

$2.14 E+01$

4.13E+03

3.31E-02

4.92E+02

1.56E-01

2.43E-01

2.35E+02

3.87E-04

4.34E-05

Total Ci/pkg. =

Total Ci/category =
Total Ci (SNF Total Ci

+ DHLW Per Category

6.75E-04 2.38E-01

$1.26 E-05 \quad 4.42 E-03$

2.01E-01 7.08E+01

$3.19 E+01 \quad 1.12 E+04$

6.07E-01 2.14E+02

$1.84 \mathrm{E}+05 \quad 6.48 \mathrm{E}+07$

$1.86 \mathrm{E}+05 \quad 6.56 \mathrm{E}+07$

4.63E+00 1.63E+03

8.18E-01 2.88E+02 .

2.22E-04 7.83E-02

$2.18 E+01 \quad 7.69 E+03$

1.23E+04 4.32E+06

$1.23 E+04 \quad 4.33 E+06$

7.46E-02 2.63E+01

$1.44 \mathrm{E}+00 \quad 5.06 \mathrm{E}+02$

$1.58 \mathrm{E}-04 \quad 5.58 \mathrm{E}-02$

$1.45 E+04 \quad 5.10 E+06$

7.61E-01 2.68E+02

$1.87 \mathrm{E}+05 \quad 6.59 \mathrm{E}+07$

$1.77 \mathrm{E}+05 \quad 6.23 \mathrm{E}+07$

7.53E+04 2.65E+07

7.53E+04 2.65E+07

$1.27 \mathrm{E}+05 \quad 4.48 \mathrm{E}+07$

2.13E+03 7.51E+05

1.24E-10 4.37E-08

5.18E-09 1.82E-06

6.43E-04 2.26E-01

3.48E-05 1.22E-02

8.89E-05 3.13E-02

2.66E-05 9.35E-03

$6.95 \mathrm{E}-04 \quad 2.45 \mathrm{E}-01$

6.43E-03 2.26E+00

3.83E-03 1.35E+00

8.42E-02 2.96E+01

6.46E-04 2.27E-01

5.91E-03 2.08E+00

2.60E-02 $9.16 E+00$

1.86E-01 6.56E+01

$3.43 E+03 \quad 1.21 E+06$

4.11E+02 1.45E+05

$3.51 E+02 \quad 1.23 E+05$

$6.99 E+03 \quad 2.46 E+05$

4.11E-02 1.45E+01

9.82E+02 3.46E+05

$1.01 E+00 \quad 3.55 E+02$

3.15E-01 1.11E+02

$2.38 E+02 \quad 8.39 E+04$

1.63E-03 5.73E-01

2.54E-04 8.95E-02

$1.07 E+06$

$3.75 \mathrm{E}+08$ 
Table 3-6. Total Curies (DOE SNF and DHLW) by Category (continued)

COMPLEX CUM TSPA Category

$\begin{array}{llll}\text { SNF, not HLW } & & 12 & 1 \times 3 \times 15^{\prime} \\ \text { INEEL } & \text { packages } & & \\ \text { SRS } & & 69 & \\ \text { Hanford } & & \\ \text { Total } & & 69 \\ & \text { SNF (Ci/pkg) } & \end{array}$

\section{Isotopes}

$\begin{array}{lr}\text { C 14 } 14 & 6.87 E-01 \\ \text { CL 36 } & 1.53 E-02 \\ \text { NI 59 } & 5.26 E-02 \\ \text { NI 63 } & 6.41 E+00 \\ \text { SE 79 } & 2.53 E-01 \\ \text { SR90 } & 2.63 E+03 \\ \text { Y90 } & 2.63 E+03 \\ \text { ZR93 } & 5.91 E-01 \\ \text { NB 93M } & 3.17 E-01 \\ \text { NB 94 } & 1.60 E-02 \\ \text { TC 99 } & 2.35 E+00 \\ \text { RU106 } & \\ \text { RH106 } & \\ \text { PD107 } & \\ \text { SN126 } & 2.47 E-03 \\ \text { I129 } & 2.84 E-01 \\ \text { CS134 } & 1.14 E-02 \\ \text { CS135 } & \\ \text { CS137 } & 2.07 E-01 \\ \text { BA137M } & 2.56 E+03 \\ \text { CE144 } & 2.56 E+03\end{array}$

CE144

PR144

PM147

SM151 9.32E+01

PB210 8.08E-05

RA226 4.63E-05

RA228 7.30E-02

AC227 4.41E-01

TH229 1.87E-01

TH230 7.07E-03

TH232 8.66E-02

PA231 1.11E+00

U233 1.21E+02

U234 $6.04 E+00$

U235 4.06E-04

U236 8.35E-04

U238 1.32E-05

NP237 7.28E-04

PU238 2.84E+00

PU239 1.84E-01

PU240 1.05E-01

PU241 2.50E+01

PU242 2.35E-04

AM241 8.57E-01

AM242M 8.99E-03

AM243 1.71E-03

CM244 1.65E-01

CM245 3.41E-05

CM246 2.25E-06

\begin{tabular}{|c|c|c|c|}
\hline DHLW (Ci/can) & $\begin{array}{l}\text { Total Ci DHLW } \\
\text { per package }\end{array}$ & $\begin{array}{l}\text { Total Ci (SNF } \\
\text { + DHLW) }\end{array}$ & $\begin{array}{l}\text { Total Ci } \\
\text { Per Category }\end{array}$ \\
\hline $0.00 E+00$ & $0.00 E+00$ & 6.87E-01 & $4.74 E+01$ \\
\hline $0.00 E+00$ & $0.00 E+00$ & 1.53E-02 & $1.06 \mathrm{E}+00$ \\
\hline 3.31E-02 & 1.49E-01 & $2.02 E-01$ & $1.39 \mathrm{E}+01$ \\
\hline $3.58 E+00$ & $1.61 E+01$ & $2.25 E+01$ & $1.55 \mathrm{E}+03$ \\
\hline 1.01E-01 & 4.54E-01 & 7.07E-01 & $4.88 E+01$ \\
\hline $3.04 E+04$ & 1.37E+05 & $1.39 E+05$ & $9.62 E+06$ \\
\hline $3.08 E+04$ & 1.39E+05 & $1.41 E+05$ & $9.74 E+06$ \\
\hline 7.71E-01 & 3.47E+00 & $4.06 E+00$ & $2.80 E+02$ \\
\hline 1.35E-01 & $6.09 E-01$ & $9.26 \mathrm{E}-01$ & $6.39 E+01$ \\
\hline 3.33E-05 & $1.50 E-04$ & $1.61 E-02$ & $1.11 E+00$ \\
\hline $3.63 E+00$ & $1.63 E+01$ & $1.87 E+01$ & $1.29 E+03$ \\
\hline $2.05 E+03$ & $9.21 E+03$ & $9.21 E+03$ & $6.35 E+05$ \\
\hline 2.05E+03 & $9.22 E+03$ & $9.22 E+03$ & $6.36 E+05$ \\
\hline $1.24 E-02$ & 5.56E-02 & $5.81 E-02$ & $4.01 E+00$ \\
\hline 2.39E-01 & $1.08 E+00$ & $1.36 E+00$ & $9.39 E+01$ \\
\hline 2.09E-08 & $9.39 E-06$ & $1.14 E-02$ & 7.86E-01 \\
\hline $2.42 E+03$ & $1.09 E+04$ & $1.09 E+04$ & $7.50 E+05$ \\
\hline $1.27 E-01$ & $5.70 E-01$ & $7.76 E-01$ & $5.36 E+01$ \\
\hline $3.05 E+04$ & 1.37E+05 & 1.40E+05 & $9.63 E+06$ \\
\hline $2.88 E+04$ & $1.30 E+05$ & $1.32 E+05$ & $9.11 E+06$ \\
\hline 1.25E+04 & $5.65 E+04$ & $5.65 E+04$ & $3.90 E+06$ \\
\hline $1.25 E+04$ & $5.65 E+04$ & $5.65 E+04$ & $3.90 E+06$ \\
\hline $2.12 E+04$ & $9.55 E+04$ & $9.55 E+04$ & $6.59 E+06$ \\
\hline $3.09 E+02$ & 1.39E+03 & $1.48 E+03$ & $1.02 E+05$ \\
\hline $0.00 E+00$ & $0.00 E+00$ & 8.08E-05 & 5.57E-03 \\
\hline $0.00 E+00$ & $0.00 E+00$ & 4.63E-05 & 3.20E-03 \\
\hline $1.07 E-04$ & 4.82E-04 & 7.34E-02 & 5.07E +00 \\
\hline $5.80 E-06$ & 2.61E-05 & 4.41E-01 & 3.05E+01 \\
\hline $1.48 E-05$ & $6.67 E-05$ & $1.87 E-01$ & $1.29 E+01$ \\
\hline 4.24E-06 & 1.91E-05 & $7.09 E-03$ & 4.89E-01 \\
\hline $1.16 \mathrm{E}-0.4$ & 5.21E-04 & 8.72E-02 & $6.01 E+00$ \\
\hline 1.07E-03 & $4.82 E-03$ & 1.12E $\div 00$ & $7.71 E+01$ \\
\hline $6.38 E-04$ & $2.87 E-03$ & 1.21E+02 & $8.38 E+03$ \\
\hline $1.27 E-02$ & $5.73 E-02$ & $6.10 E+00$ & $4.21 E+02$ \\
\hline $8.70 E-05$ & $3.91 E-04$ & 7.98E-04 & $5.50 E-02$ \\
\hline $4.76 E-04$ & $2.14 E-03$ & 2.98E-03 & 2.05E-01 \\
\hline 4.15E-03 & 1.87E-02 & 1.87E-02 & $1.29 E+\infty 0$ \\
\hline $3.02 E-02$ & 1.36E-01 & 1.37E-01 & $9.43 E+00$ \\
\hline $5.58 E+02$ & $2.51 E+03$ & $2.51 E+03$ & 1.73E+05 \\
\hline $5.20 \mathrm{E}+00$ & $2.34 E+01$ & $2.36 E+01$ & $1.63 E+03$ \\
\hline 3.57E+00 & 1.61E+01 & $1.62 E+01$ & $1.12 E+03$ \\
\hline $6.89 \mathrm{E}+02$ & $3.10 E+03$ & 3.13E+03 & $2.16 E+05$ \\
\hline $5.52 E-03$ & $2.49 E-02$ & $2.51 E-02$ & $1.73 E+00$ \\
\hline $8,20 E+01$ & $3.69 E+02$ & $3.70 E+02$ & $2.55 E+04$ \\
\hline $2.60 \mathrm{E}-02$ & 1.17E-01 & $1.26 \mathrm{E}-01$ & $8.69 E+00$ \\
\hline $4.05 E-02$ & $1.82 \mathrm{E}-01$ & $1.84 E-01$ & $1.27 E+01$ \\
\hline $3,92 E+01$ & $1.77 E+02$ & 1.77E+02 & $1.22 E+04$ \\
\hline $6.44 E-05$ & 2.90E-04 & $3.24 E-04$ & $2.24 E=02$ \\
\hline $7.24 \mathrm{E}-06$ & $3.26 E-05$ & $3.48 E-05$ & $2.40 E-03$ \\
\hline & $T$ & $7.98 E+05$ & \\
\hline & ategor & $5.51 E+07$ & \\
\hline
\end{tabular}

Prepared by Lenny Storz 9/4/98
Page 12 
3. System Characterization: Waste Package

Table 3-6. Total Curies (DOE SNF and DHLW) by Category (continued)

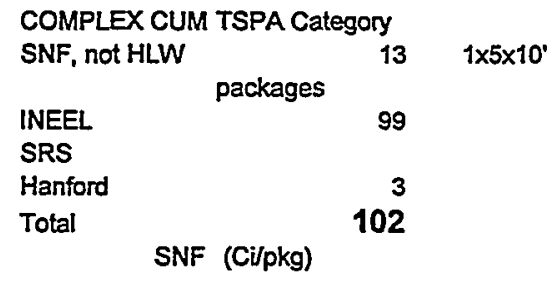

$\begin{array}{lc}\text { Isotopes } & \\ \text { C 14 } & 8.31 E-02 \\ \text { CL 36 } & 2.50 E-03 \\ \text { N1 59 } & 4.86 E-01 \\ \text { NI 63 } & 6.00 E+01 \\ \text { SE 79 } & 1.26 E-02 \\ \text { SR90 } & 2.84 E+03 \\ \text { Y90 } & 2.84 E+03 \\ \text { ZR93. } & 7.59 E-02 \\ \text { NB 93M } & 6.90 E-03 \\ \text { NB 94 } & 4.53 E-03 \\ \text { TC 99 } & 4.24 E-01 \\ \text { RU106 } & \\ \text { RH106 } & \\ \text { PD107 } & 5.84 E-04 \\ \text { SN126 } & 1.17 E-02 \\ \text { I129 } & 7.06 E-04 \\ \text { CS134 } & \\ \text { CS135 } & 3.14 E-02 \\ \text { CS137 } & 2.99 E+03 \\ \text { BA137M } & 2.99 E+03 \\ \text { CE144 } & \\ \text { PR144 } & \\ \text { PM147 } & \\ \text { SM151 } & \\ \text { PB210 } & \\ \text { RA226 } & 2.34 E+01 \\ \text { RA228 } & 1.32 E-12 \\ \text { AC227 } & 2.20 E-12 \\ \text { TH229 } & 3.43 E-09 \\ \text { TH230 } & 5.51 E-08 \\ \text { TH232 } & 1.12 E-09 \\ \text { PA231 } & 3.74 E-09 \\ \text { U233 } & 1.01 E-08 \\ \text { U234 } & 8.80 E-07 \\ \text { U235 } & 5.49 E-06 \\ \text { U236 } & 2.04 E-04 \\ \text { U238 } & 9.64 E-03 \\ \text { NP237 } & 1.29 E-02 \\ \text { PU238 } & 5.67 E-03 \\ \text { PU239 } & 1.87 E-03 \\ \text { PU240 } & 2.06 E+00 \\ \text { PU241 } & 4.45 E+00 \\ \text { PU242 } & 1.73 E+00 \\ \text { AM241 } & 1.29 E+02 \\ \text { AM242M } & 2.39 E-04 \\ \text { AM243 } & 1.98 E-01 \\ \text { CM244 } & 2.19 E-03 \\ \text { CM245 } & 2.42 E-04 \\ \text { CM246 } & 6.56 E-03 \\ & 1.34 E-07 \\ & \end{array}$

\begin{tabular}{|c|c|c|c|}
\hline DHLW (Ci/can) & $\begin{array}{l}\text { Total Ci DHLW } \\
\text { per package }\end{array}$ & $\begin{array}{l}\text { Total Ci (SNF } \\
\text { + DHLW) }\end{array}$ & $\begin{array}{l}\text { Total Ci } \\
\text { Per Category }\end{array}$ \\
\hline $0.00 E+00$ & $0.00 E+00$ & 8.31E-02 & 8.47E+00 \\
\hline $0.00 \mathrm{E}+00$ & $0.00 E+00$ & 2.50E-03 & 2.55E-01 \\
\hline $3.31 E-02$ & 1.66E-01 & $6.52 E-01$ & $6.65 \mathrm{E}+01$ \\
\hline $3.58 E+00$ & $1.79 E+01$ & $7.79 E+01$ & $7.95 \mathrm{E}+03$ \\
\hline $1.01 E-01$ & 5.05E-01 & 5.17E-01 & $5.28 E+01$ \\
\hline $3.04 E+04$ & $1.52 E \div 05$ & $1.55 E+05$ & $1.58 E+07$ \\
\hline $3.08 \mathrm{E}+04$ & $1.54 E \div 05$ & $1.57 E+05$ & $1.60 E+07$ \\
\hline 7.71E-01 & $3.86 E+00$ & $3.93 E+00$ & $4.01 E+02$ \\
\hline 1.35E-01 & $6.77 E-01$ & 6.84E-01 & $6.98 E+01$ \\
\hline 3.33E-05 & 1.66E-04 & 4.70E-03 & 4.79E-01 \\
\hline $3.63 \mathrm{E}+00$ & $1.82 E+01$ & $1.86 E+01$ & $1.90 E+03$ \\
\hline $2.05 E+03$ & $1.02 E+04$ & $1.02 E+04$ & $1.04 E+06$ \\
\hline $2.05 E+03$ & $1.02 E+04$ & $1.02 E+04$ & $1.04 E+06$ \\
\hline $1.24 \mathrm{E}-02$ & $6.18 E-02$ & $6.24 E-02$ & $6.37 E+00$ \\
\hline $2.39 E-01$ & $1.20 \mathrm{E}+00$ & $1.21 E+00$ & $1.23 E+02$ \\
\hline 2.09E-06 & 1.04E-05 & 7.17E-04 & 7.31E-02 \\
\hline $2.42 E+03$ & 1.21E+04 & $1.21 E+04$ & $1.23 E+05$ \\
\hline $1.27 \mathrm{E}-01$ & $6.33 \mathrm{E}-01$ & $6.64 \mathrm{E}-01$ & $6.77 E+01$ \\
\hline $3.05 E+04$ & 1.52E+05 & $1.55 E+05$ & $1.58 E+07$ \\
\hline $2.88 E+04$ & $1.44 E+05$ & $1.47 E+05$ & $1.50 \mathrm{E}+07$ \\
\hline $1.25 E+04$ & $6.27 \mathrm{E}+04$ & $6.27 E+04$ & $6.40 E+06$ \\
\hline $1.25 E+04$ & 6.27E $\div 04$ & $6.27 E+04$ & $6.40 \mathrm{E}+06$ \\
\hline $2.12 E+04$ & $1.06 \mathrm{E}+05$ & $1.06 E+05$ & $1.08 E+07$ \\
\hline $3.09 E+02$ & $1.54 E+03$ & $1.57 E+03$ & $1.60 \mathrm{E}+05$ \\
\hline $0.00 E+00$ & $0.00 E+00$ & 1.32E-12 & $1.35 E-10$ \\
\hline $0.00 E+00$ & $0.00 E+00$ & 2.20E-12 & $2.25 \mathrm{E}-10$ \\
\hline 1.07E-04 & 5.36E-04 & $5.36 \mathrm{E}-04$ & $5.47 \mathrm{E}-02$ \\
\hline $5.80 \mathrm{E}-06$ & 2.90E-05 & $2.91 E-05$ & 2.96E-03 \\
\hline $1.48 E-05$ & 7.41E-05 & $7.41 \mathrm{E}-05$ & $7.55 \mathrm{E}-03$ \\
\hline $4.24 \mathrm{E}-06$ & 2.12E-05 & $2.12 E-05$ & $2.16 \mathrm{E}-03$ \\
\hline 1.16E-04 & 5.79E-04 & 5.79E-04 & $5.91 E-02$ \\
\hline $1.07 \mathrm{E}-03$ & 5.36E-03 & $5.36 \mathrm{E}-03$ & 5.47E-01 \\
\hline $6.38 \mathrm{E}-04$ & $3.19 E-03$ & $3.20 E-03$ & $3.26 E-01$ \\
\hline $1.27 \mathrm{E}-02$ & $6.37 \mathrm{E}-02$ & $6.39 E-02$ & $6.52 E+00$ \\
\hline 8.70E-05 & 4.35E-04 & $1.01 \mathrm{E}-02$ & $1.03 E+00$ \\
\hline 4.76E-04 & 2.38E-03 & $1.53 \mathrm{E}-02$ & $1.56 E+00$ \\
\hline 4.15E-03 & $2.08 \mathrm{E}-02$ & $2.64 \mathrm{E}-02$ & $2.70 E+00$ \\
\hline $3.02 E-02$ & 1.51E-01 & $1.53 \mathrm{E}-01$ & $1.56 E+01$ \\
\hline $5.58 \mathrm{E}+02$ & $2.79 E+03$ & $2.79 E+03$ & $2.85 E+05$ \\
\hline $5.20 \mathrm{E}+00$ & $2.60 \mathrm{E}+01$ & $3.05 E+01$ & $3.11 E+03$ \\
\hline $3.57 E+00$ & $1.78 \mathrm{E}+01$ & $1.96 E+01$ & $2.00 E+03$ \\
\hline $6.89 E+02$ & $3.44 E+03$ & $3.57 E+03$ & $3.64 E+05$ \\
\hline $5.52 \mathrm{E}-03$ & 2.76E-02 & 2.79E-02 & $2.84 E+00$ \\
\hline $8.20 E+01$ & $4.10 E+02$ & $4.10 E+02$ & $4.18 E+04$ \\
\hline $2.60 \mathrm{E}-02$ & 1.30E-01 & 1.32E-01 & $1.35 E+01$ \\
\hline 4.05E-02 & $2.02 \mathrm{E}-01$ & 2.03E-01 & $2.07 E+01$ \\
\hline $3.92 E+01$ & $1.96 \mathrm{E} \div 02$ & $1.96 E+02$ & $2.00 E+04$ \\
\hline 6.44E-05 & $3.22 E-04$ & 3.22E-04 & $3.29 \mathrm{E}-02$ \\
\hline \multirow[t]{3}{*}{ 7.24E-06 } & $3.62 E-05$ & 3.62E-05 & $3.69 E-03$ \\
\hline & Total & 8.87E+05 & \\
\hline & Total & $9.04 E+07$ & \\
\hline
\end{tabular}


Table 3-6. Total Curies (DOE SNF and DHLW) by Category (continued)

Commercial SN TSPA Category PWR

4820 packages

SNF (Ci/pkg)

\begin{tabular}{|c|c|c|}
\hline Isotopes & Ci/package & Ci/Category \\
\hline C 14 & $1.23 E+01$ & $5.93 E+04$ \\
\hline CL 36 & $1.03 E-01$ & 4.96E+02 \\
\hline NI 59 & $2.49 E+01$ & $1.20 E+05$ \\
\hline NI 63 & $3.57 E+03$ & $1.72 E+07$ \\
\hline SE 79 & $4.38 E+00$ & 2.11E+04 \\
\hline SR9O & $4.75 E+05$ & $2.29 E+09$ \\
\hline Y90 & $4.75 E+05$ & $2.29 E+09$ \\
\hline ZR93 & $2.24 E+01$ & $1.08 E+05$ \\
\hline NB 93M & $1.43 E+01$ & $6.89 E+04$ \\
\hline NB 94 & 1.12E+01 & $5.40 \mathrm{E}+04$ \\
\hline TC 99 & $1.38 E+02$ & $6.65 E+05$ \\
\hline RU106 & & $0.00 E+00$ \\
\hline RH106 & & $0.00 E+00$ \\
\hline PD107 & $1.23 E+00$ & $5.93 E+03$ \\
\hline SN126 & $8.41 E+00$ & $4.05 E+04$ \\
\hline 1129 & 3.39E-01 & $1.63 E+03$ \\
\hline $\operatorname{cs} 134$ & & $0.00 \mathrm{E}+00$ \\
\hline CS135 & $4.96 \mathrm{E}+00$ & $2.39 E+04$ \\
\hline CS137 & $6.93 E+05$ & $3.34 E+09$ \\
\hline BA137M & $6.93 E+05$ & $3.34 E \div 09$ \\
\hline CE144 & . & $0.00 E+00$ \\
\hline PR144 & & $0.00 E+00$ \\
\hline PM147 & & $0.00 E+00$ \\
\hline SM151 & $3.75 E+03$ & $1.81 E+07$ \\
\hline PB210 & 2.42E-06 & 1.17E-02 \\
\hline RA226 & 1.17E-05 & $5.64 E-02$ \\
\hline RA228 & 1.83E-09 & 8.82E-06 \\
\hline AC227 & $1.26 E-04$ & $6.07 E-01$ \\
\hline TH229 & $3.07 E-06$ & $1.48 E-02$ \\
\hline TH230 & 2.36E-03 & $1.14 E+01$ \\
\hline TH232 & 3.00E-09 & $1.45 E-05$ \\
\hline PA231 & $2.88 \mathrm{E}-04$ & $1.39 E+00$ \\
\hline U233 & 5.33E-04 & $2.57 \mathrm{E}+00$ \\
\hline U234 & $1.23 E+01$ & $5.93 E+04$ \\
\hline U235 & 1.63E-01 & $7.86 E+02$ \\
\hline U236 & $2.72 E+00$ & 1.31E+04 \\
\hline U238 & $2.81 E+00$ & 1.35E+04 \\
\hline NP237 & $4.39 \mathrm{E}+00$ & $2.12 E+04$ \\
\hline PU238 & $3.41 E+04$ & $1.64 E+08$ \\
\hline PU239 & $3.54 E+03$ & $1.71 E+07$ \\
\hline PU240 & $5.19 E+03$ & $2.50 E+07$ \\
\hline PU241 & $5.25 E+05$ & $2.53 E+09$ \\
\hline PU242 & $1.91 E+01$ & $9.21 E+04$ \\
\hline AM241 & $2.95 E+04$ & $1.42 E+08$ \\
\hline AM242M & 2.10E+02 & $1.01 E+06$ \\
\hline AM243 & $2.42 E+02$ & 1.17E+06 \\
\hline CM244 & $1.70 E+04$ & $8.19 E+07$ \\
\hline CM245 & $3.49 E+00$ & $1.68 E+04$ \\
\hline \multirow[t]{2}{*}{ CM246 } & 7.36E-01 & $3.55 E+03$ \\
\hline & $2.96 E+06$ & $1.43 E+10$ \\
\hline
\end{tabular}


3. System Characterization: Waste Package

Table 3-6. Total Curies (DOE SNF and DHLW) by Category (continued)

Commercial SN TSPA Category

BWR

2859 packages

SNF (Ci/pkg)

Isotopes

Ci/package

C 14

CL 36

NI 59

NI 63

SE 79

SR9O

Y9O

ZR93

NB 93M

NB 94

TC 99

RU106

RH106

PD107

SN126

1129

CS134

CS135

CS137

BA137M

CE144

PR144

PM147

SM151

PB210

RA226

RA228

AC227

TH229

TH230

TH232

PA231

U233

U234

U235

U236

U238

NP237

PU238

PU239

PU240

PU241

PU242

AM241

AM242M

AM243

CM244

CM245

CM246

Total:
8.55E-02

1.37E+01

$1.86 E+03$

$3.06 E+00$

$3.27 E+05$

$3.27 E+05$

1.85E+01

1.19E+01

7.29E-01

$9.87 E+01$

8.96E-01

$5.90 E+00$

2.41E-01

$3.82 E+00$

$4.86 E \div 05$

4.86E+05

2.70E +03

1.86E-06

8.92E-06

1.29E-09

9.44E-05

1.94E-06

1.77E-03

2.10E-09

2.17E-04

3.24E-04

$9.01 \mathrm{E}+00$

1.25E-01

$1.88 E+00$

$2.48 E+00$

$2.70 E \div 00$

2.11E+04

$2.45 E+03$

$3.66 E+03$

$4.08 E+05$

1.50E+01

2.30E+04

$1.80 E+02$

$1.78 E+02$

1.13E+04

$2.08 E+00$

4.06E-01

2.10E+06
Ci/Category

$3.35 \mathrm{E}+04$

2. $44 \mathrm{E}+02$

$3.92 E+04$

5.32E+06

8.75E+03

9.35E+08

$9.35 E+08$

$5.29 \mathrm{E}+04$

$3.40 \mathrm{E}+04$

$2.08 \mathrm{E}+03$

$2.82 E+05$

$0.00 E+00$

$0.00 E+00$

$2.56 \mathrm{E}+03$

1.69E+04

$6.89 E+02$

$0.00 E+00$

$1.09 E+04$

1.39E+09

1.39E+09

$0.00 E+00$

$0.00 E+00$

$0.00 E+00$

$7.72 \mathrm{E}+06$

5.32E-03

2.55E-02

3.69E-06

2.70E-01

5.55E-03.

5.06E+00

$6.00 \mathrm{E}-06$

6.20E-01

9.26E-01

2.58E+04

$3.57 \mathrm{E}+02$

5.37E+03

$7.09 \mathrm{E}+03$

$7.72 E+03$

$6.03 \mathrm{E}+07$

$7.00 \mathrm{E}+06$

1.05E+07

1.17E+09

$4.29 \mathrm{E}+04$

$6.58 \mathrm{E}+07$

$5.15 E+05$

$5.09 \mathrm{E}+05$

$3.23 E+07$

$5.95 \mathrm{E}+03$

$1.16 E+03$

$6.01 E+09$ 


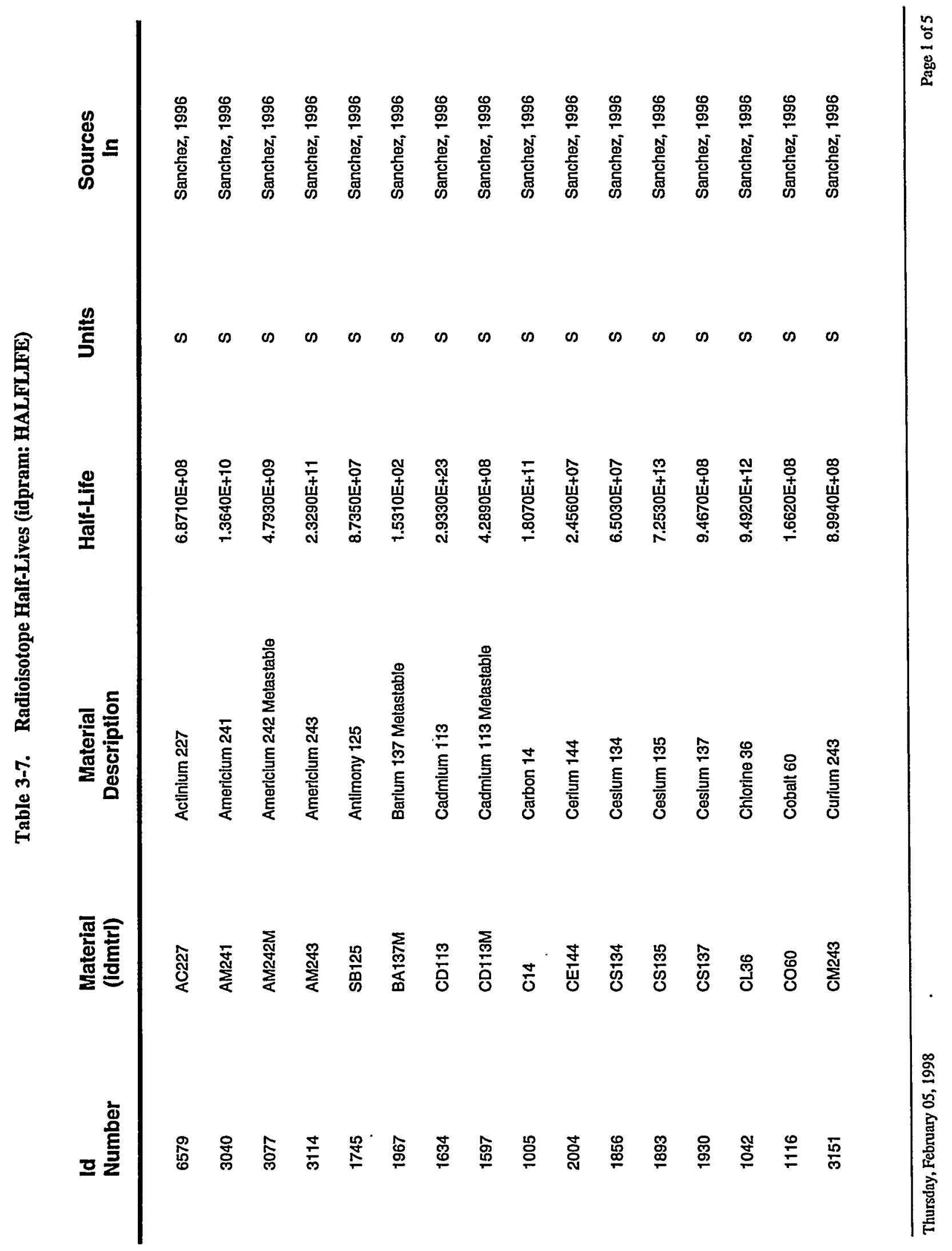




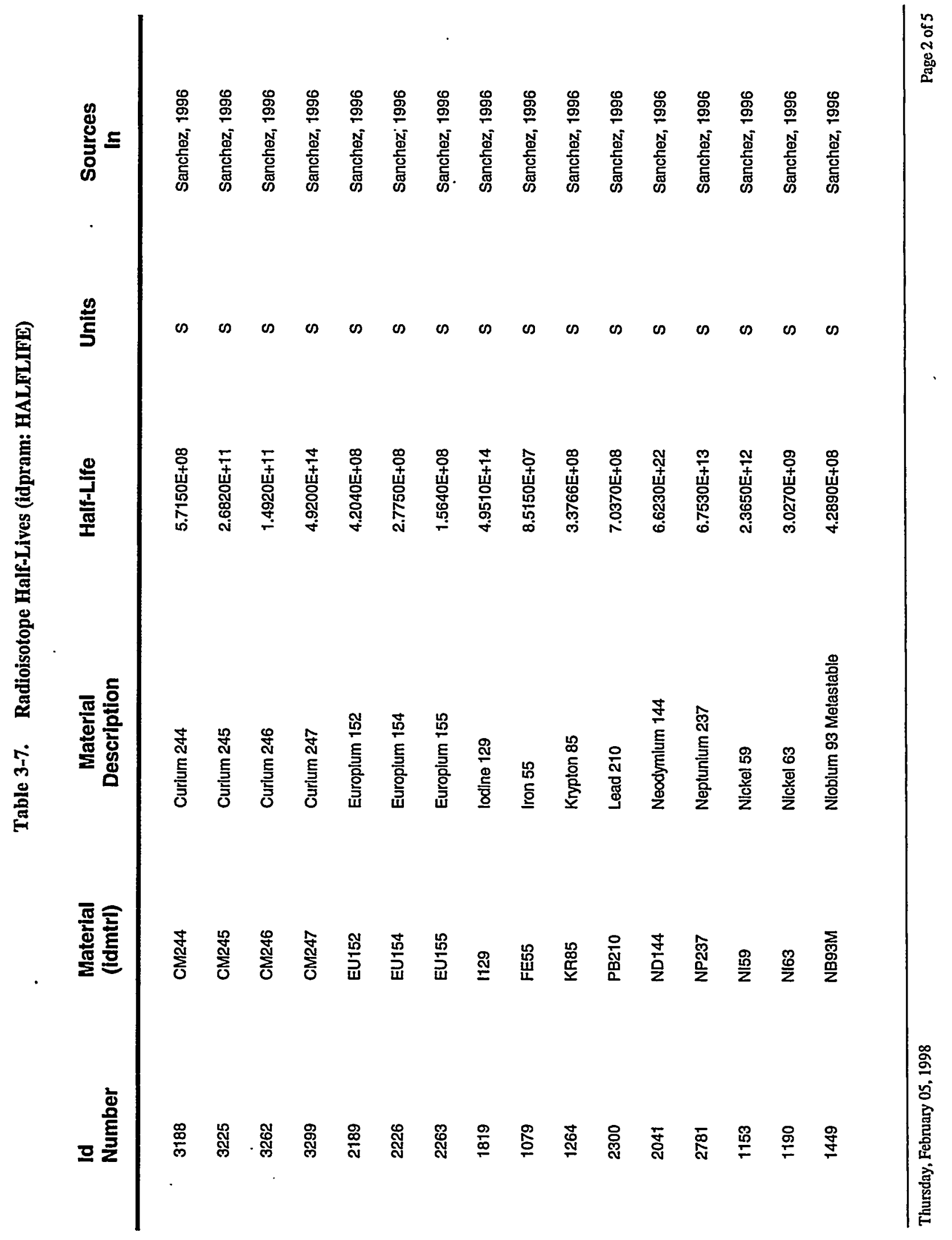




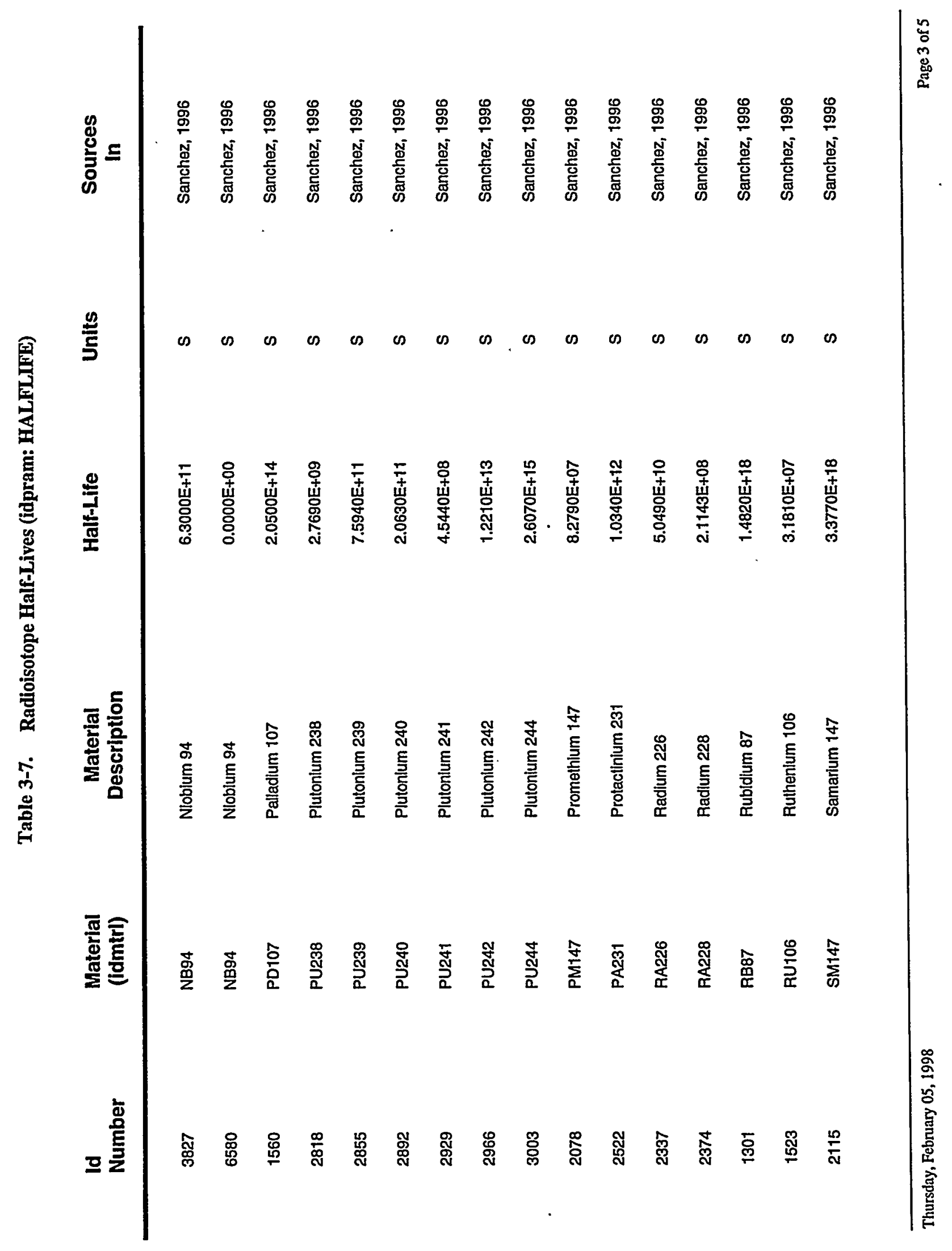




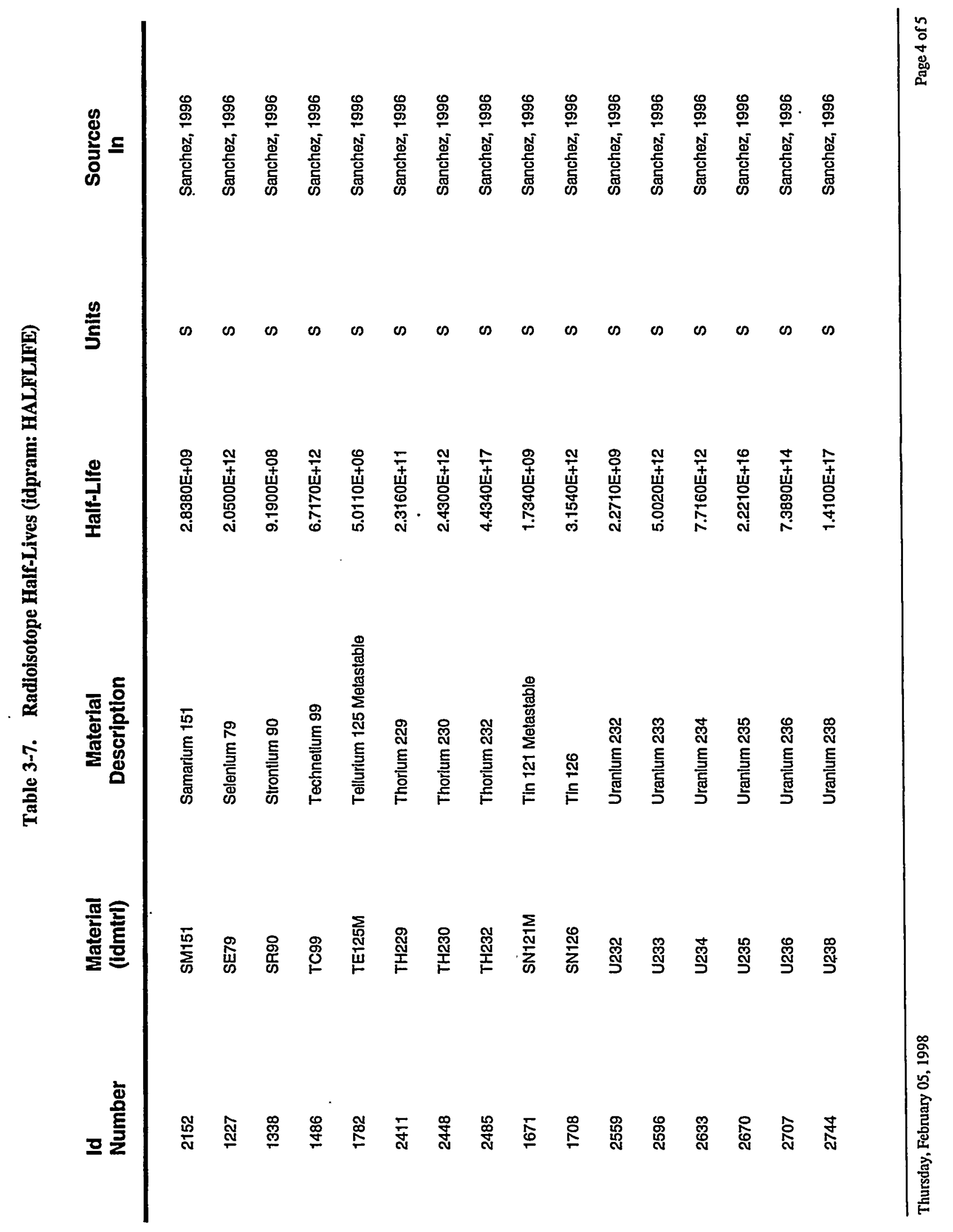




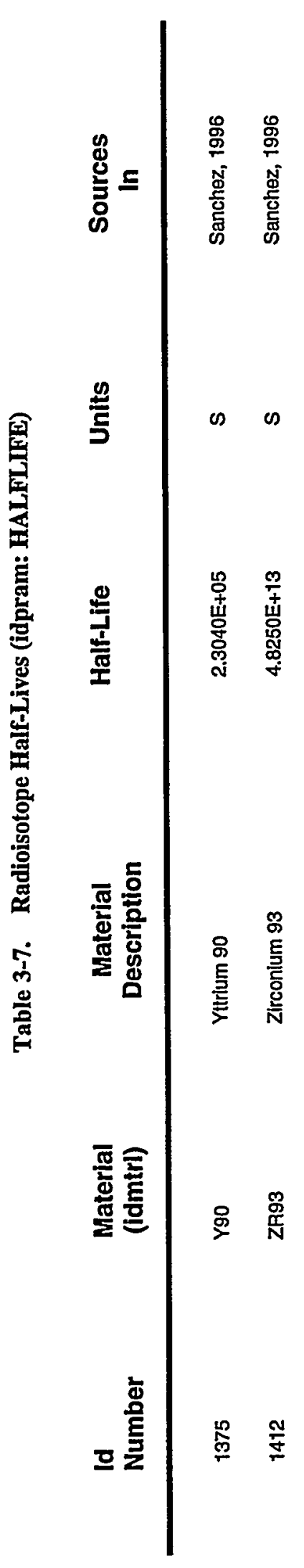

$n$
0
0
8
8
0

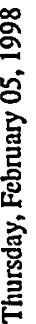




\title{
4. System Characterization: Geologic Barrier
}

\author{
K. N. Gaither, A. M. Parsons, and R. P. Rechard
}

The geologic barrier is one of two major components of the disposal system that must be characterized to provide an accurate simulation of waste performance after disposal. (The engineered barrier, another major component, comprises the waste packages and repository design, which are discussed in Chapters 3 and 5, respectively.) Characterizing the geologic barrier requires an understanding of the dynamic, large-scale natural forces that work through geologic time shaping the past, present, and future of the potential repository site.

This chapter provides a context for the data used to characterize the geologic barrier in the 1997 PA by (a) discussing previous studies, from which the current understanding of geologic conditions at Yucca Mountain is drawn; (b) providing a brief synopsis of the regional setting to explain what characteristics of the site are likely to be most relevant to containment and flow and transport of radioisotopes; (c) detailing the characteristics of the site important to the $1997 \mathrm{PA}$; (d) describing the major assumptions with regard to the geologic barrier; and (e) listing the parameters used to define the geologic barrier in the 1997 PA.

\subsection{Data Sources and Previous Studies}

Many previous publications contain comprehensive site descriptions that have evolved during site investigation (DOE, 1995; Luckey et al., 1996; Flint et al., 1996; Scott and Bonk, 1984; Wilson et al., 1994; Winograd and Thordarson, 1975; Younker et al., 1992). The 1988 Site Characterization Plan (SCP) (DOE, 1988b) contains extensive background information for virtually all aspects of the geologic barrier subsystem. In this chapter, brief, summary level descriptions of geologic barrier site data are included, where appropriate, to develop a picture of site complexity and to illuminate choices made when developing conceptual models. The 1997 PA used published reports and YMP data sources for geologic data without attempting to provide quality assurance (QA), assuming that the researchers publishing the reports and collecting the data documented the QA status of the material.

\subsection{Regional Setting and Climate}

This section summarizes the regional data, which is used in the $1997 \mathrm{PA}$ as a basis for (a) boundary conditions for site models, (b) analogues to envision changing conditions, and (c) a picture of large-scale processes that control the local-scale effects at the site. Many models attempt to simulate processes that are small on a geological scale but are driven by regional, even continental, scale climatic and crustal dynamics. Regional data are valuable because, in building conceptual models of a site as it is today and as it might be in the future, geologists and others rely on their broader knowledge of well-documented conditions and processes that have occurred'in similar settings and at other geologic times.

\subsubsection{Regional Geologic Setting}

The potential repository at Yucca Mountain is located about $137 \mathrm{~km}$ by air northwest of Las Vegas in Nye County, Nevada, on and adjacent to the southwest portions of the Nevada Test Site (NTS) (Lynch et al., 1991) (Figure 4-1). General features that make the site potentially suitable for a repository are its distance from large population concentrations; the possibility of long-term institutional control; its dry climate and deep water table; its tectonic and seismic stability; and its physically and chemically stable host lithology. These features enhance the containment potential of the repository, lengthen the period in which migrating radioisotopes could reach an exposed population through groundwater, and reduce the number of potentially exposed individuals. Another factor affecting the poten- 


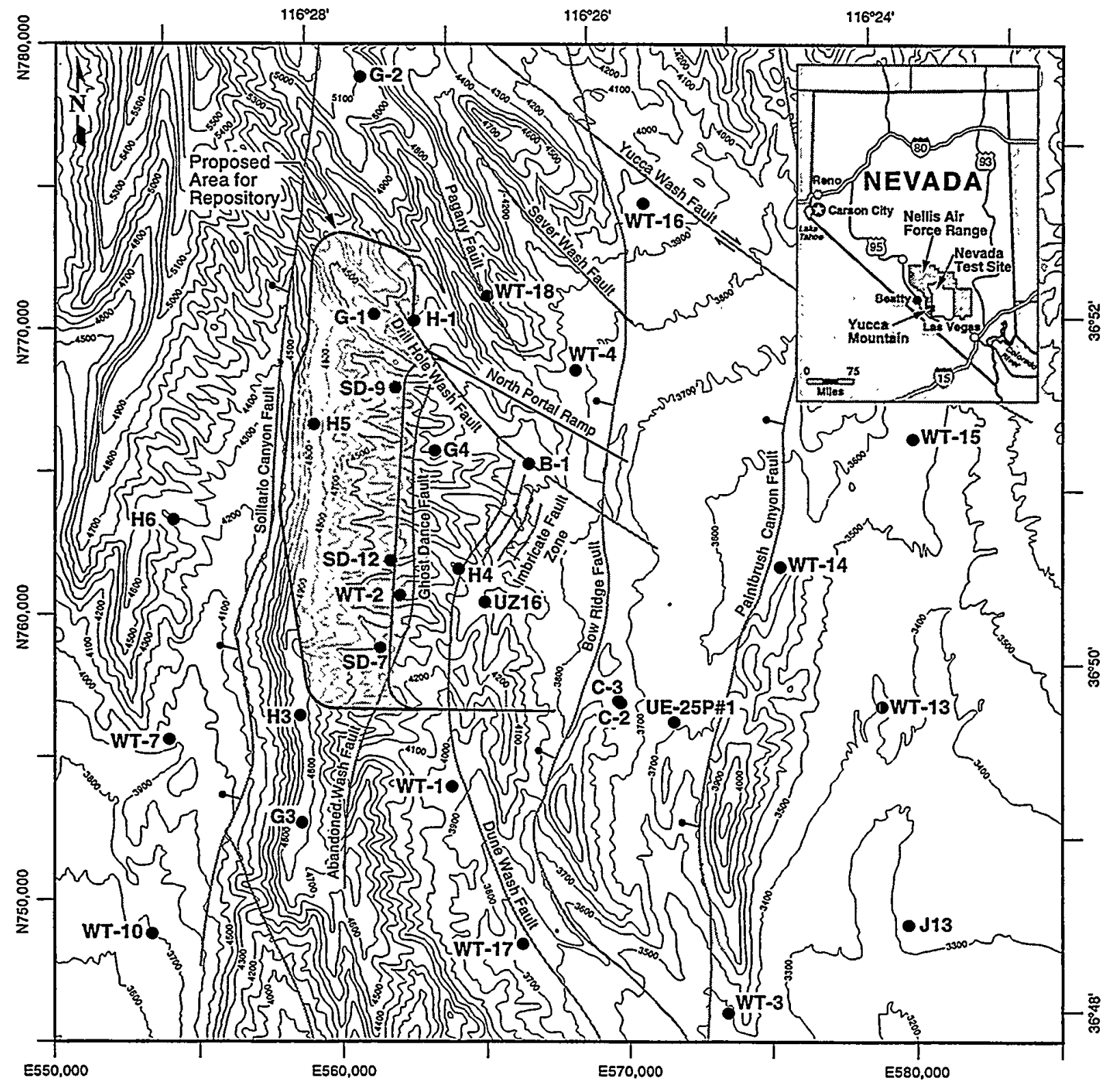

Contour interval $=100 \mathrm{ft}$
- Fault line includes both exposed and approximate trace of fault
Ball and bar on downward side of fault
$\rightleftharpoons$ Strike slip fault
$-\quad$ Drill hole location and number

TRI-6342-5473-5

Figure 4-1. Potential repository at Yucca Mountain, located northwest of Las Vegas on and adjacent to southwest portions of Nevada Test Site. 
tial for exposure of individuals in the future is the lack of unique economically valuable resources in the site area. Although some economic resources are found in the region, such as zeolites and very pure silica, this topic has been covered in detail in the SCP (DOE, 1988b, Sec. 1.7), is being addressed by other studies, and so is not addressed by the present study.

Yucca Mountain is a small part of the huge, ancient Cordilleran mountain system that began rising in the late Precambrian and now spans half the world from the Aleutian Islands $17,700 \mathrm{~km}(11,000 \mathrm{mi})$ to the tip of South America (Clark and Stearn, 1968). Yucca Mountain is located in an interior range of the Cordilleran called the Basin and Range Province in the Great Basin subprovince, an area which includes virtually the entire state of Nevada (Figure 4-2). The complexly faulted and folded Basin and Range Province was formed by late Mesozoic and early Cenozoic crustal extension, thrusting, and volcanism, which occurred during uplift of the earth's crust around a sinking geosynclinal depositional feature to the west. On a broad scale, the mountains of the Basin and Range Province are blocks cut by north-south trending Cenozoic age normal faults into ranges that stand above the surrounding desert plains.

The southern Great Basin has large zones of strike-slip faults that trend mainly northwest or northeast. The northwest trending zones include the Las Vegas Valley shear zone, Death Valley-Furnace Creek system, and the Walker Lane, in which Yucca Mountain is located (Figure 4-3). To the south and east of Yucca Mountain, the Walker Lane deformation is part of an extensional pattern with northwest shear trends dying out into north-to-northeast trending shears as part of an adjustment of the larger Great Basin structural grain into the Garlock Fault. The Walker Lane belt structural regime has not only changed the orientation of basins and ranges from the larger regional grain, but certain blocks have been rotated even farther from regional trends. Yucca Mountain has been rotated 30 degrees clockwise since middle Miocene time, probably by a couplet of right-lateral faults (Scott and Rosenbaum, 1986) as have other areas of the Walker Lane (DOE, 1988b, p. 1-115).

Normal (extensional) and strike-slip faulting dominated the Great Basin's structural history during the middle and late Tertiary as the earth's crust extended an estimated 10 to 300 percent in the area (DOE, 1988b, p. 1-328). Today, lateral crustal extension is still ongoing. Yucca Mountain normal faulting occurred from the Middle Miocene onward and accompanied early volcanic activity. Both northeast and northwest trending Southern Great Basin strikeslip faults occur in the Yucca Mountain area. Knowledge of these deep structural patterns that allowed crustal extension accompanied by normal and strike-slip faulting may be significant because of the impact on future surface faulting and ground motion. More than one conceptual model could be used to explain these patterns, including listric faults and en echelon faults, which are still under study. These fault-related conceptual models do not impact the current study directly, and they are not discussed further in the present performance assessment.

The most important faults in the 1997 PA are those associated with normal fault zones, as shown in the generalized geologic map (Figure 4-4) and the schematic cross section (Figure 4-5). Figure 4-4 shows the location of the schematic cross section and the north-south trending normal faults from Windy Wash on the northwest to Paintbrush Canyon on the east as well as the northwest structural trend that parallels Yucca Wash and Drill Hole Wash north of the potential repository area.

Tectonic Setting. The existing stress field at Yucca Mountain is a result of regional stress patterns developed about 4 to 10 million years ago and is relevant to engineering considerations for the potential repository. The least principal stress orientation for the southern Great Basin is about $\mathrm{N} 50^{\circ} \mathrm{W}$, an orientation also seen in measurements at the NTS (Carr, 1974). Ratios between the magnitude of least principal horizontal stress and vertical principal stress have been studied and provide information used in design and construction of the potential repository and for predictions of fault behavior during future tectonic events (DOE, 1988b, p. 1-333).

The USGS has been studying the tectonic setting of the potential repository through its DOE Yucca Mountain Tectonics Program, and through the probabilistic seismic hazard analysis (PSHA) that it manages for the DOE. These and other data sources have been used to analyze the possibility of disruption of the potential repository by seismicity or volcanic intrusion with a focus on two general aspects of the problem: frequency of occurrence and potential effects on the repository (McGuire et al., 1990, 1992; Barnard et al., 1992; Wilson et al., 1994; Wescott et al., 1995; EPRI, 1996). TSPA-1995 stated that in all of these studies the probability-weighted releases associated with these processes are insignificant compared to those associated with repository-induced releases (M\&O, 1995a, p. 1-4). 


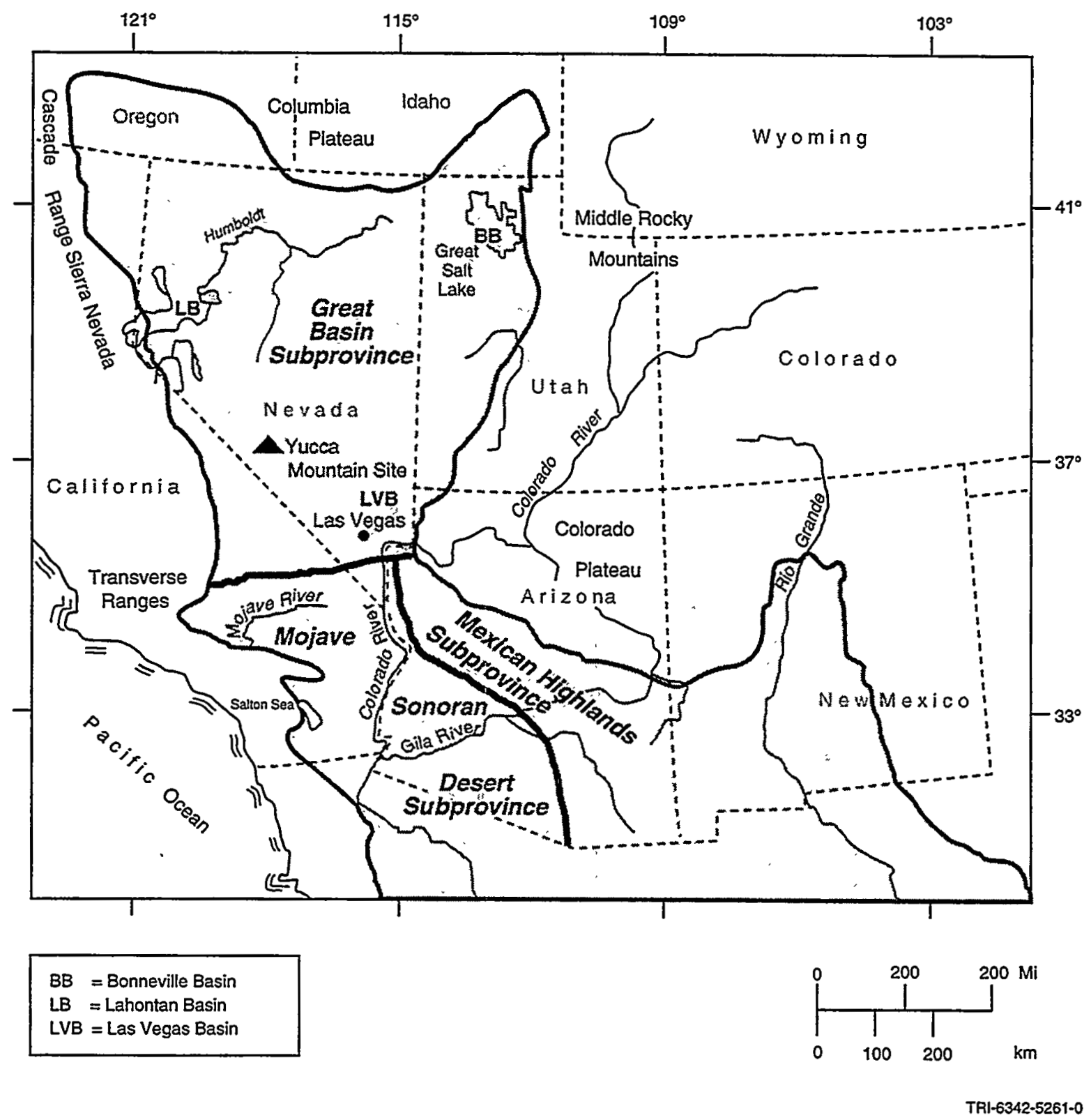

Figure 4-2. Boundaries of the Basin and Range physiographic province including three of the larger subprovinces (after DOE, 1988b, Figure 1-2). 


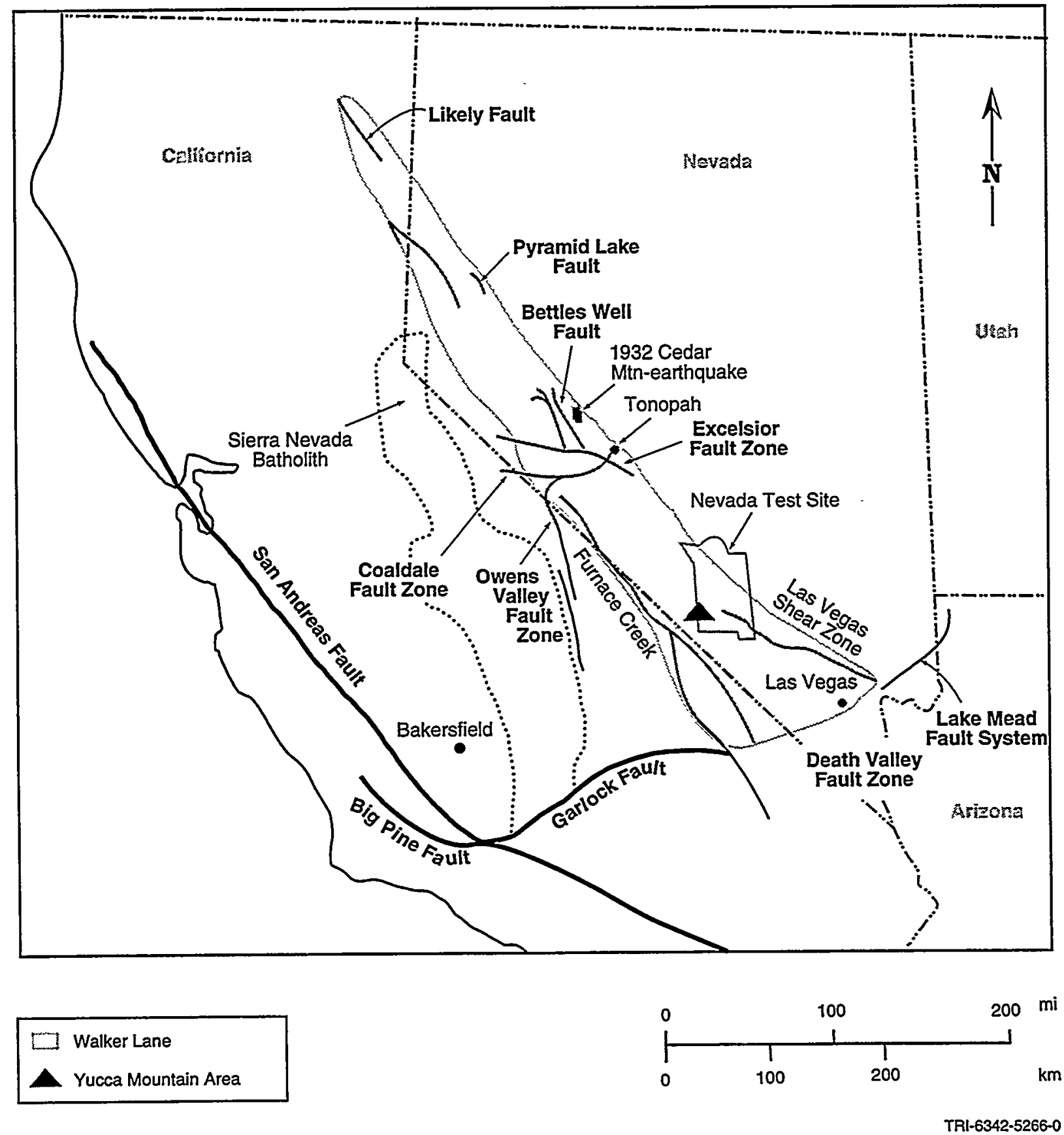

Figure 4-3. Walker Lane and major associated faults (from DOE, 1988b, after Stewart, 1985). 


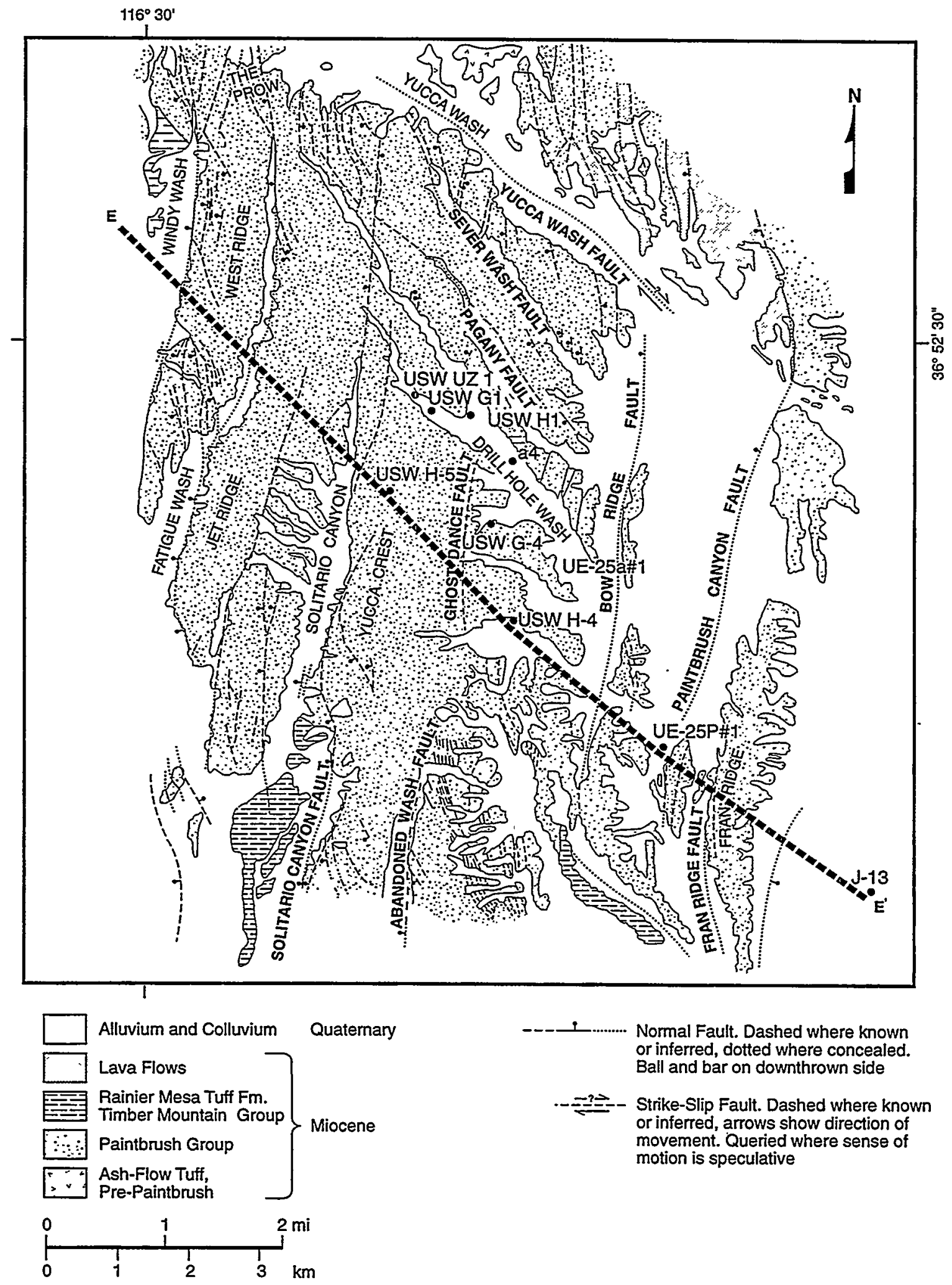

TR1-6342-5265-0

Figure 4-4. Generalized geologic map of Yucca Mountain showing major faults and stratigraphic units and location of generalized structure cross section E-E' (from DOE, 1988b, modified after Scott and Bonk, 1984). 

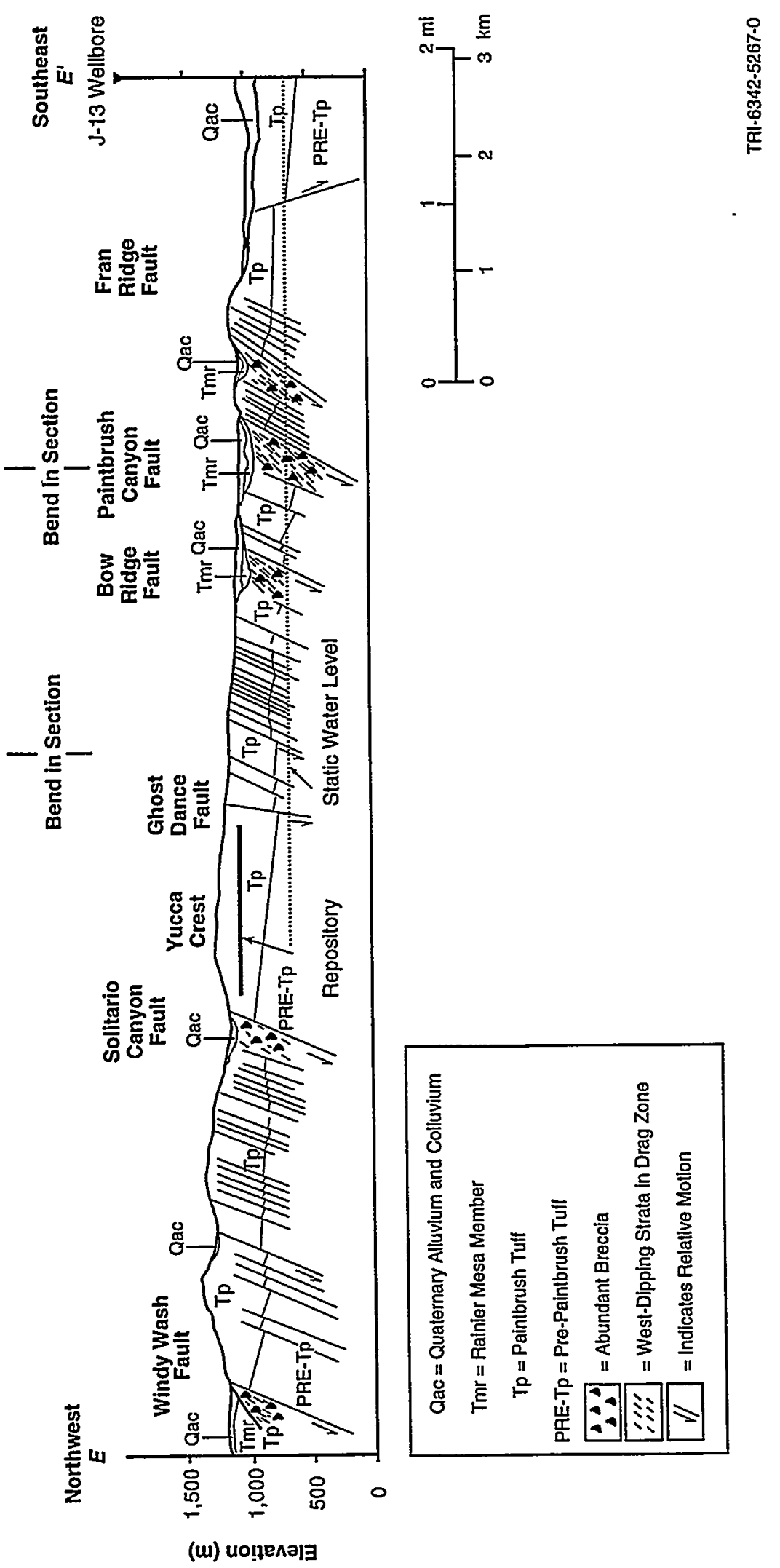

ذิำ

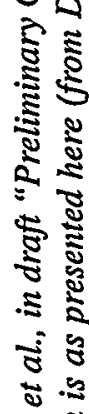

วิธี

혼

ث

.

윰

$\infty$

$\approx$

\&

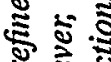

के

$\approx \Sigma$

廿.

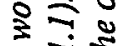

ㅊำ

ญ

2.

ริ

.5

ป ญे

ㅎํำ

$8 \%$

ڤ

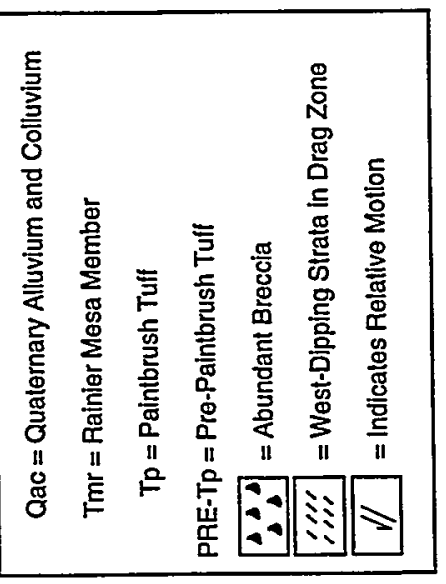

ठิ

जे

.5

కั้

ธั

氛

ธิ

융

边宁

.

๘

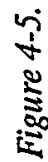




\section{System Characterization: Geologic Barrier}

High geothermal heat flow is more typical of the southern Great Basin than of the rest of the United States, but the area north of and next to Yucca Mountain has lower heat flow than is average for the southern Great Basin (DOE, 1988b, p. 1-305 and 1-333). Geothermal gradients vary significantly from borehole to borehole at Yucca Mountain. Given present technology and economics, geothermal energy is not considered a significant potential resource in the area. Further information regarding geothermal conditions at Yucca Mountain can be found in Sass et al. (1988). Ambient surface temperature was used for 1997 PA modeling, and geothermal gradients were not applied.

Geomorphic and Topographic Setting. Geomorphology influences repository performance by affecting precipitation, runoff, infiltration, and erosion, among other factors. Geomorphic data are also used for surface fault mapping. Topography creates changes in precipitation by means of elevation, e.g., moisture condenses as rising air is cooled, and the resulting effects have been noted as a factor in infiltration rates at Yucca Mountain (Flint et al., in draft "Conceptual and Numerical Model of Infiltration for the Yucca Mountain Area, Nevada"). In addition, the uplift of the Sierra Nevada mountain range to the west creates a rainshadow contributing to aridity in the area of Yucca Mountain that will continue for tens of thousands of years.

Topography in the Yucca Mountain area developed from faulting and erosion of an extensive Tertiary volcanic plateau and is characterized by flat valleys flanking steep-sided mountains. The north-trending, linear crests of these mountains are the exposed edges of faulted, rotated blocks of predominantly volcanic rock. Geomorphic data indicate that Yucca Mountain is relatively stable and that overall rates of wasting will not exceed 1 to $2 \mathrm{~m}$ over the next 10,000 yr during which climate and tectonics are not projected to vary enough to affect erosion. Future erosion is likely to be highest in localized areas of washes hit by short, high velocity flow after intense local storms. Erosion rates estimated in these types of areas on the east face of Yucca Mountain averaged over the last 0.15 to 0.3 yr have ranged as high as $375 \mathrm{~cm} /$ per 10,000 yr. The SCP (DOE, 1988b) contains a detailed discussion of this subject, which is of interest in postclosure performance but was not given detailed treatment in the 1997 PA.

Stratigraphic Setting. Yucca Mountain's youngest rocks are pyroclastic tuffs deposited during the Miocene epoch of the Tertiary period when extensive volcanism blanketed the Paleozoic carbonates with igneous deposits after subsidence of Cordilleran structural deformation in the mid Cenozoic. Volcanic activity occurred between 7 and 15 million years ago in the southwestern Nevada volcanic field and roughly centered around the Timber Mountain Caldera, which covered a large area with silicic lavas and ash-flow and ash-fall tuffs (Carr, 1988). The tuffs, which were the focus of study in the 1997 PA are (top to bottom) the Paintbrush Group, Calico Hills Formation, and Crater Flat Group. The roots of Yucca Mountain are comprised of Mississippian period and older Paleozoic era shallow oceanic carbonates deposited on the submerged continental margin (Sinnock, 1982). Precambrian marine transgressive rocks underlie the Paleozoic in the Yucca Mountain area. For more comprehensive information on the stratigraphy of the area see Flint et al. (1996), Buesch et al. (1996), Scott and Bonk (1984), Winograd and Thordarson (1975), and DOE (1988, Section 1.2).

The Paintbrush Group, comprised of four formations, forms the outcropping section at Yucca Mountain and is the host rock for the potential repository. The Group's siliceous tuffs were deposited by pyroclastic ash flow and fall with some thin, tuffaceous sedimentary beds deposited during breaks in volcanism. Textural and geochemical alteration that occurred after deposition produced features that have a strong impact on flow and transport. For PA modeling, the thermomechanical and geochemical properties and effects on the hydrology of the lithologic section are of more interest than the strictly geologic stratigraphic and petrologic details. For this reason, the 1997 PA developed hydrologic modeling units that represent the geologic section in the conceptual model. Sixteen modeling units were developed, and their interaction with fractures during flow was modeled using a composite porosity (equivalent continuum) model, which is discussed in detail in later sections of this report (see Chapter 8).

\subsubsection{Regional Hydrology}

The basins of the Basin and Range Province are typified by internal surface drainage, often to playas, with few interbasin streams. Precipitation that falls on the closed surface basins and moves below the evapotranspiration zone can enter the regional groundwater system and eventually be transported to a point of discharge by movement between basins. Regional groundwater flow in the vicinity of Yucca Mountain includes several topographic basins 
and groundwater subbasins. The SCP (DOE, 1988b, Chapter 3) and Waddell et al. (1984) describe eight hydrographic regions, with three groundwater subbasins from the eight as being of particular interest.

Figure 4-6 shows major inflows and outflows of the three subbasins. The saturated zone at Yucca Mountain is an unconfined aquifer that is part of the Alkali Flat-Furnace Creek groundwater basin which is part of the larger (47,000 $\mathrm{km}^{2}$ ) Death Valley groundwater flow system (Luckey et al., 1996). Groundwater flow from Yucca Mountain is generally to the south to southeast, which is why saturated zone modeling is often focused on a path in this direction relative to the repository.

Six aquifers exist in the potential repository vicinity with the principal ones being the valley-fill and lower carbonate aquifers. Figure 4-7 shows the relationship of the aquifers to the complete geologic column of the area with hydrogeologic units are from Winograd and Thordarson. Not all of the geologic formations, aquifers, or aquitards (confining units) are present below the potential repository. The bedded tuff aquifer rests on the lower portion of the tuff aquitard in the area of the potential repository, because the intervening section is missing. The water table below the potential repository occurs at about $730 \mathrm{~m}$ above sea level in the tuff aquitard.

The valley-fill aquifer is unsaturated near Yucca Mountain, but is a principal source of water in the Amargosa Desert area to the south. Another aquifer, the lava-flow aquifer, lies below the valley-fill but is not present at Yucca Mountain. Only the lower portion of the welded tuff aquifer, comprised of the Timber Mountain Group and upper Paintbrush Group, is present at Yucca Mountain. It is unsaturated there, but exists as an aquifer to the east at Fortymile Wash and to the south at Crater Flat. Below the welded tuff aquifer is the lava flow aquitard (upper Wahomonie Formation), which is absent at Yucca Mountain.

The zones corresponding to the welded tuff aquifer and the tuff aquitard of Winograd and Thordarson (1975) in Figure 47 are designated by Luckey et al. (1996) as the upper volcanic aquifer (welded tuff aquifer), upper volcanic confining unit, and lower volcanic aquifer. They provide a correlation of these three zones to the modeling units of Ortiz et al. (1985) and cite the occurrence of the three zones in wellbores in the Yucca Mountain area. The Luckey et al. (1996) terminology is used here as are their definitions of these units. Table 4-1 summarizes the correlation of these units, including 1997 PA modeling units (described further in Section 4.5.1).

Table 4-1. Comparison of Luckey et al. (1996) Volcanic Aquifer and Confining Units to Formal Geologic Units, Ortiz et al. (1985), and 1997 PA Modeling Units

\begin{tabular}{|c|c|c|c|}
\hline \multirow{2}{*}{ Geologic Unit } & \multicolumn{2}{|c|}{ Modeling Units } & \multirow{2}{*}{$\begin{array}{c}\text { Definition } \\
\text { (Luckey et al., 1996) }\end{array}$} \\
\hline & Ortiz et al. (1985) & 1997 PA & \\
\hline $\begin{array}{l}\text { Topopah Spring Tuff Formation } \\
\text { (upper, densely welded) }\end{array}$ & $\begin{array}{l}\text { Lower PTn, TSw1, } \\
\text { TSw2 }\end{array}$ & $\begin{array}{l}\text { Unsaturated zone } \\
\text { model: Lower PTn, TSv, } \\
\text { TSun, TSul, TSmn, TSII, } \\
\text { TSIn; } \\
\text { Saturated zone model: } \\
\text { TSwc }\end{array}$ & $\begin{array}{l}\text { Upper volcanic aquifer } \\
\text { (welded tuff aquifer) }\end{array}$ \\
\hline $\begin{array}{l}\text { Topopah Spring Tuff Formation } \\
\text { (basal vitrophyre), underlying } \\
\text { bedded tuff, Calico Hills Formation, } \\
\text { upper nonwelded Prow Pass Tuff } \\
\text { Formation }\end{array}$ & TSw3, CHn1, CHn2 & $\begin{array}{l}\text { TSlv, CHnv, upper } \\
\text { CHnz }\end{array}$ & $\begin{array}{l}\text { Upper volcanic confining } \\
\text { unit (welded tuff aquifer, } \\
\text { bedded tuff aquifer, } \\
\text { tuff aquitard) }\end{array}$ \\
\hline $\begin{array}{l}\text { Prow Pass Tuff Formation (except } \\
\text { as above), Bullfrog Tuff Formation, } \\
\text { Tram Tuff Formation }\end{array}$ & $\begin{array}{l}\text { CHn3, PPw, CFun, } \\
\text { BFw, CFmn1, } \\
\text { CFMn2, CFMn3, TRw }\end{array}$ & $\begin{array}{l}\text { lower } \mathrm{CHnz}, \mathrm{PPw} \text {, } \\
\text { CFun, BFw, CFmn, } \\
\text { TRw }\end{array}$ & $\begin{array}{l}\text { Lower volcanic aquifer } \\
\text { (tuff aquitard) }\end{array}$ \\
\hline
\end{tabular}

The upper volcanic aquifer (welded tuff aquifer) is the densely welded part of the Topopah Spring Tuff Formation and is unsaturated at the potential repository, but is saturated to the east and south (Figure 4-7). It produces water in well J-13 with a lesser contribution from the upper volcanic confining unit (Figure 41; see also Section 4.3.3). The 


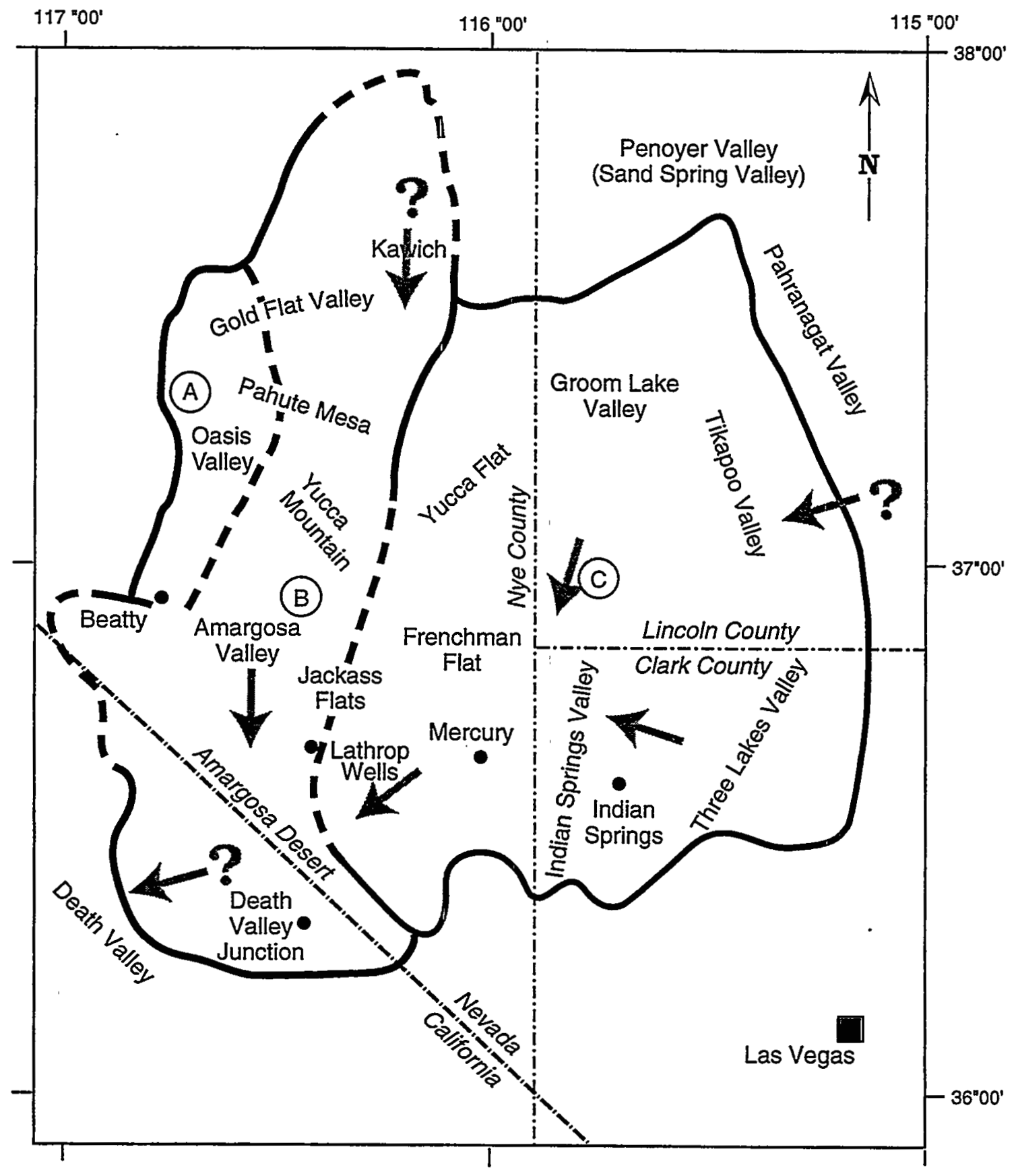

General Direction of Regional Groundwater Flow (Question mark indicates uncertainty)

A. Oasis Valley Subbasin

B. Alkali Flat-Fumace Creek Ranch Subbasin

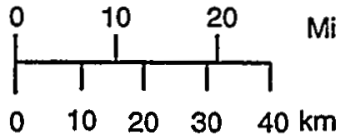

C. Ash Meadows Subbasin

TRI-6342-5260-0

Figure 4-6. Hydrogeologic flow patterns of the three groundwater subbasins (of the Death Valley System) in the region of Yucca Mountain (from DOE, 1988b, modified from Rush [1971], Blankennagel and Weir [1973], Winograd and Thordarson [1975], Dudley and Larson [1976], Waddell [1982], and Waddell et al. [1984]). 


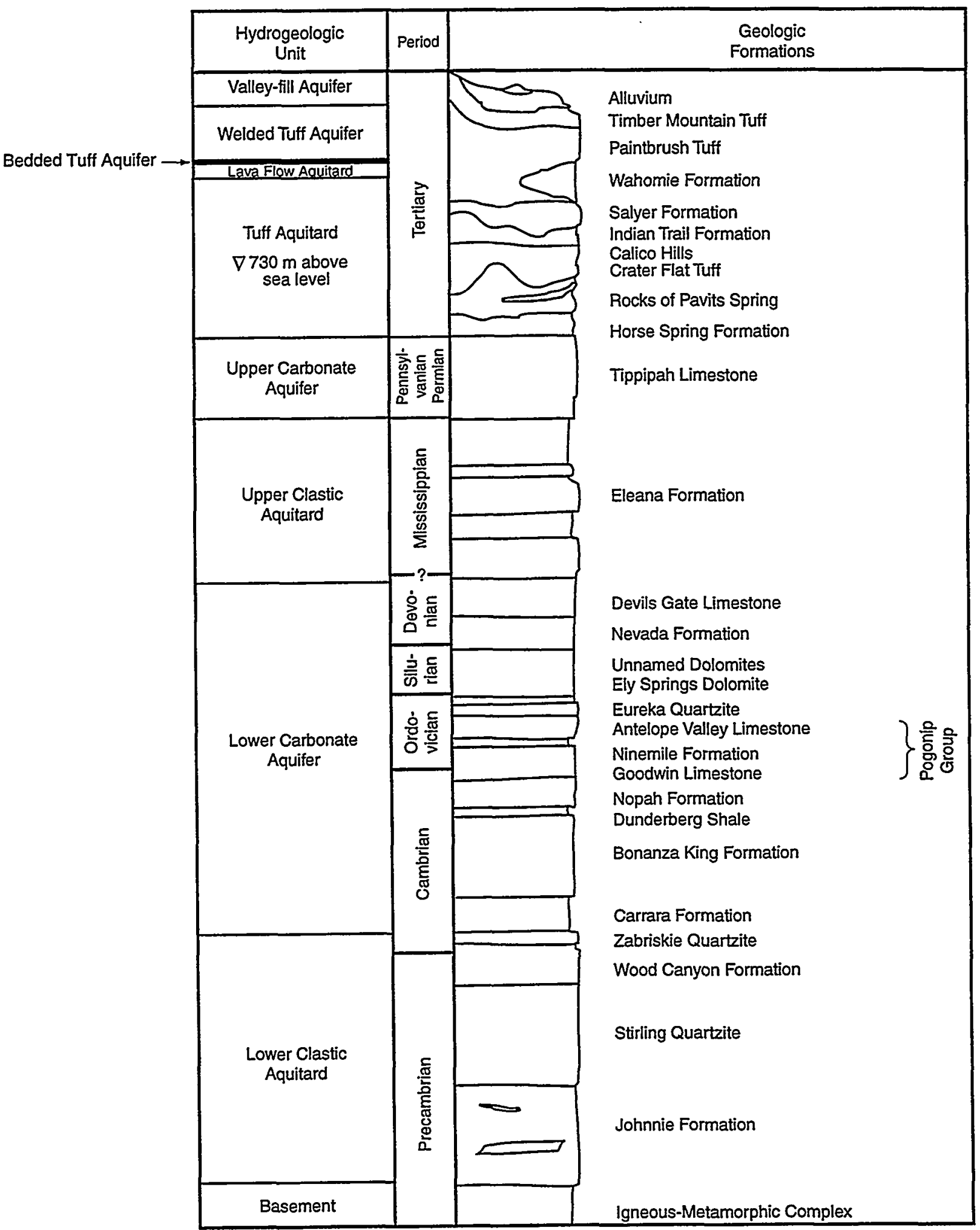

TRI-6342-5293-0

Figure 4-7. Stratigraphy in the potential repository area at Yucca Mountain with designations of aquifers and aquitards, or confining units, based on Winograd and Thordarson (1975) (after Parsons et al., 1991, modified from Sinnock, 1982). The $\nabla$ symbol indicates the elevation of the water table. 
upper volcanic confining unit consists of the basal vitrophyre of the Topopah Spring Tuff where it is not fractured or fractures are closed by fill; the bedded tuff lying below the Topopah Spring; the Calico Hills Formation; and the uppermost nonwelded Prow Pass Tuff Formation. The upper volcanic confining unit is unsaturated beneath much of the south Yucca Mountain area, but is saturated in well USW G-2 at the north end of the mountain (Figure 4-1).

The lower volcanic aquifer consists of most of the Prow Pass Tuff and the Bullfrog Tuff and Tram Tuff, all in the Crater Flat Group. This aquifer lies below all of Yucca Mountain and is saturated in most areas. The 1997 PA unsaturated zone model, which placed the water table mainly in the PPw and CFun units with the far east end in the CHnv unit, represented the top of the lower volcanic aquifer of Luckey et al. (1996). The saturated zone model covered a larger area than did the $2 \mathrm{D}$ cross-section of the unsaturated zone model, but in the area of the cross-section the correspondence to saturated zone units for location of the water table is a reasonable fit. Luckey et al. (1996) noted that the lower volcanic aquifer is densely fractured, though less so than the upper volcanic aquifer, and had reduced permeability caused by secondary alteration. They observed that during hydraulic testing only a few fractures were productive, and it was difficult to predict which zone would be productive.

The upper carbonate aquifer and upper clastic aquitard have not been found below the potential repository location at Yucca Mountain, and the Tertiary tuffs rest unconformably on the Paleozoic unit designated as the lower carbonate aquifer by Winograd and Thordarson (1975). In most YMP studies, this unit is referred to simply as the carbonate aquifer, and it is the regional aquifer providing the most likely path through which released radionuclides might migrate to other saturated units and the accessible environment. Only one well, UE-25P\#1 (Figure 4-1) penetrates to the carbonate aquifer; it reaches only the top of the aquifer. Little is known about the Paleozoic section under Yucca Mountain beyond the data that Winograd and Thordarson (1975) compiled. They noted that the lower carbonate aquifer was up to about $4570 \mathrm{~m}(15,000 \mathrm{ft})$ thick, but is deeply eroded in most areas, and regionally, has a saturated thickness of a few hundred to several thousand feet, with the greater saturated thickness likely in the Yucca Mountain region. From studies of the carbonates in outcrop, they described the rocks as having low intercrystalline porosity with isolated vugs as large as $1.0 \mathrm{~cm}(0.4 \mathrm{in}$.), but not interconnected. The rocks were highly fractured by joints and faults. Groundwater flow through the carbonate aquifer is through secondary openings developed along fractures. However, the carbonate aquifer is not modeled in the $1997 \mathrm{PA}$ as the saturated zone model goes only as deep as the lower volcanic aquifer.

\subsubsection{Regional Geochemistry}

Geochemical processes that affect transport or retardation of radioisotopes include sorption, precipitation or dissolution, and physical flow processes like diffusion, dispersion, or filtration of particulates. Changes in groundwater geochemistry that impact container corrosion and radioisotope sorption and solubility receive particular emphasis in the $1997 \mathrm{PA}$. Many aspects of the geochemistry of repository host rock are relevant to the performance assessment, including contribution to water geochemistry, chemical interaction or stability in containment, and alteration products from natural diagenesis or waste heat initiated diagenesis. Geochemistry as related to the potential repository site is discussed in Section 4.3.3.

\subsubsection{Regional Climate}

Climate has many complex interactions with the containment function of the geologic barrier and is a prime determinant of site hydrology and site vegetation. Climate cycles and daily weather combine with geomorphology, soil development, and vegetation to influence surface hydrology. Surface hydrology impacts runoff, infiltration, percolation, and recharge, which become input for parameters important to flow and transport of radioisotopes. The effect of climate variation on the potential repository and the method by which it was modeled is discussed in Section 4.3.4. 


\subsection{Site Characterization}

As discussed in Section 4.2, site characterization includes the geology, hydrology, geochemistry, and climate at the site. With regard to the geologic barrier, the 1997 PA focuses on answering questions related to containment and isolation, including how the near-field natural environment interacts with the waste and the waste canisters. Therefore, this discussion centers on those factors that most directly affect these processes. The following discussion of the structure, stratigraphy, and lithology touches lightly on classical geologic descriptions. The emphasis is on geologic criteria as expressed in modeling units so that the manner in which the 1997 PA modeled the geology is clear. Also, abundant site characterization material is available in other publications, which are cited as appropriate (e.g., DOE, 1988b).

\subsubsection{Site Geology}

The regional geologic setting described in Section 4.2 places Yucca Mountain in a broad belt of highly deformed continental crust that has been subjected to a succession of stress fields created by the interaction of major continental and oceanic plates. The 1997 PA was primarily concerned with the impact of structural features on containment, flow, transport, and thermal and mechanical interactions with the heat generated by waste. Thus, questions examined by the 1997 PA included

- Are fractures (faults and joints) permeable or do they act as permeability barriers for the fiow of liquid, gas, and heat?

- Does permeability of fractures change between the saturated and unsaturated zones; and how are these effects best modeled?

- What is the best way to conceptualize the interaction between tuff matrix porosity and fracture permeability?

- What is the best way to conceptualize major faults, as zones or discrete points of permeability change?

- What is the best approach to modeling dipping beds and fault planes?

- What is the role of fractures in the conceptualization of dripping models for groundwater falling on containers?

- What is the role of fractures in the conceptual model of infiltration and percolation?

In generating answers to these questions for the $1997 \mathrm{PA}$, site data for the geologic barrier were used to define parameters for the models. These parameters are listed in Section 4.5. The sections below describe the relationship between actual site characteristics and conceptual models used in the 1997 PA. Details with regard to the actual parameters themselves, e.g., bulk density ranges, can be found in the parameter lists (Section 4.5 ) and are only briefly touched upon here.

Geologic Framework. The 1997 PA used the YMP Integrated Site Model (ISM 2.0) (Clayton et al., 1997) as a reference for development of the geologic framework for modeling both the saturated and unsaturated zones. ISM 2.0 comprises two types of information-the geologic framework model, which captures the geometry produced by the combination of structure and stratigraphy, and several rock properties models, which contain rock properties such as porosity, geochemistry, and water saturation.

Faults. The tuffs at Yucca Mountain are fractured by several types of processes. Tectonic fractures, which include both joints and faults, arose from stress fields in the earth's crust and by stress field changes caused by unloading of overburden. Joints also developed as the hot ash deposits cooled. Properties of fractures that impact flow and transport include continuity across bedding planes, interconnectedness of fractures, correlation lengths of connected clusters of fractures, and fracture plane geometries and surface characteristics. A discrete mapping of each type of fracture would be ideal, because tectonic features developed in response to large-scale forces have different patterns of continuity and connectivity than more localized joint patterns formed in response to cooling. However, analysts cannot readily access information that distinguishes faults from joints or the trends of different joint sets. Studies that have characterized the different kinds of fractures at Yucca Mountain in detail include Sweetkind et al., 1997; Sweetkind and Williams-Stroud, 1996; and Rautman and Engstrom, 1996. 
In ISM 2.0 (Clayton et al., 1997), which was used by the 1997 PA, joints are not discretely mapped, and faults in the subsurface are mapped by downward projection of major surface faults. During construction of ISM 2.0, not all site data have been used, and for some important hydrogeologic features, data are very sparse. For example, in the area near the repository only faults with over $30.5 \mathrm{~m}$ of vertical displacement and a $3200-\mathrm{m}$ surface trace length, plus the Ghost Dance fault (which passes through the repository area), have been included in the model. Thus, smaller faults and buried faults were excluded. The significance of these criteria is that there is no hydrologic evidence to show that faults that are buried and/or smaller than those captured in the model do not have an influence on containment, flow, and transport.

Structure in the area of Yucca Mountain is dominated by north-trending, down-to-the west normal faults, including some prominent ones that can be mapped for kilometers along strikes and hundreds of smaller associated faults (Figures 4-4 and 4-5). These major normal faults dip steeply west, are typically 1 to $2 \mathrm{~km}$ apart, and have vertical offsets of more than $100 \mathrm{~m}$. Another, smaller scale group of normal faults strikes north to northwest, is closely spaced, has typically less than $3 \mathrm{~m}$ of offset, and forms an imbricate pattern (DOE, 1988b, Section 1.3). This group exists in the southern area of Yucca Mountain, but was not included in the models for the current study.

A substantial northwest-southeast-trending surface drainage, Drill Hole Wash, cuts across the general east trending surface drainage pattern at the northern boundary of the potential repository area, and may be controlled by a northwest-southeast trending structural zone with strike-slip motion (Wilson et al., 1994; Luckey et al., 1996; Scott et al., 1984). This northwest-southeast trending structural feature is paralleled by Pagany Wash Fault, Sever Wash Fault, and Yucca Wash, each located successively further north from Drill Hole Wash. These northwest-trending washes are associated with strike-slip faults with near vertical fault planes, less than $100 \mathrm{~m}$ of right-lateral offset, and brecciated zones about $20 \mathrm{~m}$ wide. In the saturated zone model, this northwest fault trend was conceptualized as a hydraulic barrier zone.

Structural dip in the large fault blocks in the Yucca Mountain area follows a pattern with strata dipping from $5^{\circ}$ to $30^{\circ}$ eastward. In the area of the potential repository, Tiva Canyon Tuff dips are $5^{\circ}$ to $8^{\circ}$ eastward and occurred in the Yucca Mountain fault block as it rotated after faulting. Both the unsaturated and saturated zone models for the current study used a dip of $4.6^{\circ}$ eastward for the modeling units. There is also a structural dip of $1^{\circ}$ to $2^{\circ}$ to the south at Yucca Mountain, which is captured in the three-dimensional saturated zone model as $<1^{\circ}$ of dip.

Representation of Faults in Models. Fault (or fracture) zones in the site geology were represented on the modeling grid by cells with permeability values different from cells to either side; these areas may also represent areas of physical offset of modeling units. Note, however, that it is misleading to label these areas as faults, because the model grids in the current study were composed of stratigraphic and structural composites and were not constructed as true geologic cross sections or plan view maps. The areas where major faults occur were considered permeability barriers in the saturated zone and both permeable zones and permeability barriers in the unsaturated zone.

In addition, it is misleading to label these areas as faults in the 1997 PA, because all permeability changes, such as hydrologic barriers in the saturated zone, cannot be explained as the result of a discrete fault plane. The large faults are actually zones of faulting with smaller faults that parallel them, forming splays as the fault break adjusted to differing stress or lithologic conditions. Permeability changes in the subsurface can occur as a result of lithologic change or mineralogic change within the same lithology. Bodvarsson et al., eds. (1997) specifically notes the effects of zeolites in causing lateral diversion of flow in the CHn unit, citing the work of Carey et al. (1997). Current subsurface mapping is insufficient to determine the complex interaction of all geologic features in the development of flow barriers, or permeable zones. Few models, including the $1997 \mathrm{PA}$, have attempted to model significant, large-scale permeability barriers using methods other than the effect of fault zones. However, as a practical matter, the designation "fault" or the more general term "fracture" has been used in several places in the discussion of permeability barriers and permeable zones to help the reader readily connect them to a real geologic feature that the conceptual model associated with an observed effect.

For the 1997 PA, the influence of fault zones on the conceptual models and the resulting effect on elements of the modeling grid are captured in Table 4-2. Fault zones were treated somewhat differently in the unsaturated and saturated zone models, beginning with differences as the result of the unsaturated zone model being $2 \mathrm{D}$ and the saturated zone model being $3 \mathrm{D}$. The area covered by the $2 \mathrm{D}$ cross-section crossed only two major fault zones: the Solitario 
Table 4-2. Treatment of Fractures in the 1997 PA Conceptual Models

\begin{tabular}{lll}
\hline & \multicolumn{1}{c}{ Unsaturated Zone Model } & \multicolumn{1}{c}{ Saturated Zone Model } \\
\hline Fault Zone Effects Modeled & Solitario Canyon; Ghost Dance & $\begin{array}{l}\text { Solitario Canyon; Bow Ridge; } \\
\text { Northwest-Southeast trending } \\
\text { fault group }\end{array}$ \\
$\begin{array}{l}\text { Conceptual Model of Effect of } \\
\text { Fault Zone on Flow }\end{array}$ & $\begin{array}{l}\text { Both permeable; lateral perme- } \\
\text { ability increase at discrete point } \\
\text { (single grid cell) }\end{array}$ & $\begin{array}{l}\text { All impermeable; lateral perme- } \\
\text { ability decrease at discrete point } \\
\text { (single grid cell) }\end{array}$ \\
$\begin{array}{l}\text { Dip of Fault Zone Plane } \\
\text { Modeling Unit Offsets }\end{array}$ & $\begin{array}{l}\text { Vodeling units offset across fault } \\
\text { Vertical }\end{array}$ \\
Eone & $\begin{array}{l}\text { Modeling units not offset across } \\
\text { fault zone }\end{array}$ \\
Effect on infiltration or flux & None & \\
\hline
\end{tabular}

Canyon on the west and the Ghost Dance on the east, and both were included in the model (see Figure 4-5). In the unsaturated zone 2D model, the Ghost Dance fault zone was treated as a permeable zone based on the work of LeCain (1997) and Exploratory Studies Facility (ESF) studies (Bodvarsson et al., eds., 1997), which have shown fracture zones to be permeable to air in the unsaturated zone. The Solitario Canyon Fault zone was treated as a less permeable zone in relation to the modeling grid cells to either side of it.

In the saturated zone, the focus was on representing as closely as possible the observed potentiometric gradient as constrained by hydraulic head measurements taken in wellbores (see Chapter 9 for more information). Fault zones were invoked as the features that created the barriers that caused the observed abrupt head changes in the potentiometric gradient and were used to locate grid cells that occurred at points of abrupt hydraulic conductivity (and therefore permeability) changes. The area covered by the 3D saturated zone model encompassed several large fault zones (Figure 4-1): the north-south trending normal faults of Solitario Canyon, Abandoned Wash-Ghost Dance, Bow Ridge, and Paintbrush, and the northwest-southeast trending strike-slip faults associated with Drill Hole Wash, Pagany Wash, Sever Wash, and Yucca Wash. Only the Solitario Canyon, a portion of the Bow Ridge, and a zone representing the effects of the northwest-southeast trending group of faults were modeled as permeability barriers. The rest of the fault zones do not appear explicitly as an area of permeability change in the model grid and are treated as permeable zones.

Stratigraphy and Rock Properties. Yucca Mountain is a highland about 6 to $10 \mathrm{~km}$ wide and about $40 \mathrm{~km}$ long with the crest altitude ranging from 1500 to $1930 \mathrm{~m}$, which places the crest about $650 \mathrm{~m}$ above the surrounding lowlands (Parsons et al., 1991). The mountain's present geomorphology evolved from faulting and erosion of a thick (1000 to $3000 \mathrm{~m}$ ) deposit of silicic volcanic Miocene rocks that now dip $5^{\circ}$ to $10^{\circ}$ to the east at the site of the potential repository. The volcanic sequence consists of a series of welded and nonwelded ash-flow and ash-fall tuffs and lavas, and volcanic breccia interbedded with thin sedimentary tuffaceous units deposited during the time between volcanic eruptions. The Cenozoic era Tertiary volcanics unconformably overly much older Paleozoic and Precambrian era clastics and carbonates. Figure 4-8 shows the stratigraphic units in the vicinity of Yucca Mountain. For more detailed depositional and lithologic descriptions, see the studies cited in Section 4.2.1, particularly the SCP (DOE, 1988b).

The portion of the geologic section at Yucca Mountain modeled in the 1997 PA is comprised of volcanic pyroclastic tuffs and the tuffaceous sedimentary units deposited during breaks in volcanic deposition. To provide a sense of relationship between actual stratigraphy and modeling units, Figure 4-9 compares three stratigraphic geologic columns of formal and informal geologic stratigraphy (Ortiz et al., 1985; Sawyer et al., 1994; and ISM 2.0 [Buesch et al., 1996 and Moyer and Geslin, 1995]) and three modeling unit columns (Ortiz et al., 1985; Rechard, ed., 1995; and the 1997 PA). The beds that are intersected by the potential repository configuration modeled in the 1997 PA are marked on the column to the far right. The potential repository lies in the TSmn layer. 


\begin{tabular}{|c|c|c|}
\hline Geologic Period & \multicolumn{2}{|c|}{ Statigraphic Unit } \\
\hline Quaternary & \multicolumn{2}{|c|}{ Alluvium } \\
\hline \multirow{11}{*}{ Tertiary } & $\begin{array}{c}\text { Timber Mountain } \\
\text { Group }\end{array}$ & Ranier Mesa Tuff \\
\hline & \multirow{4}{*}{$\begin{array}{l}\text { Paintbrush } \\
\text { Group }\end{array}$} & Tiva Canyon Tuff \\
\hline & & $\begin{array}{l}\text { Yucca Mountain } \\
\text { Tuff }\end{array}$ \\
\hline & & Pah Canyon Tuf \\
\hline & & $\begin{array}{c}\text { Topapah Spring } \\
\text { Tuff }\end{array}$ \\
\hline & \multicolumn{2}{|c|}{ Calico Hills Formation } \\
\hline & \multirow{3}{*}{$\begin{array}{c}\text { Crater Flat } \\
\text { Group }\end{array}$} & Prow Pass Tuff \\
\hline & & Bullfrog Tuff \\
\hline & & Tram Tuff \\
\hline & \multicolumn{2}{|c|}{ Lithic Ridge Tuff } \\
\hline & \multicolumn{2}{|c|}{ Older tuffs, lavas, and breccias } \\
\hline $\begin{array}{l}\text { Early Permian and } \\
\text { Pennsyivanian }\end{array}$ & \multicolumn{2}{|c|}{ Tippipah Limestone } \\
\hline $\begin{array}{l}\text { Mississippian and } \\
\text { Late Devonian }\end{array}$ & \multicolumn{2}{|c|}{ Eleana Formation } \\
\hline $\begin{array}{l}\text { Devonian to } \\
\text { Cambrian }\end{array}$ & \multicolumn{2}{|c|}{$\begin{array}{l}\text { Undifferentiated, primarily } \\
\text { carbonate rocks }\end{array}$} \\
\hline Cambrian & \multicolumn{2}{|c|}{$\begin{array}{l}\text { Undifferentiated, primarily } \\
\text { clastic rocks }\end{array}$} \\
\hline
\end{tabular}

TRI-6342-5286-0

Figure 4-8. Selected stratigraphic units in the vicinity of Yucca Mountain that are important to the hydrology (from Luckey et al., 1996; Tertiary nomenclature modified from Sawyer et al., 1994). 
When classifying modeling units, many YMP studies (Ortiz et al., 1985; Altman et al., 1996; Wilson et al., 1994; Bodvarsson et al., eds., 1997; and others) use terms related to welding, vitrification, zeolitization, and the presence and abundance of lithophysae as a means of distinguishing units. Development of these tuff properties is related to the mineralogy of the deposits, their mode of deposition, syndepositional cooling effects, and postdepositional diagenesis, and these subjects are discussed in this section. Note that stratigraphic columns often lack measured thicknesses of units because units vary in thickness over the study area. Also, as shown in the first two columns of Figure 4-9, formal stratigraphic terminology changed since Ortiz et al. (1985), with units that were previously defined as members now designated as formations, along with other minor differences.

All units in the far right column (Figure 4-9b) were modeled in the 1997 PA except the beds above the Tiva Canyon Formation. The Paleozoic carbonates under the thick tuff section (see Figure 4-8) were not modeled. For unsaturated zone modeling, the bulk of the stratigraphic section of interest at Yucca Mountain was deposited by volcanic pyroclastic activity. Caprock units of the Tiva Canyon Tuff and Topapah Spring Tuff Formations of the Paintbrush Group were deposited near the tops of the ash flows and are low porosity units. They have a different mineral composition from the rest of unit as a whole and are thought to represent deposits from a deeper magma of a different composition than that of the initial deposits. The caprock units are more mafic, being quartz latite composition rather than rhyolite as is the bulk of the underlying tuffs. The hydrologic significance of this unit is that the caprock may act as a barrier to infiltration of meteoric water except where it is breached by joints or faults (Rautman and Flint, 1992). The 1997 PA did not model the effects of outcropping caprock units. Figure 4-5 shows the outcrop of the Paintbrush Group, the overlying Timber Mountain Group, and Pre-Paintbrush tuffs in the repository area.

Pyroclastic ash-flow deposits are volcanic hot, gaseous, particulate density currents. Though individual ash-flow and ash-fall tuffs can be quite thick (100 to $300 \mathrm{~m}$ ), in general, eruptive events associated with the Timber Mountain Caldera complex north of Yucca Mountain spread each tuff layer out over distances of kilometers producing a high ratio of horizontal to vertical dimension in the resulting rock units. In the hiatus between large-volume ash-flows, thinner beds composed of tuffaceous and reworked material were emplaced a few meters to tens of meters thick (Rautman, 1995). Syndepositional welding, fracturing, hydrothermal alteration, and gas bubble formation and postdepositional faulting, jointing, and diagenesis produced additional heterogeneities of relevance to modeling unit development.

Welding in Tiva Canyon and Topopah Spring Tuffs. Both the Tiva Canyon Tuff and Topopah Spring Tuff Formations exhibit vertical layering related to the cooling history of a rapidly deposited ash-flow tuff. A simple cooling unit develops when pyroclastic deposits occur in such rapid succession that each new layer is emplaced before the previous one has cooled, so the entire sequence tends to cool as a single unit. Figure 4-10 shows idealized welding zones in a simple cooling unit that develops a more or less systematic pattern of zones with a differing degree of welding; in these zones, greater density and lesser porosity are associated with greater welding. Because the different areas of the flow cool and therefore, weld deferentially, the basal beds are more porous and less densely welded in many ash-flow tuffs. Basal beds cool relatively quickly, losing heat to the cool ground surface upon which they are deposited. Subsequent portions of the ash deposit are insulated from the cooler ground surface, cool more slowly, have the weight of the succeeding ash layers upon them, and show more plastic deformation and welding of glass shards. This effect diminishes from the interior of the deposit both upward to the top of the deposit and downward to the base, causing the top layer of the depositional unit to resemble the bottom in that it has a relatively higher porosity and less (or no) welding compared to the mid-section. After deposition and cooling, welded rocks are more likely to fracture and to sustain a discrete break than are nonwelded rocks; by fracturing, low porosity welded sections can become more permeable to groundwater flow. Rautman (1995) noted that welded and nonwelded rocks may exhibit significantly different in-situ water saturations, which can impact thermal conductivity. Another feature of intensely welded zones is the development of vitrophyres.

The Topopah Spring Tuff in the potential repository area exhibits vertical changes in texture and mineralogy that appear to be the result of deposition from three eruptive events (three simple cooling unit sequences). As described in the SCP (DOE, 1988b, p. 1-62), the first eruptive event deposited the basal vitrophyre (TSlv) through the lower lithophysal (TS1l) subunits; the second event deposited the middle nonlithophysal (TSmn) and upper lithophysal (TSul); and the third eruptive event deposited a nonlithophysal zone with a thin vitrophyre cap (TSun and TSv). Note that rock material deposited at the base of an eruptive event generally has few lithophysae, and the lithophysae tend to be more numerous toward the upper part of a cooling unit, so that associating cooling unit zonation with rock texture 
Formal

Stratigraphy

Ortiz et al. (1985)

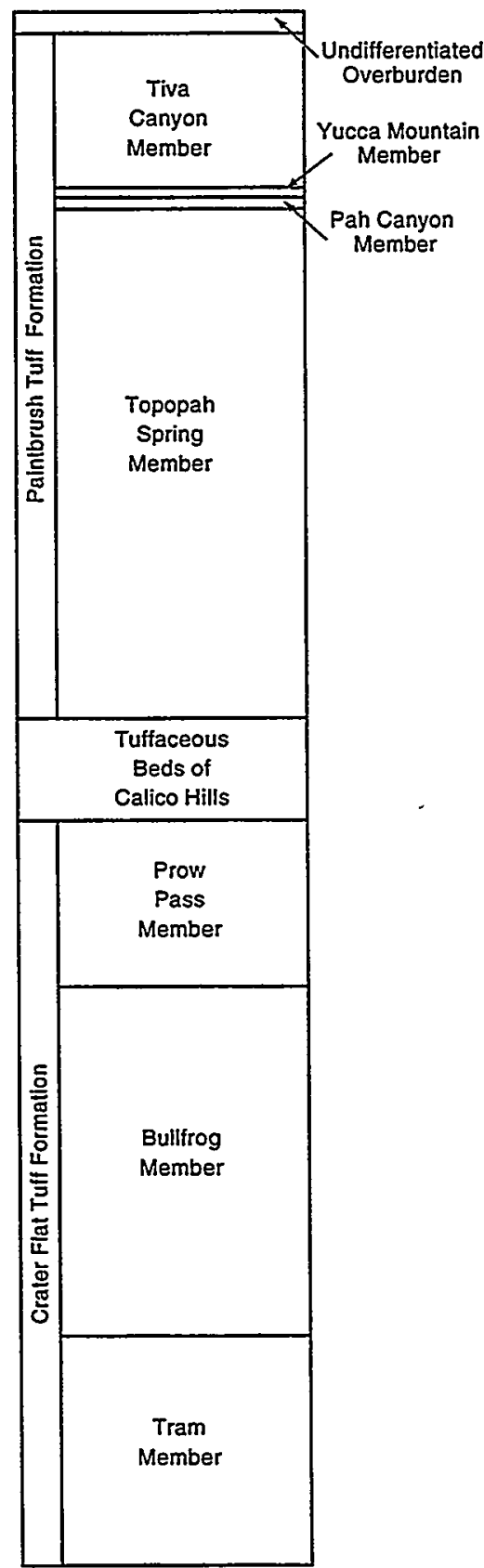

Formal

Stratigraphy

Sawyer et al. (1994)

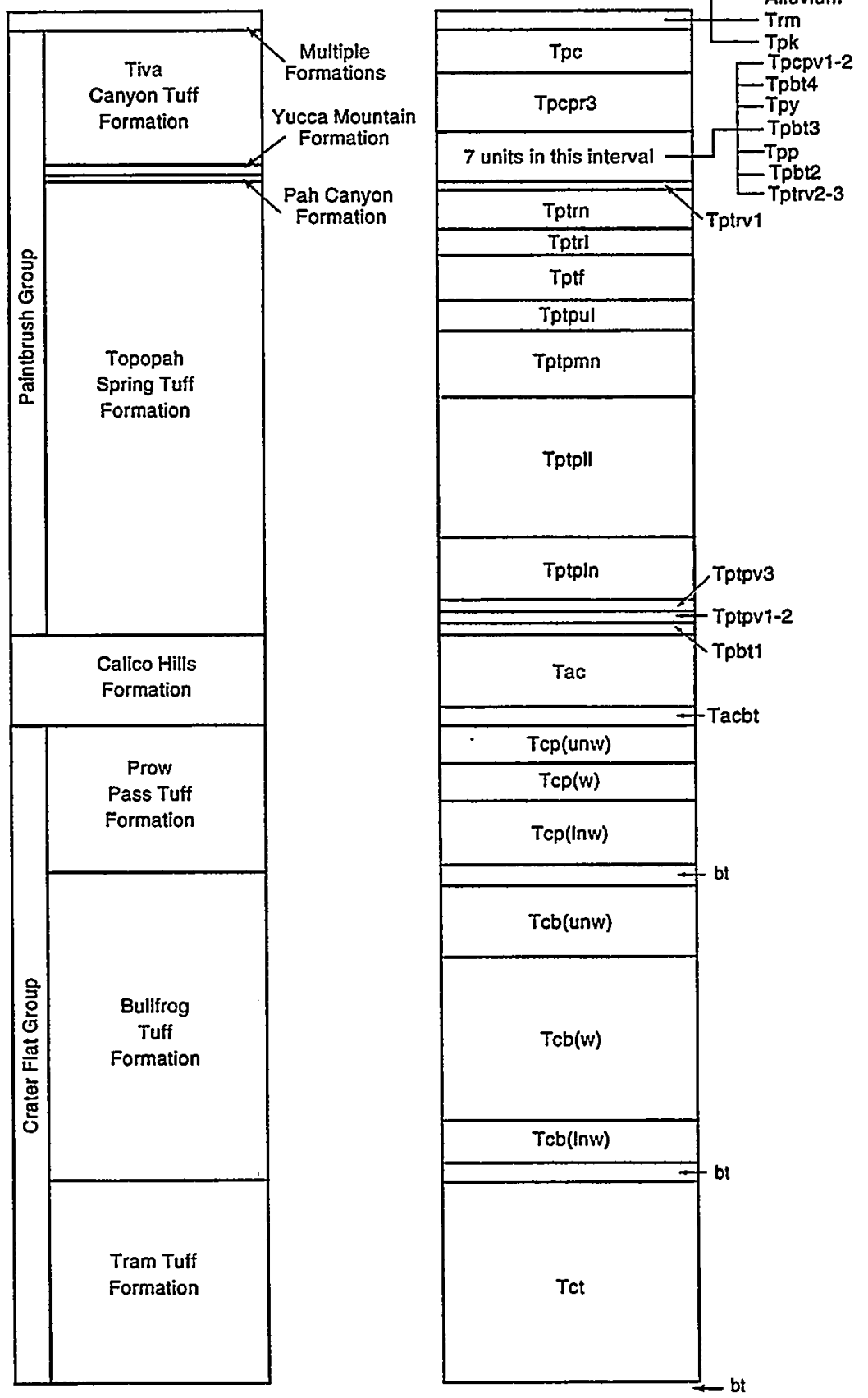

Formal and informal

Stratigraphy

ISM 2.0*

Buesch et al. (1996)

Moyer \& Geslin (1995)

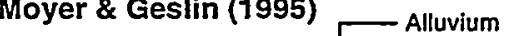

-Abbreviations for the ISM 20 column follow the symbol hierarchy: $T=T$ ertiary Period rocks: with stratigraphic group designated by the second letter ( $p=P a i n t b r u s h$. $c=$ rever letter; single or double letters in the fifth and sixth symbol position representing the zones of welding and crystallization; and intervals denoted by the numerical letter; single or double letters in the fith and sioth in the seventh or eighth position.

TRI-6342-5282-0(A)

(a)

Figure 4-9. Comparison of (a) formalinformal stratigraphic layering with (b) modeling unit layering for Yucca Mountain from various studies. The modeling units for the 1997 PA are shown in the column at the far right. Abbreviations for these units are defined in "Development of Thermal/Hydrological Modeling Units." 
Reference

Stratigraphy

(Modeling Units)

Ortiz et al. (1985)

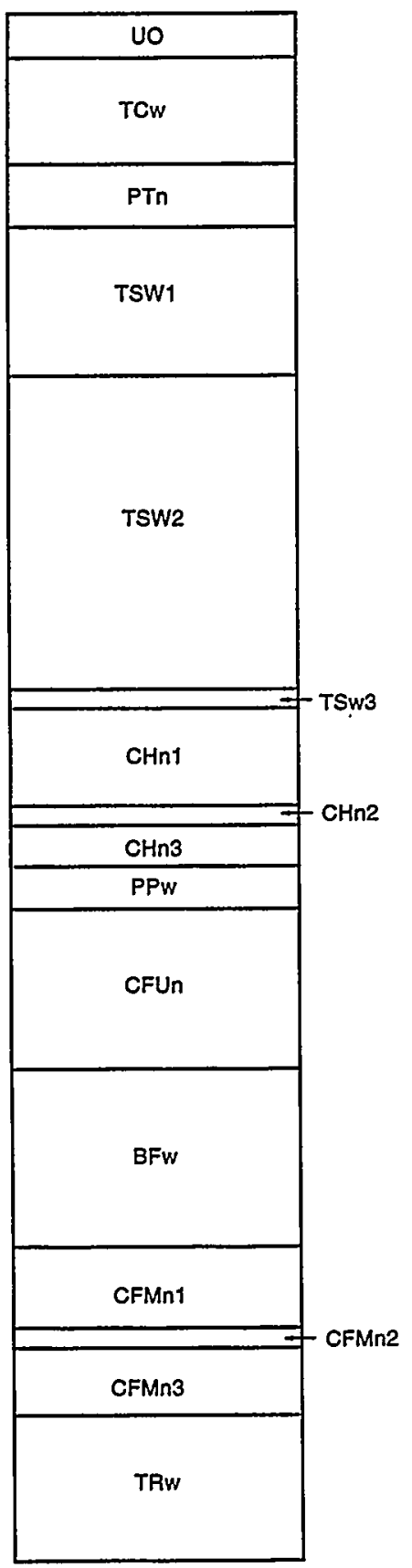

Modeling Units

1994 DSNF/DHLW PA

Rechard (1995)

\begin{tabular}{|c|}
\hline Alluvium \\
\hline TCw \\
\hline PTn \\
\hline TSW1 \\
\hline TSW2 \\
\hline TRw \\
\hline BFw \\
\hline CHnv \\
\hline
\end{tabular}

Modeling Units 1997 DSNFIDHLW PA Unsaturated Zone

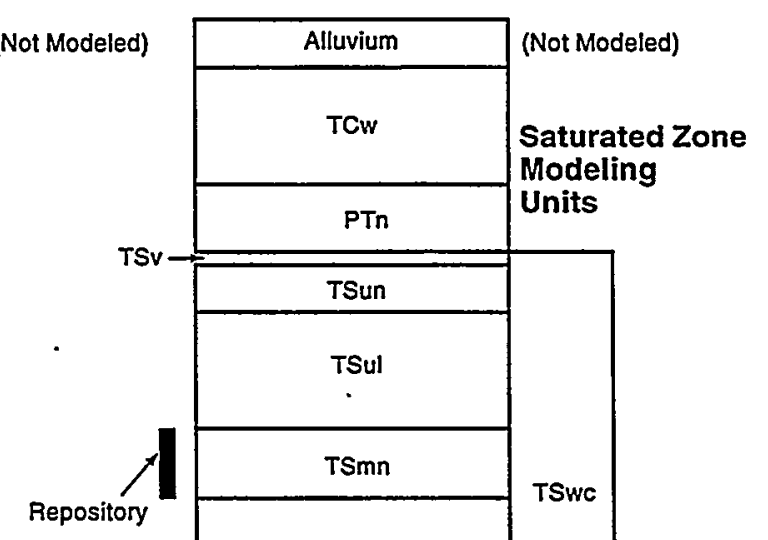

TSII

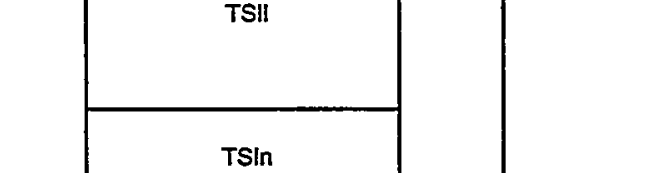

TSiv

\begin{tabular}{|c|c|}
\hline CHnv & CHSlv \\
\hline CHnz & CHnz \\
\hline & \\
\hline
\end{tabular}

(b)

Figure 4-9. Comparison of (a) formalinformal stratigraphic layering with (b) modeling unit layering for Yucca Mountain from various studies. The modeling units for the 1997 PA are shown in the column at the far right. Abbreviations for these units are defined in "Development of Thermal/Hydrological Modeling Units." (continued) 
4. System Characterization: Geologic Barrier

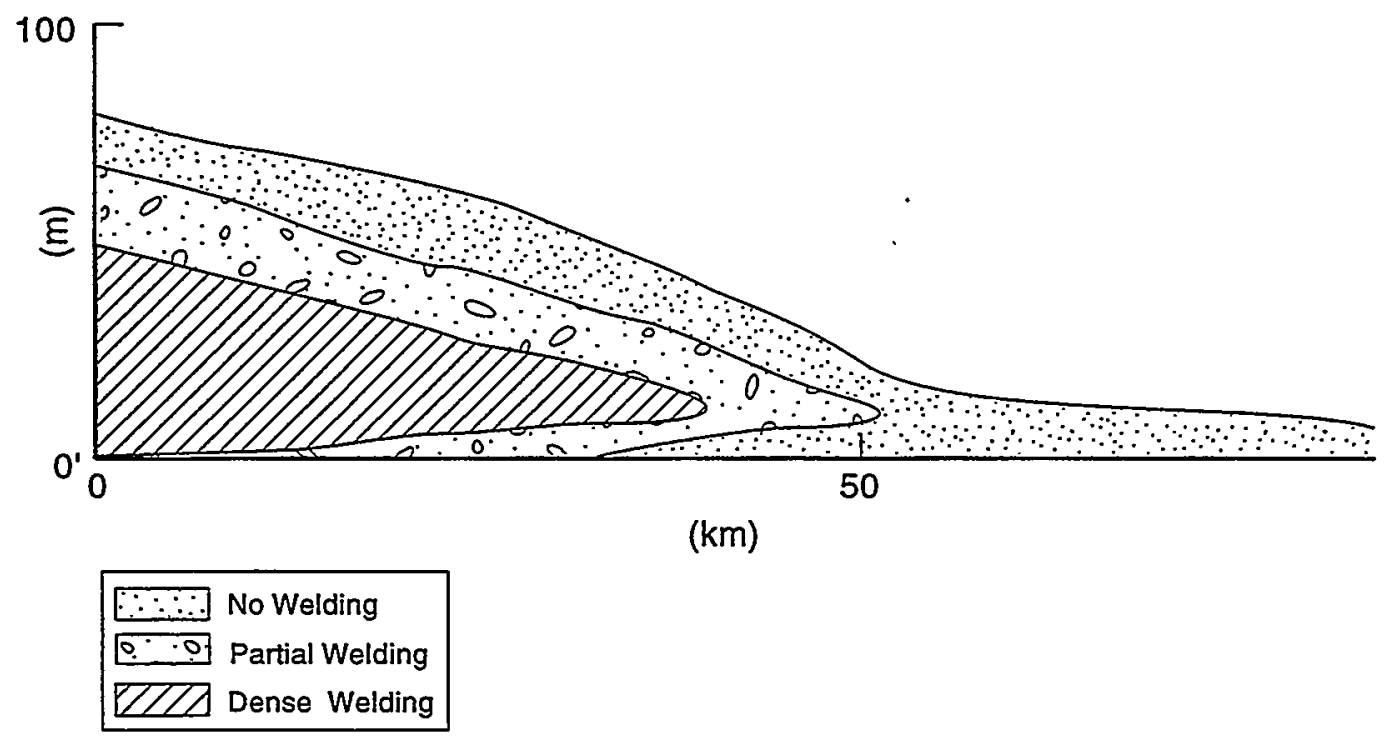

TRI-6342-5284-0

Figure 4-10. Idealized lateral and vertical configuration of welding zones in a simple cooling unit (after Fisher and Schmincke, 1984). 
explains the distribution of lithophysae in the section. The geochemistry associated with cooling unit zonation is discussed in Section 4.3.3.

Vitrification and Zeolitization. Pyroclastic ash-flow deposits are composed of crystalline minerals, glass that occurs as shards and as foamy pumice, and lithic fragments picked up from various sources (Fisher and Schmincke, 1984). Ash-flow tuff, by definition, is more than $50 \%$ ash-sized $(<2 \mathrm{~mm})$ particles, of which glass shards are the majority. Vitrophyres form when glass (which is non-crystalline by definition) is preserved in a metastable form as a result of extreme welding of hot, plastic glass shards. Vitrophyres have relatively low porosity and high particle density. Devitrification is the altering of thermodynamically unstable glass by nucleation and growth of mainly quartz crystallites and both sodium-rich and potassium-rich alkali feldspar (Cas and Wright, 1987). Volcanic glass, such as vitrophyre, provides the source material from which zeolites are formed by diagenesis, so vitric layers become zeolitized over long exposure to groundwater in the saturated zone. Zeolitization alters a metastable glass that lacks crystalline structure to a crystalline, aluminosilicate framework mineral with completely different properties than those of the glass. Compared to vitrophyres, zeolites are chemically more stable, more porous, with lighter bulk density, and they act as molecular sieves on liquids passing through them.

Lithophysae. Textural features of ash-flow tuffs include spherulites, which are radiating aggregates of alkali feldspar (with or without crystobalite and tridymite) that develop during devitrification and can produce a granular-looking texture. Large spherulites with large internal cavities, or vugs, are called lithophysae (Cas and Wright, 1987). The presence of lithophysae in the repository host rock has an important impact on heat conductance because it slows down the dissipation of heat by reducing bulk density and thermal conductivity, adding the insulating effects of airfilled vesicles. The 1997 PA used a finer division of modeling units representing the Topopah Spring Tuff section than was used by the $1994 \mathrm{PA}$ in order to capture a finer resolution of vertical heterogeneity with regard to the relative abundance of lithophysae (see Figure 4-9). This refinement of units was based on Rautman (1995) with emphasis on the use of data from wellbore UZ-16. Delineation of modeling units based on the presence or absence of lithophysae has pitfalls in that use of the term "lithophysal" as a descriptor by various investigators in the YMP can have significantly different meanings. The differences in definition include variations in the quantity of lithophysae that must be present to call the zone lithophysal as well as variations in the size of vesicles that are called lithophysae. Lack of consistency in geologic descriptive protocols has presented problems, rendering some data misleading.

Bedded Tuffs. The term "bedded tuff" is used in the YMP for sedimentary units formed during a hiatus in pyroclastic activity by wind or water erosion of the surface of the most recent major ash-flow tuff. They are relatively thin beds compared to the massive tuffs, but can be thick in some areas. These units are defined by Buesch et al. (1996) as "...tuffaceous beds interstratified with major ignimbrites, but not identified with any established formation," and are named with reference to the overlying tuff deposit, as in Tpbt4, which is the Pre-Tiva Canyon bedded tuff (Figure 4-9a, third column). Ortiz et al. (1985) also considered such units as the basal beds of the overlying tuff, as shown by their definition of the $\mathrm{CHn} 2$ unit (see Figure 4-9b, first column). Because these are informal units, they are not shown in the formal stratigraphy columns in Figure 4-9a (first and second columns), although they are shown in the ISM 2.0 formal and informal stratigraphy column (Figure 4-9a, third column)-the units ending in the initials "bt" mean bedded tuff. In the 1997 PA, bedded tuffs are grouped with the corresponding unit above (e.g., CHnz in Figure 4-9b, third column).

Many features of tuffs described in this section developed in response to physical processes that changed gradually as opposed to abruptly, such as the thermal zonation in a thick simple cooling unit. For this reason, gradational changes in rock property values from one layer to the next is the rule rather than the exception for Yucca Mountain tuffs. Gradational changes occur in porosity, grain density, and other rock properties in the Tiva Canyon Tuff Formation and Topapah Spring Tuff Formation (host rock for the potential repository) as a result of the depositional nature of the units, their cooling history, and subsequent diagenesis. The 1997 PA modeling units are laterally homogeneous, and, because the focus of this PA is on the potential repository "footprint" area in the unsaturated zone, this method of defining the units adequately represents textural changes related to cooling unit history that impact thermal, flow, and transport processes.

Development of Thermal/Hydrologic Modeling Units. In developing its modeling units, the 1997 PA focused on containment, flow, and transport processes as well as thermal and mechanical processes, and employed data on the rock properties that were believed to have the greatest effects on these processes. 
The process of flow is an example of how primary and secondary rock properties can be selectively grouped and modeled to represent the range of possibilities for a process. The flow of water, on the ground surface or in the subsurface, follows the path of least resistance, meaning the path of relatively highest permeability, lowest pressure, least chemical retardation, mechanical resistance, and so on. In the subsurface; any given path of least resistance exists due to different combinations of many rock properties, e.g., permeability, which in turn, is affected by a combination of other properties such as porosity, tortuosity, capillary pressure, and fracturing. Therefore, analysts seeking to define the process of flow through Yucca Mountain and the repository chose to define the mountain in their models as a series of layers (modeling units) with different rock properties (porosity, etc.) that are relevant to the process of flow. The parameter list used by a particular code contains the properties that affect the process (Section 4.5). The same logic applies in development of modeling units for models that capture response to heat, geochemical retardation, or combinations of several properties.

Both the 1994 and 1997 PAs built modeling units based on a combination of lithologic, thermal, mechanical, and hydrologic properties. The correlation between formal stratigraphic units and modeling units for Ortiz et al. (1985), the $1994 \mathrm{PA}$, and the $1997 \mathrm{PA}$ is shown in Figure 4-9b. Ortiz et al. (1985) was the earliest widely cited study that developed modeling units. Called "reference stratigraphy," unit characteristics were based on a combination of rock properties that controlled a process of interest, such as groundwater flow, as opposed to the standard approach of distinguishing geologic layering based on formal stratigraphic classification criteria. Ortiz et al. (1985) cited Lappin et al. (1982, p. 20-24) as originating the protocol of using process-controlling groups of rock properties to define stratigraphy for core descriptions during lithologic logging of wells. Subsequent YMP studies have used a similar approach for modeling units with some studies building directly on the reference stratigraphy of Ortiz et al. (1985). Buesch et al. (1996) in their description of formal and informal lithologic units of the Paintbrush Group also showed the correlation of these units to Ortiz et al. (1985) thermal/mechanical units. The 1994 PA adopted in large part the Ortiz et al. (1985) units with some modifications. The 1997 PA built on the 1994 PA units with further refinements in the Topopah Spring section, based on more recent data. Therefore, as a starting point, it is useful to describe the definitions and assumptions by which Ortiz et al. (1985) developed their reference stratigraphy (referred to as modeling units in the 1997 PA). In addition, subsequent assumptions by which these initial units were modified from the 1994 PA to those used in the 1997 PA are discussed.

Though actual site data were used by the 1997 PA and other YMP researchers as input for modeling units, the unit definitions and contacts were based on qualitative assessments of changes in processes in relation to changes in rock properties. Therefore, units are not defined by a distinctive range of values for each rock property of interest, though it is likely, for instance, that porosity and permeability values are higher in nonwelded units versus welded units, because during welding pore spaces are closed up. Ortiz et al. (1985) began with a stratigraphy based on porosity and grain density using work by Nimick et al. (1984). They associated the rock properties of vitrified (glassy, noncrystalline structure) versus devitrified (crystalline mineral structure) and welded versus nonwelded with the rock's grain density (which affects thermal processes) and porosity (which affects thermal, flow, and transport processes). Ortiz et al. (1985) made the following qualitative assumptions, based on observations of data, which were then adopted by the 1994 and 1997 PAs:

- Devitrified tuff: High density, low porosity

- Vitric, welded tuff: Low density, low porosity

- Vitric, nonwelded tuff: Low density, high porosity

- Zeolitized tuff: Low density (higher than vitric), high porosity

- Nonwelded: Extensively zeolitized, except upper Paintbrush nonwelded.

The reference stratigraphy definitions of Ortiz et al. (1985) are provided in Table 4-3 and were also used as thermal/hydrologic modeling unit definitions by the 1994 PA (with the exception of units TSv through TSln). Ortiz et al. (1985) divided the bulk of the Topopah Spring into two zones, the upper lithophysae-rich (more than approximately $10 \%$ by volume) TSw1 unit and the lower lithophysae-poor (less than approximately $10 \%$ by volume) TSw 2 unit. For modeling of the unsaturated zone in the 1997 PA, these two units were divided into the TSv, TSun, TSul, TSmn, TSIl, and TSIn to further refine the layering of lithophysal zones, which were inferred to have effects on thermal responses of the rock units. The source of these designations is a combination of ISM 2.0 units (Clayton et al., 1997) and Rautman (1995). Note that for the saturated zone model for the 1997 PA, the unit TSwc is the equivalent of unsaturated zone units TSv, TSun, TSul, TSmn, TSll and TSIn. 
Table 4-3. 1997 PA Thermal/Hydrologic Modeling Units modified from Ortiz et al. (1985) ${ }^{\mathrm{a}}$

\begin{tabular}{|c|c|c|}
\hline $\begin{array}{l}\text { Thermal/Hydrologic } \\
\text { Modeling Unit Name }\end{array}$ & Abbreviation ${ }^{b}$ & Definition \\
\hline $\begin{array}{l}\text { Alluvium (Ortiz et al. } \\
\text { Undifferentiated Over- } \\
\text { burden) }\end{array}$ & Alluvium & $\begin{array}{l}\text { Alluvium; colluvium; nonwelded, vitric ashflow tuff of the Tiva Canyon } \\
\text { Tuff Formation of the Paintbrush Group; any other tuff units that strati- } \\
\text { graphically overlie the welded, devitrified Tiva Canyon Tuff Formation; } \\
\text { not modeled by } 1994 \text { or } 1997 \text { PAs }\end{array}$ \\
\hline $\begin{array}{l}\text { Tiva Canyon, welded } \\
\text { unit }\end{array}$ & TCW & $\begin{array}{l}\text { Moderately to densely welded, devitrified ash-flow tuff of the Tiva Can- } \\
\text { yon Tuff Formation of the Paintbrush Group. }\end{array}$ \\
\hline $\begin{array}{l}\text { Upper Paintbrush, non- } \\
\text { welded unit }\end{array}$ & PTn & $\begin{array}{l}\text { Partially welded to nonwelded, vitric and occasionally devitrified tuffis of } \\
\text { the lower Tiva Canyon Tuff, Yucca Mountain Tuff, Pah Canyon Tuff, and } \\
\text { upper Topopah Spring Tuff Formations of the Paintbrush Group. In } 1997 \\
\text { PA, sedimentary, bedded, tuffaceous units deposited prior to deposition } \\
\text { of each of these formations were also included in the PTn; Yucca Moun- } \\
\text { tain and Pah Canyon are locally welded north of the repository area }\end{array}$ \\
\hline $\begin{array}{l}\text { Topopah Spring, } \\
\text { vitrophyre unit }\end{array}$ & TSv & $\begin{array}{l}\text { Upper, densely welded vitric portion of the Topopah Spring Tuff Forma- } \\
\text { tion of the Paintbrush Group; formerly basal section of PTn in } 1994 \text { PA, } \\
\text { correlative to ISM 2.0 Tptrv1 }\end{array}$ \\
\hline $\begin{array}{l}\text { Topopah Spring, upper, } \\
\text { nonlithophysal unit }\end{array}$ & TSun ${ }^{c}$ & $\begin{array}{l}\text { Upper, nonlithophysal portion of the Topopah Spring Tuff Formation of } \\
\text { the Paintbrush Group; correlative to ISM 2.0 Tptrn }\end{array}$ \\
\hline $\begin{array}{l}\text { Topopah Spring, upper } \\
\text { lithophysal unit }\end{array}$ & TSull $^{e}$ & $\begin{array}{l}\text { Upper, lithophysal portion of the Topopah Spring Tuff Formation of the } \\
\text { Paintbrush Group; correlative to ISM 2.0 Tptrl, Tptf, Tptpul }\end{array}$ \\
\hline $\begin{array}{l}\text { Topopah Spring, middle } \\
\text { nonlithophysal unit }\end{array}$ & $T S m n^{c}$ & $\begin{array}{l}\text { Middle, nonlithophysal portion of the Topopah Spring Tuff Formation of } \\
\text { the Paintbrush Group; correlative to ISM 2.0 Tptpmn }\end{array}$ \\
\hline $\begin{array}{l}\text { Topopah Spring, lower } \\
\text { lithophysal unit }\end{array}$ & $T S \|^{\mathrm{C}}$ & $\begin{array}{l}\text { Lower, lithophysal portion of the Topopah Spring Tuff Formation of the } \\
\text { Paintbrush Group; correlative to ISM 2.0 Tptpll }\end{array}$ \\
\hline $\begin{array}{l}\text { Topopah Spring, lower } \\
\text { nonlithophysal }\end{array}$ & $\operatorname{TS} \ln ^{c}$ & $\begin{array}{l}\text { Lower, nonlithophysal portion of the Topopah Spring Tuff Formation of } \\
\text { the Paintbrush Group; correlative to ISM 2.0 Tptpln }\end{array}$ \\
\hline $\begin{array}{l}\text { Topopah Spring, } \\
\text { welded unit, vitrophyre }\end{array}$ & TSIV ${ }^{c}$ & $\begin{array}{l}\text { Vitrophyre near the base of the Topopah Spring Tuff Formation of the } \\
\text { Paintbrush Group; potentially zeolitized. In Ortiz et al., this unit is } \\
\text { related to TSw3 unit; in } 1994 \text { PA, this unit is related to TSwv unit }\end{array}$ \\
\hline $\begin{array}{l}\text { Calico Hills and Lower } \\
\text { Paintbrush, nonwelded } \\
\text { unit }\end{array}$ & $\mathrm{CHnv}$ & $\begin{array}{l}\text { Nonwelded ashflows, bedded and reworked tuffs of the lower Topopah } \\
\text { Spring Tuff Formation of the Paintbrush Group and the upper part of the } \\
\text { Calico Hills Formation; potentially zeolitized. In Ortiz et al. this unit is } \\
\text { named CHn1. In the } 1997 \text { PA, zeolitized, in general, also means vitric, } \\
\text { and vitric was added to the definition. }\end{array}$ \\
\hline $\begin{array}{l}\text { Calico Hills and Lower } \\
\text { Paintbrush, non- } \\
\text { welded unit }\end{array}$ & $\mathrm{CHnz}$ & $\begin{array}{l}\text { Basal bedded and reworked zone of the Calico Hills Formation; poten- } \\
\text { tially zeolitized (CHn2 in Ortiz et al.); and upper partially welded ash- } \\
\text { flows of the Prow Pass Tuff Formation of the Crater Flat Group; } \\
\text { potentially zeolitized (CHn3 in Ortiz et al.) }\end{array}$ \\
\hline Prow Pass, welded unit & PPW & $\begin{array}{l}\text { Moderately welded, devitrified ashflows of the Prow Pass Tuff Formation } \\
\text { of the Crater Flat Group }\end{array}$ \\
\hline \multicolumn{3}{|c|}{$\begin{array}{l}\text { a Ortiz et al. (1985) designated zeolitic zones based on Vaniman et al. (1984). } \\
\text { b Abbreviations are constructed as follows: Capital letters are the two- or three-letter abbreviations of the most closely related for- } \\
\text { mal geologic stratigraphic unit; following is lower case, one-letter abbreviation of the general degree of welding with } w=\text { =welded to } \\
\text { moderately welded and } n=\text { nonwelded to partially welded; following are numbers that designate distinctive subunits, where appli- } \\
\text { cable. Note that for TS units, a one-letter abbreviation follows, with } v=\text { =vitric; } u=u p p e r ; m=\text { middle; l=lower; following that is the } \\
\text { second lower case, one-letter abbreviation with } n=\text { =nonlithophysal; } l=l \text { lithophysal. } \\
\text { c In the } 1994 \text { PA, these units were grouped as TSw1 and TSw2 (see Figure 4-9b). }\end{array}$} \\
\hline
\end{tabular}


Table 4-3. 1997 PA Thermal/Hydrologic Modeling Units modified from Ortiz et al. (1985) ${ }^{a}$ (Continued)

\begin{tabular}{|c|c|c|}
\hline $\begin{array}{l}\text { Thermal/Hydrologic } \\
\text { Modeling Unit Name }\end{array}$ & Abbreviation ${ }^{b}$ & Definition \\
\hline $\begin{array}{l}\text { Upper Crater Flat, } \\
\text { nonwelded unit }\end{array}$ & CFun & $\begin{array}{l}\text { Zeolitic, nonwelded to partially welded ashflows and bedded, reworked } \\
\text { portions of the lower Prow Pass Tuff Formation and the upper Bullfrog } \\
\text { Tuff Formation of the Crater Flat Group; potentially zeolitized }\end{array}$ \\
\hline Bullfrog, welded unit & BFW & $\begin{array}{l}\text { Moderately to densely welded, devitrified ashflows of the Bullfrog Tuff } \\
\text { Formation of the Crater Flat Group }\end{array}$ \\
\hline $\begin{array}{l}\text { Middle Crater Flat, } \\
\text { nonwelded unit }\end{array}$ & CFmn & $\begin{array}{l}\text { Zeolitic, partially welded to nonwelded ashflows and basal bedded, } \\
\text { reworked portions of the Bullfrog Tuff Formation of the Crater Flat Group } \\
\text { (CFMn1 and CFMn2 in Ortiz et al.); and zeolitic, partially welded ash- } \\
\text { flows of the upper portion of the Tram Tuff Formation of the Crater Flat } \\
\text { Group (CFMn3 in Ortiz et al.); in the } 1997 \text { PA, hydrologic flow and trans- } \\
\text { port properties are assumed to be identical to CFun }\end{array}$ \\
\hline Tram, wèlded unit & TRw & $\begin{array}{l}\text { Moderately welded, devitrified ashflows of the Tram Tuff Formation of the } \\
\text { Crater Flat Group }\end{array}$ \\
\hline \multicolumn{3}{|c|}{$\begin{array}{l}\text { Ortiz et al. (1985) designated zeolitic zones based on Vaniman et al. (1984). } \\
\text { Abbreviations are constructed as follows: Capital letters are the two- or three-letter abbreviations of the most closely related for- } \\
\text { mal geologic stratigraphic unit; following is lower case, one-letter abbreviation of the general degree of welding with w=welded to } \\
\text { moderately welded and } n=\text { nonwelded to partially welded; following are numbers that designate distinctive subunits, where appli- } \\
\text { cable. Note that for TS units, a one-letter abbreviation follows, with } v=\text { vitric; } u=\text { upper; } m=\text { middle; l=lower; following that is the } \\
\text { second lower case, one-letter abbreviation with n=nonlithophysal; /=lithophysal. } \\
\text { In the } 1994 \text { PA, these units were grouped as TSw1 and Tsw2 (see Figure 4-9b). }\end{array}$} \\
\hline
\end{tabular}

Grouping Units by Zeolitization. Ortiz et al. (1985) designated a horizontal surface representing "the upper limit of prevalent zeolites" that cut the reference stratigraphy at a point above the current water table, generally below TSw2 (see Figure 4-9b), but varying in elevation somewhat from borehole to borehole. They noted that, generally speaking, zeolites formed in units that were originally vitric and porous (non- to partially welded) and were exposed to groundwater for some length of time, with devitrified tuffs remaining largely unzeolitized. They listed TCw, TSw1, TSw2, PPw, BFw, and TRw as not susceptible to zeolitization, whereas units PTn, TSw3 (TSwv), CHn1, CHn2, CHn3, CFUn, CFMn1, CFMn2, and CFMn3, according to Ortiz et al., can be potentially zeolitized. PTn, according to Ortiz et al., has not been observed as extensively zeolitized at Yucca Mountain.

Grouping by Sorption Factors. The 1994 PA (Rechard, ed., 1995, Table 5-2) incorporated additional modifiers for modeling units using the rock properties: zeolitic (Z), vitric (V), and devitrified (D). The basis of this system was a combination of observations by Ortiz et al. (1985) and Wilson et al. (1994). In TSPA-93, sorption was observed to be dependent upon the rock type and thus the sorption coefficient, $K_{D}$ varied depending on the mineralogy of the substrate. TSPA-93 defined sorption only as deposition on the surface of a solid, as opposed to the more general definition that includes absorption. High $\mathrm{K}_{\mathrm{D}}$ values represent a greater capacity for retardation of transport of radionuclides than lower $\mathrm{K}_{\mathrm{D}}$ values. TSPA-93 used expert elicitation for the sorption coefficient distributions, $\mathrm{K}_{\mathrm{D}}$, for four materials per radionuclide of interest: devitrified tuff (D), vitric tuff (V), zeolitic tuff (Z), and iron oxide (Fe). Iron oxide was added to represent degraded container material, because actinides are sorbed strongly by iron oxides. Wilson et al. (1994) stated that the mineralogy of the different strata of the same rock group is very similar and sorption coefficients can be grouped in terms of these rock types, citing Thomas (1987) in support of this approach. For further details see Wilson et al. (1994, Chapter 9). Table 4-4 gives the source of the modifiers represented by the abbreviations $\mathrm{D}, \mathrm{V}$, and $\mathrm{Z}$ as used in the 1994 and 1997 PAs.

Considerations with Regard to Permeability Data. To establish rock properties such as permeability, zeolitization, or other properties impacting flow and transport, data for porosity, welding, fractures, and other rock properties must be assessed, which can be difficult because of a lack of uniformity of approach in collecting the data among studies. In the 1997 PA, this iteration was modeled by means of a combination of data sources for rock properties that impact permeability. 
Table 4-4. Designation of Rock Type Grouping for the 1997 PA Modeling Units for Purposes of Association with $K_{\mathbf{D}}$ Value Ranges from Wilson et al. (1994)

\begin{tabular}{|c|c|c|c|}
\hline & $\begin{array}{l}\text { Thermal/Hydrologic } \\
\text { Modeling Unit }\end{array}$ & $\begin{array}{l}\text { KD/Rock Type } \\
\text { Designation }\end{array}$ & Source \\
\hline \multicolumn{2}{|c|}{ TCW } & $D$ & Ortiz et al., 1985 \\
\hline \multicolumn{2}{|c|}{ PTn } & $\mathrm{v}$ & Ortiz et al., 1985 \\
\hline \multicolumn{2}{|c|}{ TSV } & $V^{b}$ & Ortiz et al., 1985 \\
\hline \multicolumn{2}{|r|}{ TSun, TSul, TSmn, TSIl, TSIn ${ }^{c}$} & $\mathrm{D}$ & Wilson et al., 1994 \\
\hline \multicolumn{2}{|c|}{ TSIv } & V & Wilson et al., 1994 \\
\hline \multicolumn{2}{|c|}{ CHnv } & V & Wilson et al., 1994 \\
\hline \multicolumn{2}{|c|}{$\mathrm{CHnz}$} & Z & Wilson et al., 1994 \\
\hline \multicolumn{2}{|c|}{ PPW } & D & Wilson et al., 1994 \\
\hline \multicolumn{2}{|c|}{ CFUn } & $Z$ & Ortiz et al., 1985 \\
\hline \multicolumn{2}{|c|}{ BFw } & $D$ & Ortiz et al., 1985 \\
\hline \multicolumn{2}{|c|}{ CFMn } & Z & Ortiz et al., 1985 \\
\hline \multicolumn{2}{|c|}{ TRw } & D & Ortiz et al., 1985 \\
\hline \multicolumn{4}{|c|}{$\begin{array}{l}\text { a Note the PTn was erroneously listed as D in Rechard, ed., 1995, Table 5-2. } \\
\text { b TSv was the vitric zone at the base of PTn in Ortiz et al. (1985), but is a separate zone } \\
\text { for the } 1997 \text { PA. } \\
\text { c These units were represented by TSw1 and TSw2 in the 1994 PA. }\end{array}$} \\
\hline
\end{tabular}

In the 1997 PA, porosity data were used to estimate thermal conductivity and bulk density. Figure 4-11 illustrates the method of estimating. Matrix porosity data (middle column) was constructed by combining data from several wellbores; these data are related to the 1997 PA modeling units (right column). In addition, the breakout of porosity data as calculated from a density $\log$ in wellbore UZ-16 is presented (left column). Combining wellbore data to form a composite section (middle column) was necessary because data from all strata could not be collected in one area. For example, at the approximate location of the composite column, near wellbore USW G-4, all TS subunits were not present; therefore values for the TS subunits were taken from UZ-16 data and placed in the column at the USW G-4 location. The composite section was hung using the elevation datum for USW G-4; note, however, that the addition of the extra strata means that elevations for the composite column do not correspond with actual elevations at USW G-4, but are an artifact of compositing. Nevertheless, these elevations and the composite thicknesses were used in fitting the composite column to the modeling grid for the unsaturated zone (see Chapter 8). A comparison of the results with the ISM 2.0 framework showed a reasonably similar correspondence of the units. Note also that the proportionate thickness of units in the1994 PA came from a vertical column taken in the center of the repository area as it was proposed at that time; for the $1997 \mathrm{PA}$, a composite column was constructed from the wellbore data previously cited. Therefore, the two are slightly different in this respect (see Figure 4-9b).

Rautman (1995), the source of the porosity data in Figure 4-11, noted that the data represented a number of original analyses performed with a variety of different measurement techniques, which meant that $20 \%$ porosity from one source may not correspond to the same measurement from another source. Because this problem cannot be totally avoided, Rautman made adjustments to minimize the impact. In using porosity models to get to values for thermal conductivity, Rautman also had to distinguish welded from nonwelded rocks. After collecting the porosity data in a histogram, he noted a distinct bimodal distribution with $10 \%$ to $12 \%$ porosity for welded tuffs and $25 \%$ to $35 \%$ porosity for more lithologically diverse nonwelded materials (Rautman, 1995, p. 18). Overall, a threshold value of $20 \%$ separated the majority of samples designated as welded from nonwelded, so Rautman used a threshold value of 


\section{System Characterization: Geologic Barrier}

22.5\% for this distinction when relating this property to thermal conductivity. This distinction can be seen in Figure 4-11.

The 1997 PA used Rautman's (1995) data (and other sources as cited) to infer hydraulic conductivity and thermal conductivity properties. Also, the porosity data from wellbore UZ-16 were compared with a density $\log$ from the wellbore to infer the presence of lithophysae (see left column of Figure 4-11), where vapor phase alteration occurs. Vapor phase alteration zones and lithophysae can appear on plots of core porosity data as zones of high (but nonuniform) porosity values in an otherwise relatively low porosity zone (Rautman, Sandia National Laboratories, personal communication, 1997). For example, when welded zones such as the Topopah Spring Tuff (TS) units include zones of erratically high porosity values (unit TSII; Figure 4-11), they are assumed to represent zones with lithophysae. If it is assumed that the density log measures bulk density and, when converted to porosity, measures total porosity, and that core porosity measures mainly matrix porosity, then the zones in which the two measurements diverge represent areas of porosity sources that are of a larger scale than the matrix porosity. The 1997 PA assumed that these larger scale porosity features were lithophysae and designated these zones in the Topopah Spring Tuff as lithophysal or nonlithophysal accordingly.

\subsubsection{Hydrology}

Hydrologic flow, the movement of liquid (water) and gases through rock material and structural discontinuities, is a physical process governed by gravity and the geometry of the geologic system. A description of the groundwater flow system requires information about both stratigraphy and structure, because both have a combined effect on the geometry of permeable pathways and impermeable barriers. Hydraulic gradient works to move water through the geometry created by the structure and stratigraphy from areas of relatively higher potential to areas of relatively lower potential. Hydraulic head measurements in wellbores and potentiometric gradient maps are used as calibration tools to ensure that models are compatible with the current data.

The following discussion supports an understanding of conceptual model development for the 1997 PA and focuses on descriptions of flow and transport in the unsaturated and saturated zones. Table 4-5 presents a comparison of how hydrologic processes were modeled in the 1994 and 1997 PAs.

Hydraulic Properties of Tuff. Fridrich et al. (1994) observed the following with regard to the hydraulic properties of tuffs. Hydraulic properties of tuff depend upon their primary fabric including welding (dense welding decreases primary porosity), presence of lithophysae (increases porosity), style of crystallization (finely crystalline has lower porosity), bedding, and primary cooling fractures. The hydraulic properties are further altered by subsequent tectonic fracturing (adds permeability, more so to densely welded tuffs), lithostatic loading (can close fractures), overburden removal (causes fracturing, which increases permeability), and diagenetic alteration (zeolitization reduces porosity). Fridrich et al. (1994) stated that densely welded tuffs are generally the most hydraulically conductive layers in the system, because they are intensely fractured and are less susceptible to zeolitic alteration. In contrast, nonwelded tuffs are the least conductive, because they have little primary fracturing and have been altered to zeolites in the saturated zone. The zones of highest permeability follow the zones where brittle, welded tuffs with coarse fabrics are subjected to major tectonic stresses, such as in the area of large fault zones.

Effect of Zeolites. The presence of diagenetic zeolites, which lowers permeability in tuffs, has been cited as a possible factor in lateral diversion of groundwater flow and perching of groundwater by Bodvarsson et al., eds., (1997). In addition, zeolites are widely recognized as an agent of mechanical filtration and sorption of molecules moving in solution, with the potential to impact groundwater transport processes. The sorptive properties of zeolites that might impact groundwater transport are discussed in Section 4.3.3, Geochemistry. Because groundwater follows the path of least resistance (i.e., relatively higher permeability) as it moves in a gradient toward areas of relatively lower potential, any strata or zone that has significant reduced permeability can cause lateral diversion or perching. Lithologic zones characterized as "perching" layers by Bodvarsson et al., eds. (1997) occur in the TSw basal vitrophyre and the $\mathrm{CHn}$, causing lateral diversion of groundwater. They also observed that lateral flow north of Drill Hole Wash follows a southeasterly direction based on the vitrophyre top surface elevation and that in the lower Prow Pass Tuff, low permeability zeolitic rocks could also divert flow. 
Porosity Plot from

Density Geophysical Log Curve

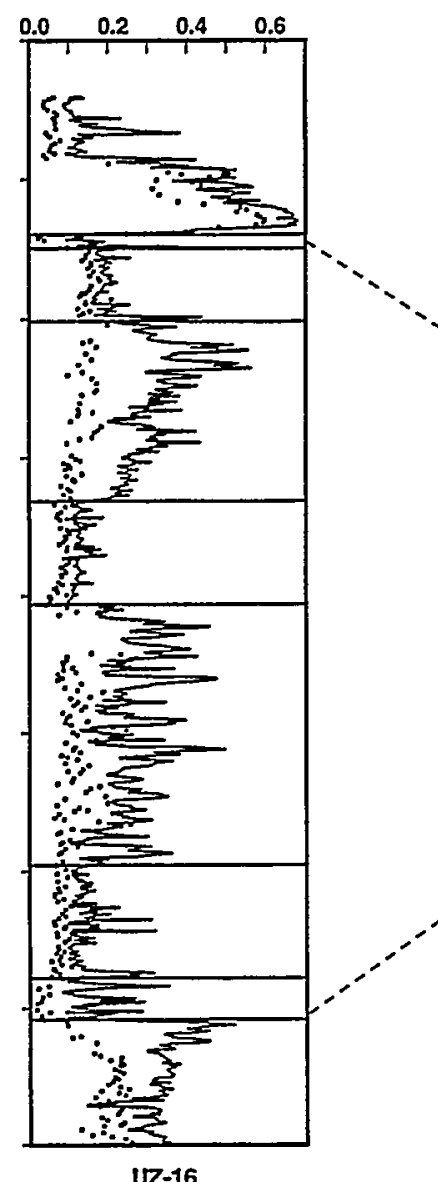

UZ-16

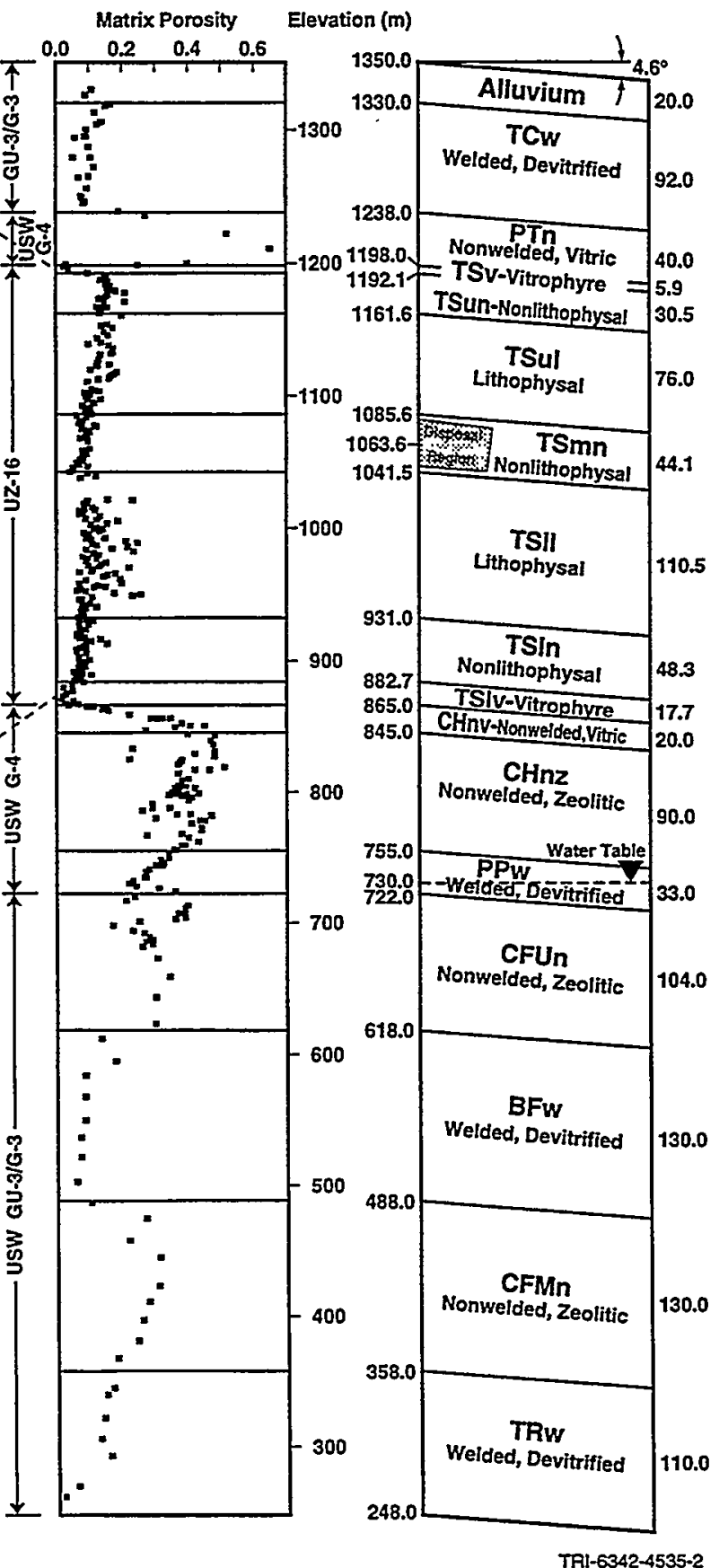

Figure 4-11. 1997 PA modeling unit column (far right) showing wellbores used for composite and a plot of core porosity values from those wellbores (middle). At far left is a breakout of the TS section from wellbore UZ-16 showing a comparison of core porosity (represented by dots) and porosity plotted from a geophysical density log (line plot) for the TS subunits. Stratigraphic dip to the east of $4.6^{\circ}$ has been added to the modeling unit column. 
Table 4-5. Comparison of Modeling Approach for Hydrologic Systems and Processes for 1994 and 1997 DOE SNF/DHLW PAS

\begin{tabular}{|c|c|c|}
\hline \multirow{2}{*}{$\begin{array}{l}\text { Phenomenon } \\
\text { Modeled }\end{array}$} & \multicolumn{2}{|c|}{ Modeling Simplification } \\
\hline & $1994 \mathrm{PA}$ & $1997 \mathrm{PA}$ \\
\hline $\begin{array}{l}\text { Two-phase flow and heat trans- } \\
\text { port with phase changes }\end{array}$ & $\begin{array}{l}\text { 2D, two-phase flow coupled with heat } \\
\text { conduction with water phase change }\end{array}$ & Same \\
\hline $\begin{array}{l}\text { Large sorption of selected radio- } \\
\text { nuclides on tuff }\end{array}$ & $\begin{array}{l}\text { Four } \mathrm{K}_{\mathrm{D}} \text { (sorption coefficient) value } \\
\text { groups, three for devitrified, vitrified, } \\
\text { zeolitic rock groups }\end{array}$ & Same \\
\hline $\begin{array}{l}\text { Exchange of }{ }^{14} \mathrm{CO}_{2} \text { with } \mathrm{H}_{2} \mathrm{CO}^{3} \\
\text { in solution }\end{array}$ & Approximated with effective $K_{D}$ & Not modeled \\
\hline \multicolumn{3}{|l|}{ Fracture and matrix flow } \\
\hline Unsaturated zone & 2D, two phase, composite porosity & Same \\
\hline Saturated zone & 3D, single-phase, dual porosity & Same \\
\hline $\begin{array}{l}\text { Climate change with glacial cycle } \\
\text { causing temporal variation in } \\
\text { infiltration }\end{array}$ & $\begin{array}{l}\text { Cosinusoidal increase and decrease } \\
\text { in infiltration }\end{array}$ & Same \\
\hline $\begin{array}{l}\text { Infiltration varied spatially with } \\
\text { elevation and geology }\end{array}$ & Not modeled & $\begin{array}{l}\text { Infiltration parameters enhanced in } \\
\text { two grid cell columns at point of high- } \\
\text { est elevation and at point over poten- } \\
\text { tial repository }\end{array}$ \\
\hline $\begin{array}{l}\text { Fast-path flow of infiltrating water } \\
\text { in unsaturated zone }\end{array}$ & Not modeled & Not modeled \\
\hline \multicolumn{3}{|l|}{ Effect of structural features on flow } \\
\hline Unsaturated zone & No structure modeled & $\begin{array}{l}\text { Two fault zones as increased perme- } \\
\text { ability zones and bed offset; } 4.6^{\circ} \text { east } \\
\text { dip }\end{array}$ \\
\hline Saturated zone & $\begin{array}{l}\text { Four fault zones modeled as low per- } \\
\text { meability zones; no bed offset in area } \\
\text { of fault zones }\end{array}$ & $\begin{array}{l}\text { Effects of north-south and northwest- } \\
\text { southeast structural trends as hydro- } \\
\text { logic barriers, no bed offset; } 4.6^{\circ} \text { east } \\
\text { dip and } 0.1^{\circ} \text { south dip }\end{array}$ \\
\hline Effect of lithologic heterogeneity & $\begin{array}{l}12 \text { modeling units in unsaturated } \\
\text { zone, internally homogeneous } \\
\text { Topopah Spring modeled as } 3 \text { units }\end{array}$ & $\begin{array}{l}12 \text { modeling units in unsaturated } \\
\text { zone, internally homogeneous } \\
\text { Topopah Spring modeled as } 7 \text { layers, } \\
6 \text { different units }\end{array}$ \\
\hline
\end{tabular}

Lateral Flow. Lateral flow has implications for modeling the movement of released radionuclides from the potential repository. YMP researchers have considered the possibility that lateral flow in the PTn layer in the unsaturated zone above the repository could reduce flux through the potential repository by rerouting flux around it. It has also been considered that lateral flow could actually reduce travel time to the water table if it caused groundwater to bypass a low permeability (slow travel time) zone and hit a "fast path" downward at a fault zone (Bodvarsson et al., eds., 1997, 2.2.7). This situation could also cause groundwater to bypass the impacts of sorption, if the low permeability zone were zeolitized. Past modeling studies have shown that the largest vector of groundwater flow is downward and vertical (Rechard, ed., 1995; Altman et al., 1996) even when zeolitized zones are represented in the model by a reduction of hydraulic conductivity (Altman et al., 1996). However, Altman et al. (1996) did not calibrate their model to include perched water zones as in the analysis of Bodvarsson et al., eds. (1997). a-TSPA-97 (M\&O, 1997) modeled several 2D vertical columns and a horizontal (lateral flow) column for their study, but did not investigate 
lateral flow in a 3D system. The 1997 PA did not calibrate the unsaturated or satirated zone models in a manner that would support detailed study of lateral flow (such as calibration to perched zones); it assumed that vertical flow dominated as indicated in the 1994 PA.

Hydraulic Gradients. Fridrich et al. (1994), citing Robison (1984), noted that below Yucca Mountain there are three hydraulic gradients: a large hydraulic gradient, 0.15 or greater, which trends northeast-southwest north of the potential repository, separating water table altitudes of $1030 \mathrm{~m}$ to $750 \mathrm{~m}$ northwest to southeast; a moderate gradient, approximately 0.015 , north of the large hydraulic gradient; and a very small gradient, 0.0001 , covering most of the area southeast of the large hydraulic gradient. Overall, the gradient is for flow toward the southeast in the area. The potentiometric surface is shown with 1984 data (Figure 4-12) and 1993 data (Figure 4-13). Various studies have noted that the large hydraulic gradient has significance in the saturated zone hydrologic system and have theorized its cause, but no single theory explaining its origin has been widely accepted (Luckey et al., 1996; Fridrich et al., 1994; Ervin et al., 1994). A 45-m step in the water table trending north-northwest in the area of the surface expression of the Solitario Canyon Fault zone separates higher heads to the west of that zone from lower ones to the east of it. This step is possible evidence that the fault zone causes a permeability barrier in the saturated zone (Luckey et al., 1996).

The saturated zone in the 1997 PA was calibrated using the potentiometric gradient and head values measured in wellbores, and used permeability barriers to separate three general head trends. The model contained nine modeling units (Figure 4-9b; see also Chapter 9) and four barrier zones that were modeled as discrete (one grid cell) low permeability features. At the northwest corner of the grid, the approximate regional potentiometric value from Luckey et al. (1996, Figure 8) was used for calibration as were wellbores WT-6 and G-2. These points lie in an area of relatively higher head values across the northern part of the saturated zone study area, separated from a lower head zone to the south by two low permeability zones and from lower heads on the east side of the study area by a low permeability zone representing the possible influence of the Solitario Canyon Fault. The large hydraulic gradient lies in this northerly area of the model.

Two northwest-southeast trending low permeability zones in the model grid represent the possible influence of the structural trends of the same orientation from Drill Hole Wash to Yucca Wash. These two zones are joined just above wellbore WT- 4 by a north-trending low permeability area, roughly corresponding to the location of the Bow Ridge structural feature. The conceptual model supporting low permeability zones in the area of fault zones allows for this effect to arise from a zone of low permeability fault planes or from juxtapositioning of low permeability strata across from permeable strata. The 1997 PA modeling grid did not place low permeability grid cells in areas where other major surface faults would be projected from surface expression to the saturated zone level. By inference, these areas are conceptualized as permeable zones. The placement of low permeability grid cells and permeable zones in the 1997 PA is somewhat different from the method used in the 1994 PA (Rechard, ed., 1995), but not conceptually different.

In addition, to the gradients noted above, vertical gradients have been found in the Yucca Mountain area based on measurements at ten sites as noted by Luckey et al. (1996). In some wellbores, potentiometric levels were found to be higher in lower intervals of the Tertiary volcanics than in the upper levels. Potentiometric measurements in wellbore UE-25P\#1 in 1984 (Craig and Johnson, 1984) indicated that levels in the Paleozoic carbonate aquifer were about $21 \mathrm{~m}$ higher than in the Tertiary lower volcanic aquifer. Vertical hydraulic gradients allow for the potential for upward groundwater flow from deeper aquifers, which creates the possibility that the saturated zone flow system could act as a barrier to radionuclide transport in these areas. In the Yucca Mountain area, data from five wellbores indicate an areally extensive upward gradient between the carbonate aquifer and the volcanic aquifers.

Groundwater Temperature. Ambient groundwater temperatures under central and southern Yucca Mountain are anomalously low, with a heat flow value one-half of that typical of the southern Great Basin (Sass et al., 1988). Sass et al. attributed at least $80 \%$ of this effect to downwelling in the saturated zone. Weeks (1987) and Galloway et al. (1991) support the concept that the remainder of cooling comes from unsaturated zone processes of evaporative cooling with wind-, barometric-, and thermally-driven air circulation through the mountain. At the water table, there are linear zones of elevated groundwater temperature (at least $10^{\circ}$ higher than the lows in the area) trending generally north, which coincide roughly to a downward projection of the Solitario Canyon, Abandoned Wash, Bow Ridge, and Paintbrush Fault zones (Fridrich et al., 1994, Figure 8). Fridrich et al. cited (1989) to suggest that these hydrostruc- 


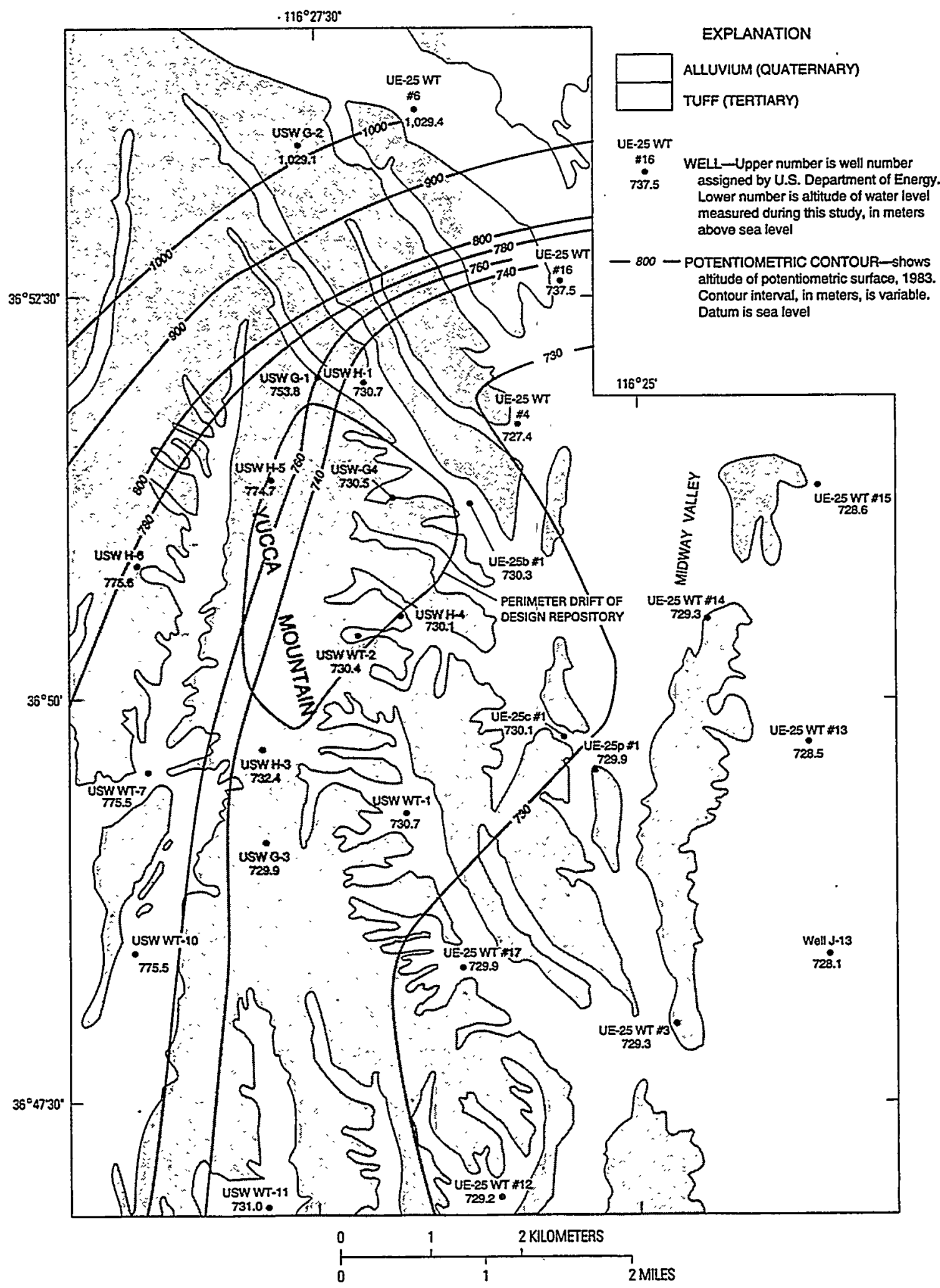

Figure 4-12. Preliminary potentiometric surface, Yucca Mountain (from Luckey et al., 1996, after Robison, 1984, Figure 2). 


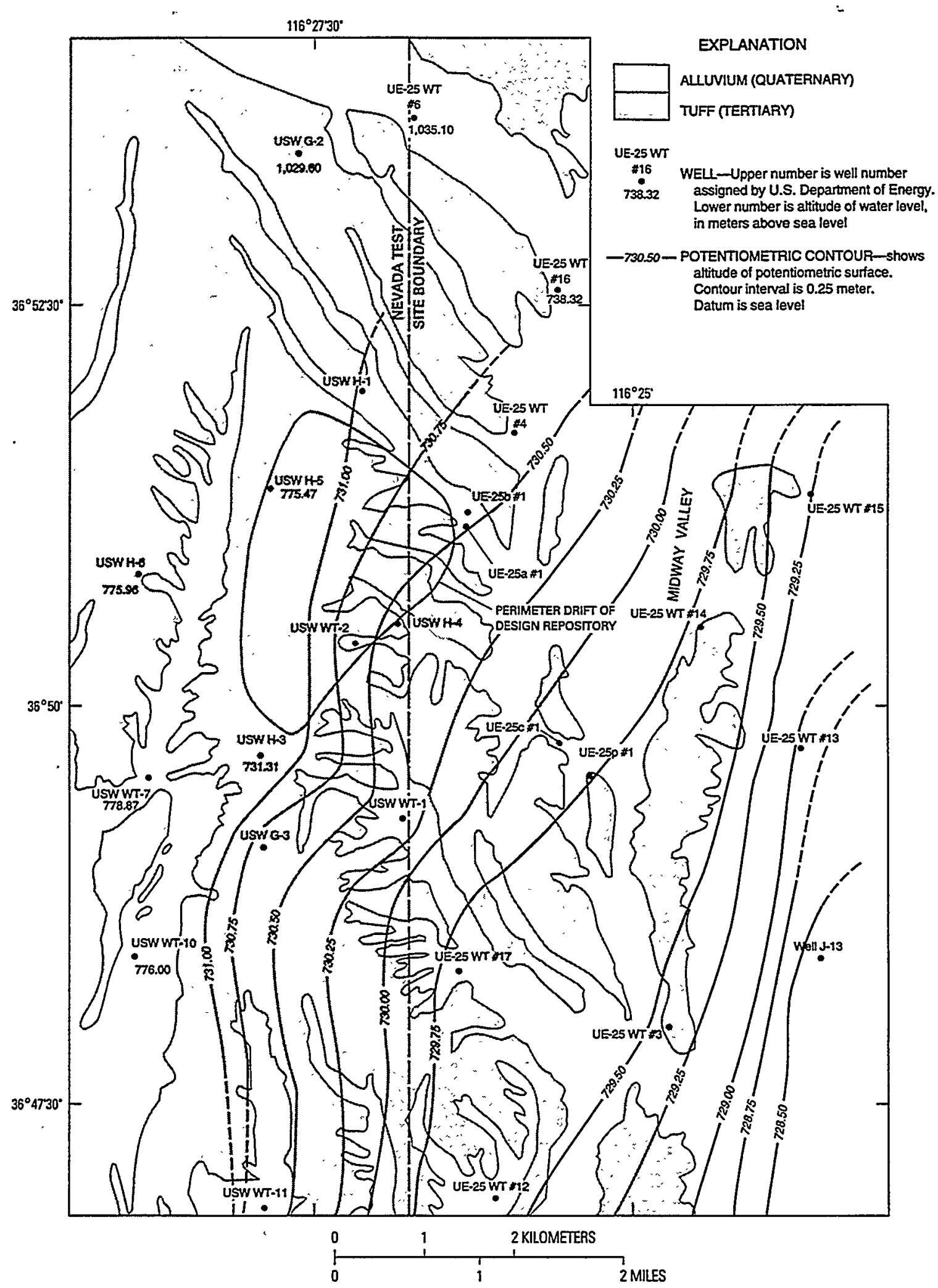

Figure 4-13. Revised poteniometric surface of an area of small hydraulic gradient, Yucca Mountain (from Luckey et al., 1996, after Ervin et al., 1993, Figure 1). 
tural features may form pathways for upwelling water under Yucca Mountain, but Fridrich et al. proposed a mechanism of upwelling caused by pressure rather than thermal buoyancy. This situation has implications for modeling of the disposal system regarding the decision to treat the fault zones as saturated zone barriers to flow or permeable zones, because indications of permeability to flow are mixed when thermal data are compared to potentiometric gradient data. Modeling thermal effects in the hydrologic system remains under study in the YMP, but because precise calibration of the model to thermal observations in the flow system was not a focus of the 1997 PA, it was not considered in this study. The saturated zone model did not handle heat at all. Ambient atmospheric temperature was input in the unsaturated zone, with the focus of thermal modeling in relation to heat from the source term.

\subsubsection{Geochemistry}

The 1997 PA focused on near-field geochemical processes. In the near-field, geochemistry plays an important role with regard to waste package corrosion and waste mobilization. The 1997 PA built on results of the 1994 PA, which indicated that (Rechard, ed., 1995, Table 12-13):

- Inclusion of radionuclide sorption on tuff in the unsaturated and saturated zones greatly diminishes the release of radionuclides.

- Only minute amounts of uranium and neptunium reached the water table in $10,000 \mathrm{yr}$, because they were present in high quantity initially and were only moderately sorbed. Plutonium did not leave the waste disposal region or reach the water table because of sorption on tuff. Between 10,000 and 50,000 yr, additional amounts of uranium $\left({ }^{233} \mathrm{U},{ }^{234} \mathrm{U}\right)$ and large amounts of neptunium $\left({ }^{233} \mathrm{~Np}\right)$ reached the water table.

- High silica concentration in groundwater reduced the solubility of uranium in groundwater.

Oxidation potential $(\mathrm{Eh})$ and therefore, modeling transport of $\mathrm{O}_{2}$ from the surface to the repository horizon is an important focus of the 1997 analysis. The BRAGFLO_T code modeled oxygen transport with varying oxygen content to compare outcomes related to oxygen depletion, especially from rusting of waste containers. Other geochemical parameters used in the 1997 PA were treated as constants and most were taken from Wilson et al. (1994). For the 1997 PA, the silica concentration in groundwater was not varied, because its effect was modeled in the 1994 PA. Based on new data, solubility of ${ }^{237} \mathrm{~Np}$ was decreased by two orders-of-magnitude for the $1997 \mathrm{PA}$.

Geochemistry/Mineralogy of the Host Rock. The Paintbrush Group makes up the bulk of the outcropping section at Yucca Mountain and comprises the thickest part of the unsaturated zone. As shown in Figure 4-9a, the Topopah Spring Tuff Formation is the thickest Paintbrush Group formation. The mineralogy of the entire unsaturated zone section has an impact on the groundwater geochemistry of infiltrating and percolating water. Topopah Spring Tuff mineralogy and other rock properties also impact thermal and mechanical effects in the potential repository area (see "Geologic Framework" in Section 4.3.1). In the potential repository area at Yucca Mountain the following generalizations apply to the Topopah Spring Tuff lithology, which is the source of some rock properties for the TS modeling units (DOE, 1998b, p. 1-64).

In the lower three-fourths of the formation phenocrysts (large, early-formed crystals) are $<2 \%$ of the rock and are sanidine, plagioclase (andesine to oligoclase), with minor quantities of quartz, biotite, amphibole, iron-titanium oxides, allanite, and zircon. The texture changes in the upper fourth of the formation where phenocrysts make up $22 \%$ of the composition with clinopyroxene as a minor addition. Groundmass texture (crystals $<4 \mathrm{~mm}$ ) comprises the bulk of the formation and has a composition that includes a range of 76 to $78 \mathrm{wt} \% \mathrm{SiO}_{2}$ with $\mathrm{Al}_{2} \mathrm{O}_{3}$ next in abundance at just under 12 to $13 \mathrm{wt} \%$ and other minerals as shown in Table 4-6. The values in Table 4-6 are from an analysis of samples from USW G-4 (Broxton et al., 1986) from a vitric unit with a composition that was representative of the majority of units in the well. The values in Table 4-6 are similar to those shown in the SCP (DOE, 1988b, Table 1-5, p. 1-69), which gives a breakdown of composition by subunits (lower nonlithophysal, etc.). A third textural and mineralogic phase is vapor-phase, which results from the presence of gases concentrated in the upper part of a cooling unit. Vapor-phase minerals in the lithologic zones correlative to the TS modeling units are mostly cristobalite and alkali feldspars. Devitrification of metastable vitric (glassy) material occurs soon after deposition and continues over time to produce cristobalite, feldspar, and tridymite. Secondary alteration of the tuff results in anhydrous minerals such as feldspar, calcite, and quartz, or hydrous smectites, zeolites, and manganese minerals (Parsons et al., 1991). 
Table 4-6. Composition of Representative Subunit of the Topopah Spring Formation from Wellbore USW/G-4

\begin{tabular}{|c|c|}
\hline $\begin{array}{lc} & \begin{array}{c}\text { Topopah Spring Tuff } \\
\text { (TSv modeling unit }\end{array} \\
\text { Oxides } & \text { Percent Weight } \\
\end{array}$ & \\
\hline $\mathrm{SiO}_{2} \quad 74.00$ & \\
\hline $\mathrm{Al}_{2} \mathrm{O}_{3}$ & \\
\hline $\begin{array}{ll}\mathrm{K}_{2} \mathrm{O} & 4.00\end{array}$ & $T$ \\
\hline $\mathrm{Na}_{2} \mathrm{O} \quad 3.40$ & a Mt. \\
\hline$\ldots$ & \\
\hline $\begin{array}{ll}\mathrm{MgO} & 0.31\end{array}$ & \\
\hline $\mathrm{Fe}_{2} \mathrm{O}_{3} \quad 1.07^{\mathrm{b}}$ & \\
\hline $\mathrm{TiO}_{2} \quad 0.10$ & \\
\hline $\begin{array}{ll}\mathrm{MnO} & 0.08\end{array}$ & \\
\hline Loss on ignition $\quad 3.79$ & \\
\hline$\quad 0.01$ & \\
\hline $0.000465^{\mathrm{c}}$ & \\
\hline 99.82 & $\begin{array}{c}0.0010 .010 .11110 \quad 100 \\
\text { Percent Weight }\end{array}$ \\
\hline
\end{tabular}

a Broxton et al., 1986, App. A; USW G-4 at $1279 \mathrm{ft}$ depth evaluated by x-ray fluorescence.

b Caporuscio and Vaniman, 1985, estimates Fe(II) oxide in minerals in Yucca Mountain to vary between 0.16 and $0.33 \%$, which is higher than found by Delany (1985) (see Table III).

c Broxton et al., 1986, App C; USW GU-3 at $1322 \mathrm{ft}$ depth (TS modeling unit) evaluated by neutron activation; uranium content increases with depth and reaches values as high as 0.0012 . 
Overall, the Topopah Spring Tuff formation is mostly rhyolite (quartz phenocrysts with biotite the dominant mafic mineral), with an upper caprock of latite (lacking quartz phenocrysts and having a variety of mafic minerals). The mineral assemblage noted in the previous paragraph is implied to accompany the presence of lithophysae in a modeling unit designation. The presence of lithophysae is modeled as increasing the insulating capability of the rock around the potential repository, because of its air pockets, which are less thermally conductive than denser rock material.

Retardation Processes. Geochemical retardation processes include sorption, precipitation, and slow migration of radionuclides, thereby allowing longer times for decay during travel. Correlation of sorptive behavior with mineralogy was identified early on in site characterization studies (DOE, 1988b, Chapter 4). Sorption of alkali metals (cesium) and alkaline earths (strontium, barium, radium) is found in the presence of minerals with exchangeable cations such as zeolites and potentially, smectite clays. The term "zeolites" includes a large group of hydrous silicates called framework aluminosilicates with $\mathrm{Na}$ and $\mathrm{Ca}$, and highly variable amounts of water in the voids of the framework. They adsorb molecules that have interacting effects, because the aluminosilicate framework has a net negative charge balanced out by interchangeable cations (Breck, 1983). Zeolites also separate molecules by size and geometry in a manner related to the size and geometry of the zeolite framework.

Silicic volcanic glass of the type comprising much of Yucca Mountain is the most common source of the aluminosilicate material from which most zeolites evolve in sedimentary and volcanic rocks. Occurrence is mainly in subsurface fractures and vesicle fillings in areas exposed to meteoric water infiltration. In a hydrologic system such as that at Yucca Mountain, zeolitization can affect thousands of vertical feet of tuff and produce chemical zonation by development of different types of zeolites. At Yucca Mountain there are also other sorptive minerals, the clay minerals smectites, that are widespread throughout the units underlying the Topopah Spring Tuff.

Geochemistry/Groundwater. Groundwater geochemistry is linked to processes involving the entire hydrologic system of the Yucca Mountain area, and is the product of highly complex, imperfectly understood interactions. As an example for the unsaturated zone, the recently completed draft of the comprehensive study for the Site-Scale Unsaturated Zone Model for the VA (Bodvarsson et al., eds., 1997) noted the following major problems that contribute considerable uncertainty to any quantitative hydrochemical analysis: episodic storms with high water input, changing climate with changing precipitation, spatial distribution uncertainties related to incomplete data on structural and hydrologic heterogeneity at Yucca Mountain, and the meaning of chlorine-36 data. These are only a small part of the uncertainties that impact the assumptions made by the $1997 \mathrm{PA}$.

Precipitation. Groundwater chemistry begins with precipitation chemistry that is analyzed from data routinely collected at four sites in the state of Nevada and summarized in Bodvarsson et al., eds. (1997, Chapter 14 and Appendix A). Bodvarsson et al., eds. (1997) rioted that these results show precipitation varies widely in total dissolved solids content from location to location and from year to year in a given location, but that the dominant neutral salt components in Nevada precipitation are calcium sulfate, ammonium nitrate, and sodium chloride. Most of these salts originate in precipitation or as dust and are concentrated through evaporation and evapotranspiration at or near the surface. They also observed that by the time groundwater has percolated to the Topopah Spring Tuff, it is saturated in the primary mineral components of the tuff, which include sanidine, cristobalite and minor plagioclase. Their studies of pore water as it migrates into the Calico Hills Tuff Formation have shown changing levels of $\mathrm{Mg}, \mathrm{Ca}$, and $\mathrm{Na}$, which probably occurred as ion exchange with the zeolites clinoptilolite and mordenite, with $\mathrm{Mg}$ possibly lost by precipitation of a hydrated magnesium silicate in the Topopah Spring Tuff. They also noted that Opal-CT and calcite are typical diagenetic precipitates on fracture surfaces and on the walls of lithophysae citing the work of Fabryka-Martin et al. (1996). These observations are part of what the authors call the "...first stage of an attempt to integrate the hydrochemistry of the hydrology of the vadose zone at Yucca Mountain..." confirming the idea that the groundwater geochemical system in the unsaturated zone is very dynamic and still not well characterized. Uncertainties in many process models for vadose zone interactions are impacted by incomplete understanding of vadose zone geochemistry.

The natural radiotracer ${ }^{36} \mathrm{Cl}$ has been used in unsaturated zone studies to estimate pore water age, and its presence in fractures deep in the unsaturated zone has been analyzed as evidence to support the "fast path" infiltration concept. Bodvarsson et al., eds. (1997) state that there is strong evidence of the presence of "bomb-pulse" ${ }^{36} \mathrm{Cl}$, meaning anomalously high concentrations of ${ }^{36} \mathrm{Cl}$ as a result of atmospheric weapons testing during the fifties, associated with ESF tunnel wall fractures. Recent studies by Fabryka-Martin et al. (1996) and Levy et al. (1997), which 
also found elevated levels of ${ }^{3} \mathrm{H}$, support this observation from which it can be inferred that surface waters have moved to significant depths through fractures over the last $40 \mathrm{yr}$. They also found no intact rock samples with these elevated levels of ${ }^{36} \mathrm{Cl}$. In the ESF, the Drill Hole Wash Fault and Ghost Dance Fault had ${ }^{36} \mathrm{Cl}$ levels that indicated that they were high permeability zones. Study continues on the significance of ${ }^{36} \mathrm{Cl}$ and the meaning of these analyses, as some researchers are not convinced that the concentrations observed are necessarily related to atmospheric testing. However, the current YMP emphasis on developing appropriate process models to capture the effects of "fast-path" infiltration is largely fueled by "bomb-pulse" data.

Saturated Zone Groundwater Chemistry. Groundwater chemistry is also an important indicator by which the regional saturated zone flow pattern is being defined. Winograd and Thordarson's (1975) comprehensive study of the hydrochemistry of the South-Central Great Basin found three general geochemical types of groundwater in the area of the Nevada Test Site: sodium and potassium bicarbonate; calcium and magnesium bicarbonate; and mixed calcium magnesium sodium bicarbonate from mixing of the first two types. They also note two more restricted geochemical facies associated with "wet" playas and some springs. In general, groundwater chemistry reflects the interaction of water and the reactive components of the soil cover and rock through which it passes. If water moves only through silica rich tuff or tuffaceous alluvium, it has a sodium-bicarbonate nature; if it passes only through the lower carbonate aquifer or valley fill rich in carbonates, it has a calcium and magnesium bicarbonate nature. Winograd and Thordarson (1975) stated that saturated zone groundwater at Yucca Mountain probably comes from subsurface flow from the north derived from recharge at higher altitudes and flows southward from the Yucca Mountain area toward the Amargosa Desert (Figure 4-6).

Table 4-7 shows the results of analysis of groundwater samples from four wells in the Yucca Mountain area (well locations are shown on Figure 4-1). The first nine rows of the table show element concentrations, the next seven rows show anion concentrations, and the $\mathrm{pH}$ and $\mathrm{Eh}$ are indicated in the last two rows. The dominant cations in the groundwater are sodium, calcium, potassium, and magnesium. Collected water samples from Wellbore UE-25P\#1 in the carbonate aquifer show high cation concentration (sodium and calcium) and a high content of the bicarbonate anion. Total dissolved solids for UE-25P\#1 are $1000 \mathrm{mg} / \mathrm{l}$, while samples from other wells vary between 200 and $400 \mathrm{mg} / 1$ (Ogard and Kerrisk, 1984). Wellbore USW G-4, which is located on Yucca Crest just south of the potential repository location, shows a high sodium content like UE-25P\#1, but a much lower calcium content. This wellbore also contains the most reducing Eh and the highest $\mathrm{pH}$ among the wells listed in the table. Most of the water from the wells were analyzed as having a pH close to neutral. The groundwater compositions of wellbore G-4, located just east of the potential repository area, and J-13, located farther southeast, are very similar to each other. In general groundwater at Yucca Mountain is oxidizing (DOE, 1988b, p. 4-146). However, Table 4-7 illustrates that water from the Tram Tuff aquifer (TRw modeling unit) in wellbore USW H-3 is reducing and could reduce radionuclides such as uranium to their more insoluble oxidation states. In simulations, most movement of radionculides in the aquifer occurs in units closer to the water table (Rechard, ed., 1995), meaning that the reducing potential of deep waters may not be significant. More important, the extent of the reducing potential for this well and others must still be confirmed through further in situ measurements. Water conditions bounded by samples from wellbores J-13 and UE-25P\#1 were used to define distributions for the solubility of radioisotopes in an oxic environment.

Solubilities. Solubility is a function of the groundwater chemistry and temperature (also pressure, theoretically, but not practically). The groundwater chemistry is expressed as the $\mathrm{pH}^{*}, \mathrm{Eh}^{\dagger}$, and dominate species (e.g., cations and anions) in the water. For an actinide in a particular oxidation state, the solubility tends to decrease with increasing $\mathrm{pH}$. Also, the solubility of a particular actinide tends to increase with increasing oxidation states, and the solubilities of some actinides are quite similar at the same oxidation state. Generally, the actinides can exist in the $+3,+4,+5$, and +6 oxidation states (e.g., plutonium can exist in all oxidation states). Uranium commonly exists as +4 (U(IV)) and $+6(U(V I))$.

Observed solubilities of both U(IV) and U(VD) in a natural, somewhat reducing environment are low, averaging around $10^{-5} \mathrm{mM}$ (Cramer and Smellie, eds., 1994) (Figure 4-14). However, in carbonate-saturated waters in an oxi-

- $\mathrm{pH}$ is defined as the negative $\log _{10}$ of the hydrogen ion concentration $\left[\mathrm{H}^{+}\right]$, so that $\mathrm{pH} 5$ means a hydrogen ion concentration of $10^{-5}$ moles liter. $\mathrm{pH}<7$ is acidic, $\mathrm{pH}=7$ is neutral, $\mathrm{pH}>7$ is basic.

$t$ Eh is the redox potential and is a measure of the ability of a solution to oxidize or reduce species in solution. Eh is defined by the Nernst equation for a half-reaction written as a reduction chemical reaction in comparison to the standard hydrogen reaction. 
4. System Characterization: Geologic Barrier

Table 4-7. Composition of Water Samples from Four Wellbores in Vicinity of Yucca Mountain

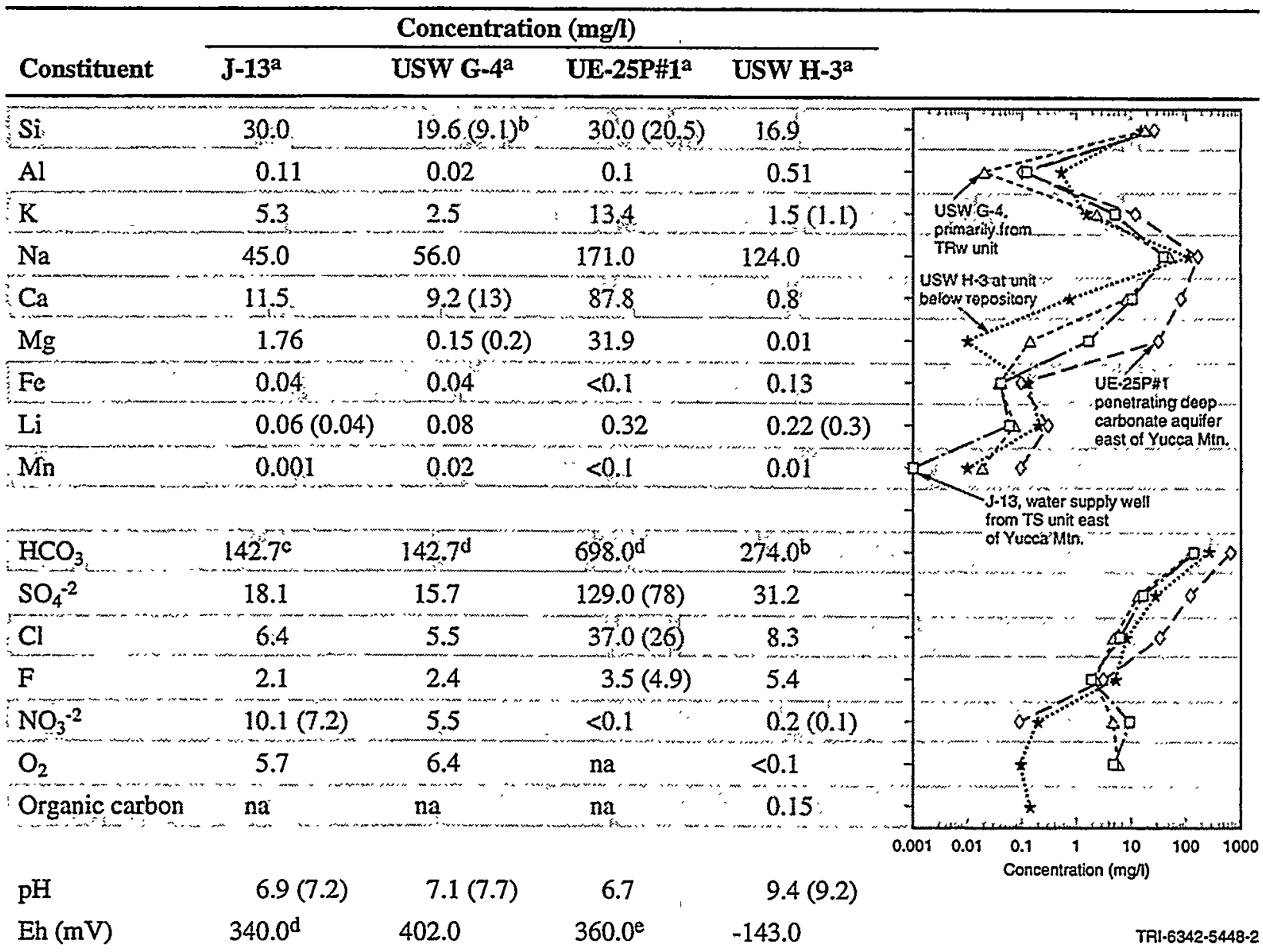

a Ogard and Kerrisk, 1984.

b Values reported by USGS (McKinley et al., 1991) when data differ by more than 0.1 order of magnitude.

c Estimated from alkalinity.

d Wilson et al., 1994, Table 9-1; the reported source is Ogard and Kerrisk (1984) but they do not report a value.

e Ogard and Kerrisk (1984) note that water might have contained air from drilling process. 
dizing environment, the solubilities of uranium are higher $\left(>10^{-3} \mathrm{mM}\right.$ ) (Samama, 1986). In the tuff environment, high silica concentrations are found in groundwater percolating through the rock (Nitsche et al., 1993; Duffy, 1993), and the dominant species limiting the concentration (and thereby solubility) of radionuclides at Yucca Mountain is thought to be silica $\left(\mathrm{SiO}_{2}\right)$ (Bruton and Shaw, 1988; Bruton et al., 1990). The average concentration of uranium controlled by the uranyl silicate, uranophane, is slightly higher. Leslie et al. (1993) reports an average solubility around $\sim 10^{-2} \mathrm{mM}\left(2.3 \times 10^{-3} \mathrm{~kg} / \mathrm{m}^{3}\right)$ at Nopal I and in laboratory experiments.

In conjunction with performance assessments for the Yucca Mountain Project, analysts have combined expert opinion with parameter values from calculations that account for differences in values for parameters in a repository environment that influence the solubility. Expert opinion defined the range of solubility by providing distributions for the solubility of uranium and plutonium in an oxic environment, assuming water conditions are bound by J-13 and UE-25P\#1 well water in the underlying aquifer (Wilson et al., 1994, p. 9-6). The expert panel values for uranium ranged from $10^{-5}$ to $10^{1} \mathrm{mM}$ (Figure 4-14).

The values were substantiated by means of EQ3/6 (version EQ3/6-V7-REL-V7.2a [Wolery, 1992a; 1992b; Wolery and Daveler, 1992]), a code for simulating interactions of groundwater and solid mineral phases in the natural environment assuming thermodynamic equilibrium. Similar to the assumptions used in the expert opinion solicitation, calculations were performed assuming only oxic conditions. The unsaturated zone was conceptualized as an open system with $\mathrm{O}_{2}$ and $\mathrm{CO}_{2}$ in equilibrium with the atmosphere (fugacities were fixed at $10^{-0.7}$ and $10^{-3.5}$, respectively [Delany and Wolery, 1984]). The J-13 well water represented the groundwater. A saturation-limited model (Kerrisk, 1984) was used, which permitted radionuclide concentrations in the waters to reach maximum concentrations. In the calculations, the solubility of uranium was found to be highly sensitive to $\mathrm{pH}$ (run at 5.9,7,8, and 9) and temperature changes (run at $25^{\circ} \mathrm{C}, 60^{\circ} \mathrm{C}$, and $100^{\circ} \mathrm{C}$ ). The solubility of $\mathrm{U}(\mathrm{VI})$ also increased with the formation of carbonate species, as noted by Nitsche et al. (1993) in experiments using J-13 well water. When the silica concentration was constant in the solution, the uranium solubility ranged from $10^{-5.6}$ to $10^{-4.4} \mathrm{mM}$ (based on $\mathrm{pH}$, temperature, and carbonate present). When silica was allowed to deplete in the solution, the uranium solubility ranged from $10^{-4.1}$ to $10^{-1.75} \mathrm{mM}$. Under oxic conditions, the solubility of plutonium was controlled by equilibrium with $\mathrm{PuO}_{2}$ solid and found to be extremely low: $10^{-9.5}$ to $10^{-7} \mathrm{mM}$, in agreement with the studies by Allard (1982). Because $\mathrm{PuO}_{2}$ forms slowly, the solubility of plutonium was also calculated by suppressing the formation of $\mathrm{PuO}_{2}$. In this case, the solubility ranged between $10^{-5.5}$ to $10^{-3.5} \mathrm{mM}$. These calculated extremes were larger than the extremes estimated by the expert panel for plutonium (Wilson et al., 1994, p. 9-6) (Figure 4-14).

For the solubility of $\mathrm{Np}$, we used a range of $1.6 \times 10^{-10}$ to $3.9 \times 10^{-6} \mathrm{M}$ with a loguniform probability distribution for the 1997 PA. This range is based on laboratory studies of the reaction of spent fuel or borosilicate glass with simulated Yucca Mountain groundwater under static or flowing conditions by Rai et al. (1982), Wilson (1990a, 1990b), Wilson and Bruton (1990), Finn et al. (1995), and Gray and Wilson (1995). These investigators directly determined the quantities of $\mathrm{Np}$ released, or provided information from which the quantities of $\mathrm{Np}$ released were calculated assuming congruent dissolution.

This range is significantly lower than the range of $1.00 \times 10^{-8.00}$ to $1.00 \times 10^{-2.00} \mathrm{M}$ used with a $\beta$ or a cumulative probability distribution for the 1994 PA (Rechard, ed., 1995) because it does not include the high dissolved Np concentrations observed in the laboratory studies by Nitsche et al. $(1993,1994)$. We excluded Nitsche's data because his oversaturation experiments do not simulate expected $\mathrm{Np}$ concentrations during release from spent fuel and glass in the potential Yucca Mountain repository.

Nitsche et al. (1993) carried out oversaturation experiments by adding the $\mathrm{Np}(\mathrm{V})$ species $\mathrm{NpO}_{2}{ }^{+}$at high concentrations (on the order of $10^{-3}$ or even $10^{-2} \mathrm{M}$ ) to J-13 groundwater at a pH of 5.9, 7.0, or 8.5 and a temperature of $25^{\circ} \mathrm{C}, 60^{\circ} \mathrm{C}$, or $90^{\circ} \mathrm{C}$. J-13 simulates groundwater from the saturated zone beneath the repository. After a few months, the $\mathrm{Np}$ concentrations decreased to (apparent) steady-state values of $(4.4 \pm 0.7) \times 10^{-5}$ to $(6.4 \pm 0.4) \times 10^{-3} \mathrm{M}$ (depending on the $\mathrm{pH}$ and temperature) as phases such as $\mathrm{Na}_{0.6} \mathrm{NpO}_{2}\left(\mathrm{CO}_{3}\right)_{0.8}: 2.5 \mathrm{H}_{2} \mathrm{O}$, $\mathrm{NaNpO}_{2}\left(\mathrm{CO}_{3}\right): 2 \mathrm{H}_{2} \mathrm{O}$, and $\mathrm{Np}_{2} \mathrm{O}_{5}$ precipitated. Nitsche et al. (1994) used similar methods and conditions, but substituted UE-25P\#1 for J-13, started the experiments at somewhat lower $\mathrm{Np}$ concentrations (on the order of $10^{-5}$ to $10^{-3}$ $\mathrm{M})$, and omitted the $90^{\circ} \mathrm{C}$ runs. UE-25P\#1 also simulates groundwater from the saturated zone, but has an ionic strength about an order of magnitude higher than J-13. In these experiments, the Np concentrations decreased to 


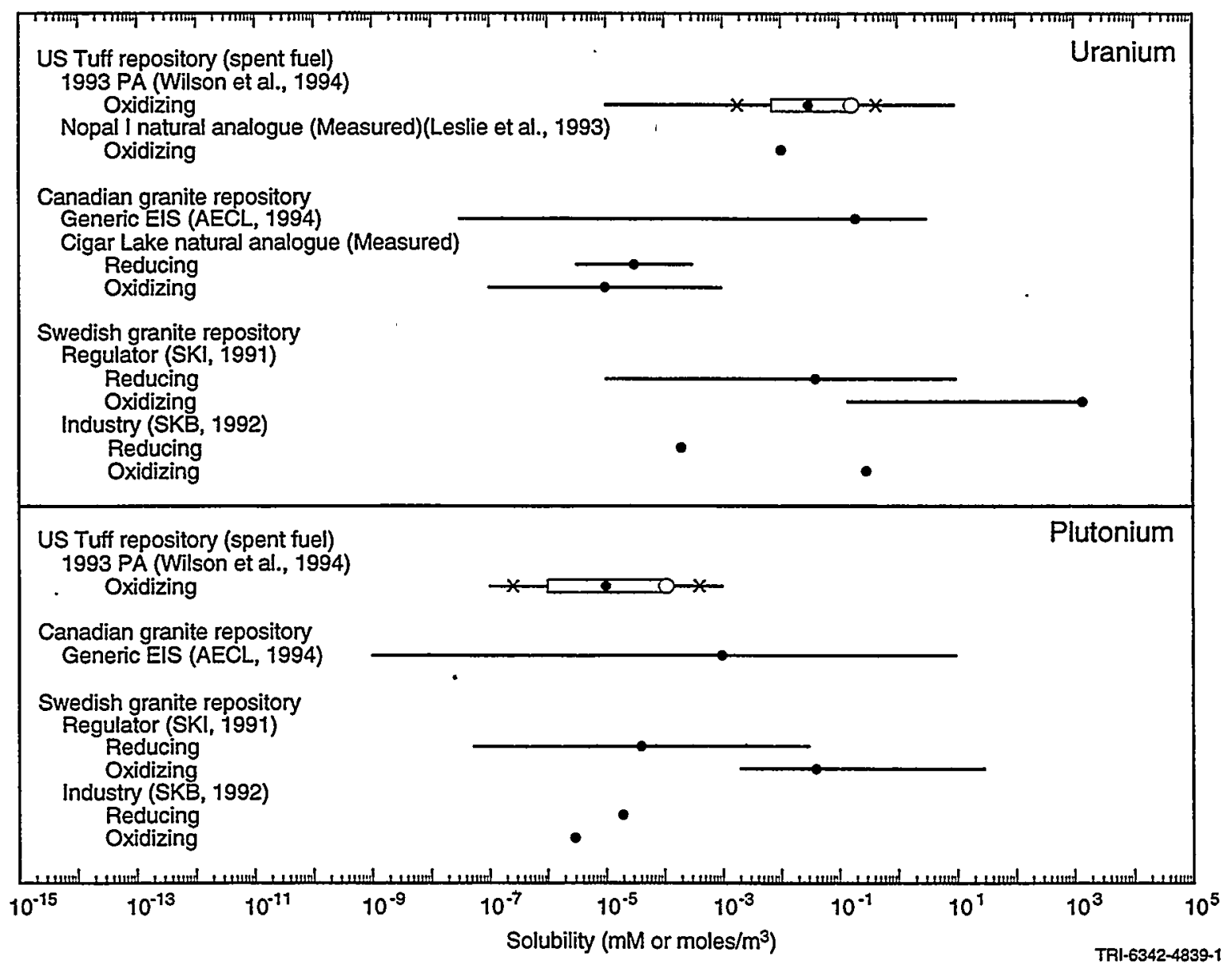

Figure 4-14. Assumed solubility of uranium and plutonium under oxic and reduced conditions in J-13 and UE25P\#1 well water at Yucca Mountain, Nevada. For purposes of comparison, the solubilities of uranium and plutonium used in other analyses are also shown. 
(apparent) steady-state values of $(7.0 \pm 0.6) \times 10^{-6}$ to $(2.9 \pm 0.6) \times 10^{-3} \mathrm{M}$ due to the precipitation of "sodium neptunyl(V) carbonate hydrates." Nitsche et al. (1994) did not specify the exact composition of these precipitates.

Experimental chemists and geochemists frequently determine the solubility of a solid phase by approaching the same, steady-state, dissolved concentration of an element in the solid from conditions of oversaturation and undersaturation. Nitsche et al. (1993, p. 9) stated "The approach from oversaturation consists of adding an excess amount of the element in soluble form to the ... solution and ... monitoring the precipitation of insoluble material until equilibrium is reached. The solid formed must then be isolated and characterized. The approach from undersaturation consists of dissolving a well-defined solid in a ... solution until equilibrium is reached. In both cases, the [dissolved] concentration is measured as a function of time until equilibrium is reached." Nitsche et al. (1993, p. 10) also stated "For unknown systems, one should first perform experiments approaching steady-state concentration from oversaturation and ... characterize the solids. This has the advantage of not specifying the solid that controls solubility but of allowing the system under investigation to determine the solid that will precipitate. These solids should be synthesized for use in confirmation experiments that approach steady state from undersaturation." Nitsche et al. $(1994$, p. 8) essentially restated this as "The approach from undersaturation consists of dissolving the same well-defined solid [precipitated in an oversaturation run] ... until equilibrium is reached."

No chemist nor geochemist would argue with the need to demonstrate solubility equilibrium by approaching the same, steady-state, dissolved concentration from conditions of oversaturation and undersaturation. However, Nitsche et al. $(1993,1994)$ did not carry out any undersaturation experiments. Therefore, it is possible that the Np concentrations would be significantly lower than those reported by Nitsche $(1993,1994)$ throughout much of the period of performance considered by the 1997 PA. Furthermore, use of the same solids precipitated in these oversaturation experiments for a confirmatory undersaturation run, or for predicting $\mathrm{Np}$ concentrations during dissolution of spent fuel or high-level-waste glass in the potential Yucca Mountain repository, is inappropriate.

$\mathrm{Np}(\mathrm{V})$ solids such as $\mathrm{Na}_{0.6} \mathrm{NpO}_{2}\left(\mathrm{CO}_{3}\right)_{0.8}: 2.5 \mathrm{H}_{2} \mathrm{O}, \mathrm{NaNpO}_{2}\left(\mathrm{CO}_{3}\right): 2 \mathrm{H}_{2} \mathrm{O}$, and $\mathrm{Np}_{2} \mathrm{O}_{5}$ are unstable with respect to the $\mathrm{Np}(\mathrm{IV})$ solid $\mathrm{NpO}_{2}$ under any conditions expected in Yucca Mountain. Therefore, the steady-state $\mathrm{Np}$ concentrations observed by Nitsche et al. $(1993,1994)$ in their oversaturation experiments would be metastable solubilities even if they had observed the same concentrations in undersaturation runs carried out under the same conditions. Np is present in spent fuel as $\mathrm{NpO}_{2}$, probably in solid solution with $\mathrm{UO}_{2}$ in the matrix. Because the solubilities of $\mathrm{NpO}_{2}$ and other $\mathrm{Np}(\mathrm{IV})$ solids are orders of magnitude lower than those of $\mathrm{Np}(\mathrm{V})$ solids under the same conditions, dissolution of $\mathrm{NpO}_{2}$ in spent fuel would result in $\mathrm{Np}$ concentrations orders of magnitude lower than those observed by Nitsche et al. (1993, 1994). In fact, the Np concentrations for spent fuels and glass under static and flowing conditions ranged from $1.6 \times 10^{-10}$ to $3.9 \times 10^{-6} \mathrm{M}$ (see above). These concentrations are too low for nucleation and growth of $\mathrm{Na}_{0.6} \mathrm{NpO}_{2}\left(\mathrm{CO}_{3}\right)_{0.8}: 2.5 \mathrm{H}_{2} \mathrm{O}, \mathrm{NaNpO}_{2}\left(\mathrm{CO}_{3}\right): 2 \mathrm{H}_{2} \mathrm{O}$, and $\mathrm{Np}_{2} \mathrm{O}_{5}$, and other phases precipitated in the studies of Nitsche et al. $(1993,1994)$.

Transport Processes. Geochemical processes by which radionuclides may be transported away from the repository to the accessible environment include adsorption on natural colloids or the formation of radiocolloids. Naturally occurring colloids in groundwater arise from smectites, vermiculites, illites, kaolinite, and chlorite. Radiocolloids (colloids containing radionuclides) may arise from a variety of sources including corrosion of canister material and degradation of engineered backfills (DOE, 1988b, Chapter 4). Colloidal systems are large molecules or small particles suspended in a solvent with at least one dimension within the size range $10^{-9}$ to $10^{-6} \mathrm{~m}$ (Parsons et al., 1991). They function well as adsorbents due to their large surface area, which has a relatively high electrical charge that facilitates ionic exchange. Colloids may also coalesce or precipitate. They behave in a manner different from dissolved species, and some investigators (Apps et al., 1982; Bonano and Beyeler, 1985; Champ et al., 1982) have concluded that radionuclides can be transported faster as colloids than as a dissolved species. However, their large size may contribute to their being retarded by physical filtering in a zeolitic zone or in a matrix with small pores, which means that migration through fractures is the most likely pathway for colloids (Tsang and Mangold, 1984). The implication here is that if transport in the unsaturated or saturated zones is thought to be predominantly through the matrix, retardation of colloidal transport is more likely to occur than if transport is predominantly through fractures.

Sorption. As more fully described below, sorption is the accumulation of material at the interface of another material. Adsorption of fissile material can occur in several locations throughout the disposal system. As an illustra- 


\section{System Characterization: Geologic Barrier}

tion, three locations are considered: corrosion products (primarily rust) of a container, devitrified tuff, and zeolitic tuff.

Sorption refers to the reversible accumulation of material at the interface of another material. Actinide adsorption commonly occurs on clays, organic matter, and iron hydroxides; in the natural environment, these materials often have uranium concentrations higher than background levels. Adsorption subsumes several different mechanisms (e.g., surface complexation and ion exchange) ${ }^{\ddagger}$; hence the factors influencing adsorption are the factors that influence the various mechanisms that contribute collectively to adsorption. ${ }^{* *}$ Generally adsorption is dependent upon the nature of the contaminant (e.g., molecular size, weight, and charge) and surface (e.g., functional organic groups), the concentration of the contaminant in solution, and the surface area and density of active adsorptive sites on the immobile solid. Given a specific adsorptive surface and contaminant, factors infiuencing adsorption include $\mathrm{pH}$, Eh, temperature, and pressure as they influence dominant species [e.g., U(IV) or U(VI)] of the adsorbate, the condition of the adsorbent surface, and the ionic strength through its influence on the diffuse double layer or competition for adsorptive sites.

For both ion exchange and surface complexation, only a limited number of areas ("adsorptive sites") exist on a surface area. Although the number of active sites can be far less than a theoretically or physically estimated number of sites, because the size of the adsorbing material can vary greatly, the estimate does provide an upper bound.

For modeling, adsorption in geologic media is most often expressed as the distribution of material between liquid phase (here, the mobile uranium and plutonium radioisotopes in natural water) and the immobile host rock, in this case, volcanic tuff. The coefficient expressed by the linear, Freundlich isotherm model is the "distribution coefficient" $\left(K_{D}\right)$ (see Appendix A):

$$
\mathrm{x} / \mathrm{m}=\mathrm{K}_{\mathrm{D}} \mathrm{C}_{\mathrm{e}}
$$

where

$$
\begin{aligned}
& \mathrm{x}=\text { the mass of attached adsorbate on the solid, } \\
& \mathrm{m}=\text { the mass of immobile solid, } \\
& \mathrm{C}_{\mathrm{e}}=\text { the equilibrium concentration of the adsorbate in the solution. }
\end{aligned}
$$

The Freundlich isotherm was one of the first developed and has been frequentiy used for adsorption of cation species in low concentrations in liquids where the kinetics of adsorption are fast. However, the validity of the Freundlich isotherm can be compromised when used in a geologic system, because (1) multiple adsorption sites are available since tuff is composed of several minerals, (2) the character of the exposed minerals can change spatially, and, (3) chemical equilibrium between species of $\mathrm{Pu}(\mathrm{III})$ and $\mathrm{Pu}(\mathrm{IV})$ or $\mathrm{U}(\mathrm{IV})$ and U(VI) can potentially take hundreds of years.

Various surface complexation models such as double layer, triple layer, and constant capacitance models have been proposed (Kent et al., 1988). Although these models represent a more robust modeling approach because chemical properties of the adsorbate and adsorbent are evaluated, they have several fitting parameters that must be determined for the chemical system. In addition, these models require detailed modeling of the water chemistry. Mechanistic modeling has been applied in industrial situations for trace element adsorption onto iron oxyhydroxide (e.g., Papelis and Leckie, 1988). However, these models are only slowly replacing the linear Freundlich isotherm model for geologic situations because data for evaluating the fitting parameters of the complexation models are not readily available nor are species data for detailed modeling of the water chemistry.

\# Although precipitation can also retain radioisotopes on the surface, herein, precipitation is not considered a mechanism of adsorption. It is considered separately below because its behavior is different at various concentrations. Specifically, precipitation retains a species only when the aqueous species exceeds its solubility limit; thus, precipitation provides an upper limit to the amount of species that can be transported away. In contrast, the activity (concentration) of the radioisotopes determines the amount adsorbed for the adsorption mechanisms mentioned.

** Because adsorption was attributed initially to only one of a few possible mechanisms, rather than all, geochemists prefer to use the more inclusive term "sorption." 
In this report, the analyses have relied upon ranges and distributions for the distribution coefficient $\left(K_{D}\right)$ from YMP expert judgment to properly reflect the uncertainty in the data from experiments (Wilson et al., 1994) (Figure 4-15). Both Jacobsson and Rundberg (1997) and Langmuir (1978) report very high distribution coefficients for uranium on alumina and goethite, respectively, in the absence of carbonate. ${ }^{\text {it }}$ However the presence of carbonate drops the adsorption three orders of magnitude (Hsi and Langmuir, 1985), especially at a higher $\mathrm{pH}$ as might occur due to the presence of concrete, and is reflected in the estimates for rust from Wilson et al. (1994). For Np, we used separate $\mathrm{K}_{\mathrm{D}} \mathrm{s}$ for $\mathrm{Np}$ in Fe-bearing corrosion products, zeolitic tuffs, and nonzeolitic (vitric and devitrified) tuffs for the 1997 PA.

For Fe-bearing corrosion products, we used a range of 0 to $2000 \mathrm{ml} / \mathrm{g}$ with a uniform probability distribution. The upper limit of this range is based on a recent batch-sorption study with zeolitic tuff in contact with pure, synthetic hematite $\left(\mathrm{Fe}_{2} \mathrm{O}_{3}\right)$ in contact with J-13 groundwater at a pH of 7.4 to 7.6 (see Triay et al., 1996a, p. 19). These values are identical or very similar to the average J-13 field $\mathrm{pH}$ of 7.4 reported by Harrar et al. (1990). Triay et al. (1996a) also reported that decreasing the $\mathrm{pH}$ of J-13 in contact with hematite from a range of 7.4 to 7.6 to a range of 6.7 to 6.9 decreases the $N p K_{D}$ from a range of about 100 to $2000 \mathrm{ml} / \mathrm{g}$ to a range of 200 to $900 \mathrm{ml} / \mathrm{g}$. However, it is unclear whether this difference is significant or simply a result of the lack of precision in quantifying $K_{D} S$ when the dissolved concentration of ${ }^{237} \mathrm{~Np}$ after a sorption experiment is low. The lower limit of this range is based on the observation by Triay et al. (1996a) that "Although the ... sorption of $\mathrm{Np}$... onto pure hematite is large, [Np] sorption onto devitrified tuffs, which appear to have traces of hematite $(1 \% \pm 1)$, is essentially zero. This result could be due to differences in the surface of pure hematite compared to hematite in tuff. It could also be due to passivation of the hematite surfaces in the tuff by elements (such as the rare earths) that have a higher affinity for hematite than neptunium ... and, thus occupy the sorption sites."

For zeolitic tuffs, we used a range of 0 to $3 \mathrm{ml} / \mathrm{g}$ with a uniform probability distribution. The upper limit of this range is based on batch-sorption experiments with zeolitic tuff in contact with J-13 groundwater at a pH of 7.0 to 7.3 (Triay et al., 1996a). Furthermore, a column-transport study with crushed zeolitic tuff supports a value of $3 \mathrm{ml} / \mathrm{g}$ for this rock under in situ conditions (Triay et al., 1996b). However, Triay et al. (1996a) also reported that increasing the $\mathrm{pH}$ of J-13 in contact with zeolitic tuff from about 7 to 8.5 decreases the $\mathrm{Np} \mathrm{K}_{\mathrm{D}}$ from about 3 to $1.5 \mathrm{ml} / \mathrm{g}$. Robinson et al. (1996) attributed this decrease to complexation of the dissolved $\mathrm{Np}(\mathrm{V})$ species $\mathrm{NpO}_{2}{ }^{+}$by $\mathrm{CO}_{3}{ }^{2-}$ or $\mathrm{OH}^{-}$: clinoptilolite (the main mineral in zeolitic tuffs) sorbs $\mathrm{NpO}_{2}^{+}$(the dominant dissolved $\mathrm{Np}$ species under acidic and neutral conditions), but does not sorb species such as $\mathrm{NpO}_{2}\left(\mathrm{CO}_{3}\right)^{-}, \mathrm{NpO}_{2}\left(\mathrm{CO}_{3}\right)_{2}^{3-}, \mathrm{NpO}_{2}(\mathrm{OH})^{\circ}$, and $\mathrm{NpO}_{2}(\mathrm{OH})_{2}{ }^{-}$, (the dominant Np species under basic conditions). Furthermore, Robinson et al. (1996) concluded that in saturated-zone groundwater such as UE-25P\#1, complexation of $\mathrm{NpO}_{2}{ }^{+}$by $\mathrm{CO}_{3}{ }^{2-}$ and $\mathrm{OH}^{-}$becomes significant at $\mathrm{pH}$ values about one unit lower than in J-13. Reactions between groundwater and cementitious materials in the drifts (drift liners, inverts, etc.) could increase the $\mathrm{pH}$ of unsaturated-zone fluids from neutral or nearly neutral to basic or very basic values; these basic conditions could persist along a significant portion of the flow path before dilution by uncontaminated groundwater or reaction with tuffs neutralizes them. Therefore, we used a lower limit of $0 \mathrm{ml} / \mathrm{g}$ and a uniform distribution for the range in zeolitic tuffs.

For nonzeolitic tuffs, we used a value of $0 \mathrm{ml} / \mathrm{g}$ for the $\mathrm{Np} \mathrm{K}_{\mathrm{D}}$. This value is based on the conclusion by Triay et al. (1996a) that "The sorption of $\mathrm{Np}(\mathrm{V})$... in J-13 water onto devitrified and vitric tuffs, albite, and quartz is essentially zero."

\subsubsection{Climate}

The effect of climate on the disposal system is related to its impact on the hydrologic system, the atmospheric influences on the unsaturated zone, and the impact of the glacial cycle on increasing precipitation and possibly raising the water table in the future. In addition, climatic factors can affect biosphere modeling. The overall suitability of the potential repository site is closely related to the site's dry climate, subsequent low infiltration rates, deep water table, and low population density which, in part, is related to sparse water resources.

it Samama (1986, Figure 6-1) reports a $\mathrm{K}_{\mathrm{D}}$ of $15 \mathrm{~m}^{3} / \mathrm{kg}$ for a natural goethite-rich sample in $1 \mathrm{mM} \mathrm{NaCl}$ solution, but this value is still not enough to cause criticality. 


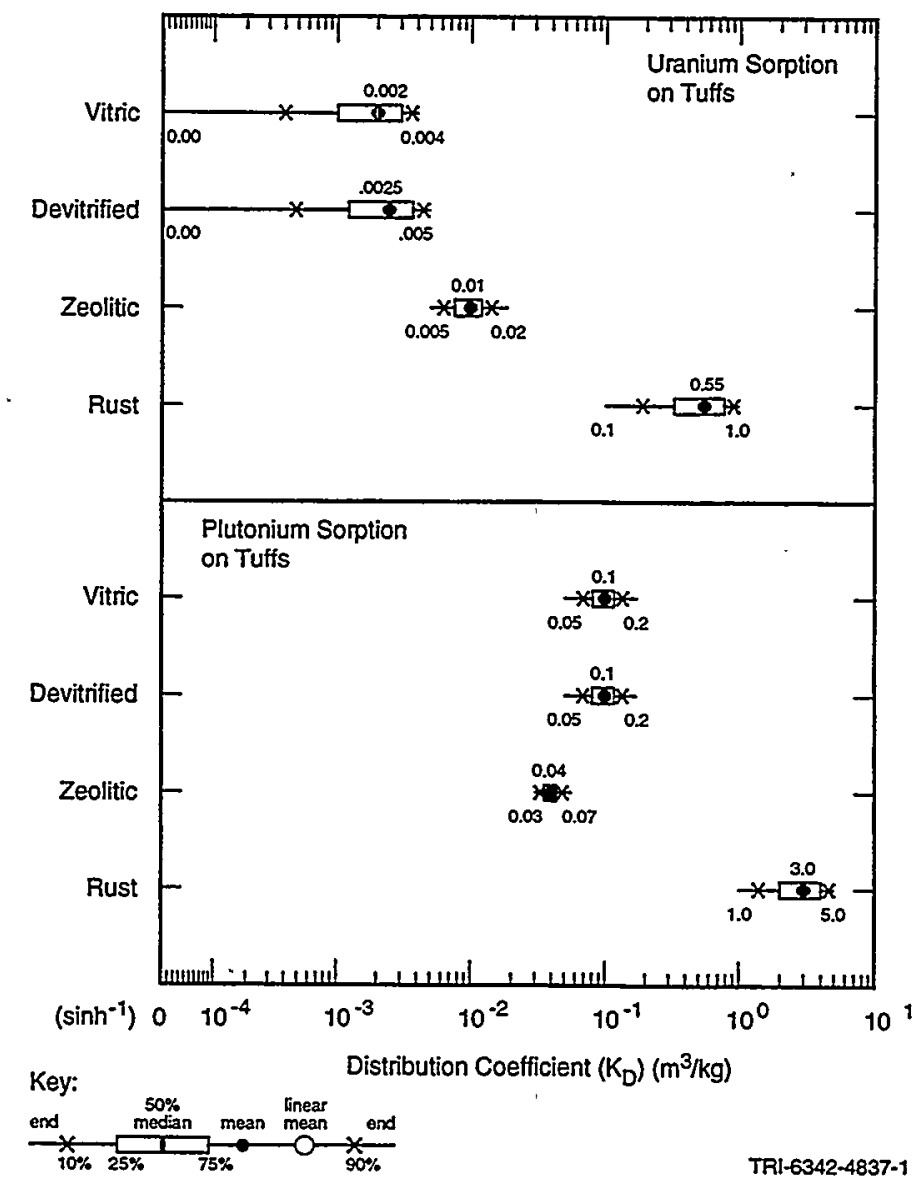

Figure 4-15. Estimates of distribution coefficients for uranium and plutonium adsorption on rust (i.e., goethite) and volcanic tuff at Yucca Mountain, Nevada, in J-13 well water. 
The potential repository site at Yucca Mountain is located in an area that is arid to semi-arid and has long, hot, dry summers and short, mild winters with thunderstorms periodically from April through October. Winds are generally gentle, but can be strong in the spring and during storms. Annual precipitation is low overall; although it increases at higher elevations, it is still limited. Summer temperatures are also cooler at higher elevations. In general, humidity is low, evaporation is high, and vegetation is sparse and mostly xeric. Yucca Mountain's climate fits the general climatological classification of midlatitude desert (modified Keoppen system per Critchfield, 1983). In midlatitude deserts there are large fluctuations in temperature annually and diurnally with significant variability in precipitation from year to year (DOE, 1988b, Chapter 5). Table 4-8 provides some representative average, minimum, and maximum values for basic meteorologic measurements either at Yucca Mountain or from nearby stations. Figure 4-16 shows the location of weather stations in the vicinity of Yucca Mountain, including at Yucca Flat, which is cited as having weather representative of that at Yucca Mountain.

Table 4-8. Climate and Meteorological Conditions Typical for Yucca Mountain ${ }^{\mathrm{a}}$

\begin{tabular}{|c|c|}
\hline Temperature & $\begin{array}{l}\text { Average annual daily maximum, } 22.5^{\circ} \mathrm{C}\left(72.5^{\circ} \mathrm{F}\right) \\
\text { Average daily minimum, } 3^{\circ} \mathrm{C}\left(37.4^{\circ} \mathrm{F}\right) \\
\text { Daily maximum average }(\text { July }), 35.6^{\circ} \mathrm{C}\left(96.1^{\circ} \mathrm{F}\right) \\
\text { Daily minimum average (December), } 6.7^{\circ} \mathrm{C}\left(19.9^{\circ} \mathrm{F}\right)\end{array}$ \\
\hline Relative Humidity & $\begin{array}{l}\text { Minimum monthly average, } 25 \% \text { (June or July) } \\
\text { Maximum monthly average, } 55 \% \text { (December) } \\
\text { Annual average, } 53 \% \text { ( } 4 \text { a.m.) }\end{array}$ \\
\hline Wind & $\begin{array}{l}\text { Speed } 5.4 \mathrm{~m} / \mathrm{s}(12 \mathrm{mph}) \text { with diurnal variation related to heating, } \\
\text { southerly to northerly predominant with terrain affecting local direction }\end{array}$ \\
\hline Pressure $^{b}$ & Annual average, $26.1 \mathrm{in}$. \\
\hline Precipitation & $\begin{array}{l}\text { Annual average } 133 \mathrm{~mm} \\
\text { Daily maximum } 89 \mathrm{~mm}^{\mathrm{c}}\end{array}$ \\
\hline \multicolumn{2}{|c|}{$\begin{array}{l}\text { Some data are from weather stations in the vicinity of Yucca Mountain, especially at Yucca Flat, where } \\
\text { longer term data have been collected. DOE (1988b) is the source of these data, except as cited. } \\
\text { Pressure is measured using wet bulb depression correction. } \\
\text { Flint et al., draft of "Conceptual and Numerical Model of Infiltration for Yucca Mountain Area, Nevada." }\end{array}$} \\
\hline
\end{tabular}

Climate and Unsaturated Zone Aeration. As a result of climatic factors of barometric pressure and related wind changes, vapor and air movement occurs in the thick unsaturated zone at Yucca Mountain. It impacts thermal gradients in both the unsaturated and saturated zones as well as the supply of atmospheric gases. Air movement through Yucca Mountain has been observed in the ESF, and LeCain (1997) observed air movement in boreholes that he attributed mainly to air flow through fractures. The 1994 PA considered air flow as a pathway for migration of radionuclide gas, including ${ }^{14} \mathrm{C}$, which replaces nonradioactive carbon in carbon dioxide. It is not considered as a pathway in the $1997 \mathrm{PA}$, because current regulatory guidance regarding dose calculation methods renders negligible the impact of the low amount of gaseous radionuclide migration that has been modeled by previous studies ([M\&O, 1995, p. 7-20]). The implications of air flow to geothermal gradient are discussed in the Section 4.3.2.

Air flow from the atmosphere is the primary source of $\mathrm{O}_{2}, \mathrm{~N}_{2}$, and water vapor, which affects several important 1997 PA modeling considerations. In the unsaturated zone BRAGFLO_T handles two phase flow and calculates $\mathrm{O}_{2}$, $\mathrm{N}_{2}$, and water vapor over time near a waste package. See Chapter 7 for details of how these processes were modeled. The flow of oxygen, which comes primarily from air movement in the mountain, is important in corrosion calculations, which assume inputs of atmospheric carbon dioxide, and nitrogen and use atmospheric pressure values. Atmospheric gases, especially the availability of oxygen, are important factors in modeling geochemical interactions amount groundwater, waste, and waste containers. Modeling the near-field impact of these factors is a focus for the $1997 \mathrm{PA}$ and is discussed in detail Chapter 7. 
4. System Characterization: Geologic Barrier .

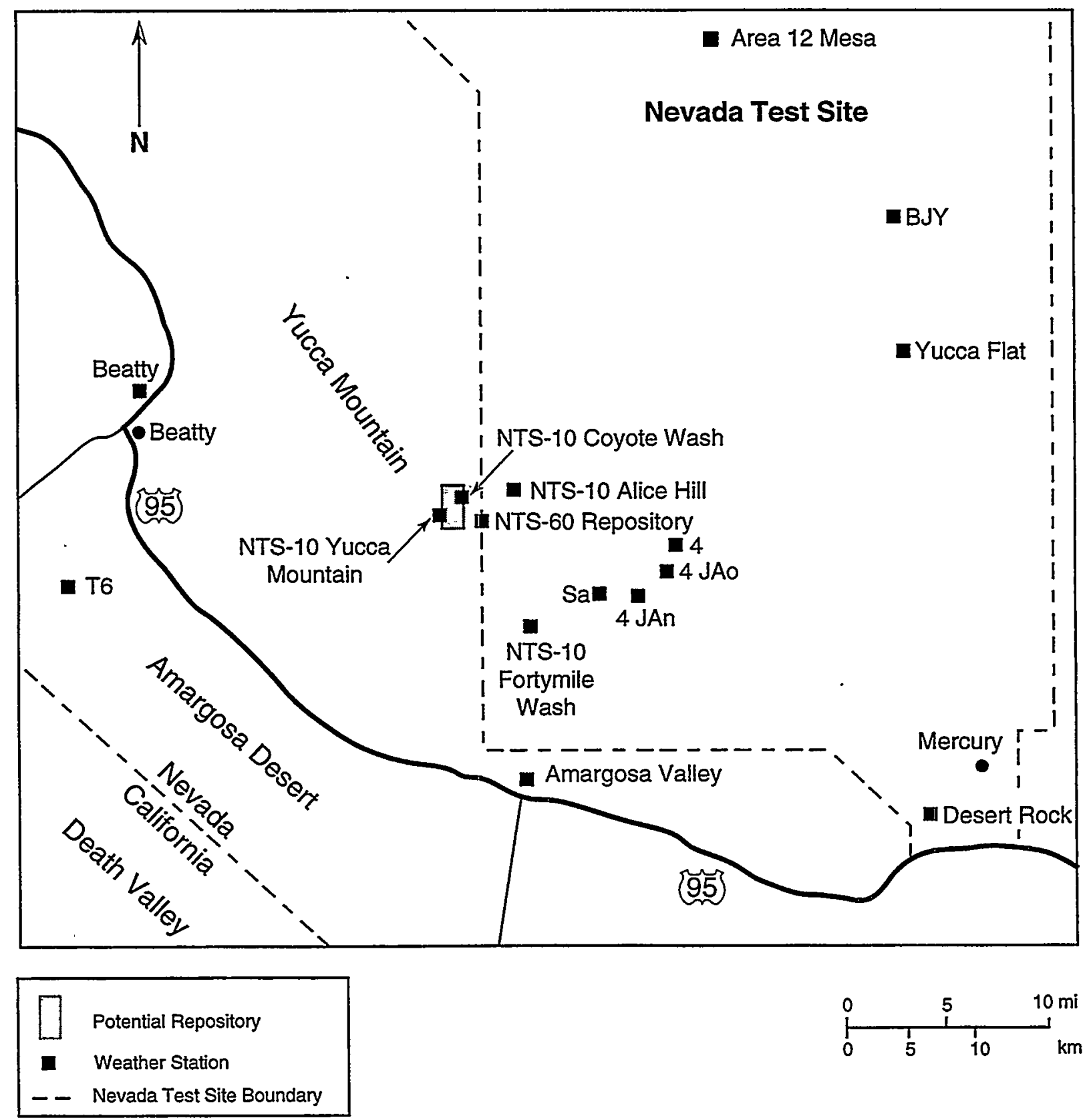

TRI-6342-5292-0

Figure 4-16. Location of weather stations in the vicinity of Yucca Mountain, including Yucca Flat, which is cited as having weather representative of that at Yucca Mountain. 
Glacial Cycle. Major variations in Earth's climate are related to glacial cycles about 100,000-yr long wherein continental glaciation appears to increase and decrease as the amount of solar radiation received by Earth increases and decreases (Imbrie and Imbrie, 1979). Until 400,000 years ago, it appears that the glacial cycles were about 41,000 -yr long, a timing that seems to be better correlated to changes in insolation than does the 100,000 -yr cycle. Forester et al. (1996) state that the last glacial period was about 40 to $10 \mathrm{ka}$. TSPA-1995 (M\&O, 1995a) stated that given the long periods of time modeled, in some cases up to $1,000,000 \mathrm{yr}$, it is likely that changes in climate will occur resulting in changes that will affect net infiltration and percolation flux in the unsaturated zone, elevation of the water table, and changes in advective flux in the saturated zone. The same study also noted that, at the time of publication, process model results were not available on the potential effects of these changes (M\&O, 1995a).

In general, the most common approach to representation of wetter glacial periods in modeling is to increase the infiltration rate (Rechard, ed., 1995; Bodvarsson et al., eds., 1997; Altman et al., 1996). a-TSPA-1997 did not vary climate in their model (M\&O, 1997). As explained in Chapter 8, the 1997 PA modeled increased precipitation indirectly by modeling the increased infiltration at the surface of the two-dimensional cross-section. The added moisture then percolated slowly through the strata. For infiltration, the $1997 \mathrm{PA}$ used the same uniform distribution of periods as in Rechard, ed. (1995), which is uniform from 40,000 to $160,000 \mathrm{yr}$, with a mean of $100,000 \mathrm{yr}$. The terminology for glacial periods is "wet climate" and for interglacial periods, it is "dry climate." Considerable variation in temperature probably exists within the 100,000-yr cycles (Boulton and Payne, 1992), so it is understood that the 100,000-yr cycle with uniform rates of change in temperature is an idealization of reality.

Climate and Biosphere Modeling. Both climate and meteorological data are of importance in biosphere modeling; however, the 1997 PA's conceptual model for exposure was based only on today's climate, populations, and farming practices. No variation with changing climate was modeled. The biosphere transport and dose model included a ranching family raising cattle and crops, using a well contaminated by the maximum expected radionuclide concentration, and having a stock pond that waters the cattle and can be a source of contaminated dust. The dust from the dried-up pond is transported $500 \mathrm{~m}$ in the model (see Chapter 10).

LaPlante et al. (1995) provides much of the information regarding pathways used by the GENII-S code, which calculates radiation doses to individuals or populations from acute or chronic radionuclide releases to air, water, or soil. They stated that for an analogous determination of local water uses and agricultural practices, the Amargosa Valley region, approximately $32 \mathrm{~km}(20 \mathrm{mi})$ south of the Yucca Mountain potential repository site, is a reasonable focal point. For data relevant to the Yucca Mountain area they used NOAA and NCDC meteorological information from the Desert Rock station and EPA compilations of wind data from the Yucca Flat station (Figure 4-16). 


\subsection{Summary of Assumptions for Geologic Barrier Conceptual Model}

Table 4-9. Summary of Geologic Barrier Conceptual Models including Geology, Hydrology, Geochemistry and Climate

\begin{tabular}{|c|c|c|}
\hline $\begin{array}{l}\text { System } \\
\text { or Process }\end{array}$ & $\begin{array}{l}\text { Unsaturated Zone } \\
\text { Conceptual Model Assumptions }\end{array}$ & $\begin{array}{l}\text { Saturated Zone } \\
\text { Conceptual Model Assumptions }\end{array}$ \\
\hline $\begin{array}{l}\text { Fractures } \\
\text { In modeled } \\
\text { area of Yucca } \\
\text { Mountain }\end{array}$ & $\begin{array}{l}\text { Solitario Canyon and Ghost Dance Faults } \\
\text { have effect on flow; welded lithologies more } \\
\text { densely fractured than nonwelded; no } \\
\text { separation of effects of joints and faults }\end{array}$ & $\begin{array}{l}\text { Solitario Canyon, northern Bow Ridge, and } \\
\text { northwest trending Fault zones have effect } \\
\text { on flow; other assumptions same as UZ }\end{array}$ \\
\hline $\begin{array}{l}\text { Effect on flow, } \\
\text { permeability }\end{array}$ & $\begin{array}{l}\text { Solitario Canyon Fault, lower permeability } \\
\text { zone; Ghost Dance higher permeability } \\
\text { zone }\end{array}$ & $\begin{array}{l}\text { Faults listed above lower permeability, other } \\
\text { faults in model no effect on flow, permeable }\end{array}$ \\
\hline $\begin{array}{l}\text { Effect on } \\
\text { stratigraphy }\end{array}$ & Model layers offset across faults & No offset of model layers across faults \\
\hline Dip of fault planes & Zones representing fault planes vertical & Zones representing fault planes vertical \\
\hline Structural Dip & $\begin{array}{l}2 D \text { model, } 4.6 \text { degrees east tilt to model } \\
\text { layers }\end{array}$ & $\begin{array}{l}\text { 3D model, } 4.6 \text { degrees east dip, }<1 \text { degree } \\
\text { south tilt to model layers }\end{array}$ \\
\hline $\begin{array}{l}\text { Stratigraphy } \\
\text { Paintbrush } \\
\text { Group, Calico } \\
\text { Hills Formation, } \\
\text { Crater Flat } \\
\text { Group }\end{array}$ & $\begin{array}{l}\text { Simplified to } 12 \text { layers; See Figure } 4-9 \mathrm{~b} \text { and } \\
\text { Table } 4-3\end{array}$ & $\begin{array}{l}\text { Simplified to } 9 \text { layers; See Figure } 4-9 b \text { and } \\
\text { Table 4-3 }\end{array}$ \\
\hline $\begin{array}{l}\text { Geometry and } \\
\text { composition }\end{array}$ & $\begin{array}{l}\text { 2D layers high width to thickness ratio; vary } \\
\text { in texture (welding, lithophysae) and } \\
\text { composition (vitric, devitrified, zeolitic) } \\
\text { laterally homogeneous except for effect of } \\
\text { fault zones }\end{array}$ & Same as unsaturated zone, but 3D \\
\hline $\begin{array}{l}\text { Mineralogy, } \\
\text { density and } \\
\text { texture of tuff }\end{array}$ & $\begin{array}{l}\text { Modeling unit layers designations of } D, V \text {, } \\
\text { and } Z \text { assigned varying } K_{d} \text { (retardation } \\
\text { value); vitric, welded=low density, low } \\
\text { porosity; vitric, nonwelded=low density; } \\
\text { high porosity; devitrified=high density; low } \\
\text { porosity; zeolitized=low density (higher } \\
\text { than vitric), high porosity }\end{array}$ & Same as unsaturated zone \\
\hline $\begin{array}{l}\text { Groundwater } \\
\text { Geochemistry }\end{array}$ & $\begin{array}{l}\mathrm{CO}_{2}, \mathrm{O}_{2}, \mathrm{CO}_{3}, \mathrm{SiO}_{2} \mathrm{Eh}, \mathrm{pH}, \mathrm{Ca} \text {, and other } \\
\text { cations and anions important to near-field } \\
\text { reactions with canisters, waste }\end{array}$ & $\begin{array}{l}\text { Radionuclide concentration received from } \\
\text { unsaturated zone is only geochemistry mod- } \\
\text { eled }\end{array}$ \\
\hline Flow & $\begin{array}{l}\text { Slow flow=longer time for radionuclide } \\
\text { decay, decreasing exposure; radionuclides } \\
\text { filtered by flow through small pores, frac- } \\
\text { tures; percolation flux affects heat distribu- } \\
\text { tion; infiltration rate tied to glacial cycle, } \\
\text { altitude; fracture flow constitutes fast path } \\
\text { (not simulated in model) }\end{array}$ & $\begin{array}{l}\text { Saturated zone lies in upper volcanic aquifer; } \\
\text { regional carbonate aquifer not modeled; } \\
\text { recharge from regional volcanic aquifer simu- } \\
\text { lated by boundary heads matching potentio- } \\
\text { metric gradient; regional potentiometric } \\
\text { gradient moves flow to south/southeast; mod- } \\
\text { eled three different zones of potential to rep- } \\
\text { resent different local gradients; slow } \\
\text { flow=longer time for radionuclide decay }\end{array}$ \\
\hline Transport & $\begin{array}{l}\text { Radionuclide transport/precipitation/ sorp- } \\
\text { tion depend on } \mathrm{pH} \text {, silica, colloids, zeolites } \\
\text { and clays }\end{array}$ & $\begin{array}{l}\text { Radionuclide transport affected by } D, V, Z=K_{d} \\
\text { of layers }\end{array}$ \\
\hline
\end{tabular}


Table 4-9. Summary of Geologic Barrier Conceptual Models including Geology, Hydrology, Geochemistry and Climate (Continued)

\begin{tabular}{|c|c|c|}
\hline $\begin{array}{l}\text { System } \\
\text { or Process }\end{array}$ & $\begin{array}{l}\text { Unsaturated Zone } \\
\text { Conceptual Model Assumptions }\end{array}$ & $\begin{array}{c}\text { Saturated Zone } \\
\text { Conceptual Model Assumptions }\end{array}$ \\
\hline $\begin{array}{l}\text { Climate } \\
\text { Atmospheric } \\
\text { influence }\end{array}$ & $\begin{array}{l}\text { Air flows readily through unsaturated zone; } \\
\text { Input of } \mathrm{O}_{2} \text { keeps water oxic (Eh); Input of } \\
\mathrm{O}_{2}, \mathrm{~N}_{2} \text {, water vapor affect corrosion rates of } \\
\text { canisters; ambient temperature }\left(26^{\circ} \mathrm{C}\right) \text { input } \\
\text { to geochemical modeling; migration of gas- } \\
\text { eous radionuclides to accessible environ- } \\
\text { ment above site not modeled }\end{array}$ & Not modeled \\
\hline $\begin{array}{l}\text { Glacial cycle, } \\
\text { precipitation }\end{array}$ & $\begin{array}{l}\text { Modeled precipitation/infiltration cycle with } \\
\text { uniform distribution of periods from } 40,000 \\
\text { to } 160,000 \text { years, mean of } 100,000 \text { years; } \\
\text { glacial=wet climate, interglacial=dry }\end{array}$ & $\begin{array}{l}\text { Concentration of radionuclides impacted by } \\
\text { infiltration rate; no intake of infiltration inde- } \\
\text { pendent of radionuclide concentration }\end{array}$ \\
\hline $\begin{array}{l}\text { Human expo- } \\
\text { sure }\end{array}$ & $\begin{array}{l}\text { Surface water conditions impact forage, } \\
\text { animals, crop types, irrigation patterns; } \\
\text { winds disperse contaminated dust }\end{array}$ & $\begin{array}{l}\text { Radionuclide concentration at potential } \\
\text { repository, } 2.4 \mathrm{~km} \text { and } 5 \mathrm{~km} \text { boundaries } \\
\text { carried in well water used by farm family and } \\
\text { stockpond for ranchers }\end{array}$ \\
\hline
\end{tabular}


4. System Characterization: Geologic Barrier

\subsection{Modeling Parameters for Geologic Barrier}

Tables 4-10 through 4-23 list the parameters for the units in geologic barrier used by BRAGFLO_T. Tables 4-24 through 4-41 list the parameters for units in the geologic barrier used by STAFF3D. 


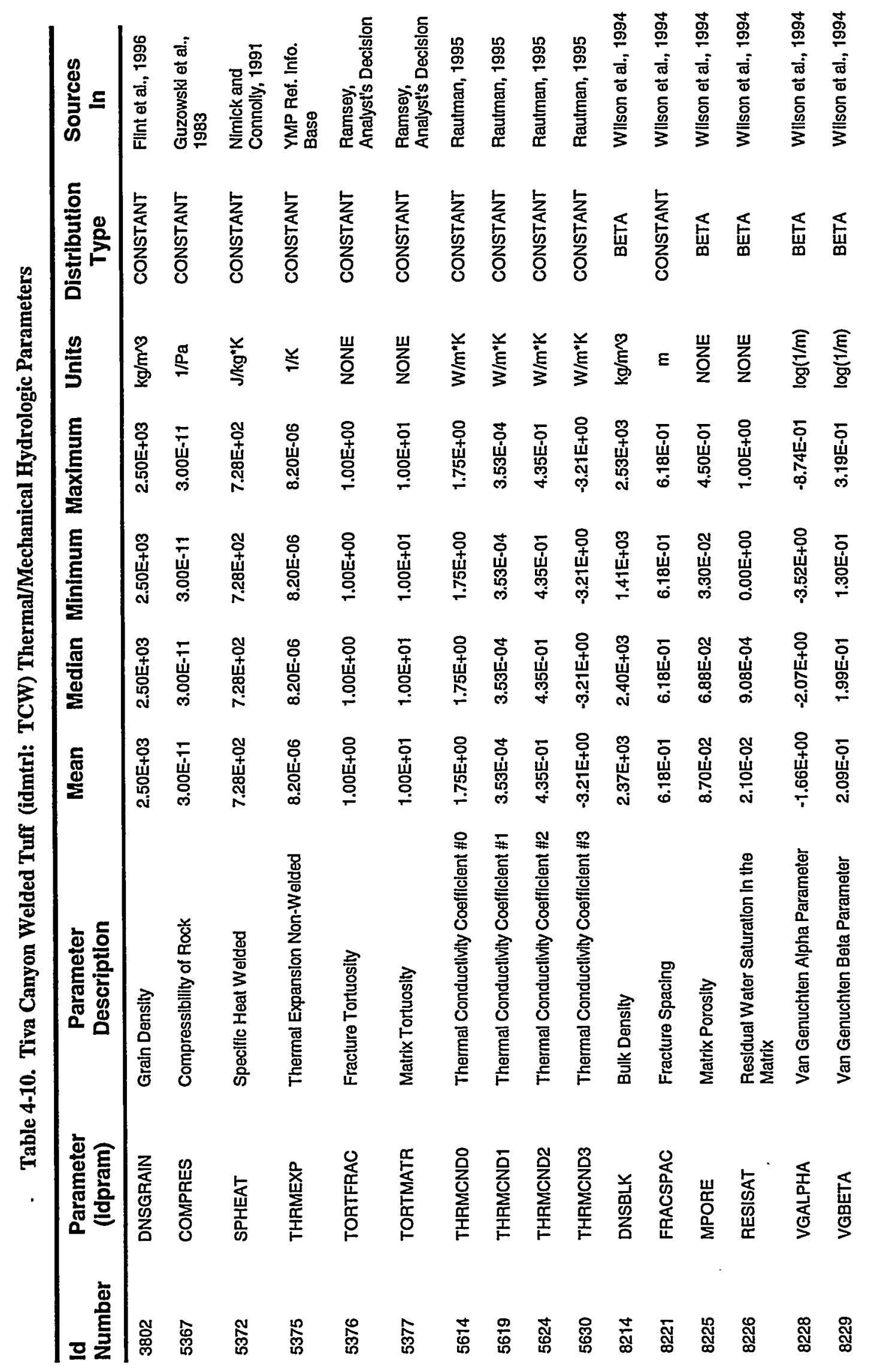

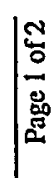




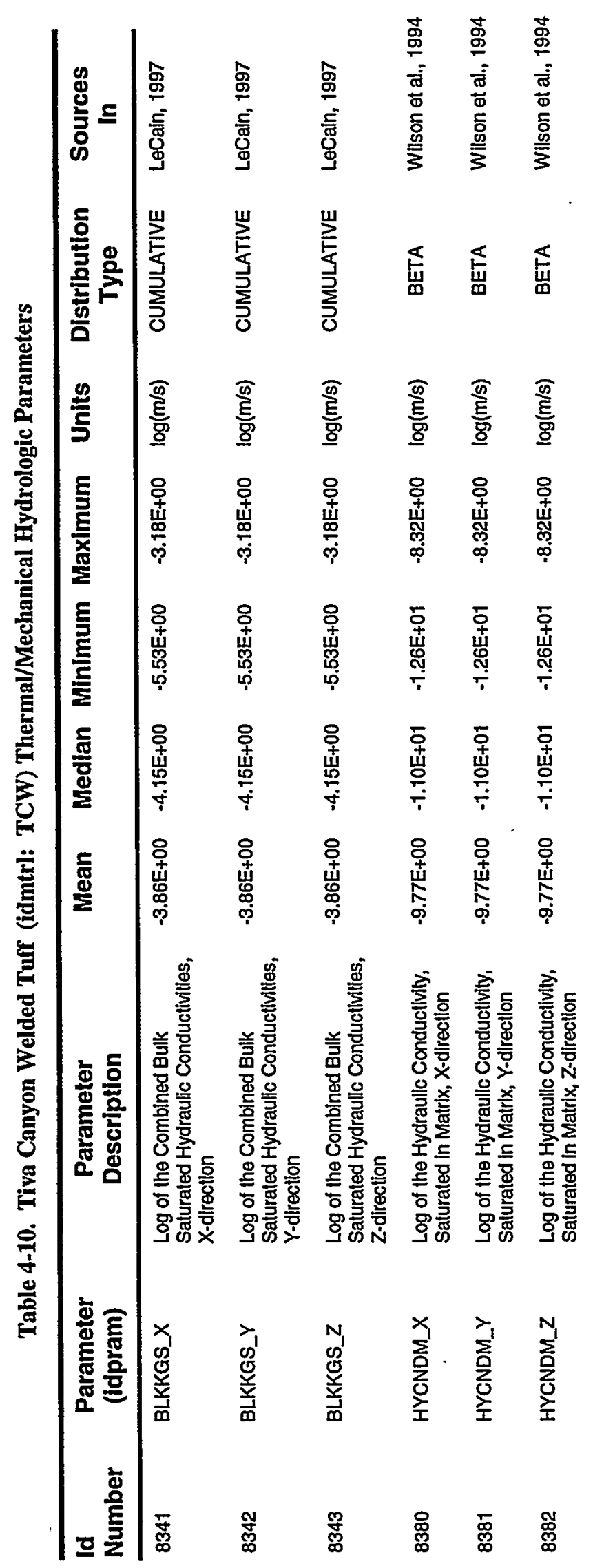




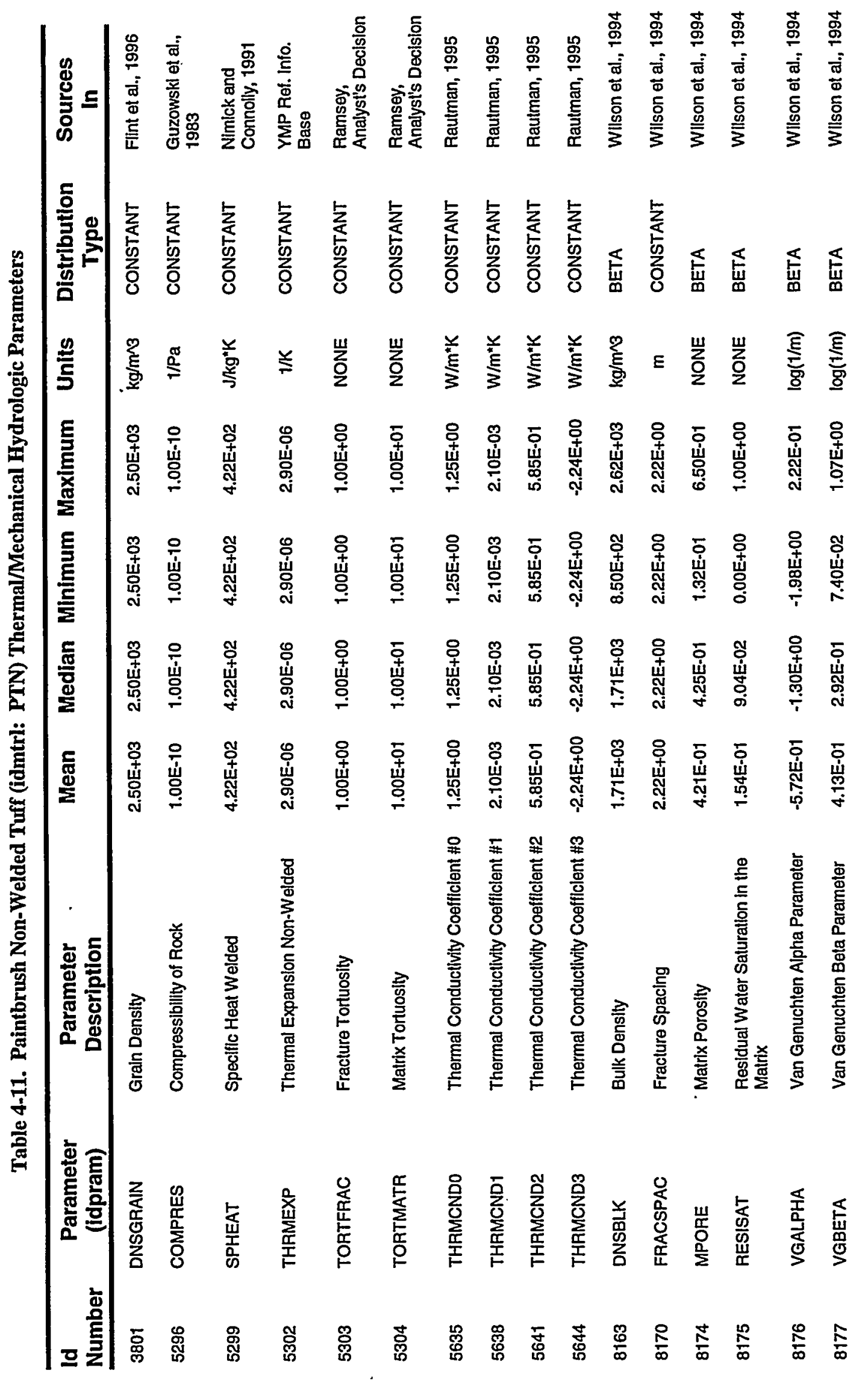

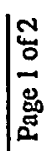




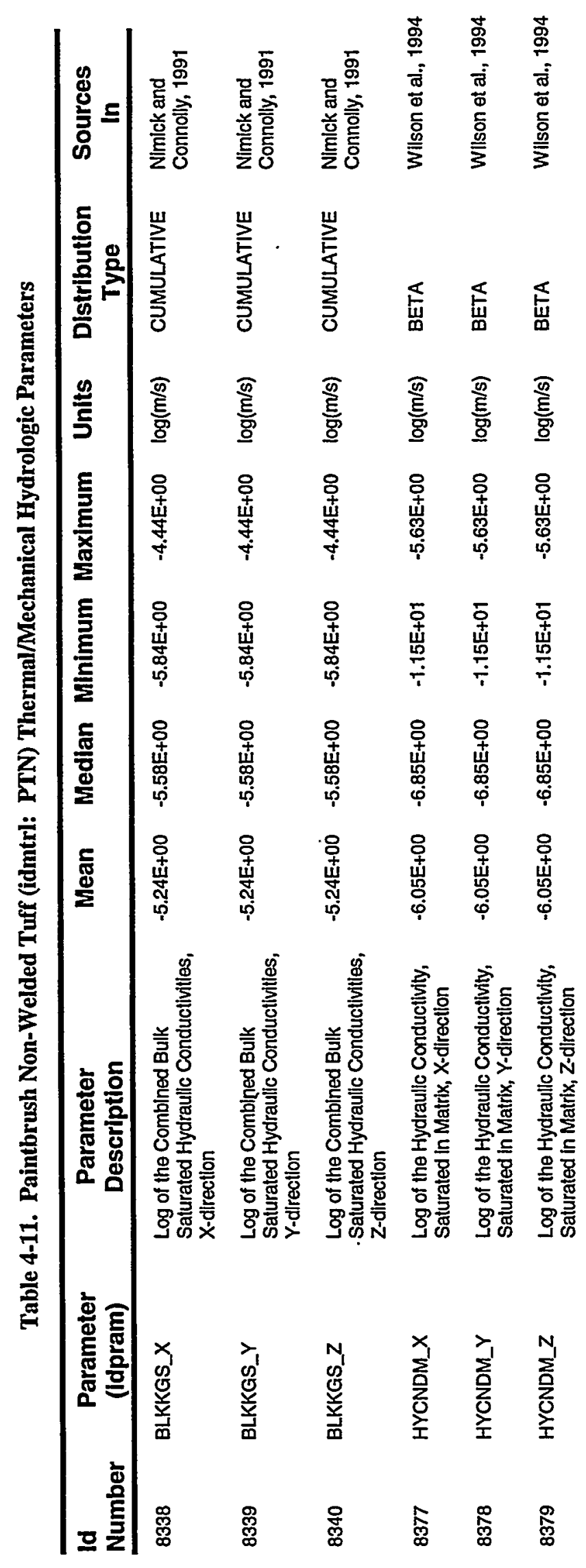




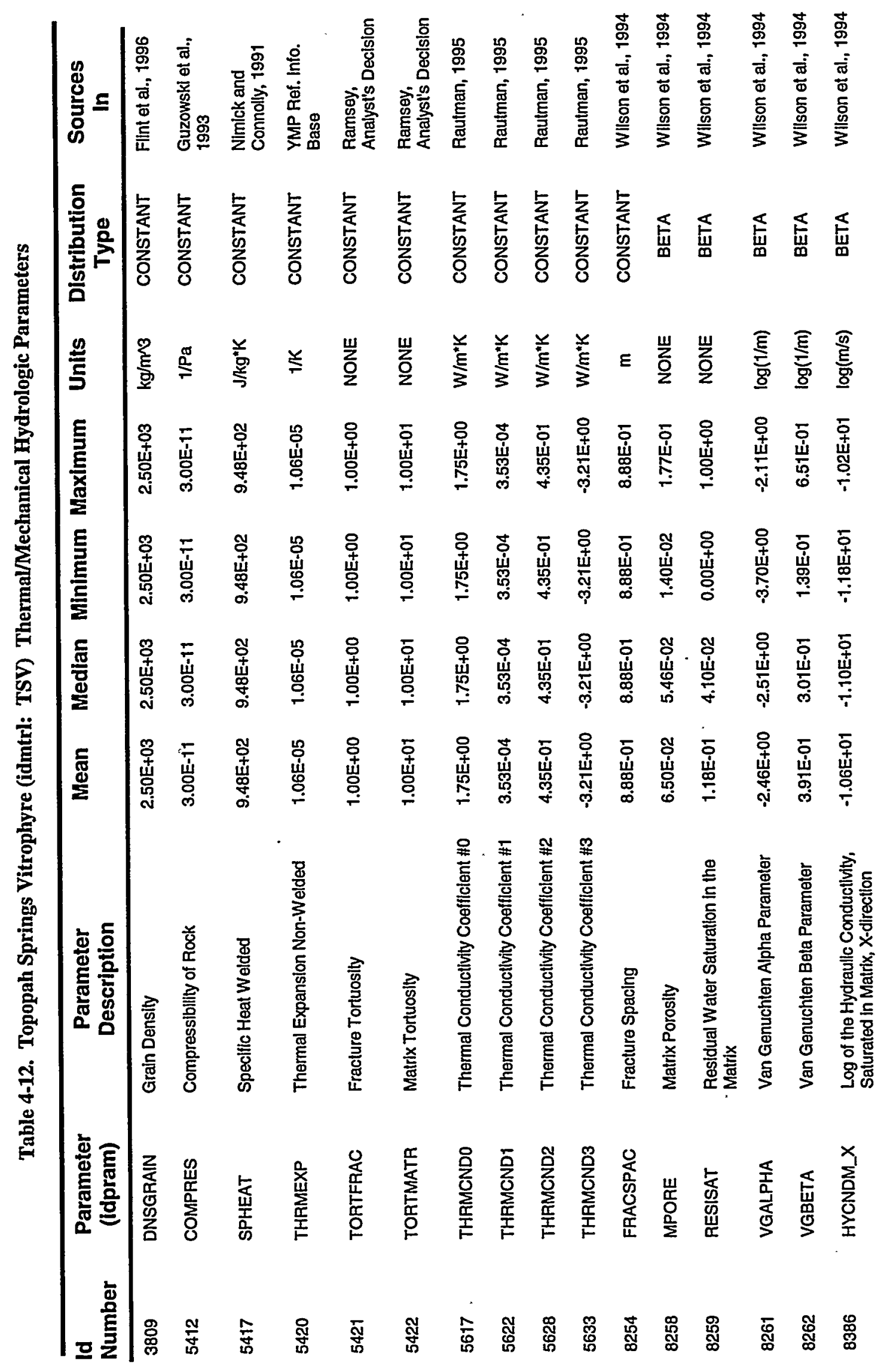




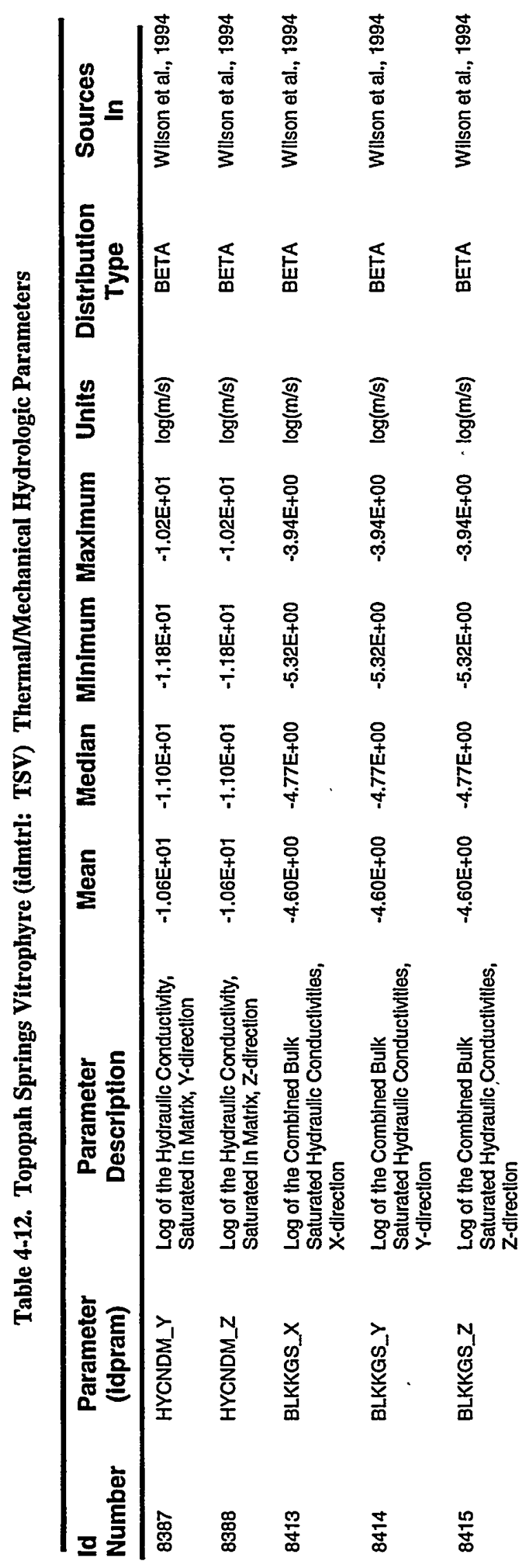




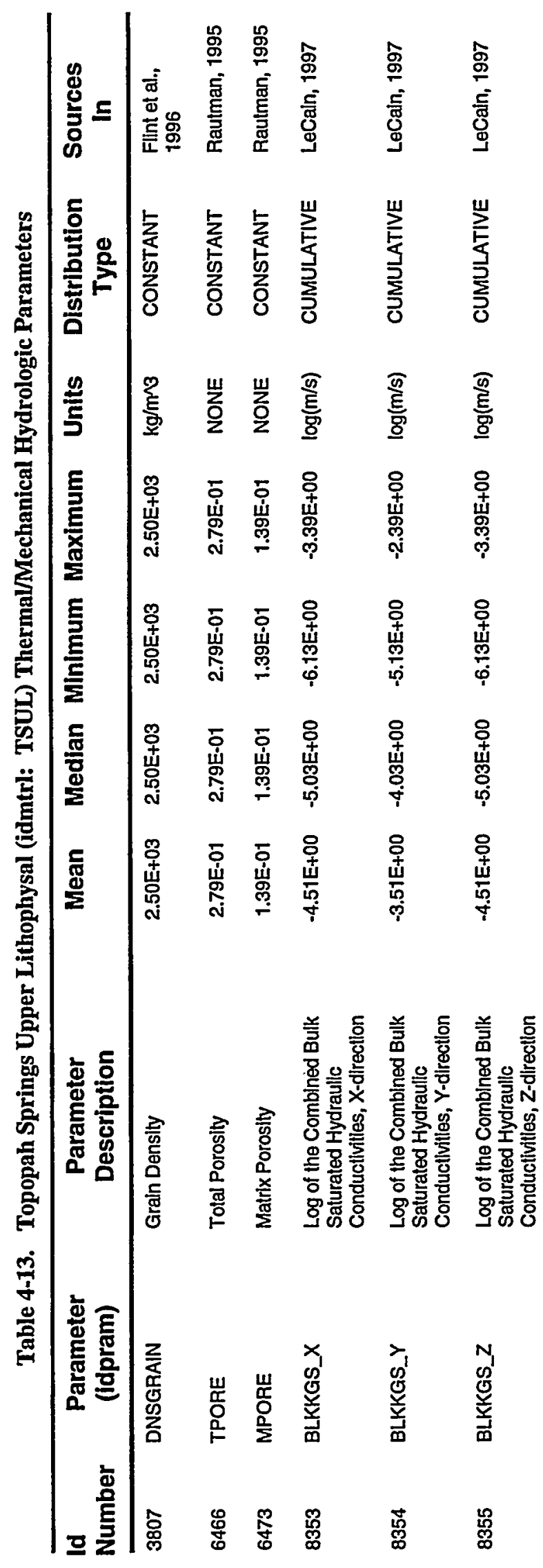




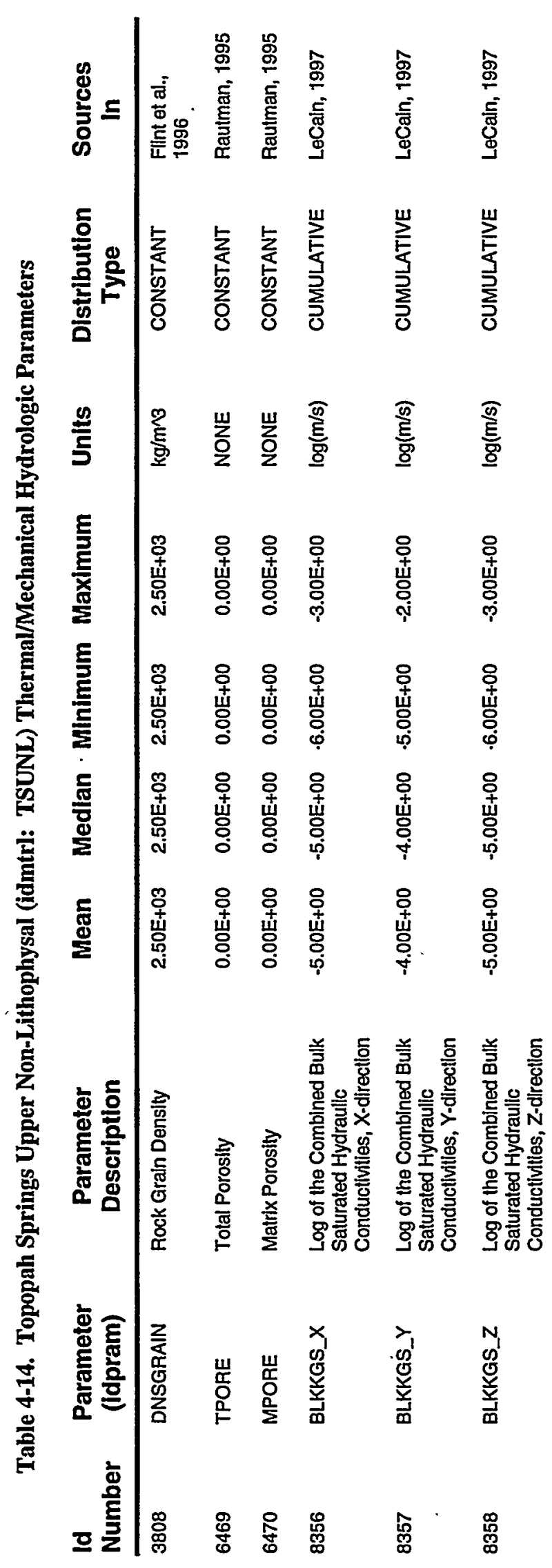




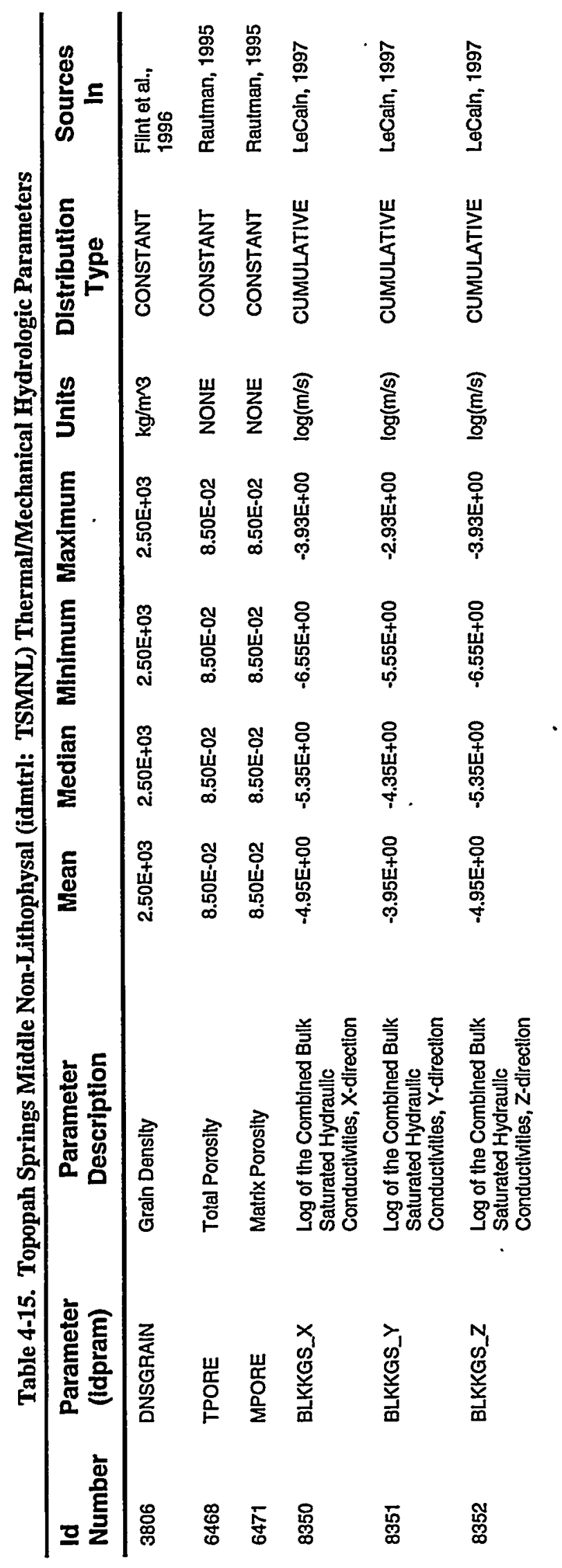




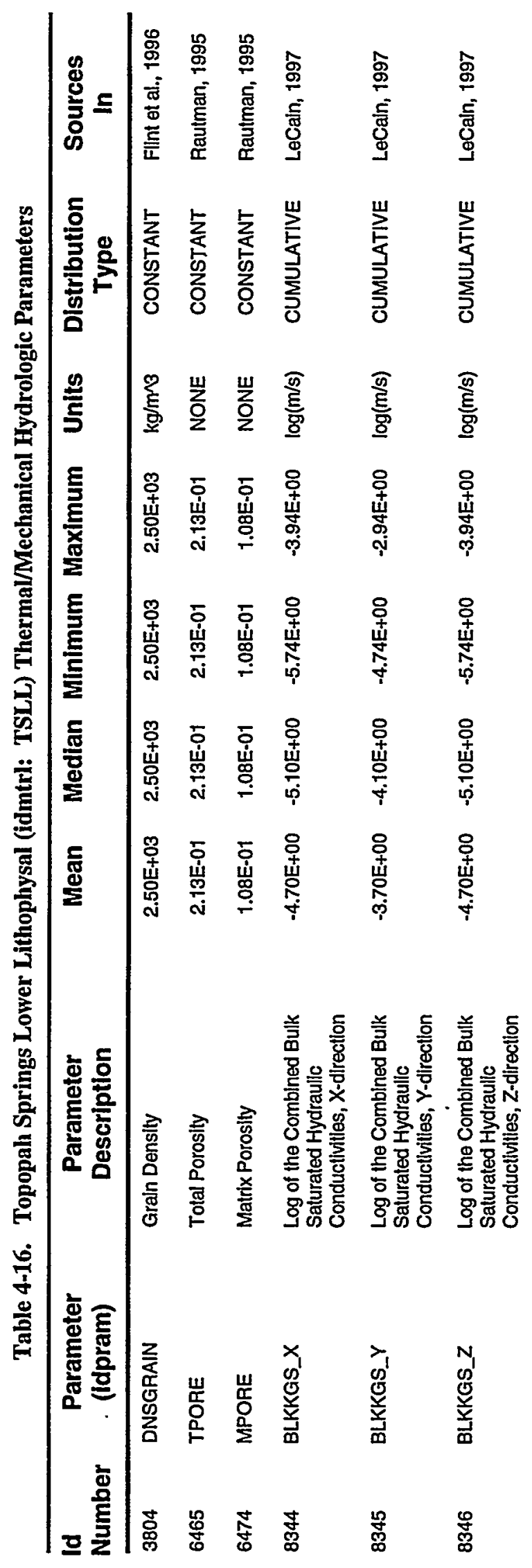




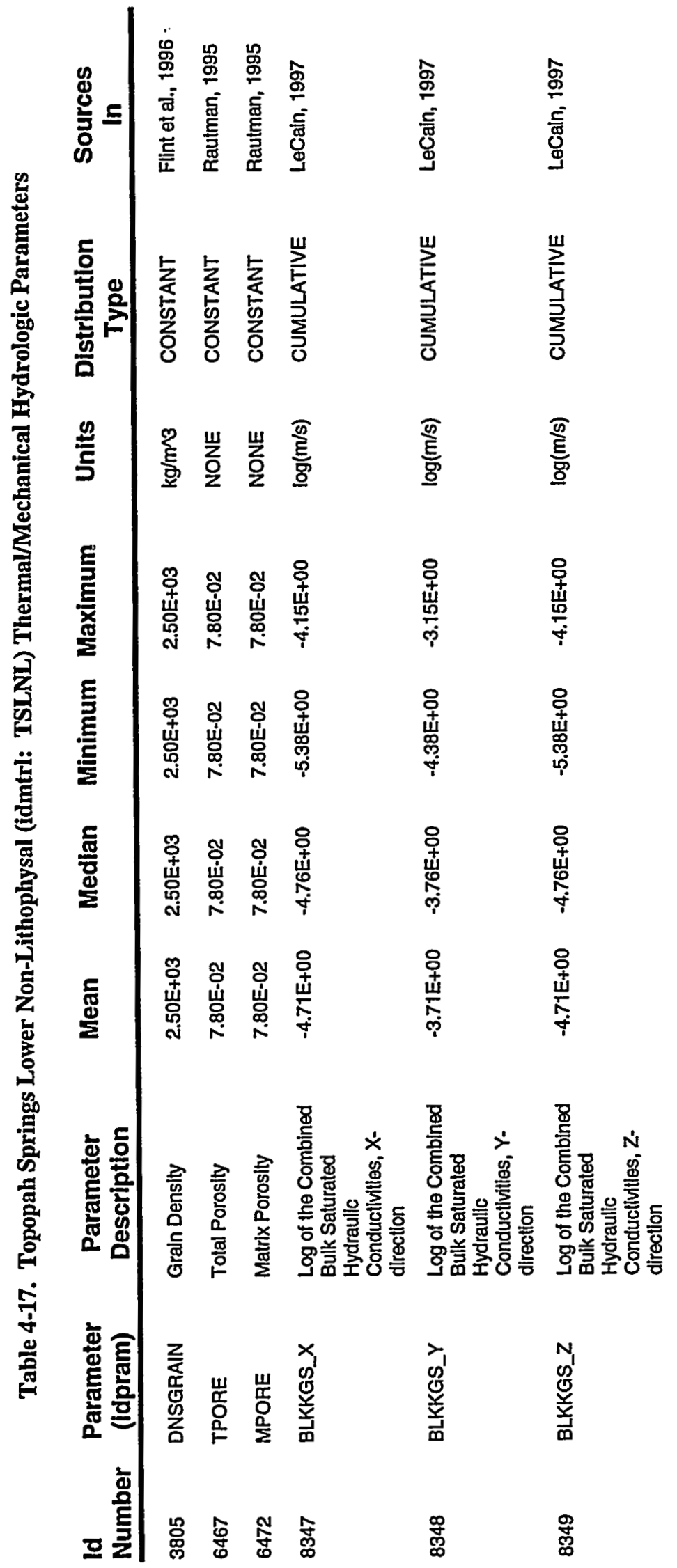

站 


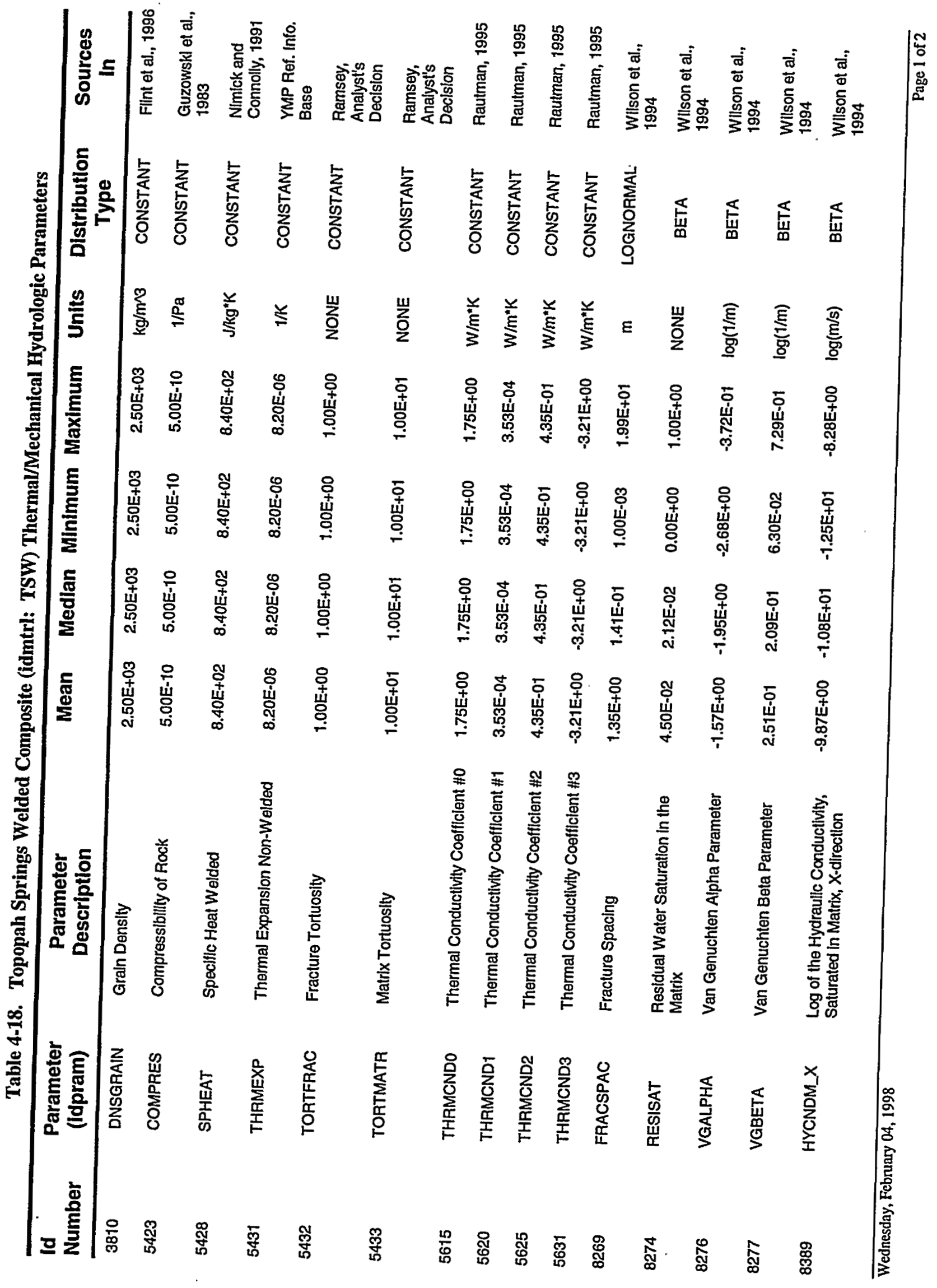



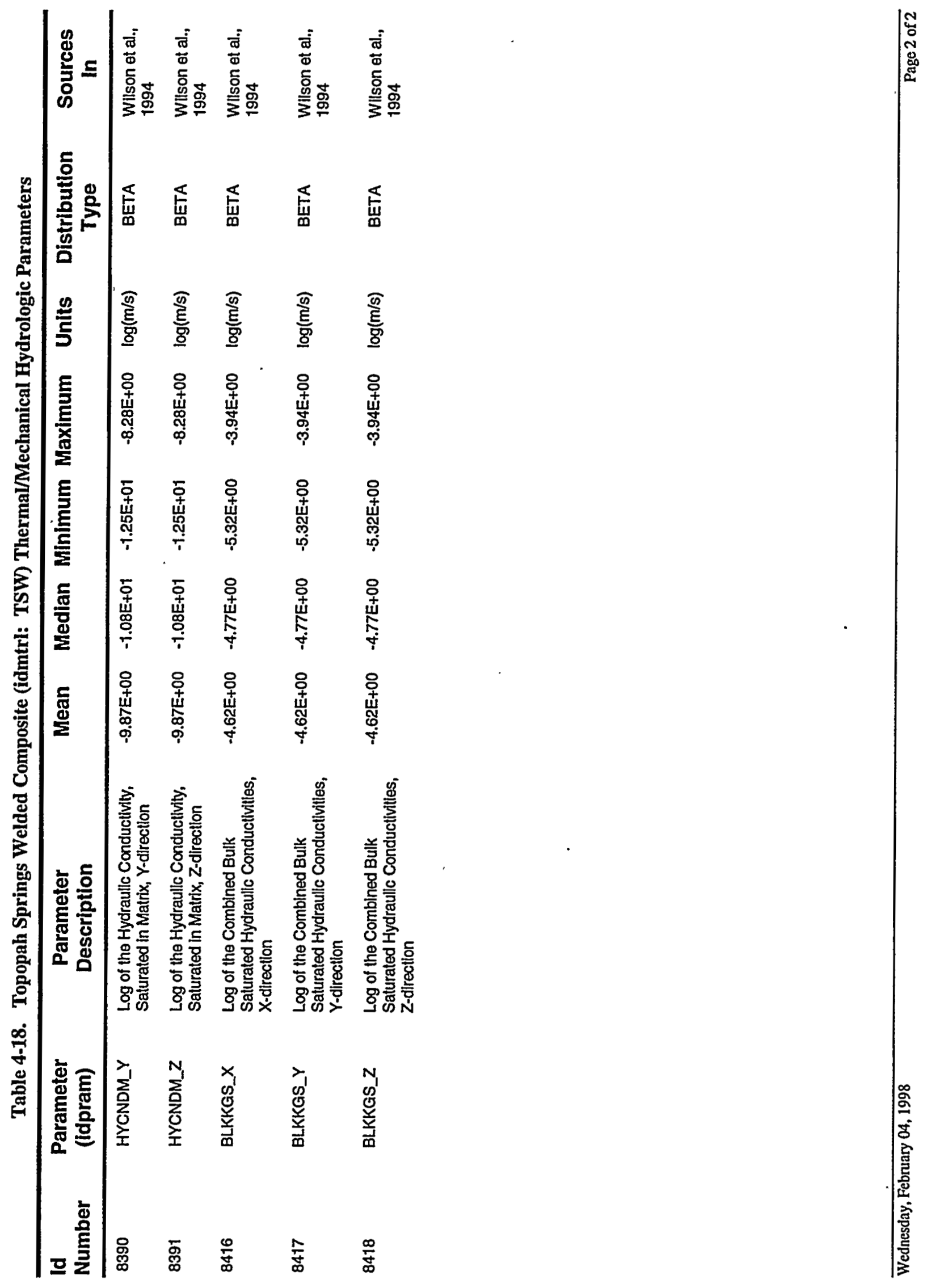


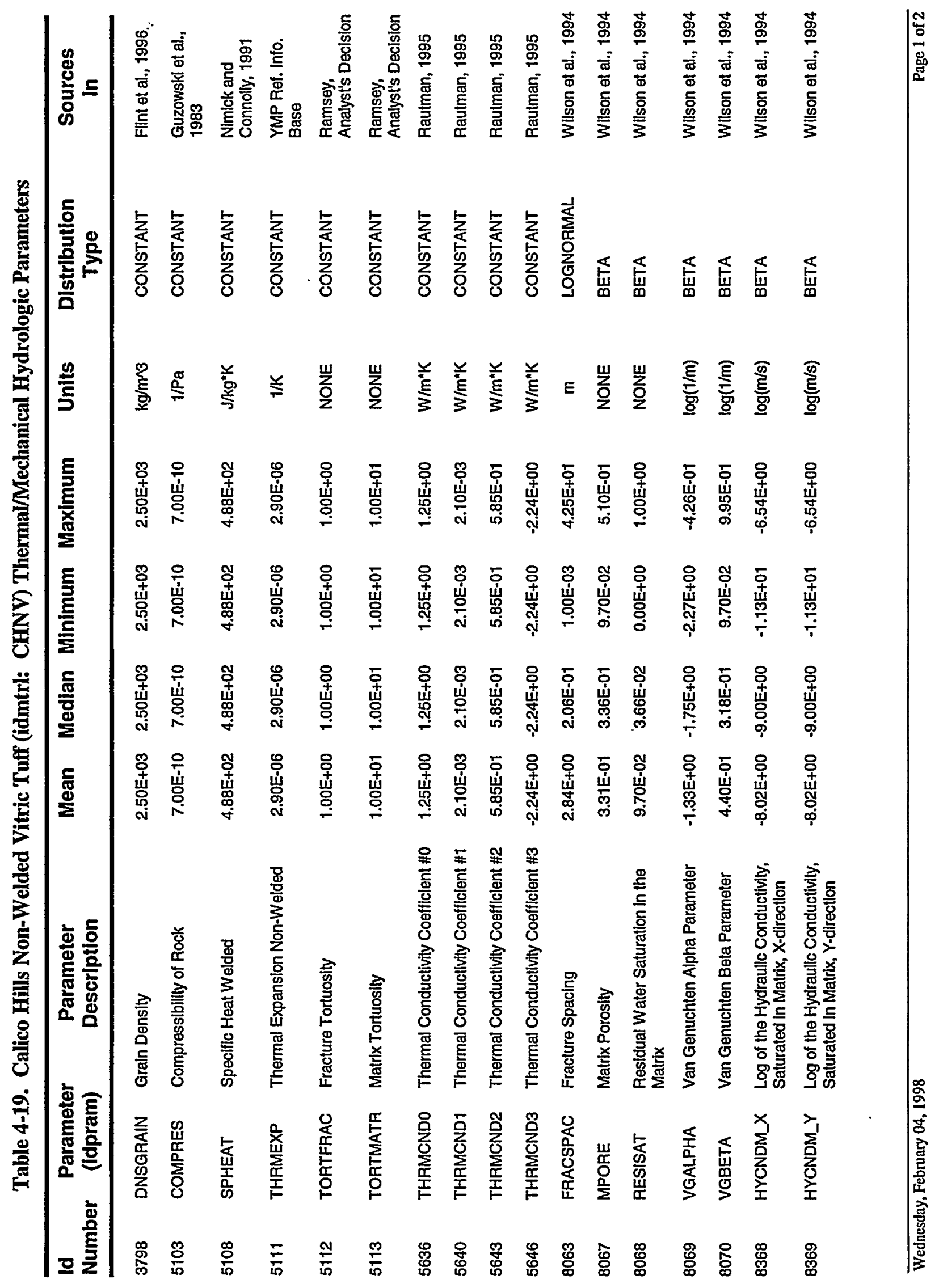




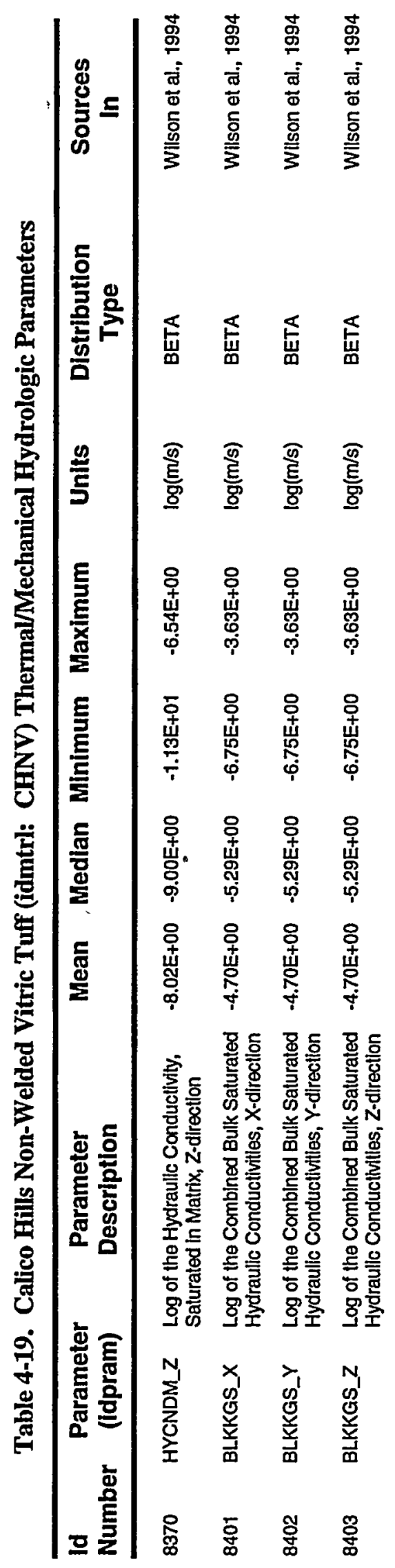




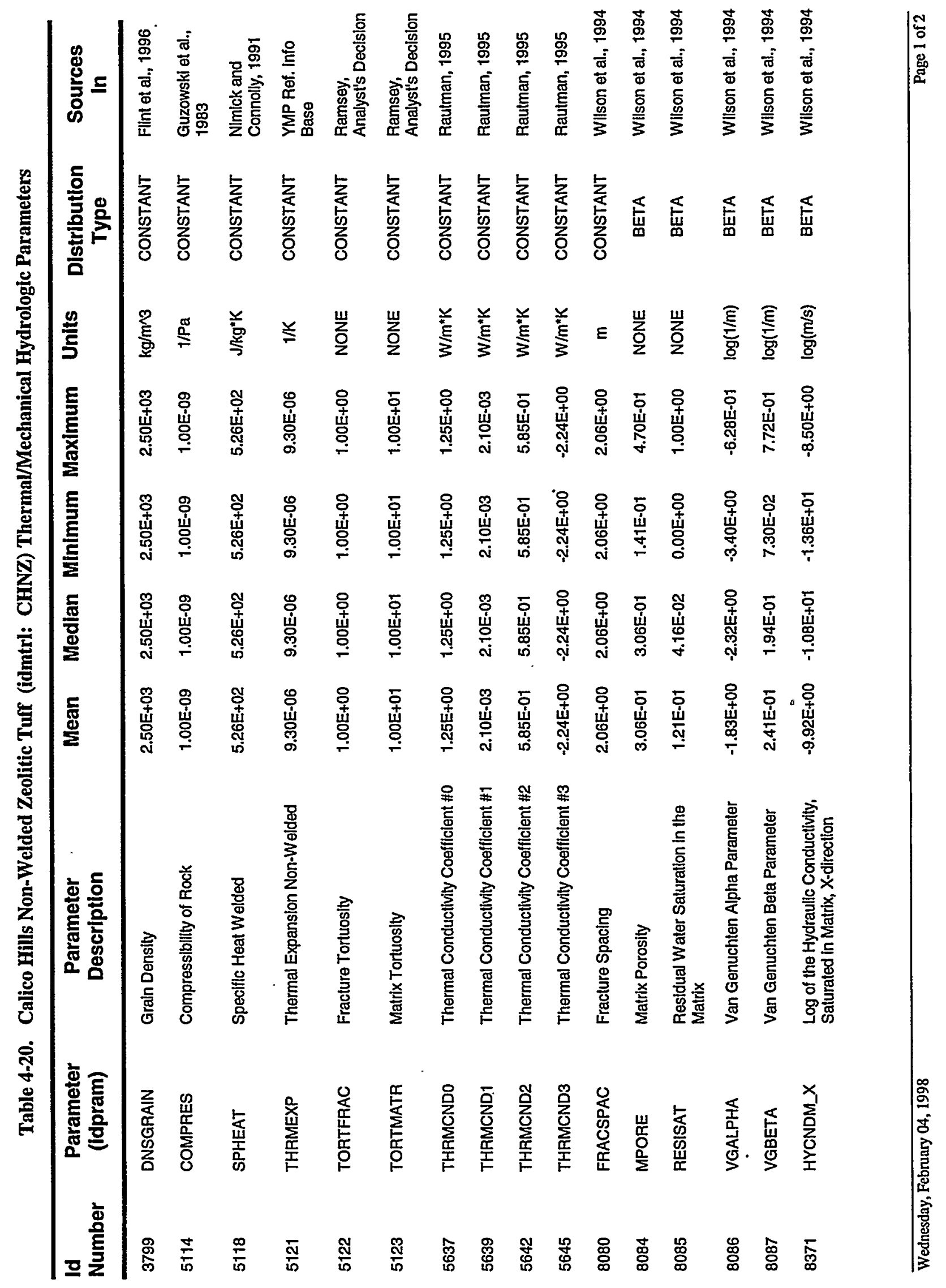




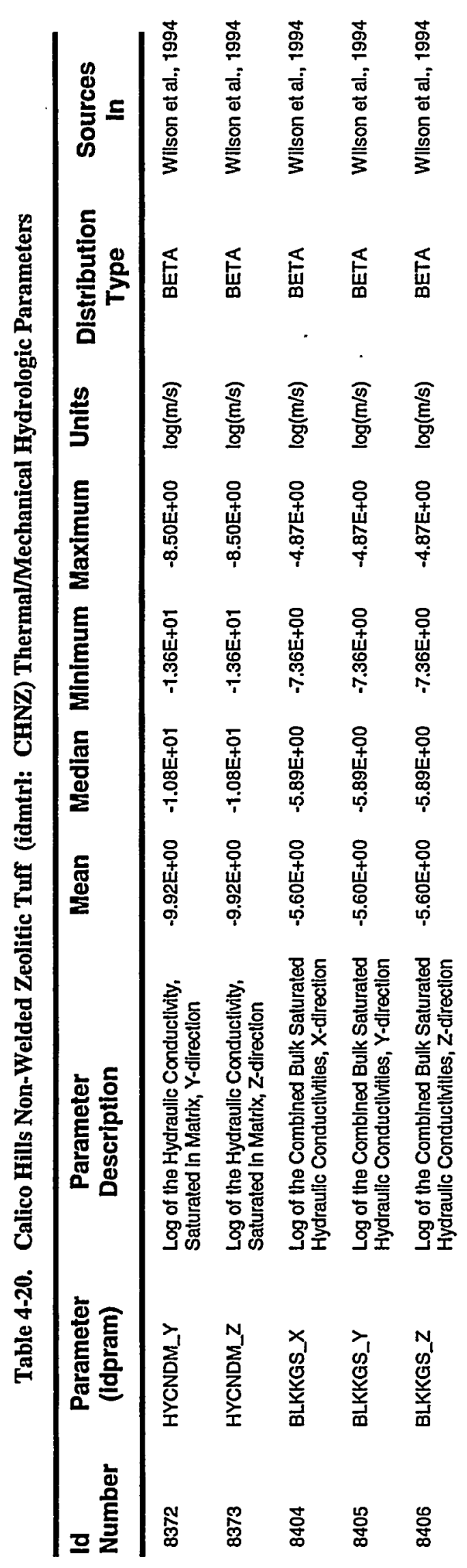

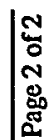




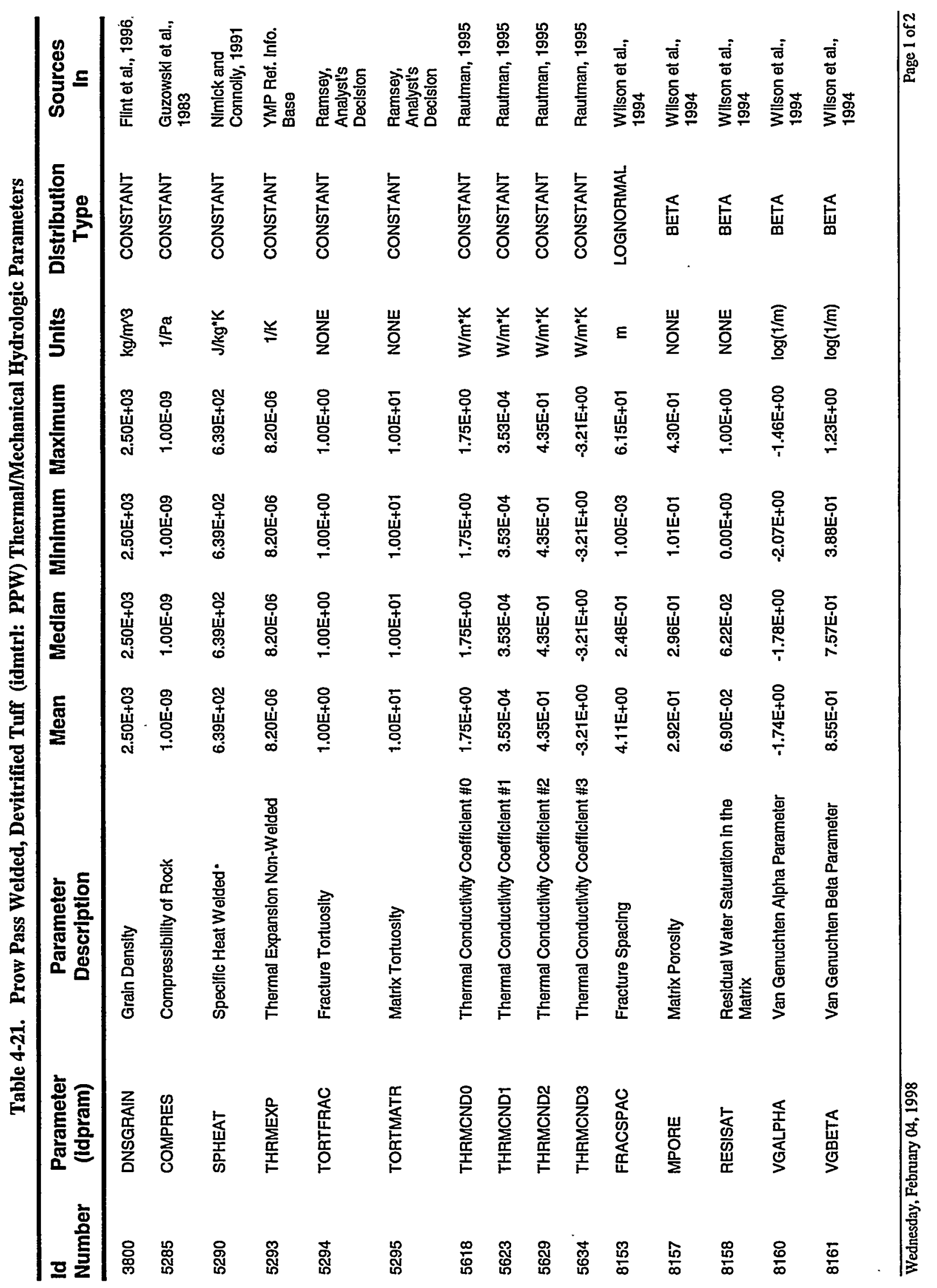




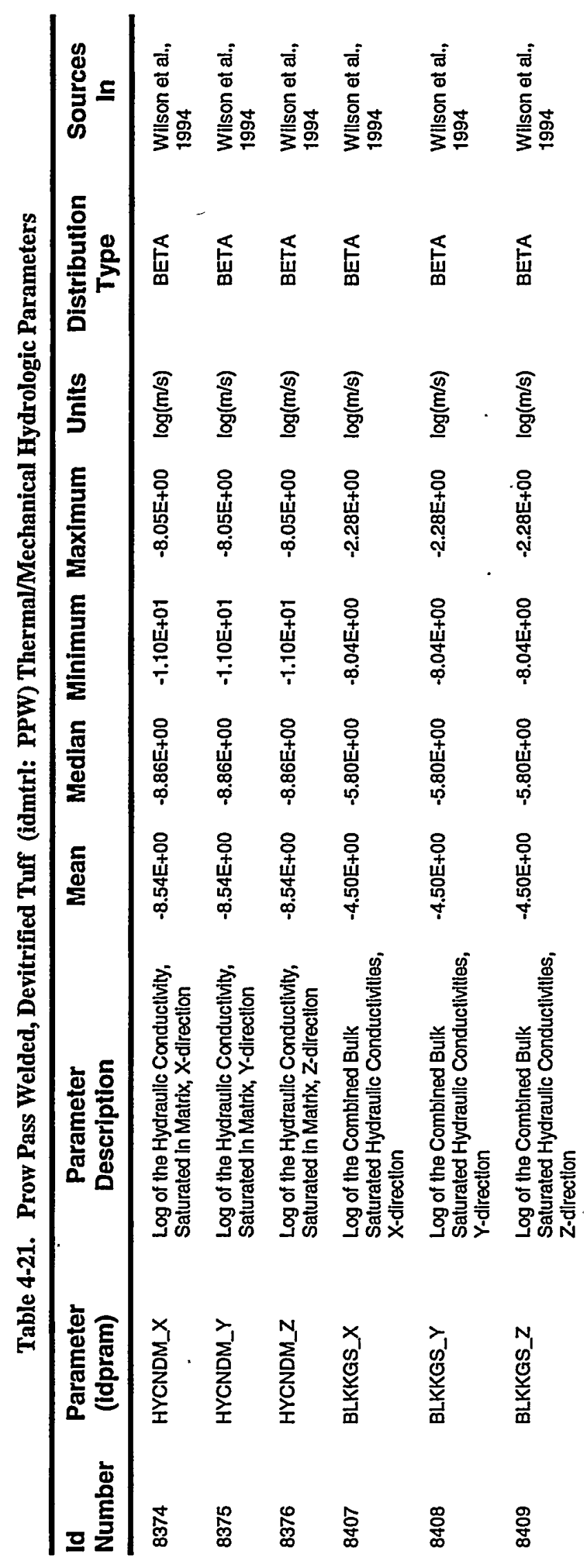




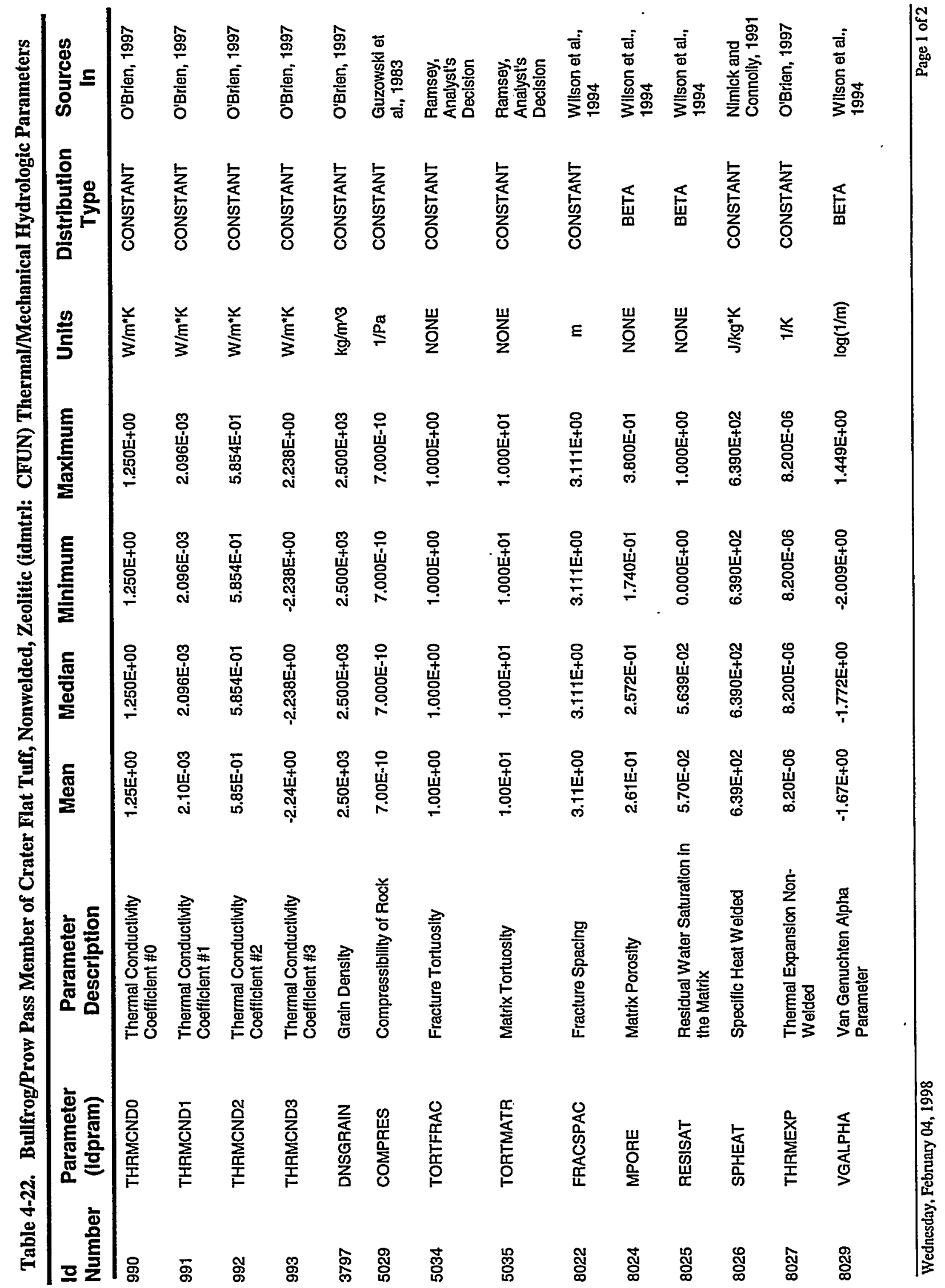




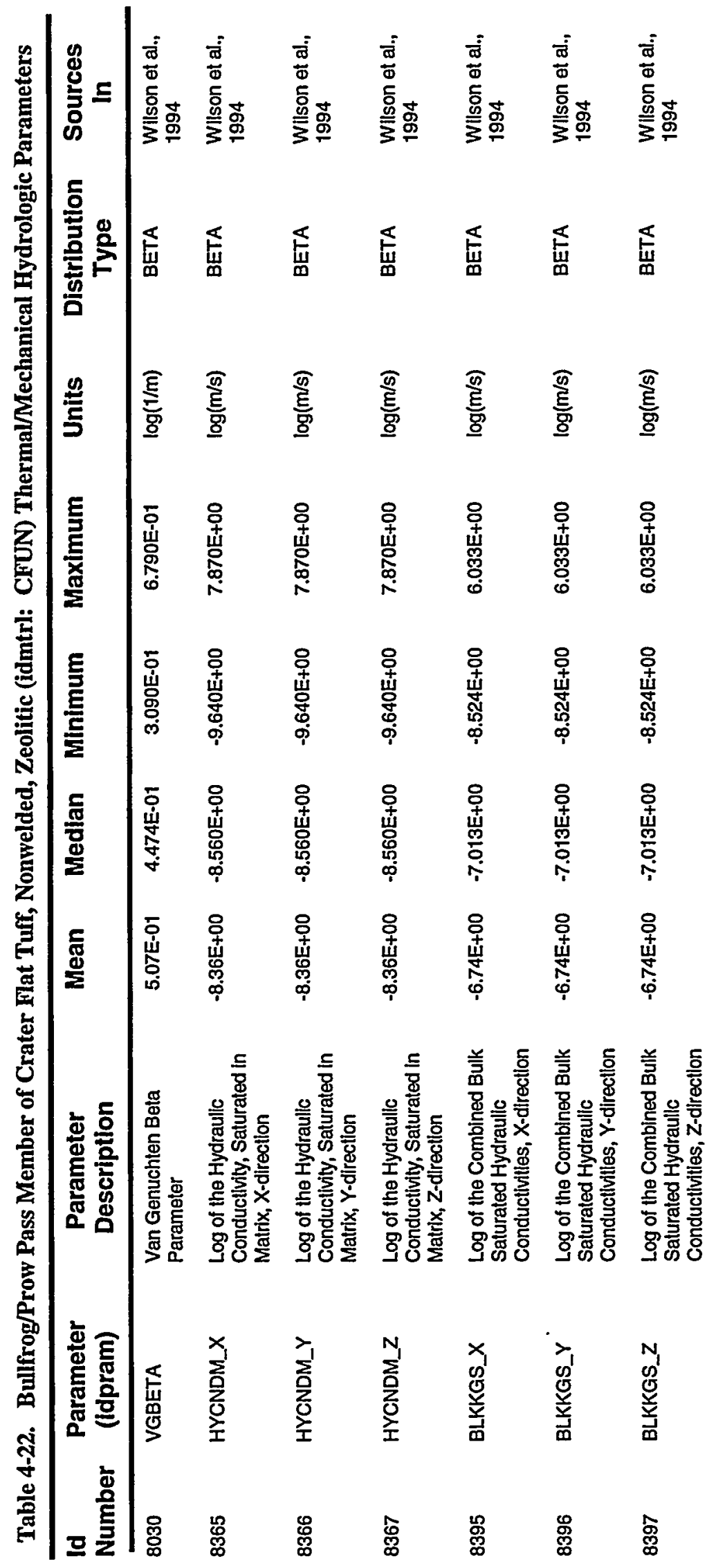




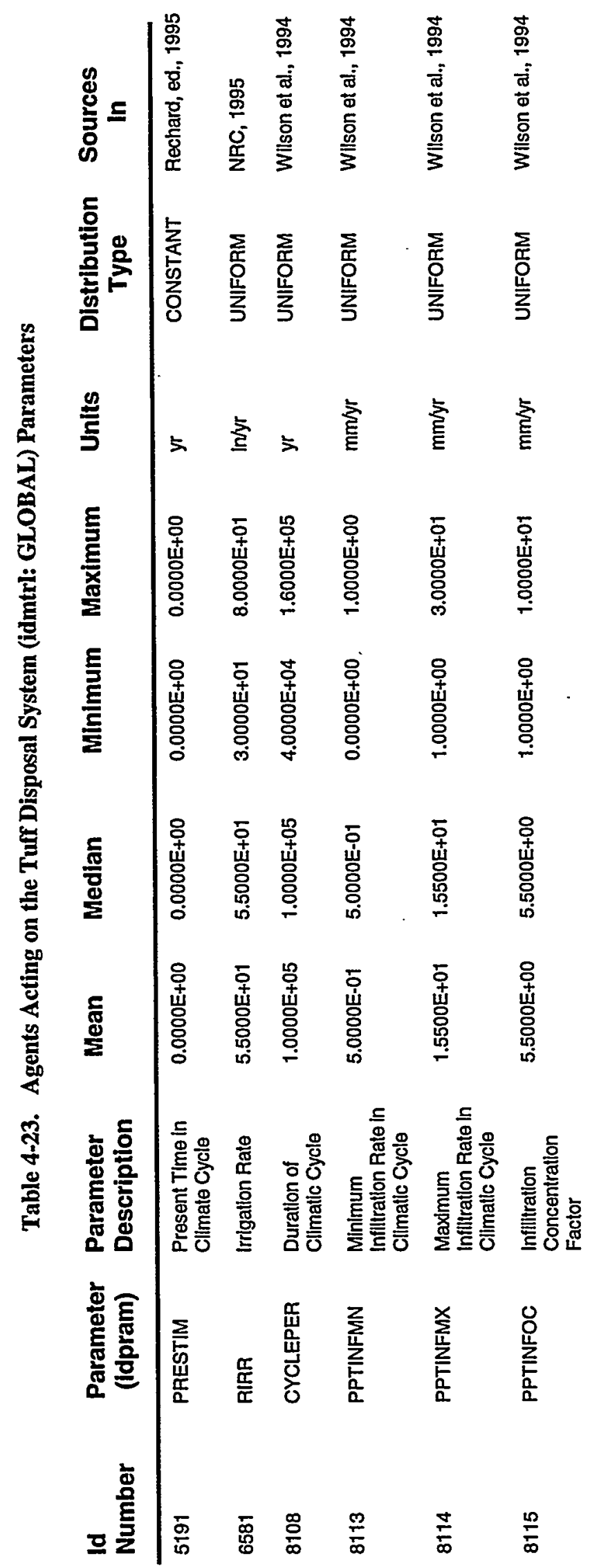




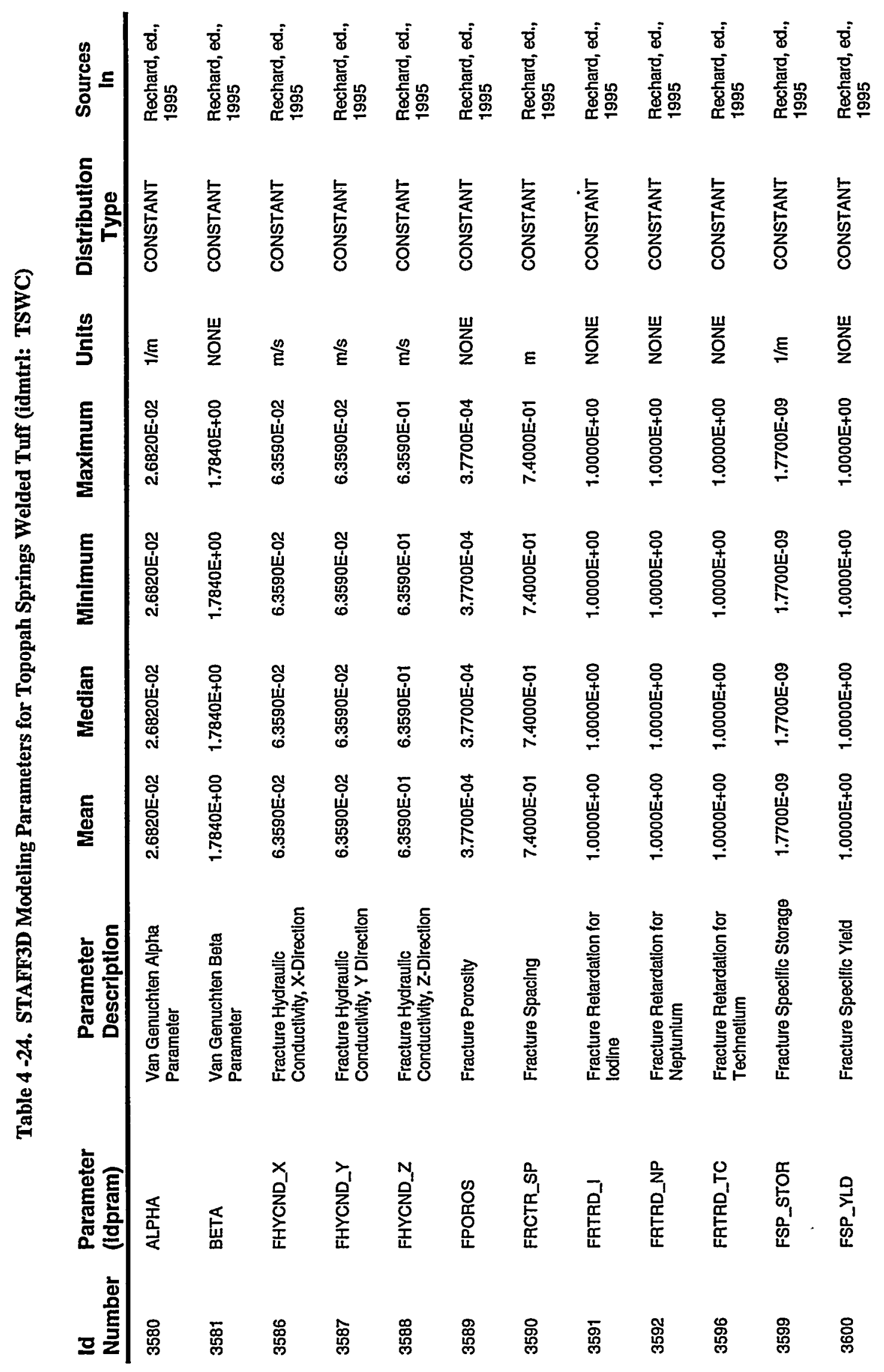

$\mid \begin{aligned} & m \\ & 0 \\ & 0 \\ & 0 \\ & 0\end{aligned}$ 

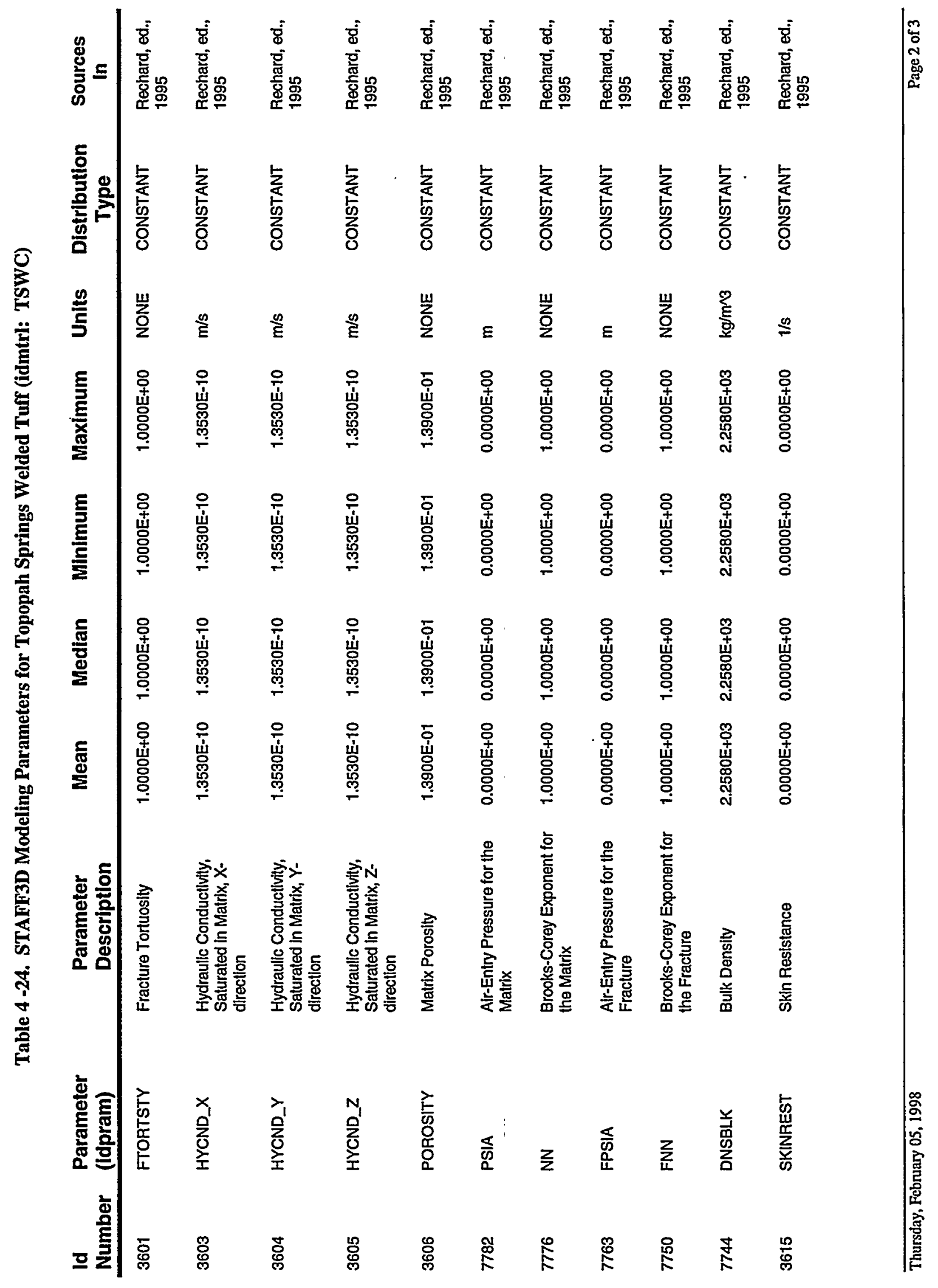


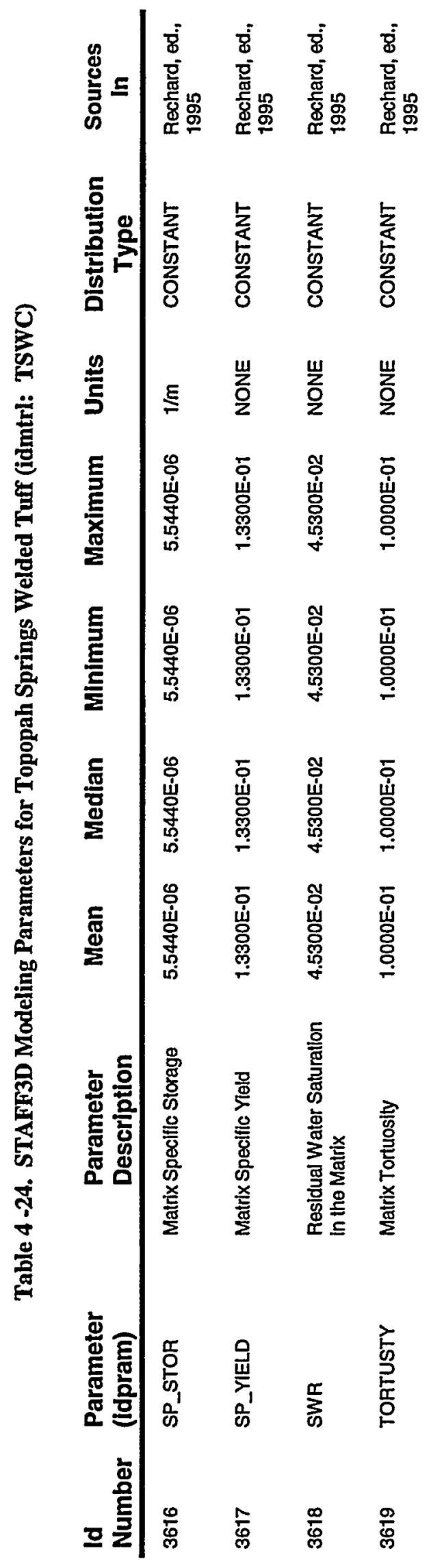




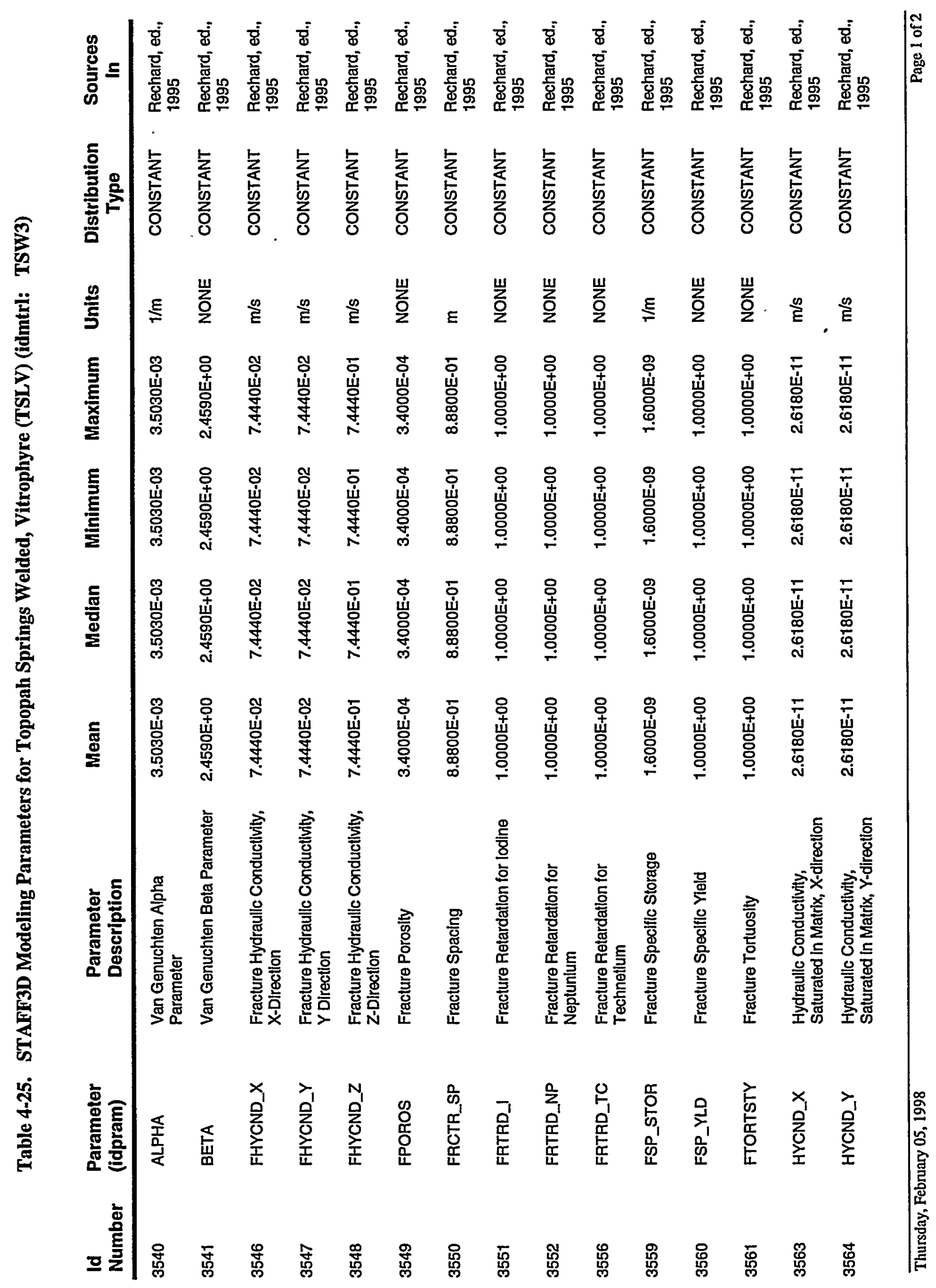




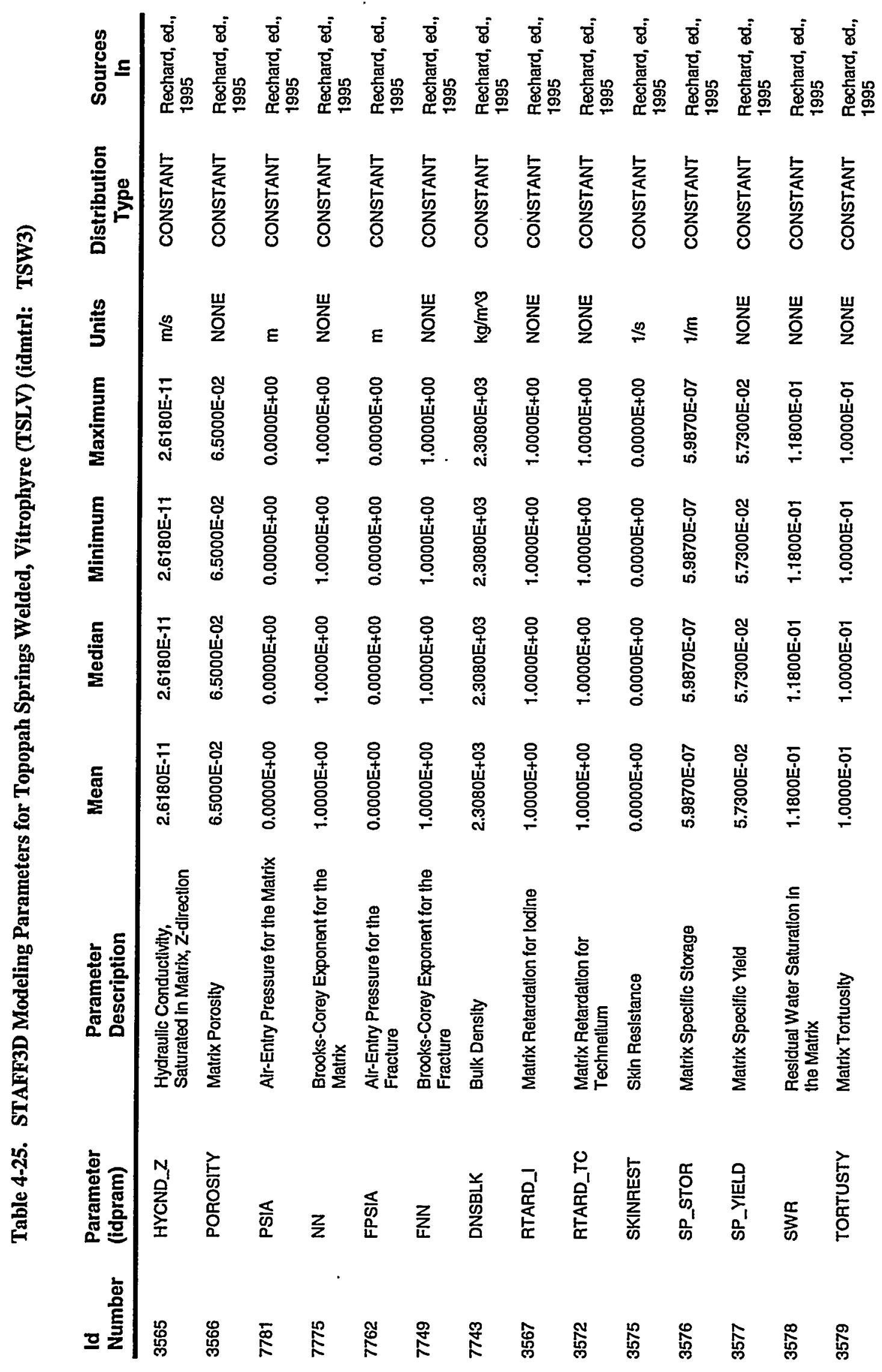

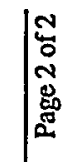




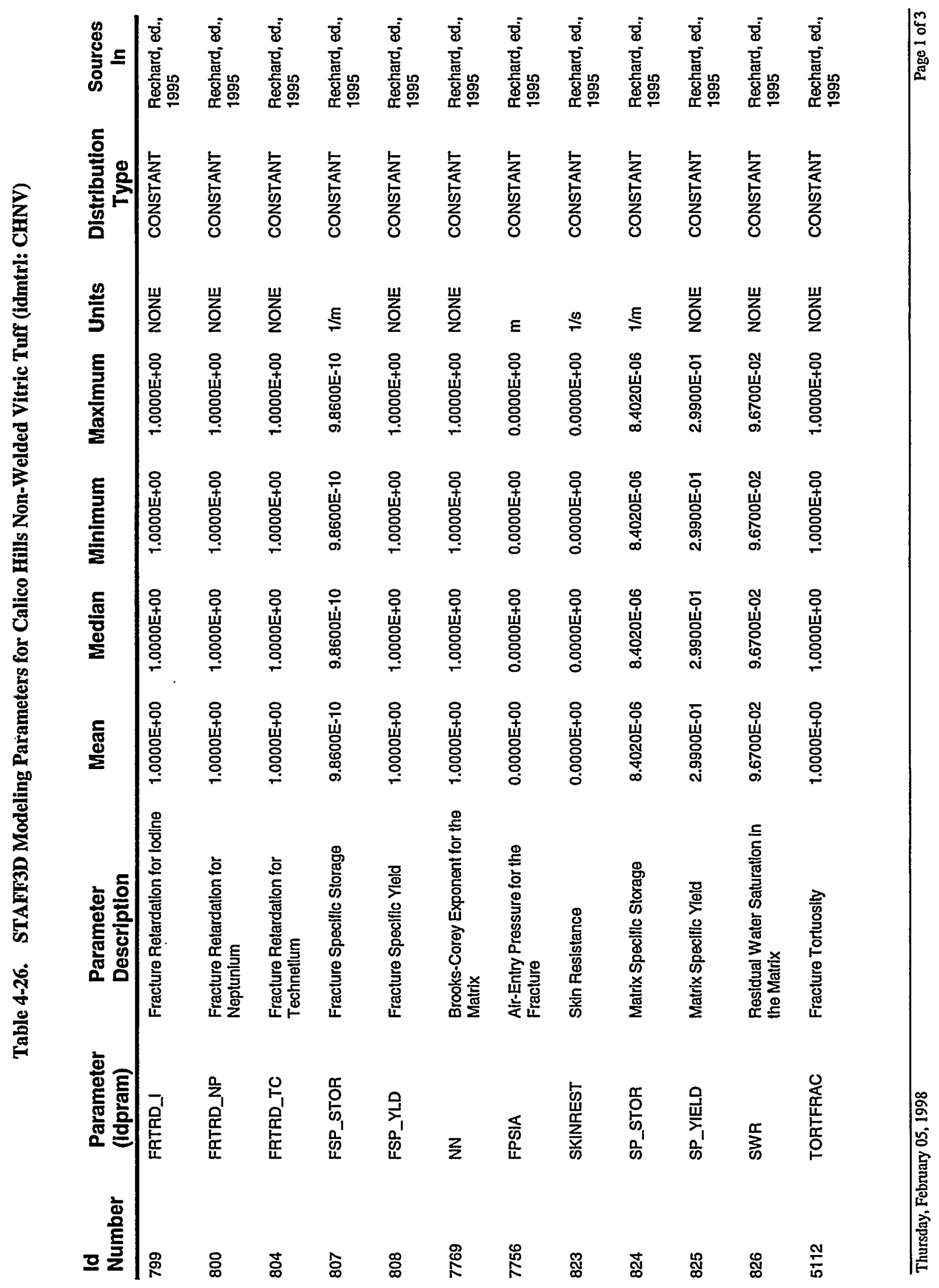




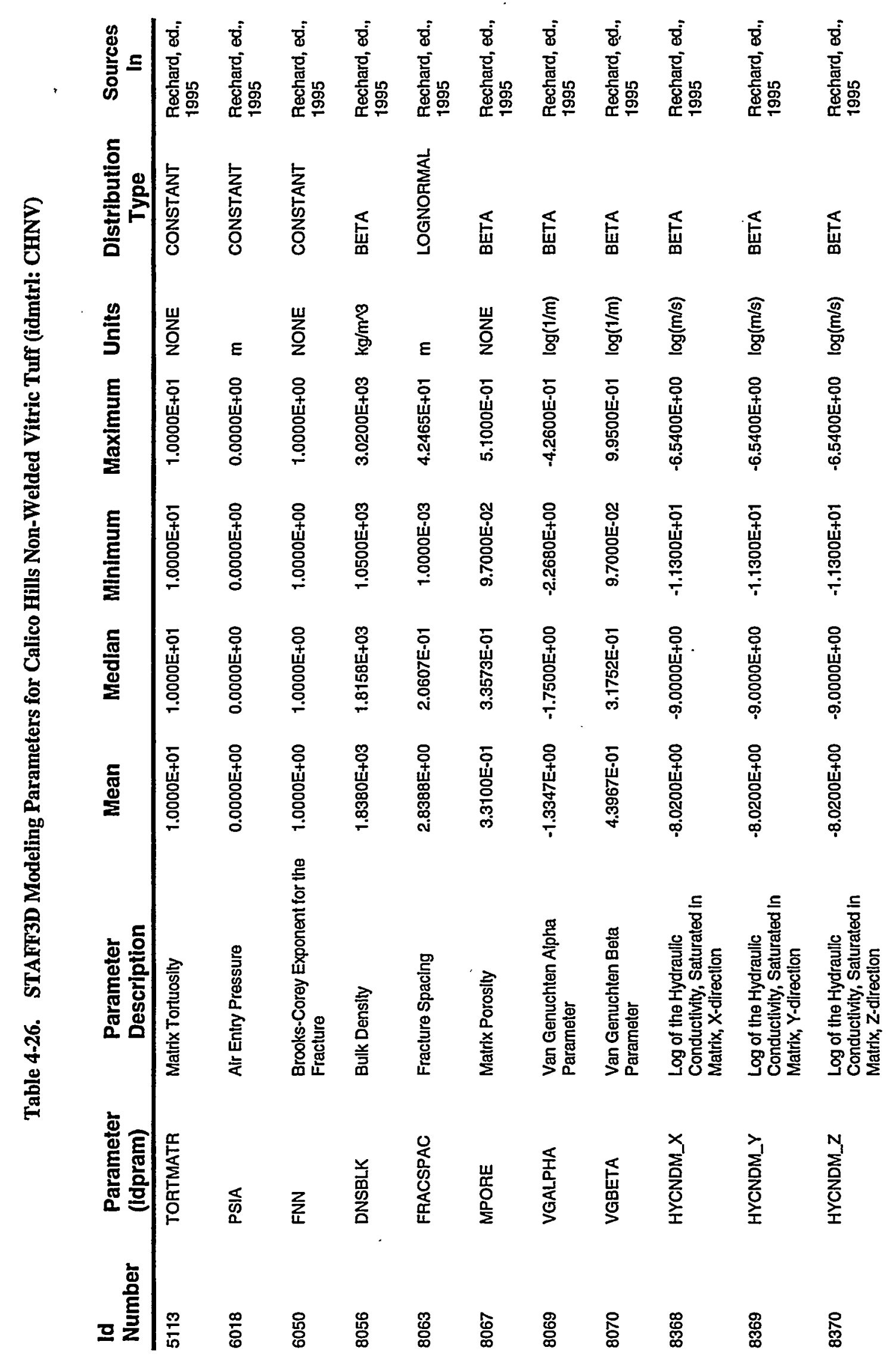

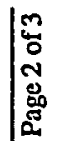



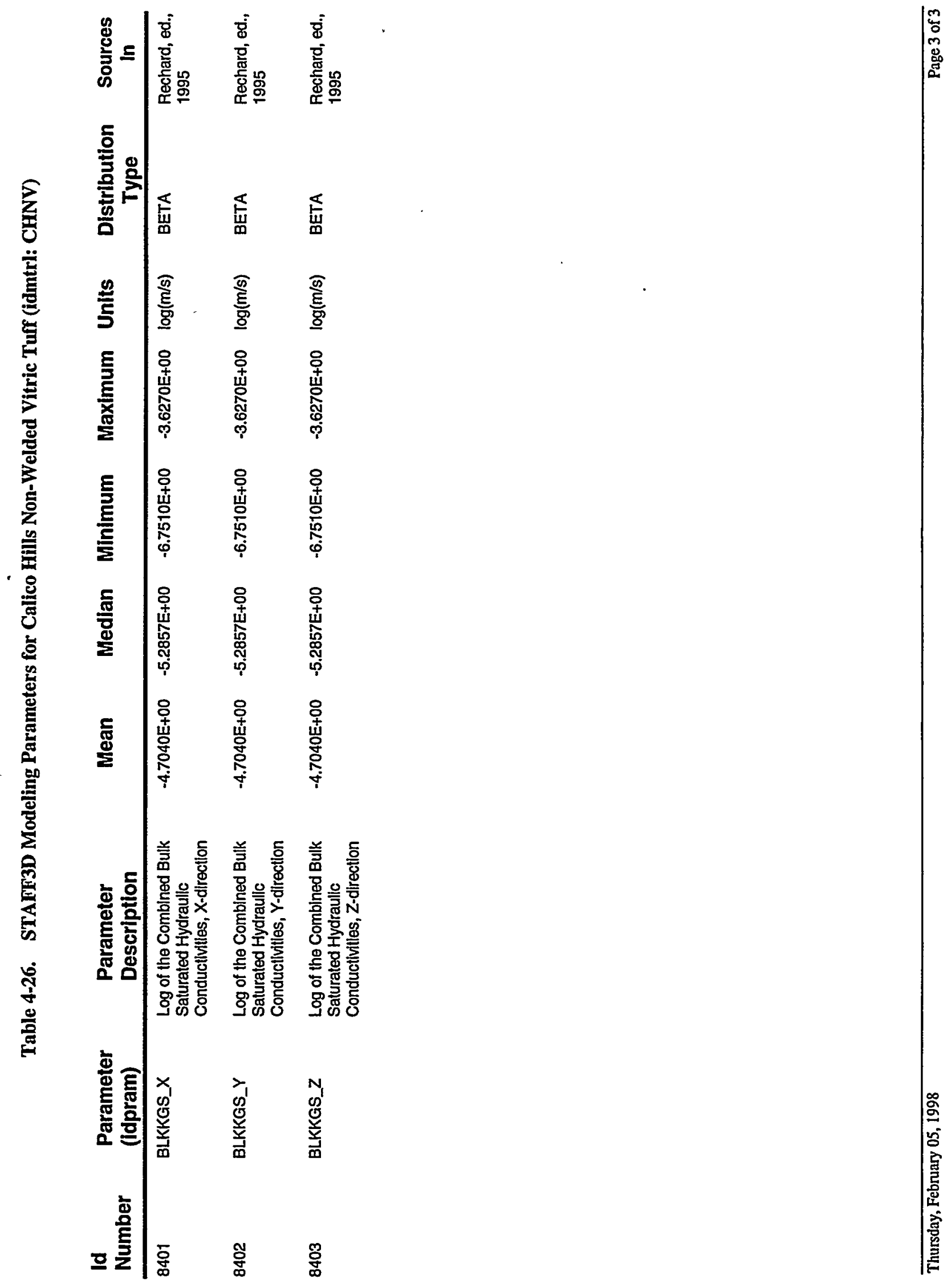


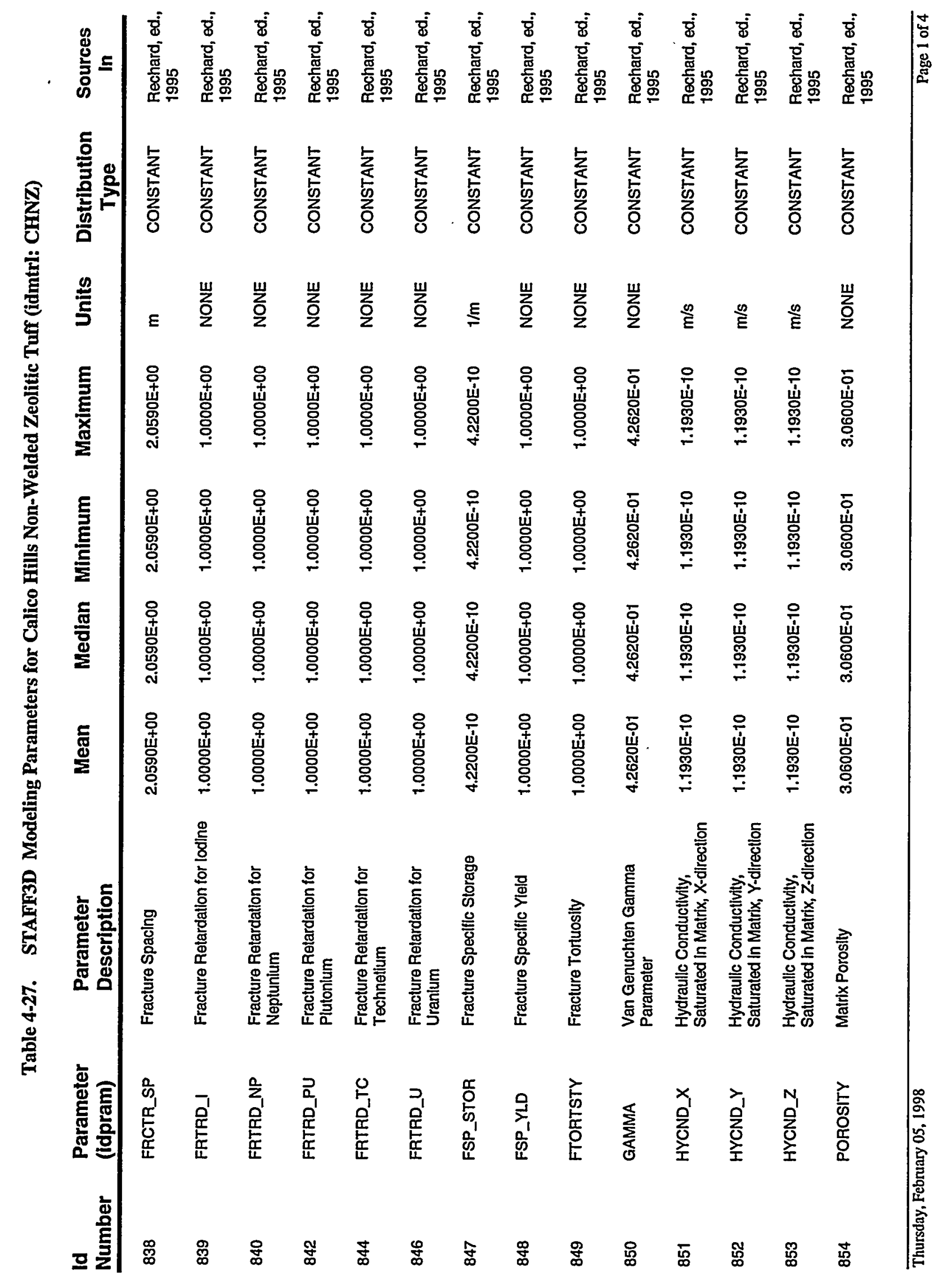




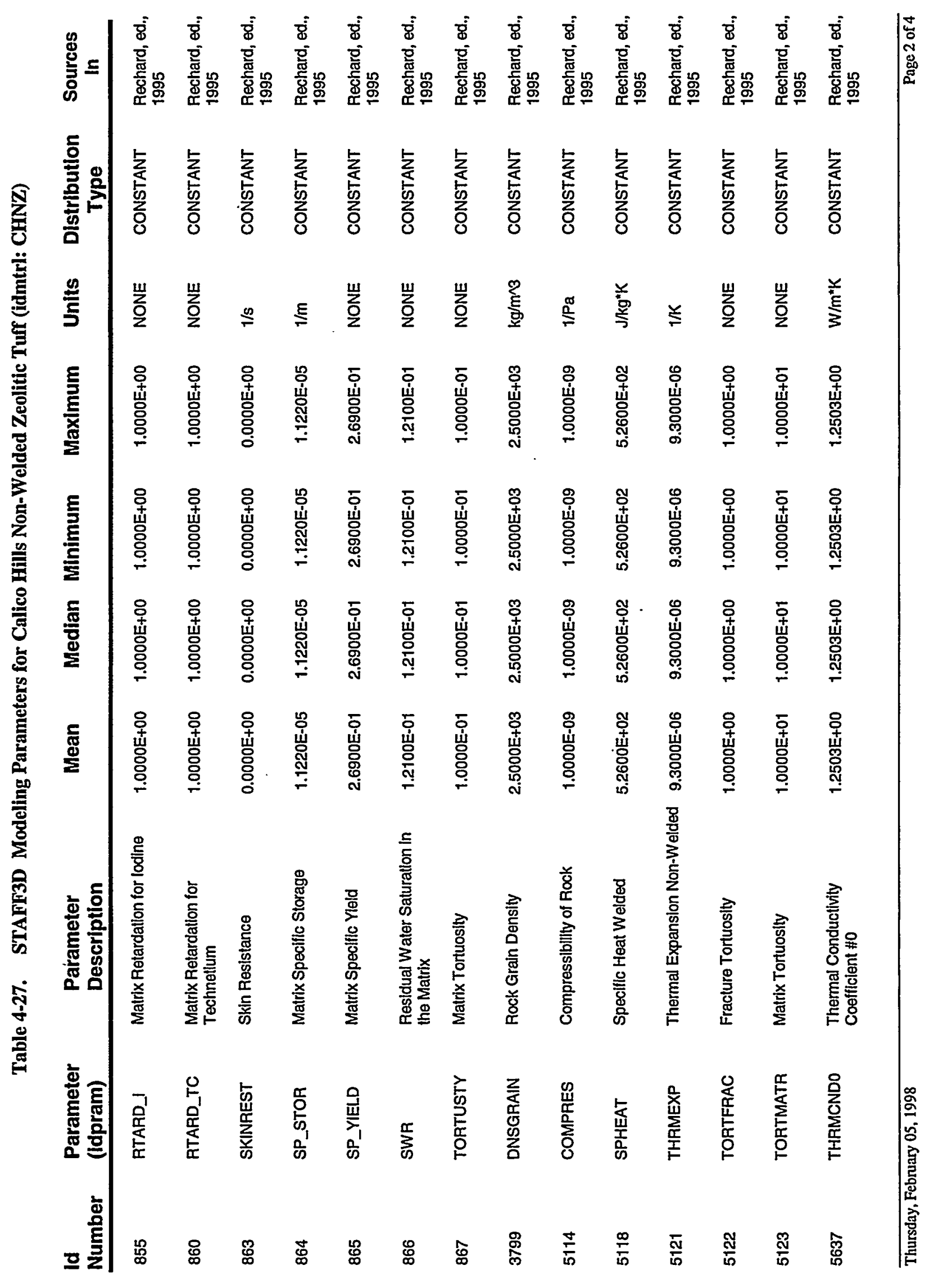




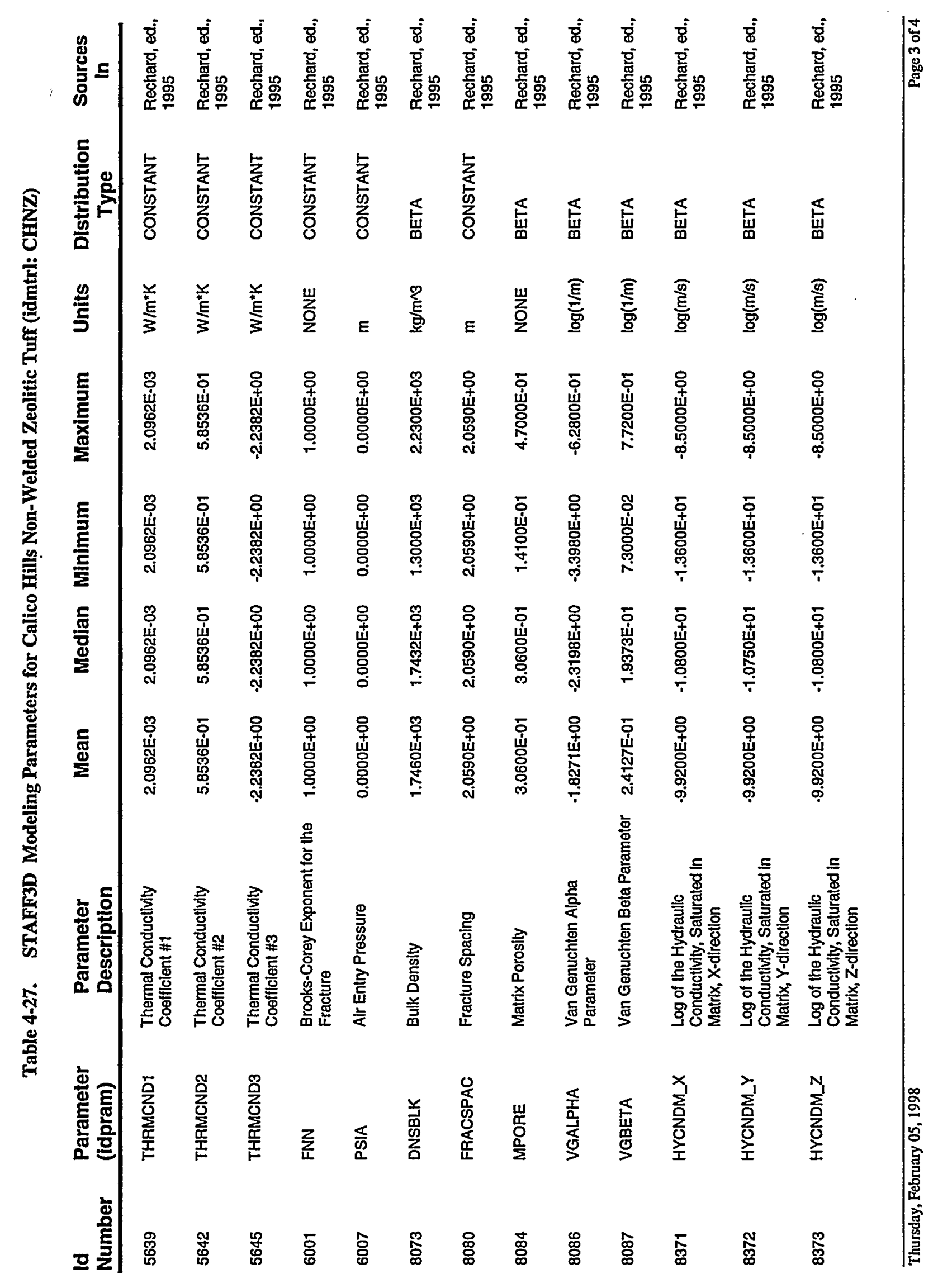



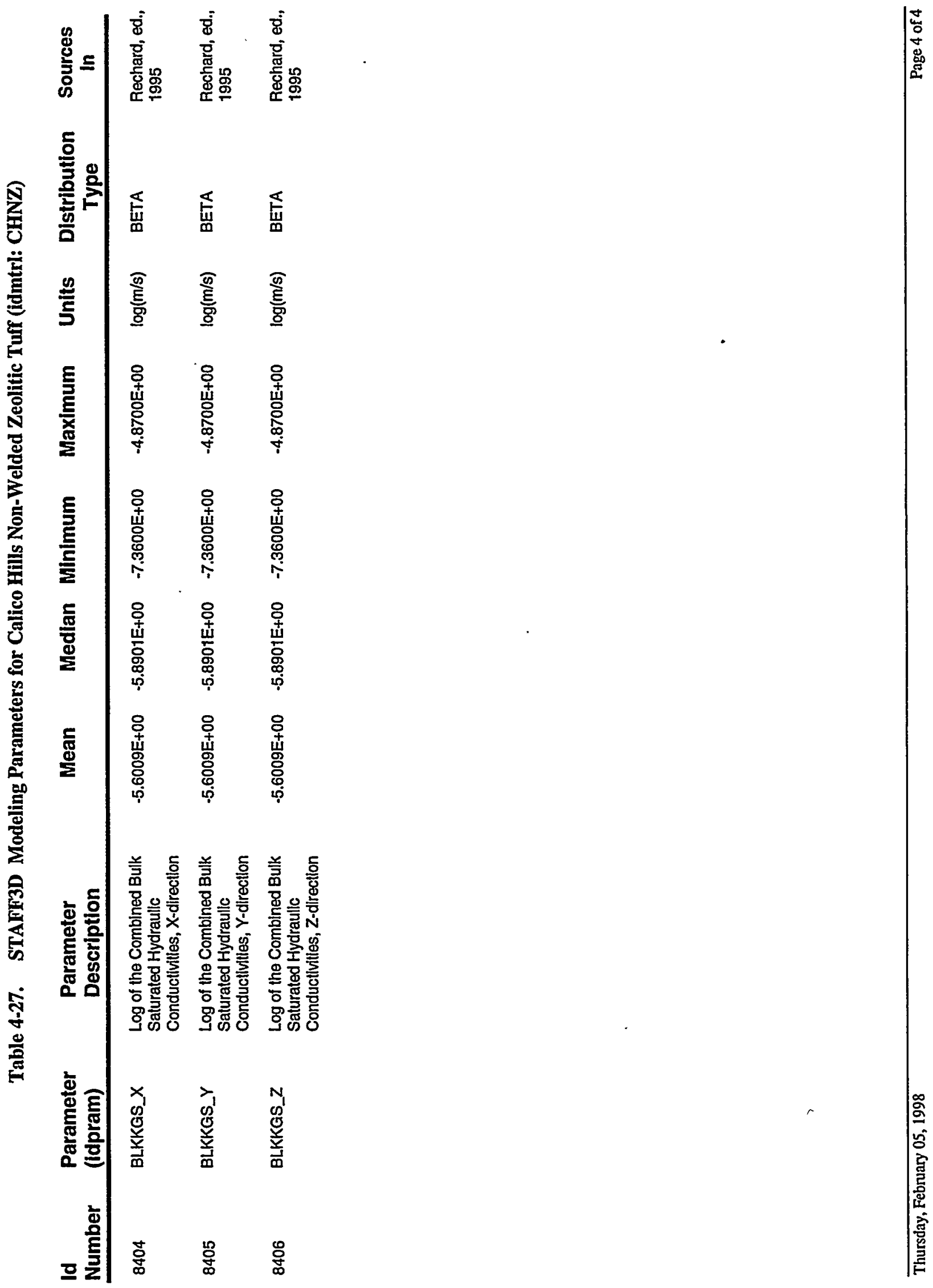


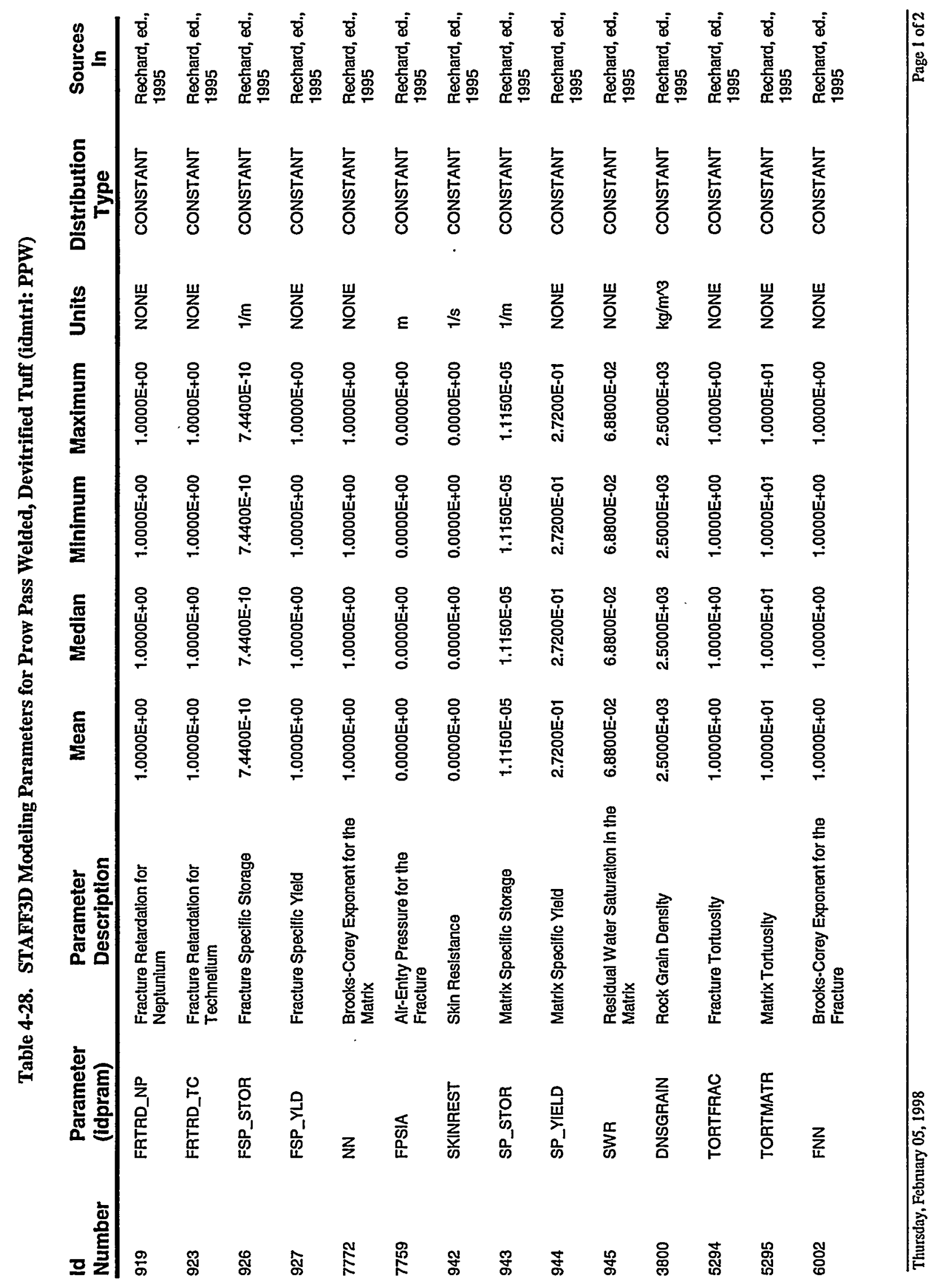




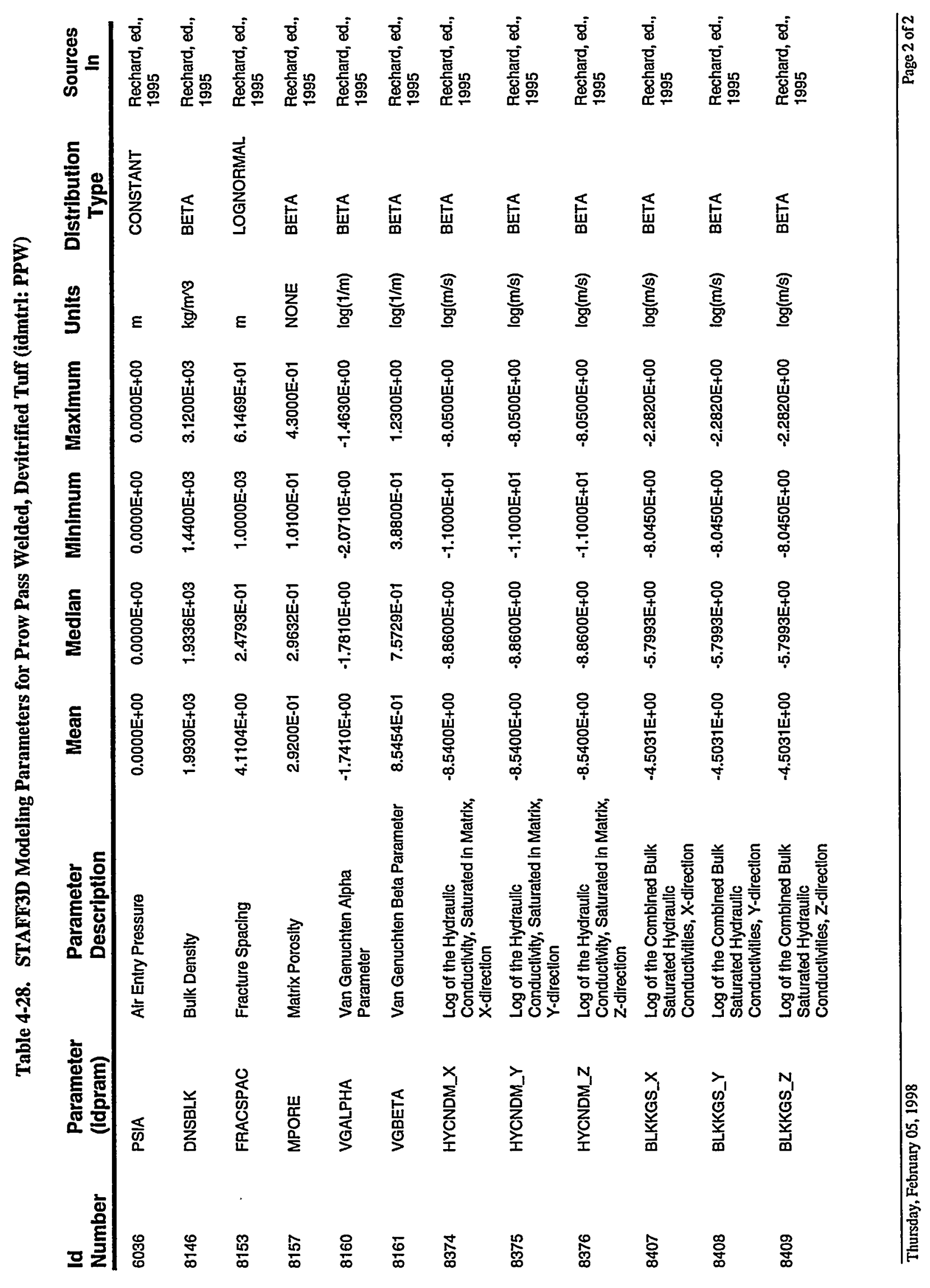




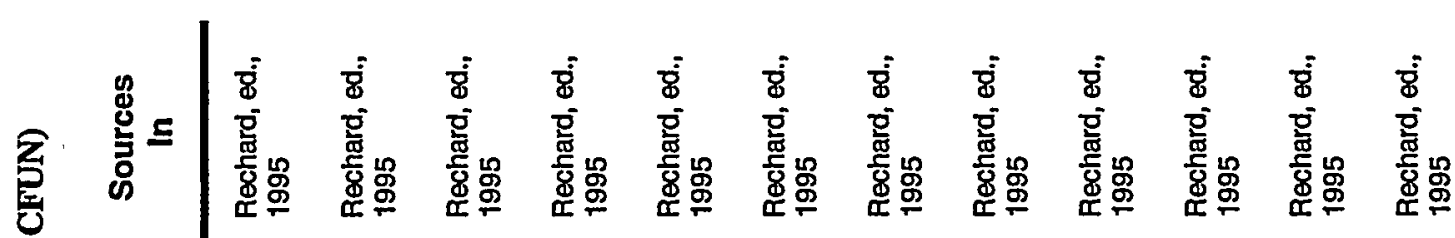

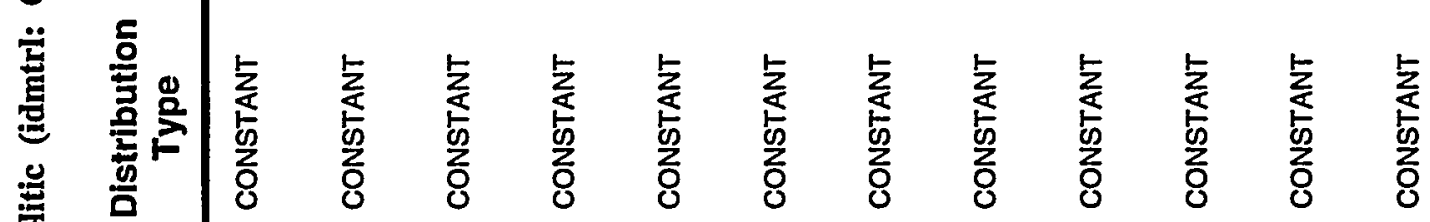

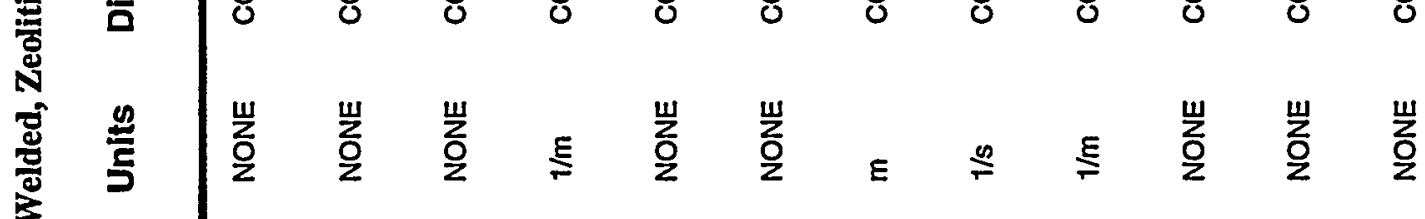

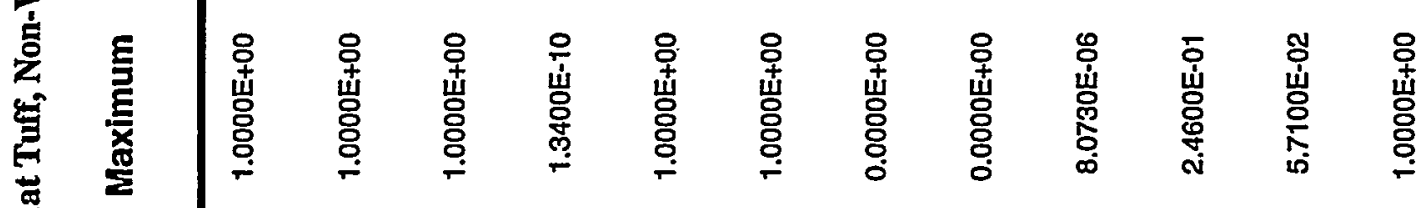

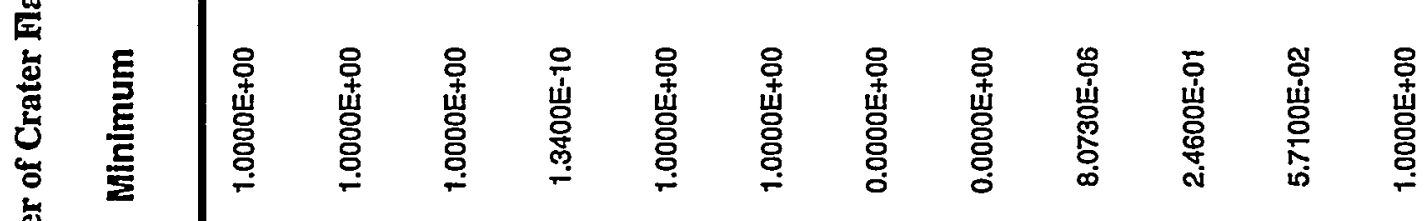

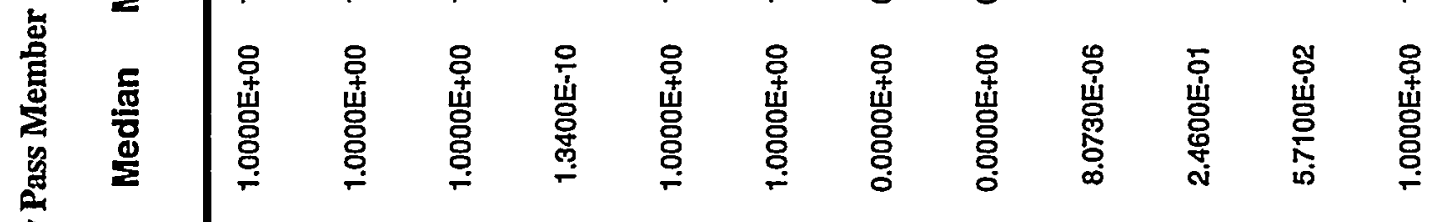

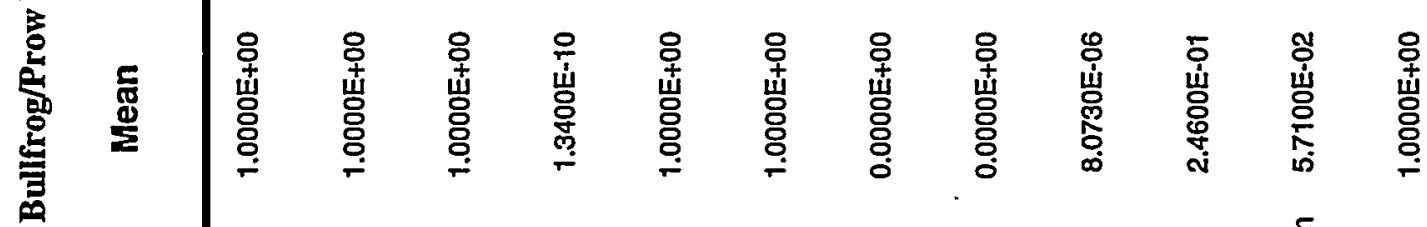

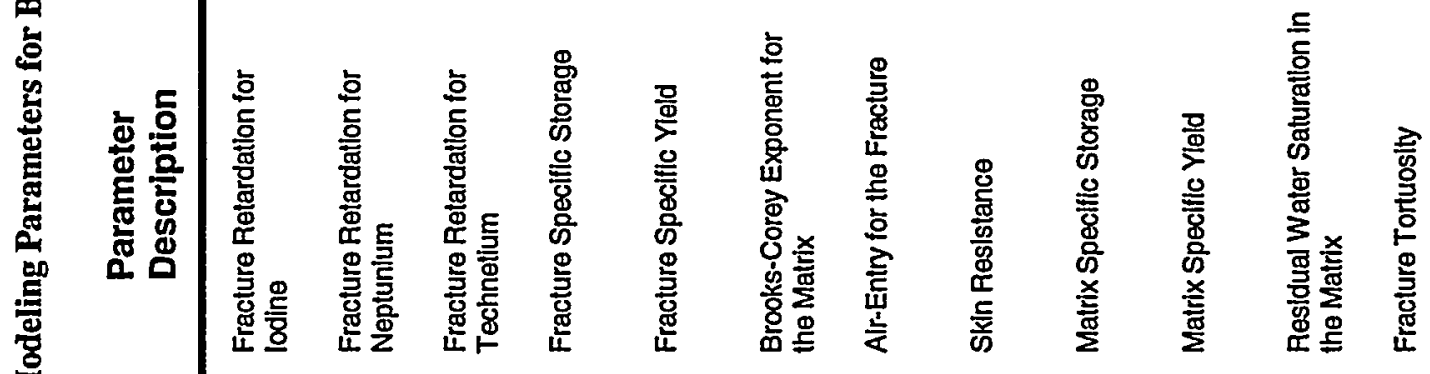

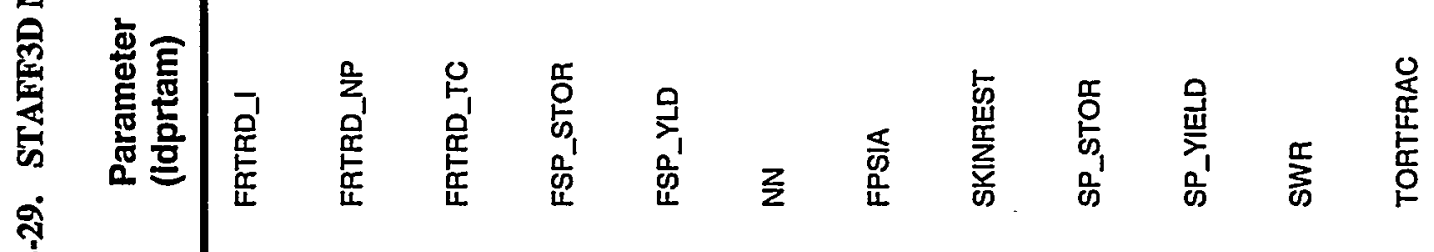

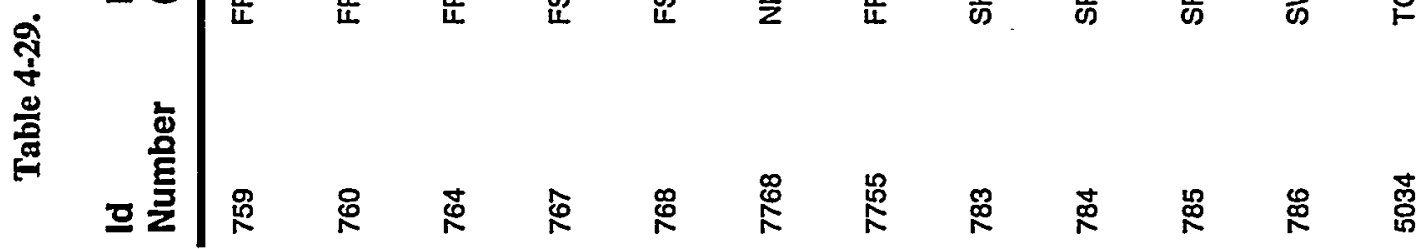




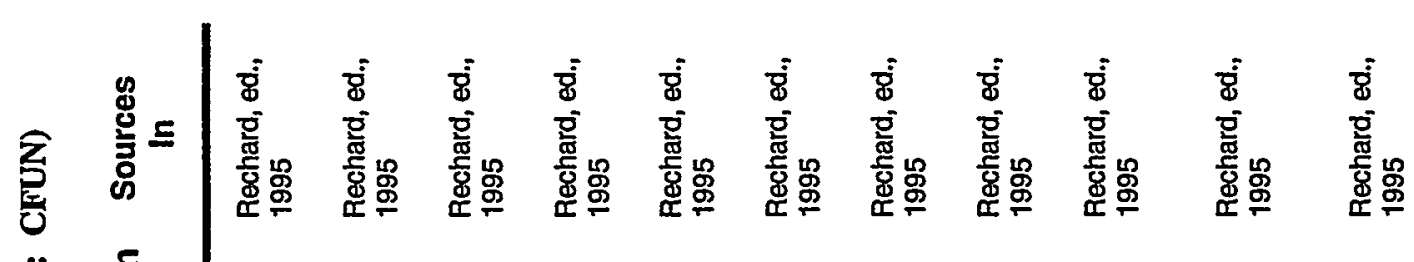

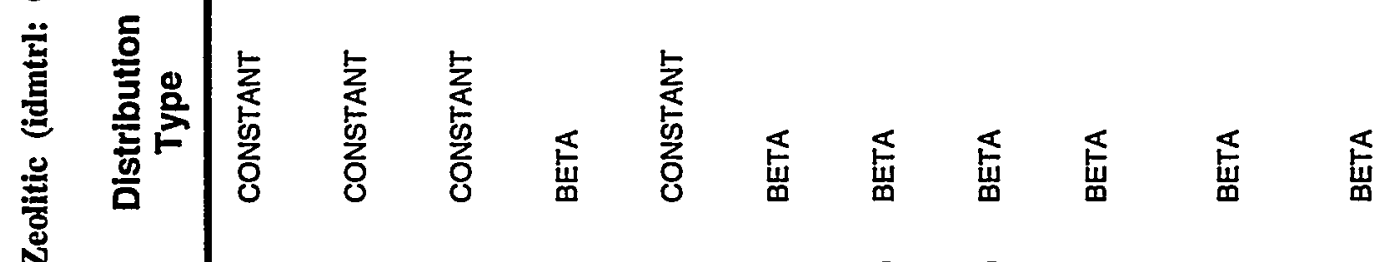

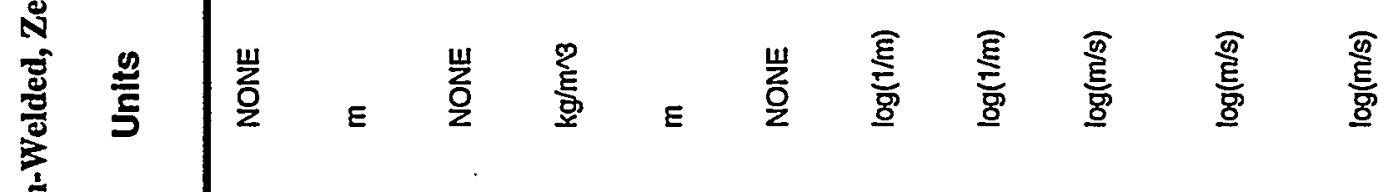

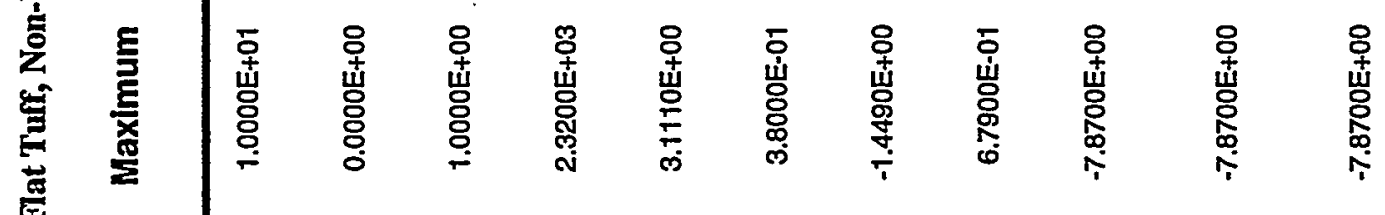

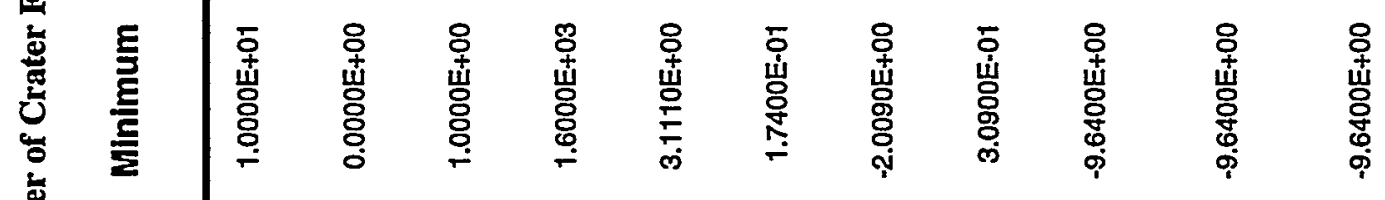

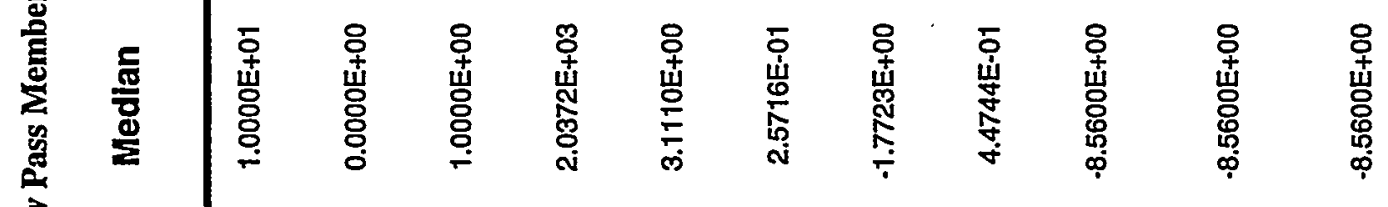

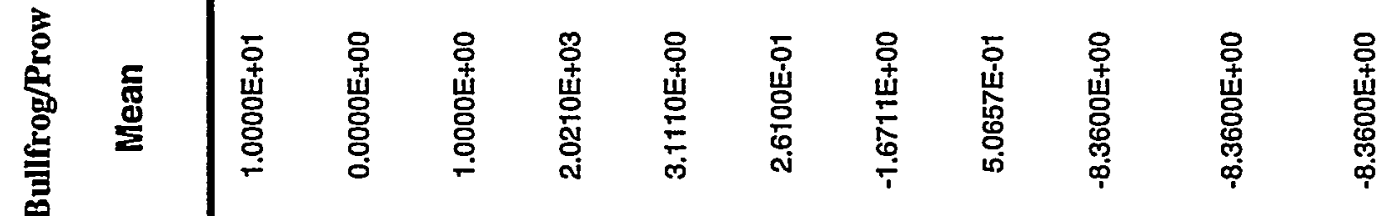

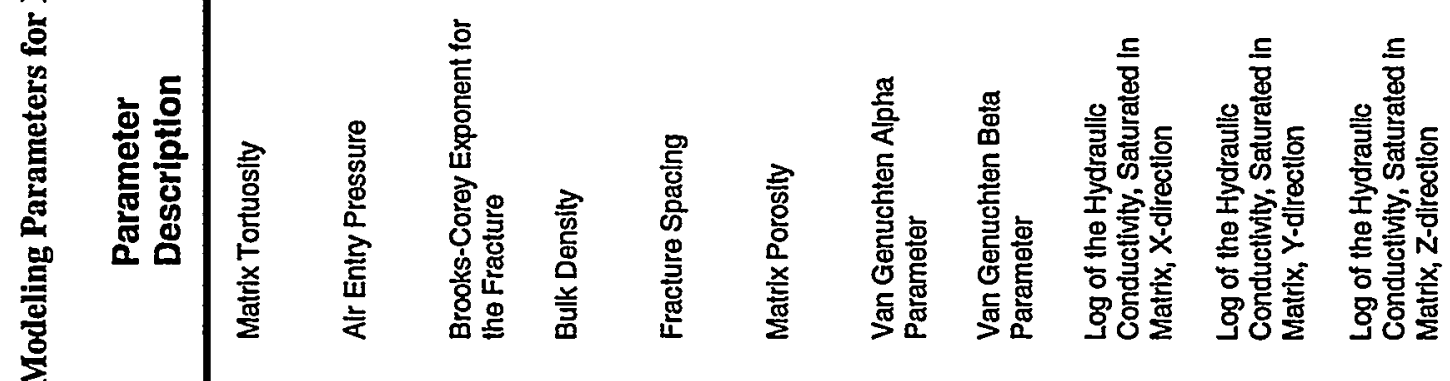

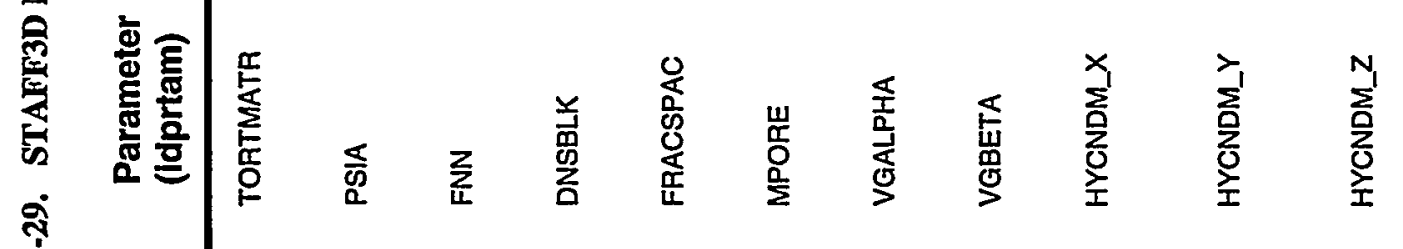

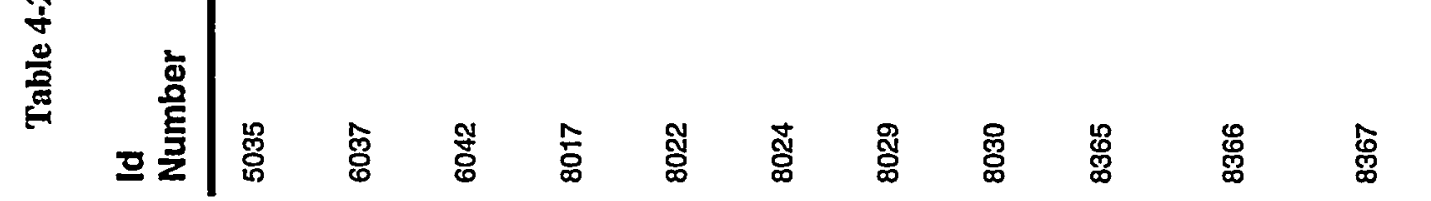



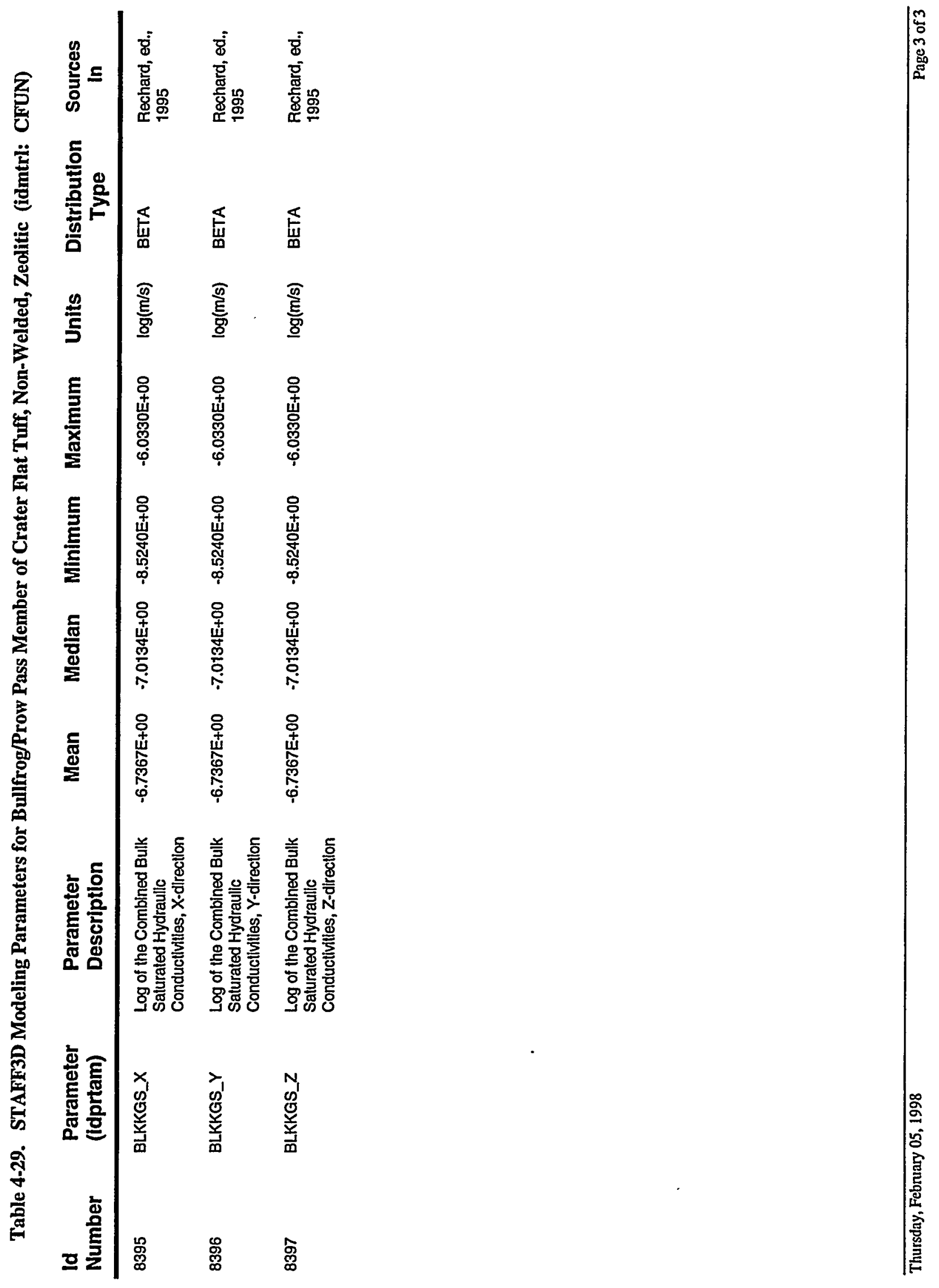


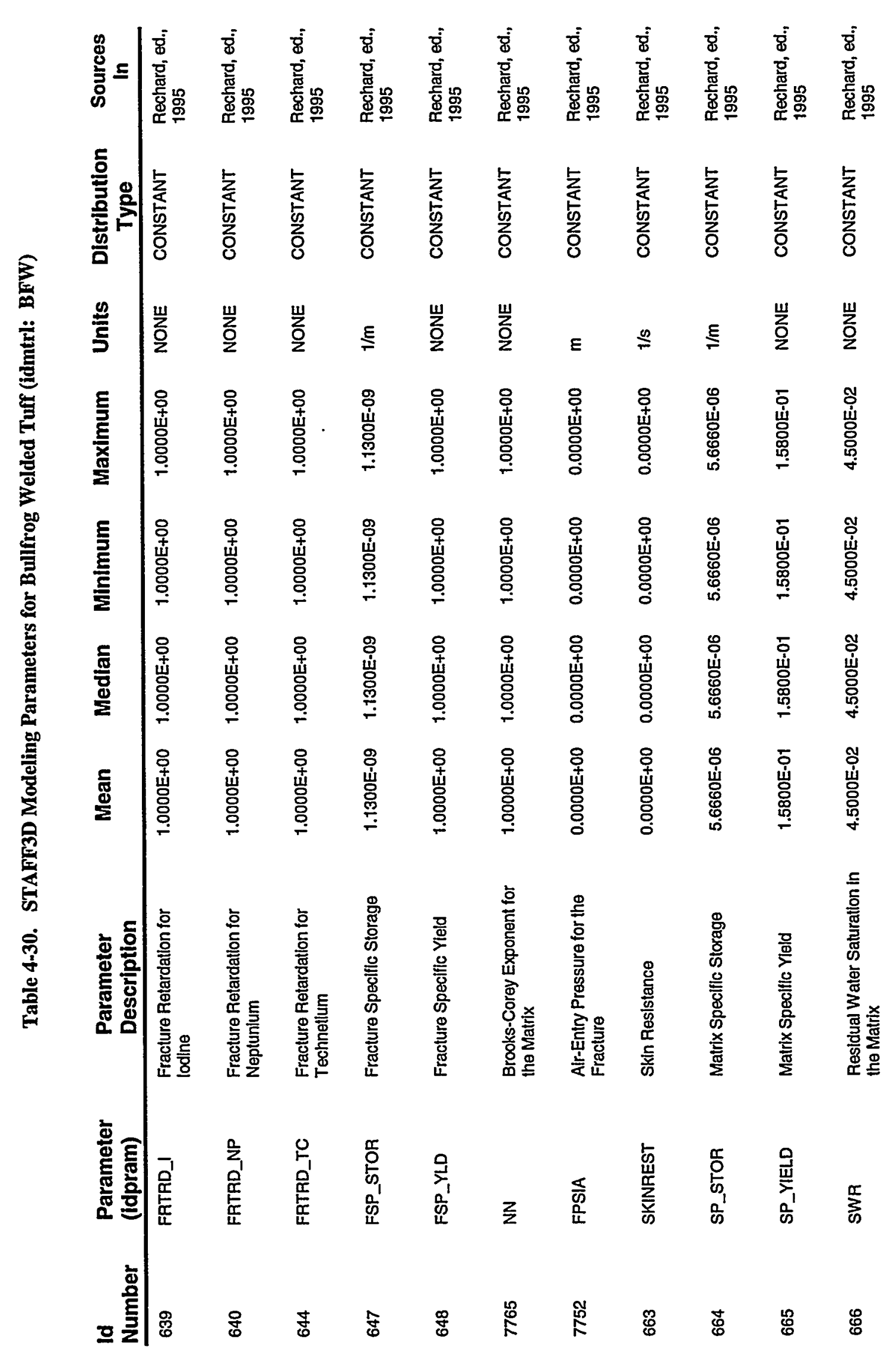

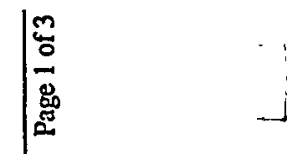




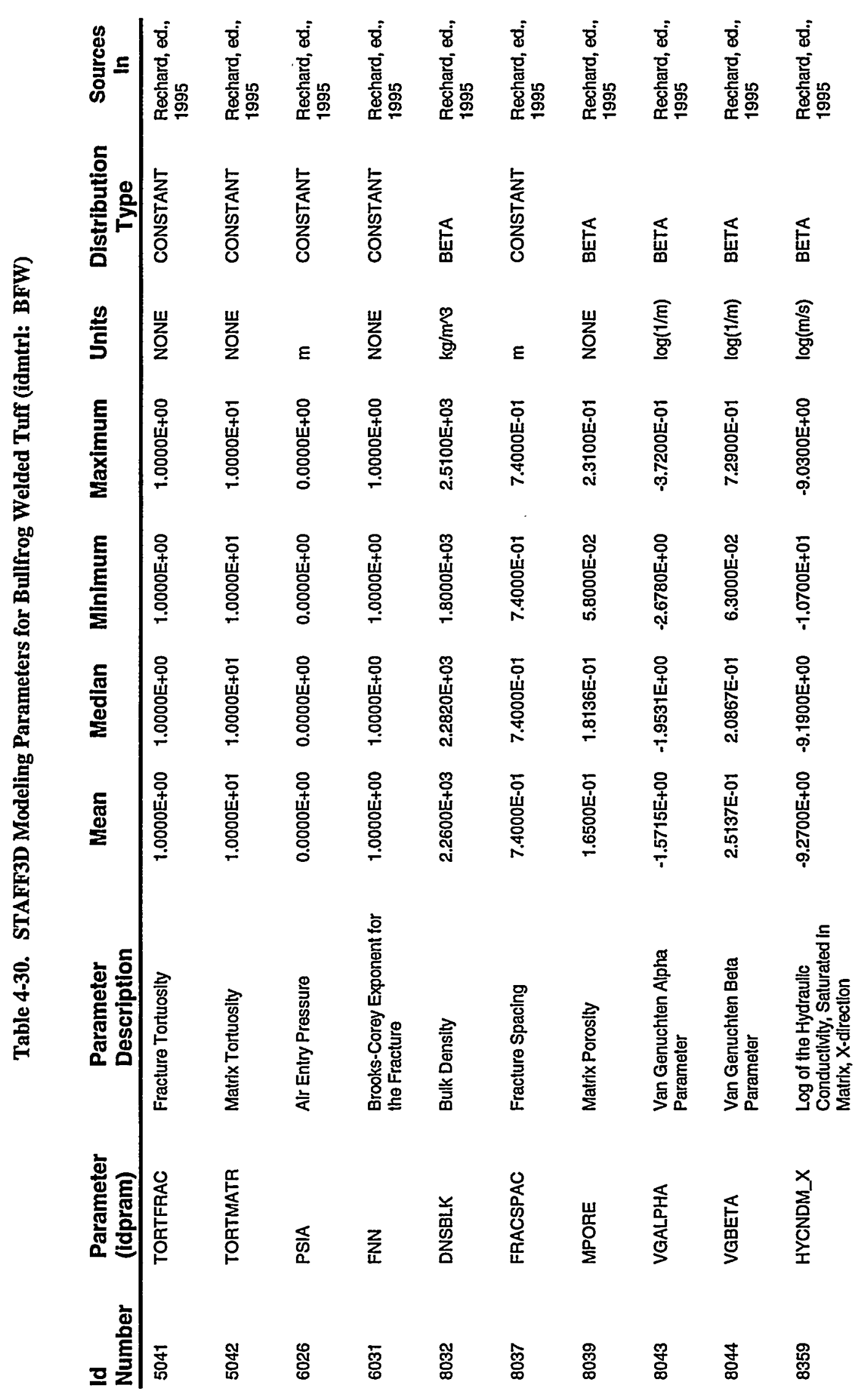

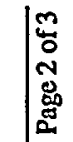




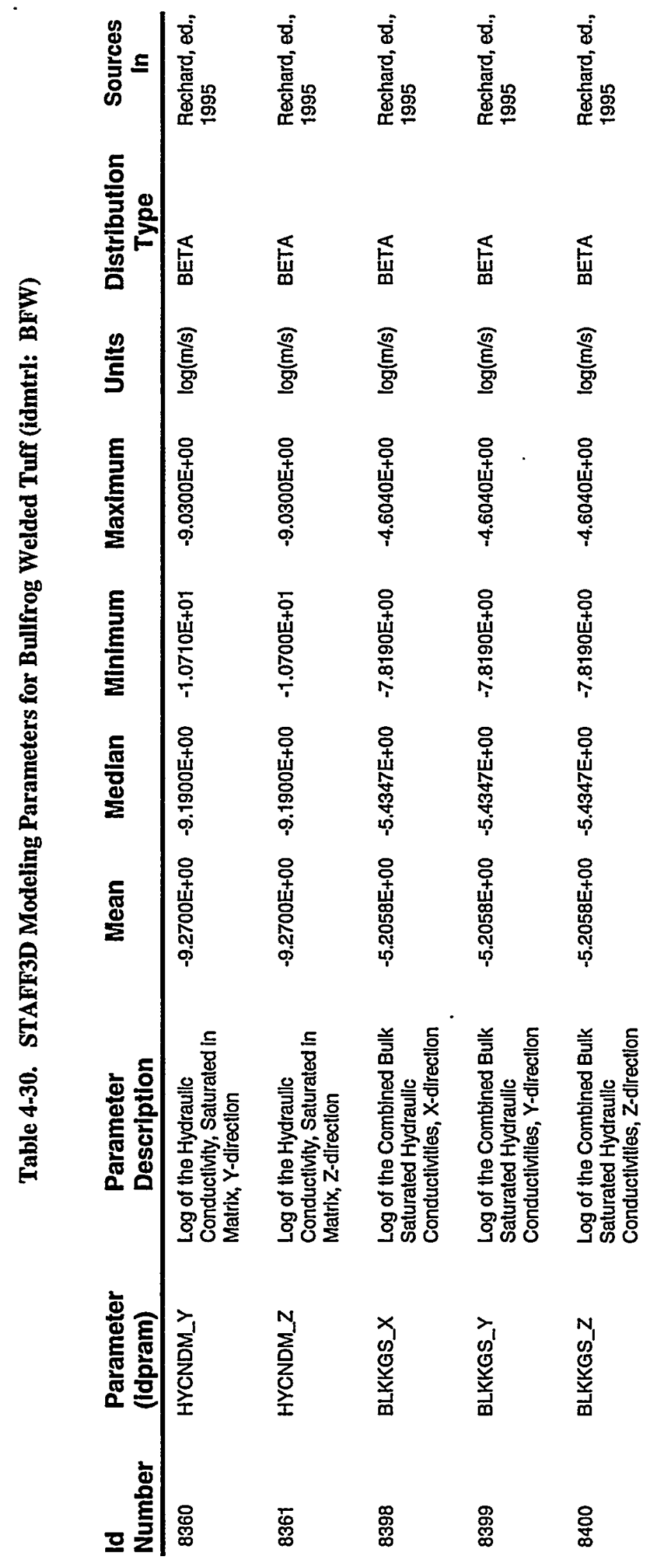




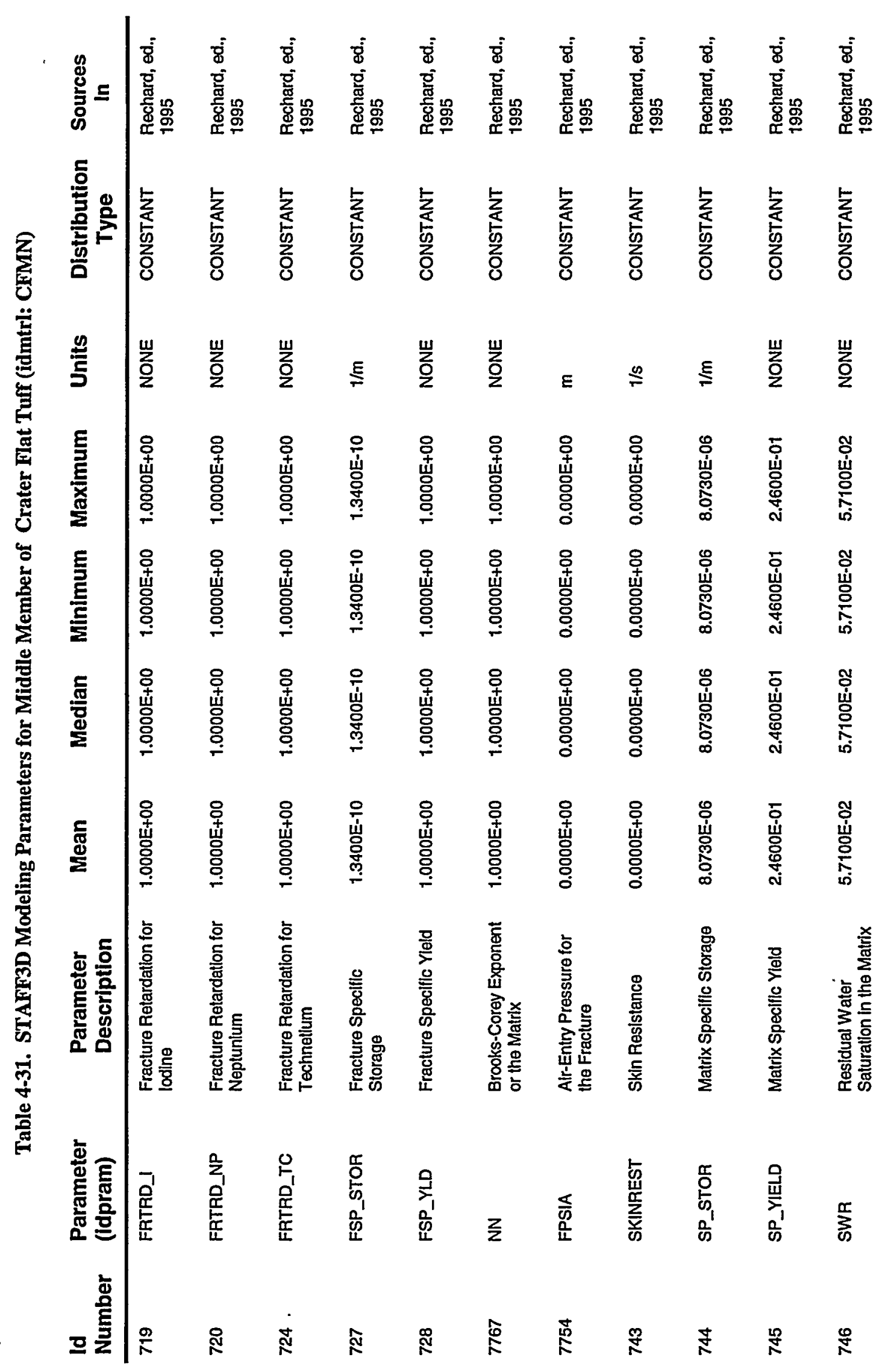




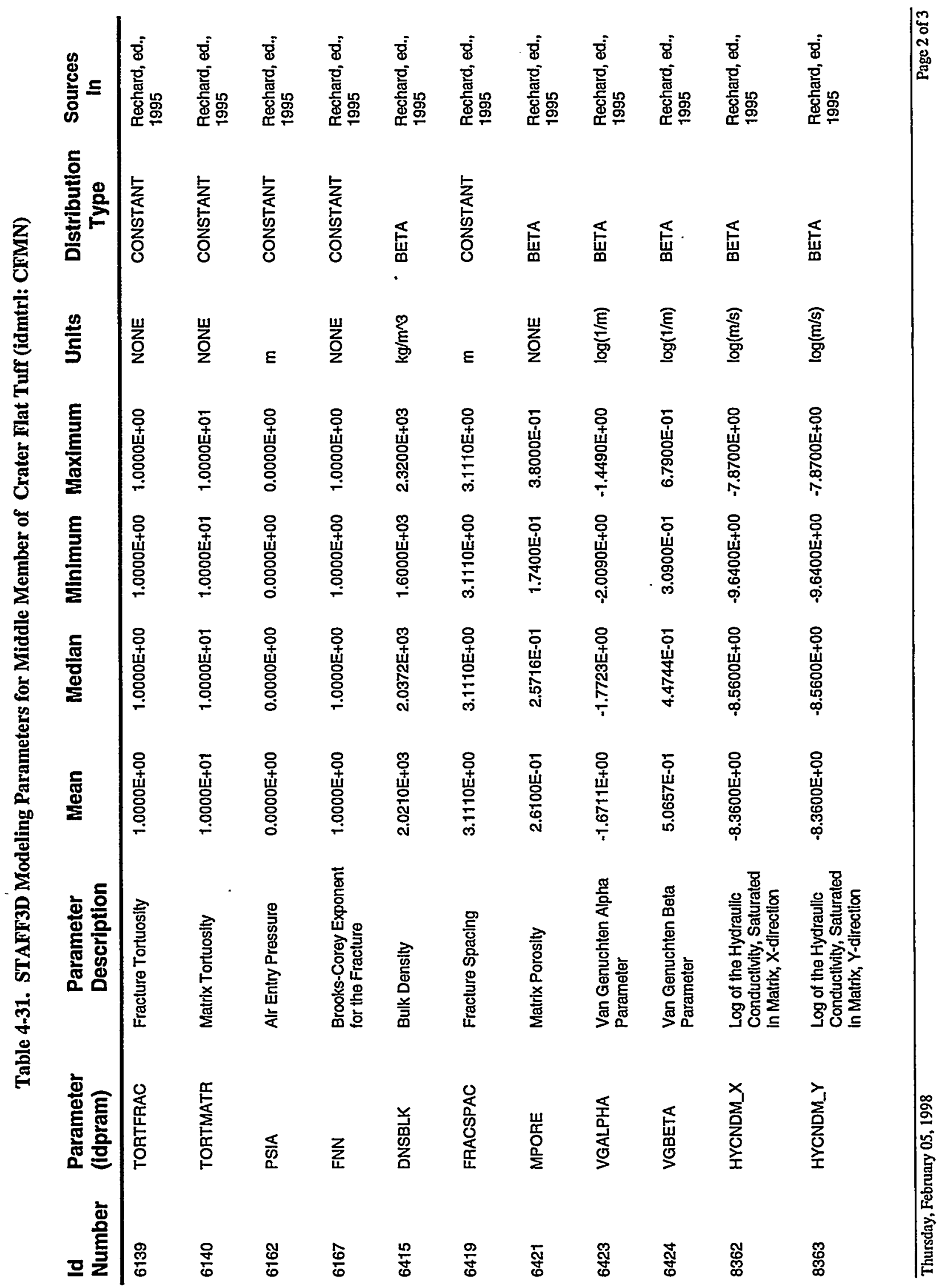



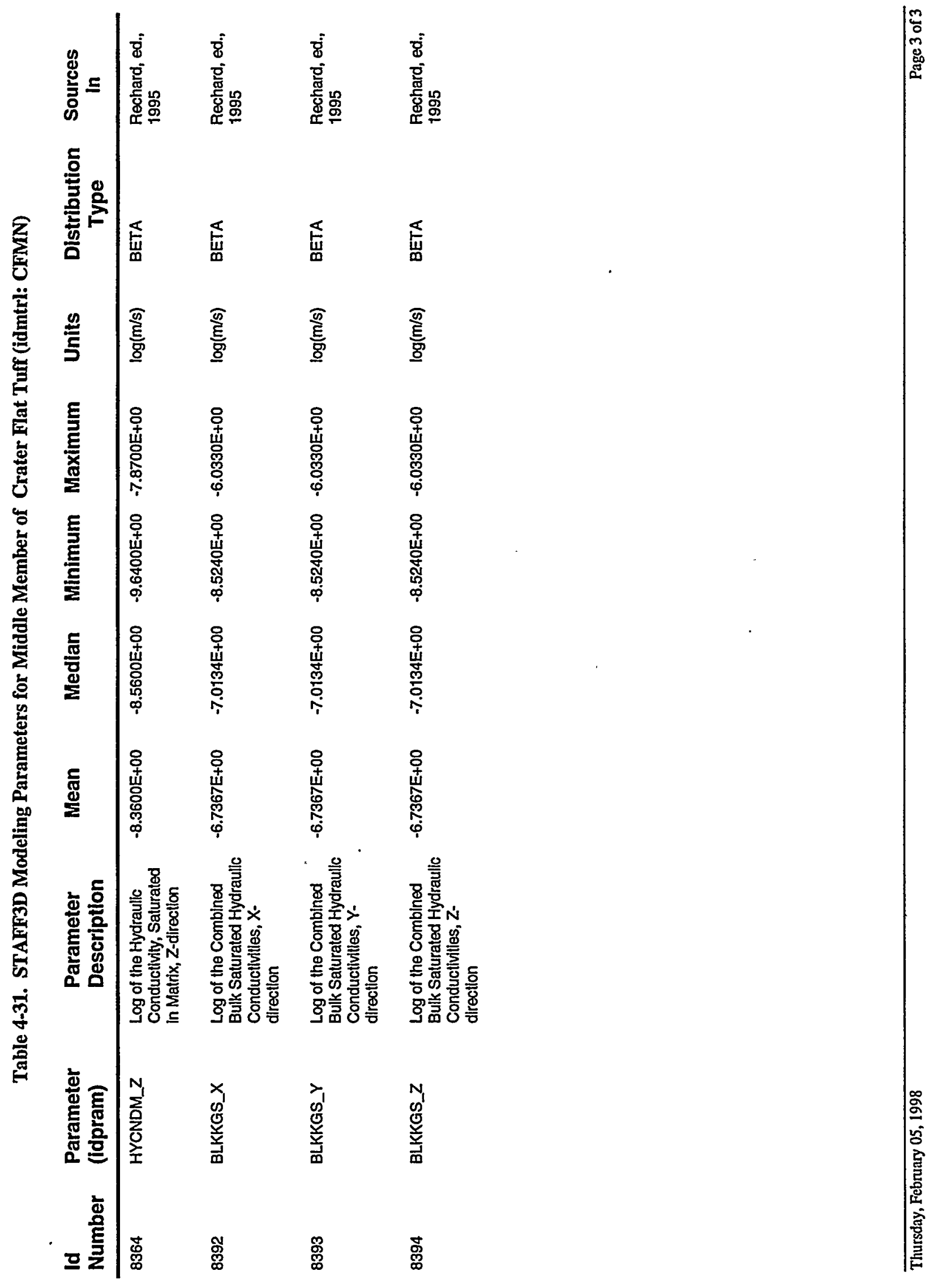


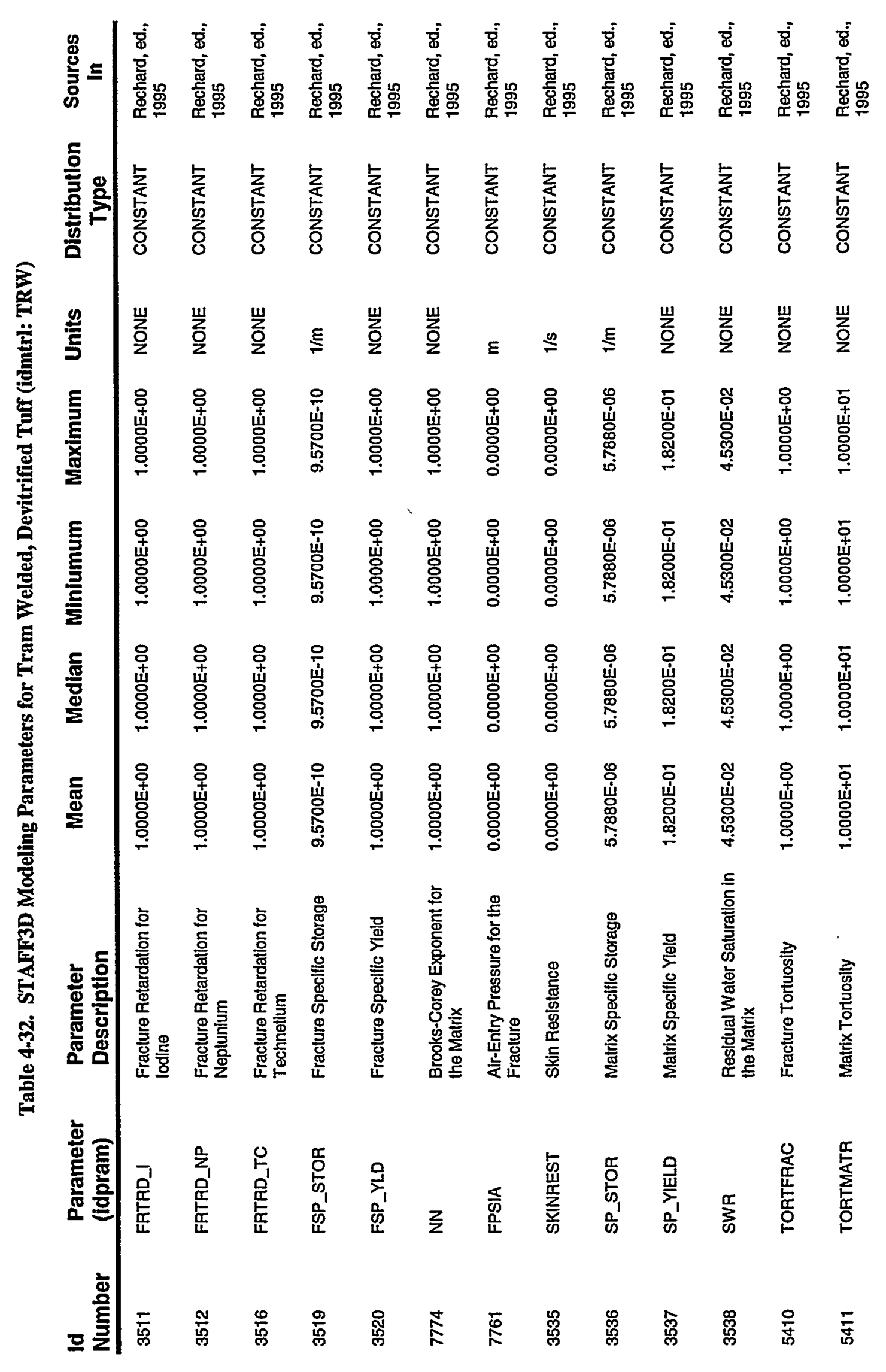

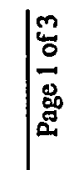




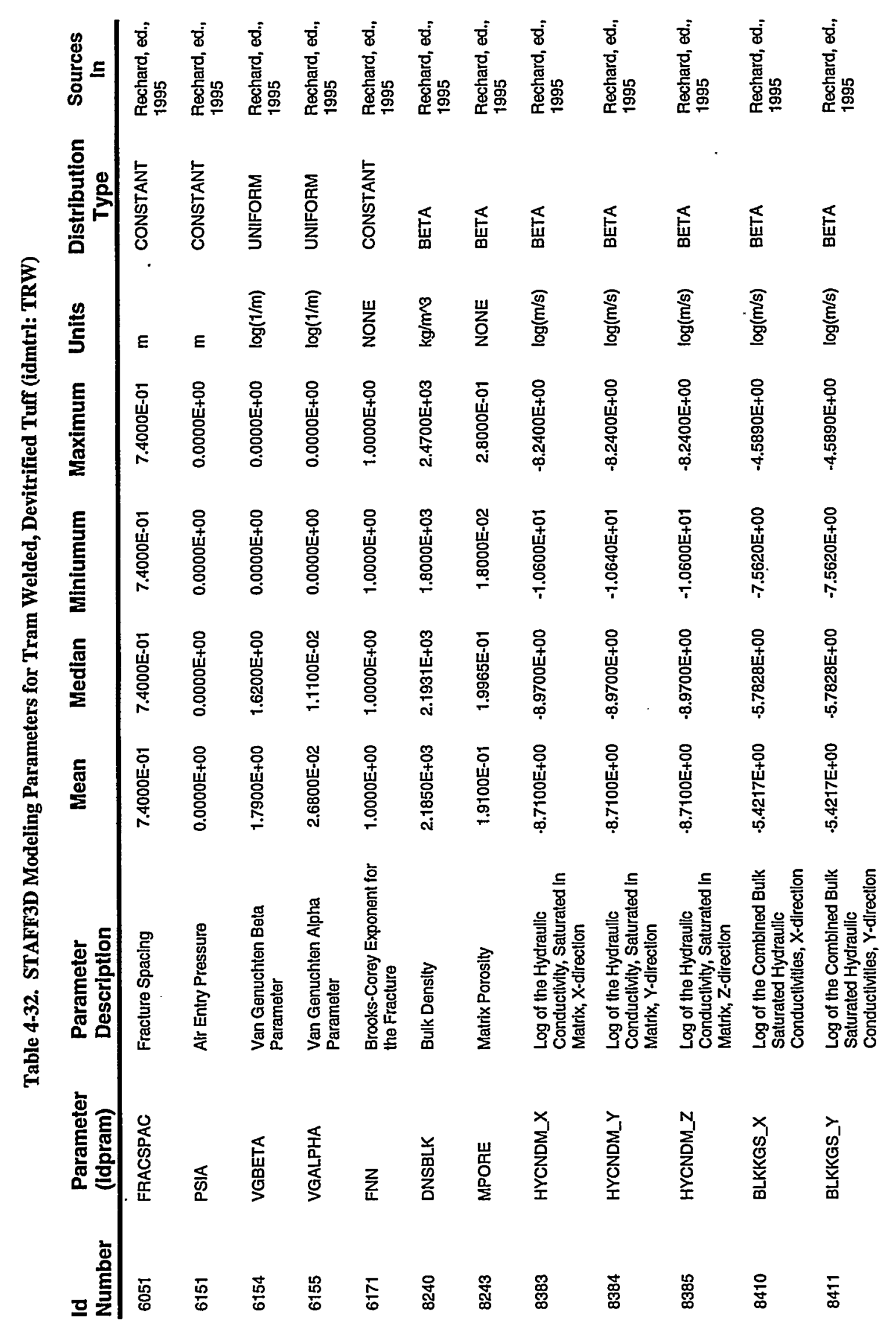




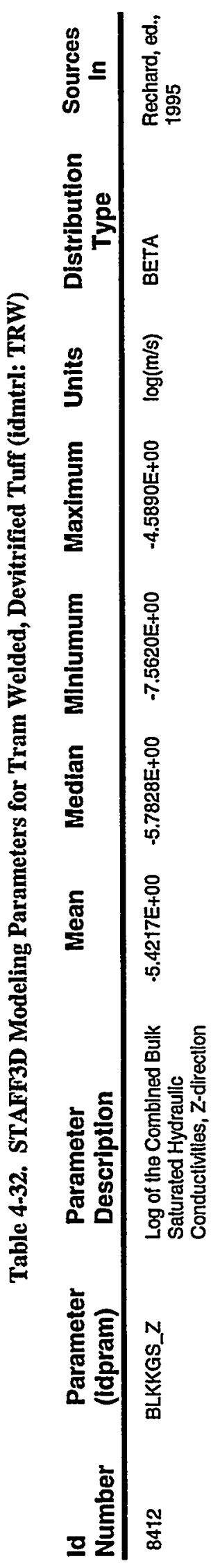




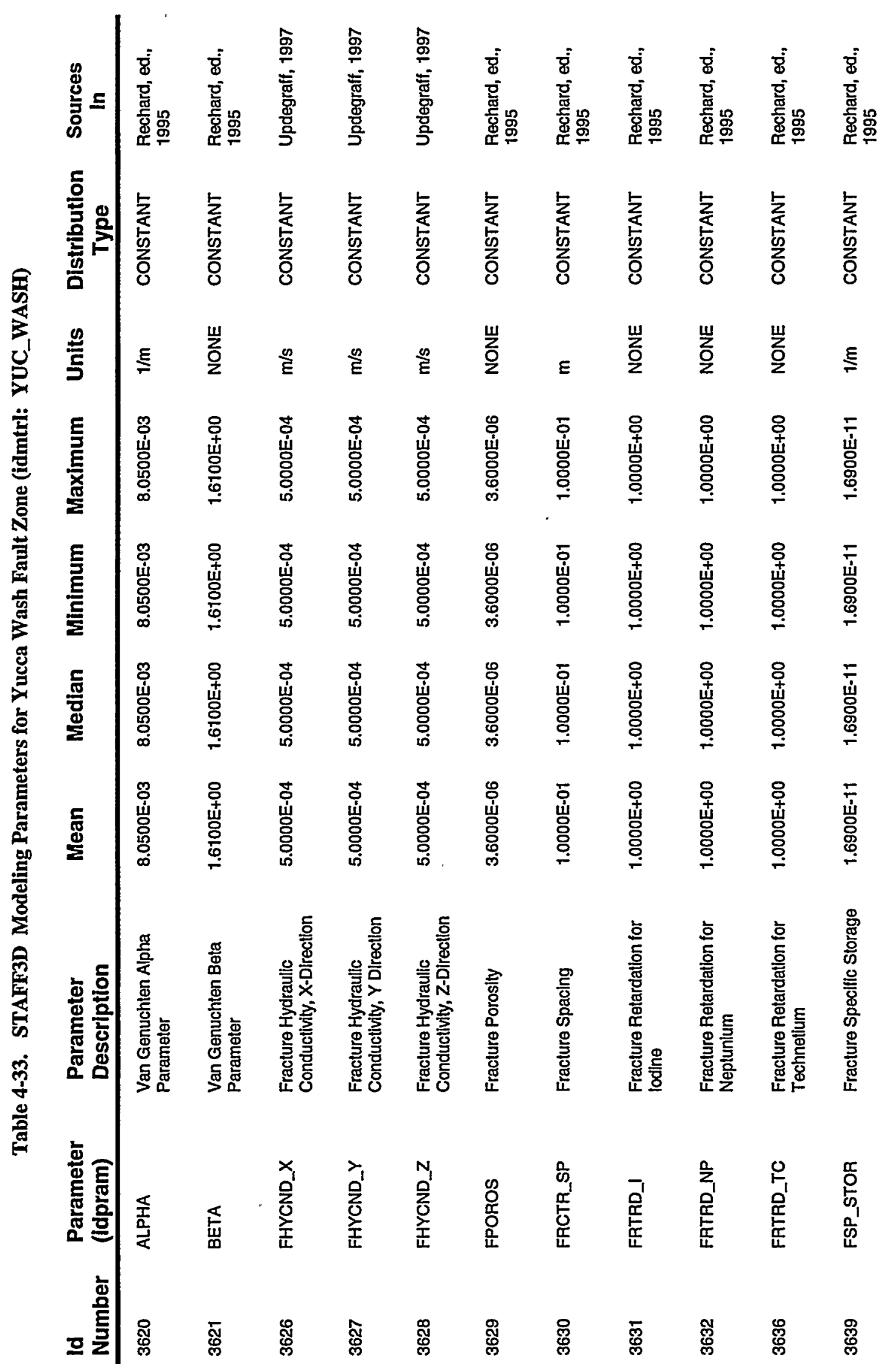




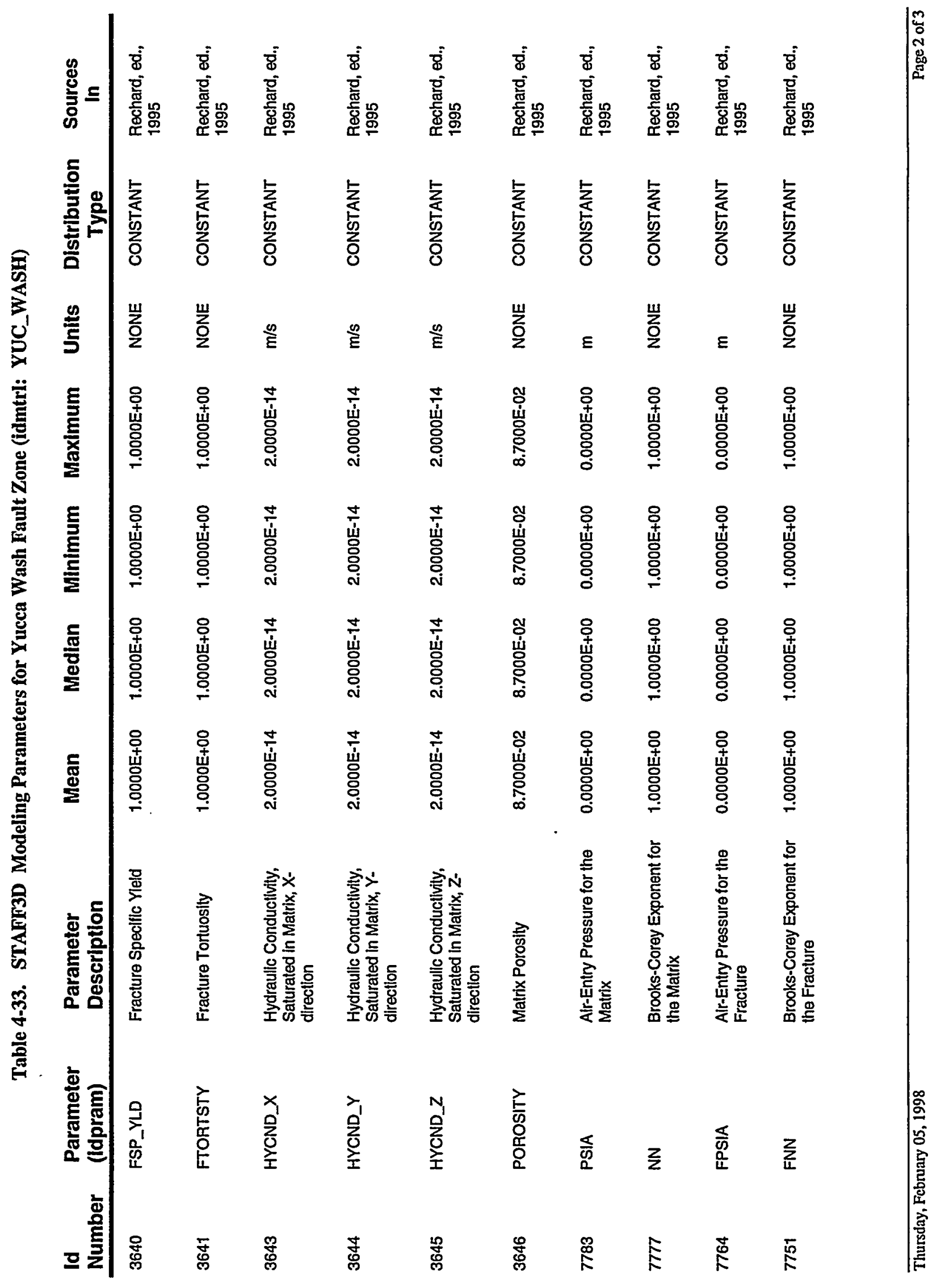



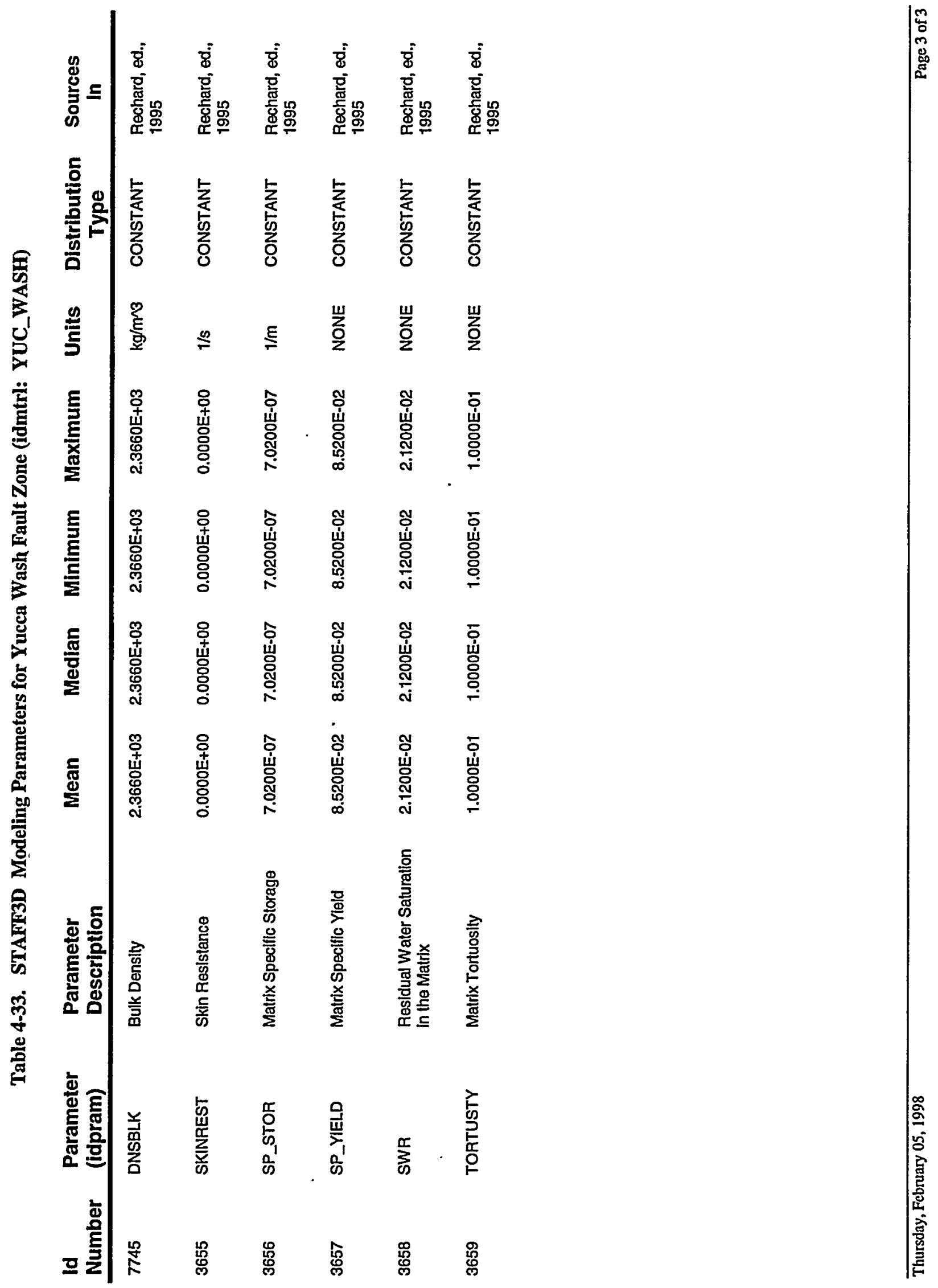


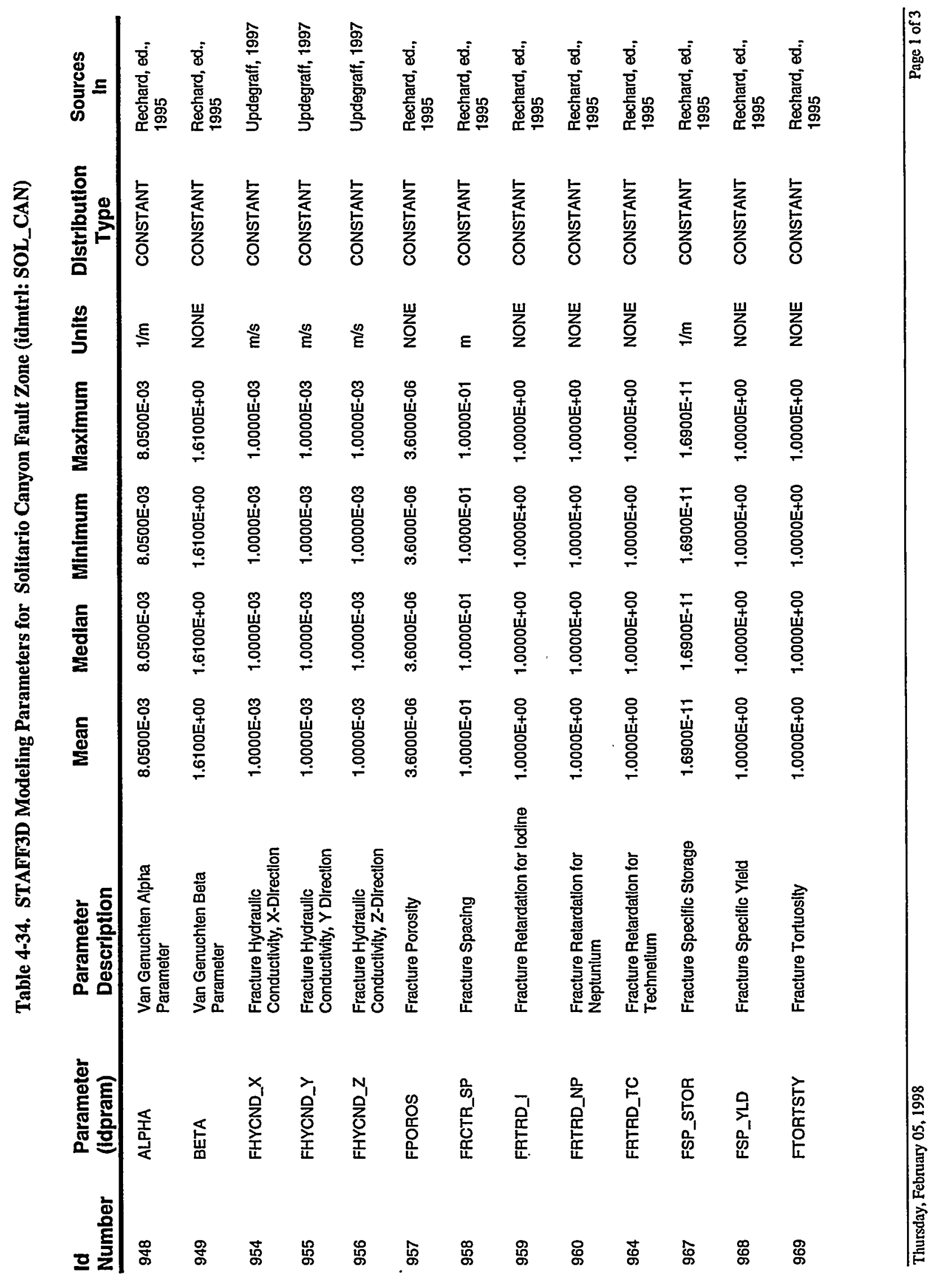




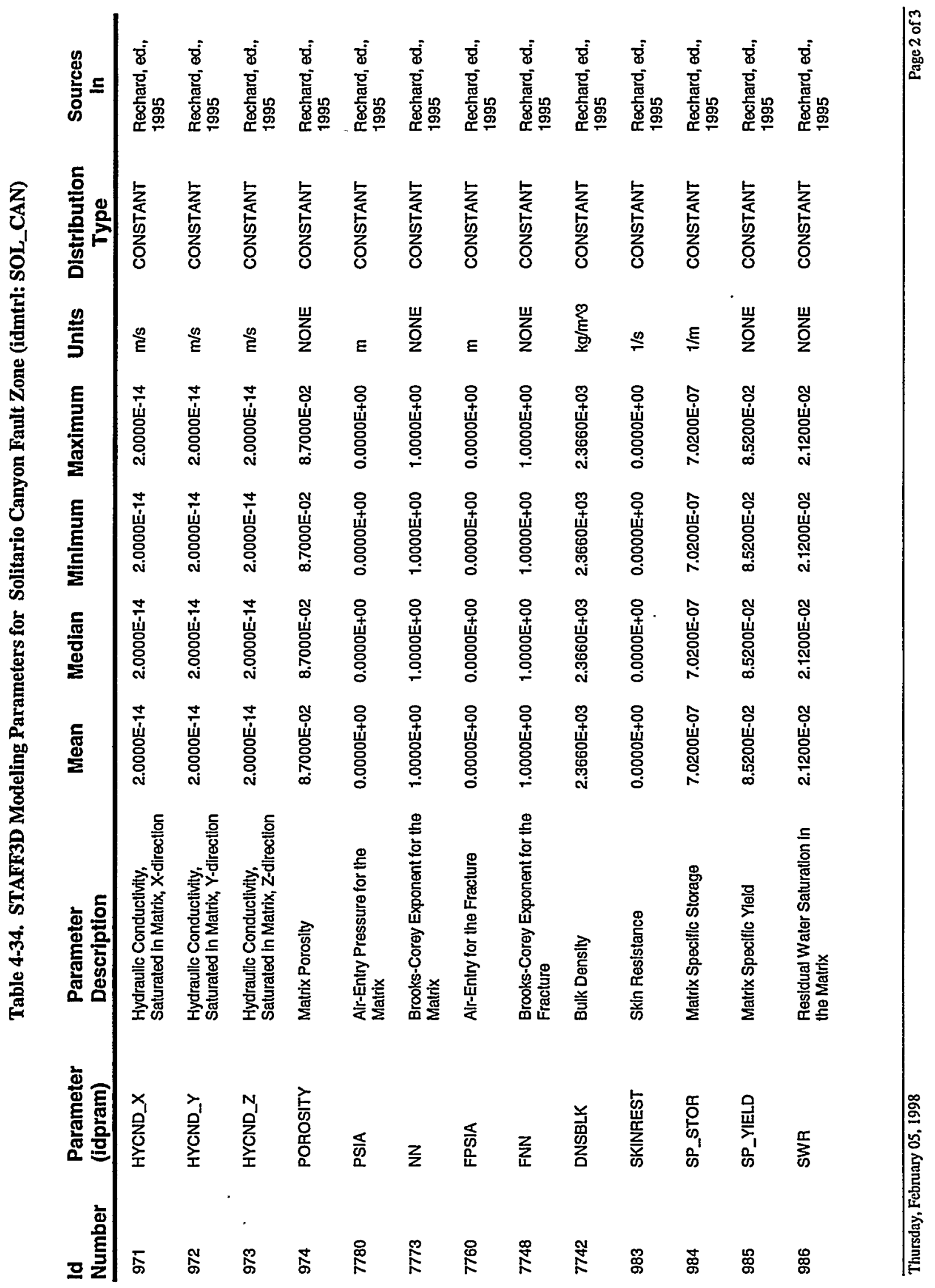



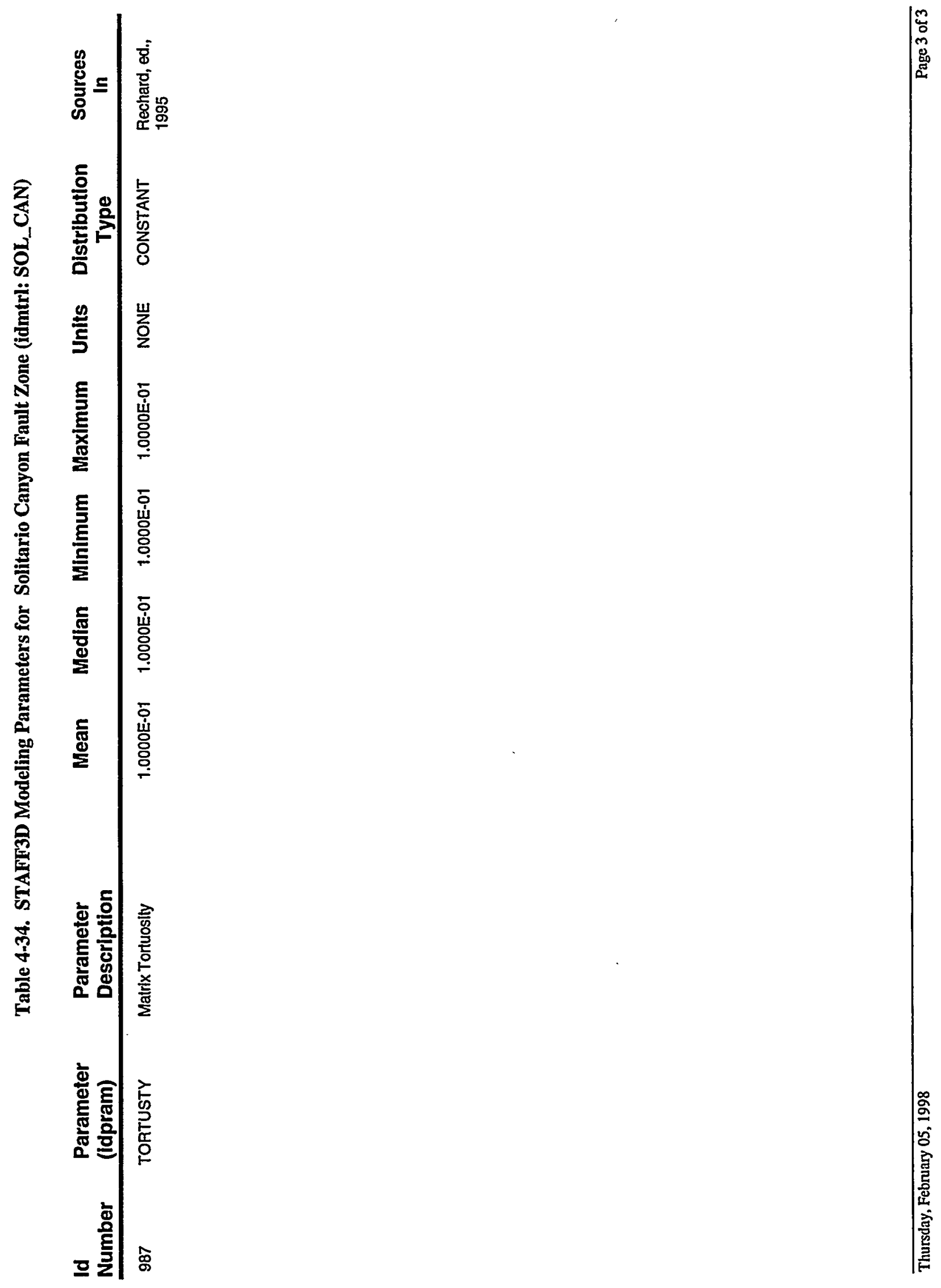

4-102 


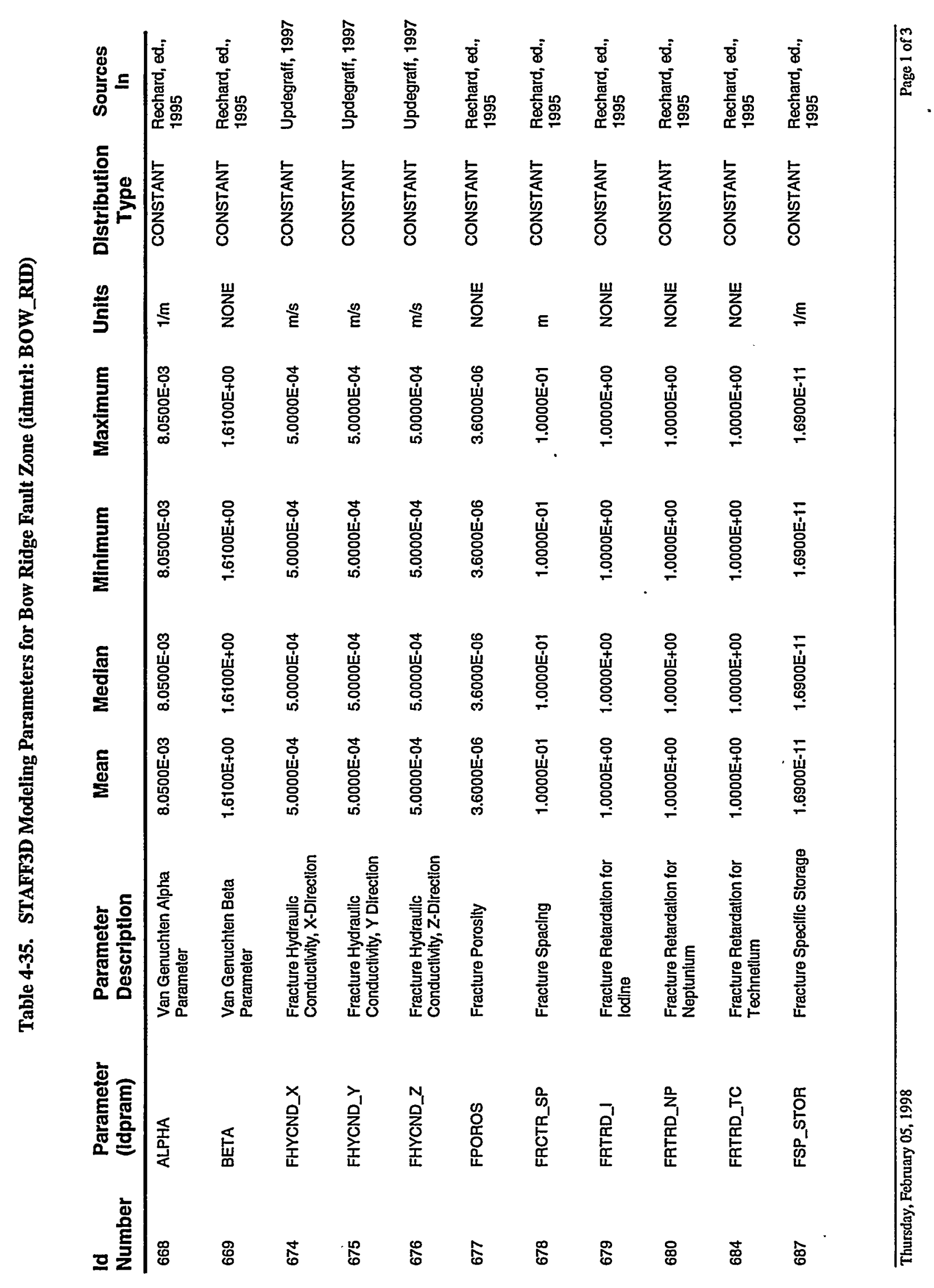




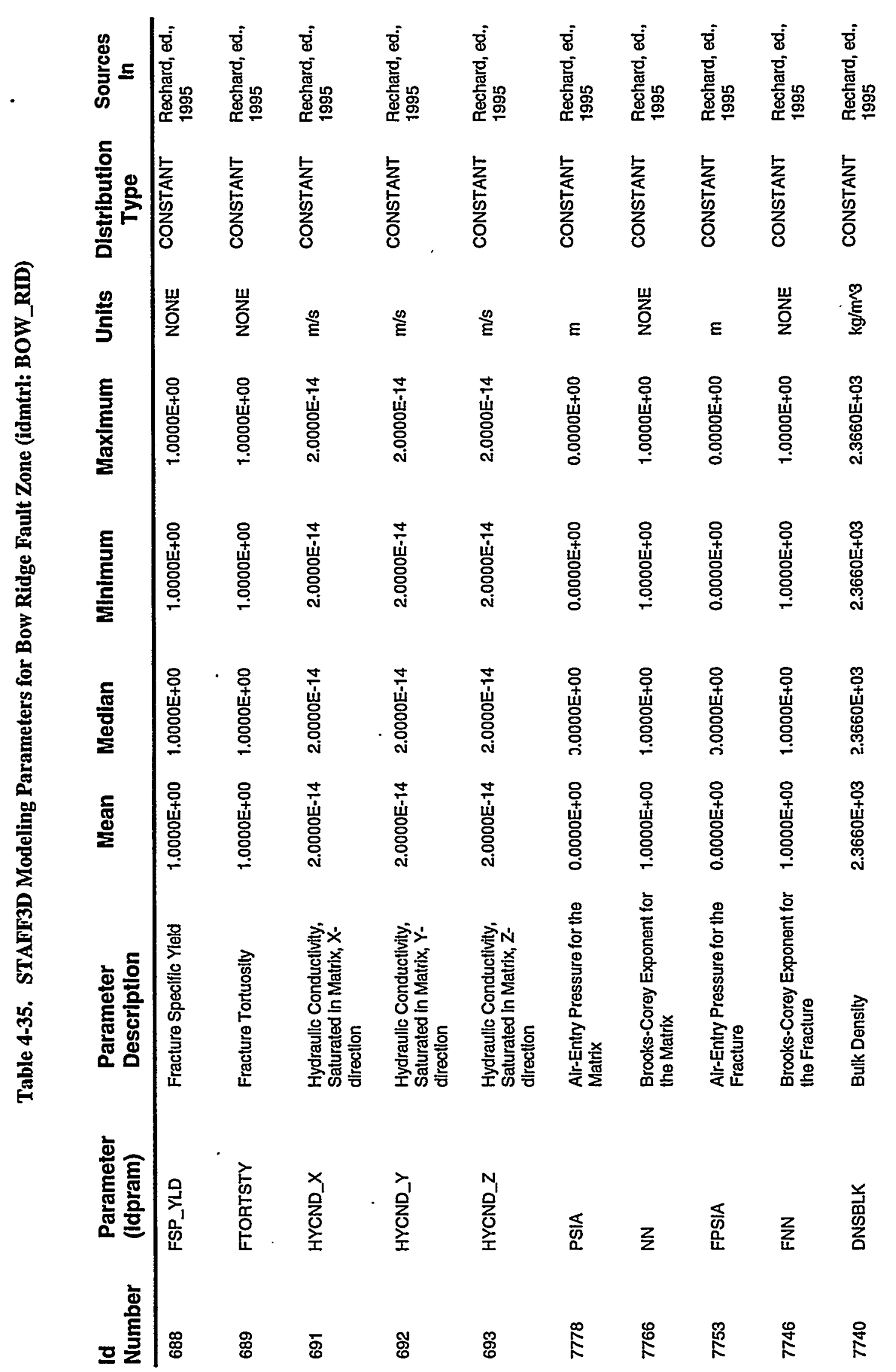

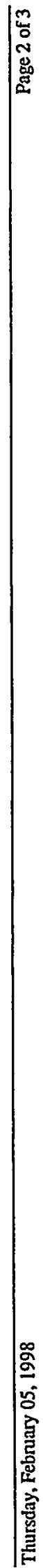




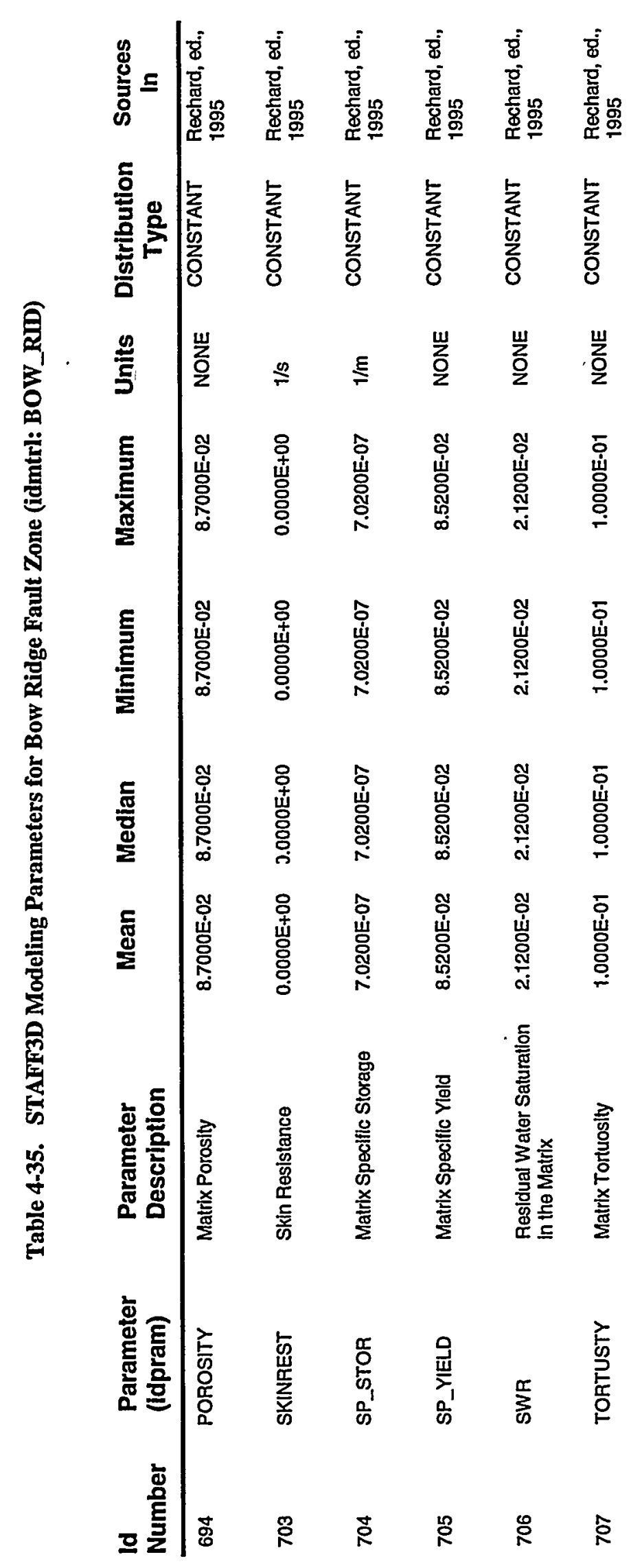




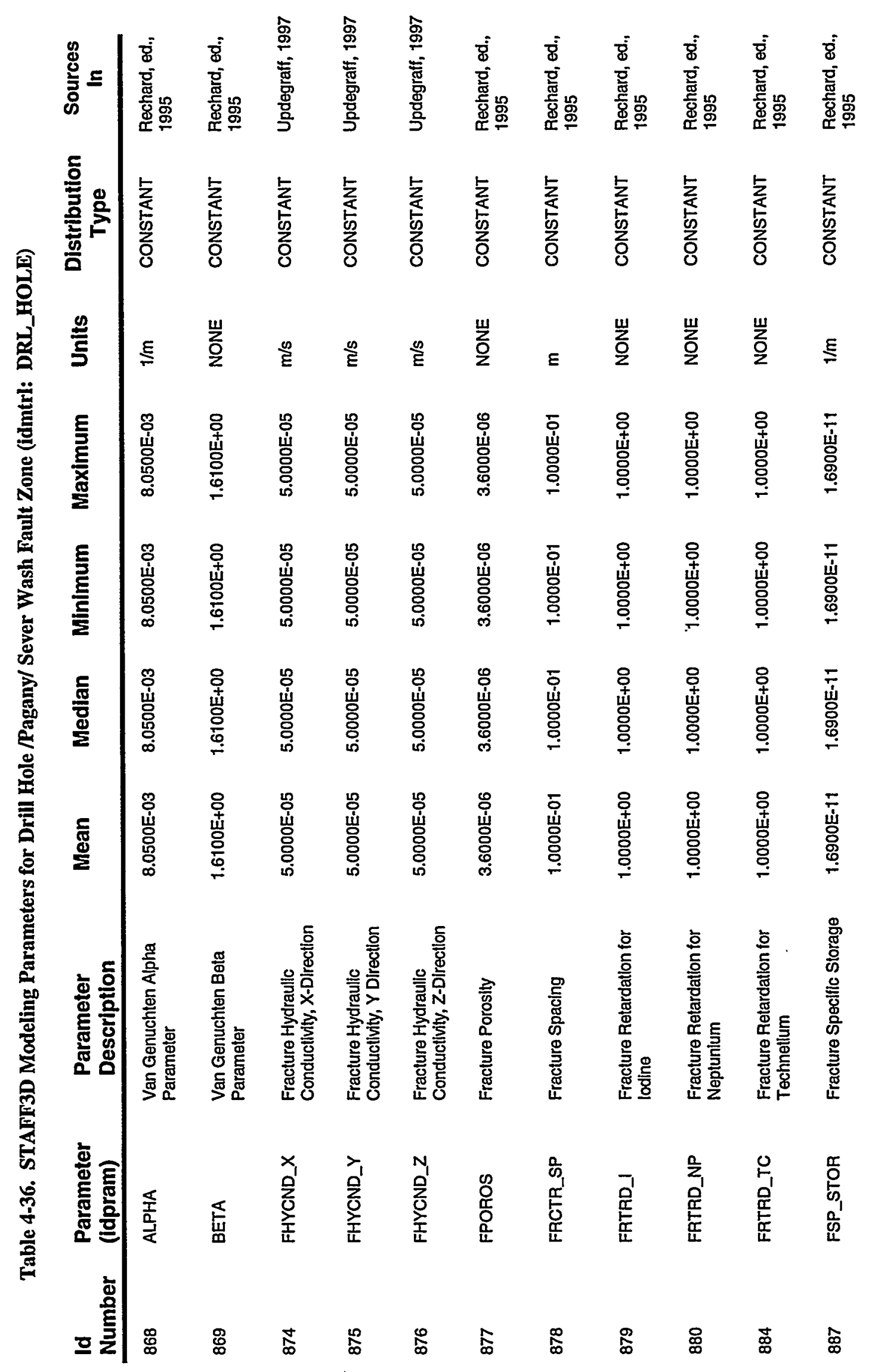

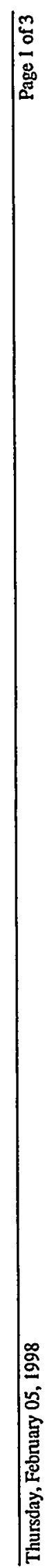




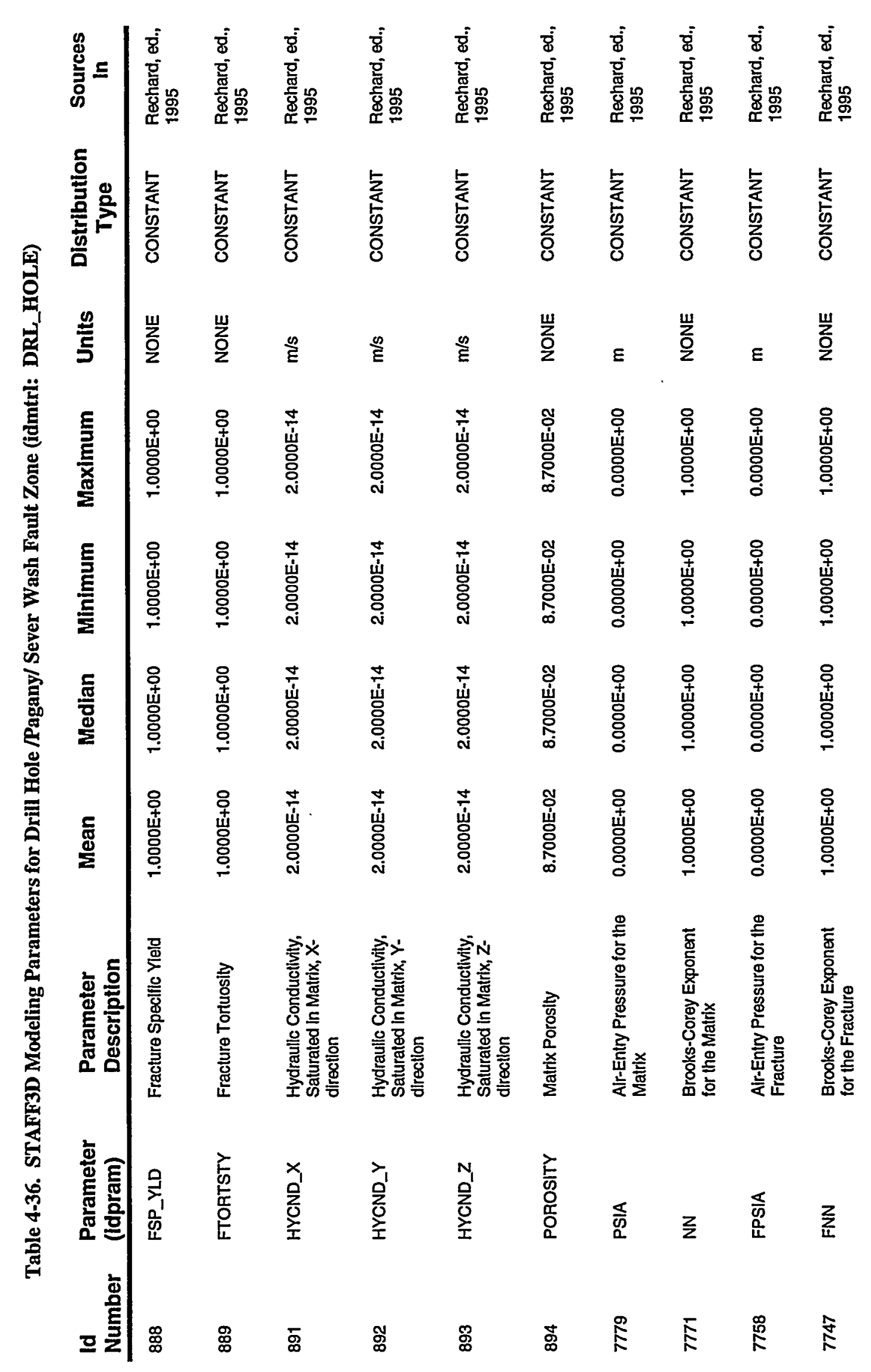

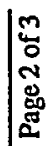




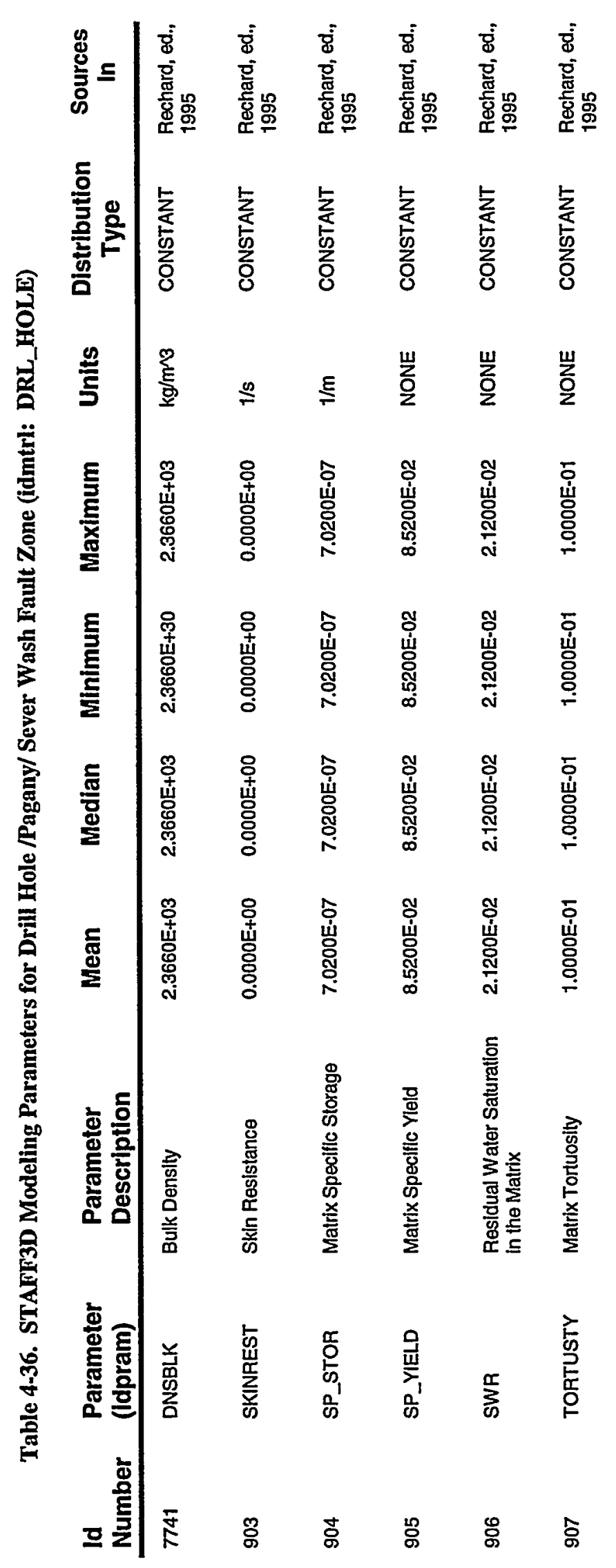




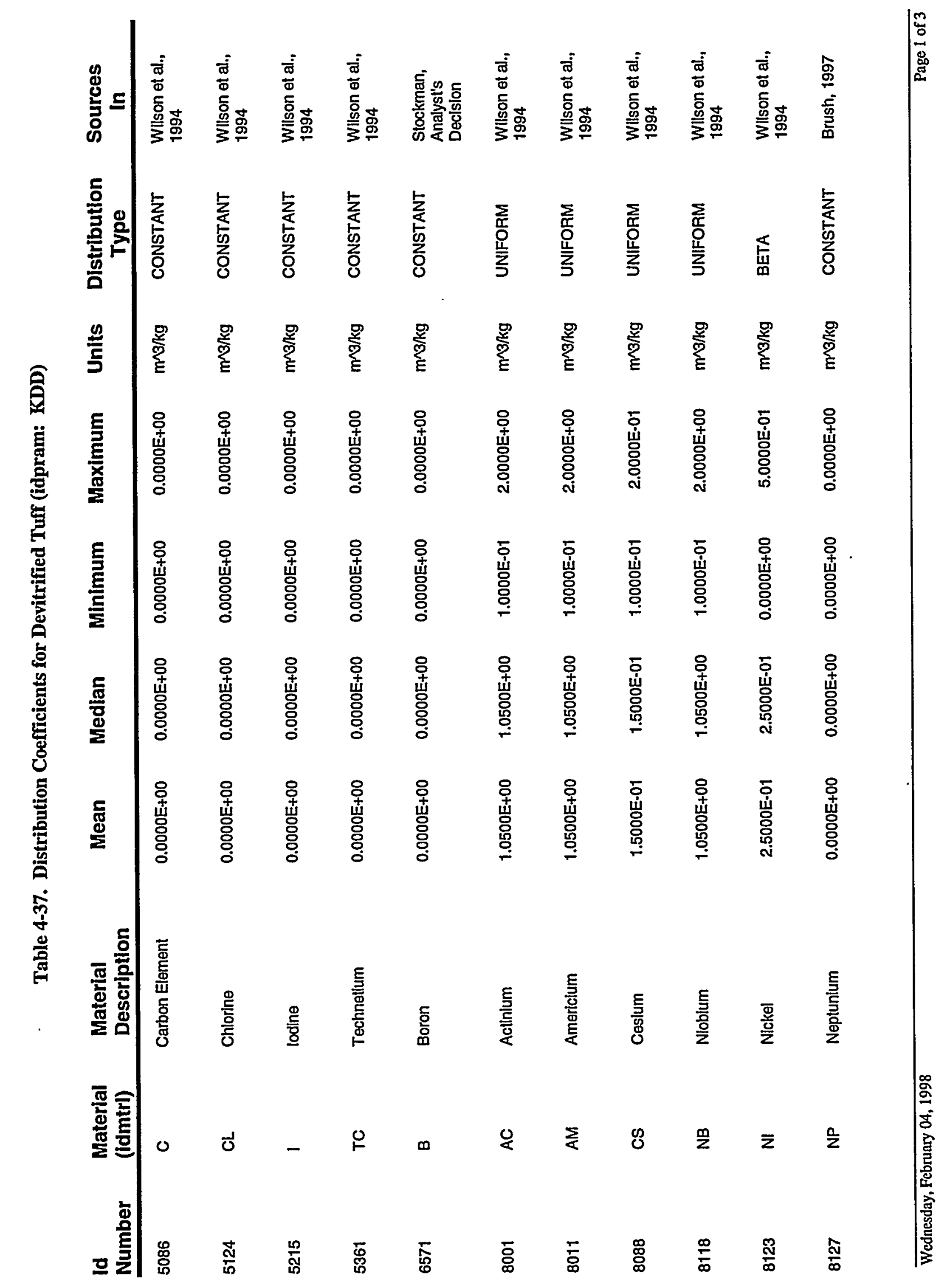




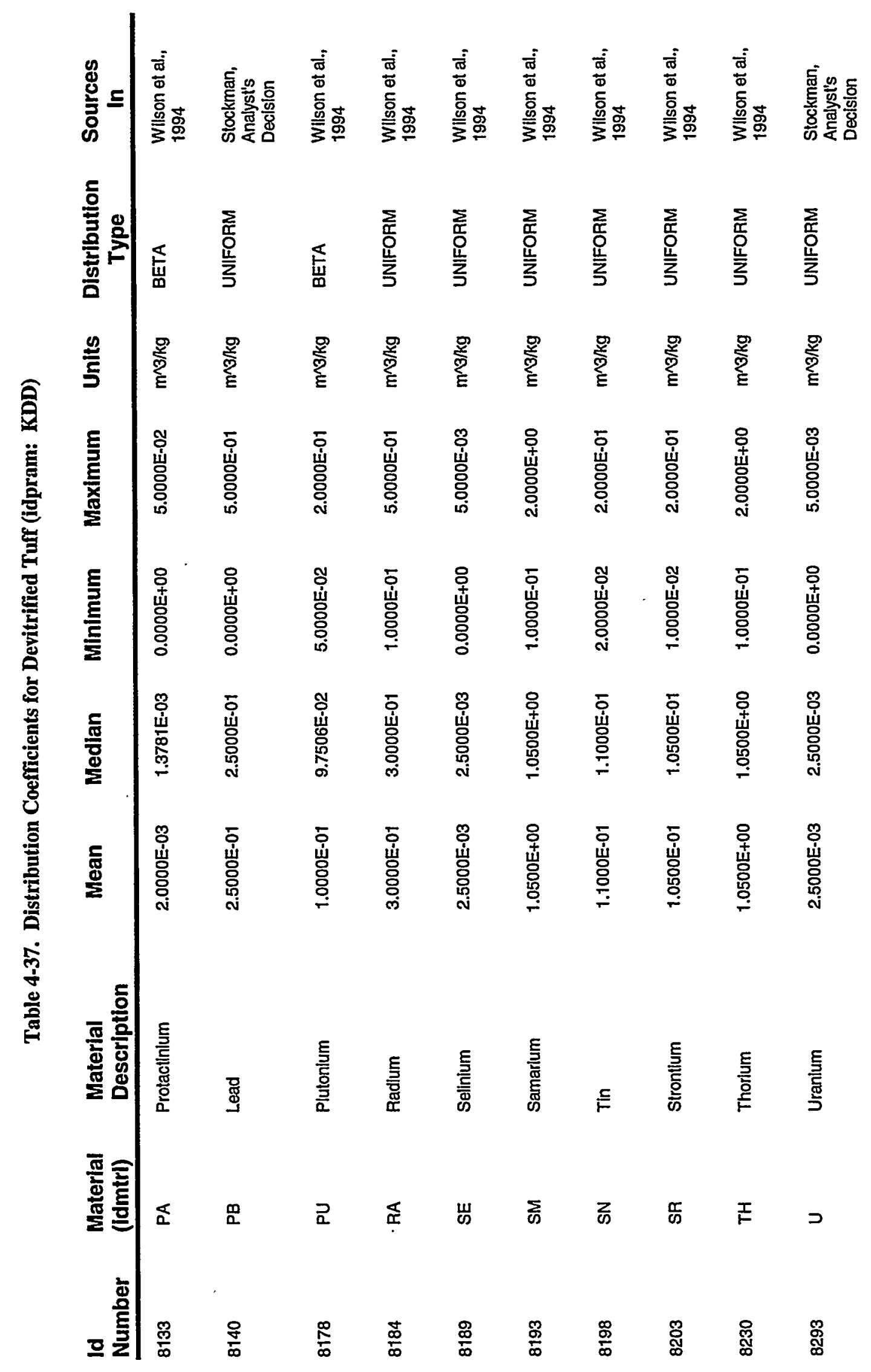

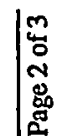




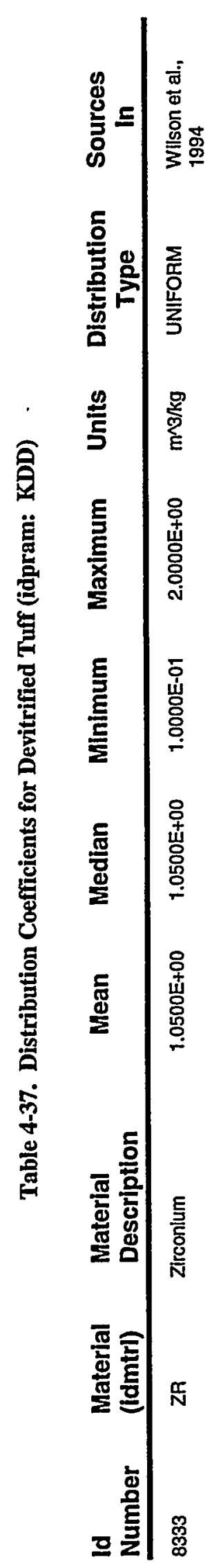




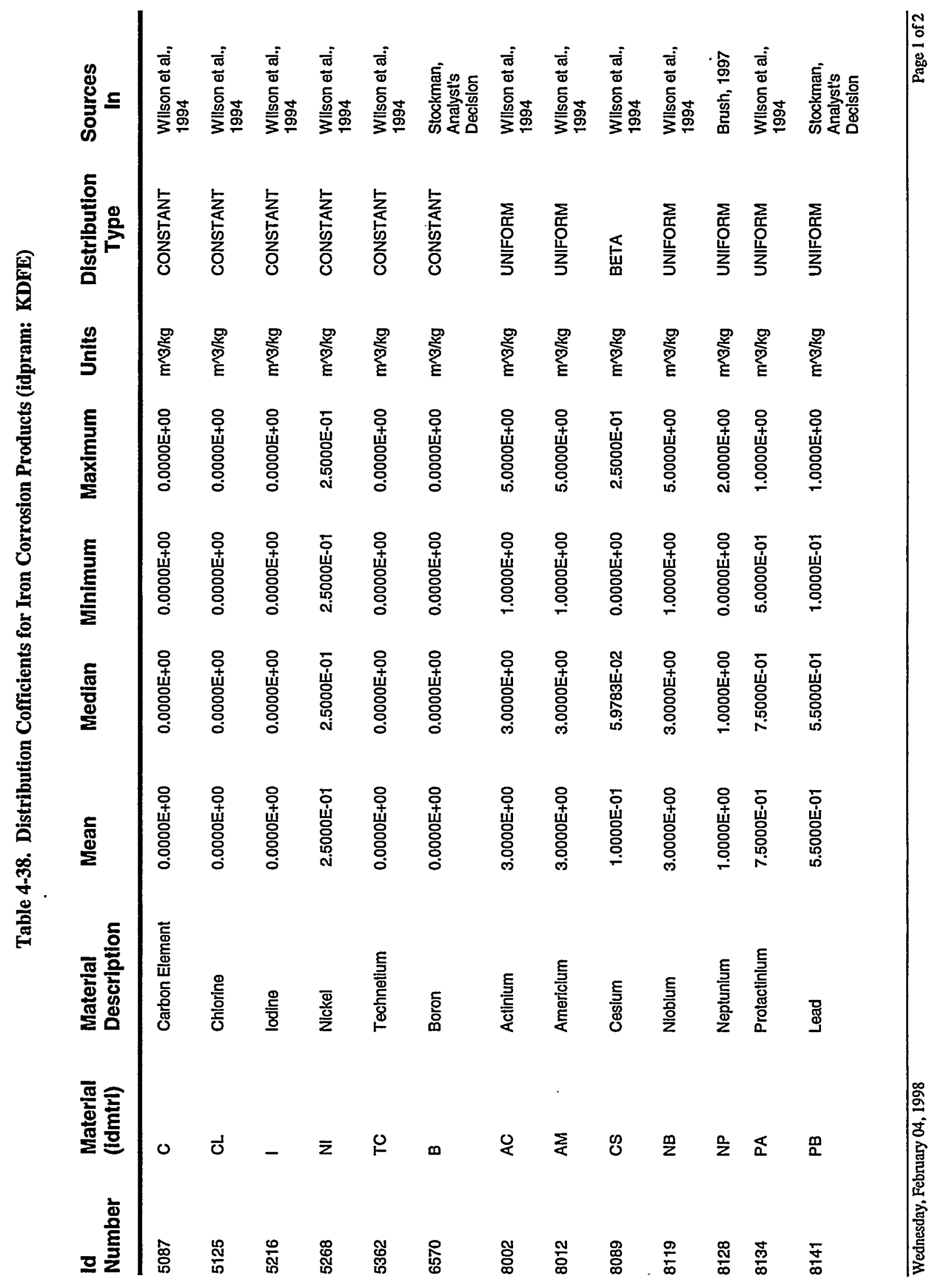




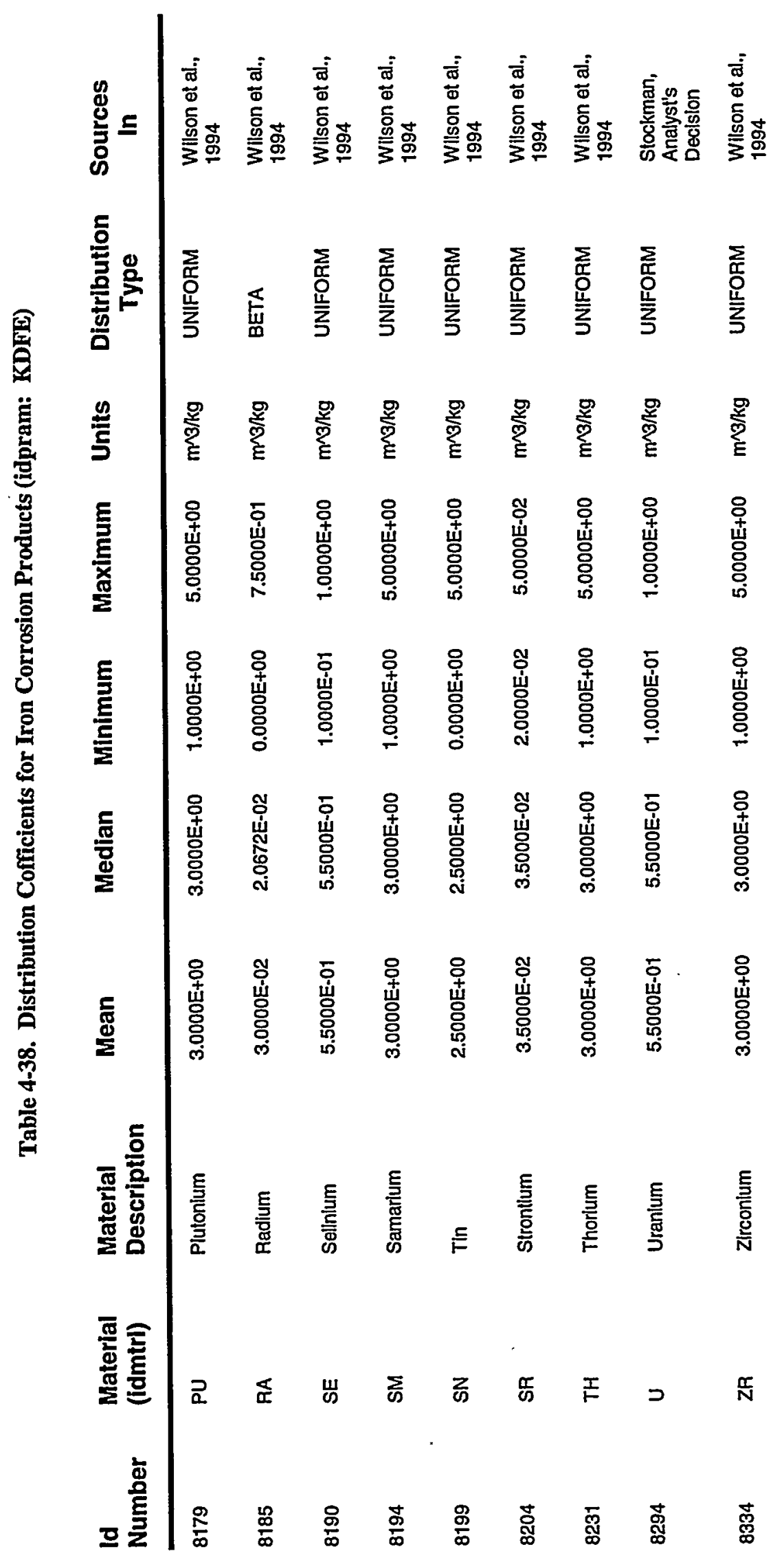

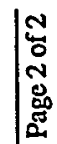




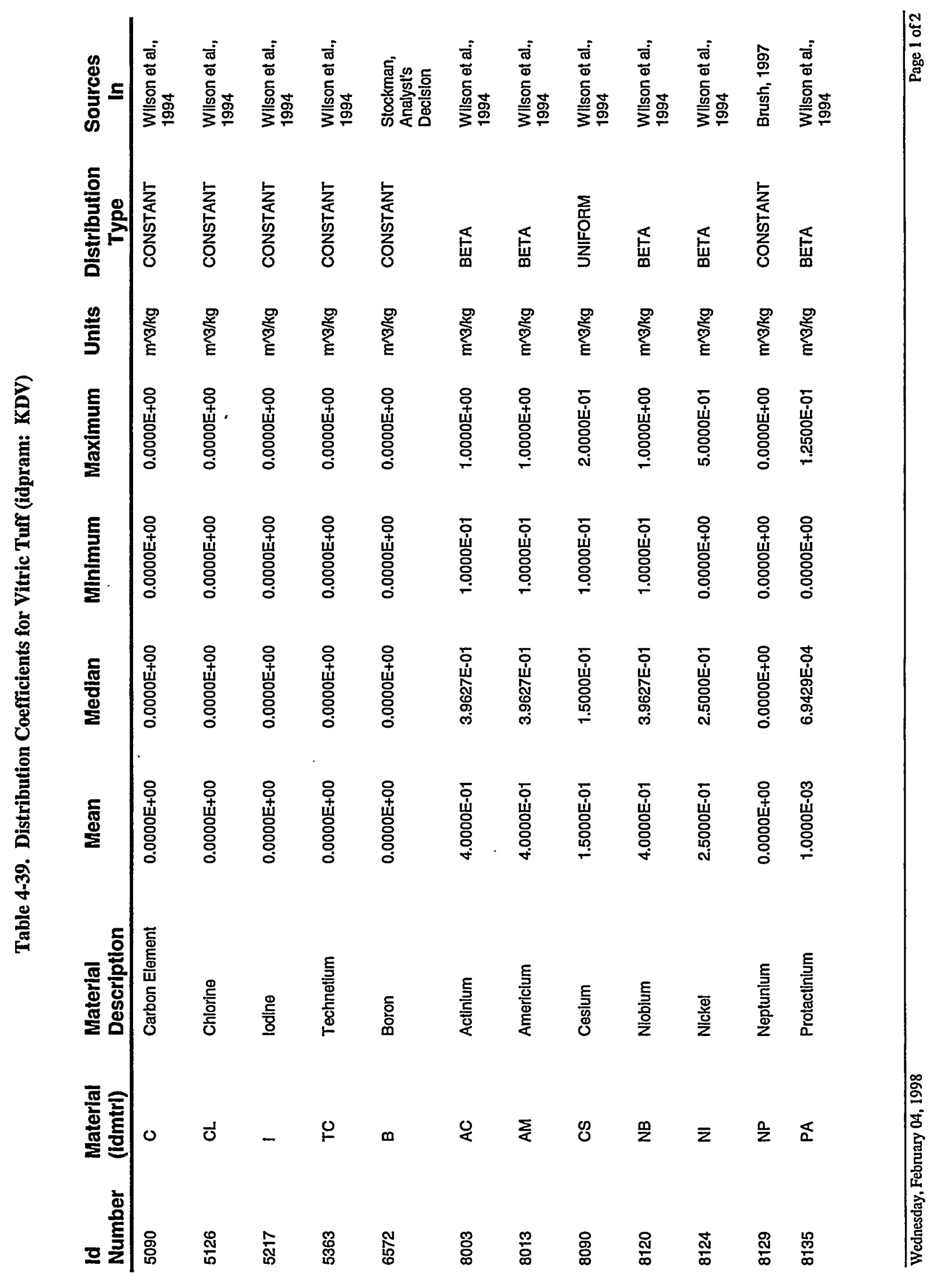




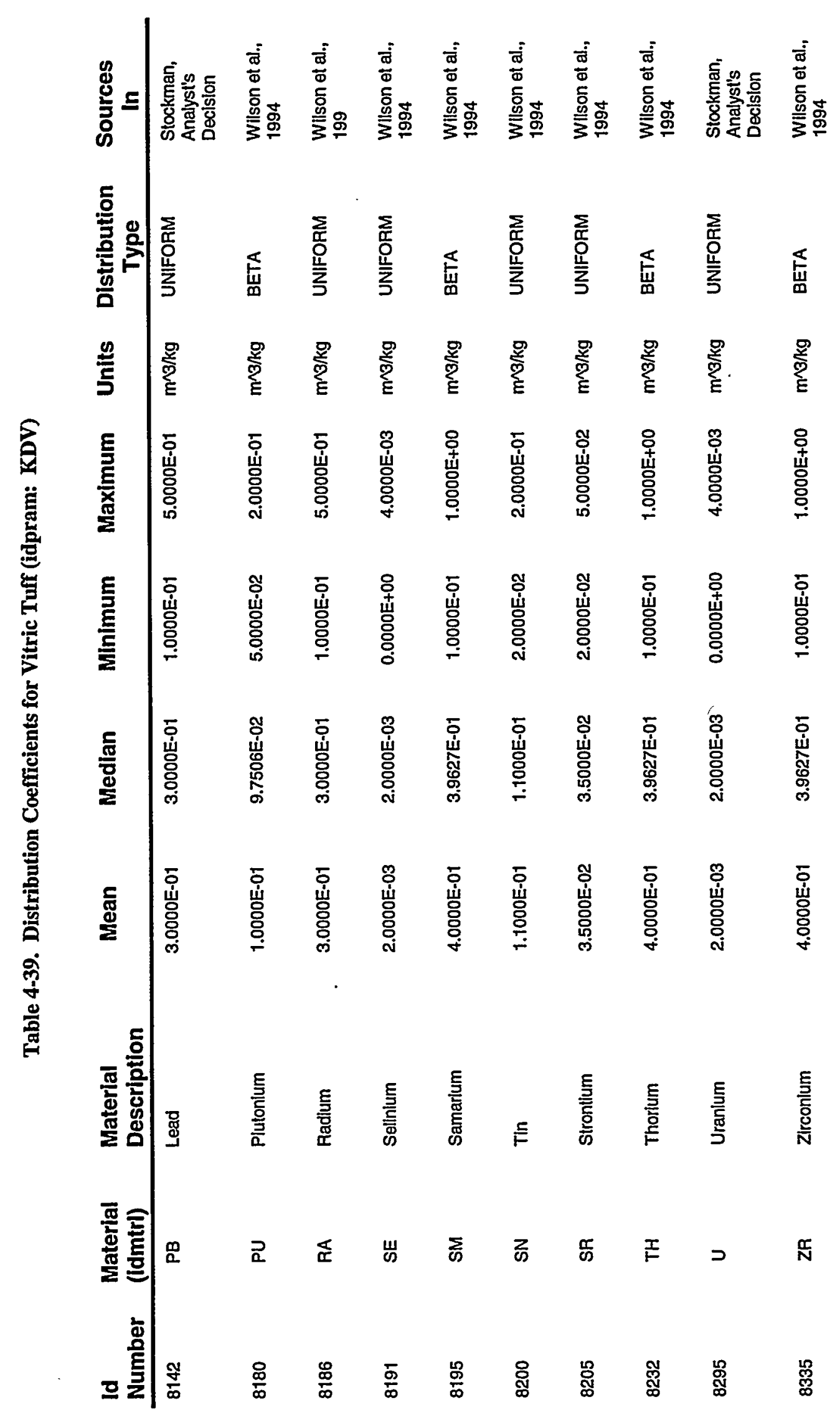

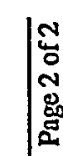




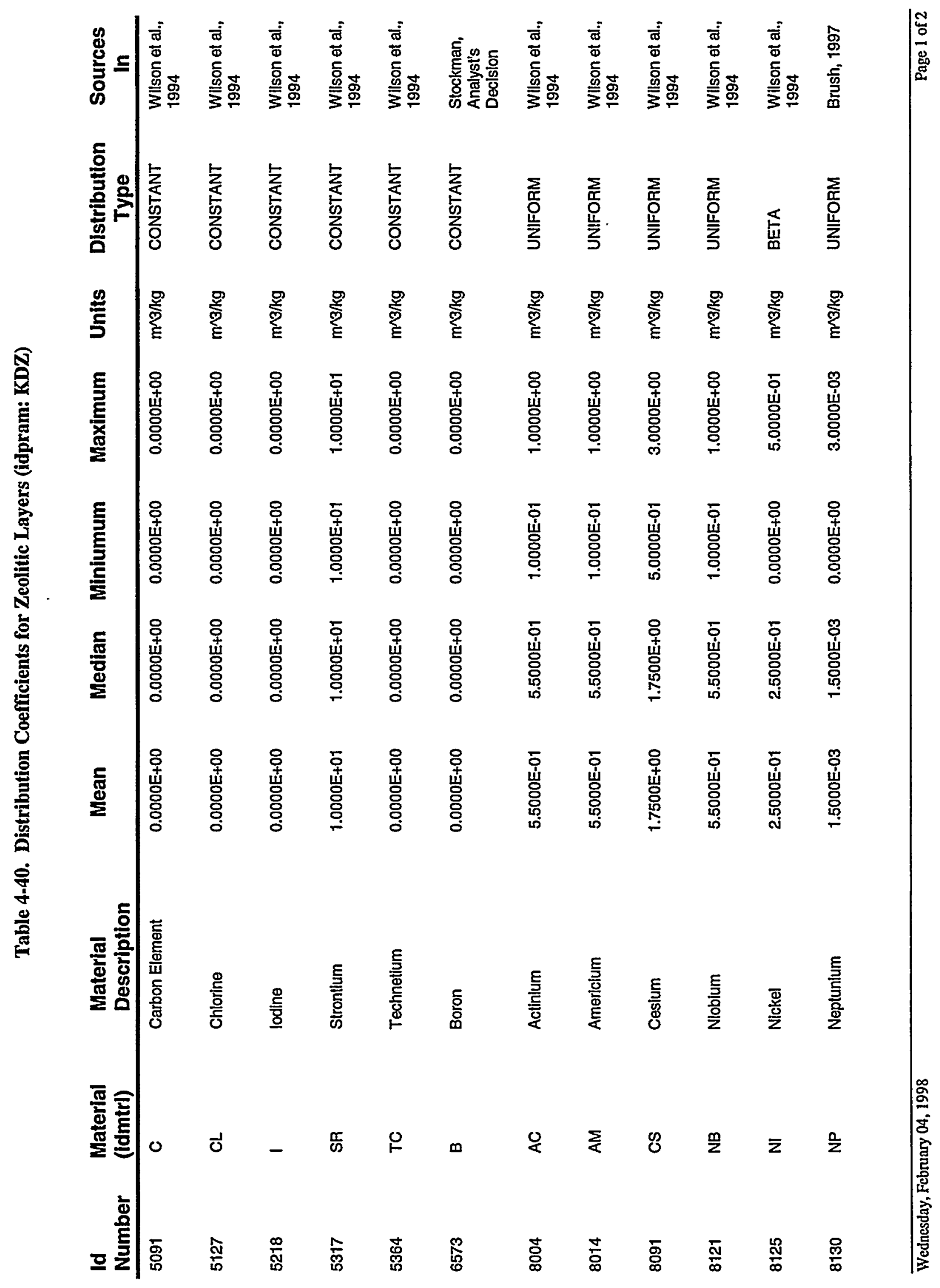




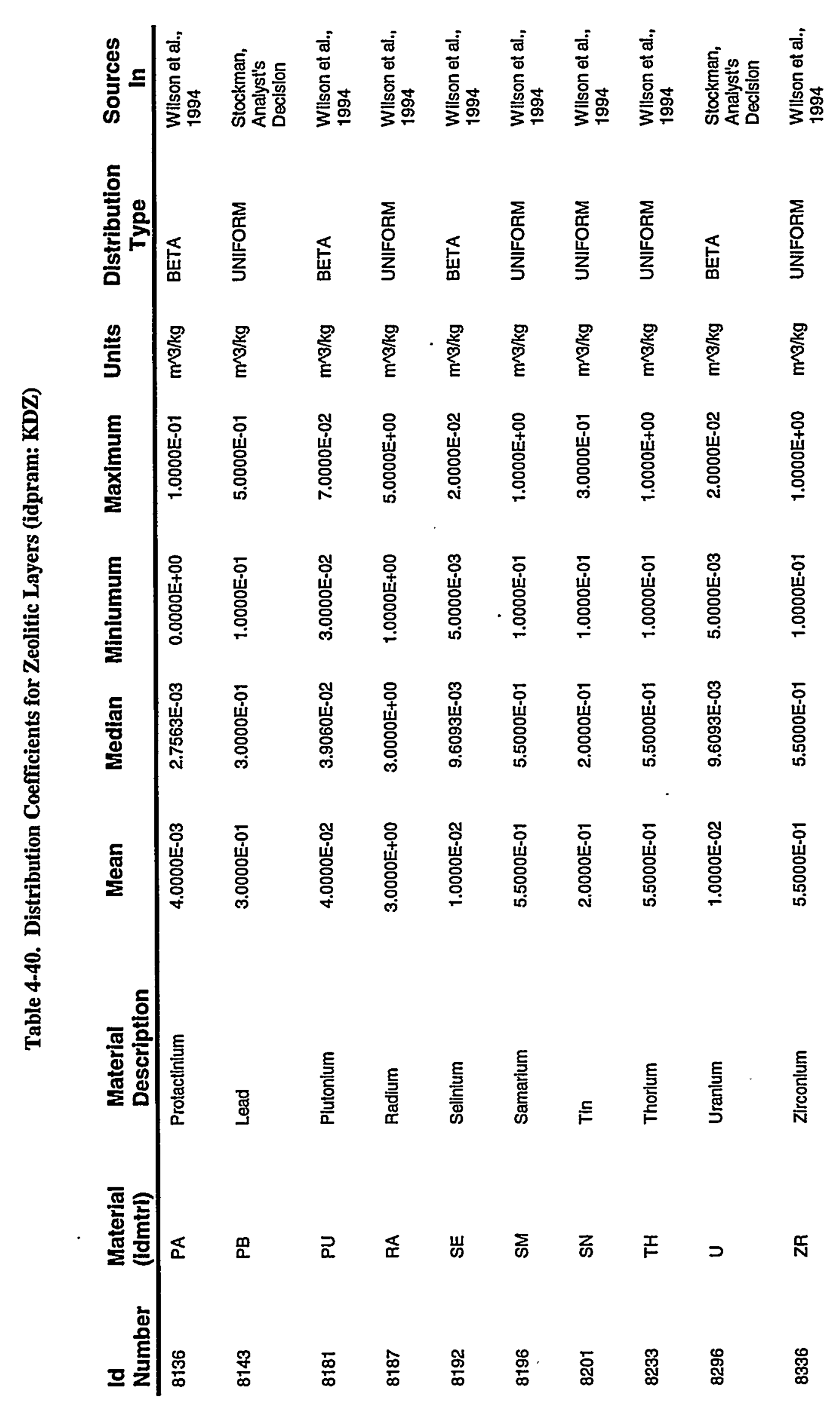

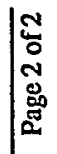




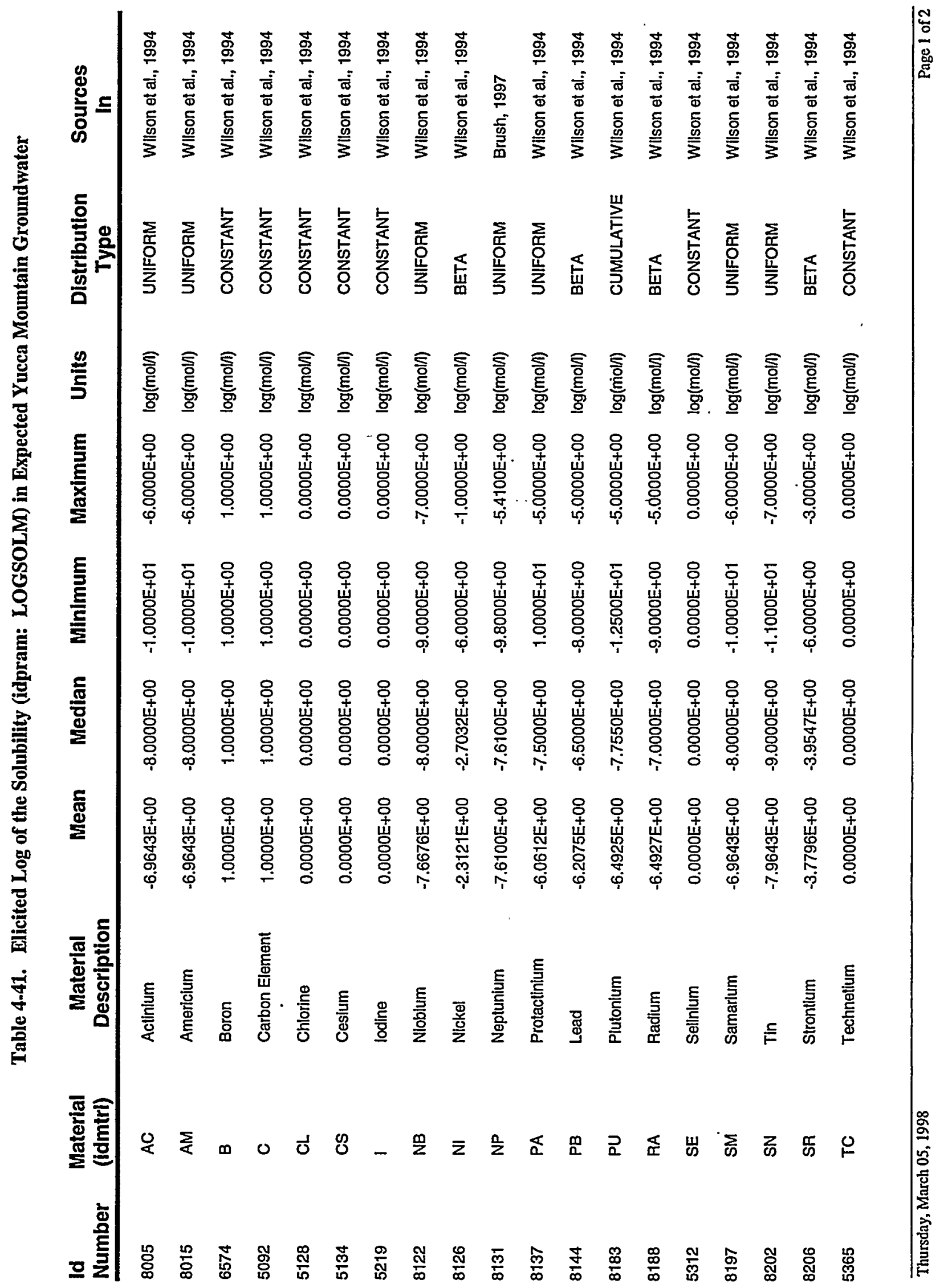




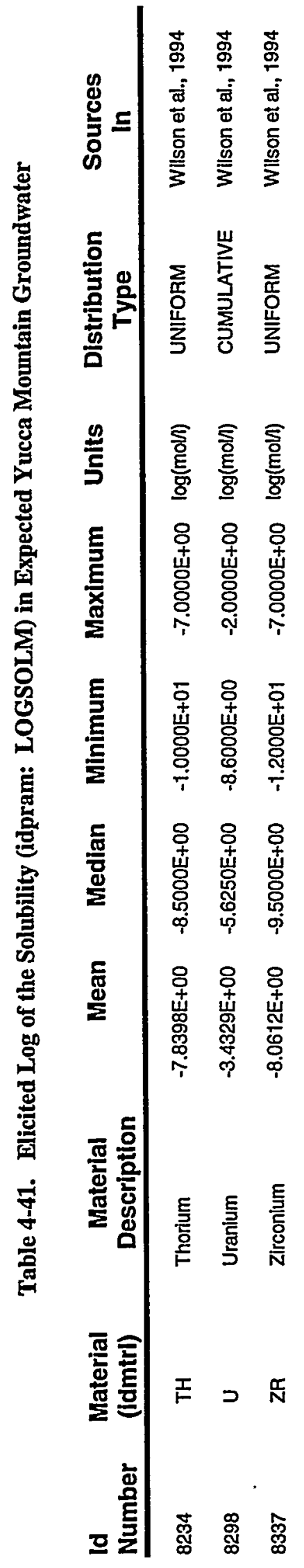




\title{
5. System Characterization: Repository Design
}

\author{
R. D. McCurley
}

Repository design is characterized, along with the waste package (see Chapter 3), as part of the engineered barrier subsystem. (The geologic barrier, the second major component of system characterization, is described in Chapter 4.) Repository design includes subsurface facility layout, emplacement of waste packages, and backfill.

\subsection{Subsurface Facilities}

The disposal region of the repository has been sized to accommodate 75,336 MTHM of spent fuel or equivalent high-level waste. The repository in the $1997 \mathrm{PA}$ is based on the most current data for the potential repository at Yucca Mountain (M\&O, 1995a; M\&O, 1996). The modeled repository is designed to accommodate the "hot" repository layout, which represents high areal mass loading. In TSPA-1995, six potential emplacement areas were identified (M\&O, 1995a, p 3-21), based on design considerations such as the decision to use the tunnel boring machine for construction and the possibility of using low or high areal mass loading. Sensitivity analyses performed for TSPA-1995 indicated that the hot repository concept would be considered as the base case (M\&O, 1996, p. 2-1). With this layout, all the waste is located in one area, labeled "primary area, upper block" in the TSPA-1995 report (M\&O, 1995a, p. 3-21); it is this area that is modeled in the 1997 PA (Figure 5-1). The waste packages are placed relatively closely together so that the accumulated heat from all the packages creates repository-scale thermal effects in the host rock.

The potential repository is situated $260 \mathrm{~m}$ below the surface (top of alluvium to repository floor). It is a tunnel and pillar configuration for in-drift (horizontal) emplacement, with the entire repository consisting of disposal tunnels (Figure 5-2). Three main drifts, 7.62 in width, traverse the length of the repository, providing access to the disposal tunnels (Figure 5-2). A tunnel boring machine is assumed for excavation of the rooms and drifts and is the preferred mining scheme for the YMP (M\&O, 1995a). The design of the repository is governed by the total waste volume $(75,336 \mathrm{MTHM})$ and the emplacement of the waste packages. Because of the difference in the amount of waste being modeled in the 1997 PA compared to that in the 1994 PA (75,336 MTHM vs. 12,000 MTHM), the repository area is more than 10 times larger in this performance assessment $\left(3.12 \times 10^{6} \mathrm{~m}^{2}\right.$ in the $1997 \mathrm{PA}$ vs. $2.56 \times 10^{5} \mathrm{~m}^{2}$ in the 1994 PA). General dimensions of the repository as modeled in the 1997 PA are provided in Table 5-1.

Table 5-1. Dimensions of Subsurface Facilities for the 1997 PA

\begin{tabular}{|c|c|}
\hline Region & Dimensions \\
\hline Disposal Tunnels & $\begin{array}{l}\text { Length: } 1236 \mathrm{~m}^{\star} \\
\text { Diameter: } 5.3 \mathrm{~m}^{\star \star} \\
\text { Total: } 87 \text { tunnels }\end{array}$ \\
\hline Pillars & $\begin{array}{l}\text { Length: } 1236 \mathrm{~m} \\
\text { Width: } 23.3 \mathrm{~m}\end{array}$ \\
\hline Access Drifts & Width: $7.62 \mathrm{~m}$ \\
\hline Repository Area (total) & $3.12 \times 10^{6} \mathrm{~m}^{2}$ \\
\hline Waste Disposal Area (excavated) & $6.08 \times 10^{5} \mathrm{~m}^{2}$ \\
\hline $\begin{array}{l}\text { Includes two 2-m access tunnels } \\
* \text { Without invert; see Section 5.2.2. }\end{array}$ & \\
\hline
\end{tabular}




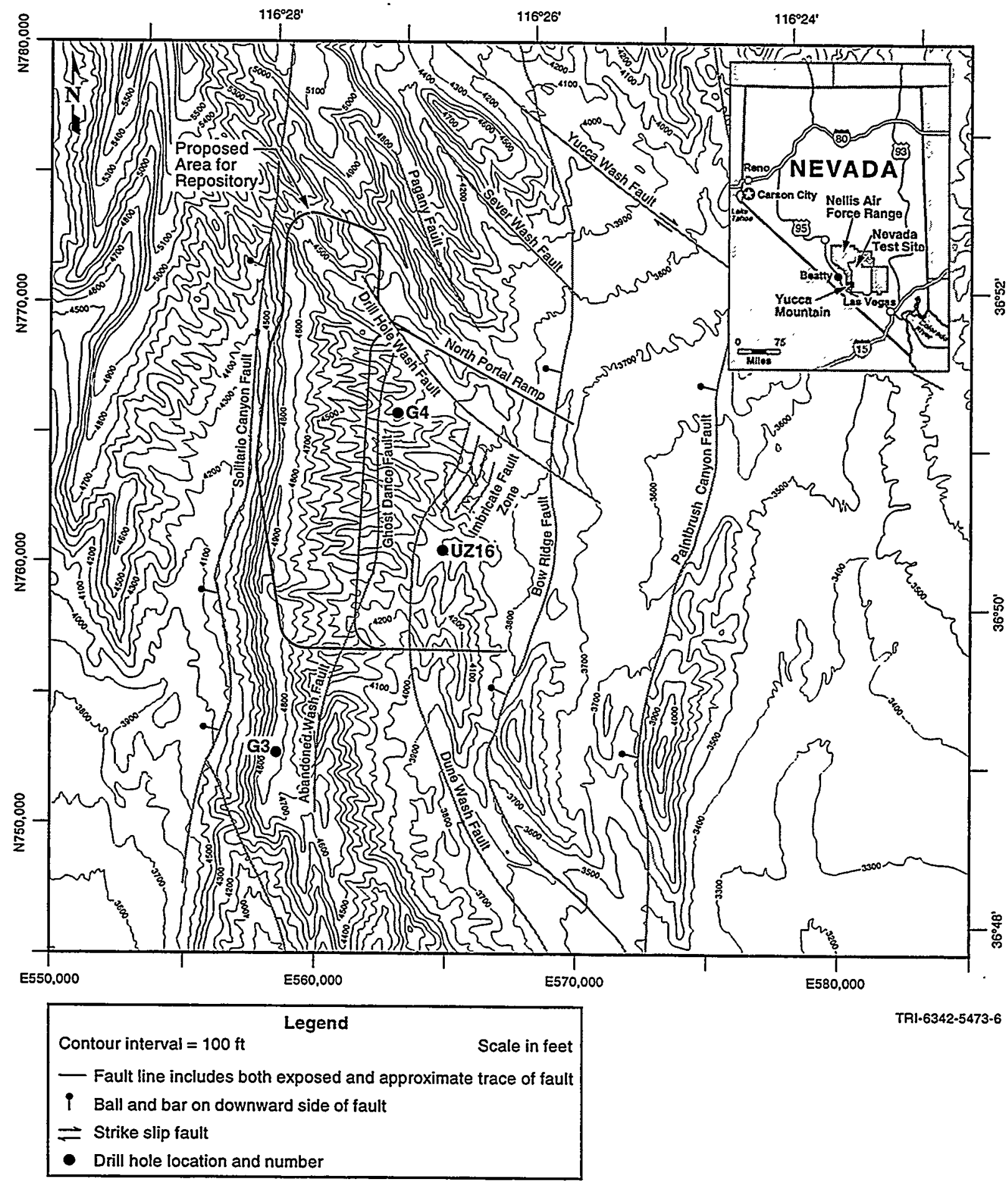

Figure 5-1. Location of potential repository at Yucca Mountain, Nevada. 


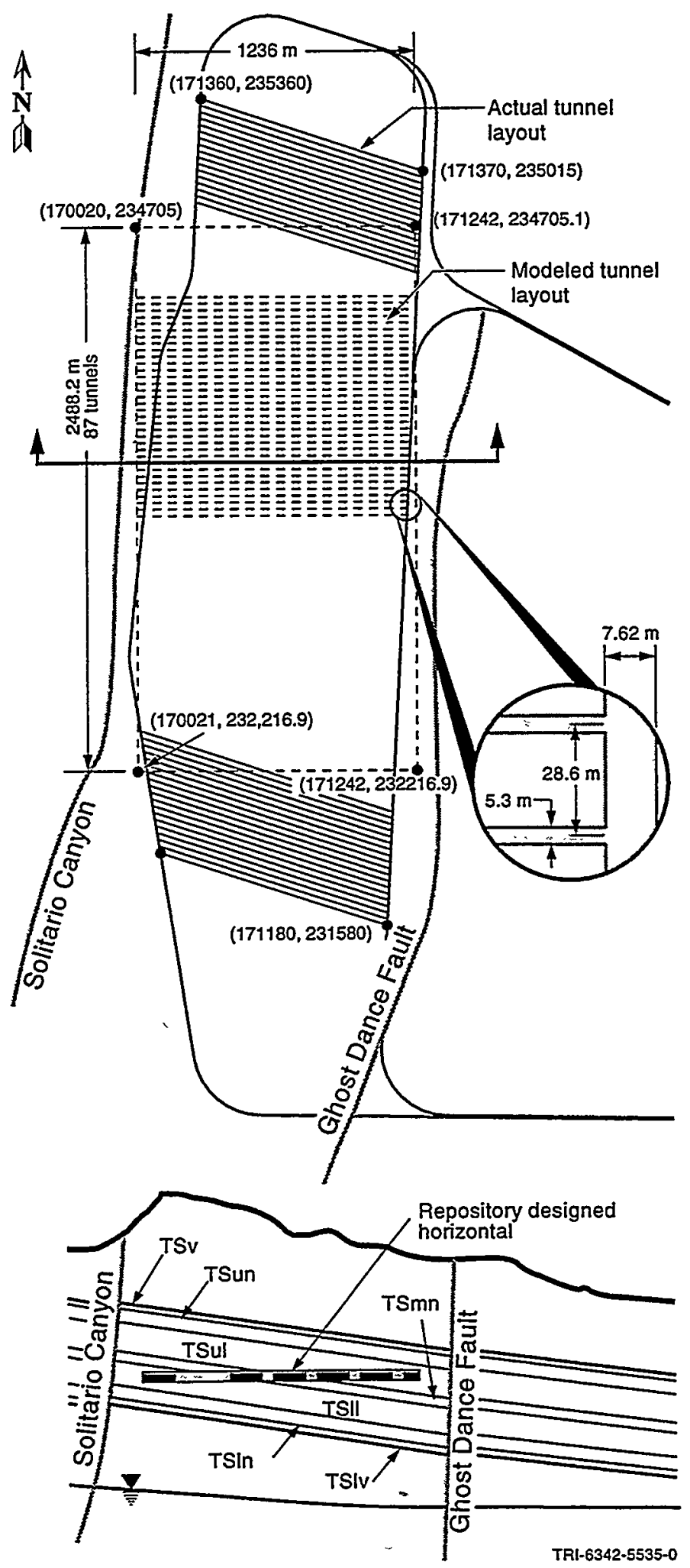

Figure 5-2. Layout of disposal tunnels and access drifts for potential repository in tuff with in-drift emplacement. 


\subsection{Emplacement of Waste Packages}

For this performance assessment, center in-drift on pedestal (CIDP) emplacement is modeled, in which waste packages are emplaced along the centerline of an excavated drift. A 0.2 -m-thick precast cement liner is installed in the disposal tunnels. With the CIDP option (Figure 5-3), waste packages are placed by means of a remote controlled rail-mounted gantry crane on permanent pedestals, composed of carbon steel, that are prepositioned in the drift (M\&O, 1995a, p. 3-7). The drift diameter of $5.3 \mathrm{~m}^{\prime}$ (before installation of the liner) provides a reasonable operating clearance and additional space for ground support and excavation misalignment tolerance. The materials beneath the emplacement pedestal have not yet been determined; current options under consideration by the YMP include no backfill, a layering of tuff with a gravel-like consistency, or a sand-like material with specific chemical properties. In the 1997 PA, no gravel or other material is placed beneath the pedestal; only the concrete liner is assumed.

Table 5-2 provides a listing of the number of waste packages required to dispose of the spent fuel and high-level waste. (See also Chapter 3 for additional information about waste volumes and types.) .

\subsubsection{Waste Package Spacing}

Three factors potentially affect the spacing of waste packages within the disposal tunnels and the distance between tunnels: (1) ability to bore closely spaced borehole and/or tunnel closely spaced areas, (2) potential for neutron interaction between containers, which would enhance the possibility of a critical condition, and (3) thermal limits placed on the waste form and/or adjacent host rock. In the $1997 \mathrm{PA}$, with regard to item 1, the tunnel boring machine is assumed for construction. With regard to item 2, results of the previous performance assessment (1994 $\mathrm{PA}$ ) indicated that neutron interaction would be minimal because of the large amount of material present in the lids of the disposal containers, so no special spacing was considered.

With regard to thermal limits (item 3), the design goal in the 1997 PA was to use the hot repository concept, which is defined by the YMP as density of heavy-metal mass, which, for commercial spent nuclear fuel, is roughly equivalent to calculating areal power density. The design-basis MTHM density used by the YMP is $2.1 \mathrm{MTHM} / \mathrm{m}^{2}$ ( $85 \mathrm{MTHM} / \mathrm{acre}$ ) or $18.7 \mathrm{~W} / \mathrm{m}^{2}{ }^{*}$. This goal is achieved by controlling the spacing among nearest-neighbor waste packages. Note that TSPA-1995 initially used $83 \mathrm{MTHM} / \mathrm{acre}^{\dagger}$ as the maximum for the hot repository concept (M\&O, 1995a). A recommended thermal limit by the YMP is to maintain a temperature below $115^{\circ} \mathrm{C}$ at the interface of two hydrologic modeling units TSlv and CHn (see Chapter 4). This limit was originally proposed in conceptual designs of the Yucca Mountain repository to prevent alteration of zeolites in the hydrologic modeling unit, $\mathrm{CHnz}$, which is directly below $\mathrm{CHn}$; aiteration of zeolites may reduce their ability to absorb radioisotopes (MacDougall et al., 1987). Results of sensitivity analyses performed for TSPA-1995 indicated that the initial value of $83 \mathrm{MTHM} / \mathrm{acre}$ could be increased to $85 \mathrm{MTHM} / \mathrm{acre}$ without exceeding the $115^{\circ} \mathrm{C}$ limit.

The hot repository concept was selected as part of the base case for TSPA-1995 (M\&O, 1996, p. 2-1). The purpose of designing a hot repository is to dry out the host rock for hundreds of years. The waste packages have a heat output at the time of emplacement that depends primarily on the burnup and decay of the commercial spent fuel. (The heat output from DOE SNF and DHLW containers is also considered but is relatively low.) The accumulated heat from all the packages creates repository-scale thermal effects (e.g., heating and dryout) in the surrounding rock. The amount of heat produced varies with time and is a function of the time that the radioisotopes have decayed (fuel "age") and the original irradiation of the fuel in the nuclear reactors (fuel "burnup"). The heat rates and MTHM totals for the spent fuel modeled in the 1997 PA are shown in Table 5-2.

\footnotetext{
- There is a correlation between MTHM and thermal power for commercial fuel, but because heat output from waste packages depends on the burnup and decay of the fuel, describing a hot repository in terms of MTHM is a less useful definition for DOE-owned fuel. Because of its experimental nature, DOE-owned fuel is more varied in age and so does not have similar bumup values among containers. Therefore, the 1997 PA focuses on achieving a specific thermal power $\left(W / \mathrm{m}^{2}\right)$ as its means of measuring a hot repository.

Actual units used by the YMP were 83 MTU/acre (TRW, 1995, p. 3-3).
} 

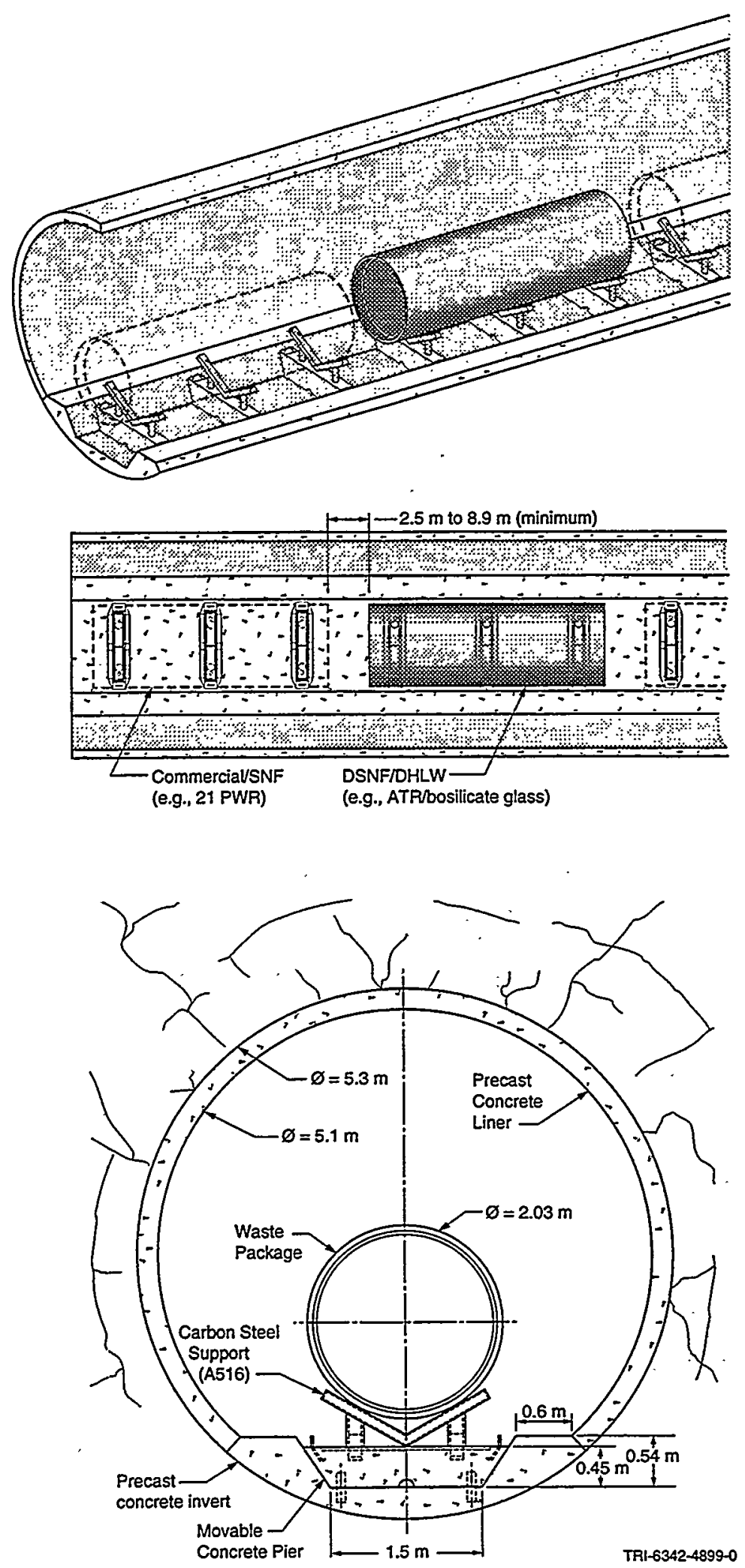

Figure 5-3. Disposal tinnel and emplacement of waste package for horizontal, in-drift emplacement. 
5. System Characterization: Repository Design

Table 5-2. Quantity of Waste Packages and Heat Rates for the Spent Nuclear Fuel and High-Level Waste Modeled in the 1997 Performance Assessment

\begin{tabular}{|c|c|c|c|c|c|c|c|}
\hline $\begin{array}{l}\text { Spent } \\
\text { Fuel } \\
\text { Category }\end{array}$ & $\begin{array}{l}\text { Quantity } \\
\text { of Waste } \\
\text { Packages }\end{array}$ & $\begin{array}{c}\text { Container } \\
\text { Configuration } \\
\text { (DOE SNF } \times \\
\text { DHLW) }\end{array}$ & $\begin{array}{c}\text { DOE SNF } \\
\text { MTHM/pkg }\end{array}$ & $\begin{array}{c}\text { DHLW } \\
\text { MTHM/pkg }\end{array}$ & $\begin{array}{l}\text { Length of } \\
\text { Container } \\
(\mathrm{m})\end{array}$ & $\begin{array}{c}\text { Percentage } \\
\text { of Total } \\
\text { Power }\end{array}$ & $\begin{array}{c}\text { Power/ } \\
\text { Pkg } \\
\text { (W) }\end{array}$ \\
\hline 1 & 118 & $4 \times 0$ & 18.07 & 0.0 & 5.30 & $\overline{0.1}$ & 793 \\
\hline 2 & 9 & $1 \times 5$ & $4.51 \times 10^{-3}$ & 2.5 & 5.30 & 0.0 & 343 \\
\hline 3 & 55 & $1 \times 4$ & 7.652 & 2.0 & 3.79 & 0.0 & 154 \\
\hline 4 & 203 & $1 \times 5$ & 0.393 & 2.5 & 3.79 & 0.2 & 637 \\
\hline 5 & 595 & $1 \times 4$ & 0.148 & 2.0 & 3.79 & 0.3 & 292. \\
\hline 6 & 750 & $1 \times 5$ & $1.2 \times 10^{-2}$ & 2.5 & 5.30 & 0.5 & 405 \\
\hline 7 & 225 & $1 \times 5$ & $5.65 \times 10^{-2}$ & 2.5 & 5.30 & 0.1 & 344 \\
\hline 8 & 545 & $1 \times 5$ & $4.52 \times 10^{-2}$ & 2.5 & 5.30 & 0.2 & 295 \\
\hline 9 & 103 & $1 \times 5$ & $1.56 \times 10^{-2}$ & 2.5 & 5.30 & 0.0 & 280 \\
\hline 10 & 5 & $1 \times 4$ & $1.14 \times 10^{-2}$ & 2.0 & 5.30 & 0.0 & 317 \\
\hline 11 & 352 & $1 \times 4$ & $1.12 \times 10^{-2}$ & 2.0 & 5.30 & 0.2 & 287 \\
\hline 12 & 69 & $1 \times 3$ & 0.80 & 1.5 & 5.30 & 0.0 & 195 \\
\hline 13 & 102 & $1 \times 5$ & $2.17 \times 10^{-2}$ & 2.5 & 3.79 & 0.0 & 177 \\
\hline 14 & 4820 & $21-P^{2} R^{c}$ & 8.62 & 0.0 & 5.30 & 69.6 & 9570 \\
\hline 15 & 2859 & 44-BWR ${ }^{d}$ & 7.57 & 0.0 & 5.30 & 28.7 & 6650 \\
\hline \multicolumn{8}{|c|}{$\begin{array}{l}\text { Mass of heavy metal based on radioisotope inventory presented in Chapter } 3 \text {, since this determines the power per } \\
\text { container. } \\
\text { b All DHLW was estimated as having } 0.5 \text { MTHM per short handling container and } 0.75 \text { MTHM per long handling con- } \\
\text { tainer based on a planning method developed for the DOE in the } 1980 \text { s; and the } 1987 \text { estimated inventory. This is } \\
\text { in contrast to the } 1994 \text { PA which evaluated equivalent MTHM based on instructions in } 40 \text { CFR 191, Appendix A, } \\
\text { and the } 1992 \text { estimated inventory. } \\
\text { c Category } 14 \text { includes } 21-P W R \text { and12-PWR packages, but the 21-PWR package is modeled. } \\
\text { d Category } 15 \text { includes 44-BWR and 24-BWR packages, but the 44-BWR package is modeled. }\end{array}$} \\
\hline
\end{tabular}

In this performance assessment, the waste is uniformly distributed throughout the potential repository, with DOE-owned waste comingled with commercial waste. The DOE-owned waste, which is cooler than the commercial waste (see Table 5-2), is placed relatively closely to the commercial waste (see Figure 5-4). The intention is to produce a temperature profile throughout the repository that is fairly uniform. Figure 5-4 illustrates the spacing of the DOE-owned and commercial waste per 77-m section of the repository used for modeling; this spacing pattern is repeated throughout each $1236-\mathrm{m}$ disposal tunnel.

\subsubsection{Disposal Tunnel Spacing}

The disposal tunnels are spaced $28.6 \mathrm{~m}$ apart (center to center) (Figure 5-2), which is based on the potential repository layout described in TSPA-1995 (M\&O, 1995a). Based on heat loads of the waste used in the 1997 PA, a spacing of $28.6 \mathrm{~m}$ between the 5.3-m-wide tunnels maintains a heat load equivalent to $85 \mathrm{MTHM} / \mathrm{acre}$ for commercial fuel. The disposal tunnels are assumed to be lined with a $0.2-\mathrm{m}$-thick precast concrete liner. A thick segment on the base of the tunnel forms an invert into which a minimum of 3 piers can be set for container support (Figure 5-3). 


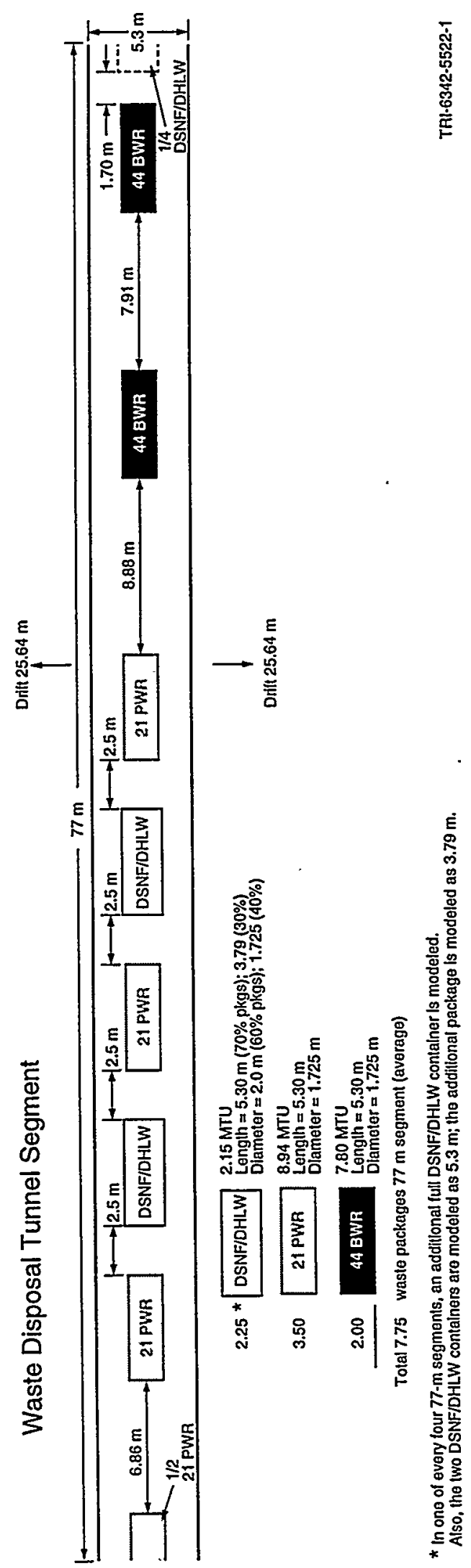

ป⿱艹 
5. System Characterization: Repository Design

\subsubsection{Enclosed Area}

The combination of waste package and tunnel spacing resulted in an actual areal density of $2.035 \times 10^{-2} \mathrm{MTHM}$ $\mathrm{m}^{2}$ (82.34 MTHM/acre), and an actual areal power density of $18.74 \mathrm{~W} / \mathrm{m}^{2}$ in the year 2030 . The resulting enclosed area of the disposal region is $5.79 \times 10^{5} \mathrm{~m}^{2}$ with an extraction ratio of $19 \%$. Table $5-3$ provides repository design parameters.

Table 5-3. Repository Design Geometry Parameters

\begin{tabular}{ll}
\hline \multicolumn{1}{c}{ Parameter } & \multicolumn{1}{c}{ Unit } \\
\hline Disposal Tunnel & $5.3 \mathrm{~m}$ \\
Diameter & $1236 \mathrm{~m}$ \\
Length of tunnel & 87 \\
Total number of disposal tunnels & $1.07 \times 10^{5} \mathrm{~m}$ \\
Length available for waste disposal & $7.62 \mathrm{~m}$ \\
Access tunnel, diameter & \\
Repository & $1251 \mathrm{~m}$ \\
Length & $2465 \mathrm{~m}$ \\
Width (parallel to tunnels) & $3.23 \times 10^{6} \mathrm{~m}^{2}$ \\
Area & $19 \%$ \\
Extraction ratio (excavated area/repository area) & \\
Waste Packages & $6018.2 \mathrm{~W}$ \\
Average power per waste package & 124 \\
Maximum number per tunnel & 84 \\
Minimum number per tunnel & $1.0711 \times 10^{5} \mathrm{~m} \mathrm{~m}^{2}$ \\
Total length of all waste packages & $1.1583 \times 10^{5} \mathrm{~m}{ }^{2}$ \\
Total projected area & \\
Power Density & $2.10 \times 10^{-2} \mathrm{MTHM} / \mathrm{m}^{2}$ \\
Design-basis MTHM density & $(85 \mathrm{MTHM} / \mathrm{acre})$ \\
Actual MTHM density & $2.035 \times 10^{-2} \mathrm{MTHM} / \mathrm{m}^{2}$ \\
Areal power density (actual) & $(82.34 \mathrm{MTHM} / \mathrm{acre})$ \\
Repository power density (total power of all waste divided by repository area) & $18.74 \mathrm{~W} / \mathrm{m}^{2}$ \\
\hline & $21.16 \mathrm{~W} / \mathrm{m}^{2}$ \\
\hline
\end{tabular}

\subsection{Backfill}

As used in this report, backfill is the material that seals the excavated openings of the potential repository. Special categories of backfill include (a) seals for providing immediate sealing of access drifts and ramps and (b) backfill, which is placed directly around the waste package. In the 1997 PA, only the ramps to the disposal area were assumed to be backfilled and effectively sealed.

Backfill was not used in the disposal tunnels. An air gap was selected in place of backfill. The radiative heat transfer through the air gap is more effective than the heat transfer through the crushed-tuff backfill. The trapped air 
in crushed-tuff backfill, which has a low thermal conductivity, would act as an insulator thus increasing centerline temperatures. Also the gap provides a capillary break, which does not eliminate dripping but does limit contact of water percolating through the matrix.

\subsection{Summary of Assumptions for Repository Design Conceptual Model}

- Although the repository is only roughly rectangular with disposal tunnels at an angle to the access drifts, the repository is modeled as rectangular with tunnels.

- Hot repository concept (85 MTHM/acre) is modeled.

- Center in-drift on pedestal (horizontal waste) emplacement is modeled.

- Disposal tunnels are $1236 \mathrm{~m}$ long.

- Waste is uniformly distributed throughout potential repository with DOE-owned waste and commercial waste comingled.

- Total number of disposal tunnels equals 87.

- No backfill is modeled in disposal tunnel. 


\title{
6. Analysis Design
}

\author{
R. P. Rechard
}

Analysis design involves (a) selecting the features, events, and processes that provide detail about what may happen to the disposal system in the future, (b) creating conceptual models based on the disposal system characterization (described in Chapters 3, 4, and 5) and the selected features, events, and processes, and (c) incorporating those conceptual models into the performance assessment calculations.

\subsection{Selected Features, Events, and Processes}

The selected features, events, and processes were drawn largely from previous studies, e.g., TSPA-1993 (Wilson et al., 1994). Because the focus of the 1997 PA is the performance of DOE-owned spent nuclear fuel and high-level waste, some combinations of events and features were not evaluated, e.g., volcanism was not considered based on results from a previous performance assessment (Wilson et al., 1994), which showed a probability of occurrence to be just above $10^{-4}$ in $10,000 \mathrm{yr}$ in conjunction with insignificant releases.

The basic features, events, and processes selected for modeling in this performance assessment are briefly summarized below. The features are described more fully in Chapters 3, 4, and 5 . As noted below, no specific events were considered in the 1997 PA. The incorporation of the features and processes is discussed in the chapters on consequence modeling (Chapters 7, 8, 9, and 10).

\subsubsection{Features}

The basic features explored in the $1997 \mathrm{PA}$ are (1) areas of concentrated infiltration above the repository; (2) a highly fractured, tilted pancake stratigraphy; (3) a large unsaturated zone that holds a repository; (4) horizontal emplacement of waste packages; and (5) an underlying aquifer. The features are described in Chapters 3,4 , and 5 .

\subsubsection{Event}

No events are considered in the 1997 PA because neither human intrusion nor criticality is specifically modeled. However, related criticality calculations are being performed by Sandia National Laboratories as part of the broader DOE program for developing a safe, cost-effective technical strategy for the interim management and ultimate disposition of DOE-owned spent fuel and high-level waste.

\subsubsection{Processes}

Basic processes are explored for the geologic barrier, waste form, waste container, and repository.

Geologic Barrier. For the geologic barrier, processes considered are two-phase flow coupled with heat conduction, allowing possible phase change in a fractured, porous matrix, and infiltration variations from climate change. The transport process of radionuclides in the liquid phase with large degrees of sorption is also included, as is transport of $\mathrm{O}_{2}$ from the surface to the repository horizon, which is an important focus of this analysis.

Waste Form. For the waste form, the process considered is control of radionuclide release by solubility; matrix alteration or immediate release of some radionuclides in gaps and grain boundaries of spent fuel; and degradation of the cladding (except for fuel with initially failed cladding) and the matrix containing radionuclides. 
Waste Package and Repository. For the waste package and repository, processes considered are $\mathrm{O}_{2}$ consumption from several layers because of general oxic corrosion, radionuclide release through container controlled by localized corrosion, and advection and diffusion through container breach into the host rock.

\subsection{Selection of Computational Codes}

\subsubsection{Grouping of Codes}

In a performance assessment, many categories of software are needed to complete the task. These categories basically reflect the fundamental steps of a performance assessment (see Chapter 1, Figure 1-1). The categories that must be considered in a performance assessment are modeling setup, consequence modeling, probability modeling, assessment of results, and sensitivity analysis. For the $1997 \mathrm{PA}$, the consequence model was divided into four major groups of computational models (submodels), which are discussed in the indicated chapters:

- Corrosion and source term submodel (Chapter 7)

- Unsaturated zone, flow and transport submodels (Chapter 8)

- Saturated zone, flow and transport submodels (Chapter 9)

- Biosphere transport submodel (Chapter 10).

These modeling categories were created to address particular aspects of the calculational design. The corrosion group of computational codes was formed in response to the large amount of data required to simulate container corrosion and subsequent escape of radioisotopes. The flow and transport categories were grouped by unsaturated and saturated conditions, which are convenient subdivisions of the geologic barrier. Finally, the biosphere codes were grouped to determine human dose; the results address the performance criteria with regard to acceptable dose limit.

Also, in addition to identifying and selecting features, events, and processes, the degree of model simplification must be considered during design of the conceptual model and the selection of the computational codes. In general the degree of simplification in the models is addressed in the 1997 PA by designing the analysis to complement a-TSPA-1997. The latter uses a level of simplification in which abstractions of results from detailed phenomenological models are used, which allows simulations to be set up quickly and geared toward fast run times. The 1997 PA uses detailed phenomenological simulation codes. The codes for the 1997 PA and the basic features they model are shown in Figure 6-1. The codes are based on mathematical models with potentially spatially distributed model parameters and use partial differential equations that are solved numerically on coarse grids. The performance criteria were evaluated directly from multiple execution of the simulation codes and thus provided a direct connection between the input data and the performance criteria. (When model abstraction is used for the simplification, as in a-TSPA-1997, the connection between data and results can become somewhat obscure.)

The submodels for the 1997 PA and how they are linked are shown in Figure 6-2. The linkage of this software is by means of the Software Configuration Management System (SCMS), which uses identical concepts but has replaced CAMCON (Rechard, ed., 1992; Rechard et al., 1993), a system originally developed for code linkage. (Information about support codes that are used with the main modeling codes can be found in Rechard, ed., 1995, Appendix G.) The categories of software required by the 1997 PA are described in the following sections.

\subsubsection{Modeling Setup}

The main function of the modeling setup (Figure 6-2) is to assemble modeling parameter input, which includes (1) gathering the data collected during site characterization; (2) selecting parameter values and distributions ( $x=x_{1}$ ..., $\mathrm{x}_{\mathrm{nV}}$ ) consistent with the manner of use in models; (3) placing parameter values in secondary data base for use by models; (4) selecting subset of parameters to vary (parameter uncertainty); and (5) sampling with Latin Hypercube sampling (LHS) (Iman and Shortencarier, 1984). 


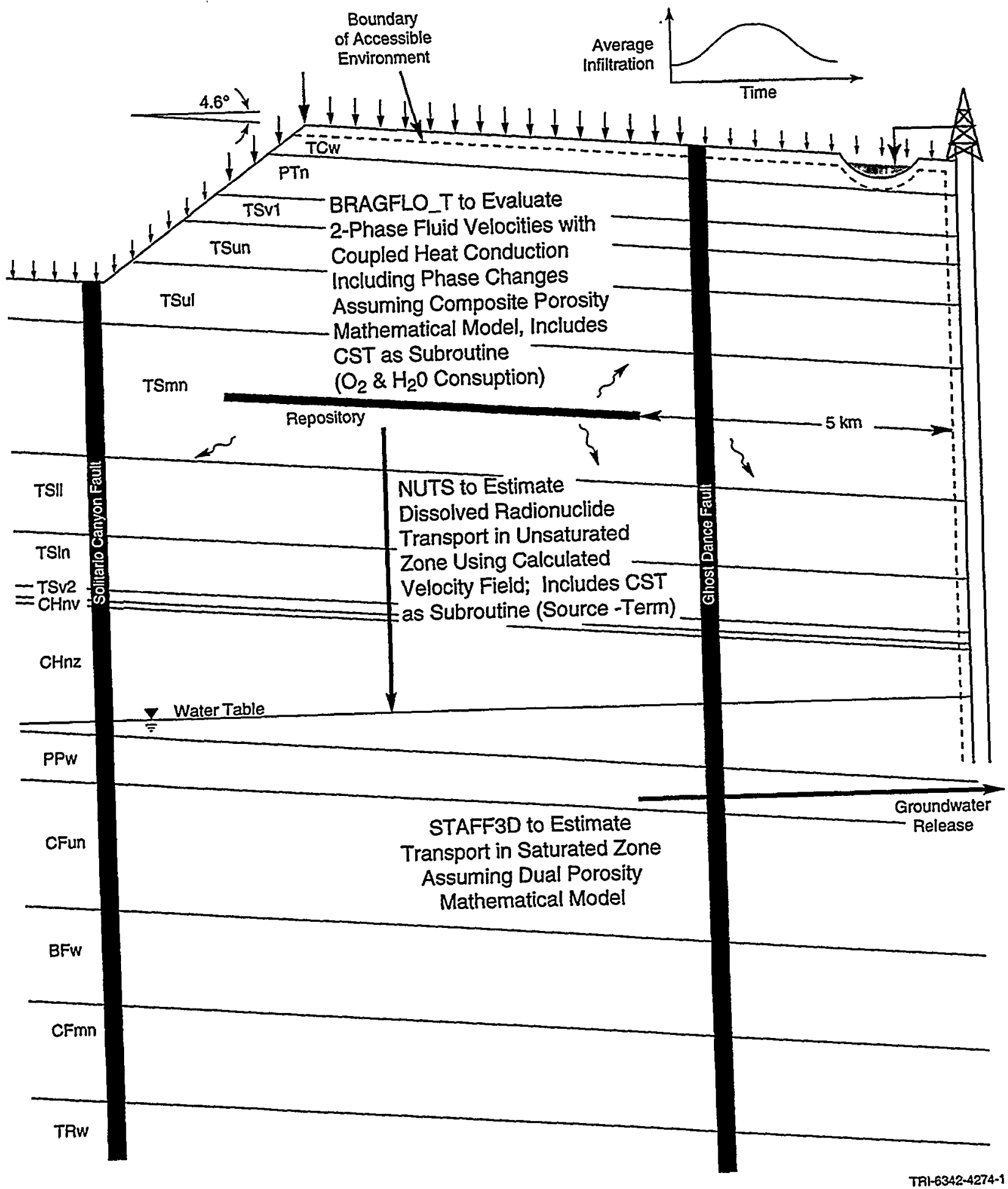

Figure 6-1. General conceptual model for tuff disposal system. The schematic includes the main features and the corresponding computational models (codes). 


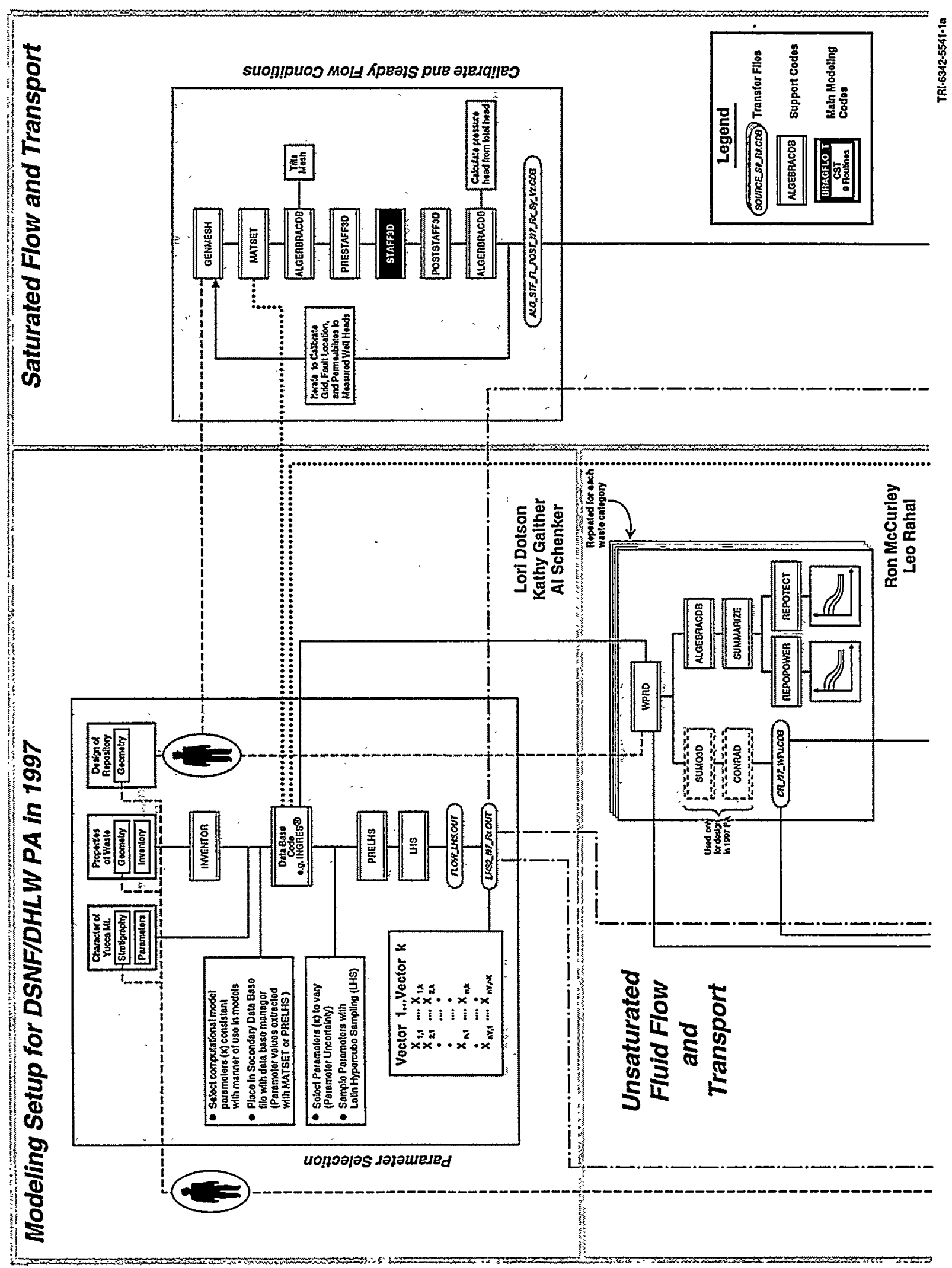




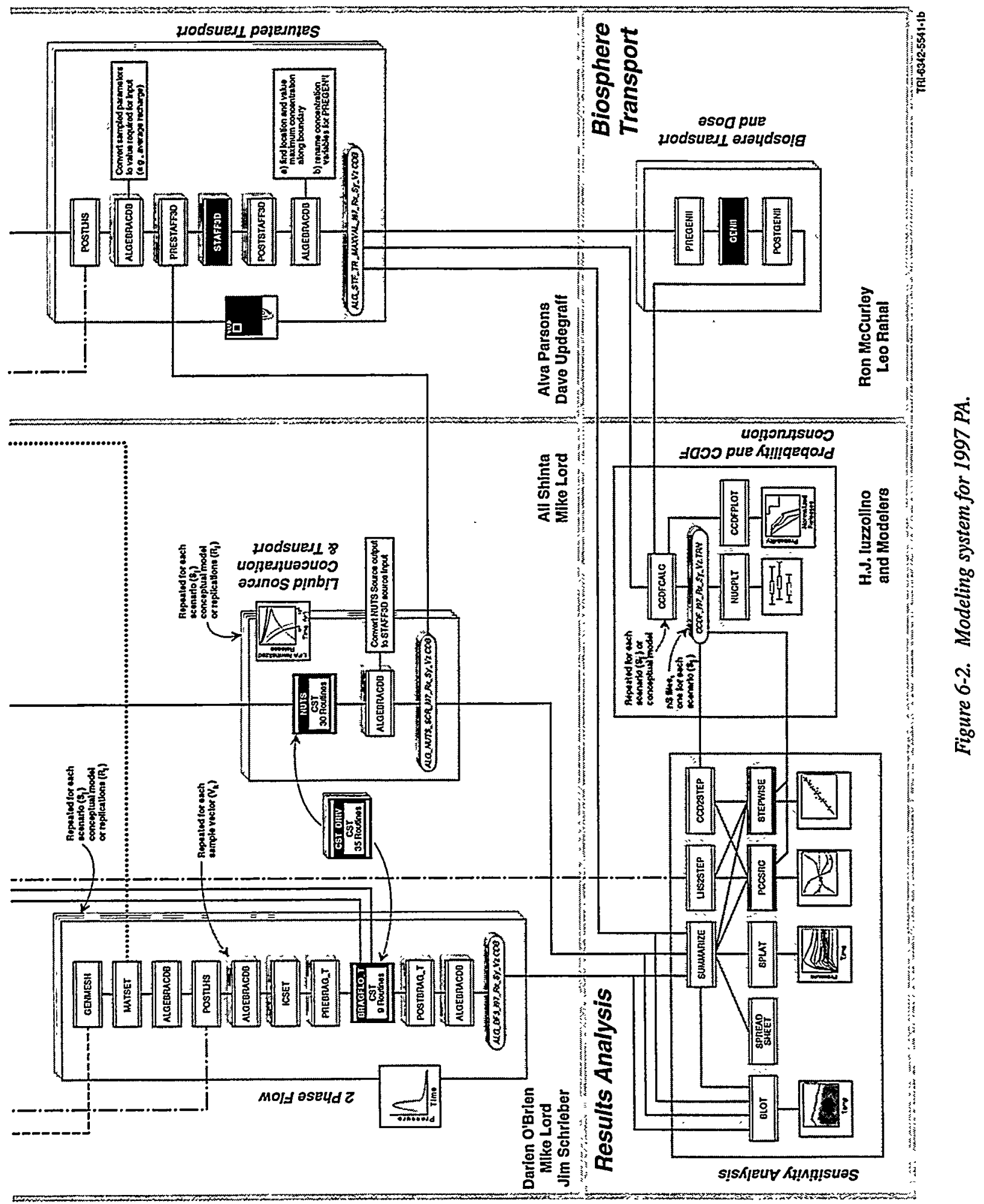


The software codes used for the modeling setup are INVENTOR, INGRES, PRELHS, and LHS.

1. INVENTOR (QA Version 2.70)

Function: INVENTOR gathers and assembles radioisotope inventory data for INGRES@.

2. INGRES®

Function: Generally, INGRES $\circledast$ stores and manipulates data.

3. PRELHS (QA Version 2.20)

Function: Generally, PRELHS translates from the secondary database to LHS. Specifically, PRELHS extracts parameter distribution data requested by the user from the secondary database file, TUFF.SDB, and sets up the LHS input file.

4. LHS (QA Version 2.50)

Function: LHS samples distributions of input parameters using either normal Monte Carlo sampling or efficient Latin hypercube sampling. LHS also permits correlations (restricted pairings) between parameters. Latin hypercube sampling reduces the number of sample vectors $[n K]$ required to about $4 / 3 \cdot n_{\alpha}$, where $n_{\alpha}$ is the number of varying parameters (Iman and Shortencarier, 1984).

\subsubsection{Corrosion and Source Term Consequence Modeling}

This software code, CST, evaluates (1) the amount of $\mathrm{O}_{2}$ and $\mathrm{H}_{2} \mathrm{O}$ consumed during general corrosion and alteration of layers and matrices of the containers and nuclear waste, and (2) release of radioisotopes to the host rock as the containers fail. The first function, $\mathrm{O}_{2}$ and $\mathrm{H}_{2} \mathrm{O}$ consumption, is conducted inside BRAGFLO_T and consists of nine CST routines. The second function, radioisotope release, is conducted inside NUTS and consists of 30 CST routines.

\subsubsection{Power Output of Waste}

A small group of analytic codes is used to evaluate (1) the power output of waste packages over time, WPRD, (2) the general temperature of the repository over time to aid in design, SUMQ_3D, and (3) the variation of temperature near a container, CONRAD, to aid in design. The results of this analysis become input for BRAGFLO_T, discussed in Section 6.2.5.

1. WPRD (QA Version 2.31)

Function: WPRD calculates the power output of waste packages over time.

2. SUMQ_3D (QA Version 2.10)

Function: Calculates temperature change at a point of interest from a three-dimensional layout of linear heat sources (waste packages).

3. CONRAD (QA Version 2.20)

Function: Calculates the temperature distribution for 1D spherical or cylindrical conduction and radiative heat transfer with a decaying central heat source. Permits spatially varying thermal properties and spatially varying heat generation. 


\subsubsection{Unsaturated Zone, Flow Consequence Modeling}

This software group evaluates radioisotope movement in the unsaturated zone of the disposal system. BRAGLO_T and NUTS are the primary modeling codes, solving the heat conduction and two-phase flow equations on a coarse two-dimensional finite-difference grid.

1. GENMESH (QA Version 6.08)

Function: In general, GENMESH generates a rectilinear finite element or finite difference mesh. Specifically, GENMESH constructs a right-handed, Cartesian, rectangular one-, two-, or three-dimensional finite-difference grid from a user input file specifying the geometry. In addition to setting the node coordinates and mesh connectivity, GENMESH sets material regions, identifies (flags) nodes or elements for boundary conditions, and sets elevations of elements. All information is then stored as a computational database file, CAMDAT.

2. MATSET (QA Version 9.00)

Function: The code sets material names, material property names, and values, and attribute names and values in CAMDAT.

3. POSTLHS (QA Version 4.07)

Function: The code writes LHS output to CAMDAT.

4. ALGEBRACDB (QA Version 2.35)

Function: The code generates additional data (or removes unnecessary data) in CAMDAT by algebraically manipulating data already stored. With ALGEBRA, an analyst can generate pertinent data external to a code by combining data already stored in CAMDAT rather than by modifying a code and thereby invoking a new quality assessment.

5. BCSET (QA Version 2.09)

Function: BCSET sets nodal boundary flags, element boundary flags, and values at boundary flags. The flags are those defined either in the mesh generation module or by BCSET in a user input file.

6. ICSET (QA Version 2.22)

Function: Generally, ICSET sets up initial conditions. Specifically, ICSET sets CAMDAT analysis array variables: history, global, nodal, and/or element variable values, at the first time step obtained from a user file. In addition, any nodal or element variables (existing or new) can be linearly interpolated by specifying interpolation tables in the ICSET input text file.

7. PREBRAG_T (QA Version 3.08)

Function: PREBRAG_T translates the information in the CAMDAT file format into the BRAGFLO_T input file format.

8. BRAGFLO_T (QA Version 3.10)

Function: Generally, BRAGFLO_T models components of gas and liquid flow using an implicit mathematical formulation based on the pressure, temperature, and saturation state variables of each component and a finite-difference solution technique. The mathematical model of BRAGFLO_T describes the simultaneous flow of two phases through a porous heterogeneous reservoir. BRAGFLO_T's fully implicit finite difference formulation makes it well suited for simulating convergent flow. This version of BRAGFLO_T includes reaction (corrosion) source and sink terms to the gas and water components through the CST subroutines. Reactions are completed when the reactants (e.g., iron) are consumed. The BRAGFLO_T formulation is designed to be robust and numerically stable over a wide range of conditions and input property values, making it well-suited to perform as a component of a probabilistic computation system. BRAGFLO_T differs from BRAGFLO, its parent code, primarily by the addition of the energy equation for temperature and 
6. Analysis Design

the compositional treatment of the gas phase. However, omission of some features and addition of others means that, except for the solver, the codes share few common subroutines.

9. POSTBRAG_T (QA Version 4.01)

Function: POSTBRAG_T translates binary output from BRAGFLO_ $T$ into the CAMDAT file format.

\subsubsection{Unsaturated Zone, Transport Modeling}

1. MATSET

\section{ALGEBRACDB}

3. NUTS (QA Version 2.05)

Function: NUTS (NUclides Transport System) is a three-dimensional, multicomponent, finite-difference, dual-permeability simulator to track the movement of radionuclides in the repository and surrounding formations. The model simulates radioactive chain decay during radioisotope transport and can determine migration rate, distance, concentration, and level of radioactivity. Three types of sorption isotherms are considered - linear, Fruendlich, and Langmuir equilibrium isotherms - in addition to hydrodynamic dispersion. Solubility limit of the waste components and their precipitation during migration are included. The precipitate is allowed to undergo decay and redissolve in the liquid if the concentration drops below the solubility limit. The system of partial differential equations is solved implicitly and sequentially to determine the contribution from parent radionuclide decay to the immediate daughter.

Two types of input are required: flow field and radioisotopes. The flow field input is taken directly from BRAGFLO_T's output. The radioisotope input provides component properties in addition to data required for dispersion and sorption, such as molecular dispersion, dispersivities, rock densities, and sorption coefficients. The current version calls CST routines to determine radioisotope concentrations.

\subsubsection{Saturated Zone, Flow and Transport Consequence Modeling}

The software code, STAFF3D (Huyakorn et al., 1986; 1992), models the transport of radioisotopes in the saturated zone of the disposal system. The source term considered is radioisotopes that reach the water table. STAFF3D is similar to that used in the 1994 PA.

\section{ALGEBRACBD}

2. PRESTAFF3D (QA Version C-1.00ZO)

Function: PRESTAFF3D creates an input file for STAFF3D by translating data from the CAMDAT database.

\section{STAFF3D (QA Version C-1.01Z0)}

Function: STAFF3D is a finite element code designed to simulate confined and unconfined groundwater flow and single or multiple component solute transport in fractured or porous aquifers in two or three dimensions. Fractured porous media are represented using either discrete-fracture or dual-porosity approaches.

4. POSTSTAFF3D (QA Version C-1.00ZO)

Function: POSTSTAFF3D places total head and interstitial velocity components or solute concentrations (nodal variable) from STAFF3D output in CAMDAT. 


\subsubsection{Biosphere Consequence Modeling}

This software code, GENII-A (Napier et al., 1988a,b,c), evaluates the migration of radioisotopes that reach the accessible environment and eventually come in contact with humans through food or water consumption.

1. PREGENII (QA Version 6.30)

Function: PREGENII creates activity concentrations in various media (air, soil, groundwater) in the accessible environment using results from CAMDAT, which were generated in other performance assessment analyses. The concentrations are input to GENII-A. The input is downloaded to the PC from the mainframes via user interface.

2. GENII-A (QA Version 2.10)

Function: GENII-A determines internal and external radiation doses to humans using radioisotope activity concentrations (e.g., $\mathrm{Ci} / \mathrm{kg}$ ) in various media together with transfer factors, internal and external dose factors, and selected exposure pathway specifications. The media are groundwater, air, soil, or surface water; the transfer factors are related to food intake; and the pathway specifications can be factors such as inhalation, duration of exposure, and amounts of dietary products consumed. The activity concentrations can be input directly to the various media or calculated inside GENII-S via some transport mechanisms, such as movement through air and deposition. However, GENII-A does not calculate groundwater transport.

3. POSTGENII (QA Version 4.20)

Function: The code transfers GENII output to CAMDAT.

\subsubsection{Probability Modeling and CCDF Construction}

In the $1997 \mathrm{PA}$, the probability model was used to estimate the likelihoods of uncertain parameter values and to construct the CCDF for doses.

1. CCDFCALC (QA Version 4.28)

Function: In general, CCDFCALC preprocesses radionuclide time histories for CCDFs. Specifically, for each vector of all scenarios, CCDFCALC accesses the appropriate CAMDAT database file, integrates to find cumulative releases for each radionuclide, and calculates the EPA sum. The results are written to a transfer file used by CCDFPLOT or NUCPLOT.

2. NUCPLOT (QA Version 1.19)

Function: Interactively plots Tukey Box plots showing distribution of individual radionuclide release or normalized discharge.

3. CCDFPLOT (QA Version 4.21)

Function: Interactively plots the CCDF data read from the CCDFCALC transfer file.

\subsubsection{Sensitivity Analysis}

As the name implies, this group of software evaluates parameter importance through sensitivity analysis by determining the partial correlation coefficients and standardized regression coefficients. The main codes are STEPWISE (Iman et al., 1980) and PCCSRC (Iman and Helton, 1985). No changes occurred from the 1994 PA.

1. SUMMARIZE (QA Version 2.20)

Function: SUMMARIZE reads specified variable values from multiple CAMDAT databases (one for each vector) or transfer files. The data are then written to a text transfer file suitable for input to an $X-Y$ plot program. 
2. SPLAT (QA Version 1.02)

Function: Interactive $\mathrm{x}$-, $\mathrm{y}$-graphing package.

3. LHS2STEP (QA Version 1.03)

Function: LHS2STEP reads an LHS sampled output file containing the dependent variables and writes an output file for either STEPWISE or PCCSRC.

4. PCCSRC (QA Version 2.21)

Function: PCCSRC evaluates variable importance by reporting the partial correlation coefficients (PCC) and standardized regression coefficients (SRC) on either the rank or raw data. Provided useful regression models can be developed, the absolute values of the standardized regression coefficients (or mathematically related partial correlation coefficients) can be used to rank variable importance.

5. CCD2STEP (QA Version 1.08)

Function: CCD2STEP reads CCDFCALC files containing radionuclide release data for the dependent variables and writes an output file for either STEPWISE or PCCSRC.

6. STEPWISE (QA Version 2.21)

Function: STEPWISE evaluates variable importance by developing regression models between the observed response and input variables using a forward, backward, or stepwise regression procedure on the raw or ranked data. Provided useful regression models can be developed, the absolute values of the standardized regression coefficients (or mathematically related partial correlation coefficients) can be used to rank variable importance.

\subsection{Parameter Database}

The parameter database for the 1997 PA, called INEEL97, is a relational database stored in INGRES®, a commercial (off-the-shelf) relational database application. The database was designed to store specific information about parameters of interest in the 1997 PA calculations. A parameter defines a constant (i.e., gravity), a geologic (i.e., Tiva Canyon Welded), or a chemical (i.e., radionuclide inventory) attribute. A naming convention was constructed to identify a parameter, utilizing a combination of a material name coupled with a property name, creating a unique material: property name that describes a particular parameter. A parameter is then assigned an identification number for tracking purposes. The database contains information, including distribution type, mean value, median value, standard deviation, minimum value, maximum value, units, and source information for these parameters. The codes used in the 1997 PA calculation retrieve relevant information directly from the database, so every effort is made to ensure the integrity of the parameter information. For this reason, INEEL97 is a controlled database and an official project record. Strict control of read and write access and audit trails ensures the integrity and traceability of information into and out of the database.

\subsection{Sampled Parameters}

Model parameters $\left(x=x_{1} \ldots, x_{n v}\right)$ are the fundamental entities of computational probability and consequence models. For this performance assessment, they are all scalar quantities and are all coefficients in the mathematical models underlying the computational models.

\subsubsection{Parameters Selected for Sampling}

A parameter was initially selected as imprecisely known in the 1997 PA if (1) the parameter had proved to be sensitive in the 1994 PA (Rechard, ed., 1995) or (2) the parameter was thought to be at least moderately sensitive in the updated iteration of the consequence model for the 1997 PA. To keep the calculations tractable, this initial list 
was then reduced to 63 parameters, 33 of which were used in the source-term submodel (Table 6-1). The assumed distributions for the 63 parameters are shown in Figures 6-3 through 6-17.

Parameters sampled in the engineered barrier were alteration rates of waste-immobilizing matrices; alteration rates of layers of the container; solubilities of neptunium, plutonium, and uranium; sorption coefficient on rust of container; effective area for diffusion of contaminants; fraction released immediately from matrix; fraction of containers with failed Incoloy 625 layer; fraction of containers with rubble; and area above container that contributes water for corrosion and transport through the container.

Parameters sampled in the geologic barrier were bulk hydraulic conductivity, matrix hydraulic conductivity, and fracture spacing for Topopah Spring, Calico Hills, and Prow Pass. Also sampled were the sorption coefficients for plutonium and uranium in the devitrified tuff; neptunium, plutonium, and uranium in the zeolitic tuff; and the dispersivity fraction.

Climate change was simulated by sampling on minimum and maximum infiltration rates and the "wet" cyclic period. Also, a factor that concentrated a portion of infiltration into one element of the two-dimensional mesh was sampled. The parameter sampled for biosphere transport was irrigation rate.

\subsubsection{Parameters Held Constant}

A total of approximately 3024 parameters were required by the consequence and probability models for the disposal system. The dimensions of important engineered and geologic features of the system are not considered as part of the total number of parameters.

Because only 63 parameters were varied, the majority of modeling parameters were held constant, usually at the mean value (values associated with the source term model, CST, were held constant at the median value). Yet ranges and distributions were estimated for 269 of these parameters to examine the potential validity of the single value assigned. The tables listing the model parameters are presented in Chapters 3,4 , and 5 for the waste form and package, geologic barrier, and repository, respectively. 
Table 6-1. Parameters Varied at Repository for 1997 Performance Assessment Calculations

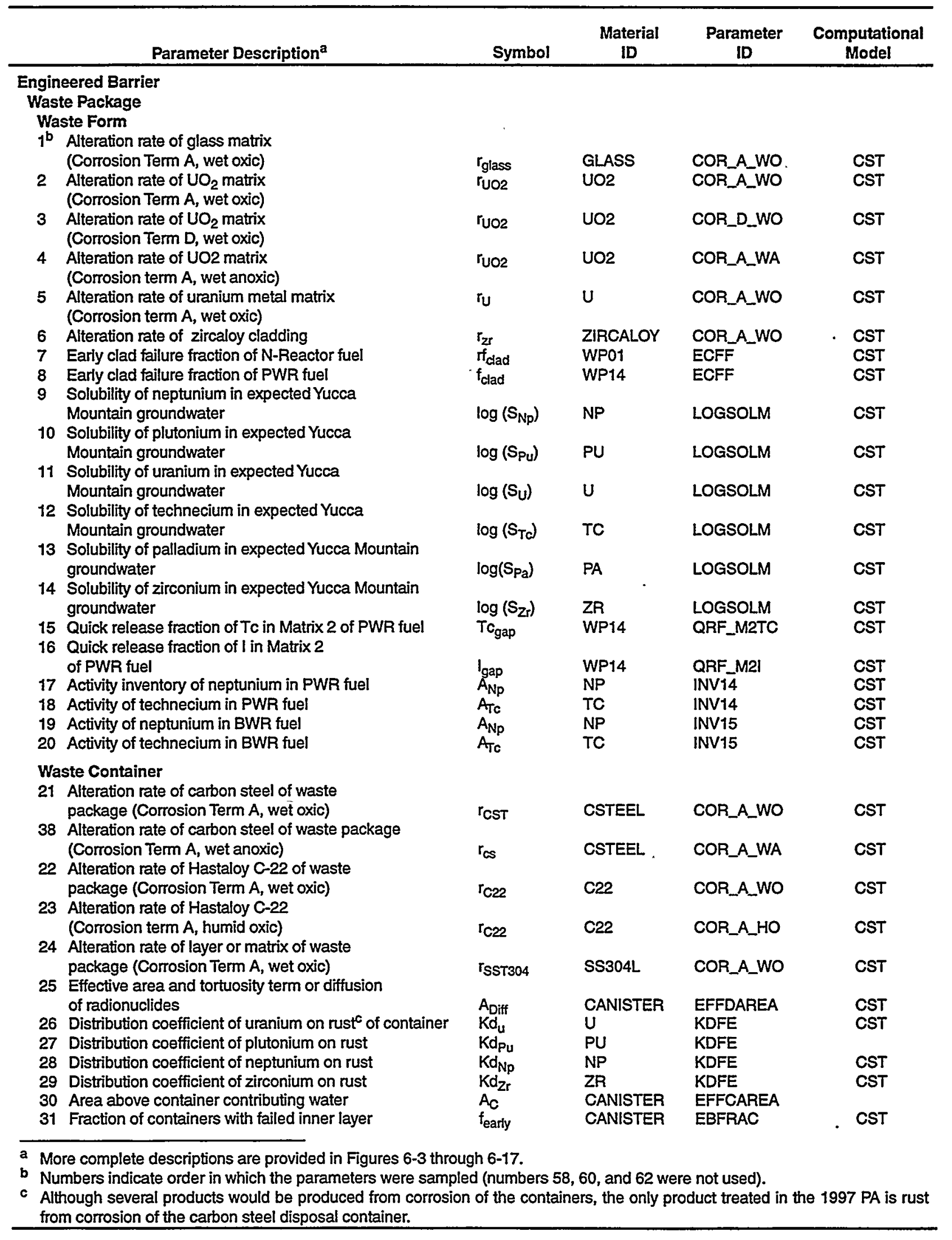


Table 6-1. Parameters Varied at Repository for 1997 Performance Assessment Calculations (Continued)

\begin{tabular}{|c|c|c|c|c|}
\hline Parameter Description ${ }^{a}$ & Symbol & $\begin{array}{l}\text { Material } \\
\text { ID }\end{array}$ & $\begin{array}{l}\text { Parameter } \\
\text { ID }\end{array}$ & $\begin{array}{c}\text { Computational } \\
\text { Model }\end{array}$ \\
\hline $\begin{array}{l}\text { Repository } \\
32 \text { Fraction of containers with rubble }\end{array}$ & $f_{\text {rubble }}$ & CANISTER & BACFRAC & CST \\
\hline $\begin{array}{l}\text { Dose } \\
33 \text { Irrigation rate } \\
34 \text { Consumption of drinking water } \\
35 \text { Consumption of vegetables } \\
36 \text { Consumption of milk } \\
37 \text { Consumption of beef }\end{array}$ & $\begin{array}{l}r_{\text {iir }} \\
r_{\text {h20 }} \\
r_{\text {veg }} \\
r_{\text {milk }} \\
r_{\text {beef }}\end{array}$ & $\begin{array}{l}\text { GLOBAL } \\
\text { GLOBAL } \\
\text { GLOBAL } \\
\text { GLOBAL } \\
\text { GLOBAL }\end{array}$ & $\begin{array}{l}\text { RIRR } \\
\text { RH2O } \\
\text { RVEG } \\
\text { RMILK } \\
\text { RBEEF }\end{array}$ & $\begin{array}{l}\text { GENII } \\
\text { GENII } \\
\text { GENII } \\
\text { GENII } \\
\text { GENII }\end{array}$ \\
\hline $\begin{array}{l}\text { Geologic Engineered Barrier } \\
\text { Unsaturated Zone } \\
\text { Hydrologic/Bulk } \\
39 \text { Bulk, saturated hydraulic conductivity of TSw layers } \\
40 \text { Bulk, saturated hydraulic conductivity of CHnv layers } \\
41 \text { Bulk, saturated hydraulic conductivity of CHnz layers } \\
42 \text { Bulk, saturated hydraulic conductivity of PPw layers }\end{array}$ & $\begin{array}{l}\mathrm{Kb}_{T S W} \\
\mathrm{~Kb}_{\mathrm{CHnV}} \\
\mathrm{Kb}_{\mathrm{CHnz}} \\
\mathrm{Kb}_{\mathrm{PPw}}\end{array}$ & $\begin{array}{l}\text { TSW } \\
\text { CHNV } \\
\text { CHNZ } \\
\text { PPW }\end{array}$ & $\begin{array}{l}\text { BLKKGS_X } \\
\text { BLKKGS_X } \\
\text { BLKKGS_X } \\
\text { BLKKGS_X }\end{array}$ & $\begin{array}{l}\text { BRAGFLO_T } \\
\text { BRAGFLO_T } \\
\text { BRAGFLO_T } \\
\text { BRAGFLO_T }\end{array}$ \\
\hline $\begin{array}{l}\text { Hydrologic/Matrix } \\
43 \text { Hydraulic conductivity on TSw modeling unit } \\
44 \text { Hydraulic conductivity on CHnv modeling unit } \\
45 \text { Hydraulic conductivity on CHnz modeling unit } \\
46 \text { Hydraulic conductivity on PPw modeling unit } \\
47 \text { Van Genuchten beta coefficient of TSw modeling unit } \\
48 \text { Van Genuchten beta coefficient for CHnv modeling unit } \\
49 \text { Van Genuchten beta coefficient for CHnz modeling unit } \\
50 \text { Van Genuchten beta coefficient for PPw modeling unit }\end{array}$ & $\begin{array}{l}K_{\text {TSw }} \\
K_{\text {CHnv }} \\
K_{\text {CHna }} \\
K_{\text {PPw }} \\
\beta_{T S w} \\
\beta_{C H m} \\
\beta_{C H n z} \\
\beta_{\text {PPw }}\end{array}$ & $\begin{array}{l}\text { TSW } \\
\text { CHNV } \\
\text { CHNZ } \\
\text { PPW } \\
\text { TSW } \\
\text { CHNV } \\
\text { CHNZ } \\
\text { PPW }\end{array}$ & $\begin{array}{l}\text { HYCNDM_X } \\
\text { HYCNDM_X } \\
\text { HYCNDM_X } \\
\text { HYCNDM_X } \\
\text { VGBETA } \\
\text { VGBETA } \\
\text { VGBETA } \\
\text { VGBETA }\end{array}$ & $\begin{array}{l}\text { BRAGFLO_T } \\
\text { BRAGFLO_T } \\
\text { BRAGFLO_T } \\
\text { BRAGFLO_T } \\
\text { BRAGFLO_T } \\
\text { BRAGFLO_T } \\
\text { BRAGFLO_T } \\
\text { BRAGFLO_T }\end{array}$ \\
\hline $\begin{array}{l}\text { Hydrologic/Fracture } \\
51 \text { Spacing of fractures on TSw modeling unit } \\
52 \text { Spacing of fractures on CHnv modeling unit } \\
53 \text { Spacing of fractures on CHnz modeling unit } \\
54 \text { Spacing of fractures on PPW modeling unit }\end{array}$ & $\begin{array}{l}2 B_{T S W} \\
2 B_{C H n v} \\
2 B_{C H n z} \\
2 B_{P P w}\end{array}$ & $\begin{array}{l}\text { TSW } \\
\text { CHNV } \\
\text { CHNZ } \\
\text { PPW }\end{array}$ & $\begin{array}{l}\text { FRACSPAC } \\
\text { FRACSPAC } \\
\text { FRACSPAC } \\
\text { FRACSPAC }\end{array}$ & $\begin{array}{l}\text { BRAGFLO_T } \\
\text { BRAGFLO_T } \\
\text { BRAGFLO_T } \\
\text { BRAGFLO_T }\end{array}$ \\
\hline $\begin{array}{l}\text { Saturated and Unsaturated Zones } \\
\text { Transport } \\
55 \text { Distribution coefficients for neptunium - vitrified tuff } \\
56 \text { Distribution coefficients for uranium - devitrified tuff } \\
57 \text { Distribution coefficients for plutonium - devitrified tuff } \\
59 \text { Distribution coefficients for uranium - zeolitic tuff } \\
61 \text { Dispersivity fraction }\end{array}$ & $\begin{array}{l}K d v_{N p} \\
K d d_{U} \\
K d d_{P u} \\
K d z_{U} \\
\alpha_{F}\end{array}$ & $\begin{array}{l}\text { NP } \\
U \\
\text { PU } \\
U \\
\text { GLOBAL }\end{array}$ & $\begin{array}{l}\text { KDV } \\
\text { KDD } \\
\text { KDD } \\
\text { KDZ } \\
\text { DISPPERC }\end{array}$ & $\begin{array}{l}\text { NUTS } \\
\text { \& STAFF3D } \\
\text { NUTS } \\
\text { \& STAFF3D } \\
\text { STAFF3D }\end{array}$ \\
\hline $\begin{array}{l}\text { Agents Acting Upon Disposal System } \\
\text { Climate Change } \\
63 \text { Minimum infiltration rate } \\
64 \text { Maximum infiltration rate } \\
65 \text { Duration of climatic cycle } \\
66 \text { Infiltration concentration factor }\end{array}$ & $\begin{array}{l}\mathfrak{j}_{\text {infil }} \\
A_{\text {infl }} \\
T_{\text {infil }} \\
f_{\text {infll }}\end{array}$ & $\begin{array}{l}\text { GLOBAL } \\
\text { GLOBAL } \\
\text { GLOBAL } \\
\text { GLOBAL }\end{array}$ & $\begin{array}{l}\text { PPTINFMN } \\
\text { PPTINFMX } \\
\text { CYCLEPER } \\
\text { PPTINFOC }\end{array}$ & $\begin{array}{l}\text { BRAGFLO_T } \\
\text { BRAGFLO_T } \\
\text { BRAGFLO_T } \\
\text { BRAGFLO_T }\end{array}$ \\
\hline \multicolumn{5}{|c|}{$\begin{array}{l}\text { a More complete descriptions are provided in Figures 6-3 through 6-17. } \\
\text { b Numbers indicate order in which the parameters were sampled (numbers } 58,60 \text {, and } 62 \text { were not used). } \\
\text { c Although several products would be produced from corrosion of the containers, the only product treated in the } 1997 \text { PA is rust } \\
\text { from corrosion of the carbon steel disposal container. }\end{array}$} \\
\hline
\end{tabular}


6. Analysis Design
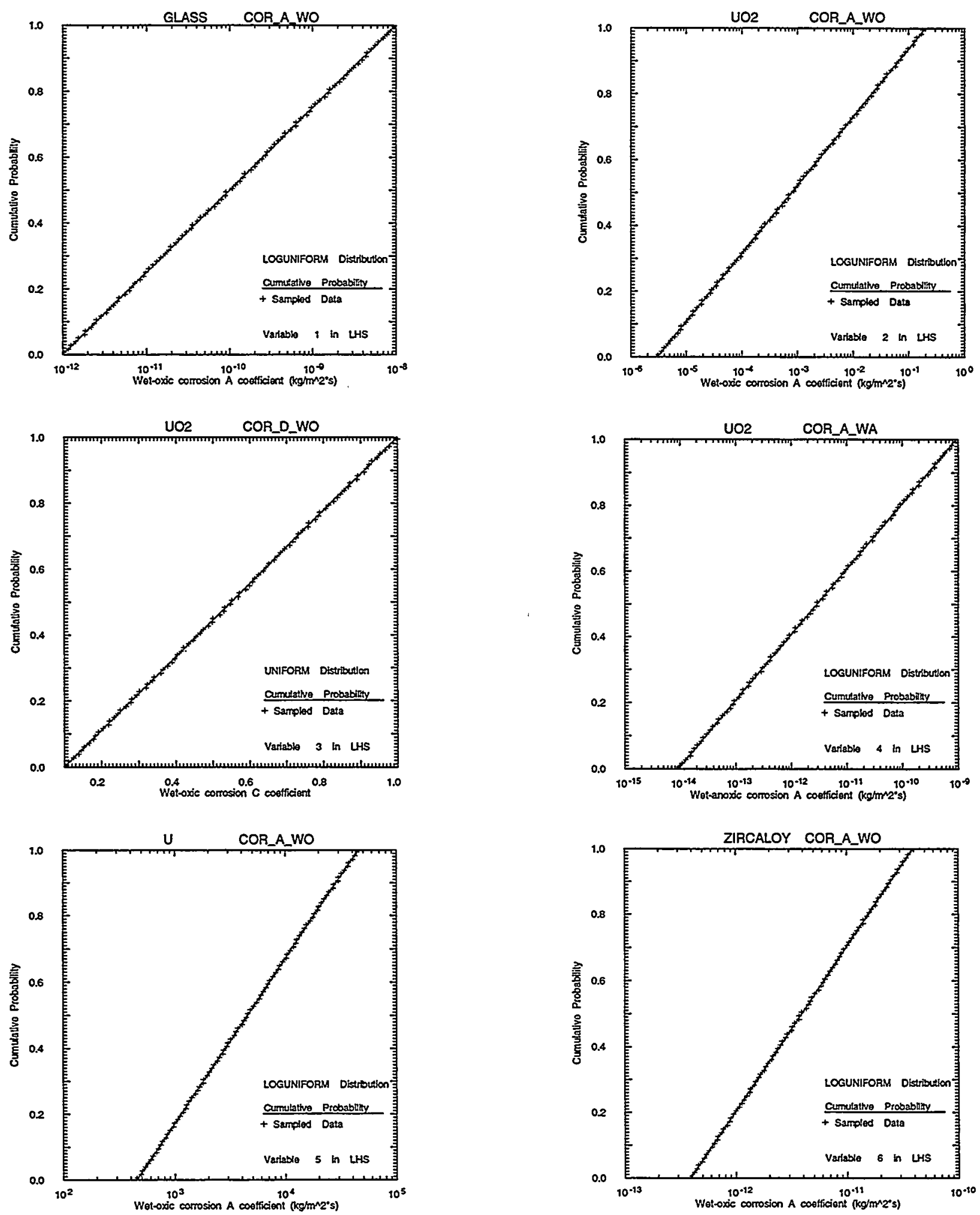

TR1-6342-5579-1

Figure 6-3. Probability distributions of alteration rate of waste and cladding. Distributions include alteration rates per surface area (constant in front of exponential term of Arrhenius rate equation) of glass, $\mathrm{UO}_{2}$, uranium metal, and Zircaloy cladding. 

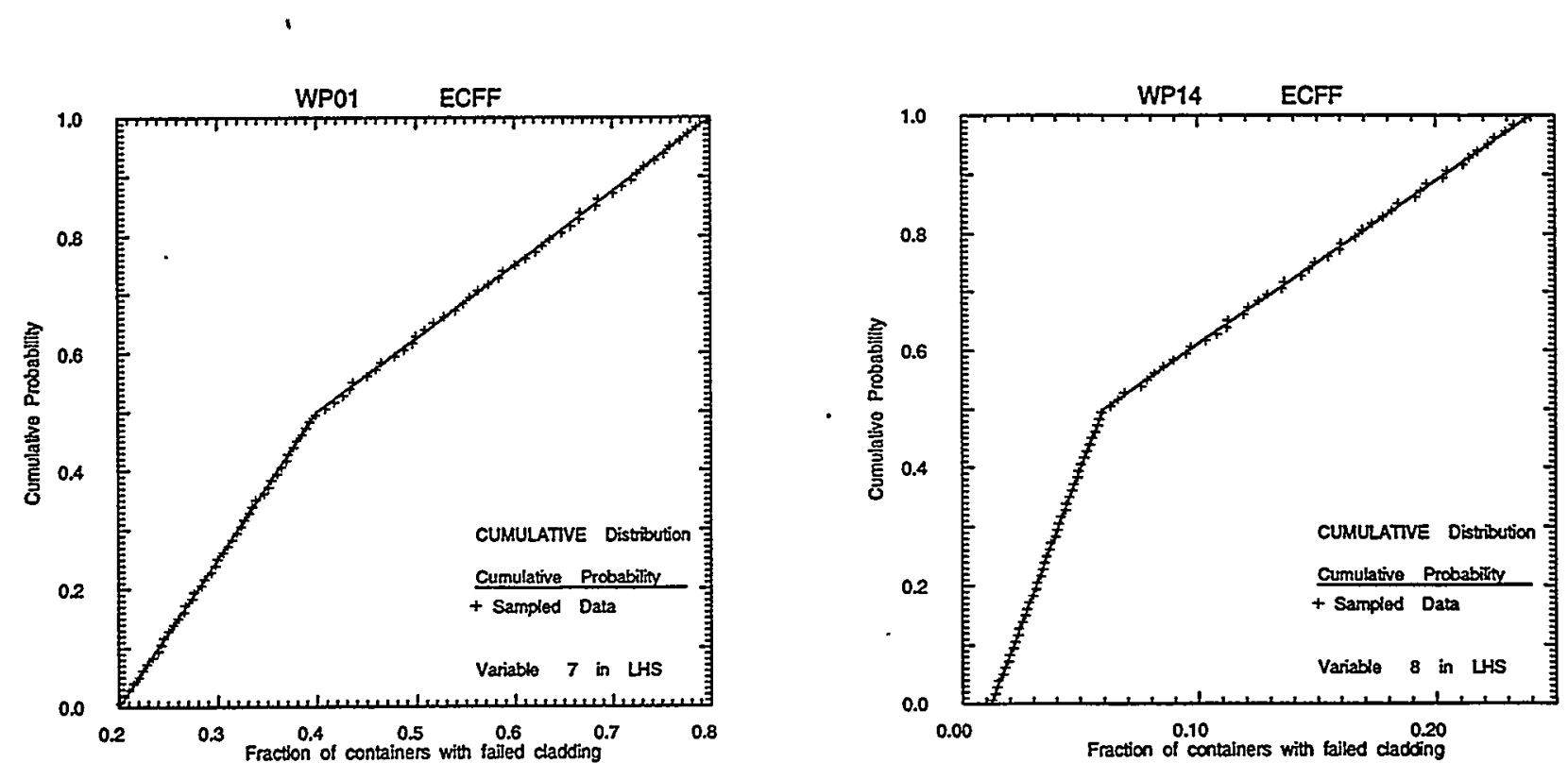

TRI-6342-6019-0

Figure 6-4. Early clad failure fractions for N-Reactor (Category 1) and commercial PWR (Category 14) fuel. 

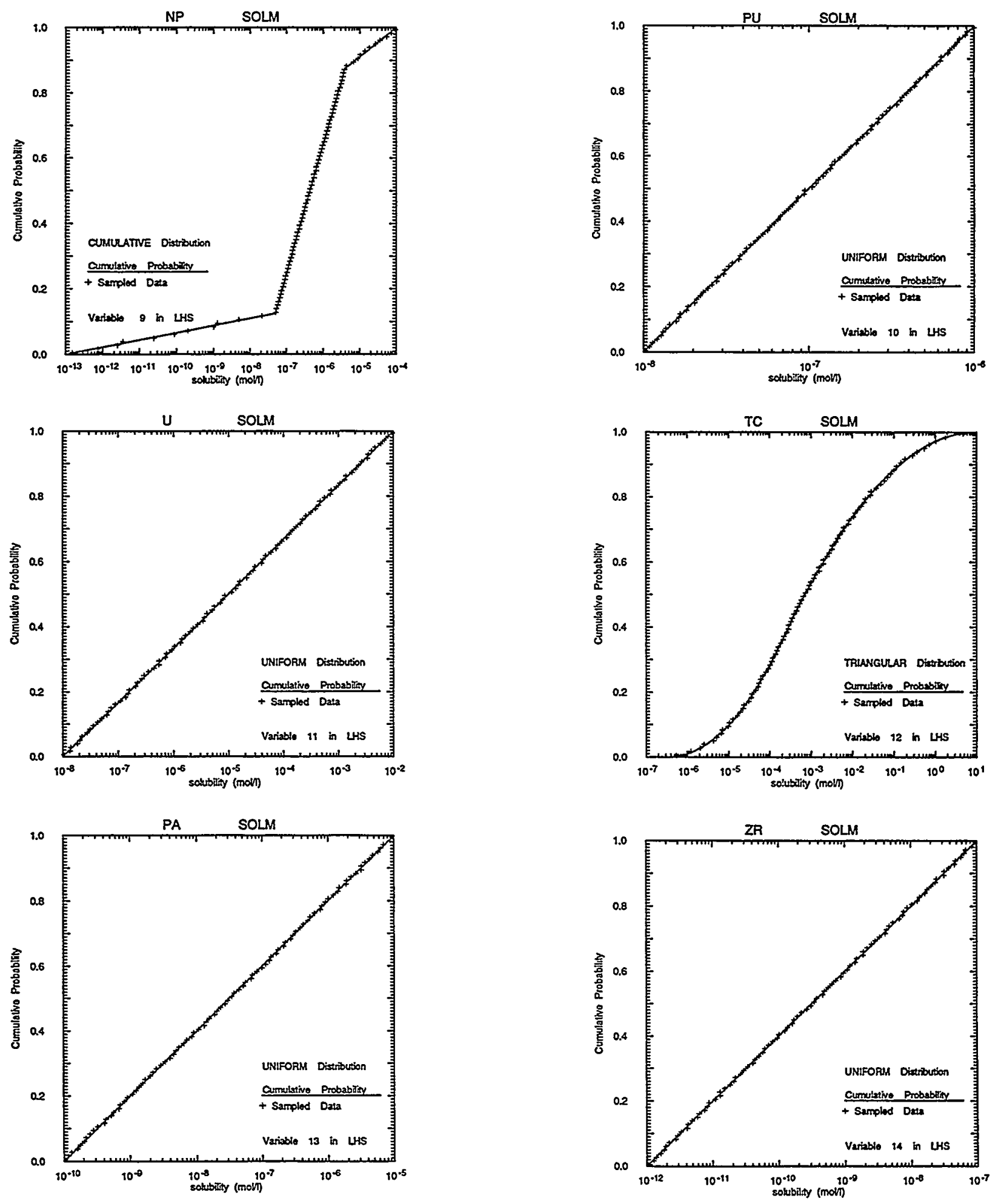

TRI-6342-5580-1

Figure 6-5. Probability distributions for solubility of radioisotopes in expected Yucca Mountain groundwater. 

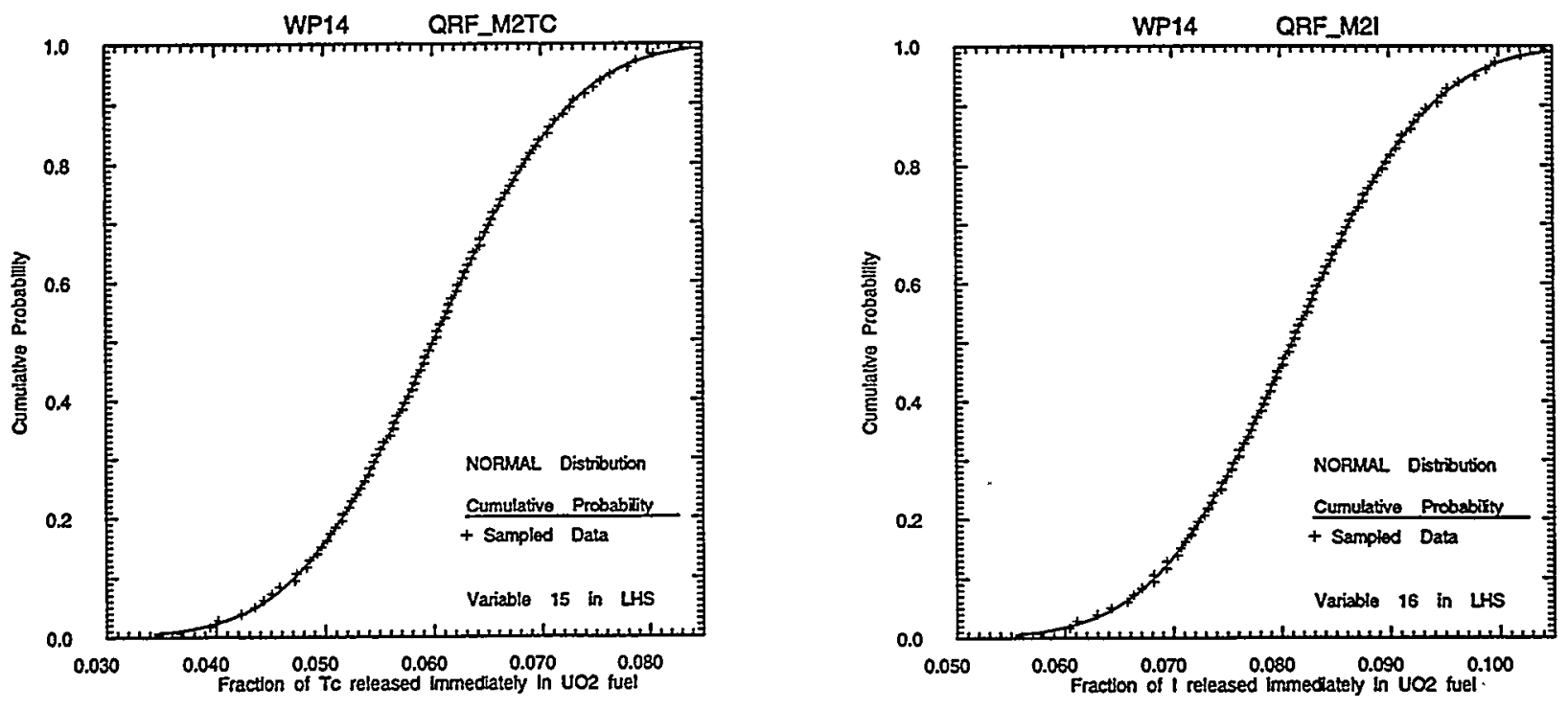

TRI-6342-6022-0

Figure 6-6. Probability distributions for quick release fraction in matrix 2 of PWR fuel (Category 14). Distributions include fraction of technetium-99 and iodine-129 present in grain boundaries of uranium dioxide, which is released immediately when cladding fails. 

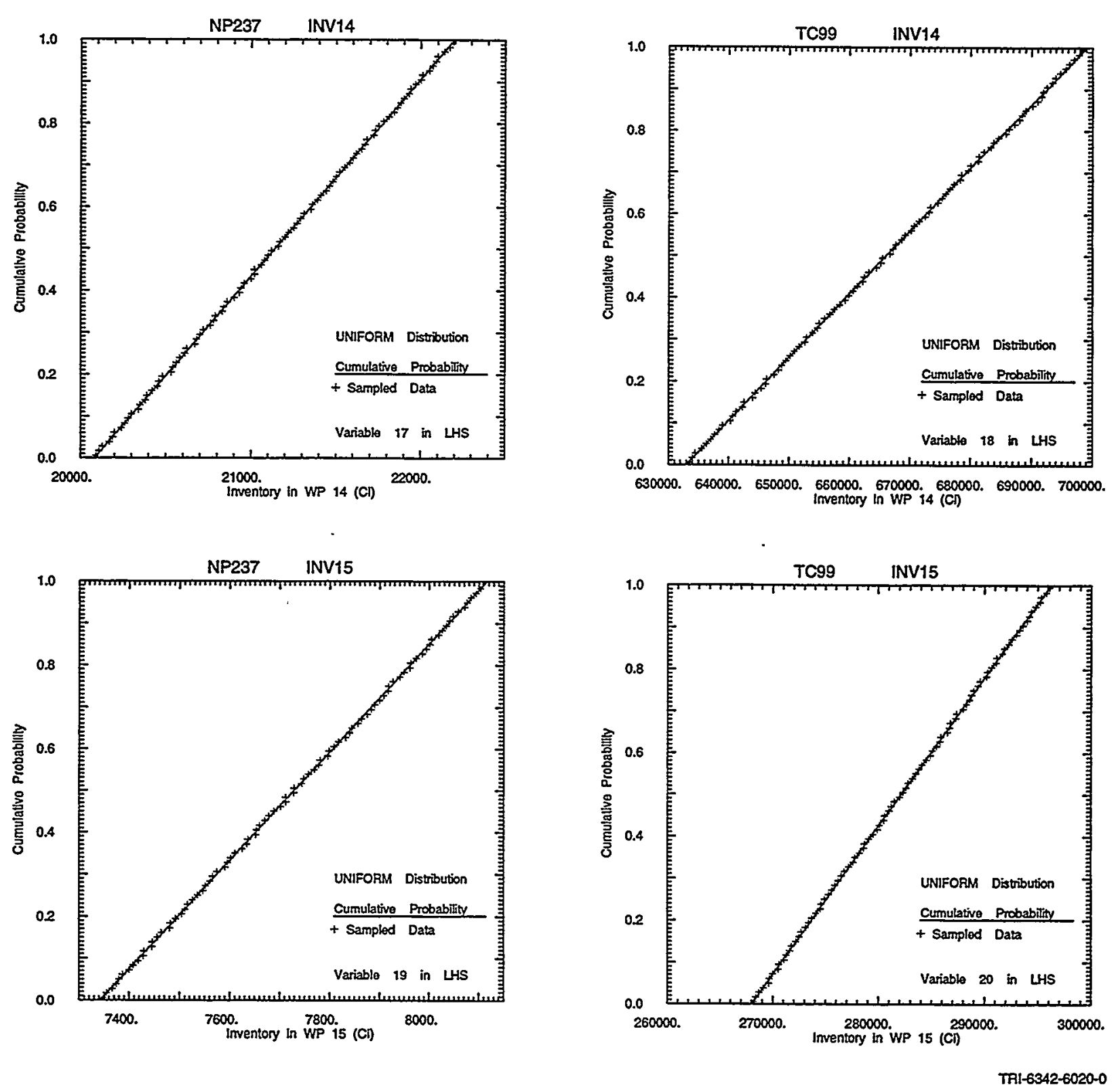

Figure 6-7. Activity inventory of neptunium and technetium in PWR (Category 14) and BWR (Category 15) fuels. 

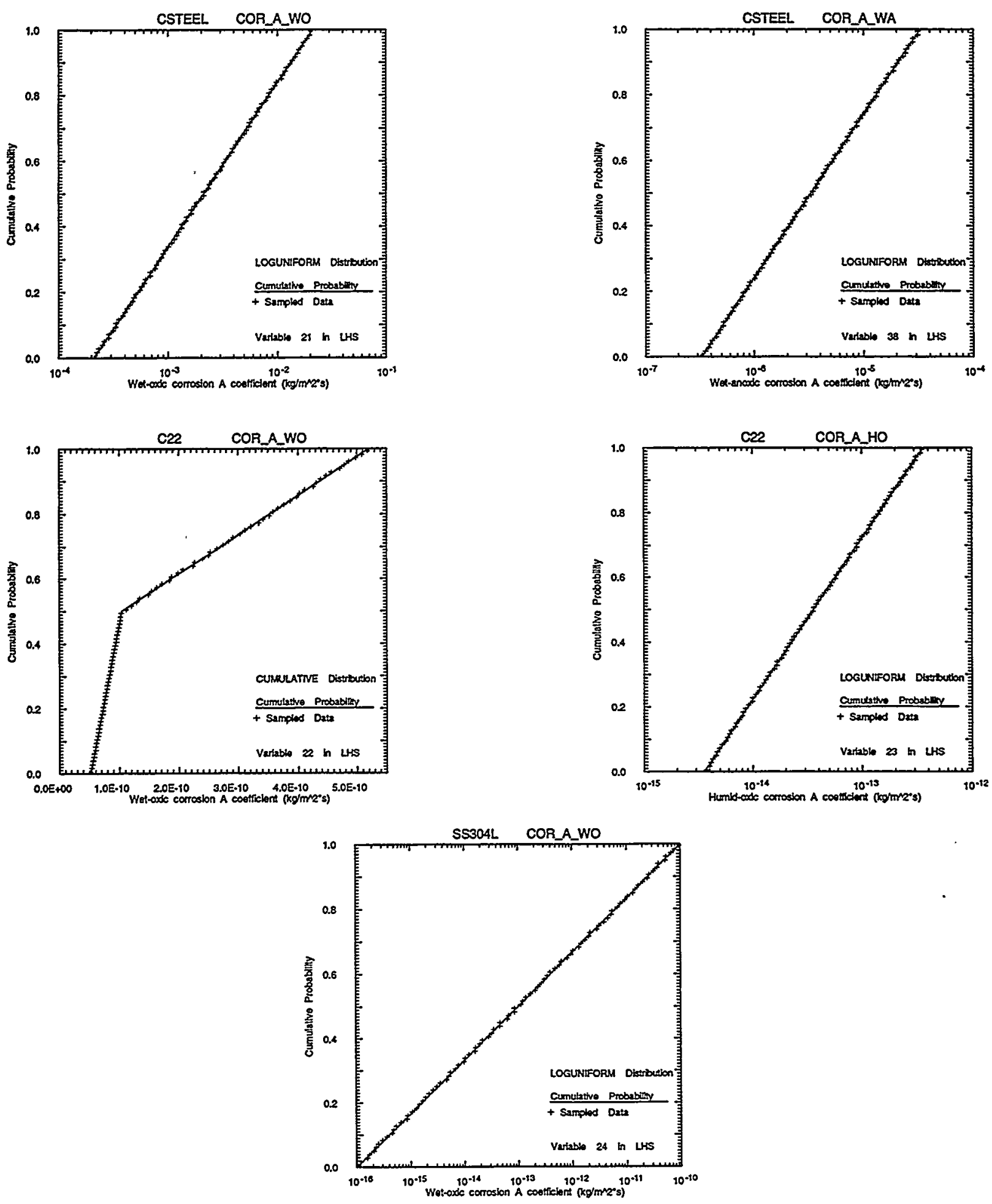

TRI-6342-5582-1

Figure 6-8. Probability distributions of alteration rate of waste package layer. Distributions include alteration rates per surface area (constant in front of exponential term of Arrhenius rate equation) of carbon steel, Hastaloy C-22, and stainless steel. 

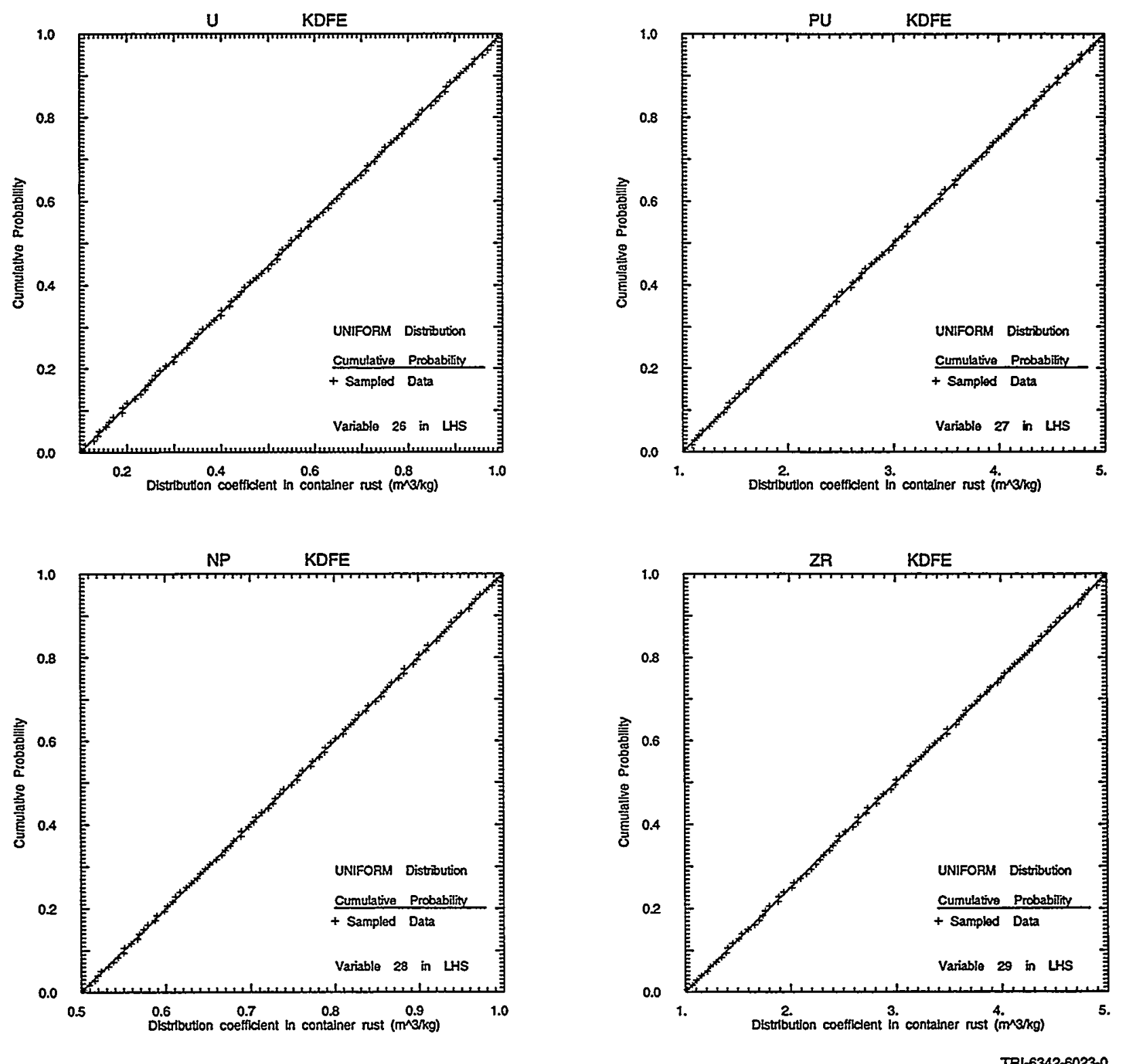

Figure 6-9. Probability distributions of radioisotopes (uranium, plutonium, neptunium, and zirconium) on rust of outer carbon steel container. 

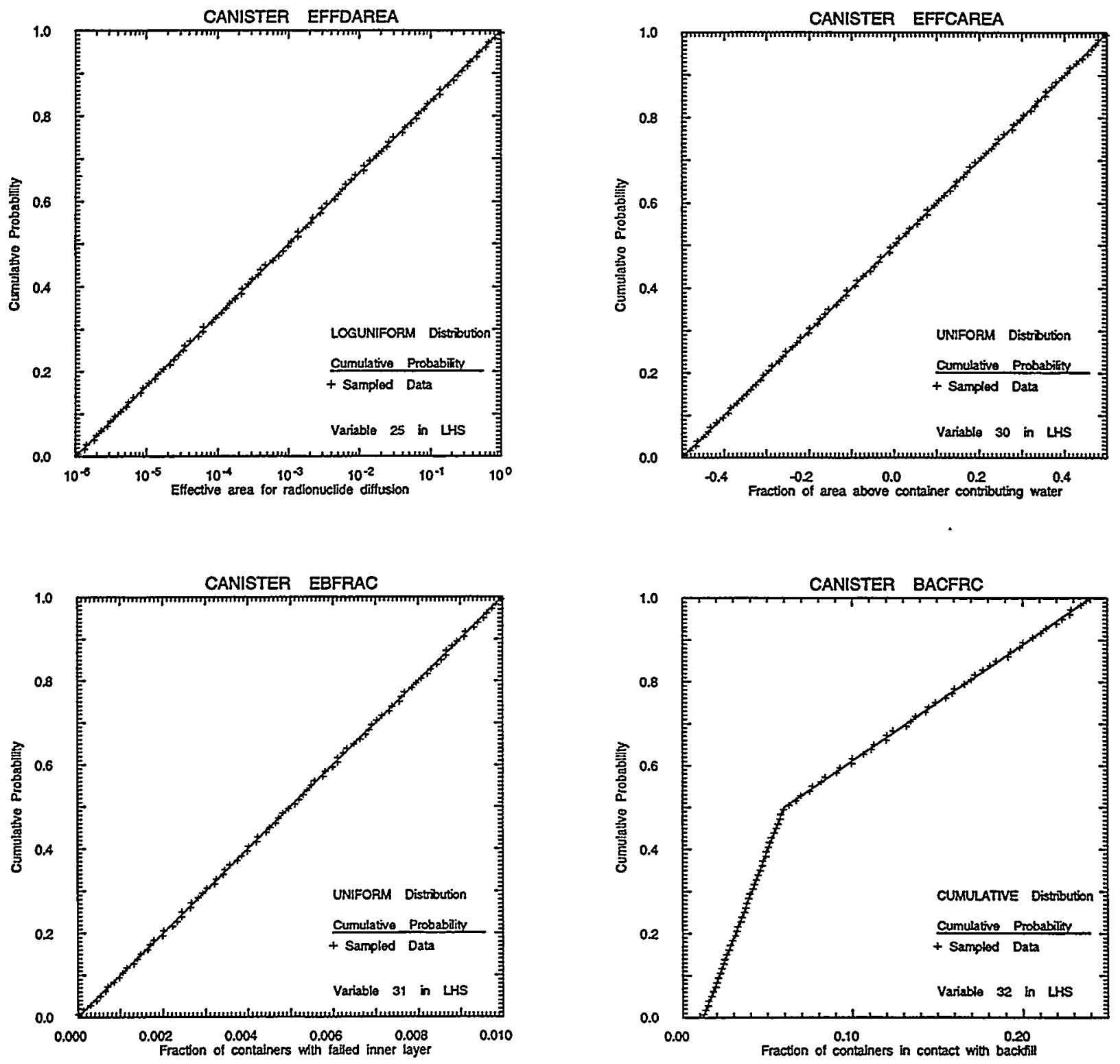

TRI-6342-5583-1

Figure 6-10. Probability distributions of diffusion of radionuclides through the breached containers, area above the container that contributes water (e.g., drips), that in turn contacts the container surface, fraction of containers with failed inner layer, and fraction of containers with rubble. 

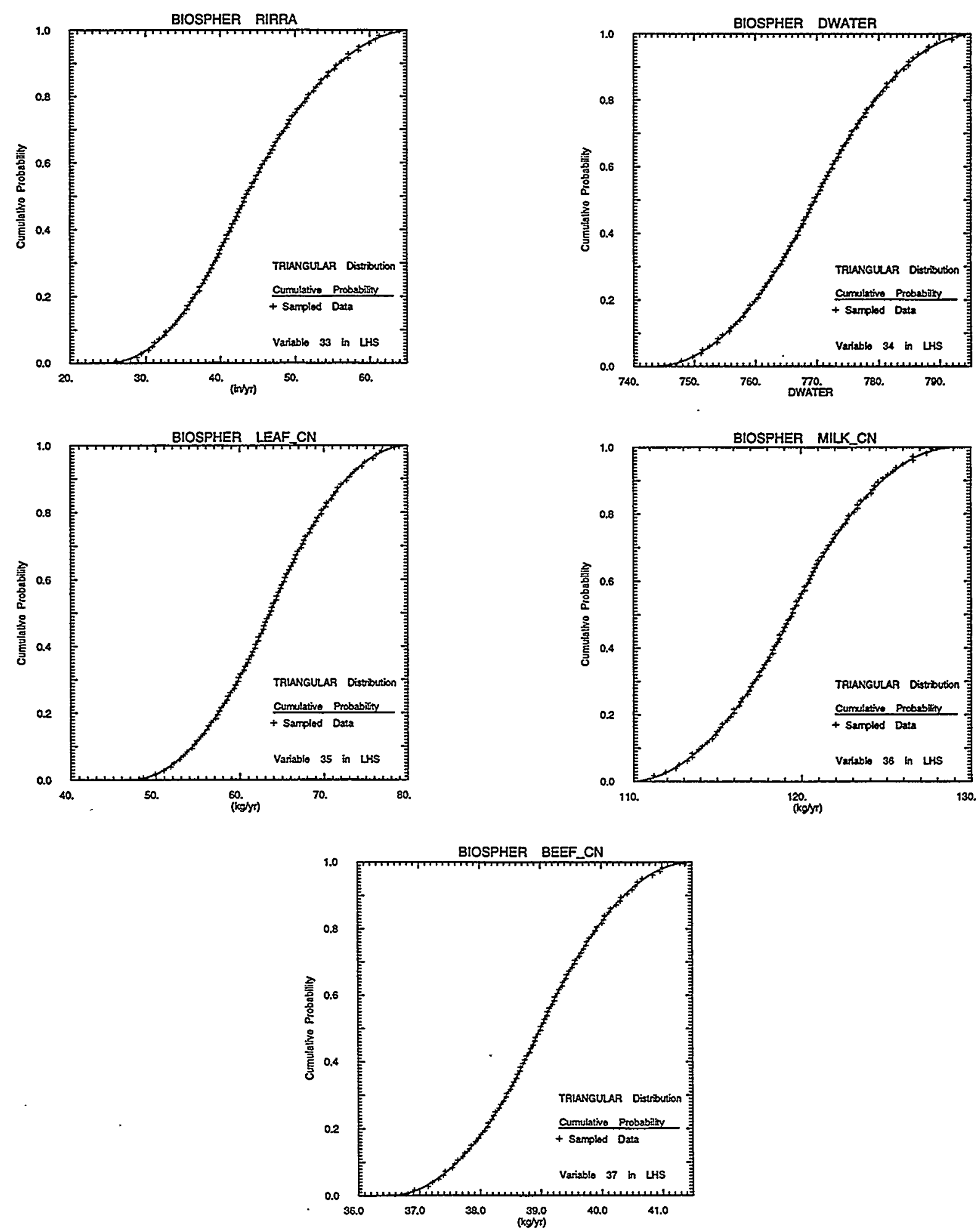

TR1-6342-5578-1

Figure 6-11. Probability distributions for biosphere parameters, including irrigation rate, and consumption of drinking water, vegetables, milk, and beef. 

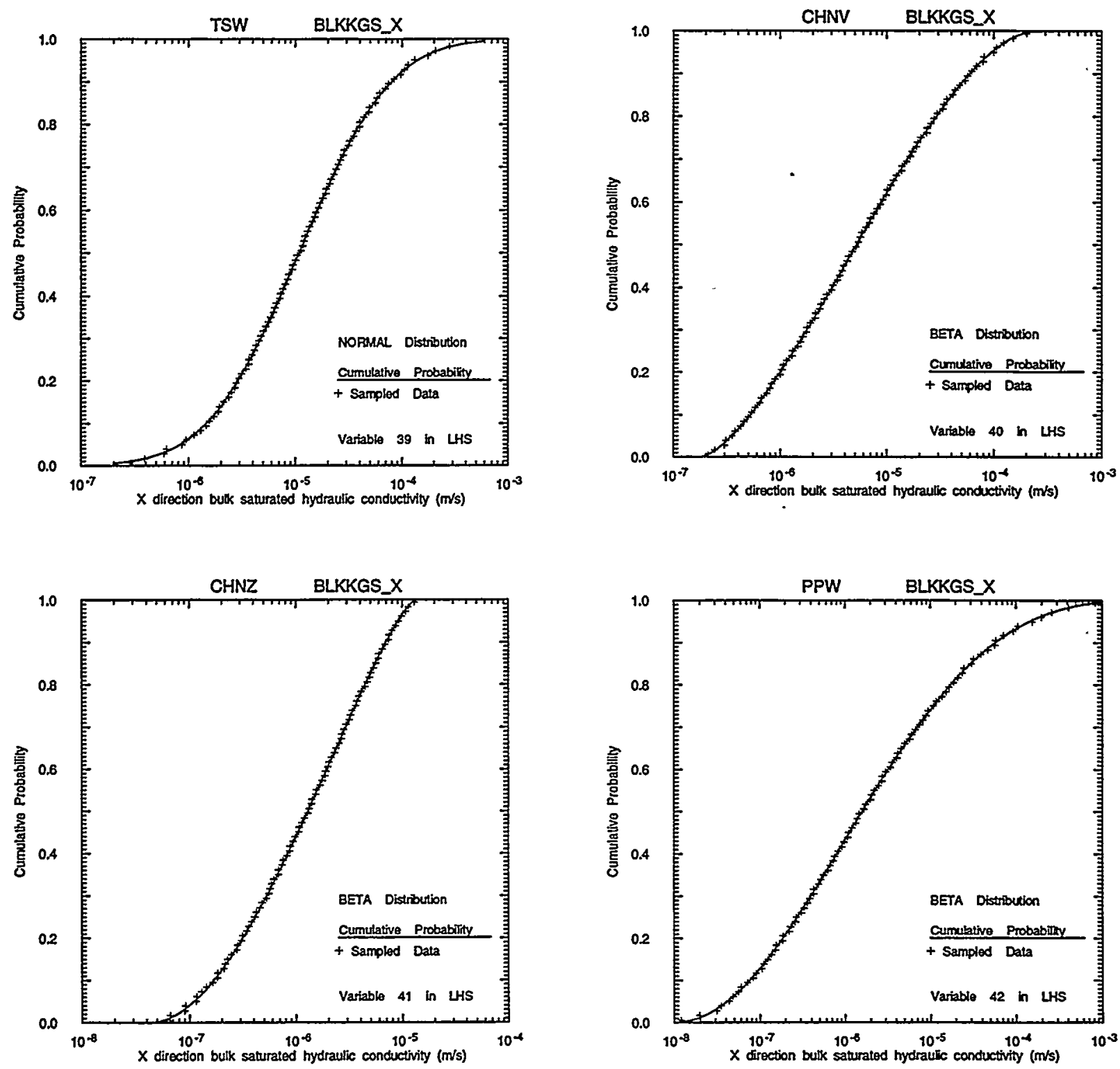

TRI-6342-5572-1

Figure 6-12. Probability distributions of bulk, saturated hydraulic conductivity of modeling layers TSw, CHnv, $C H n z$, and $P P w$. 


\section{Analysis Design}
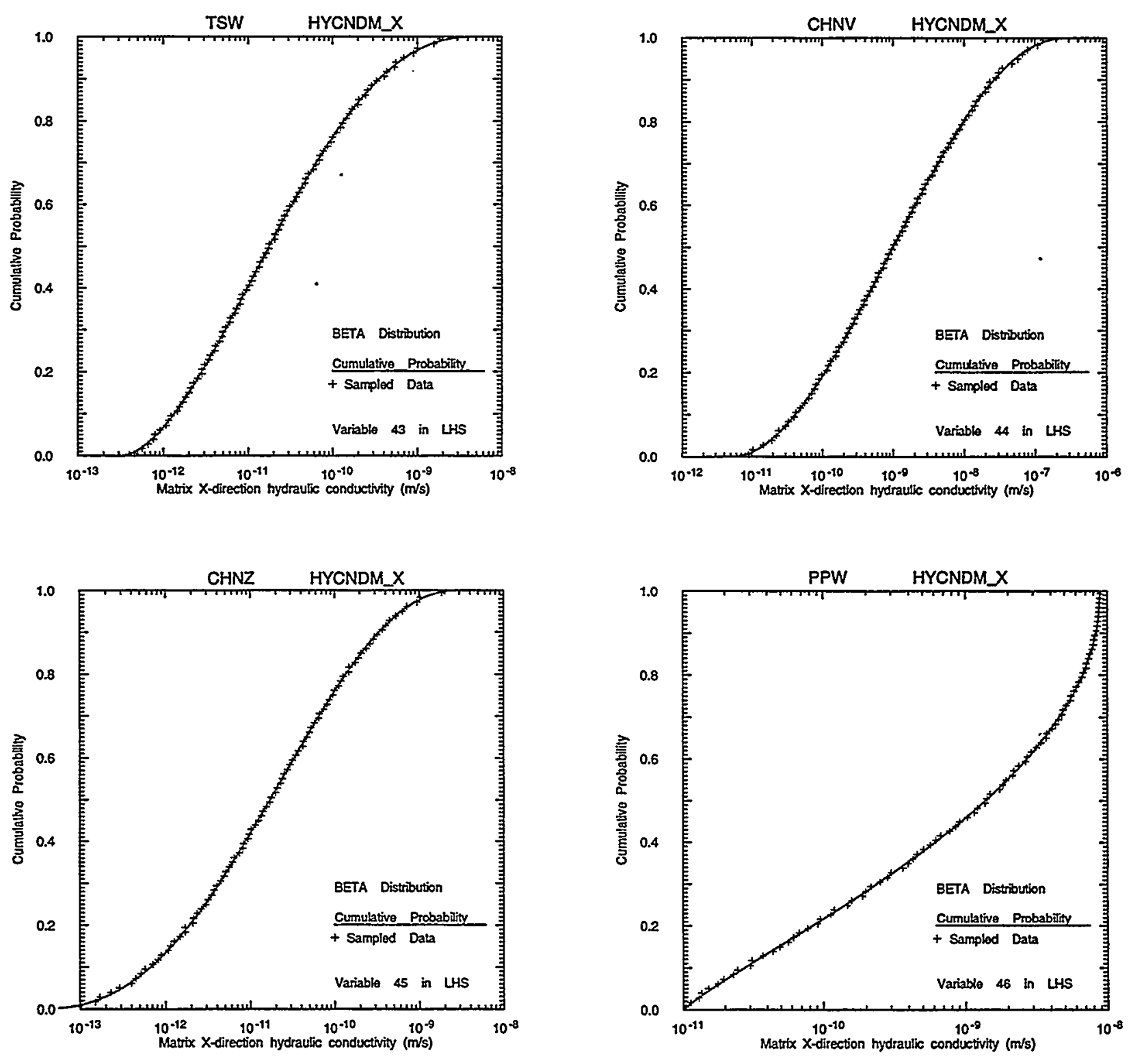

TR1-6342-5573-1

Figure 6-13. Probability distributions of hydraulic conductivity of layers. Distributions include matrix conductivity assigned to modeling layers TSw, CHnv, CHnz, and PPw. 

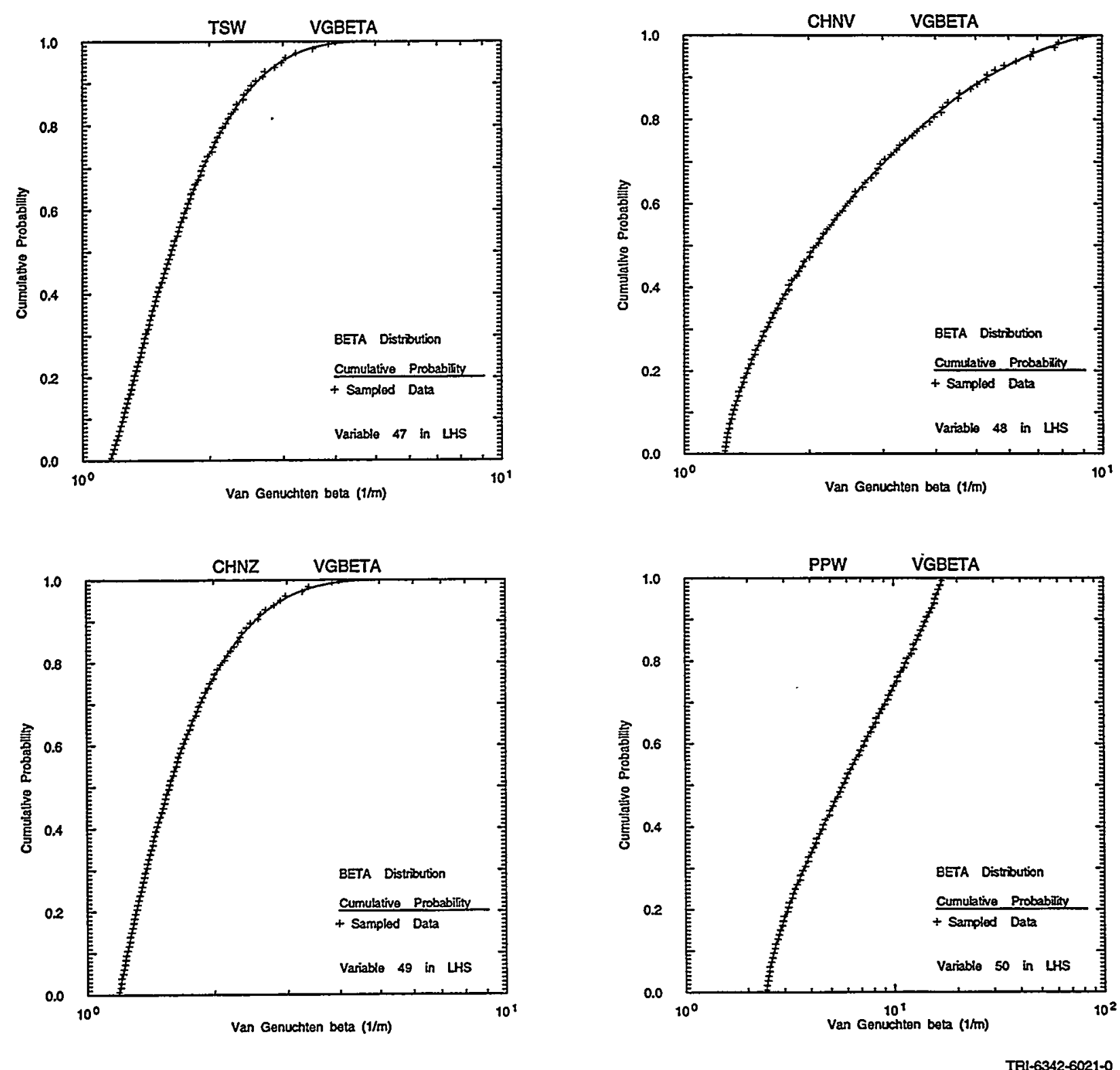

Figure 6-14. Distribution of Van Genuchten value for TSw, CHnw, CHnz, and PPw. 

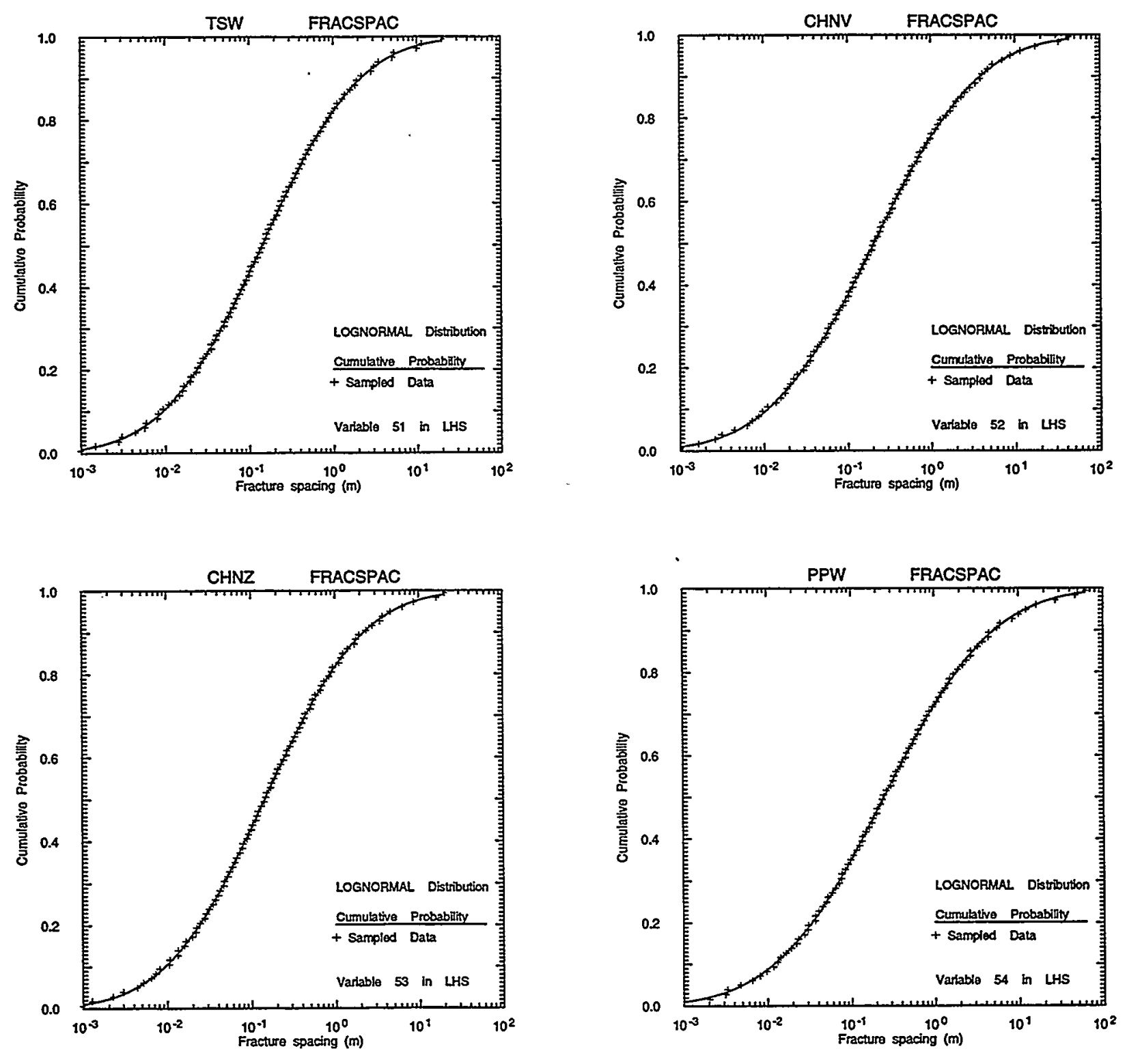

TR1-6342-5574-1

Figure 6-15. Probability distributions of spacing of fractures assigned to modeling layers TSw, CHm, CHnz, and PPw. 

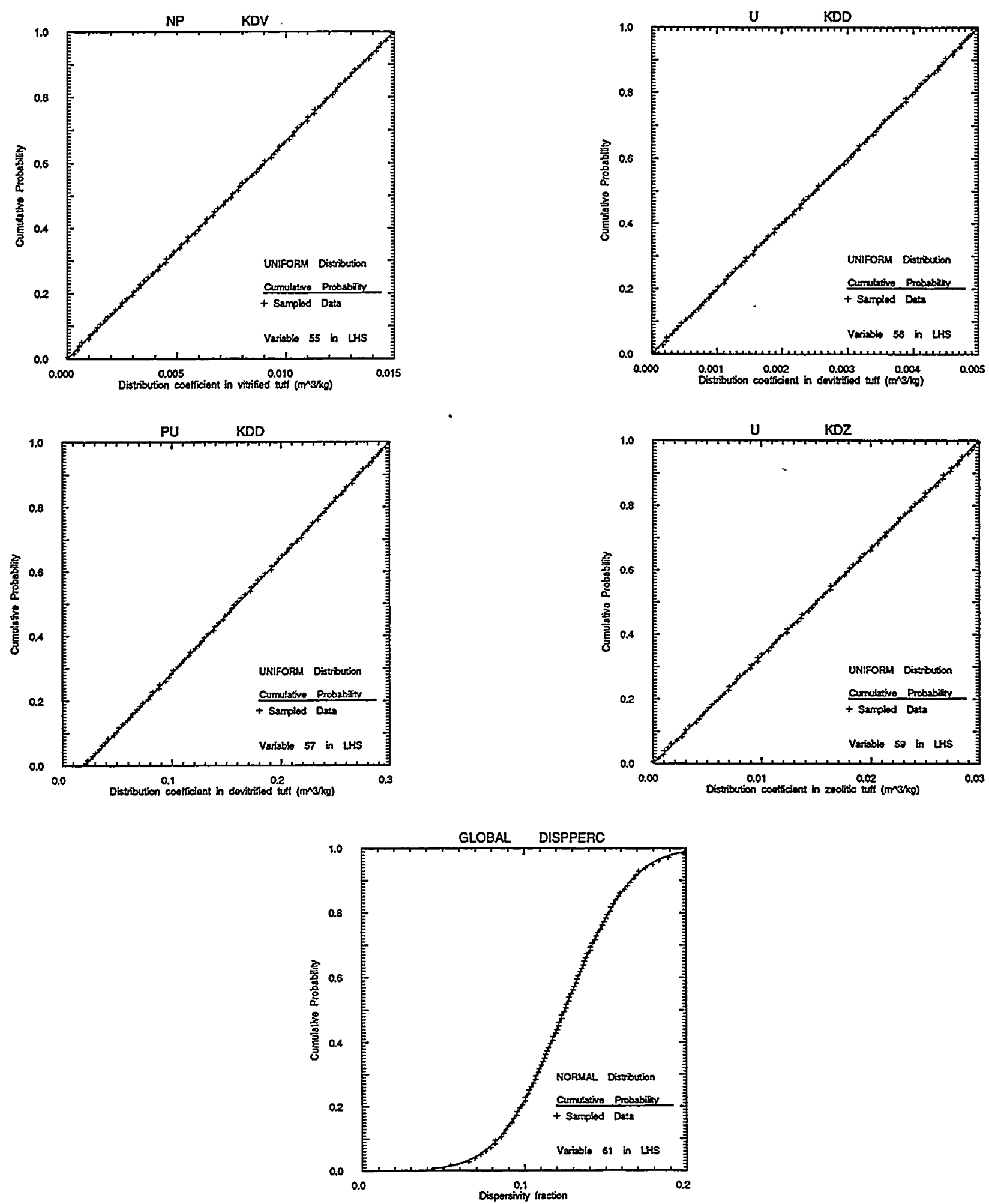

TRI-6342-5575-1

Figure 6-16. Probability distributions for distribution coefficients for neptunium for vitrified tuff, uranium and plutonium for devitrified tuff, uranium for zeolitic tuff, and for dispersivity fraction. 


\section{Analysis Design}
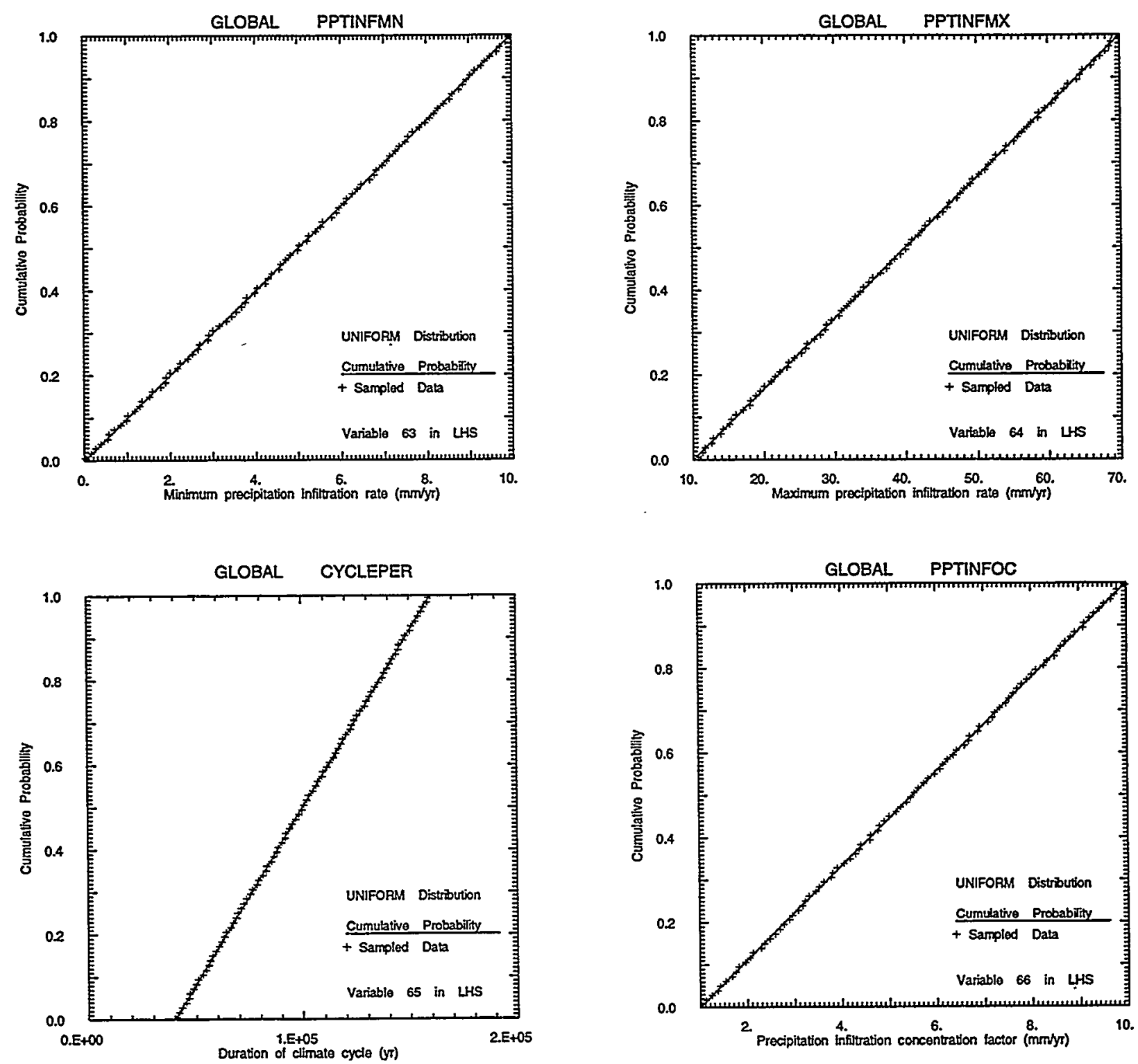

TA1-6342-5577-1

Figure 6-17. Probability distributions for global parameters for climate change. Distributions include infiltration rate above repository for current dry climate, increase in infiltration rate during wet climate, period of climatic change, and potential concentration of infiltration because of surface features above the repository. 


\title{
7. Consequence Modeling: Corrosion and Source-Term Model
}

\author{
C. T. Stockman, L. H. Brush, and L. J. Storz
}

The Corrosion and Source-Term (CST) model comprises a corrosion submodel and a source-term submodel. The corrosion submodel predicts: (1) the rates at which corrosion will penetrate the waste packages in a potential Yucca Mountain repository, thereby exposing radionuclides in the waste to mobilization by aqueous solutions and possible transport to the accessible environment; and (2) the effects of corrosion on the gas (especially oxygen) and water contents of the near field (that portion of the repository significantly affected by the waste). For the 1993 and the 1994 PA calculations, Stockman (1993) and Stockman et al. (1995) referred to the corrosion submodel as GASSNF. The source-term submodel predicts: (1) radioactive decay and ingrowth; (2) the distribution of radionuclides among aqueous and solid phases within the waste packages; and (3) the transport of radionuclides from the waste packages to the intact host rock (welded tuff of the Topopah Spring Formation). Stockman (1993) referred to the source-term submodel used for the 1993 PA as CONCSNF; Stockman et al. (1995) referred to the new code written for the 1994 PA as USATCONC.

\subsection{Corrosion Submodel}

\subsubsection{Overview of Waste Packages, Corrosion, and Corrosion Submodel}

The waste packages to be emplaced in the potential Yucca Mountain repository comprise several layers (see Chapter 3, Section 3.6). For example, the 4820 waste packages containing commercial pressurized-water-reactor (PWR) spent fuel (Category 14) and the 2859 packages of commercial boiling-water-reactor (BWR) spent fuel (Category 15), by far the largest categories in terms of spent-fuel mass consist of (for the 1997 PA calculations): (1) a 10-cm-thick outer package (also referred to as outer shell) and outer lid of ASTM A516 Grade 60 steel, a carbon steel (see "ASTM A516 Grade 60 Steel Disposal Container" in Section 7.3.1 for composition); (2) a 2-cm-thick inner package and inner lid of Inconel 625 (see "Inconel 625 Disposal Container" in Section 7.3.1 for composition); (3) an array of steel spacers; (4) a set of steel tubes (the BWR waste packages do not contain these tubes); and (5) uranium dioxide $\left(\mathrm{UO}_{2}\right)$ spent fuel pellets enclosed in Zircaloy-2 cladding (see "Zircaloy-2 Cladding" in Section 7.3.1) and separated by steel or borated-steel baskets, depending on the extent of spent-fuel burnup (fuel with low burnup require borated-steel baskets to poison thermal neutrons; for high-burnup fuel, borated steel is unnecessary). Figures 3-29 and 3-30 in Chapter 3 show the waste packages for commercial PWR and BWR spent fuel, respectively. On the other hand, the 118 waste packages containing spent fuel from N-Reactor (Category 1), the largest DOE-owned spent fuel category, consist of: (1) a 10-cm-thick, ASTM A516 Grade 60 steel outer package and outer lid; (2) a 2-cm-thick, Inconel-625 inner package and inner lid; (3) four handling containers, 9.5-mm-thick multi-canister overpack (MCO) of 304L stainless steel (see "304L Stainless Steel Handling Containers," Section 7.3.1), and (4) two layers of metallic $U$ in three layers of Zircaloy-2 cladding. Figure 3-16 in Chapter 3 shows the waste package for $N$ Reactor spent fuel. The main difference between waste packages containing $\mathrm{N}$-reactor spent fuel and those with other categories of DOE SNF are that the waste packages for the other categories include, in addition to one handling container of spent fuel, three, four, or five handling containers of DHLW in which fission products removed from spent fuel by reprocessing are vitrified in borosilicate glass.

Materials that oxidize rapidly in corrosive environments, such as ASTM A516 Grade 60 steel, are referred to as corrosion-allowance materials, and require relatively thick layers $10 \mathrm{~cm}$ in the case of the disposal containers described above) to serve as effective constituents of waste packages. Those that oxidize slowly, such as Inconel 625, are referred to as corrosion-resistant, and require only thin layers $(2 \mathrm{~cm}$ in this case) to be effective. Materials resist corrosion because: (1) they are thermodynamically stable with respect to oxidation (noble); (2) they are thermodynamically unstable, but passivate (develop a protective coating, usually an oxide, that resists further attack), or (3) they are unstable, but corrode very slowly because the activation energy for the reaction is high, or because reactants or products migrate to or from the corroding surface very slowly. 
The corrosion submodel of CST predicts the sequential failure of both the corrosion-allowance and the corrosion-resistant materials described above. In a sense, the corrosion submodel treats corrosion of these materials like peeling the layers of skin off an onion (in the case of general corrosion, described in Section 7.1.4) or cutting through layers of skin (localized corrosion, also described in Section 7.1.4). For the 1997 PA, the corrosion submodel did not necessarily include every layer in a waste package. For the commercial PWR and BWR spent fuels (Categories 14 and 15), for example, it included the 10-cm-thick, ASTM A516 Grade 60 steel outer package and outer lid; the 2-cm-thick, Inconel-625 inner package and inner lid; the $\mathrm{UO}_{2}$ spent fuel, the Zircaloy-2 cladding, and the steel or borated-steel baskets. (However, it assumed that the corrosion reaction and the corrosion rate for these baskets are identical to those for 304L stainless steel.) It did not include the ASTM A516 Grade 60 steel spacers nor the tubes. For N-Reactor spent fuel (Category 1), the corrosion submodel included all of the layers described above, except the two inner layers of Zircaloy-2 cladding. Waste package layers are listed in Chapter 3, Table 3-6.

In addition to predicting the sequential failure of the corrosion-allowance and corrosion-resistant materials in the multi-layer waste packages described above, the corrosion submodel includes early failure of some of these waste packages. In this report, early failure refers to exposure of radionuclides in the waste to the near-field environment at the time of emplacement in the potential Yucca Mountain repository due to manufacturing defects, damage during transportation, or damage during emplacement. For the 1997 PA, we assumed that EBFRAC (the parameter that specifies the early failure fraction or early breach fraction of the waste packages) has a range of 0 to 0.01 and a uniform probability distribution (see Chapter 6, Figure 6-10). Furthermore, we assumed that this parameter pertains only to the failure of the 2-cm-thick, Inconel-625 inner package and lid; not the 10-cm-thick, ASTM A516 Grade 60 steel outer package and lid, the $0.635-\mathrm{cm}$-thick, 304L stainless steel handling container, the steel or borated-steel spacers, tubes, and baskets, nor the fuel materials.

\subsubsection{Contact with Groundwater}

The disposal tunnels in which the waste packages described above are emplaced include an air gap between the waste package and the intact host rock. For the 1997 PA, we assumed that three or, perhaps, four steel supports on a concrete pier on a pre-cast, concrete invert hold each waste package at the time of emplacement and, possibly, for a significant period of time thereafter (see Figure 7-1). The air gap will (1) ensure effective radiative transfer of heat from the waste packages to the tuff; and (2) prevent transport of groundwater from the tuff to the waste packages by capillarity (also referred to capillary suction or wicking). The air gap will not, of course, prevent groundwater from dripping on the waste packages if the $\mathrm{H}_{2} \mathrm{O}$ content of the tuff increases to values high enough to initiate fracture flow, nor will it prevent inundation of the disposal tunnels in the much less likely event of complete saturation of the tuff.

We assumed that, during the regulatory period (100,000 yr for the 1997 PA), rubble from the back (top) and ribs (sides) of the disposal tunnels will, at least to some extent, eliminate the air gap between the intact host rock and the waste packages. If sufficient rubble accumulates, capillarity might transport groundwater from the partially saturated tuff through the rubble to the waste packages if the $\mathrm{H}_{2} \mathrm{O}$ content of the tuff is high enough for fracture flow.

For the 1997 PA, whether or not sufficient rubble accumulates for capillarity determined: (1) the mode of contact between a waste package and groundwater; (2) the threshold value of the $\mathrm{H}_{2} \mathrm{O}$ content of fractures required to initiate this contact; and (3) how much $\mathrm{H}_{2} \mathrm{O}$ was available for corrosion in each time step. For a waste package with an air gap, dripping was the mode of groundwater contact; the threshold value of the intact-host-rock fracture $\mathrm{H}_{2} \mathrm{O}$ content required to initiate dripping was relatively high; and only the $\mathrm{H}_{2} \mathrm{O}$ above that required to initiate dripping was available for corrosion in each time step. For a waste package with rubble, capillarity was the mode of groundwater contact; the threshold value of the intact-tuff or rubble fracture $\mathrm{H}_{2} \mathrm{O}$ content required to initiate capillarity was relatively low; and all of the $\mathrm{H}_{2} \mathrm{O}$ above that required to initiate capillarity was available for corrosion in each time step. Because the threshold value of the fracture $\mathrm{H}_{2} \mathrm{O}$ saturation required to initiate dripping was somewhat higher than that required for capillarity, somewhat more $\mathrm{H}_{2} \mathrm{O}$ was available for corrosion of waste packages with rubble than for waste packages with an air gap in each time step. For wet oxic and wet anoxic corrosion (see Section 7.1.5, Corrosion Reactions), the $\mathrm{H}_{2} \mathrm{O}$-saturation-dependence function $\mathrm{SD}$ incorporates the effects of the fracture $\mathrm{H}_{2} \mathrm{O}$ content in the equations used to predict the rates of most of the corrosion reactions in the corrosion submodel (see Section 7.1.6, Corrosion Rates). 

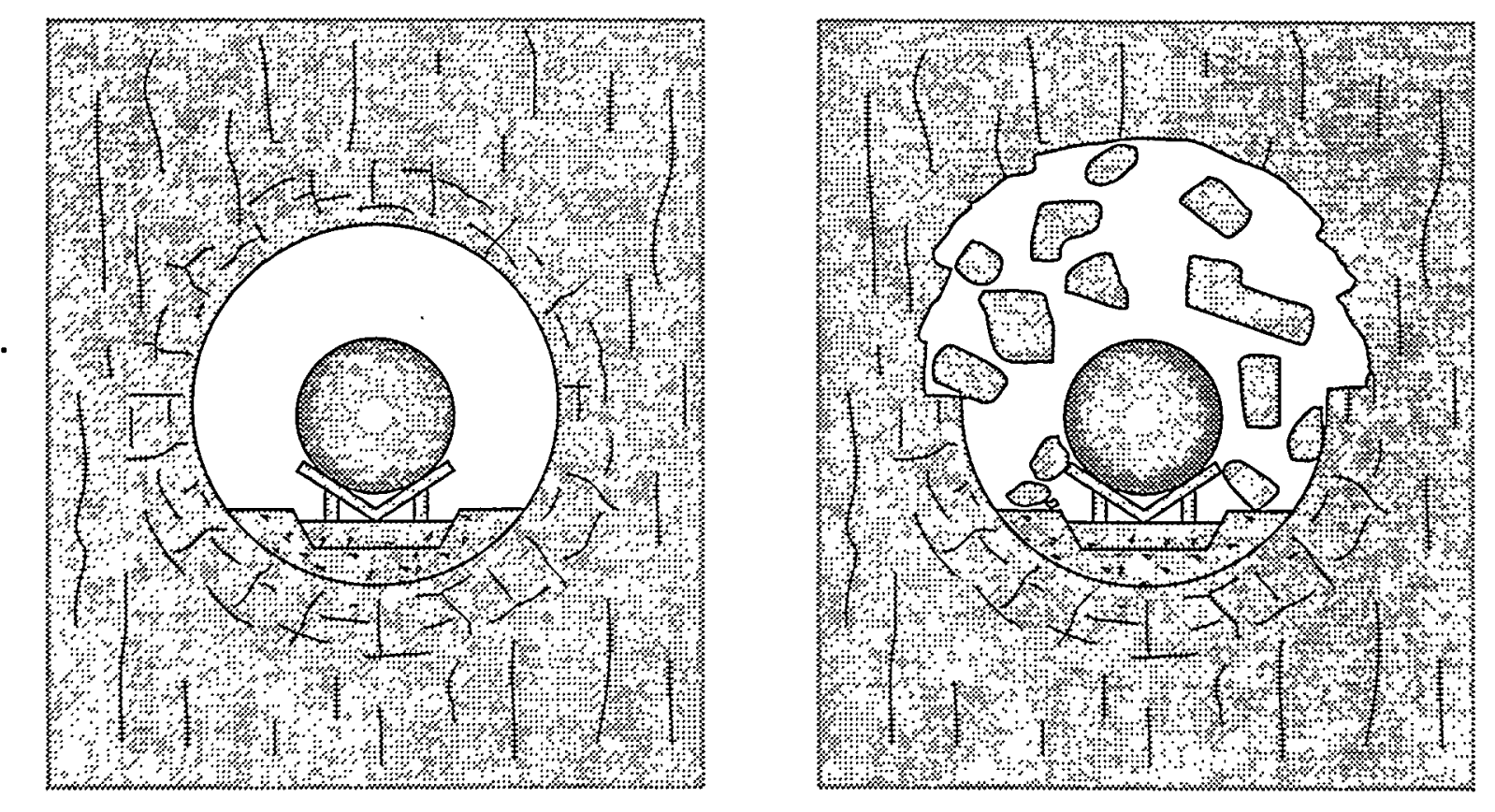

TRI-6342-5090-1

Figure 7-1. Emplaced waste package with air gap (left) and host rock rubble (right). 
For the 1997 PA, we assumed that BACFRC (Backfill Fraction), the parameter that specifies the fraction of the waste packages with sufficient rubble for capillarity, has a range of 0 to 1 and a uniform probability distribution (see Chapter 6, Figure 6-10). (Stockman, 1993, named this parameter BACFRC for the 1993 PA because she assumed that all of the waste packages in a bedded salt or granitic repository would include a bentonite backfill.) For any given realization (vector) of the PA calculations, the value of BACFRC was constant rather than time-dependent.

We did not include other possible modes of contact between waste packages and groundwater, such as inundation of the waste packages by groundwater or condensation of $\mathrm{H}_{2} \mathrm{O}$ on the waste packages, because we consider them much less likely than capillarity or dripping.

\subsubsection{Container Groups}

The corrosion submodel allows the user to define container groups. This submodel then applies pertinent equations and/or parameters to all of the waste packages within these groups as it loops over the waste packages during each time step. The order of nesting for these loops is: (1) the waste grid blocks; (2) the container groups within each waste grid block; (3) the waste package categories within each container group; (4) the matrices exposed to the nearfield environment within each waste package category. The user-input name for container groups is ICG (integers required to specify Container Group).

For the 1997 PA we defined container groups that specify: (1) whether the waste packages fail at the time of emplacement in the potential Yucca Mountain repository (see Section 7.1.1); and (2) whether there is an air gap between the waste package and the intact host rock and, hence, the mode of contact between a waste package and groundwater (Section 7.1.2). Table 7-1 shows the container groups specified for the 1997 PA. Although we used only the two criteria described above to define container groups, it is possible to use additional criteria to define them.

Table 7-1. Container Groups (ICGs) Specified for the 1997 PA

\begin{tabular}{ccc}
\hline & \multicolumn{2}{c}{ Early Failure of Waste Package? } \\
\cline { 2 - 3 } Rubble Present & No & Yes \\
\hline No & $I C G=1$ & $I C G=2$ \\
Yes & $I C G=3$ & $I C G=4$ \\
\hline
\end{tabular}

\subsubsection{Types of Corrosion}

Two types of corrosion can occur in the potential Yucca Mountain repository: (1) general corrosion, in which corrosion occurs at a uniform or nearly uniform rate at all of the surfaces exposed to oxidants (Section 7.1.5); (2) localized corrosion, in which corrosion occurs at significantly higher rates in crevices, pits, or cracks with significantly different concentrations of the reactants (especially $\mathrm{O}_{2}$ ) or products (especially metal ions) of the reactions responsible for corrosion. Crevices or pits could form at the sites of manufacturing defects in or mechanical damage to the materials used to fabricate waste packages. Stress-corrosion cracking, a form of localized corrosion, occurs when the susceptible material corrodes while under a tensile stress. Tensile stresses can be active, such as those caused by lithostatic loads, or residual, such as those imposed by welding or other manufacturing processes.

The corrosion submodel includes both general and localized corrosion. For ASTM A516 Grade 60 steel, the first layer to be exposed, uniform corrosion will dominate. In the case of the Inconel 625 layer, the second material that will be exposed to corrosion, pitting will probably determine the rate at which this layer is breached. However, the corrosion submodel also includes uniform corrosion of Inconel 625 to predict the effects of this type of corrosion on the $\mathrm{H}_{2} \mathrm{O}$, gas, and $\mathrm{O}_{2}$ contents of the near field. The parameter FRCPEN, defined as the mass fraction of a layer corroded uniformly at the time of penetration of that layer by localized attack, specifies the relative importance of the 
two types of corrosion for any given material. If FRCPEN is 0 , localized corrosion penetrates the layer prior to any general corrosion; if FRCPEN is 1, no localized corrosion occurs.

\subsubsection{Corrosion Reactions}

Corrosion of most of the metallic and nonmetallic constituents of the waste packages in the potential Yucca Mountain repository (disposal containers, handling containers, spacers, tubes, baskets, fuel cladding, and spent fuel) occurs when oxidants react with them to form corrosion products that no longer effectively isolate the waste from the near-field. Potential oxidants include oxygen $\left(\mathrm{O}_{2}\right)$, water $\left(\mathrm{H}_{2} \mathrm{O}\right)$, peroxide $\left(\mathrm{O}_{2}{ }^{2-}\right)$, sulfate $\left(\mathrm{SO}_{4}{ }^{2-}\right)$, sulfide $\left(\mathrm{S}^{2-}\right)$, etc. (Alteration of borosilicate glass will not, for the most part, involve oxidation.) In addition to the inorganic oxidants listed above, microorganisms might also facilitate corrosion by a process referred to as microbially induced corrosion (MIC).

The corrosion submodel explicitly includes corrosion reactions in which the oxidant is $\mathrm{O}_{2}$ (referred to hereafter as oxic corrosion) or $\mathrm{H}_{2} \mathrm{O}$ (anoxic corrosion). In the case of corrosion of $\mathrm{UO}_{2}$ under anoxic conditions, the corrosion submodel implicitly includes $\mathrm{O}_{2}{ }^{2-}$ and other products of the radiolysis of $\mathrm{H}_{2} \mathrm{O}$ by using an overall reaction in which $\mathrm{H}_{2} \mathrm{O}$ appears as the oxidant, but by using corrosion rates measured in the presence of these radiolysis products. The corrosion submodel does not explicitly or implicitly include other oxidants such as $\mathrm{O}_{2}{ }^{2-}$ and sulfide $\mathrm{S}^{2-}$, nor $\mathrm{MIC}$.

The corrosion submodel includes corrosion by $\mathrm{O}_{2}$ dissolved in the gaseous phase (referred to as humid oxic corrosion), or by $\mathrm{O}_{2}$ dissolved in the aqueous phase (wet oxic corrosion). For both types of oxic corrosion, the overall reaction is:

$$
\text { metal (or oxide) }+\left(\mathrm{S}_{\mathrm{O}_{2}}\right) \cdot \mathrm{O}_{2}=\left(\mathrm{S}_{\mathrm{rust}}\right) \cdot \text { solid corrosion product(s) }+\left(\mathrm{S}_{\mathrm{gas}}\right) \cdot \operatorname{gas}(\mathrm{es})
$$

in which $\mathrm{S}_{\mathrm{O}_{2}}, \mathrm{~S}_{\text {rust }}$, and $\mathrm{S}_{\text {gas }}$ are stoichiometric factors used to balance the reaction for one mole of metal (ASTM A516 Grade 60 steel, Inconel $625,304 \mathrm{~L}$ stainless steel, $\mathrm{U}$, etc.) or oxide $\left(\mathrm{UO}_{2}\right)$. The corrosion submodel uses these factors to compute the quantities of $\mathrm{O}_{2}$, rust (defined by this submodel as the $\mathrm{Fe}(\mathrm{III})$-bearing corrosion products), and gas (defined as any gaseous species except $\mathrm{H}_{2} \mathrm{O}$ vapor and $\mathrm{O}_{2}$ ) consumed or produced per unit mass of material corroded during each time step. (The source-term submodel of CST includes sorption of radionuclides by $\mathrm{Fe}_{2} \mathrm{O}_{3}$, for which we have sorption data. It does not include sorption by the Fe(II) corrosion products formed under anoxic conditions, $\mathrm{Fe}(\mathrm{OH})_{2}$ and $\mathrm{Fe}_{3} \mathrm{O}_{4}$, nor by $\mathrm{Al}_{2} \mathrm{O}_{3}, \mathrm{Cr}_{2} \mathrm{O}_{3}$, and $\mathrm{NiO}$, for which we have no data.) We assumed that, for any given metallic or nonmetallic waste package material, the humid and wet oxic corrosion reactions are stoichiometrically identical. However, we did not assume that these reactions occur at the same rate under humid and wet conditions (see Section 7.1.6 and parameters listed in Section 7.5). As written, Reaction 7-1 forms anhydrous corrosion products. Formation of hydrous corrosion products is also possible, of course, but we omitted them and the potential reactant $\mathrm{H}_{2} \mathrm{O}$ to avoid confusion with $\mathrm{S}_{\mathrm{H}_{2} \mathrm{O}}$, the stoichiometric factor for water in the overall anoxic-corrosion reaction (see below). Table 7-2 lists the oxic-corrosion reactions and the oxic stoichiometric factors used in the corrosion submodel. The parameters pertaining to humid oxic corrosion in the data base include the designator HO; those pertaining to wet anoxic corrosion include WO. Therefore, for all of the materials in Table 7-2, IDPRAM (the parameter identifier) for $\mathrm{SO}_{2}$ (the stoichiometric factor for $\mathrm{O}_{2}$ consumption by corrosion of ASTM A516 Grade 60 steel under humid oxic conditions) is SO2HO.

The mine air trapped in the disposal tunnels when they are filled and sealed will contain about $20 \% \mathrm{O}_{2}$. Oxic corrosion will consume this $\mathrm{O}_{2}$ prior to significant corrosion of the waste packages. However, transport of $\mathrm{O}_{2}$ from the far field could result in additional oxic corrosion thereafter. The gaseous phase in the voids in the unsaturated zone surrounding the tunnels contains $\mathrm{O}_{2}$, which could diffuse into the near field at significant rates as oxic corrosion consumes $\mathrm{O}_{2}$ and creates a concentration gradient. Furthermore, advection of the gaseous phase could transport $\mathrm{O}_{2}$ at significant rates during the thermal period (the first few hundred years after waste emplacement, during which radioactive decay of fission products such as ${ }^{137} \mathrm{Cs}$ and ${ }^{90} \mathrm{Sr}$ increase the temperature of the near field significantly). Finally, groundwater containing dissolved $\mathrm{O}_{2}$ could also transport $\mathrm{O}_{2}$ and facilitate additional oxic corrosion. If the overall $\mathrm{O}_{2}$ transport rate is sufficient, oxic corrosion could proceed at relatively high rates well after the $\mathrm{O}_{2}$ initially present in the disposal tunnels is consumed. On the other hand, oxic corrosion could consume $\mathrm{O}_{2}$ faster than transport replenishes it, thus limiting the extent of oxic corrosion. 
Table 7-2. Humid and Wet Oxic Corrosion Reactions, and Stoichiometric Factors Used in CST for the 1997 PA Calculations

\begin{tabular}{|c|c|c|c|c|}
\hline Reaction & $\mathrm{SH}_{2} \mathrm{O}$ & $\mathrm{SO}_{2}$ & $S_{\text {rust }}$ & $S_{\text {gas }}{ }^{A}$ \\
\hline \multicolumn{5}{|c|}{ Disposal Container, Handling Container, Basket, and Cladding Materials: } \\
\hline \multicolumn{5}{|c|}{ ASTM A516 Grade 60 steel disposal container: ${ }^{B}$} \\
\hline $\mathrm{Fe}+0.75 \mathrm{O}_{2}=\mathrm{Fe}_{2} \mathrm{O}_{3}$ & 0 & -0.75 & 1 & 0 \\
\hline Inconel 625 disposal container: ${ }^{C}$ & & & & \\
\hline $\begin{array}{c}\mathrm{Ni}_{0.69} \mathrm{Cr}_{0.22} \mathrm{Mo}_{0.09}+0.09 \mathrm{PbO}+0.645 \mathrm{O}_{2}= \\
0.69 \mathrm{NiO}+0.11 \mathrm{Cr}_{2} \mathrm{O}_{3}+0.09 \mathrm{MoPbO}_{4}\end{array}$ & 0 & -0.645 & 0 & 0 \\
\hline \multicolumn{5}{|l|}{ 304L stainless steel handling container: ${ }^{D}$} \\
\hline $\begin{array}{l}\mathrm{Fe}_{0.7} \mathrm{Cr}_{0.2} \mathrm{Ni}_{0.1}+0.725 \mathrm{O}_{2} \\
\quad=0.35 \mathrm{Fe}_{2} \mathrm{O}_{3}+0.1 \mathrm{Cr}_{2} \mathrm{O}_{3}+0.1 \mathrm{NiO}\end{array}$ & 0 & -0.725 & 0.35 & 0 \\
\hline
\end{tabular}

\section{Borated-steel baskets: ${ }^{\mathrm{E}}$}

Assumed to have the same stoichiometry as stainless steel $304 \mathrm{~L}$, and to react at the same rate (deactivated for the 1997 PA calculations).

Aluminum cladding: $F$
$\mathrm{Al}+0.75 \mathrm{O}_{2}=0.5 \mathrm{Al}_{2} \mathrm{O}_{3}$
0
$-0.75$
0
0

BISO cladding: ${ }^{\mathrm{G}}$

Neither reaction and stoichiometric factors nor kinetic parameters included in the 1997 PA.

Graphite binder/moderator: ${ }^{H}$

$\mathrm{C}+\mathrm{O}_{2}=\mathrm{CO}_{2}$

0

$-1$

0

1

TRISO cladding: ${ }^{G}$

Reaction and stoichiometric factors not included in the 1997 PA, but kinetic parameters included.

Zircaloy 2 cladding:

$\mathrm{Zr}+\mathrm{O}_{2}=\mathrm{ZrO}_{2}$

0

$-1$

0

0

A. Stoichiometric factor for gaseous phase includes all gases except $\mathrm{H}_{2} \mathrm{O}$ vapor and $\mathrm{O}_{2}$.

B. Assumed to comprise 100\% Fe (see "ASTM A516 Grade 60 Steel Disposal Container" in Section 7.3.1 for actual composition).

C. Assumed to comprise $69 \% \mathrm{Ni}, 22 \% \mathrm{Cr}$, and $9 \% \mathrm{Mo}$ (see "Inconel 625 Disposal Container" in Section 7.3.1 for actual composition). This reaction is the weighted average of the reactions for each of its pure components: $\mathrm{Ni}+0.5 \mathrm{O}_{2}=\mathrm{NiO} ; \mathrm{Cr}+0.75 \mathrm{O}_{2}$ $=0.5 \mathrm{Cr}_{2} \mathrm{O}_{3} ;$ and $\mathrm{Mo}+\mathrm{PbO}+1.5 \mathrm{O}_{2}=\mathrm{MoPbO}_{4}$.

D. Assumed to comprise $70 \% \mathrm{Fe}, 20 \% \mathrm{Cr}$, and $10 \% \mathrm{Ni}$ (see "304L Stainless Steel Handling Containers" in Section 7.3 .1 for actual composition). This reaction is the weighted average of the reactions: $\mathrm{Fe}+0.75 \mathrm{O}_{2}=0.5 \mathrm{Fe}_{2} \mathrm{O}_{3} ; \mathrm{Cr}+0.75 \mathrm{O}_{2}=$ $0.5 \mathrm{Cr}_{2} \mathrm{O}_{3}$; and $\mathrm{Ni}+0.5 \mathrm{O}_{2}=\mathrm{NiO}$. Composition, reaction stoichiometry, and reaction rate for $316 \mathrm{~L}$ stainless steel assumed to be the same as those for $304 \mathrm{~L}$ stainless steel.

E. Composition, stoichiometry, and kinetic parameters assumed to be the same as those for $304 \mathrm{~L}$ stainless steel.

F. Assumed to comprise $100 \%$ Al (actual composition, if different, unavailable).

$G$. Not an acronym. Failed cladding consisting of two layers of pyrolytic $C$.

H. Used as the binder (and other structural members) and moderator for Fort St. Vrain and Peach Bottom uranium-thorium carbide spent fuel.

1. Not an acronym. Assumed failed (but actually intact or mainly intact) cladding consisting of an outer layer of pyrolytic $\mathrm{C}, \mathrm{a}$ middle layer of $\mathrm{SiC}$, and an inner layer of pyrolytic $\mathrm{C}$. The SiC layer significantly increases the corrosion resistance of TRISO relative to that of BISO. TRISO is the only cladding for which we took credit in the $1997 \mathrm{PA}$.

J. Assumed to comprise $100 \% \mathrm{Zr}$ (see "Zircaloy-2 Cladding" in Section 7.3 .1 for actual composition). 
Table 7-2. Humid and Wet Oxic Corrosion Reactions, and Stoichiometric Factors Used in CST for the 1997 PA Calculations (Continued)

\begin{tabular}{lcccc}
\hline \multicolumn{1}{c}{ Reaction } & $\mathrm{SH}_{2} \mathrm{O}$ & $\mathrm{SO}_{2}$ & $\mathrm{~S}_{\text {rust }}$ & $\mathrm{S}_{\text {gas }}{ }^{\mathrm{A}}$ \\
\hline & Fuel Materials: & & & \\
Category 1, metallic uranium: & & & & \\
$\mathrm{U}+2 \mathrm{H}_{2} \mathrm{O}+1.5 \mathrm{O}_{2}=\mathrm{UO}_{3}: 2 \mathrm{H}_{2} \mathrm{O}$ & -2 & -1.5 & 0 & 0 \\
Category 2, uranium-zirconium alloy: ${ }^{\mathrm{N}}$ & & & &
\end{tabular}

Assumed to have the same stoichiometry as Category 1 (U), and to react at the same rate (deactivated for the 1997 PA calculations).

Category 3, uranium-molybdenum alloy: ${ }^{M}$

Assumed to have the same stoichiometry as Category $1(\mathrm{U})$, but to react at ten times the rate.

Categories 4, 5, 14, and 15 , uranium dioxide: ${ }^{\mathrm{N}}$

$\mathrm{UO}_{2}+2 \mathrm{H}_{2} \mathrm{O}+0.5 \mathrm{O}_{2}=\mathrm{UO}_{3}: 2 \mathrm{H}_{2} \mathrm{O}$

$-2$

$-0.5$

0

0

Category 6 , uranium-aluminum alloy: ${ }^{\circ}$

Assumed to have the same stoichiometry as Category 1 (U), and to react at the same rate (deactivated for the 1997 PA calculations).

Category 7, uranium silicide alloy: $P$

Assumed to have the same stoichiometry as Category $1(\mathrm{U})$, but to react at one-tenth the rate.

Categories 8 and 9 , thorium-uranium carbide: ${ }^{Q}$

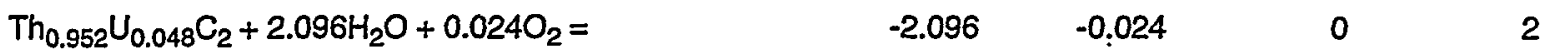
$0.952 \mathrm{ThO}_{2}+0.048 \mathrm{UO}_{3}: 2 \mathrm{H}_{2} \mathrm{O}+\mathrm{C}_{2} \mathrm{H}_{2}+\mathrm{H}_{2}$

Category 10, uranium-plutonium carbide: ${ }^{R}$

Assumed to have the same stoichiometry as Categories 8 and $9\left(T_{0.952} \mathrm{U}_{0.048} \mathrm{C}_{2}\right)$ and to react at the same rate (deactivated for the 1997 PA calculations).

Category 11, mixed oxide fuel: $\mathrm{S}$

Assumed to have the same stoichiometry as Categories $4,5,14$, and $15\left(\mathrm{UO}_{2}\right)$ and to react at the same rate (deactivated for the 1997 PA calculations).

K. N Reactor, failed Zircaloy-2 cladding.

L. Composition unavailable. Chicago Pile 5, assumed failed (but actually intact or mainly intact) Zircaloy-2 cladding.

M. Composition unavailable. Fermi Reactor, assumed failed (but actually intact) Zircaloy-2 cladding.

N. Category 4: Shippingport, assumed failed (but actually intact or mainly intact) Zircaloy-2 cladding; Category 5: Three-Mile Island Unit 2, failed Zircaloy-2 cladding; Category 14: commercial PWR fuel, assumed failed (but actually intact or mainly intact) Zircaloy-2 cladding; Category 15: commercial BWR fuel, assumed failed (but actually intact or mainly intact) Zircaloy2 cladding.

O. Composition unavailable. Advanced Test Reactor, failed aluminum cladding.

P. Composition unavailable. Materials Testing Reactor, assumed failed (but actually intact) aluminum cladding.

Q. Category 8: Fort St. Vrain, intact or mainly intact TRISO cladding (the only cladding for which we took credit in the 1997 PA; Category 9: Peach Bottom, assumed failed (but actually $65 \%$ intact) BISO cladding. Thorium-uranium carbide comprises 95.2\% $\mathrm{ThC}_{2}$ and $4.8 \% \cup \mathrm{UC}_{2}$. We did not have a formula for the thorium-uranium carbide from Peach Bottom at the time of the 1997 PA, so we assumed it has the same composition as that from Fort St. Vrain. This reaction is the weighted average of the reactions: $\mathrm{ThC}_{2}+2 \mathrm{H}_{2} \mathrm{O}=\mathrm{ThO}_{2}+\mathrm{C}_{2} \mathrm{H}_{2}+\mathrm{H}_{2}$ and $\mathrm{UC}_{2}+4 \mathrm{H}_{2} \mathrm{O}+0.5 \mathrm{O}_{2}=\mathrm{UO}_{3}: 2 \mathrm{H}_{2} \mathrm{O}+\mathrm{C}_{2} \mathrm{H}_{2}+\mathrm{H}_{2}$. Note that this fuel is labeled Th-U here because of its content; elsewhere in this report it is cited as U-Th, per Appendix A.

R. Composition unavailable. Sodium Reactor Experiment, assumed failed (but actually intact or mainly intact) stainless-steel cladding.

S. Assumed to comprise $\left(\mathrm{U}_{x} \mathrm{Pu}_{1-x}\right) \mathrm{O}_{2}$ (actual composition unavailable). Fast Flux Test Facility, assumed failed (but actually intact or mainly intact) stainless-steel cladding. 
Table 7-2. Humid and Wet Oxic Corrosion Reactions, and Stoichiometric Factors Used in CST for the 1997 PA Calculations (Continued)

\begin{tabular}{lcccc}
\hline \multicolumn{1}{c}{ Reaction } & $\mathrm{SH}_{2} \mathrm{O}$ & $\mathrm{SO}_{2}$ & $\mathrm{~S}_{\text {rust }}$ & $\mathrm{S}_{\text {gas }^{\mathrm{A}}}$ \\
\hline Category 12, thorium-uranium Qxide: & ${ }^{\mathrm{A}}$ & & & \\
$\mathrm{ThO}_{2, \text { solid }}=\mathrm{Th}(\mathrm{IV})$, aqueous & 0 & 0 & 0 & 0 \\
Category 13, uranium-zirconium hydride: $\mathrm{U}$ & & & &
\end{tabular}

Assumed to have the same stoichiometry as Category 1 (U), but to react at one-tenth the rate of Categories 4,5 , 14 , and $15\left(\mathrm{UO}_{2}\right)$.

Defense high-level waste:

Reaction and stoichiometric factors not included in the 1997 PA, but kinetic parameters included.

T. Assumed to comprise $\mathrm{ThO}_{2}$ (actual composition unavailable). Shippingport Light Water Breeder Reactor, assumed failed (but actually intact or mainly intact) Zircaloy-2 cladding. Thorium-uranium oxide assumed to comprise $100 \% \mathrm{ThO}_{2}$. Assumed instantaneous, equilibrium dissolution with no effect on the $\mathrm{H}_{2} \mathrm{O}$, gas, or $\mathrm{O}_{2}$ budget, and solubility-limited transport out of the waste package. Aqueous speciation of Th not considered.

U. Composition unavailable. General Atomic, Training, Research, and Production, assumed failed (but actually intact or mainly intact) stainless-steel cladding.

Therefore, the corrosion submodel also explicitly includes corrosion reactions in which the oxidant is $\mathrm{H}_{2} \mathrm{O}$ present as an aqueous phase (wet anoxic corrosion) but not as $\mathrm{H}_{2} \mathrm{O}$ vapor (humid anoxic corrosion). For wet anoxic corrosion, the overall reaction is:

$$
\text { metal (or oxide })+\left(\mathrm{S}_{\mathrm{H}_{2} \mathrm{O}}\right) \cdot \mathrm{H}_{2} \mathrm{O}=\left(\mathrm{S}_{\text {rust }}\right) \cdot \text { solid corrosion product }(\mathrm{s})+\left(\mathrm{S}_{\text {gas }}\right) \cdot \text { gas }(\mathrm{es}) \text {, }
$$

in which $\mathrm{S}_{\mathrm{H}_{2} \mathrm{O}}, \mathrm{S}_{\text {rust, }}$, and $\mathrm{S}_{\text {gas }}$ are the stoichiometric factors. In Reaction 7-2, metallic or nonmetallic constituents of the waste packages react with the oxygen contained in $\mathrm{H}_{2} \mathrm{O}$, thereby liberating gaseous $\mathrm{H}_{2}$ (see, for example, Haberman and Frydrych, 1988; Simpson and Schenk, 1989; Brush, 1990; Telander and Westerman, 1993; 1997). The corrosion submodel does not include humid anoxic corrosion because, based on results obtained by Telander and Westerman (1993; 1997) for brines, we assume that the concentration of $\mathrm{H}_{2} \mathrm{O}$ in the gaseous phase will probably be too low to oxidize waste package constituents at significant rates. Table 7-3 lists the anoxic-corrosion reactions and the anoxic stoichiometric factors used in the corrosion submodel. For rust, it uses the oxic stoichiometric factor because we assume that the anoxic, Fe-bearing corrosion products $\left(\mathrm{Fe}(\mathrm{OH})_{2}\right.$ and $\left.\mathrm{Fe}_{3} \mathrm{O}_{4}\right)$ will eventually oxidize to the oxic, $\mathrm{Fe}$-bearing corrosion product $\left(\mathrm{Fe}_{2} \mathrm{O}_{3}\right)$ due to transport of $\mathrm{O}_{2}$ into the near field (see above). The parameters pertaining to wet anoxic corrosion in the data base include the designator WA. Therefore, IDPRAM for $\mathrm{S}_{\text {gas }}$, the stoichiometric factor for gas $\left(\mathrm{H}_{2}\right.$, in this case) production by corrosion of ASTM A516 Grade 60 steel under wet anoxic conditions, is SGASWA.

\subsubsection{Corrosion Rates}

Rate Equations for General Corrosion. The corrosion submodel uses one equation, written slightly differently for different conditions, to predict the rates of most of the corrosion reactions in Tables 7-2 and 7-3. For the 1997 PA, the corrosion submodel used the following equations for humid oxic, wet oxic, and wet anoxic corrosion, respectively:

$$
\begin{gathered}
m_{i}=A \cdot e^{-B / T} \cdot\left(\left(t_{j+1}\right)^{C}-t_{j}^{C}\right) \cdot X O_{2} D \cdot S A \\
m_{i}=A \cdot e^{-B / T} \cdot\left(\left(t_{j}+1\right)^{C}-t_{j}{ }^{C}\right) \cdot X O_{2} D \cdot S D \cdot B P \cdot S A \\
m_{i}=A \cdot e^{-B / T} \cdot\left(\left(t_{j+1}\right)^{C}-t_{j}{ }^{C}\right) \cdot\left(1-5 \cdot X O_{2}\right) \cdot S D \cdot B P \cdot S A
\end{gathered}
$$


Table 7-3. Wet Anoxic Corrosion Reactions and Stoichiometric Factors Used in CST for the 1997 PA Calculations

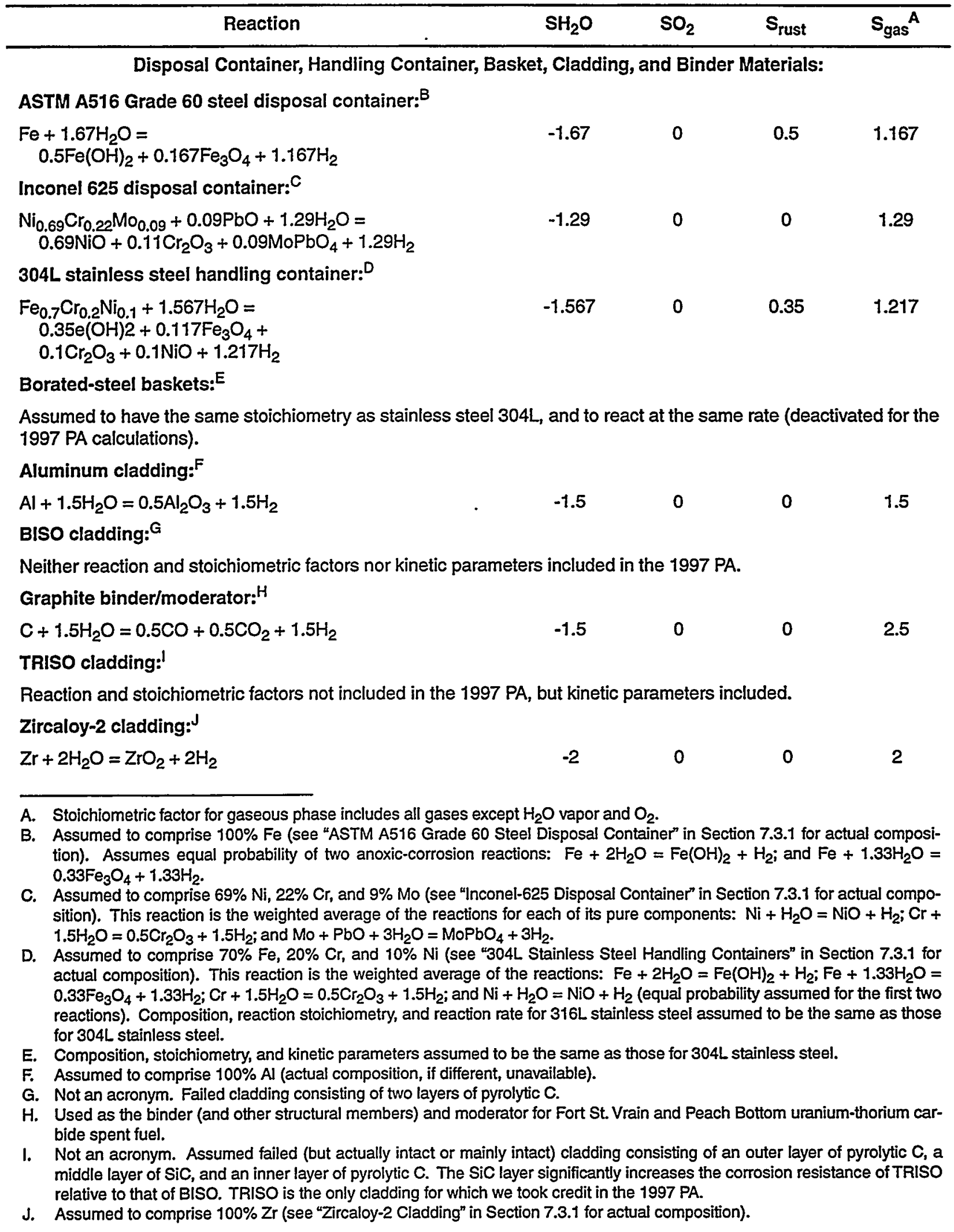


Table 7-3. Wet Anoxic Corrosion Reactions and Stoichiometric Factors Used in CST for the 1997 PA Calculations (Continued)

\begin{tabular}{llllll}
\hline Reaction & $\mathrm{SH}_{2} \mathrm{O}$ & $\mathrm{SO}_{2}$ & $\mathrm{~S}_{\text {rust }}$ & $\mathrm{S}_{\text {gas }}{ }^{A}$ \\
\hline
\end{tabular}

Fuel Materials:

Category 1, metallic uranium: ${ }^{\mathrm{K}}$

$\mathrm{U}+2 \mathrm{H}_{2} \mathrm{O}=\mathrm{UO}_{2}+2 \mathrm{H}_{2}$

0

2

Category 2, uranium-zirconium alloy: $\mathrm{L}$

Assumed to have the same stoichiometry as Category 1 (U), and to react at the same rate (deactivated for the 1997 PA calculations).

Category 3, uranium-molybdenum alloy: ${ }^{M}$

Assumed to have the same stoichiometry as Category $1(\mathrm{U})$, but to react at ten times the rate.

Categories $4,5,14$, and 15 , uranium dioxide: ${ }^{\mathrm{N}}$

$\mathrm{UO}_{2}+3 \mathrm{H}_{2} \mathrm{O}=\mathrm{UO}_{3}: 2 \mathrm{H}_{2} \mathrm{O}+\mathrm{H}_{2}$

$-3$

0

0

1

Category 6, uranium-aluminum alloy: ${ }^{\circ}$

Assumed to have the same stoichiometry as Category 1 (U), and to react at the same rate (deactivated for the 1997 PA calculations).

Category 7 , uranium silicide alloy: ${ }^{P}$

Assumed to have the same stoichiometry as Category $1(U)$, but to react at one-tenth the rate.

Categories 8 and 9 , thorium-uranium carbide: ${ }^{Q}$

$\mathrm{Th}_{0.952} \mathrm{U}_{0.048} \mathrm{C}_{2}+2 \mathrm{H}_{2} \mathrm{O}=$

$-2 \quad 0 \quad 0$

2

Th0.952U0.048 $\mathrm{O}_{2}+\mathrm{C}_{2} \mathrm{H}_{2}+\mathrm{H}_{2}$

Category 10, uranium-plutonium carbide: ${ }^{R}$

Assumed to have the same stoichiometry as Categories 8 and $9\left(T_{0.952} \mathrm{U}_{0.048} \mathrm{C}_{2}\right)$, and to react at the same rate (deactivated for the 1997 PA calculations).

Category 11, mixed oxide fuel: $\mathrm{S}$

Assumed to have the same stoichiometry as Categories $4,5,14$, and $15\left(\mathrm{UO}_{2}\right)$, and to react at the same rate (deactivated for the 1997 PA calculations).

K. N Reactor, failed Zircaloy-2 cladding.

L. Composition unavailable. Chicago Pile 5, assumed failed (but actually intact or mainly intact) Zircaloy-2 cladding.

M. Composition unavailable. Fermi Reactor, assumed failed (but actually intact or mainly intact) Zircaloy-2 cladding.

N. Category 4: Shippingport, assumed failed (but actually intact or mainly intact) Zircaloy-2 cladding; Category 5: Three-Mile Island Unit 2, failed Zircaloy-2 cladding; Category 14: commercial PWR fuel, assumed failed (but actually intact or mainly intact) Zircaloy-2 cladding; Category 15: commercial BWR fuel, assumed failed (but actually intact or mainly intact) Zircaloy2 cladding.

O. Composition unavailable. Advanced Test Reactor, failed aluminum cladding.

P. Composition unavailable. Materials Testing Reactor, assumed failed (but actually intact or mainly intact) aluminum cladding.

Q. Category 8: Fort St. Vrain, intact or mainly intact TRISO cladding (the only cladding for which we took credit in the 1997 PA; Category 9: Peach Bottom, assumed failed (but actually $65 \%$ intact) BisO cladding. Thorium-uranium carbide comprises 95.2\% $\mathrm{ThC}_{2}$ and $4.8 \% \mathrm{UC}_{2}$. We did not have a formula for the thorium-uranium carbide from Peach Bottom at the time of the $1997 \mathrm{PA}$, so we assumed it has the same composition as that from Fort St. Vrain. This reaction is the weighted average of the reactions: $\mathrm{ThC}_{2}+2 \mathrm{H}_{2} \mathrm{O}=\mathrm{ThO}_{2}+\mathrm{C}_{2} \mathrm{H}_{2}+\mathrm{H}_{2}$ and $\mathrm{UC}_{2}+4 \mathrm{H}_{2} \mathrm{O}+0.5 \mathrm{O}_{2}=\mathrm{UO}_{3}: 2 \mathrm{H}_{2} \mathrm{O}+\mathrm{C}_{2} \mathrm{H}_{2}+\mathrm{H}_{2}$. Note that this fuel is labeled Th-U here because of its content; elsewhere in this report it is cited as $U-T h$, per Appendix A.

R. Composition unavailable. Sodium Reactor Experiment, assumed failed (but actually intact or mainly intact) stainless-steel cladding.

S. Assumed to comprise $\left(\mathrm{U}_{\mathrm{x}} \mathrm{Pu}_{1-\mathrm{x}}\right) \mathrm{O}_{2}$ (actual composition unavailable). Fast Flux Test Facility, assumed failed (but actually intact or mainly intact) stainless-steel cladding. 
Table 7-3. Wet Anoxic Corrosion Reactions and Stoichiometric Factors Used in CST for the 1997 PA Calculations (Continued)

\begin{tabular}{lcccc}
\multicolumn{1}{c}{ Reaction } & $\mathrm{SH}_{2} \mathrm{O}$ & $\mathrm{SO}_{2}$ & $\mathrm{~S}_{\text {rust }}$ & $\mathrm{S}_{\text {gas }}{ }^{A}$ \\
\hline Category 12, thorium-uranium oxide: $^{\mathrm{T}}$ & & & & \\
$\mathrm{ThO}_{2}$, solid $=\mathrm{Th}(\mathrm{IV})_{\text {aqueous }}$ & 0 & 0 & 0 & 0 \\
Category 13, uranium-zirconium hydride: & & & &
\end{tabular}

Assumed to have the same stoichiometry as Category $1(\mathrm{U})$, but to react at one-tenth the rate of Waste Categories $4,5,14$, and $15\left(\mathrm{UO}_{2}\right)$.

Defense high-level waste:

Reaction and stoichiometric factors not included in the 1997 PA, but kinetic parameters included.

T. Assumed to comprise $\mathrm{ThO}_{2}$ (actual composition unavailable). Shippingport Light Water Breeder Reactor, assumed failed (but actually intact or mainly intact) Zircaloy-2 cladding. Thorium-uranium oxide assumed to comprise $100 \% \mathrm{ThO}_{2}$. Assumed instantaneous, equilibrium dissolution with no effect on the $\mathrm{H}_{2} \mathrm{O}$, gas, or $\mathrm{O}_{2}$ budget, and solubility-limited transport out of the waste package. Aqueous speciation of Th not considered

U. Composition unavailable. General Atomic, Training, Research, and Production, assumed failed (but actually intact or mainly intact) stainless-steel cladding.

In these equations, $m_{i}$ is the mass (in $\mathrm{kg}$ ) of layer $\mathrm{i}$ corroded in each time step; $\mathrm{A}$ is the pre-exponential parameter $\left(\mathrm{kg} / \mathrm{m}^{2} \cdot \mathrm{s}\right)$ in the familiar Arrhenius function used to predict the effects of temperature on reaction rates; B is the Arrhenius activation-energy parameter $(\mathrm{K}) ; \mathrm{T}$ is the temperature $(\mathrm{K}) ; \mathrm{t}_{j}$ is the time $(\mathrm{s})$ at the start of time step $j ; \mathrm{t}_{j+1}$ is the time at the start of the next time step; $C$ is a dimensionless time-dependence parameter; $X$ is the dimensionless mole fraction of $\mathrm{O}_{2}$ in the gaseous phase; $\mathrm{D}$ is a dimensionless $\mathrm{O}_{2}$-dependence parameter; $\mathrm{SD}$ is a dimensionless $\mathrm{H}_{2} \mathrm{O}$-saturation-dependence function; $\mathrm{BP}$ is a dimensionless term used to prevent numerical instability in BRAGFLO_T near the boiling point of $\mathrm{H}_{2} \mathrm{O}$; and $\mathrm{SA}$ is the surface area $\left(\mathrm{m}^{2}\right)$. The corrosion submodel used Equation 7-3a, 7-3b, or 7-3c when CORMOD was set to 0 . For those materials that corrode according to Equation 7-3a, 7-3b, or 7-3c, IDPRAM for A is COR_A_HO; IDPRAM for B is COR_B_WO; IDPRAM for C is COR_C_WA; etc.

Arrhenius Function. For those materials for which experimental data on the effects of temperature on corrosion were available, we used Excel 7.0 to fit the parameters $A$ and $B$ to the data. First, we used the equation

$$
B=\left[\left(T_{1} \cdot T_{2}\right) /\left(T_{2}-T_{1}\right)\right] \cdot \ln \left(m_{1} / m_{2}\right)
$$

to solve for $B$ : In this equation, $m_{1}$ and $m_{2}$ are the corrosion rates measured at temperatures $T_{1}$ and $T_{2}$, respectively, and $\mathrm{ln}$ is the natural logarithm. We then used the equation

$$
\mathrm{A}=\mathrm{m} / \mathrm{e}^{\mathrm{B} / \mathrm{T}}
$$

to solve for $\mathrm{A}$. In this equation, $\mathrm{m}$ is the corrosion rate measured at temperature $\mathrm{T}$.

If there are no data on the effects of temperature, we set $B$ equal to 0 and the exponential term assumed a value of unity.

The corrosion submodel obtains the temperature for each waste grid block from $\underline{B}$ rine and $\underline{\text { Gas }}$ Elow - Thermal (BRAGFLO_T) prior to each time step, or reads the temperature for each waste package from a temperature-versustime file from Conduction and Radiation (CONRAD). (The temperatures obtained from BRAGFLO_T are averages for each grid block. Therefore, these temperatures are lower than those predicted by CONRAD for the waste packages.) For the $1997 \mathrm{PA}$, the corrosion submodel obtained temperatures from CONRAD.

Time-Dependence Function. The rates of many corrosion reactions decrease as the corrosion products thicken and retard the transport of reactants and products to and from the corroding surfaces. Most rate equations, however, typically express this decrease as a parabolic or cubic function of time, not thickness or some other measure of reac- 
tion progress. For the 1997 PA, we set COR_C_HO, COR_C_WO, and COR_C_WA for all materials equal to 1. Therefore, all of the corrosion rates were independent of time. Because many of these rates would actually decrease as corrosion proceeds, this was probably conservative. For future calculations, we might express these decreases as a function of reaction progress.

$\mathrm{O}_{2}$-Dependence Function. The rates of humid oxic and wet oxic corrosion reactions might decrease if the $\mathrm{O}_{2}$ content of the near field decreases (see Section 7.1.5, Corrosion Reactions). For the 1997 PA, the corrosion submodel used $\mathrm{X}_{\mathrm{O}_{2}}^{\mathrm{D}}$, in which $\mathrm{X}$ is the mole fraction of $\mathrm{O}_{2}$ in the gaseous phase and $\mathrm{D}$ is an experimentally obtained parameter, to predict the effects of $\mathrm{O}_{2}$ concentration on oxic corrosion rates. For those materials for which experimental data on the effects of $\mathrm{O}_{2}$ on corrosion were available, we used Excel 7.0 to fit the parameter $\mathrm{D}$ to the data. If there were no data on the effects of $\mathrm{O}_{2}$, we set $\mathrm{D}$ equal to 0 and the $\mathrm{O}_{2}$-dependence term assumed a value of unity.

$\mathrm{H}_{2} \mathrm{O}$-Saturation-Dependence Function. The corrosion submodel uses the term SD to incorporate the effects of the $\mathrm{H}_{2} \mathrm{O}$ content of fractures in intact host rock and rubble on wet oxic and wet anoxic corrosion (Equations 7-3b and 7-3c). The user specifies the form of this function by selecting a value of 1,2 , or 3 for IBRE (integers required to select Breach Flag).

If IBRE is set to 1 , all of the layers in the waste packages fail at the time of emplacement $(t=0)$.

If IBRE is 2, the corrosion submodel uses the following equation to gradually initiate wet oxic or wet anoxic corrosion as the $\mathrm{H}_{2} \mathrm{O}$ content of fractures increases above the threshold required for dripping or capillarity:

$$
S D=1-e^{(-\alpha \cdot(S a-S t))} \text {. }
$$

In this equation, $\alpha$ is $\ln \left(0.01 /\left(S_{99}-S_{t}\right)\right)$, in which $S_{99}$ is the dimensionless fracture $\mathrm{H}_{2} \mathrm{O}$ content at which the wet oxic or wet anoxic corrosion rate is $99 \%$ of its value at $100 \%$ saturation; $\mathrm{S}_{\mathrm{t}}$ is the threshold fracture $\mathrm{H}_{2} \mathrm{O}$ saturation (the dimensionless $\mathrm{H}_{2} \mathrm{O}$ content required to initiate fracture flow); and $\mathrm{S}_{\mathrm{a}}$ is the actual fracture $\mathrm{H}_{2} \mathrm{O}$ saturation (the actual dimensionless $\mathrm{H}_{2} \mathrm{O}$ content obtained from BRAGFLO_T). Stockman et al. (1995) attempted to use a step function to initiate wet oxic or wet anoxic corrosion when the $\mathrm{H}_{2} \mathrm{O}$ content of the fractures is sufficient for dripping or capillarity. Because the step function caused numerical instability in BRAGFLO_T, they added this exponential equation to the corrosion submodel.

If IBRE is 3, the corrosion submodel used the linear equation:

$$
S D=\left(S_{a}-S_{t}\right) /\left(S_{f}-S_{t}\right)
$$

in which $\mathrm{S}_{\mathrm{f}}$ is the full fracture $\mathrm{H}_{2} \mathrm{O}$ saturation (the dimensionless $\mathrm{H}_{2} \mathrm{O}$ content above which additional $\mathrm{H}_{2} \mathrm{O}$ will not in and of itself increase fracture flow).

For the $1997 \mathrm{PA}$, we used Equation 7-7 for SD in all cases for which Equations 7-3b or 7-3c were used to predict wet oxic or wet anoxic corrosion rates. BRAGFLO_T used a constant (non-sampled) value of 0.04 for $S_{p}$ the residual fracture $\mathrm{H}_{2} \mathrm{O}$ saturation (the dimensionless $\mathrm{H}_{2} \mathrm{O}$ content below which $\mathrm{H}_{2} \mathrm{O}$ will not drain from the fractures). For the container groups with an air gap, we set TSA (the IDPRAM for $S_{t}$ for these container groups) to the value of $S_{p}$ or 0.04 ; and we set FSA (the IDPRAM for $S_{f}$ ) to $2 \cdot S_{5}$ or 0.08 . For the container groups with rubble, we set TSB (the IDPRAM for $S_{t}$ for these container groups) to 0.00 ; and we set FSB (the IDPRAM for $S_{f}$ ) to $2 \cdot S_{p}$ or 0.08 . Figure 7-2 plots Equation 7-7 for the values of $S_{p} S_{t}$, and $S_{f}$ used in the 1997 PA.

The corrosion submodel uses the function $\left(\left(105^{\circ} \mathrm{C}-\mathrm{T}\right) / 5\right)$, in which $\mathrm{T}$ is the temperature (in $\left.{ }^{\circ} \mathrm{C}\right)$, for the term $\mathrm{BP}$ in Equations 7-3b and 7-3c when $100 \# \mathrm{~T} \# 105^{\circ} \mathrm{C}$. For $\mathrm{T}<100^{\circ} \mathrm{C}, \mathrm{BP}=1$; and for $\mathrm{T}>105^{\circ} \mathrm{C}, \mathrm{BP}=0$. This function gradually reduce the rates of wet oxic and wet anoxic corrosion from their full values to zero at or close to the boiling point of $\mathrm{H}_{2} \mathrm{O}$. Previous experience had shown that use of a step function prevented $\mathrm{BRAGFLO} T$ from converging.

Surface-Area Term. For the 545 waste packages containing thorium-uranium carbide spent fuel $\left(\mathrm{Th}_{0.952} \mathrm{U}_{0.048} \mathrm{C}_{2}\right)$ from the Fort St. Vrain reactor (Category 8 ), the 103 waste packages containing thorium-uranium carbide spent fuel (composition unspecified) from the Peach Bottom reactor (Category 9), and the 5 waste packages 
7.1 Corrosion Submodel

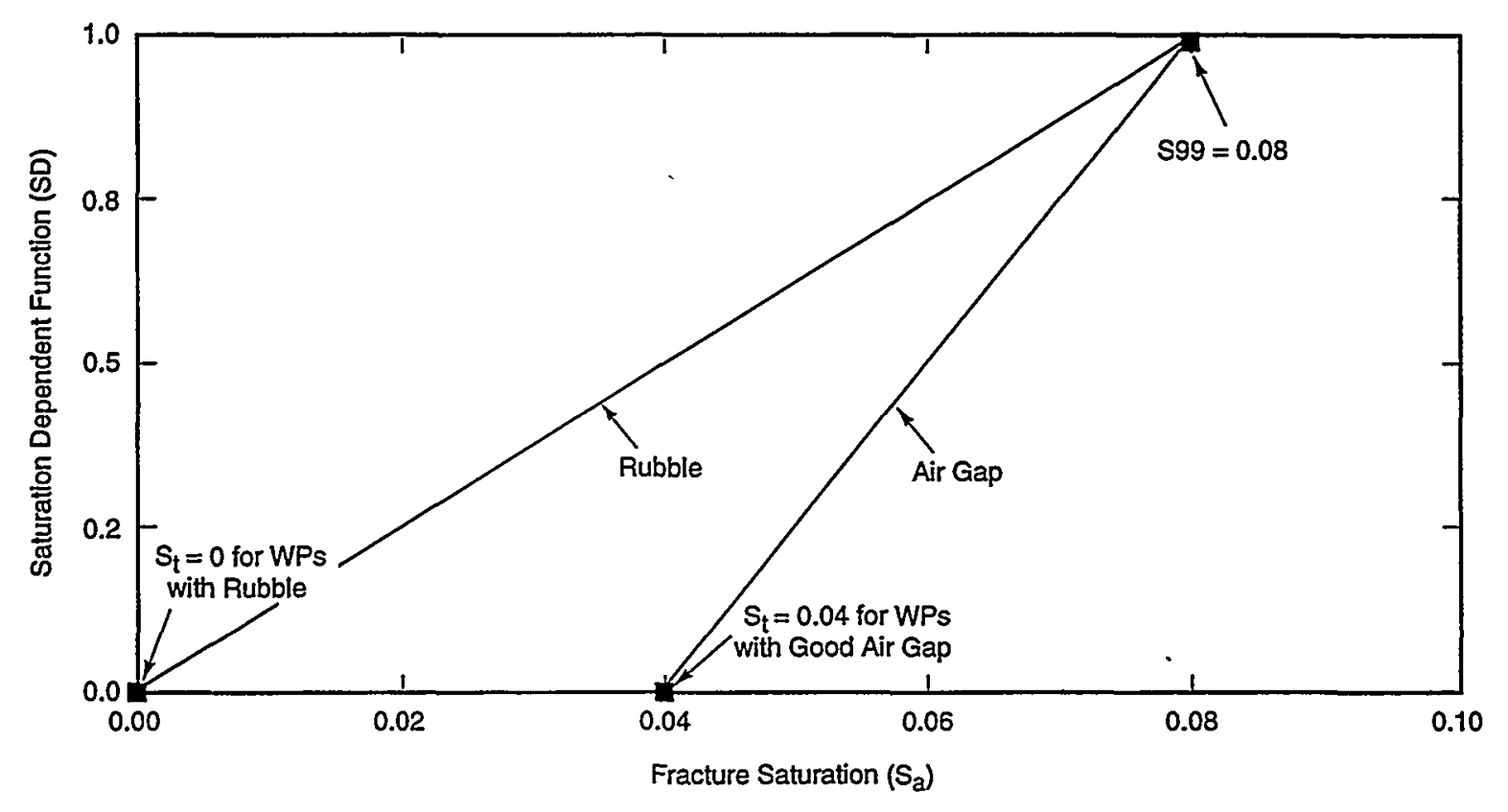

TRI-6342-5091-1

Figure 7-2. Step function equation plotted with values for waste packages with and without an intact air gap. 


\section{Consequence Modeling: Corrosion and Source-Term Model}

containing uranium-plutonium carbide spent fuel (composition unspecified) from the Sodium Reactor Experiment (Category 10), we could not obtain estimates of the surface areas of the spent fuels from personnel at INEEL for the 1997 PA. Furthermore, we could not obtain an estimate of the surface area of the TRISO cladding from INEEL personnel. Because Equations 7-3a, 7-3b, and 7-3c all include the surface-area term SA $\left(\mathrm{m}^{2}\right)$, we assumed that the surface area of these materials was $1 \mathrm{~m}^{2}$ per waste package and deactivated Equation 2 of Stockman et al. (1995), the corrosion equation used for the 1994 PA when surfaces areas were unknown and CORMOD was set to 1.

Solubility-Limited Dissolution. For the 69 waste packages containing Shippingport Light Water Breeder Reactor uranium-thorium oxide spent fuel (Category 12), we assumed instantaneous solubility equilibrium between $\mathrm{ThO}_{2}$ in the fuel and dissolved Th(IV) species. (CST does not predict the aqueous speciation of any of the radionuclides in the waste.) Because we could not obtain the composition of this material for the 1997 PA from INEEL personnel, we assumed that it was pure $\mathrm{ThO}_{2}$. Because $\mathrm{ThO}_{2}$, a Th(IV)-bearing solid, will dissolve to form aqueous Th(IV) species, this reaction has no effect on the near-field $\mathrm{H}_{2}$ nor $\mathrm{O}_{2}$ contents. Furthermore, we assumed that dissolution of $\mathrm{ThO}_{2}$ will not affect the near-field $\mathrm{H}_{2} \mathrm{O}$ content. Finally, we assumed solubility-limited advective and/or diffusive transport of ${ }^{229} \mathrm{Th},{ }^{230} \mathrm{Th}$, and ${ }^{232} \mathrm{Th}$ out of these waste packages. The corrosion submodel assumes instantaneous solubility equilibrium and solubility-limited transport out of these waste packages when CORMOD is set to 2. (Actually, the source-term submodel, not the corrosion model, predicts solubility-limited dissolution; see Section 7.2. This discussion appears here for the sake of completeness.)

Lamont's Equation. Finally, the corrosion submodel includes Lamont's equation,

$$
\mathrm{M}=\left(\mathrm{AT}^{2}+\mathrm{BT}+\mathrm{C}\right) \mathrm{SA},
$$

to predict the corrosion rate of ASTM A516 Grade 60 steel. In Equation 7-8, M is the mass (in $\mathrm{kg}$ ) of a layer corroded in each time step; $A, B$, and $C$ are parameters obtained by fitting a quadratic equation to temperature-dependent corrosion data, and $\mathrm{T}$ is the temperature. Andrews et al. (1994) obtained this equation from A. Lamont during an expert elicitation on corrosion for INTERA, Inc.'s 1993 PA of the potential Yucca Mountain repository (Andrews et al., 1994). We have never used Lamont's equation for any of the DOE SNF/DHLW PA calculations. The CORMIOD setting for this equation is 2 .

Calculation of Overall Rates. For any material, the corrosion submodel can select the maximum corrosion rate calculated for wet anoxic, humid oxic, and wet oxic conditions to predict the failure time of each layer of the waste packages. (The corrosion submodel provides these failure times to the source-term submodel.) The corrosion submodel can select the $\mathrm{H}_{2} \mathrm{O}$, gas, and $\mathrm{O}_{2}$ consumption and production rates calculated for the fastest corrosion reactions to predict the overall consumption and production rates. Stockman et al. (1995) used this approach for the 1994 PA.

For the $1997 \mathrm{PA}$, the corrosion submodel added the corrosion rates calculated for wet anoxic, humid oxic, and wet oxic conditions to predict failure times. However, the equations described in "Rate Equations for General Corrosion" include terms, such as BP in Equations 7-3b and 7-3c, and $\left(1-5 \cdot \mathrm{X}_{\mathrm{O}_{2}}\right)$ in Equation 7-3c, that in some cases significantly reduce these rates or set them cqual to 0 when conditions differ from those for which they are predicted. The corrosion submodel added the $\mathrm{H}_{2} \mathrm{O}$, gas, and $\mathrm{O}_{2}$ consumption and production rates for wet anoxic, humid oxic, and wet oxic corrosion, subject to any terms that reduce or eliminate them, to predict the effects of corrosion on the gas and water contents of the near field.

In either case (maximum rate or sum of the rates), the corrosion submodel calculates the mass of material corroded from each layer exposed to the near-ficld environment, then calculates the quantities of $\mathrm{O}_{2}, \mathrm{H}_{2} \mathrm{O}$, and gas consumed or produced by the corrosion of each of these layers (see Section 7.1.3, Container Groups, for the order of nesting). It adds the $\mathrm{O}_{2}, \mathrm{H}_{2} \mathrm{O}$, and gas consumed or produced from all of the corroding layers in each waste grid block, and divides the sums by the length of the time step. It then passes the consumption or production rates for each waste grid block to BRAGFLO_T. The corrosion submodel also calculates the mass of $\mathrm{Fe}_{2} \mathrm{O}_{3}$ and provides it to the source-term submodel for predictions of radionuclide sorption. 


\subsection{Source-Term Submodel}

The source-term submodel of CST predicts: (1) radioactive decay and ingrowth of both transported and nontransported radionuclides; (2) the effects of dissolution-precipitation and sorption-desorption reactions on the distribution of radionuclides among aqueous and solid phases within the waste packages; and (3) advective and diffusive transport of radionuclides from the waste packages to the intact host rock. After corrosion of the fuel materials (see Section 7.1), these chemical and transport processes will move radionuclides to new locations, such as groundwater, the lattices of solids formed by the corrosion of spent fuels, and sorption sites on $\mathrm{Fe}_{2} \mathrm{O}_{3}$, both within the waste packages and outside of the waste packages but within the disposal tunnels. Because the repository designs considered for the 1993 PA included backfill between the waste packages and the intact host rock, Stockman (1993) and Stockman et al. (1995) referred to the waste packages and thcir immediate surroundings, including any backfill or air gap, as waste emplacement packages (WEPs). In this report, we refer to them simply as waste packages.

Stockman et al. (1995) embedded the corrosion submodel GASSNF in the source-term submodel USATCONC when they rewrote the source-term submodel for the 1994 PA. (They rewrote the source term model because they could not use CONCSNF, developed by Stockman, 1993, for the $\mathrm{H}_{2} \mathrm{O}$ - or brine-saturated conditions anticipated for the granitic or bedded-salt repository considered for the $1993 \mathrm{PA}$, for the unsaturated tuff repository considered in 1994.) Stockman et al. (1995) embedded the corrosion submodel in the source-term submodel because: (1) the corrosion routines run so quickly that executing them within the source-term submodel is almost as fast as running them separately and reading the failure times of the layers of the waste packages and the masses of $\mathrm{Fe}_{2} \mathrm{O}_{3}$ produced from a transfer file; (2) gas consumption or production will not significantly affect the flow of $\mathrm{H}_{2} \mathrm{O}$ within the disposal tunnels, inclusion of the corrosion routines in the source-term submodel allows the user to vary the corrosion parameters without having to re-run the source-term calculations; and (3) the standalone version of the source-term model is more complete (essentially equivalent to CST) with the corrosion routines, thus facilitating sensitivity studies.

\subsubsection{Radioactive Decay and Ingrowth}

The source-term submodel predicts the radioactive decay and ingrowth of the radioisotopes specified by the user. For the 1997 PA, we selected 41 of the radioisotopes in the waste inventory (see Appendix A) for the source-term calculations. However, we selected only three of these radioisotopes for Nuclide Transport Systems (NUTS), the model used to predict radionuclide transport from the disposal tunnels to the underlying water table. Table 3-5 in Chapter 3 lists the radionuclides included in the heat-production calculations, the source-term submodel, and NUTS. We selected only three radioisotopes for the transport calculations because (1) the virtual memory of the computers used for the 1997 PA limited the number of radioisotopes that could be included when the source-term submodel was run in NUTS; (2) the regulations for the potential Yucca Mountain repository do not specify release limits for short-lived radioisotopes, which do not accumulate in large quantities; and (3) previous PA calculations had shown that many of the radioisotopes in the waste inventory did not significantly affect the overall performance of the repository because, for example, they are present in small quantitics at the time of emplacement, have low solubilities, or have high distribution coefficients $\left(\mathrm{K}_{\mathrm{D}} \mathrm{s}\right)$, etc. (see Andrews et al., 1994; Wilson et al., 1994; Rechard, ed., 1995; M\&O, 1995a).

NUTS predicts only the radioactive decay and ingrowth of the three transported radioisotopes. However, some of the non-transported radionuclides included in the heat-production and source-term calculations decay to transported daughters. For example ${ }^{241} \mathrm{Am}$, a non-transported radionuclide, decays to ${ }^{237} \mathrm{~Np}$, a transported radionuclide. Furthermore, ${ }^{237} \mathrm{~Np}$ can significantly affcct the overall performance of the potential Yucca Mountain repository. Therefore, the source-term submodel predicts the decay and ingrowth of non-transported radionuclides both before and after they are released from the waste packages. In particular, the source-term submodel predicts the post-release decay of non-transported radionuclides within each waste grid block, and provides to NUTS the transported daughters produced during each time step. This minimizes the error caused by transporting only a subset of the radionuclides used for the heat-production and sourcc-term calculations. 


\subsubsection{Distribution of Radioisotopes within the Waste Packages}

The source-term submodel also predicts the effects of dissolution and precipitation, and sorption and desorption on the distribution of radioisotopes within the disposal tunnels.

Locations of Radioisotopes. The source-term submodel includes four types of aqueous and solid phases among which radioisotopes will be distributed alicr corrosion exposes spent fuels and borosilicate glass to the near-field environment (Figure 7-3): (1) matrices (mostly spent fuels and borosilicate glass); (2) unbound solids produced by corrosion of spent fuels and borosilicate glass: (3) groundwater (including dissolved but not colloidal radionuclides); and (4) $\mathrm{Fe}_{2} \mathrm{O}_{3}$.

Matrices. The source-term submodel assumes that, at the time of emplacement, most of the radioisotopes in the waste will reside in matrices such as spent fucls and borosilicate glass. These bound radioisotopes will be homogeneously distributed in these materials at that timc. When the protective layers surrounding the spent fuels and borosilicate glass fail, exposing these materials to the near-field environment, the bound radioisotopes will move from these matrices to the unbound solids at the same rates as the matrices corrode (see Section 7.1.6, Corrosion Rates).

On the other hand, some of the radioisotopes, referred to as the quick-release fraction, will reside on grain boundaries in the spent fuels, or in the gap between the spent fuels and the cladding at the time of emplacement. The source-term submodel assumes that, when the layers surrounding the spent fuels fail, the quick-release fraction will immediately move to the unbound solids.

For the $1997 \mathrm{PA}$, we numbered the matrices as shown in Table 7-4. Note that the waste package identification numbers used by the corrosion submodel arc identical to the fuel categories described in Chapter 3. We included the graphite binder/moderator as Matrix 2 for Calegories 8 and 9 because about $60 \%$ of the ${ }^{14} \mathrm{C}$ in the waste inventory will reside in this matrix at the time of emplacement. We included the borated-steel baskets as Matrix 1 for Categories 14 and 15 to predict the release of $B$ for the criticality calculations.

Unbound Solids. After the source-term submodel transfers radionuclides from the grain boundaries in the spent fuels, the gap between the spent fuels and thc cladding, or the spent-fuel and borosilicate-glass matrices to the unbound solids, these radionuclides will cquilibrate among the unbound solids, the groundwater in each waste package, and the $\mathrm{Fe}_{2} \mathrm{O}_{3}$ formed by oxic corrosion of metallic $\mathrm{Fe}$ in each waste package. The source-term submodel assumes instantaneous, reversible solubility cquilibria between radioisotopes in the unbound solids and the groundwater in each waste package, and instantancous, reversible, linear sorption equilibria between radioisotopes in the groundwater and on $\mathrm{Fe}_{2} \mathrm{O}_{3}$ (see "Dissolution-Precipitation and Sorption-Desorption Reactions" in this section).

Groundwater. Predictions of the distribution of radioisotopes among the unbound solids, the groundwater, and the $\mathrm{Fe}_{2} \mathrm{O}_{3}$ in each waste package depend, among other factors, on the total volume of groundwater (V) that will contact the unbound solids in each waste package in a time step. To obtain V, the source-term submodel adds the volume of residual groundwater $\left(\mathrm{V}_{\text {res }}\right)$ and the volume of groundwater that flows through a waste package in a time step $\left(\mathrm{V}_{\text {flow }}\right)$. We assume that residual groundwalcr is always present in the waste packages after they fail so we can predict diffusive transport out of the waste packagcs when the flow rate is zero. For the 1997 PA, we assumed that VOLRES, the user-input name for $V_{\text {res }}$, was $10 \mathrm{ml}$, and used the following equation to calculate $V_{\text {flow: }}$ :

$$
V_{\text {flow }}=Q_{c i c} \cdot \operatorname{CSA} \cdot 10^{E C A} \cdot\left(\left(t_{j+1}\right)-t_{j}\right)
$$

In this equation, $Q_{e c}$ (obtained from BRAGFLO_T at the start of each time step) is the volumetric flow rate for the fractures and the matrix in each waste grid block divided by the cross-sectional area of that grid block (in m/s); CSA is the cross-sectional area of a waste packagc: ECA is the effective-collection-area term ( $\mathrm{m}^{2}$ ) (see below); $t_{j}$ is the time at the start of time step $j$, and $t_{j+1}$ is thc lime at the start of the next time step. We used Equation 7-9 for waste packages with an air gap and for those with rubble. 


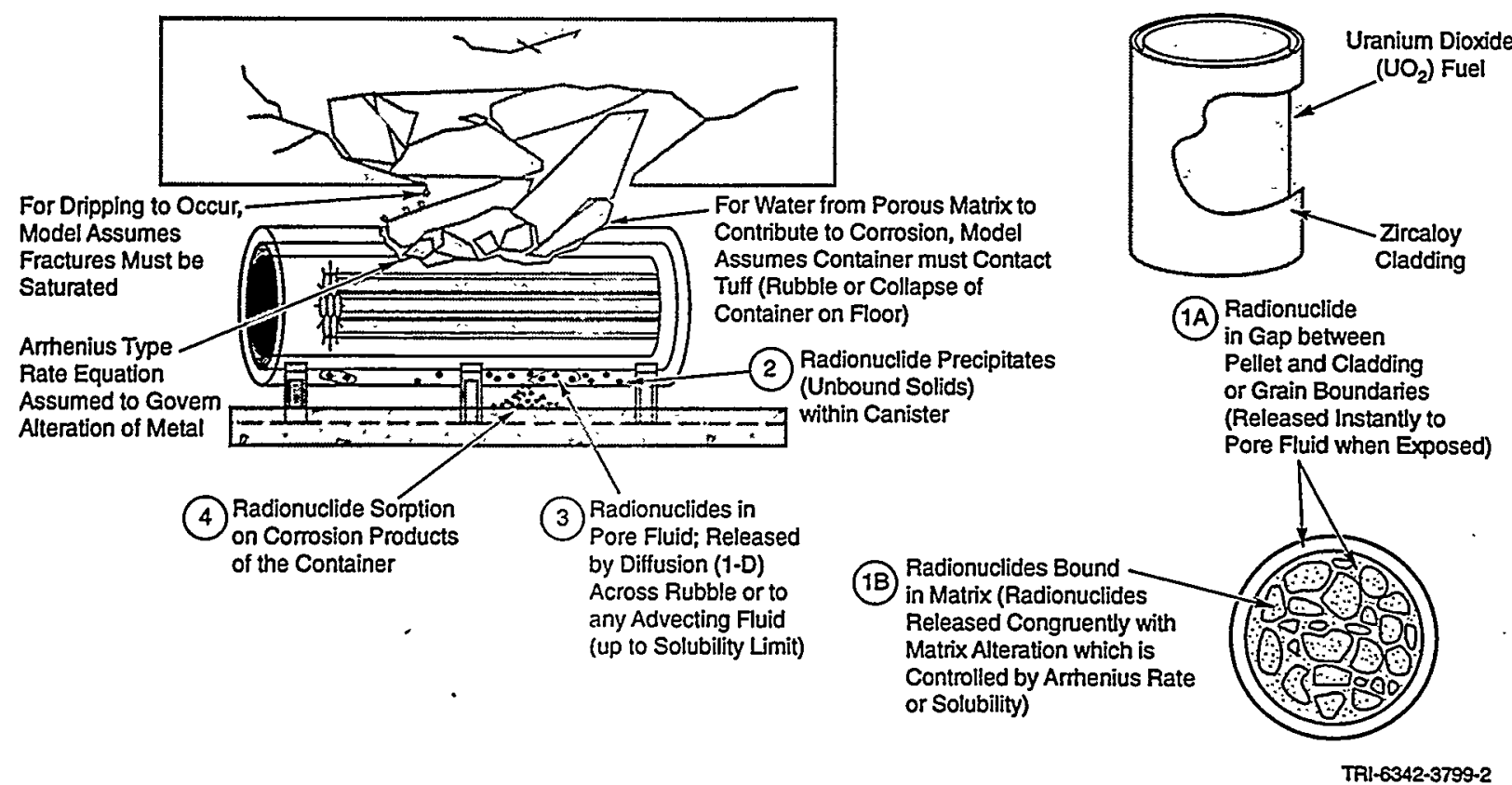

Figure 7-3. Locations of radioisotopes in waste package. The source-term submodel monitors these locations as protective layers are breached. 
Table 7-4. Matrix Identification Numbers Specified for the 1997 PA

\begin{tabular}{cllc}
\hline $\begin{array}{c}\text { Waste Package } \\
\text { ID Number }\end{array}$ & \multicolumn{1}{c}{ Matrix 1 } & \multicolumn{1}{c}{ Matrix 2 } & Matrix 3 \\
\hline WP01 & Uranium metal & \multicolumn{1}{c}{ - } & - \\
WP02 & Borosilicate glass & Uranium-zirconium & - \\
WP03 & Borosilicate glass & Uranium-molybdenum & - \\
WP04 & Borosilicate glass & Uranium dioxide & - \\
WP05 & Borosilicate glass & Uranium dioxide & - \\
WP06 & Borosilicate glass & Uranium-aluminum & - \\
WP07 & Borosilicate glass & Uranium silicide & - \\
WP08 & Borosilicate glass & Graphite & Uranium-thorium carbide \\
WP09 & Borosilicate glass & Graphite & Uranium-thorium carbide \\
WP10 & Borosilicate glass & Uranium-plutonium carbide & - \\
WP11 & Borosilicate glass & Mixed oxide fuel & - \\
WP12 & Borosilicate glass & Uranium-thorium oxide & - \\
WP13 & Borosilicate glass & Uranium-zirconium hydride & - \\
WP14 & Borated-steel & Uranium dioxide & - \\
WP15 & Borated-steel & Uranium dioxide & - \\
WP16 & Borosilicate glass & & - \\
\hline
\end{tabular}

Equation 7-9 probably overestimated groundwater-flow rates or the waste packages in the 1997 PA. For waste packages with an air gap, dripping is the mode of groundwater contact (see Section 7.1.2, Contact with Groundwater). However, $Q_{e c}$ includes both fracture and matrix flow. Stockman et al. (1995) stated that "... there is no mechanism for matrix flow to enter [waste packages] with an intact air gap." Furthermore, according to Stockman et al. (1995), it is possible "... that fracture flow will follow the [back and ribs] and not drip onto the canister." Moreover, it is even possible that Equation 7-9 overestimated the flow rate for waste packages with rubble because, as Stockman et al. (1995) pointed out, "... it is uncertain that the matrix-parcel interface would allow matrix flow to leave the matrix and enter the parcel." Finally, we assumed that, once a waste package fails, groundwater will instantaneously fill it, and that groundwater will contact all of the waste before flowing out of the waste package.

Although we define ECA as the effective-collection-area term, this sampled parameter actually included several related uncertainties for the 1997 PA. Among them were: (1) the effects of fracture-matrix interactions on groundwater flow through intact host rock, disposal tunnels, and waste packages; (2) the effects of waste-induced heating of the near field on groundwater flow; (3) the effects of rubble on groundwater contact with the waste packages; (4) the number, locations, and sizes of holes in failed waste packages; and (5) the nature of groundwater flow through the waste. For the $1997 \mathrm{PA}$, we used a range of -0.5 to 0.5 and a uniform probability distribution for EFFCAREA, the IDPRAM for ECA (see Chapter 6, Figure 6-9). We obtained this range and probability distribution from Wilson et al. (1994).

$\mathrm{Fe}_{2} \mathrm{O}_{3}$. The source-term submodel predicts the sorption of radionuclides from groundwater in each waste package by the $\mathrm{Fe}_{2} \mathrm{O}_{3}$ formed by oxic corrosion of metallic $\mathrm{Fe}$ in $\mathrm{C}$ steel, stainless steel, and borated steel. Stockman et al. (1995) referred to $\mathrm{Fe}_{2} \mathrm{O}_{3}$ as rust. The source-term submodel does not predict sorption by the $\mathrm{Fe}$ (II) corrosion products formed under anoxic conditions, $\mathrm{Fe}(\mathrm{OH})_{2}$ and $\mathrm{Fe}_{3} \mathrm{O}_{4}$, nor by $\mathrm{Al}_{2} \mathrm{O}_{3}, \mathrm{Cr}_{2} \mathrm{O}_{3}$, and $\mathrm{NiO}$, because we have no sorption data for these corrosion products.

We assume that all of the $\mathrm{Fe}_{2} \mathrm{O}_{3}$ produced by oxic corrosion remains within the waste packages. 
Dissolution-Precipitation and Sorption-Desorption Reactions. After the source-term submodel moves the quick-release fraction and the bound radionuclides from the matrices to the unbound solids (see Figure 7-3), it assumes that dissolution-precipitation rcactions establish instantaneous, reversible equilibria between radioisotopes in the unbound solids and the groundwater in cach waste package. The source-term submodel does not include specific dissolution-precipitation reactions similar to those included in the comosion submodel (see Tables 7-2 and 7-3). Rather, it assumes that unspecified reactions produce dissolved radionuclides at the concentrations (solubilities) specified by the user. Furthermore, the corrosion submodel does not predict the aqueous speciation of the radioisotopes, nor the effects of variation in chemical conditions on speciation and, hence, solubilities. However, we implicitly include these variations and their effects by sampling radionuclide solubilities over (sometimes wide) ranges. Moreover, the assumption that equilibrium is attaincd instantaneously will result in overestimates of the dissolved radionuclide concentrations if the groundwater flow ratcs through the waste packages are fast relative to the dissolution rates. Clearly, the rates at which radionuclides will be released from the waste packages will depend on both their solubilities and the groundwater flow rates. Furthermore, sorption of radionuclides will also affect their release rates.

Therefore, the corrosion submodel also includes instantaneous, reversible sorption-desorption reactions. It does not specify sorption-desorption reactions, the speciation of the sorbates, nor the nature of the sorbent sites. Instead, it uses the equation

$$
m_{i, \text { solids }}=K_{D} \cdot c_{i, a q} \cdot m_{\text {solids }}
$$

to calculate the quantity of radioisotope sorbed. In this equation, $\mathrm{m}_{\mathrm{i} \text {, solids }}$ is mass of the radioisotope $\mathrm{i}$ sorbed, $\mathrm{K}_{\mathrm{D}}$ is the distribution coefficient (defined below), $c_{i . a q}$ is the dissolved concentration of radioisotope $i$, and $m_{\text {solids }}$ is the mass of the solids. Equation 7-10 is based on the traditional definition of the $\mathrm{K}_{\mathrm{D}}$, the slope of the linear portion of the function obtained by plotting $C_{i}$, solids (the concentration of radioisotope $i$ associated with the solids) on the ordinate and $\mathrm{C}_{\mathrm{i}, \mathrm{aq}}$ (the dissolved concentration of the same radioisotope) on the abscissa. The source-term submodel includes sorption of radioisotopes by $\mathrm{Fe}_{2} \mathrm{O}_{3}$, for which we have $\mathrm{K}_{\mathrm{D}} \mathrm{s}$, but not by $\mathrm{Fe}(\mathrm{OH})_{2}, \mathrm{Fe}_{3} \mathrm{O}_{4}, \mathrm{Al}_{2} \mathrm{O}_{3}, \mathrm{Cr}_{2} \mathrm{O}_{3}$, and $\mathrm{NiO}$, for which we have no $\mathrm{K}_{\mathrm{D}}$ s. The assumption that sorption is reversible will result in overestimates of the dissolved radioisotope concentrations if sorption is irreversiblc. However, the assumption of instantaneous equilibrium will result in underestimates if the groundwater flow rates are fast relative to the sorption rates.

The source-term submodel defines the solubility of each radioisotope (and the quantity of each radioisotope sorbed) as the solubility of the element of which the radionuclide is an isotope (or the amount of the element sorbed) times the mole fraction of the element contributcd by each radionuclide. Because different isotopes of the same element have different half-lives and, hence, dccay at different rates, the source-term submodel predicts the isotopic composition (including stable isotopes) of cach clement every time step. CST includes stable isotopes in these predictions.

If there is a sufficient quantity of a radioisotope to saturate the groundwater and the sorption sites on the $\mathrm{Fe}_{2} \mathrm{O}_{3}$ in a given waste package, the source-term submodcl uses Equations 7-10 and the solubility and the $K_{D}$ sampled for the vector to predict the quantity of the radioisolope removed from the unbound solids. If the quantity of a radioisotope is insufficient, it dissolves all of the radioisotope from the unbound solids without saturating the groundwater with respect to radioisotope, and uses Equation 7-10 to predict the amount sorbed.

We assume that different isotopes of the same element have the solubility and the same $\mathrm{K}_{\mathrm{D}}$. This assumption is reasonable in view of the small differences helwcen the masses of different isotopes of these elements.

\subsubsection{Transport of Radioisotopes from the Waste Packages to the Intact Host Rock}

The source-term submodel includes advcctive and diffusive transport. Currently, these processes are not coupled. 


\section{Consequence Modeling: Corrosion and Source-Term Model}

The source-term submodel uses the equation

$$
m_{i, a d v}=c_{i, a q} \cdot V_{\text {flow }}
$$

to predict the quantities of radioisotopes transported from the waste packages to the intact host rock by advection. In this equation, $m_{i}$, adv is the mass $(\mathrm{kg})$ of the radioisotope $i$ released in a time step; $c_{i}$ a is the dissolved concentration of radioisotope $i$, and $V_{\text {flow }}$ is the volume of groundwater that flows through a waste package in a time step (see "Groundwater" in Section 7.2.2).

For the $1997 \mathrm{PA}$, we assumed that, once advection removes radioisotopes from the waste packages, they will move to the intact host rock instantaneously. We assumed no sorption of radioisotopes by $\mathrm{Fe}_{2} \mathrm{O}_{3}$ produced by corrosion of the three or four steel supports beneath each waste package, $\mathrm{Fe}$ (II)-bearing corrosion products from these supports, the concrete piers and inverts, nor any rubble present. Furthermore, we assumed instantaneous transport from the waste packages to the intact host rock.

The air gap surrounding the waste packages will eliminate diffusive transport pathways from the waste packages to the intact host rock. However, if sufficicnt rubble accumulates during the 100,000-yr regulatory period, and if the $\mathrm{H}_{2} \mathrm{O}$ content of this rubble is high enough, it might establish such pathways. Therefore, the source-term model uses the equation

$$
M_{i, \text { dif }}=D_{i, \text { mol }} \cdot E D A \cdot\left(S A_{w p} / L\right) \cdot\left(c_{i, w p}-c_{i, i h r}\right) \cdot\left(\left(t_{j}+1\right)-t_{j}\right)
$$

to predict diffusive transport of radioisotopes from waste packages with rubble to the intact host rock. In this equation, $M_{i}$, dif is the quantity (in moles) of radioisotope $i$ that a layer corroded in each time step; $D_{i, \text { mol }}$ is the free-solution tracer diffusion coefficient (also referred to as the free-water diffusion coefficient) of radioisotope $i$ (we assume that different isotopes of the same element have the same $\left.D_{i, m o l}\right) ; E D A$ is the effective-diffusion-area term $\left(\mathrm{m}^{2}\right)$ (see below); $\mathrm{SA}_{\mathrm{wp}}$ is the surface area of the wastc package $\left(\mathrm{m}^{2}\right) ; \mathrm{L}$ is the shortest distance between the waste package and the intact host rock at the time of emplacement $(m) ; c_{i}$, wp is the concentration of radioisotope $i$ within the waste package; $c_{i, i h r}$ is the concentration of radioisolope $i$ within the intact host rock; $t_{j}$ is the time at the start of time step $j$, and $\mathrm{t}_{\mathrm{j}+1}$ is the time at the start of the next time step.

The term EDA in Equation 7-12 is analogous to the term ECA in Equation 7-9 (see "Groundwater" in Section 7.2.2) because, although we define it as the effective-diffusion-area term, this sampled parameter also included several related uncertainties for the 1997 PA. These included: (1) the number, locations, and sizes of holes in failed waste packages; (2) the nature of contact between the rubble and the waste packages, especially the holes; and (3) the diffusive characteristics of the rubble, including its saturation and tortuosity. For the $1997 \mathrm{PA}$, we used a range of $1.00 \times 10^{-6}$ to $1.00 \times 10^{0}$ and a loguniform probability distribution for EFFDAREA, the IDPRAM for EDA (see Chapter 6, Figure 6-9). Clearly the ranges and distributions chosen for ECA and EDA determine the relative importance of advective and diffusive transport from the waste packages to the intact host rock.

The corrosion submodel uses the concentralion gradient from each waste package to the intact host rock at the start of a time step, $c_{i \text {, wp }}-c_{i, i n r}$ in Equation 7-12, to predict the maximum diffusive release of each radioisotope for that time step. If the quantity of a radioisotope in the unbound solids, dissolved in the residual groundwater, and sorbed by $\mathrm{Fe}_{2} \mathrm{O}_{3}$ exceeds the maximum diffusive release predicted for that time step, the corrosion submodel moves the maximum predicted quantity from the waste package to the intact host rock prior to re-equilibrating the radioisotope among the unbound solids, groundwater, and $\mathrm{Fe}_{2} \mathrm{O}_{3}$. If the maximum exceeds the quantity of the radioisotope in a waste package, the source-term submodcl rcleases all of the radioisotope. Because it does not reduce the concentration gradient as diffusion increases the radioisotope concentration in the intact host rock, this might overestimate the diffusive release. Furthermore, the corrosion submodel does not include sorption of radioisotopes by the rubble, nor does it delay release by the time requircd for cliffusive transport through the rubble.

After advection or diffusion transports radioisotopes to the intact host rock, NUTS predicts their movement from the disposal tunnels to the underlying watcr tablc. However, NUTS initially assumes that all radioisotopes that have reached the intact host rock are homogencously distributed throughout each waste grid block, including the disposal tunnels. 


\subsection{Kinetic Parameters Used for the 1997 PA}

This section describes the material-specilic kinetic parameters used in the corrosion and source-term submodels for the 1997 PA. Section 7.1.5, Corrosion Reactions, and Tables 7-2 and 7-3 provide the material-specific reactions and stoichiometric factors used in the corrosion submodel for the 1997 PA. Waste package parameters are provided in Chapter 3, Section 3.6 and Section 7.5.

\subsubsection{Disposal Container, Handling Container, Basket, Cladding, and Binder Materials}

ASTM A516 Grade 60 Steel Disposal Container. The 10-cm-thick, corrosion-allowance, ASTM A516 Grade 60 steel of the disposal container constitutes the outer layer (Layer 1) of the waste packages (see Chapter 3). ASTM A516 Grade 60 steel comprises $0.27 \%$ C, 0.60 to $1.20 \% \mathrm{Mn}, 0.035 \% \mathrm{P}, 0.040 \% \mathrm{~S}, 0.15$ to $0.30 \% \mathrm{Si}$, balance Fe. However, we assumed that it consisted of $100 \% \mathrm{Fe}$ (see, for example, Tables 7-2 and 7-3).

We used a setting of 0 for CORMOD in the 1997 PA. This activated Equation 7-3a for humid oxic conditions, Equation 7-3b for wet oxic conditions, and Equation 7-3c for wet anoxic conditions.

Humid Oxic Corrosion. We used the samc range and probability distribution for COR_A_HO, and the same constant value for COR_B_HO in the 1997 PA that we used for wet oxic corrosion (see Table 7-2). We used these values because we had no data on the corrosion of ASTM A516 Grade 60 steel under humid oxic conditions. Therefore, we assumed that it would corrode at the same rates under these conditions that were used for wet oxic conditions (see "Wet Oxic Corrosion" in this section).

We did not use the data of Haberman and Frydrych (1988) on the corrosion of ASTM A216 Grade WCA steel at $90^{\circ} \mathrm{C}, 150^{\circ} \mathrm{C}$, and $200^{\circ} \mathrm{C}$ to estimate COR_A_HO and COR_B_HO for the 1997 PA because they did not study the corrosion of ASTM A516 Grade 60 steel under humid conditions.

We used a value of $1.00 \times 10^{0}$ for COR_C_HO in the 1997 PA because we had no data on the effects of time on the corrosion of ASTM A516 Grade 60 steel under humid oxic conditions.

We used a value of $1.00 \times 10^{\circ}$ for COR_D_HO in the 1997 PA because we assumed that the corrosion rate of ASTM A516 Grade 60 steel is linearly proportional to the $\mathrm{O}_{2}$ concentration of the gaseous phase.

We used a value of $9.00 \times 10^{-1}$ for FRCPENHO for the 1997 PA because the thin film of $\mathrm{H}_{2} \mathrm{O}$ expected on the surfaces of ASTM A516 Grade 60 steel under these conditions will not promote as much pitting as the aqueous phase under wet oxic or wet anoxic conditions. Therefore, we believe general corrosion will be more important than localized corrosion under humid oxic conditions. This is the only material for which we used different values of FRCPEN (see "Wet Oxic Corrosion" and "Wet Anoxic Corrosion" in this section). However, we should have used a value of $2.50 \times 10^{-1}$ to be consistent with the value of this parameter under wet oxic and wet anoxic conditions. A consistent value of FRCPEN is necessary because the corrosion submodel added the corrosion rates calculated for humid oxic, wet oxic, and wet anoxic conditions in the 1997 PA instead of choosing the maximum rate calculated for each of these conditions as in earlier PAs. The eflccts. il any, of using a different value of FRCPENHO on these calculations is unclear.

We accidentally interchanged the humid oxic and wet anoxic kinetic parameters for ASTM A516 Grade 60 steel for the $1997 \mathrm{PA}$, and did not have time to repeat the calculations with the correct values.

Wet Oxic Corrosion. We used a range of $2.10 \times 10^{-4}$ to $2.10 \times 10^{-2} \mathrm{~kg} / \mathrm{m}^{2} \cdot \mathrm{s}$, and a loguniform probability distribution for COR_A_WO, and a constant valuc of $2.53 \times 10^{3} \mathrm{~K}$ for COR_B_WO in the 1997 PA (see Table 7-5). We used data from Kirby (1979) on the effects of temperature on the corrosion of steel to obtain COR_A_WO and COR_B_WO. We used Excel 7.0 to fit thesc parameters, the constants A and B in the Arrhenius expression (see "Arrhenius Function" in Section 7.1), to 12 mils (thousandths of an inch) per year (mpy), the corrosion rate for $20^{\circ} \mathrm{C}$ in Figure 1 of Kirby (1979), and to $50 \mathrm{mpy}$. the upper end of the range of 40 to $50 \mathrm{mpy}$ for $80^{\circ} \mathrm{C}$ in that figure. This 
7. Consequence Modeling: Corrosion and Source-Term Model

Table 7-5. Kinetic Parameters Used for ASTM A516 Grade 60 Steel in the 1997 PA

\begin{tabular}{|c|c|c|c|}
\hline Parameter & Humid Oxic Corrosion & Wet Oxic Corrosion & Wet Anoxic Corrosion \\
\hline $\begin{array}{l}A\left(\mathrm{~kg} / \mathrm{m}^{2} \cdot \mathrm{s}\right) \text { in } \\
\text { Eqs. } 7-3 a, b, c\end{array}$ & $\begin{array}{c}\text { COR_A_HO= } \\
2.10 \times 10^{-4} \text { to } \\
2.10 \times 10^{-2}, \text { loguniform }\end{array}$ & $\begin{array}{c}\text { COR_A_WO = } \\
2.10 \times 10^{-4} \text { to } \\
2.10 \times 10^{-2}, \text { loguniform }\end{array}$ & $\begin{array}{c}\text { COR_A_WA }= \\
6.64 \times 10^{-7} \text { to } \\
6.64 \times 10^{-5}, \text { loguniform }\end{array}$ \\
\hline $\begin{array}{l}\text { B (Kelvin) in } \\
\text { Eqs. } 7-3 a, b, c\end{array}$ & $\begin{array}{c}\text { COR_B_HO } \\
2.53 \times 10^{3}\end{array}$ & $\begin{array}{c}\text { COR_B_WO } \\
2.53 \times 10^{3}\end{array}$ & $\underset{2.53 \times 10^{3}}{\text { COR_B_WA }}=$ \\
\hline $\begin{array}{l}C \text { (dimensionless) in } \\
\text { Eqs. } 7-3 a, b, c\end{array}$ & $\underset{1.00 \times 10^{0}}{\text { COR_C_HO }}=$ & $\begin{array}{c}\text { COR_C_WO } \\
1.00 \times 10^{0}\end{array}$ & $\underset{1.00 \times 10^{0}}{\text { COR_C_WA }}=$ \\
\hline $\begin{array}{l}\mathrm{D} \text { (dimensionless) in } \\
\text { Eqs. } 7-3 \mathrm{a}, \mathrm{b}\end{array}$ & $\begin{array}{l}\text { COR_D_HO }= \\
1.00 \times 10^{0}\end{array}$ & $\begin{array}{c}\text { COR_D_WO }= \\
1.00 \times 10^{0}\end{array}$ & Not applicable \\
\hline $\begin{array}{c}\text { FRCPEN } \\
\text { (dimensionless) }^{\mathrm{a}}\end{array}$ & $\begin{array}{l}\text { FRCPENHO = } \\
9.00 \times 10^{-1}\end{array}$ & $\begin{array}{l}\text { FRCPENWO }= \\
2.5 \times 10^{-1}\end{array}$ & $\begin{array}{l}\text { FRCPENWA = } \\
2.5 \times 10^{-1}\end{array}$ \\
\hline
\end{tabular}

a The mass fraction of a layer corroded uniformly at the time of penetration of that layer by localized attack.

yielded values of $2.10 \times 10^{-3} \mathrm{~kg} / \mathrm{m}^{2} \cdot \mathrm{s}$ and $2.53 \cdot 10^{3} \mathrm{~K}$ for $\mathrm{A}$ and $\mathrm{B}$, respectively. We then assumed a range of $2.10 \times 10^{-4}$ to $2.10 \times 10^{-2} \mathrm{~kg} / \mathrm{m}^{2} \cdot \mathrm{s}$ and a loguniform probability distribution for COR_A_WO.

We did not use the data of Haberman and Frydrych (1988) to estimate the effects of temperature on the corrosion rate of ASTM A516 Grade 60 steel for the 1997 PA, because they studied the corrosion of ASTM A216 Grade WCA steel in magnesium- (Mg-) containing brines. We believe that their results include the effects of $\mathrm{Mg}$ hydrolysis and concomitant acidification, which were probably significant in their high-temperature experiments but would not occur in the low-Mg groundwater expected in the potential Yucca Mountain repository:

We used the same values for COR_C_WO in the 1997 PA as in the 1994 PA. Stockman et al. (1995) described the methods used to establish these valucs.

We used a value of $1.00 \times 10^{0}$ for COR_D_HO in the 1997 PA because we assumed that the corrosion rate of ASTM A516 Grade 60 steel is linearly proportional to the $\mathrm{O}_{2}$ concentration of the gaseous phase.

We used a value of $2.50 \times 10^{-1}$ for FRCPENWO for the $1997 \mathrm{PA}$. We used the pitting factor of four from Andrews et al. (1994) to obtain this valuc. A pitting factor of four means that penetration by pitting is four times faster than general corrosion, and is equivalent to a FRCPEN of 0.25 .

- Wet Anoxic Corrosion. We used a range of $6.64 \times 10^{-7}$ to $6.64 \times 10^{-5} \mathrm{~kg} / \mathrm{m}^{2} \cdot \mathrm{s}$ for COR_A_WO in the 1997 PA (see Table 7-5). We used predictions by Asano ct al. (1992) of the effects of dissolved $\mathrm{O}_{2}$ on the corrosion of mild steel to obtain this range. However, we calculated this range incorrectly. We should have used the correct range of $3.29 \times 10^{-7}$ to $3.29 \times 10^{-5} \mathrm{~kg} / \mathrm{m}^{2} \cdot \mathrm{s}$ (see below).

Asano et al. (1992) predicted general corrosion rates of 0.285 and $0.000447 \mathrm{~mm} / \mathrm{yr}$ for mild steel under wet oxic and wet anoxic conditions, respectively (comparc Equations 6 and 7 on p. 1660 of their paper). For oxic conditions, they assumed neutral or nearly neutral conditions, a temperature of $25^{\circ} \mathrm{C}$, and a dissolved $\mathrm{O}_{2}$ concentration of $2.53 \times 10^{-7} \mathrm{~mol} / \mathrm{cm}^{3}$ (the solubility of $\mathrm{O}_{2}$ in purc $\mathrm{H}_{2} \mathrm{O}$ at $25^{\circ} \mathrm{C}$ ), and calculated the corrosion rate from the current density for the cathodic (reduction) reaction:

$$
2 \mathrm{H}_{2} \mathrm{O}+\mathrm{O}_{2}+4 \mathrm{e}^{-}=4 \mathrm{OH}^{-}
$$

proceeding at a rate limited by the rate of $\mathrm{O}_{2}$ diffusion through pure $\mathrm{H}_{2} \mathrm{O}$. For anoxic conditions, they assumed $\mathrm{O}_{2-}$ free $\mathrm{H}_{2} \mathrm{O}$ at the same $\mathrm{pH}$ and temperaturc and calculated the corrosion rate from the current density for the cathodic reaction: 


$$
2 \mathrm{H}^{+}+2 \mathrm{e}^{-}=\mathrm{H}_{2} \text {. }
$$

We then divided $0.000447 \mathrm{~mm} / \mathrm{yr}$ by $0.285 \mathrm{~mm} / \mathrm{yr}$, the rates calculated by Asano et al. (1992) for Reactions 7-14 and 7.13 respectively, and obtained a reduction factor of 0.00157 , or $10^{-2.80}$. Next, we multiplied this factor times the range for COR_A_WO, $2.10 \times 10^{-4}$ to $2.10 \times 10^{-2} \mathrm{~kg} / \mathrm{m}^{2} \cdot \mathrm{s}$, to obtain the range for COR_A_WA given above. Finally, we assumed the same loguniform probability distribution for COR_A_WA that we assumed for COR_A_WO (see "Wet Oxic Corrosion" in this section). We did not include the effects of possible $\mathrm{pH}$ variations in this range and distribution (see, for example, Marsh and Taylor, 1988; Asano et al., 1992; and Telander and Westerman, 1997).

We did not use the data of Haberman and Frydrych (1988) to estimate the effects of temperature on the corrosion ASTM A516 Grade 60 steel for the 1997 PA (see "Humid Oxic Corrosion" in this section).

We used a value of $1.00 \times 10^{\circ}$ for COR_C_WA because at the time of the 1997 PA we had no data on the effects of time on the corrosion ASTM A516 Grade 60 steel.

We used a value $2.50 \times 10^{-1}$ for FRCPENWA to be consistent with the value of this parameter under wet oxic conditions (see "Wet Oxic Corrosion" in this section). However, we used a different value for FRCPENHO (see "Humid Oxic Corrosion" in this section). (This is the only material for which we used different values of FRCPEN.)

We accidentally interchanged the humid oxic and wet anoxic kinetic parameters for ASTM A516 Grade 60 steel for the $1997 \mathrm{PA}$, and did not have time to repeat the calculations with the correct values.

Inconel 625 Disposal Container. The 2-cm-thick, corrosion-resistant, Inconel 625 layer of the disposal container constitutes the second layer of the waste packages (see Chapter 3). Inconel 625 comprises $0.05 \% \mathrm{C}, 21.5 \% \mathrm{Cr}$, $2.5 \% \mathrm{Fe}, 0.2 \% \mathrm{Mn}, 9.0 \% \mathrm{Mo}, 4 \% \mathrm{Nb}, 63 \% \mathrm{Ni}, 0.2 \% \mathrm{Si}$, and $0.2 \% \mathrm{Ti}$ (Braithwaite and Molecke, 1980). However, we assumed that it consisted of $22 \% \mathrm{Cr}, 9 \% \mathrm{Mo}$, and $69 \% \mathrm{Ni}$ (see Tables 7-2 and 7-3). The composition of Inconel 625 is similar to that of Incoloy 825, the material specified for the corrosion-resistant disposal containers for the 1994 PA (see Stockman et al., 1995). Therefore, wc uscd the same values of COR_A_HO, COR_B_HO, COR_C_HO, FRCPENHO, COR_A_WO, COR_B_WO, COR_C_WO, and FRCPENWO for the 1997 PA that Stockman et al. (1995) used in the 1994 PA.

We used a setting of 0 for CORMOD in the 1997 PA. This activated Equation 7-3a for humid oxic conditions, Equation $7-3 \mathrm{~b}$ for wet oxic conditions, and Equation $7-3 \mathrm{c}$ for wet anoxic conditions.

Humid Oxic Corrosion. We used the same values for COR_A_HO, COR_B_HO, COR_C_HO, and FRCPENHO in the 1997 PA (see Table 7-6) as in the 1994 PA. Stockman et al. (1995) described the methods used to establish these values.

We used a value of 0 for COR_D_HO hccause at the time of the 1997 PA we had no data on the effect of $\mathrm{O}_{2}$ concentration on the corrosion of Inconel 625.

Wet Oxic Corrosion. We used the same range and probability distribution for COR_A_WO, and the same constant values for COR_B_WO, COR_C_WO. and FRCPENWO in the 1997 PA (see Table 7-6) as in the 1994 PA. Stockman et al. (1995) described the methods uscd to establish these values.

We used a value of 0 for COR_D_WO bccause at the time of the 1997 PA we had no data on the effect of $\mathrm{O}_{2}$ concentration on the corrosion of Inconel 625.

Wet Anoxic Corrosion. We used a value of 0 for COR_A_WA and COR_B_WA in the 1997 PA. We used this value because we had no data on the corrosion of Inconel 625 under wet anoxic conditions. Therefore, we assumed that it would not corrode at significant rates undcr these conditions. However, we should have used a range of values for COR_A_WA that included $0 \mathrm{~kg} / \mathrm{m}^{2} s$ as a lower limit and some yet-to-be-defined, non-zero upper limit (see below).

We assumed that Inconel 625 would not corrode under wet anoxic conditions because $\mathrm{Ni}$, the most abundant constituent of this alloy, is stable in the presence of $\mathrm{H}_{2} \mathrm{O}$ under at least some anoxic conditions (see, for example, Pour- 
Table 7-6. Kinetic Parameters Used for Inconel-625 in the 1997 PA

\begin{tabular}{|c|c|c|c|}
\hline Parameter & Humid Oxic Corrosion & Wet Oxic Corrosion & Wet Anoxic Corrosion \\
\hline $\begin{array}{l}A\left(\mathrm{~kg} / \mathrm{m}^{2} \bullet \mathrm{s}\right) \text { in } \\
\text { Eqs. } 7-3 a, b, c\end{array}$ & $\underset{4.56 \times 10^{-3}}{\text { COR_A_HO }}=$ & $\begin{array}{c}\text { COR_A_WO = } \\
5.60 \times 10^{13} \text { to } 5.60 \times 10^{15} \\
\text { loguniform }\end{array}$ & COR_A_WA $=0$ \\
\hline $\begin{array}{c}\text { B (Kelvin) in Eqs. } \\
7-3 a, b, c\end{array}$ & $\begin{array}{l}\text { COR_B_HO= } \\
1.3155 \times 10^{4}\end{array}$ & $\begin{array}{c}\text { COR_B_WO }= \\
1.9639 \times 10^{4}\end{array}$ & COR_B_WA $=0$ \\
\hline $\begin{array}{c}C \text { (dimensionless) in } \\
\text { Eqs. } 7-3 a, b, c\end{array}$ & $\begin{array}{c}\text { COR_C_HO } \\
1.00 \times 10^{0}\end{array}$ & $\begin{array}{c}\text { COR_C_WO } \\
1.00 \times 10^{0}\end{array}$ & $\begin{array}{c}\text { COR_C_WA } \\
1.00 \times 10^{0}\end{array}$ \\
\hline $\begin{array}{c}D \text { (dimensionless) in } \\
\text { Eqs. } 7-3 a, b\end{array}$ & COR_D_HO $=0$ & COR_D_WO $=0$ & Not applicable \\
\hline $\begin{array}{c}\text { FRCPEN } \\
\text { (dimensionless) }^{a}\end{array}$ & $\begin{array}{c}\text { FRCPENHO }= \\
3.00 \times 10^{-2}\end{array}$ & $\begin{array}{c}\text { FRCPENWO }= \\
3.00 \times 10^{-2}\end{array}$ & $\begin{array}{c}\text { FRCPENWA = } \\
3.00 \times 10^{-2}\end{array}$ \\
\hline
\end{tabular}

baix, 1966, pp. 330-342). The Eh-pH diagram for Ni at $25^{\circ} \mathrm{C}$ (Figure 1, p. 333 of Pourbaix, 1966) implies that the stability fields of $\mathrm{Ni}$ and $\mathrm{H}_{2} \mathrm{O}$ overlap within a region bounded by the lower stability limit of $\mathrm{H}_{2} \mathrm{O}$ and a line parallel to the lower stability limit of $\mathrm{H}_{2} \mathrm{O}$ but about $100 \mathrm{mV}$ above it between $\mathrm{pH}$ values of about 9 and 12 . (Geochemists typically accept the definition of Garrels and Christ, 1965, that a solid element or compound is stable within those areas of an Eh-pH diagram in which the total activity of dissolved species, in this case $\mathrm{Ni}$, in equilibrium with the Nibearing solid is $10^{-6} \mathrm{M}$ or less.) Therefore. Pourbaix (1966, p. 333) concluded that "Nickel can be considered to be a slightly noble metal, as its domain of thermodynamic stability has a small zone in common with that of water, ..." Furthermore, Pourbaix $\left(1966\right.$, p. 333) implics that the corrosion product $\mathrm{Ni}(\mathrm{OH})_{2}$ will passivate $\mathrm{Ni}$ between $\mathrm{pH}$ values of 9 and 12 if the Eh exceeds that of the lower stability limit of $\mathrm{H}_{2} \mathrm{O}$ by more than $100 \mathrm{mV}$, and that the corrosion product $\mathrm{Ni}_{3} \mathrm{O}_{4}$ will passivate $\mathrm{Ni}$ over a significantly wider range of $\mathrm{pH}$ if the Eh exceeds that of the lower stability limit of $\mathrm{H}_{2} \mathrm{O}$ by more than about $900 \mathrm{mV}$ (compare Figure 1, p. 333, and Figure 2, p. 334). Moreover, Pourbaix (1966, pp. 256-271) implies that corrosion products such as $\mathrm{Cr}(\mathrm{OH})_{3}$ and $\mathrm{Cr}_{2} \mathrm{O}_{3}$ will passivate $\mathrm{Cr}$, the second-most abundant constituent of Inconel 625 , in the absence of chloride ion $\left(\mathrm{Cl}^{-}\right)$. However, if $\mathrm{Cr}(\mathrm{OH})_{3}{ }^{\bullet} \mathrm{nH}_{2} \mathrm{O}$ is the corrosion product and $\mathrm{Cl}^{-}$is present, the conditions under which passivation of $\mathrm{Cr}$ will occur are much more restricted than those under which passivation of $\mathrm{Ni}$ will occur (compare Figure 5, p. 265, and Figure 2, p. 334).

However, we should have used a range that included non-zero values of COR_A_WA because: (1) there exists a wide range of conditions $(\mathrm{Eh}>-400$ to -500$) \mathrm{mV}, \mathrm{pH}<9$ ) under which $\mathrm{Ni}$ will corrode and neither $\mathrm{Ni}(\mathrm{OH})_{2}$ nor $\mathrm{Ni}_{3} \mathrm{O}_{4}$ will passivate $\mathrm{Ni}$ under wet anoxic conditions (see Figure 1, p. 333, and Figure 2, p. 334, of Pourbaix, 1966), and these conditions could occur in the potential Yucca Mountain repository if near-field $\mathrm{O}_{2}$ consumption occurs faster than transport replenishes it; (2) $\mathrm{Cl}^{-}$could be present in sufficient quantities, or $\left.\mathrm{Cr}(\mathrm{OH})\right)_{3} \bullet^{\circ \mathrm{nH}_{2}} \mathrm{O}$ could be the corrosion product, thus restricting significantly the range of conditions under which $\mathrm{Cr}$ passivation will occur; and (3) Pourbaix (1966, pp. 272-279) implies that Mo, the third-most abundant constituent of Inconel 625, will corrode under most wet anoxic conditions.

We used a value of $1.00 \times 10^{\circ}$ for COR_C_WA because at the time of the 1997 PA we had no data on the effects of time on the corrosion of Inconel 625.

We used a value $3.00 \times 10^{-2}$ for FRCPENWA to be consistent with the value of this parameter under humid oxic and wet oxic conditions (see "Humid Oxic Corrosion" and "Wet Oxic Corrosion" in this section). (For the 1997 PA, the corrosion submodel added the corrosion raics calculated for humid oxic, wet oxic, and wet anoxic conditions instead of choosing the maximum rate calculated for each of these conditions.)

304L Stainless Steel Handling Container. The 6.35- or 9.5-mm-thick, 304L stainless steel handling containers constitute the third layer of the waste packages (sce Chapter 3). 304L stainless steel comprises \#0.08\% C, 19\% Cr, $\# 2.0 \% \mathrm{Mn}, 10 \% \mathrm{Ni}$, \#1.0\% Si, balancc Fc (see Braithwaite and Molecke, 1980). However, we assumed it consisted 
of $70 \% \mathrm{Fe}, 20 \% \mathrm{Cr}$, and $10 \% \mathrm{Ni}$ (see Tables $7-2$ and 7-3). In Category 1 (metallic $\mathrm{U}$ fuel from $\mathrm{N}$ Reactor), the handling container is a multi-canister overpack (MCO) (Layer 3), which comprises 9.5-mm-thick 304L stainless steel. There are four MCOs per waste package (Chapter 3). In Categories 2 through 13, there is one 6.35-mm-thick 304L stainless-steel handling container (Layer $3 \mathrm{a}$. 3b, or 3c) of spent fuel codisposed with three (Category 12), four (Categories 3,5,10,11), or five (Categories 2, 4.6, 7,8,9, and 13) 9.5-mm-thick 304L stainless steel handling containers (Layer 3d) containing borosilicate glass with DHLW per waste package (Chapter 3). For the 1997 PA, we designated the handling container of spent fuel as Layer $3 a$ in Categories 3, 5, 10, and 11 (codisposed with 4 handling containers of borosilicate glass), as Layer $3 \mathrm{~b}$ in Catcgories 2, 4, 6, 7, 8, 9, and 13 (codisposed with 5 handling containers of glass), and as Layer $3 \mathrm{c}$ in Category 12 (codisposed with 3 handling containers containing glass). In Categories 14 and $15\left(\mathrm{UO}_{2}\right.$ from commercial PWRs and BWRs, respectively), there are no 304L stainless steel handling containers.

Stainless steel also constitutes the cladding for Categories 10 (U-Pu carbide fuel from the Sodium Reactor Experiment), 11 (mixed-oxide fuel from the Fast Flux Test Facility), and 13 (U-Zr hydride fuel from the General Atomic Training, Research and Production Rcactor). The stainless steel cladding in these categories is intact or mainly intact, but we assumed that it will have failed by the time of exposure to the near-field environment for the 1997 PA.

Although 304L stainless steel resists gencral corrosion, it is subject to localized corrosion, such as pitting and stress corrosion cracking, at any temperalurc unless the humidity is low enough to prevent the formation of a surficial film of $\mathrm{H}_{2} \mathrm{O}$ (relative humidity less than about $70 \%$ or $80 \%$ ), and no corrosive chemicals, such as dissolved or precipitated salts or $\mathrm{SO}_{2}$ fumes, are present.

We used a setting of 0 for CORMOD in the 1997 PA. This activated Equation 7-3a for humid oxic conditions, Equation 7-3b for wet oxic conditions, and Equation 7-3c for wet anoxic conditions.

Humid Oxic Corrosion. We used the same range and probability distribution for COR_A_HO, and the same constant values for COR_B_HO, COR_C_HO. and FRCPENHO in the 1997 PA (see Table 7-7) as in the 1994 PA. Stockman et al. (1995) described the methods used to establish these values.

Table 7-7. Kinetic Parameters Used for 304L Stainless Steel in the 1997 PA

\begin{tabular}{|c|c|c|c|}
\hline Parameter & Humid Oxic Corrosion & Wet Oxic Corrosion & Wet Anoxic Corrosion \\
\hline $\begin{array}{l}A\left(\mathrm{~kg} / \mathrm{m}^{2} \cdot \mathrm{s}\right) \text { in } \\
\text { Eqs. } 7-3 a, b, c\end{array}$ & $\begin{array}{c}\text { COR_A_HO }= \\
1.00 \times 10^{-16} \text { to } \\
1.00 \times 10^{-10}, \text { loguniform }\end{array}$ & $\begin{array}{c}\text { COR_A_WO }= \\
1.00 \times 10^{-16} \text { to } \\
1.00 \times 10^{-10}, \text { loguniform }\end{array}$ & $\begin{array}{c}\text { COR_A_WA }= \\
1.00 \times 10^{-13} \text { to } \\
1.00 \times 10^{-9}, \text { loguniform }\end{array}$ \\
\hline $\begin{array}{l}B \text { (Kelvin) in } \\
\text { Eqs. } 7-3 a, b, c\end{array}$ & COR_B_HO $=0$ & COR_B_WO $=0$ & COR_B_WA $=0$ \\
\hline $\begin{array}{l}C \text { (dimensionless) in } \\
\text { Eqs. } 7-3 a, b, c\end{array}$ & $\underset{1.00 \times 10^{0}}{\text { COR_C_HO }}=$ & $\begin{array}{c}\text { COR_C_WO } \\
1.00 \times 10^{\circ}\end{array}$ & $\underset{1.00 \times 10^{\circ}}{\text { COR_C_WA }}=$ \\
\hline $\begin{array}{c}D \text { (dimensionless) in } \\
\text { Eqs. } 7-3 a, b\end{array}$ & COR_D_HO $=0$ & COR_D_WO =0 & Not applicable \\
\hline $\begin{array}{c}\text { FRCPEN } \\
\text { (dimensionless) }^{a}\end{array}$ & FRCPENHO $=\mathrm{C}$ & FRCPENWO $=0$ & FRCPENWA $=0$ \\
\hline
\end{tabular}

We used a value of 0 for COR_D_HO beciluse at the time of the 1997 PA we had no data on the effect of $\mathrm{O}_{2}$ concentration on the corrosion of 304L stainless sicel. 
Wet Oxic Corrosion. We used the samc range and probability distribution for COR_A_WO, and the same constant values for COR_B_WO, COR_C_WO. and FRCPENWO in the 1997 PA (see Table 7-7) as in the 1994 PA. Stockman et al. (1995) described the methods used to establish these values.

We used a value of 0 for COR_D_WO because at the time of the 1997 PA we had no data on the effect of $\mathrm{O}_{2}$ concentration on the corrosion of $304 \mathrm{~L}$ stainless stcel.

Wet Anoxic Corrosion. We used the sanc range and probability distribution for COR_A_WA in the 1997 PA (see Table 7-7) as in the 1995 PA. Because the 1995 PA was never completed nor documented, we cannot explain this range and distribution. Therefore, we should have used the same range and probability distribution for this parameter that we used for humid oxic and wet oxic corrosion. We should have done so because we had no data on the corrosion of 304L stainless steel under wct anoxic conditions. Consequently, we should have assumed that it would corrode at the same rates under these conditions that we used for humid oxic and wet oxic conditions (see "Humid Oxic Corrosion" and "Wet Oxic Corrosion" in this section).

We used a value of 0 for COR_B_WA because at the time of the 1997 PA we had no data for the effects of temperature on this corrosion of $304 \mathrm{~L}$ stainless stcel.

We used a value of $1.00 \times 10^{\circ}$ for COR_C_WA in the 1997 PA because we had no data on the effects of time on the corrosion of $304 \mathrm{~L}$ stainless steel.

We used a value of 0 for FRCPENWA in the 1997 PA to be consistent with the value of this parameter under humid oxic and wet oxic conditions (see "Humid' Oxic Corrosion" and "Wet Oxic Corrosion" in this section). (For the 1997 PA, the corrosion submodel added the corrosion rates calculated for humid oxic, wet oxic, and wet anoxic conditions instead of choosing the maximum rate calculated for each of these conditions.)

Borated-Steel Baskets. Borated steel is present in Categories 14 and 15 as baskets for this low burnup fuel $\left(\mathrm{UO}_{2}\right.$ fuel from commercial PWRs and BWRs. respectively). (Fuel with low burnup requires borated-steel baskets to poison thermal neutrons; for high burnup fucl, borated steel is unnecessary.)

We deactivated the parameters for borated steel in the 1997 PA calculations. We did so because we assumed that borated steel will corrode by the same reactions as those predicted for 304L stainless steel (Tables 7-2 and 7-3), and that it will corrode at the same rates (see Tablc 7-7).

Aluminum Cladding. Al constitutes the cladding for Category 6 (Al-U alloy fuel from the Advanced Test Reactor) and Category 7 (U-Si alloy fuel from the Matcrials Testing Reactor). Because we did not know the alloy(s) used for these claddings, we assumed it compriscl 100\% Al (see Tables 7-2 and 7-3). The Al cladding in Category 6 has failed. The Al cladding in Category 7 is intact or mainly intact, but for the 1997 PA we assumed that it will have failed by the time of exposure to the near-field environment. However, we included corrosion reactions (Tables 7-2 and 7-3) and corrosion rates (Table 7-8) for the Al cladding.

We used a setting of 0 for CORMOD in the 1997 PA. This activated Equation 7-3a for humid oxic conditions, Equation 7-3b for wet oxic conditions, and Equation 7-3c for wet anoxic conditions.

Humid Oxic Corrosion. We used the same values for COR_A_HO and COR_B_HO in the 1997 PA (see Table 7-8) as in the 1994 PA. Stockman et al. (1995) described the methods used to establish these values.

We used a value of $1.00 \times 10^{0}$ for COR_C_HO because at the time of the 1997 PA we had no data on the effects of time on the corrosion of $\mathrm{Al}$.

We used a value of 0 for COR_D_HO in the 1997 PA because we had no data on the effect of $\mathrm{O}_{2}$ concentration on the corrosion of $\mathrm{Al}$. 
Table 7-8. Kinetic Parameters Used for Aluminum in the 1997 PA

\begin{tabular}{|c|c|c|c|}
\hline Parameter & Humid Oxic Corrosion & Wet Oxic Corrosion & Wet Anoxic Corrosion \\
\hline $\begin{array}{l}A\left(\mathrm{~kg} / \mathrm{m}^{2} \cdot \mathrm{s}\right) \text { in } \\
\text { Eqs. } 7-3 a, b, c\end{array}$ & COR_A_HO $=2.36 \times 10^{18}$ & $\begin{array}{c}\text { COR_A_WO }= \\
2.00 \times 10^{-8}\end{array}$ & $\begin{array}{c}\text { COR_A_WA }= \\
1.00 \times 10^{-10} \text { to } \\
1.00 \times 10^{-8}, \text { loguniform }\end{array}$ \\
\hline $\begin{array}{l}\text { B (Kelvin) in } \\
\text { Eqs. } 7-3 a, b, c\end{array}$ & $\begin{array}{c}\text { COR_B_HO }= \\
2.20 \times 10^{4}\end{array}$ & COR_B_WO $=0$ & COR_B_WA $=0$ \\
\hline $\begin{array}{c}C \text { (dimensionless) in } \\
\text { Eqs. } 7-3 a, b, c\end{array}$ & $\underset{1.00 \times 10^{0}}{\text { COR_C_HO }}=$ & $\underset{1.00 \times 10^{0}}{\text { COR_C_WO }}=$ & $\begin{array}{c}\text { COR_C_WA } \\
1.00 \times 10^{0}\end{array}$ \\
\hline $\begin{array}{c}D \text { (dimensionless) in } \\
\text { Eqs. } 7-3 a, b\end{array}$ & COR_D_HO $=0$ & COR_D_WO $=0$ & Not applicable \\
\hline $\begin{array}{c}\text { FRCPEN } \\
\text { (dimensionless) }^{a}\end{array}$ & FRCPENHO $=0$ & FRCPENWO $=0$ & FRCPENWA $=0$ \\
\hline iss fra & uniformly at the & enetration of that laye & ized attack. \\
\hline
\end{tabular}

We used a value of 0 for FRCPENHO in the 1997 PA because the Al cladding in Category 6 has failed, and because we assumed that the $\mathrm{Al}$ cladding in Calegory 7 will have failed by the time of exposure to the near-field environment.

Wet Oxic Corrosion. We used the same values for COR_A_WO, COR_B_WO, and COR_C_WO in the 1997 PA (see Table 7-8) as in the 1994 PA. Stockman ct al. (1995) described the methods used to establish these values.

We used a value of 0 for COR_D_WO because at the time of the 1997 PA we had no data on the effect of $\mathrm{O}_{2}$ concentration on the corrosion of $\mathrm{Al}$.

We used a value of 0 for FRCPENWO in the 1997 PA to be consistent with the value of this parameter under humid oxic conditions (see "Humid Oxic Corrosion" in this section). (For the 1997 PA, the corrosion submodel added the corrosion rates calculated for humid oxic, wet oxic, and wet anoxic conditions instead of choosing the maximum rate calculated for each of these conditions.)

Wet Anoxic Corrosion. We used the same range and probability distribution for COR_A_WA in the 1997 PA (see Table 7-8) as in the 1995 PA. Although the 1995 PA was never completed nor documented, we suspect that C. T. Stockman based this range and distribution on the statement by Godard (1960) that "... deaeration of Kingston tap water prevents the initiation of pitting that would otherwise occur and it seems reasonable to assume that deaeration will have a similar influence in other waters." We believe that this statement, while useful qualitatively, does not provide sufficient justification for assigning a quantitative range to COR_A_WA, especially a range that includes values lower than the constant values used for humicl oxic and wet oxic conditions (compare COR_A_HO, COR_A_WO, and COR_AWA in Table 7-8). Therefore, we should have used the same constant value for this parameter that we used for wet oxic corrosion. We should have done so because we had no data on the corrosion of Al under wet anoxic conditions. Therefore, we should have assumed that it will corrode at the same rates under these conditions that we used for wet oxic conditions in the 1997 PA (sce "Wet Oxic Corrosion" in this section).

We used a value of 0 for FRCPENWA in the 1997 PA to be consistent with the value of this parameter under humid oxic and wet oxic conditions (see "Humid Oxic Corrosion" in this section).

BISO Cladding. BISO (not an acronym) comprises two layers of pyrolytic C. BISO constitutes the cladding for Category 9 ( $\mathrm{Th}_{0.952} \mathrm{U}_{0.048} \mathrm{C}_{2}$ fuel from the Pcach Bottom Reactor).

We did not include corrosion rates for the BISO cladding in the 1997 PA because it has failed. 
Graphite Binder/Moderator. Graphite constitutes the binder (and other structural members) and moderator for Categories 8 and $9\left(\mathrm{Th}_{0.952} \mathrm{U}_{0.048} \mathrm{C}_{2}\right.$ fuel from the Fort St. Vrain and Peach Bottom Reactors, respectively).

We used a setting of 0 for CORMOD in the 1997 PA. This activated Equation 7-3a for humid oxic conditions, Equation 7-3b for wet oxic conditions, and Equation 7-3c for wet anoxic conditions.

Humid Oxic Corrosion. We used the same values for COR_A_HO, COR_B_HO, COR_C_HO, and FRCPENHO in the 1997 PA (see Table 7-9) as in the 1994 PA. Stockman et al. (1995) described the methods used to establish these values.

Table 7-9. Kinetic Parameters Used for Graphite in the 1997 PA

\begin{tabular}{|c|c|c|c|}
\hline Parameter & Humid Oxic Corrosion & Wet Oxic Corrosion & Wet Anoxic Corrosion \\
\hline $\begin{array}{l}A\left(\mathrm{~kg} / \mathrm{m}^{2} \cdot \mathrm{s}\right) \text { in } \\
\text { Eqs. } 7-3 a, b, c\end{array}$ & $\underset{4.79 \times 10^{1}}{\mathrm{COR} A \_\mathrm{HO}}=$ & $\begin{array}{c}\text { COR_A_WO } \\
4.79 \times 10^{1}\end{array}$ & $\begin{array}{c}\text { COR_A_WO = } \\
1.00 \times 10^{-22} \text { to } \\
1.00 \times 10^{-18}, \text { loguniform }\end{array}$ \\
\hline $\begin{array}{l}B \text { (Kelvin) in } \\
\text { Eqs. } 7-3 a, b, c\end{array}$ & $\underset{1.72 \times 10^{4}}{\text { COR_B_HO }}=$ & $\underset{1.72 \times 10^{4}}{C O R \_B \_W O}=$ & COR_B_WA $=0$ \\
\hline $\begin{array}{l}C \text { (dimensionless) in } \\
\text { Eqs. } 7-3 a, b, c\end{array}$ & $\begin{array}{c}\text { COR_C_HO } \\
1.00 \times 10^{0}\end{array}$ & $\underset{1.00 \times 10^{\circ}}{\text { COR_C_WO }}=$ & $\begin{array}{c}\text { COR_C_WA } \\
1.00 \times 10^{0}\end{array}$ \\
\hline $\begin{array}{c}D \text { (dimensionless) in } \\
\text { Eqs. } 7-3 a, b\end{array}$ & COR_D_HO $=0$ & COR_D_WO $=0$ & Not applicable \\
\hline $\begin{array}{c}\text { FRCPEN } \\
\text { (dimensionless) }^{a}\end{array}$ & FRCPENHO $=0$ & FRCPENWO $=0$ & FRCPENWA $=0$ \\
\hline n of & ded uniformly at the & penetration of that layer $b$ & lized attack. \\
\hline
\end{tabular}

We used a value of 0 for COR_D_HO because at the time of the 1997 PA we had no data on the effect of $\mathrm{O}_{2}$ concentration on the corrosion of graphitc.

Wet Oxic Corrosion. We used the same values for COR_A_WO, COR_B_WO, COR_C_WO, and FRCPENWO in the 1997 PA (see Table 7-9) as in the 1994 PA. Stockman et al. (1995) described the methods used to establish these values.

We used a value of 0 for COR_D_HO because we had no data on the effect of $\mathrm{O}_{2}$ concentration on the corrosion of graphite at the time of the 1997 PA.

Wet Anoxic Corrosion. We used the same range and probability distribution for COR_A_WA.and the same constant value for COR_B_WA in the 1997 PA (see Table 7-9) as in the 1995 PA. Because the 1995 PA was never completed nor documented, we cannot explain this range and distribution. Therefore, we should have used the same constant values for these parameters that we used for humid oxic and wet oxic corrosion. We should have used these values because we had no data on the corrosion of graphite under wet anoxic conditions. Consequently, we should have assumed that it will corrode at the same rates under these conditions that we used for humid oxic and wet oxic conditions in the 1997 PA (see "Humid Oxic Corrosion" and "Wet Oxic Corrosion" in this section).

We used a value of $1.00 \times 10^{0}$ for COR_C_WA in the 1997 PA because we had no data on the effects of time on the corrosion of graphite.

We used a value of 0 for FRCPENWA in the 1997 PA to be consistent with the value of this parameter under humid oxic and wet oxic conditions (sec "Humid Oxic Corrosion" and "Wet Oxic Corrosion" in this section). (For the 1997 PA, the corrosion submodcl added the corrosion rates calculated for humid oxic, wet oxic, and wet anoxic conditions instead of choosing the maximum rate calculated for each of these conditions.) 
TRISO Cladding. TRISO (not an acronym) comprises an outer layer of pyrolytic $\mathrm{C}$, a middle layer of $\mathrm{SiC}$, and an inner layer of pyrolytic $\mathrm{C}$. The $\mathrm{SiC}$ layer significantly increases the corrosion resistance of TRISO relative to that of BISO (see "BISO Cladding"). TRISO constitutes the cladding for Category 8 ( $\mathrm{Th}_{0.952} \mathrm{U}_{0.048} \mathrm{C}_{2}$ fuel from the Fort St. Vrain Reactor). The TRISO cladding is the only cladding for which we did not assume failure by the time of exposure to the near-field environment. We did not include corrosion reactions for TRISO in the 1997 PA because we assumed that the corrosion of this matcrial would not affect the quantities of $\mathrm{O}_{2}, \mathrm{H}_{2} \mathrm{O}$, rust, or gas (defined as any gaseous species except $\mathrm{H}_{2} \mathrm{O}$ vapor and $\mathrm{O}_{2}$ ) present in the near field. However, we did include corrosion rates for TRISO (see Table 7-10).

Table 7-10. Kinetic Parameters Used for TRISO in the 1997 PA

\begin{tabular}{|c|c|c|c|}
\hline Parameter & Humid Oxic Corrosion & Wet Oxic Corrosion & Wet Anoxic Corrosion \\
\hline $\begin{array}{l}A\left(\mathrm{~kg} / \mathrm{m}^{2} \cdot \mathrm{s}\right) \text { in } \\
\text { Eqs. } 7-3 a, b, c\end{array}$ & $\begin{array}{c}\text { COR_A_HO = } \\
3.00 \times 10^{-12} \text { to } \\
3.00 \times 10^{-10}, \text { loguniform }\end{array}$ & $\begin{array}{c}\text { COR_A_WO = } \\
1.77 \times 10^{-11} \text { to } \\
1.77 \times 10^{-9}, \text { loguniform }\end{array}$ & $\begin{array}{c}\text { COR_A_WA }= \\
1.77 \times 10^{-11} \text { to } \\
1.77 \times 10^{-9}, \text { loguniform }\end{array}$ \\
\hline $\begin{array}{c}B \text { (Kelvin) in } \\
\text { Eqs. } 7-3 a, b, c\end{array}$ & COR_B_HO $=0$ & COR_B_WO $=0$ & COR_B_WA $=0$ \\
\hline $\begin{array}{c}C \text { (dimensionless) in } \\
\text { Eqs. 7-3a, b, c }\end{array}$ & $\begin{array}{c}\text { COR_C_HO } \\
1.00 \times 10^{0}\end{array}$ & $\underset{1.00 \times 10^{0}}{\text { COR_C_WO }}=$ & $\begin{array}{c}\text { COR_C_WA }= \\
1.00 \times 10^{0}\end{array}$ \\
\hline $\begin{array}{c}D \text { (dimensionless) in } \\
\text { Eqs. } 7-3 a, b\end{array}$ & COR_D_HO $=0$ & COR_D_WO $=0$ & Not applicable \\
\hline $\begin{array}{c}\text { FRCPEN } \\
\text { (dimensionless) }^{a}\end{array}$ & $\begin{array}{c}\text { FRCPENHO }= \\
1.00 \times 10^{0}\end{array}$ & $\begin{array}{l}\text { FRCPENWO }= \\
1.00 \times 10^{0}\end{array}$ & $\begin{array}{c}\text { FRCPENWA }= \\
1.00 \times 10^{0}\end{array}$ \\
\hline
\end{tabular}

We used a setting of 0 for CORMOD in the 1997 PA. This activated Equation 7-3a for humid oxic conditions, Equation 7-3b for wet oxic conditions. and Equation 7-3c for wet anoxic conditions.

Humid Oxic Corrosion. We used a range of $3.00 \times 10^{-12} \mathrm{~s}^{-1}$ to $3.00 \times 10^{-10} \mathrm{~s}^{-1}$ and a loguniform probability distribution for COR_A_HO in the 1997 PA (see Table 7-10). This is the same range and distribution used in the 1995 PA. Because the 1995 PA was never completed nor documented, we describe the methods used to establish this range and distribution below (see "Wet Oxic Corrosion" in this section). Although Stockman used the same methods to establish the range and distribution for COR_A_HO in the 1995 PA that she used to establish the range and distribution for COR_A_WO and COR_A_WA, she either changed COR_A_WO and COR_A_WA from a range $3.00 \times 10^{-12} \mathrm{~s}^{-1}$ to $3.00 \times 10^{-10} \cdot \mathrm{s}^{-1}$ and a loguniform probability distribution to a range of $1.77 \times 10^{-11}$ to $1.77 \times 10^{-9}$ $\mathrm{kg} / \mathrm{m}^{2} \bullet \mathrm{s}$ and a loguniform probability distribution but forgot to change COR_A_HO; or did not change COR_A_HO for some forgotten reason. In either calse, we should have used a range of $2.06 \times 10^{-12}$ to $2.06 \times 10^{-10} \mathrm{~kg} / \mathrm{m}^{2} \cdot \mathrm{s}$ and a loguniform probability distribution (sce "Wet Oxic Corrosion" in this section).

We used the same values for COR_B_HO, COR_C_HO, and FRCPENHO in the 1997 PA (see Table 7-10) as in the 1994 PA. Stockman et al. (1995) described the methods used to establish these values.

We used a value of 0 for COR_D_HO because at the time of the $1997 \mathrm{PA}$ we had no data on the effect of $\mathrm{O}_{2}$ concentration on the corrosion of TRISO.

Wet Oxic Corrosion. We used the same range and probability distribution for COR_A_WO (and COR_A_WA) in the 1997 PA (see Table 7-10) as in the 1995 PA. Because the 1995 PA was never completed nor documented, we describe the methods used to establish this range and distribution (see below). However, we should have used a range of $2.06 \times 10^{-12}$ to $2.06 \times 10^{-10} \mathrm{~kg} / \mathrm{m}^{2} \cdot$ s and a loguniform probability distribution (see below). 
Stockman et al. (1995) established a constant value of $3.00 \times 10^{-12} \mathrm{~kg} / \mathrm{m}^{2} \cdot \mathrm{s}$ for COR_A_HO (and COR_A_WO) in the 1994 PA. First, they assumed that the TRISO cladding would not fail until 10,000 yr after exposure of this material to the near-field environment in the potential Yucca Mountain repository. (Because all of the calculations for the 1994 PA predicted the performance of the potential repository for $10,000 \mathrm{yr}$ after exposure, this in turn ensured that the Th-U carbide fuel in this category was never exposed to the near-field during these calculations and, hence, that no radionuclides were released from this waste.) Next, they divided 1 by 10,000 yr to obtain $1.00 \times 10^{-4} \mathrm{yr}^{-1}$, converted $1.00 \times 10^{-4} \mathrm{yr}^{-1}$ to $3.17 \times 10^{-12} \mathrm{~s}^{-1}$, and rounded this to $3.00 \times 10^{-12} \mathrm{~s}^{-1}$. For the $1995 \mathrm{PA}$, Stockman started with a value of $3.17 \times 10^{-12} \mathrm{~s}^{-1}$ (the value of COR_A_HO and COR_A_WO established for the $1994 \mathrm{PA}$ prior to rounding). Next, she assumed a range of $3.17 \times 10^{-12}$ to $3.17 \times 10^{-10} \mathrm{~s}^{-1}$ and a loguniform probability distribution for COR_A_WO (and COR_A_HO and COR_A_WA) to breach the TRISO cladding and release radionuclides from these waste packages prior to $10,000 \mathrm{yr}$ in most, if not all, of the vectors. She made this assumption because: (1) she believed that the constant value used in the $1994 \mathrm{PA}$, and the prediction that no TRISO cladding would fail in $10,000 \mathrm{yr}$, were too optimistic; and (2) she wanted to determine the sensitivity of the overall performance of the repository to the durability of the TRISO cladding. Stockman then established a range of $1.77 \times 10^{-11}$ to $1.77 \times 10^{-9} \mathrm{~kg} / \mathrm{s}$ for COR_A_WO (and COR_A_WA) by multiplying $3.17 \times 10^{-12}$ to $3.17 \times 10^{-10} \mathrm{~s}^{-1}$ by $56 \mathrm{~kg}$, the mass of TRISO per waste package in the Th-U carbide from Fort St. Vrain, and assuming that $56 \mathrm{~kg}$ of TRISO has a surface area of $1.00 \mathrm{~m}^{2}$ to obtain a range of $1.77 \times 10^{-11}$ to $1.77 \times 10^{-9} \mathrm{~kg} / \mathrm{m}^{2} \cdot \mathrm{s}$ for COR_A_WO (and COR_A_WA). Finally, Stockman assumed a loguniform probability distribution for COR_A_WO (and COR_A_WA).

For the $1997 \mathrm{PA}$, our assumption of a range of $3.00 \times 10^{-12}$ to $3.00 \times 10^{-10} \mathrm{~s}^{-1}$ was as good as any. However, instead of multiplying the range of $3.17 \times 10^{-12}$ to $3.17 \times 10^{-10} \mathrm{~s}^{-1}$, (the preliminary values of COR_A_WO, COR_A_HO, and COR_A_WA established for the 1995 PA) by $56 \mathrm{~kg}$ (the mass of TRISO per waste package in the Th-U carbide from Fort St. Vrain in the $1995 \mathrm{PA}$ ) and assuming that $56 \mathrm{~kg}$ of TRISO has a surface area of $1.00 \mathrm{~m}^{2}$ to obtain a range of $1.77 \times 10^{-11}$ to $1.77 \times 10^{-9} \mathrm{~kg} / \mathrm{m}^{2} \cdot \mathrm{s}$, we should have multiplied $3.17 \times 10^{-12}$ to $3.17 \times 10^{-10} \mathrm{~s}^{-1}$ by $0.65 \mathrm{~kg}$ of TRISO per waste package (the value obtained from INEEL personnel for the $1997 \mathrm{PA}$ ), and assumed that $0.65 \mathrm{~kg}$ of TRISO has a surface arca of $1.00 \mathrm{~m}^{2}$ to obtain a range of $2.06 \times 10^{-12}$ to $2.06 \times 10^{-10} \mathrm{~kg} / \mathrm{m}^{2} \cdot \mathrm{s}$ for COR_A_WO (and COR_A_HO and COR_A_WA). Finally, we should have continued to assume a loguniform probability distribution for this range.

Use of a mass of $0.65 \mathrm{~kg}$ of TRISO per waste package and the assumption that $0.65 \mathrm{~kg}$ of TRISO has a surface area of $1.00 \mathrm{~m}^{2}$ would have been consistent with the approach we suggested to establish COR_A_HO, COR_A_WO, and COR_A_WA for the Th-U carbide fuels (see "Humid Oxic Corrosion" in this section). However, we strongly suspect that the value of $0.65 \mathrm{~kg}$ of TRISO per waste package is incorrect. Perhaps a better approach would be to use a value of the mass of TRISO per wastc package from Lotts et al. (1992) to establish COR_A_HO,COR_A_WO, and COR_A_WA for this material.

We used a value of 0 for COR_D_WO because at the time of the 1997 PA we had no data on the effect of $\mathrm{O}_{2}$ concentration on the corrosion of TRISO.

Wet Anoxic Corrosion. We used the same range and probability distribution for COR_A_WA (and COR_A_WO) in the 1997 PA (see Table 7-10) as in the 1995 PA (see "Wet Oxic Corrosion" in this section). However, we should have used a range of $2.06 \times 10^{-12}$ to $2.06 \times 10^{-10} \mathrm{~kg} / \mathrm{m}^{2} \cdot$ s and a loguniform probability distribution (see "Wet Oxic Corrosion" in this section).

We used a value of 0 for COR_B_WA because at the time of the 1997 PA we had no data for the effects of temperature on the corrosion of TRISO.

We used a value of $1.00 \times 10^{0}$ for COR_C_WA in the 1997 PA because we had no data on the effects of time on the corrosion of TRISO.

We used a value of $1.00 \times 10^{0}$ for FRCPENWA in the 1997 PA to be consistent with the value of this parameter under humid oxic and wet oxic conditions (see "Humid Oxic Corrosion" and "Wet Oxic Corrosion" in this section). (For the $1997 \mathrm{PA}$, the corrosion submodel added the corrosion rates calculated for humid oxic, wet oxic, and wet anoxic conditions instead of choosing the maximum rate calculated for each of these conditions.) 
Zircaloy-2 Cladding. Zircaloy-2 constitutes the cladding for Categories 1 through 5, 12, 14, and 15. Zircaloy-2 comprises $0.12 \% \mathrm{C}, 0.1 \% \mathrm{Cr}, 0.05 \% \mathrm{Ni}, 1.5 \% \mathrm{Sn}$, and $98.2 \% \mathrm{Zr}$ (see Braithwaite and Molecke, 1980). However, we assumed it consisted of $100 \% \mathrm{Zr}$ (sce Tables 7-2 and 7-3). The Zircaloy-2 cladding in Categories 1 and 5 has failed. The Zircaloy-2 cladding in Categorics 2, 3, 4, 12, 14, and 15 is intact or mainly intact, but we assumed that it will have failed by the time of exposure to the near-field environment for the 1997 PA. However, we included corrosion reactions (Tables 7-2 and 7-3) and corrosion rates (Table 7-11) for all of the Zircaloy-2 cladding.

Table 7-11. Kinetic Parameters Used for Zircaloy-2 in the 1997 PA

\begin{tabular}{|c|c|c|c|}
\hline Parameter & Humid Oxic Corrosion & Wet Oxic Corrosion & Wet Anoxic Corrosion \\
\hline $\begin{array}{l}A\left(\mathrm{~kg} / \mathrm{m}^{2} \cdot \mathrm{s}\right) \text { in } \\
\text { Eqs. } 7-3 a, b, c\end{array}$ & $\underset{6.00 \times 10^{-2}}{\text { COR_A_HO }}=$ & $\begin{array}{c}\text { COR_A_WO }= \\
1.00 \times 10^{-12}\end{array}$ & $\underset{1.00 \times 10^{-12}}{\text { COR_A_WA }}=$ \\
\hline $\begin{array}{c}\text { B (Kelvin) in } \\
\text { Eqs. } 7-3 a, b, c\end{array}$ & $\begin{array}{l}\text { COR_B_HO }= \\
1.1173 \times 10^{4}\end{array}$ & COR_B_WO $=0$ & COR_B_WA $=0$ \\
\hline $\begin{array}{l}C \text { (dimensionless) in } \\
\text { Eqs. } 7-3 a, b, c\end{array}$ & $\underset{1.00 \times 10^{0}}{\text { COR_C_HO }}=$ & $\underset{1.00 \times 10^{0}}{\text { COR_C_WO }}=$ & $\begin{array}{c}\text { COR_C_WA } \\
1.00 \times 10^{\circ}\end{array}$ \\
\hline $\begin{array}{c}D \text { (dimensionless) in } \\
\text { Eqs. } 7-3 a, b\end{array}$ & COR_D_HO $=0$ & COR_D_WO $=0$ & Not applicable \\
\hline $\begin{array}{c}\text { FRCPEN } \\
\text { (dimensionless) }\end{array}$ & FRCPENHO $=0$ & FRCPENWO $=0$ & FRCPENWA $=0$ \\
\hline a The mass fraction of a & orroded uniformly at the & penetration of that layer by & ized attack. \\
\hline
\end{tabular}

We used a setting of 0 for CORMOD in the 1997 PA. This activated Equation 7-3a for humid oxic conditions, Equation 7-3b for wet oxic conditions, and Equation 7-3c for wet anoxic conditions.

Humid Oxic Corrosion. We used the same values for COR_A_HO, COR_B_HO, and COR_C_HO in the 1997 PA (see Table 7-11) as in the 1994 PA. Stockman et al. (1995) described the methods used to establish these values.

We used a value of 0 for COR_D_HO because we had no data on the effect of $\mathrm{O}_{2}$ concentration on the corrosion of Zircaloy-2 at the time of the 1997 PA.

We used a value of 0 for FRCPENHO in the 1997 PA because the Zircaloy-2 cladding in Categories 1 and 5 has failed, and because we assumed that the Zircaloy-2 cladding in Categories 2, 3, 4,12,14, and 15 will have failed by the time of exposure to the near-field environment.

Wet Oxic Corrosion. We used the same values for COR_A_WO, COR_B_WO, and COR_C_WO in the 1997 PA (see Table 7-11) as in the 1994 PA. Stockman et al. (1995) described the methods used to establish these values.

We used a value of 0 for COR_D_WO because at the time of the 1997 PA we had no data on the effect of $O_{2}$ concentration on the corrosion of Zircaloy-2.

We used a value of 0 for FRCPENWO in the 1997 PA to be consistent with the value of this parameter under humid oxic conditions (see "Humid Oxic Corrosion" in this section). (For the 1997 PA, the comosion submodel added the corrosion rates calculated for humid oxic, wet oxic, and wet anoxic conditions instead of choosing the maximum rate calculated for each of these conditions.)

Wet Anoxic Corrosion. We used the same value of $1.00 \times 10^{-12} \mathrm{~kg} / \mathrm{m}^{2} \cdot \mathrm{s}$ for COR_A_WA in the 1997 PA that we used for COR_A_WO (see "Wet Oxic Corrosion" in this section). We used this value because we assumed that the Zircaloy-2 cladding will react too slowly to affect the quantities of $\mathrm{O}_{2}, \mathrm{H}_{2} \mathrm{O}$, rust, or gas (defined as any gaseous species except $\mathrm{H}_{2} \mathrm{O}$ vapor and $\mathrm{O}_{2}$ ) present in the near field. The value we used for COR_A_WO and COR_A_WA 
for Zircaloy-2 is even lower than the value of $3.00 \times 10^{-12} \mathrm{~kg} / \mathrm{m}^{2} \cdot \mathrm{s}$ used by Stockman et al. (1995) for TRISO (see "Humid Oxic Corrosion" in the TRISO Cladding section). Their value ensured that the TRISO cladding did not fail during the 10,000-yr PA calculations carried out in 1994. Furthermore, because we used a value of 0 for FRCPENHO, FRCPENWO, and FRCPENWA for Zircaloy-2 in the 1997 PA (see "Humid Oxic Corrosion" in this section), our use of a low value for COR_A_WA did not affect the time at which this cladding failed.

We used a value of 0 for COR_B_WA because at the time of the 1997 PA we had no data for the effects of temperature on the corrosion of Zircaloy-2.

We used a value of $1.00 \times 10^{\circ}$ for COR_C_WA in the 1997 PA because we had no data on the effects of time on the corrosion of Zircaloy-2.

We used a value of 0 for COR_D_WA because we had no data on the effect of $\mathrm{O}_{2}$ concentration on Zircaloy-2 corrosion.

We used a value of 0 for FRCPENWA in the 1997 PA to be consistent with the value of this parameter under humid oxic conditions (see "Humid Oxic Corrosion" in this section).

\subsubsection{Fuel Materials}

Metallic Uranium. U constitutes the fuel for Category 1 (N Reactor). The Zircaloy-2 cladding in Category 1 has failed. .

We used a setting of 0 for CORMOD in the 1997 PA. This activated Equation 7-3a for humid oxic conditions, Equation 7-3b for wet oxic conditions, and Equation 7-3c for wet anoxic conditions.

Humid Oxic Corrosion. We used the same values for COR_A_HO, COR_B_HO, and COR_C_HO in the 1997 PA (see Table 7-12) as in the 1994 PA. Stockman et al. (1995) described the methods used to establish these values.

Table 7-12. Kinetic Parameters Used for Metallic Uranium in the 1997 PA

\begin{tabular}{|c|c|c|c|}
\hline Parameter & Humid Oxic Corrosion & Wet Oxic Corrosion & Wet Anoxic Corrosion \\
\hline $\begin{array}{l}\mathrm{A}\left(\mathrm{kg} / \mathrm{m}^{2} \cdot \mathrm{s}\right) \text { in } \\
\text { Eqs. } 7-3 a, b, c\end{array}$ & $\underset{1.35 \times 10^{2}}{\text { COR_A_HO }}=$ & $\begin{array}{c}\text { COR_A_WO }= \\
2.85 \times 10^{13}\end{array}$ & $\begin{array}{c}\text { COR_A_WA }= \\
9.40 \times 10^{3}\end{array}$ \\
\hline $\begin{array}{l}B \text { (Kelvin) in } \\
\text { Eqs. } 7-3 a, b, c\end{array}$ & $\underset{7.24 \times 10^{3}}{\text { COR_B_HO }}=$ & $\underset{1.659 \times 10^{4}}{\text { COR_B_WO }}=$ & $\begin{array}{c}\text { COR_B_WA } \\
7.97 \times 10^{3}\end{array}$ \\
\hline $\begin{array}{c}C \text { (dimensionless) in } \\
\text { Eqs. } 7-3 a, b, c\end{array}$ & $\underset{1.00 \times 10^{0}}{\text { COR_C_HO }}=$ & $\begin{array}{c}\text { COR_C_WO } \\
1.00 \times 10^{0}\end{array}$ & $\underset{1.00 \times 10^{0}}{\text { COR_C_WA }}=$ \\
\hline $\begin{array}{c}D \text { (dimensionless) in } \\
\text { Eqs. } 7-3 a, b\end{array}$ & $\underset{1.00 \times 10^{0}}{\text { COR_D_HO }}=$ & $\begin{array}{l}\text { COR_D_WO } \\
1.00 \times 10^{\circ}\end{array}$ & Not applicable \\
\hline $\begin{array}{c}\text { FRCPEN } \\
\text { (dimensionless) }^{a}\end{array}$ & FRCPENHO $=0$ & FRCPENWO $=0$. & FRCPENWA $=0$ \\
\hline he mass fraction of & orroded uniformly at the & penetration of that layer $b$ & ized attack. \\
\hline
\end{tabular}

We used a value of $1.00 \times 10^{\circ}$ for COR_D_HO in the 1997 PA because we assumed that the corrosion rate of $U$ is linearly proportional to the $\mathrm{O}_{2}$ concentration of the gaseous phase.

The value of FRCPENHO used in the 1997 PA was irrelevant because $U$ is the innermost material of the waste packages in Category 1. 
Wet Oxic Corrosion. We used values of $2.85 \times 10^{13} \mathrm{~kg} / \mathrm{m}^{2} \cdot \mathrm{s}$ and $1.659 \times 10^{4} \mathrm{~K}$ for COR_A_WO and COR_B_WO, respectively, in the 1997 PA (sce Table 7-12). We used data from Wilkinson (1962) on the effects of temperature and the composition of the gaseous-phase on the corrosion of $U$ to obtain COR_A_WO and COR_B_WO. We used Excel 7.0 to fit these parameters, the constants A and B in the Arrhenius expression (see "Arrhenius Function" in Section 7.1.6), to $0.0005 \mathrm{mg} / \mathrm{cm}^{2} \cdot \mathrm{hr}$, the average rate of loss of $\mathrm{U}$ in air-saturated $\mathrm{H}_{2} \mathrm{O}$ at $50^{\circ} \mathrm{C}$ in Table 15 of Wilkinson (1962), and to $0.010 \mathrm{mg} / \mathrm{cm}^{2} \cdot \mathrm{hr}$, the average rate air-saturated $\mathrm{H}_{2} \mathrm{O}$ at $70^{\circ} \mathrm{C}$ in that table. This yielded the values of COR_A_WO and COR_B_WO given above.

We used a value of $1.00 \times 10^{\circ}$ for COR_C_WO because at the time of the 1997 PA we had no data on the effects of time on the corrosion of $U$.

We used a value of $1.00 \times 10^{0}$ for COR_D_HO in the 1997 PA because we assumed that the corrosion rate of $U$ is linearly proportional to the $\mathrm{O}_{2}$ concentration of the gaseous phase.

The value of FRCPENWO used in the 1997 PA was irrelevant because $U$ is the innermost material of these waste packages.

Wet Anoxic Corrosion. We used the same values for COR_A_WA, and COR_B_WA in the 1997 PA (see Table 7-12) that Stockman et al. (1995) used for COR_A_WO and COR_B_WO in the 1994 PA. Stockman et al. fitted these parameters, the constants $A$ and $B$ in the Arrhenius expression (see "Arrhenius Function" in Section 7.1.6), to

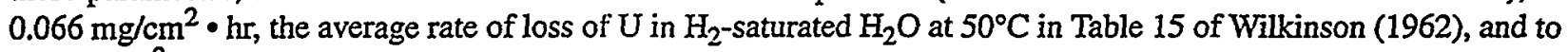
$1.0 \mathrm{mg} / \mathrm{cm}^{2} \cdot \mathrm{hr}$, the average rate in $\mathrm{H}_{2}$-saturated $\mathrm{H}_{2} \mathrm{O}$ at $90^{\circ} \mathrm{C}$ in that table. However, the $\mathrm{H}_{2}$-saturated conditions under which these data were obtained are more appropriate to wet anoxic corrosion than to wet oxic corrosion. Therefore, we used them for COR_A_WA and COR_B_WA in the 1997 PA instead of for COR_A_WO and COR_B_WO.

We used a value of $1.00 \times 10^{0}$ for COR_C_WA because at the time of the 1997 PA we had no data for the effects of time on the corrosion of $U$.

The value of FRCPENWA used in the 1997 PA was irrelevant because $U$ is the innermost material of these waste packages.

Uranium-Zirconium Alloy. U-Zr alloy constitutes the fuel for Category 2 (Chicago Pile 5). We did not have a formula for this alloy at the time of the 1997 PA. The Zircaloy-2 cladding in Category 2 is intact or mainly intact, but we assumed that it will have failed by the timc of exposure to the near-field environment for the 1997 PA.

We deactivated the parameters for U-Zr alloy in the 1997 PA calculations. We did so because we assumed that $\mathrm{U}-\mathrm{Zr}$ alloy would corrode by the same reactions as those predicted for $U$ (Tables 7-2 and 7-3), and that it would corrode at the same rates (see Table 7-12).

Uranium-Molybdenum Alloy. U-Mo alloy constitutes the fuel for Category 3 (Fermi Reactor). We did not have a formula for this alloy at the time of the 1997 PA. The Zircaloy-2 cladding in Category 3 is intact or mainly intact, but we assumed that it will have failed by the time of exposure to the near-field environment for the 1997 PA.

We used values of COR_A_HO, COR_A_WO, and COR_A_WA equal to ten times the values used for U, and values of all of the other kinetic parameters equal to those for $U$ (compare Tables 7-12 and 7-13). We did so because we assumed that U-Mo alloy will corrode by the same reactions as those predicted for U (Tables 7-2 and 7-3), but that it will corrode at ten times the rate.

Uranium Dioxide. $\mathrm{UO}_{2}$ constitutes the fucl for Categories 4 (Shippingport), 5 (Three Mile Island), 14 (commercial PWRs) and 15 (commercial BWRs). The Zircaloy-2 cladding in Category 5 has failed. The Zircaloy-2 cladding in Categories 4, 14, and 15 is intact or mainly intact, but we assumed that it will have failed by the time of exposure to the near-field environment for the $1997 \mathrm{PA}$. 
7. Consequence Modeling: Corrosion and Source-Term Model

Table 7-13. Kinetic Parameters Used for Uranium-Molybdenum Alloy in the 1997 PA

\begin{tabular}{|c|c|c|c|}
\hline Parameter & Humid Oxic Corrosion & Wet Oxic Corrosion & Wet Anoxic Corrosion \\
\hline $\begin{array}{l}A\left(\mathrm{~kg} / \mathrm{m}^{2} \cdot \mathrm{s}\right) \text { in } \\
\text { Eqs. 7-3a, b, c }\end{array}$ & $\underset{1.35 \times 10^{3}}{\text { COR_A_HO }}=$ & $\begin{array}{c}\text { COR_A_WO }= \\
2.85 \times 10^{14}\end{array}$ & $\begin{array}{c}\text { COR_A_WA }= \\
9.40 \times 10^{4}\end{array}$ \\
\hline $\begin{array}{l}B \text { (Kelvin) in } \\
\text { Eqs. } 7-3 a, b, c\end{array}$ & $\underset{7.24 \times 10^{3}}{\text { COR_B_HO }}=$ & $\begin{array}{c}\text { COR_B_WO } \\
1.659 \times 10^{4}\end{array}$ & $\underset{7.97 \times 10^{3}}{\text { COR_B_WA }}=$ \\
\hline $\begin{array}{c}C \text { (dimensionless) in } \\
\text { Eqs. } 7-3 a, b, c\end{array}$ & $\underset{1.00 \times 10^{0}}{\text { COR_C_HO }}=$ & $\underset{1.00 \times 10^{0}}{\text { COR_C_WO }}=$ & $\underset{1.00 \times 10^{0}}{\text { COR_C_WA }}=$ \\
\hline $\begin{array}{c}D \text { (dimensionless) in } \\
\text { Eqs. } 7-3 a, b\end{array}$ & $\begin{array}{l}\text { COR_D_HO } \\
1.00 \times 10^{0}\end{array}$ & $\begin{array}{l}\text { COR_D_WO } \\
1.00 \times 10^{\circ}\end{array}$ & Not applicable \\
\hline $\begin{array}{c}\text { FRCPEN } \\
\text { (dimensionless) }^{\mathrm{a}}\end{array}$ & FRCPENHO $=0$ & FRCPENWO $=0$ & FRCPENWA $=0$ \\
\hline The mass fraction & orroded uniformly at th & penetration of that layer by & zed attack. \\
\hline
\end{tabular}

We used a setting of 0 for CORMOD in the 1997 PA. This activated Equation 7-3a for humid oxic conditions, Equation 7-3b for wet oxic conditions, and Equation 7-3c for wet anoxic conditions.

Humid Oxic Corrosion. We used the same values for COR_A_HO, COR_B_HO, and COR_C_HO in the 1997 PA (see Table 7-14) as in the 1994 PA. Stockman et al. (1995) described the methods used to establish these values.

Table 7-14. Kinetic Parameters Used for Uranium Dioxide in the 1997 PA

\begin{tabular}{|c|c|c|c|}
\hline Parameter & Humid Oxic Corrosion & Wet Oxic Corrosion & Wet Anoxic Corrosion \\
\hline $\begin{array}{l}A\left(\mathrm{~kg} / \mathrm{m}^{2} \cdot \mathrm{s}\right) \text { in } \\
\text { Eqs. } 7-3 a, b, c\end{array}$ & $\begin{array}{c}\text { COR_A_HO }= \\
1.72 \times 10^{-4}\end{array}$ & $\begin{array}{c}\text { COR_A_WO }= \\
3.00 \times 10^{-6} \text { to } \\
2.00 \times 10^{-1}, \text { loguniform }\end{array}$ & $\begin{array}{l}\text { COR_A_WA } \\
1.00 \times 10^{-11}\end{array}$ \\
\hline $\begin{array}{l}\text { B (Kelvin) in } \\
\text { Eqs. 7-3a, b, c }\end{array}$ & $\underset{5.989 \times 10^{3}}{\text { COR_B_HO }}$ & $\underset{3.65 \times 10^{3}}{\text { COR_B_WO }}=$ & COR_B_WA $=0$ \\
\hline $\begin{array}{l}C \text { (dimensionless) in } \\
\text { Eqs. 7-3a, b, c }\end{array}$ & $\underset{1.00 \times 10^{0}}{\text { COR_C_HO }}=$ & $\begin{array}{c}\text { COR_C_WO } \\
1.00 \times 10^{0}\end{array}$ & $\underset{1.00 \times 10^{0}}{\text { COR_C_WA }}=$ \\
\hline $\begin{array}{c}D \text { (dimensionless) in } \\
\text { Eqs. 7-3a, b }\end{array}$ & $\begin{array}{l}\text { COR_D_HO } \\
1.00 \times 10^{\circ}\end{array}$ & $\begin{array}{c}\text { COR_D_WO= } \\
1.00 \times 10^{-1} \text { to } \\
1.00 \times 10^{0} \text {, uniform }\end{array}$ & Not applicable \\
\hline $\begin{array}{c}\text { FRCPEN } \\
\text { (dimensionless) }^{\mathrm{a}}\end{array}$ & FRCPENHO $=0$ & FRCPENWO $=0$ & FRCPENWA $=0$ \\
\hline
\end{tabular}

We used a value of $1.00 \times 10^{0}$ for COR_D_HO in the 1997 PA because we assumed that the corrosion rate of $\mathrm{UO}_{2}$ is linearly proportional to the $\mathrm{O}_{2}$ concentration of the gaseous phase.

The value of FRCPENHO used in the 1997 PA was irrelevant because $\mathrm{UO}_{2}$ is the innermost material of the waste packages in Categories 4, 5, 14, and 15 .

Wet Oxic Corrosion. We used a range of $3.00 \times 10^{-6}$ to $2.00 \times 10^{-1} \mathrm{~kg} / \mathrm{m}^{2} \cdot \mathrm{s}$ and a loguniform probability distribution for COR_A_WO in the 1997 PA (see Table 7-14). The M\&O (1995a) used experimental data from Gray et al. 
(1992) and Steward and Gray (1994) to develop the following equation to predict spent fuel alteration in a potential Yucca Mountain repository for TSPA-1995:

$$
\log \left(\mathrm{R}_{\mathrm{SF}}\right)=\mathrm{a}_{0}+\mathrm{a}_{1} / \mathrm{T}+\mathrm{a}_{2} \cdot \log \left(\mathrm{CO}_{3}\right)+\mathrm{a}_{3} \cdot \mathrm{pH}+\varepsilon
$$

In this equation, $\mathrm{R}_{\mathrm{SF}}$ is the spent-fuel alteration rate (in $\mathrm{mg} / \mathrm{m}^{2} \cdot$ day), $\mathrm{T}$ is the temperature $(\mathrm{K}) ; \mathrm{CO}_{3}$ is the total carbonate $\left(\mathrm{H}_{2} \mathrm{CO}_{3}+\mathrm{HCO}_{3}{ }^{-}+\mathrm{CO}_{3}{ }^{2-}\right)$ concentration of the groundwater $(\mathrm{M}), \mathrm{pH}$ is the negative logarithm of the activity of $\mathrm{H}+$ (dimensionless), $\mathrm{a}_{0}, \mathrm{a}_{1}, \mathrm{a}_{2}$, and $\mathrm{a}_{3}$ are constants, and $\varepsilon$ is a term representing uncertainty not included in the preceding terms. First, we obtained a value of $3.65 \times 10^{3} \mathrm{~K}$ for COR_B_WO directly from $\mathrm{a}_{1}$ in M\&O (1995a). Next, we obtained a preliminary range of $3.00 \times 10^{-6}$ to $9.00 \times 10^{-3} \mathrm{~kg} / \mathrm{m}^{2} \cdot \mathrm{s}$ for $\mathrm{A}$ by using this value of $\mathrm{B}$, Steward and Gray's (1994) range of 0.2 to $20 \mathrm{mM}$ for the total carbonate concentration, our estimated range of 4 to 10 for the $\mathrm{pH}$, and $\mathrm{a}_{0}, \mathrm{a}_{2}$, and $\mathrm{a}_{3}$ from $\mathrm{M} \& \mathrm{O}(1995 \mathrm{a})$. Finally, we multiplied $9.00 \times 10^{-3} \mathrm{~kg} / \mathrm{m}^{2} \cdot \mathrm{s}$, the maximum value of this preliminary range, by a factor obtained by dividing $4.00 \times 10^{-3} \mathrm{~m}^{2} / \mathrm{g}$ by $1.00 \times 10^{-1} \mathrm{~m}^{2} / \mathrm{g}$, Gray and Wilson's (1995) range of specific surface areas for commercial spent-fuel, to obtain a final range of $3.00 \times 10^{-6}$ to $2.00 \times 10^{-1}$ $\mathrm{kg} / \mathrm{m}^{2} \cdot \mathrm{s}$ for COR_A_HO. We assumed a loguniform probability distribution for this parameter.

We used a value of $1.00 \times 10^{\circ}$ for COR_C_WO because at the time of the 1997 PA we had no data on the effects of time on the corrosion of $\mathrm{UO}_{2}$.

We used a range of $1.00 \times 10^{-1}$ to $1.00 \times 10^{0}$ for COR_D_WO in the $1997 \mathrm{PA}$ to determine the sensitivity of $\mathrm{UO}_{2}$ corrosion to the dissolved $\mathrm{O}_{2}$ concentration. We would have preferred to have used a range of 0 to $1.00 \times 10^{0}$ or $1.00 \times 10^{-2}$ to $1.00 \times 10^{0}$ for COR_D_WO, but we thought these ranges would cause numerical instability in BRAGFLO_T.

The value of FRCPENWO used in the 1997 PA was irrelevant because $\mathrm{UO}_{2}$ is the innermost material of these waste packages.

Wet Anoxic Corrosion. We used a value of $1.00 \times 10^{-11} \mathrm{~kg} / \mathrm{m}^{2} \cdot \mathrm{s}$ for COR_A_WA in the 1997 PA. First, we assumed that all of the spent-fuel dissolution and unirradiated $\mathrm{UO}_{2}$ observed by Steward and Gray (1994) at their lowest $\mathrm{O}_{2}$ concentrations $(0.3$ and $0.2 \%$ ) resulted from oxidation of U(IV)-bearing solids to U(VI)-bearing solids and dissolved $\mathrm{U}(\mathrm{VI})$ species by oxidizing species formed by $\alpha$ radiolysis of $\mathrm{H}_{2} \mathrm{O}$. The highest rate reported by Steward and Gray (1994) for spent fuel at 0.3 and $0.2 \% \mathrm{O}_{2}$ was $2.83 \mathrm{mg} / \mathrm{m}^{2}$ - day, equivalent to $7.86 \times 10^{-10} \mathrm{~kg} / \mathrm{m}^{2} \cdot \mathrm{s}$. They obtained this rate at their lowest temperatures $\left(23^{\circ} \mathrm{C}\right.$ and $\left.25^{\circ} \mathrm{C}\right)$, their highest total carbonate concentration $(20 \mathrm{mM})$, and a pH of 8.0 (see Run 14 in Table 1 of Steward and Gray, 1994). Next, we estimated that the spent fuel in Category 14 will be exposed to the near-field environment $1020 \mathrm{yr}$ after emplacement in the potential Yucca Mountain repository. (Category 14 comprises commercial spent fuel from PWRs, the spent fuel most similar to that used by Steward and Gray, 1994.) We then estimated that, by 1020 yr after emplacement, the rate of $\alpha$ radiolysis of $\mathrm{H}_{2} \mathrm{O}$ from this fuel (and the concentrations of oxidizing species) will be $1 / 65$ ( 0.0154 times) the rate at $10 \mathrm{yr}$ (the approximate age of the spent fuel used by Steward and Gray, 1994). Finally, we multiplied $7.86 \times 10^{-10} \mathrm{~kg} / \mathrm{m}^{2} \cdot \mathrm{s}$ by 0.0154 and obtained $1 \times 10^{-11} \mathrm{~kg} / \mathrm{m}^{2} \cdot \mathrm{s}$ after rounding to one significant figure.

We used a value of 0 for COR_B_WA because at the time of the 1997 PA we had no data on the effects of temperature on the corrosion of $\mathrm{UO}_{2}$ under anoxic conditions.

We used a value of $1.00 \times 10^{\circ}$ for COR_C_WA in the 1997 PA because we had no data on the effects of time on $\mathrm{UO}_{2}$ corrosion.

The value of FRCPENWA used in the 1997 PA was irrelevant because $\mathrm{UO}_{2}$ is the innermost material of these waste packages.

Uranium-Aluminum Alloy. U-Al alloy constitutes the fuel for Category 6 (Advanced Test Reactor). We did not have a formula for this alloy at the time of the 1997 PA. The Al cladding in Category 6 has failed. 
We deactivated the parameters for U-Al alloy in the 1997 PA calculations. We did so because we assumed that U-Al alloy would corrode by the same reactions as those predicted for U (Tables 7-2 and 7-3), and that it would corrode at the same rates (see Table 7-12).

Uranium Silicide Alloy. U-Si alloy constitutes the fuel for Category 7 (Materials Testing Reactor). We did not have a formula for this alloy at the time of the $1997 \mathrm{PA}$. The Al cladding in Category 7 is intact or mainly intact, but we assumed that it will have failed by the time of exposure to the near-field environment for the 1997 PA.

We used values of COR_A_HO, COR_A_WO, and COR_WA equal to one-tenth the values used for $U$, and values of all of the other kinetic parameters equal to those for $U$ (compare Tables 7-12 and 7-15). We did so because we assumed that U-Si alloy will corrode by the same reactions as those predicted for U (Tables 7-2 and 7-3), but that it would corrode at one-tenth the rate.

Table 7-15. Kinetic Parameters Used for Uranium Silicide Alloy in the 1997 PA

\begin{tabular}{|c|c|c|c|}
\hline Parameter & Humid Oxic Corrosion & Wet Oxic Corrosion & Wet Anoxic Corrosion \\
\hline $\begin{array}{l}A\left(\mathrm{~kg} / \mathrm{m}^{2} \cdot \mathrm{s}\right) \text { in } \\
\text { Eqs. } 7-3 a, b, c\end{array}$ & $\begin{array}{c}\text { COR_A_HO } \\
1.35 \times 10^{1}\end{array}$ & $\begin{array}{c}\text { COR_A_WO_W } \\
2.85 \times 10^{12}\end{array}$ & $\begin{array}{c}\text { COR_A_WA }= \\
9.40 \times 10^{2}\end{array}$ \\
\hline $\begin{array}{l}\text { B (Kelvin) in } \\
\text { Eqs. } 7-3 a, b, c\end{array}$ & $\underset{7.24 \times 10^{3}}{\text { COR_B_HO }}=$ & $\underset{1.659 \times 10^{4}}{\text { COR_B_WO }}=$ & $\underset{7.97 \times 10^{3}}{\text { COR_B_WA }}=$ \\
\hline $\begin{array}{l}C \text { (dimensionless) in } \\
\text { Eqs. } 7-3 a, b, c\end{array}$ & $\underset{1.00 \times 10^{0}}{\text { COR_C_HO }}=$ & $\begin{array}{c}\text { COR_C_WO } \\
1.00 \times 10^{\circ}\end{array}$ & $\underset{1.00 \times 10^{0}}{\text { COR_C_WA }}=$ \\
\hline $\begin{array}{l}D \text { (dimensionless) in } \\
\text { Eqs. } 7-3 a, b\end{array}$ & $\underset{1.00 \times 10^{0}}{\text { COR_D_HO }}=$ & $\underset{1.00 \times 10^{0}}{\text { COR_D_WO }}=$ & Not applicable \\
\hline $\begin{array}{c}\text { FRCPEN } \\
\text { (dimensionless) }^{\mathrm{a}}\end{array}$ & FRCPENHO = 0 & FRCPENWO $=0$ & FRCPENWA $=0$ \\
\hline \multicolumn{4}{|c|}{ The mass fraction of a layer corroded uniformly at the time of penetration of that layer by localized attack. } \\
\hline
\end{tabular}

Thorium-Uranium Carbide. Th-U carbide constitutes the fuel for Categories 8 (Fort St. Vrain) and 9 (Peach Bottom). ${ }^{*}$ The composition of the former fuel is $\mathrm{Th}_{0.952} \mathrm{U}_{0.048} \mathrm{C}_{2}$. We did not have a formula for the latter fuel at the time of the 1997 PA, so we assumed it had the same composition. The TRISO cladding in Category 8 is intact or mainly intact. (TRISO is the only cladding for which we have not assumed failure by the time of exposure to the near-field environment.) The BISO cladding in Category 9 is $65 \%$ intact, but we assumed that all of it will have failed by the time of exposure to the near-field environment.

We used a setting of 0 for CORMOD in the 1997 PA. This activated Equation 7-3a for humid oxic conditions, Equation 7-3b for wet oxic conditions, and Equation 7-3c for wet anoxic conditions.

Humid Oxic Corrosion. We used the same values for COR_A_HO, COR_B_HO, and COR_C_HO in the 1997 PA (see Table 7-16) as in the 1994 PA. Stockman et al. (1995) described the methods used to establish these values. However, we should have used values of $8.43 \times 10^{-8} \mathrm{~kg} / \mathrm{m}^{2} \cdot \mathrm{s}$ and $2.92 \times 10^{-8} \mathrm{~kg} / \mathrm{m}^{2} \cdot \mathrm{s}$ for COR_A_HO for Categories 8 and 9, respectively (see below).

Stockman et al. (1995) established a constant value of $1.00 \times 10^{-9} \mathrm{~s}^{-1}$ for COR_A_HO (and COR_A_WO) in the 1994 PA. At the request of INEEL personnel, they assumed that all of the Th-U carbide would dissolve in $17 \mathrm{yr}$ after exposure to the near-field environment regardless of conditions, divided 1 by $17 \mathrm{yr}$ to obtain $0.0588 \mathrm{yr}^{1}$, converted $0.0588 \mathrm{yr}^{-1}$ to $1.86 \times 10^{-9} \mathrm{~s}^{-1}$, and rounded this to $1.00 \times 10^{-9} \mathrm{~s}^{-1}$ (the "nearest order of magnitude"). For the 1995 $\mathrm{PA}$, which was never completed nor documented, Stockman established a value of $1.00 \times 10^{-6} \mathrm{~kg} / \mathrm{m}^{2} \cdot \mathrm{s}$ for

- Note that this fuel is labeled Th-U carbide here because of its content; elsewhere it is cited at U-Th carbide, per Appendix A. 
COR_A_WO (and COR_A_WA) by multiplying $1.86 \times 10^{-9} \mathrm{~s}^{-1}$ (the value of COR_A_HO and COR_A_WO established for the $1994 \mathrm{PA}$ prior to rounding) by $560 \mathrm{~kg}$, the mass of radioisotopes in Th-U carbide per waste package for Categories 8 (Fort St. Vrain) and 9 (Peach Bottom) from Lotts et al. (1992), rounding the product to $1.00 \times 10^{-6} \mathrm{~kg} / \mathrm{s}$, and assuming that this $560 \mathrm{~kg}$ of fuel has a surface area of $1.00 \mathrm{~m}^{2}$ to obtain $1.00 \times 10^{-6} \mathrm{~kg} / \mathrm{m}^{2} \cdot \mathrm{s}$. (For the 1994 and 1995 PAs, Categories 8 and 9 constituted one category.) Stockman then either changed COR_A_WO and COR_A_WA from $1.00 \times 10^{-9} \mathrm{~m}^{-2} \cdot \mathrm{s}^{-1}$ to $1.00 \times 10^{-6} \mathrm{~kg} / \mathrm{m}^{2} \cdot \mathrm{s}$, but forgot to change COR_A_HO; or did not change COR_A_HO for some forgotten reason.

For the $1997 \mathrm{PA}$, we continued to assume that all of the Th-U carbide fuel will dissolve in $17 \mathrm{yr}$ after exposure to the near-field environment regardless of conditions. However, instead of multiplying $1.86 \times 10^{-9} \mathrm{~m}^{-2} \cdot \mathrm{s}^{-1}$ (the value of COR_A_HO and COR_A_WO established for the 1994 PA prior to rounding) by $560 \mathrm{~kg}$ (the mass of radioisotopes in Th-U carbide per waste package for Categories 8 and 9 used in the 1995 PA), we should have multiplied $1.86 \times 10^{-9} \mathrm{~m}^{-2} \cdot \mathrm{s}^{-1}$ by $45.3 \mathrm{~kg}$, the mass of radioisotopes of $\mathrm{Th}, \mathrm{U}$, and $\mathrm{Pu}$ in $\mathrm{Th}_{0.952} \mathrm{U}_{0.048} \mathrm{C}_{2}$ in the year 2035 , to obtain $8.43 \times 10^{-8} \mathrm{~kg} / \mathrm{m}^{2} \cdot \mathrm{s}$, for Category 8 , and we should have multiplied $1.86 \times 10^{-9} \mathrm{~m}^{-2} \cdot \mathrm{s}^{-1}$ by $15.7 \mathrm{~kg}$, the mass of radioisotopes of Th, $U$, and $P u$ in Th-U carbide in the year 2035 , to obtain $2.92 \times 10^{-8} \mathrm{~kg} / \mathrm{m}^{2} \cdot \mathrm{s}$ for Category 9 . We obtained values of 45.3 and $15.7 \mathrm{~kg}$ by dividing 24,680 and $1,615 \mathrm{~kg}$, the total inventory of radioisotopes of Th, $\mathrm{U}$, and $\mathrm{Pu}$ in Categories 8 and 9 in the year 2035, respectively (see Table 3-3), by 545 and 103 waste packages, the number of packages in Categories 8 and 9 , respectively.

We could not obtain estimates of the surface areas of the Th-U carbide spent fuels from INEEL personnel for the 1997 PA (see "Surface-area term"). Therefore, we assumed that the surface area of these materials was $1.00 \mathrm{~m}^{2}$ per waste package. However, we should have used values of 2.89 and $1.00 \mathrm{~m}^{2}$ per waste package for Categories 8 and 9 , respectively. We should have assumed that the surface area of Category 9 is $1.00 \mathrm{~m}^{2}$ per waste package, divided $45.3 \mathrm{~kg}$ by $15.7 \mathrm{~kg}$ to obtain 2.89 , the ratio of the mass of radioisotopes of $\mathrm{Th}, \mathrm{U}$, and $\mathrm{Pu}$ in $\mathrm{Th}_{0.952} \mathrm{U}_{0.048} \mathrm{C}_{2}$ in Category 8 in the year 2035 to the mass of radioisotopes of Th, U, and Pu in Th-U carbide in Category 9 in 2035, and multiplied 2.89 by $1.00 \mathrm{~m}^{2}$, the surface area per waste package assumed for Category 9 , to obtain $2.89 \mathrm{~m}^{2}$ per waste package for Category 8 .

We used a value of 0 for COR_D_HO because at the time of the 1997 PA we had no data on the effect of $\mathrm{O}_{2}$ concentration on the corrosion of Th- $U$ carbide.

The value of FRCPENHO used in the 1997 PA was irrelevant because Th-U carbide is the innermost material of the waste packages in Categories 8 and 9.

Wet Oxic Corrosion. We used a value of $1.00 \times 10^{-6} / \mathrm{m}^{2} \cdot \mathrm{s}$ for COR_A_WO for Categories 8 and 9 in the 1997 PA (see Table 7-16). However, we should have used values of $8.43 \times 10^{-8} \mathrm{~kg} / \mathrm{m}^{2} \cdot \mathrm{s}$ and $2.92 \times 10^{-8} \mathrm{~kg} / \mathrm{m}^{2} \cdot \mathrm{s}$ for Categories 8 and 9, respectively (see "Humid Oxic Corrosion" in this section). We should have used these values because we assumed that all of the Th-U carbide fuel would dissolve in $17 \mathrm{yr}$ after exposure to the near-field environment regardless of conditions.

Furthermore, we assumed that the surface area of these materials was $1.00 \mathrm{~m}^{2}$ per waste package. However, we should have used values of 2.89 and $1.00 \mathrm{~m}^{2}$ per waste package for Categories 8 and 9, respectively (see "Humid Oxic Corrosion" in this section).

We used the same values for COR_B_WO and COR_C_WO in the 1997 PA (see Table 7-16) as in the 1994 PA. Stockman et al. (1995) described the methods used to establish these values.

We used a value of 0 for COR_D_WO because at the time of the 1997 PA we had no data on the effect of $\mathrm{O}_{2}$ concentration on the corrosion of thorium-uranium carbide.

The value of FRCPENWO used in the 1997 PA was irrelevant because thorium-uranium carbide is the innermost material of these waste packages.

Wet Anoxic Corrosion. We used a value of $1.00 \times 10^{-6} / \mathrm{m}^{2} \cdot \mathrm{s}$ for COR_A_WA for Categories 8 and 9 in the 1997 $\mathrm{PA}$ (see Table 7-16). However, we should have used values of $8.43 \times 10^{-8} \mathrm{~kg} / \mathrm{m}^{2} \cdot \mathrm{s}$ and $2.9 \times 10^{-8} \mathrm{~kg} / \mathrm{m}^{2} \cdot \mathrm{s}$ for Cate- 
Table 7-16. Kinetic Parameters Used for Uranium-Thorium Carbide in the 1997 PA

\begin{tabular}{|c|c|c|c|}
\hline Parameter & Humid Oxic Corrosion & Wet Oxic Corrosion & Wet Anoxic Corrosion \\
\hline $\begin{array}{l}A\left(\mathrm{~kg} / \mathrm{m}^{2} \bullet \mathrm{s}\right) \text { in } \\
\text { Eqs. } 7-3 a, b, c\end{array}$ & $\begin{array}{c}\text { COR_A_HO } \\
1.00 \times 10^{-9}\end{array}$ & $\begin{array}{c}\text { COR_A_WO } \\
1.00 \times 10^{-6}\end{array}$ & $\begin{array}{c}\text { COR_A_WA }= \\
1.00 \times 10^{-6}\end{array}$ \\
\hline $\begin{array}{l}\text { B (Kelvin) in } \\
\text { Eqs. 7-3a, b, c }\end{array}$ & COR_B_HO $=0$ & COR_B_WO $=0$ & COR_B_WA $=0$ \\
\hline $\begin{array}{c}C \text { (dimensionless) in } \\
\text { Eqs. } 7-3 a, b, c\end{array}$ & $\underset{1.00 \times 10^{0}}{\text { COR_C_HO }}=$ & $\begin{array}{c}\text { COR_C_WO } \\
1.00 \times 10^{0}\end{array}$ & $\underset{1.00 \times 10^{0}}{\text { COR_C_WA }}=$ \\
\hline $\begin{array}{c}D \text { (dimensionless) in } \\
\text { Eqs. } 7-3 a, b\end{array}$ & COR_D_HO $=0$ & COR_D_WO $=0$ & Not applicable \\
\hline $\begin{array}{c}\text { FRCPEN } \\
\text { (dimensionless) }^{2}\end{array}$ & FRCPENHO $=0$ & FRCPENWO $=0$ & FRCPENWA $=0$ \\
\hline mass fraction of & rroded uniformly at th & penetration of that layer by & ized attack. \\
\hline
\end{tabular}

gories 8 and 9, respectively (see "Humid Oxic Corrosion" in this section). We should have used these values because we assumed that all of the Th-U carbide fuel will dissolve in $17 \mathrm{yr}$ after exposure to the near-field environment regardless of conditions.

Furthermore, we assumed that the surface area of these materials was $1.00 \mathrm{~m}^{2}$ per waste package. However, we should have used values of 2.89 and $1.00 \mathrm{~m}^{2}$ per waste package for Categories 8 and 9, respectively (see "Humid Oxic Corrosion" in this section).

We used a value of 0 for COR_B_WA because at the time of the 1997 PA we had no data on the effects of temperature on the corrosion of Th-U carbide under anoxic conditions.

We used a value of $1.00 \times 10^{\circ}$ for COR_C_WA in the 1997 PA because we had no data on the effects of time on Th-U carbide corrosion.

The value of FRCPENWA used in the 1997 PA was irrelevant because Th- $U$ carbide is the innermost material of these waste packages.

Uranium-Plutonium Carbide. U-Pu carbide constitutes the fuel for Category 10 (Sodium Reactor Experiment). We did not have a formula for this alloy at the time of the 1997 PA. The stainless-steel cladding in Category 10 is intact or mainly intact, but we assumed that it will have failed by the time of exposure to the near-field environment for the 1997 PA.

We deactivated the parameters for U-Pu carbide in the 1997 PA calculations. We did so because we assumed that $\mathrm{U}-\mathrm{Pu}$ carbide would corrode by the same reactions as those predicted for $\mathrm{Th}_{0.952} \mathrm{U}_{0.048} \mathrm{C}_{2}$ (Tables 7-2 and 7-3), and that it would corrode at the same rates (see Table 7-16). However, we should have used a value of $2.12 \times 10^{-8} \mathrm{~kg} /$ $m^{2} \cdot s$ for COR_A_HO, COR_A_WO, and COR_A_WA for Category 10 (see below).

For the 1997 PA, we assumed that U-Pu carbide would corrode at the same rates as Th-U carbide (see "Humid Oxic Corrosion" in this section and Table 7-16). Stockman et al. (1995) assumed that all of the Th-U carbide would dissolve in $17 \mathrm{yr}$ after exposure to the near-field environment regardless of conditions, obtained a value of $1.86 \times 10^{-9} \mathrm{~m}^{-2} \cdot \mathrm{s}^{-1}$ for COR_A_HO and COR_A_WO, and rounded it to $1.00 \times 10^{-9} \mathrm{~m}^{-2} \cdot \mathrm{s}^{-1}$ for the 1994 PA. Stockman established a value of $1.00 \times 10^{-6} \mathrm{~kg} / \mathrm{m}^{2} \cdot \mathrm{s}$ for COR_A_WO and COR_A_WA for the 1995 PA by multiplying $1.86 \times 10^{-9} \mathrm{~m}^{-2} \cdot \mathrm{s}^{-1}$ (the value of COR_A_HO and COR_A_WO established for the 1994 PA prior to rounding) by $560 \mathrm{~kg}$, the mass of radioisotopes in Th-U carbide per waste package, and rounding the product to $1.00 \times 10^{-6} \mathrm{~kg} / \mathrm{m}^{2} \cdot \mathrm{s}$. Stockman then changed COR_A_WO and COR_A_WA from $1.00 \times 10^{-9} \mathrm{~m}^{-2} \cdot \mathrm{s}^{-1}$ to $1.00 \times 10^{-6} \mathrm{~kg} / \mathrm{m}^{2} \cdot \mathrm{s}$, but did not change COR_A_HO. 
For the $1997 \mathrm{PA}$, we continued to assume that all of the Th-U and U-Pu carbide fuels will dissolve in $17 \mathrm{yr}$ after exposure to the near-field environment regardless of conditions. However, instead of multiplying $1.86 \times 10^{-9}$ $\mathrm{m}^{-2} \cdot \mathrm{s}^{-1}$ (the value of COR_A_HO and COR_A_WO established for the 1994 PA prior to rounding) by $560 \mathrm{~kg}$ (the mass of radioisotopes in Th-U carbide per waste package for Categories 8 and 9 used in the 1995 PA), we should have multiplied $1.86 \times 10^{-9} \mathrm{~m}^{-2} \cdot \mathrm{s}^{-1}$ by $11.4 \mathrm{~kg}$, the mass of radioisotopes of $U$ and $\mathrm{Pu}$ in U-Pu carbide in the year 2035 , to obtain $2.12 \times 10^{-8} \mathrm{~kg} / \mathrm{m}^{2} \cdot \mathrm{s}$ for Category 10 . We obtained a value of 11.4 by dividing $57 \mathrm{~kg}$, the total inventory of radioisotopes of $U$ and Pu in Category 10 in the year 2035 (see Table 3-3) by 5 waste packages, the number of packages in Category 10.

We could not obtain an estimate of the surface areas of the U-Pu carbide spent fuel from INEEL personnel for the 1997 PA (see "Surface-Area Term"). Therefore, we assumed that the surface area of these materials was $1.00 \mathrm{~m}^{2}$ per waste package. However, we should have used a value of 2.89 and $1.00 \mathrm{~m}^{2}$ per waste package for Waste Categories 8 and 9, respectively. We should have assumed that the surface area of Category 9 is $1.00 \mathrm{~m}^{2}$ per waste package, divided $11.4 \mathrm{~kg}$ by $15.7 \mathrm{~kg}$ to obtain 0.726 , the ratio of the mass of radioisotopes of $U$ and Pu in U-Pu carbide in Category 10 in the year 2035 to the mass of radioisotopes of Th, $U$, and $\mathrm{Pu}$ in Th- $U$ carbide in Category 9 in 2035, and multiplied 0.726 by $1.00 \mathrm{~m}^{2}$, the surface area per waste package assumed for Category 9 , to obtain $0.726 \mathrm{~m}^{2}$ per waste package for Category 10.

Mixed-Oxide Fuel. $\left(\mathrm{U}_{\mathrm{x}} \mathrm{Pu}_{1-\mathrm{x}}\right) \mathrm{O}_{2}$ constitutes the fuel for Category 11 (Fast Flux Test Facility). We used the formula $\left(\mathrm{U}_{\mathrm{x}} \mathrm{Pu}_{1-\mathrm{x}}\right) \mathrm{O}_{2}$ for mixed-oxide fuel because at the time of the $1997 \mathrm{PA}$ we had no data on the relative quantities of $\mathrm{U}$ and $\mathrm{Pu}$ in this fuel. The stainless-steel cladding in Category 11 is intact or mainly intact, but we assumed that it will have failed by the time of exposure to the near-field environment for the $1997 \mathrm{PA}$.

We deactivated the parameters for $\left(\mathrm{U}_{\mathrm{x}} \mathrm{Pu}_{1-\mathrm{x}}\right) \mathrm{O}_{2}$ in the $1997 \mathrm{PA}$ calculations. We did so because we assumed that $\left(\mathrm{U}_{\mathrm{x}} \mathrm{Pu}_{1-\mathrm{x}}\right) \mathrm{O}_{2}$ will corrode by the same reactions as those predicted for $\mathrm{UO}_{2}$ (Tables 7-2 and 7-3), and that it will corrode at the same rates (see Table 7-14).

Thorium-Uranium Oxide. $\left(\mathrm{Th}_{\mathrm{x}} \mathrm{U}_{1-\mathrm{x}}\right) \mathrm{O}_{2}$ constitutes the fuel for Category 12 (Shippingport Light Water Breeder Reactor). ${ }^{\dagger}$ We assumed that $\mathrm{x}=1$ and used the formula $\mathrm{ThO}_{2}$ for this fuel because at the time of the $1997 \mathrm{PA}$ we had no data on the relative quantities of Th and $U$. The Zircaloy- 2 cladding in Category 12 is intact or mainly intact, but we assumed that it will have failed by the time of exposure to the near-field environment for the $1997 \mathrm{PA}$.

This is the only material for which we used a setting of 2 for CORMOD in the 1997 PA. Therefore, the sourceterm model assumed instantaneous solubility equilibrium between $\mathrm{ThO}_{2}$ in the fuel and unspecified dissolved $\mathrm{Th}(\mathrm{IV})$ species. (CST does not predict the aqueous speciation of any of the radionuclides in the waste.) (Furthermore, the corrosion submodel assumed that the dissolution of $\mathrm{ThO}_{2}$ did not affect the near-field $\mathrm{H}_{2} \mathrm{O}$, gas, nor $\mathrm{O}_{2}$ contents.) Finally, the source-term model assumed solubility-limited advective and/or diffusive transport of ${ }^{229} \mathrm{Th},{ }^{230} \mathrm{Th}$, and ${ }^{232} \mathrm{Th}$ out of these waste packages.

Uranium-Zirconium Hydride. U-Zr hydride constitutes the fuel for Category 13 (General Atomic Training, Research, and Production Reactor). We did not have a formula for this alloy at the time of the 1997 PA. The stainless-steel cladding in Category 13 is intact or mainly intact, but we assumed that it will have failed by the time of exposure to the near-field environment for the $1997 \mathrm{PA}$.

We used values of COR_A_HO, COR_A_WO, and COR_WA equal to one-tenth the values used for $\mathrm{UO}_{2}$, and values of all of the other kinetic parameters equal to those for $\mathrm{UO}_{2}$ (compare Tables 7-14 and 7-16). We did so because we assumed that $U-\mathrm{Zr}$ hydride would corrode by the same reactions as those predicted for $U$ (Tables 7-2 and 7-3), but that it would corrode at one-tenth the rate of $\mathrm{UO}_{2}$.

Borosilicate Glass. In Waste Packages 2 through 13, handling containers of borosilicate glass with DHLW are codisposed with handling containers of spent fuel. There are three (Category 12), four (Categories 3, 5, 10,11), or five (Categories 2, 4, 6,7,8,9, and 13) handling containers of borosilicate glass and one handling container of spent

$\dagger$ Note that this fuel is labeled Th-U oxide here because of its content; elsewhere it is cited as U-Th oxide, per Appendix A. 
fuel per waste package (see Chapter 3). The handling containers of the glass comprise 9.5 -mm-thick $304 \mathrm{~L}$ stainless steel.

We used a setting of 0 for CORMOD in the 1997 PA. This activated Equation 7-3a for humid oxic conditions, Equation 7-3b for wet oxic conditions, and Equation 7-3c for wet anoxic conditions.

Humid Oxic Corrosion. We used the same value for COR_A_HO in the 1997 PA (see Table 7-17) as in the 1995 PA. Because the 1995 PA was never completed nor documented, we cannot explain this value. Therefore, we should have used the same range and probability distribution for $C O R \_A \_H O$ that we used for wet oxic corrosion. We used this range and distribution because we had no data on the corrosion of borosilicate glass under humid oxic conditions at the time of the $1997 \mathrm{PA}$. Therefore, we should have assumed that it would corrode at the same rates under these conditions that we used for wet oxic and wet anoxic conditions in the 1997 PA (see "Wet Oxic Corrosion" in this section).

Table 7-17. Kinetic Parameters Used for Uranium-Zirconium Hydride in the 1997 PA

\begin{tabular}{|c|c|c|c|}
\hline Parameter & Humid Oxic Corrosion & Wet Oxic Corrosion & Wet Anoxic Corrosion \\
\hline $\begin{array}{l}A\left(\mathrm{~kg} / \mathrm{m}^{2} \cdot \mathrm{s}\right) \text { in } \\
\text { Eqs. } 7-3 a, b, c\end{array}$ & $\underset{1.72 \times 10^{-5}}{\text { COR_ACHO }}=$ & $\begin{array}{c}\text { COR_A_WO= } \\
3.00 \times 10^{-7} \text { to } \\
2.00 \times 10^{-2}, \text { loguniform }\end{array}$ & $\begin{array}{c}\text { COR_A_WA }= \\
1.00 \times 10^{-12}\end{array}$ \\
\hline $\begin{array}{l}\text { B (Kelvin) in } \\
\text { Eqs. } 7-3 a, b, c\end{array}$ & $\begin{array}{c}\text { COR_B_HO } \\
5.989 \times 10^{3}\end{array}$ & $\begin{array}{c}\text { COR_B_WO } \\
3.65 \times 10^{3}\end{array}$ & COR_B_WA $=0$ \\
\hline $\begin{array}{l}C \text { (dimensionless) in } \\
\text { Eqs. } 7-3 a, b, c\end{array}$ & $\underset{1.00 \times 10^{0}}{\text { COR_C_HO }}=$ & $\underset{1.00 \times 10^{0}}{\text { COR_C_WO }}=$ & $\underset{1.00 \times 10^{0}}{\text { COR_C_WA }}=$ \\
\hline $\begin{array}{c}D \text { (dimensionless) in } \\
\text { Eqs. } 7-3 a, b\end{array}$ & $\underset{1.00 \times 10^{0}}{\text { COR_D_HO }}=$ & $\begin{array}{c}\text { COR_D_WO }= \\
1.00 \times 10^{-1} \text { to } \\
1.00 \times 10^{0} \text {, uniform }\end{array}$ & Not applicable \\
\hline $\begin{array}{c}\text { FRCPEN } \\
\text { (dimensionless) }^{\mathrm{a}}\end{array}$ & FRCPENHO $=0$ & FRCPENWO $=0$ & FRCPENWA $=0$ \\
\hline fract & 78 & enetration of that layer $b$ & ized attack. \\
\hline
\end{tabular}

We used a value of 0 for COR_D_HO because we had no data on the effect of $\mathrm{O}_{2}$ concentration on the corrosion of borosilicate glass.

The value of FRCPENHO used in the 1997 PA was irrelevant because borosilicate glass is the innermost material of the handling containers containing DHLW in Waste Packages 2 through 13. However, we believe a value of 0 is appropriate for FRCPENHO because thermal stress that occurs during cooling of borosilicate glass after vitrification of DHLW will probably fracture the glass extensively.

Wet Oxic Corrosion. We used the same range and probability distribution for COR_A_WO, and the same constant same values for COR_B_HO and COR_C_HO in the 1997 PA (see Table 7-18) as in the 1994 PA. Stockman et al. (1995) described the methods used to establish these values.

We used a value of 0 for COR_D_WO because at the time of the 1997 PA we had no data on the effect of $\mathrm{O}_{2}$ concentration on the corrosion of borosilicate glass.

The value of FRCPENWO used in the 1997 PA was irrelevant because borosilicate glass is the innermost material of the handling containers containing DHLW in these waste packages. However, we believe a value of 0 is appropriate for FRCPENWO (see "Humid Oxic Corrosion" in this section). 
Table 7-18. Kinetic Parameters Used for Defense High Level Waste Glass in the 1997 PA

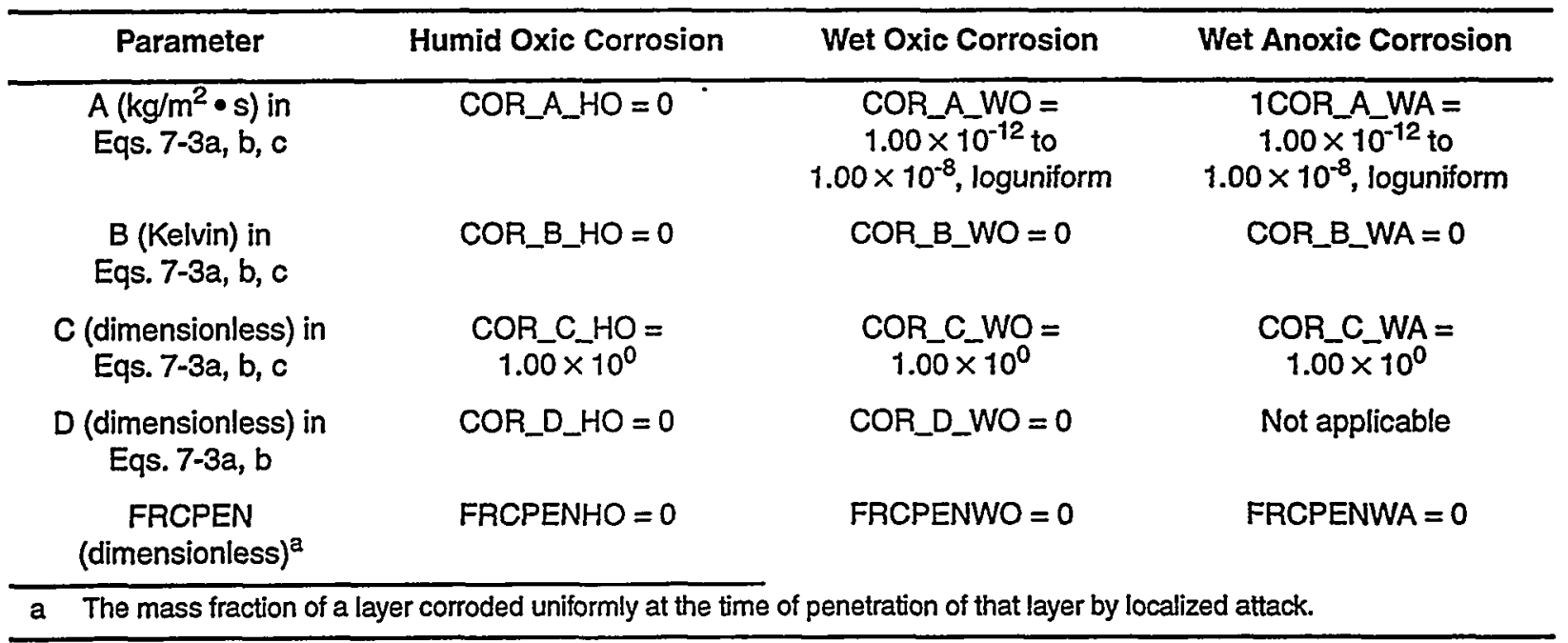

Wet Anoxic Corrosion. We used the same range and probability distribution for COR_A_WA in the 1997 PA (see Table 7-18) as in the 1995 PA. Because the 1995 PA was never completed nor documented, we cannot explain this range and distribution. Therefore, we should have used the same range and distribution for COR_A_WA that we used for wet oxic corrosion. We should have used this range and distribution because we had no data on the corrosion of borosilicate glass under wet anoxic conditions. Consequently, we should have assumed that it would corrode at the same rates under these conditions that we used for wet oxic conditions in the 1997 PA (see "Wet Oxic Corrosion" in this section).

The value of FRCPENWA used in the 1997 PA was irrelevant because borosilicate glass is the innermost material of the handling containers containing DHLW in these waste packages. However, we believe a value of 0 is appropriate for FRCPENWA (see "Humid Oxic Corrosion" in this section). 


\subsection{Summary of Assumptions for Corrosion and Source-Term Conceptual Models}

\section{Corrosion Submodel}

- Sequential failure of corrosion-allowance and corrosion-resistant materials occurs in multilayer packages.

- Early failure occurs with some waste packages, i.e., exposure of radionuclides in the waste to the near-field environment at the time of emplacement due to manufacturing defects, damage during transportation, or damage during emplacement (range of 0 to 0.01 with uniform probability distribution); early failure pertains only to failure of 2-cm-thick Inconel 625 layer.

- Three or four steel supports on a concrete pier on a pre-cast concrete invert hold each waste package at time of emplacement and, possibly, for a significant time thereafter.

- An air gap around waste package (1) ensures effective radiative transfer of heat from waste packages to tuff and (2) prevents transport of groundwater from tuff to the waste packages by capillarity.

- During 100,000-yr regulatory period, rubble will eliminate air gap to some extent between intact host rock and waste packages. If sufficient rubble accumulates and $\mathrm{H}_{2} \mathrm{O}$ content is high enough for fracture flow, capillarity might transport groundwater from partially saturated tuff through rubble to the waste packages.

- Whether or not sufficient rubble accumulates for capillarity determines: (1) mode of contact between waste package and groundwater; (2) threshold value of the $\mathrm{H}_{2} \mathrm{O}$ content of fractures required to initiate this contact, and (3) how much $\mathrm{H}_{2} \mathrm{O}$ was available for corrosion in each time step. Fraction of waste packages with sufficient rubble has a range of 0 to 1 and a uniform probability distribution.

- No other possible modes of contact between waste packages and groundwater were included.

- Oxic corrosion (humid and wet) and anoxic corrosion are considered. For any given metallic or nonmetallic waste package material, humid and wet oxic corrosion reactions are stoichiometrically identical but rates differ.

- Humid anoxic corrosion was not included because concentration of $\mathrm{H}_{2} \mathrm{O}$ in gaseous phase was assumed to be too low to oxidize waste package constituents at significant rates.

\section{Source-Term Submodel}

- Because some non-transported radionuclides decay to transported daughters, decay and ingrowth of nontransported radionuclides are predicted both before and after they are released from waste packages.

- Radioisotopes can be located in matrices, unbound solids, groundwater, and $\mathrm{Fe}_{2} \mathrm{O}_{3}$.

- At time of emplacement, most radioisotopes are assumed to reside in matrices and are homogeneously distributed; these move to unbound solids at same rates as the matrices corrode.

- Some radioisotopes (quick-release fraction) reside on grain boundaries in spent fuel or in gap between spent fuels and cladding; these immediately move to unbound solids.

- Instantaneous, reversible solubility equilibria is assumed between radioisotopes in unbound solids and groundwater in each waste package; instantaneous, reversible, linear sorption equilibria is assumed between radioisotopes in groundwater and on $\mathrm{Fe}_{2} \mathrm{O}_{3}$.

- Residual groundwater is assumed to be always present in waste packages after they fail.

- Different isotopes of the same element have the same solubility and $\mathrm{K}_{\mathrm{D}}$.

- Advective and diffusive transport are included but not coupled.

- Once advection removes radioisotopes from waste packages, they move to intact host rock immediately.

- No sorption of radioisotopes was assumed by $\mathrm{Fe}_{2} \mathrm{O}_{3}$ from corrosion of steel supports beneath waste package.

- Diffusion pathways could occur if sufficient rubble accumulates. 


\subsection{Modeling Parameters for Corrosion and Source-Term Submodels}

Tables 7-19 through 7-34 list the parameters used for the materials in the waste packages for the preliminary performance assessment. The parameter values used in the final 1997 PA are provided in Appendix $D$. 


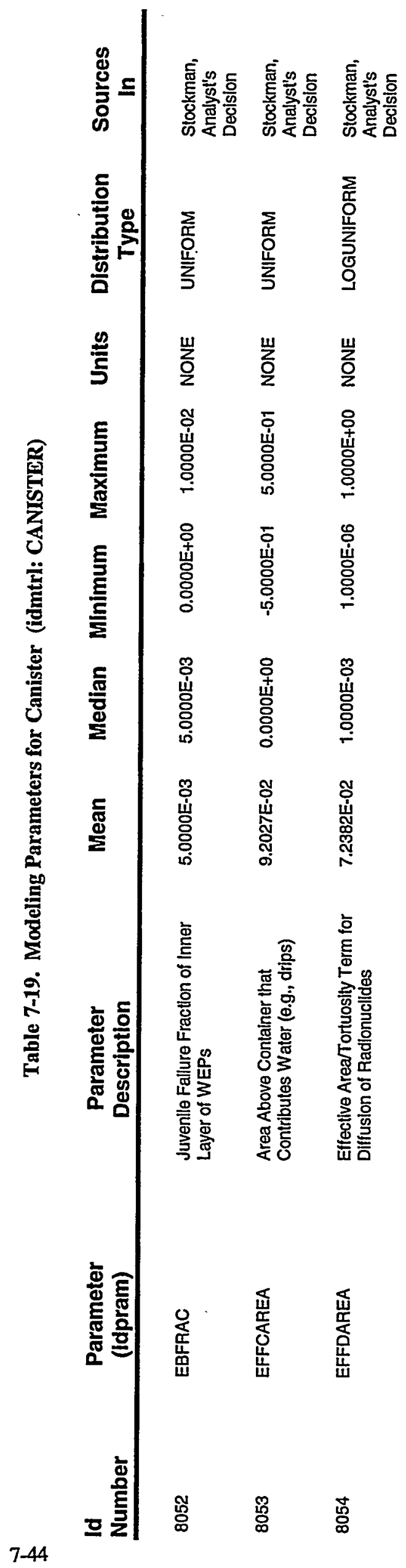

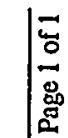




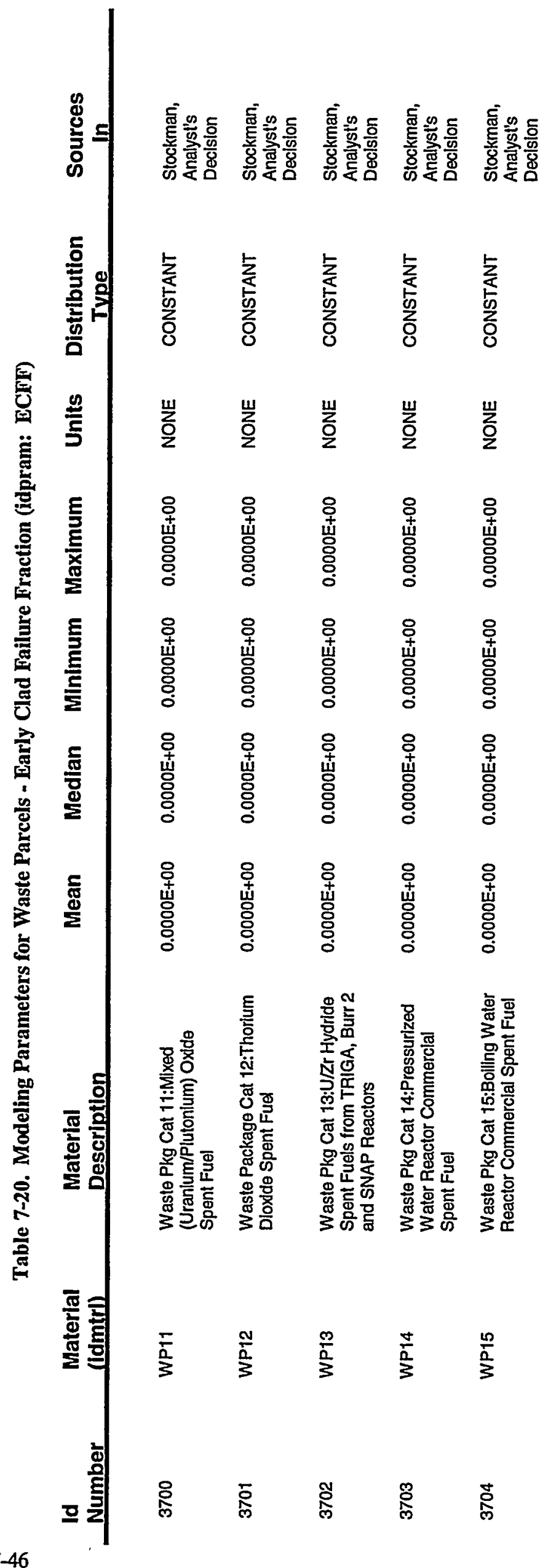




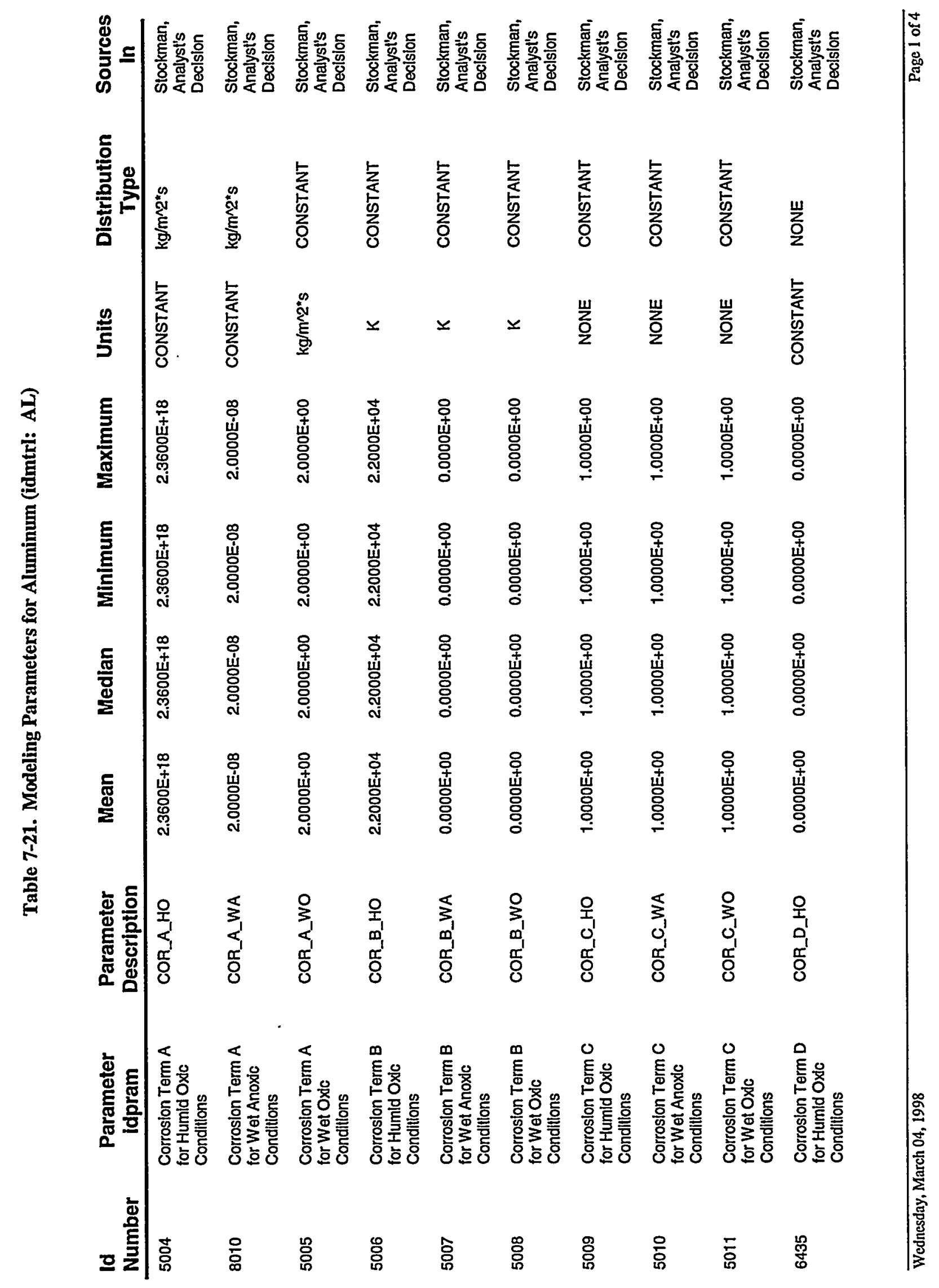




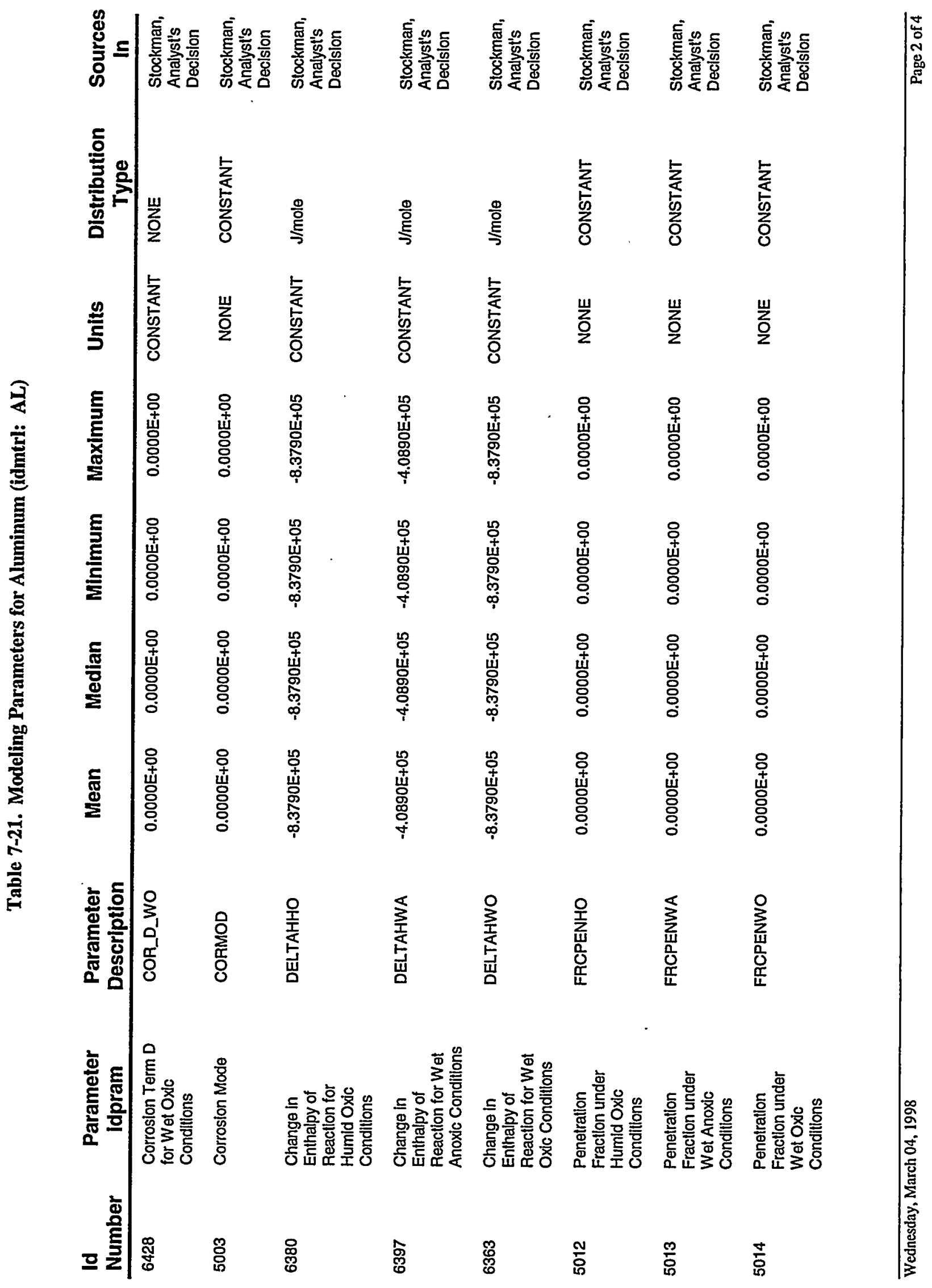




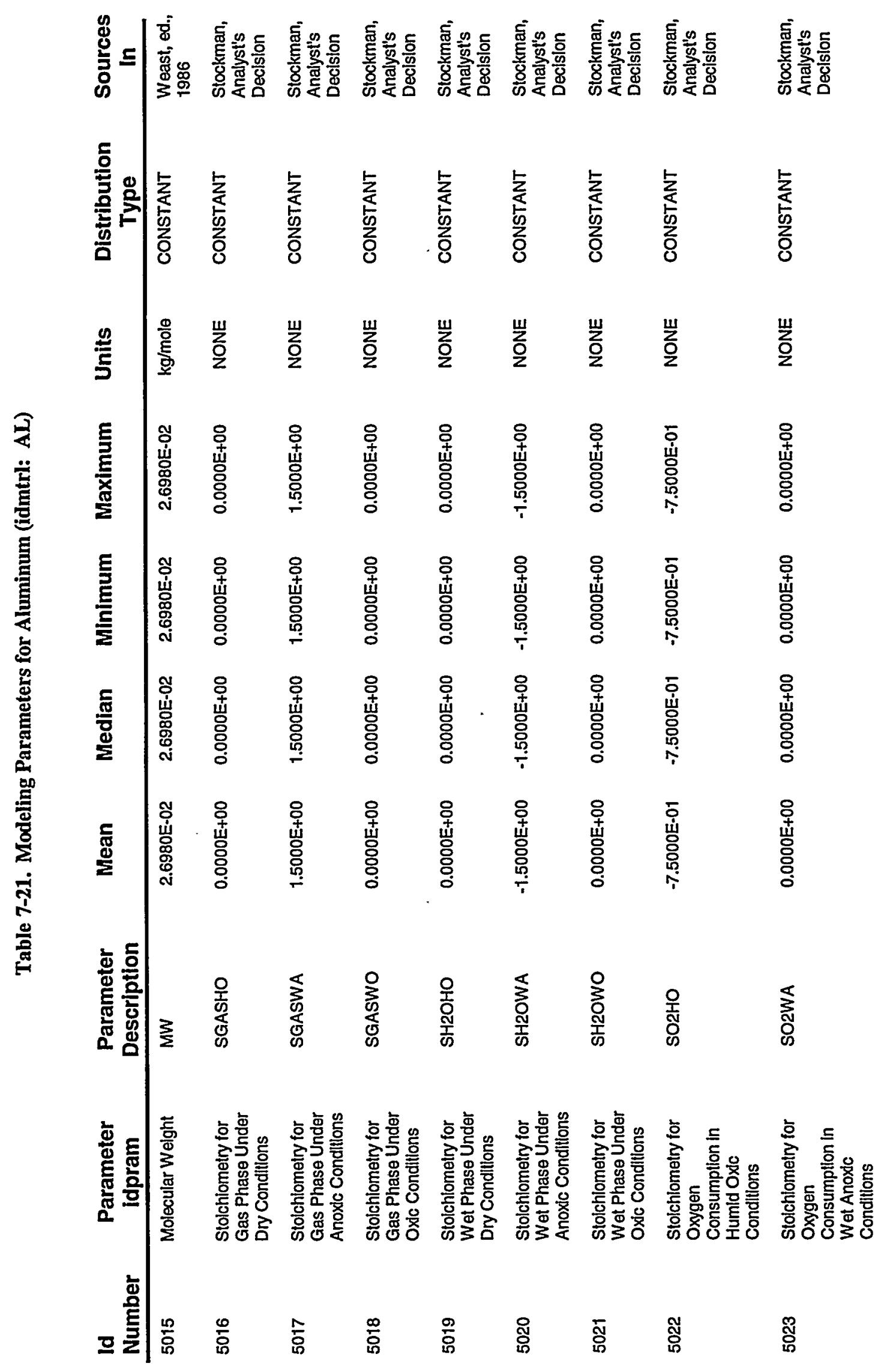



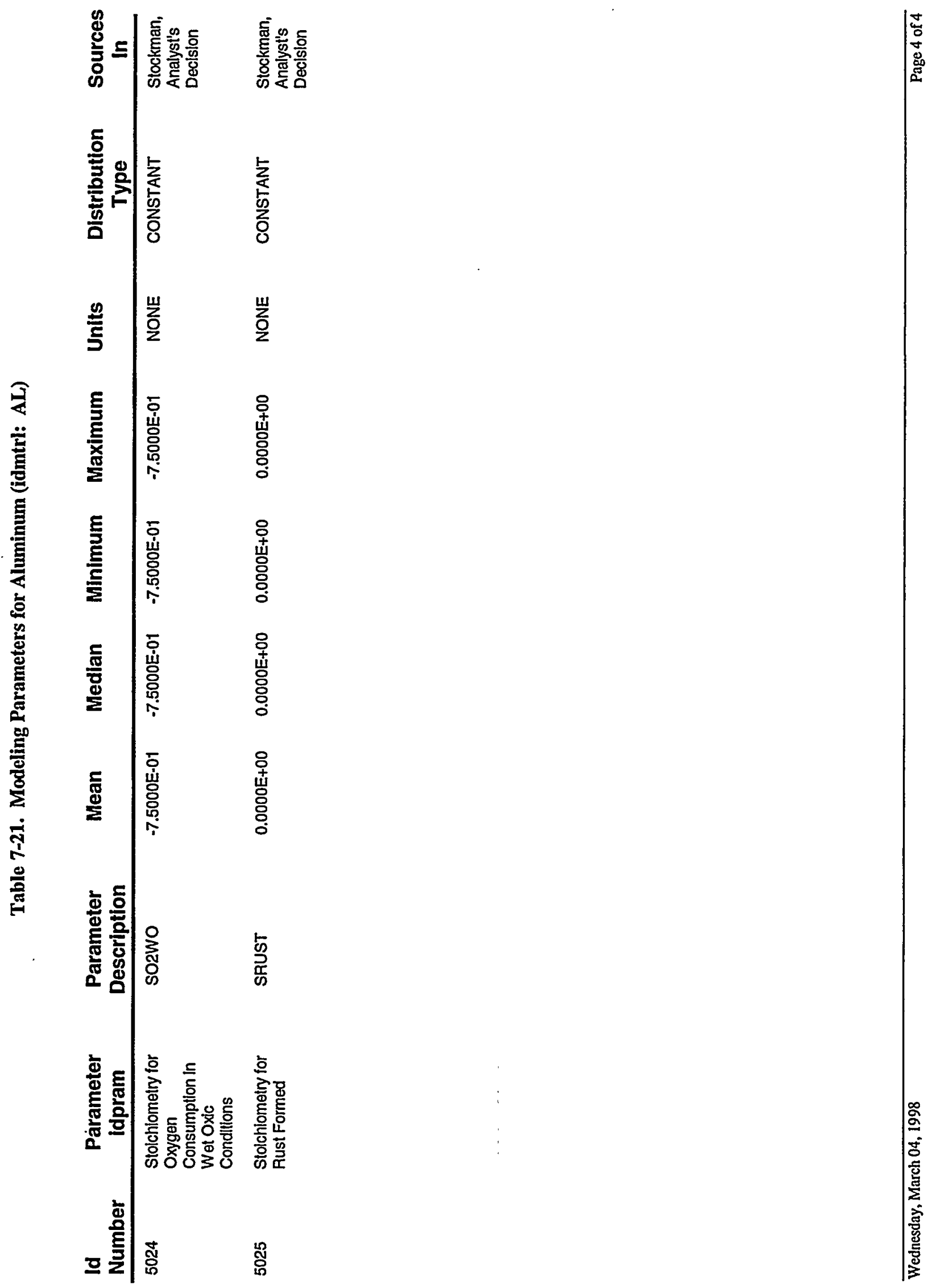


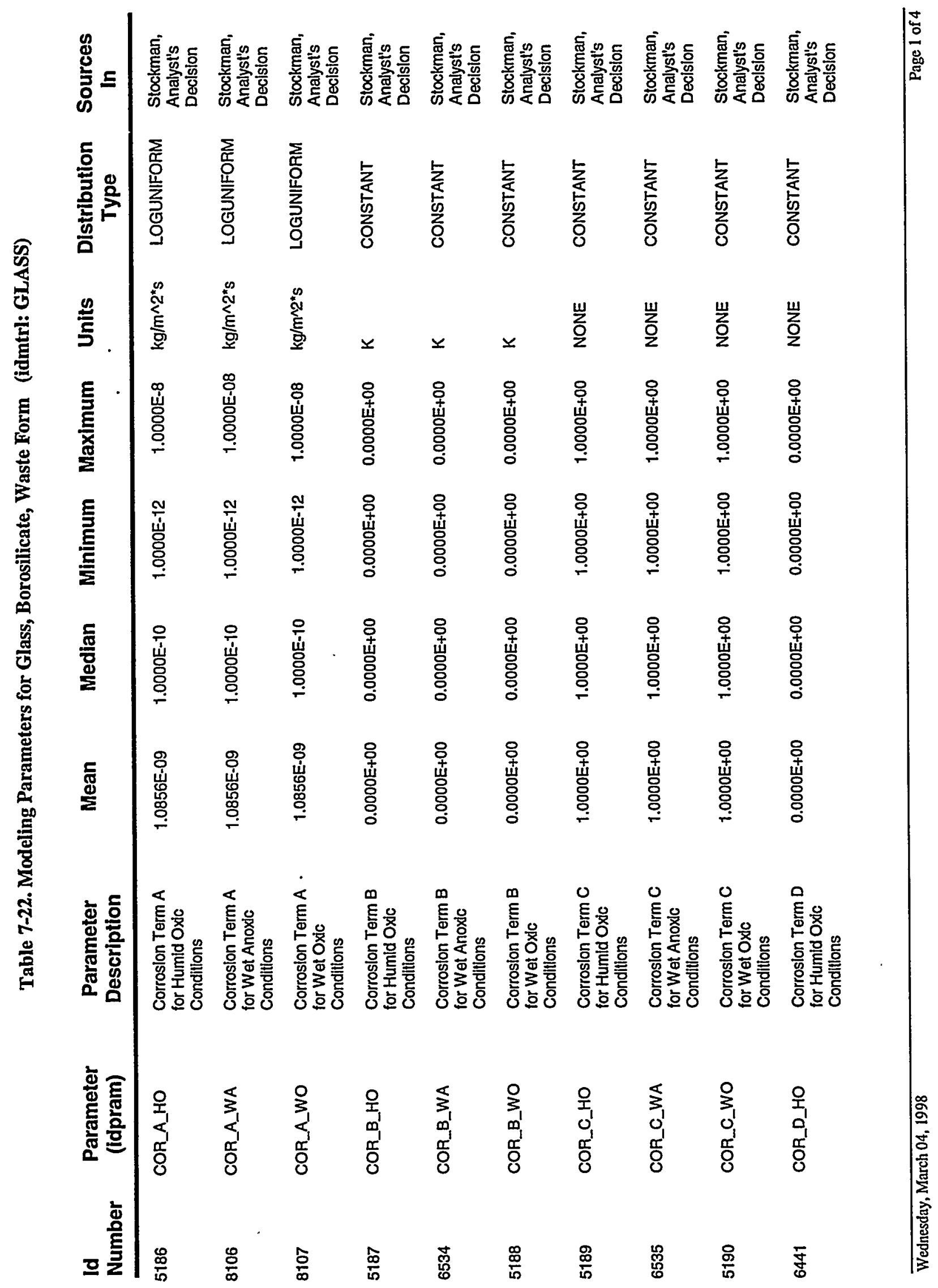




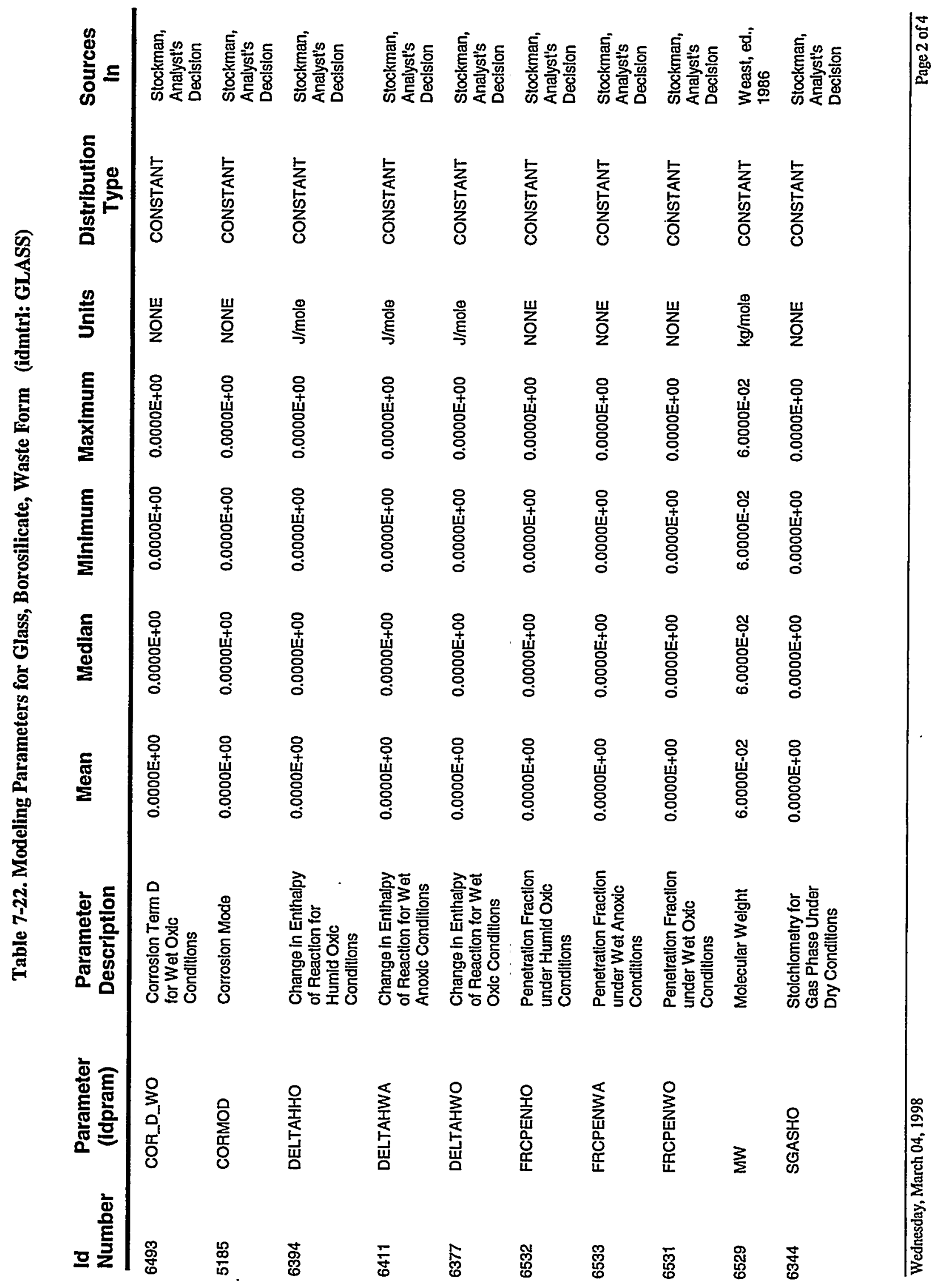




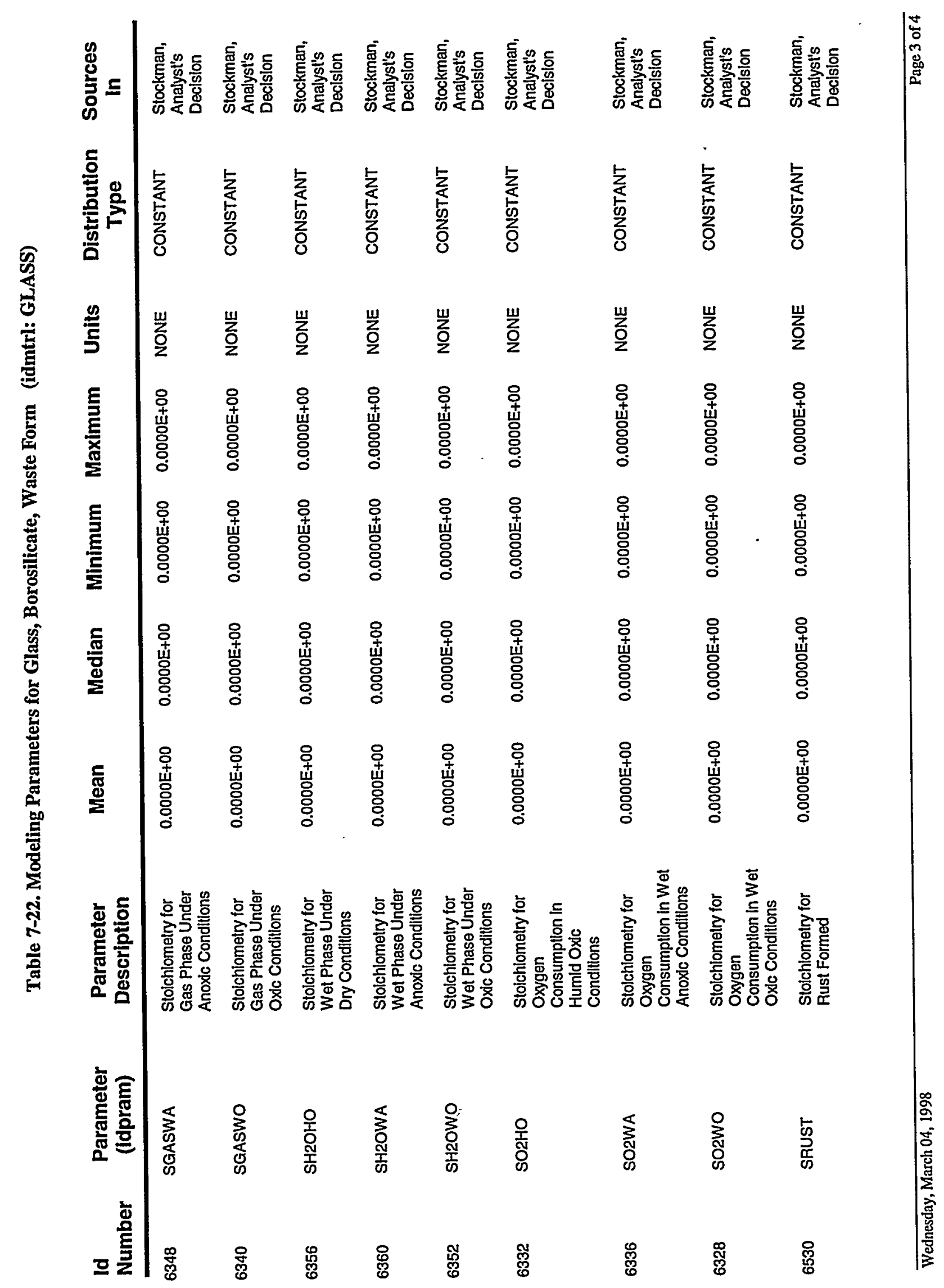



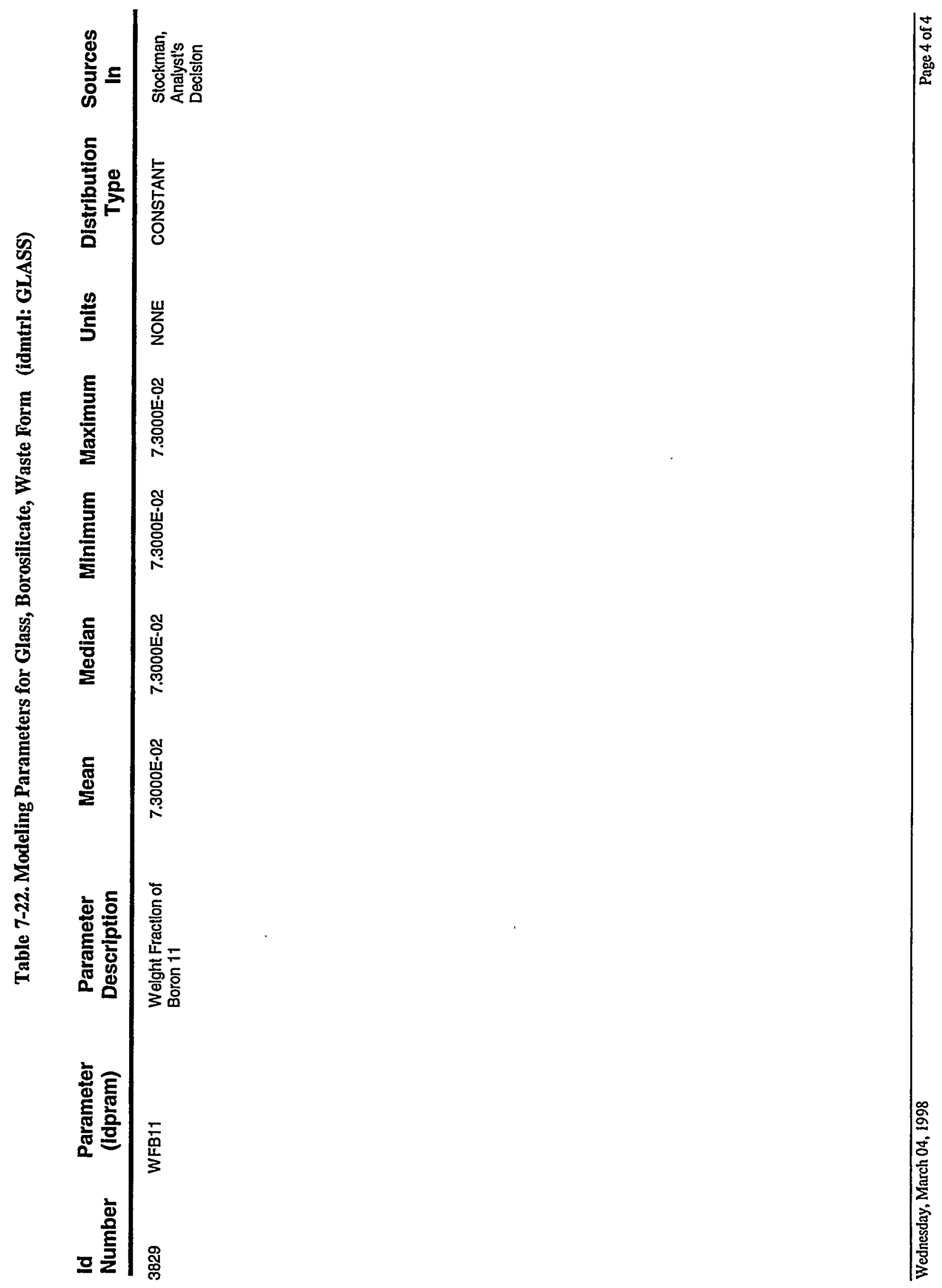


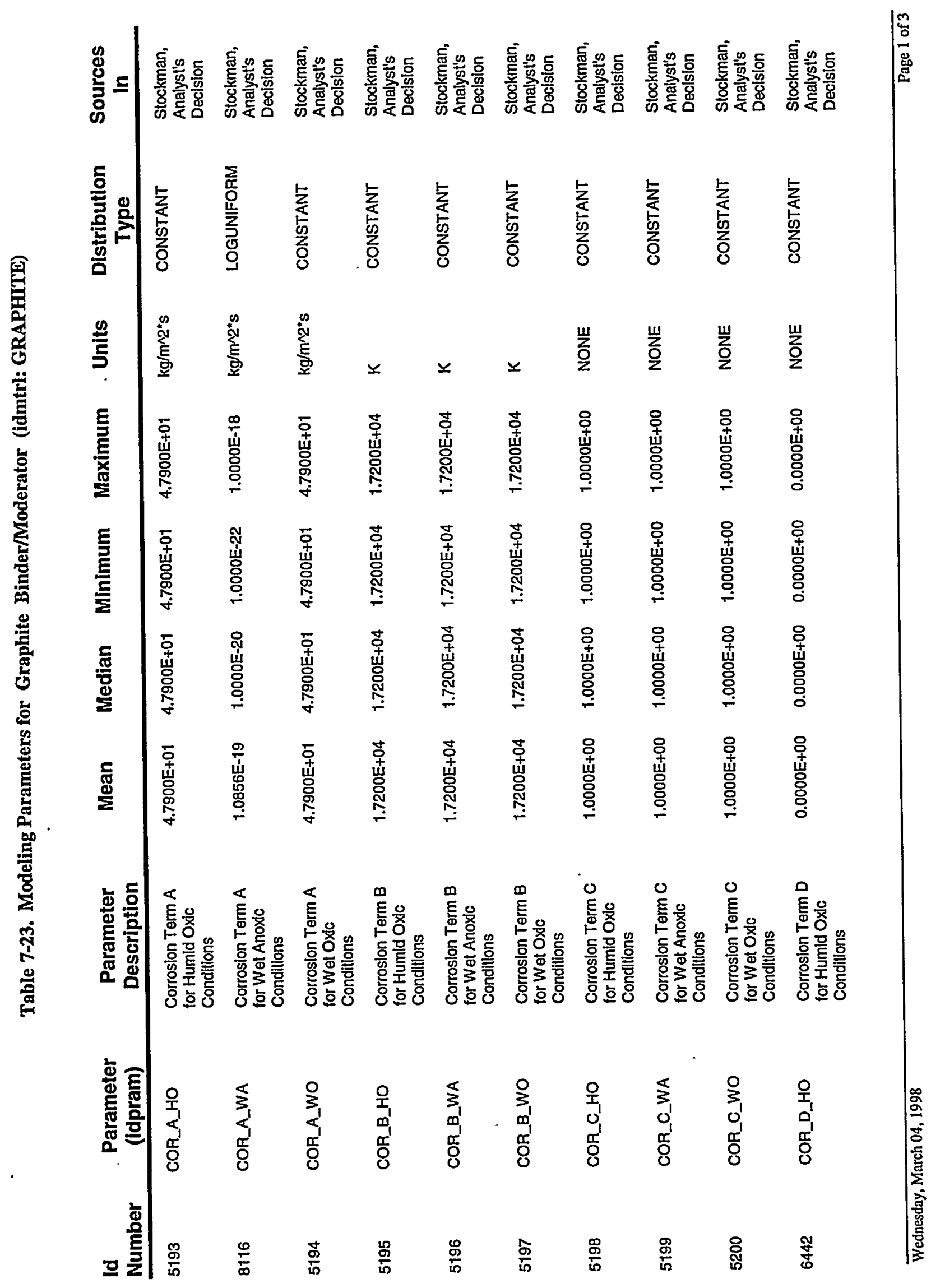




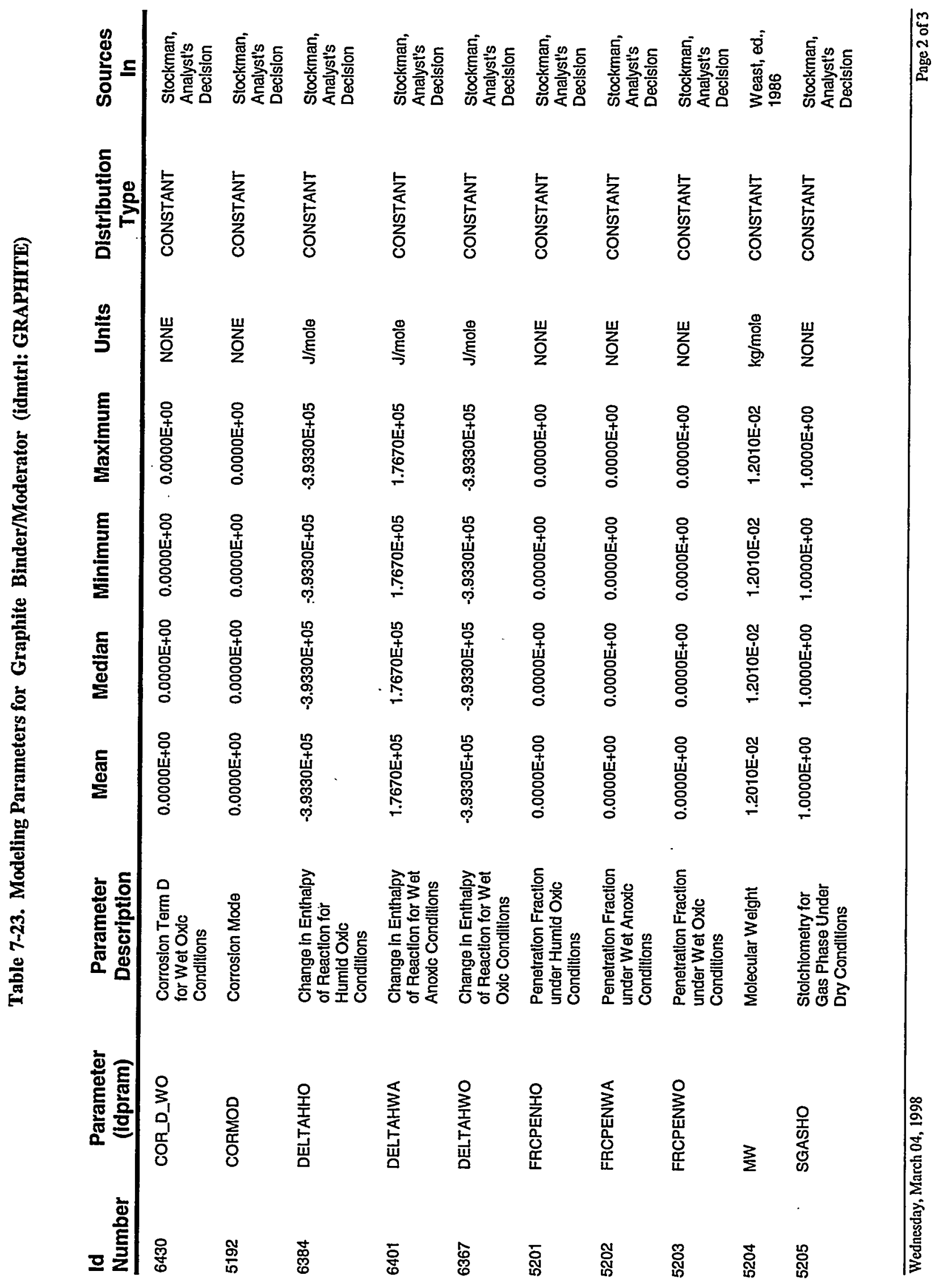




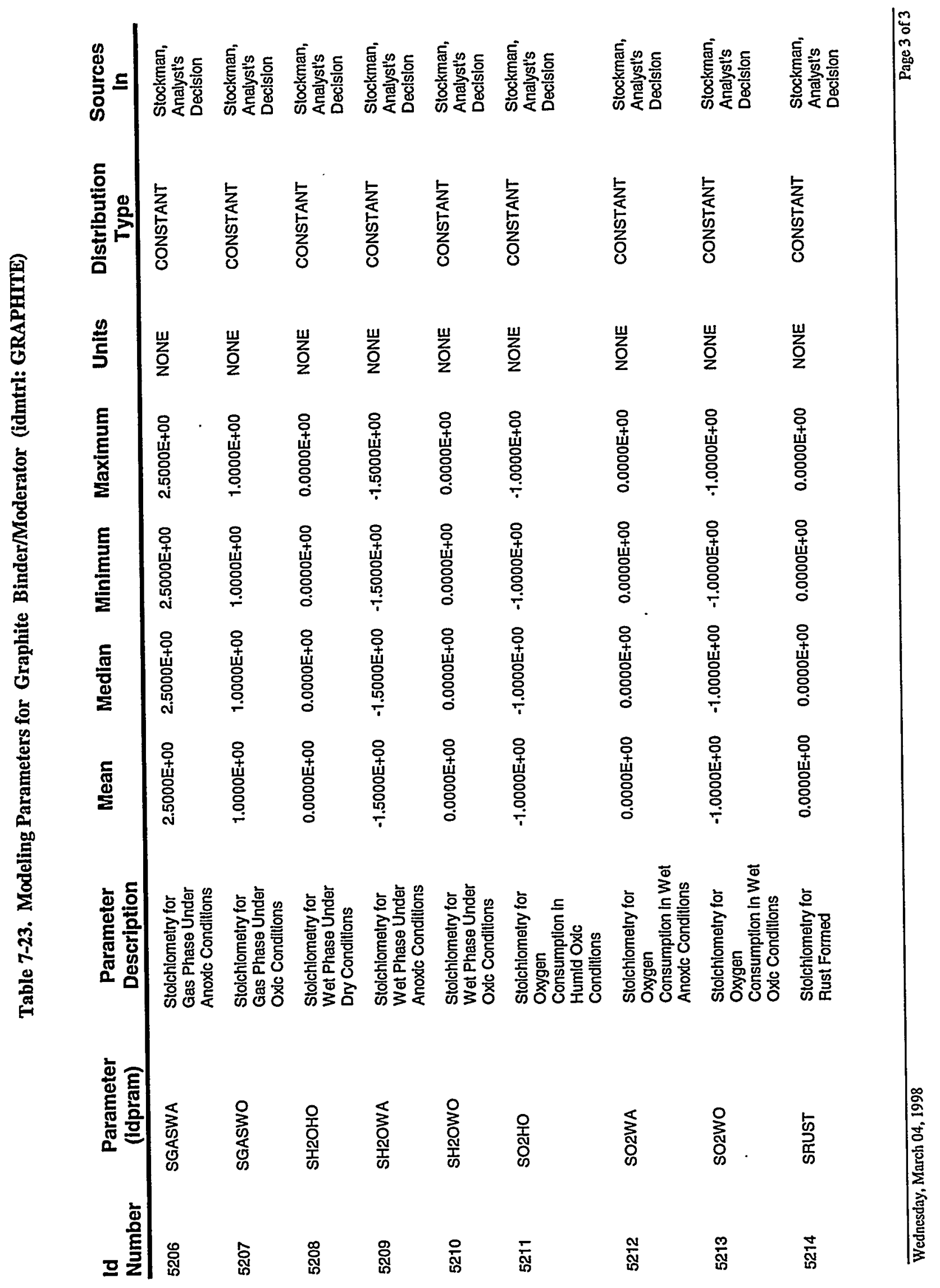




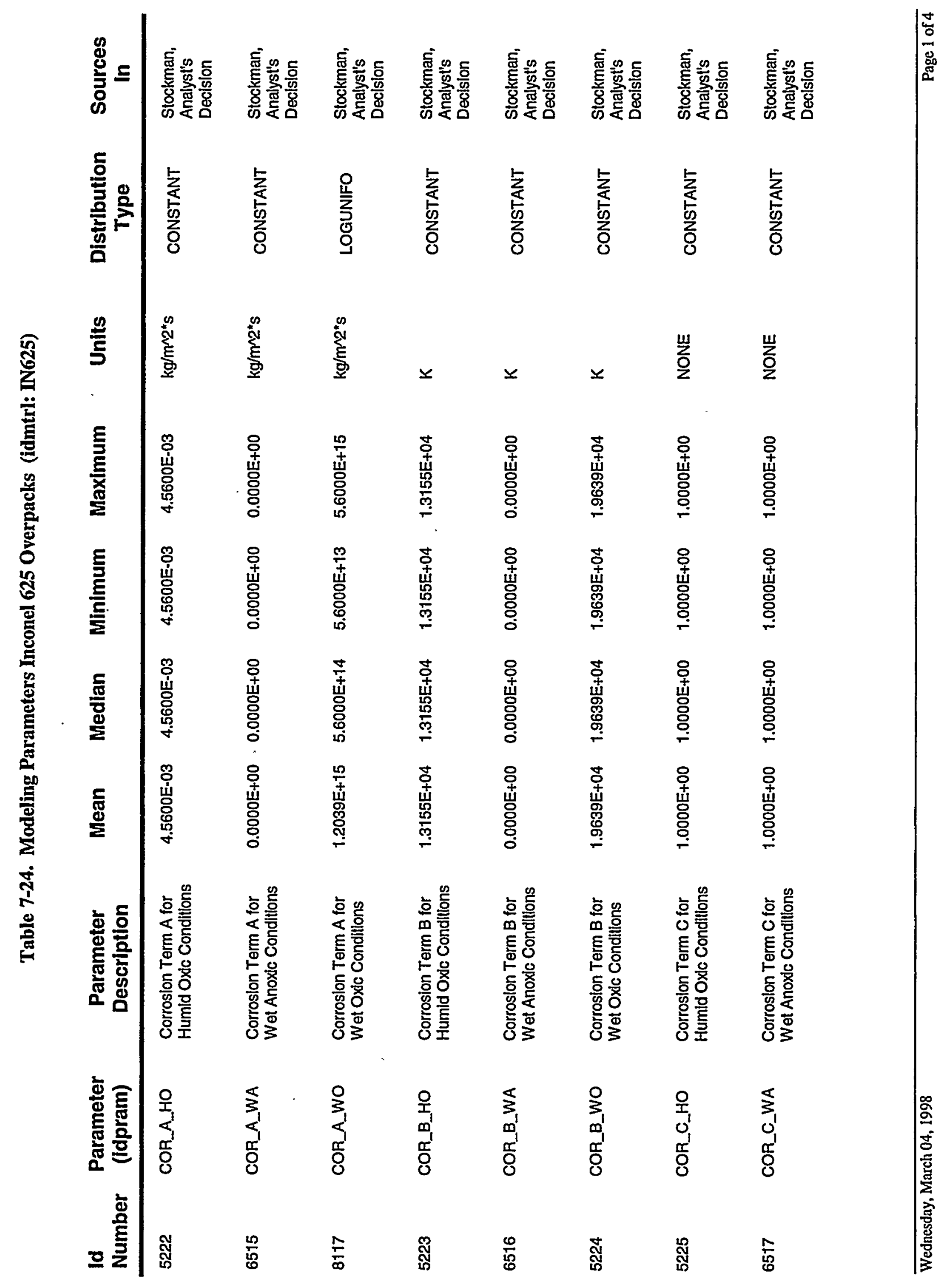




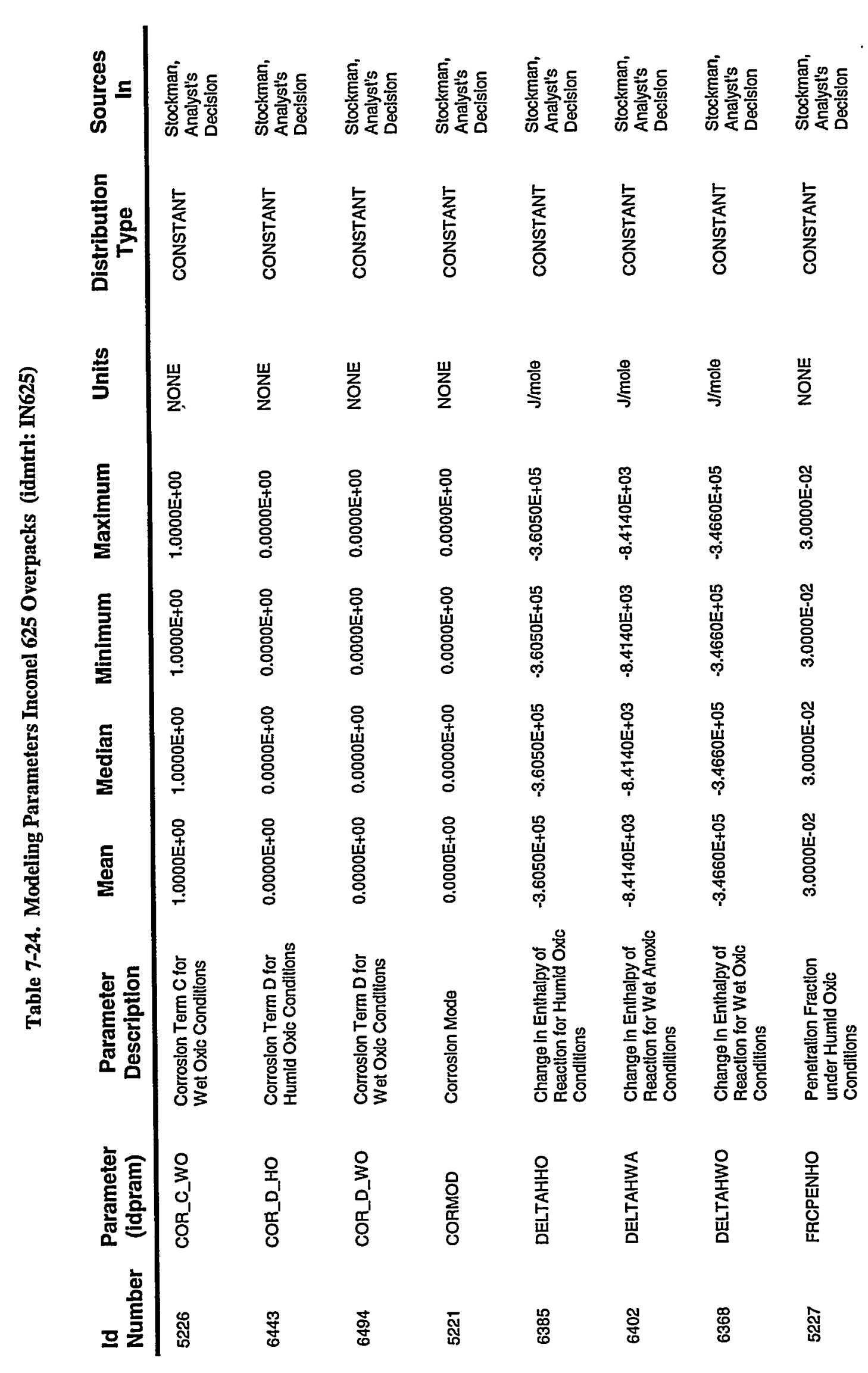




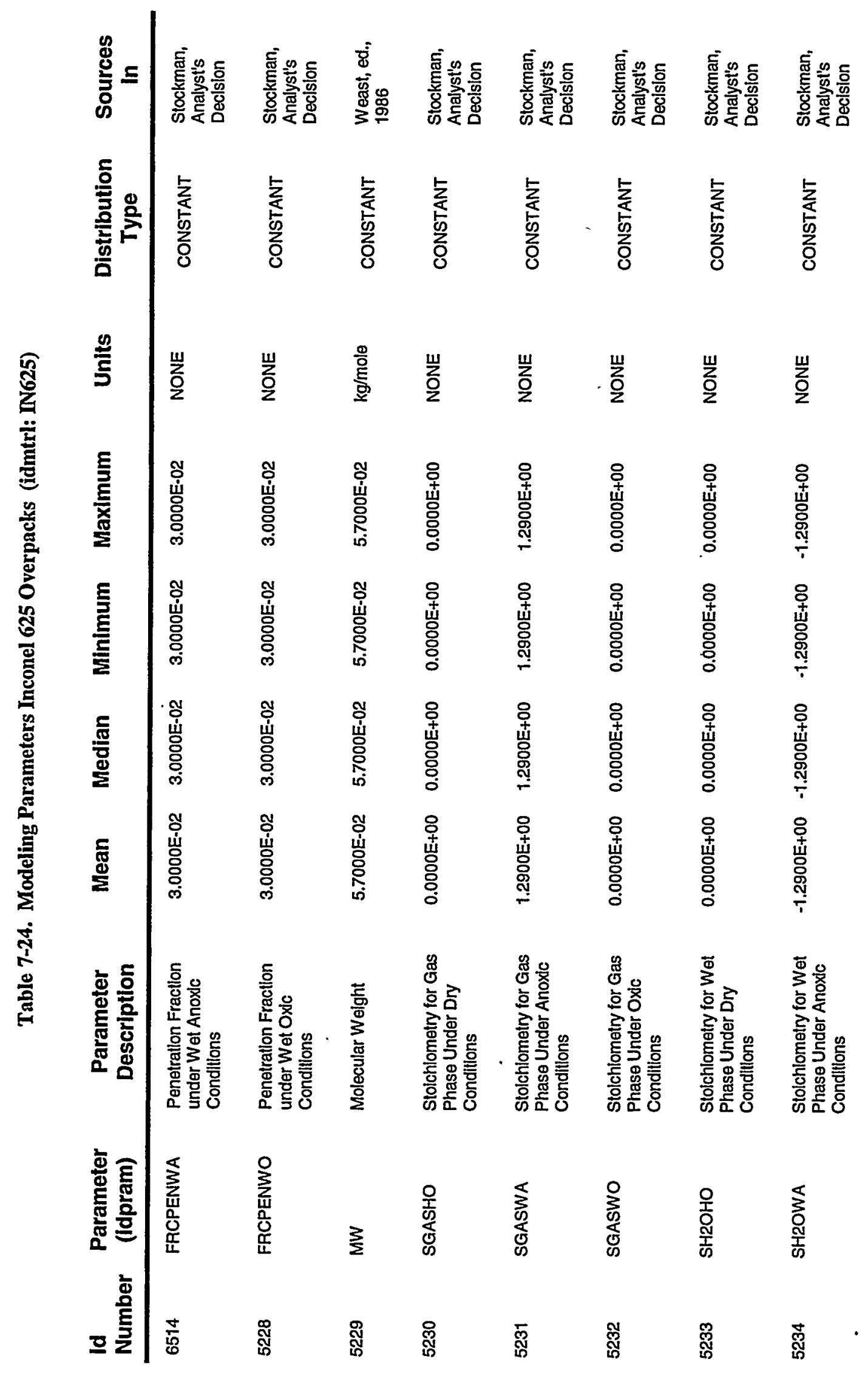




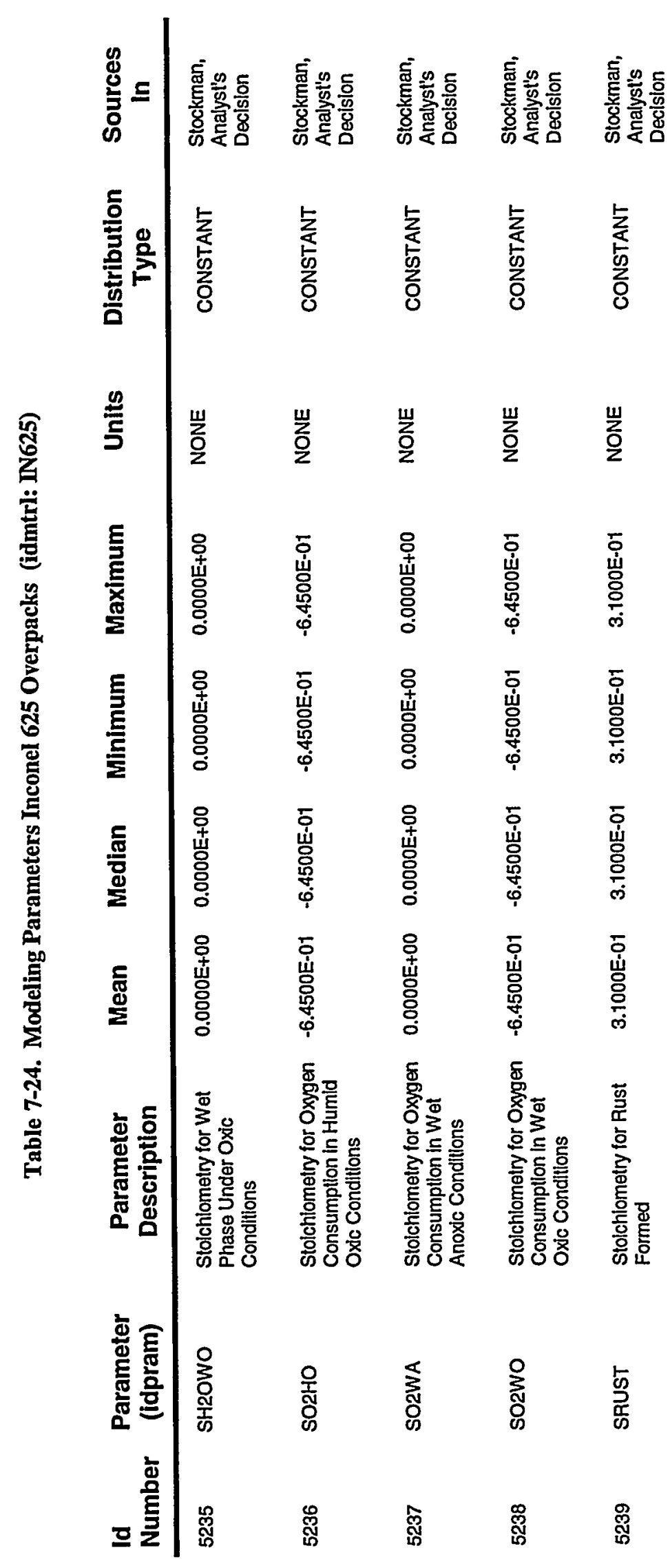

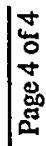




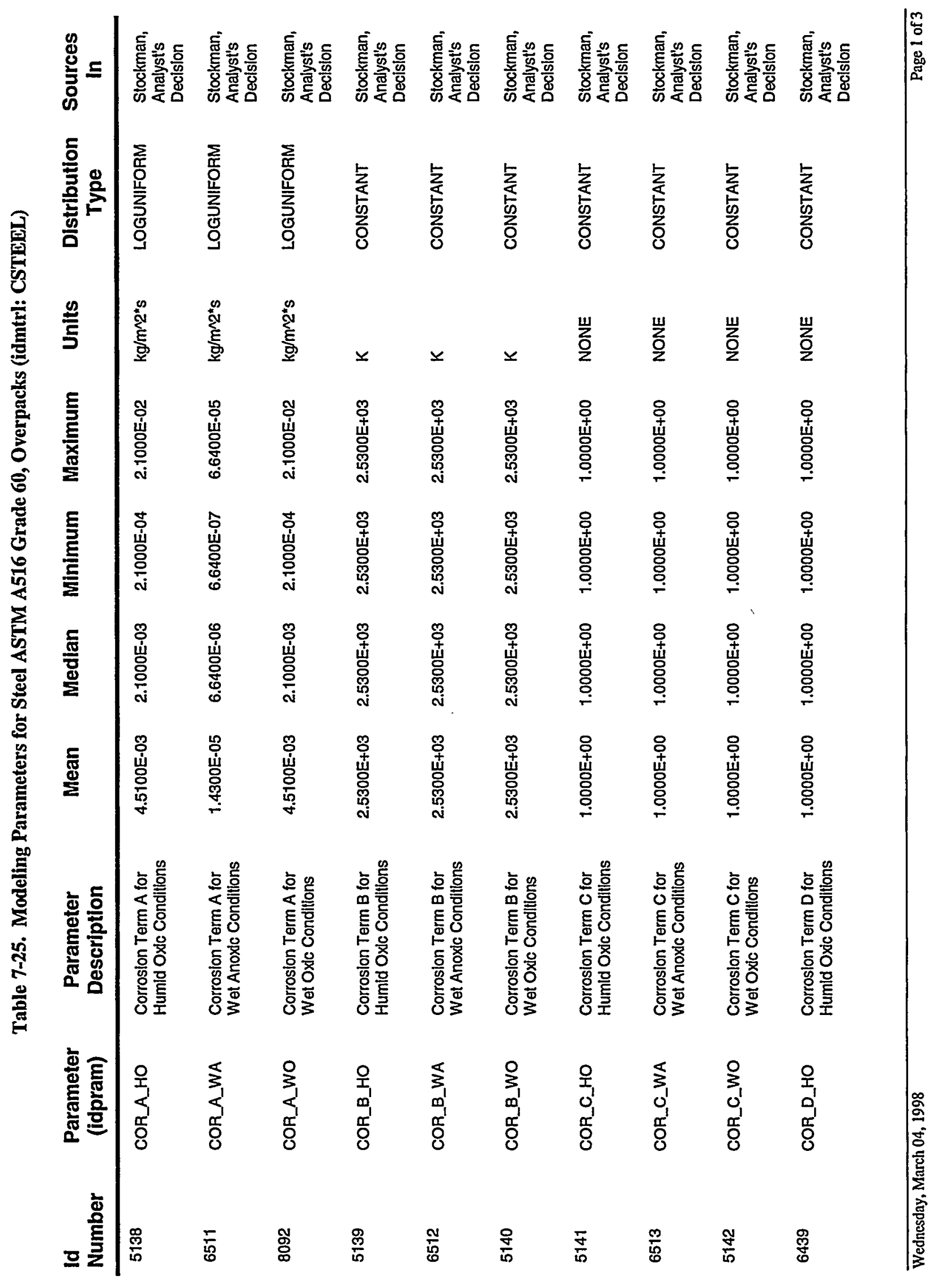




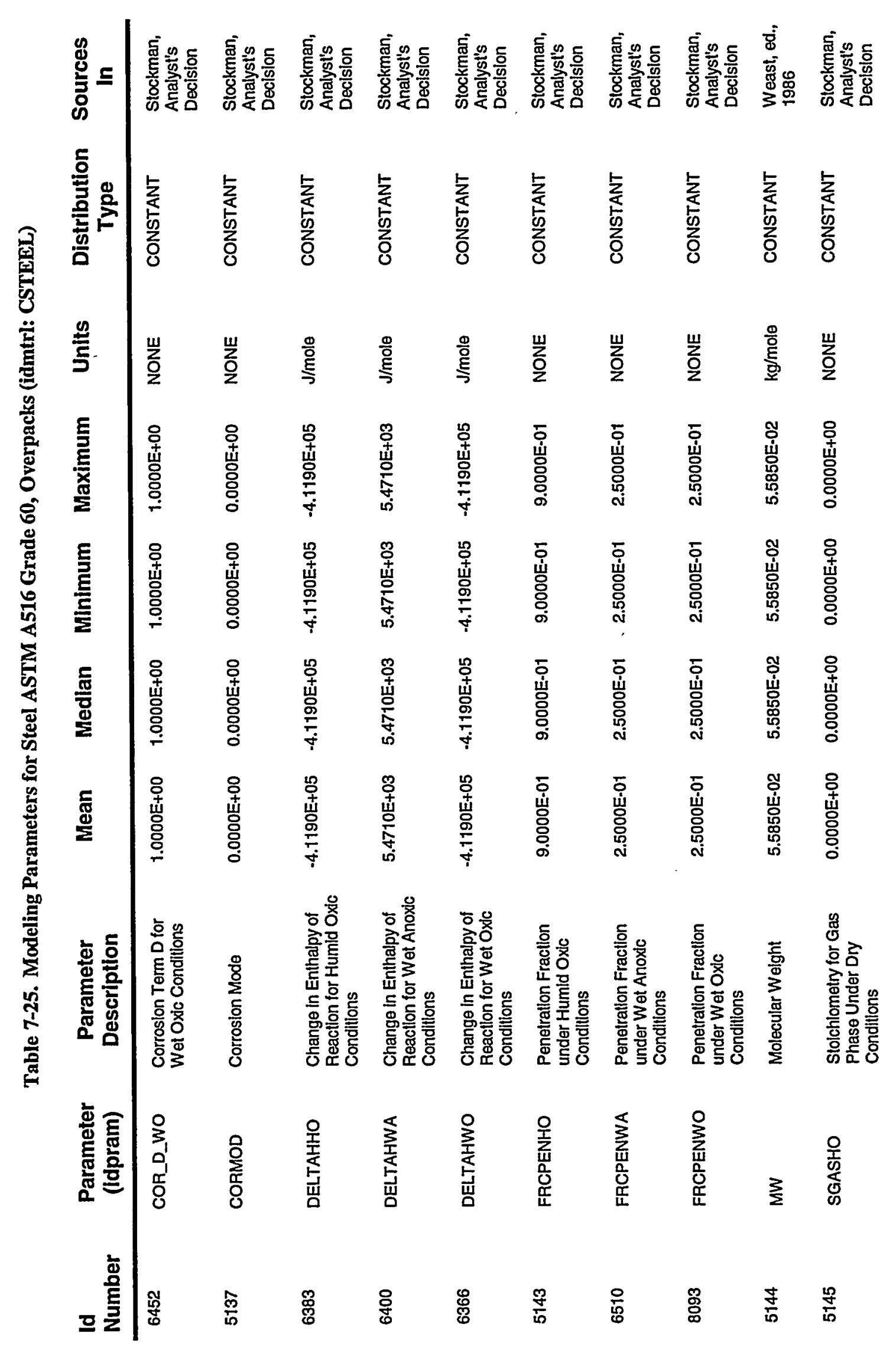

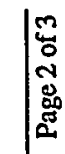




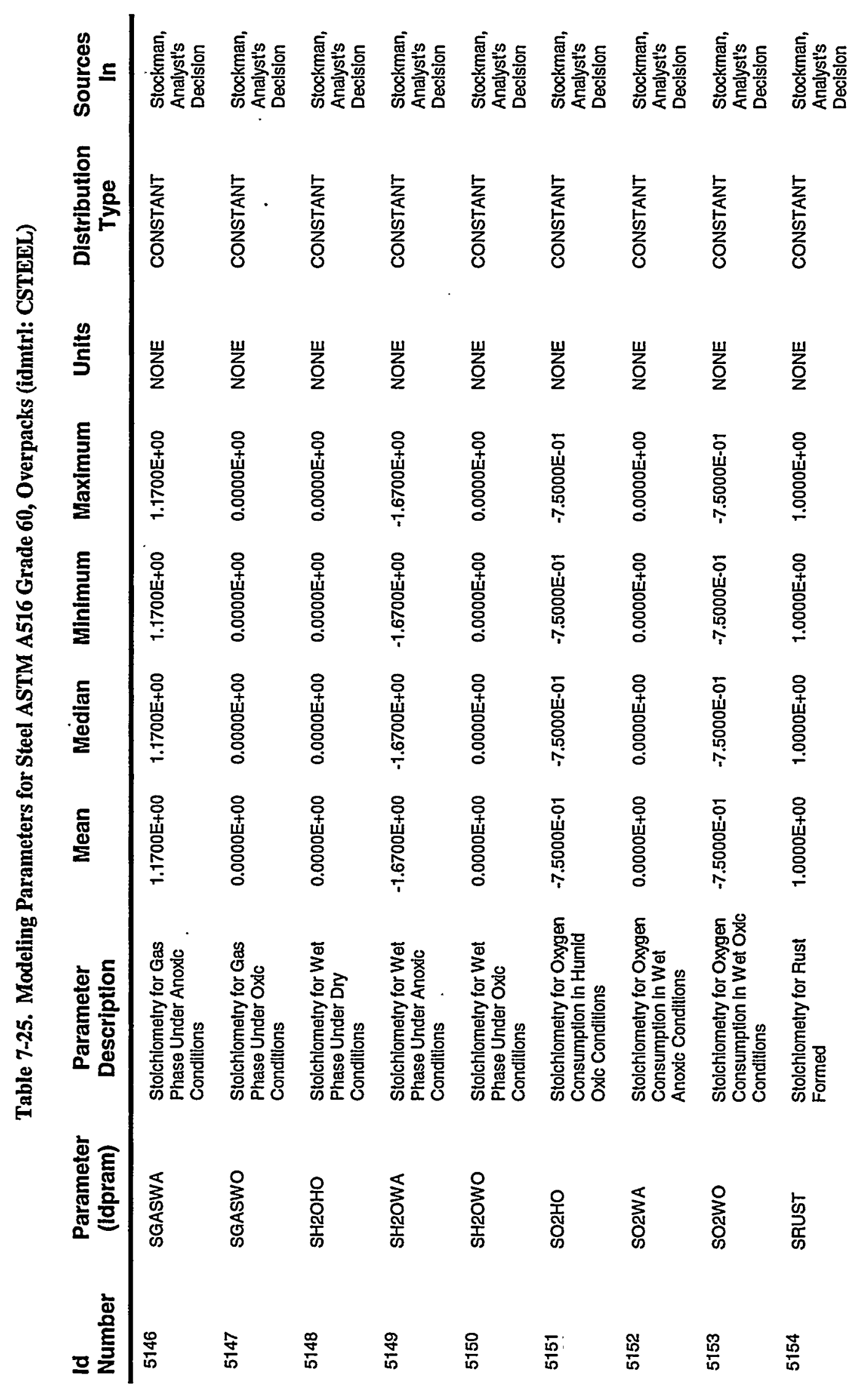



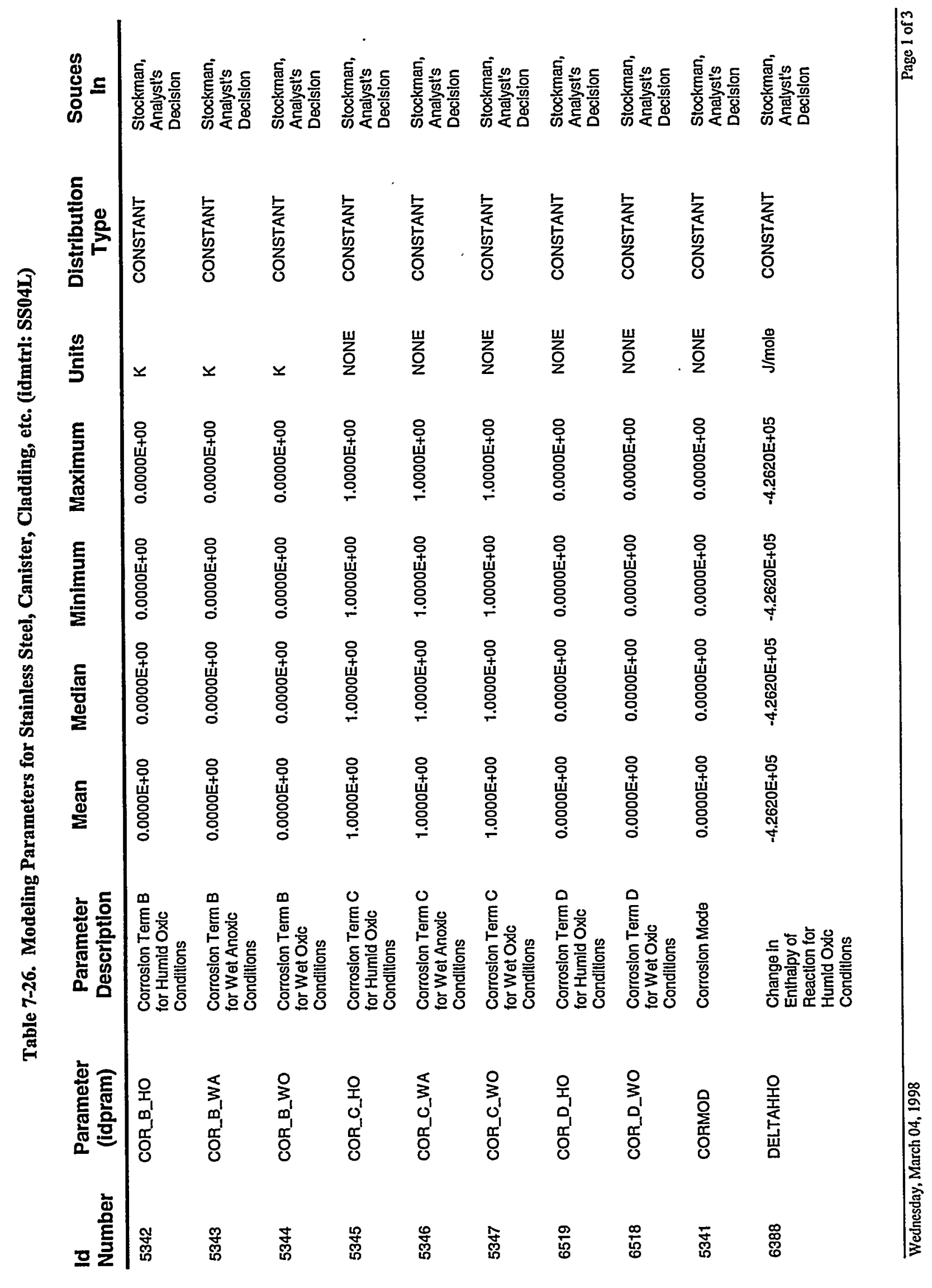


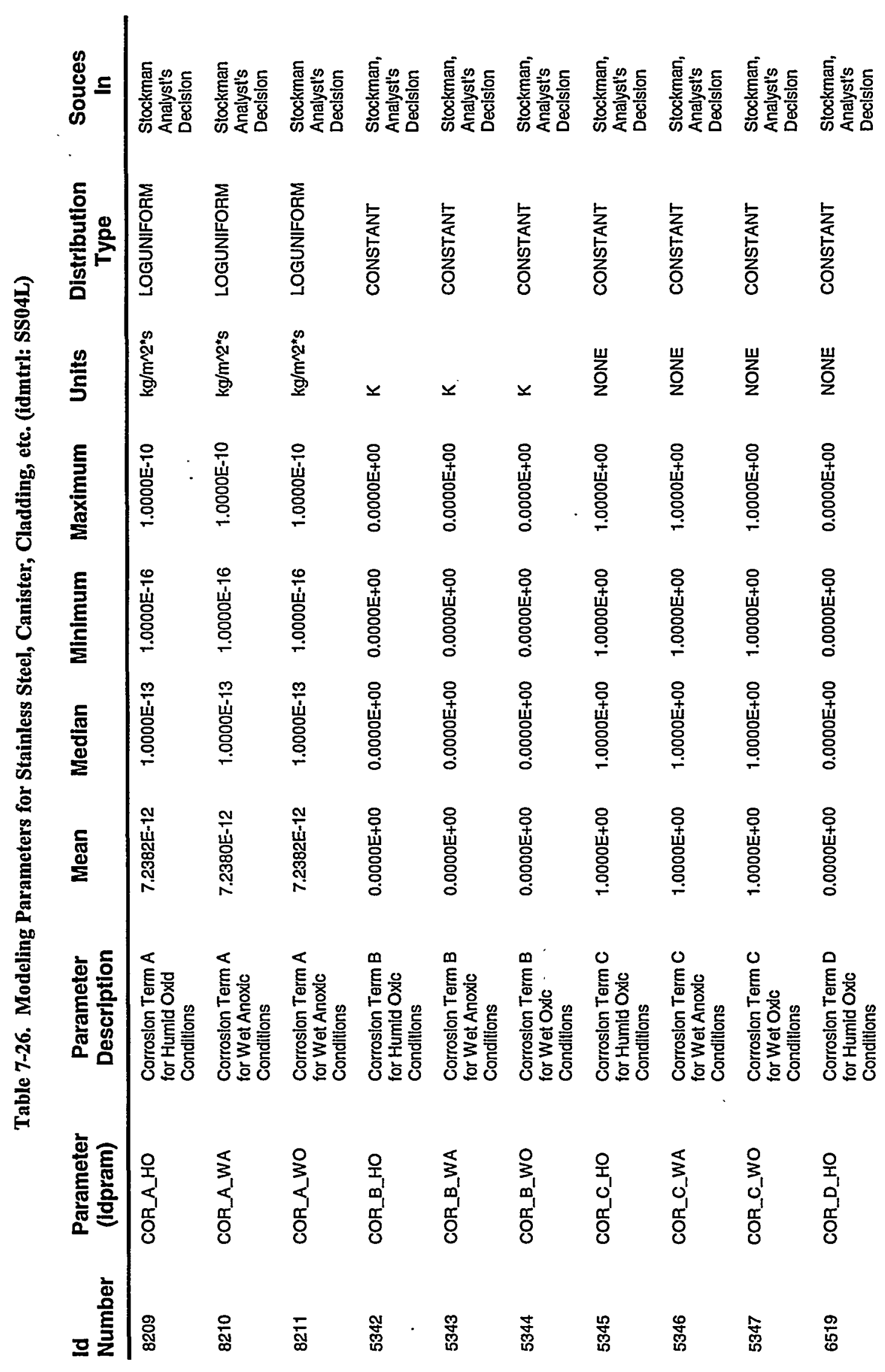




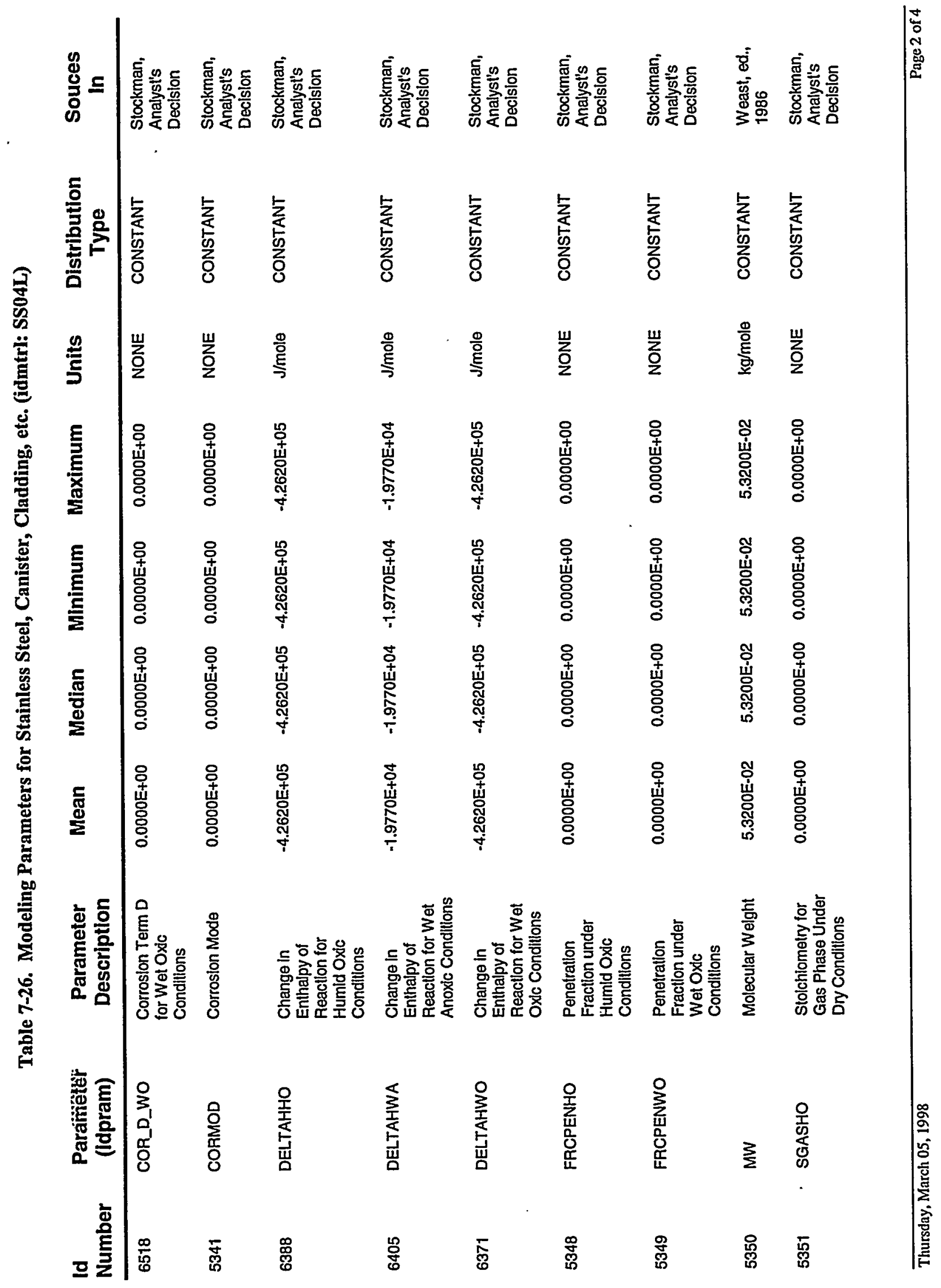




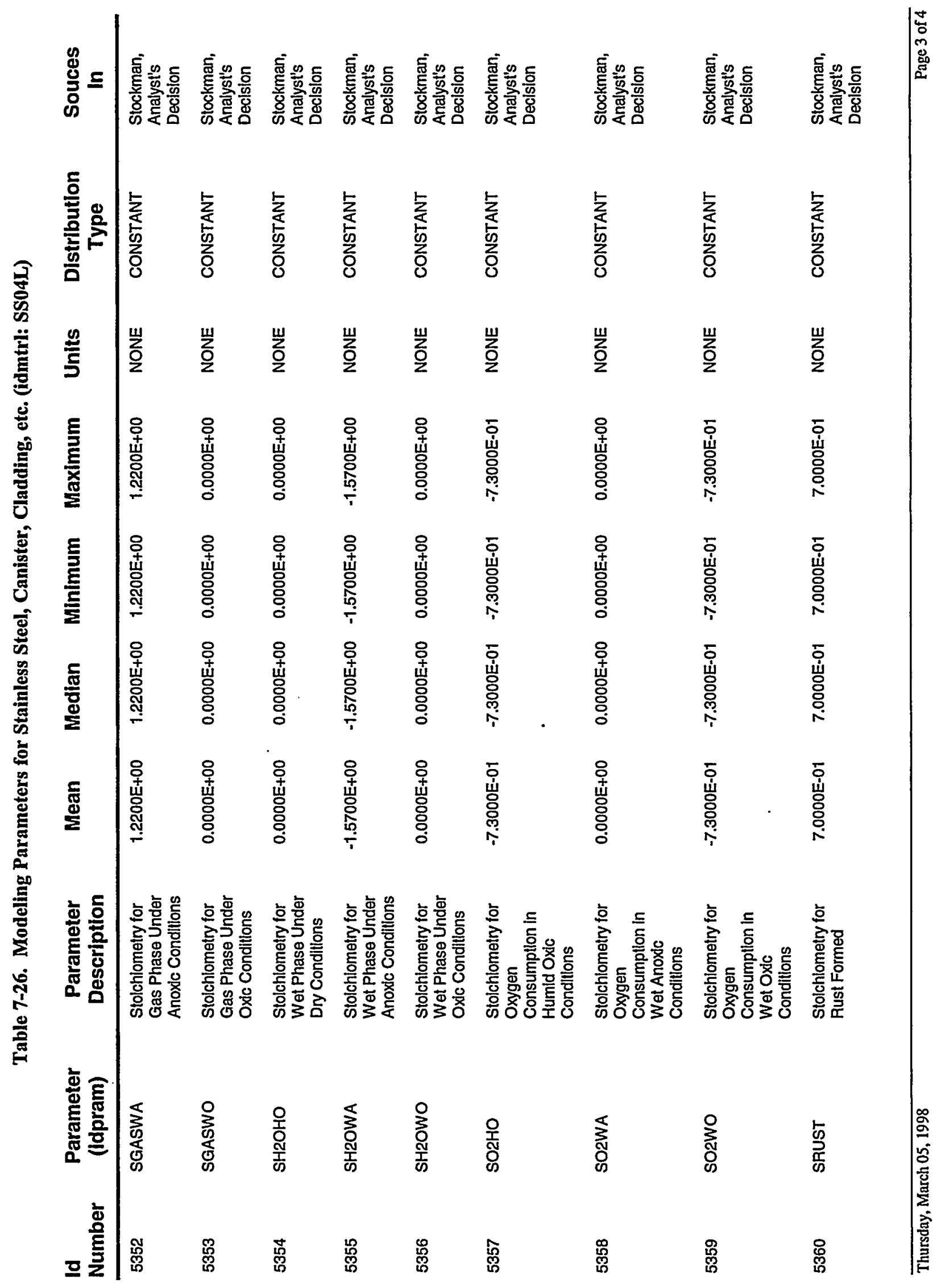



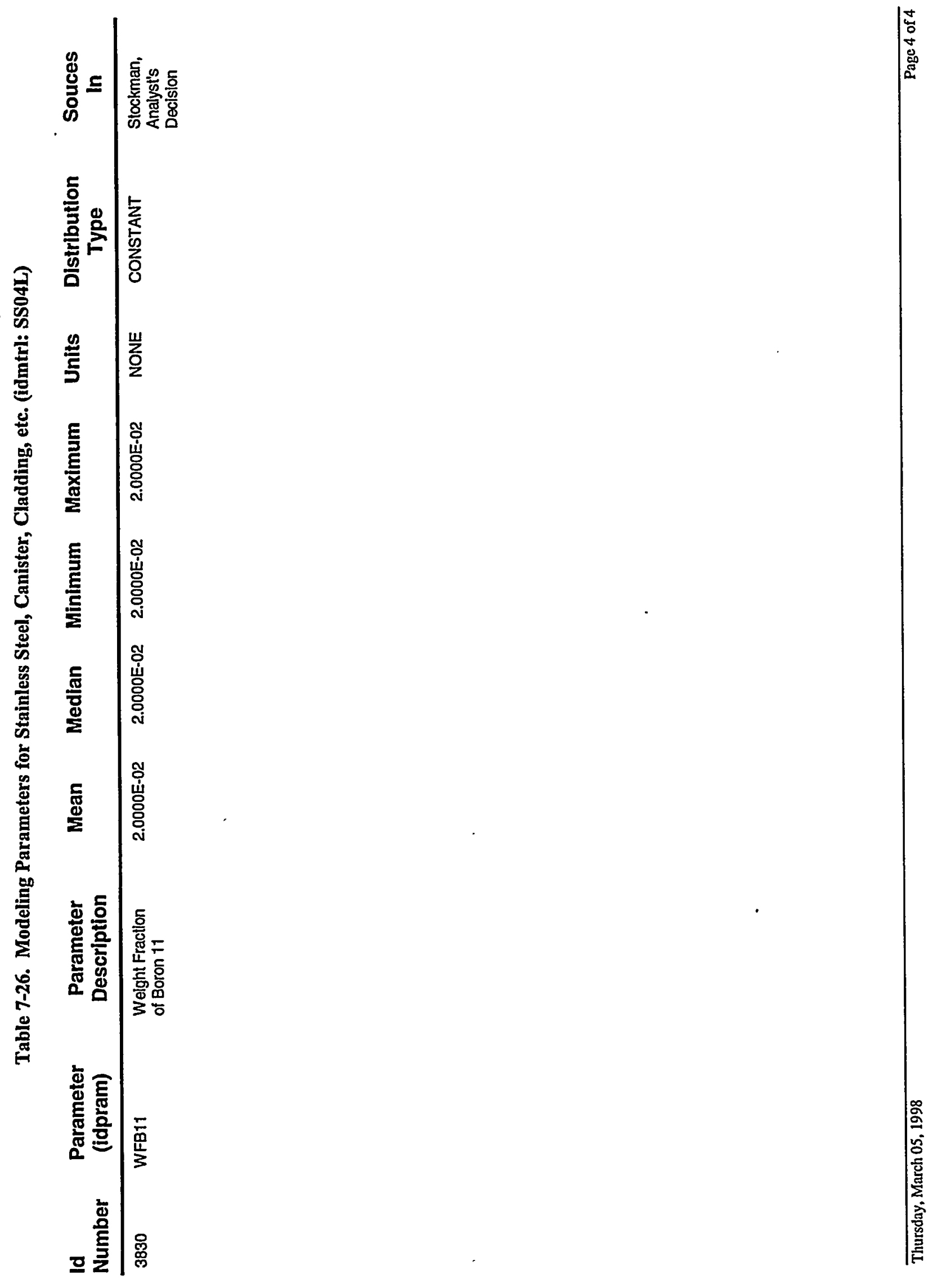


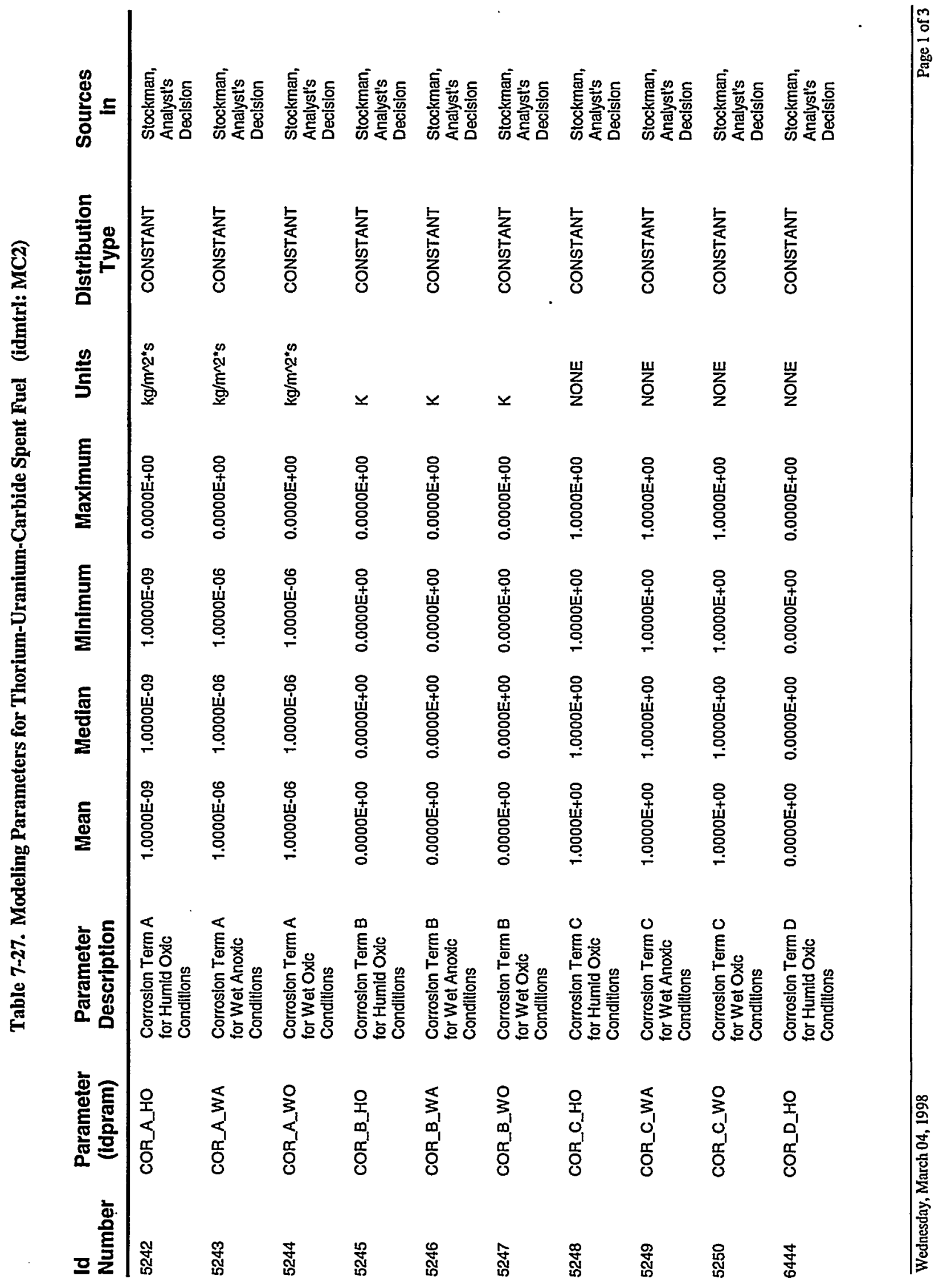




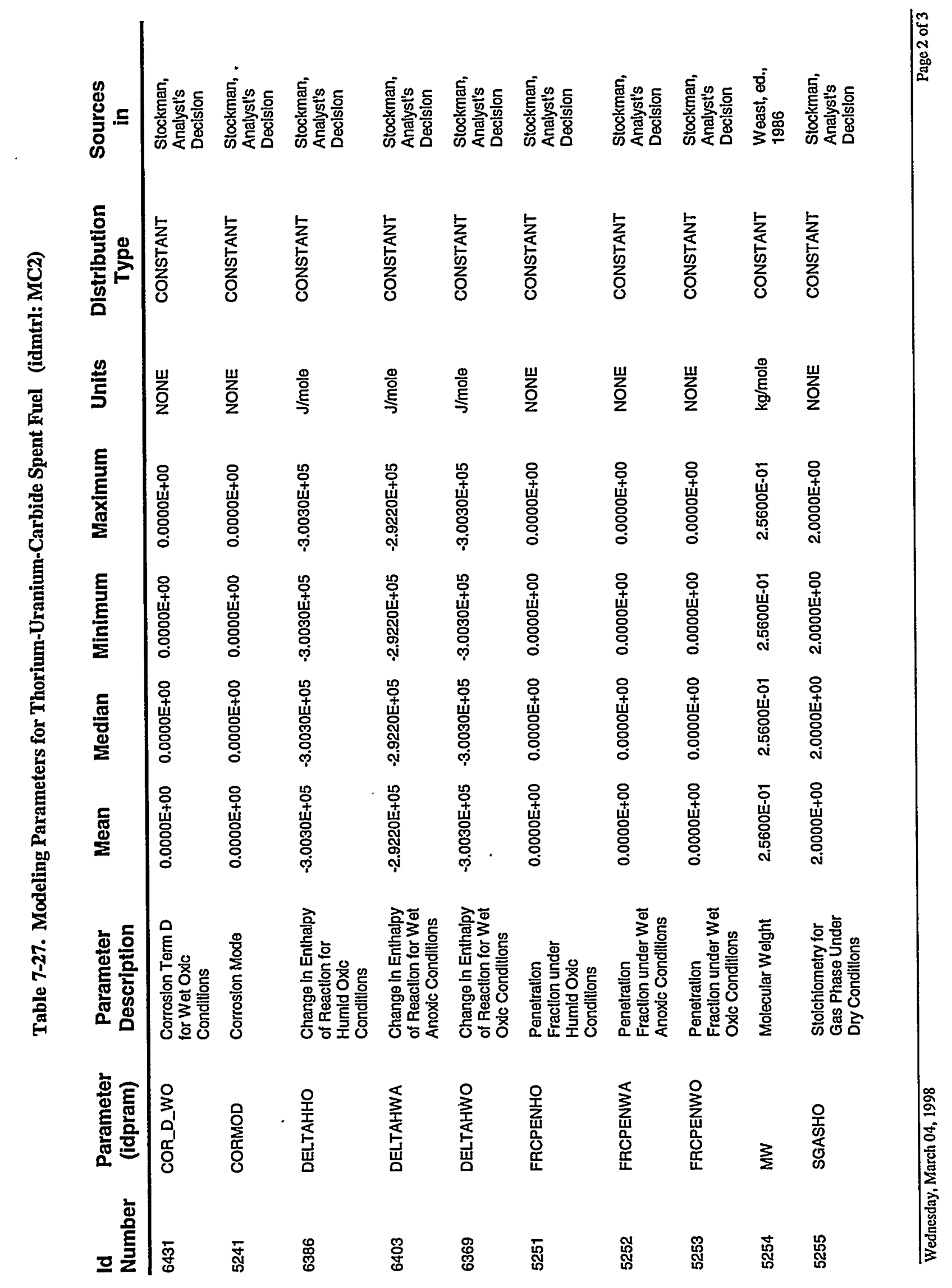




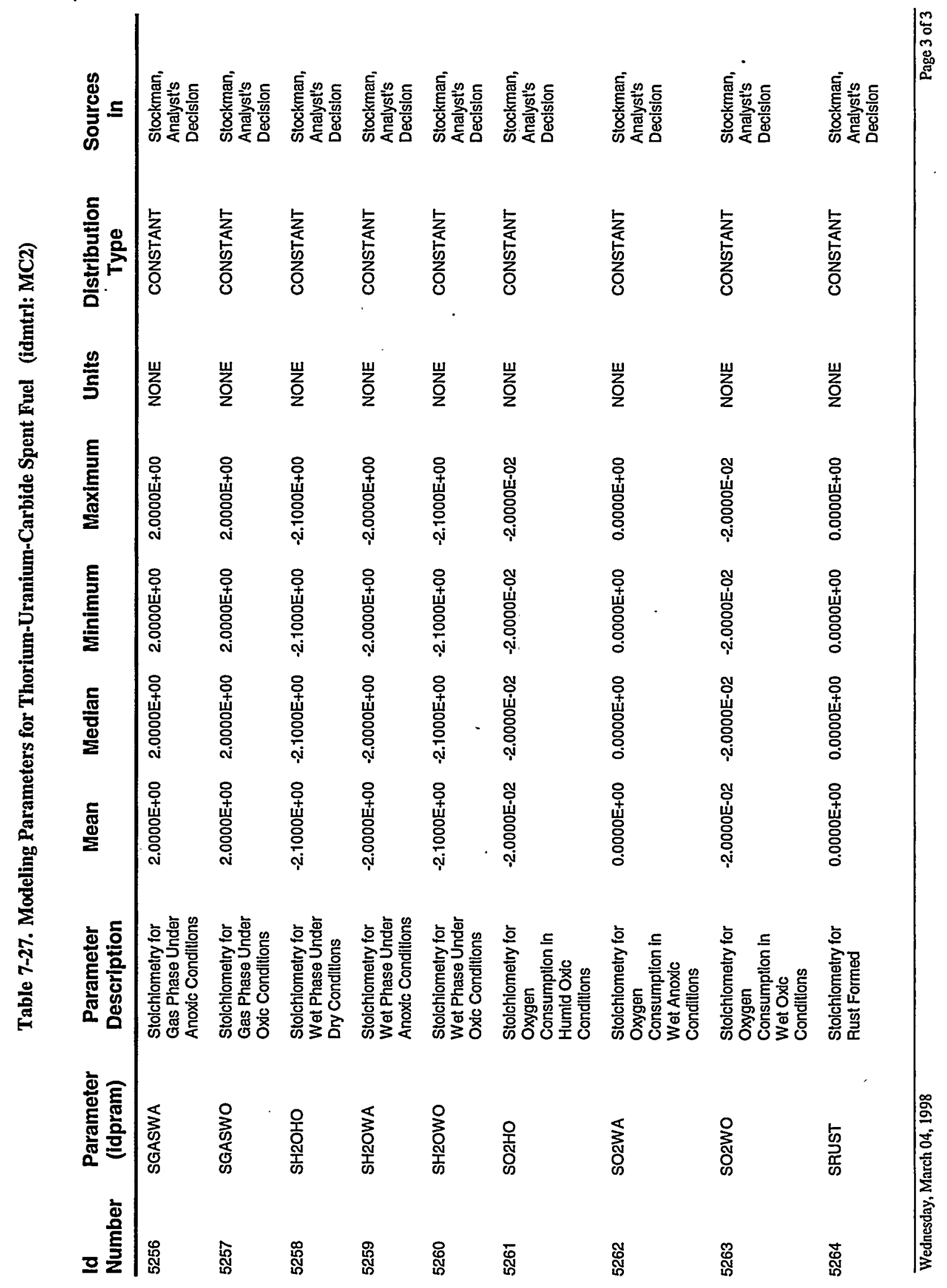




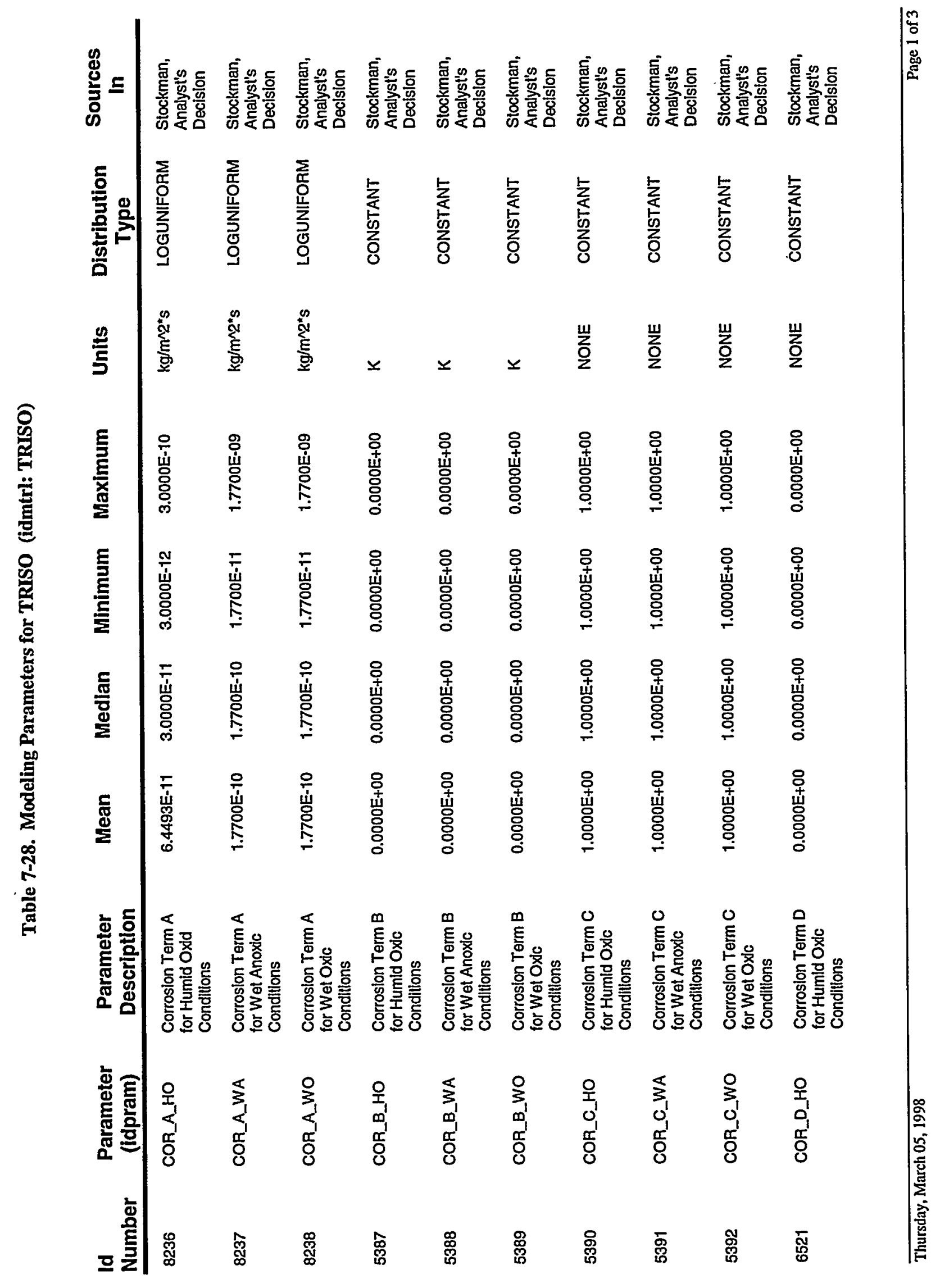




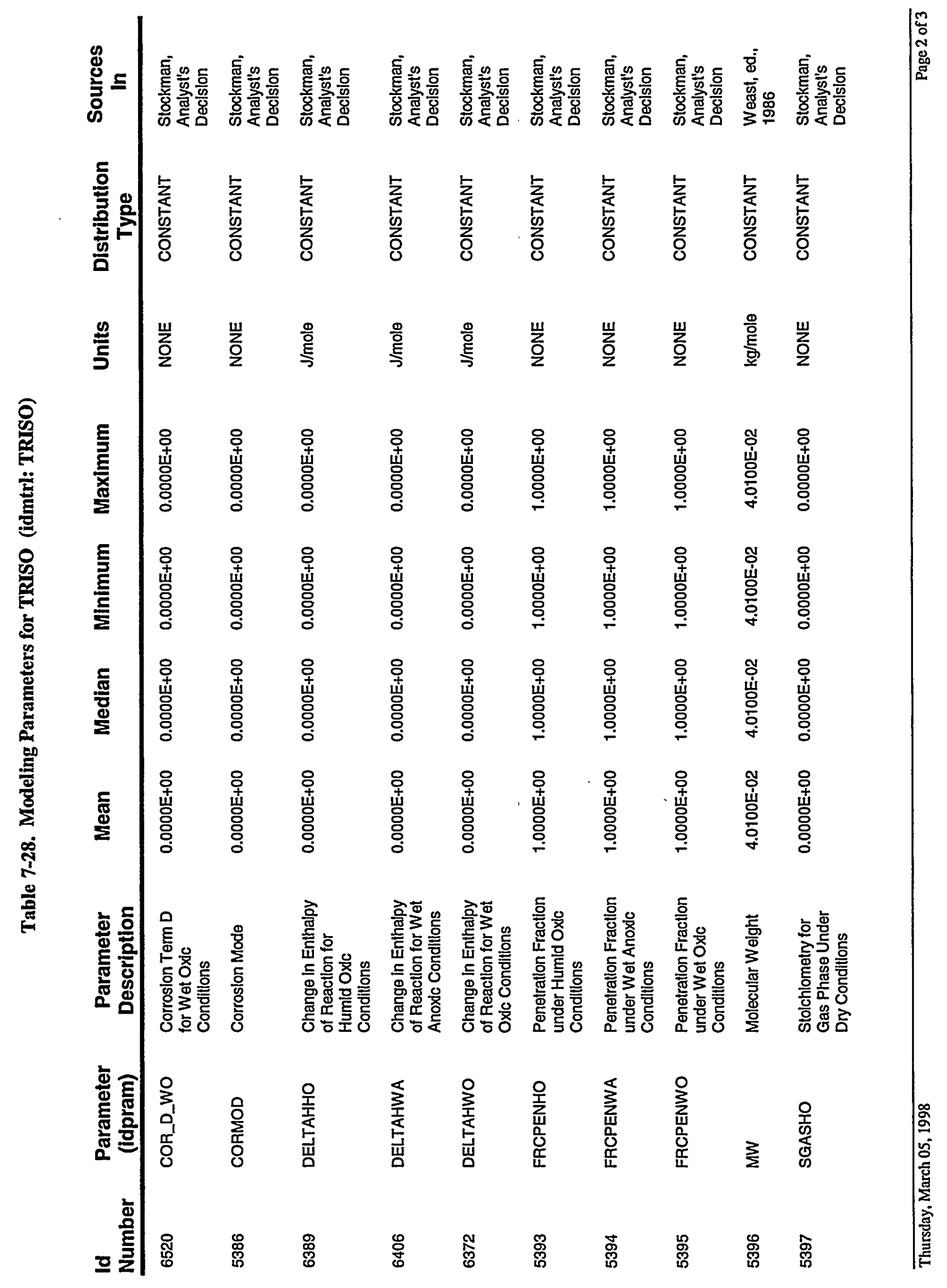




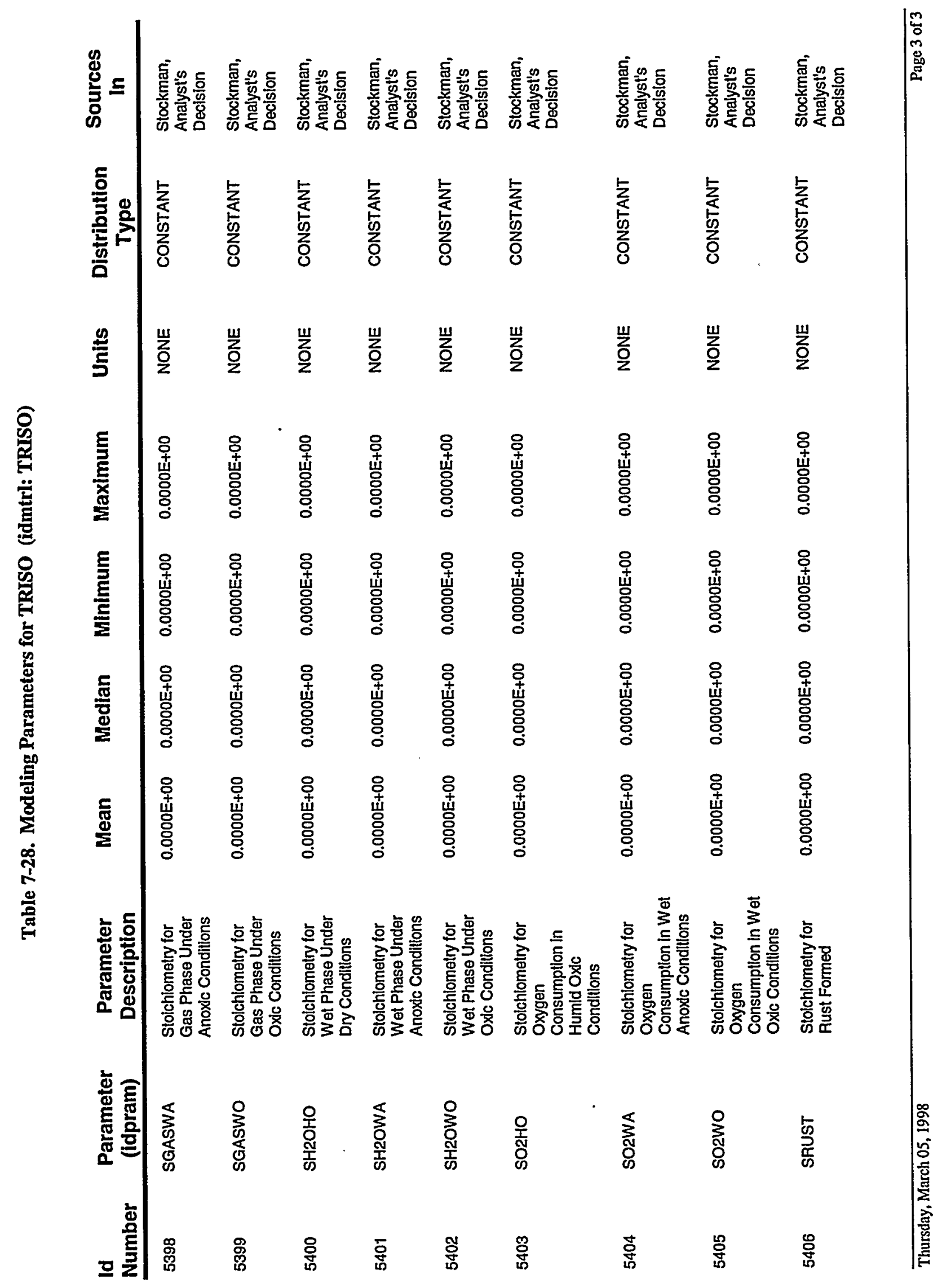




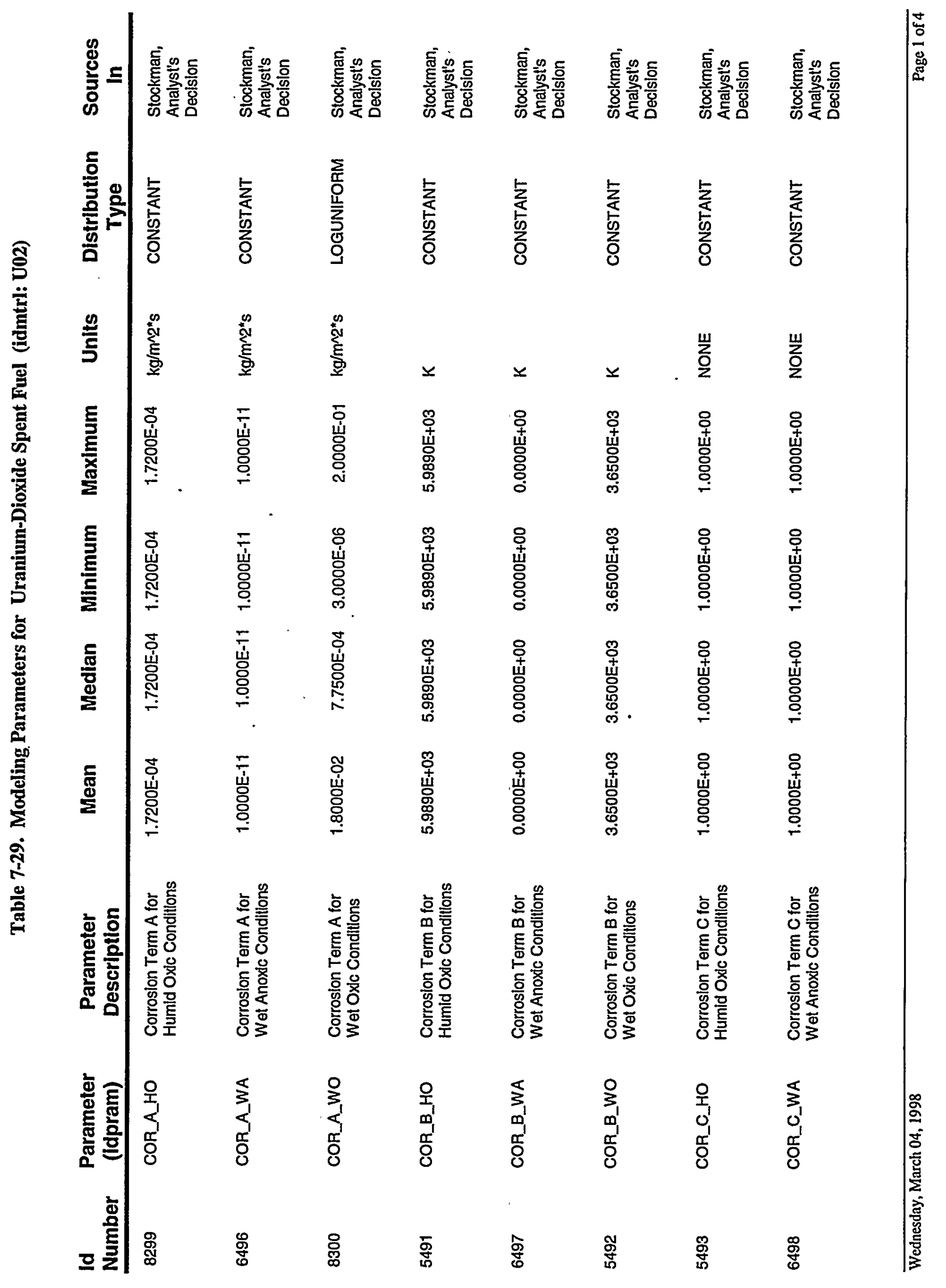




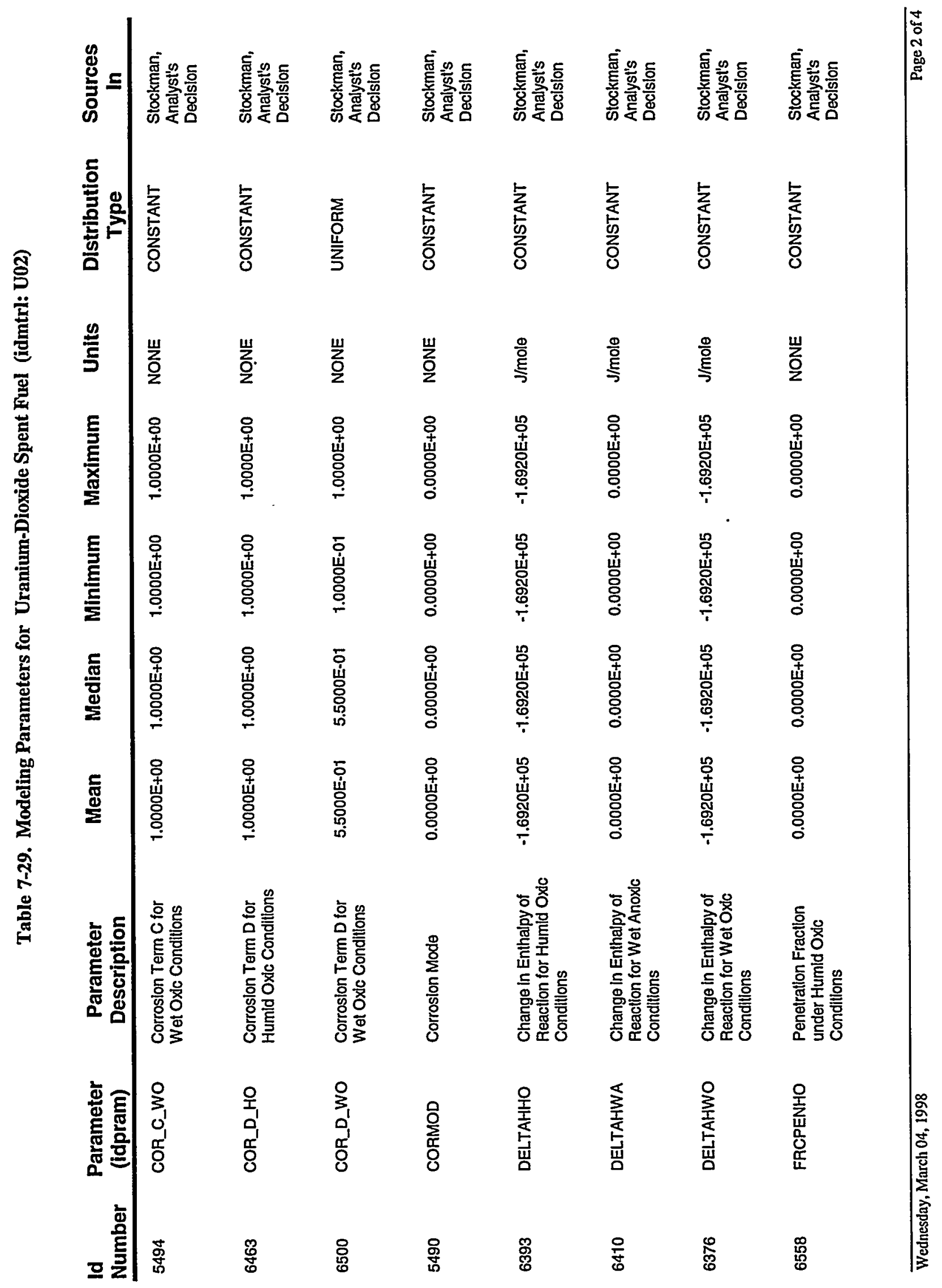




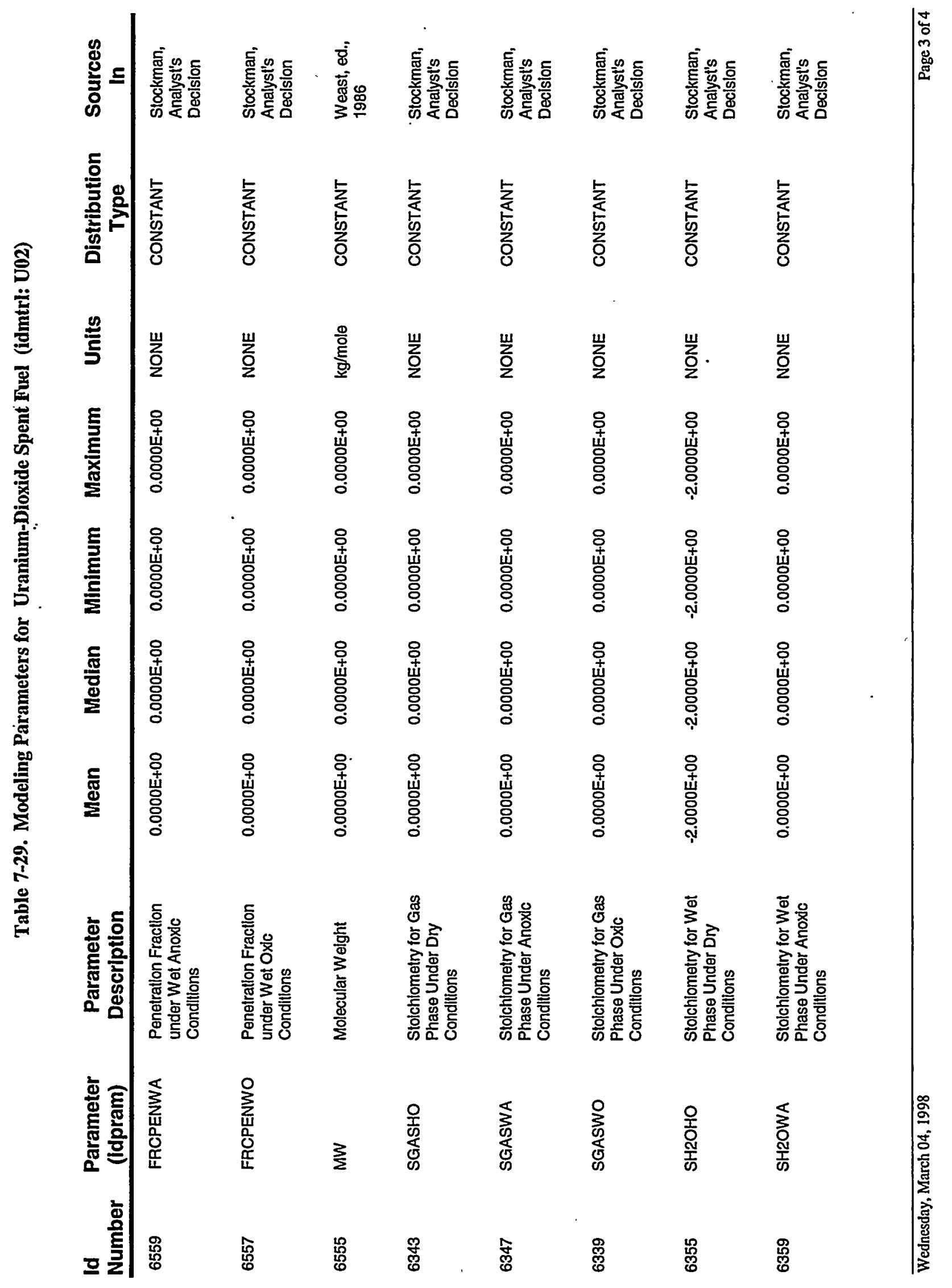




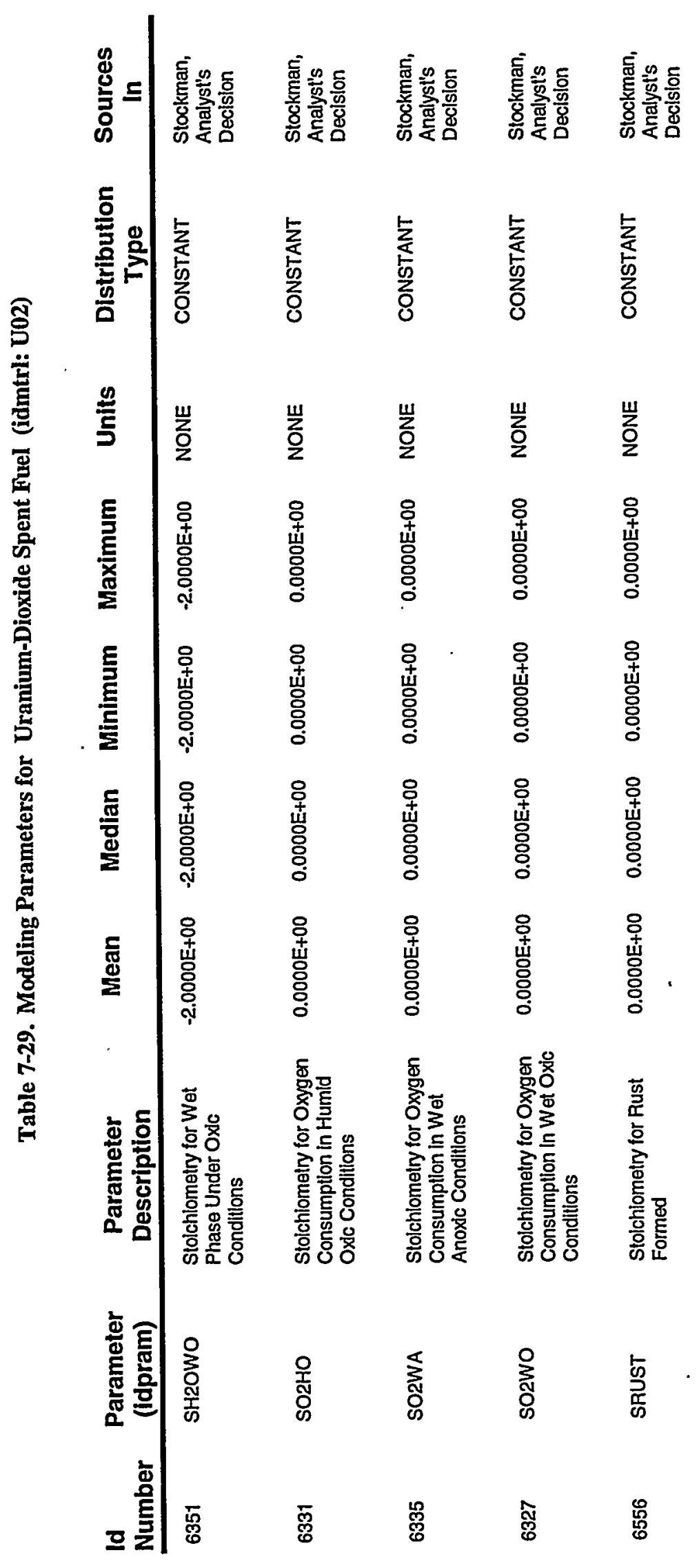




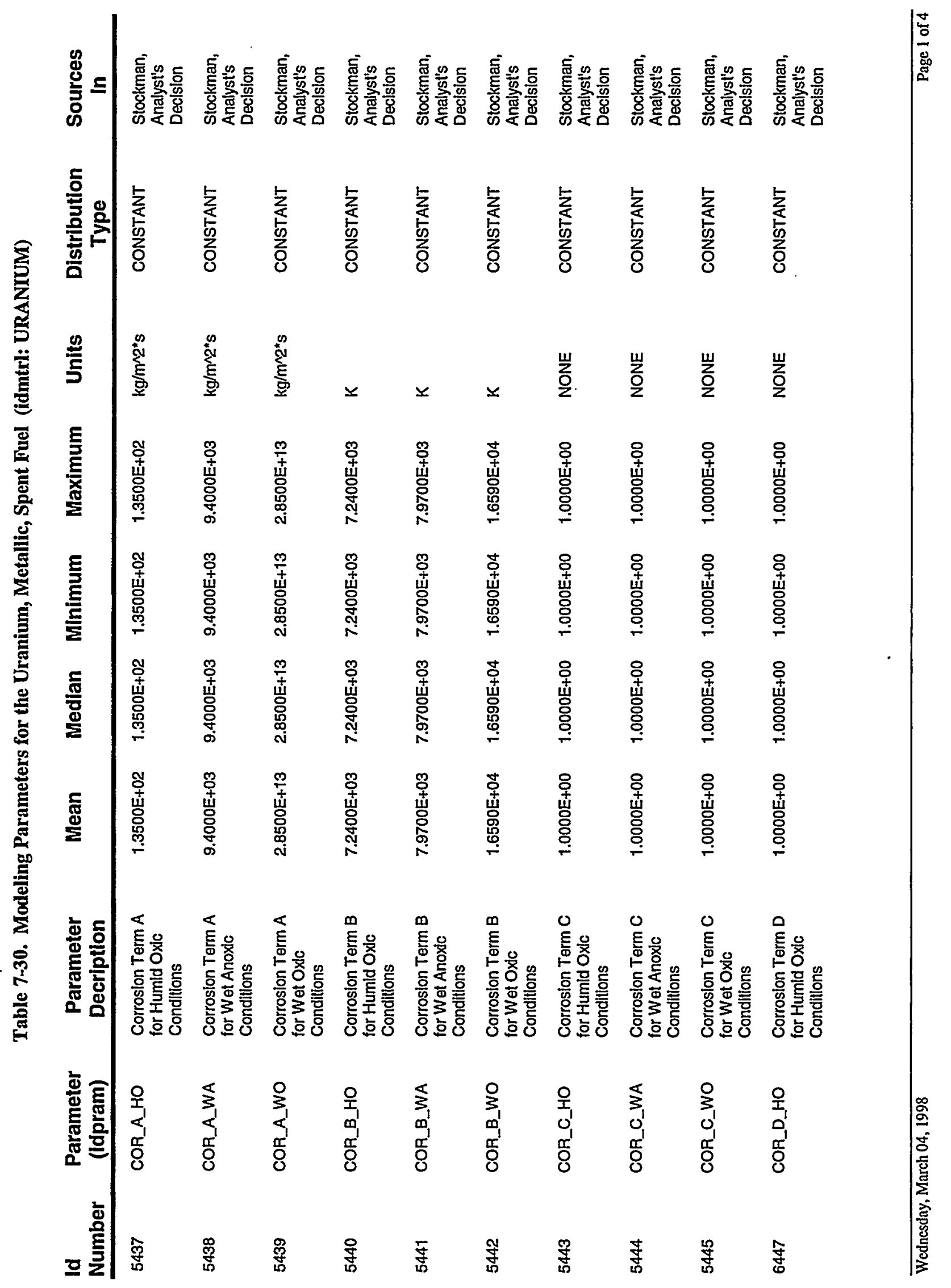




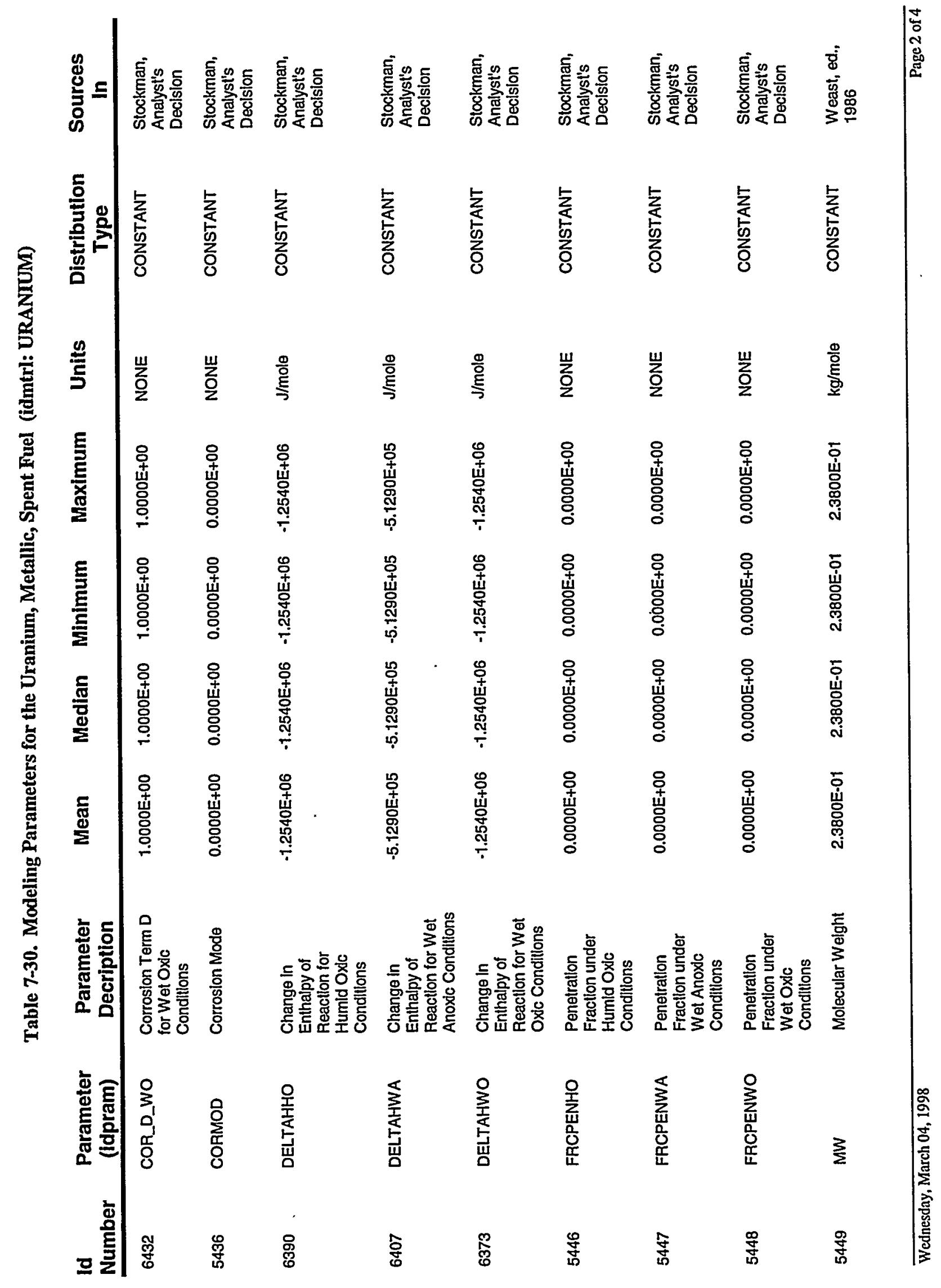




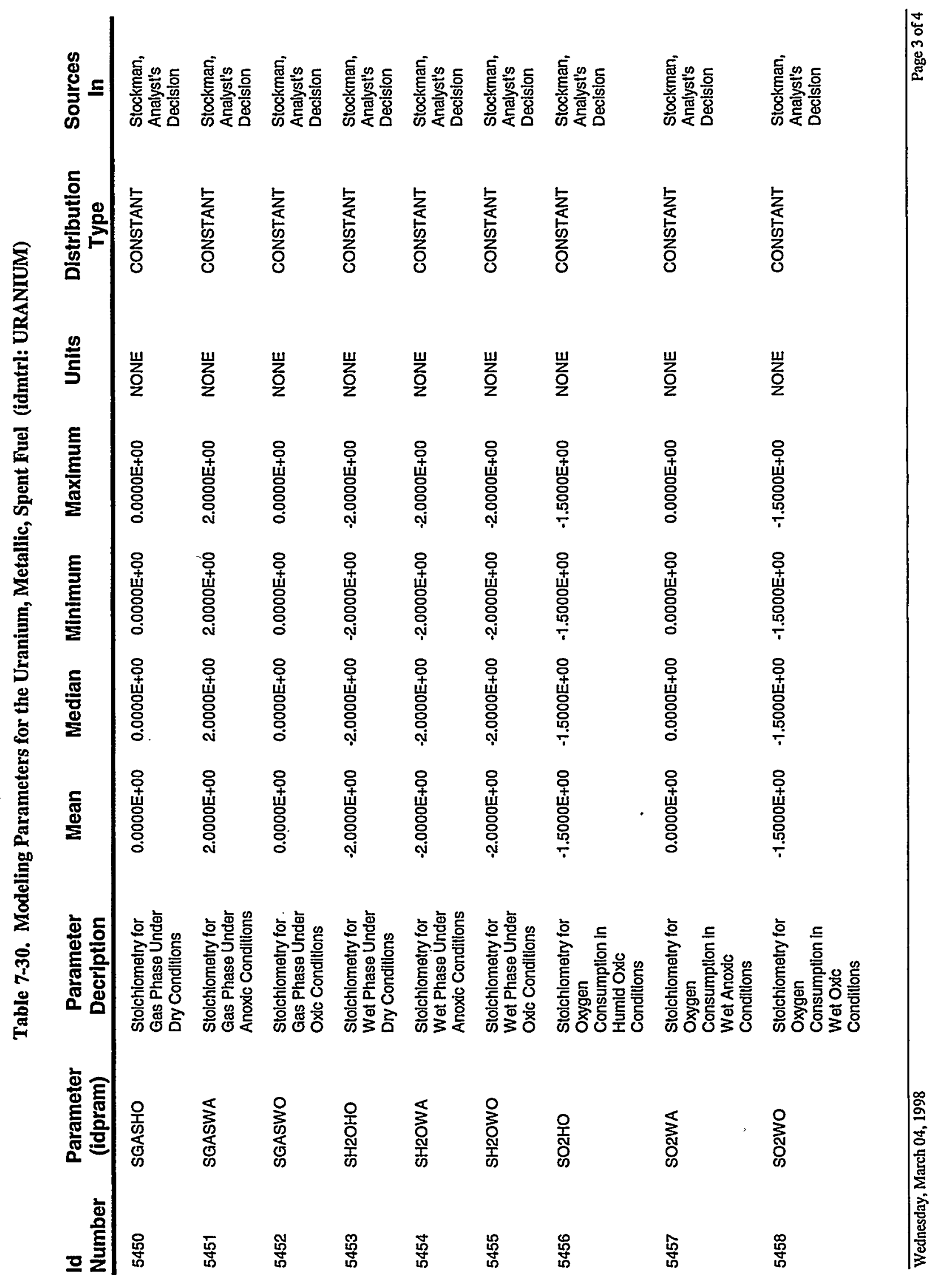




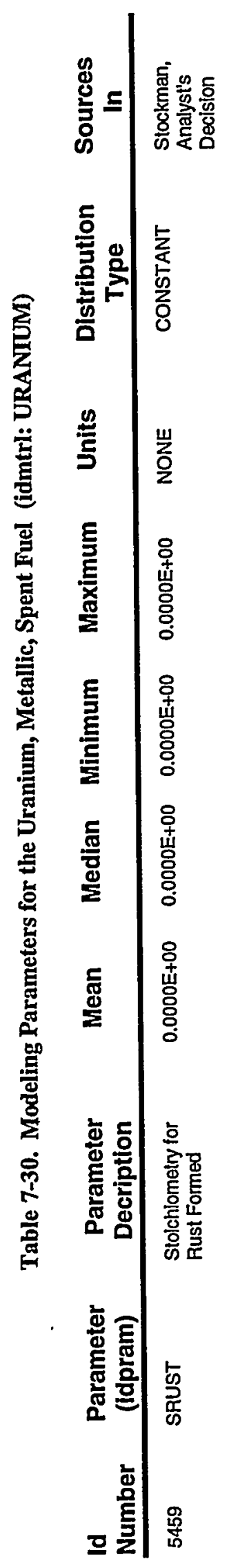

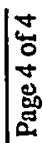




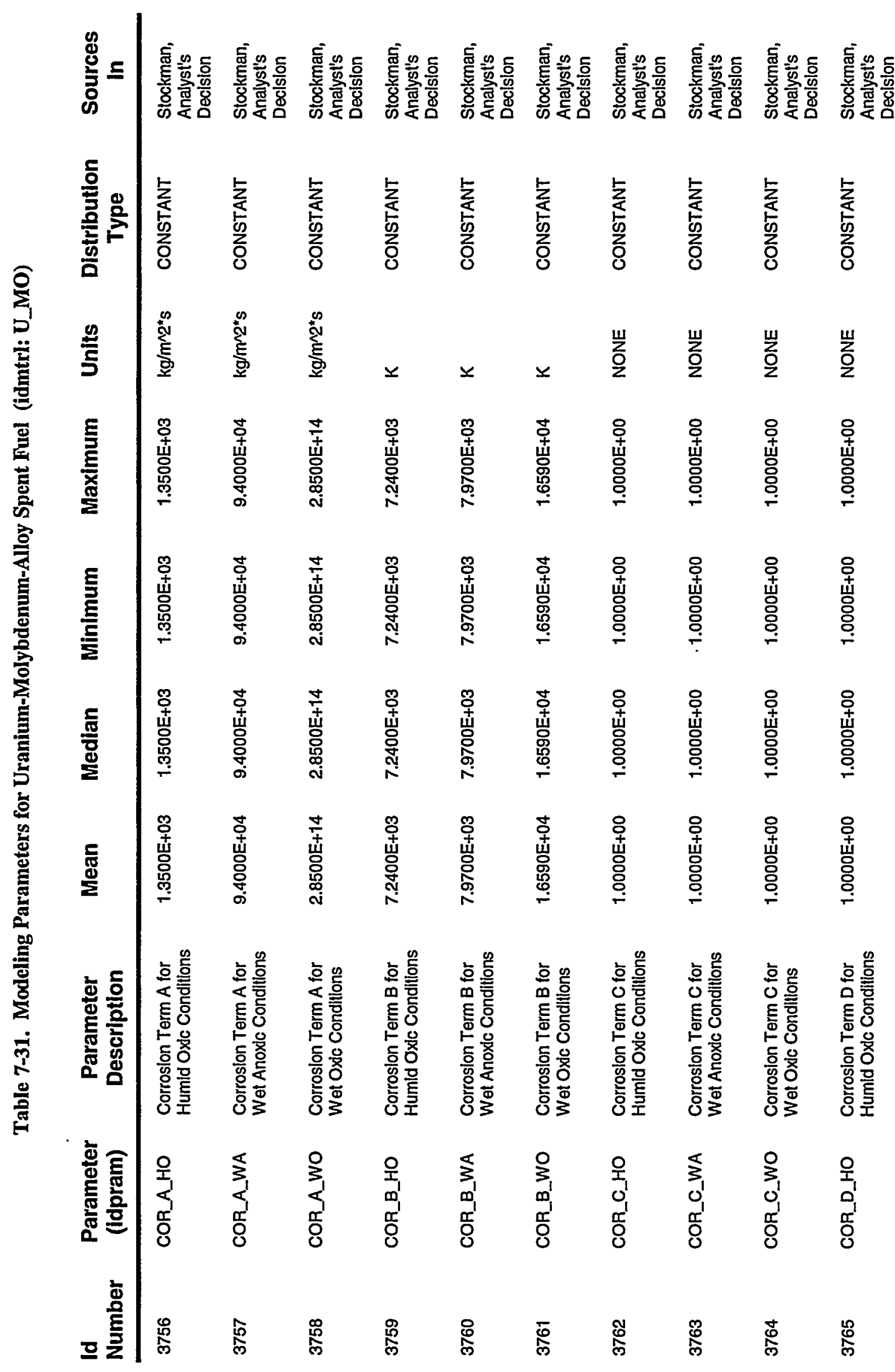




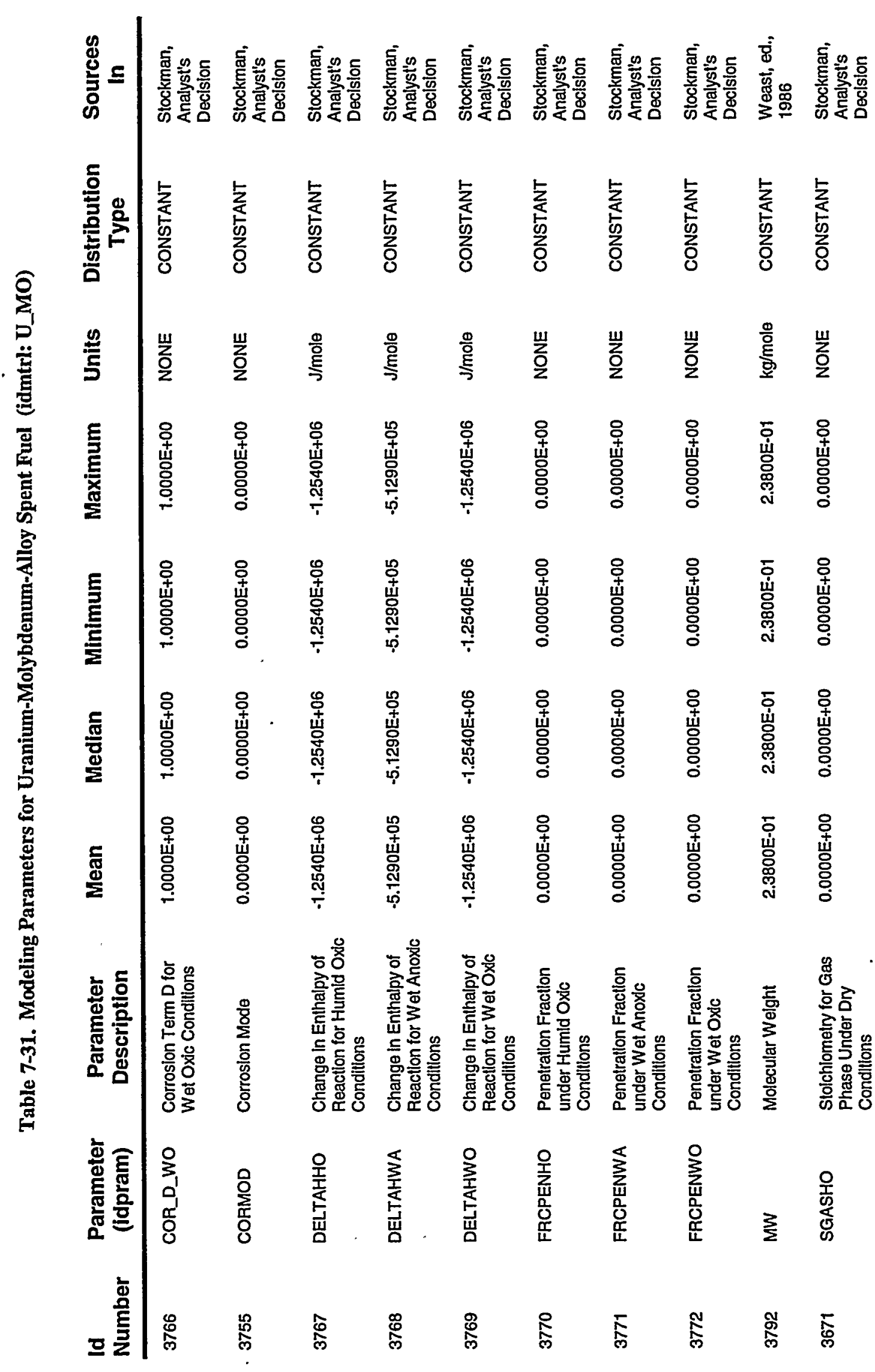

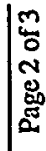




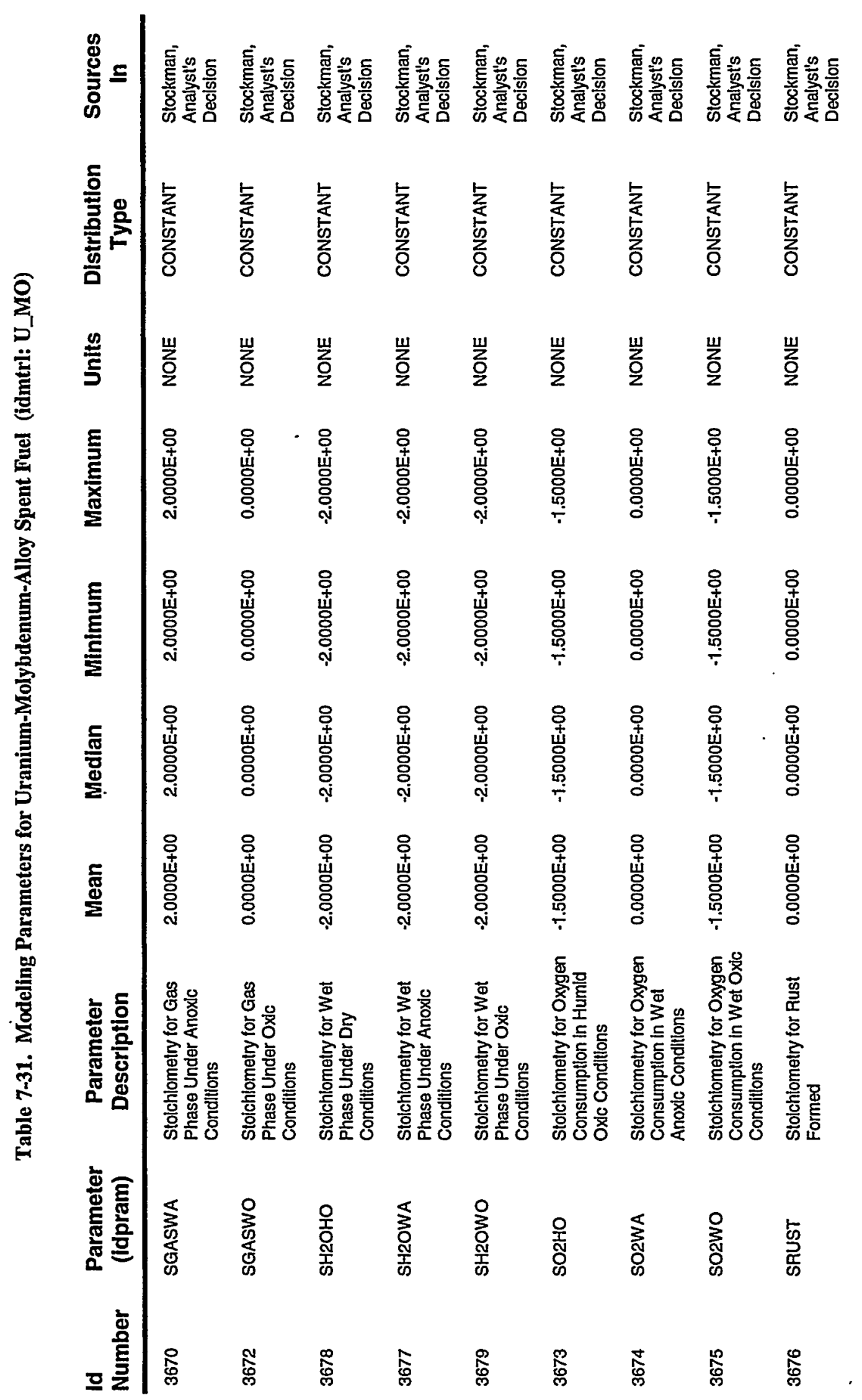

$\mid \begin{aligned} & 0 \\ & 0 \\ & 0 \\ & 0 \\ & 2 \\ & 2 \\ & \\ & \end{aligned}$ 


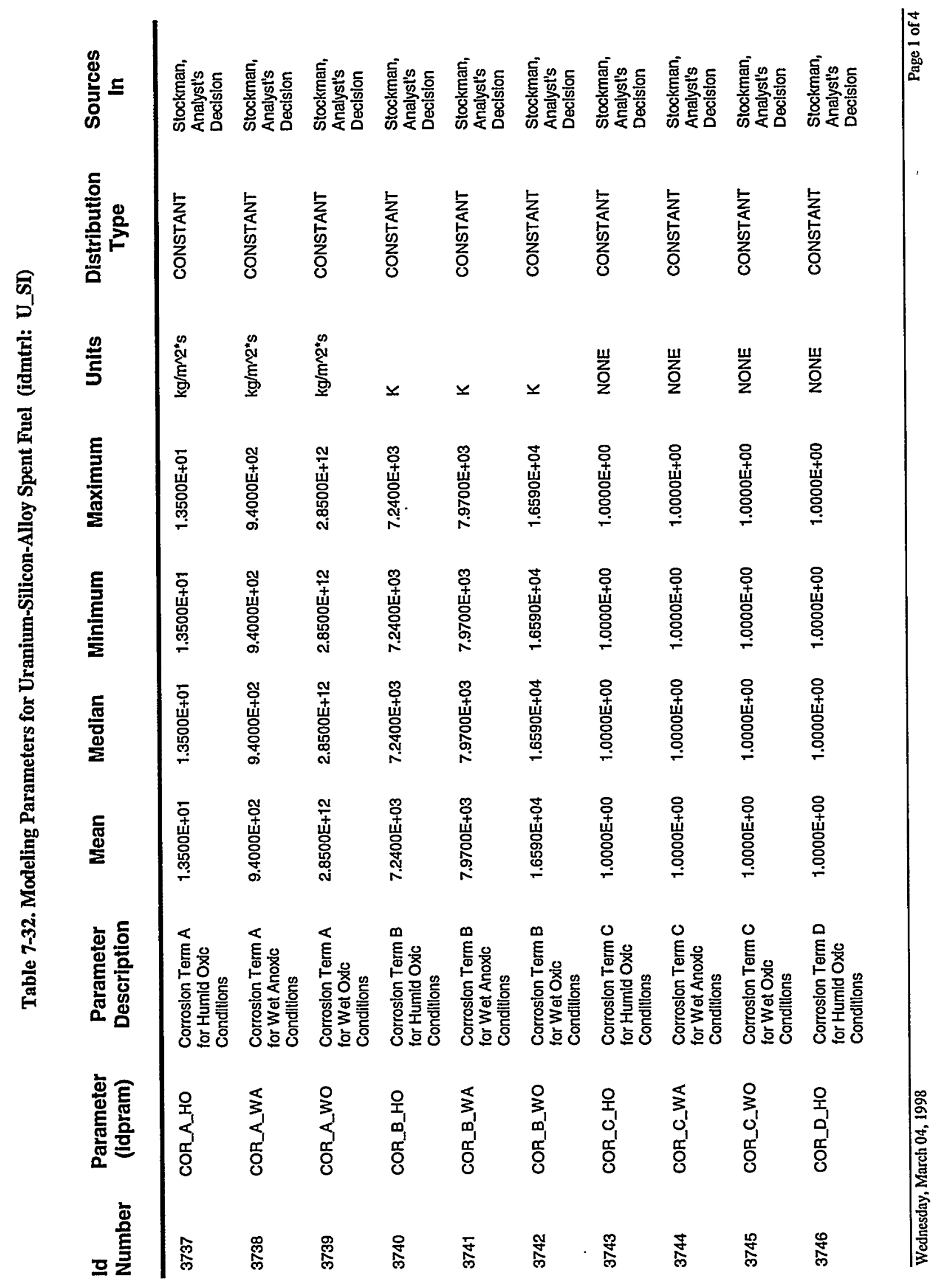




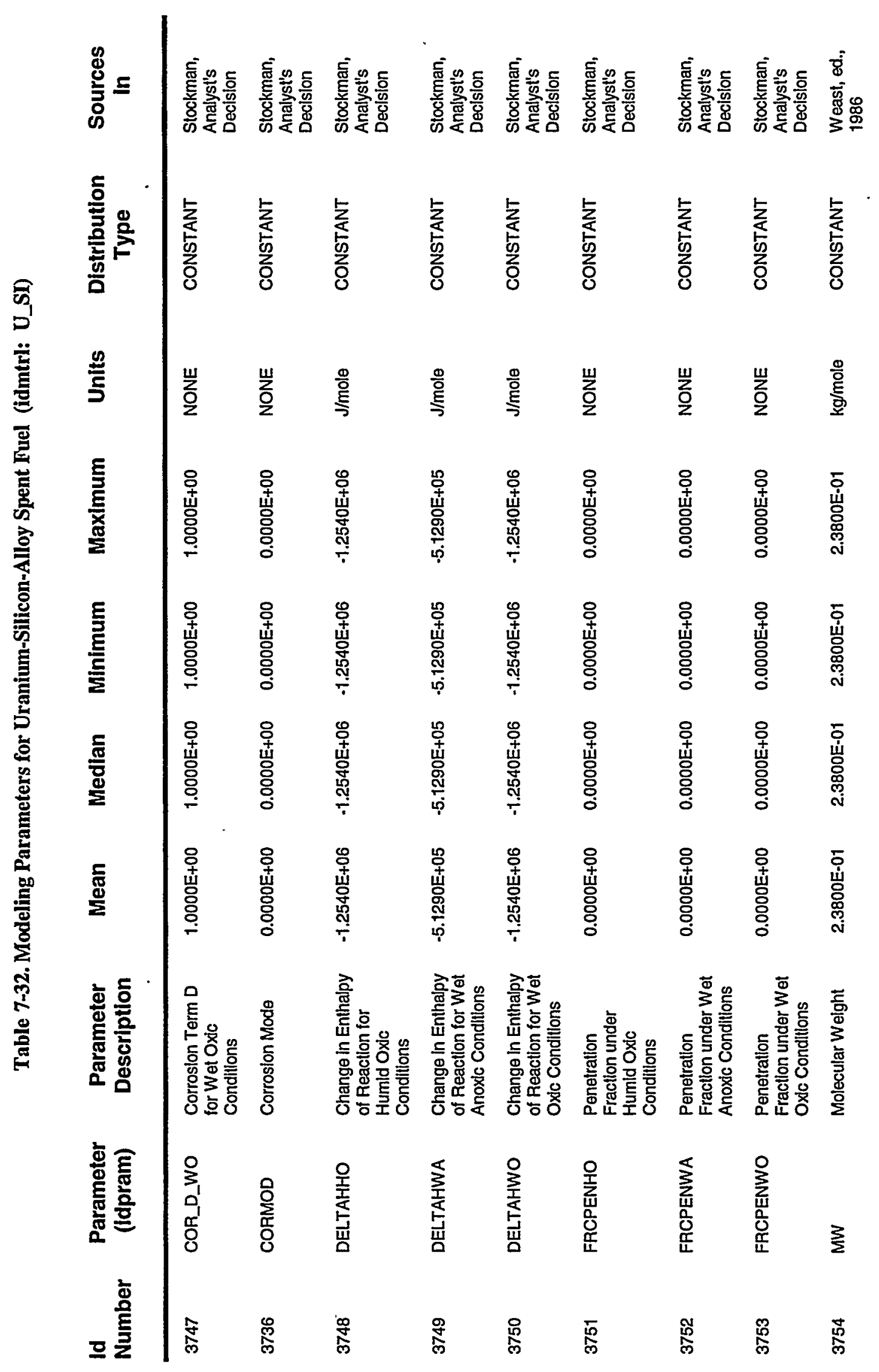




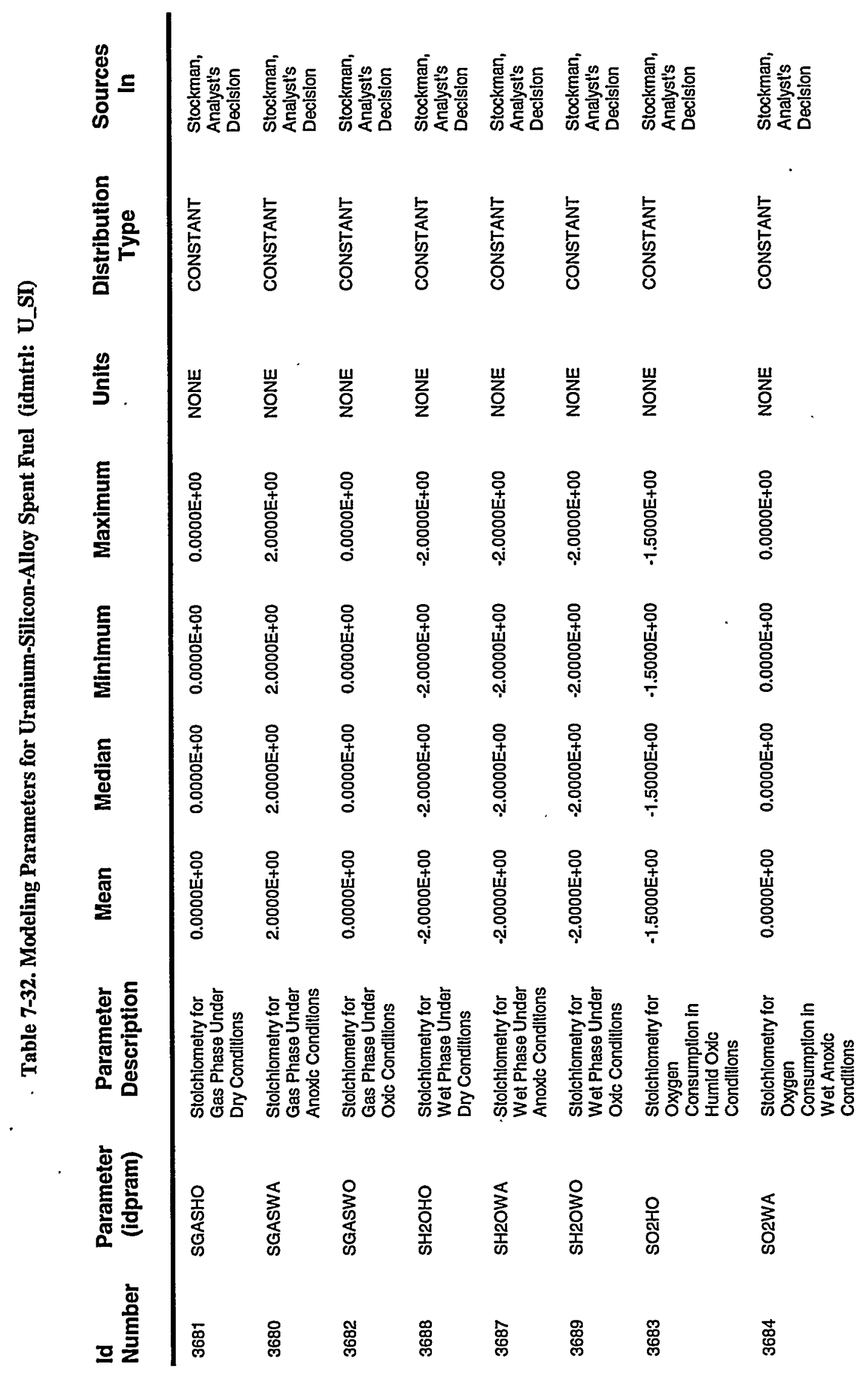



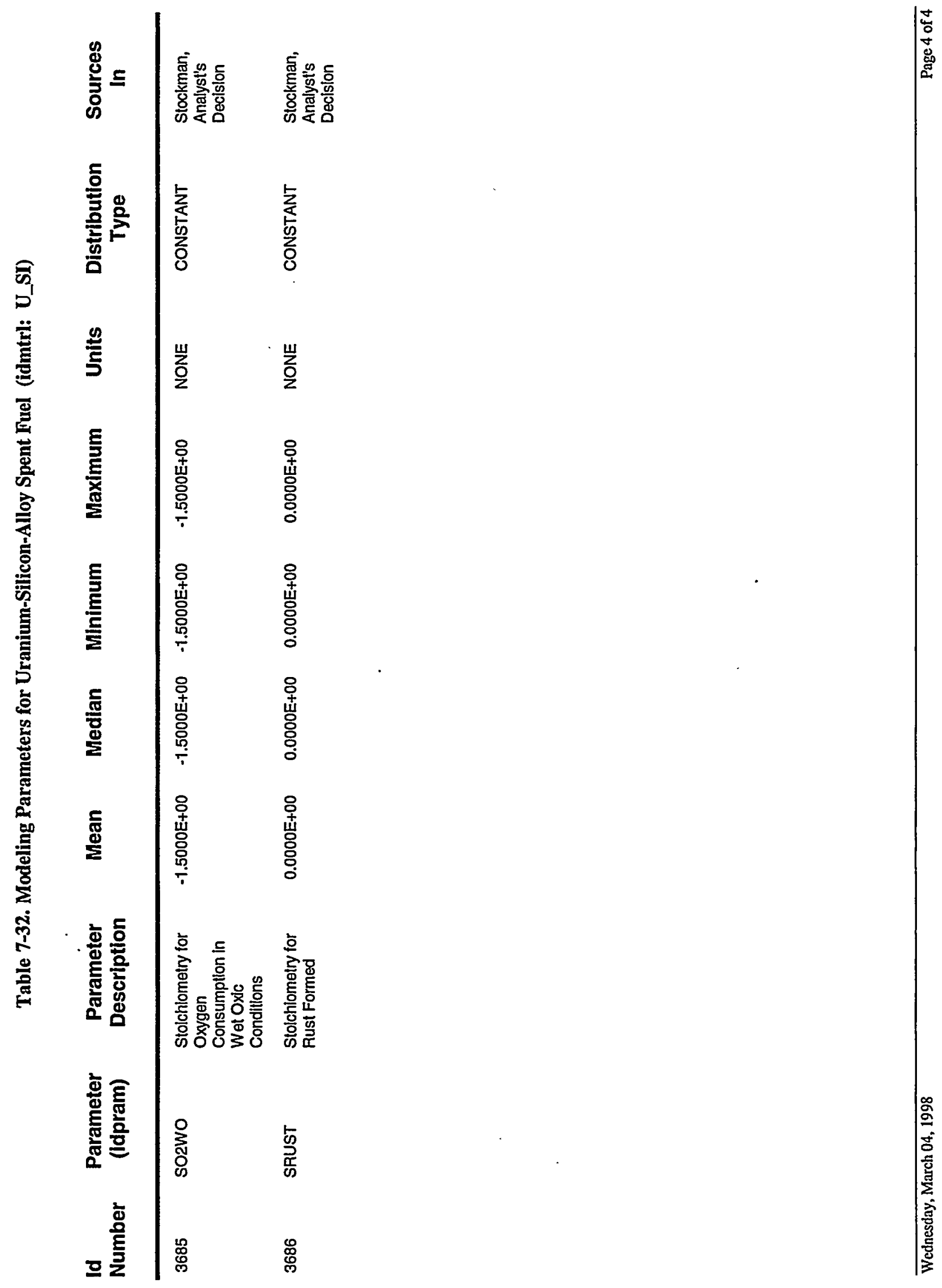


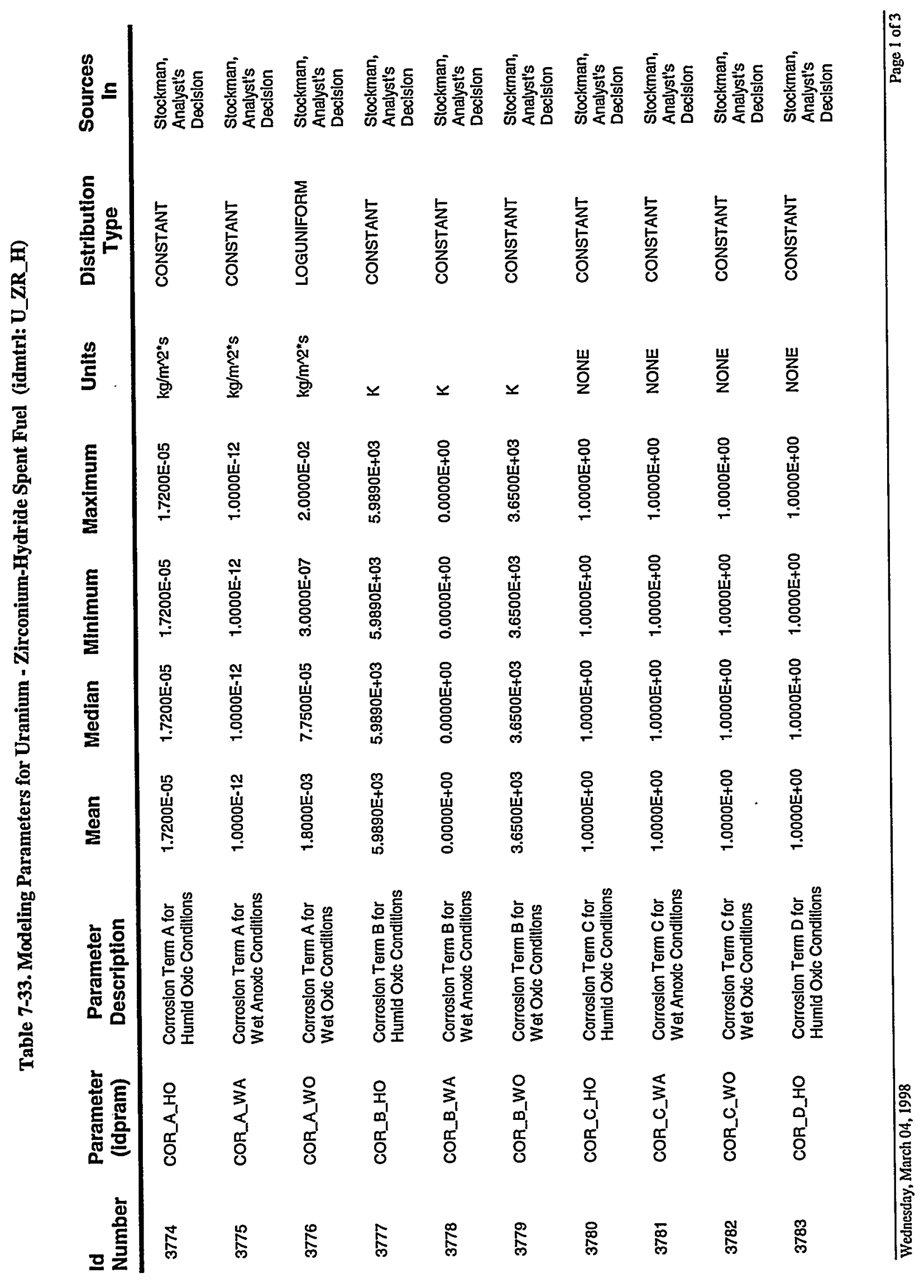




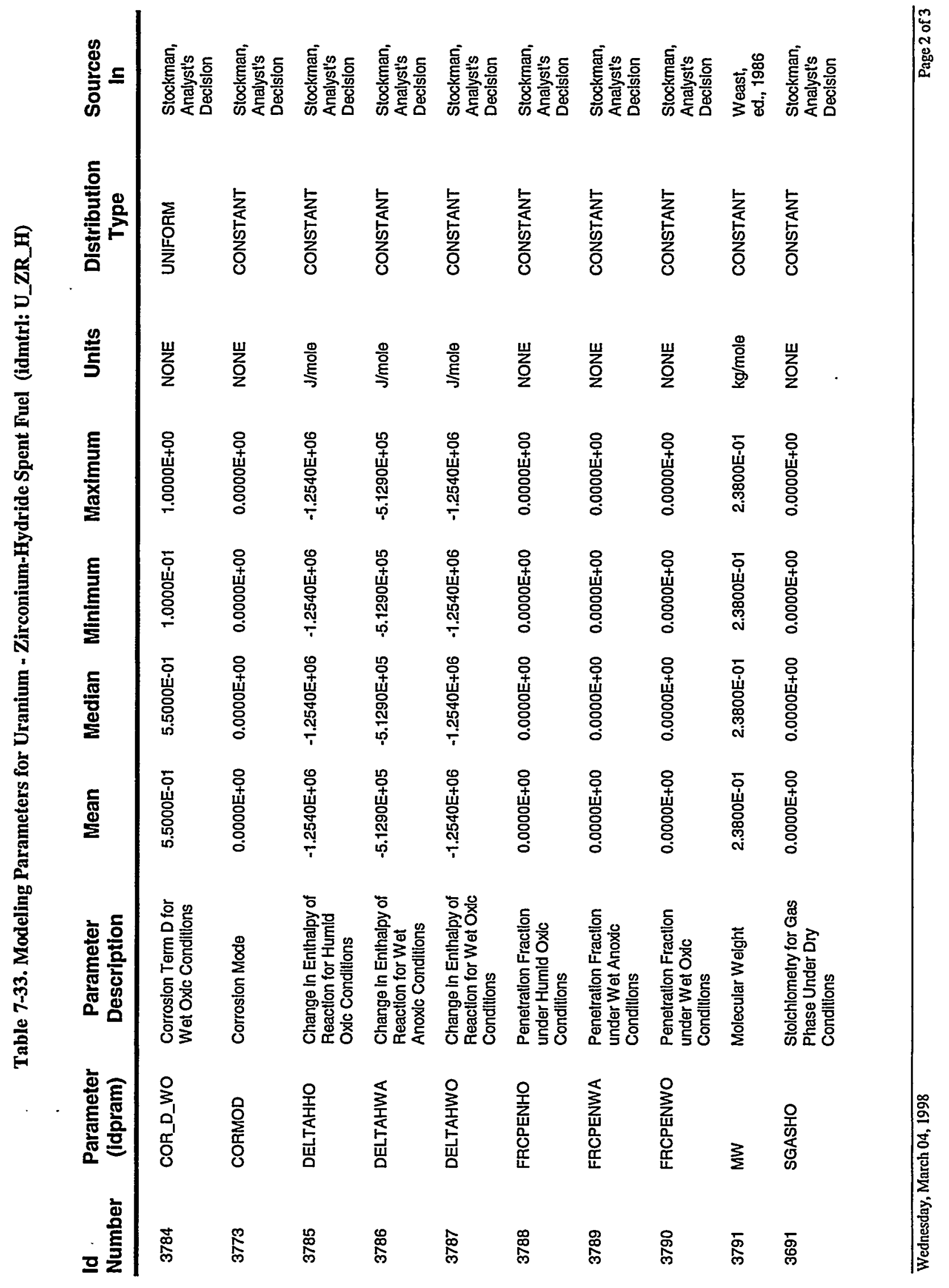




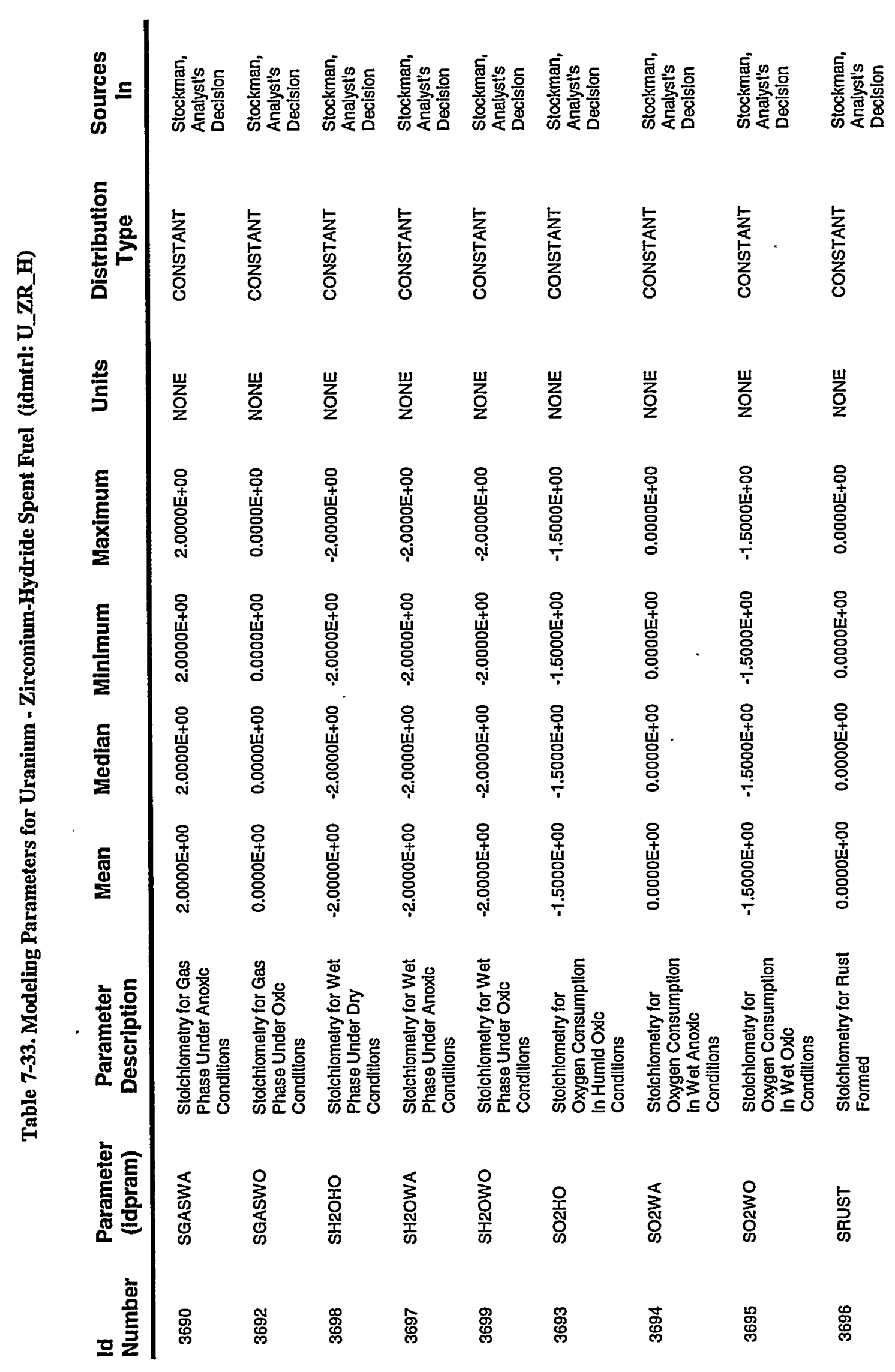

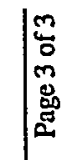




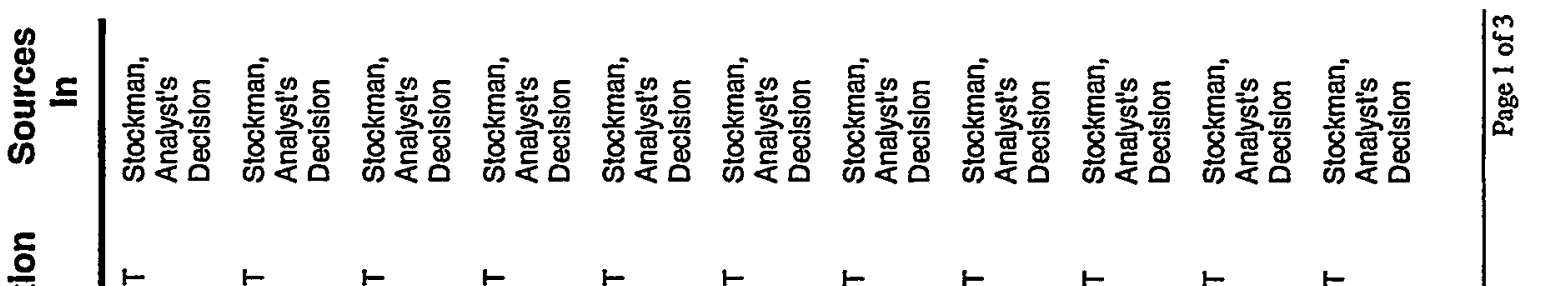

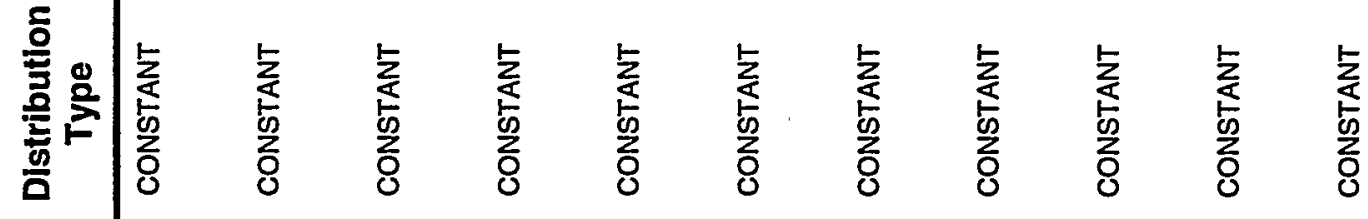

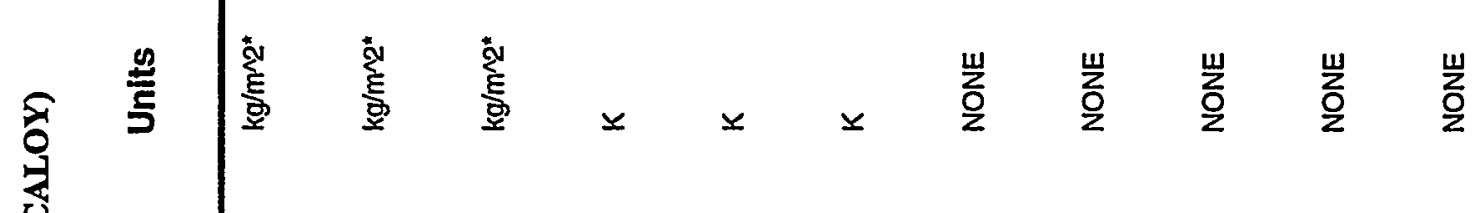

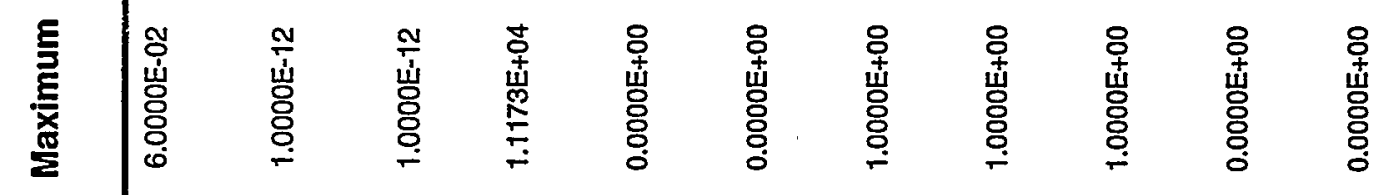

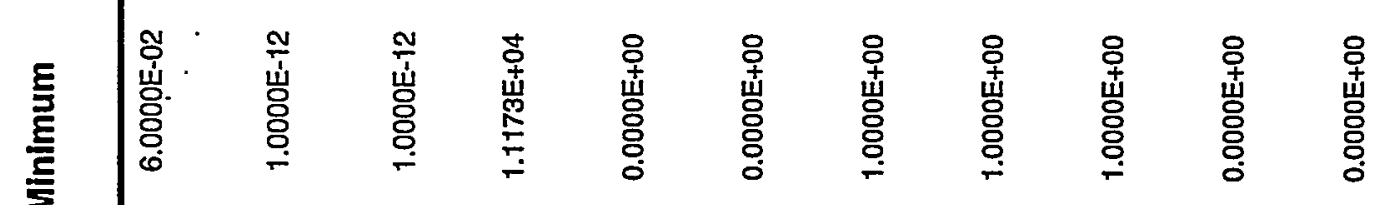

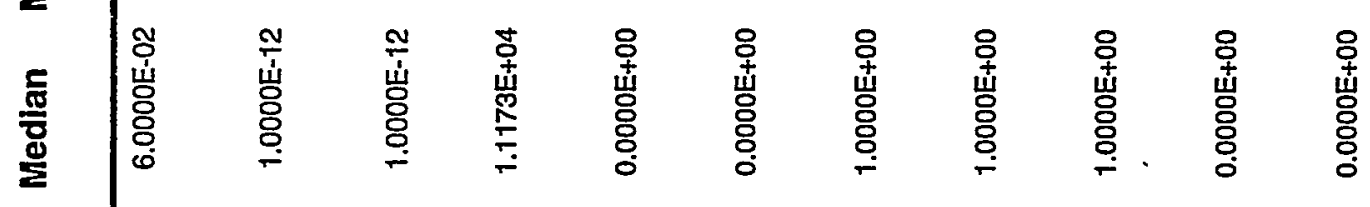

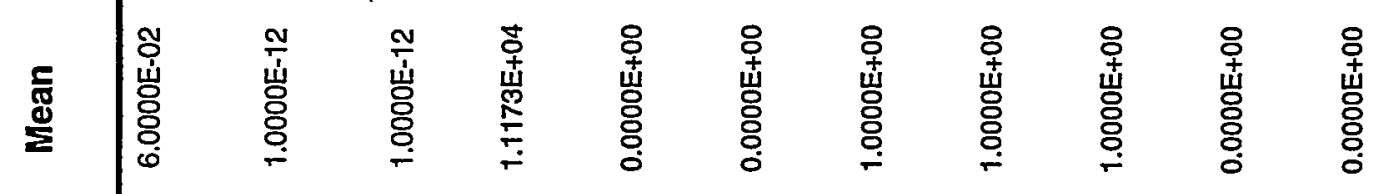

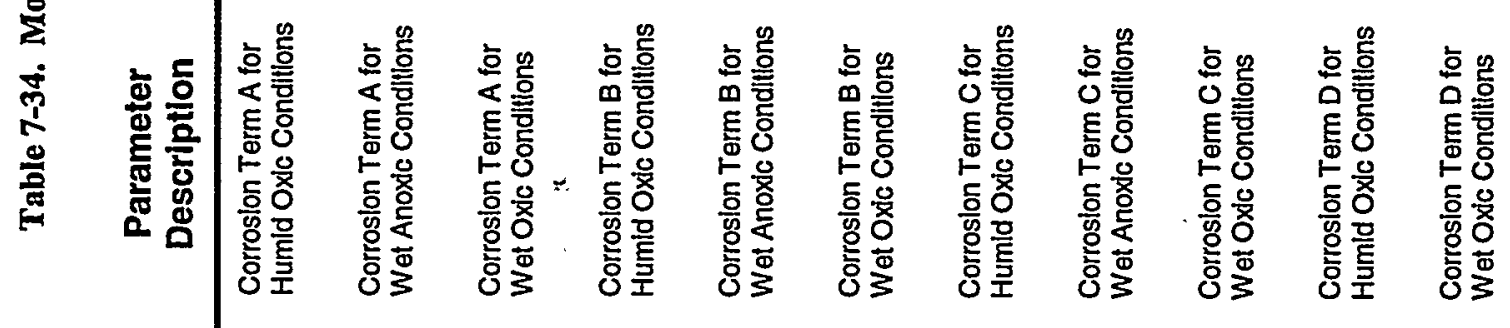

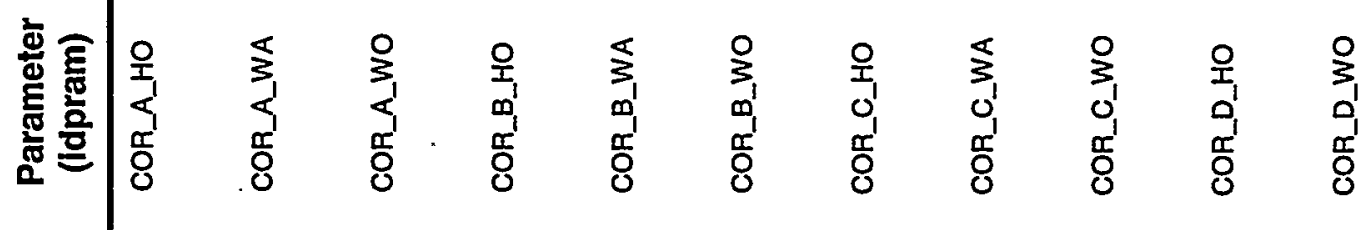

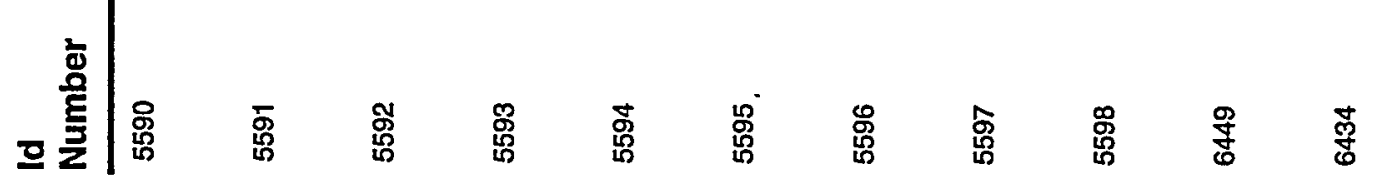




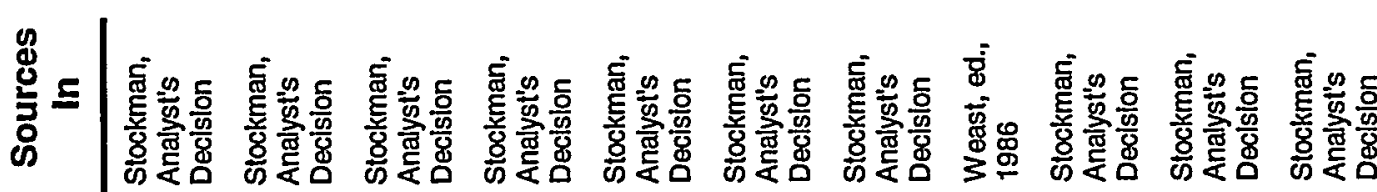

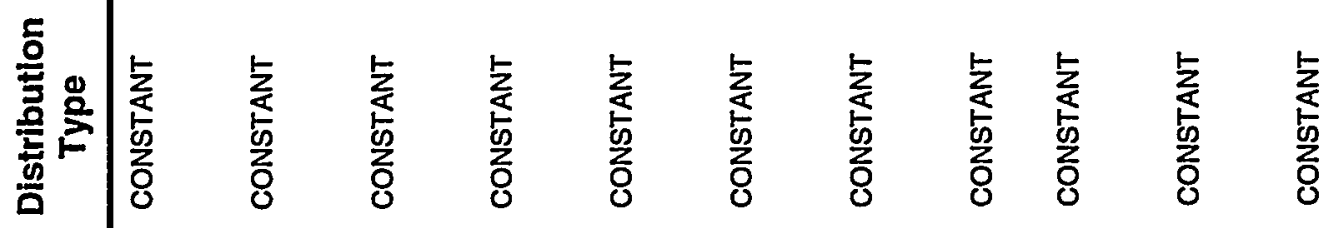

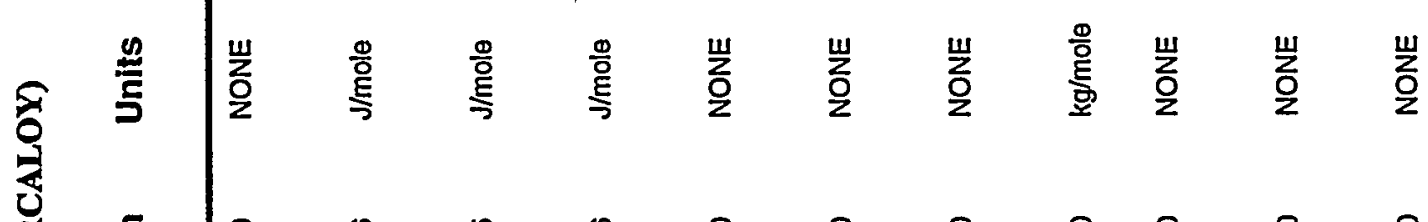

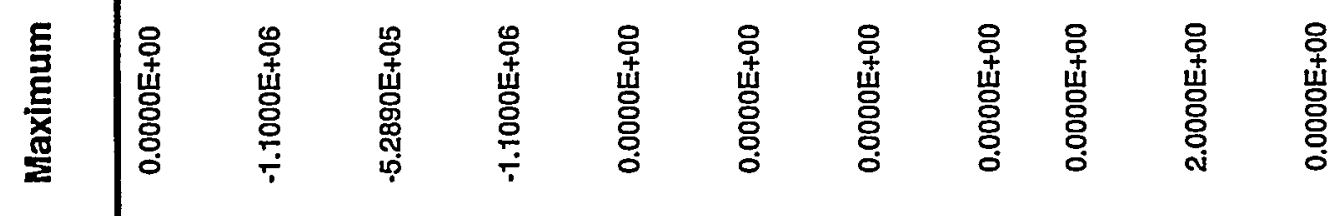

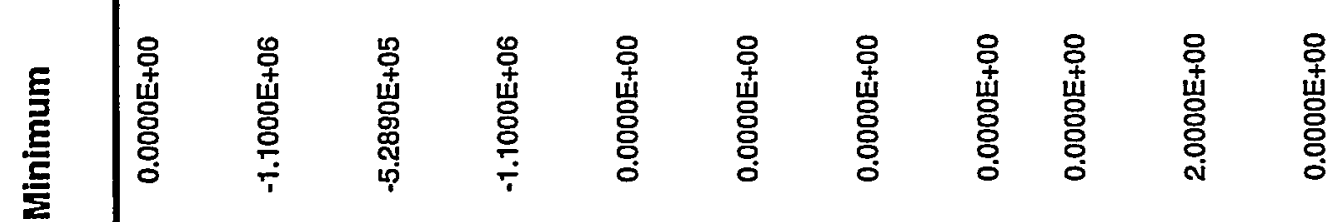

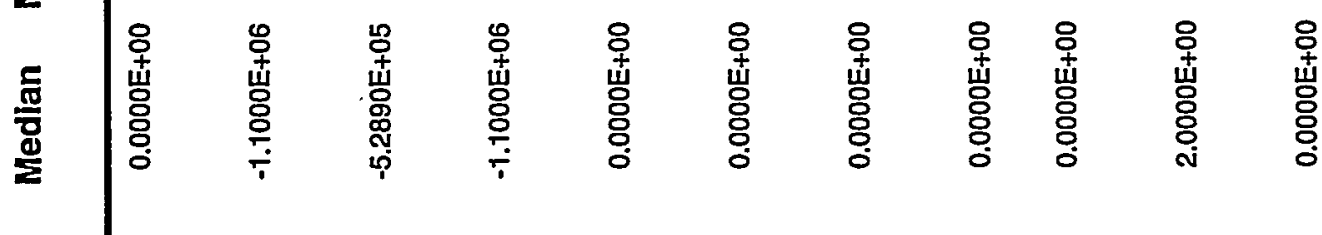

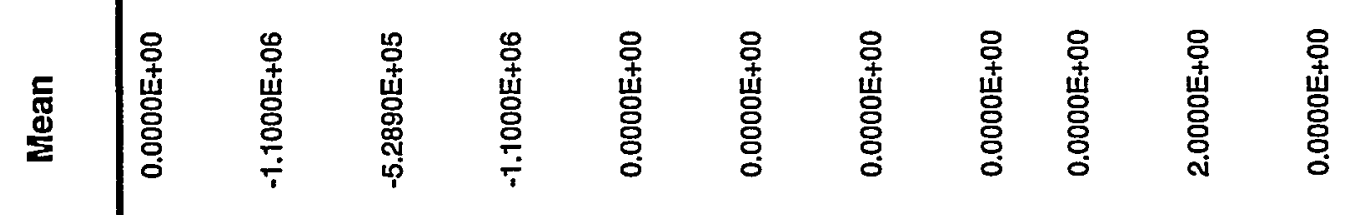

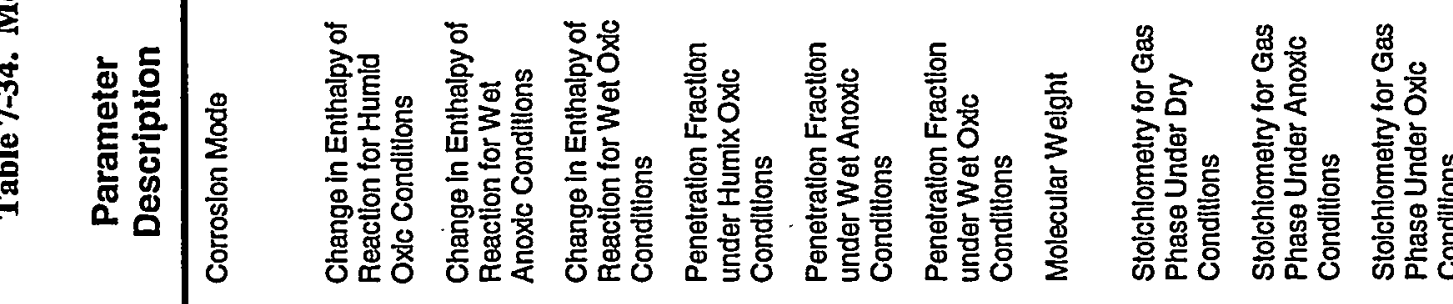

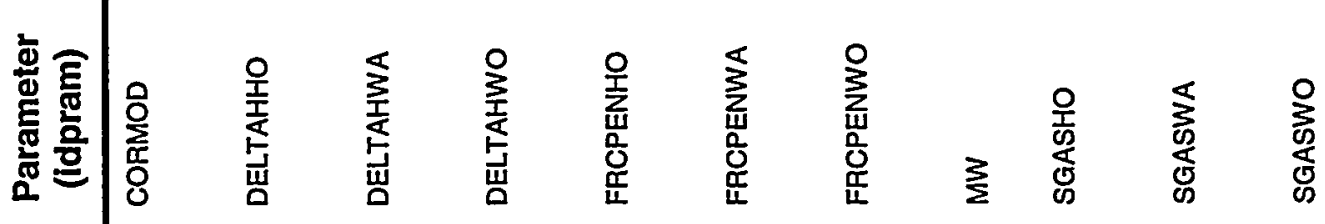

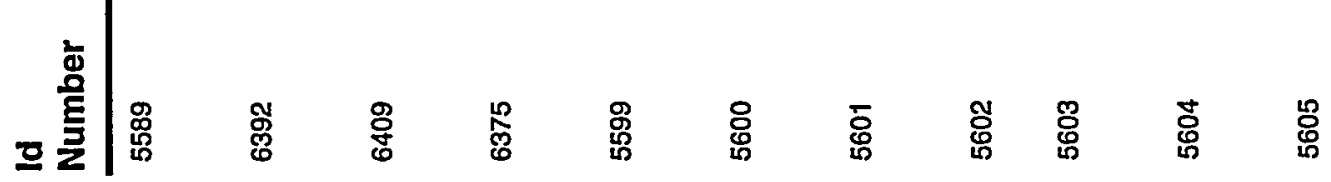



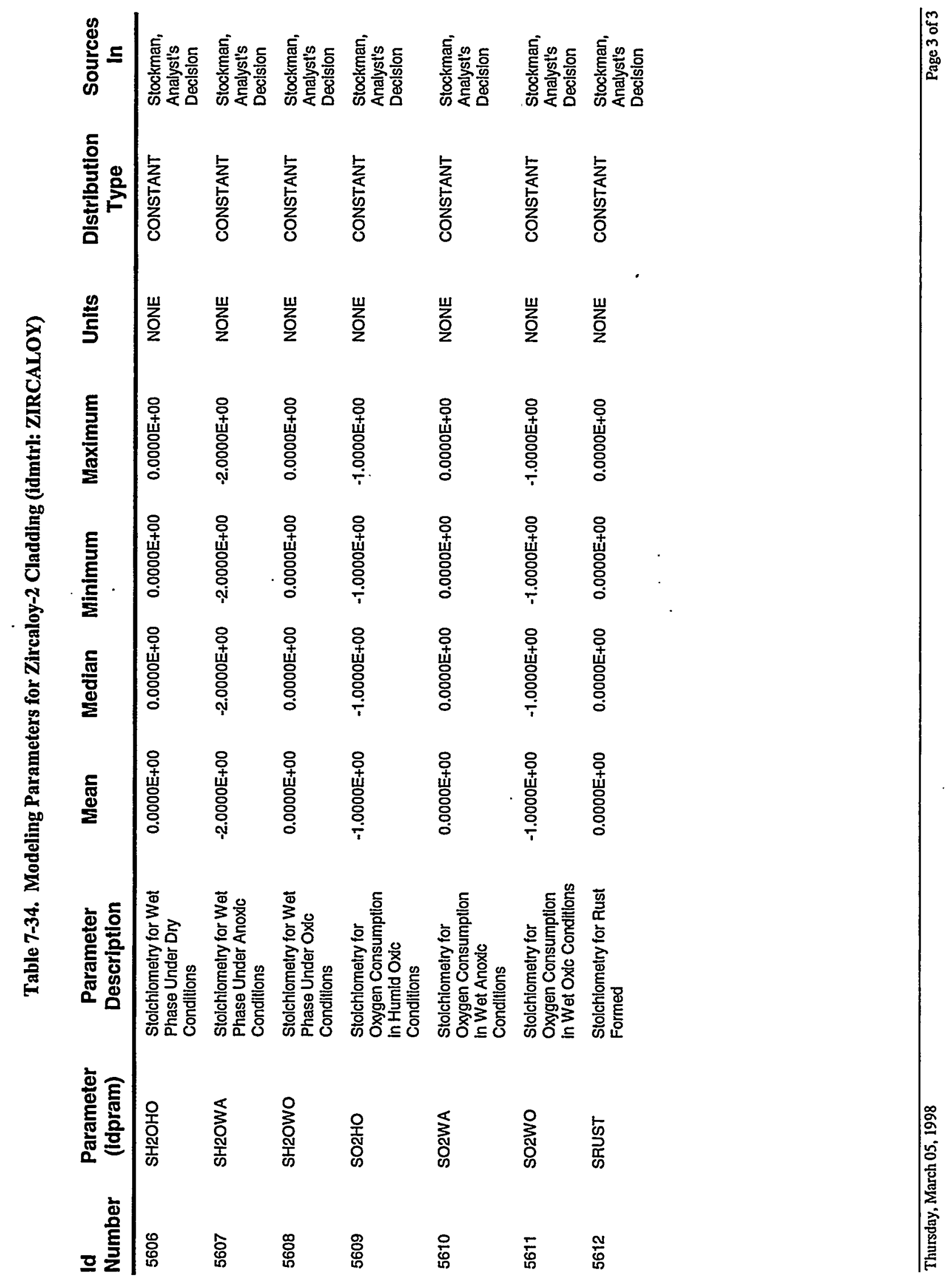


\title{
8. Consequence Modeling: Unsaturated Zone Flow and Transport
}

\author{
D. G. O’Brien, M. E. Lord, J. D. Schreiber, R. D. McCurley, and A. A. Shinta
}

The 1997 performance assessment is designed to study the movement of radioactive waste by means of a series of flow and transport numerical models in a Monte Carlo analysis. The principal models for the unsaturated zone include BRAGFLO_T (flow) and NUTS (transport), both of which are described in this chapter. STAFF3D, the principal code for the saturated zone, is described in Chapter 9. Other important models and submodels used in the 1997 PA are CST, CONRAD, and SUMQ_3D. CST is described in Chapter 7; CONRAD and SUMQ_3D are described in Section 8.2.

In a Monte Carlo analysis, parameter uncertainty and sensitivity to the solution are investigated by performing a number of simulations with different sets of input parameters. In this analysis, 52 simulations were performed consisting of 50 sampling realizations, plus two parameter sets that contained only mean or median value parameters.

\subsection{Repository Fluid Flow and Temperature Modeling}

\subsubsection{Mathematical Model Description}

BRAGFLO_T (ver. 3.10) is the numerical simulator used to compute the flow of water, nitrogen, oxygen, and heat in the 1997 PA. BRAGFLO_T is an enhanced version of the isothermal multiphase flow model, BRAGFLO (WIPP Performance Assessment Dept., 1992, Appendix A). The enhancements include the addition of the energy balance equation and the incorporation of thermal effects on both fluid and rock properties. The code also contains submodels that predict gas and water consumption/production as the result of waste package corrosion, and a submodel that predicts the energy released as the result of radioactive decay of the waste. Version 3.10 includes many features and solution techniques used in TOUGH2 (Pruess, 1991), such as an effective continuum approximation for modeling fractured porous media, vapor pressure lowering due to capillary pressure, and diffusive mass flux in the gas phase.

The code uses a finite-difference formulation to solve four partial differential equations (PDEs) that describe the mass and energy balance of a three-component, two-phase system. Fick's Law and a multiphase extension of Darcy's Law are used to describe fiuid flow. Heat is transported by conduction and convection, the latter including both sensible and latent heat. The following are the governing partial differential equations solved by BRAGFLO_T:

Component mass balance equations $(i=1,2,3)$ :

$$
\begin{aligned}
& \nabla \cdot\left[\frac{\alpha y_{i} \rho_{n} k_{m n} k}{\mu_{n}}\left(\nabla P_{n}+\rho_{n} g \nabla Z\right)+\frac{\alpha x_{i} \rho_{w} k_{m w} k}{\mu_{w}}\left(\nabla P_{w}+\rho_{w} g \nabla Z\right)+\frac{\alpha \rho_{n} \phi S_{n} D_{i j}}{\tau} \nabla y_{i}\right] \\
& +\alpha\left[y_{i}^{s} q_{n}^{s}+x_{i}^{s} q_{w}^{s}+y_{i}^{c} q_{n}^{c}+x_{i}^{c} q_{w}^{c}\right]=\alpha \frac{\partial}{\partial t}\left[y_{i} \phi \rho_{n} S_{n}+x_{i} \phi \rho_{w} S_{w}\right]
\end{aligned}
$$

Energy balance equation:

$$
\begin{aligned}
& \nabla \cdot(\alpha \lambda \nabla T)+\nabla \cdot\left[\frac{\alpha h_{n} \rho_{n} k_{r n} k}{\mu_{n}}\left(\nabla P_{n}+\rho_{n} g \nabla Z\right)+\frac{\alpha h_{w} \rho_{w} k_{n w} k}{\mu_{w}}\left(\nabla P_{w}+\rho_{w} g \nabla Z\right)\right] \\
& +\alpha\left[h_{n}^{s} q_{n}^{s}+h_{w}^{s} q_{w}^{s}+h_{n}^{c} q_{n}^{c}+h_{w}^{c} q_{w}^{c}\right]=\alpha \frac{\partial}{\partial t}\left[\phi\left(U_{n} \rho_{n} S_{n}+U_{w} \rho_{w} S_{w}\right)+(1-\phi) \rho_{r} U_{r}\right]
\end{aligned}
$$




\section{Consequence Modeling: Unsaturated Zone Flow and Transport}

where

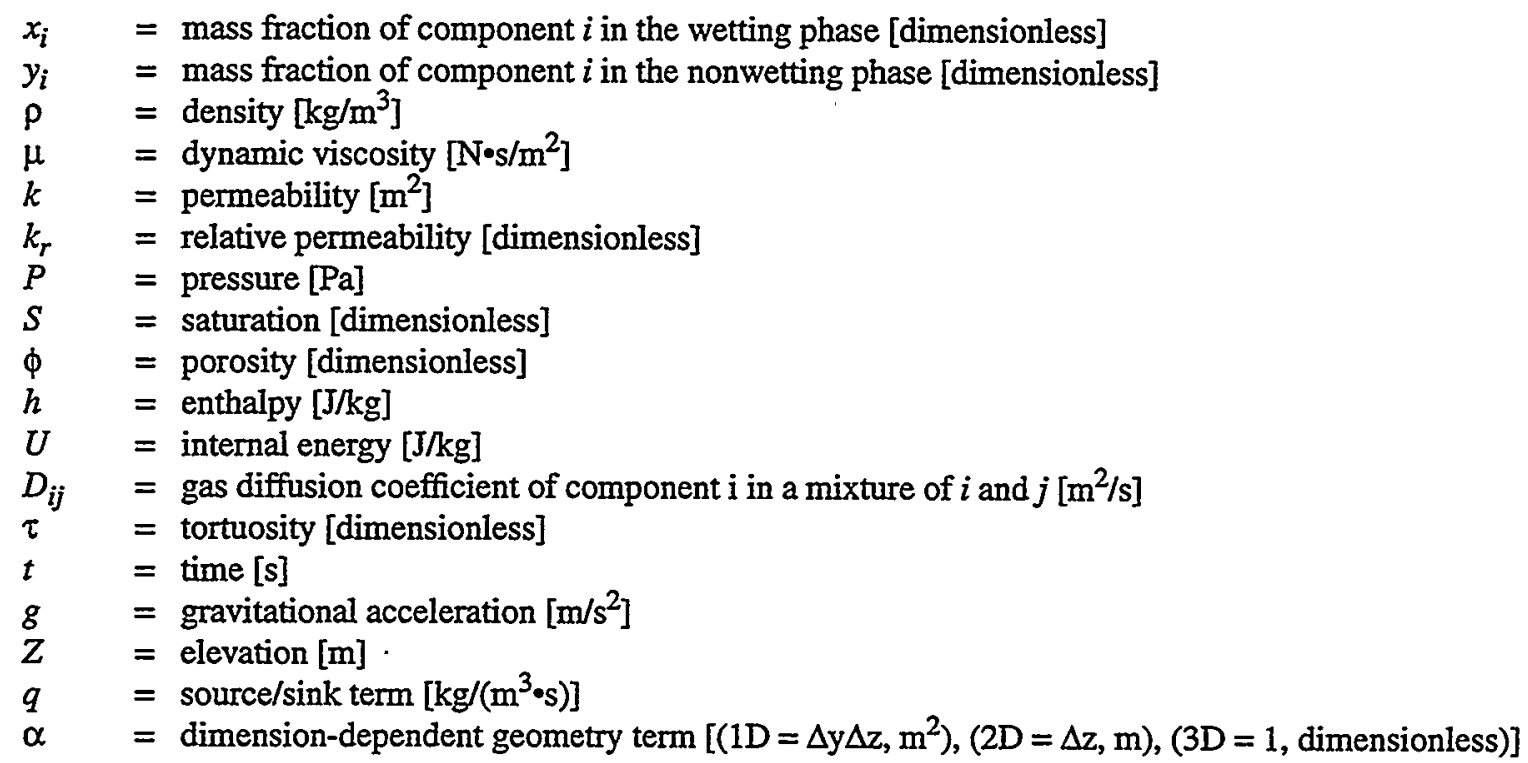

In Equation 8.1-1, the mass balance is compositional with respect to component $i$, where $i$ is equal to 1 for the condensible water component, 2 for the nitrogen component, and 3 for the oxygen component. Subscripts $w$ and $n$ refer to the wetting and nonwetting phases, respectively, while subscript $r$ refers to the rock. Superscripts are used to distinguish the origin of a source/sink term; $s$ refers to wells, and $c$ to chemical reactions.

Complex numerical models, such as BRAGFLO_T (3.10), are typically verified by comparing the results of the simulator with both analytical solutions and similar numerical models. An earlier version of BRAGFLO_T (2.00) was verified using an analytical solution to the heat pipe problem (Udell and Fitch, 1985) and TOUGH (Pruess et al., 1990), a comparable numerical simulator. Verification results were presented in Rechard, ed. (1995).

Effective Continuum. To simulate the thermohydrologic processes of a fractured porous medium, an effective continuum approximation (Pruess et al., 1990; Peters and Klaveter, 1988) was added to BRAGFLO_T. The model is based on the assumption that a state of local thermodynamic equilibrium (phase pressures and temperatures) exists between the matrix and fracture network. This assumption greatly simplifies the mathematical description of a fractured porous medium by cutting the number of unknowns in half. Consequently, the fractured porous medium can be represented by a single effective continuum with one set of thermodynamic variables describing the state of the system.

Peters and Klaveter (1988) present a detailed discussion of the mathematical simplifications needed to derive the effective continuum formulation for Richards equation (Richards, 1931). Pruess et al. (1990) extended the work to multiphase flow. The effective continuum implemented in BRAGFLO_T is very similar to that of Pruess et al. (1990); however, there are slight differences, warranting a brief description of the formulation used. A technique is also presented to extract the matrix and fracture flow fields from the effective continuum solution.

To implement the effective continuum model, parameters and characteristic curves representative of the fractured porous medium must be obtained. These parameters and expressions are derived from the properties of the rock matrix and the interconnected fractures. Designating matrix parameters with the subscript $m$, and fracture parameters with the subscript $f$, the total volume of the system, $V$, is

$$
V=V_{m}+V_{f}
$$

The effective matrix porosity, $\bar{\phi}_{m}$ (matrix pore volume divided by the total volume), is calculated from the matrix porosity, $\phi_{m}$ (matrix pore volume divided by the matrix volume). 


$$
\bar{\phi}_{m}=\phi_{m} \frac{V_{m}}{V}
$$

Similarly, the effective fracture porosity, $\bar{\phi}_{f}$, is computed as,

$$
\bar{\phi}_{f}=\phi_{f} \frac{V_{f}}{V}
$$

The effective total porosity, $\phi$, defined as the total pore volume divided by the total volume, is then the sum of the effective matrix and effective fracture porosities.

$$
\phi=\bar{\phi}_{m}+\bar{\phi}_{f}
$$

The effective continuum wetting phase saturation, $S_{w}$ is computed in a like manner in terms of the wetting phase matrix and fracture saturations, $S_{w, m}$ and $S_{w, f}$

$$
S_{w}=\frac{S_{w, m} \phi_{m} V_{m}+S_{w, f} \phi_{f} V_{f}}{\phi_{m} V_{m}+\phi_{f} V_{f}}
$$

The matrix and fracture network can be conceptualized as a parallel flow system in each of the principle coordinate directions. Thus, the effective continuum absolute permeability is calculated in each coordinate direction, and is expressed in terms of the matrix and fracture permeabilities, $k_{m}$ and $k_{f}$ and the cross-sectional areas of each, $A_{m}$ and $A_{f}$ (Amyx et al., 1960).

$$
k=\frac{A_{m} k_{m}+A_{f} k_{f}}{A_{m}+A_{f}}
$$

Including the relative permeability of the matrix and the fracture, $k_{r, m}$ and $k_{r, f}$, an analogous averaging formula describes the effective permeability to the phase $\beta, k_{e \beta}$ ( $\beta=$ wetting, or nonwetting phase),

$$
k_{e \beta}=\frac{A_{m} k_{r \beta, m} k_{m}+A_{f} k_{r \beta, f} k_{f}}{A_{m}+A_{f}}
$$

The effective continuum relative permeability to phase $\beta, k_{r \beta}$, is derived from Equations 8.1-8 and 8.1-9,

$$
k_{r \beta}=\frac{k_{e \beta}}{k}=\frac{A_{m} k_{r \beta, m} k_{m}+A_{f} k_{r \beta, f} k_{f}}{A_{m} k_{m}+A_{f} k_{f}}
$$

The effective continuum relative permeability is a function of the relative permeabilities in the matrix and the fracture, which are functions of their respective wetting phase saturations. Therefore, to evaluate Equation 8.1-10, the wetting phase saturation in the matrix and the fracture must be obtained. This is accomplished by imposing the assumption of local thermodynamic equilibrium, which establishes the constraint that,

$$
P_{c, m}\left(S_{w, m}\right)=P_{c, f}\left(S_{w, f}\right)
$$

The matrix and fracture wetting phase saturations are then computed by simultaneous solving Equations $8.1-7$ and 8.1-11. 
Boundary Heat Loss. Heat is transported across specified problem domain boundaries using a one-dimensional analytical solution to the heat equation,

$$
\frac{\partial T}{\partial t}=k \frac{\partial^{2} T}{\partial x^{2}}
$$

where

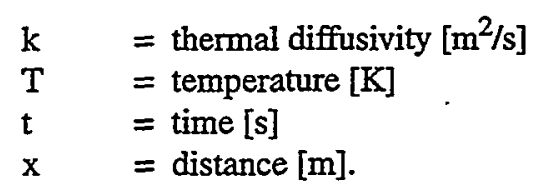

The boundary condition is

$$
T(0, t)=F(t), t \geq 0 \text { (given temperature history), }
$$

and initial condition is

$$
T(x, 0)=T_{1}, x \geq 0 \text { (given initial temperature). }
$$

The diffusivity is expressed in terms of the formation properties as

where

$$
\mathrm{k}=\mathrm{K} / \mathrm{\rho c}
$$

$\mathrm{K}=$ thermal conductivity $[\mathrm{J} / \mathrm{s} / \mathrm{m} / \mathrm{K}]$

$\rho \quad=$ density $\left[\mathrm{kg} / \mathrm{m}^{3}\right]$

c $\quad=$ specific heat $[\mathrm{J} / \mathrm{kg} / \mathrm{K}]$

Let

$t_{i}=$ ith time in simulation, $i=1, \ldots, n+1$

$\mathrm{T}_{\mathrm{i}} \quad=$ temperature at ith time

$\Delta \mathrm{T}_{\mathrm{i}}=\mathrm{T}_{\mathrm{i}+\mathrm{i}}-\mathrm{T}_{\mathrm{i}}$ (temperature increment over ith time step).

Note $T_{1}$ is temperature at initial time $t_{1}$.

The solution of Equation 8.1-12 with the above initial and boundary conditions was derived by Carslaw and Jaeger (1959). The result of their work is

$$
T-T_{1}=\sum_{i=1}^{n} \Delta T_{i} e r f c\left[\frac{x}{2 \sqrt{k\left(t-t_{i}\right)}}\right]
$$

where erfc is the complementary error function. The temperature gradient at the boundary $x=0$ is computed to be

$$
\frac{\partial T}{\partial \mathrm{x}}(0, t)=-\frac{1}{\sqrt{\pi k}} \sum_{i=1}^{n} \Delta T_{i} / \sqrt{t-t_{i}}, t_{n} \leq t \leq t_{n+1} .
$$

The cumulative heat flux over the time step is obtained from

$$
Q_{\text {boundary }}=-\int_{t_{n}}^{t_{n}+1} K A \frac{\partial T}{\partial x}(0, t) d t
$$


and is found to be

$$
Q_{\text {boundary }}=E(n)-E(n-1)
$$

where

$$
E(n)=\frac{2 K A}{\sqrt{\pi k}} \sum_{i=1}^{n} \Delta T_{i} \sqrt{t_{n+1}-t_{i}}
$$

Capillary Pressure. At the boundary between two phases, an imbalance in molecular forces creates a component of force acting tangential to the interface between the two phases, referred to as the interfacial tension. Interfacial tension acting on a curved surface is balanced, at equilibrium, by pressure differences between the two phases. The difference in pressure is called the capillary pressure, $P_{c}$, and is defined as,

$$
P_{c}=P_{n}-P_{w}
$$

Though capillary effects dominate $P_{c}$ at high to moderately low wetting phase saturations, wetting phase adsorption to the solid phase dominates $P_{c}$ at low saturations (Melrose, 1991). To model both the capillary and phase adsorption effects, two mathematical expressions were selected; the van Genuchten (1980) model and the Ross et al. (1992) exponential model. In a manner very similar to that proposed by Rossi and Nimmo (1994), the two mathematical models were combined in a piecewise fashion, as follows:

$$
\begin{gathered}
S_{w}<S_{w j} \quad P_{c 1}=P_{m} \operatorname{Exp}\left(\frac{-S_{w}}{C}\right) \\
S_{w}>S_{w j} \quad P_{c 2}=P_{o}\left(S_{e}^{[n /(1-n)]}-1\right)^{1 / n}
\end{gathered}
$$

The exponential model, Equation 8.1-19, describes phase adsorption effects at low wetting phase saturations. The parameters, $P_{m}$ and $C$, are constants, where $P_{m}$ is the maximum possible capillary pressure at zero wetting phase saturation. The exponential model is joined to the van Genuchten model, Equation 8.1-20, at the junction saturation, $S_{w j}$. The parameters $P_{o}$ and $n$, are constants dependent on the rock type. $S_{e}$ is the "effective" wetting phase saturation defined by,

$$
S_{e}=\frac{S_{w}-S_{w r}}{1-S_{w r}}
$$

where, $S_{w r}$ is the residual wetting phase saturation. Luckner et al. (1989) define $S_{w r}$ as the point "when all or parts of the connecting films become so thin, and hence so strongly adsorbed onto the solid phase, that the wetting fluid loses its capability to respond to hydraulic gradients." Therefore, $S_{w j}$ is always greater than $S_{w r}$ but only by a small margin.

Making the piecewise function both continuous and smooth at $S_{w j}$, the following constraints are imposed;

$$
\begin{aligned}
P_{c 1}\left(S_{w j}\right) & =P_{c 2}\left(S_{w j}\right) \\
\frac{\partial}{S_{w}} P_{c 1}\left(S_{w j}\right) & =\frac{\partial}{S_{w}} P_{c 2}\left(S_{w j}\right)
\end{aligned}
$$

Distributions of the van Genuchten parameters $P_{o}$ and $n$, are known from the extensive research already conducted at Yucca Mountain. $P_{m}$ is assumed to be constant and formation independent as suggested by Rossi and Nimmo (1994). The remaining unknowns, $C$ and $S_{w j}$, are found by solving the nonlinear Equations 8.1-22 and 8.1-23 simultaneously. 
During the solution process, it was necessary to use a value of $10^{9} \mathrm{~Pa}$ for $P_{m}$ to ensure that a value of $C$ and $S_{w j}$ could be solved that satisfied the conditions in Equations 8.1-22 and 8.1-23 for all sample sets.

The most important benefit of the piecewise capillary pressure model is that it allows water/vapor phase changes to take place at, above, and below $S_{w r}$ The original van Genuchten model cannot handle conditions of $S_{w} \leq S_{w r}$ This difference is shown graphically in Figure 8-1, where the van Genuchten and the piecewise model are plotted using median parameter values of the TSw matrix (Table 8-1).

The median value capillary pressure functions for both the matrix and the fracture in the TSw are plotted in Figure 8-2. Notice that the capillary pressure of the matrix greatly exceeds the capillary pressure of the fracture over most of the diagram. Furthermore, the assumption of local equilibrium imposed as part of the effective continuum approximation inherently implies capillary pressure equilibrium between the matrix and the fracture. A primary limitation of this work is that in order to activate fracture flow, $S_{w}>S_{w r}$ the matrix must first be highly water saturated. Therefore, the possibility of water preferentially flowing through the fracture network in unsaturated rock (i.e., the WEEPS model) is not modeled in the 1997 PA.

Relative Permeability. The relative permeability functions used in this study were presented by Luckner et al. (1989) and based on the theory of Mualem (1976). They are;

$$
\begin{aligned}
& k_{r w}=S_{e}^{1 / 2}\left[1-\left(1-S_{e}^{1 / m}\right)^{m}\right]^{2} \\
& k_{r n}=\left(S_{e}^{*}\right)^{1 / 3}\left[1-\left(1-S_{e}^{*}\right)^{1 / m}\right]^{2 m}
\end{aligned}
$$

where, $k_{r w}$ and $k_{r m}$ are the wetting and nonwetting phase relative permeabilities, respectively, $m$ is derived from the van Genuchten parameter $n, m=1-1 / \mathrm{n}$, and $S_{e}^{*}$, is the nonwetting phase effective saturation,

$$
S_{e}^{*}=\frac{S_{n}-S_{n r}}{1-S_{n r}}
$$

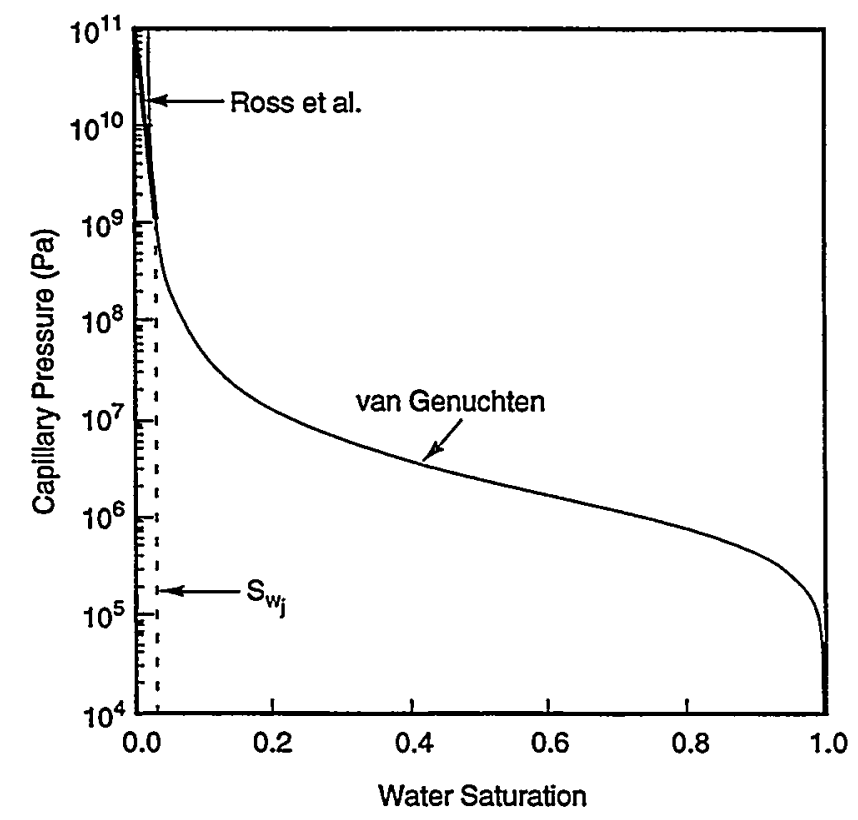

TRI-6342-5044-0

Figure 8-1. Comparison of van Genuchten and piecewise models. 
Table 8-1. TSw Median Capillary Pressure and Relative Permeability Parameters

\begin{tabular}{ccc}
\hline & Matrix & Fracture \\
\hline $\mathrm{n}$ & 1.62 & 3.0 \\
$P_{\mathrm{o}}(\mathrm{Pa})$ & $8.806 \times 10^{5}$ & $1246 \times 10^{3}$ \\
$\mathrm{~S}_{\mathrm{wr}}$ & 0.02124 & 0.04 \\
$\mathrm{~S}_{\mathrm{nr}}$ & 0.02124 & 0.04 \\
$\mathrm{~S}_{\mathrm{wj}}$ & 0.03320 & 0.04139 \\
$P_{m}(\mathrm{~Pa})$ & $1 \times 10^{11}$ & $1 \times 10^{11}$ \\
$\mathrm{C}$ & $7.379 \times 10^{-3}$ & $2.772 \times 10^{-3}$ \\
\hline
\end{tabular}

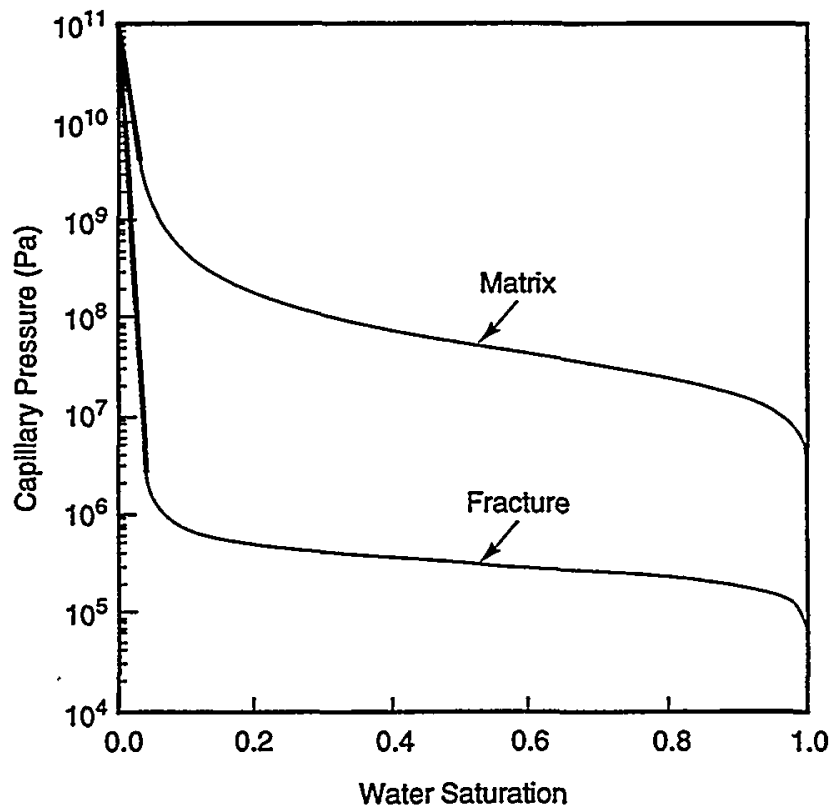

TRI-6342-5045-0

Figure 8-2. Median value capillary pressure functions for both matrix and fracture in TSw.

$S_{n}$ is the nonwetting phase saturation, and $S_{n r}$ is the nonwetting phase residual saturation. Median value wetting and nonwetting relative permeability functions of the TSw matrix are shown in Figure 8-3.

Comparison of BRAGFLO_T and TOUGH2 Formulation. The effective continuum properties of porosity, saturation, permeability and relative permeability described above are the same parameters described by Ho (March $22,1994)$, implemented in the code TOUGH2. This section demonstrates that these two sources are equivalent in their treatment of the effective continuum properties.

Consider an elemental volume of rock, which would represent a grid block volume in the numerical code, to consist of the fracture volume, the matrix bulk volume, and the matrix pore volume. Now introduce the notation

$V_{\text {tot }}=$ total bulk volume,

$V_{\text {por }}=$ matrix pore volume, 
8. Consequence Modeling: Unsaturated Zone Flow and Transport

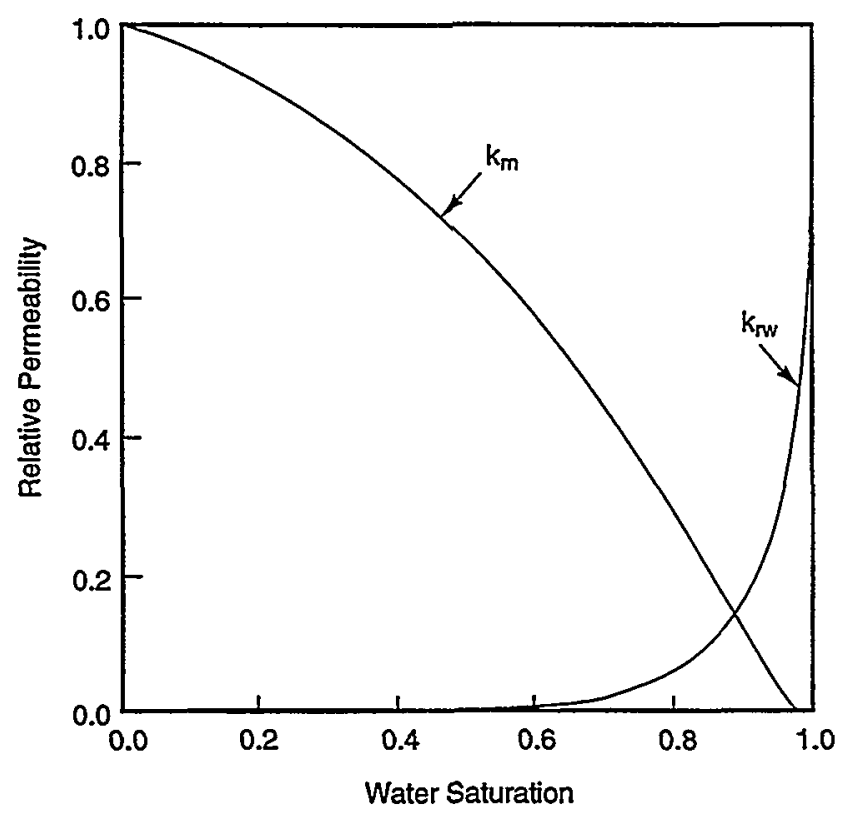

Figure 8-3. Median value wetting and nonwetting relative permeability functions of the TSw matrix.

$V_{\text {mat }}=$ matrix bulk volume,

$V_{\text {frac }}=$ fracture volume.

Ho (1994, equation 1) describes the effective continuum porosity by

$$
\phi_{e f f}=\phi_{f}+\left(1-\phi_{f}\right) \phi_{m}
$$

from which we compute

$$
\phi_{\text {eff }}=\frac{V_{f}}{V_{\text {tot }}}+\left(1-\frac{V_{f}}{V_{\text {tot }}}\right) \frac{V_{\text {por }}}{V_{\text {tot }}}=\frac{V_{f}}{V_{\text {tot }}}+\frac{V_{\text {mat }}}{V_{\text {tot }}} \frac{V_{\text {por }}}{V_{\text {tot }}}=\frac{V_{f}}{V_{\text {tot }}}+\frac{V_{\text {por }}}{V_{\text {tot }}}
$$

The effective porosity as defined in Equation 8.1-6 is

$$
\phi_{e f f}=\bar{\phi}_{m}+\bar{\phi}_{f}=\frac{V_{f}}{V_{t o t}}+\frac{V_{p o r}}{V_{t o t}}
$$

which shows that the effective porosity is equivalent in the two treatments.

The effective continuum saturation from TOUGH2 (Ho, 1994, Eq. 2) is

$$
S_{e f f}=\frac{S_{f} \phi_{f}+S_{m}\left(1-\phi_{f}\right) \phi_{m}}{\phi_{f}+\left(1-\phi_{f}\right) \phi_{m}}
$$


The fracture/matrix weighting factors are, respectively,

$$
\begin{gathered}
\frac{\phi_{f}}{\phi_{f}+\left(1-\phi_{f}\right) \phi_{m}}=\frac{\phi_{f}}{\phi_{f}+\left(1-\phi_{f}\right) \phi_{m}} \frac{V_{t o t}}{V_{t o t}}=\frac{V_{f}}{V_{f}+V_{p o r}} \\
\frac{\left(1-\phi_{f}\right) \phi_{m}}{\phi_{f}+\left(1-\phi_{f}\right) \phi_{m}}=\frac{\left(1-\phi_{f}\right) \phi_{m}}{\phi_{f}+\left(1-\phi_{f}\right) \phi_{m}} \frac{V_{t o t}}{V_{t o t}}=\frac{V_{p o r}}{V_{f}+V_{p o r}}
\end{gathered}
$$

Similarly, in Equation 8.1-7, the fracture/matrix weighting factors for the effective continuum saturation are, respectively,

$$
\begin{gathered}
\frac{\phi_{f} V_{f}}{\phi_{m} V_{m a t}+\phi_{f} V_{f}}=\frac{V_{f}}{V_{p o r}+V_{f}} \\
\frac{\phi_{m} V_{\text {mat }}}{\phi_{m} V_{\text {mat }}+\phi_{f} V_{f}}=\frac{V_{p o r}}{V_{p o r}+V_{f}}
\end{gathered}
$$

Since the respective weights are the same, the BRAGFLO_T and TOUGH2 effective saturations are equivalent.

The effective formation permeability is computed in TOUGH2 with fracture/matrix weighting, respectively,

$$
\begin{gathered}
\phi_{f}=\frac{V_{f}}{V_{t o t}} \\
1-\phi_{f}=\frac{V_{\text {mat }}}{V_{t o t}}
\end{gathered}
$$

The BRAGFLO_T effective formation permeability is computed in terms of the flow areas (Equation 8.1-8). If $\mathrm{h}$ denotes the element height, then the fracture/matrix weights are

$$
\begin{aligned}
& \frac{A_{m}}{A_{m}+A_{f}}=\frac{A_{m}}{A_{m}+A_{f}} \frac{h}{h}=\frac{V_{m a t}}{V_{\text {tot }}} \\
& \frac{A_{f}}{A_{m}+A_{f}}=\frac{A_{f}}{A_{m}+A_{f}} \frac{h}{h}=\frac{V_{f}}{V_{\text {tot }}}
\end{aligned}
$$

Therefore, the effective permeability in BRAGLO_T is equivalent to the treatment in TOUGH2. The relative permeabilities use the same weighting as the permeabilities. Thus the effective continuum relative permeability treatment in BRAGFLO_T is equivalent to the treatment in TOUGH2.

Thus, all effective continuum parameters as implemented in BRAGFLO_T are equivalent to the respective parameters in the TOUGH2 code. 


\section{Consequence Modeling: Unsaturated Zone Flow and Transport}

Now consider the dependence of the effective continuum parameters on the fracture/matrix parameters. Mean values for fracture/matrix properties in the welded Topopah Springs member were assumed as:

\begin{tabular}{lcc}
\hline \multicolumn{1}{c}{ Parameter } & Fracture & Matrix \\
\hline porosity & 0.00039 & 0.139 \\
permeability & $1.54 \mathrm{E}-17$ & $6.997 \mathrm{E}-9$ \\
van Genuchten & 3.0 & 1.7839 \\
$\mathrm{~S}_{\mathrm{wr}}$ & 0.04 & 0.045 \\
$\mathrm{~S}_{\mathrm{gr}}$ & 0.04 & 0.045 \\
\hline
\end{tabular}

The resulting van Genuchten relative permeability to water is shown in Figure 8-4a and the relative permeability to gas is shown in Figure 8-4b. These figures demonstrate that the fracture permeability completely dominates the relative permeability behavior, since the effective relative permeabilities overlay the fracture relative permeabilities in the figures.

The effective continuum saturation is dominated by the matrix saturation, which is shown in Figure 8-4c, where effective continuum saturation is shown as a function of fracture saturation for a family of matrix saturations $(\mathrm{Sm}=0 ., 0.2,0.4,0.6,0.8,1.0)$. The effective continuum saturation is essentially determined by the matrix saturation, which is accounted for by the very low ratio of fracture to matrix porosity. This implies that while the effective continuum may contain high water saturation, the fracture may be dry. The fracture saturation should control dripping from the tunnel ceiling to the waste. Therefore, the wet corrosion rate is linked to the fracture saturation as discussed in Chapter 7.

\subsubsection{Applied Model}

Model Geometry and Spatial Discretization. In the unsaturated zone, fluid and heat flow are modeled in two dimensions using the computational mesh depicted in Figure 8-5. This diagram is essentially a vertical cross-section through the repository and surrounding geologic strata. Each square of the computational mesh represents a grid block in the finite-difference spacial discretization. Integers on the drawings (shown in reverse type) represent the two-dimensional element indices. The grid blocks, as shown, are not to scale. Instead, each cell is displayed with unit dimensions. The actual grid block dimensions are shown below the grid for the $\mathrm{x}$-direction, above the grid for the z-direction, and to the right of the grid for the y-direction.

Fluid and energy transport are modeled from the surface to the water table. The computational mesh is based on the stratigraphic column of Yucca Mountain, shown in Chapter 4 (Figure 4-9b) with the exception that the 20-m-thick alluvium is not modeled. All geologic units are assumed to be homogenous and of constant thickness. The modeled cross section extends from west of the Solitario Canyon Fault passing near the location of well H-6 to east of the Ghost Dance Fault passing near the location of well SD-12 (see Figure 4-1 in Chapter 4). The model grid has been superimposed over the actual stratigraphy in the cross section of Figure 8-6. An enlarged illustration of the grid is shown in Figure 8-7. The mesh extends horizontally $1690.5 \mathrm{~m}$ beyond the edge of the repository in both the west and east directions. The vertical relief of the mesh does not overlay perfectly on the geologic cross section because the stratigraphic column of Yucca Mountain used to develop the computational mesh is a composite thickness representation.

In the $1997 \mathrm{PA}$, the modeling unit layers, which represent stratigraphy, exhibit surface topography and stratigraphic dip at $4.6^{\circ}$ from west to east. Assigned rock properties vary between 12 hydrologic units (TCw, PTn, TSv, Tsun, TSul, TSmn, TSll, TSIn, CHnv, CHnz, PPw and CFun) and are laterally homogeneous within each unit. The repository is also dipping in the same direction as the geologic layers. Interaction of the water table with the unsaturated zone is included, and the water table is held at a constant elevation of $730 \mathrm{~m}$, positioning it near the bottom of the Prow Pass nonwelded zeolitic member (CFun). 


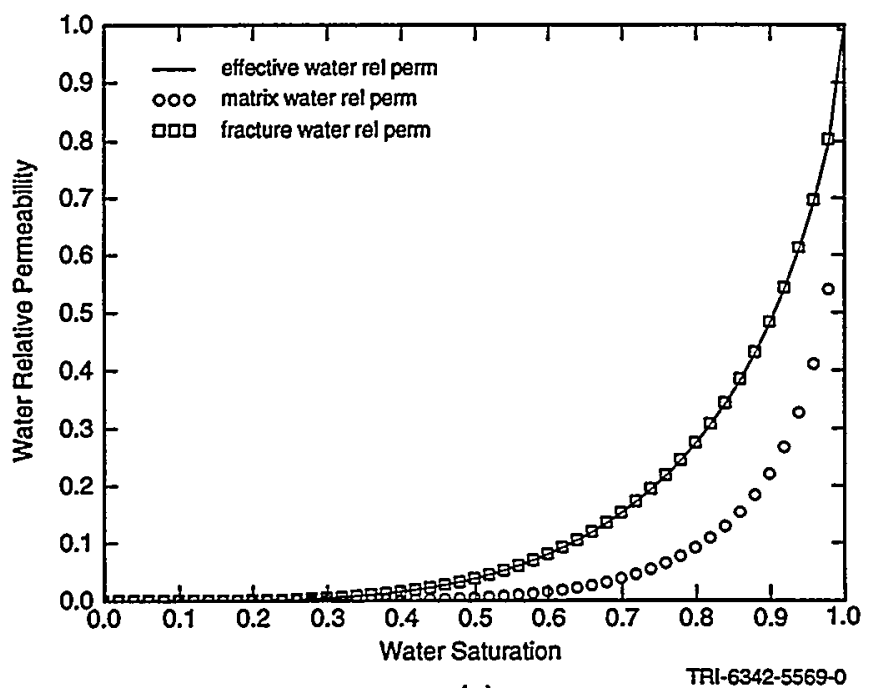

(a)

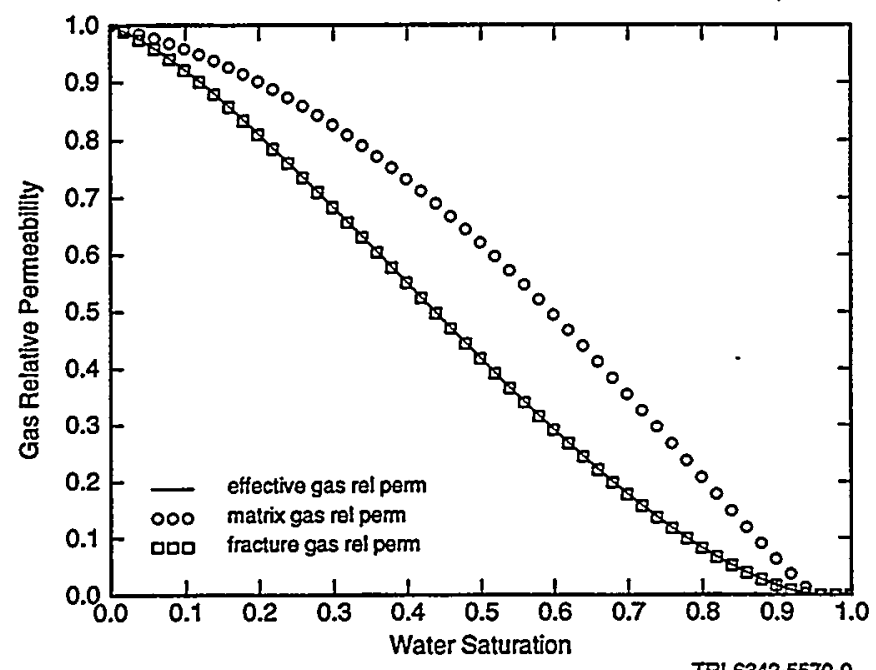

(b)

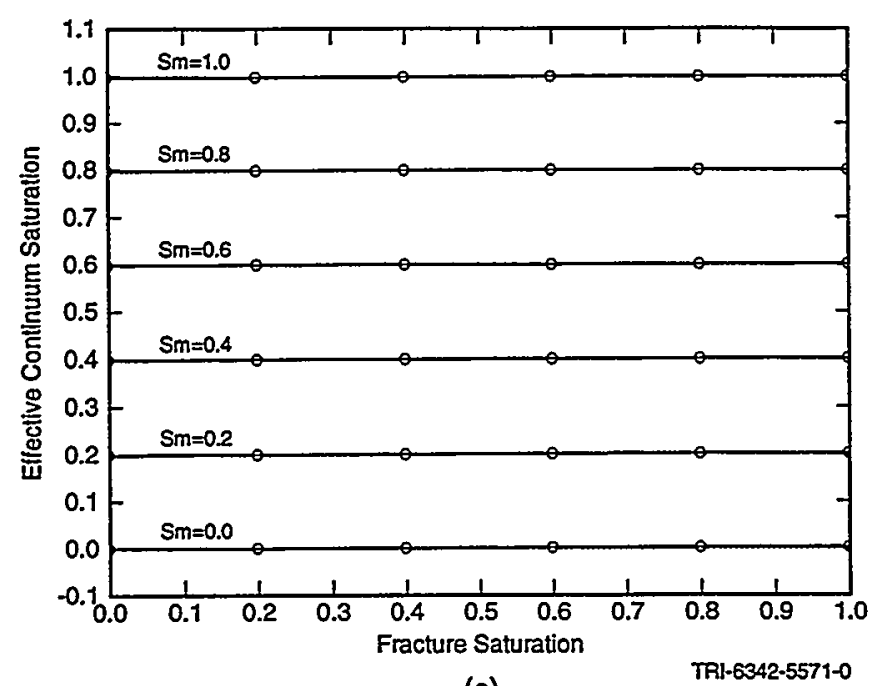

(c)

Figure 8-4. Plots indicating (a) water saturation vs. water relative permeability, (b) water saturation vs. gas relative permeability, and (c) fracture saturation vs. effective continuum saturation. 


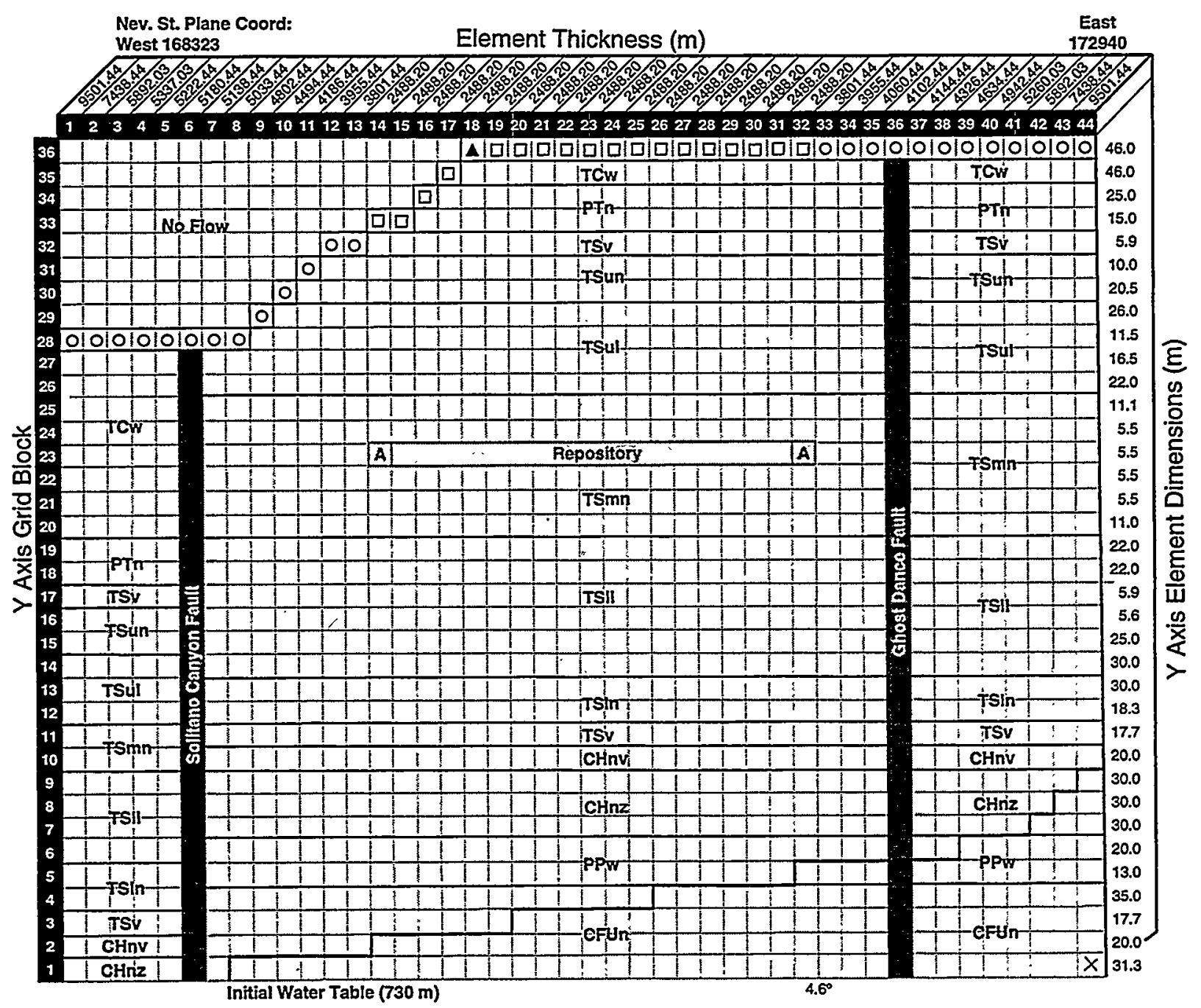

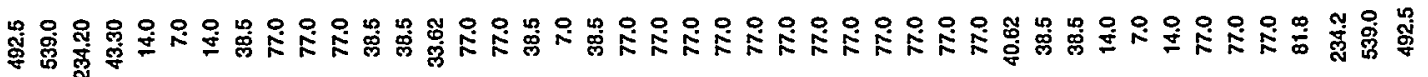
$X$ Axis Element Dimensions (m)

Average Water Infiltration
$\square$ Repository Water Infiltration
$\Delta$ Enhanced Water Infiltration
$\times$ Constant Water Pressure
A Repository and Access Tunnels

TRI-6342-4542-1

Figure 8-5. BRAGFLO_T computational mesh. 
Cross Section Throu

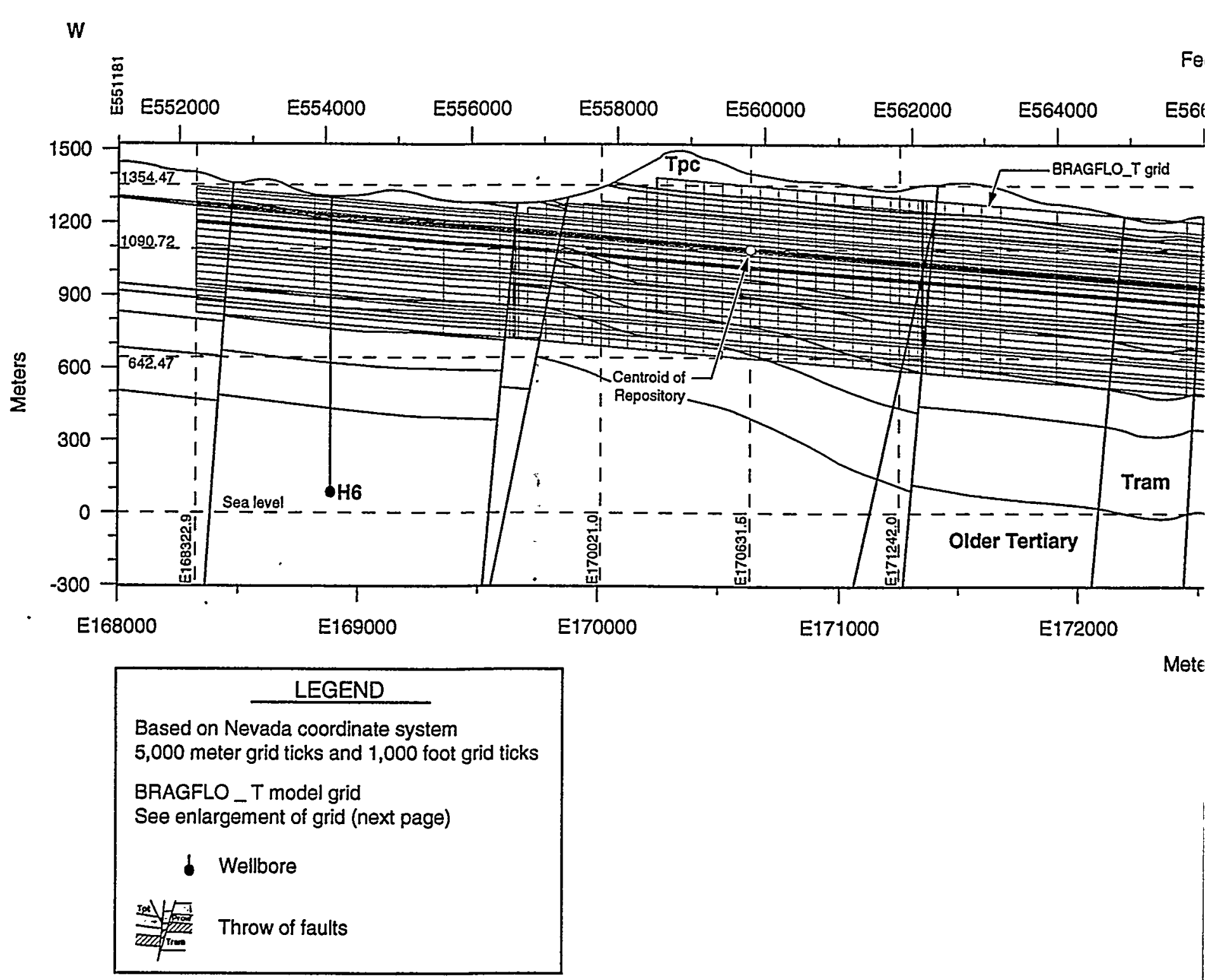

N232654 (m)

Based on Nevada coordinate system

5,000 meter grid ticks and 1,000 foot grid ticks

BRAGFLO_T model grid

See enlargement of grid (next page)

b Wellbore

Throw of faults 


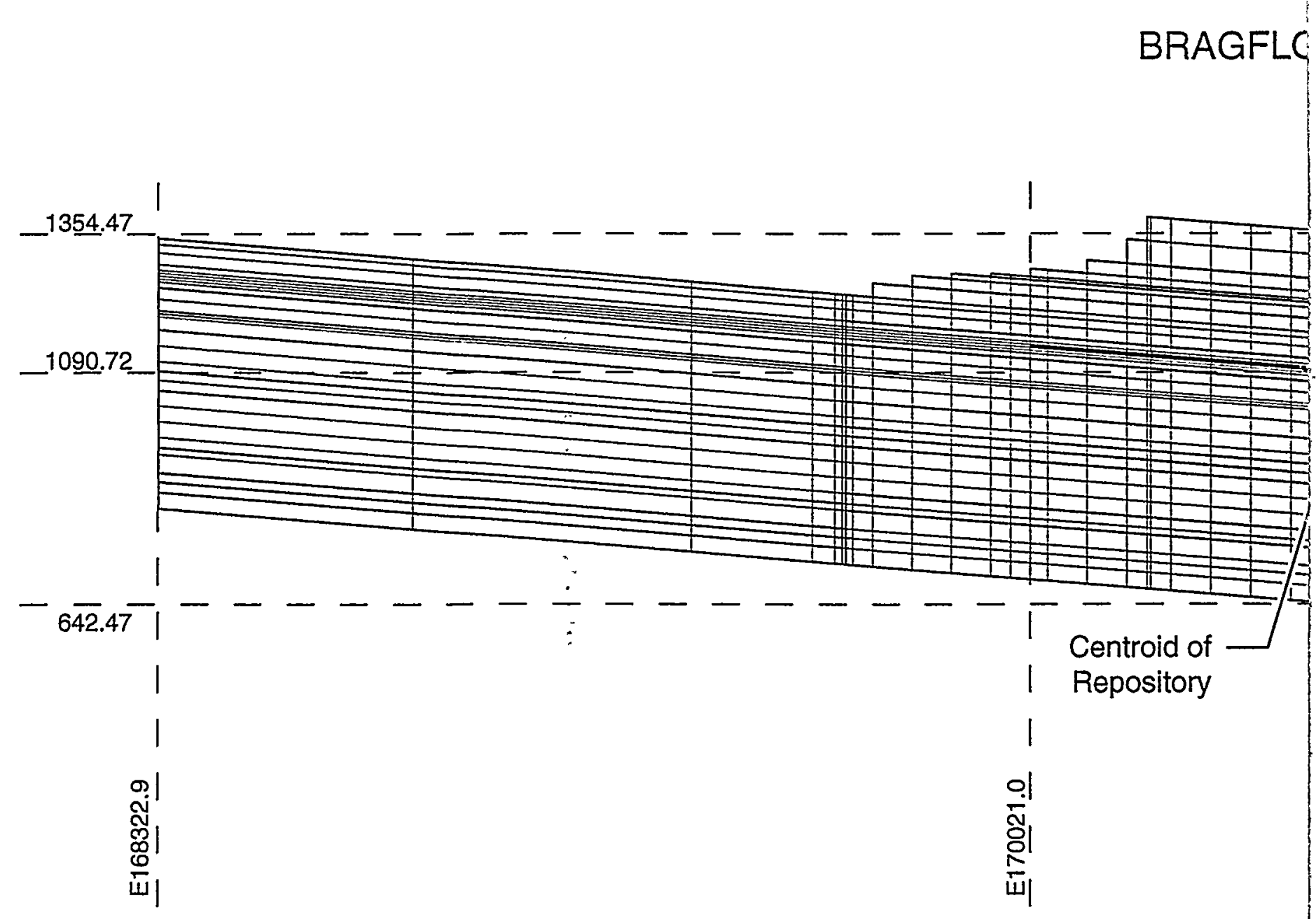

Scale in meters

Figure 8-7. BRAGFLO_T model grid. 


\section{_T Grid}

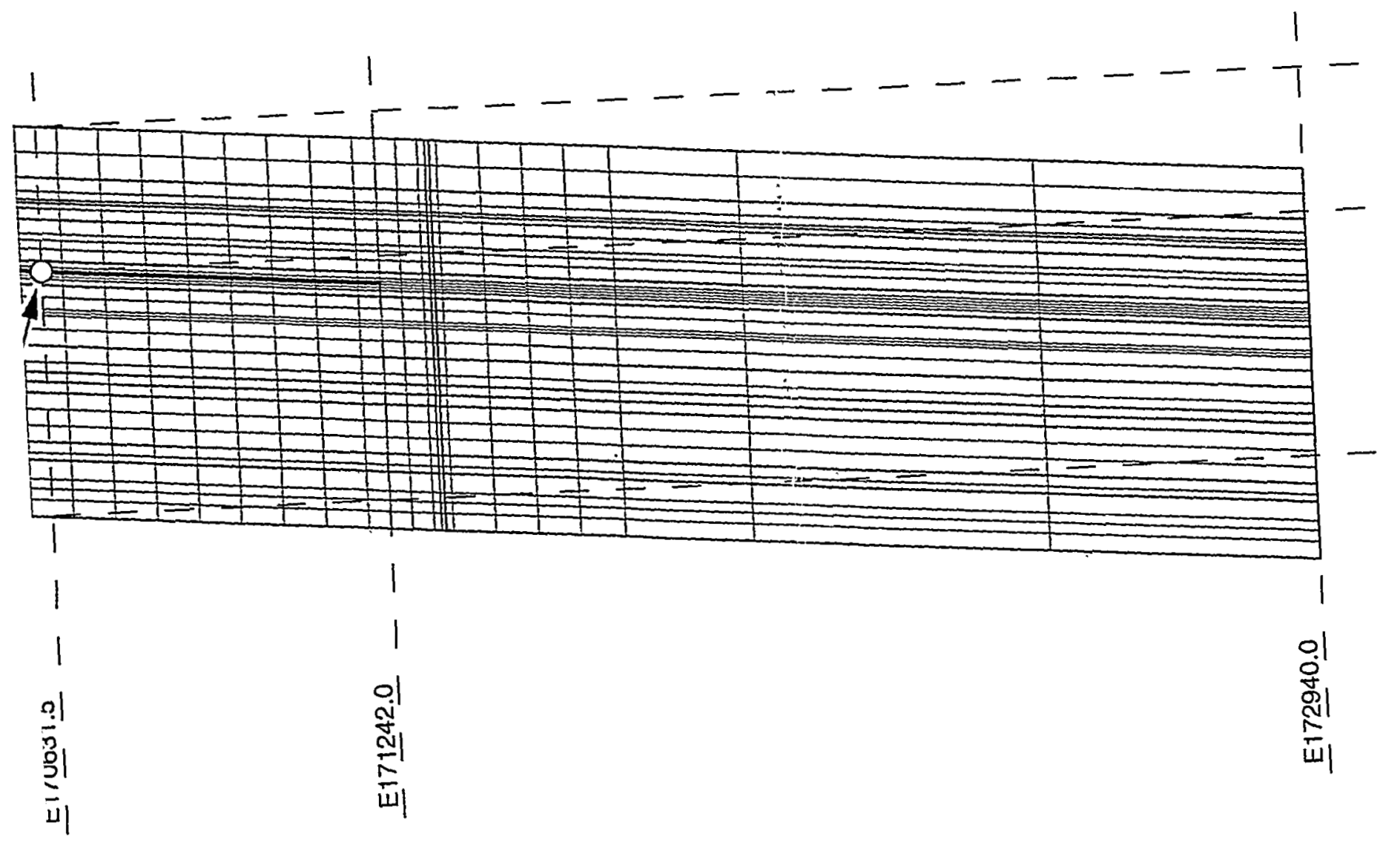

TR1-6342-5568-1 
Flaring. The front and top view of the 2D model cross section is presented in Figure 8-8. Notice that rectangular elements are used within the repository, and rectangular flaring of elements is used for the $\mathrm{z}$ direction.

The thickness of the rectangular elements increases as the distance from the repository increases, to preserve volume. This technique of modeling geometry has been used previously by the WIPP PA Department to model fluid flow (Rechard et al., 1990, p. 37; Rechard et al., 1993, p. 12-12).

Model Description. As shown in Figures 8-5 and 8-6, the size of the grid is 44 blocks along the $x$-axis direction (west to east) and 36 blocks vertically along the $y$-axis direction. Because the model is $2 \mathrm{D}$, only 1 grid cell is used in the z-axis direction (north to south). The western edge of the grid is at Nevada State Plane (NSP) Coordinate $168322.88 \mathrm{~m}$ and the eastern edge of the grid is at NSP Coordinate $172940.12 \mathrm{~m}$. The grid blocks that contain the repository extend along the $x$-axis direction from the $14^{\text {th }}$ to the $32^{\text {nd }}$ cell inclusive (19 cells total) and are in the $23^{\text {rd }}$ cell in the $y$-axis direction. Each repository cell is $5.5 \mathrm{~m}$ in vertical height and $2488.2 \mathrm{~m}$ thick (north to south). The north to south dimension was computed by taking the number of disposal tunnels in the entire repository (87) and multiplying by the spacing between tunnel centers along the north to south direction $(28.6 \mathrm{~m})$.

The two grid cells on either edge of the repository identified as " $\mathrm{A}$ " in Figure 8-5 include access tunnels in addition to the waste region. Each access tunnel has a length of $7.62 \mathrm{~m}$ (west to east). The total west to east length of the repository by itself is $1221 \mathrm{~m}$ so the total length across the $14^{\text {th }}$ to the $32^{\text {nd }}$ grid cell along the $x$-axis direction is $(7.62+1221+7.62)=1236.24 \mathrm{~m}$. The western edge of the repository (inciuding the access tunnel) for the $14^{\text {th }}$ cell is at NSP Coordinate: $170013.38 \mathrm{~m}$. The eastern edge of the repository (including the access tunnel) is at NSP Coordinate: $171249.62 \mathrm{~m}$. The centroid of the repository is located at an elevation of $1090.72 \mathrm{~m}$ at NSP Coordinate $170631.45 \mathrm{~m}$ and is located in grid cell $(x=24, y=23)$. The base of the model below the centroid of the repository is at an elevation (accounting for the $4.6^{\circ}$ west to east dip) of $642.47 \mathrm{~m}$. The base of the model was extended deeper in order to insure that the $730 \mathrm{~m}$ elevation of the water table was fully captured below the lateral extent of the repository.

Grid blocks within the repository include waste, tuff, and void space. To simulate the thermal hydrologic behavior of these components in a single element, volumetrically averaged properties are used. As discussed in Section 8.1.1, an effective continuum approximation consisting of a two-component, matrix/fracture system is used to simulate the geologic barrier. The same approach could be extended to a three- or, in this case, a four-component system. However, decoupling the flow field for the radionuclide transport calculations would then be extremely difficult, so a two-component system was maintained. To account for the void space between the waste packages and the rock matrix, this volume is incorporated into the fracture continuum by increasing the fracture porosity. The volume occupied by the waste packages is included in the matrix continuum and simply modeled as tuff matrix.

Initial Conditions. The initial thermodynamic state of the system can be defined by the initial temperature and initial fluid phase pressures. The effects of thermal gradients are considered negligible, so the initial temperature was set equal to the repository temperature, $299.15 \mathrm{~K}\left(26^{\circ} \mathrm{C}\right)$, at all locations. Below the water table, the initial water pressure is calculated assuming hydrostatic equilibrium. Above the water table, the initial gas pressure is assumed to be atmospheric, $10^{5} \mathrm{~Pa}$. Water pressure in the unsaturated zone is interpolated from point values. The point values are computed from sampled matrix capillary pressure parameters and the initial water saturation values. The water pressure, $P_{w}$ is calculated from the relationship,

$$
P_{w}=P_{g}-P_{c}\left(S_{w}\right)
$$

where $P_{g}$ is the initial gas pressure, and $P_{c}\left(S_{w}\right)$ is the capillary pressure.

This approach can result in water pressure profiles that are severely out of equilibrium and unrealistic. To compensate for this possibility, each simulation is run 1000 yr before inserting the waste. The 1000 -yr period permits the initial pressures and saturations to approach steady-state conditions in each parameter sample set.

Boundary Conditions. BRAGFLO_T assumes "no flow" (Neuman type with a flux rate of zero) boundary conditions for both mass and energy. Dirichlet (constant head) and Neuman (specified flux) boundary conditions can be imposed on the mass balance, though, by using constant pressure and specified flux wells in elements adjacent to the 


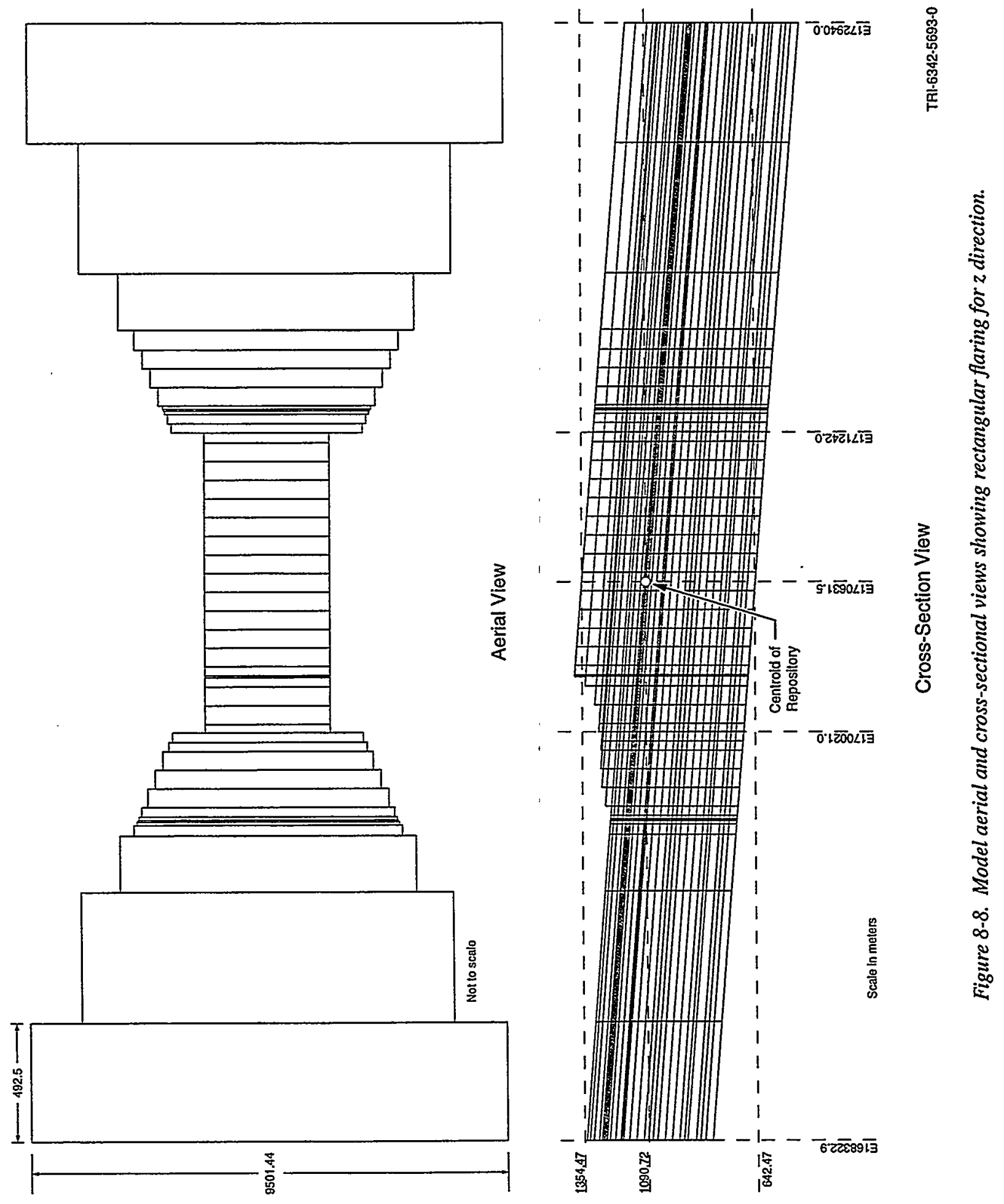


boundary. Neuman boundary conditions can be imposed on the energy balance using the boundary heat loss equation discussed in Section 8.1.1.

A constant water and gas pressure Dirichlet boundary condition was used at the eastern base of the CFun to maintain the water table in a relatively stable position. This boundary condition prevents unrealistic increases in the water table elevation caused by the accumulation of infiltrating water. A constant head boundary condition is also used in the top of the TCW in the gas phase. A Dirichlet gas pressure $\left(\mathrm{N}_{2}\right.$ and $\left.\mathrm{O}_{2}\right)$ is set at the topographic high (mountain peak) and maintained at $10^{5} \mathrm{~Pa}$ to simulate a constant atmospheric pressure condition at the surface. Temperature is initially set at $299.15 \mathrm{~K}$.

Surface water infiltration is modeled in the top of the TCw using specified flux water injection wells. The injection rate is a function of time as shown in Figure 8-9.

The parameters of the infiltration equation are sampled, so each sample set has a different injection rate. The values computed by the cosine function represent the average "regional" infiltration rate and are used in all injection wells not located directly above the repository. Circular symbols show the location of these wells in Figure 8-5. Above the repository, the surface infiltration rate is nonuniform. A single well (solid triangle) injects water at a rate 1 to 10 times (sample parameter) the average injection rate, simulating a rainfall catchment or any other mechanism of "locally" enhancing the surface infiltration. The injection rate of the remaining wells above the repository (square symbols) is slightly less than the average rate, computed such that the total infiltration rate above the repository is equal to the average "regional" rate.

Heat is permitted to flow across problem domain boundaries using Equation 8.1-17, in the boundary heat loss routine. Use of the boundary heat loss routine in the z-direction allows heat to flow in all three dimensions from the repository. The three-dimensional representation should produce good results particularly in the center of the repository.

Input Data. For all modeling parameters that were not sampled, the mean value of that parameter is used in all 52 sample sets. In the Topopah Spring member hydraulic units, the vertical permeability in the $y$-axis direction was taken to be 10 times the horizontal permeability in the $\mathrm{x}$-axis direction based upon a vertical to horizontal anisotropy ratio of 10:1 reported by LeCain (1997).

Fracture porosities for all hydrologic units are based upon the Snow parallel plate theory (Snow, 1968).

A comparison of the formation effective permeability between the 1997 PA and the 1994 PA is shown in Figure 8-10. This figure shows that the enhanced vertical fracture permeability in the Topopah Springs units increases the effective vertical permeability approximately one order of magnitude. Also, the Ghost Dance fault, which was not modeled in the $1994 \mathrm{PA}$, provides a high permeability fast path for fluid circulation. The treatment of the enhanced vertical fracture permeability offers the water phase a greater potential for vertical flow and the gas phase greater potential for circulation within the vicinity of the waste.

Fault Treatment. The Solitario Canyon Fault and the Ghost Dance Fault were assigned the lower and upper range, respectively, of the mean permeabilities for all hydraulic units that were intersected by the faults. The Solitario Canyon Fault blocks were assigned the lower mean permeability range because measurements of the water table elevations on the west and east side of the fault are different, suggesting a sealing nature. Similarly the Ghost Dance Fault blocks were assigned the higher mean permeability range because there have been no appreciable changes in water table elevations to the west and east of the fault. Also, grid cells on the west side of the Solitario Canyon Fault have been displaced vertically from those on the east side of the fault so additional flow variations in the horizontal direction occur because of the permeability variations of juxtaposed grid cells. The grid cells on the west and east side of the Ghost Dance Fault were not offset. 


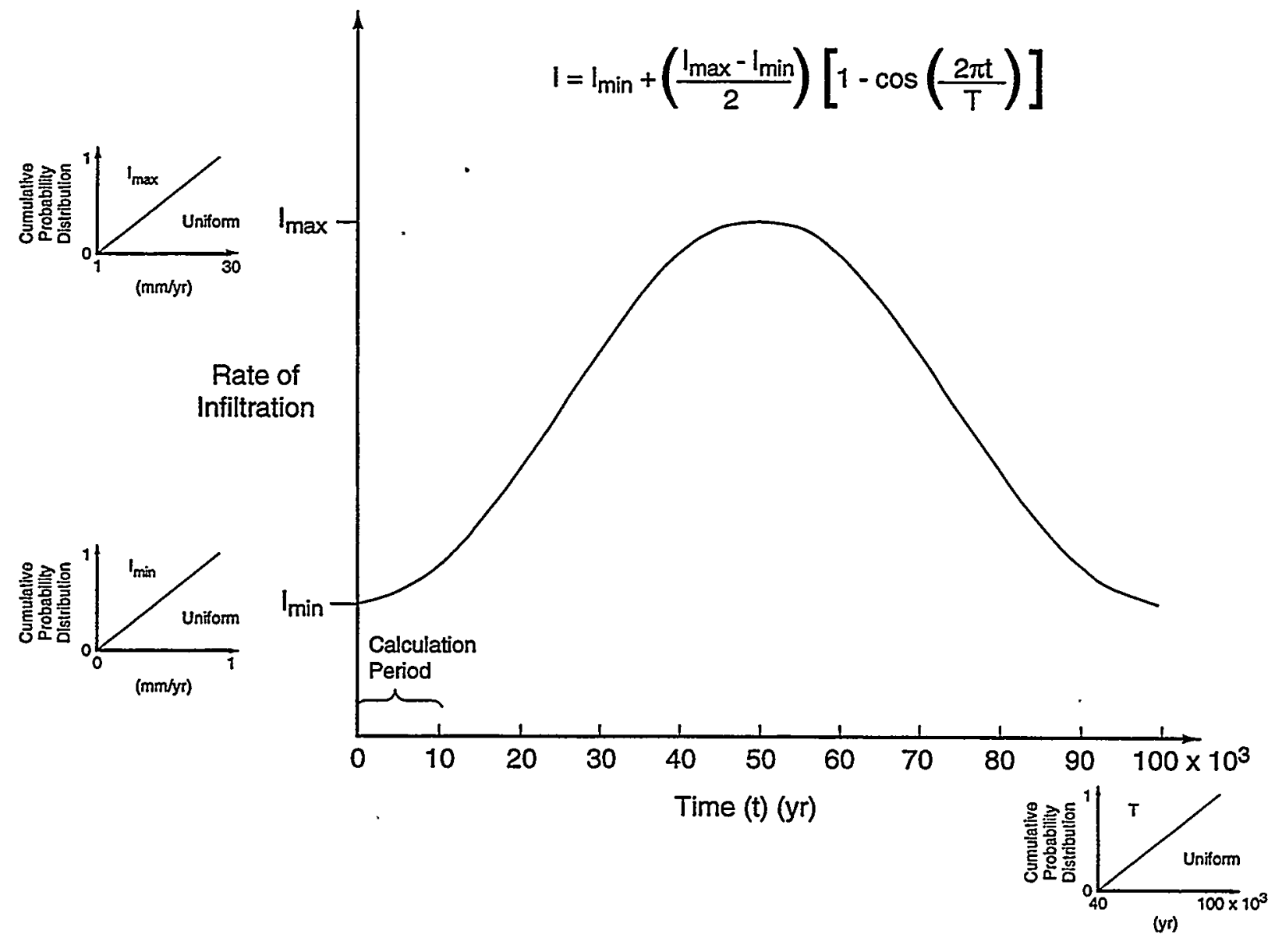

TRI-6342-4291-1

Figure 8-9. Cosine function representing annual infiltration fluctuations in next 100,000 yr. 
8.1 Repository Fluid Flow and Temperature Modeling

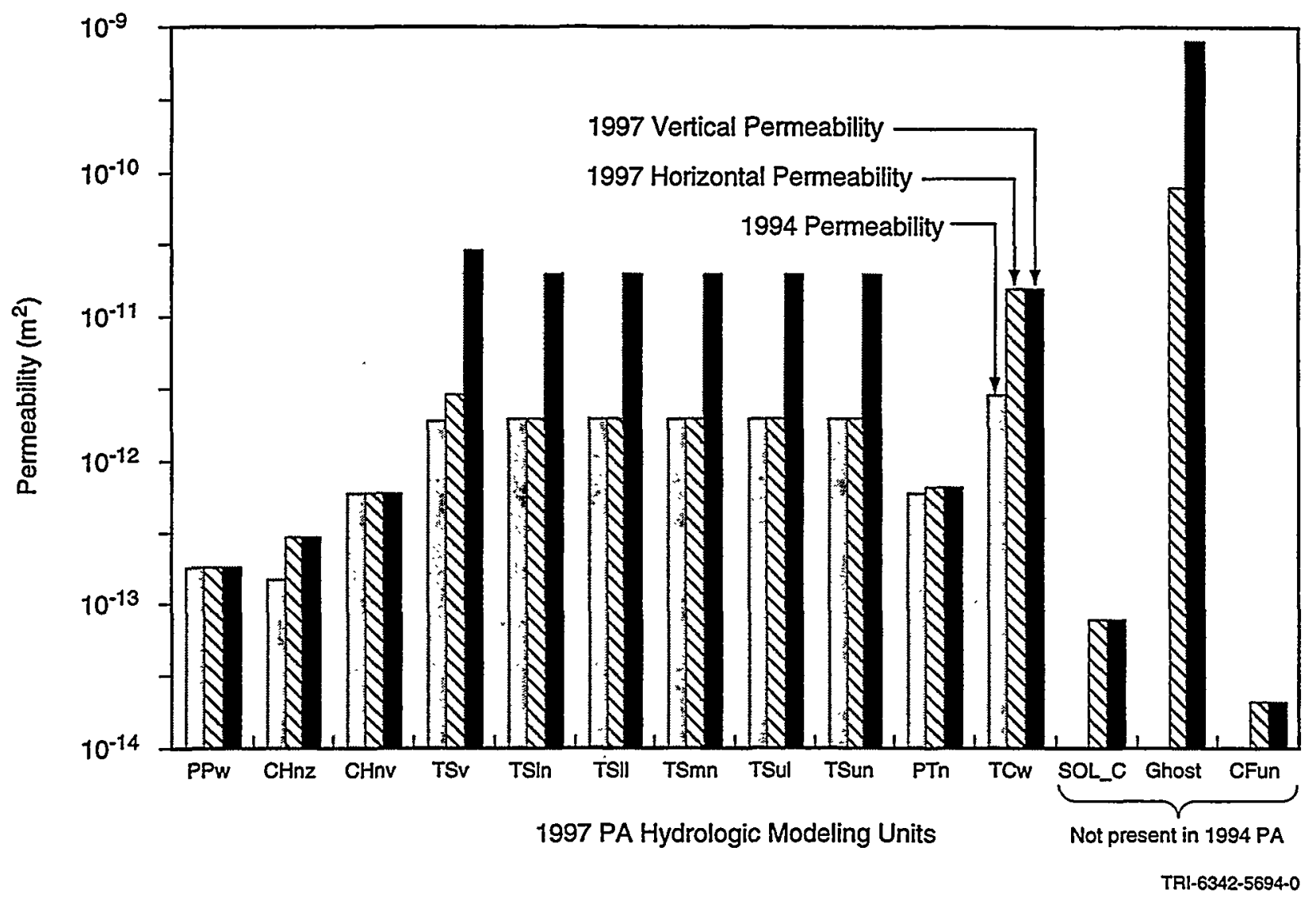

Figure 8-10. Comparison of formation effective permeability between the 1997 and 1994 PAs. 


\subsubsection{Intermediate Results}

The flow field and temperature results of 47 simulations together with the mean and median runs are presented as Figures 8-11 through 8-15. The results for a given parameter are presented on a single plot. A general trend in behavior can be seen when all simulations are compared in this way. The resulting parameter plots represent the most probable system response, given the underlying modeling assumptions and existing data. In the discussion that follows, general trends and behavior patterns are discussed in detail.

The repository and surrounding formations are represented spatially by a finite difference grid. The resulting hydro/thermodynamic properties such as temperature, pressures, and saturations are evaluated at grid block centers and represent average values of the system within the grid block element. Repository variables may fluctuate significantly within an element. Temperature is of particular concern, because the waste packages will generally be much hotter than the average grid block temperature.

In an attempt to approach steady state conditions before inserting the waste in the model, a 1000-yr equilibrium period was run (see "Initial Conditions" in Section 8.1.2). The results, as presented, do not include the equilibrium portion of the simulation. Instead, the state of the system at the end of the 1000 -yr equilibration period is treated, and referred to, as the initial conditions.

Cumulative water flow and flow rates in the vertical direction are computed by integrating the flow field over the repository footprint, that is, over the formation location vertically above or below the repository and within the lateral extent of the repository.

The repository temperature, matrix and fracture water saturation, fluid pressures, and oxygen mass fraction within the gas phase are shown at both the center and edge of the repository in Figures 8-11 through 8-15.

Repository Temperature. Heat production is greatest after initial waste disposal. As the waste decays, the energy output declines. The response to this type of thermal loading is shown in Figures 8-11a and 8-11b. The repository temperature increases from an initial value of $26^{\circ} \mathrm{C}$ to boiling at $100^{\circ} \mathrm{C}$. After all the water is boiled away, the temperature will continue to increase with water in the superheated phase. With heat load decreasing with time, the temperatures will decrease while steam condenses back to the liquid phase. The transition from boiling to superheated steam occurs at different times depending on the initial water saturation. The heat dissipation is greatest near the edge of the repository where it is demonstrated that many of the runs never reach superheated steam condition.

Heat produced by the waste is transported away from the repository convectively through fluid advection and through the conduction of heat within the rock matrix. Flow properties of the geologic barrier, which are sampled, result in very different fiow fields, and thus different amounts of convectively transported heat. The thermal conductivity and specific heat of the rock are not sampled though, so each simulation has approximately the same amount of energy transported by conduction.

Repository Water Saturation. Initial matrix water saturation varies from approximately 0.03 to 0.06 within the repository. Initial fracture water saturation varies from approximately 0.3 to 0.6 . The saturation history at both the center and edge of the repository is shown in Figures 8-11c and 8-11d. Within a few years the elevated temperatures with resulting vaporization decreases the water saturations to zero at the repository center. The saturation then rises with cooling and resulting condensation. Also, it is expected that water will resaturate the repository by flow from surrounding formations. At the repository edge, the matrix water saturation quickly recovers from the initial drop, and then in most cases increases to levels that exceed the initial saturation. This is classical heat pipe behavior, where water vaporizes near the energy source, is transported in the gas phase, and then condenses in cooler regions of the repository and surrounding rock.

The fracture water saturation Figures 8-11e and 8-11f show that the fractures at the center of the repository dry out at early time because of the heating, but after about $1000 \mathrm{yr}$ the fractures are recharged from water infiltrating from the surface. Behavior at the edge of the repository is similar but with more variability. 


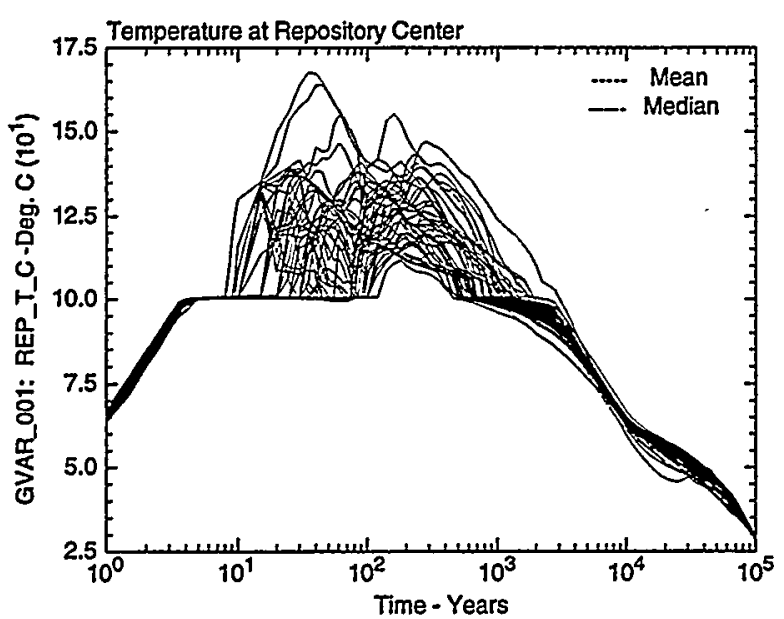

(a) Center

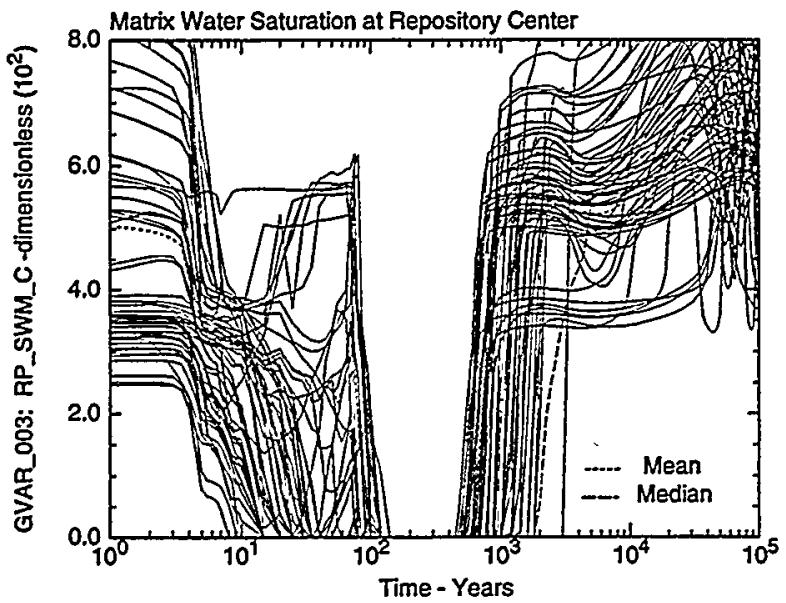

(c) Center

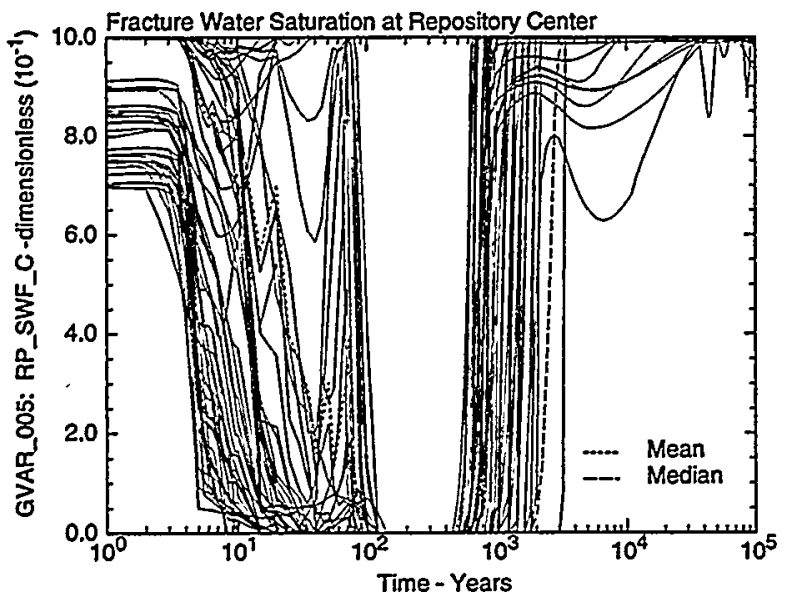

(e) Center

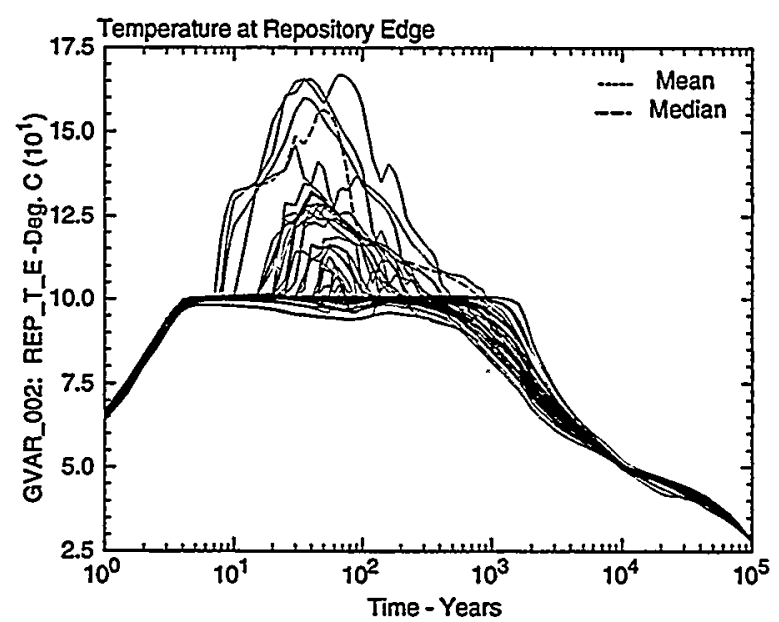

(b) Edge

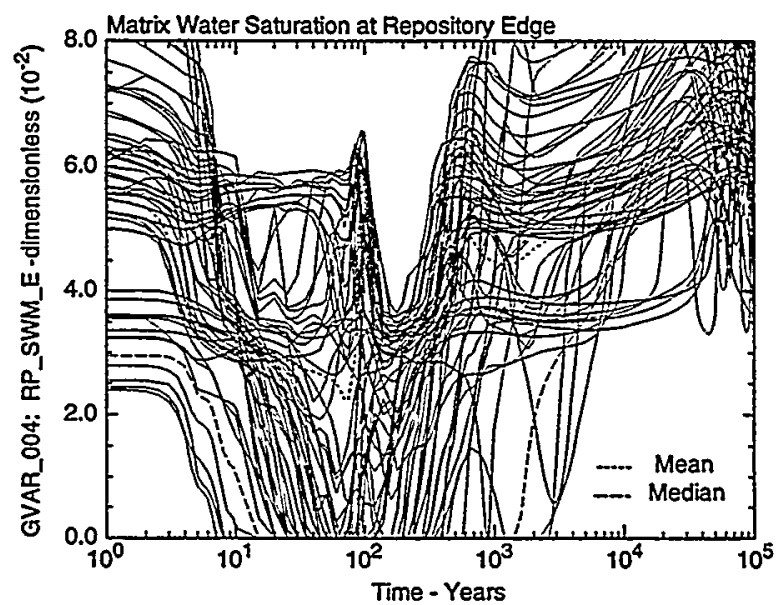

(d) Edge

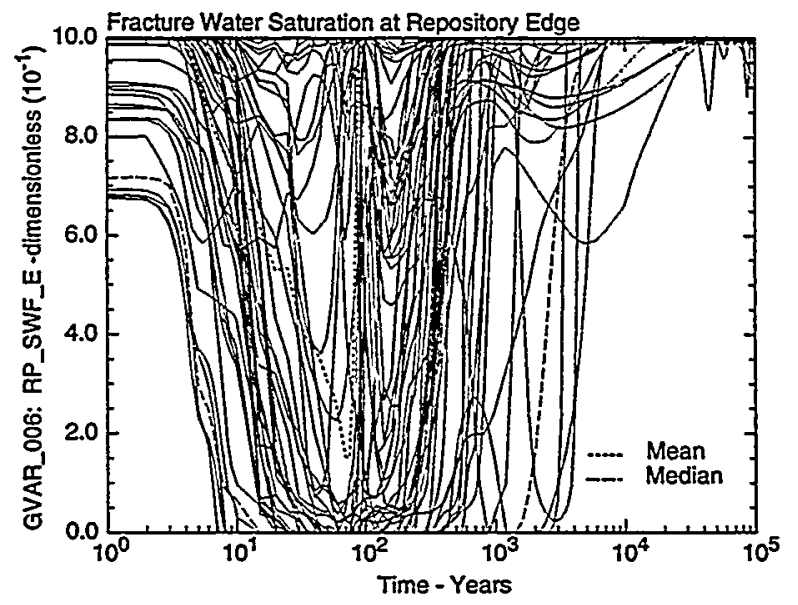

(f) Edge

TRI-6342-5589-1

Figure 8-11. Repository temperature, matrix water saturation, and fracture water saturation at the center and down-dip edge of the repository. 


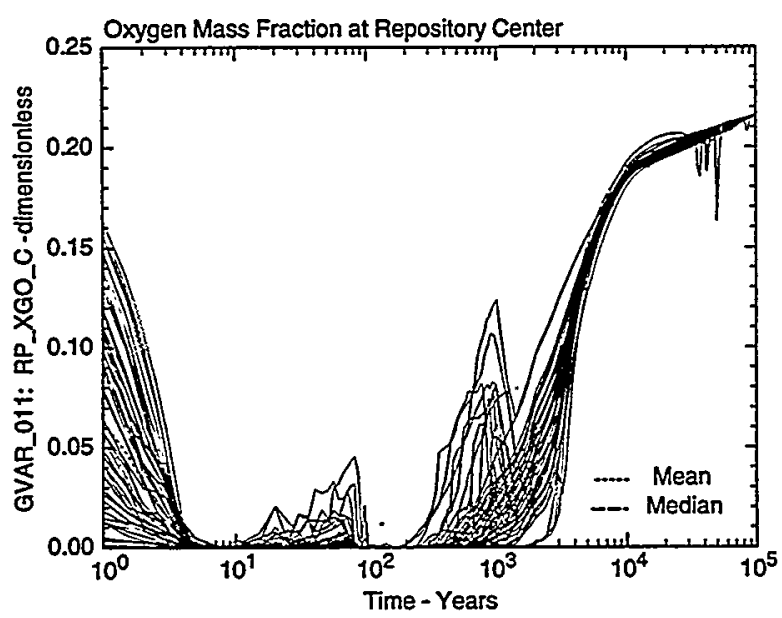

(a) Center

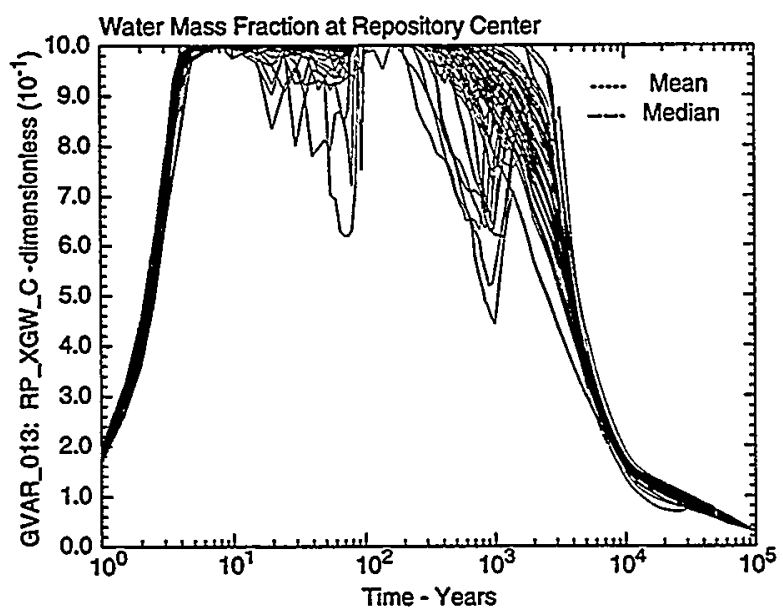

(c) Center

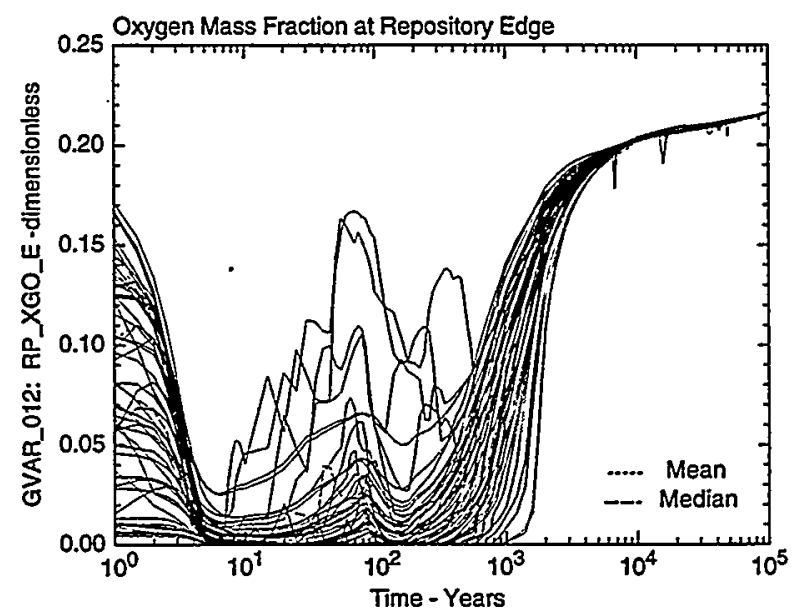

(b) Edge

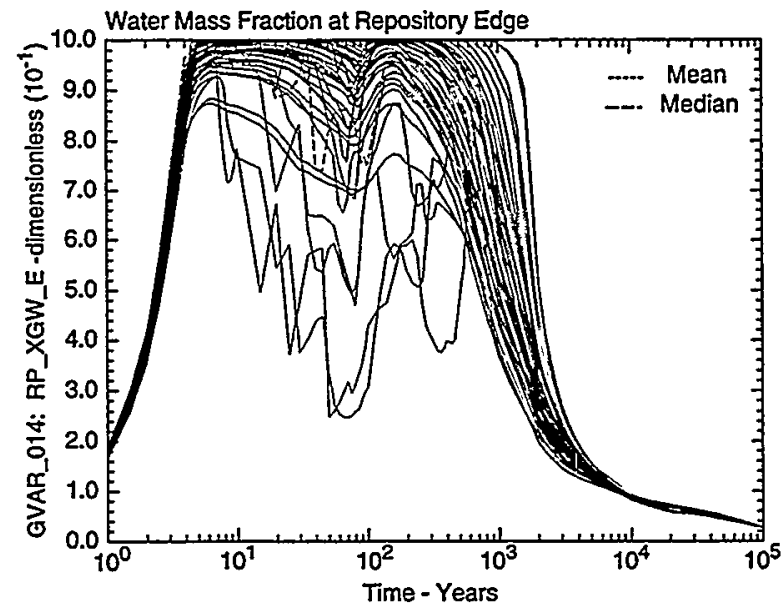

(d) Edge

TA1-6342-5590-1

Figure 8-12. Repository oxygen mass fraction and water mass fraction at the center and down-dip edge of the repository. 


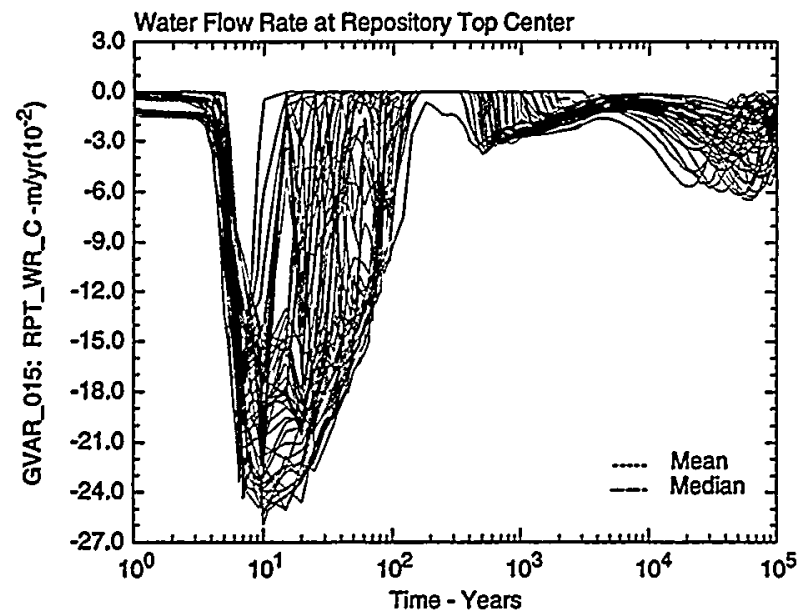

(a) Top Center

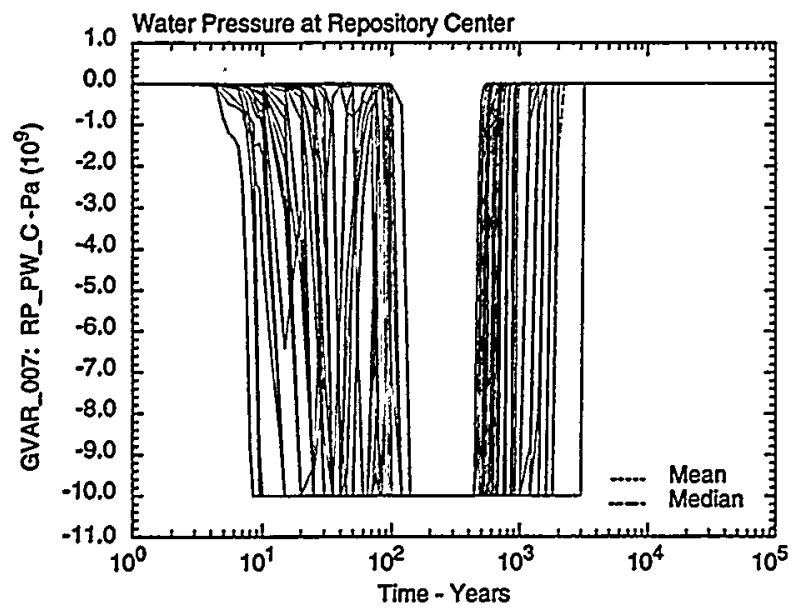

(c) Center

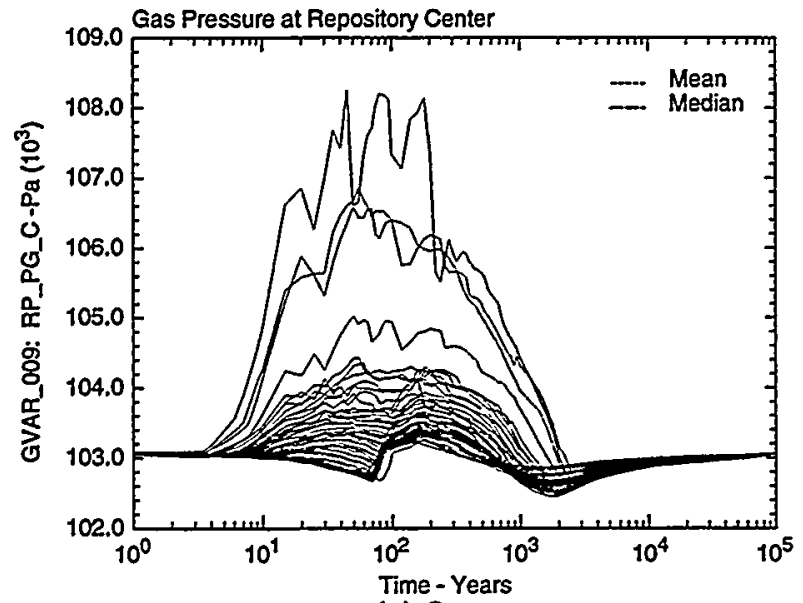

(e) Center

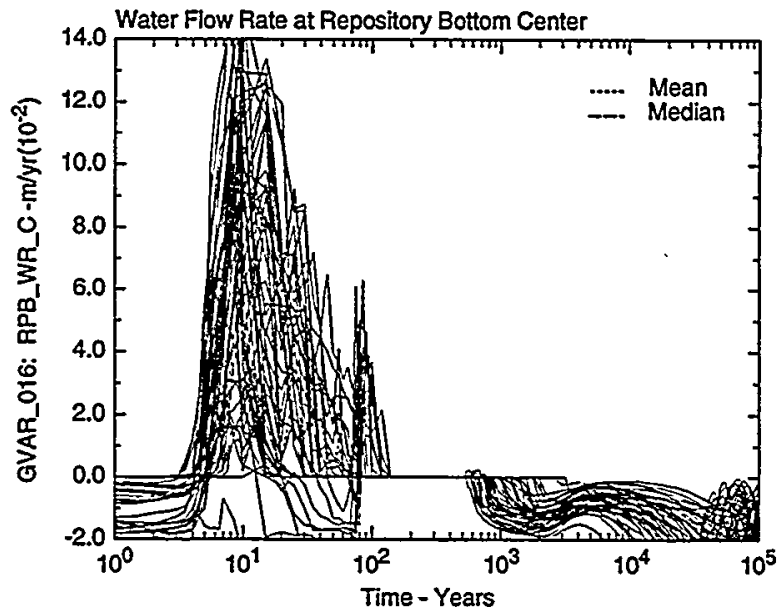

(b) Bottom Center

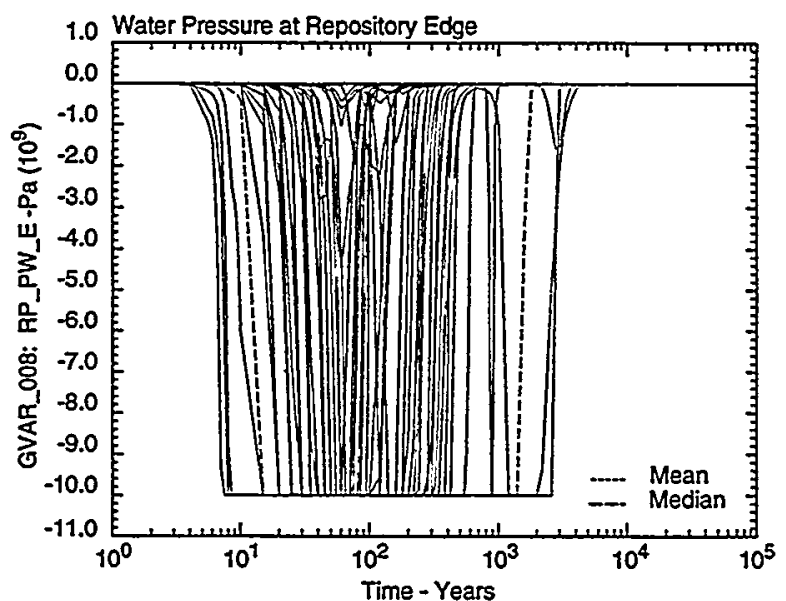

(d) Edge

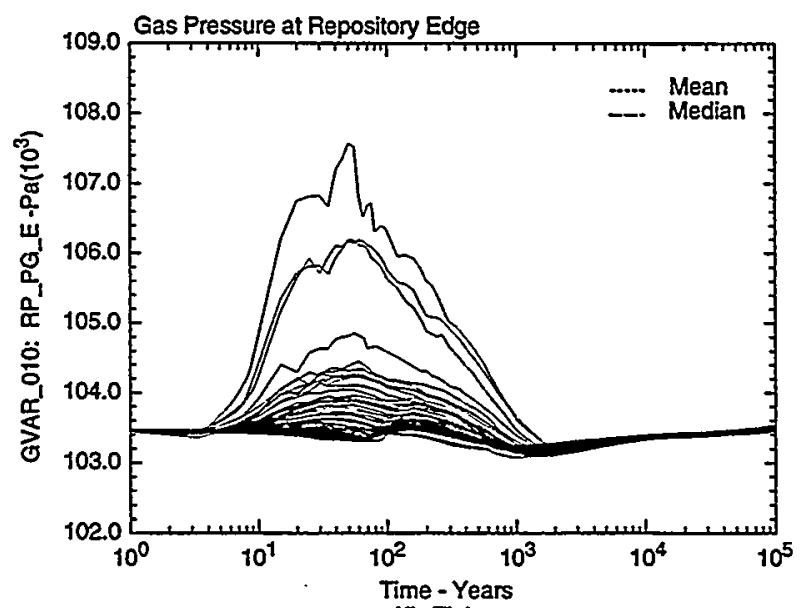

(f) Edge

TRI-6342-5591-1

Figure 8-13. Vertical water flow rate at the center of the repository, and water pressure and gas pressure at the center and down-dip edge of the repository. 

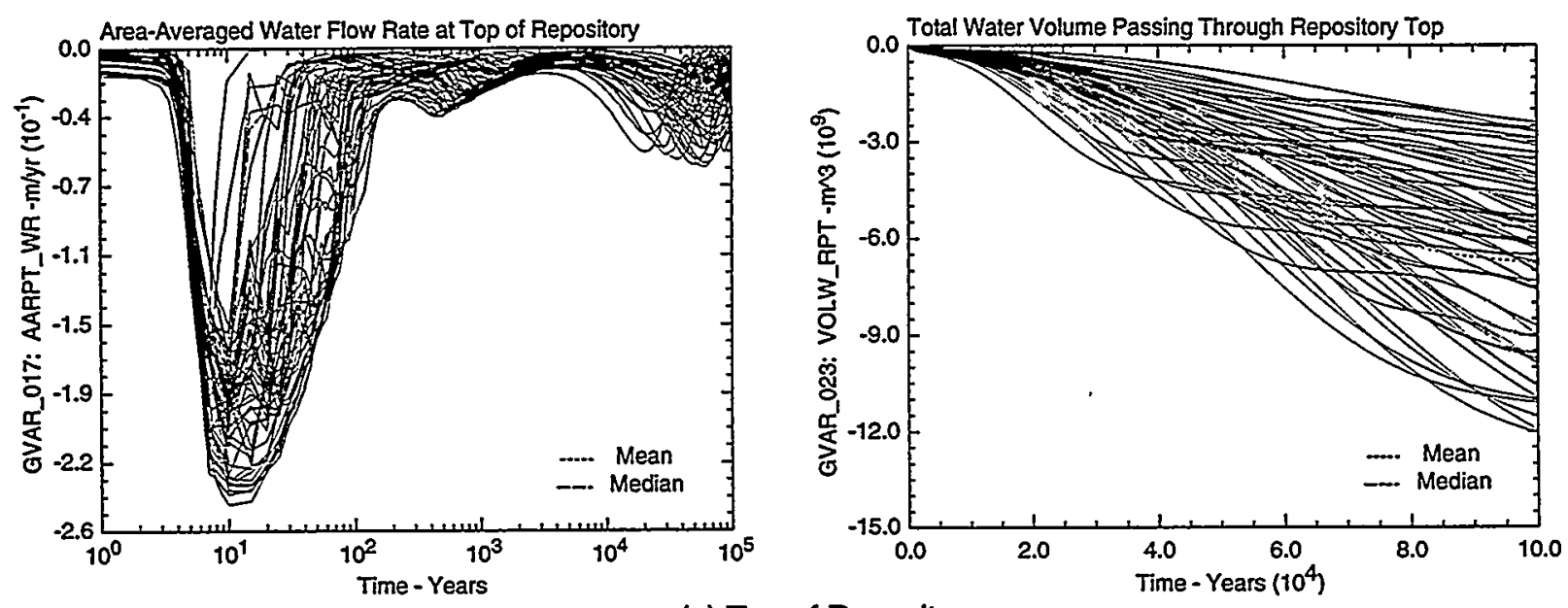

(a) Top of Repository
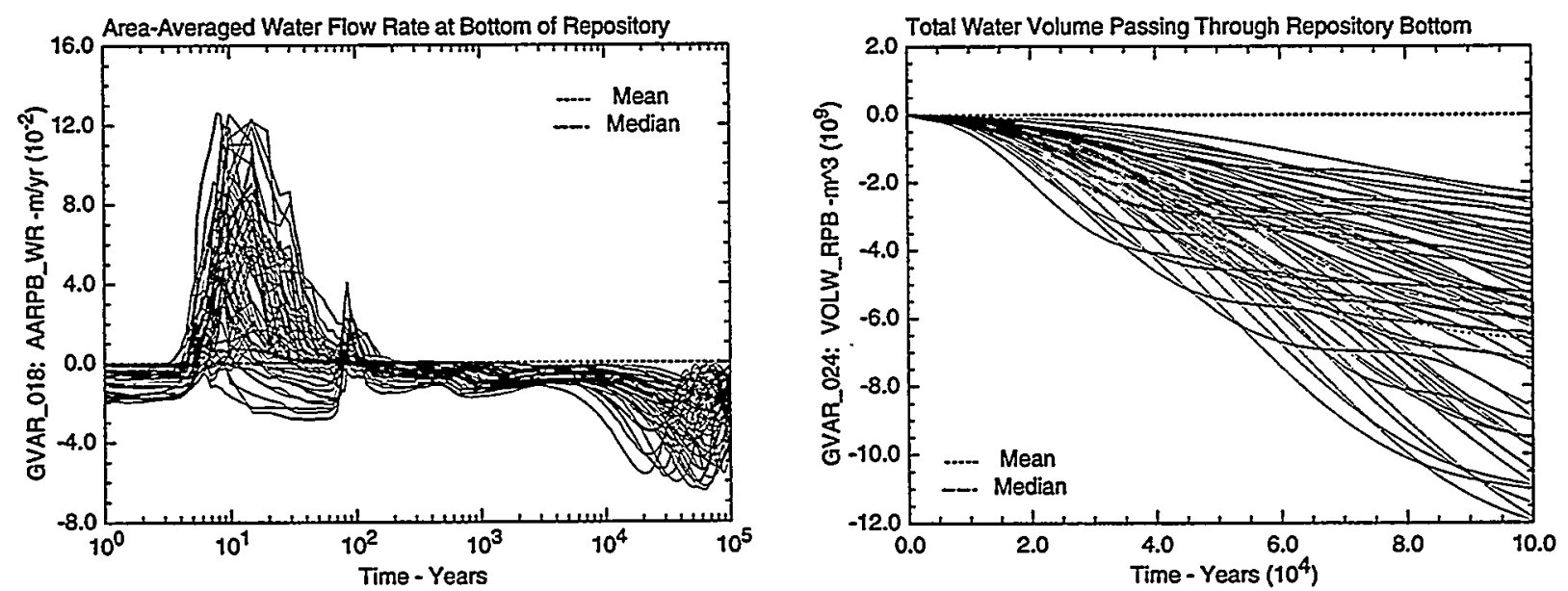

(b) Bottom of Repository
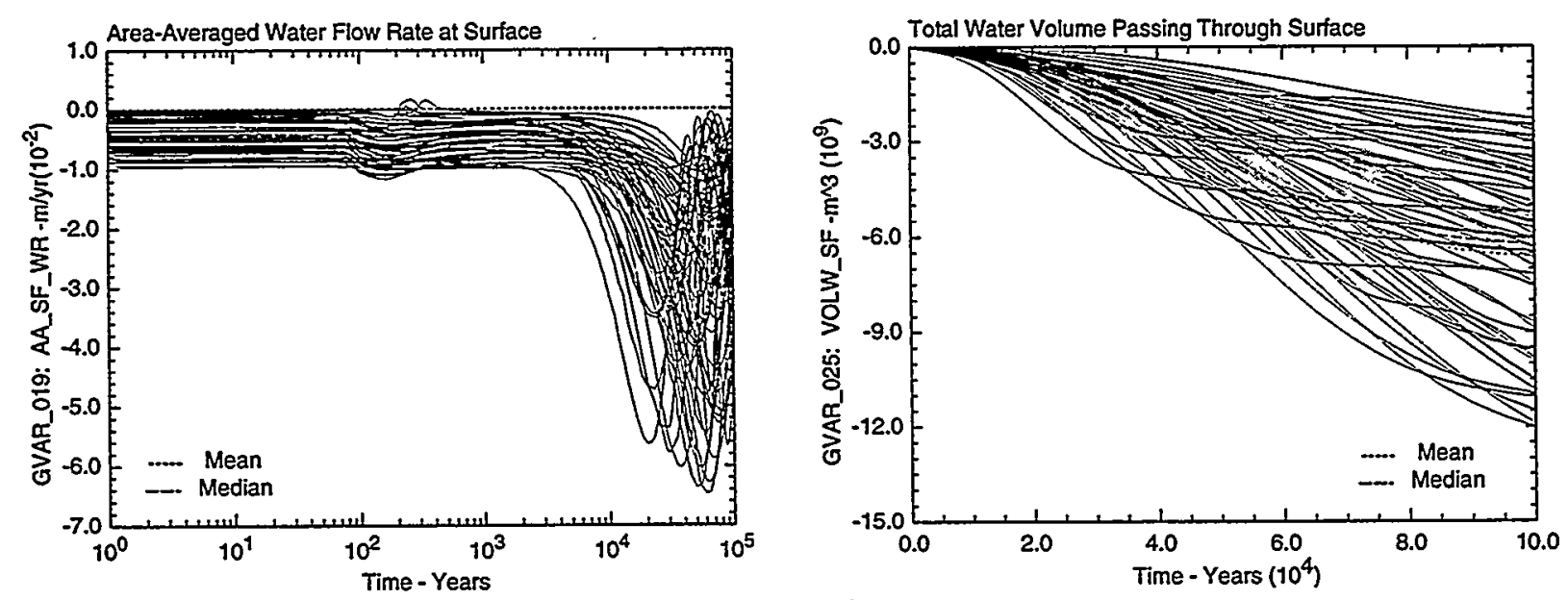

(c) Surface

TRI-6342-5592-1

Figure 8-14. Infiltration rate and cumulative volume of water passing through the top and bottom surfaces of the repository and the ground surface. 

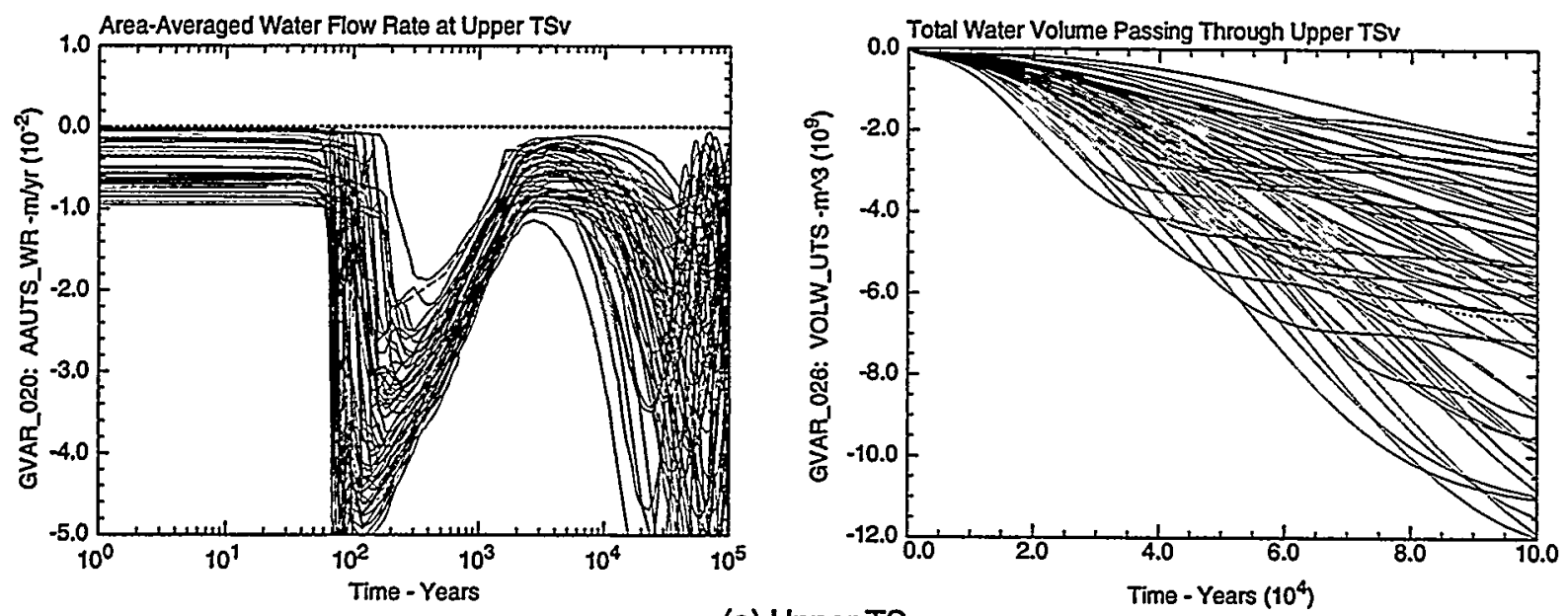

(a) Upper TSv
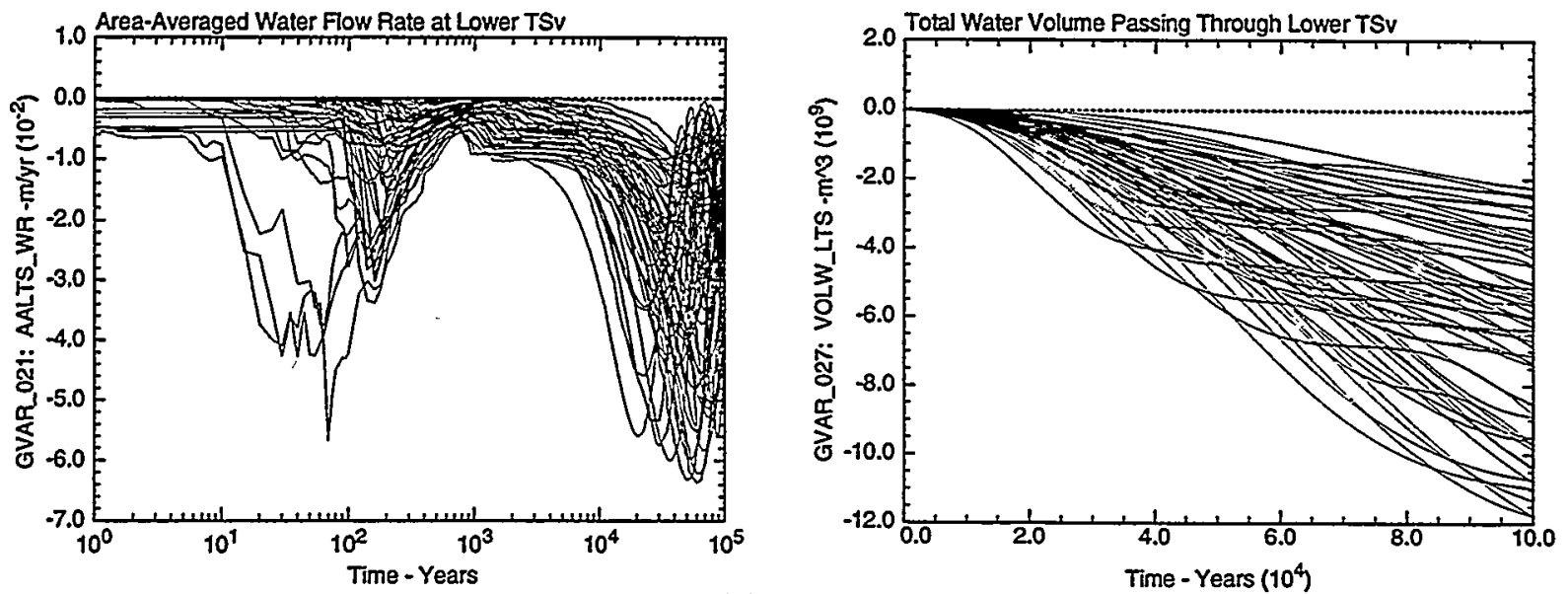

(b) Lower TSV
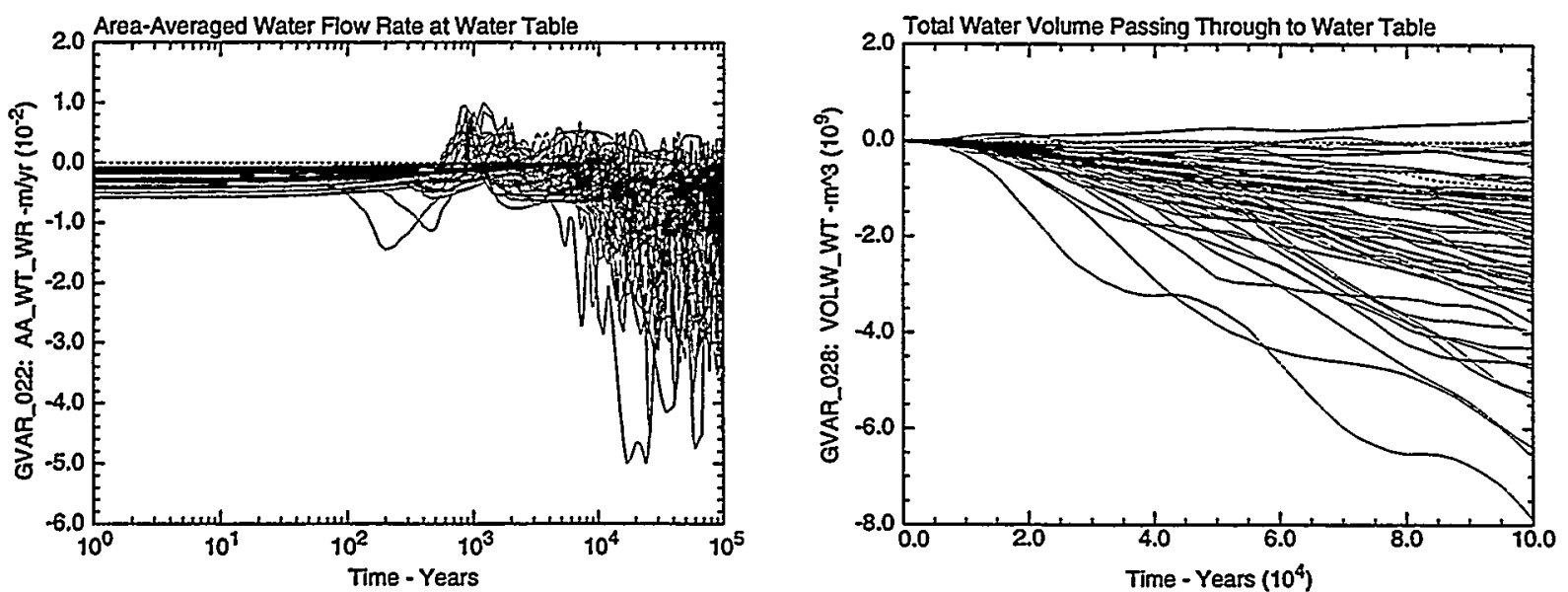

(c) Water Table

TRI-6342--5593-1

Figure 8-15. Infiltration rate and cumulative volume of water passing through the upper TSv and lower TSv layers and into the water table. 
Repository Pressure. Following the 1000-yr equilibration period, the repository water pressure in most simulations is slightly negative. Water pressure changes resulting from vaporization are accompanied by a drop in water pressure due to capillary effects, as shown in Figures 8-13c and 8-13d. At later time, greater water saturation results in water pressures near gas pressure. Gas pressures are near atmospheric and show only small variation due to temperature changes as shown in Figures 8-13e and 8-13f.

Repository Gas Composition. The gas phase is a mixture of water vapor, nitrogen and oxygen. At early time, when the temperature increases and liquid water is vaporized into the gas phase, the noncondensible gases are displaced from the repository as shown by the oxygen mass fraction in Figures 8-12a and 8-12b. As the repository cools, oxygen returns to the repository by diffusion and advection, eventually reaching levels approximating the initial state. The water mass fraction in the gas phase in Figures $8-12 \mathrm{c}$ and $8-12 \mathrm{~d}$ shows the displacement of the noncondensible gases during the early time and subsequent reduction at later times due to condensation and temperature decrease.

Repository Fluid Flow. Vertical liquid water at the top center of the repository is shown in Figure 8-13a, and flow through the bottom of the repository is shown in Figure 8-13b. At the top of the repository, negative flow is into the repository while at the bottom of the repository positive flow is into the repository. The vaporization process at early time produced a drop in liquid pressure and simultaneous increase in gas pressure. The drop in liquid pressure induced the flow of liquid water towards the repository.

Water Flux. The water flux rates at the top of the repository are shown in Figure 8-14a and cumulative water that infiltrates at the top of the repository is shown in Figure 8-14b (left). The behavior is the same as that reported at the top center of the repository. Similarly, flow rates and cumulative flow over the bottom of the repository are shown in 8-14b. The water flux rate and cumulative water fiux at the upper TSv, lower TSv, and at the water table are shown in Figures 8-15a, 8-15b, and 8-15c. The cumulative water flowing through each of the reported horizons, including the water table, is very similar. This suggests that the downward flow of water is somewhat uniform from the upper formations to the water table.

Oxygen Availability. The availability of oxygen for source term oxic corrosion is dependent on several factors. The oxygen bulk density is the product

$$
\rho_{o 2}=y_{o 2} S_{g} \rho_{g} \phi
$$

where

$y_{o 2}=$ mass fraction of oxygen in the gas phase [oxygen mass/gas mass]
$S_{g}=$ gas saturation [gas pore volume/total pore volume]
$\rho_{g}=$ gas density [gas mass/gas volume]
$\phi \quad=\quad$ porosity [pore volume/bulk volume].

The quantity of oxygen available depends on the following:

- the composition of gases within the gas phase, $y_{02}$.

- the distribution of phases within the two phase system, $S_{g}$,

- the gas density, $\rho_{g}$, which is pressure and temperature dependent,

- porosity, $\phi$, which for a slightly compressible formation should be nearly constant.

Figure 8-16a shows the oxygen density at the center of the repository as a function of time for the mean run. Also for the mean run, Figure 8-16b shows the water, nitrogen, and oxygen mass fraction at the center of the waste region for all time $[0,100000 \mathrm{yr}]$. Although not shown, the gas saturation for the mean run at this location varies, approximately $0.7<S_{g}<1.0$; the gas density varies by a factor of about 2 between the hot early time and the cooler late time; while the porosity is essentially constant. Comparison of Figures 8-16a and 8-16b shows that the variability in the oxygen density is described primarily by the oxygen mass fraction. During early time $[0,500 \mathrm{yr}]$ the steam generated displaces the water phase with gas saturation becoming 1.0, while within the gas phase the noncondensible gases are displaced by the water vapor. As the region cools, the mass faction of water decreases because of condensation and the nitrogen and oxygen are recharged. 


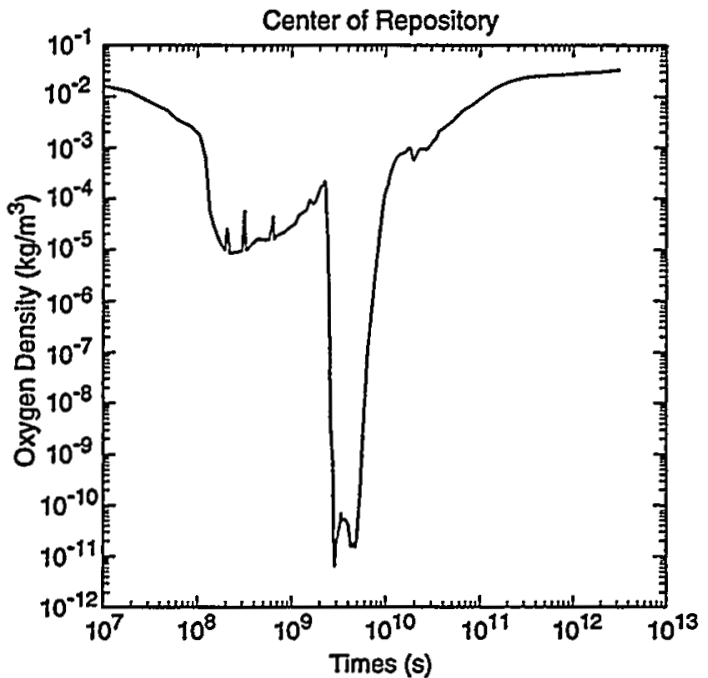

(a)

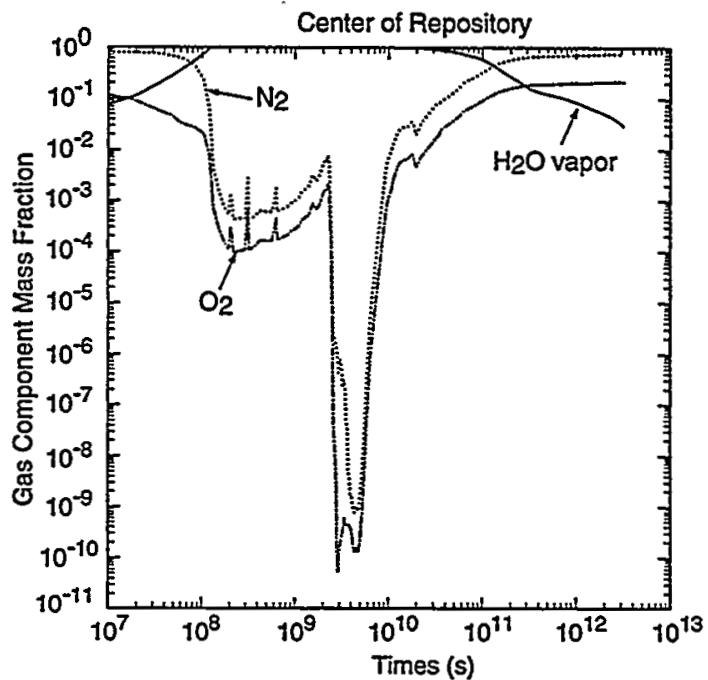

(b)

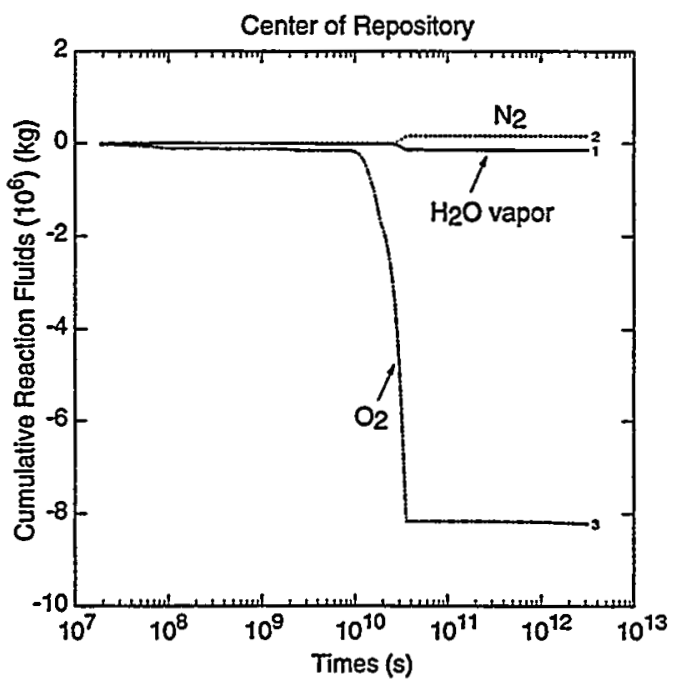

(c)

TRI-6342-5654-2

Figure 8-16. Oxygen availability as (a) oxygen density at center of repository, (b) water, nitrogen, and oxygen mass fraction at center of repository, and (c) cumulative reaction fluids over time. 
The cumulative mass of reaction fluids over time is shown in Figure 8-16c. This plot shows the oxygen and water consumption. All products of reaction are lumped into the nitrogen component. Most of the oxic corrosion occurs between $1.0 \times 10^{10}$ and $2.0 \times 10^{10} \mathrm{~s}$ (approximately 300 to $600 \mathrm{yr}$ ). If the oxygen mass fraction at this time interval is inspected in Figure 8-16b, it is noted that the oxygen consumption from corrosion does not significantly affect the oxygen in place. Sufficient oxygen is available to support the oxic corrosion.

\subsection{Thermal Calculations for Waste Package in Near Field}

The heat generated by the waste within the waste disposal packages is significant to the repository environment. A repository design consideration is the spacing of waste packages so that local temperatures from the wastes do not exceed the limits of either the waste forms or the host rock that surrounds the packages. Values for the heat generated by the wastes are also essential to computations of local hydrologic response, which affects corrosion rates of the packages.

For the 1997 PA, activity inventories and decay parameters (e.g. half-lives) are used to obtain an initial thermal power and a thermal power time history for each category of waste and for the entire waste inventory. Further thermal conduction and radiative transfer calculations generated temperatures in the disposal packages per waste category. These data are intended to be used by the source term submodel, CST (Chapter 7), in conjunction with thermal data calculated by BRAGFLO_T. The thermal data described here were used to scale the thermal values generated by BRAGFLO_T as a means of obtaining increased resolution regarding temperatures within and on the surface of the packages.

\subsubsection{Code Descriptions for Thermal Conduction Calculations}

To determine time-dependent temperature distributions near a waste package and within the repository, the 1997 $P A$ ran two thermal conduction codes-CONRAD and SUMQ_3D. As described below, CONRAD, a modified version of the heat-conduction code, COND_1D (used in the 1993 PA), was run to determine waste package and nearfield temperatures. Two waste packages each per type (i.e., Waste Packages 1 to 15) were selected for the calculations, with one package located near the center of the repository and the other at the repository edge. CONRAD treats thermal conduction in an axisymmetric geometry and allows for radiation heat transport across an air gap. SUMQ_3D was used to compute the far-field, transient thermal response within the repository. To establish the heat generated by the waste, the near-field package temperatures computed by CONRAD were corrected by adding the temperature from the SUMQ_3D computations in the repository at a selected location near the appropriate package. These modified results can be combined with temperatures computed by BRAGFLO_ $T$ to obtain more accurate repository temperatures for the near field from the individual waste packages. Specific details about BRAGFLO_T are provided in Section 8.1 .

CONRAD. CONRAD is an explicit finite-difference code that is designed to study one-dimensional thermal conduction and thermal radiation in spherical or cylindrical coordinates. It solves coupled conductive and radiative heat transfer equations. CONRAD permits different material properties, time dependent external and internal thermal sources, and radiative heat transport across an air gap. CONRAD is used to determine the temperature field within and adjacent to a single package.

CONRAD is based on the solution to the thermal conduction equation (Scipio, 1967):

$$
\nabla \bullet(K \nabla T)+Q=\rho c \frac{\partial T}{\partial t}
$$

where

$\mathrm{Q}=$ heat rate within the medium,

C = specific heat, 


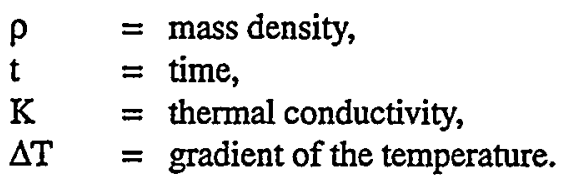

Equation 8.2-1 is solved explicitly using finite-difference approximations to the derivatives along a nonuniform, one-dimensional grid. Decaying heat sources are permitted.

Heat was assumed to be transferred across the air gap that forms between the package and the host rock by thermal radiation only. The values are calculated by solving the equation (Jakob and Hawkins, 1957):

where

$$
q_{1-2}=\frac{\sigma A_{1}}{\frac{1}{\varepsilon_{1}}+\frac{A_{1}}{A_{2}}\left(\frac{1}{\varepsilon_{2}}-1\right)}\left(T_{1}^{4}-T_{2}^{4}\right)
$$

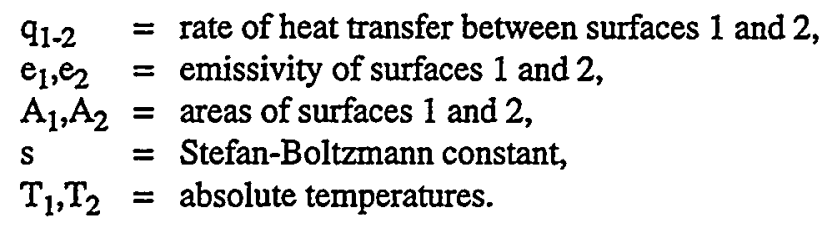

All packages were circular in cross-section and separated from the tuff by an approximate 1.69-m air gap (for standard packages that were $1.725 \mathrm{~m}$ in diameter) or 1.55 - $\mathrm{m}$ air gap (for super packages that were $2 \mathrm{~m}$ in diameter). The thermal conduction and radiation properties considered are provided in Table 8-2.

Table 8-2. Thermal Conduction and Radiation Properties

\begin{tabular}{ll}
\hline \multicolumn{1}{c}{ Description } & \multicolumn{1}{c}{ Unit } \\
\hline Thermal conductivity of welded tuff & $2.33 \mathrm{~W} /(\mathrm{mK})$ \\
Diffusivity of welded tuff & $9.716 \times 10^{-7} \mathrm{Wm} / \mathrm{J}$ \\
Thermal conductivity of container & $41.0 \mathrm{~W} /(\mathrm{mK})$ \\
Diffusivity of container & $4.375 \times 10^{-7} \mathrm{Wm}^{2} / \mathrm{J}$ \\
Emissivity of container wall & 0.8 \\
Emissivity of welded tuff & 0.8 \\
Stefan-Boltzmann constant & $5.617 \times 10^{-8} \mathrm{~W} /\left(\mathrm{m}^{2} \mathrm{~K}^{4}\right)$ \\
Initial temperature & $26^{\circ} \mathrm{C}$ \\
\hline
\end{tabular}

Local transient temperatures were computed for each type of waste package (i.e., Waste Packages 1 through 15) with CONRAD. Two waste packages each per type were selected for the calculations, with one package located near the center of the repository and the other at the repository edge.

SUMQ 3D. SUMQ_3D was used to compute the far-field, transient thermal response within the repository that determined the far-field boundary location for CONRAD. SUMQ_3D generates time-dependent temperatures for the total contribution of all packages in the repository. SUMQ_3D is designed to determine the temperature at any point and at any time within an arbitrary array of decaying thermal line sources of finite length. The code is based on the superposition of a large number of decaying point source solutions in an infinite medium. 
The governing equations for SUMQ_3D are based upon analytical work discussed in Carslaw and Jaeger (1959). The temperature change at any point in a field away from a constant thermal source and at any time, assuming constant conductivity and diffusivity, is governed by the equation:

$$
\Delta T=\frac{\bar{q}}{4 \pi K r} \operatorname{erfc}\left[\frac{r}{\sqrt{4 k t}}\right]
$$

where

$$
\begin{aligned}
\Delta \mathrm{T} & =\text { temperature change } \\
\bar{q} & =\text { source strength, } \\
\mathrm{K} & =\text { thermal conductivity } \\
\mathrm{r} & =\text { radius from source } \\
\mathrm{k} & =\text { thermal diffusivity } \\
\mathrm{t} & =\text { time. }
\end{aligned}
$$

When the source decays with time (which was the solution method implemented in the $1997 \mathrm{PA}$ ), the governing equations must be written in integral form as

$$
\Delta T=\frac{k}{4 K(\pi k)^{\frac{3}{2}}} \int_{\sqrt{\frac{1}{t}}}^{\infty} \bar{q}\left(t-\frac{1}{\tau^{2}}\right) \exp \left(-\frac{r^{2} \tau^{2}}{4 k}\right) d \tau
$$

To model the time-dependent thermal source of nuclear waste, the solutions to an array of point sources (Equation 8.2-3) are configured to approximate a field of packages of finite length and summed to determine the temperature change at any point and at any time. Typically, a package is represented by 50 point sources. SUMQ_3D can model many thousands of packages, although fewer can be used to determine the temperature change at a particular point. Thus, for a 1000-package array, Equation 8.2-3 would be solved 50,000 times and the results summed to obtain the temperature at a single point. When thermal decay is taken into account, Equation 8.2-3 must be integrated numerically. Simpson's rule was used for the numerical integration.

Thermal Calculations. Two distinct types of SUMQ_3D calculations were run for the 1997 PA. The first, as described above, was used to modify the near-field package temperatures calculated with CONRAD. The second type of calculation compared the CONRAD temperature computations with SUMQ_3D and BRAGFLO_T.

SUMQ $3 D$ and CONRAD Calculations. Temperatures at the center and surface of the waste packages were calculated by CONRAD (see above), and then adjusted by adding the difference between the temperature calculated by SUMQ_3D at a selected location and the corresponding temperature calculated by CONRAD. The location was chosen as a point lying radially $2.55 \mathrm{~m}$ (approximate radius of a waste package) away from the center of the appropriate waste package on a line perpendicular to the centerline of the disposal tunnel. This point was chosen because of the radiation connection between it and the package surface (as modeled by CONRAD). Or more succinctly,

$$
T_{C I J}^{M}=T_{C I J}+\left(T_{S J}^{\prime}-T_{C J}^{\prime}\right)
$$

where

$T_{s}^{\prime}$ and $T_{c}^{\prime}$ are temperatures evaluated $2.55 \mathrm{~m}$ away from the centerline of the package under consideration; the subscripts, $s$ and c, stand for SUMQ_3D and CONRAD evaluations, respectively; and $T_{C I}^{M}$ is the modified CON$R A D$ package temperature ( $I=1,2$ for the center and surface of the package, respectively).

These adjustments were made for each waste package type at the center and edge of the repository $(\mathrm{J}=1$ and 2), and then the results interpolated for locations between these locations. The temperatures were adjusted in an attempt to account for the radiation connection between waste packages and the three-dimensional nature of heat transport in and near the repository (not modeled by CONRAD). 
To calculate with sufficient detail the temperatures near packages located at the center and edge of the repository, the SUMQ_3D calculation assumed that packages were placed in disposal tunnels in the configuration shown in Chapter 5, Figure 5-4.

SUMQ_3D and BRAGFLO_T Calculations. The second type of thermal calculation, which was run to compare temperature computations with those of BRAGFLO_T, used a mixture of waste with a single decay curve representing the entire inventory (identical to the power curve used by BRAGFLO_T). In this calculation, a representative SUMQ 3D near-field repository zonal temperature was calculated for zones with dimensions similar to those used in BRAGFLO_T in the repository. The waste (and thus the heat source) is distributed uniformly along each tunnel (individual packages were not modeled). This model is consistent with the distribution that BRAGFLO_T uses for the heat source.

Thus, the adjusted CONRAD temperatures described above can be further modified by considerations involving the representative temperatures calculated by SUMQ_3D and BRAGFLO_T. A straightforward modification might be to adjust the CONRAD temperatures by adding the difference between BRAGFLO_T and SUMQ_3D (as calculated using the mixture):

$$
T_{C I J}^{B}=T_{C I J}^{M}+\left(\bar{T}_{B J}-\bar{T}_{S J}\right)
$$

where $\bar{T}_{B J}, \bar{T}_{S J}$ are representative temperatures computed in the repository by BRAGFLO_T and SUMQ_3D, respectively. $T_{C I J}^{B}$ is the container temperature calculated by CONRAD and modified by BRAGFLO_T results (in addition to SUMQ_3D results, as described above); $I=1,2$ for center and surface of container, and $J=1,2$ for repository center and edge. Finally, the $T_{C I J}^{B}$ can be interpolated across the length of the repository.

The SUMQ 3D temperature histories calculated here are shown in Figure 8-17 for 10,000 yr (top) and $1000 \mathrm{yr}$ (bottom). SUMQ_3D predicts a peak repository temperature of about $150^{\circ} \mathrm{C}$ near the center and $\sim 130^{\circ} \mathrm{C}$ near the edge of the repository.

Table 8-3. Repository Design Summary for 1997 PA

\begin{tabular}{clccc}
\hline $\begin{array}{c}\text { Waste } \\
\text { Package ID\# }\end{array}$ & \multicolumn{1}{c}{ Spent Nuclear Fuel } & $\begin{array}{c}\text { Includes } \\
\text { DHLW }\end{array}$ & $\begin{array}{c}\text { No. of } \\
\text { Containers Used }\end{array}$ & $\begin{array}{c}\text { Power/Pkg } \\
\text { (W) }\end{array}$ \\
\hline 1 & Uranium metal & No & 118 & 793 \\
2 & Uranium-zirconium alloy & Yes & 9 & 343 \\
3 & Uranium-molybdenum alloy & Yes & 55 & 154 \\
4 & Uranium oxide-intact clad & Yes & 203 & 637 \\
5 & Uranium oxide-failed clad & Yes & 595 & 292 \\
6 & Uranium aluminum alloy & Yes & 750 & 405 \\
7 & Uranium silicide & Yes & 225 & 344 \\
8 & Uranium-thorium carbide-intact clad & Yes & 545 & 295 \\
9 & Uranium-thorium carbide-failed clad & Yes & 103 & 280 \\
10 & Uranium/plutonium carbide & Yes & 5 & 317 \\
11 & Mixed oxide fuel & Yes & 352 & 287 \\
12 & Uranium-thorium oxide & Yes & 69 & 195 \\
13 & Uranium/zirconium hydride & Yes & 102 & 177 \\
14 & Commercial fuel, PWR & No & 4820 & 9570 \\
15 & Commercial fuel, BWR & No & 2859 & 6650 \\
& & & Total Power & $6.62 \times 10^{7}$ \\
\hline
\end{tabular}


8. Consequence Modeling: Unsaturated Zone Flow and Transport

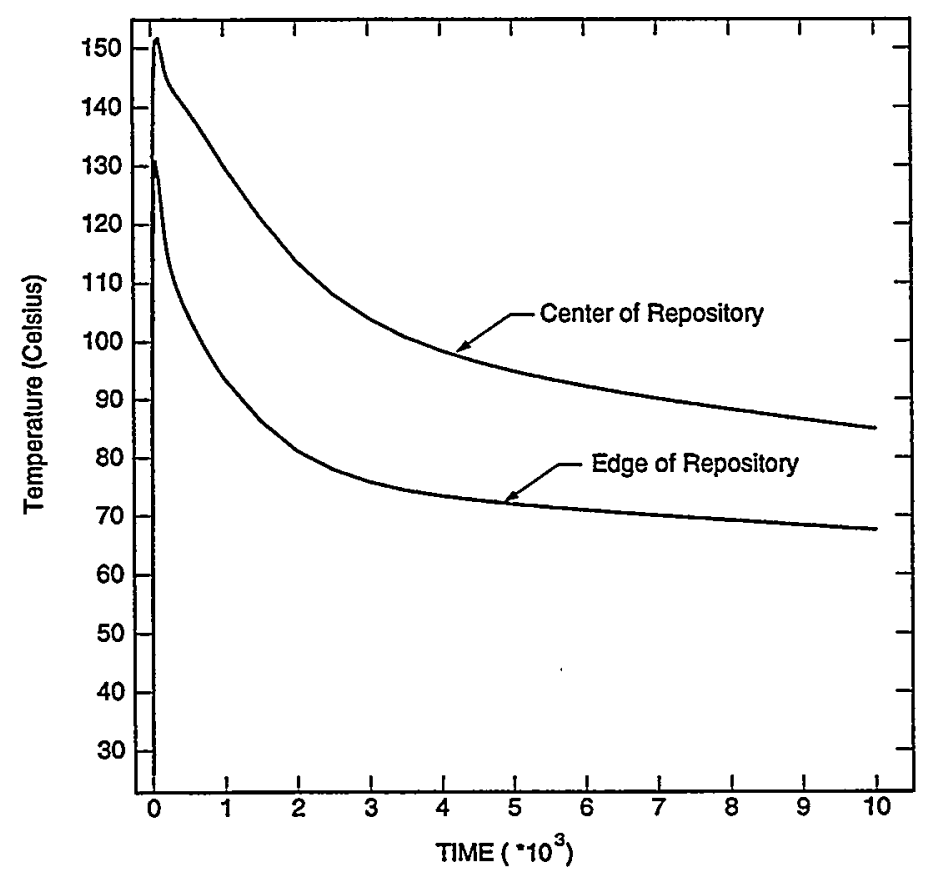

(a) $10,000 \mathrm{yr}$

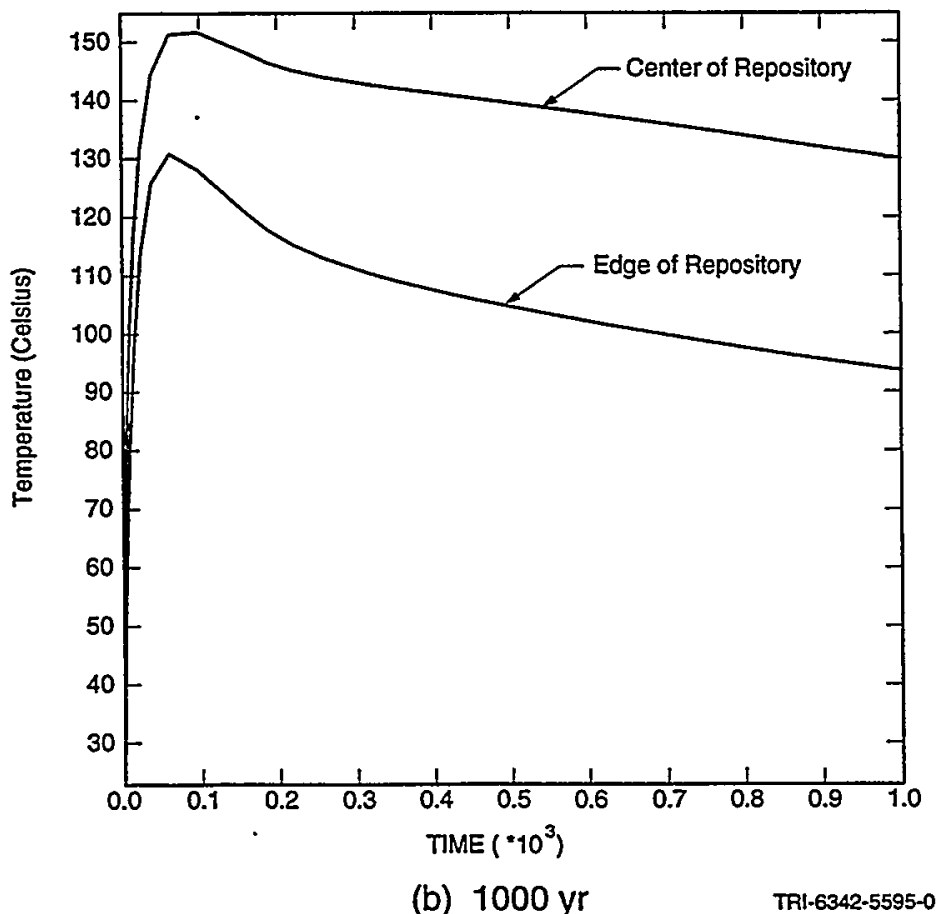

Figure 8-17. SUMQ_3D thermal histories at (a) 10,000 yr and (b) $1000 \mathrm{yr}$. 
Configuration. For both of the SUMQ_3D calculations, 87 tunnels were modeled, the center-to-center spacing between disposal tunnels was $28.6 \mathrm{~m}$, and the waste was distributed to maintain a uniform initial thermal power and power density (see Table 8-3 and Figure 5-4 in Chapter 5), except where detailed power distributions were needed for the package calculations. All packages were circular in cross-section. The package surfaces were separated from the tuff by an air gap determined by the package radius (assumed here as $2.55 \mathrm{~m}$ ) and the radius of the tunnel.

The disposal tunnels were each assumed to be a 1232-m-long linear heat source. ${ }^{*}$ The thermal power from each disposal tunnel equals $1 / \mathrm{N}$ of the repository total, where $\mathrm{N}$ represents the number of waste disposal tunnels (see Figure 8-18). The thermal conduction and radiation properties are the same as those provided in Rechard, ed., (1995, Table 12-1).

\subsubsection{Computed Thermal Histories}

SUMQ_3D was used to compute the thermal response for waste packages out to $10,000 \mathrm{yr}$. Local transient temperatures were computed for each waste package type with CONRAD as modified by SUMQ 3D. Figures 8-19, 8-20, and 8-21 provide the temperature response for the center of the DOE SNF/DHLW, PWR, and BWR packages at the edge and center of the repository.

For the $1997 \mathrm{PA}$, most temperature histories for the packages conform to a pattern of a rapid rise in temperature, followed by a gradual decrease in temperature out to $10,000 \mathrm{yr}$. The early, rapid rise in temperature (tens of yearspeaking around $50 \mathrm{yr}$ at the center and $25 \mathrm{yr}$ at the edge of the repository) is the result of local heating effects within the package and from nearby packages. A secondary pulse that increases the temperature gradient (making it more horizontal) generally occurs later in time (hundreds of years) and is the result of heat from the cumulative effect of all packages in the repository. The PWR fuel (Waste Package 14) has the highest peak temperature of nearly $190^{\circ} \mathrm{C}$ (unmodified by BRAGFLO_T computations) at the repository center (about $175^{\circ} \mathrm{C}$ at the edge) with the package surface slightly cooler than the package center. At the center of the repository, the BWR fuel (Waste Package 15) is hotter than DOE SNF/DHLW fuel; however this condition is not true at the edge of the repository. The DOE SNF/ DHLW temperatures shown are for packages emplaced between two PWR packages (only $2.5 \mathrm{~m}$ separates them). Because the DOE SNF/DHLW package is heated by the PWR packages, the peak for the DOE SNF/DHLW package (at $\sim 160^{\circ} \mathrm{C}$ ) is almost as hot as the BWR package $\left(\right.$ at $\sim 170^{\circ} \mathrm{C}$ ) at the repository center, the DOE SNF/DHLW package is hotter $\left(140^{\circ} \mathrm{C}\right.$ vs. $125^{\circ} \mathrm{C}$ for DOE SNF/DHLW and BWR, respectively) at the repository edge. These results suggest that the BWR package must also be heated by the PWR package. The temperature of the BWR package is less than that of the DOE SNF/DHLW package at the repository edge, perhaps because no PWR packages lie beyond the BWR package at the edge of the repository (see Chapter 5, Figure 5-4 for package disposal layout). The repository temperatures predicted by SUMQ_3D are somewhat higher than those predicted by BRAGFLO_T. Higher temperatures were expected, however, because among other modeling differences, SUMQ_3D does not account for convective heat transfer, a major source of heat loss from the repository.

\subsection{Radionuclide Transport Modeling in Unsaturated Zone}

For the $1997 \mathrm{PA}$, liquid phase radionuclide transport in the unsaturated zone was performed with the NUTS (NUclide Transport System) finite-difference program. The flow fields were calculated with the BRAGFLO_T code (discussed in Section 8.1) and then passed to the NUTS model as input. NUTS, which was originally developed for use by the WIPP Project, was selected for the 1997 PA because of its capability for performing transport calculations in the unsaturated zone in multi-porous media (dual-porosity/dual-permeability). In NUTS, the transport calculation is decoupled from fluid flow. The underlying assumption allowing for decoupling of the flow and transport simulation is that solute concentrations are small and do not affect fluid density or thermal properties. The radionuclide source term for NUTS was provided by CST, discussed in Chapter 7. The release rate from the source submodel is implemented as a point source (injection) in the repository computational nodes.

\footnotetext{
The 1236-m length for the disposal tunnel include $4 \mathrm{~m}$ of access tunnels (see Section 5.2.3). Because this portion is not heat-producing, the 1232 -m length was used in the heat calculations.
} 
8. Consequence Modeling: Unsaturated Zone Flow and Transport
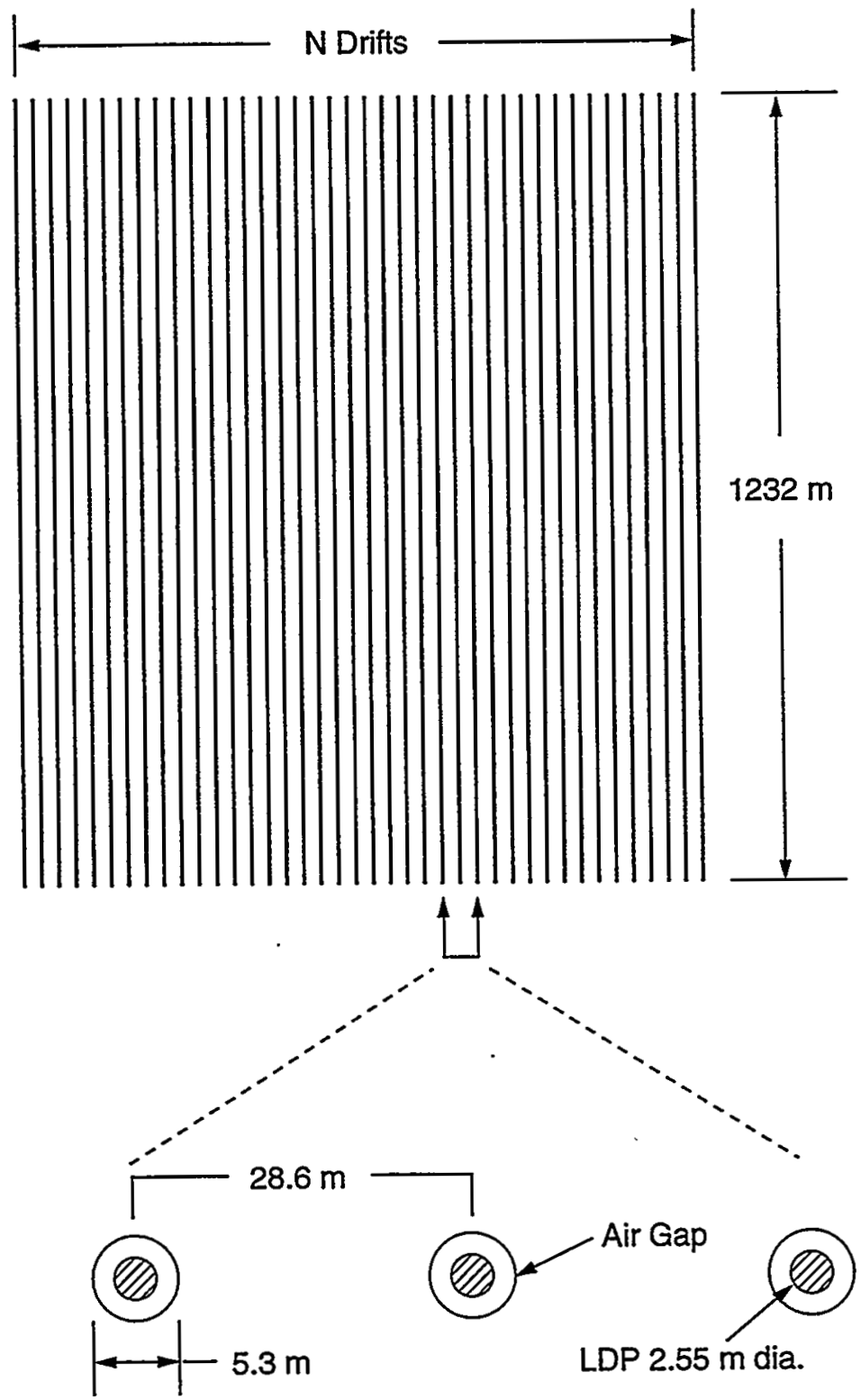

TR1- $6342-5585-0$

Figure 8-18. Repository layout for thermal calculations. 

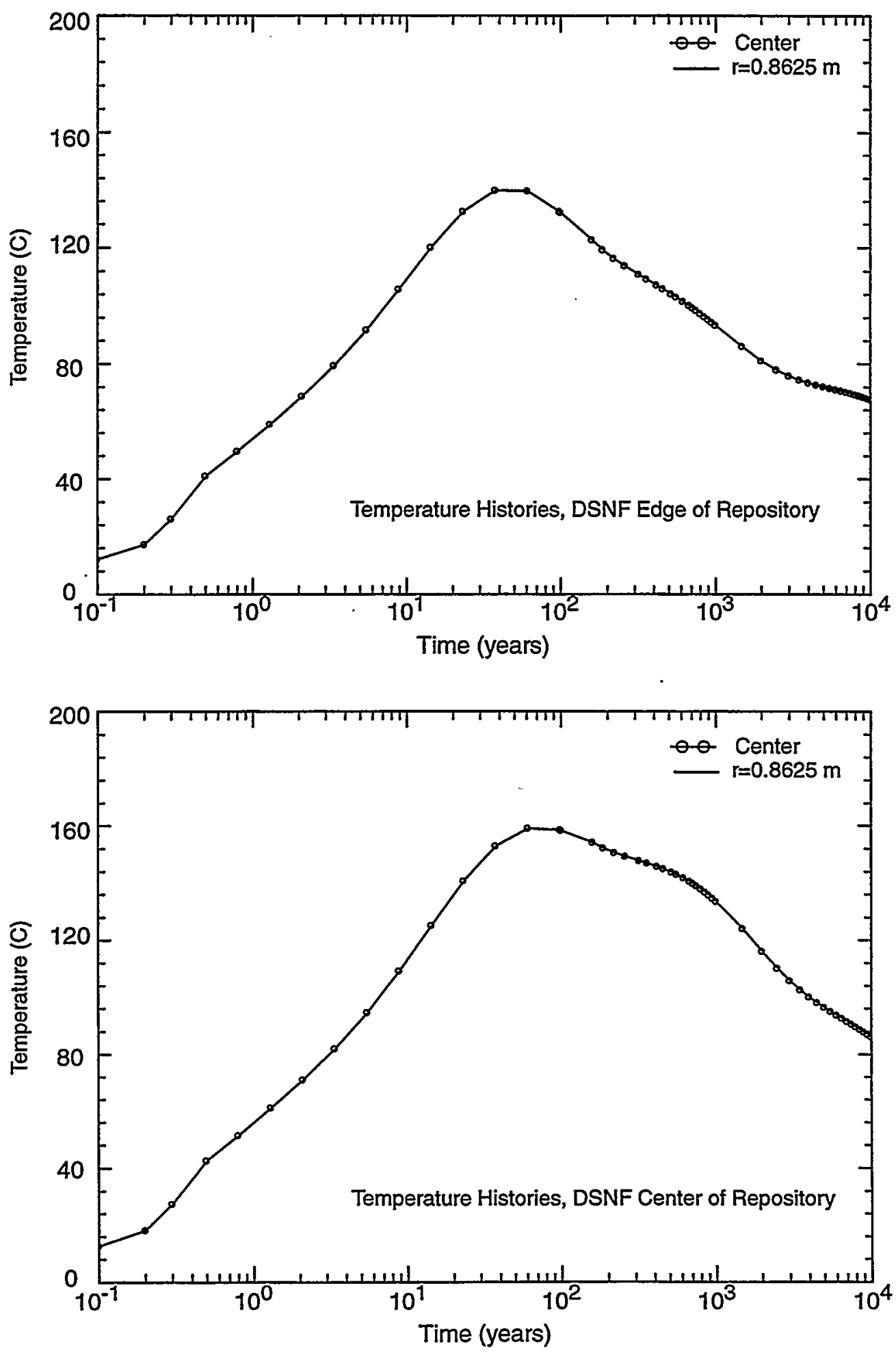

TRI-6342-5586-0

Figure 8-19. Temperature history for DOE SNF/DHLW package at edge and center of repository. 
8. Consequence Modeling: Unsaturated Zone Flow and Transport
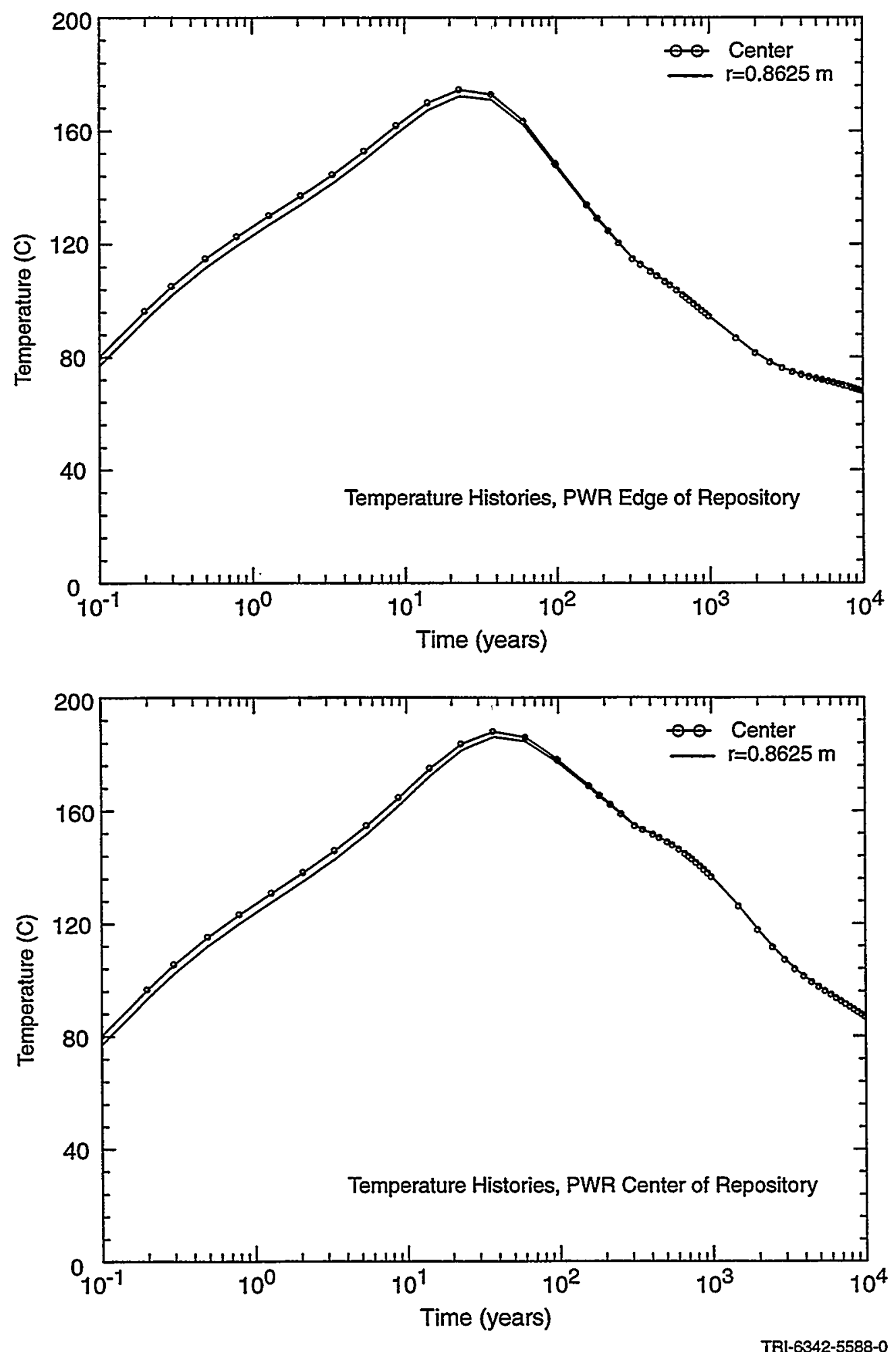

Figure 8-20. Temperature history for PWR package at edge and center of repository. 

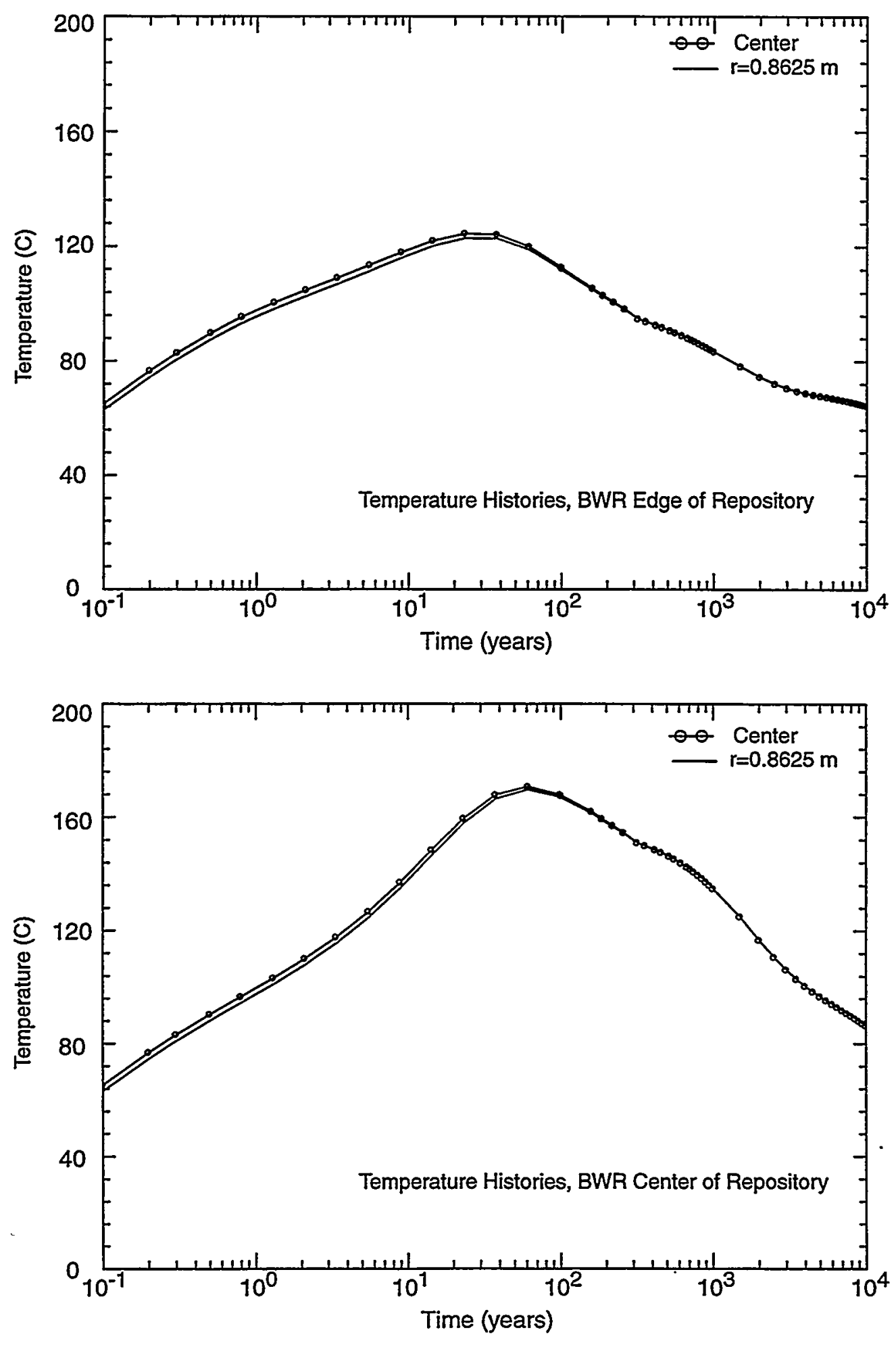

TRI-6342-5587-0

Figure 8-21. Temperature history for BWR package at edge and center of repository. 


\subsubsection{Mathematical Model Description}

NUTS is a multidimensional, multicomponent transport code with single-porosity, dual-porosity, and dual-permeability capabilities. The model is comprehensive and capable of determining the migration rate, distance, concentration, and level of radioactivity of radionuclides transported from the repository and in the surrounding formations.

\section{Transport in the Liquid Phase.}

Transport Mass Balance Equation. In standard dual permeability, the partial differential equations that represent the transport in a fractured porous medium consist of fracture and matrix equations coupled by a transfer function between the two media (Kazemi et al., 1976; Hill and Thomas, 1985; Litvak, 1986; Coats, 1989). The multidimensional difference form of the equations governing the transport of radionuclides in the liquid phase in the dual-permeability formulation (Bear et al., 1993; Shinta and Kazemi, 1993) are as follows:

Fracture:

$$
\begin{aligned}
& \Delta_{x}\left[\phi_{f} S_{w f} K_{w f} \Delta_{x} C_{n w f}\right]+\Delta_{x}\left[T_{w f} C_{n w f}\left(\Delta_{x} P_{w f}-\gamma_{w f} \Delta_{x} D_{f}\right)\right]+C_{n w f}^{*} Q_{w f}-\tau_{w m / f} C_{n w m / f}- \\
& \phi_{m} S_{w m} K_{w m / f}\left(C_{n w f}-C_{n w m}\right)=\frac{V_{R}}{\Delta t} \Delta_{t}\left[\phi_{f} S_{w f} C_{n w f}+\left(1-\phi_{t}\right) \rho_{s} C_{n s f}\right]+ \\
& V_{R}\left[\phi_{f} S_{w f} C_{n w f}+\left(1-\phi_{t}\right) \rho_{s} C_{n s f}\right] \lambda_{n}-V_{R} \sum_{j=1}^{J}\left\{\left\{\phi_{f} S_{w f} C_{n w f p j}+\left(1-\phi_{t}\right) \rho_{s} C_{n s f p j}\right\} \lambda_{n p j}\right\} \\
& n=1,2, \ldots, \ldots N
\end{aligned}
$$

Matrix:

$$
\begin{aligned}
& \Delta_{x}\left[\phi_{m} S_{w m} K_{w m} \Delta_{x} C_{n w m}\right]+\Delta_{x}\left[T_{w m} C_{n w m}\left(\Delta_{x} P_{w m}-\gamma_{w m} \Delta_{x} D_{m}\right)\right]+C_{n w m}^{*} Q_{w m}+\tau_{w m / f} C_{n w m / f}+ \\
& \phi_{m} S_{w m} K_{w m / f}\left(C_{n w f}-C_{n w m}\right)=\frac{V_{R}}{\Delta t} \Delta_{t}\left[\phi_{m} S_{w m} C_{n w m}+\left(1-\phi_{t}\right) \rho_{s} C_{n s m}\right]+ \\
& V_{R}\left[\phi_{m} S_{w m} C_{n w m}+\left(1-\phi_{t}\right) \rho_{s} C_{n s m}\right] \lambda_{n}-V_{R} \sum_{j=1}^{J}\left\{\left\{\phi_{m} S_{w m} C_{n w m p j}+\left(1-\phi_{t}\right) \rho_{s} C_{n s m p j}\right\} \lambda_{n p j}\right\} \\
& n=1,2, \ldots . ., N
\end{aligned}
$$

where

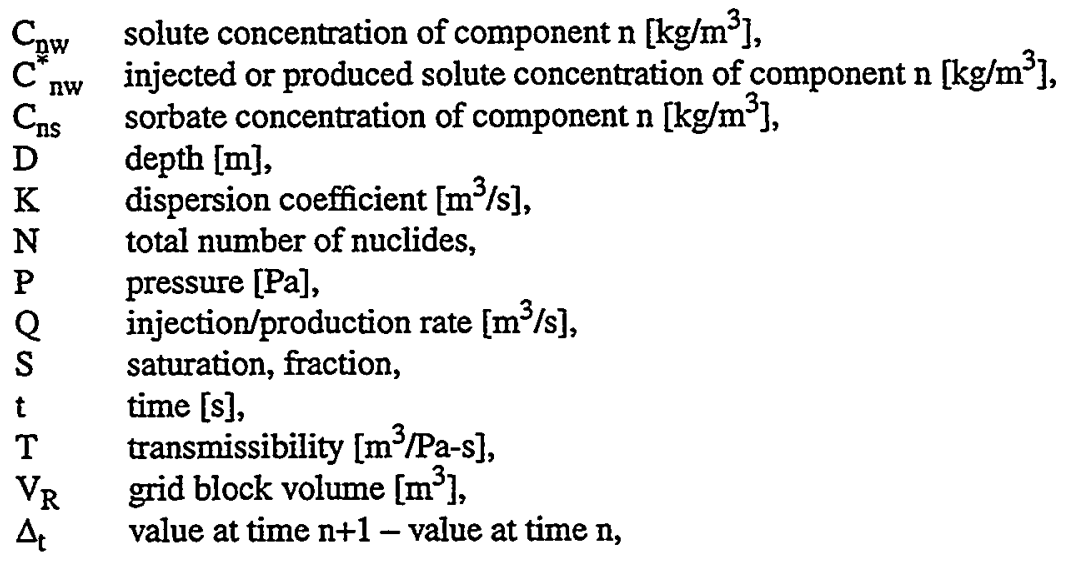


$\Delta_{\mathrm{x}} \quad$ finite difference operator in $\mathrm{x}$-direction,

$\gamma \quad$ static pressure gradient $[\mathrm{Pa} / \mathrm{m}]$,

$\lambda$ decay constant $\left[\mathrm{s}^{-1}\right]$,

$\phi$ porosity,

$\rho \quad$ density $\left[\mathrm{kg} / \mathrm{m}^{3}\right]$,

$\tau \quad$ liquid matrix fracture transfer function $\left[\mathrm{m}^{3} / \mathrm{s}\right]$,

Subscripts:

f fracture

m matrix

$\mathrm{m} / \mathrm{f}$ matrix/fracture

n component number

pj parent number

s solid phase (rock)

t total (fracture + matrix)

w water phase

For compactness purposes in the above formulations, only $\Delta x$ which represents a central finite-difference operator in $\mathrm{x}$-direction, is written. Similar operators can be included for $\mathrm{y}$ - and $\mathrm{z}$-direction. The number of continua in the above formulae is limited to two, i.e., matrix and fracture. Therefore, it is possible to perform single-porosity, dualporosity, and dual-permeability simulations. In the single-porosity, the calculations can be run either in the fracture or in the matrix continuum. Depending on the flow flux field and whether matrix-matrix flow is assumed, NUTS behaves as dual-porosity when the fracture is the main path for the transport (continuous continuum) and the matrix is dispersed in the fracture as a sink/source term, and a dual-permeability model when appreciable transport can be attributed to the matrix flow. In both dual-porosity and dual-permeability models, matrix-fracture transfer is the coupling transport term between the two continua.

Convective Transport. The second and fourth terms in Equations 8.3-1 and 8.3-2 are the contribution from the convective transport. In the NUTS conceptualization, mass transfer across grid block interfaces is governed by a Darcian transient flow of the liquid phase consistent with BRAGFLO_T. However, the transfer at the fracture/matrix interface is dominated by a semi-steady-state Darcian flow represented by $\tau_{\mathrm{wm} / \mathrm{f}}$. The magnitude of these flow terms and their idealizations are provided by BRAGFLO_T as volumetric rates.

The x-component of fracture and matrix transmissibilities of the liquid phase (water) $T_{w f x}$ and $T_{w m x}$ are defined as follows:

$$
T_{w f x}=\frac{\Delta y \Delta z}{\Delta x}(k \phi)_{f x}\left(\frac{k_{r}}{\mu}\right)_{w f}
$$

and

$$
T_{w m x}=\frac{\Delta y \Delta z}{\Delta x} k_{m x}\left(\frac{k_{r}}{\mu}\right)_{w m}
$$

The fracture/matrix transfer function $\tau$ for the water is given by

$$
\tau_{w}=\sigma V_{R} k_{m}\left(\frac{k_{r}}{\mu}\right)_{w m / f}\left[P_{w f}-P_{w m}-\gamma_{w m / f}\left(D_{f}-D_{m}\right)\right],
$$

where

$$
\begin{array}{ll}
\mathrm{D} & \text { depth }[\mathrm{m}], \\
\mathrm{K} & \text { permeability }\left[\mathrm{m}^{2}\right], \\
\mathrm{k}_{\mathrm{r}} & \text { relative permeability [dimensionless], }
\end{array}
$$




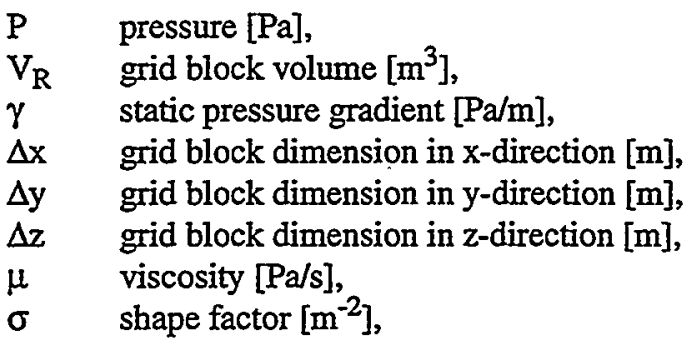

Dispersive Transport. When a contaminant is transported in porous media, the front spreads out beyond the abrupt interface expected only from the convective part of the flow. Among the processes that lead to a smear (Sshape) front are the sorption-desorption process and mixing or dispersion. Dispersion phenomena are classified into

1. Mechanical dispersion, which results from the variation of velocity distribution along the tortuous path of the pore spaces. Heterogeneity introduced by local variations of permeability can also contribute to this kind of dispersion.

2. Molecular diffusion caused by differential tracer concentration. The difference in concentration could be a consequence of any chemical process capable of changing the concentration of the transported nuclide, such as deposition, sorption, solution ion exchange, chemical reaction, and radioactive decay.

In practical application of solute transport, it is customary to include hydrodynamic dispersion (both molecular diffusion and mechanical dispersion). Fick's second law of dispersion is utilized in NUTS to describe the dispersion contribution to mass transfer in both fracture and matrix (term $I$ in Equations 8.3-1 and 8.3-2). Dispersion here is treated as a tensor with the assumption that the dispersivity corresponds to an isotropic porous media. Bear (1988) has shown that dispersion is a second order tensor and can be represented by the following matrix,

$$
K=\left|\begin{array}{lll}
K_{x x} & K_{x y} & K_{x z} \\
K_{y x} & K_{y y} & K_{y z} \\
K_{z x} & K_{z y} & K_{z z}
\end{array}\right|
$$

The above tensor is symmetric and has the property of $K_{x y}=K_{y x}, K_{x z}=K_{z x}$, and $K_{y z}=K_{z y}$ If the coordinate system coincides with the principal axes of dispersion (the medium is assumed to be isotropic with regards to dispersivity), off-diagonal values will be zero in the tensor matrix. Therefore, the dispersion coefficients for the $x$-direction in the fracture and the matrix (Bear et al., 1993) are

$$
K_{f x}=\frac{\Delta y \Delta z}{\Delta x}\left\{\frac{D_{m}^{*}}{\tau *_{f}}+\frac{1}{\phi_{f} S_{w f}\left|\bar{p}_{w f}\right|}\left[\alpha_{L f} v_{w f x}^{2}+\alpha_{T f}\left(v_{w f y}^{2}+v_{w f z}^{2}\right)\right]\right\}
$$

and

$$
K_{m x}=\frac{\Delta y \Delta z}{\Delta x}\left\{\frac{D_{m}^{*}}{\tau *_{m}}+\frac{1}{\phi_{m} S_{w m}\left|\vec{v}_{w m}\right|}\left[\alpha_{L m} v_{w m x}^{2}+\alpha_{T m}\left(v_{w m y}^{2}+v_{w m z}^{2}\right)\right]\right\}
$$

where the terms between brackets are the $\mathrm{x}$-component of the dispersivity tensor and,

$$
\begin{array}{ll}
D_{m}^{*} & =\text { molecular diffusion coefficient }\left[\mathrm{m}^{2} / \mathrm{s}\right] \\
\tau^{*} & =\text { tortuosity [dimensionless], } \\
\alpha_{L} & =\text { longitudinal dispersivity }[\mathrm{m}], \\
\alpha_{\tau} & =\text { transverse dispersivity [m] }
\end{array}
$$


and the velocity vector $\vec{v}$ is given by

$$
|\vec{v}|=\sqrt{v_{x}^{2}+v_{y}^{2}+v_{z}^{2}}
$$

where $v_{x}, v_{y}$ and $v_{z}$ are the velocities [m/s] in $\mathrm{x}, \mathrm{y}$, and $\mathrm{z}$ directions, respectively. Similar formulations can be written for $y$ and $z$ components of the dispersion tensor.

Dispersion in the fracture and matrix is generally small and can be neglected if the convective part of the transport is dominant. However, for transport in the repository, dispersion could be the major transport term, particularly away from the radioactive source. In either case, dispersion can be a significant means of mass transfer between fracture and matrix (term 5 in Equations 8.3-1 and 8.3-2). The dispersion coefficient for fracture/matrix flow (Bear et al., 1993) is given by

$$
K_{w m / f}=\sigma V_{R}\left\{\frac{D{ }_{m}}{\tau{ }_{m}}+\alpha_{m} \frac{\left|v_{w m / f}\right|}{\phi_{m} S_{w m / f}}\right\}
$$

where,

$v_{w m / f}=$ velocity of liquid transfer $(\mathrm{m} / \mathrm{s})$

$S_{w m / f}=$ upstream weighted fracture or matrix saturation [dimensionless]

The shape factor $\sigma$ above is defined in general from as

where

$$
\sigma=\Sigma \frac{A_{m}}{V_{m} d}
$$

$$
\begin{aligned}
& A_{m} \quad=\text { the surface area of the face subjected to flow }\left[\mathrm{m}^{2}\right] \text {, } \\
& V_{m} \quad=\text { the volume of the matrix block }\left[\mathrm{m}^{3}\right] \text {, } \\
& d \quad=\text { the distance between the face and the center of the matrix block [m]. }
\end{aligned}
$$

For cubical matrix block, the shape factor (Kazemi et al., 1976) is therefore,

$$
\sigma=4\left(\frac{1}{L_{x}^{2}}+\frac{1}{L_{y}^{2}}+\frac{1}{L_{z}^{2}}\right),
$$

where $L_{x}, L_{y}$ and $L_{z}$ are the block dimension in $\mathrm{x}, \mathrm{y}$, and $\mathrm{z}$ coordinates.

Sorption. Many chemicals might undergo sorption to the solid surface of the matrix rock. This surface phenomenon takes place because of the mass transfer between the solute and the solid surface and is controlled by the chemical properties of both the solid and the solute. Sorption as a retarding factor in the transport front propagation is expressed in NUTS through the variables $\mathrm{C}_{\mathrm{sm}}$ and $\mathrm{C}_{\mathrm{sf}}$, which represent the sorbed concentration of the radionuclide per unit rock weight. It is customary to relate $C_{s m}$ and $C_{s f}$ to the solute concentration by some sort of empirical relations called isotherms. As the name implies, these isotherms are no more than the sorbate concentration versus the solute concentration at a constant temperature. The linear sorption isotherm is used in the 1997 PA in which the sorbate concentration is related to the solute concentration (Freeze and Cherry, 1979) by

$$
C_{s}^{n+1}=k_{d} C_{w}^{n+1} \text {, }
$$

where

$$
\begin{array}{ll}
n+1 & =\text { implicitness of the concentration (current time step), } \\
\mathrm{k}_{\mathrm{d}} & =\text { partition equilibrium constant. }
\end{array}
$$




\section{Consequence Modeling: Unsaturated Zone Flow and Transport}

Decay. NUTS simulates decay of first-order chains of the form $A \rightarrow B \rightarrow C \ldots \rightarrow N$. The properties of the modeled radionuclides are provided in the input and the differential form of the decay is used (as opposed to the Bateman's equations). The second and third terms of the right-hand side of Equations 8.3-1 and 8.3-2 are the disappearance of radionuclide $n$ by decay and appearance of $n$ from decay of the preceding chain member, respectively. Examination of these terms shows that both sorbed and dissolved radionuclides contribute to the decay terms. Because of the implicit treatment of the parent contribution into the immediate daughter, the partial differential equations are solved sequentially from the top of each chain.

Solubility Limit and Precipitation. When groundwater comes in contact with released waste, dissolution of radionuclides begins and continues until equilibrium concentrations are attained or all radionuclides are consumed. The capacity of the groundwater to dissolve radionuclides is controlled by temperature, pressure, and chemical composition. Consequently, there is a solubility limit for each element in the waste. NUTS requires the solubility limit of the elements and allocates the solubility limit for the isotopes according to their mole fractions. The amount of radionuclides in excess of their solubility limit precipitates in the grid block. Precipitate in NUTS undergoes decay, and precipitation is treated as a reversible process. Once the solute concentration drops below the solubility limit, groundwater is allowed to redissolve from the precipitate a mass equivalent of the solubility limit (or lower) of that solute, depending on the abundance of the precipitate.

Source/Sink Terms. Source/sink terms are included in the 1997 PA as follows:

1. Variable strength radioactive source from CST for each computational grid block containing a waste.

2. Constant pressure (Dirichlet boundary condition) sinks from BRAGFLO_T at the upper boundary of the simulated domain (emission into the atmosphere).

Temperature Dependency. It is optional in NUTS to consider the temperature dependency of certain parameters, such as solubility of radionuclide, molecular diffusion, and the solute-solid partition coefficients (equilibrium constants). Discussion of implementation and derivation of these parameters will follow in Section 8.3.4.

\subsubsection{Transport in the Gas Phase}

It is essential in gas transport to consider the chemical interaction between the gas and the porous medium (and its content) in which it flows. This interaction or phase equilibrium can be classified as gas-solid phase equilibrium described by sorption-desorption process and gas-liquid phase equilibrium.

The description of multiphase equilibrium in porous media is complex and requires a compositional simulator that strongly couples the transport with flow equations. This kind of treatment arises because some mixtures like $\mathrm{CO}_{2}$ $+\mathrm{H}_{2} \mathrm{O}$, can be observed under certain conditions of pressure, temperature and composition, as a single phase, liquid or vapor, two-phase (vapor-liquid or liquid-liquid), or three-phase (vapor-liquid-liquid). To describe such a system appropriately and predict the number of phases in equilibrium, transport in the liquid and gas phases should be determined simultaneously. In this case, the solute will be partitioned according to its equilibrium constant between the phases in equilibrium.

However, this compositionally equilibrated system requires not only a detailed description of the pressure, temperature and initial composition fields in the computational domain, but also is very demanding with regard to computation time and resources. Consequently, because of data limitations, NUTS considers the porous medium and its fluid content as a retardation factor for the constituents transported by the gas phase (Doctor et al., 1992). The following phenomena are included in NUTS:

1. Solid-gas phase equilibrium is considered by the same linear equilibrium sorption isotherm described above.

2. Liquid-gas equilibrium is modeled through gas solubility in the water as a function of temperature. The equation that describes this equilibrium is 


$$
K_{L G}=\frac{C_{L}}{C_{G}}
$$

where $C_{L}$ and $C_{G}$ are the concentration of the solute in the liquid and gas phases, respectively. The value of the ratio is represented by a linear function of temperature

$$
\frac{C_{L}}{C_{G}}=A-B T
$$

where $A$ and $B$ are the intercept and the slope, respectively. This linear representation is valid only for a limited range of temperature and at low pressure where composition dependency is usually weak.

Because the migration rate in the liquid phase is much slower than in the gas phase, and because the gas and the liquid phases transport are decoupled in NUTS, liquid transport of a constituent abundantly transported by the gas phase will not be tracked. With the above mentioned assumptions, the transport in the gas phase of a dual-permeability system can be described by the following equations:

Fracture:

$$
\begin{aligned}
& \Delta_{x}\left[\phi_{f} S_{G f} K_{G f} \Delta_{x} C_{n G f}\right]+\Delta_{x}\left[T_{G f} C_{n G f}\left(\Delta_{x} P_{G f}-\gamma_{G f} \Delta_{x} D_{f}\right)\right]+C_{n G f}^{*} Q_{G f}-\tau_{G m / f} C_{n G m / f}- \\
& \phi_{m} S_{G m} K_{G m / f}\left(C_{n G f}-C_{n G m}\right)=\frac{V_{R}}{\Delta t} \Delta_{t}\left[\phi_{f} S_{G f} C_{n G f}+\left(1-\phi_{t}\right) \rho_{s} C_{n s f}\right. \\
& \left.+\phi_{f}\left(1-S_{G f}\right) K_{L G} C_{n G f}\right]+V_{R}\left[\phi_{f} S_{G f} C_{n G f}+\left(1-\phi_{t}\right) \rho_{s} C_{n s f}+\phi_{f}\left(1-S_{G f}\right) K_{L G} C_{n G f}\right] \lambda_{n} \\
& -V_{R} \sum_{j=1}^{J}\left\{\left\{\phi_{f} S_{G f} C_{n G f p j}+\left(1-\phi_{t}\right) \rho_{s} C_{n s f p j}+\phi_{f}\left(1-S_{G f}\right) K_{L G} C_{n G f p j}\right\} \lambda_{n p j}\right\} n=1,2, \ldots, . ., N
\end{aligned}
$$

Matrix:

$$
\begin{aligned}
& \Delta_{x}\left[\phi_{m} S_{G m} K_{G m} \Delta_{x} C_{n G m}\right]+\Delta_{x}\left[T_{G m} C_{n G m}\left(\Delta_{x} P_{G m}-\gamma_{G m} \Delta_{x} D_{m}\right)\right]+C_{n G m}^{*} Q_{G m}-\tau_{G m / f} C_{n G m / f}+ \\
& \phi_{m} S_{G m} K_{G m / f}\left(C_{n G f}-C_{n G m}\right)=\frac{V_{R}}{\Delta t} \Delta_{t}\left[\phi_{m} S_{G m} C_{n G m}+\left(1-\phi_{t}\right) \rho_{s} C_{n s m}\right. \\
& \left.+\phi_{m}\left(1-S_{G m}\right) K_{L G} C_{n G m}\right]+V_{R}\left[\phi_{m} S_{G m} C_{n G m}+\left(1-\phi_{t}\right) \rho_{s} C_{n s m}+\phi_{m}\left(1-S_{G m}\right) K_{L G} C_{n G m}\right] \lambda_{n} \\
& -V_{R} \sum_{j=1}^{J}\left\{\left\{\phi_{m} S_{G m} C_{n G p m j}+\left(1-\phi_{t}\right) \rho_{s} C_{n s m p j}+\phi_{m}\left(1-S_{G m}\right) K_{L G} C_{n G m p j}\right\} \lambda_{n p j}\right\} n=1,2, \ldots . ., N
\end{aligned}
$$

where subscript $G$ refers to the gas phase, and $K_{L G}$ is the gas-liquid equilibrium constant. The rest of the nomenclatures are defined in Equations 8.3-1 and 8.3-2.

\subsubsection{Assumptions and Limitations}

Assumptions and limitations of NUTS calculations are as follows:

1. Decoupling of flow and transport equations is permitted because of the dilute solute concentration (does not affect fluid density, viscosity, etc.).

2. Local equilibrium exists between interacting phases. 
3. The radionuclides transported by this model are thermodynamically stable within their solubility limit. No gas phase or radionuclides-rich liquid phase is allowed. However, precipitation is possible beyond the solubility limit.

4. Radionuclides are in equilibrium with the precipitate. The precipitation is a reversible process controlled by the solubility limit as an upper bound.

5. Sorption obeys only equilibrium isotherms (linear isotherm is used in these calculations).

6. Dispersion is assumed to obey Fick's second law with dispersitivites corresponding to an isotropic porous medium so that it can be represented by two constants in the principal directions of flow.

7. Colloidal transport is not considered in this model.

8. Solubility of each isotope is evaluated as:

$$
(\text { Solubility })_{\text {isotope }}=(\text { Mole Fraction })_{\text {isotope }} \times(\text { Solubility })_{\text {element }}
$$

9. Gas-liquid equilibrium follows the linear relationship represented by Equation 8.3-14.

\subsubsection{Implementation of Mathematical Model (Computational Model)}

Numerical Approach. The transport equations (Equations 8.3-1 and 8.3-2) have $N{ }^{*} \mathrm{G}$ unknowns for single porosity and $N * 2 G$ unknowns for dual-porosity and dual-permeability formulations, where $G$ is the number of grid blocks in the simulated spatial domain. The system of partial differential equations is strongly coupled due to the contribution of the parent decay to the immediate daughter (the last terms in Equations 8.3-1 and 8.3-2). The sequential method is used to solve the system implicitly. In the sequential method, the solution proceeds progressively from the top of each radioactive chain. Therefore, the contribution to any daughter from parent decay will be available. This method was chosen to avoid inverting a big (for large number of radionuclides) and very sparse matrix in which the bands are not well structured.

Standard Gaussian elimination or conjugate gradient methods can be used to invert the numerical matrix. In the first method, two techniques are applied to reduce the numerical matrix to a more manageable size. In the first technique, an optimum dimension (in standard ordering) is chosen to define the connectivity of the grid blocks. The guideline of this choice is the minimum number of grid blocks. Hence, for a two-dimensional problem in $x$ and $y$, the numbering will start from $\min (x, y)$ and proceed to the next dimension. The second technique is included by straightening the numerical matrix diagonals and limiting the calculation in the entries between the uppermost and the lowermost diagonals. Therefore, for a single-phase system in two dimension, a pentagonal matrix of $\mathrm{IBW}^{*} \mathrm{G}$ is inverted instead of $\mathrm{G}^{*} \mathrm{G}$ matrix, where $\mathrm{IBW}$ is the band width.

Algorithms for Temperature-Dependent Parameters. With the lack of experimental data on most of the parameters discussed in this section, a preliminary derivation and treatment of temperature dependency will be suggested here. The main objective behind this section is to raise the question of whether it is important to include this obvious dependency, and to suggest some simplified thermodynamic relations to implement it. With the availability of experimental data, one can test these formulations and fine tune them if necessary.

Solubility Limit. Experimental results (Nitsche et al., 1992; Wilson, 1993) showed that the extent to which solid radionuclides can dissolve in water varies with temperature and the chemical composition of the water (e.g., $\mathrm{pH}$ of the solution). The equation of equilibrium of radionuclide $i$ can be written as

$$
f_{i}^{s}=f_{i}^{1}=\gamma_{i} x_{i} f_{i}^{o}
$$


where $f_{i}^{s}$ is the fugacity of the pure solid phase, $f_{i}^{1}$ is the fugacity of the solute in the liquid solution, $\gamma_{i}$ is the activity coefficient of the liquid phase, and $f_{i}^{o}$ is standard state fugacity to which $\gamma_{i}$ refers. From Equation 8.3-17, the solubility $x_{i}$ can be evaluated

$$
x_{i}=\frac{f_{i}^{s}}{\gamma_{i} f_{i}^{o}}
$$

Following Prausnitz et al. (1986), one can show that the fugacity ratio in Equation 8.3-18 can be found from the following relationship

$$
\ln \frac{f_{i}^{1}}{f_{i}^{o}}=\frac{\Delta H^{f}}{R T_{t}}\left(\frac{T_{t}}{T}-1\right)-\frac{\Delta c_{p}}{R}\left(\frac{T_{t}}{T}-1\right)+\frac{\Delta c_{p}}{R} \ln \frac{T_{t}}{T}
$$

provided that the temperature of the solution is not far from the triple point, where,

$$
\begin{array}{ll}
\Delta H^{f} & =\text { enthalpy of diffusion, } \mathrm{J} \text { mole } \\
R & =\text { gas constant, } \mathrm{J} \text { mole } \\
R & \mathrm{~K} \\
\Delta c_{p} & =\text { heat capacity at constant pressure, } \text { mole }^{-1} \mathrm{~K}^{-1} \\
T & =\text { temperature, } \mathrm{K} \\
T_{t} & =\text { triple point temperature, } \mathrm{K}
\end{array}
$$

However, in this approach, the activity coefficient $\gamma_{i}$ data are not available. Unless the solution is assumed to be ideal $\left(\gamma_{i}=1\right)$, this lack of activity coefficient experimental data will be a significant limitation.

Another way to implement the temperature effect on radionuclide solubility is possible by writing the equilibrium equation (Equation 8.3-17) as

$$
f_{i}^{s}=\phi_{i} x_{i} P
$$

where $\phi_{i}$ is the fugacity coefficient of the solute $i$ and can be obtained from any equation of state (EOS) and $P$ is the pressure $(\mathrm{Pa})$. The pure solid fugacity $f_{i}^{s}$ can be derived from the basic definition of the fugacity to be

$$
f_{i}^{s}=P_{i}^{s} \phi_{i}^{s} \exp \left(\int_{P_{i}^{s}}^{P} \frac{v_{i}^{s} \partial P}{R T}\right)
$$

where,

$$
\begin{array}{ll}
P_{i}^{s} & \text { saturation (vapor) pressure, } \mathrm{Pa} \\
\phi_{i}^{s} & \text { fugacity coefficient at saturation pressure [dimensionless] } \\
v_{i}^{s} & \text { radionuclide molar volume }\left[\mathrm{m}^{3} \mathrm{~mole}^{-1]}\right.
\end{array}
$$

For the solid radionuclides which is incompressible, $f_{i}^{s}$ is fairly approximated by $P_{i}^{s}$. However, the fugacity coefficient calculation requires sufficient binary solubilities (radionuclide + water) at different temperatures to obtain the binary interaction coefficients used by the EOS.

In general most of the experimental data needed for such a phase equilibrium calculation are not available. Therefore, a statistical correlation in terms of temperature and $\mathrm{pH}$ is adopted for the NUTS calculations. The polynomial (Andrews et al., 1994) describing this temperature dependency of the solubility is

$$
\ln (S)=c_{o}+\frac{c_{1}}{T}+\frac{c_{2}}{T^{2}}+c_{3} T+c_{4} \ln (T)+c_{5} p H
$$


where,

$S$ Solubility of the radionuclide, $\mathrm{gm} / \mathrm{m}^{3}$

$c_{0-5}$ Polynomial parameters (constants)

Molecular Diffusion. Experimentally, diffusion is proportional to the concentration difference across a well defined boundary, or more conveniently, to the concentration gradient, $\partial C / \partial \mathrm{x}$, where $C$ is concentration in $\mathrm{kg} / \mathrm{m}^{3}$ of the solution. The diffusion rate is also proportional to the cross-sectional area $\mathrm{A}$ across which diffusion is realized. If the diffusion rate is written as $\partial \mathrm{m} / \partial \mathrm{t}$, the mass transferred across the boundary per second, one can arrive to Fick's law of diffusion

$$
\frac{\partial m}{\partial t}=-D A \frac{\partial C}{\partial x}
$$

where $D$ is the proportionality constant referred to as molecular diffusion. $D$ can be recognized as the amount of the solute that diffuses across a unit cross sectional area in one second under the influence of unit concentration gradient. Let us consider diffusion across a distance $\partial \mathrm{x}$ over which the concentration changes from $\mathrm{C}$ to $\mathrm{C}-\partial \mathrm{C}$. The driving force is the difference in molar Gibb's free energy $(\mathrm{g})$ of the solute at the two concentrations which can be written as

$$
g_{c-\partial c}-g_{c}=R T \ln \left(\frac{C-\partial C}{C}\right)
$$

If we rewrite Equation 8.3-24 for one macroscopic particle (Barrow, 1973), then the energy difference per particle (G) is

$$
G_{c-\partial c}-G_{c}=\Delta G=\frac{R T}{N} \ln \left(1-\frac{\partial C}{C}\right)
$$

For a small value of $\partial \mathrm{C} / \mathrm{C}$ the following approximation is valid,

$$
\ln \left(1-\frac{\partial C}{C}\right)=\frac{\partial C}{C}
$$

therefore,

$$
\Delta G=-\frac{R T}{N} \frac{\partial C}{C}
$$

where $\mathrm{N}$ is the number of particles per mole. Gibb's free energy change corresponds to the work done in the transfer of one particle across the distance $\partial \mathrm{x}$ and can be written as: $\partial \mathrm{G}=\mathrm{Fd} * \partial \mathrm{x}$, where $\mathrm{Fd}$ is the driving force, therefore

$$
F d=\frac{\partial G}{\partial x}=-\frac{R T}{N} \frac{1}{C} \frac{\partial C}{\partial x}
$$

The force Fd will be balanced by frictional forces Ff when a certain velocity $v$ is reached. If the particle is assumed to have a spherical shape of radius $r$, then the frictional forces exerted by the fluid of viscosity $\mu$ is defined by Stoke's law as

$$
F f=6 \pi r \mu v=6 \pi r \mu \frac{\partial x}{\partial t}
$$

The diffusion velocity increases until Fd = Ff. By equating Equations 8.3-28 and 8.3-29 one can arrive at

$$
C \frac{\partial x}{\partial t}=-\frac{R T}{6 \pi r \mu N} \frac{\partial C}{\partial x}
$$


In the above equation $C \frac{\partial x}{\partial t}$ is equivalent to $\frac{\partial m}{A \partial t}$ in Equation 8.3-23 since both of them refers to the mass transferred across the boundary cross sectional area $A$ in the time $\partial t$ (note that $C \partial x=\frac{\partial m}{A}$ ). Therefore comparing Equation 8.3-30 with Fick's law (Equation 8.3-23) will lead to

$$
D=\frac{R T}{6 \pi r \mu N}
$$

The major assumption in the above equation is that the particles are spherical and they obey Stoke's law. However, regardless of the shape of the particles, the importance of this equation lies in the fact that $D \propto \frac{T}{\mu}$ therefore, if we have an experimentally measured value of $D$ at specified temperature and viscosity, then one can write

$$
\frac{D_{m}^{*}(T)}{D_{m}^{o}}=\frac{R T /(6 \pi r \mu(T) N)}{R T^{o} /\left(6 \pi r \mu^{o} N\right)}
$$

and therefore,

$$
D_{m}^{*}(T)=D_{m}^{o} \frac{T}{T^{o}} \frac{\mu^{o}}{\mu(T)}
$$

where,

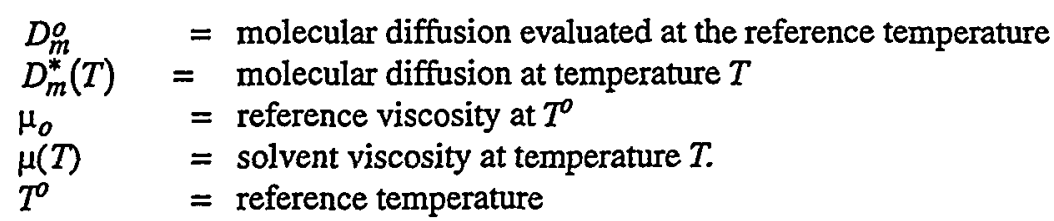

The above derivation is conducted for the liquid phase. However, it is possible from the kinetic-molecular theory of gases to prove that the proportionality in Equation 8.3-33 is also valid for the gas phase.

Sorption. The equilibrium between the solute and the solid phase is dependent on temperature, pressure, and composition of both the solute and the mineral of the rock surrounding the path of the fluid flow. At equilibrium, Gibb's free energy of sorption is

$$
\Delta g=-R T \ln K_{d} .
$$

However, from basic thermodynamics

$$
\Delta g=\Delta H-T \Delta S,
$$

where

$\begin{array}{ll}\mathrm{g} & \text { molar Gibbs free energy } \\ \mathrm{H} & \text { molar enthalpy } \\ \mathrm{K}_{\mathrm{d}} & \text { solid-solute equilibrium constant } \\ \mathrm{R} & \text { universal gas constant } \\ \mathrm{S} & \text { molar entropy } \\ \mathrm{T} & \text { temperature }\end{array}$




\section{Consequence Modeling: Unsaturated Zone Flow and Transport}

Equating and rearranging Equations 8.3-34 and 8.3-35 will lead to

$$
\ln K d=\frac{1}{R}\left[\frac{-\Delta H}{T}+\Delta S\right]
$$

The change in the enthalpy, $\Delta \mathrm{H}$ and the entropy, $\Delta \mathrm{S}$ of sorption to go from the reference temperature $T^{o}$ to the operating temperature $T$ is

$$
\Delta H=\Delta H^{o}+\Delta c_{p}^{o}\left(T-T^{o}\right)
$$

and

$$
\Delta S=\Delta S^{o}+\Delta c_{p}^{o} \ln \frac{T}{T^{o}}
$$

In Equations 8.3-37 and 8.3-38, the heat capacity at constant pressure $\Delta c_{p}{ }^{\circ}$ is assumed to be temperature independent. Substituting Equations 8.3-37 and 8.3-38 in Equation 8.3-36, will result in

$$
\ln K d=\frac{1}{R}\left\{-\frac{\Delta H^{o}}{T}+\Delta S^{o}+\Delta c_{p}^{o}\left[\ln \frac{T}{T^{o}}+\frac{T^{o}}{T}-1\right]\right\}
$$

The third term on the right-hand side of Equation 8.3-39 is small and can be neglected to simplify the equation to

$$
\ln K d=\frac{1}{R}\left[-\frac{\Delta H^{o}}{T}+\Delta S^{o}\right]
$$

where $\Delta H^{\circ}$ and $\Delta S^{\circ}$ are the enthalpy and entropy of sorption at reference temperature, $T^{\circ}$, respectively. If we write Equation 8.3-40 for two temperatures $T_{1}$ and $T_{2}$ and eliminate $\Delta S^{\circ} / R$ from the two equations, we can find

$$
\ln \left(\frac{K d_{2}}{K d_{1}}\right)=\frac{\Delta H^{\circ}}{R}\left[\frac{1}{T_{1}}-\frac{1}{T_{2}}\right]
$$

Equation 8.3-41 if rearranged will lead to

$$
K d_{2}=K d_{1} \quad \exp \left[\frac{\xi\left(T_{2}-T_{1}\right)}{T_{2} T_{1}}\right] \quad \text { where } \xi=\frac{\Delta H^{o}}{R}
$$

In the above formulation, $K d_{1}$, and $K d_{2}$ are partition equilibrium constants at temperatures $T_{1}$ and $T_{2}$, respectively, $R$ is the gas constant, and $\Delta H^{\circ}$ is the enthalpy of sorption at reference temperature $T^{\circ}$.

\subsubsection{Applied Model}

Spatial Discretization and Model Geometry. The geometry of the transport model is identical to that of BRAGFLO_T. The three-dimensional spatial domain is described by two dimensions with element flaring. Upstream weighted concentration in five-point finite-difference approximation is used to discretize the differential equations. The central finite-difference operator is used in spatial discretization. The resultant system of discretized equations in a more compact form is

$$
A x=B
$$


where the coefficient matrix $A$ is a banded pentagonal matrix and $\mathrm{B}$ is the right hand side vector. The solution vector $\mathrm{x}$ is therefore,

$$
x=A^{-1} B
$$

where the inverse matrix $A^{-1}$ is evaluated by Gaussian elimination.

Temporal Discretization. The backward finite-difference operator is utilized in temporal discretization. In order to develop a fully implicit system of equations, each time-dependent variable is dealt with implicitly. Because of the decoupled structure of this calculation (transport follows the flow), communication between NUTS and BRAGFLO_T is achieved by the overall modeling system, CAMCON. BRAGFLO_T output in the database provides NUTS with the required time-dependent parameters. These parameters are velocity at the grid block interfaces, porosities, water or gas saturations, production/injection rates, temperatures, and viscosities. For consistency purposes, the time step size in NUTS is also set to be identical with that of BRAGFLO_T.

Initial and Boundary Conditions. The objective of most numerical solutions is to determine unknown functions that satisfy a given set of partial differential equations in a given domain and at given points at the boundary of that domain. Equations 8.3-1 and 8.3-2 are second order partial differential equations and, therefore, two boundary and/or initial conditions are required to solve each equation. The initial concentrations in both fracture and matrix are initialized to zero, i.e., $C_{n}(x, y, 0)=0$. In the source submodel, however, the boundary conditions are treated differently, as mentioned in Chapter 7.

Because the external boundary conditions in the flow model (BRAGFLO_T) are set to be no-flow boundaries, the advective part of the differential equation will consistently be zero. However, the dispersive term of the equation is independent of the advection flux (depends on concentration gradient) and its value needs to be specified at the boundaries. For the two-dimension spatial domain described earlier, a non-transporting (zero concentration gradient) boundary is assumed.

Interfacing with the Source Submodel (CST). The source submodel, CST is included in NUTS as an internal point source for all the nodes that represent the potential repository. CST calculates the transport inside the waste package and in the possible rubble surrounding the waste package. In the liquid phase transport, the source submodel is called within the main time loop of NUTS. The mass $(\mathrm{kg} / \mathrm{s})$ transported by CST out of its boundary is reinjected in NUTS as a source term for the transport in the spatial domain. See Chapter 7 for more information about CST.

Material Properties. For equivalent continuum simulation runs in NUTS, two sets of material properties are required: equivalent rock properties and radionuclide properties. Equivalent rock properties include porosity, grain density, tortuosity, longitudinal dispersivity, and transverse dispersivity. Radionuclide properties include half-life, molecular or atomic weight, element solubility, specific activity, chains description, molecular diffusion, and liquidsolid equilibrium constants. The remaining properties are read from the data base. The transverse dispersivities used in this year's calculation are sampled parameters that range from $5 \%$ to $20 \%$ of the longitudinal dispersivity. Linear partition coefficients $\left(\mathrm{K}_{\mathrm{D}}\right)$ are used. The only sampled transport parameters, besides the dispersivity percentage mentioned above, are $K_{D}$ 's for neptunium in zeolitic tuff. The mean value is used for the rest of the unsampled properties.

A total of three radioisotopes were transported in the liquid phase $\left({ }^{237} \mathrm{~Np},{ }^{99} \mathrm{Tc},{ }^{129} \mathrm{~T}\right)$; no radionuclides are transported in the gas phase. 


\title{
9. Consequence Modeling: Saturated Zone Flow and Transport
}

\author{
A. M. Parsons and C. D. Updegraff
}

Groundwater flow and radionuclide transport modeling in the saturated zone beneath the repository was performed with the STAFF3D (Solute Transport And Fracture Flow in 3 Dimensions) finite-element program. STAFF3D can calculate either Darcy fluid flow or radionuclide transport in either two or three dimensions with variably saturated conditions and with either a single porosity or dual porosity (Bear and Braester, 1972) idealization. In this performance assessment, the dual porosity conceptual model was used for both the flow and transport with water table conditions. The groundwater flow capability of STAFF3D was used to generate the velocity field for STAFF3D's transport capability. A variable fluid density capability is also available but was not used.

In the dual-porosity approach, the system is assumed to contain numerous fractures, and the domains of both the fractures and porous rock matrix can be represented as two overlapping continua, each filling the entire space. Advective transport occurs only in the fracture domain. Solute exchange between the fracture and matrix occurs due to diffusion at the fracture matrix interface. For this analysis, the flow and transport were uncoupled and run separately based on the assumption that solute concentrations are small and do not affect fluid density or flow properties. The radionuclide source term was implemented in this analysis at several interior grid points using time-dependent, mass flux boundary conditions or injection wells. For this performance assessment, the mass flux rate histories were estimated using the NUTS code as described in Chapter 8. Fluid flow associated with the mass injection from NUTS was assumed to have a negligible effect on the aquifer flow; only the solute mass was injected into the saturated zone flow system.

For reference, Table 9-1 summarizes conceptual models of the saturated zone in related studies. Assumptions for the saturated zone conceptual model can be found in Chapter 4, Table 4-9.

\subsection{Fluid Flow Model}

\subsubsection{Mathematical Model Description}

STAFF3D is a finite-element code designed to simulate variably saturated groundwater flow and solute transport in fractured or granular aquifers with water table boundary conditions (Huyakorn et al., 1992) in either two or threedimensions. The original 2D version was developed through a joint effort by HydroGeoLogic, Inc. and the International Ground Water Modeling Center of the Holcomb Research Institute. Improved versions of the code have since become commercially available through HydroGeoLogic: the current 3D version is Version 2.0. Changes to the code were made by Sandia to accommodate input/output requirements and to tailor the code inputs to the secondary database for the Waste Isolation Pilot Plant (Rechard et al., 1989); these changes also apply to the 1997 PA.

\section{Mass Balance Equation for Flow.}

Fracture Domain. The governing three-dimensional equation for saturated-unsaturated flow in the fracture domain can be written per unit total (bulk) volume as

$$
\begin{gathered}
\frac{\partial}{\partial x_{i}}\left[\phi_{f} K_{i j} k_{r w} \frac{\partial h}{\partial x_{j}}\right]=\phi_{f} \eta \frac{\partial h}{\partial t}-q-\Lambda \\
\eta=S_{w} S_{s}+S_{y} \frac{d S_{w}}{d h} \\
S_{s}=\rho_{w} g\left(\phi \beta_{c}+\alpha_{c}\right)
\end{gathered}
$$


9. Consequence Modeling: Saturated Zone Flow and Transport

Table 9-1. Summary of Previous Conceptual Models for Flow and Transport in the Saturated Zone at Yucca Mountain

\begin{tabular}{|c|c|c|c|c|}
\hline Investigator(s) & Domain Size & Dimensions & Flow & Transport \\
\hline $\begin{array}{l}\text { Rice (1984). Calculated regional } \\
\text { ground-water flow system flux }\end{array}$ & $\begin{array}{l}230 \mathrm{~km} \times 270 \mathrm{~km} \\
\text { ( } 3 \text { ground-water } \\
\text { basins) - } 1 \text { layer }\end{array}$ & 2 & $\begin{array}{l}\text { Steady state, } \\
\text { porous medium }\end{array}$ & none \\
\hline $\begin{array}{l}\text { Waddell (1982). Calculated } \\
\text { regional ground-water flow } \\
\text { system flux }\end{array}$ & $\begin{array}{l}150 \mathrm{~km} \times 170 \mathrm{~km} \\
\text { (3 ground-water } \\
\text { basins) - } 1 \text { layer }\end{array}$ & 2 & $\begin{array}{l}\text { Steady state, } \\
\text { porous medium }\end{array}$ & none \\
\hline $\begin{array}{l}\text { Czarnecki and Waddell (1984). } \\
\text { Evaluated sensitivity to boundary } \\
\text { flux changes }\end{array}$ & $\begin{array}{l}50 \mathrm{~km} \times 100 \mathrm{~km} \\
\text { (one groundwater } \\
\text { basin) - } 1 \text { layer }\end{array}$ & 2 & $\begin{array}{l}\text { Steady state, } \\
\text { porous medium }\end{array}$ & none \\
\hline $\begin{array}{l}\text { Czarnecki (1985). Estimated } \\
\text { variations in water table elevation }\end{array}$ & $\begin{array}{l}50 \mathrm{~km} \times 100 \mathrm{~km} \\
\text { (one groundwater } \\
\text { basin) - } 1 \text { layer }\end{array}$ & 2 & $\begin{array}{l}\text { Steady state, } \\
\text { porous medium }\end{array}$ & none \\
\hline $\begin{array}{l}\text { Barr and Miller (1987). } \\
\text { Evaluated structural control on } \\
\text { hydraulic gradient. }\end{array}$ & $\begin{array}{l}8 \mathrm{~km} \times 20 \mathrm{~km} \text { (con- } \\
\text { strained by } \\
\text { Czarnecki [1985] } \\
\text { subregion) - } 1 \text { layer }\end{array}$ & $2^{a}$ & $\begin{array}{l}\text { Steady state, } \\
\text { porous medium }\end{array}$ & none \\
\hline $\begin{array}{l}\text { Ahola and Sagar (1993). } \\
\text { Determined the impact of } \\
\text { disruptive conditions on } \\
\text { saturated zone flow system and } \\
\text { changes in water table elevation }\end{array}$ & $\begin{array}{l}250 \mathrm{~km} \times 250 \mathrm{~km} \\
\text { regional and } \\
50 \mathrm{~km} \times 50 \mathrm{~km} \\
\text { subregional - } \\
1 \text { layer }\end{array}$ & 2 & $\begin{array}{l}\text { Steady state, } \\
\text { porous medium } \\
\text { (PORFLOW) }\end{array}$ & none \\
\hline $\begin{array}{l}\text { Wilson et al. (1994). Provided } \\
\text { two calibrated models that } \\
\text { represent alternate conceptual } \\
\text { models of saturated flow system. }\end{array}$ & $\begin{array}{l}8 \mathrm{~km} \times 8 \mathrm{~km} \mathrm{x} \\
200 \mathrm{~m}-4 \text { layers }\end{array}$ & $\begin{array}{l}3 \text { flow, } 1 \\
\text { transport }\end{array}$ & $\begin{array}{l}\text { Steady state flow } \\
\text { through fractured } \\
\text { porous media } \\
\text { (STAFF3D) }\end{array}$ & $\begin{array}{l}\text { 1-D flow tube } \\
\text { consistent with } \\
3-D \text { velocity vec- } \\
\text { tors }\end{array}$ \\
\hline $\begin{array}{l}\text { Rechard, ed. (1995). Evaluated } \\
\text { performance of different waste } \\
\text { treatment and disposal options in } \\
\text { hypothetical repository }\end{array}$ & $\begin{array}{l}8 \mathrm{~km} \times 13 \mathrm{~km} \times 400 \\
\text { to } 900 \mathrm{~m}-5(?) \\
\text { layers }\end{array}$ & 3 & $\begin{array}{l}\text { Dual porosity, } \\
\text { domains of frac- } \\
\text { tures and porous } \\
\text { rock represented } \\
\text { as two overlapping } \\
\text { continua. Advec- } \\
\text { tive flow occurs } \\
\text { only in fractures } \\
\text { (STAFF3D) }\end{array}$ & $\begin{array}{l}\text { Dual porosity, } \\
\text { solute exchange } \\
\text { between matrix } \\
\text { and fracture only } \\
\text { due to matrix } \\
\text { diffusion }\end{array}$ \\
\hline $\begin{array}{l}\text { Altman et al. (1996). Modeled } \\
\text { ground-water flow to provide } \\
\text { estimates of pre-waste-emplace- } \\
\text { ment groundwater travel time. }\end{array}$ & $\begin{array}{l}\text { about } 15 \mathrm{~km} \times \\
13 \mathrm{~km} \times 250 \mathrm{~m} \\
\text { extended bound- } \\
\text { aries of Wilson et } \\
\text { al. (1994) }-5 \text { layers }\end{array}$ & 3 & $\begin{array}{l}\text { Steady state, } \\
\text { (STAFF3D). Used } \\
\text { particle tracker for } \\
\text { travel time }\end{array}$ & none \\
\hline $\begin{array}{l}\text { Ho et al. (unpublished report). } \\
\text { Gained insight into the thermo- } \\
\text { hydrologic response of the sys- } \\
\text { tem following emplacement of } \\
\text { heat-generating waste. }\end{array}$ & $\begin{array}{l}\text { Same domain as } \\
\text { Altman et al. (1996) } \\
\text { - } 5 \text { layers }\end{array}$ & 3 & $\begin{array}{l}\text { Transient analy- } \\
\text { sis, simulated heat } \\
\text { conduction in the } \\
\text { unsat zone and } \\
\text { fully coupled } \\
\text { groundwater flow } \\
\text { and heat trans- } \\
\text { port, (FEHMN) }\end{array}$ & none \\
\hline
\end{tabular}

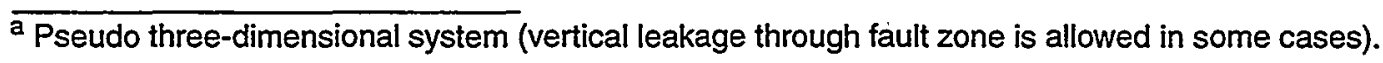


Table 9-1. Summary of Previous Conceptual Models for Flow and Transport in the Saturated Zone at Yucca Mountain

\begin{tabular}{|c|c|c|c|c|}
\hline Investigator(s) & Domain Size & Dimensions & Flow & Transport \\
\hline $\begin{array}{l}\text { Czarnecki et al. (1996). Among } \\
\text { other purposes, develop a } \\
\text { calibrated flow model that } \\
\text { provides a means for subsequent } \\
\text { flow, heat, and radionuclide } \\
\text { transport modeling. }\end{array}$ & $\begin{array}{l}15 \mathrm{~km} \times 15 \mathrm{~km} \times \\
\text { variable depth }\end{array}$ & 3 & $\begin{array}{l}\text { Nonisothermal, } \\
\text { multiphase flow } \\
\text { and transport } \\
\text { through heteroge- } \\
\text { neous, fractured } \\
\text { porous media, } \\
\text { (FEHMN) }\end{array}$ & work in progress \\
\hline $\begin{array}{l}\text { TRW Environmental Safety } \\
\text { Systems, Inc. (Nov 1995). TSPA- } \\
1995 \text { for incorporating revised } \\
\text { site and design information from } \\
\text { TSPA-1993. }\end{array}$ & $\begin{array}{l}50 \mathrm{~m} \text { mixing depth, } \\
5 \mathrm{~km} \text { accessible } \\
\text { environment and } \\
30 \mathrm{~km} \text { dose expo- } \\
\text { sure point }\end{array}$ & $\begin{array}{l}\text { Used 2-D } \\
\text { fluxes from } \\
\text { TSPA-1993; } \\
\text { 1-D transport }\end{array}$ & $\begin{array}{l}\text { Analytic solution } \\
\text { for dilution, } \\
\text { "stirred-tank" mix- } \\
\text { ing model }\end{array}$ & \\
\hline $\begin{array}{l}\text { D'Agnese et al. (1996). } \\
\text { Characterizes the regional } \\
\text { hydrogeology and documents a } \\
\text { numerical simulation of present } \\
\text { gw flow system. }\end{array}$ & $\begin{array}{l}\text { Approx. } 200 \mathrm{~km} \times \\
250 \mathrm{~km} \times 2750 \mathrm{~m}- \\
3 \text { layers }\end{array}$ & 3 & $\begin{array}{l}\text { Incorporated all } \\
\text { available data, } \\
\text { used GIS and } \\
\text { GSIS to build } \\
\text { model, flow calcs } \\
\text { with MODFLOP }\end{array}$ & None \\
\hline
\end{tabular}

where,

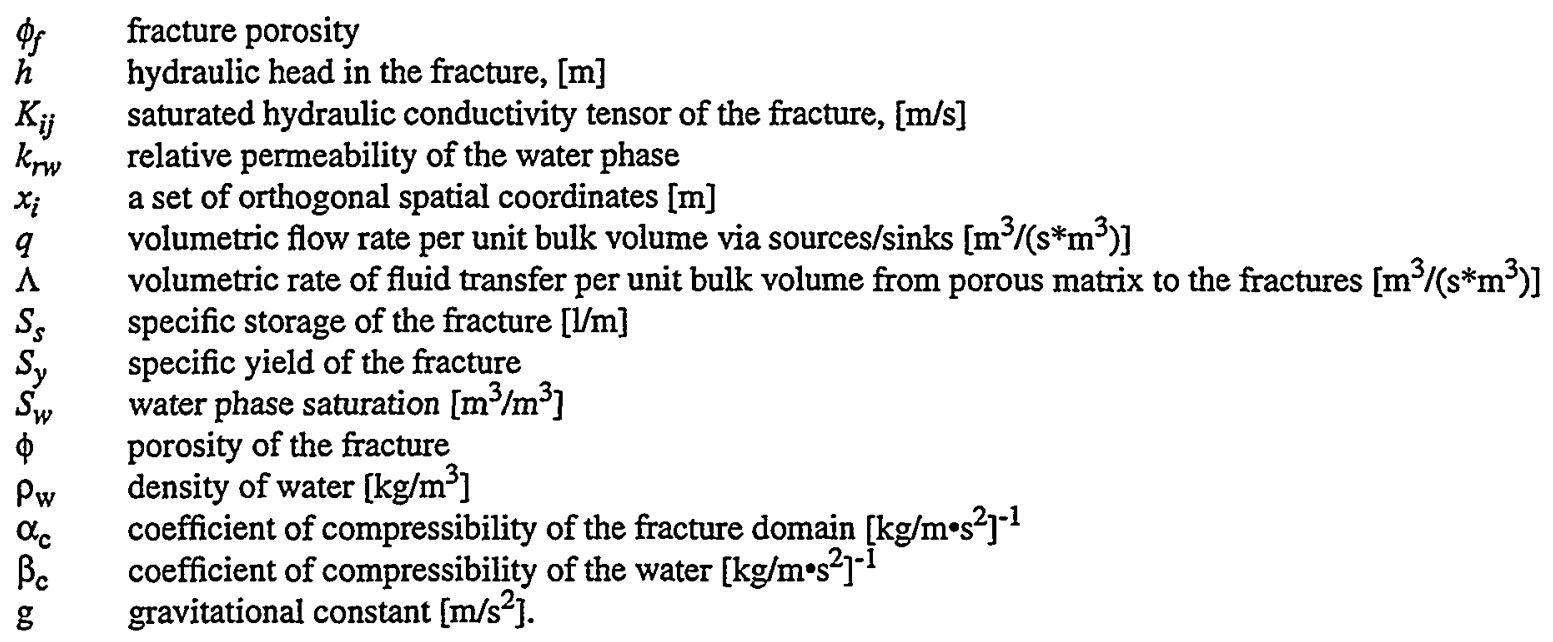

To solve the variably saturated flow problem, it is also necessary to specify the relationship of relative permeability versus water phase saturation and pressure head versus water phase saturation. Two alternative functional relations can be used. These are given by (Brooks and Corey, 1966):

$$
k_{r w}=S_{e}^{n}
$$

and (van Genuchten et al., 1977);

$$
k_{r w}=S_{e}^{1 / 2}\left[1-\left(1-S_{e}^{1 / \gamma}\right)^{\gamma}\right]^{2}
$$




$$
S_{e}=\left(S_{w}-S_{w r}\right) /\left(1-S_{w r}\right)
$$

where, $\mathrm{n}$ and $\gamma$ are empirical parameters, $S_{e}$ is the effective water saturation, and $S_{w r}$ is the residual water saturation.

The relationship of pressure head, $\Psi[\mathrm{m}]$, versus water saturation is described by the following function (van Genuchten et al., 1977; Mualem, 1976):

$$
\frac{S_{w}-S_{w r}}{1-S_{w r}}= \begin{cases}\frac{1}{\left[1+\left(\alpha\left|\psi-\psi_{a}\right|\right)^{\beta}\right]^{\gamma}} & \text { for } \psi<\psi_{a}<0 \\ 1 & \text { for } \psi \geq \psi_{a}\end{cases}
$$

where,

$$
\begin{array}{ll}
\alpha & =\text { empirical parameter }[1 / \mathrm{m}] \\
\beta & =\text { empirical parameter, } \\
\psi_{\mathrm{a}} & =\text { air entry pressure head, } \\
\gamma & =\text { usually } 1-1 / \beta .
\end{array}
$$

The Brooks-Corey and van Genuchten functions for moisture retention and relative permeability characteristics can be measured in the laboratory for a given soil.

After the distributions for head and saturation have been determined, Darcy velocity components within the fracture domain, $V_{i}[\mathrm{~m} / \mathrm{s}]$, are calculated from:

$$
V_{i}=-K_{i j} k_{r w} \frac{\partial h}{\partial x_{j}}
$$

Porous Matrix Domain. The $\Lambda$ term in Equation 9.1-1 represents the interaction between the porous rock matrix and fractures. As the hydraulic head is reduced in the fractures, fluid flows from the matrix into the fractures. The flux is a function of the head in the fractures and the head in the porous rock. The hydraulic head distribution in the matrix blocks can be determined by solving a simple one-dimensional flow problem, assuming the hydraulic conductivity of the matrix blocks is small compared with the fracture hydraulic conductivity. For the slab block idealization (Chapter 8), the equation is as follows:

$$
\frac{\partial}{\partial \chi}\left(K^{\prime} \frac{\partial h^{\prime}}{\partial \chi}\right)=S_{s}^{\prime} \frac{\partial h^{\prime}}{\partial t}
$$

subject to,

where,

$$
\begin{array}{ll}
h^{\prime}=h_{o}^{\prime} & \text { at } t=0 \\
K^{\prime} \frac{\partial h^{\prime}}{\partial \chi}=0 & \text { at } \chi=0 \\
h^{\prime}=h-\varsigma K^{\prime} \frac{\partial h^{\prime}}{\partial \chi} & \text { at } \chi=B
\end{array}
$$

$h^{\prime} \quad=$ hydraulic head in the matrix block [m],

$K^{n} \quad=$ hydraulic conductivity of the matrix block [m/s],

$S_{s}^{\prime} \quad=$ specific storage coefficient of the matrix block $[1 / \mathrm{m}]$,

$\varsigma \quad=$ skin resistance parameter (ratio of skin thickness to skin hydraulic conductivity, set to 0 in this analysis) [s],

$\chi \quad=$ spatial coordinate measured from center of stab block $[\mathrm{m}]$,

$B \quad=$ half-width of slab block [m]. 
The volumetric rate of fluid entering the fracture per unit volume of rock matrix is $\mathrm{V}_{\mathrm{n}} \sigma$, where

$$
V_{n}=-\left.K^{\prime} \frac{\partial h^{\prime}}{\partial \chi}\right|_{B}
$$

is the boundary condition of the slab block equation, $\mathrm{V}_{\mathrm{n}}$ is the Darcy velocity or specific flux at the interface, and $\sigma$ is the specific surface for a slab block $[1 / \mathrm{m}]$. The volumetric rate of fluid entering the fracture per unit bulk volume, $\Lambda$ (the coupling term), is therefore

$$
\Lambda=V_{n} \sigma\left(1-\phi_{f}\right)=\frac{V_{n}}{B} .
$$

Assumptions and Limitations of Mathematical Model. The assumptions and limitations of the model are as follows:

- Flow obeys Darcy's law.

- Hydraulic conductivities in the porous matrix block are small compared to hydraulic conductivities in the fractures.

- Temperature effects on properties are negligible; ambient groundwater temperature and heat from repository are not accounted for.

Numerical Approach. The finite-element approximation technique applied to the flow equations for the fracture domain uses the 3D Galerkin finite-element method (Huyakorn and Pinder, 1983; Huyakorn and Thomas, 1984; Huyakorn et al., 1986; Panday et al., 1993). The standard Galerkin approximation technique is used for the one-dimensional matrix flow.

The overall solution procedure is as follows. Matrix computations are performed for heads in both the fracture and porous matrix domains using heads from the previous time step as initial conditions for the current time step. Tridiagonal sets of algebraic equations for the porous matrix blocks are generated and decomposed using the Thomas algorithm to obtain the relationship between the fluid fiux from the porous matrix and the heads. Using an implicit treatment of the fluid flux terms contributed by the porous matrix, a set of algebraic equations for the fracture domain is generated and solved for nodal values of head in the fractures using direct banded or ORTHOMIN and Preconditioned Conjugant Gradient (PCG) solvers (Huyakorn et al., 1991). The nodal values are then used to evaluate the fluid fluxes from the porous matrix blocks. Knowing these fluid fluxes, the nodal head values in the porous matrix can be determined by performing the back substitution step of the Thomas algorithm.

Verification. For a previous analysis (1994 PA), STAFF3D was verified with analytical solutions for both saturated and unsaturated flow. Several of the analytical problems described in the STAFF3D manual have been run and the reported STAFF3D results were reproduced. Analytical flow problems verified include:

- Well fiow in a confined aquifer with parallel fractures (Neuman and Witherspoon, 1969).

- Radial well flow in a confined aquifer with parallel fractures (Bear, 1979).

- Flow to parallel drains in an unconfined aquifer (Bear, 1979).

- Two-dimensional flow in a rectangular soil slab (comparison with VAM3DCG results).

Also, a benchmarking exercise was done for the 1997 PA to ensure that the 1994 PA results could be reproduced using the same grid, boundary conditions, and input parameters.

\subsubsection{Applied Model}

Model Geometry and Spatial Discretization. The saturated zone underlying the potential repository site was discretized into a local three-dimensional grid oriented to the north and east to correlate with the Nevada State Plane coordinates. The grid extends $8,000 \mathrm{~m}$ in the $\mathrm{x}$ direction (east), and $13,000 \mathrm{~m}$ in the $\mathrm{y}$ (north) direction to cover an 


\section{Consequence Modeling: Saturated Zone Flow and Transport}

area of $1.04 \times 10^{8} \mathrm{~m}^{2}$ (Figure 9-1). This grid was chosen to incorporate pressure head data from 28 wells drilled in the Yucca Mountain region, which were used for model calibration. A "pancake" layering in the $z$ direction was developed to include properties of the hydrologic units described in Chapter 4. Orthogonal grid-block dimensions varied from $46 \mathrm{~m} \times 120 \mathrm{~m} \times 16.5 \mathrm{~m}$ at the water table beneath the repository region, to $650 \mathrm{~m} \times 1164.9 \mathrm{~m} \times 210 \mathrm{~m}$ in the TRw unit near the upper right corner of the domain. Additionally, four vertical permeability barriers were included to act as transmissibility barriers for the flow calibration. In all, the grid consists of $30 \times 37 \times 22$ grid lines, or 24,420 nodes and 21,924 elements.

Geologic Layer Description and Material Properties. The objective of the aquifer modeling is to approximate flow and transport behavior in the saturated zone within tuffaceous rock beneath the potential waste disposal repository at Yucca Mountain. This conceptual model is an approximation of the Yucca Mountain aquifer system, with enough features to adequately describe local flow and transport, and thus radionuclide releases for this performance assessment. The main geologic features incorporated in the model include tilted stratigraphy, vertical barrier regions, and an unconfined aquifer system.

Tilted Stratigraphy. The west-to-east dip in the local stratigraphy was achieved by taking a "best fit" plane of the vertical coordinates (mean sea level) at the top of the TSwc unit at wells G-1, WT-2, B-1, and H-5. These wells are located just north, south, east, and on the western edge of the repository location. The resulting plane has a $4.6^{\circ}$ dip west-to-east, and a slight $0.1^{\circ}$ dip north-to-south. The remaining geological units were "hung" with a constant thickness from the TSwc unit. The material properties used in the dual porosity flow analysis are summarized in Chapter 4, Tables 4-24 through 4-36. The vertical fracture hydraulic conductivity was reduced by a factor of ten to achieve better calibration with the measured well data, except for the TSlv and TSwc layers where the vertical hydraulic conductivity was increased by a factor of 10 . The unit thicknesses are the same as those shown in Chapter 4 (Figure 4-11), with the TRw layer having a thickness of $210 \mathrm{~m}$. Because this model was used in saturated zone transport, only the layers that come in contact with the aquifer are modeled. Thus, units TCW and PTn were excluded.

Vertical Fault Regions and Variable Water Table. Examination of the well data revealed several regions of anomalous water table levels (Table 9-2). In the area around the repository site, the water table stays constant at about 730 $\mathrm{m}$, with a slight decrease from west to east. North of the repository area, however, wells G-2 and WT-6 show a water table level of $1029 \mathrm{~m}$ and $1035 \mathrm{~m}$. Wells drilled to the west of the repository area (WT-7, WT-10, and H-6) show a piezometric head of $776 \mathrm{~m}$, and well WT-16 recorded the water table at $738 \mathrm{~m}$. Numerous theories have been presented in the Yucca Mountain literature to explain this water table behavior (a complete list of references are presented in TSPA-1993 [Wilson et al., 1994]). For this study, a simplified approach using permeability barriers was used to obtain a reasonable fit of the well data. A partially sealing barrier was inserted between areas of significantly differing piezometric heads. The barrier locations correspond roughly to surface outcrops of some of the major faults in the Yucca Mountain area, namely, Solitario Canyon to the west, Paintbrush to the east, Bow Ridge between them as north-south trending faults, and Drill Hole Wash, Sever Wash, Pagany, and Yucca Wash faults trending northwestsoutheast. The Ghost Dance fault was not included in this conceptual model, because the well data indicates it to be nonsealing. These zones are used as permeability barriers to flow only, and as such have no dip or strike offset. The water table was allowed to fluctuate as governed by the percolation model summarized in Chapter 6 , and imposed on the flow field as a uniform Neumann boundary conditions along the top plane of nodes. The "focus factor" used in the unsaturated zone model to simulate an enhanced flow region above the repository was not included in this saturated zone model.

Flow Properties. The flow parameters used in the flow runs are presented in the parameter tables located in Chapter 4, Section 4.5. For the STAFF3D flow runs, three parameters related to climatic variations-PPTINFMN, PPTINFMX, and CYCLEPER-were sampled (Table 4-23). For the remaining flow parameters, the median parameter values were used except for bulk gas hydraulic conductivity, BLKKGS; fracture spacing, FRACSPAC; and matrix hydraulic conductivity, HYCNDM_X, which used the mean values. In most cases, the mean and median flow parameter values are the same. The nonsampled flow parameter values are presented in Chapter 4, Tables 4-24 to 4-36. The sampling and subsequent flow modeling with these parameters provided a unique flow field for each of the 50 vectors simulated.

Note that the saturated zone model used the TSwc unit in place of the six units (TSv, TSun, TSul, TSmn, TSll, TSin) that were modeled for the unsaturated zone. See also Chapter 4. 


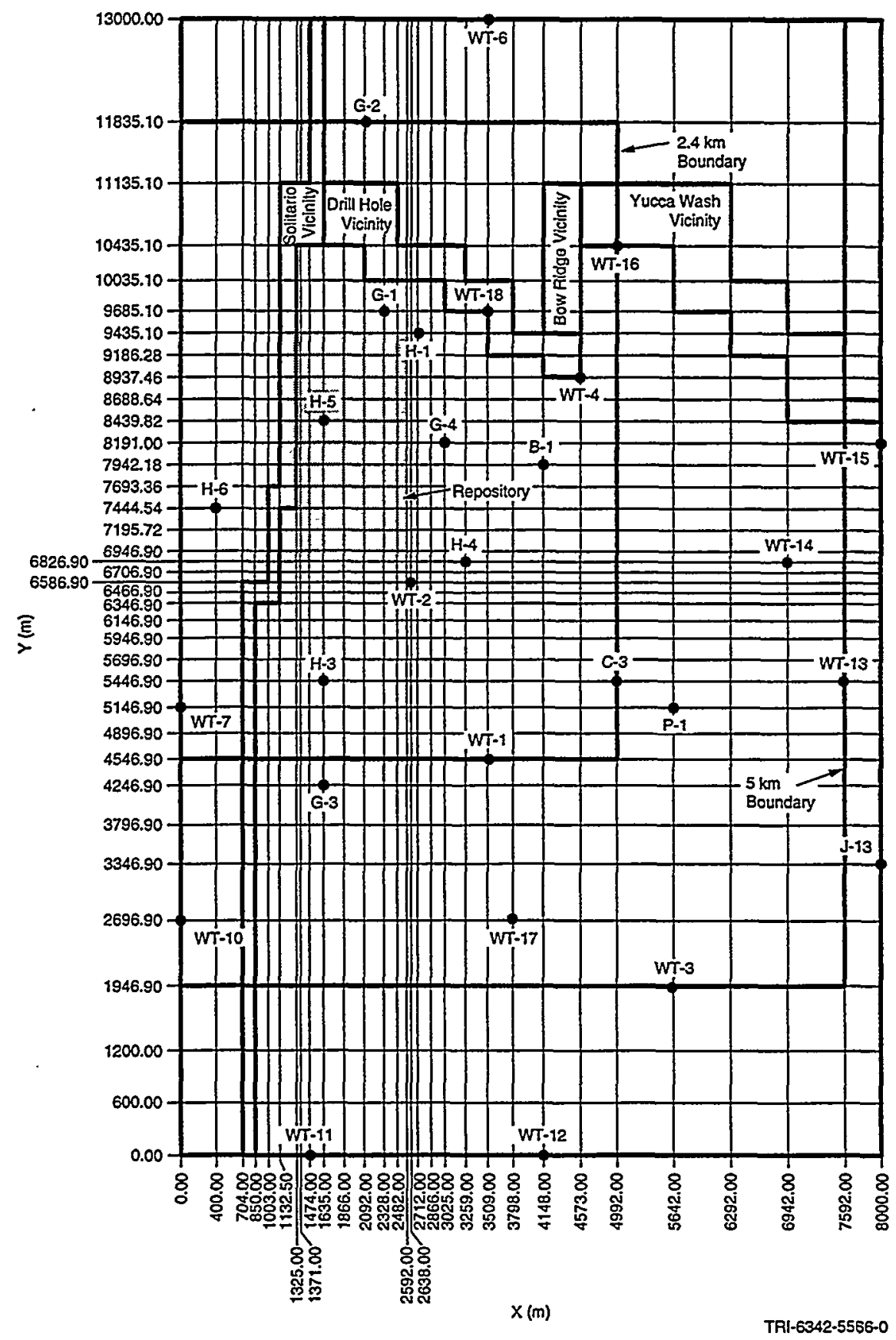

Figure 9-1. X-Y "surface" view of STAFF3D grid used for aqueous flow and transport 
9. Consequence Modeling: Saturated Zone Flow and Transport

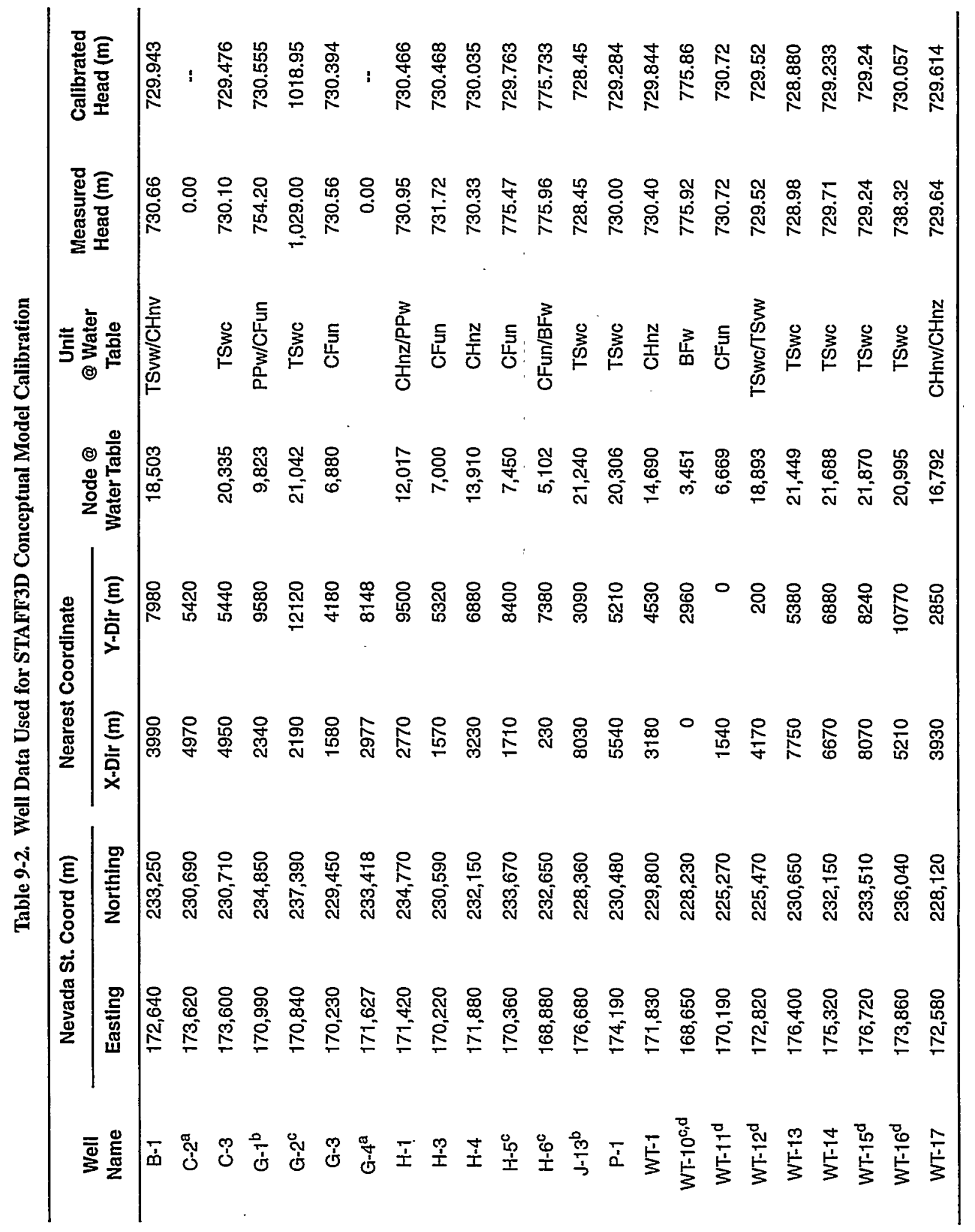




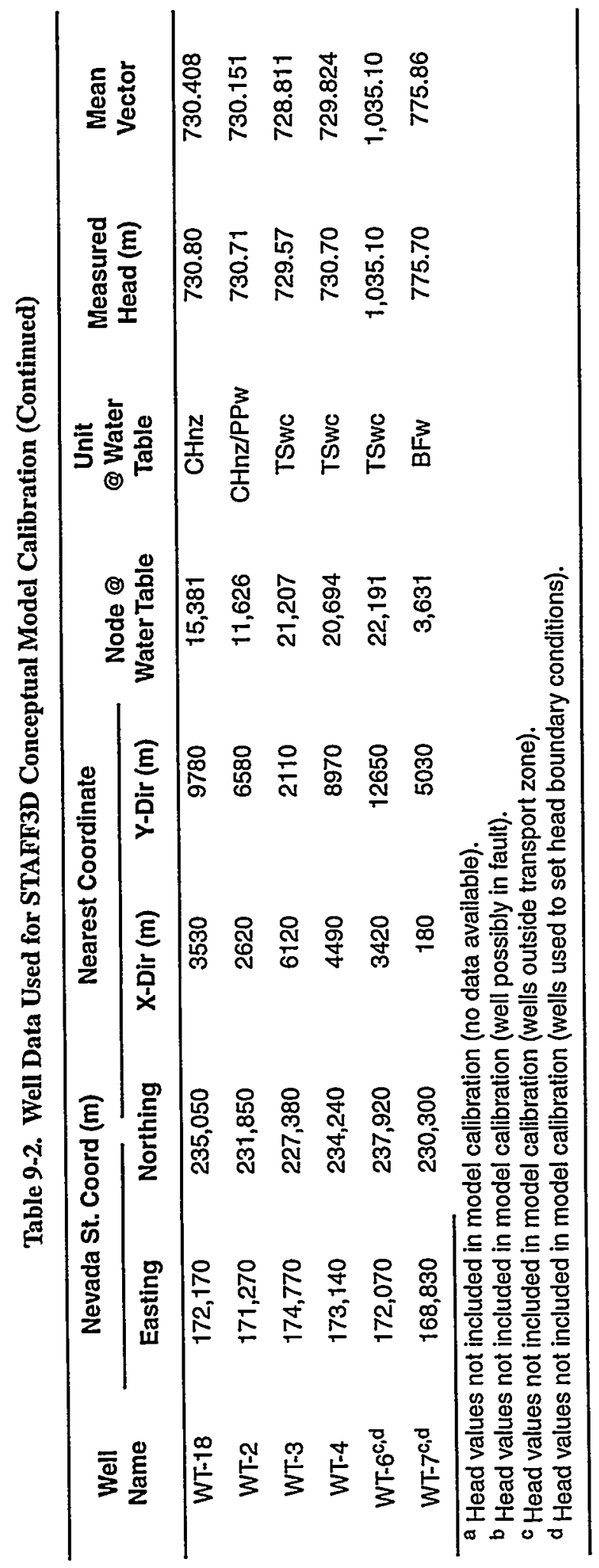


An average recharge rate over the 100,000-yr simulation period was generated from the sampled transport parameters, PPTINFMIN, PPTINFMX, and CYCLEPER. The average recharge rate was related to the climate parameters by:

$$
I=\left(I_{\max }+I_{\min }\right) / 2+\left[\left(I_{\max }-I_{\min }\right) T \sin \left(2 \pi T_{\text {end }} / T\right)\right] / 2 \pi T_{\text {end }}
$$

where $I$ is the average infiltration rate, $I_{\min }$ is the minimum infiltration rate (PPTINFMN) $[\mathrm{m} / \mathrm{s}], I_{\max }$ is the maximum infiltration rate (PPTINFMX) $[\mathrm{m} / \mathrm{s}], T$ is the cycle period (CYCLEPER) [yr], and $T_{\text {end }}$ is the simulation time $(100,000 \mathrm{yr})$. The average infiltration rate has an impact on the velocity field.

For the flow calculations, STAFF3D uses the hydraulic conductivity of the fractures instead of a bulk hydraulic conductivity. The bulk gas hydraulic conductivity of the rock is presented in Tables 4-26 through 4-32 (Chapter 4) for the units CHnv, CHnz, PPw, CFun, BFw, CFmn, and TRw. The cubic law for fracture transmissivity (Freeze and Cherry, 1979) is used to relate the bulk hydraulic conductivity to the horizontal hydraulic conductivity of the fractures through the relationships:

$$
b=\left[12 \mu f_{s}\left(K_{b}-K_{m}\right) /(\rho g)\right]^{1 / 3}
$$

and

where

$$
K_{f}=b^{2} \rho g l(12 \mu)
$$

$K_{b}, K_{f}, K_{m}=$ bulk gas hydraulic conductivity $\left(10^{\mathrm{BLKKGS}}\right)$, fracture horizontal hydraulic conductivity, and matrix hydraulic conductivity $\left(10^{\mathrm{HYDCND}} \mathrm{X}\right)$, respectively $[\mathrm{m} / \mathrm{s}]$

$f_{s} \quad=$ distance between fractures (FRACSPAC) $[\mathrm{m}]$

$g \quad=$ gravitational constant $\left[\mathrm{m} / \mathrm{s}^{2}\right]$

$\rho=$ density of water $\left[\mathrm{kg} / \mathrm{m}^{3}\right]$

$\mu \quad=$ dynamic viscosity of water $[\mathrm{Pa} \cdot \mathrm{s}]$

$b=$ fracture aperture $[\mathrm{m}]$.

In Equation 9.1-13, the matrix hydraulic conductivity must be subtracted from the bulk hydraulic conductivity, because the gas hydraulic conductivity is determined from a combination of both fracture and matrix flow. The fracture porosity, $\phi_{f}$ can then be calculated from

$$
\phi_{f}=b / f_{s}
$$

The vertical fracture hydraulic conductivity was set to one-tenth the horizontal fracture for all layered units except the TSlv ${ }^{\dagger}$ and TSwc units. In these two units, the vertical fracture hydraulic conductivity was set to ten times the horizontal fracture hydraulic conductivity.

Flow Model Calibration: Initial and Boundary Conditions. An iterative process was used to calibrate model results to measured well data. Well locations are shown in Figure 9-1. In the first step of this process, fixed head (Dirichlet) boundary conditions were derived by linearly interpolating Dirichlet conditions for the north, east, and south grid boundaries using measured total head values from wells located on the grid boundaries (WT-6, WT-11, WT-12, WT-15, and J-13). An estimated total head value was selected for the northeast corner of $800 \mathrm{~m}$ based on an interpretation of the water table contour map for Yucca Mountain presented in Fridrich et al. (1994). The west grid boundary remained at a constant head of $775.86 \mathrm{~m}$, which is the average head of wells H-6, WT-7, and WT-10. In the second step, values for fracture hydraulic conductivities in the fault regions were assumed based on data from Rechard, ed. (1995) and Wilson et al. (1994). The model was then run under dual porosity, steady-state conditions using the flow properties of the various non-fault hydrologic units as described above (see Tables 4-24 to 4-32) and a constant recharge rate of $0.5 \mathrm{~mm} / \mathrm{yr}$. For these calibration runs, an initial condition of $730.5 \mathrm{~m}$ was assumed for all interior nodes in the mesh. The resulting values for head were compared to measured well data; the head contour map

In the 1997 PA database, the TSlv unit is labeled as TSw3. 
based on well data is shown in Chapter 4, Figure 4-13. If the match was poor, new values for fault hydraulic conductivities were assumed, and the second part of the iterative procedure was repeated. A reasonable match of calculated head to measured well data was achieved (see Table 9-2) and a fairly good match to the hydraulic head gradient was obtained (see Figure 9-2 for hydraulic heads in several units).

The values for wells located on the model grid boundaries (J-13, WT-6, WT-11, WT-12, and WT-15) match exactly because heads in these wells were used to set the Dirichlet boundary conditions. The agreement between the measured and calculated data agree to within $0.75 \mathrm{~m}$ for most wells south and east of the repository location, where the majority of radionuclide transport occurs. However, a difference of $45 \mathrm{~m}$ exists for well $\mathrm{H}-5$ along the west edge of the repository location. The total head measured in $\mathrm{H}-5$ is more similar to the head measurements west of the Solitario Canyon Fault than those east of the fault zone; H-5 may actually be completed west of the fault zone. Other models have incorporated additional geologic structures in an attempt to reconcile large differences in heads measured in wells H-5 and G-4 (Altman et al., 1996). A difference of several meters also exists between the measured and calculated heads for well G-2, located north of the repository in the zone of a large hydraulic gradient, and for wells G-1 and WT-16, both of which are north of the repository near the fault zones. Because these well locations are outside the area in which transport occurs, the fact that the heads did not match very well was not considered important.

The calibrated heads for all well locations are less than the measured values (Table 9-2). The reason for this is unclear, but could be related to the hydraulic conductivities assigned to the fault zones. Increasing hydraulic conductivities in the fault zones seemed to increase the calibrated heads so that they matched the observed values better. However, this distorted the head field in a manner that was not consistent with the measured head field shown in Chapter 4, Figure 4-13. In general, increasing the fault zone hydraulic conductivities caused steeper gradients in the area of faults and caused the head field to turn to the east along the northern fault zones so that flow was more southerly than observed in Figure 4-13. Decreasing the hydraulic conductivities in the fault zones causes a northerly flow near the intersection of the Solitario Canyon Fault zone and the southern model boundary, which was also inconsistent with Figure 4-13. The final assigned fault zone hydraulic conductivities were approximately four orders of magnitude larger than those used in Rechard, ed. (1995). However, when bulk fracture hydraulic conductivities are considered, the bulk fault zone hydraulic conductivities are still four to five orders of magnitude smaller than those of the layered units and must still be considered as sealing.

The calibrated hydraulic heads for the BFw, CFun, PPw, and CHnz units are shown on Figure 9-2. These units all intersect the water table at some point near the repository and so were used for comparison with the water table map (Chapter 4, Figure 4-13). Because the units dip to the east at a steeper gradient than the water table, none of the heads in these units serves as a water table proxy. However, they can be used for general comparison with the water table map (Figure 4-13). The head fields are fairly similar for the four units and show a hydraulic gradient similar to that observed on Figure 4-13. The hydraulic gradient match is important because transport velocities are determined from the hydraulic gradients rather than from the heads. An interesting observation is that there is a slight upward flow occurring in the transport zone. This would seem to indicate that, at least in the lower units, the flow through the fault zones has a stronger influence on the calibrated heads than the natural recharge.

The most important part of the flow field is the portion south, east, and downgradient from the potential repository location. If isotropic hydraulic conductivities are assumed, the apparent direction of calibrated groundwater flow is east-southeast from the repository (Figure 9-2), which is consistent with the current understanding of groundwater flow away from the repository (see Chapter 4, Figure 4-13). The wells that are not in good agreement with the groundwater flow calibration (G-1, G-2, H-5, and WT-16) are located north and west of the repository (Figure 9-1) and, therefore, do not have a large impact on flow and transport from the repository.

Because the main objective of this model is to evaluate flow and transport in the saturated zone, a simple BrooksCorey model was used ( $n=1$, or $K_{r w}=S_{\text {weff }}$ ) for the STAFF3D unsaturated zone relative permeability flow behavior in the fractures. STAFF3D neglects unsaturated matrix flow in its dual-porosity formulation, hence the capillary pressure behavior in the fractures was set equal to that in the matrix by using the same van Genuchten parameters for capillary pressure. Doing so forced flow equilibrium between the fractures and matrix so that velocity fields could be obtained that were able to be compared with the unsaturated zone model. 


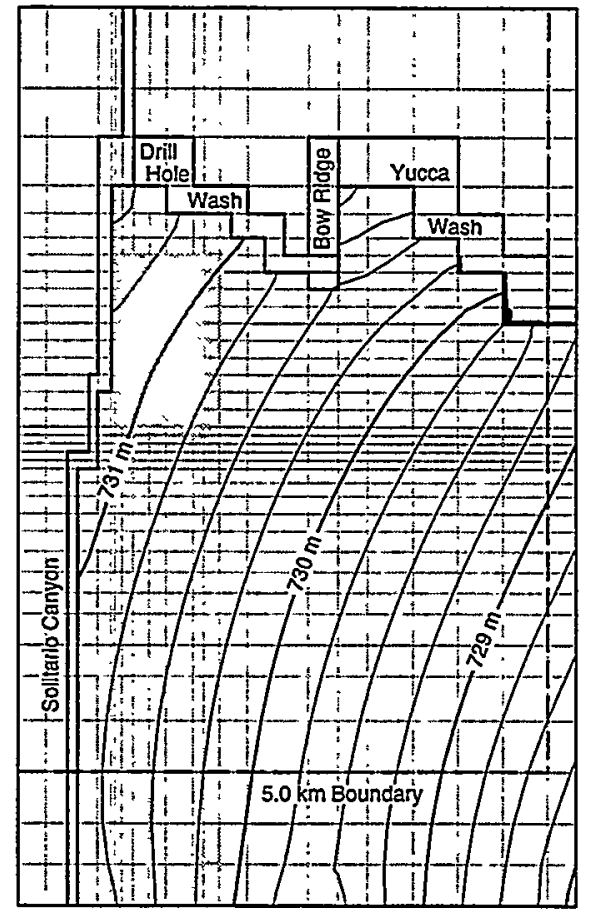

(a) BFW

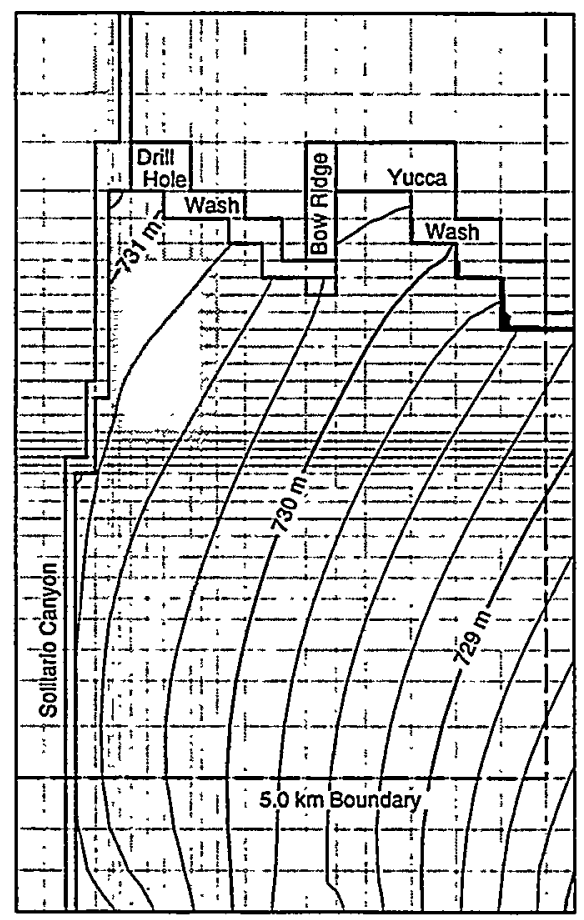

(c) PPw

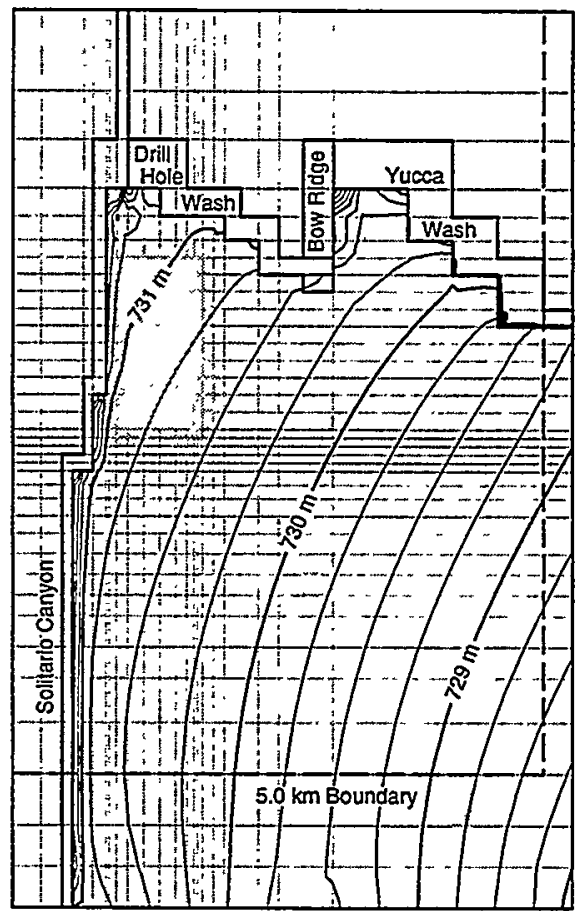

(b) CFun

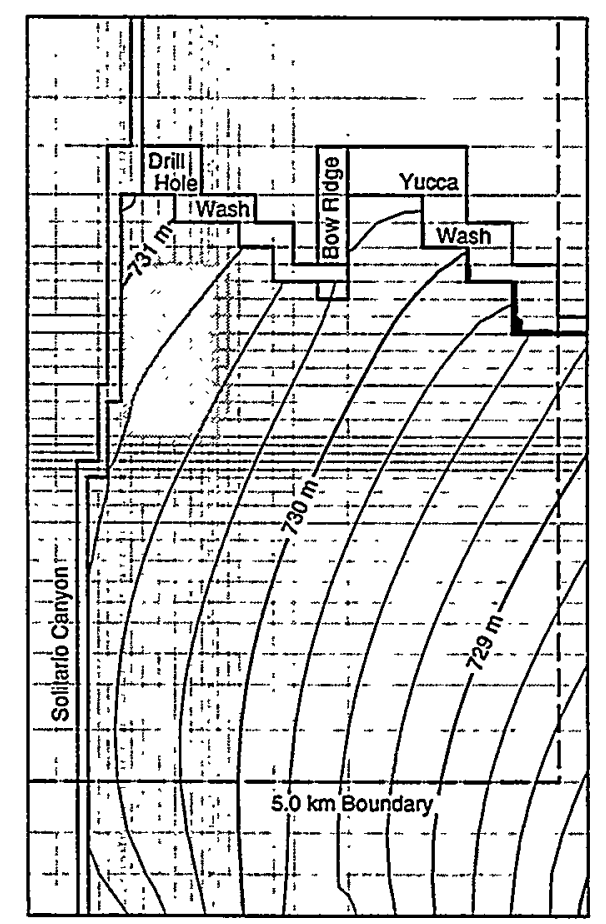

(d) $\mathrm{CHinz}$

TRl-6342-5642-0

Figure 9-2. Calculated hydraulic head field for units intersecting water table near repository. (a) $B F w,(b) C F u n$, (c) $P P$ w, and (d) CHnz. 


\subsection{STAFF3D Transport Model}

\subsubsection{Mathematical Model Description}

Mass Balance Equation for Dual Porosity Transport.

Fracture Domain. The unsaturated, solute transport mass balance equation assuming constant fluid density, variable porosity and saturation, linear equilibrium sorption, and a fluid and solute source used to model transport in the fractures per unit fracture volume is as follows (see Figure 9-3):

$$
\begin{aligned}
& \nabla \cdot\left(D_{l J}\right) \cdot \nabla c_{l}-V \cdot \nabla c_{l} \\
& =S_{w} R_{l} \frac{\partial}{\partial t}\left(c_{l}\right)+\lambda_{l} \phi S_{w} R_{l} c_{l}-\lambda_{p} \phi S_{w} R_{p} c_{p}-\frac{q^{\prime}}{\phi_{f}}\left(c_{l}^{*}-c_{l}\right)-\Gamma_{l}
\end{aligned}
$$

where (SI units are shown),

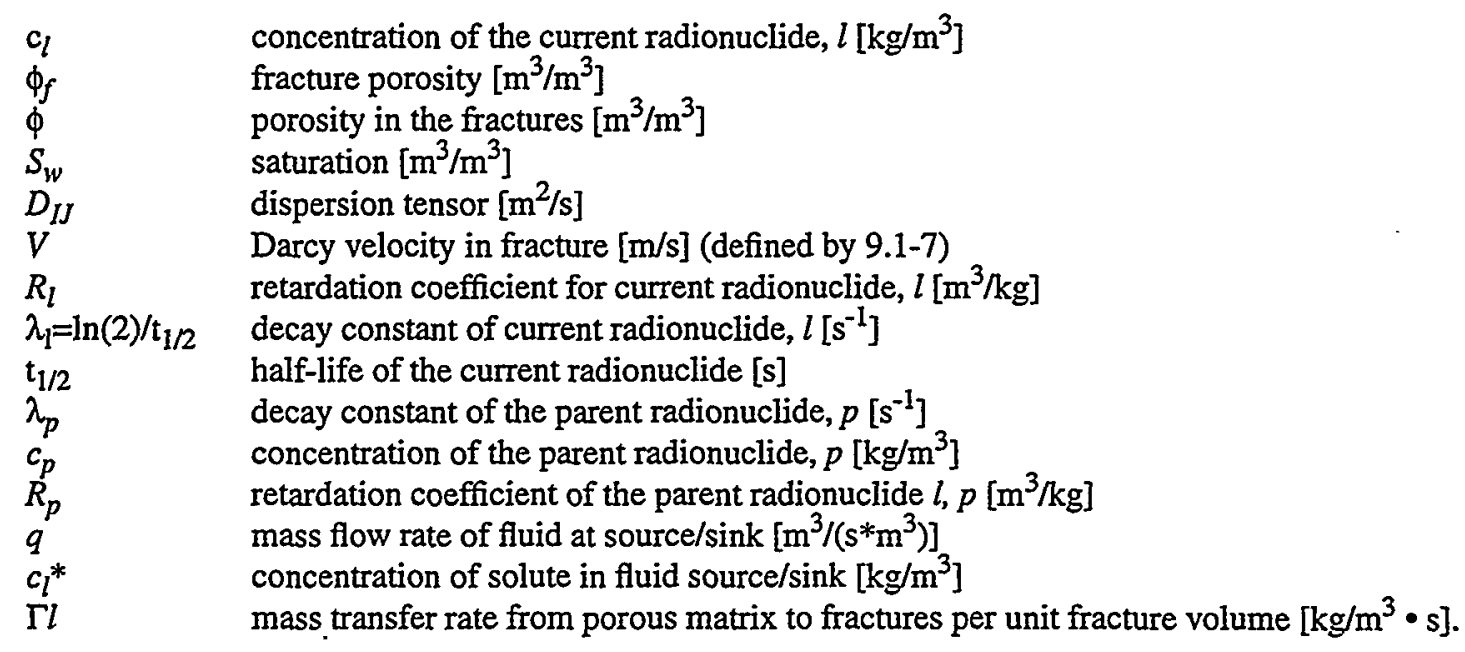

The elements of the dispersion tensor, $D_{\mathrm{IJ}}$, are defined as (Scheidegger, 1960),

$$
\begin{aligned}
& D_{11}=\frac{\alpha_{L} V_{1}^{2}+\alpha_{T} V_{2}^{2}}{|V|}+D_{1}^{*},\left[\mathrm{~m}^{2} / \mathrm{s}\right] \\
& D_{12}=\left(\alpha_{L}-\alpha_{T}\right) \frac{V_{1} V_{2}}{|V|},\left[\mathrm{m}^{2} / \mathrm{s}\right] \\
& D_{22}=\frac{\alpha_{L} V_{2}^{2}+\alpha_{T} V_{1}^{2}}{|V|}+D_{2}^{*},\left[\mathrm{~m}^{2} / \mathrm{s}\right]
\end{aligned}
$$

where $\alpha_{\mathrm{L}}$ and $\alpha_{\mathrm{T}}[\mathrm{m}]$ are the longitudinal and transverse dispersivities, and $D_{1}{ }^{*}$ and $D_{2}{ }^{*}\left[\mathrm{~m}^{2} / \mathrm{s}\right]$ are the effective coefficients of molecular diffusion, including tortuosity effects $\left(D_{1}^{\circ} \tau\right)$ where $D_{1}^{\circ}\left[\mathrm{m}^{2} / \mathrm{s}\right]$ is the free water molecular diffusion of species $l$, and $\tau$ [dimensionless] is the tortuosity defined as [L/Le] ${ }^{2}$, where $L[\mathrm{~m}]$ designates straight line length and $\mathrm{Le}[\mathrm{m}]$ designates actual path length for fluid.

The decay constant is 
9. Consequence Modeling: Saturated Zone Flow and Transport

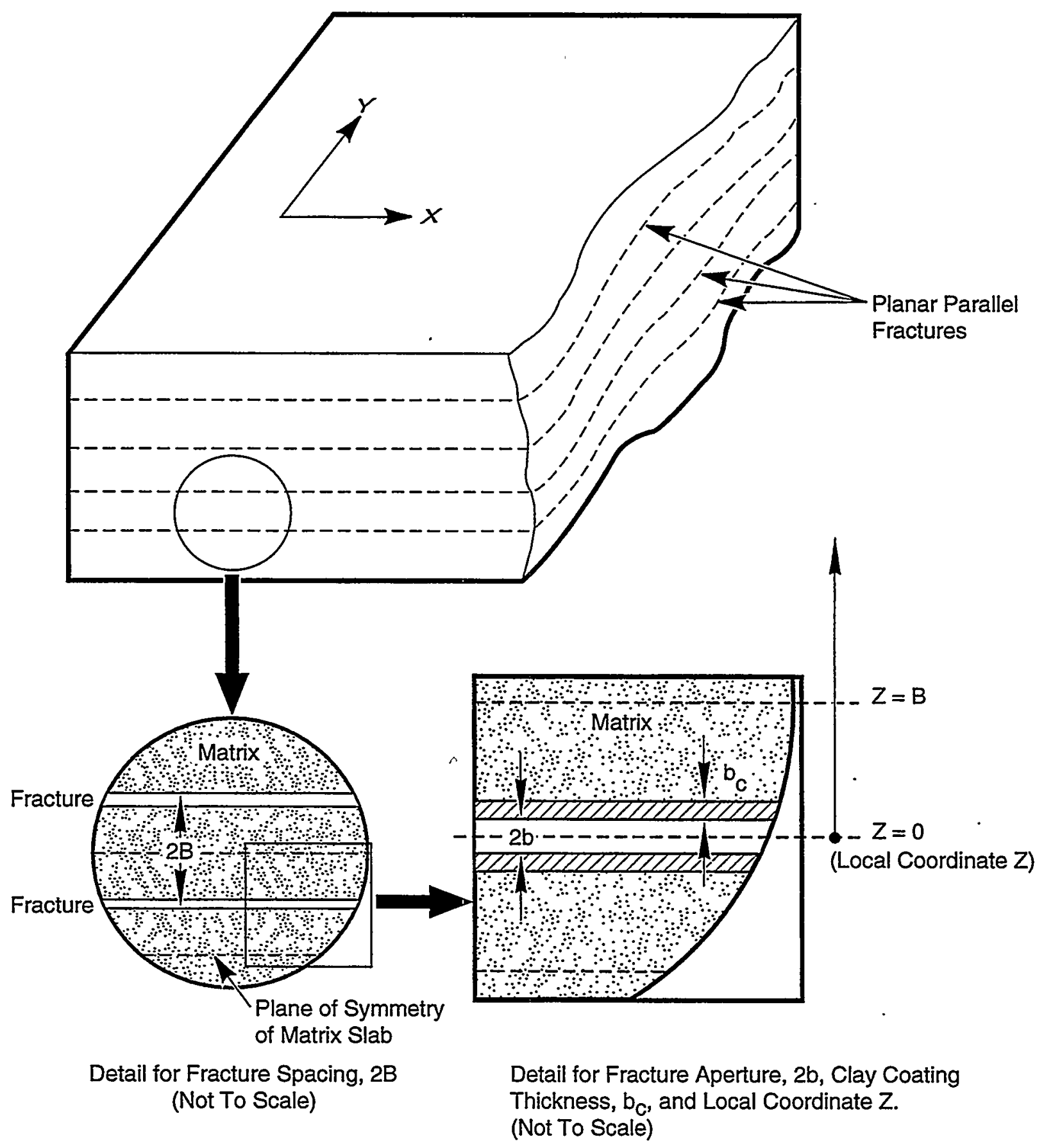

TRI- 6342-1436-0

Figure 9-3. Conceptual hydrologic model of the aquifer. 


$$
\lambda=\frac{\ln (2)}{t_{1 / 2}},\left[s^{-1}\right]
$$

where $\mathrm{t}^{1 / 2}$ is the half-life of radionuclide $l[\mathrm{~s}]$.

Matrix Domain. The one-dimensional transport equation for slab geometry and no flow conditions in the porous matrix is as follows:

$$
\frac{\partial}{\partial \chi}\left(D^{\prime} \frac{\partial c_{l}^{\prime}}{\partial \chi}\right)=\phi^{\prime} R_{l}^{\prime} \frac{\partial}{\partial t}\left(c_{l}^{\prime}\right)+\lambda_{l} \phi^{\prime} R_{l}^{\prime} c_{l}^{\prime}-\lambda_{p} \phi^{\prime} R_{p}^{\prime} c_{p}^{\prime}
$$

subject to,

$$
\begin{gathered}
c_{l}^{\prime}(B, t)=c_{l}(x, y, z, t) \\
\frac{D^{\prime} \partial c_{l}^{\prime}(o, t)}{\partial \chi}=0
\end{gathered}
$$

where,

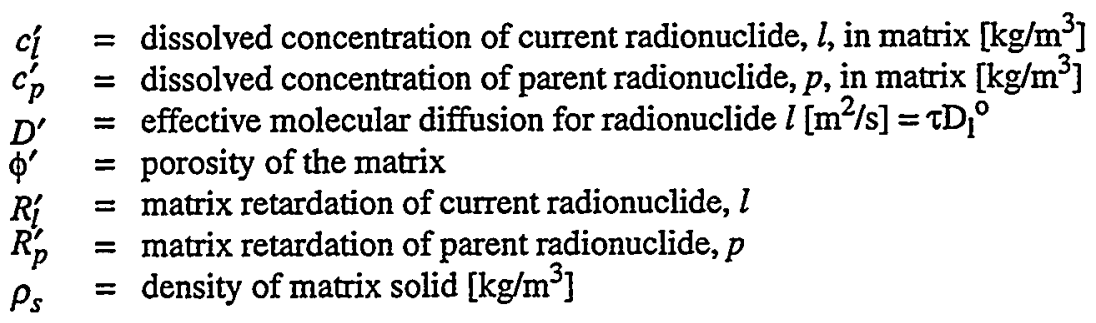

The dual porosity transport Equations 9.2-1 and 9.2-4 are coupled by equating the flux from fracture, $\Gamma_{1}$, with diffusive flux at the matrix/fracture interface as follows:

$$
\begin{gathered}
\Gamma_{l}=-\left(\frac{D^{\prime} \partial c_{l}^{\prime}(x, y, B, t)}{\partial \chi}\right)\left(1-\phi_{f}\right) \sigma \\
\Gamma_{l}=\frac{1}{b+B}\left(\left.D^{\prime} \frac{\partial c_{l}^{\prime}}{\partial \chi}\right|_{\chi=B}\right)
\end{gathered}
$$

The second $\left(1-\phi_{f}\right)$ and third $(\sigma)$ terms on the right-hand side convert the areal flux at the matrix surface to the fracture/matrix control volume of the system.

Assumptions and Limitations of Mathematical Model. The assumptions and limitations of the model are as follows:

- Dispersive transport is governed by Fick's law.

- Solute effects on fluid density are assumed negligible so flow and transport can be decoupled.

- The dispersivity is assumed to correspond to an isotropic porous medium so that it can be represented by two constants in the principle direction of flow.

- Adsorption obeys a linear equilibrium isotherm.

- There is local chemical equilibrium between the liquid and the solid.

- The fracture aperture is small compared to other dimensions in the fracture plane. 
Numerical Approach. The finite-element approximation technique applied to the transport equations for the fracture domain is an adaptive upstream-weighted residual technique (Huyakorn and Nilkuha, 1979; Huyakorn and Pinder, 1983; Huyakorn et al., 1987; Panday et al., 1993) designed to overcome oscillations of the numerical solutions when advective terms are dominant. The standard Galerkin approximation technique is used for the one-dimensional matrix transport because it does not contain any advective term.

The overall solution procedure is as follows. Matrix computations are performed for concentrations in both the fracture and porous matrix domains using concentrations from the previous time step as initial conditions for the current time step. Tridiagonal sets of algebraic equations for the porous matrix blocks are generated and decomposed using the Thomas algorithm to obtain the relationship between the solute flux from the porous matrix and the concentrations in the fractures. Using an implicit treatment of the flux terms contributed by the porous matrix, a set of algebraic equations for the fracture domain is generated and solved for nodal values of concentration in the fractures using direct banded or ORTHOMIN and Preconditioned Conjugant Gradient (PCG) solvers (Huyakorn et al., 1991). The nodal values are then used to evaluate the concentration fluxes from the porous matrix blocks. Knowing these concentration fluxes, the nodal concentration values in the porous matrix can be determined by performing the back substitution step of the Thomas algorithm.

Verification. For a previous analysis (1994 PA), STAFF3D was verified with analytical solutions for both saturated and unsaturated transport. Several of the analytical problems described in the STAFF3D manual have been run and the reported STAFF3D results were reproduced. Analytical transport problems verified include:

- Longitudinal transport in fractures and transverse matrix diffusion (Tang et al., 1981).

- One-dimensional transport of a three member radioactive decay chain (Coats and Smith, 1964; Lester et al., 1975).

- Two-dimensional transport in a soil slab (comparison with VAM3DCG results).

\subsubsection{Applied Model}

Transport Properties. The radionuclide inventory and transport parameters used in the transport runs are presented in the parameter tables located in Chapters 3 and 4, Sections 3.6 and 4.5, respectively. For the STAFF3D transport runs, two parameters-dispersivity fraction, DISPPERC, and the porous matrix partitioning coefficient for neptunium in zeolitic tuffs (CFmn, CFun, and CHnz), $\mathrm{KDZ}$-were sampled. The median values for the remaining transport parameters presented in Tables 4-24 to 4-36 were chosen for the transport runs. In most cases, these values are the same as the mean values.

Because the sampled parameters are not used directly by STAFF3D, they were converted into derived parameters. For the transport simulations, the neptunium partitioning coefficient for the zeolitic units was converted into a retardation coefficient through the relationship:

$$
R=1 \div\left(\rho_{\text {bulk }} K_{d}\right) /\left(\phi S_{w}\right)
$$

where

$$
\begin{array}{ll}
\mathrm{R} & =\text { retardation coefficient } \\
\rho_{\text {bulk }} & =\text { bulk density of the porous matrix }\left[\mathrm{kg} / \mathrm{m}^{3}\right] \\
\mathrm{K}_{\mathrm{d}} & =\text { porous matrix partitioning coefficient }\left[\mathrm{m}^{3} / \mathrm{kg}\right] \\
\phi & =\text { porosity of the porous matrix } \\
\mathrm{S}_{\mathrm{w}} \quad=\text { degree of saturation in the porous matrix. }
\end{array}
$$

Longitudinal dispersivity for each of the 50 vectors and the mean and median runs was determined by multiplying a uniformly sampled dispersivity fraction, DISPPERC, times the model's transport distance. This distance was $2400 \mathrm{~m}$ for the STAFF3D models, which is the distance from the repository edge to the 2.4-km boundary. The dis- 
persion fraction was varied from 0.05 to 0.20 ; therefore, longitudinal dispersivity for the STAFF3D transport model ranged from 120 to $480 \mathrm{~m}$. The transverse dispersivity was set to one-tenth of the longitudinal dispersivity.

The fracture porosities are the same as those used in the flow runs. The porous matrix porosities are presented in Chapter 4, Tables 4-24 to 4-36.

The material regions were subdivided into three main rock types for radionuclide partitioning: devitrified (D) for the TRw, BFw, PPw, and TSwc layers and all fault zones; vitrified (V) for the CHnv and TSlv layers; and zeolitic (Z) for the CFmn, CFun, and CHnz layers. For this analysis, only the matrix adsorption for ${ }^{237} \mathrm{~Np}$ in the zeolitic rocks was considered. ${ }^{237} \mathrm{~Np}$ was not adsorbed in the fault zones or in the vitrified and devitrified layers. ${ }^{129} \mathrm{I}$ and ${ }^{99} \mathrm{Tc}$ were not retarded in the matrix $\left(\mathrm{K}_{\mathrm{d}}=0\right)$. Retardations for transport in the fractures were set to $1.0\left(\mathrm{~K}_{d}=0\right)$ for all three isotopes and rock types. The sample range for the devitrified partitioning coefficient for ${ }^{237} \mathrm{~Np}$ is presented in Table 437. Chapter 4, and was taken from TSPA-1993.

Initial and Boundary Conditions, Coupling to NUTS Output. All initial concentrations (time zero) were set to zero. The BRAGFLO_TAUTS mesh used for unsaturated zone flow and transport is a two-dimensional, axisymmetric, fiared finite-difference model, which is completely different from the three-dimensional finite-element mesh used in STAFF3D.

A zero concentration gradient boundary condition was assumed at all external vertical grid boundaries, which allows advection at the boundaries, but not dispersion. At the top and bottom of the grid, a zero transport condition was applied. In addition, at all vertical boundaries, solute was allowed to fiow out of, but not into, the grid.

The NUTS unsaturated zone transport results were used as a time-dependent flux source to the STAFF3D model. In order to couple the mass fluxes from NUTS to the STAFF3D grid, the NUTS grid was overlain on the STAFF3D grid so that the centers of the repository in both grids coincided. The repository elements from the NUTS grid were then expanded horizontally to cover the repository nodes in the STAFF3D grid. Outside the repository area, the NUTS grid was expanded in an elliptical fashion. The first ellipse was designed so that it touched the four corners of the repository. Three subsequent ellipses were expanded in a manner that depended on the dimensions of the repository, approximately $1221 \mathrm{~m}$ in the east/west direction and $2488.2 \mathrm{~m}$ in the north/south direction. These ellipses were designed so that they were equidistant from each other. The total east/west range of the repository area and the four ellipses covered the distance across the NUTS grid, approximately $4617 \mathrm{~m}$. Mass fiuxes in the NUTS elements just above the water table were used to calculate the mass fluxes into the grid points just below the STAFF3D water table. The mass flux into each STAFF3D repository node was calculated by summing the mass fluxes from the NUTS elements under the repository and dividing by the number of STAFF3D repository nodes at the water table. The mass fluxes into each ellipse were calculated in a similar fashion. For example, the NUTS elements just above the water table for the first ellipse were determined and the mass fluxes from these elements were summed to get an ellipse total. The number of STAFF3D nodes under the first ellipse was counted and the mass flux into each node was calculated by dividing the total mass flux from the first ellipse by the number of STAFF3D nodes in the first ellipse. This process was repeated for each time step written by NUTS. As a general rule, as distance from the repository edge increased, the mass fluxes at the water table in the NUTS grid decreased. Thus, the mass fluxes produced by NUTS were redistributed as a time- and space-dependent flux source term over a portion of STAFF3D that was somewhat larger than the repository area. STAFF3D creates a lookup table of time-dependent mass fluxes for each radioisotope to interpolate for every STAFF3D time step throughout the transport calculation.

It should be noted that the elements chosen to represent the BRAGFLO_T water table for STAFF3D coupling were approximately $100 \mathrm{~m}$ above the physical water table (100\% water-saturated elements). This location was selected to avoid the slight numerical instabilities encountered at the water table due to capillary pressure and phase effects (see Section 8.1), as well as to account for any possible rise in the water table.

Dual-Porosity Transport Behavior. For the dual porosity assumption, STAFF3D calculates convective/dispersive transport in the fractures with diffusion into the matrix. The Darcy velocity components needed for the convective transport in the fracture are read in from the STAFF3D flow solution for each of the 50 vectors and the mean and median runs. Three radioisotopes were transported by STAFF3D: ${ }^{129} \mathrm{I},{ }^{237} \mathrm{~Np}$, and ${ }^{99} \mathrm{Tc}$. 
The maximum concentrations of the radioisotopes transported by STAFF3D and their location of maximum occurrence on the 5-km boundary for each vector (except for runs 23,32, and 44 where BRAGFLO-T did not run completely) for the mean and median runs are presented in Tables $9-3,9-4$, and $9-5$ for ${ }^{99} \mathrm{Tc}$, ${ }^{129} \mathrm{I}$, and ${ }^{237} \mathrm{~Np}$, respectively. (Note Tables 9-3 through 9-8 and Figures 9-5 through 9-7 are all placed at the end of this section.) Except for the three outliers produced by runs 8,9, and 31, the maximum concentrations for ${ }^{99} \mathrm{Tc}$ that appear on Table 9-3 are concentrated in the range from about $6.7 \times 10^{-7} \mathrm{~kg} / \mathrm{m}^{3}$ to $1.0 \times 10^{-5} \mathrm{~kg} / \mathrm{m}^{3}$. There does not appear to be anything significant about the five sampled parameters that accounts for the outliers. All the sampled parameters associated with the outliers are well within the range of the sampled parameters, and none is at the high or low extremes. The maxiimum time of occurrence on the $5-\mathrm{km}$ boundary ranges from a low of $30,000 \mathrm{yr}$ to a high of $60,000 \mathrm{yr}$. There does not appear to be a relationship between the maximum concentration and the time of occurrence on the boundary. The exit layer in which the maximum concentration occurs is concentrated in three levels. The various levels of the PPw (layers 9 and 10) unit are the most common exit units. However, exit units are as low as the CFun/BFw interface (layer 5) and as high as the TSwc unit (layer 20). Maximum concentrations of ${ }^{129}$ I (Table 9-4) produce results similar to the ${ }^{99} \mathrm{Tc}$ results because both are released as a pulse and neither is adsorbed.

The maximum concentrations for ${ }^{237} \mathrm{~Np}$ in Table $9-5$ range from a low of about $3.7 \times 10^{-11} \mathrm{~kg} / \mathrm{m}^{3}$ to a high of $7.9 \times 10^{-6} \mathrm{~kg} / \mathrm{m}^{3}$. There do not appear to be any outliers, nor does there appear to be any strong correlation between the maximum concentrations and the five sampled parameters. The time of occurrence of the maximum concentration on the $5-\mathrm{km}$ boundary ranges from a low of $60,000 \mathrm{yr}$ to a high of $100,000 \mathrm{yr}$. The time of occurrence may actually be greater than $100,000 \mathrm{yr}$, but because the simulations were limited to $100,000 \mathrm{yr}$, this could not be observed. There does not appear to be a relationship between the maximum concentration and the time of occurrence on the boundary. The layer in which the maximum concentration occurs is concentrated in the upper unit, the TSwc (layers 19,20 , and 21). However, the PPw unit (layers 9 and 10) serves as the exit unit in a few runs.

The results of the maximum concentration occurrence on the 5-km boundary are shown in Tables 9-6, 9-7, and 9-8 for the median and mean runs of ${ }^{99} \mathrm{Tc},{ }^{129} \mathrm{I}$, and ${ }^{237} \mathrm{~Np}$, respectively. For the mean and median runs of ${ }^{99} \mathrm{Tc}$ and ${ }^{129} \mathrm{I}$, the maximum concentration occurs at about $40,000 \mathrm{yr}$ in the PPw unit, reflecting the pulse type of input of these radioisotopes into the groundwater and the rapid movement of the pulse through the saturated zone. A point of interest is that the layer in which the maximum concentration occurs varies with time, which is probably a reflection of the differing velocities in the various units. The $\mathrm{PPw}$ unit has the highest fracture hydraulic conductivity for the mean and median runs, and this is the unit in which the maximum concentration occurs.

${ }^{237} \mathrm{~Np}$, on the other hand, has a maximum concentration occurring at $100,000 \mathrm{yr}$. Table $9-8$ indicates that the maximum ${ }^{237} \mathrm{~Np}$ concentration is actually rising at $100,000 \mathrm{yr}$ such that the maximum concentration most likely occurs after $100,000 \mathrm{yr}$. The ${ }^{237} \mathrm{~Np}$ exit unit is always the TSwc unit. ${ }^{237} \mathrm{~Np}$ is not retarded in this unit but is in some of the lower units, implying that large quantities of ${ }^{237} \mathrm{~Np}$ are not reaching the lower units where the groundwater moves rapidly. It appears that retardation has a significant impact on the movement of ${ }^{237} \mathrm{~Np}$.

Figure 9-4 shows the 100,000 -yr time history contours of ${ }^{99} \mathrm{Tc}$ concentration in the PPw unit at 10,000 -yr intervals for the median run. The PPw unit was chosen because it is the unit in which the maximum concentration occurs on the 5-km boundary even though most of the ${ }^{99} \mathrm{Tc}$ enters the groundwater through the CFun unit. The CFun unit has the slowest fracture velocity and the PPw unit has the fastest fracture velocity of all the layered units. Through the first 30,000 yr (Figures 9-4a to 9-4c), the ${ }^{99} \mathrm{Tc}$ concentration builds rapidly underneath the southeastern corner of the repository reflecting the pulse input of the ${ }^{99} \mathrm{Tc}$. The ${ }^{99} \mathrm{Tc}$ pulse enters the saturated zone between $15,000 \mathrm{yr}$ and $26,000 \mathrm{yr}$. At about $30,000 \mathrm{yr}$, the concentrations begin to decline more slowly than their buildup in the first $30,000 \mathrm{yr}$. This decline is probably a reflection of the slow release of ${ }^{99} \mathrm{Tc}$ from the porous matrix into the fractures near the southern edge of the repository and the rapid velocity in the fractures of the PPw unit. The pulse source term ends by $30,000 \mathrm{yr}$ and is no longer a source of ${ }^{99} \mathrm{Tc}$ to the groundwater. This release from the fractures continues from 30,000 to $100,000 \mathrm{yr}$.

Concentration plots of ${ }^{99} \mathrm{Tc}$ for several units at the $40,000 \mathrm{yr}$ time are shown in Figure $9-5$. The ${ }^{99} \mathrm{Tc}$ source term has ceased by this time. Therefore, this figure shows the release of ${ }^{99} \mathrm{Tc}$ from the matrix into the fractures and the subsequent movement through the various units. The impact of fracture velocity is demonstrated by the contours in the CFun and PPw units (Figures 9-5b and 9-5c). The highest concentration of ${ }^{99} \mathrm{Tc}$ is located under the southern 
boundary of the repository in the CFun unit as most of the ${ }^{99} \mathrm{Tc}$ enters this unit. A sizable amount of ${ }^{99} \mathrm{Tc}$ enters the $\mathrm{PPw}$, but its maximum concentration is about one-fourth that of the CFun. The main difference between the two units is that the contours are closed in the CFun unit while they extend past the $5-\mathrm{km}$ boundary in the PPw unit. This difference is a reflection of the higher fracture velocities in the PPw compared to those in the CFun. At the 5-km boundary, the fracture velocities are about 34 times faster in the PPw unit than in the CFun unit. The upper units also show extended contours in a manner similar to those in the PPw unit. Fracture velocities in these units are at least a factor of 3.5 faster than those in the CFun. As was noted previously and as shown in Table 9-6, the maximum concentrations of ${ }^{99} \mathrm{Tc}$ on the $5-\mathrm{km}$ boundary occur in the PPw unit. In addition, the ${ }^{99} \mathrm{Tc}$ contours are very similar between the $\mathrm{PPw}$ unit and the overlying CHnz unit. This suggests an upward vertical transport of ${ }^{99} \mathrm{Tc}$ from the PPw unit to the $\mathrm{CHnz}$ unit, because only small amounts of ${ }^{99} \mathrm{Tc}$ enter the $\mathrm{CHnz}$ unit through the water table.

Concentration plots for ${ }^{237} \mathrm{~Np}$ in the TSwc unit are shown in Figure 9-6 for the 100,000-yr simulation time at 10,000 -yr intervals. On the $5-\mathrm{km}$ boundary, the maximum ${ }^{237} \mathrm{~Np}$ concentration occurs at $100,000 \mathrm{yr}$ in this unit (Table 9-8). This is interesting because ${ }^{237} \mathrm{~Np}$ does not enter the TSwc unit directly through the source term. Instead, ${ }^{237} \mathrm{~Np}$ can enter the TSwc unit only by convection and hydrodynamic dispersion from the underlying units. In addition, the peak concentrations are lower in the TSwc than for any of the underlying units (see Figure 9-7). This suggests that hydrodynamic dispersion and vertical velocities are very important for the transport of ${ }^{237} \mathrm{~Np}$.

Concentrations of ${ }^{237} \mathrm{~Np}$ at $100,000 \mathrm{yr}$ are shown for six layers in Figure 9-7. The plots for the CFun, PPw, and CHnz layers show the most interesting results (Figures 9-7b, 9-7c, and 9-7d). Most of the ${ }^{237} \mathrm{~Np}$ enters the saturated groundwater system through the CFun layer. The ${ }^{237} \mathrm{~Np}$ contours are closed in the CFun, which indicates that ${ }^{237} \mathrm{~Np}$ does not travel very far in this unit. This condition is a reflection of both the slow fracture velocities and the adsorption of the ${ }^{237} \mathrm{~Np}$ on the porous matrix in the CFun. The slow fracture velocities are probably the dominant reason for the closed contours. The peak ${ }^{237} \mathrm{~Np}$ concentration also occurs in this unit. On the other hand, the ${ }^{237} \mathrm{~Np}$ contours are long and extended in the $\mathrm{PPw}$ unit, much like the ${ }^{99} \mathrm{Tc}$ contours appeared in this same unit. The ${ }^{237} \mathrm{~Np}$ is not adsorbed in the PPw. Because the fracture velocities are highest in this unit relative to the other layers and large amounts of ${ }^{237} \mathrm{~Np}$ enter the PPw unit at the water table, transport of the ${ }^{237} \mathrm{~Np}$ away from the repository is significant in this unit. The contours for the CHnz unit are similar to those of the PPw even though the fracture velocities in the $\mathrm{CHnz}$ are about one-eighth those in the PPw and the ${ }^{237} \mathrm{~Np}$ is adsorbed in the porous matrix. Because only small amounts of ${ }^{237} \mathrm{~Np}$ enter the $\mathrm{CHnz}$ unit at the water table, it appears that the cause of this similarity is vertical transport of the ${ }^{237} \mathrm{~Np}$ from the PPw unit to the $\mathrm{CHnz}$ through vertical velocities and hydrodynamic dispersion. This phenomenon was also observed for ${ }^{99} \mathrm{Tc}$ between these two units.

In summary, the horizontal velocity is the dominant transport mechanism for radionuclides if there is no matrix adsorption. Vertical transport by means of vertical velocity and hydrodynamic dispersion can impact the location of the maximum concentration if there is adsorption onto the porous matrix. However, adsorption is important only if radioisotopes can diffuse into the porous matrix from the fractures. 
9. Consequence Modeling: Saturated Zone Flow and Transport

Table 9-3. Technetium-99 - Maximum Concentration and Location

\begin{tabular}{|c|c|c|c|c|c|c|}
\hline Run & H-Index & J-Index & K-Index & Node & Time (yr) & Concentration $\left(\mathrm{kg} / \mathrm{m}^{3}\right)$ \\
\hline 8 & 29 & 13 & 10 & 10379 & 50000 & 3.74E-03 \\
\hline 9 & 29 & 12 & 19 & 20339 & 30000 & 2.00E-04 \\
\hline 31 & 29 & 12 & 9 & 9239 & 30000 & 3.84E-05 \\
\hline 47 & 29 & 11 & 16 & 16979 & $60000^{-}$ & 1.02E-05 \\
\hline 35 & 29 & 12 & 20 & 21449 & 30000 & $9.68 \mathrm{E}-06$ \\
\hline 15 & 29 & 12 & 19 & 20339 & 40000 & $9.25 E-06$ \\
\hline 11 & 29 & 13 & 9 & 9269 & 40000 & 8.85E-06 \\
\hline 21 & 29 & 13 & 19 & 20369 & 30000 & 8.70E-06 \\
\hline 7 & 29 & 12 & 10 & 10349 & 50000 & 7.80E-06 \\
\hline 41 & 29 & 11 & 20 & 21419 & 50000 & 7.49E-06 \\
\hline 37 & 29 & 11 & 16 & 16979 & 50000 & 7.43E-06 \\
\hline 29 & 29 & 12 & 10 & 10349 & 40000 & 7.41E-06 \\
\hline 46 & 29 & 13 & 9 & 9269 & 30000 & $6.99 E-06$ \\
\hline 34 & 29 & 13 & 9 & 9269 & 30000 & 6.62E-06 \\
\hline Median & 29 & 12 & 10 & 10349 & 40000 & $6.44 E-06$ \\
\hline 2 & 29 & 13 & 9 & 9269 & 30000 & $6.39 \mathrm{E}-06$ \\
\hline 19 & 29 & 12 & 9 & 9239 & 40000 & $6.10 \mathrm{E}-06$ \\
\hline Mean & 29 & 12 & 10 & 10349 & 40000 & $6.01 \mathrm{E}-06$ \\
\hline 18 & 29 & 12 & 9 & 9239 & 40000 & 5.65E-06 \\
\hline 38 & 29 & 13 & 10 & 10379 & 40000 & 5.64E-06 \\
\hline 3 & 29 & 13 & 10 & 10379 & 40000 & $5.28 \mathrm{E}-06$ \\
\hline 16 & 29 & 11 & 20 & 21419 & 50000 & 5.24E-06 \\
\hline 49 & 29 & 14 & 9 & 9299 & 30000 & $5.20 \mathrm{E}-06$ \\
\hline 30 & 29 & 11 & 20 & 21419 & 60000 & 4.90E-06 \\
\hline 14 & 29 & 13 & 5 & 4829 & 50000 & 4.88E-06 \\
\hline 27 & 29 & 14 & 9 & 9299 & 30000 & 4.74E-06 \\
\hline 22 & 29 & 11 & 14 & 14759 & 40000 & 4.69E-06 \\
\hline 13 & 29 & 12 & 12 & 12569 & 40000 & $4.46 \mathrm{E}-06$ \\
\hline 17 & 29 & 12 & 19 & 20339 & 30000 & $4.22 E-06$ \\
\hline 6 & 29 & 11 & 19 & 20309 & 60000 & 4.17E-06 \\
\hline 24 & 29 & 13 & 9 & 9269 & 30000 & 4.17E-06 \\
\hline 40 & 29 & 12 & 10 & 10349 & 30000 & $3.69 \mathrm{E}-06$ \\
\hline 43 & 29 & 13 & 19 & 20369 & 30000 & 3.44E-06 \\
\hline 10 & 29 & 14 & 9 & 9299 & 30000 & 3.35E-06 \\
\hline 39 & 29 & 11 & 20 & 21419 & 60000 & 3.34E-06 \\
\hline 20 & 29 & 12 & 9 & 9239 & 40000 & 3.31E-06 \\
\hline 26 & 29 & 13 & 19 & 20369 & 30000 & 3.22E-06 \\
\hline 42 & 29 & 12 & 19 & 20339 & 30000 & 2.67E-06 \\
\hline 48 & 29 & 11 & 20 & 21419 & 60000 & 2.63E-06 \\
\hline 28 & 29 & 14 & 9 & 9299 & 30000 & 2.57E-06 \\
\hline 4 & 29 & 12 & 10 & 10349 & 60000 & 2.44E-06 \\
\hline 33 & 29 & 13 & 9 & 9269 & 30000 & 2.40E-06 \\
\hline 12 & 29 & 12 & 19 & 20339 & 30000 & 2.37E-06 \\
\hline 5 & 29 & 11 & 19 & 20309 & 60000 & 2.29E-06 \\
\hline 36 & 29 & 12 & 19 & 20339 & 50000 & 1.87E-06 \\
\hline 1 & 29 & 12 & 20 & 21449 & 50000 & 1.62E-06 \\
\hline 50 & 29 & 13 & 5 & 4829 & 50000 & 1.27E-06 \\
\hline 45 & 29 & 13 & 9 & 9269 & 30000 & 9.61E-07 \\
\hline 25 & 29 & 14 & 9 & 9299 & 20000 & 6.59E-07 \\
\hline
\end{tabular}


Table 9-4. Iodine-129 - Maximum Concentration and Location

\begin{tabular}{|c|c|c|c|c|c|c|}
\hline Run & I-Index & J-Index & K-Index & Node & Time (yr) & Concentration $\left(\mathrm{kg} / \mathrm{m}^{3}\right)$ \\
\hline 8 & 29 & 13 & 10 & 10379 & 50000 & $1.36 \mathrm{E}-03$ \\
\hline 9 & 29 & 12 & 19 & 20339 & 30000 & 7.92E-05 \\
\hline 31 & 29 & 12 & 9 & 9239 & 30000 & 1.72E-05 \\
\hline 47 & 29 & 11 & 16 & 16979 & 60000 & 2.89E-06 \\
\hline 7 & 29 & 12 & 10 & 10349 & 50000 & 2.67E-06 \\
\hline 35 & 29 & 12 & 20 & 21449 & 30000 & 2.56E-06 \\
\hline 15 & 29 & 12 & 19 & 20339 & 40000 & 2.47E-06 \\
\hline 11 & 29 & 13 & 9 & 9269 & 40000 & 2.38E-06 \\
\hline 29 & 29 & 12 & 10 & 10349 & 40000 & 2.31E-06 \\
\hline 21 & 29 & 13 & 19 & 20369 & 30000 & 2.22E-06 \\
\hline 41 & 29 & 11 & 20 & 21419 & 50000 & 2.06E-06 \\
\hline 46 & 29 & 13 & 9 & 9269 & 30000 & $1.84 E-06$ \\
\hline Median & 29 & 12 & 10 & 10349 & 40000 & 1.72E-06 \\
\hline 34 & 29 & 13 & 9 & 9269 & 30000 & $1.72 E-06$ \\
\hline 19 & 29 & 12 & 9 & 9239 & 40000 & $1.70 \mathrm{E}-06$ \\
\hline 37 & 29 & 11 & 16 & 16979 & 50000 & $1.68 \mathrm{E}-06$ \\
\hline Mean & 29 & 12 & 10 & 10349 & 40000 & 1.67E-06 \\
\hline 2 & 29 & 13 & 9 & 9269 & 30000 & 1.67E-06 \\
\hline 18 & 29 & 12 & 9 & 9239 & 40000 & $1.50 \mathrm{E}-06$ \\
\hline 38 & 29 & 13 & 10 & 10379 & 40000 & 1.49E-06 \\
\hline 16 & 29 & 11 & 20 & 21419 & 50000 & 1.44E-06 \\
\hline 3 & 29 & 13 & 10 & 10379 & 40000 & 1.43E-06 \\
\hline 30 & 29 & 11 & 20 & 21419 & 60000 & $1.40 \mathrm{E}-06$ \\
\hline 49 & 29 & 14 & 9 & 9299 & 30000 & 1.33E-06 \\
\hline 22 & 29 & 11 & 14 & 14759 & 40000 & 1.33E-06 \\
\hline 14 & 29 & 13 & 5 & 4829 & 50000 & 1.25E-06 \\
\hline 6 & 29 & 11 & 19 & 20309 & 60000 & 1.25E-06 \\
\hline 27 & 29 & 14 & 9 & 9299 & 30000 & $1.20 \mathrm{E}-06$ \\
\hline 13 & 29 & 12 & 12 & 12569 & 40000 & $1.18 E-06$ \\
\hline 17 & 29 & 12 & 19 & 20339 & 30000 & $1.09 \mathrm{E}-06$ \\
\hline 24 & 29 & 13 & 9 & 9269 & 30000 & 1.08E-06 \\
\hline 40 & 29 & 12 & 10 & 10349 & 30000 & $9.50 E-07$ \\
\hline 39 & 29 & 11 & 20 & 21419 & 60000 & 9.39E-07 \\
\hline 43 & 29 & 13 & 19 & 20369 & 30000 & 8.92E-07 \\
\hline 10 & 29 & 14 & 9 & 9299 & 30000 & 8.88E-07 \\
\hline 26 & 29 & 13 & 19 & 20369 & 30000 & 8.51E-07 \\
\hline 48 & 29 & 11 & 20 & 21419 & 60000 & $7.90 \mathrm{E}-07$ \\
\hline 20 & 29 & 12 & 9 & 9239 & 40000 & 7.85E-07 \\
\hline 28 & 29 & 14 & 9 & 9299 & 30000 & 7.39E-07 \\
\hline 4 & 29 & 12 & 10 & 10349 & 60000 & $6.93 E-07$ \\
\hline 42 & 29 & 12 & 19 & 20339 & 30000 & 6.87E-07 \\
\hline 5 & 29 & 11 & 19 & 20309 & 60000 & 6.41E-07 \\
\hline 12 & 29 & 11 & 12 & 12539 & 40000 & $6.23 \mathrm{E}-07$ \\
\hline 33 & 29 & 13 & 9 & 9269 & 30000 & 6.16E-07 \\
\hline 36 & 29 & 12 & 18 & 19229 & 50000 & 5.14E-07 \\
\hline 1 & 29 & 12 & 20 & 21449 & 50000 & 4.43E-07 \\
\hline 50 & 29 & 13 & 5 & 4829 & 50000 & 3.50E-07 \\
\hline 45 & 29 & 13 & 9 & 9269 & 30000 & 2.49E-07 \\
\hline 25 & 29 & 14 & 9 & 9299 & 20000 & $1.58 \mathrm{E}-07$ \\
\hline
\end{tabular}


9. Consequence Modeling: Saturated Zone Flow and Transport

Table 9-5. Neptunium-237 - Maximum Concentration and Location

\begin{tabular}{|c|c|c|c|c|c|c|}
\hline Run & F-Index & J-Index & K-Index & Node & Time (yr) & Concentration $\left(\mathrm{kg} / \mathrm{m}^{3}\right)$ \\
\hline 42 & 29 & 13 & 22 & 23699 & 60000 & $7.89 \mathrm{E}-06$ \\
\hline 17 & 29 & 12 & 20 & 21449 & 70000 & $2.81 E-06$ \\
\hline 27 & 29 & 16 & 10 & 10469 & 90000 & 2.44E-06 \\
\hline 31 & 29 & 13 & 22 & 23699 & 70000 & 2.10E-06 \\
\hline 36 & 29 & 12 & 20 & 21449 & 60000 & $1.26 E-06$ \\
\hline 11 & 29 & 13 & 9 & 9269 & 60000 & $1.25 E-06$ \\
\hline 43 & 29 & 14 & 21 & 22619 & 50000 & $1.08 E-06$ \\
\hline 10 & 29 & 14 & 22 & 23729 & 60000 & 9.98E-07 \\
\hline 34 & 29 & 14 & 9 & 9299 & 50000 & 8.63E-07 \\
\hline 12 & 29 & 12 & 21 & 22559 & 80000 & 2.41E-07 \\
\hline Mean & 29 & 12 & 21 & 22559 & 100000 & 1.74E-07 \\
\hline 37 & 29 & 12 & 21 & 22559 & 70000 & $1.68 \mathrm{E}-07$ \\
\hline Median & 29 & 12 & 21 & 22559 & 100000 & 1.55E-07 \\
\hline 16 & 29 & 11 & 21 & 22529 & 80000 & 1.50E-07 \\
\hline 49 & 29 & 13 & 22 & 23699 & 60000 & $1.49 \mathrm{E}-07$ \\
\hline 46 & 29 & 13 & 9 & 9269 & 60000 & 1.41E-07 \\
\hline 45 & 29 & 12 & 22 & 23669 & 60000 & 1.10E-07 \\
\hline 13 & 29 & 12 & 20 & 21449 & 70000 & 1.03E-07 \\
\hline 21 & 29 & 13 & 10 & 10379 & 50000 & 8.04E-08 \\
\hline 19 & 29 & 12 & 22 & 23669 & 70000 & 5.06E-08 \\
\hline 29 & 29 & 12 & 20 & 21449 & 100000 & 4.92E-08 \\
\hline 26 & 29 & 12 & 22 & 23669 & 90000 & 4.02E-08 \\
\hline 35 & 29 & 12 & 22 & 23669 & 70000 & 2.96E-08 \\
\hline 8 & 29 & 12 & 21 & 22559 & 100000 & 2.32E-08 \\
\hline 40 & 29 & 13 & 10 & 10379 & 90000 & $2.25 \mathrm{E}-08$ \\
\hline 38 & 29 & 12 & 20 & 21449 & 60000 & $1.84 \mathrm{E}-08$ \\
\hline 33 & 29 & 13 & 22 & 23699 & 70000 & 1.72E-08 \\
\hline 2 & 29 & 12 & 20 & 21449 & 90000 & $1.64 \mathrm{E}-08$ \\
\hline 28 & 29 & 14 & 22 & 23729 & 60000 & 1.36E-08 \\
\hline 25 & 29 & 13 & 22 & 23699 & 70000 & $1.31 E-08$ \\
\hline 3 & 29 & 12 & 22 & 23669 & 70000 & $1.24 \mathrm{E}-08$ \\
\hline 22 & 29 & 11 & 20 & 21419 & 60000 & $9.77 E-09$ \\
\hline 30 & 29 & 12 & 22 & 23669 & 80000 & $5.74 \mathrm{E}-09$ \\
\hline 20 & 29 & 13 & 21 & 22589 & 60000 & 5.63E-09 \\
\hline 14 & 29 & 14 & 22 & 23729 & 60000 & 5.26E-09 \\
\hline 50 & 29 & 14 & 22 & 23729 & 60000 & 3.39E-09 \\
\hline 6 & 29 & 11 & 22 & 23639 & 100000 & 3.35E-09 \\
\hline 4 & 29 & 12 & 10 & 10349 & 90000 & $3.34 E-09$ \\
\hline 15 & 29 & 11 & 22 & 23639 & 80000 & $2.78 \mathrm{E}-09$ \\
\hline 9 & 29 & 12 & 22 & 23669 & 60000 & 1.77E-09 \\
\hline 24 & 29 & 13 & 22 & 23699 & 60000 & 1.51E-09 \\
\hline 5 & 29 & 12 & 22 & 23669 & 80000 & 1.37E-09 \\
\hline 7 & 29 & 12 & 21 & 22559 & 80000 & 1.21E-09 \\
\hline 47 & 29 & 12 & 22 & 23669 & 80000 & $6.87 E-10$ \\
\hline 1 & 29 & 12 & 22 & 23669 & 80000 & $6.77 E-10$ \\
\hline 48 & 29 & 12 & 22 & 23669 & 80000 & $6.16 \mathrm{E}-10$ \\
\hline 18 & 29 & 13 & 22 & 23699 & 70000 & $6.07 E-10$ \\
\hline 41 & 29 & 11 & 22 & 23639 & 90000 & $6.92 E-11$ \\
\hline 39 & 29 & 12 & 22 & 23669 & 70000 & $3.72 E-11$ \\
\hline
\end{tabular}


Table 9-6. Technetium-99 - Maximum Concentration at 5-km Boundary

\begin{tabular}{|c|c|c|c|c|c|c|}
\hline $\begin{array}{c}\text { Time } \\
(y r)\end{array}$ & I-Index & J-Index & K-Index & Node & Unit & $\begin{array}{c}\text { Concentration } \\
\left(\mathrm{kg} / \mathrm{m}^{3}\right)\end{array}$ \\
\hline \multicolumn{7}{|l|}{ Median } \\
\hline 0 & - & - & - & - & - & $0.00 E+00$ \\
\hline 10000 & 29 & 16 & 9 & 9359 & PPw/CFun & $4.33 E-10$ \\
\hline 20000 & 29 & 18 & 9 & 9419 & PPw/CFun & $9.00 \mathrm{E}-08$ \\
\hline 30000 & 29 & 14 & 19 & 20399 & TSwc & 4.42E-06 \\
\hline 40000 & 29 & 12 & 10 & 10349 & PPw & $6.44 E-06$ \\
\hline 50000 & 29 & 10 & 12 & 12509 & $\mathrm{CHnz}$ & $3.70 E-06$ \\
\hline 60000 & 29 & 12 & 3 & 2579 & $\mathrm{BFw} / \mathrm{CFmn}$ & 1.85E-06 \\
\hline 70000 & 29 & 11 & 3 & 2549 & BFw/CFmn & 1.36E-06 \\
\hline 80000 & 29 & 10 & 3 & 2519 & BFw/CFmn & 8.86E-07 \\
\hline 90000 & 29 & 12 & 6 & 5909 & CFun & $8.12 E-07$ \\
\hline 100000 & 29 & 11 & 6 & 5879 & CFun & 7.19E-07 \\
\hline \multicolumn{7}{|l|}{ Mean } \\
\hline 0 & - & - & - & - & - & $0.00 \mathrm{E}+00$ \\
\hline 10000 & 29 & 16 & 9 & 9359 & PPw/CFun & $3.20 \mathrm{E}-09$ \\
\hline 20000 & 29 & 17 & 9 & 9389 & PPw/CFun & 8.43E-08 \\
\hline 30000 & 29 & 14 & 19 & 20399 & TSwe & 4.47E-06 \\
\hline 40000 & 29 & 12 & 10 & 10349 & PPw & 6.01E-06 \\
\hline 50000 & 29 & 10 & 12 & 12509 & $\mathrm{CHnz}$ & 3.24E-06 \\
\hline 60000 & 29 & 12 & 3 & 2579 & BFw/CFmn & $1.59 \mathrm{E}-06$ \\
\hline 70000 & 29 & 10 & 3 & 2519 & BFw/CFmn & 1.16E-06 \\
\hline 80000 & 29 & 12 & 6 & 5909 & CFun & 7.61E-07 \\
\hline 90000 & 29 & 12 & 6 & 5909 & CFun & 6.99E-07 \\
\hline 100000 & 29 & 11 & 6 & 5879 & CFun & $6.18 \mathrm{E}-07$ \\
\hline
\end{tabular}


9. Consequence Modeling: Saturated Zone Flow and Transport

Table 9-7. Iodine-129 - Maximum Concentration at 5-km Boundary

\begin{tabular}{|c|c|c|c|c|c|c|}
\hline $\begin{array}{l}\text { Time } \\
(y r)\end{array}$ & I-Index & J-Index & K-Index & Node & Unit & $\begin{array}{c}\text { Concentration } \\
\left(\mathrm{kg} / \mathrm{m}^{3}\right)\end{array}$ \\
\hline \multicolumn{7}{|l|}{ Median } \\
\hline 0 & - & - & - & - & - & $0.00 E+00$ \\
\hline 10000 & 29 & 16 & 9 & 9359 & PPw/CFun & $1.07 E-10$ \\
\hline 20000 & 29 & 18 & 9 & 9419 & PPw/CFun & 2.30E-08 \\
\hline 30000 & 29 & 14 & 19 & 20399 & TSwc & 1.15E-06 \\
\hline 40000 & 29 & 12 & 10 & 10349 & PPw & 1.72E-06 \\
\hline 50000 & 29 & 10 & 12 & 12509 & $\mathrm{CHnz}$ & $1.02 E-06$ \\
\hline 60000 & 29 & 12 & 3 & 2579 & BFw/CFmn & 5.27E-07 \\
\hline 70000 & 29 & 11 & 3 & 2549 & BFw/CFmn & 4.01E-07 \\
\hline 80000 & 29 & 10 & 3 & 2519 & BFw/CFmn & 2.68E-07 \\
\hline 90000 & 29 & 12 & 6 & 5909 & CFun & 2.55E-07 \\
\hline 100000 & 29 & 11 & 6 & 5879 & CFun & 2.33E-07 \\
\hline \multicolumn{7}{|l|}{ Mean } \\
\hline 0 & - & - & - & - & - & $0.00 \mathrm{E}+00$ \\
\hline 10000 & 29 & 16 & 9 & 9359 & PPw/CFun & $7.75 E-10$ \\
\hline 20000 & 29 & 17 & 9 & 9389 & PPw/CFun & 2.24E-08 \\
\hline 30000 & 29 & 14 & 19 & 20399 & TSwc & $1.21 \mathrm{E}-06$ \\
\hline 40000 & 29 & 12 & 10 & 10349 & PPw & 1.67E-06 \\
\hline 50000 & 29 & 10 & 12 & 12509 & $\mathrm{CHnz}$ & $9.25 \mathrm{E}-07$ \\
\hline 60000 & 29 & 12 & 3 & 2579 & BFw/CFmn & 4.74E-07 \\
\hline 70000 & 29 & 10 & 3 & 2519 & BFw/CFmn & 3.56E-07 \\
\hline 80000 & 29 & 12 & 6 & 5909 & CFun & 2.42E-07 \\
\hline 90000 & 29 & 12 & 6 & 5909 & CFun & 2.29E-07 \\
\hline 100000 & 29 & 11 & 6 & 5879 & CFun & 2.09E-07 \\
\hline
\end{tabular}


Table 9-8. Neptunium-237 - Maximum Concentration at 5-km Boundary

\begin{tabular}{|c|c|c|c|c|c|c|}
\hline $\begin{array}{c}\text { Time } \\
(y r)\end{array}$ & I-Index & J-Index & K-Index & Node & Unit & $\begin{array}{c}\text { Concentration } \\
\left(\mathrm{kg} / \mathrm{m}^{3}\right)\end{array}$ \\
\hline \multicolumn{7}{|l|}{ Median } \\
\hline 0 & - & - & - & - & - & $0.00 \mathrm{E}+00$ \\
\hline 10000 & 29 & 16 & 21 & 22679 & TSwc & $2.08 \mathrm{E}-12$ \\
\hline 20000 & 29 & 14 & 21 & 22619 & TSwc & $7.62 E-12$ \\
\hline 30000 & 29 & 16 & 21 & 22679 & TSwc & $2.34 \mathrm{E}-10$ \\
\hline 40000 & 29 & 15 & 21 & 22649 & TSwc & 4.13E-09 \\
\hline 50000 & 29 & 14 & 21 & 22619 & TSwc & $2.56 \mathrm{E}-08$ \\
\hline 60000 & 29 & 13 & 22 & 23699 & TSwe & $6.21 E-08$ \\
\hline 70000 & 29 & 13 & 22 & 23699 & TSwc & $1.02 E-07$ \\
\hline 80000 & 29 & 12 & 22 & 23669 & TSwc & $1.30 \mathrm{E}-07$ \\
\hline 90000 & 29 & 12 & 21 & 22559 & TSwe & 1.47E-07 \\
\hline 100000 & 29 & 12 & 21 & 22559 & TSwe & $1.55 \mathrm{E}-07$ \\
\hline \multicolumn{7}{|l|}{ Mean } \\
\hline 0 & - & - & - & - & - & $0.00 E \div 00$ \\
\hline 10000 & 29 & 15 & 21 & 22649 & TSwe & $6.97 \mathrm{E}-12$ \\
\hline 20000 & 29 & 14 & 21 & 22619 & TSwe & $1.37 E-11$ \\
\hline 30000 & 29 & 16 & 21 & 22679 & TSwc & $6.95 \mathrm{E}-10$ \\
\hline 40000 & 29 & 15 & 21 & 22649 & TSwe & $9.43 E-09$ \\
\hline 50000 & 29 & 14 & 21 & 22619 & TSwe & $3.46 E-08$ \\
\hline 60000 & 29 & 13 & 22 & 23699 & TSwc & 7.21E-08 \\
\hline 70000 & 29 & 13 & 22 & 23699 & TSwc & 1.13E-07 \\
\hline 80000 & 29 & 13 & 21 & 22589 & TSwc & $1.45 \mathrm{E}-07$ \\
\hline 90000 & 29 & 12 & 22 & 23669 & TSwc & 1.66E-07 \\
\hline 100000 & 29 & 12 & 21 & 22559 & TSwe & 1.74E-07 \\
\hline
\end{tabular}




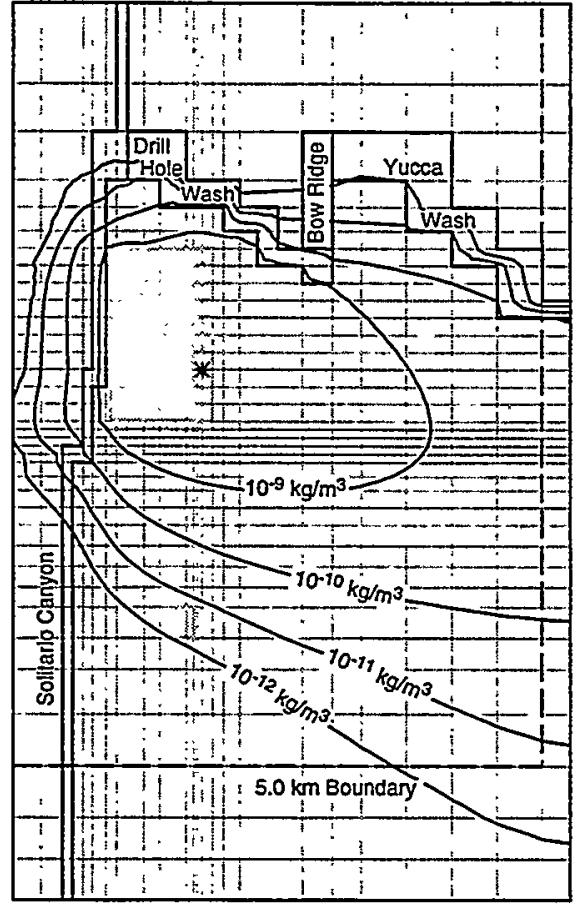

(a) 10,000 years

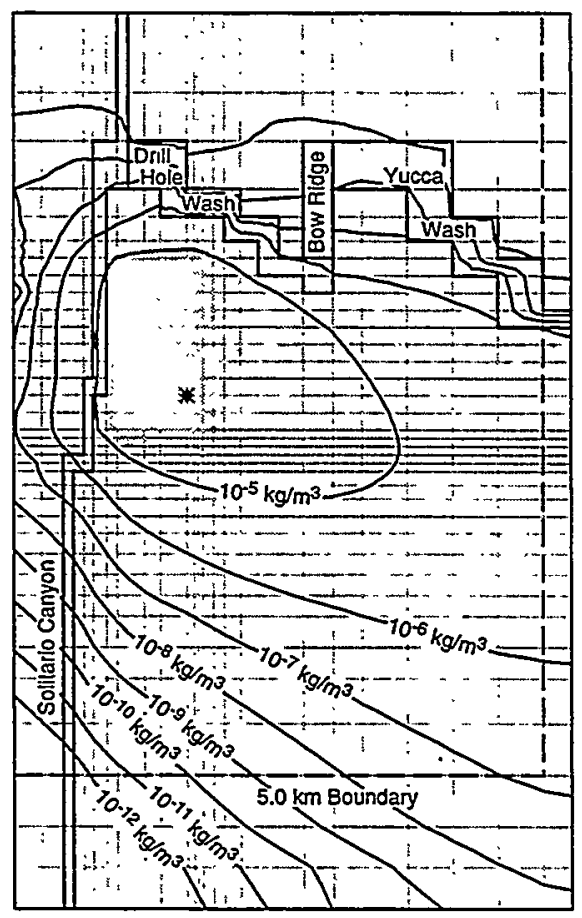

(c) 30,000 years

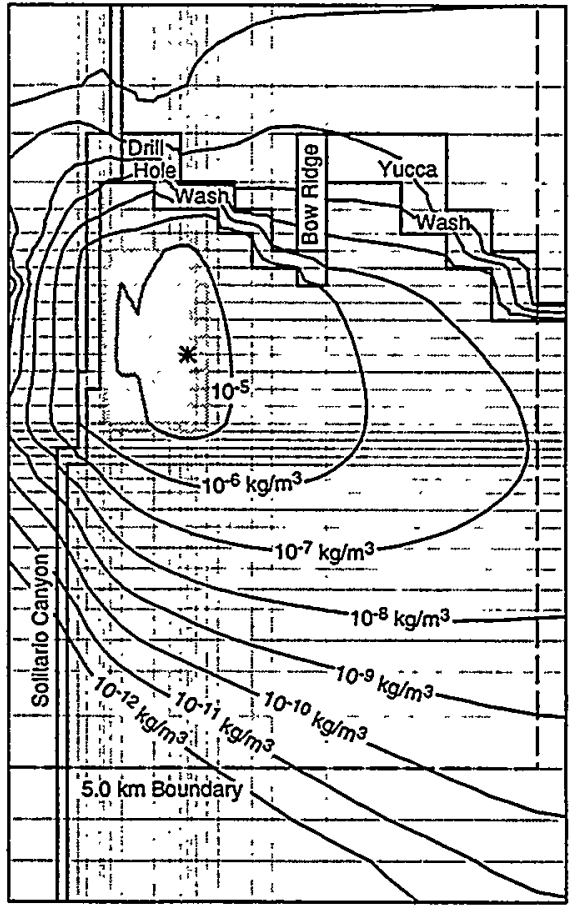

(b) 20,000 years

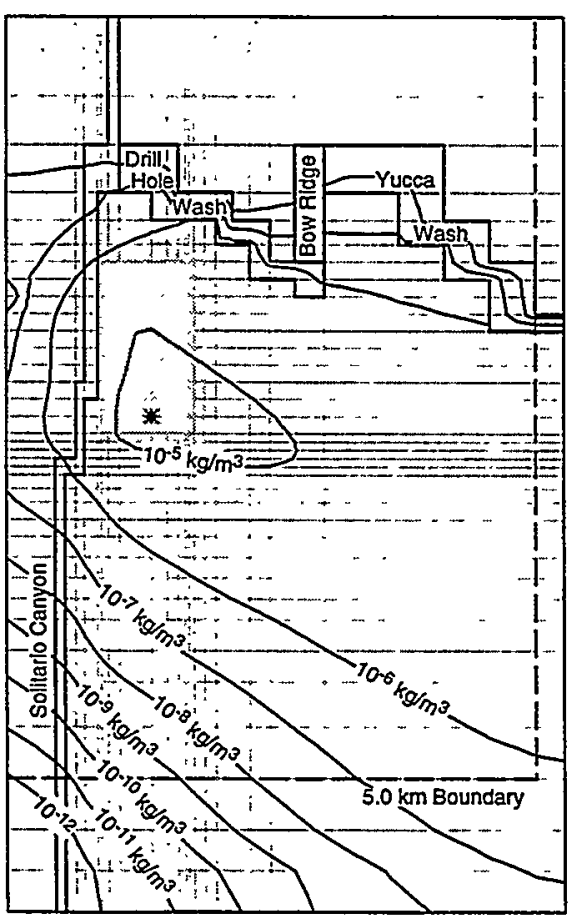

(d) 40,000 years

TRI-6342-5648-0

Figure 9-4. ${ }^{99} \mathrm{Tc}$ concentrations in the $P P w$ unit for various times. (a) $10,000 \mathrm{yr}$, (b) 20,000 yr, (c) 30,000 yr, (d) 40,000 yr, (e) 50,000 yr, (f) 60,000 yr, (g) 70,000 yr, (h) 80,000 yr, (i) 90,000 yr, and (j) 100,000 yr. 


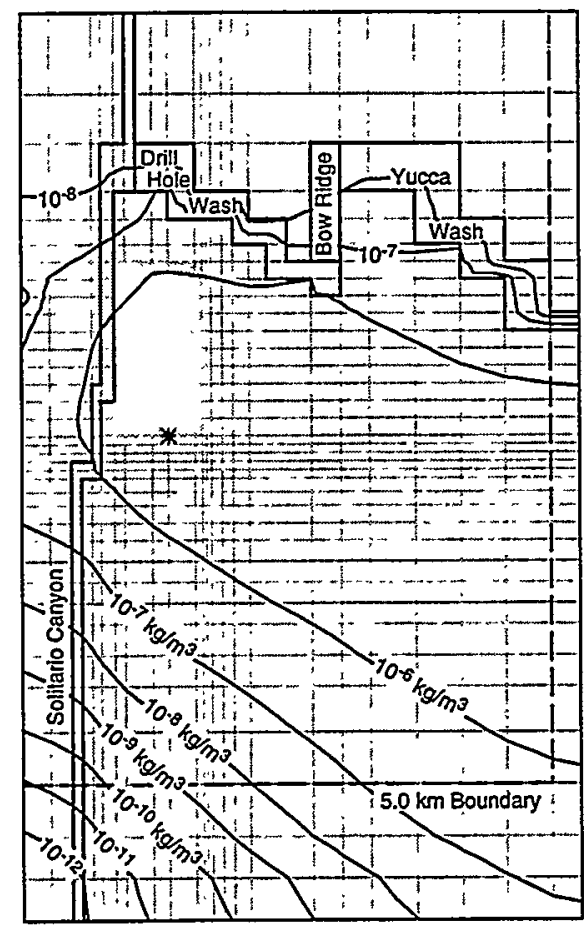

(e) 50,000 years

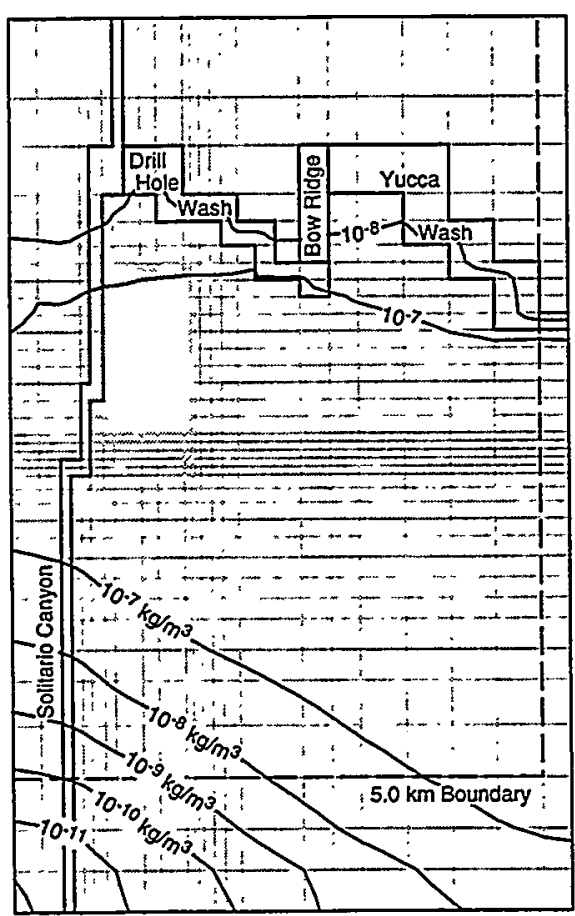

(g) 70,000 years

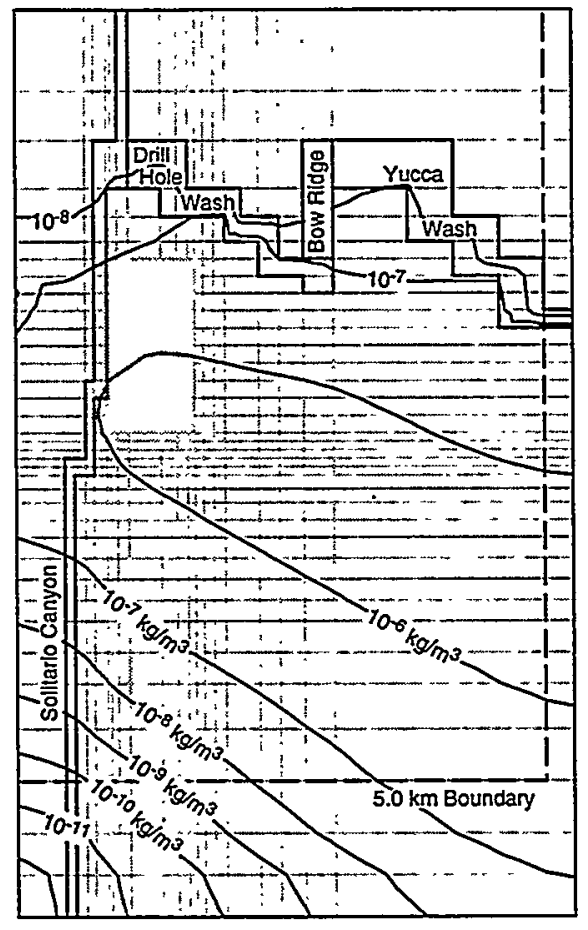

(f) 60,000 years

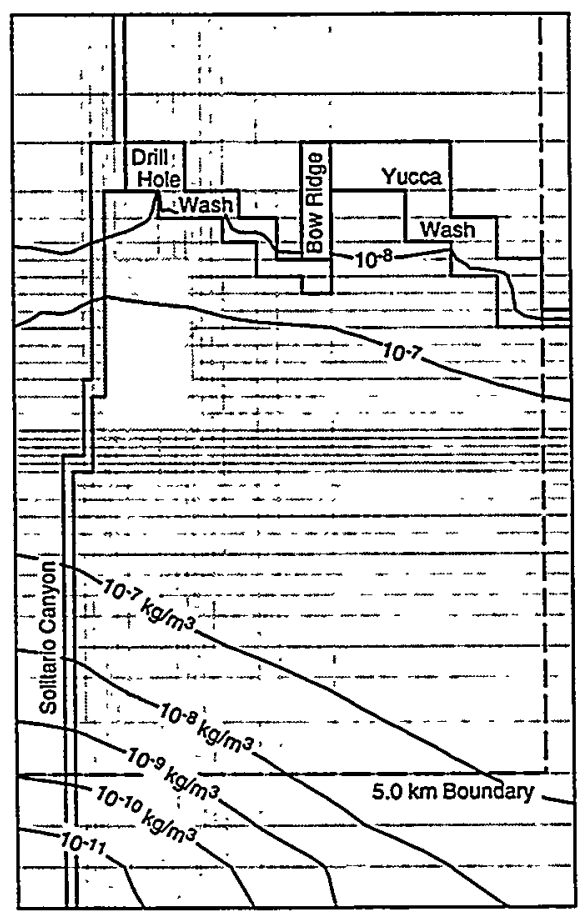

(h) 80,000 years

TRI-6342-5649-0

Figure 9-4. ${ }^{99} \mathrm{Tc}$ concentrations in the PPw unit for various times. (a) 10,000 yr, (b) 20,000 yr, (c) 30,000 yr, (d) 40,000 yr, (e) 50,000 yr, (f) 60,000 yr, (g) 70,000 yr, (h) 80,000 yr, (i) 90,000 yr, and (j) 100,000 yr (continued). 

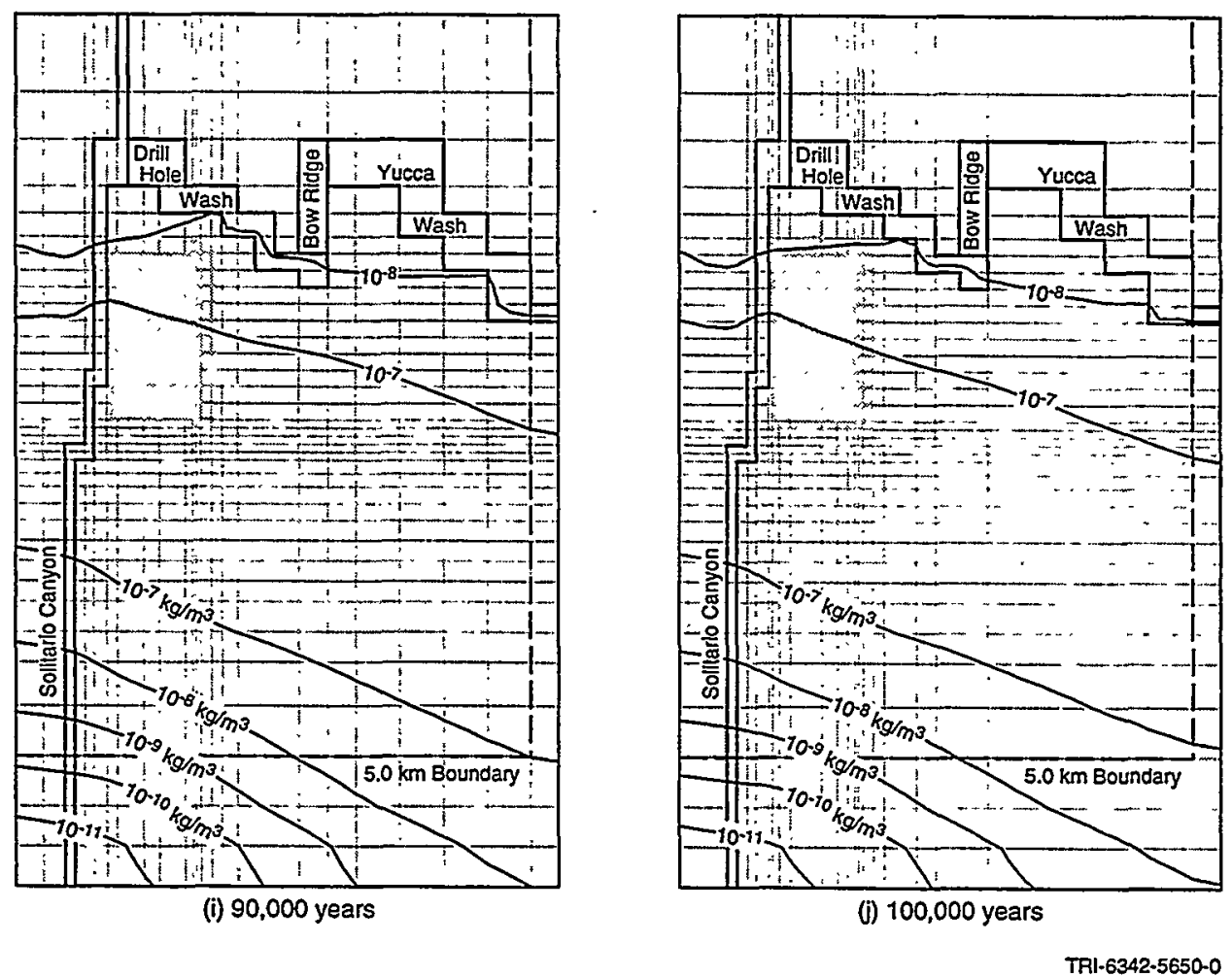

Figure 9-4. ${ }^{99} \mathrm{Tc}$ concentrations in the PPw unit for various times. (a) $10,000 \mathrm{yr}$, (b) $20,000 \mathrm{yr}$, (c) 30,000 yr, (d) 40,000 yr, (e) 50,000 yr, (f) 60,000 yr, (g) 70,000 yr, (h) 80,000 yr, (i) 90,000 yr, and (j) 100,000 yr (continued). 


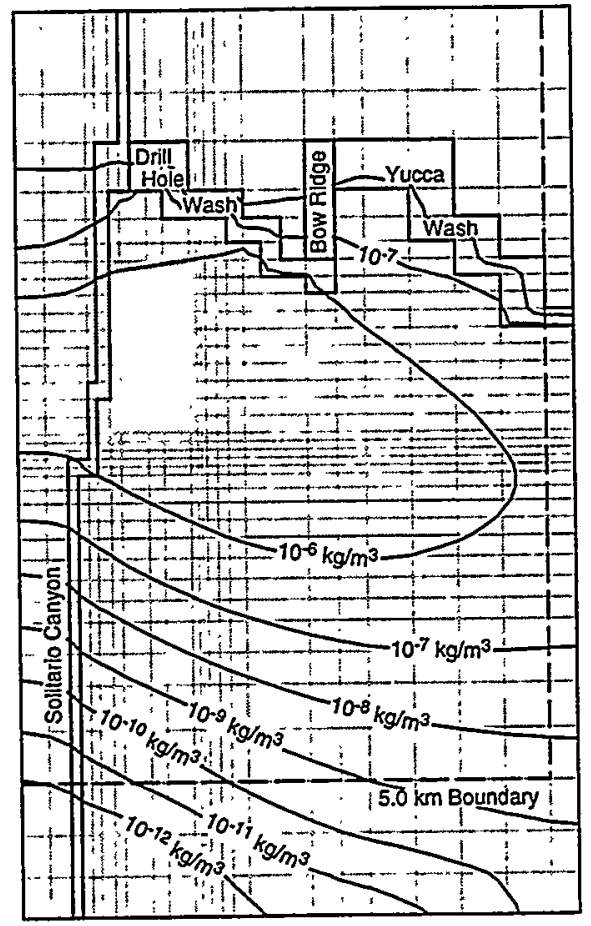

(a) BFW

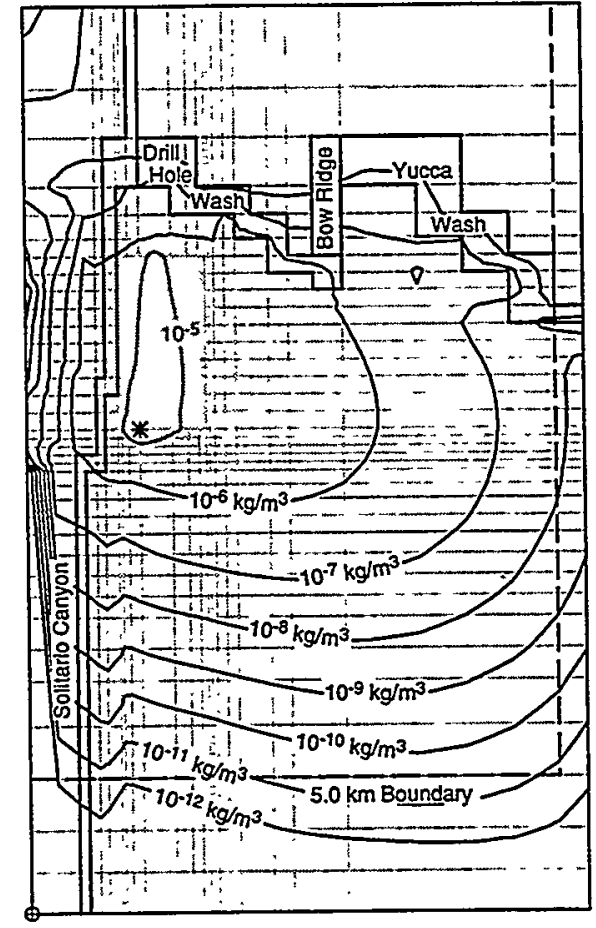

(b) CFun

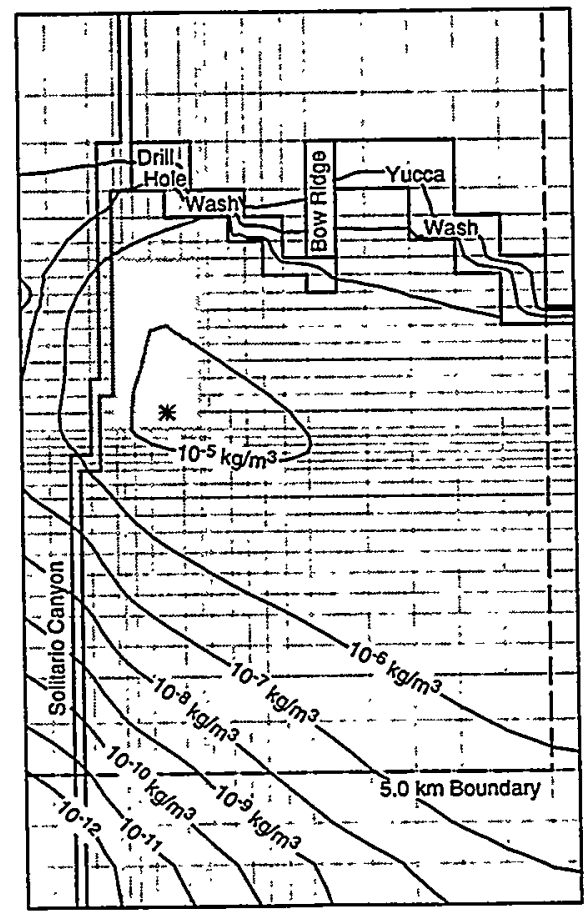

(c) PPW

TRI-6342.5651-0

Figure 9-5. ${ }^{99} \mathrm{Tc}$ concentrations at 40,000 yr for various units. (a) BFw, (b) CFun, (c) PPw, (d) CHnz, (e) CHnv/ TSlv interface, and (f) TSwc. 


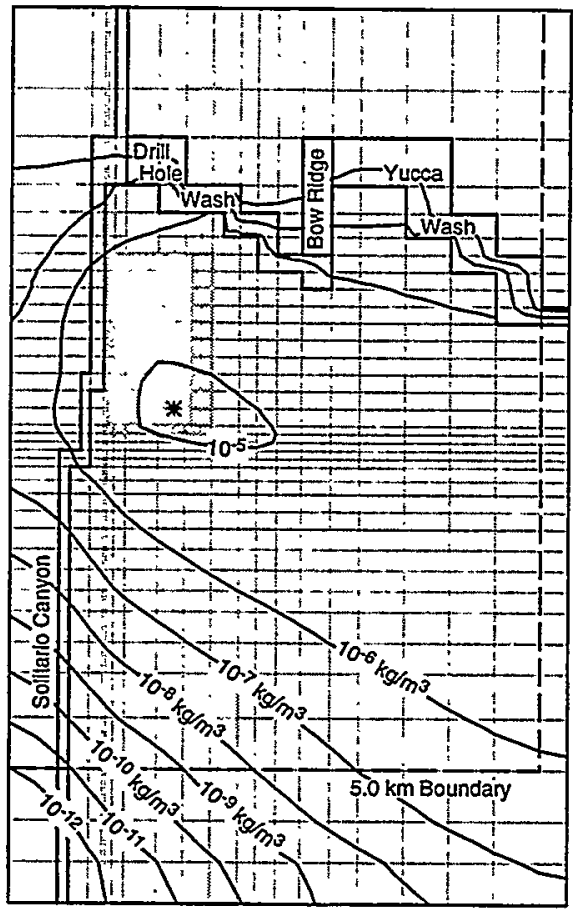

(d) $\mathrm{CHnz}$

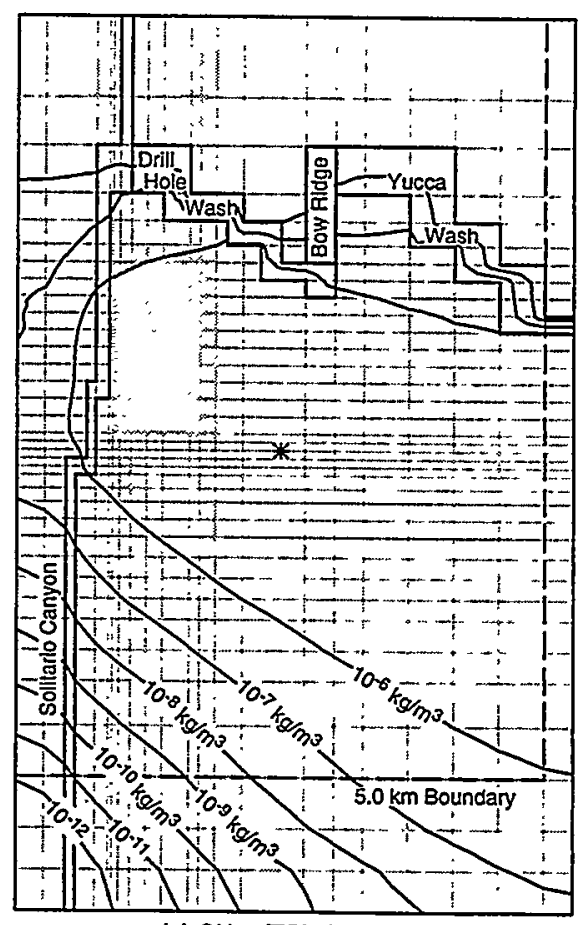

(e) CHnv/TSIv Interface

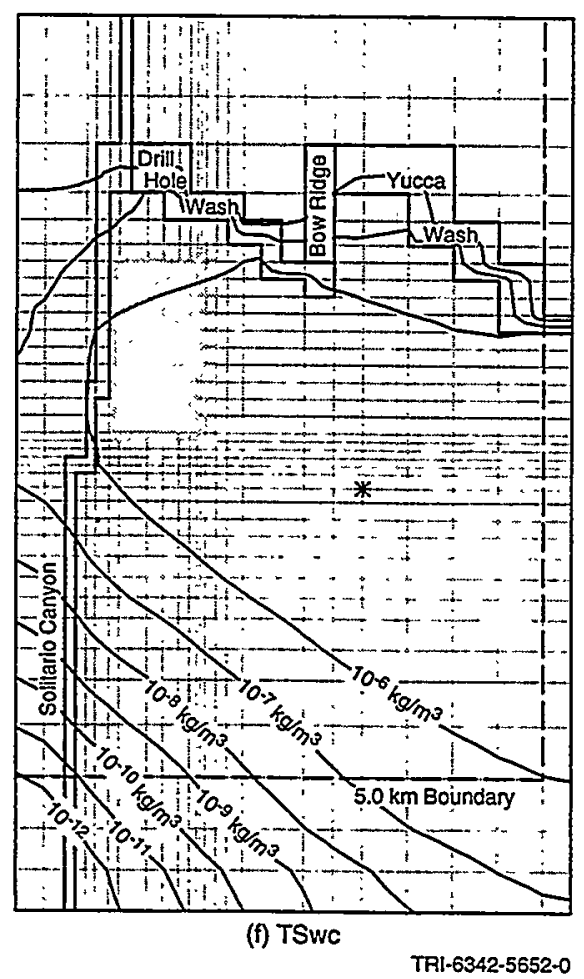

Figure 9-5. ${ }^{99}$ Tc concentrations at 40,000 yr for various units. (a) BFw, (b) CFun, (c) PPw, (d) CHnz, (e) CHnv/ TSlv interface, and (f) TSwc (continued). 


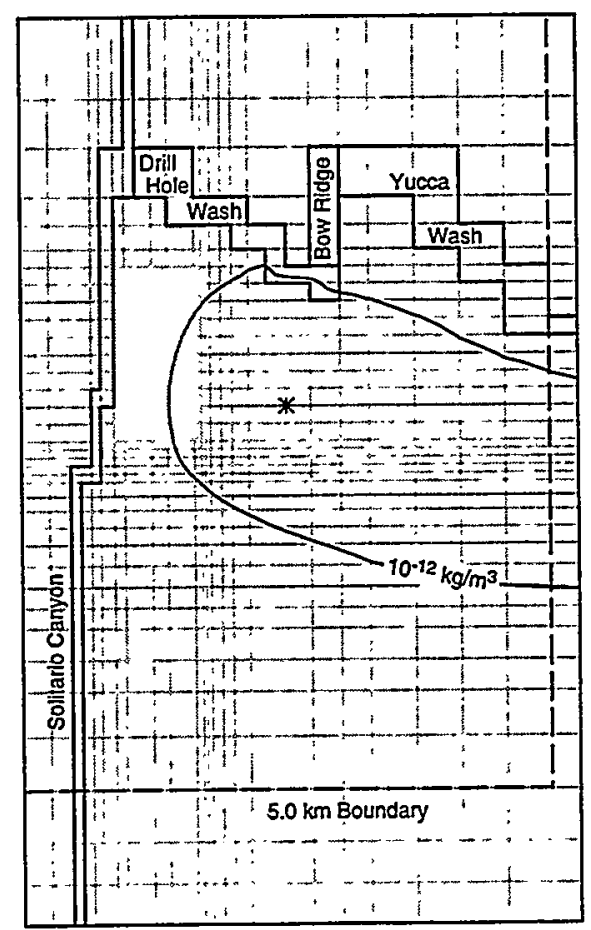

(a) 10,000 years

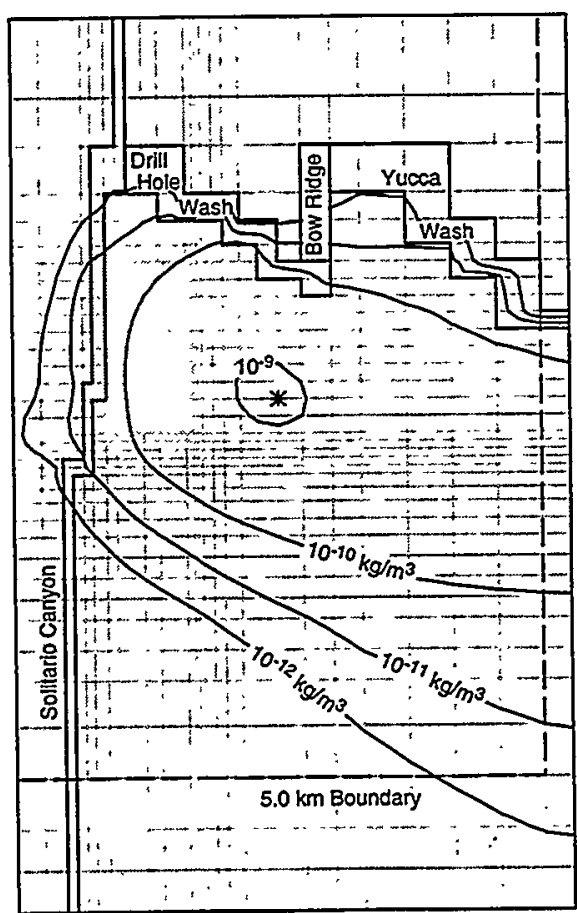

(c) 30,000 years

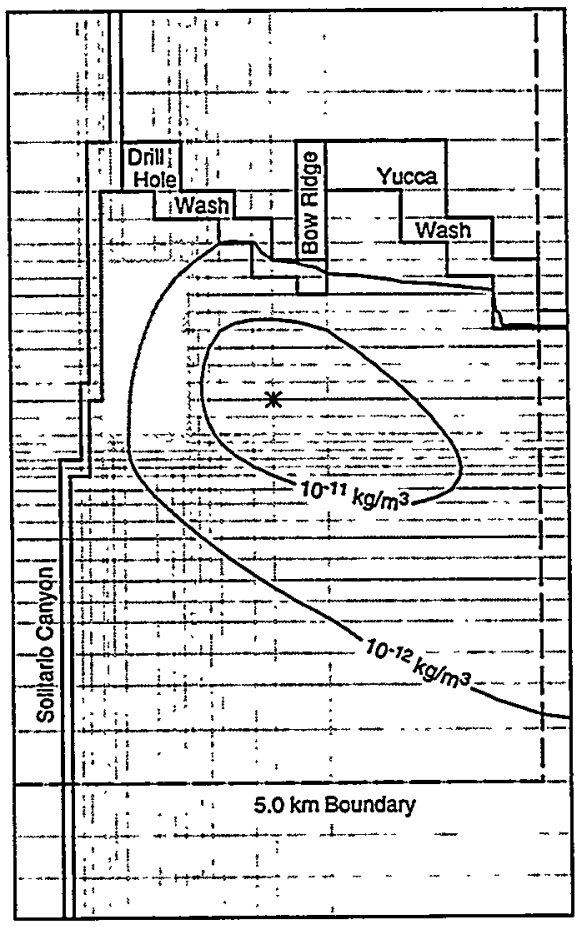

(b) 20,000 years

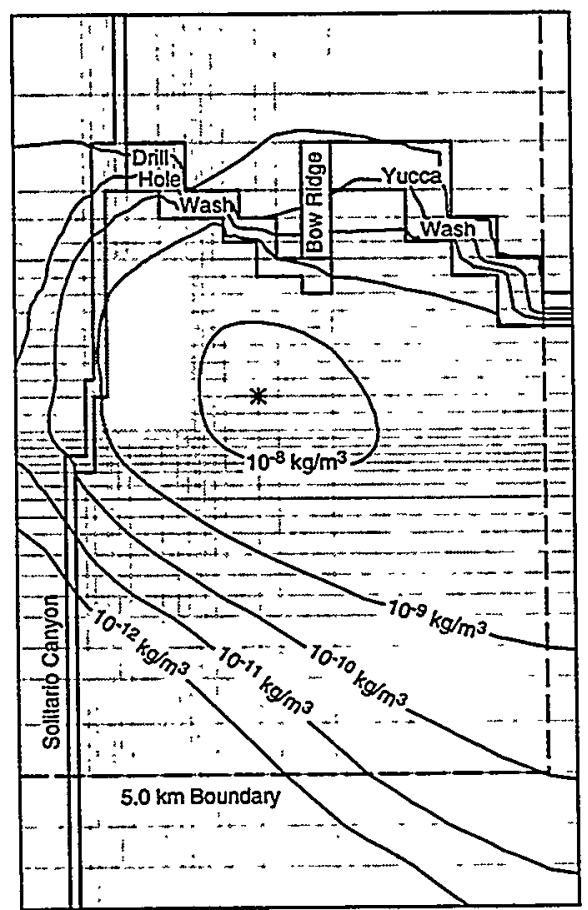

(d) 40,000 years

TRI-6342-5643-0

Figure 9-6. ${ }^{237} \mathrm{~Np}$ concentrations in the TSwc unit for various times. (a) 10,000 $y r_{,}$(b) 20,000 yr, (c) 30,000 yr, (d) 40,000 yr, (e) 50,000 yr, (f) 60,000 yr, (g) 70,000 yr, (h) 80,000 yr, (i) 90,000 yr, and (j) 100,000 yr. 


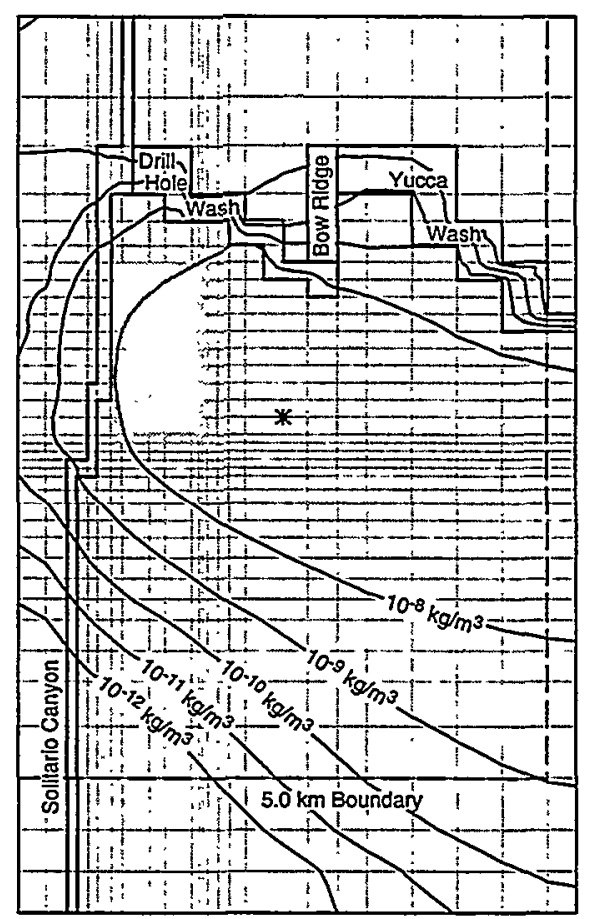

(e) 50,000 years

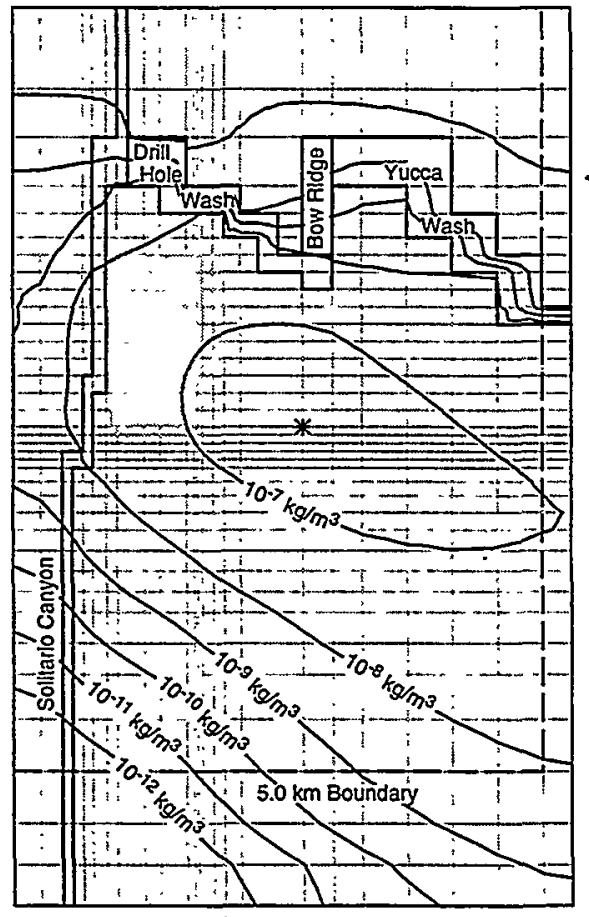

(g) 70,000 years

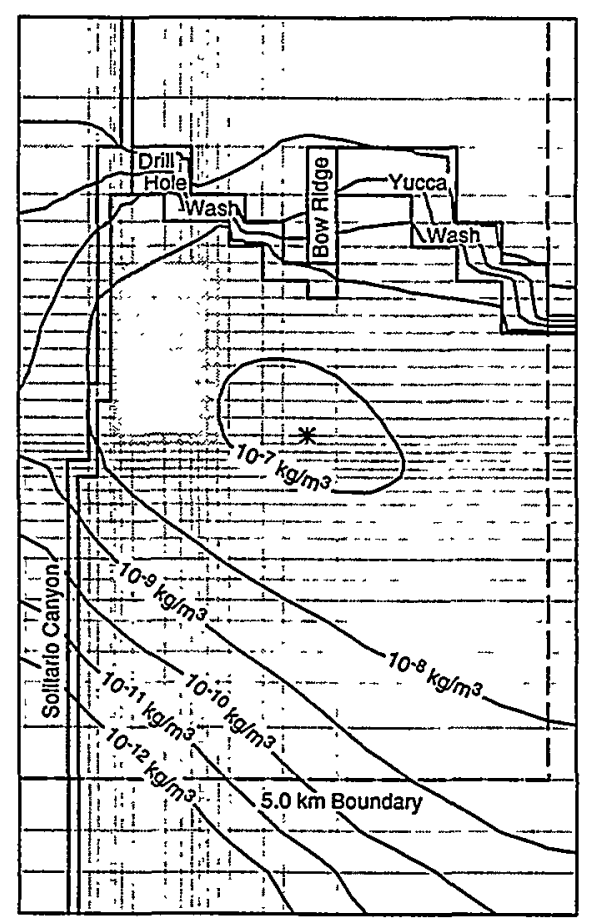

(f) 60,000 years

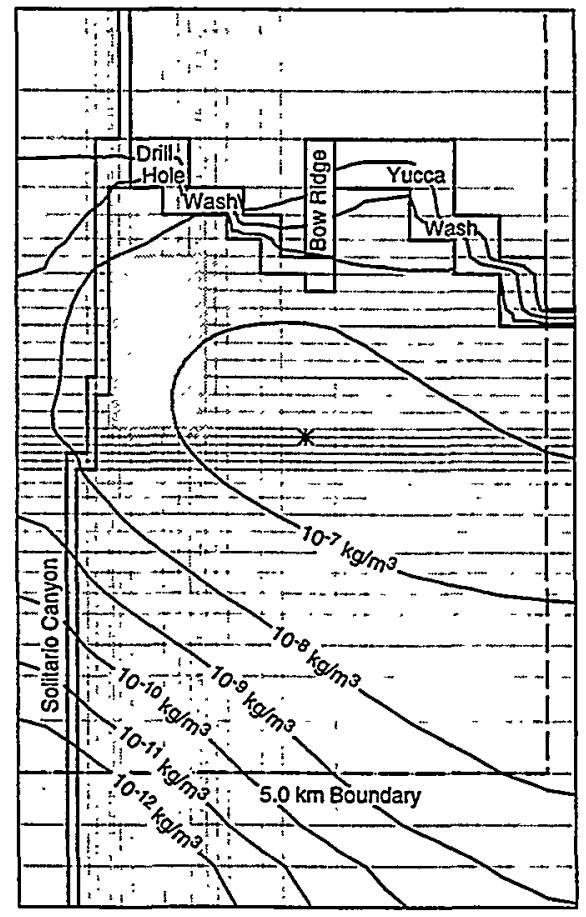

(h) 80,000 years

TRI-6342-5644-0

Figure 9-6. $\quad{ }^{237} \mathrm{~Np}$ concentrations in the TSwc unit for various times. (a) 10,000 yr, (b) 20,000 yr, (c) 30,000 $\mathrm{yr}$, (d) 40,000 yr, (e) 50,000 yr, (f) 60,000 yr, (g) 70,000 yr, (h) 80,000 yr, (i) 90,000 yr, and (j) 100,000 yr (continued). 


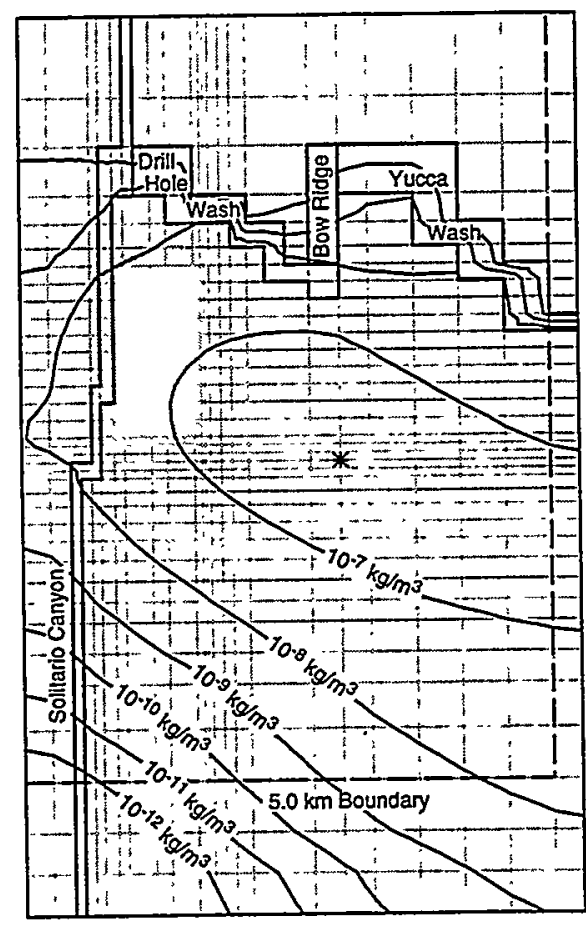

(i) 90,000 years

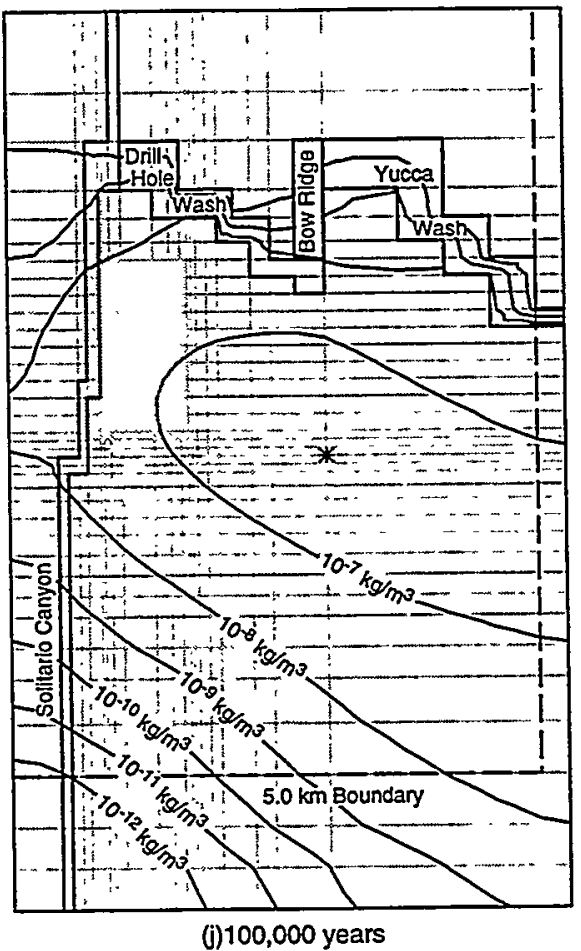

TRI-6342-5645-0

Figure 9-6. ${ }^{237} \mathrm{~Np}$ concentrations in the TSwc unit for various times. (a) 10,000 yr, (b) 20,000 yr, (c) 30,000 yr, (d) 40,000 yr, (e) 50,000 yr, (f) 60,000 yr, (g) 70,000 yr, (h) 80,000 yr, (i) 90,000 yr, and (j) 100,000 yr (continued). 


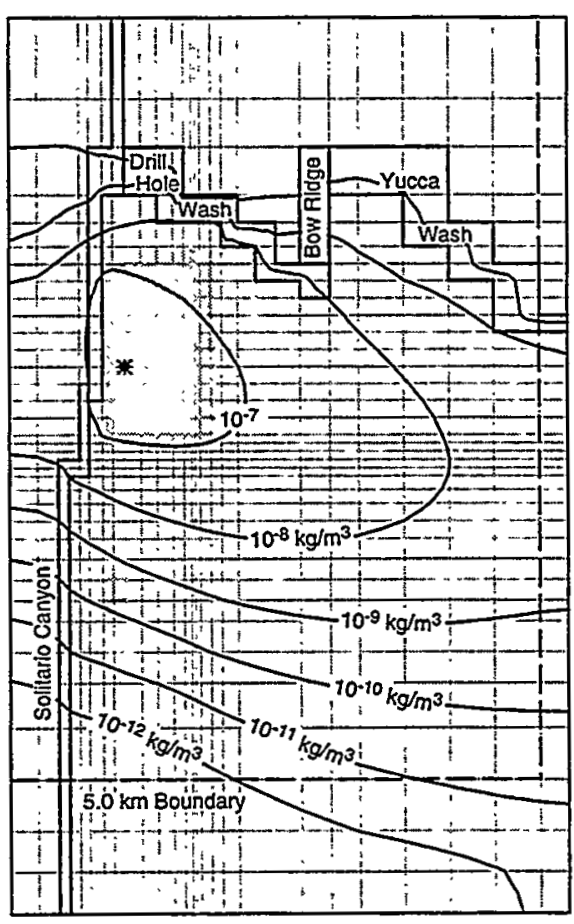

(a) BFW

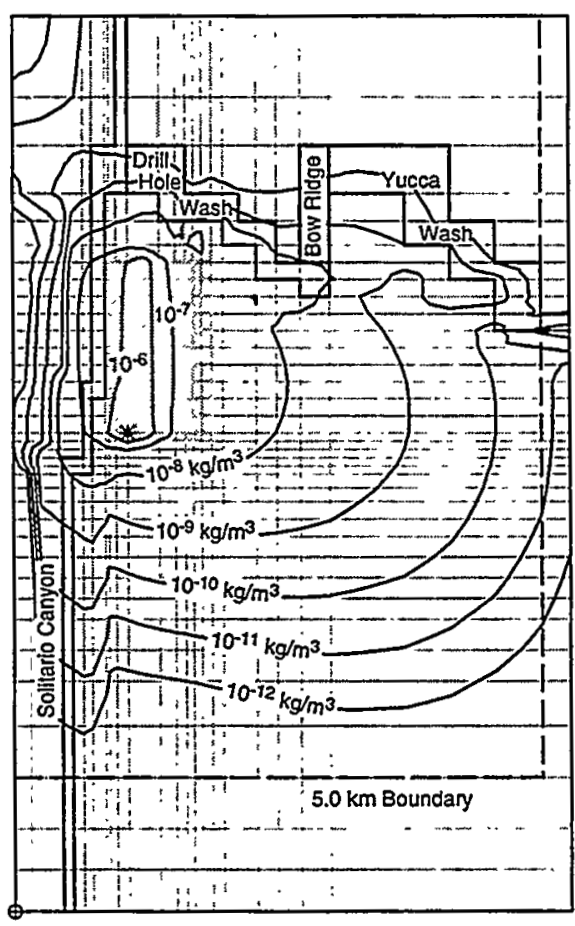

(b) CFun

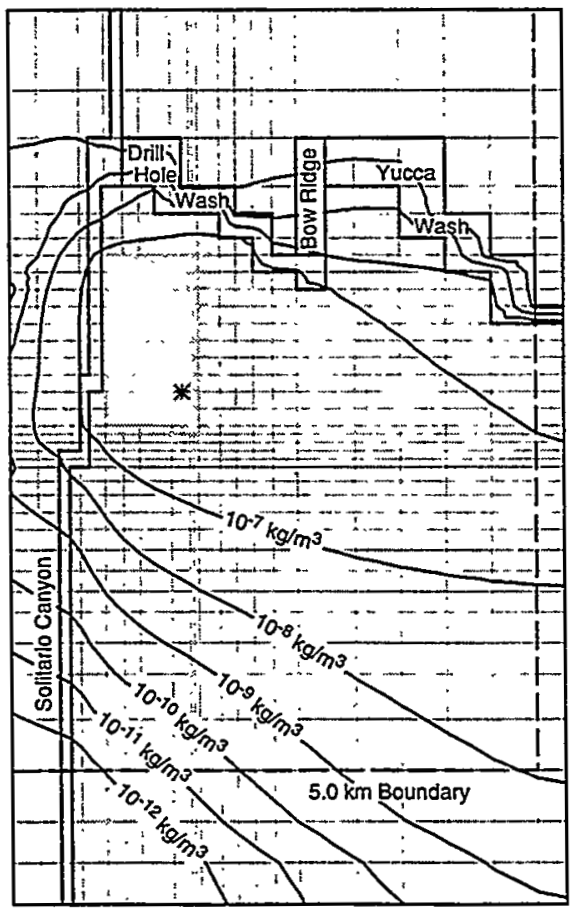

(c) PPW

TAI-6342-5646-0

Figure 9-7. $\quad{ }^{237} \mathrm{~Np}$ concentrations at 10,000 yr for various units. (a) BFw, (b) CFun, (c) $P P w$, (d) CHnz, (e) CHnv/ TSlv interface, and $(f)$ TSwc. 


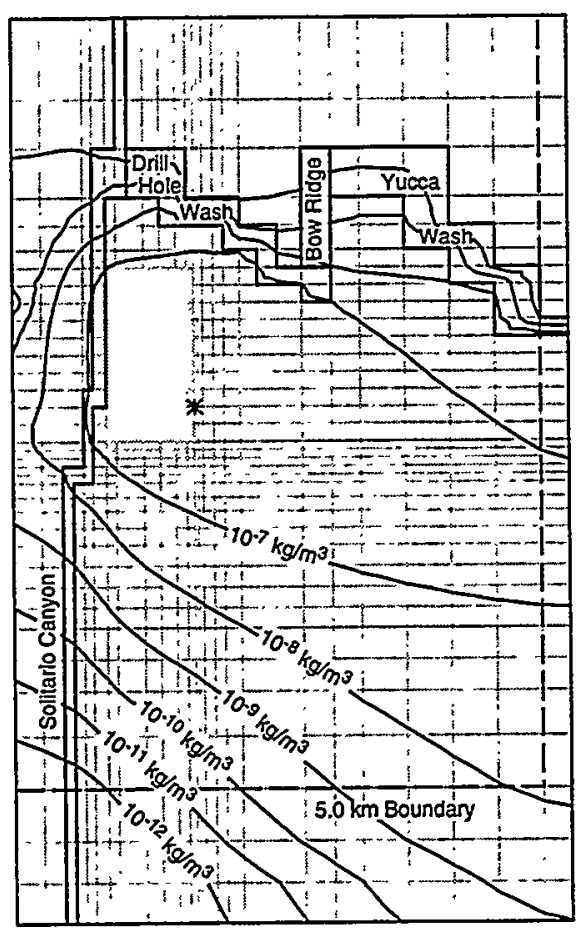

(d) $\mathrm{CHnz}$

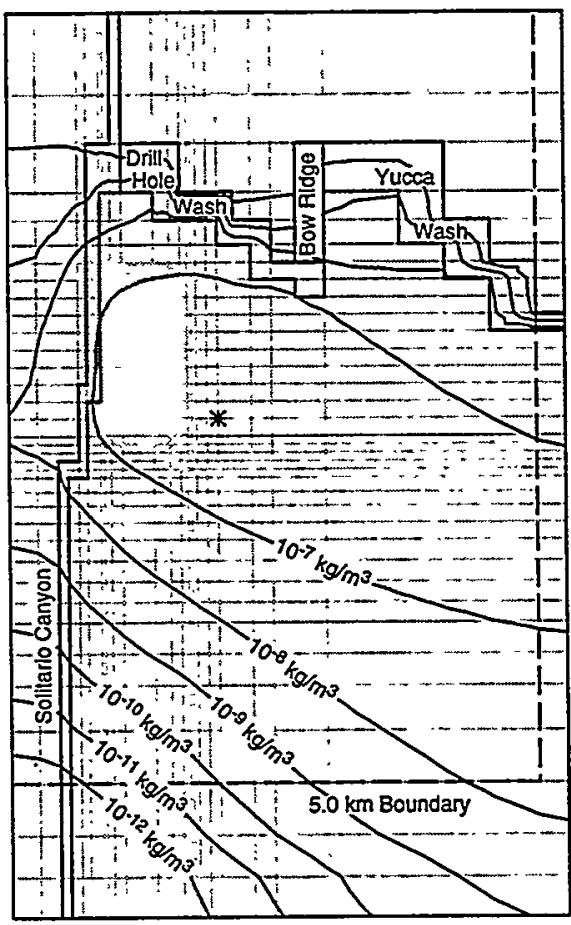

(e) CHnv/TSiv Interface

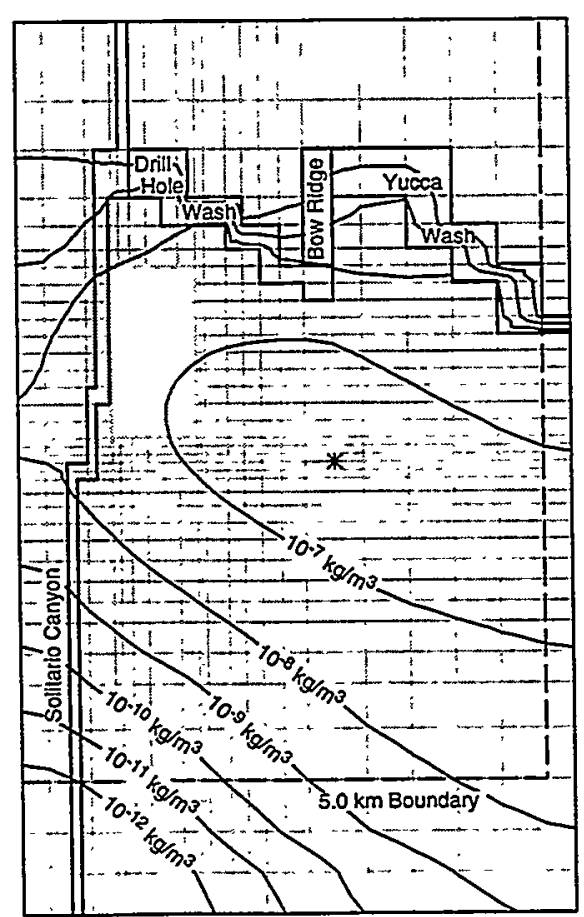

(f) TSwc

TRI-6342.5647-0

Figure 9-7. $\quad{ }^{237} \mathrm{~Np}$ concentrations at 10,000 yr for various units. (a) $B F w$, (b) CFun, (c) $P P w$, (d) CHnz, (e) CHnv/ TSlv interface, and (f) TSwc (continued). 


\title{
10. Consequence Modeling: Biosphere Transport and Dose
}

\author{
R. D. McCurley and L. J. Rahal
}

\subsection{Introduction}

In previous performance assessments, the most significant performance metric was cumulative radioactive release; this criterion was selected because of the Containment Requirements in 40 CFR 191. However, more recent guidance from the NAS (NAS/NRC, 1995) indicates that criteria similar to the Individual Protection Requirements of 40 CFR 191 should now take precedence. The soon to be promulgated 40 CFR 197 will delineate these requirements. In the $1997 \mathrm{PA}$, dose calculations were based on critical pathways, modeled with the code, GENII-A. These calculations comprise three distinct exposure scenarios. One exposure scenario dominated by drinking water was used for purposes of comparison with dose calculated in a-TSPA-1997. This chapter describes the methodology used for biosphere transport and dose in the 1997 PA, the critical pathways under consideration, the functions of GENII-A, and related parameters.

\subsubsection{Assumptions for Dose Calculations}

In response to guidance provided by the NAS (NAS/NRC, 1995), the performance metric for the 1997 PA is the maximum annual effective dose equivalent (EDE) to an individual from release of radioactivity by way of the potential repository. In this study, as in a-TSPA-1997, the individual is assumed to be the maximally exposed individual*, i.e., an individual located at a point on the accessible environment $(5-\mathrm{km})$ boundary that corresponds to the peak of the radionuclide concentration within an aquifer in the saturated zone. An additional calculation considers a likely member of a critical group, i.e., the average local resident of a similar location. Note that a difference exists between a-TSPA-1997 and the 1997 PA with regard to defining the location of peak radionuclide concentration. In the 1997 PA, STAFF3D produces a contour of concentrations in the aquifer, which indicates not only the location (in a 3-dimensional environment) in the saturated zone where the concentration is highest but also the time period at which the concentration is highest; in the $1997 \mathrm{PA}$, no additional dilution is assumed in establishing the peak concentration.

\subsubsection{Methodology}

Dose to humans was evaluated in the 1997 PA by means of the software code, GENII-A. In addition, calculations similar to the method used in a-TSPA-1997 were conducted for purposes of comparison.

Evaluating Dose with GENU-A. In the 1997 PA, the software code GENII-A (Napier et al., 1988a,b,c) was used to evaluate individual dose from the migration of radioisotopes through the biosphere that reach the accessible environment by means of a water well. The 1997 PA considers three main exposure scenarios: (1) a Ranch case, in which a rancher is exposed to radionuclides by means of beef consumption only, (2) a Farm case, in which a member of a farming family is exposed to radionuclides by means of food consumption, water consumption, and inhalation, and (3) a Small Community case, in which an average resident of a small community uses the contaminated water for drinking and consumes some foods grown locally from the Farm activities, including vegetables, fruits, dairy, and meat products.

For all cases, dose is evaluated from peak concentrations of the transported radioisotopes- ${ }^{129} \mathrm{I},{ }^{237} \mathrm{~Np},{ }^{99} \mathrm{Tc}$-at the 5- $\mathrm{km}$ boundary. The location of the highest concentrations of the radioisotopes was used to establish the well

* Dose to a maximally exposed individual is more conservative than dose to an average member of a critical group, because members of the critical group may have differing lifestyles and be situated in different locations. However, use of the critical group has also been recommended by the NAS (NAS/NRC, 1995). 
location, i.e., as if the Rancher, Farmer, or Small Community had drilled the water well into the saturated zone at the point on the $5-\mathrm{km}$ boundary that showed the highest radioisotope concentrations. (In the 1997 PA, the highest concentrations for that time period were from ${ }^{99} \mathrm{Tc}$ and ${ }^{129} \mathrm{I}$, so the same location was used for ${ }^{237} \mathrm{~Np}$ concentrations.) For these cases, dose is evaluated as a function of time for the mean and median runs. GENII-A receives the concentrations for the transported radioisotopes- ${ }^{129} \mathrm{I},{ }^{237} \mathrm{~Np}$, and ${ }^{99} \mathrm{Tc}$ - from STAFF3D.

Note that modeling these exposure cases necessarily incorporates some unrealistic assumptions. For example, both the Ranch and Farm cases assume farming and/or irrigation practices based on those currently practiced in the Amargosa Valley, about $80 \mathrm{~km}$ south of the potential repository. The principal source of water in the Amargosa Valley originates in the valley-fill aquifer (see Chapter 4, Figure 4-7), which is actually unsaturated near Yucca Mountain; therefore, the 1997 PA models the lower volcanic aquifer, which is primarily in the PPw and CFun units (see Chapter 4, Figure 4-9) and a less likely source of water for farming purposes.

Supplemental Calculations. For comparison of results with a-TSPA-1997, additional calculations were performed to compare the effective dose from saturated zone dilution to dilution as in a-TSPA-1997. The dilution methods for a-TSPA-1997 are the same as those as discussed in TSPA-1995, i.e., (a) the stirred tank model, as discussed in Section 7.6.2 of TSPA-1995 (M\&O, 1995a) and (b) the infinite homogenous aquifer with steady-state transport, as discussed in Section 7.6.3 of TSPA-1995.

Stirred Tank Model. The fluxes and concentrations in the unsaturated zone for the stirred tank model correspond to the values predicted by the NUTS code at the time and location of the peak ${ }^{99} \mathrm{Tc}$ value, that is, the values for ${ }^{129} \mathrm{I}$ and ${ }^{237} \mathrm{~Np}$ are taken at the same location and the same time. The maximum mass flux value for ${ }^{99} \mathrm{Tc}$ occurs at timestep 349 at $7625 \times 10^{11} \mathrm{~s}$ (or about $24,000 \mathrm{yr}$ ), a value of $1.4464 \times 10^{-7} \mathrm{~kg} / \mathrm{s}$. Using a saturated zone Darcy velocity of $0.31 \mathrm{~m} / \mathrm{s}$ and a screened cross-section of $4000 \mathrm{~m} \times 50 \mathrm{~m}$, the diluted concentration in the stirred tank model is

$$
\mathrm{C}_{\mathrm{sZ}}^{\mathrm{Tc} 99}=\frac{1.4464 \times 10^{-7} \mathrm{~kg} / \mathrm{s}}{(0.31 \mathrm{~m} / \mathrm{yr})(4000)(50) \cdot \mathrm{yr} / \mathrm{s} \cdot \mathrm{m}^{3} \mathrm{~s}}\left(=7.365 \times 10^{-5} \mathrm{~kg} / \mathrm{m}^{3}\right)
$$

Assuming consumption of 2 liters of drinking water per day, the 50 -yr committed dose from ${ }^{99} \mathrm{Tc}$, using an internal dose factor of $2.2 \times 10^{3} \mathrm{rems} / \mathrm{Ci}$ as calculated by GENII- $\mathrm{A}^{\dagger}$ is

$$
\text { dose }=7.365 \times 10^{-5} \mathrm{~g} / \mathrm{m}^{3} \cdot 16.96 \mathrm{Ci} / \mathrm{kg} \cdot 2.2 \times 10^{3} \mathrm{rem} / \mathrm{Ci} * 0.73=2.01 \mathrm{rem}
$$

Homogenous Infinite Aquifer Model with Steady-State Transport. The equations for this model are from TSPA1995 (M\&O, 1995a), where the dilution in the saturated zone at $5.0 \mathrm{~km}$ is calculated by

$$
S=\frac{q_{s z} I r \sqrt{\pi / \beta_{z}}}{q_{u z} A_{r e p} \operatorname{erf}\left\{\frac{1}{4 r \sqrt{\beta_{z}}}\right\}}
$$

where

$$
\begin{array}{ll}
\mathrm{S} & =\text { dilution in saturated zone at } 5.0 \mathrm{~km} \\
\mathrm{q}_{\mathrm{sz}} & =\text { saturated zone Darcy velocity } \\
\mathrm{q}_{\mathrm{uz}} & =\text { unsaturated zone Darcy velocity } \\
1 & =\text { length of source of water } \\
\mathrm{r} & =\text { distance from repository in direction of flow } \\
\mathrm{A}_{\mathrm{rep}} & =\text { contaminated flow area of repository } \\
\beta_{\mathrm{y}} \beta_{\mathrm{z}} & =\text { transverse dispersivity coefficients (longitudinal dispersivity is reflected). }
\end{array}
$$

Note that the internal dose factor for ${ }^{99} \mathrm{Tc}$, as calculated by GENII-A, is about $2.2 \times 10^{3} \mathrm{rems} / \mathrm{Ci}$, a value about $50 \%$ higher than the internal dose factor referenced by TSPA-1995 (as reported in Eckerman et al., 1988). 
Given the values for $q_{u z}, q_{s z}, 1, A_{\text {rep }}$ as reported in a-TSPA-1997 (M\&O, 1997), and $\beta_{y}=\beta_{z}=0.001$, then

$S=140.1$

Using Equation 7.6-10 of TSPA-1995 (M\&O, 1995a),

$$
\mathrm{S}=\frac{\mathrm{Cuz}_{\mathrm{uz}}}{\mathrm{C}}
$$

where,

$\mathrm{C}_{\mathrm{uz}}=$ concentration in the unsaturated zone where water flows into the saturated zone

$\mathrm{C}=$ concentration at the $5-\mathrm{km}$ boundary,

then the values of concentration for ${ }^{129} \mathrm{I},{ }^{99} \mathrm{Tc}$, and ${ }^{237} \mathrm{~Np}$ are $7.099 \times 10^{-7} \mathrm{~kg} / \mathrm{m}^{3}, 2.785 \times 10^{-6} \mathrm{~kg} / \mathrm{m}^{3}$, and $8.00 \times 10^{-10} \mathrm{~kg} / \mathrm{m}^{3}$, respectively. These values are based on corresponding peak concentrations in the unsaturated zone of $9.947 \times 10^{-5} \mathrm{~kg} / \mathrm{m}^{3}, 3.902 \times 10^{-4} \mathrm{~kg} / \mathrm{m}^{3}$, and $1.121 \times 10^{-7} \mathrm{~kg} / \mathrm{m}^{3}$ taken from NUTS (Joel Miller, Sandia National Laboratories, personal communication, December 1997).

The corresponding doses for consumption of 730 liters of drinking water per year ( 2 liters per day) are $22.9 \mathrm{mrem}, 75.8 \mathrm{mrem}$, and $2.1 \mathrm{mrem}$ for ${ }^{129} \mathrm{I},{ }^{99} \mathrm{Tc}$, and ${ }^{237} \mathrm{~Np}$, respectively. The total whole-body 50 -yr committed dose is $100.9 \mathrm{mrem}$.

\subsubsection{Criteria}

The criterion examined for the Farm and Ranch cases was the maximum annual effective dose equivalent (EDE) to an individual. For the Small Community, the criterion was that the person be a member of the most probable critical group. The 1997 PA calculated the variation over a 100,000-yr period of the committed dose for a 50-yr segment of an individual's life after one year of exposure. Although the performance measure of maximum individual dose can be represented by a complementary cumulative distribution function (CCDF), the Individual Protection Requirements from 40 CFR 191 state simply that the mean of the doses from the disposal system of a case without an anthropogenic disturbance must be below $150 \mu \mathrm{Sv}$ ( $15 \mathrm{mrem})$. Hence, for comparison, the performance measure was the mean of the doses; see also Section 10.3.1 for a description of EDE.

\subsection{Description of Critical Pathways}

In developing the model for GENII-A, critical pathways are defined, which refer to a sequence of hypothetical pathways by which radionuclides from a repository could reach humans. The sequence may consist of one pathway, e.g., in the Ranch case, only ingested meat is considered. Alternatively, there may be several simultaneous pathways, e.g., ingestion and inhalation, as for the Farm case. Figure 10-1 illustrates the pathways considered in the 1997 PA. Both cases assume that a water well is drilled at the $5-\mathrm{km}$ boundary at some time after loss of institutional control.

\subsubsection{Critical Pathway for Ranch Case}

The critical pathway (Figure 10-1a) for the Ranch case involves exposure to an individual living on a cattle ranch, who consumes meat from cattle. The cattle drink water from a tank of contaminated water that has been filled by means of a well located at the $5 \mathrm{~km}$ boundary. The cattle also consume feed grown on the ranch, which was irrigated by the same well. Meat is assumed to be the only contaminated food consumed (i.e., the rancher does not grow crops for human consumption). No other pathways are considered in this case. 


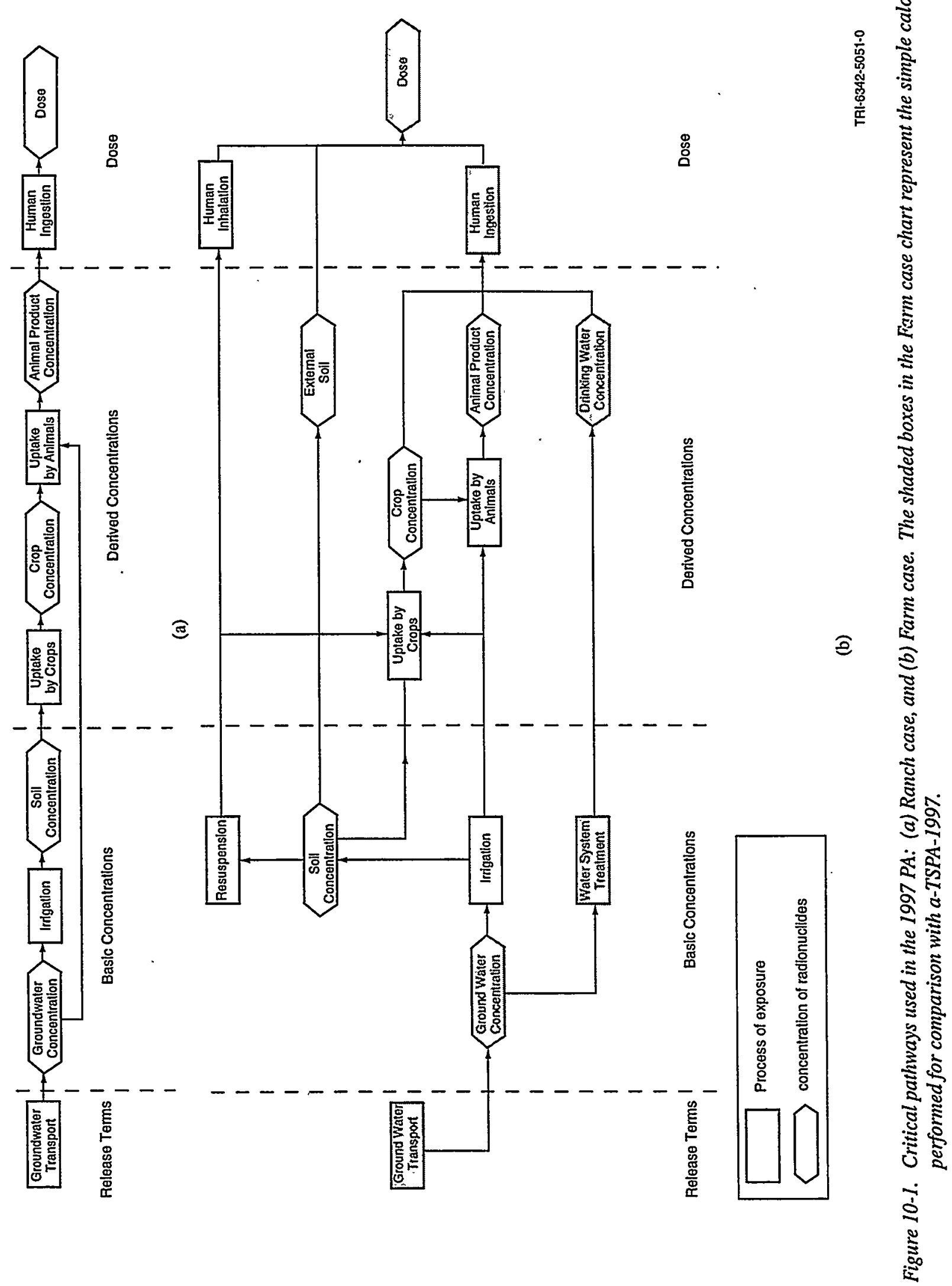


For this pathway, it was assumed that sufficient water is supplied by a well for the drinking requirements of typical cattle in the area and for irrigation purposes to grow cattle feed.

\subsubsection{Critical Pathways for Farm Case}

Farm Case. The pathway (Figure 10-1b) for the Farm case considers exposure of a member of the farm family from a well drilled into the aquifer in the saturated zone. Enough water is supplied by the well to support the requirements of a modest farm. These include growing and consumption of crops and animal products as well as drinking and irrigation from the well source. The well is located at the $5-\mathrm{km}$ boundary.

Besides water and food consumption, inhalation is also considered as a critical pathway (Figure 10-1b) in the Farm case. Contaminated soil dries, becoming dust in the air, which is inhaled by the farm family member. Thus, the concentration from inhalation is included in the total dose.

\subsubsection{Critical Pathways for Small Community}

The critical pathways for a member of the Small Community are qualitatively similar to those for the Farm family, except that inhalation is not considered. A resident in a small community consumes tap water from a well located at the $5-\mathrm{km}$ boundary as the major source of drinking water. The resident also consumes some foods that are grown locally from Farm activities, including vegetables, fruits, dairy, and meat products, as described for the Farm case. However, the resident obtains most food from sources that are not local.

\subsection{GENII-A: Dosimetry Model}

For the $1997 \mathrm{PA}$, potential doses resulting from exposure to radionuclides hypothetically released from the repository were calculated for the Ranch and Farm cases. The principal code for this calculation is the GENII-A code (Napier et al., 1988a,b,c). GENII-A, which was first developed at Pacific Northwest Laboratories, calculates radiation doses to individuals or populations from acute or chronic radionuclide releases to air, water, or soil. Dose calculations performed by GENII-A follow the ICRP guidelines presented in ICRP Publication 26 (ICRP, 1977) and Publication 30 (ICRP, 1979).

\subsubsection{Types of Doses Calculated}

Three types of doses are calculated by GENII-A: (1) internal EDEs, (2) external dose, and (3) total EDEs. GENII-A calculates all dose types described below, but only the committed EDE is reported in the 1997 PA.

Internal Effective Dose Equivalents (EDEs). Internal EDEs are either committed EDEs or cumulative EDEs. A committed EDE is the dose that is expected to occur over an individual's or a population's lifetime as a result of an intake from ingestion or inhalation of radionuclides for a period of one year. Figure 10-2 is a schematic representation of the extended internal dose expected from an initial exposure. It is referred to as an EDE because it is not an actual dose, but rather a prediction of the dose that will occur due to radionuclides present in an individual's body.

In Figure 10-2, intake represents a constant rate of radionuclide intake (from inhalation and/or ingestion) and dose rate represents the resulting dose rate to tissue or organ. The integral of the dose rate over 50 or $70 \mathrm{yr}$ gives the committed dose (i.e., the area under the dose-rate curve) resulting from one year of intake. EDEs for 50- and 70-yr commitment periods are typical for dose calculations; in GENII-A, these time periods are input by the user. The 1997 PA calculated 50-yr committed EDEs. 

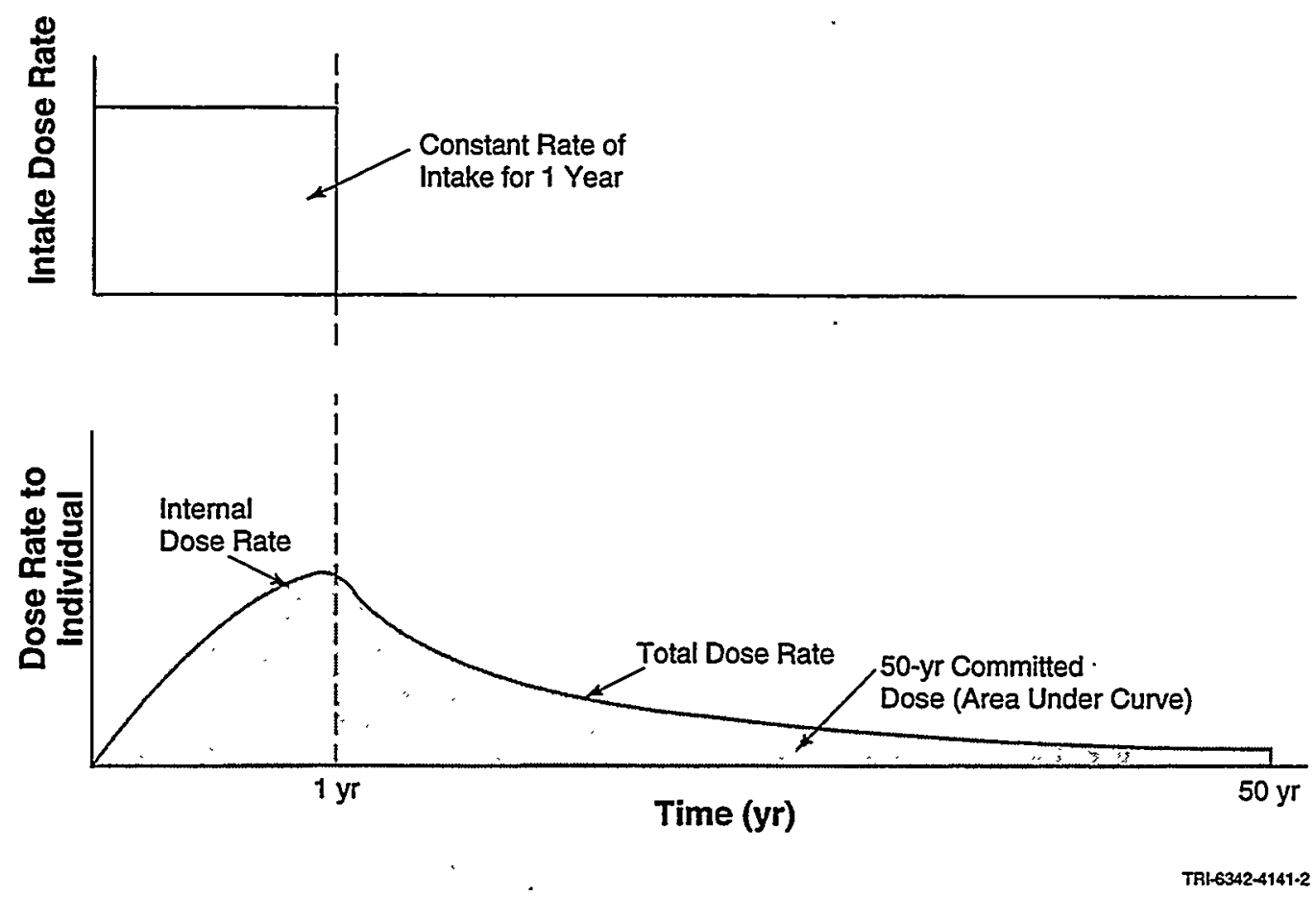

Figure 10-2. Situation showing an internal effective dose equivalent (EDE) as a committed EDE (area under curve) from 1-yr internal intake dose. The 1997 PA reports dose as the 50-yr committed EDE.

A cumulative EDE is the dose that is expected over an individual's or a population's average lifetime as a result of continued intake (greater than one year) of radionuclides. The intake period for cumulative EDE can vary from greater than one year to the expected lifetime. Although 50-yr and 70-yr EDEs are common, GENII-A accepts any time period. (Cumulative EDEs are not reported in the 1997 PA.)

External Dose. The external dose calculated by GENII-A is an actual dose that results from exposure to the source for any length of time. Exposure times are specified in the GENII-A input. (External dose is not reported in the 1997 PA.)

Total Effective Dose Equivalent (EDE). The total dose is the whole-body effective dose equivalent (WEDE), representing the sum of the internal and external doses (not reported in the 1997 PA).

\subsubsection{Data Input}

GENII-A has the potential to calculate radiation doses resulting from internal and/or external exposure to contaminated air, water, soil, or to products derived from contaminated air, water, or soil. Data may be entered into the code in one of three ways.

In the $1997 \mathrm{PA}$, data were entered as the concentration of radionuclides in the groundwater and surface soil at the exposure location. In this case, GENII-A calculates the subsequent dose to an individual at that location. (GENII-A does not have the capability to calculate groundwater transport.) With this method, data can also be input as radionuclide concentrations in the air or surface water, but these inputs were not used in the 1997 PA.

The second method of data input that is available with GENII-A (but not used in the 1997 PA) is to specify a release of radioactive material into the air, surface water, or deep soil. Given the release information, GENII-A will 
estimate the movement of the radioactive material to the exposure location and calculate the subsequent doses to an individual or population there. In the third method, the concentration of radionuclides in consumer goods derived from contaminated air, water, or soil may be specified (not used in the $1997 \mathrm{PA}$ ). For example, the radionuclide concentration in milk or drinking water may be given, which GENII-A will use to calculate the subsequent dose to an individual or population who consumes the food product(s).

The extent of the radiological damage to various organs of the human body was calculated in the 1997 PA. This damage depends, calculationally, on transfer factors that determine how much of the ingested contamination is transferred into various organs (see Table 10-1) and on factors called "internal dose factors." Internal dose factors relate radiation types $(g, B, \alpha)$ and energies, e.g., from nuclear disintegration to biological effects, measured in units of rem or sieverts (Sv) (1 Sv equals $100 \mathrm{rem}$ ). Doses in this report are in units of rem unless indicated otherwise. For the organ calculations, the various human organs are weighted as listed in Table 10-1 (ICRP, 1977):

Table 10-1. Various Human Organ Weights

\begin{tabular}{lc}
\hline \multicolumn{1}{c}{ Organ } & $\mathrm{W}_{\mathrm{j}}$ (weight by organ index) \\
\hline gonads & 0.25 \\
breast & 0.15 \\
red bone marrow & 0.12 \\
lung & 0.12 \\
thyroid & 0.03 \\
bone & 0.03 \\
remainder & 0.30 \\
\hline
\end{tabular}

\subsubsection{Calculating Dose}

GENII-A calculates both internal and external dose, although only internal dose is reported in the 1997 PA.

Internal Dose Calculations. Internal dose factors are calculated in subroutine INTDF of GENII-A. This subroutine calculates the incremental organ doses for each year following an initial intake. The following theory supporting the internal dose calculations is presented as general information for understanding the model used in the 1997 PA.

The mean absorbed dose rate on the jth organ (or tissue) $D_{R, j}^{k}$ due to radionuclide $k$ or radiation type $R$ is evaluated. This evaluation involves three primary factors: (1) the activity in source region, $\Omega$ irradiating the organ $A_{\Omega}$, (2) the mean energy emitted by radionuclide $k$ or radiation type $R\left(\Delta_{R}\right)$, and (3) the ratio of energy absorbed by the jth organ to energy emitted at the source region $\Phi_{R}$ :

$$
\overline{\bar{D}}_{R, j}^{k}=A_{\Omega}(\tau) \cdot \Delta_{R} \Phi_{R}
$$

is the mean absorbed dose rate or rate of energy sorption in organ $j$ per unit mass. Because there is more than one type of radiation, a sum over radiation types is performed:

$$
\overline{\dot{D}}_{R, j}^{k}=A_{\Omega}(\tau)\left(\sum_{R} \Delta_{R} \Phi_{R}\right) .
$$


The activity may also be present in more than one source organ. Therefore, a sum over source regions, $\Omega$, is performed:

$$
\overline{\dot{D}}_{R, j}^{k}=\sum_{\Omega} A_{\Omega}(\tau) \sum_{R} \Delta_{R} \Phi_{R}
$$

Now the quantity $S$, the mean absorbed dose rate in organ $j$ per unit of radioactivity in $\Omega$, is defined as

$$
S=\sum_{R} \Delta_{R} \Phi_{R}
$$

Therefore,

$$
\bar{D}_{R, j}^{k}=\sum_{\Omega} A_{\Omega}(\tau) S
$$

The $S$ factor can be written in terms of dose equivalent $\stackrel{\ddagger}{\ddagger}$ by inclusion of modifying factors $Q_{R}$ and $N$ where $Q_{R}$ is called the quality factor that weights the absorbed dose according to the biological effectiveness of the radiation type producing the dose and $N$ is the product of all other modifying factors. Presently, $N=1$ is assigned by the ICRP.

$$
S=\sum_{R} \Delta_{R}\left(Q_{R} \cdot N\right) \Phi_{R}
$$

ICRP (1979), in recommendations on secondary limits, defined the specific effective energy quantity $(S E E)_{R}$ as

$$
(S E E)_{R}=\sum_{R} \Delta_{R}\left(Q_{R} \cdot N\right) \Phi_{R}
$$

Therefore, based on this definition

$$
\overline{\dot{D}}_{R, j}^{k}=\sum_{\Omega} A_{\Omega}(\tau)(S E E)_{R}
$$

The absorbed dose rate to the organ is from radionuclide $R$ with radiation type $j$ given by $\bar{D}_{R, j}^{k}$. The absorbed dose rate $\bar{D}_{R, j}^{k}$ is used to calculate the committed equivalent dose (CED) over the selected commitment period $T$

$\mp \quad$ The dose equivalent of the point $P$ has been defined by the ICRP (1977) as

$$
H(P)=D(P) Q(P) N
$$

$D(P)$ is the absorbed dose expressed as

$$
D(P)=\text { limit } \Delta \mathrm{V} \rightarrow \mathrm{P} \quad\left(\frac{\Delta \vec{E}}{\Delta M}\right) .
$$

where $\Delta \bar{E}$ is the mean energy emitted by the radiation event.

Note: The absorbed dose is an integral quantity that corresponds to the deposition of energy over time. The absorbed dose is considered inadequate for the prediction of health effects that are associated with irradiation of tissue and therefore the modified quantity, dose equivalent, was defined above. The term "dose" is generally used in place of "dose equivalent." 


$$
H_{j}^{k}=\int_{0}^{T} \overline{\dot{D}}_{R, j}(\tau) \cdot W_{R} d \tau
$$

$W_{R}$ is the appropriate weighting factor for the type of radiation. The above derivation is taken from Till and Meyer, eds. (1983). The procedure used in the GENII-A code for the calculation of internal dose follows the method outlined above.

In subroutine INTDF of GENII-A, estimates of the dose equivalents in a number of target organs are determined from the activity in a given source organ $S$. For each type of radiation $R$, the dose equivalent $H_{j, R}^{k}$ in $\operatorname{target}$ organ $j$ from radionuclide $k$ in source organ (region) $S$ is the product of two factors:

- the total number of nuclear transformations of radionuclide $k$ in source organ $S$ over the period of interest following intake (the committed dose period),

- the energy absorbed per gram in target organ $j$, modified with a "quality factor" (ICRP, 1977), from radiation of type $R$ per transformation of radionuclide $k$ in source organ.

For each radiation of type $R$ from radionuclide $k$, the dose equivalent is given by the expression

$$
H^{k}{ }_{j, R}=U_{S} \cdot 1.6 \cdot 10^{-13} \cdot(S E E)_{R} \cdot 10^{3}
$$

where $U_{S}=$ the number of transformations of radionuclide $k$, in source organ $S$, over the period of integration following intake of the radionuclide (i.e., the commitment period) and the factor $1.6 \times 10^{-13}$ is the number of joules in $1 \mathrm{MeV}$. ( $S E E)_{R}$ is the specific effective energy for radiation of type $R$ (in $\mathrm{MeV} / \mathrm{gm}$ ) modified by the quality factor, which is absorbed by organ $j$ from each radionuclide transformation emitted by the source organ. The factor $10^{3}$ converts $\mathrm{gm}^{-1}$ to $\mathrm{kg}^{-1}$.

In the general case, for the intake of a mixture of radionuclides, which consist of parents with daughters, the dose in the target organ $j$ from radioactivity emitted from several different source organs is given by

$$
H_{j}=1.6 \cdot 10^{-10} \cdot \sum_{S} \sum_{k}\left[U_{S} \cdot \sum_{R}(S E E)_{R}\right]_{k}
$$

The units of dose are in Sv (sieverts). The committed effective dose equivalent (CEDE) is calculated as outlined in ICRP 26 (1977) as

$$
\mathrm{CEDE}=\sum_{j} W_{j} \cdot H_{j}
$$

where the factor $W_{j}$ is the organ weighting factor given in ICRP 26 (1977) (see Table 10-1).

The CEDE (as outlined in ICRP 26 [1977]) for the whole body, is given by:

$$
\mathrm{CEDE}=\sum_{j} W_{j} H_{j}+\text { external dose }
$$

where $j$ is an organ index, $W_{j}$ is a weighting factor for a particular organ (see Table 10-1; also ICRP, 1977), and $H_{j}$ is a "dose equivalent" for that same organ. $H_{j}$ is calculated for the period of integration shown in Figure 10-2, which illustrates conceptually a 50-yr committed dose. $H_{j}$ also depends on the radiation types and energies from radioactive 
disintegrations in the body organs. A detailed description of these equations can be found in Volume 1, Hanford Environmental Radiation Dosimetry Software System (Napier et al., 1988a).

External Dose Calculations. External dose is calculated in the EXTDF subroutine of GENII-A, which is a modification of the shielding code ISOSHLD (Engel et al., 1966), using dose conversion factors from DOE/EH-0070 (DOE, 1988c). External dose was not reported in the 1997 PA.

\subsubsection{Types of Exposure}

The types of exposure potentially available in GENII-A are listed in Table 10-2; those used in the 1997 PA are highlighted in bold. Internal radiation doses arise through inhalation or ingestion. Inhalation exposures occur when an individual or population breathes in suspended radionuclide particulates. The particulates can be part of a contaminant plume that is advecting downwind or they can be resuspended particulates that were once deposited on surface soil. Ingestion exposures occur when an individual or population consumes a contaminated food product (drinking water, leafy vegetables, meat or fish that has undergone exposure). External radiation doses occur when an individual or population is exposed to (1) contaminated surface soil (groundshine), (2) radionuclide particulates suspended in air (finite or infinite plume), or (3) contaminated non-drinking water sources (bathing, swimming, boating, shoreline activities).

Table 10-2. Types of Exposure Included in the 1997 PA

\begin{tabular}{lll}
\hline \multicolumn{1}{c}{ Internal Exposure } & \multicolumn{1}{c}{$\begin{array}{c}\text { External } \\
\text { Exposure }\end{array}$} \\
\hline Finite Plume & \multicolumn{1}{c}{ Ingestion Exposure } & Groundshine \\
Infinite Plume & Leafy Vegetables & Finite Plume \\
Resuspension & Root Vegetables & Infinite Plume \\
& Grains & Swimming \\
& Fruit & Boating \\
& Milk & Shoreline \\
& Beef & \\
& Eggs & \\
& Poultry & \\
& Aquatic Foods & \\
& Soil & \\
& Swimming Water & \\
\hline
\end{tabular}

\subsubsection{Link to 1997 PA Data (PREGENII)}

GENII-A input data are specified by the code or by the user (see Section 10.3.2, Data Input), with default data contained in a file accessed by the code. Examples of inventory parameters are source conversion and soil-source conversion factors, environmental factors addressing absolute humidity, deposition velocity/resuspension, animal consumption, animal drinking water, acute fresh forage by season, vegetable production, and weighting factors for organ doses. The specific parameters used in the 1997 PA are listed in Section 10.4. 
To provide GENII-A with the source term concentrations and input appropriate for Latin Hypercube Sampling (LHS) capabilities, a linked software tool named PREGENII was applied to create GENII-A input for critical pathways. This process is represented schematically in Figure 10-3. PREGENII is primarily a keyword-driven preprocessing program that creates an ASCII input file that the GENII-A program can use to perform simulations of radionuclide exposure pathways to humans. Additionally, PREGENII reformulates concentrations from transport calculations to make them compatible with GENII-A expectations. Although not used in the 1997 PA, PREGENII has a built-in suspension model for generating atmospheric source terms for transport to an exposure site with GENII-A.

The postprocessor, POSTGENII, transfers output data (i.e., the EDE specified by radionuclide or origin) from GENII-A by translating the output to CAMDAT by means of a user-supplied POSTGENII text input file. The translation allows the suite of processing packages available in the overall modeling system, CAMCON, to be used for displaying results (see Chapter 6).

\subsection{Conceptual Models for Exposure Cases}

\subsubsection{Model for the Ranch Case}

The Ranch case involves exposure to a rancher who settles at the $5-\mathrm{km}$ boundary at the arrival time for peak radionuclide concentrations via transport through the aquifer, i.e., many centuries after closure.

Critical Pathway. The critical pathway in this case is ingestion of contaminated beef. The rancher sinks a well into the aquifer and uses its waters to irrigate feed crops for the cattle and to supply a stock tank for the cattle; the rancher subsequently consumes meat from the cattle. GENII-A calculates radionuclide concentrations in the beef and uptake by the rancher through ingestion. The doses reported in the $1997 \mathrm{PA}$ are for meat ingestion by the rancher at the location described in Section 10.1.2.

Baseline Data for Ranch Case. To model ingestion exposures, GENII-A requires the following information: (1) the rancher's beef consumption rate, (2) the cattle's drinking rate, (3) the cattle's forage rate, (4) the irrigation rate, and (5) the concentration of radionuclides in the groundwater (input from STAFF3D via PREGENII). The parameters for the Ranch case are listed in Table 10-3.

\subsubsection{Model for Farm Case}

The Farm case considers exposure of a member of a farm family by means of water from a well drilled into the aquifer in the saturated zone. Enough water is supplied by the well to support the requirements of a modest farm. The well is located at the $5-\mathrm{km}$ boundary.

Critical Pathways. In the Farm case, crops are irrigated with the contaminated water. The farm family member consumes (a) the crops, (b) animal products that were fed with the crops, and (c) drinking water. In addition, the individual receives exposure from inhalation by means of dust from the soil that was contaminated through irrigation.

Baseline Data for Farm Case. To model the Farm critical pathways, GENII-A requires the following information: crop irrigation rates, drinking water consumption, vegetable growing time, animal growing times, leaf vegetable, root vegetable, fruit, and grain consumption; and beef, poultry, milk, and egg consumption, in addition to other data describing the source concentrations (from STAFF3D via PREGENII). The dust resuspension factor is used to calculate inhalation exposure. The parameters selected for the Farm case are listed in Tables 10-4 and 10-5. 
10. Consequence Modeling: Biosphere Transport and Dose

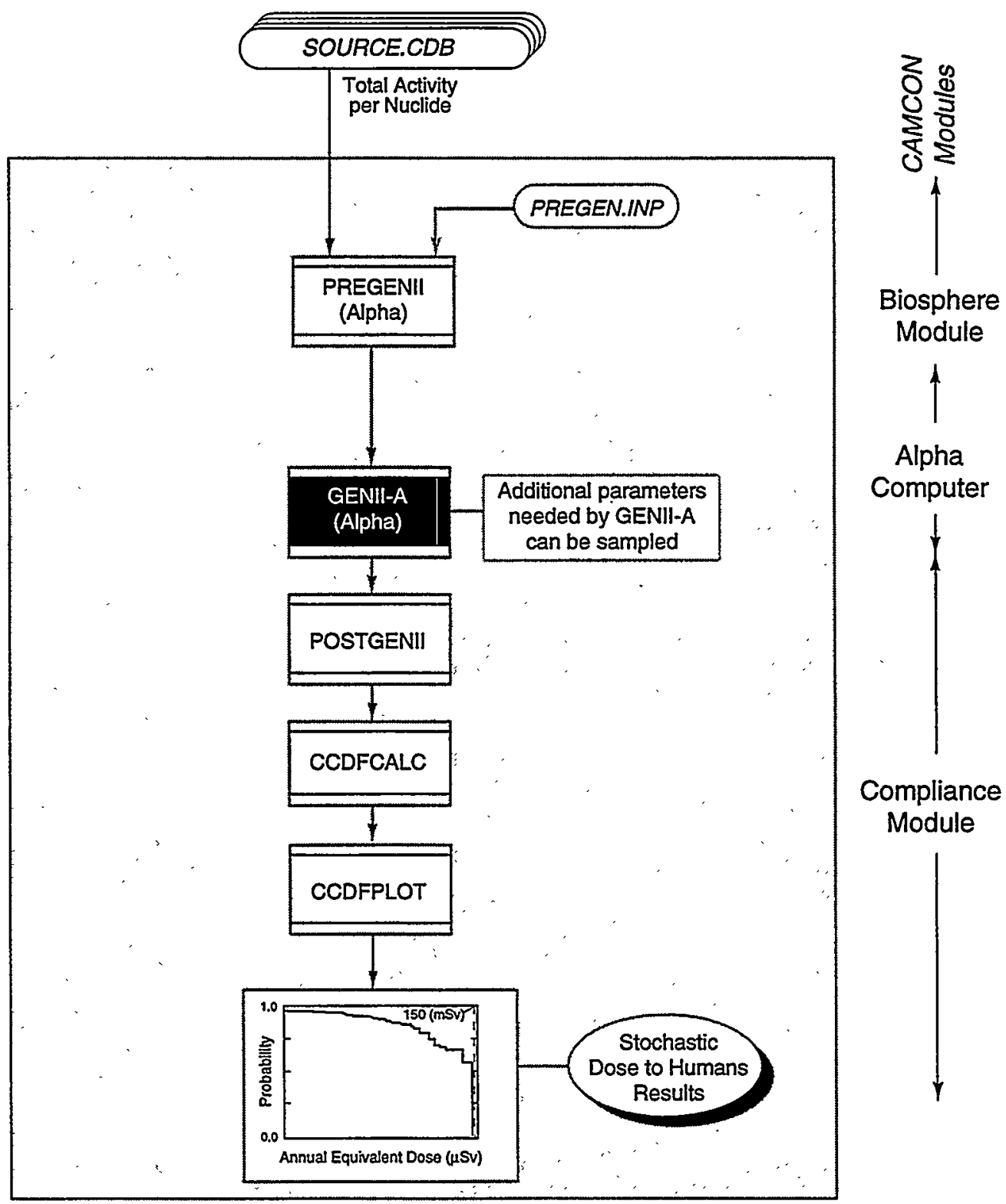

TRI-6342-4480-4

Figure 10-3. Schematic of biosphere transport modeling, including data input by means of PREGENII. 
Table 10-3. Variables for Ranch Case

\begin{tabular}{lcc}
\hline \multicolumn{1}{c}{ Variable } & Range of Values & Distribution \\
\hline Irrigation Rate & $33-88 \mathrm{in} . / \mathrm{yr}$ & Uniform \\
& $(84-224 \mathrm{~cm} / \mathrm{yr})$ & \\
Forage for Cattle & $2 \mathrm{~kg} / \mathrm{m}^{3}$ & Fixed \\
Consumption of Beef by Rancher & $100 \mathrm{~kg} / \mathrm{yr}$ & Fixed \\
Drinking Water for Cattle & 26 liters/day & Fixed \\
\hline
\end{tabular}

Table 10-4. Variables for Farm Case

\begin{tabular}{|c|c|c|c|c|c|}
\hline & Meàn & Median & Range & Distribution & Units \\
\hline Irrigation rate & 48.0 & 48.0 & $\begin{array}{l}33.0-88.0 \\
(84-224)\end{array}$ & Uniform & $\begin{array}{l}\text { in./yr } \\
(\mathrm{cm} / \mathrm{yr})\end{array}$ \\
\hline Irrigation duration & 4.0 & 4.0 & $N A^{a}$ & Fixed & mos. \\
\hline Drinking water consumption ${ }^{c}$ & 769.7 & DNA $^{\mathrm{b}}$ & DNA & DNA & liter/yr \\
\hline Leafy vegetables consumption ${ }^{c}$ & 63.6 & DNA & DNA & DNA & $\mathrm{kg} / \mathrm{yr}$ \\
\hline Root vegetables consumption ${ }^{c}$ & 28.9 & DNA & DNA & DNA & $\mathrm{kg} / \mathrm{yr}$ \\
\hline Fruit consumption ${ }^{c}$ & 59.3 & DNA & DNA & DNA & $\mathrm{kg} / \mathrm{yr}$ \\
\hline Grain consumption ${ }^{c}$ & 60.6 & 60.6 & NA & Fixed & $\mathrm{kg} / \mathrm{yr}$ \\
\hline Beef consumption ${ }^{c}$ & 39.0 & DNA & DNA & DNA & $\mathrm{kg} / \mathrm{yr}$ \\
\hline Poultry consumption ${ }^{c}$ & 15.7 & 15.7 & NA & Fixed & $\mathrm{kg} / \mathrm{yr}$ \\
\hline Milk consumption ${ }^{c}$ & 119.4 & DNA & DNA & DNA & liter/yr \\
\hline Egg consumptionc & 15.8 & 15.8 & NA & Fixed & $\mathrm{kg} / \mathrm{yr}$ \\
\hline Soil/plant transfer scale ${ }^{d}$ & 1.0 & 1.0 & $0.117-8.51$ & Lognormal & - \\
\hline Animal uptake scale ${ }^{d}$ & 1.0 & 1.0 & $0.117-8.51$ & Lognormal & - \\
\hline Inhalation exposure time $e^{d}$ & 5563 & 5563 & NA & Fixed & $\mathrm{hr} / \mathrm{yr}$ \\
\hline Soil exposure time ${ }^{d}$ & 5563 & 5563 & NA & Fixed & $h r / y r$ \\
\hline Direct resuspension factor & $1 \times 10^{-7}$ & $1 \times 10^{-7}$ & NA & Fixed & $\mathrm{m}^{-1}$ \\
\hline Mass load & $1.93 \times 10^{-5}$ & $1.93 \times 10^{-5}$ & NA & Fixed & $\mathrm{gm} / \mathrm{m}^{3}$ \\
\hline \multicolumn{6}{|c|}{$\begin{array}{ll}\text { a } & N A=\text { not applicable } \\
\text { b } & \text { DNA = data not available } \\
\text { c Mean values (and median, if distribution is fixed) from Table 2.10.4.5.1-4 in "Preliminary Biosphere TSPA-VA Chapter," } \\
\text { B00000000-01717-220-00203, February 27, 1998. } \\
\text { d Mean values from "Chapter 11, TSPA-VA Technical Basis Document, Summary and Conclusions," B00000000-01717- } \\
4301-00011 \text {, Rev 00B, August 11, 1998. }\end{array}$} \\
\hline
\end{tabular}


Table 10-5. Growing Cycle and Consumption Data for GENII-A

\begin{tabular}{lcccccc}
\hline & $\begin{array}{c}\text { Leafy } \\
\text { Vegetables }\end{array}$ & $\begin{array}{c}\text { Root } \\
\text { Vegetables }\end{array}$ & Fruits & Grains & Beef & Poultry \\
\hline Growing Time (day) & 90 & 90 & 90 & 90 & 45 & 45 \\
Yield $\left(\mathrm{kg} / \mathrm{m}^{2}\right.$ ) & 1.5 & 4 & 2 & 0.8 & - & - \\
Holdup Time (day) & 0 & 0 & 0 & 0 & 15 & 1.0 \\
\hline
\end{tabular}

\subsubsection{Model for Small Community}

The Small Community case considers exposure of a member of the most probable critical group. The resident consumes tap water from a well drilled into the aquifer in the saturated zone at the $5-\mathrm{km}$ boundary, and consumes some local food products, as described in the Farm pathway.

Critical Pathways. In the Small Community case, the average resident is exposed by means of tap water and locally grown food products.

Baseline Data for Small Community Case. To model the Small Community critical pathways, GENII-A requires the same parameters used for the Farm case, with the exception of parameters required for inhalation and external exposure (Table 10-6). ${ }^{* *}$ Since most food products used in the Small Community are imported, the amounts consumed are a fraction of what a member of the Farm family consumes.

Table 10-6. Variables for Small Community Case

\begin{tabular}{|c|c|c|c|c|c|}
\hline & Mean & Median & Range & Distribution & Units \\
\hline Irrigation rate & 48.0 & .48 .0 & $\begin{array}{l}33.0-88.0 \\
(84-224)\end{array}$ & Uniform & $\begin{array}{l}\text { in./yr } \\
(\mathrm{cm} / \mathrm{yr})\end{array}$ \\
\hline Irrigation duration & 4.0 & 4.0 & $N A^{a}$ & Fixed & mos \\
\hline Drinking water consumption $^{c}$ & 683.8 & $D_{N A}^{b}$ & DNA & DNA & liter/yr \\
\hline Leafy vegetables consumption ${ }^{c}$ & 4.39 & DNA & DNA & DNA & $\mathrm{kg} / \mathrm{yr}$ \\
\hline Root vegetables consumption ${ }^{c}$ & 2.1 & DNA & DNA & DNA & $\mathrm{kg} / \mathrm{yr}$ \\
\hline Fruit consumption ${ }^{c}$ & 4.5 & DNA & DNA & DNA & $\mathrm{kg} / \mathrm{yr}$ \\
\hline Grain consumption ${ }^{c}$ & 0.4 & 0.4 & NA & Fixed & $\mathrm{kg} / \mathrm{yr}$ \\
\hline Beef consumption ${ }^{c}$ & 0.9 & DNA & DNA & DNA & $\mathrm{kg} / \mathrm{yr}$ \\
\hline Poultry consumption ${ }^{c}$ & 0.5 & 0.5 & NA & Fixed & $\mathrm{kg} / \mathrm{yr}$ \\
\hline Milk consumption ${ }^{c}$ & 4.8 & DNA & DNA & DNA & liter/yr \\
\hline Egg consumption ${ }^{c}$ & 2.32 & 15.8 & NA & Fixed & $\mathrm{kg} / \mathrm{yr}$ \\
\hline Soil/plant transfer scale ${ }^{d}$ & 1.0 & 1.0 & $0.117-8.51$ & Lognormal & - \\
\hline Animal uptake scale ${ }^{d}$ & 1.0 & 1.0 & $0.117-8.51$ & Lognormal & - \\
\hline \multicolumn{6}{|c|}{$\begin{array}{l}\text { a } N A=\text { not applicable } \\
\text { b DNA = data not available } \\
\text { c Mean values (and median, if distribution is fixed) from Table 2.10.4.5.1-2 in "Preliminary Biosphere TSPA-VA Chap- } \\
\text { ter," B00000000-01717-220-00203, February 27, 1998. } \\
\text { d Mean values from "Chapter 11, TSPA-VA Technical Basis Document Summary and Conclusions," B00000000- } \\
01717-4301-00011, \text { Rev O0B, August 11, 1998. }\end{array}$} \\
\hline
\end{tabular}

** Parameter distributions shown in Chapter 6 were prepared in anticipation of sampling for the Small Community case, but due to time constraints were not used in the 1997 PA. 


\subsection{Biosphere Conversion Factors}

The results shown in Table 10-7 are the annual effective dose equivalents (AEDE) for each listed radionuclide for a concentration in the groundwater of $1.0 \mathrm{pCi}$ per liter. A member of the Farm family is the maximally exposed of the three groups considered (Ranch, Farm, Small Community). The Rancher has the smallest AEDEs; the Small Community resident is slightly more at risk than the Rancher, and both are significantly less at risk than a member of the subsistence Farm family.

Table 10-7. Biosphere Dose Conversion Factors for Unit Concentrations of $1 \mathrm{pCi}$ in Groundwater for Signifcant Radioisotopes Transported in 1997 PA

\begin{tabular}{cccc} 
& \multicolumn{3}{c}{ Annual Effective Dose Equivalents (mrem/yr/PCi/liter) } \\
\cline { 2 - 4 } Radioisotope & Rancher & Farmer & Small Community \\
\hline Significant Radioisotopes & & & \\
${ }^{99} \mathrm{TC}$ & $1.1 \times 10^{-3}$ & $7.5 \times 10^{-3}$ & $1.8 \times 10^{-3}$ \\
${ }^{129} \mathrm{I}$ & $1.5 \times 10^{-1}$ & 1.5 & $2.3 \times 10^{-1}$ \\
${ }^{237} \mathrm{~Np}$ & 1.6 & $1.7 \times 10^{-1}$ & 4.1 \\
Selected Additional Radioisotopes That May be Significant & \\
${ }^{240} \mathrm{Pu}$ & $2.1 \times 10^{-3}$ & $1.1 \times 10^{1}$ & 2.8 \\
${ }^{239} \mathrm{Pu}$ & $2.1 \times 10^{-3}$ & $1.1 \times 10^{1}$ & 2.8 \\
${ }^{238} \mathrm{Pu}$ & $1.9 \times 10^{-3}$ & 9.8 & 2.5 \\
${ }^{236} \mathrm{U}$ & $1.6 \times 10^{-2}$ & $8.8 \times 10^{-1}$ & $2.1 \times 10^{-1}$ \\
${ }^{226} \mathrm{Ra}$ & $2.6 \times 10^{-1}$ & 3.1 & $7.6 \times 10^{-1}$ \\
${ }^{210} \mathrm{~Pb}$ & $6.5 \times 10^{-1}$ & $1.7 \times 10^{1}$ & 4.2 \\
\hline
\end{tabular}

It is important to note that isotopes of plutonium and lead $\left({ }^{210} \mathrm{~Pb}\right)$ along with neptunium $\left({ }^{237} \mathrm{~Np}\right)$ show the largest biosphere dose conversion factors. Results from previous performance assessments have shown that up to $10,000 \mathrm{yr}$, the contribution to dose from plutonium is negligible. Also it has been shown that ${ }^{234} U$ and its daughters which include ${ }^{226} \mathrm{Ra}$ and ${ }^{210} \mathrm{~Pb}$ are more significant contributors than plutonium but not as important as ${ }^{99} \mathrm{Tc}$, ${ }^{129} \mathrm{I}$, and ${ }^{237} \mathrm{~Np}$. To assess the significance of these other radioisotopes at later times would likely require transport in the saturated zone, with simulations of times approaching one million years. 


\title{
11. Selected Overall Results
}

\author{
R. P. Rechard, H. J. Iuzzolino, D. K. Rudeen, \\ M. E. Lord, and R. D. McCurley
}

Although the results from this performance assessment encompass the interaction of the components of the disposal system, the results are organized into five areas: specific findings about the waste form, the waste container, the repository, and the geologic barrier, and general findings about the system.

\subsection{Findings for Waste Form}

The term "waste form" is used to designate the type of spent fuel (e.g., uranium metal) or high-level waste in combination with a treatment or packaging option. In the $1997 \mathrm{PA}$, the direct disposal option was considered, in which the DOE SNF is not treated and is packaged in stainless steel handling containers only.

\subsubsection{Description of Spent Nuclear Fuel}

A large portion of the total radioisotope inventory (75,336 MTHM) used in this study is commercial SNF (63,080 MTHM; 83.7\%), with only 9842 MTHM $^{*}$ (13.1\%) from DHLW and 2415 MTHM (3.2\%) from DOE SNF (Figure 11-1). The commercial spent nuclear fuel will potentially be sent from 110 different reactors; however, the radioisotope inventory supplied for this analysis was an average recently estimated by the OCRWM for the 1998 TSPA-VA. Two categories that represent seventeen PWR and five BWR classes of commercial SNF were modeled: 21-PWR Westinghouse $17 \times 17$ LOPAR fuel assemblies (Category 14) and 44-BWR General Electric $8 \times 8$ fuel assemblies (Category 15) (see Table 1-1 in Chapter 1; Chapter 3).

Thirteen categories of DOE SNF were modeled in the 1997 PA (see Table 1-1 in Chapter 1; Chapter 3). The categories were based primarily on the type of fuel matrix (but not fissile content); the type of cladding and its condition were considered as secondary criteria. Within the total SNF, $2.8 \%$ ( $88.3 \%$ of the $3.2 \%$ ) of the heavy metal mass is $\mathrm{N}$ Reactor spent nuclear fuel, which was grouped as Category 1 for the analysis. The remaining $0.4 \%$ (11.7\% of the $3.2 \%$ ) of DOE SNF represents about 250 types of experimental fuel. Twelve of the DOE SNF categories were codisposed with DHLW in borosilicate glass (Categories 2 through 13). (The DOE SNF does not include the Navy propulsion reactor spent nuclear fuel.)

\subsubsection{Parameters Sampled}

For the source-term model, the waste form information is placed into two categories: matrices and layers. Matrices incorporate the radioisotopes. The rate of matrix alteration determines the release rates of radioisotopes. Layers protect underlying layers and matrices. Penetration of a layer is dependent upon localized corrosion rate, while generation of rust adsorbents and gas and liquid consumption/production is dependent upon general corrosion rates. Four types of information are required for each layer (see Chapter 7): (a) stoichiometry of corrosion, (b) initial surface area and/or mass of each layer, (c) condition and ability of each layer to protect underlying layers and matrices, and (d) general and localized corrosion rates based on either surface area or mass of a layer. The corrosion rates can potentially depend upon oxygen (oxic/anoxic) conditions and availability of moisture (wet/humid/dry). These four types of information are also required for the matrices. In addition, calculations for the matrices require solubility of radioisotopes and inventory of radioisotopes and their location in the waste package. The locations are modeled

\footnotetext{
* The calculation of the equivalent MTHM as required in 40 CFR 191, using the remaining inventory of radioisotopes after reprocessing and decayed to time of emplacement in 2030, is 9842 . When MTHM equivalents were calculated assuming 0.5 MTHM per DHLW short handling container and 0.75 MTHM per long handling container, as was done for DOE planning documents in 1980, the value is 9409.
} 


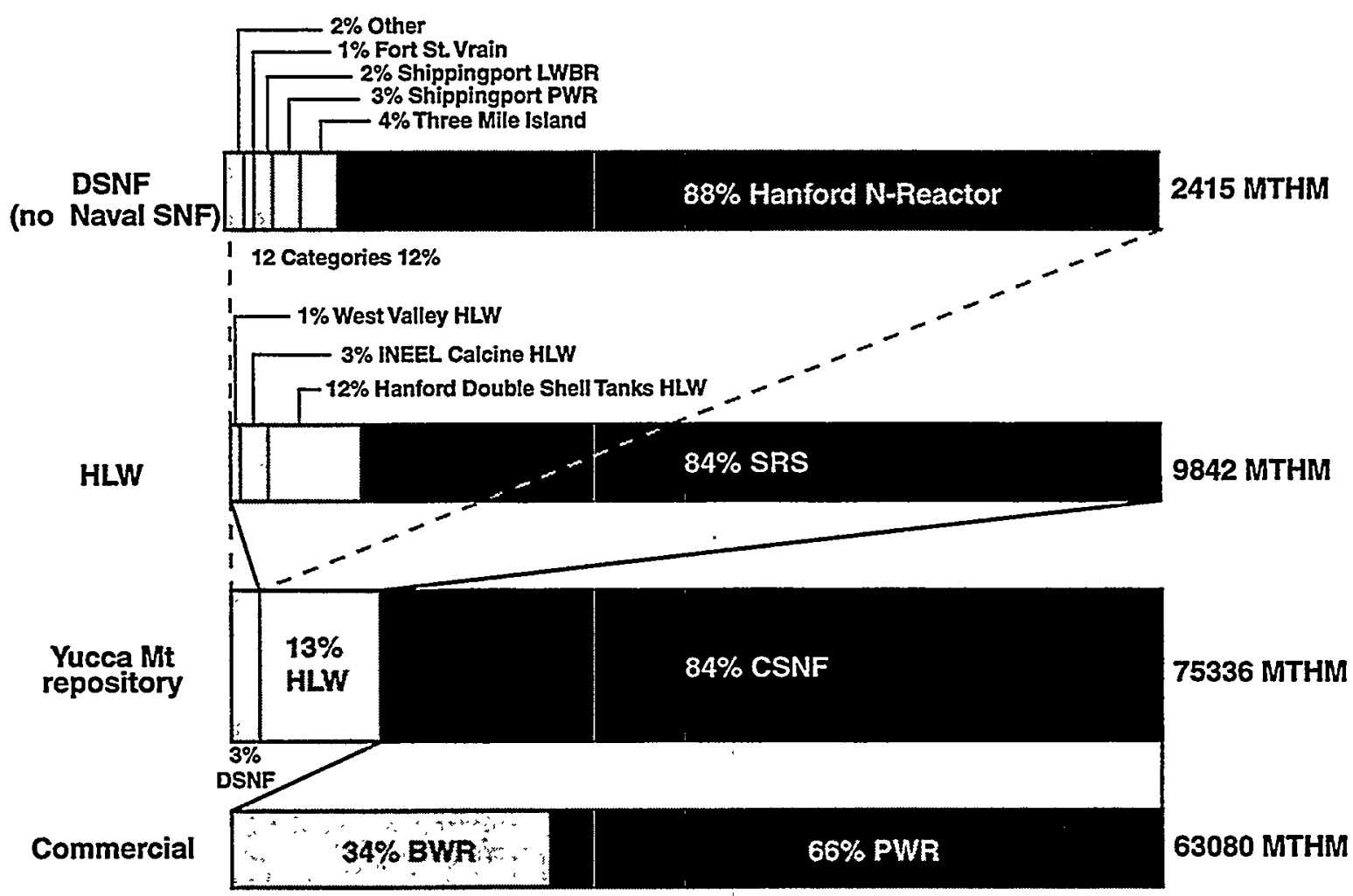

TRI-6342-5733-4

Figure 11-1. A large portion (84\%) of the total radioisotope inventory is commercial spent nuclear fuel (SNF), with only $13 \%$ defense high level waste (DHLW), and 3\% DOE SNF. Hence, the ability of DOE SNF to dominate behavior of the potential Yucca Mountain repository is limited.

either as directly in the matrix or residing in gaps between a layer (e.g., cladding) and a matrix, or as interstitial grain boundaries that permit immediate release of radioisotopes.

For the analysis reported here, 20 parameters directly influencing the radioisotope release rate were varied in the source term. The parameters chosen were based on parameters that were somewhat influential in the 1994 PA. More information on source term parameter values can be found in Chapter 7. Parameter values were generally those used in the $1994 \mathrm{PA}$. The solubility of neptunium was one parameter that was lowered from the values used in the $1994 \mathrm{PA}$ (and TSPA-1995) (Figure 11-2). The current mean is $10^{-5.4} \mathrm{M}=4.0 \times 10^{-6} \mathrm{M}\left(9.6 \times 10^{-4} \mathrm{~kg} / \mathrm{m}^{3}\right)$; the former mean was $1.1 \times 10^{-4} \mathrm{M}\left(2.7 \times 10^{-2} \mathrm{~kg} / \mathrm{m}^{3}\right)$. This reduction was due to a reevaluation of previous experiments and the decision to exclude those by Nitsche et al. (1993), which were not representative of expected repository conditions, as explained in Chapter 7.

A comparison of alteration ("corrosion") rates for the waste form shows that the wide range of corrosion rates for borosilicate uranium dioxide encompasses the point values used for other matrix materials (Figure 11-3; see also Chapter 6). Hence, release rates of radioisotopes from DOE and commercial SNF are similar as soon as the matrix material is exposed. Therefore, differences in behavior will be the result of differences in inventory (and protection from cladding, if considered) more than other characteristics. 

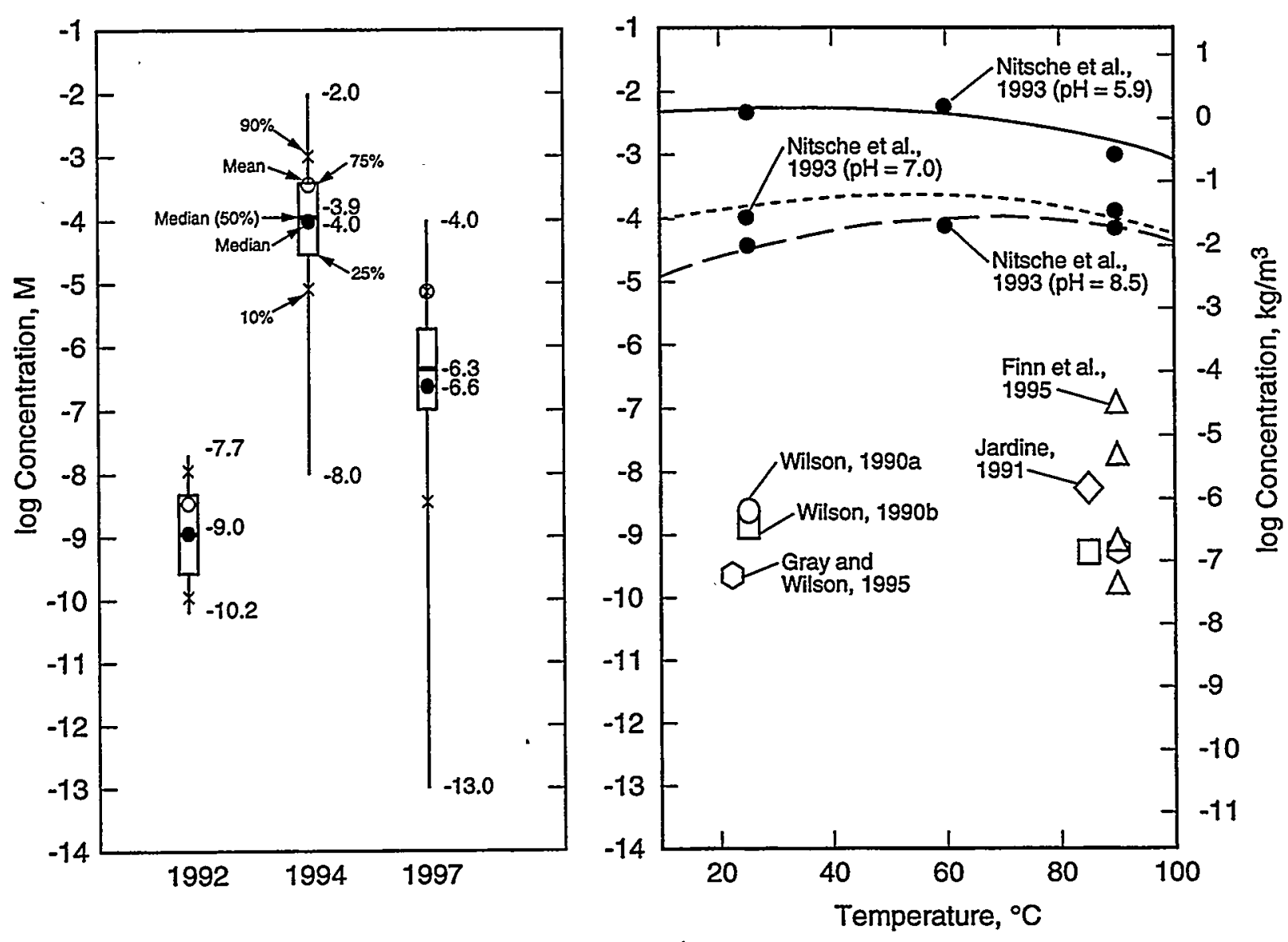

TRI-6342-5830-2

Figure 11-2. Comparison of neptunium solubilities used in various performance assessments. 


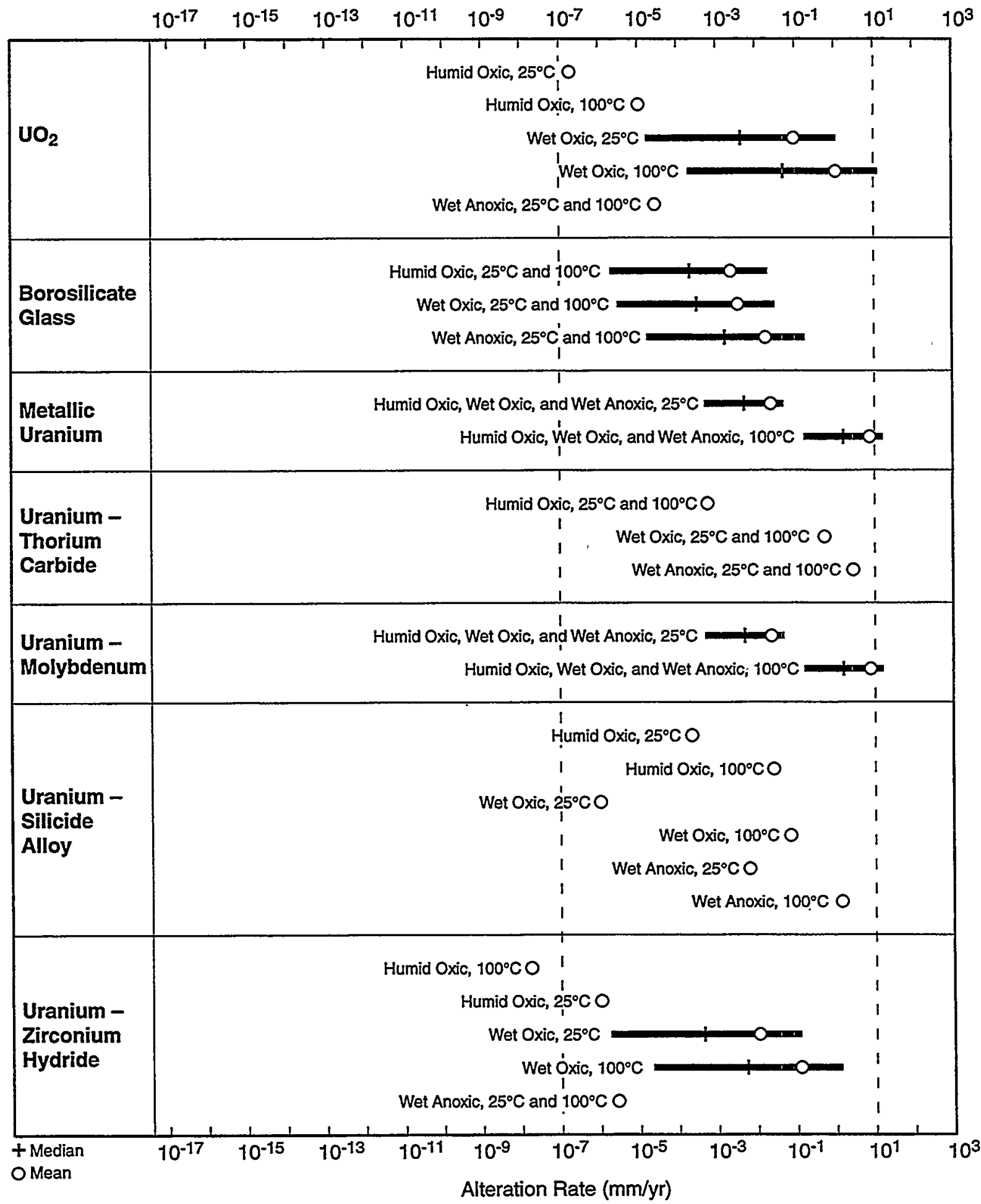

Figure 11-3. Alteration ("corrosion") rates for matrices of spent nuclear fuel (SNF) as depth altered per time, based on parameter values reported in Chapter 7 for the Arrhenius equation. The range of alteration rates for borosilicate glass and uranium dioxide $\left(\mathrm{UO}_{2}\right)$ encompasses the range for most other $\mathrm{SNF}$ materials. 


\subsubsection{Releases of ${ }^{99} \mathrm{Tc}$ and ${ }^{237} \mathrm{~Np}$ from Waste Packages}

For the deterministic runs with model parameters set at the mean values, the releases of ${ }^{99} \mathrm{Tc}$ and ${ }^{237} \mathrm{~Np}$ from the commercial SNF exceed those of DOE SNF/DHLW on both a per mass and per package basis (Figure 11-4). The release of ${ }^{99} \mathrm{Tc}$ for $\mathrm{PWR}$ is $\sim 140 \mathrm{Ci} / \mathrm{pkg}$, while the release of ${ }^{99} \mathrm{Tc}$ for $\mathrm{N}-\mathrm{Reactor}$ fuel is $\sim 27 \mathrm{Ci} / \mathrm{pkg}$. The release of ${ }^{237} \mathrm{~Np}$ for $\mathrm{PWR}$ is $\sim 7.0 \mathrm{Ci} / \mathrm{pkg}$, while the release of ${ }^{237} \mathrm{~Np}$ for $\mathrm{N}$-Reactor fuel is $\sim 0.9 \mathrm{Ci} / \mathrm{pkg}$. Hence, if the commercial SNF can meet the regulatory criteria for dose, then the DOE SNFDHLW can also meet these criteria. These results assume that the cladding of all SNF provides no protection (i.e., has failed). Thus, in contrast to an earlier study (the $1994 \mathrm{PA}$ ), the type and integrity of the cladding was not important in determining releases in the $1997 \mathrm{PA}$. Consequently, the inventory of ${ }^{99} \mathrm{Tc}$ and ${ }^{237} \mathrm{~Np}$ in each SNF category determined its behavior. Therefore, DOE SNF with poor cladding (e.g., weak aluminum cladding on the ATR fuel or the mean of $40 \%$ failed zircaloy cladding on the N-Reactor fuel) performed better than commercial SNF, because of DOE SNF's generally lower ${ }^{99} \mathrm{Tc}$ and ${ }^{237} \mathrm{~Np}$ content per package. The $680 \mathrm{Ci}$ of ${ }^{237} \mathrm{~Np}$ in the DOE SNF and DHLW represent only $2 \%$ of the total repository inventory; also, of this $2 \%$, only $23 \%$ comes from DOE SNF. Thus, the inclusion of DOE SNF and DHLW in the repository greatly helps with compliance.

The dependence of the results on the initial inventory is readily apparent in Figure 11-5, where the ranking of activity per package for each category is similar to the ranking of releases from each package shown in Figure 11-4. ${ }^{\dagger}$ Figure 11-5 also suggests that DHLW in the codisposal option is the primary source of $\mathrm{Np}$ and Tc; thus, the usefulness of grouping SNF into many diverse categories for a performance assessment is limited when cladding is not considered.

The content of fission-product radioisotopes per MTHM gives a rough estimate of the burnup of SNF. Generally, the content of ${ }^{99} \mathrm{Tc}$ and ${ }^{237} \mathrm{~Np}$ in DOE SNF is similar to commercial SNF (Figures 11-6a and b), with two DOE SNF categories containing many fewer of these radioisotopes (i.e., Fermi Reactor fuel, Category 3, and Shippingport LWBR fuel, Category 12) and two DOE SNF categories containing slightly more (i.e., Chicago Pile, Category 2, and ATR, Category 6). However, when the content of the DOE SNF is combined with that in the DHLW with the codisposal option, the overall average per MTFM is less than for commercial SNF (Figures $11-6 \mathrm{c}$ and $d$ ).

$T$ The activity release of ${ }^{237} \mathrm{~Np}$ as shown in Figure $11-4$ is slightly larger than ${ }^{237} \mathrm{~Np}$ 's initial activity in the N-Reactor container (as shown in Figure $11-5 b$ ) because of the ingrowth of ${ }^{237} \mathrm{~Np}$ from the decay of ${ }^{241} \mathrm{Am}$ and ${ }^{241} \mathrm{Pu}$, which can triple the inventory of ${ }^{237} \mathrm{~Np}$. 


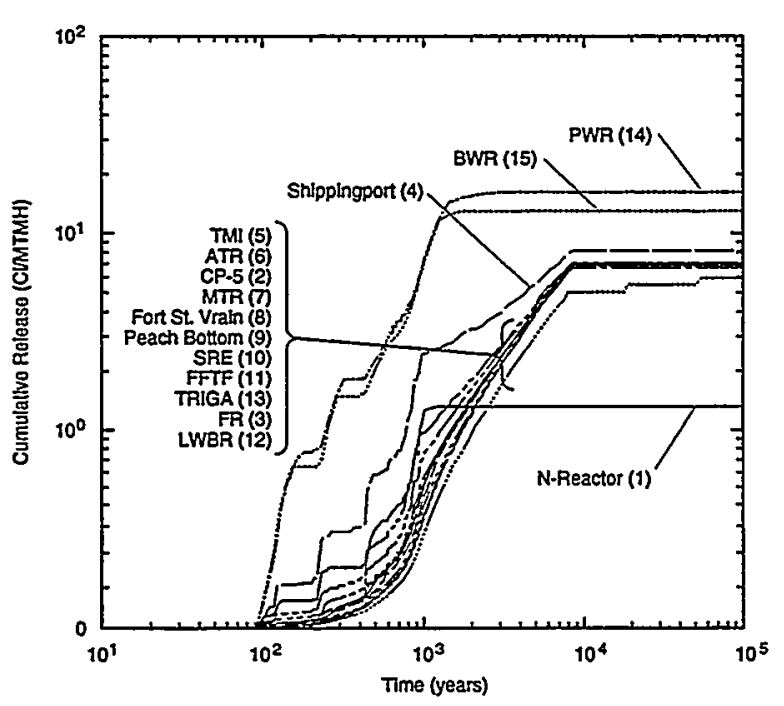

(a) Tc Release (Ci/MTHM)

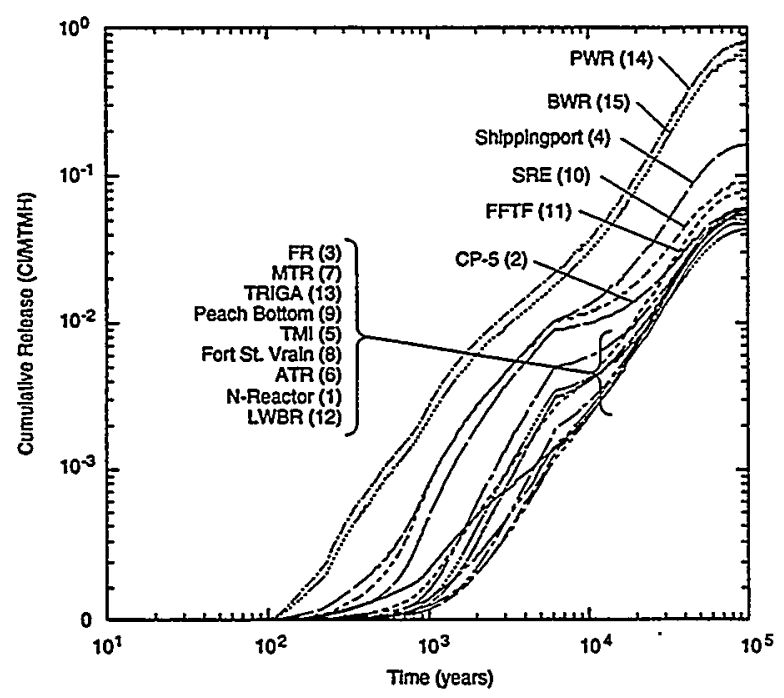

(c) Np Release (Ci/MTHIM)

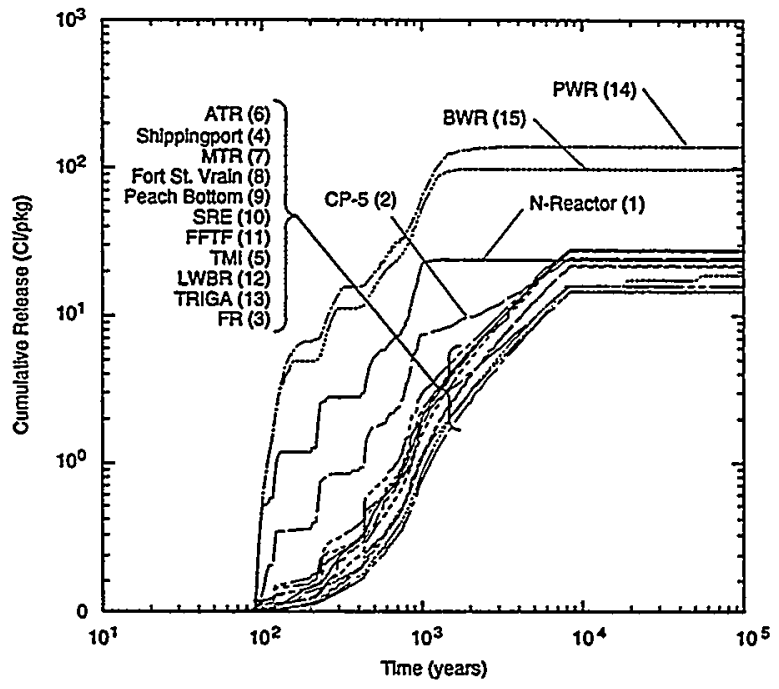

(b) Tc Release (Ci/Pkg)

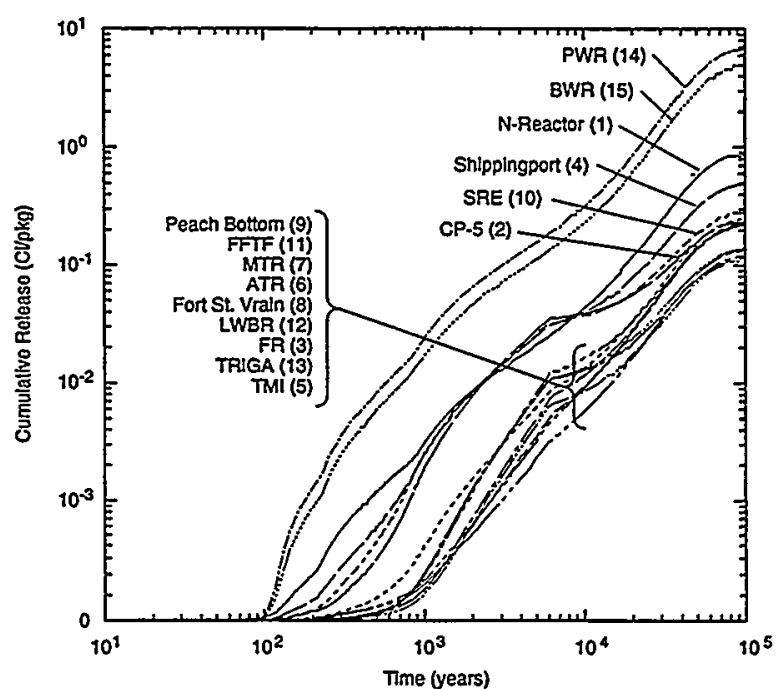

(d) Np Release (Ci/Pkg)

TRI-6342-5869-1

Figure 11-4. Fraction of releases from the waste package in the deterministic run using mean model parameter values for (a) ${ }^{99} \mathrm{TC}$ normalized by mass of heavy metal radionuclides in inventory, $(b)^{99} \mathrm{Tc}$ normalized by number of waste packages in each category, (c) ${ }^{237} \mathrm{~Np}$ normalized by mass of heavy metal radionuclides in inventory, and (d) ${ }^{237} \mathrm{~Np}$ normalized by number of waste packages in each category. Because ${ }^{99} \mathrm{TC}$ and ${ }^{237} \mathrm{~Np}$ are the most important radioisotopes for determining dose, and commercial SNF releases more of these radioisotopes both on a per package and per MTHM basis than DOE SNF, then DOE SNF can meet regulatory dose requirements if commercial SNF can meet them. 


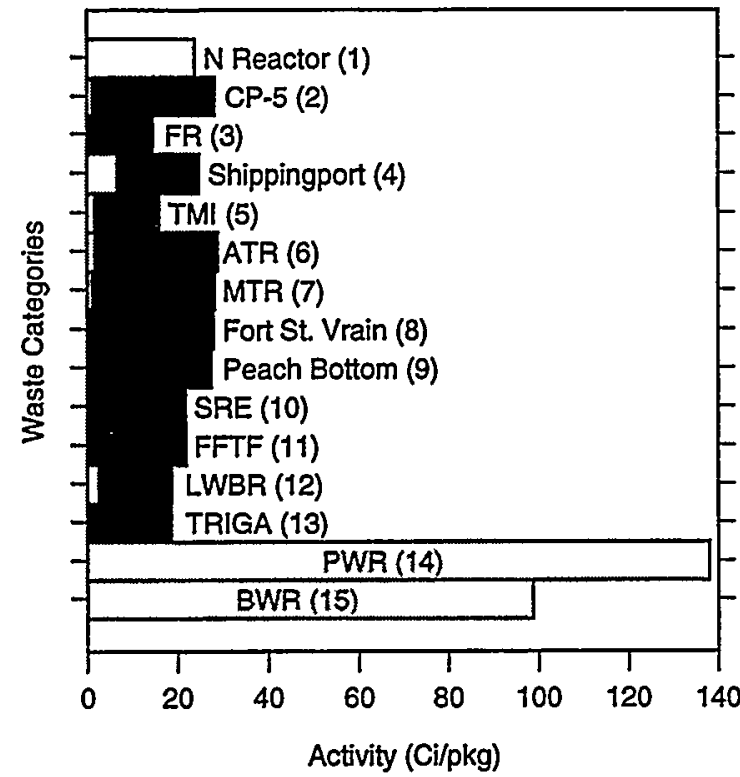

(a) ${ }^{99} \mathrm{Tc}$ activity per package

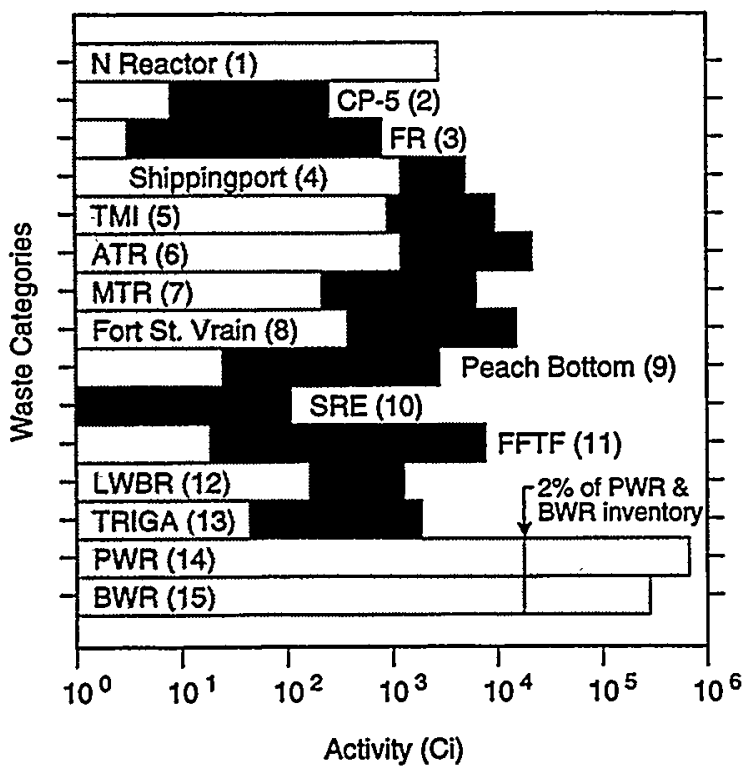

(c) Total ${ }^{99} \mathrm{Tc}$ activity in category (logarithmic scale)

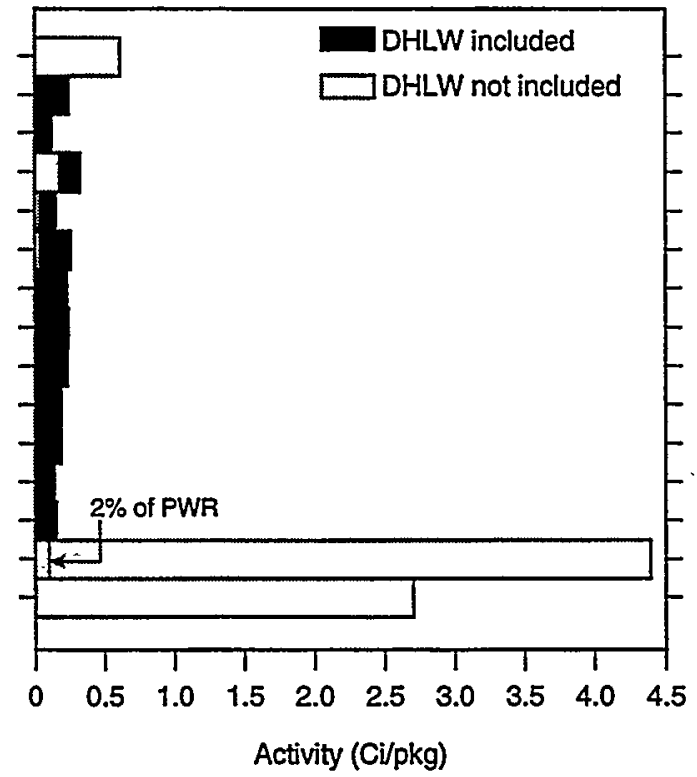

(b) ${ }^{237} \mathrm{~Np}$ initially present per package (amount nearly triples from decay of ${ }^{241} \mathrm{Am}$ and ${ }^{241} \mathrm{Pu}$ )

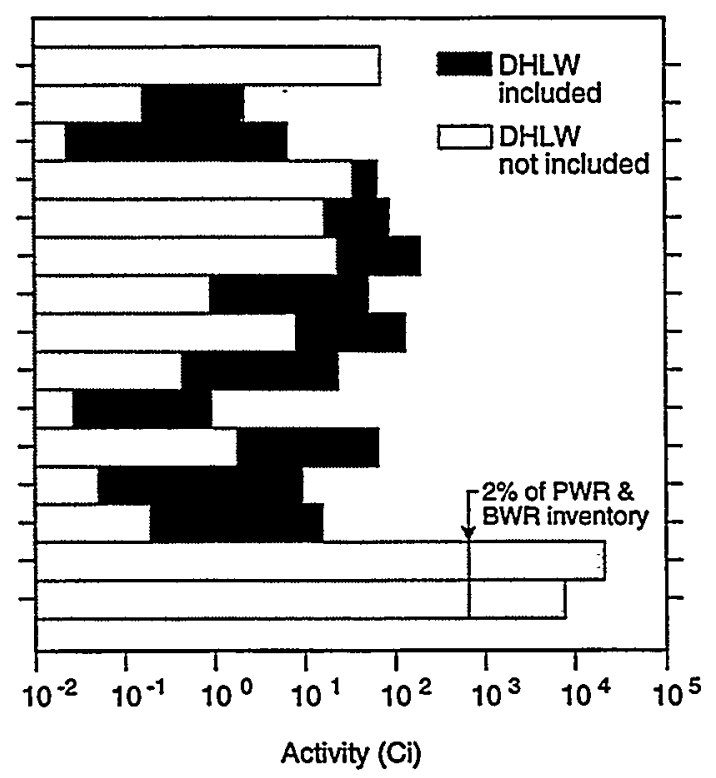

(d) Total ${ }^{237} \mathrm{~Np}$ activity in category (logarithmic scale)

TRI-6342-5828-0

Figure 1I-5. Comparison of inventory of ${ }^{99} \mathrm{TC}$ and ${ }^{237} \mathrm{~Np}$ in each SNF category shows that the inventory of DHLW in the codisposal option dominates the categories, making them appear to be similar. Hence, the PA calculations cannot readily distinguish among the behavior of various DOE SNF, which limits the usefulness of numerous and diverse categories of SNF. The influence of cladding when intact, relative to the unclad or poorly clad DOE SNF, can readily be estimated in (c) and (d), i.e., if $98 \%$ of the cladding was assumed intact (as used in the 1994 PA, for example), then the inventory of available ${ }^{99} T c$ and ${ }^{237} \mathrm{~Np}$ is similar to that of poorly clad categories of DOE SNF until the cladding fails. 


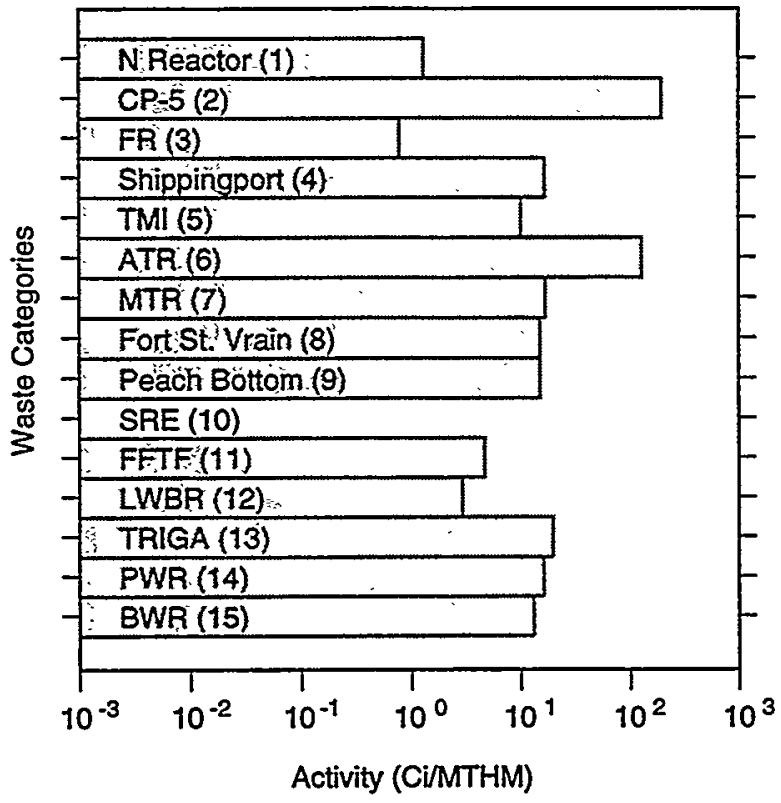

(a) ${ }^{99} \mathrm{Tc}$ content per MTHM without DHLW

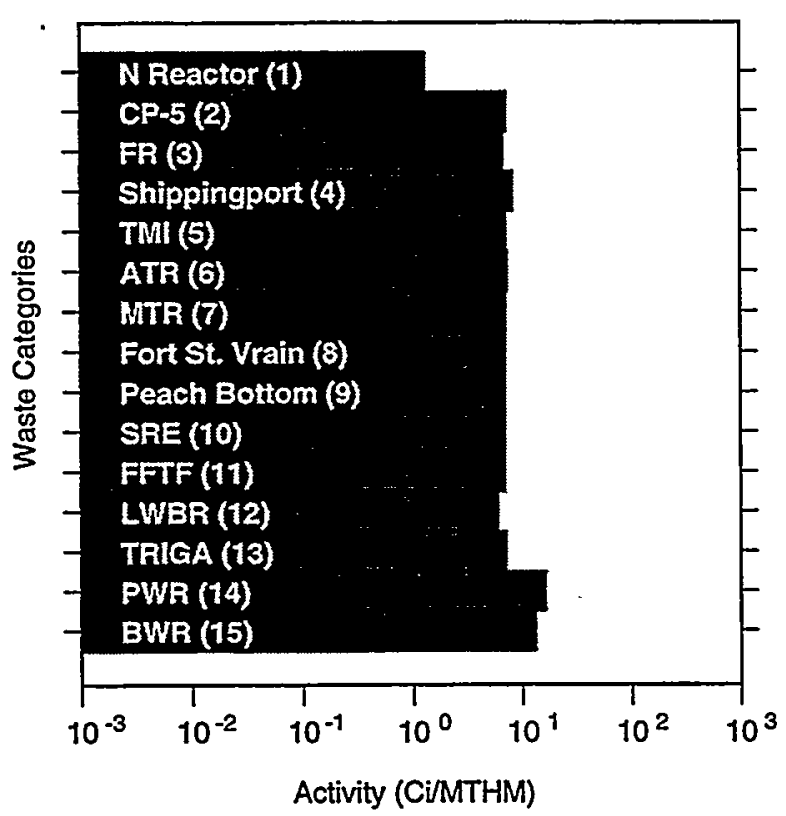

(c) ${ }^{99} \mathrm{Tc}$ content per MTHM with DHLW

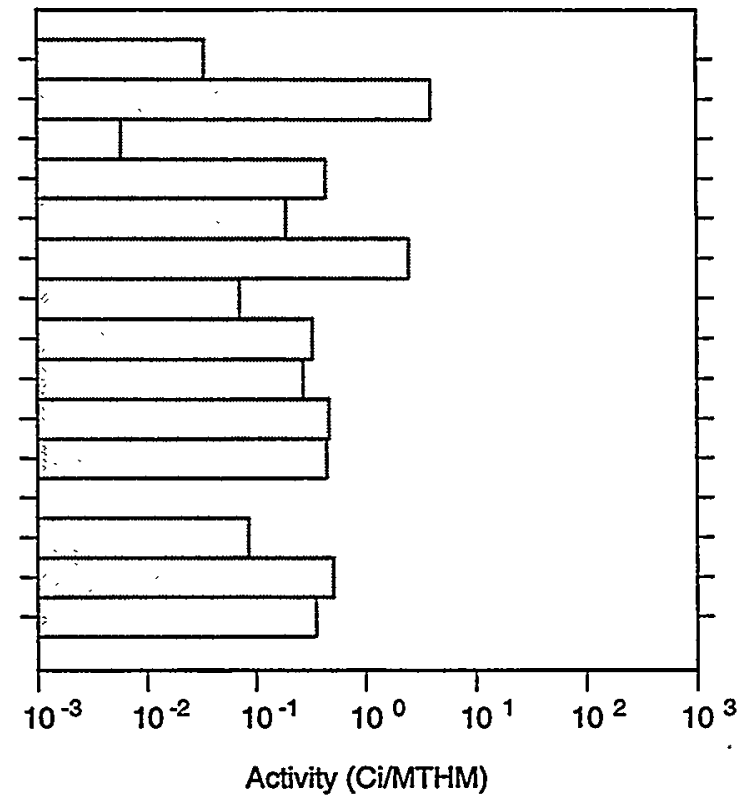

(b) ${ }^{237} \mathrm{~Np}$ content per MTHM without DHLW

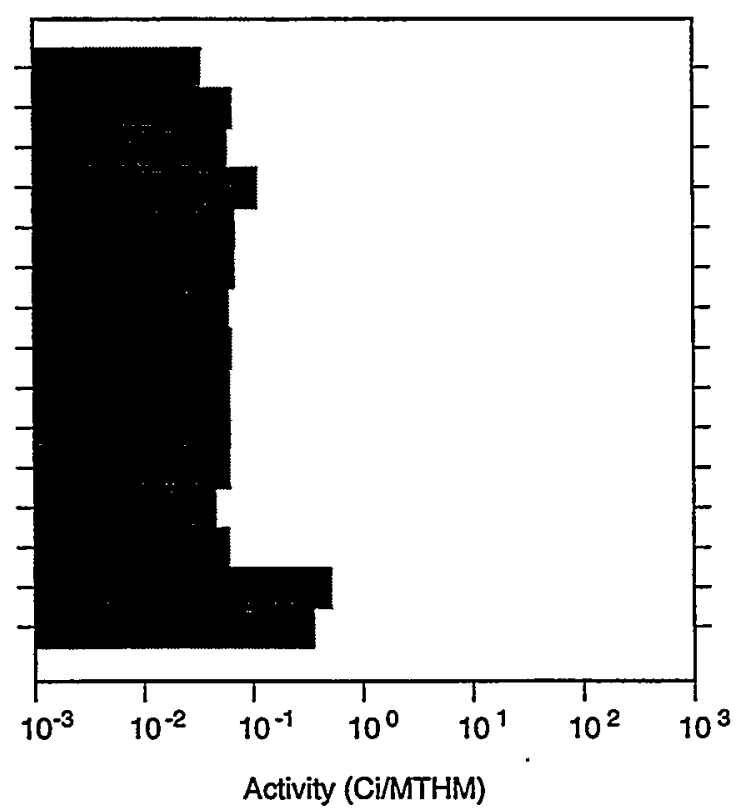

(d) ${ }^{237} \mathrm{~Np}$ content per MTHM with DHLW

TRI-6342-5832-1

Figure 11-6. Content of ${ }^{99} \mathrm{TC}$ and ${ }^{237} \mathrm{~Np}$ per MTHM for SNF categories, with and without codisposal with DHLW. Content per MTHM for (a) ${ }^{99} T c$ without DHLW, (b) ${ }^{237} \mathrm{~Np}$ without DHLW, (c) ${ }^{99} \mathrm{Tc}$ with DHLW, and (d) ${ }^{237} \mathrm{~Np}$ with DHLW. Generally, the content of ${ }^{99} \mathrm{Tc}$ and ${ }^{237} \mathrm{~Np}$ in DOE SNF is similar to commercial SNF, but when combined with DHLW, the content is somewhat less than commercial SNF. 


\subsubsection{Fraction of Waste Exposed, Altered, and Released}

As shown in Figure 11-3 (Section 11.1.2), the corrosion rates of the container materials are high when the repository is hot. Because the repository was modeled using the hot repository concept, breach of the disposal container layers-carbon steel and Incoloy 625-occurs rapidly, i.e., $100 \%$ within 1000 yr for the 1997 PA. This rapid exposure of the matrix is similar to results in the 1994 PA, except that less was exposed in the 1994 PA (30\% vs. 100\%) because the $1994 \mathrm{PA}$ included protection from cladding. The alteration rates of the fuel matrix are also temperature sensitive and thus fuel alteration occurs rapidly as well when the repository is hot. This alteration is much more rapid than in the 1994 PA where even after exposure, the amount of waste altered was only one-third of that exposed at 10,000 yr.

The alteration rate is more rapid than the rate at which radioisotopes can be carried away from the container. ${ }^{99} \mathrm{Tc}$ has a very high solubility $\left(10^{-3} \mathrm{M}\right.$ for mean run, $1 \mathrm{M}$ maximum) and so its release is only marginally controlled by its solubility (Figure 11-7a). The releases of ${ }^{99} \mathrm{Tc}$ were limited only by the amount of waste that was exposed when at the maximum solubility. Only after the repository has cooled down after $1000 \mathrm{yr}$ does the alteration rate of the matrix substantially decrease. Yet a substantial decrease in the release rate of ${ }^{99} \mathrm{Tc}$ is deferred until the inventory of radioisotopes released prior to $1000 \mathrm{yr}$ from the matrix but held within the container (through precipitation) has been removed (Figure $11-8$ ). ${ }^{237} \mathrm{~Np}$ has a very low solubility $\left(10^{-5.36} \mathrm{M}\right.$ for mean run). Hence, releases of ${ }^{237} \mathrm{~Np}$ were limited primarily by its solubility and adsorption (Figure 11-7a). (If solubility were the sole determining factor, releases of ${ }^{237} \mathrm{~Np}$ would be identical on an MTHM basis for all SNF categories; as seen in Figure 11-4c, this is not the case, but the releases are quite similar.)

The releases of both ${ }^{99} \mathrm{Tc}$ and ${ }^{237} \mathrm{~Np}$ are dominated by advective releases from water passing through the container. Diffusive releases of ${ }^{99} \mathrm{Tc}$ and ${ }^{237} \mathrm{~Np}$ are minor (Figure 11-9). 


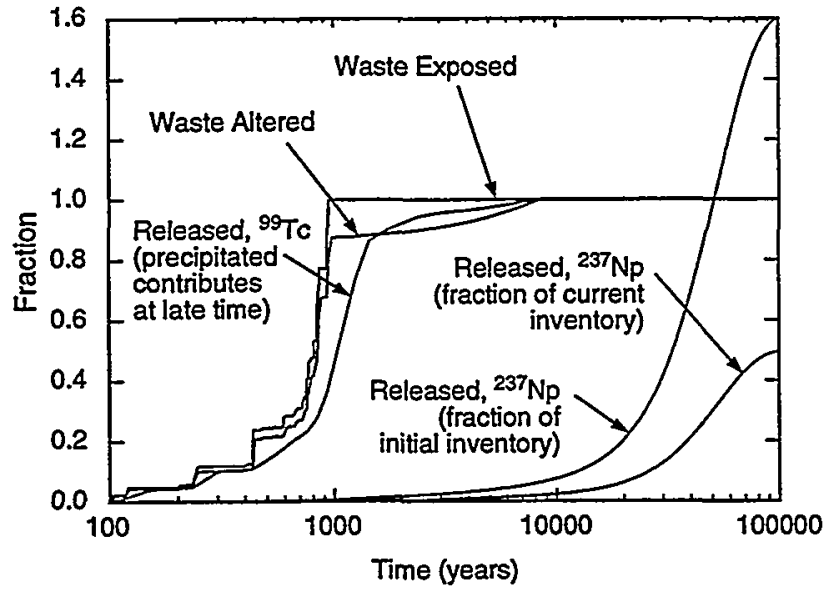

(a) $1997 \mathrm{PA}$, Mean parameters

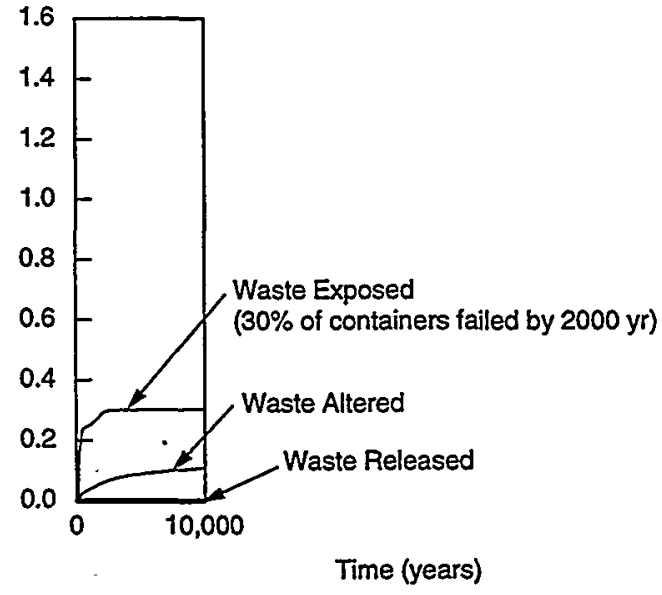

(b) 1994 PA, Mean of 36 simulations

TRI-6342-5802-5

Figure 11-7. Performance of waste package in 1997 and 1994 PAs. Fraction of waste exposed, altered, and released for (a) 1997 PA, mean parameter values, and (b) 1994 PA, mean parameter values. The major differences between the 1994 and 1997 PAs is the higher temperature of the repository in the $1997 \mathrm{PA}\left(100^{\circ} \mathrm{C}\right.$ vs. $145^{\circ} \mathrm{C}$ at center) and the factor of 10 increase in water passing through the repository in the 1997 PA. The higher temperatures increase the amount of waste exposed. (Inconel 625 does not fail rapidly at low temperatures.) The additional water increases the amount of waste exposed and released. Releases of ${ }^{99} \mathrm{Tc}$ mirror the amount of waste altered, which in turn roughly mirrors the amount of waste exposed. Release of ${ }^{237} \mathrm{~Np}$ is limited by its solubility.

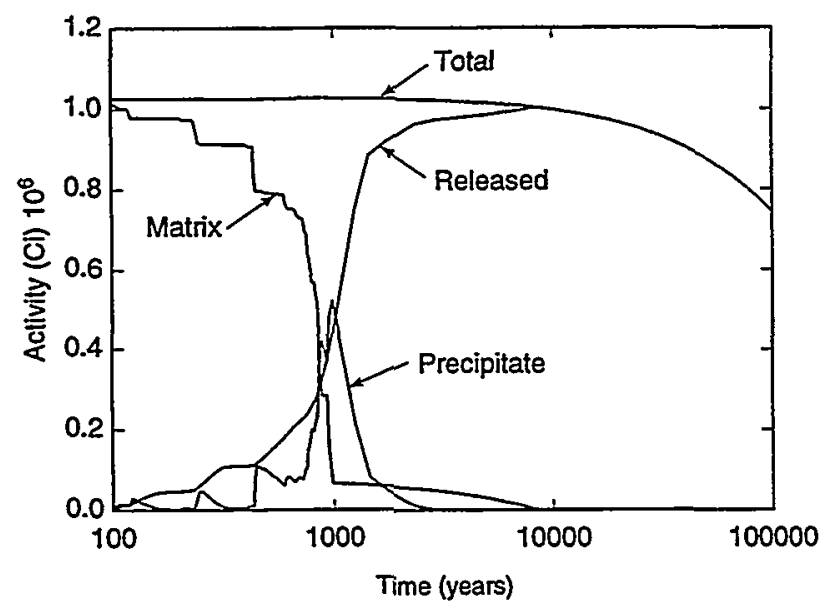

(a) ${ }^{99} \mathrm{Tc}$

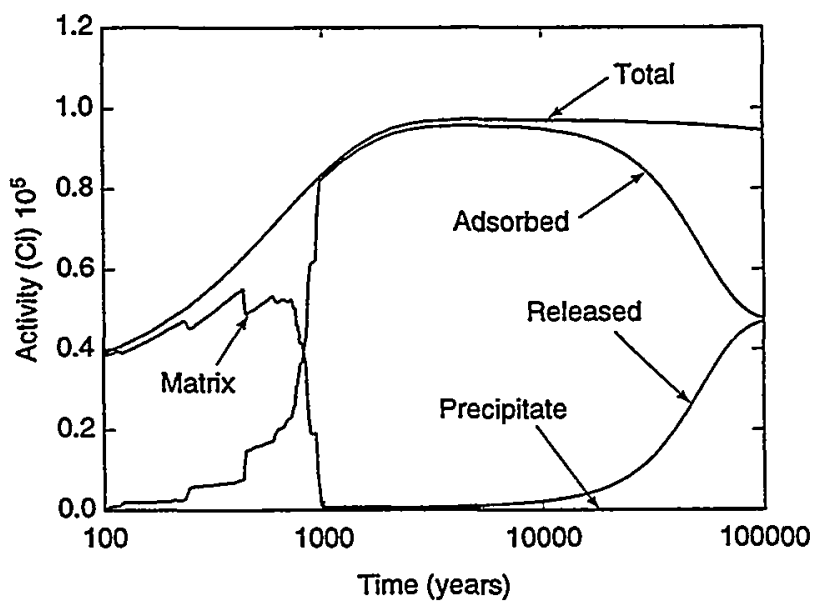

(b) ${ }^{237} \mathrm{~Np}$

TRI-6342-5870-1

Figure 11-8. Location of radioisotopes in various compartments of the source-term model for $(a){ }^{99} \mathrm{Tc}$ and (b) ${ }^{237} \mathrm{~Np}$. 


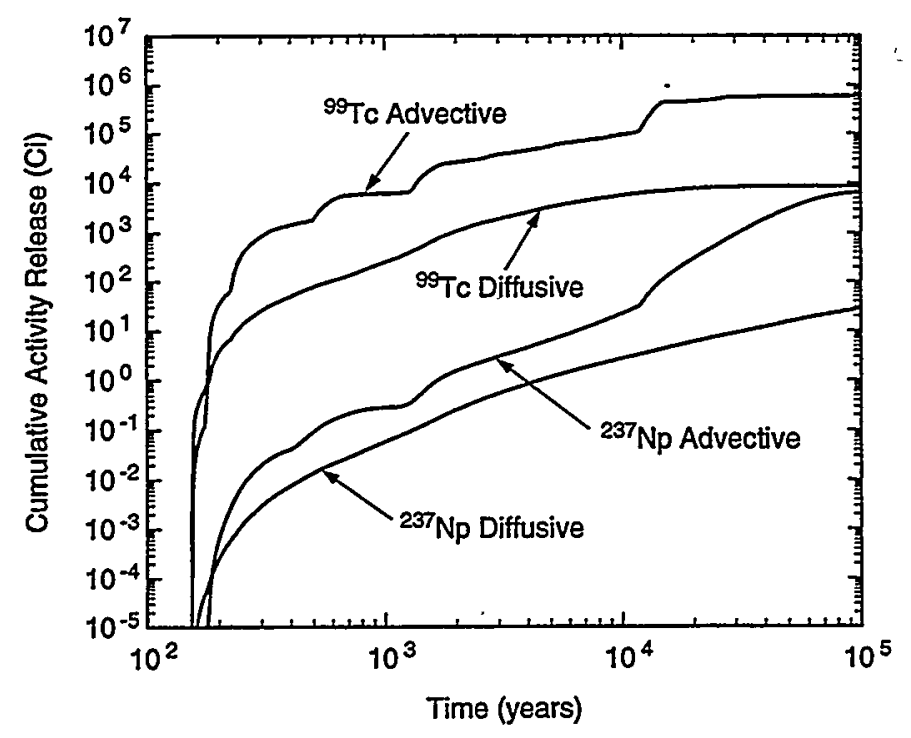

(a) 1997 PA, Median parameter values, 75,336 MTHM

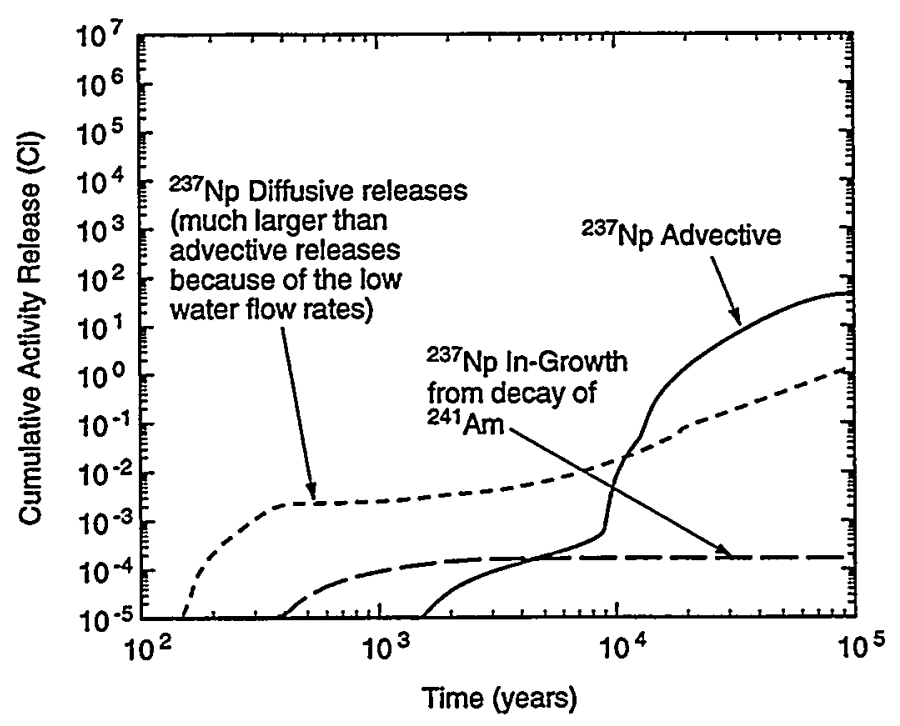

(b) 1994 PA, Median parameter values, 12,060 MTHM

TRI-6342-5811-4

Figure 11-9. Advective and diffusive repository releases of technetium and neptunium using median properties for (a) 1997 PA, 75,336-MTHM repository, and (b) 1994 PA, 12,060-MTHM repository. 


\subsubsection{Changes in Modeling of Waste Form}

The YMP may consider taking credit for cladding on the commercial SNF; hence, this section demonstrates the important infiuence of cladding.

Influence of Cladding. As argued in the $1994 \mathrm{PA}$, it can be important to include the effects of cladding for the commercial SNF when modeling the release. In this study, the inclusion of cladding was also found to be important; it greatly changed the total amount of ${ }^{99} \mathrm{Tc}$ and ${ }^{237} \mathrm{~Np}$ released, as well as the relative performance of the DOE and commercial SNF (Figure 11-10, which can be compared with Figure 11-4).

As noted in Figure 11-5, the influence of cladding, when intact, on the relative releases can be estimated from the total inventory of ${ }^{99} \mathrm{Tc}$ and ${ }^{237} \mathrm{~Np}$ (Figures $11-5 \mathrm{c}$ and $11-5 \mathrm{~d}$ ). If $98 \%$ of the cladding on commercial SNF is effective for the first $10^{5} \mathrm{yr}$ (98\% was used in the $1994 \mathrm{PA}$ ), then the inventory of ${ }^{99} \mathrm{Tc}$ and ${ }^{237} \mathrm{~Np}$ is similar to that in DHLW. Hence, the DHLW will contribute substantially to the releases of ${ }^{99} \mathrm{Tc}$ and ${ }^{237} \mathrm{~Np}$ during the period when commercial SNF cladding is intact. DOE SNF would contribute only a very small percentage to this release, except for the NReactor fuel (Category 1), as seen in Figure 11-5.

Although the vast majority of cladding on the commercial SNF is intact, the 1997 PA used an average $6 \%$ that had failed. Only the radioisotopes not protected by cladding (6\% of the commercial SNF and $40 \%$ of N-Reactor fuel, etc.) are released within $10^{5} \mathrm{yr}$, with most of the release from DHLW (Figure 11-11).

Influence of Changes in Surface Area. The surface area of the matrix containing the radioisotopes directly influences the rate of alteration of the matrix. The surface area of the matrix for the commercial SNF and N-Reactor fuel was changed to examine the influence of an order-of-magnitude increase in corrosion rates on the total and the rates of release of ${ }^{99} \mathrm{Tc}$ and ${ }^{237} \mathrm{~Np}$. The total release of $\mathrm{Tc}$ and $\mathrm{Np}$ is not affected, but the alteration of the matrix is changed. Thus, matrix alteration rates are not important for $\mathrm{Np}$; for $\mathrm{Tc}$, they are not important except at the highest solubility value of $\mathrm{Tc}$, as discussed below. The cladding is so effective as a barrier that a doubling of the corrosion rate does not noticeably increase the time of failure over a $10^{5}-\mathrm{yr}$ period (Figure 11-12).

Infiuence of Changes in Solubility. The solubility of $\mathrm{Tc}$ and $\mathrm{Np}$ were increased to the maximum values ( $1 \mathrm{M}$ and $10^{-4} \mathrm{M}$, respectively) and $\mathrm{K}_{\mathrm{D}}$ of $\mathrm{Np}$ on rust decreased to $0.025 \mathrm{~kg} / \mathrm{m}^{3}$ to study their influence on performance (Figure 11-13). The total amount of $\mathrm{Tc}$ does not change, because all radioisotopes were previously released except those that were encapsulated in matrices in which alteration was slow, but release is more rapid than before. The total amount of $\mathrm{Np}$, however, does increase from that observed in Figure 11-8.

Inffuence of Changes in DOE SNF Inventory. Of some concern is the effect of not knowing the actual inventory of DOE SNF in the to-be-written repository acceptance criteria and whether a larger inventory than the current value might significantly affect performance. At present, the accuracy of the current inventory is not known because the over 250 DOE SNFs have been grouped into broad categories. To evaluate the influence of inventory on performance, the inventories of the two most important radioisotopes, ${ }^{99} \mathrm{Tc}$ and ${ }^{237} \mathrm{~Np}$, were tripled. In this study, increasing the inventory had only a minor influence, because the DOE SNF is such a small contributor to the total mass of the repository. Only release rates of ${ }^{99} \mathrm{Tc}$ at about $1000 \mathrm{yr}$ are noticeably different (Figure 11-14). Also, it can be surmised from Figure 11-8b that changing the inventory of $\mathrm{Np}$ in the commercial SNF would have no effect on releases in the first $10^{5} \mathrm{yr}$ because solubility is controlling release. 


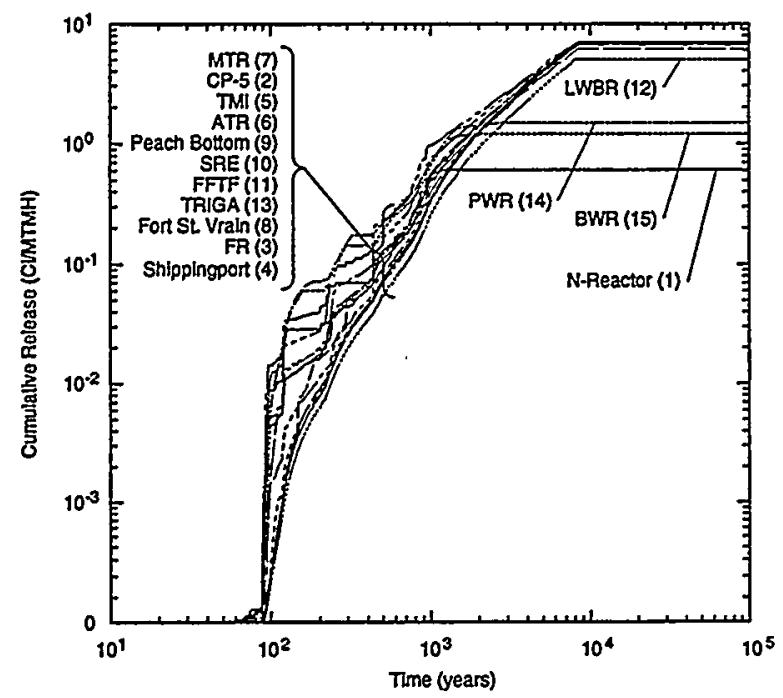

(a) Tc Release (Ci/MTHM)

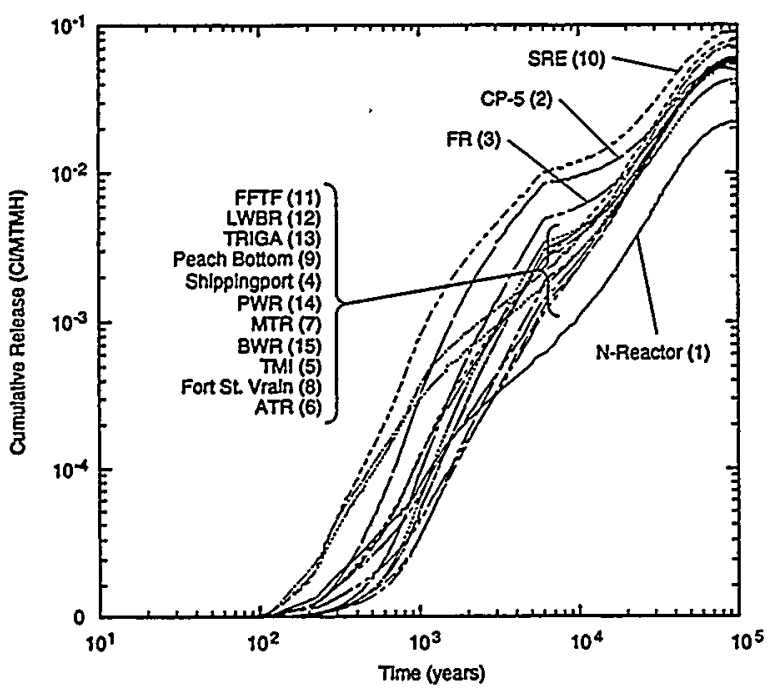

(c) Np Release (Ci/MTHM)

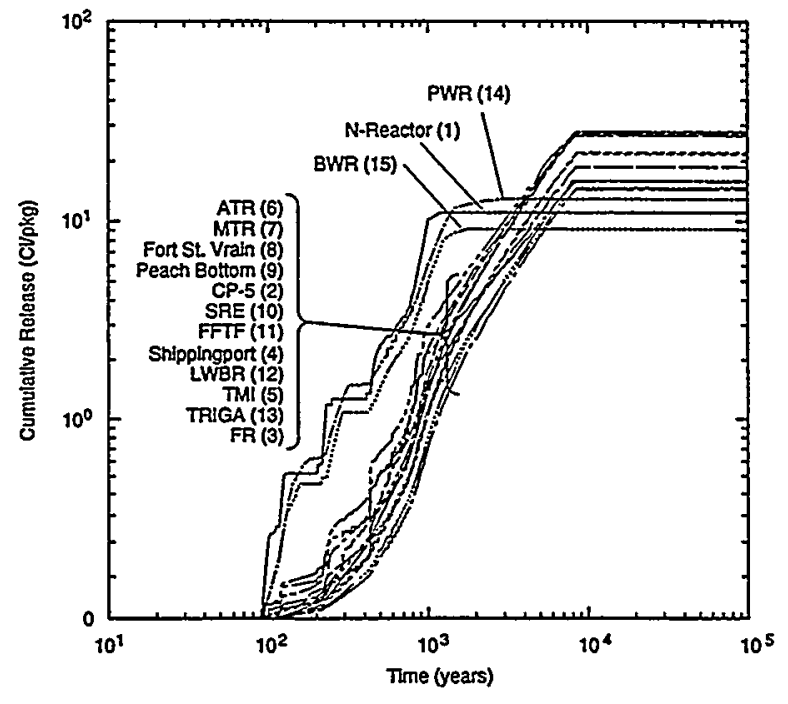

(b) Tc Release (Ci/Pkg)

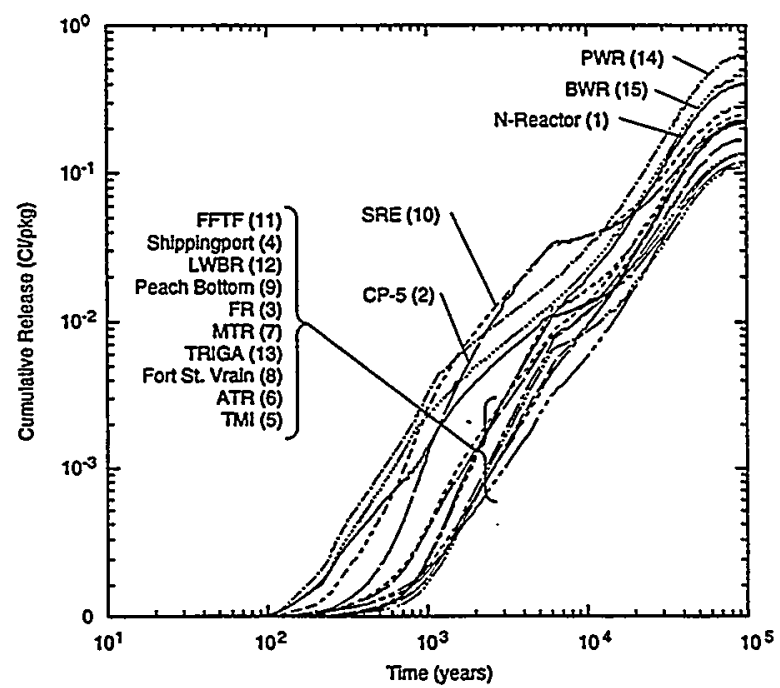

(d) Np Release (Ci/Pkg)

TRI-6342-5871-1

Figure 11-10. Releases from the waste package when cladding is included for the deterministic run using mean model parameters for $(a){ }^{99} \mathrm{Tc}$ normalized by mass of heavy radioisotopes in inventory, $(b){ }^{99} \mathrm{Tc}$ normalized by number of waste packages in each category, (c) ${ }^{237} \mathrm{~Np}$ normalized by mass of heavy radioisotopes in inventory, and (d) ${ }^{237} \mathrm{~Np}$ normalized by number of waste packages in each category. Releases from commercial SNF have been reduced by about $94 \%$ to about one order or magnitude below that released from codisposed DOE SNF (most releases from DHLW). 


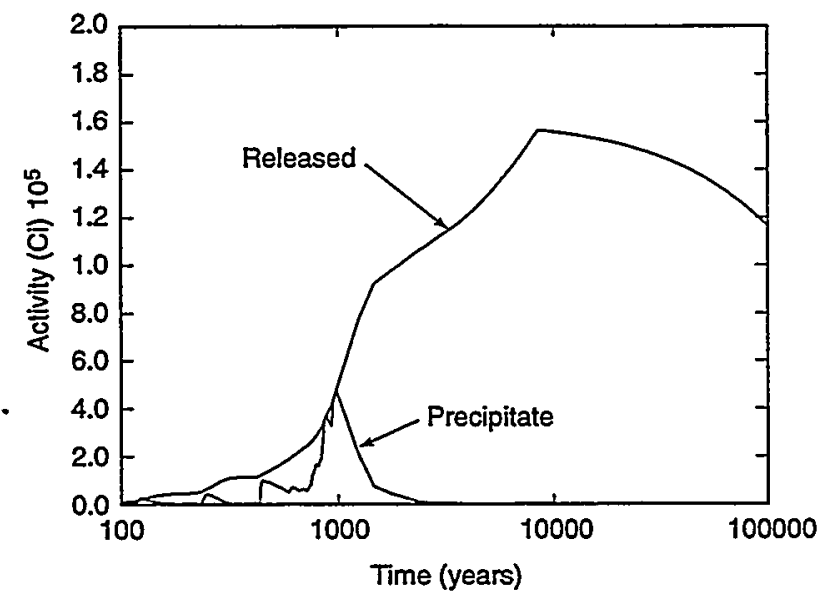

(a) ${ }^{99} \mathrm{Tc}$

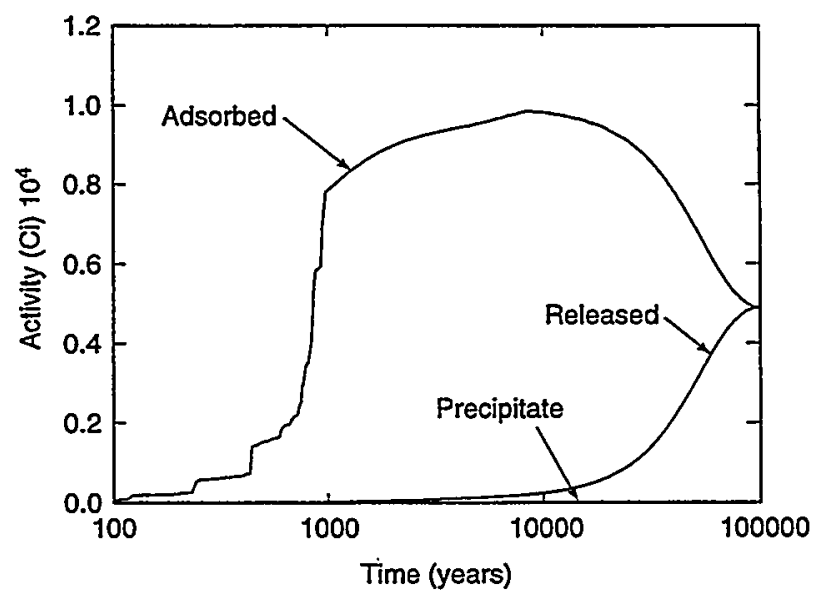

(b) ${ }^{237} \mathrm{~Np}$

TR1-6342-5872-1

Figure 11-11. Location of radioisotopes when cladding is included. Only radioisotopes not protected by cladding ( $6 \%$ of commercial SNF, $40 \%$ of $\mathrm{N}$-Reactor fuel) are released in the first $10^{5} \mathrm{yr}$. Hence, releases are reduced by about two orders of magnitude.

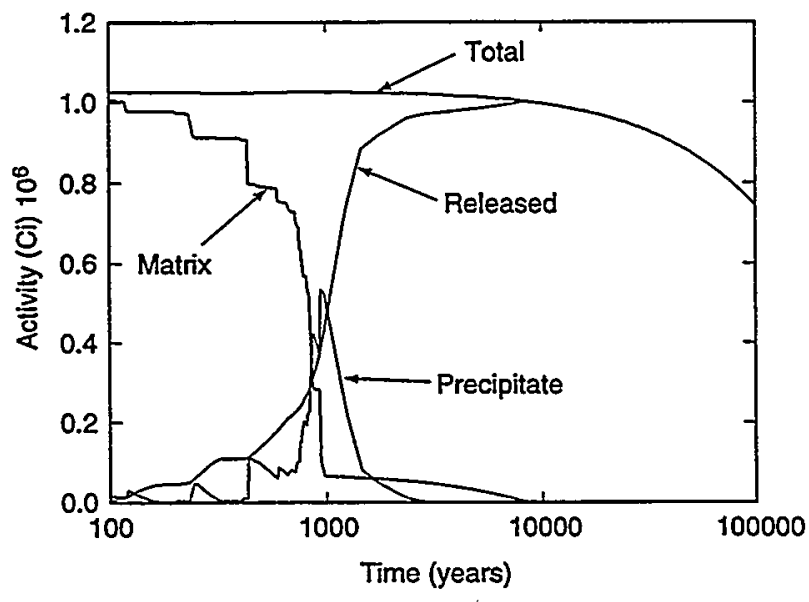

(a) ${ }^{99} \mathrm{Tc}$

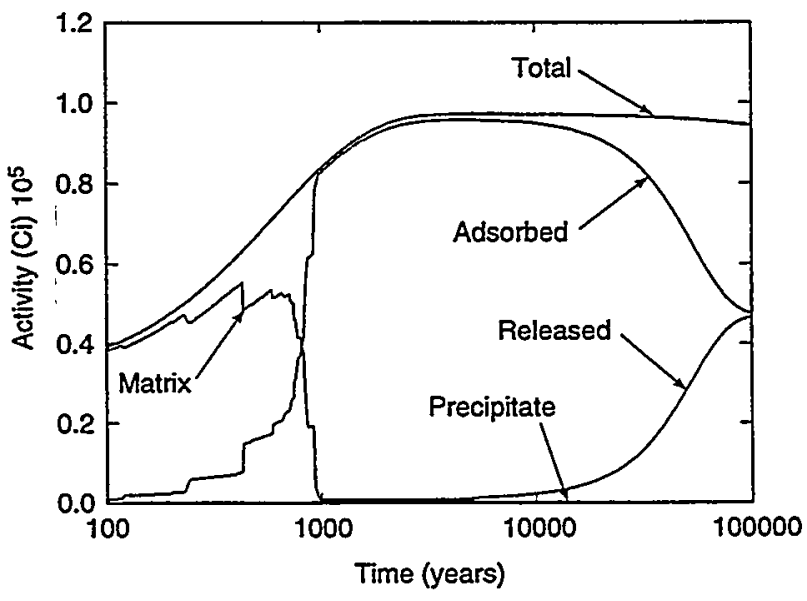

(b) ${ }^{237} \mathrm{~Np}$

TRI-6342-5873-1

Figure 11-12. Location of radioisotopes in various components of the source term model when doubling surface area of fuel matrix on commercial SNF and N-Reactor fuel in the deterministic run, using mean model parameter values for all other parameters for $(a)^{99} \mathrm{Tc}$ and $(b)^{237} \mathrm{~Np}$. 


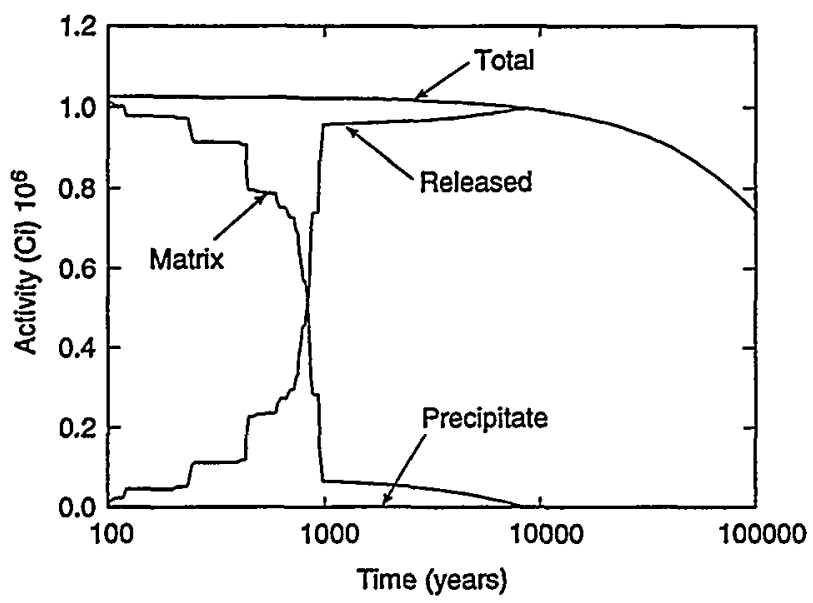

(a) ${ }^{99} \mathrm{Tc}$

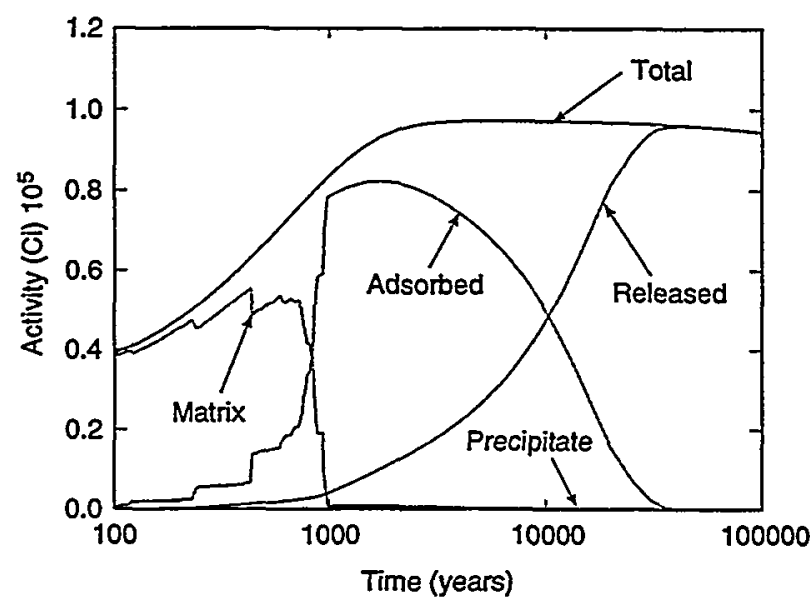

(b) ${ }^{237} \mathrm{~Np}$

TR1-6342-5877-1

Figure 11-13. Location of radioisotopes when solubility is set at maximum values (and sorption, $K_{D}$, of $N p$ on rust of $0.025 \mathrm{~kg} / \mathrm{m}^{3}$ ). (a) For $T c$, rate of release is influenced by alteration of matrix at $1 \mathrm{M}$ solubility but total release is not affected, and (b) both rate and total amount of $N p$ released increases when solubility of $\mathrm{Np}$ is increased to $10^{-4} \mathrm{M}$. 


\section{Selected Overall Results}

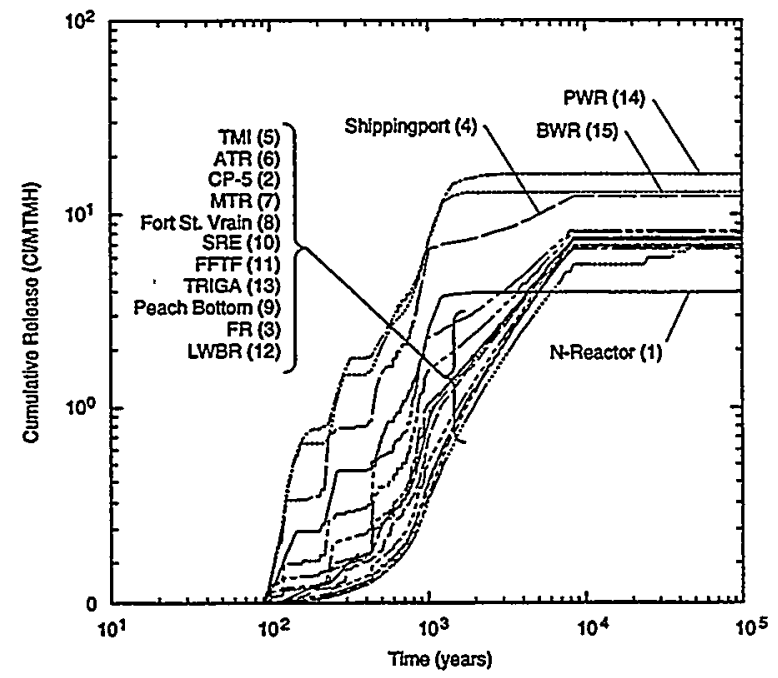

(a) Tc Release (Ci/MTHM)

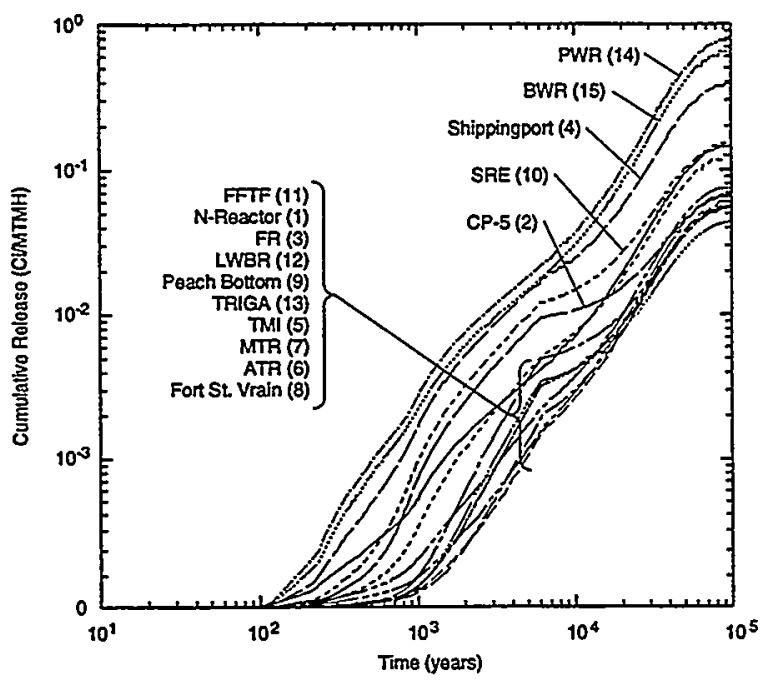

(c) Np Release (Ci/MTHM)

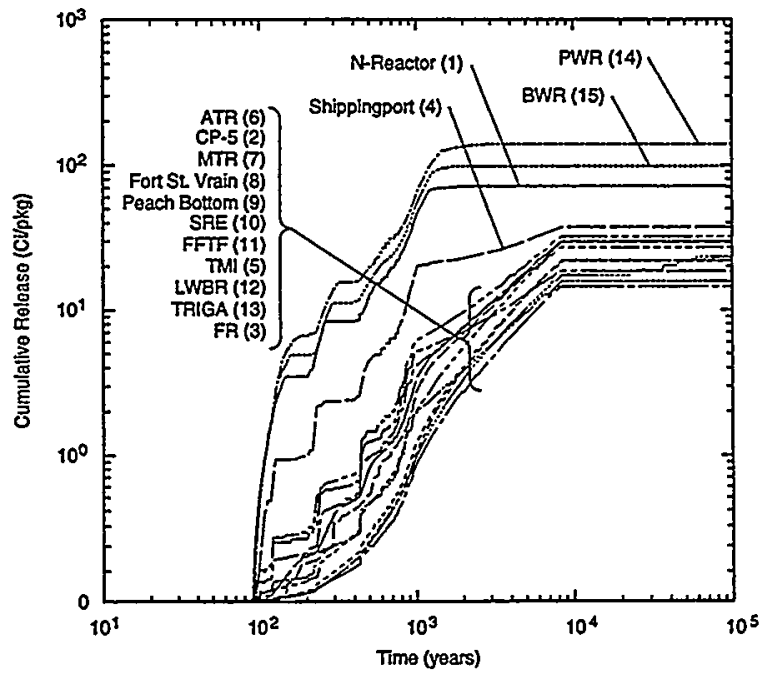

(b) Tc Release (Ci/Pkg)

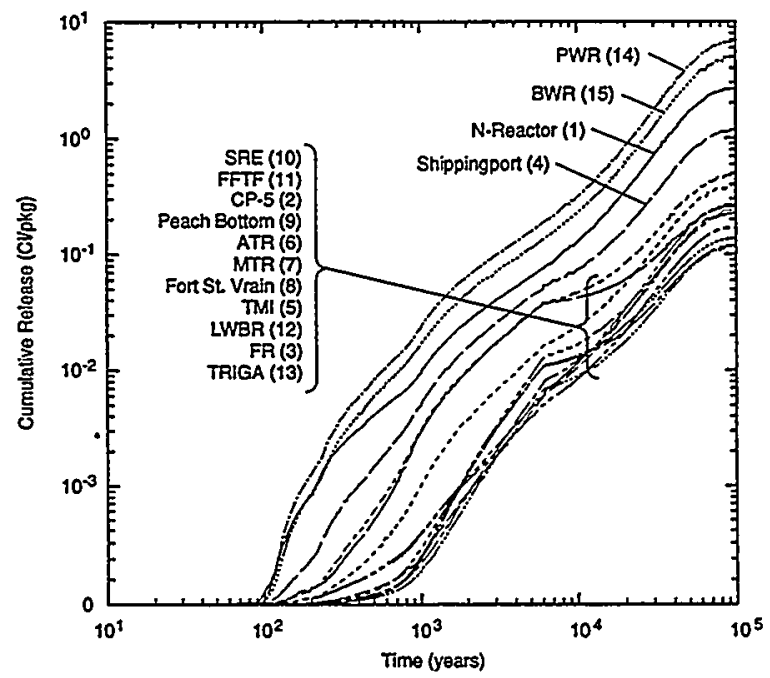

(d) Np Release (Ci/Pkg)

TRI-6342-5874-1

Figure 11-14. Fraction of releases from the waste package when the inventory of DOE SNF is tripled for the deterministic run using mean model parameter values for $(a)^{99} \mathrm{Tc}$ normalized by mass of heavy radioisotopes in inventory, (b) ${ }^{99} \mathrm{Tc}$ normalized by number of waste packages in each category, (c) ${ }^{237} \mathrm{~Np}$ normalized by mass of heavy radioisotopes in inventory, and $(d)^{237} \mathrm{~Np}$ normalized by number of waste packages in each category. 
Table 11-1 is a summary of the model results for the waste form.

Table 11-1. Summary of Model Results for Waste Form

1. Corrosion rates of borosilicate glass and various DOE SNF waste forms were similar to the possible range of $\mathrm{UO}_{2}\left(10^{-7}\right.$ to $\left.10^{1} \mathrm{~mm} / \mathrm{yr}\right)$ in commercial SNF (Figure 11-3).

2. Cumulative releases of ${ }^{99} \mathrm{Tc}^{*}$ and ${ }^{237} \mathrm{~Np}$ from commercial SNF exceed those of DOE SNF both on per MTHM and per package basis (Figure 11-4). Thus, if commercial SNF can meet regulatory performance criteria, then the DOE SNF can also meet the criteria. This result is due in large part to the lower content of Tc and Np in codisposal packages (Figures 11-4a and c, and Figure 11-6), the lower number of DOE SNF assemblies per waste package (Figures 11-4b and $d$ and Figure 11-5), and the assumption that the cladding of the commercial SNF provided no protection.

3. In contrast to the $1994 \mathrm{PA}$, the type and integrity of cladding were not important in the 1997 PA because all the cladding was assumed to have failed. As shown in the 1994 PA and in sensitivity analysis conducted herein (Figure 11-10), the low corrosion rate of zircaloy provides an important barrier; hence not including this protective layer is a very significant assumption.

4. At the highest solubility ( $1 \mathrm{M}$ ), releases of ${ }^{99} \mathrm{Tc}$ are limited only by the amount of waste that is exposed (Figure 11-7a); hence, the quick release fraction of commercial SNF is an important parameter. At average solubility, $10^{-3} \mathrm{M}, \mathrm{Tc}$ solubility controls releases and the quick release fraction is unimportant (Figure 11-8a).

5. Only at the highest solubility of $\mathrm{Tc}$ is the corrosion rate of the fuel matrix, e.g., $\mathrm{UO}_{2}$, an important parameter.

6. Even at the highest solubility $\left(10^{-4} \mathrm{M}\right)$, releases of ${ }^{237} \mathrm{~Np}$ are limited by its solubility (Figure 11-7a); hence the solubility of $\mathrm{Np}$ is a very important parameter to evaluate experimentally.

7. At the high fluid flow rate through the repository, the releases of both ${ }^{99} \mathrm{Tc}$ and ${ }^{237} \mathrm{~Np}$ are dominated by advective releases from water passing through the container (Figure 11-9a).

8. Intact zircaloy cladding does not fail within $10^{5} \mathrm{yr}$ (Figure 11-11) even when the corrosion rate is doubled (Figure 11-12).

9. Tripling the DOE SNF TC and Np inventory had only a minor influence on performance because the DOE SNF is such a small contributor to the total mass of the repository (Figure 11-14).

- As noted in Section 11.5, neptunium, technetium, and iodine radioisotopes are the primary contributors to human dose at the accessible environment since they are poorly retarded by the volcanic tuff. Because iodine behaves similarly to technetium, only neptunium and technetium are typically discussed in this table. 


\subsection{Findings for Waste Container}

\subsubsection{Description of Containers}

In the $1997 \mathrm{PA}$, the plans for the handling and disposal containers and the codisposal configurations for the DOE SNF, DHLW, and commercial SNF as of September 1997 were incorporated, although the variety of dimensions and configurations were reduced to ease the modeling burden.

Handling Containers. The DOE SNF and DHILW were modeled as being placed in handling containers and disposal containers (see Chapter 3). DOE SNF Category 1 (N-Reactor fuel) was modeled in a multi-canister overpack (MCO), which consisted of a $0.95-\mathrm{cm}$-thick, $61-\mathrm{cm}$ outer diameter stainless steel shell. DOE SNF Categories 2 through 13 were modeled in a 6.35 -mm-thick, $304 \mathrm{~L}$ stainless steel handling container. The DHLW handling container was modeled as the $0.95-\mathrm{cm}$-thick, $61-\mathrm{cm}$-diameter standard DOE canister.

Disposal Containers. Each of the 10,810 disposal containers for all waste packages included a 10-cm-thick outer carbon steel layer and a 2-cm-thick inner Inconel 625 layer. Most of the disposal containers modeled were $5.3 \mathrm{~m}$ long and $1.65 \mathrm{~m}$ in diameter. For Waste Package 1, four MCOs containing N-Reactor fuel were placed in this disposal container; for Waste Packages 2 through 13, DOE SNF/DHLW were codisposed with either 3, 4, or 5 handling containers of DHLW; for Waste Packages 14 and 15, 21-PWR and 44-BWR assemblies, respectively, were placed in the disposal container.

Container Conditions. In general, the air gap between the waste package and the intact host rock was modeled by means of an increase in porosity. During the performance period, rubble from the back (top) and ribs (sides) of the tunnels was assumed to eliminate the air gap, to some extent. If rubble were present (absence or presence of rubble was a sampled parameter) and if the $\mathrm{H}_{2} \mathrm{O}$ content of the tuff were high enough, movement of groundwater from the partially saturated tuff through the rubble to the waste packages was assumed. For the $1997 \mathrm{PA}$, four container groups were defined that specified (a) whether the inner Inconel 625 layer of the disposal container failed at the time of emplacement (yes/no) and (b) whether there was an air gap between the waste package and the intact host rock over 100,000 yr (yes/no) (see Chapter 7).

\subsubsection{Parameters Sampled}

Twelve parameters related to the disposal and handling containers were sampled. Five of these sampled parameters were related to the corrosion rate in a wet (dripping water) oxic environment for carbon steel, Hastaloy C-22, and stainless steel. Corrosion rates in other environments and layers (such as aluminum cladding, and graphite and TRISO covering) were constant. Another sampled parameter was the fraction of containers in which the inner Inconel 625 layer was flawed and thus immediately failed when exposed. Four parameters were related to the distribution coefficients for plutonium, uranium, neptunium, and zirconium on rust. The final two sampled parameters were the effective area for diffusion of radioisotopes through the breached container and the area above the container that contributed water as drips onto the container. None of these sampled parameters was found to significantly influence doses in the 1997 PA because at least $80 \%$ of the containers rapidly failed (see Figure 11-7a). A similar rapid failure occurred in the 1994 PA, but only 30\% to 40\% failed (see Figure 11-7b). In both the 1994 and 1997 PAs, the 100-mm carbon steel layer essentially provided no measurable protection except as an adsorbent for ${ }^{237} \mathrm{~Np}$, as discussed later.

The range of corrosion rates for Inconel 625 in a wet oxic environment is between $10^{-3}$ and $10^{-1} \mathrm{~mm} / \mathrm{yr}$ at $100^{\circ} \mathrm{C}$; the range at $25^{\circ} \mathrm{C}$ is 6 orders of magnitude less $\left(10^{-9}\right.$ to $\left.10^{-7} \mathrm{~mm} / \mathrm{yr}\right)$. Hence, if the Inconel 625 is exposed when the repository is wet and hot, the Inconel 625 rapidly corrodes. For comparison, the range of corrosion rates for carbon steel is only 2 orders of magnitude greater at $100^{\circ} \mathrm{C}\left(10^{-1}\right.$ and $\left.10^{1} \mathrm{~mm} / \mathrm{yr}\right)$ (Figure 11-15). The lower repository temperatures in the $1994 \mathrm{PA}$ reduced releases because the inner layer of the disposal container was less prone to failure. Substituting Hastaloy C-22 for Inconel 625 as the inner layer, as now planned by the YMP, will likely have a similar effect because Hastaloy C-22 is thought to be less prone to pitting corrosion and stress corrosion cracking at high temperatures. 


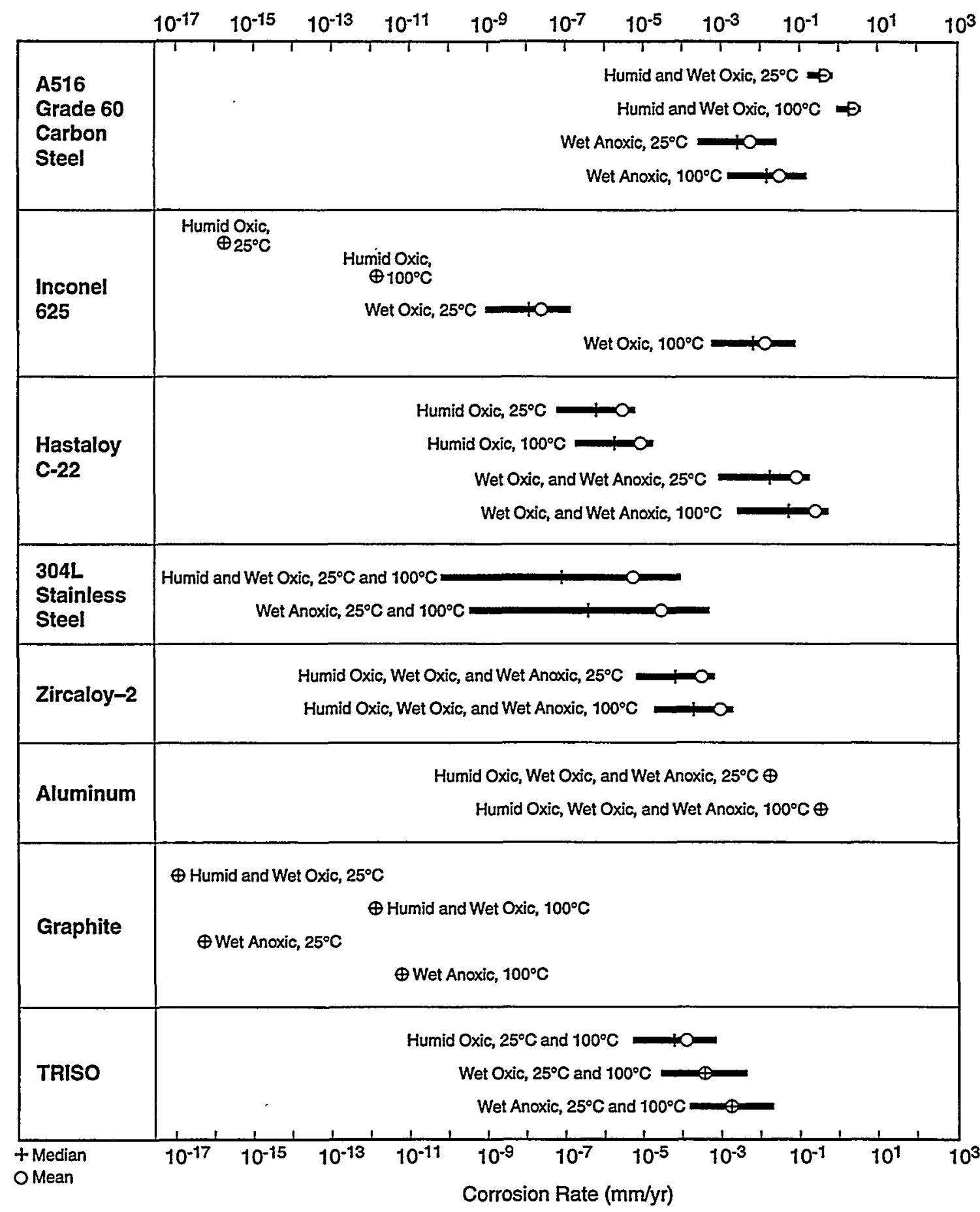

TRI-6342-5735-3

Figure 11-15. Corrosion rates for layers of waste containers and cladding on waste. The wet oxic rates for carbon steel, Inconel 625, and stainless steel were sampled in the 1997 PA. Note that these are general corrosion rates and do not account for localized pitting corrosion, which is accounted for through a reduced effective thickness. Also, note that the general corrosion rate of Inconel 625 is similar to that of carbon steel in a hot, wet environment. Thus, a cooler repository generally performs better than a hot one since, in the 1997 PA, the length of time the repository remains totally dry does not compensate for the increased corrosion. 


\subsubsection{Results for Waste Container}

The environment at the edge of the repository early on in the simulation is wet, oxic, and hot, and so containers breach at the edge within $200 \mathrm{yr}$ (Figure 11-16a). However, breach varies across the repository. In the 1994 PA, the breach and release situation was just the opposite because the temperatures were near $100^{\circ} \mathrm{C}$ in the center of the repository, but below $80^{\circ} \mathrm{C}$ at the edges (Figure $11-16 \mathrm{c}$ ). When temperature in the repository is reduced to $75^{\circ} \mathrm{C}$, the time of first breach shows a pattern similar to the 1994 PA (Figure 11-16b). The release rate of radioisotopes also varies across the repository (Figure 11-17).

In the $1997 \mathrm{PA}$, once an area in the repository cools below $100^{\circ} \mathrm{C}$, the entire carbon steel outer layer of the disposal container rapidly corrodes; at the repository center, complete corrosion of the carbon steel took about $1000 \mathrm{yr}$ in the simulation with mean parameters. The total amount of rust across the repository accumulated between 100 and $2000 \mathrm{yr}$ in this same simulation (Figure 11-18).

Integrity of the disposal container can potentially mitigate exposure from ${ }^{237} \mathrm{~Np}$. However, solubility is already limiting release of the roughly $28.9 \mathrm{kCi}$ of ${ }^{237} \mathrm{~Np}$ in the commercial SNF by a factor of 3 to $4 \mathrm{kCi}$ over $50,000 \mathrm{yr}$ (Figure 11-7c). Assuming a constant failure rate, container failure would have to be reduced from $100 \%$, as seen in the $1997 \mathrm{PA}$, to $25 \%$ in 50,000 yr to limit inventory release beyond that controlled by solubility.

The current mean ${ }^{237} \mathrm{~Np}$ solubility of $9.6 \times 10^{-4} \mathrm{~kg} / \mathrm{m}^{3}$ must be reduced to a concentration of $-10^{-9} \mathrm{~kg} / \mathrm{m}^{3}$ to stay below a 15 to $25 \mathrm{mrem} / \mathrm{yr}$ dose target (i.e., diluted $\sim 5$ orders of magnitude through a combination of dispersion and dilution with uncontaminated water when drawn from a well for irrigation). To obtain this additional 5 order of magnitude reduction solely by the disposal container would require failure of less than 1 container every $240,000 \mathrm{yr}$ (assuming $4.4 \mathrm{Ci} / \mathrm{pkg}$ of ${ }^{237} \mathrm{~Np}$ and $1.3 \times 10^{7} \mathrm{~m}^{3} / 50,000 \mathrm{yr}$ flowing through the repository) until sufficient amounts of ${ }^{237} \mathrm{~Np}$ have decayed (half-life of $2 \times 10^{6} \mathrm{yr}$ ). Such conditions could not be accomplished except by an extremely robust container. 


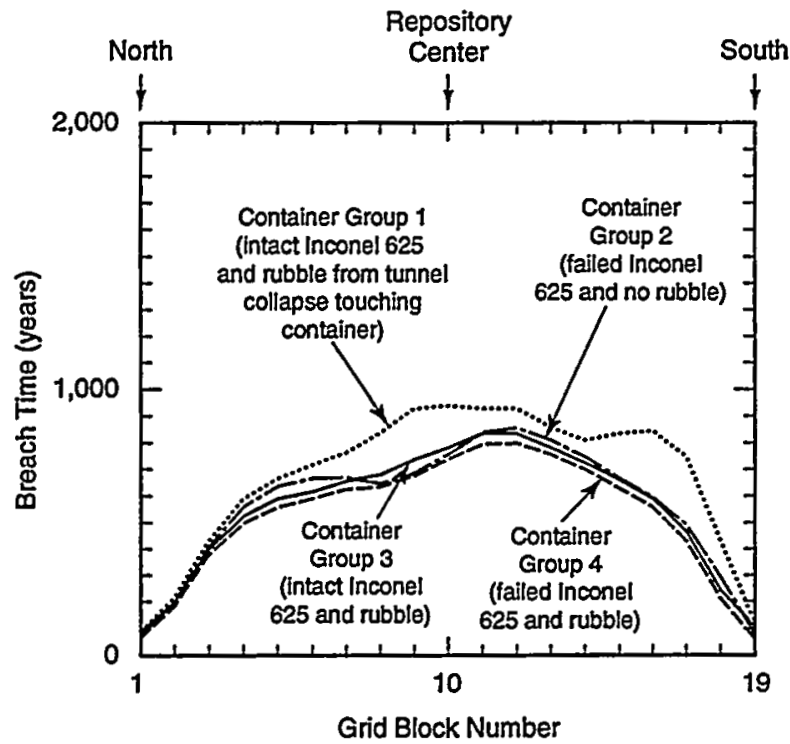

(a) 1997 PA 75,336 MTHM

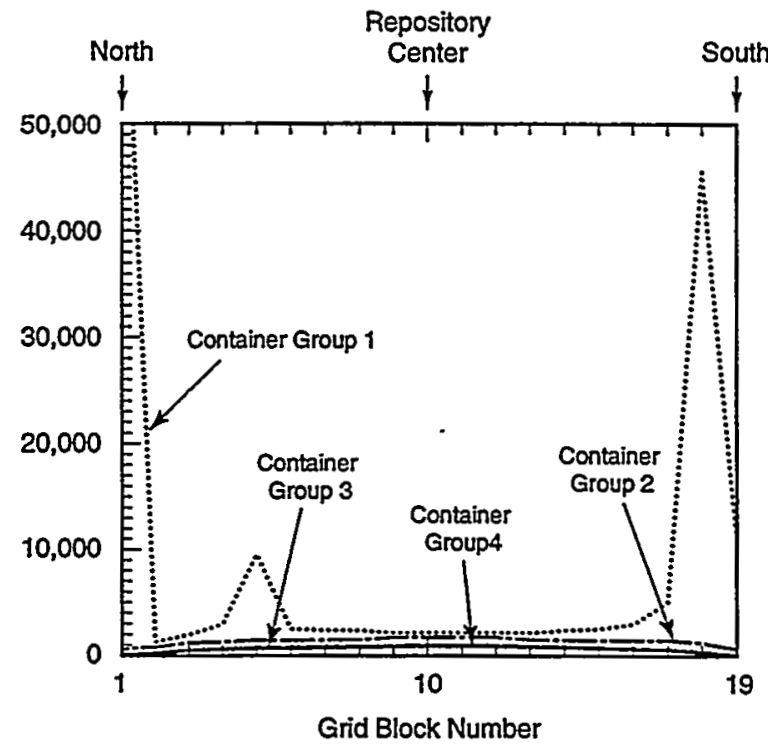

(b) 1997 PA peak temperature reduced to $75^{\circ} \mathrm{C}$.

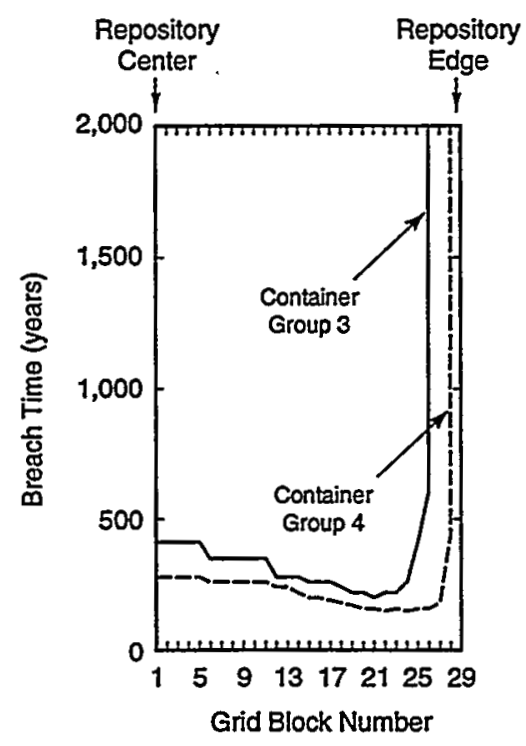

(c) 1994 PA 12,060 MTHM

TRI-6342-5810-5

Figure 11-16. Time of first breach for each container group across the repository for Waste Package I (N-Reactor) for (a) 1997 PA, 75,336-MTHM repository, (b) $1997 \mathrm{PA}$ with temperature reduced to $75^{\circ} \mathrm{C}$, and (c) 1994 PA, 12,060-MTHM repository. Container Group 1-no rubble, no early failure of Iconel 625 layer; Container Group 2-no rubble but early failure; Container Group 3-rubble present, but no early failure; Container Group 4-rubble present and early failure. In the 1994 PA, the whole repository was cooler. Temperatures were near $100^{\circ} \mathrm{C}$ in the center of the repository, which prompted rapid corrosion, but containers at the edge, where temperatures remained below $80^{\circ} \mathrm{C}$, did not breach. 


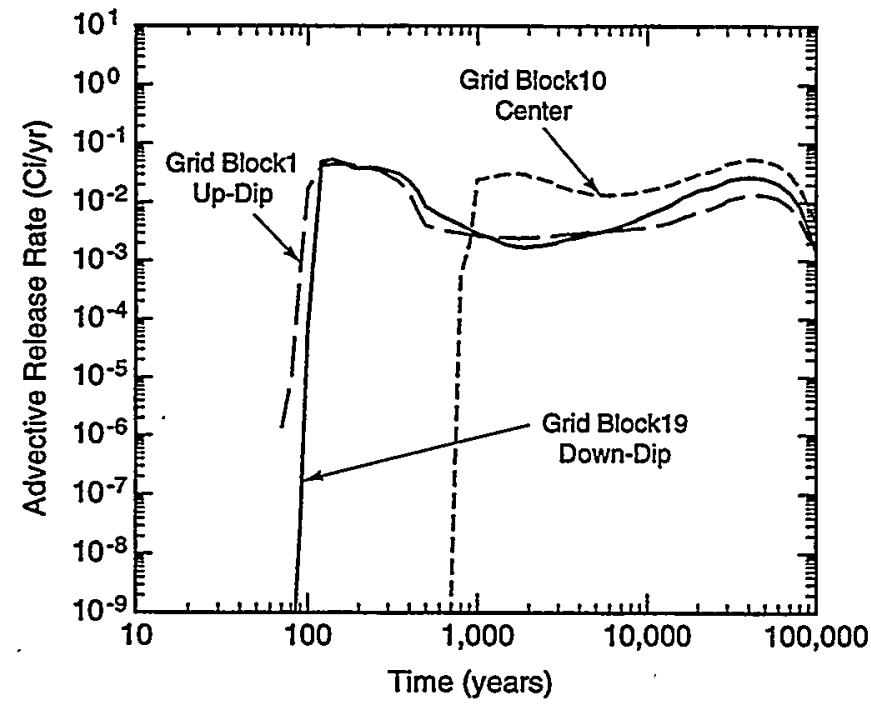

(a) Release rate of ${ }^{237} \mathrm{~Np}$ in repository in $1997 \mathrm{PA}$

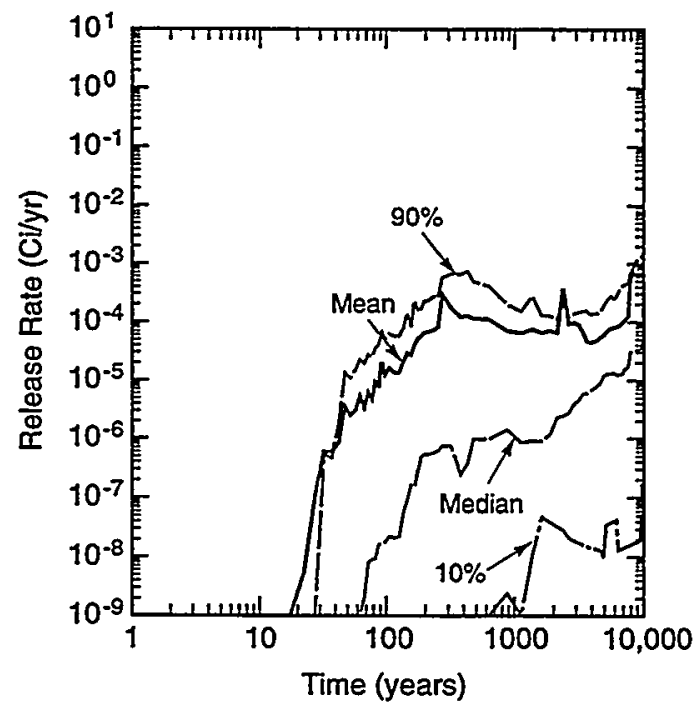

(b) Release rate of ${ }^{237} \mathrm{~Np}$ in repository in 1994 PA

TRI-6342-5809-5

Figure 11-17. Neptunium release rates from (a) deterministic run, using median properties, for 1997 PA hot repository compared to $(b)$ overall repository release rate of ${ }^{237} \mathrm{~Np}$ in $1994 \mathrm{PA}$. 


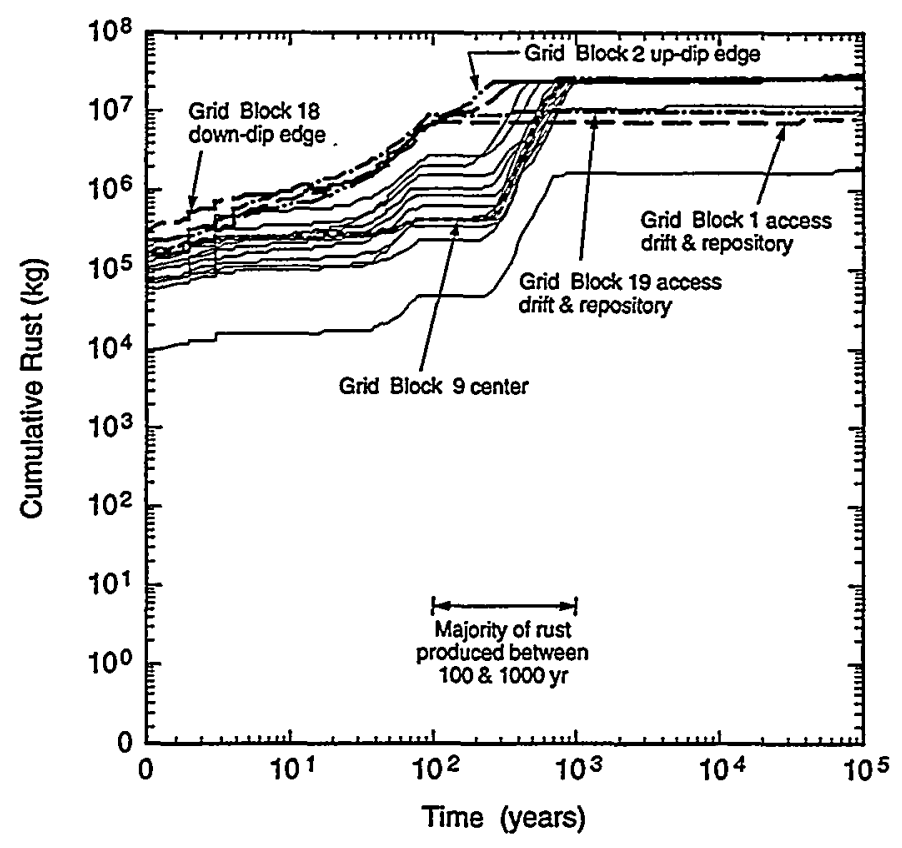

(a)

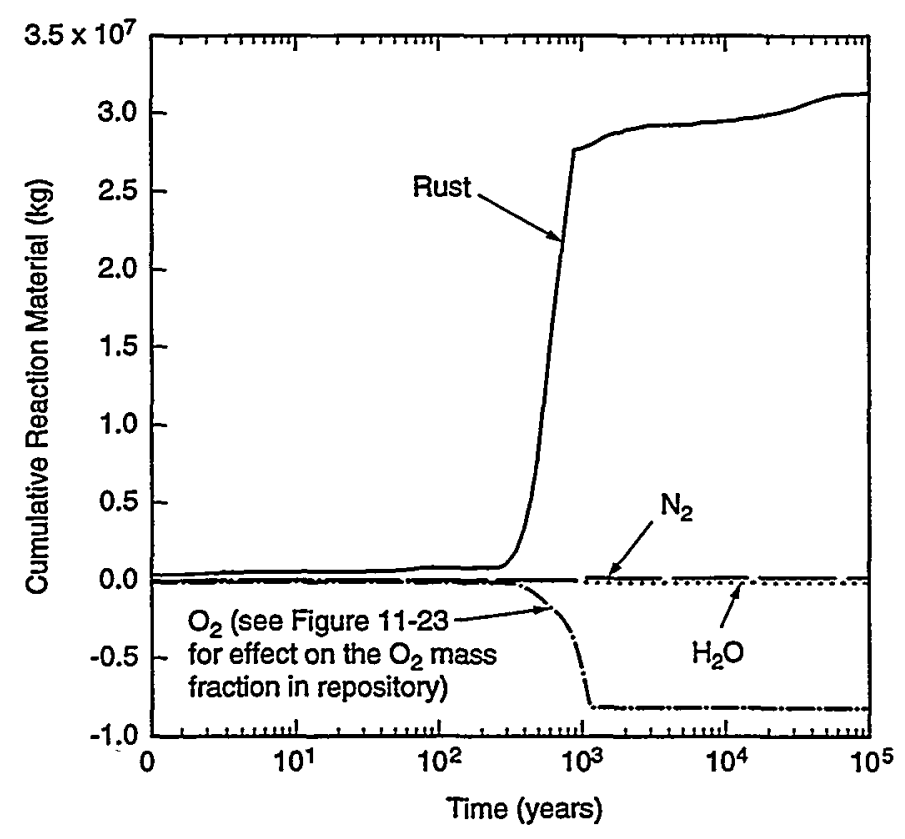

(b)

TRI-6342-5803-3

Figure 11-18. Production of reaction material within repository, using mean parameter values. (a) Production of rust in all grid blocks of repository from wet oxic corrosion of steel and (b) production of reaction material in center grid block of repository. The entire inventory of carbon steel is corroded in about $1000 \mathrm{yr}$, which appears nearly instantaneous when viewing the full 100,000-yr period. 


\subsubsection{Potential Changes in Container}

The YMP is actively considering changes in the material of the disposal container, including the use of Hastaloy C-22 in place of Inconel 625, titanium in place of carbon steel, etc. The use of Hastaloy C-22 is examined in this section.

Influence of Use of Hastaloy C-22. Because of the potential for Inconel 625 to experience localized pitting corrosion at high temperatures, the YMP will likely change to Hastaloy C-22 for the second layer of the disposal container, and thus this study examined the influence of this change on the time of breach and total amount of release using approximately the same generalized corrosion rates estimated by the YMP (i.e., the corrosion rate was assumed to be a factor of 100 faster than zircaloy) (Figure 11-15). Because the corrosion rate was much faster than zircaloy, yet the thickness of the Hastaloy C-22 layer was only a factor of 10 greater than the thickness of zircaloy cladding, the C-22 does not provide as much protection as the zircaloy cladding. However, neither the cladding nor the C-22 layer fail within the first $10^{5}$ year. The only release in this period is from faulty C-22 layers ("prebreached") (Figure 11-19).

Influence of Change in Size of Container. Consolidation of SNF is occasionally proposed to allow the YMP to use smaller packages. The time of breach of a smaller (or larger) container does not change, provided the thickness of the container layers do not change when the size is changed (or, alternately, the surface area and mass of the layer are changed at the same time). However, the location of radioisotopes within the container can change if the corrosion products of the container are fairly adsorptive (Figure 11-20). Furthermore, even at a very small $\mathrm{K}_{\mathrm{D}}\left(0.025 \mathrm{~m}^{3} / \mathrm{kg}\right)$, the timing of the release is influenced by the availability of the adsorptive rust (Figure 11-21). 


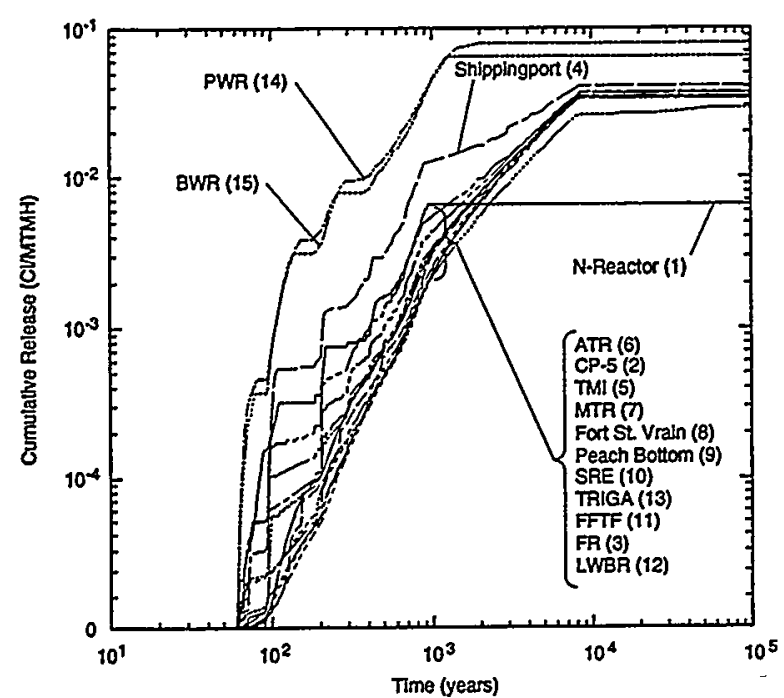

(a) Tc Release (Ci/MTHM)

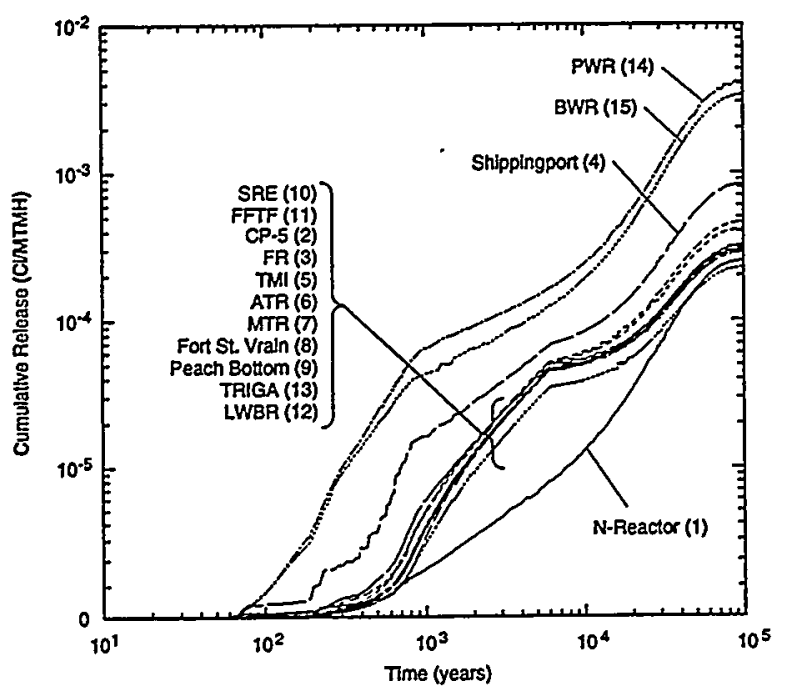

(c) Np Release (Ci/MTHM)

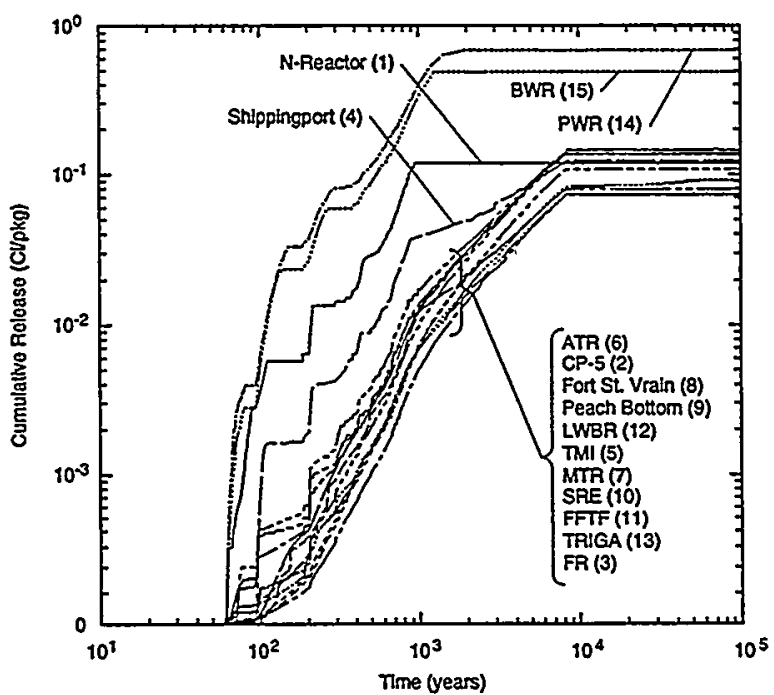

(b) Tc Release (Ci/Pkg)

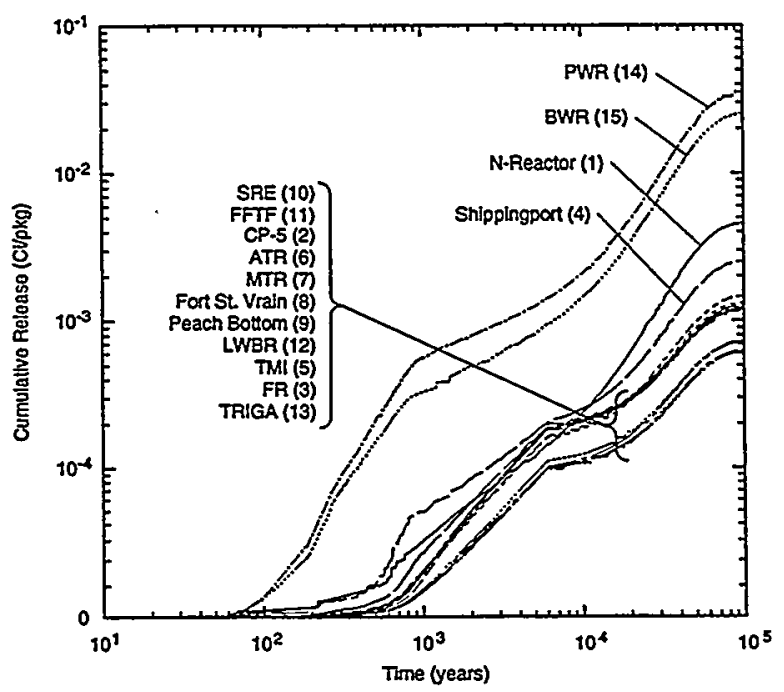

(d) Np Release (Ci/Pkg)

TRI-6342-5875-1

Figure 11-19. Fraction of release from the waste package when using Hastaloy C-22 in place of Inconel 625 in the deterministic run using mean model parameter values for $(a){ }^{99} \mathrm{TC}$ normalized by mass of heavy radioisotopes in inventory, (b) ${ }^{99} \mathrm{Tc}$ normalized by number of waste packages in each category, (c) ${ }^{237} \mathrm{~Np}$ normalized by mass of heavy radioisotopes in inventory, and (d) ${ }^{237} \mathrm{~Np}$ normalized by number of waste packages in each category. 
11. Selected Overall Results

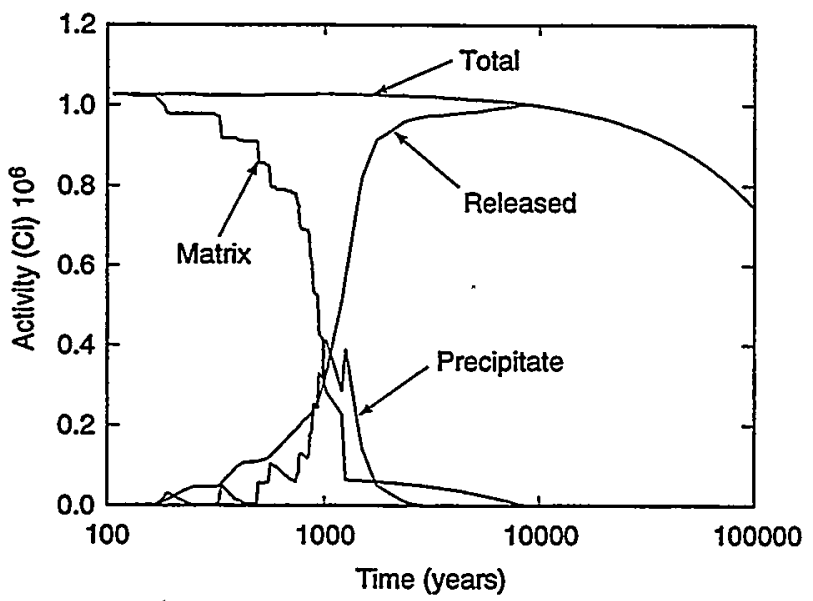

(a) ${ }^{99} \mathrm{Tc}$

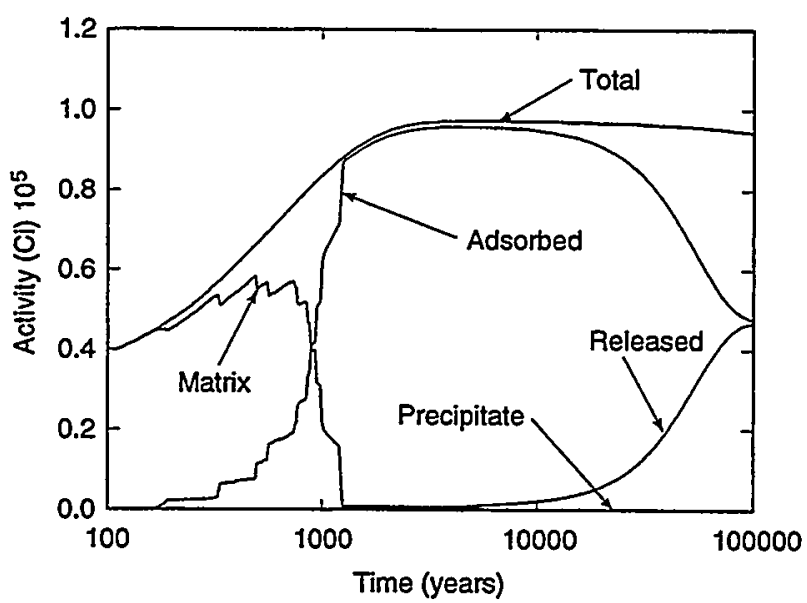

(b) ${ }^{237} \mathrm{~Np}$

TRI-6342-5876-1

Figure 11-20. Location of radioisotopes when the size of the container is halved through waste consolidation for the deterministic run using mean model parameter values for $(a)^{99} \mathrm{Tc}$ and $(b)^{237} \mathrm{~Np}$ (at $4.0 \times 10^{-6} \mathrm{M}$ solubility). Total releases are not changed.

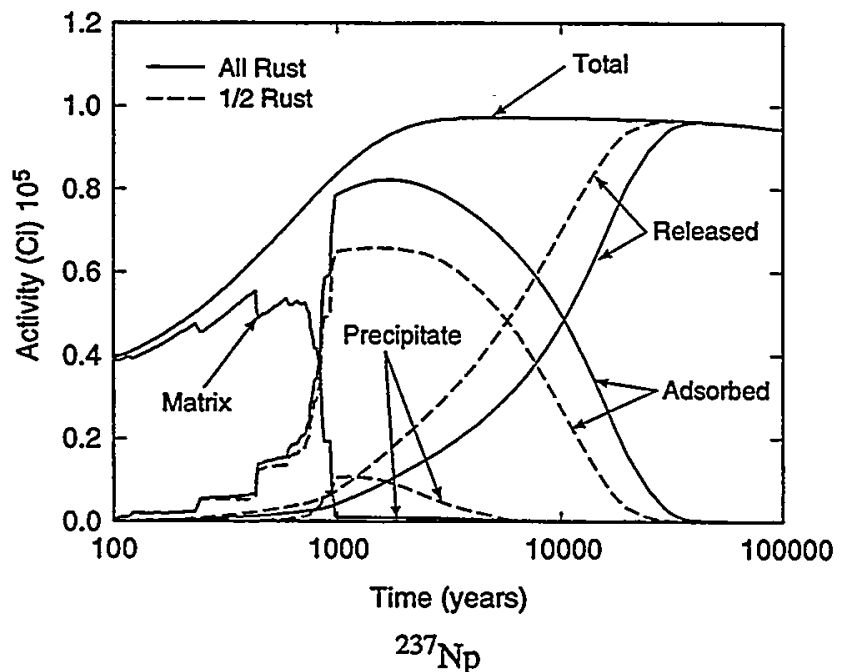

TRI-6342-5878-1

Figure 11-21. When amount of adsorptive rust is decreased by one-half using the maximum solubility of $10^{-4} M$, the time of ${ }^{237} \mathrm{~Np}$ release occurs earlier even at small $K_{D}$ values $\left(0.025 \mathrm{~m}^{3} / \mathrm{kg}\right.$ ). 
Table 11-2 summarizes the results for the waste container.

Table 11-2. Summary of Model Results for Waste Container

1. The carbon steel layer provides very little protection (similar to results in $1994 \mathrm{PA}$ ).

2. Failure of at least $80 \%$ of the containers occurs in deterministic runs in the first 3000 to $5000 \mathrm{yr}$ (see Figure 11-7 [Section 11.1]). The remaining containers do not fail before $100,000 \mathrm{yr}$.

3. Waste containers breach earliest (around $200 \mathrm{yr}$ ) at the edges of the repository (Figure 11-16a). Waste containers near the edge of the repository breach earliest because they are near $100^{\circ} \mathrm{C}$ for a longer period at early times. (This is the opposite of the $1994 \mathrm{PA}$ because the temperatures were near $100^{\circ} \mathrm{C}$ close to the center and the edges remained below $80^{\circ} \mathrm{C}$, and thus below the temperature at which inconel 625 is susceptible to pitting corrosion.)

4. The time histories of releases are somewhat different across the repository because of differences in temperature and water flux (Figure 11-17).

5. Once the repository cools below $100^{\circ} \mathrm{C}(\sim 1500 \mathrm{yr}$ after emplacement; see Section 11.3), the entire carbon steel outer disposal container rapidly corrodes between $100 \mathrm{yr}$ and $2000 \mathrm{yr}$ in the deterministic run with mean parameter values (Figure 11-18a).

6. When Inconel 625 is replaced by Hastaloy C-22, intact containers do not fail. (Hastaloy C-22 is a material with an assumed corrosion rate 100 times faster than zircaloy and not susceptible to localized corrosion.) Only those containers assumed to be faulty $(0.5 \%)$ release any radioisotopes in the first $10^{5} \mathrm{yr}$ (Figure 11-19).

7. Amount of sorptive material (e.g., amount of rust from a corroded steel container) can influence the timing of the release of ${ }^{237} \mathrm{~Np}$ (Figure 11-21). 


\subsection{Findings for Repository Design}

\subsubsection{Description}

For the $1997 \mathrm{PA}$, the potential repository modeled was sized at $1252 \mathrm{~m}$ by $2493 \mathrm{~m}$ to accommodate 75,336 MTHM of spent fuel or equivalent high-level waste. The waste was uniformly distributed throughout the repository, with the DOE-owned waste comingled with commercial waste. The repository consists of $1236-\mathrm{m}$-long tunnels bored by machine. The tunnels are surrounded and connected by access drifts, which lead to ramps that connect to the surface (M\&O, 1996). The design goal in the 1997 PA was to use the hot repository concept. A center-to-center spacing of $28.6 \mathrm{~m}$ between the $5.3-\mathrm{m}$-wide tunnels was used to maintain a heat load equivalent of $21.2 \mathrm{~W} / \mathrm{m}^{2}$ over the repository area (2.1 MTHM/m $\mathrm{m}^{2}$ or $85 \mathrm{MTHM} / \mathrm{acre}$ for commercial fuel). (The repository power density was $13.5 \mathrm{~W} /$ $\mathrm{m}^{2}$ in the $1994 \mathrm{PA}$.) The 1236 -m-long tunnels were assumed to be lined with a 0.2 -m-thick precast concrete liner. A thick segment on the base of the tunnel forms an invert into which a minimum of 3 piers can be set for container support. Only the ramps to the disposal area were assumed to be backfilled and effectively sealed. The parameter that was varied for modeling the repository was fraction of waste packages that are in contact with rubble from the tunnel roof.

\subsubsection{Results for Repository Design}

The thermal loading from combining the commercial SNF with the codisposed DOE SNF/DHLW is sufficient to vaporize water near the repository center within $4 \mathrm{yr}$ of emplacement and produce superheated steam between 80 and 200 yr. Only eight radioisotopes contribute the majority of the heat during this early time (Table 11-3). Hence, only these eight radioisotopes, plus ${ }^{237} \mathrm{~Np},{ }^{99} \mathrm{Tc}$, and a few other radioisotopes important for doses, need to be accurately evaluated by the NSNFP for the DOE/EM. The time and maximum temperature of the peak were very sensitive to the permeability of tuff directly around the repository in the unsaturated zone. Lower permeability trapped steam and increased temperatures, while higher permeability allowed steam to escape, transporting heat away from the repository. After $2000 \mathrm{yr}$, the water had completely condensed at the repository center (Figure 11-22a). At the repository edge, heat is dissipated to the surrounding formation at a faster rate than at the repository center. Hence, about twothirds of the simulations maintained water at $100^{\circ} \mathrm{C}$; one-third of the runs produced superheated steam. Consequently, more liquid water was available at the repository edge for corrosion (Figure 11-22b), resulting in the breach (but not complete corrosion) of a few disposal containers within $200 \mathrm{yr}$, as noted in previous sections.

Table 11-3. Major Contributors to Initial Power

\begin{tabular}{lcc}
\hline Radioisotope & $\begin{array}{c}\text { Power } \\
\text { (MW) }\end{array}$ & $\begin{array}{c}\text { Percent of Total } \\
(66.23 \mathrm{MW})\end{array}$ \\
\hline${ }^{137 \mathrm{~m} \mathrm{Ba}}$ & 19.02 & 28.72 \\
${ }^{90} \mathrm{Y}$ & 18.26 & 27.57 \\
${ }^{238} \mathrm{Pu}$ & 7.40 & 11.17 \\
${ }^{241} \mathrm{Am}$ & 6.88 & 10.39 \\
${ }^{137} \mathrm{Cs}$ & 4.88 & 7.37 \\
${ }^{244} \mathrm{Cm}$ & 3.94 & 5.95 \\
${ }^{90} \mathrm{Sr}$ & 3.82 & 5.77 \\
${ }^{240} \mathrm{Pu}$ & 1.09 & 1.65 \\
\hline
\end{tabular}




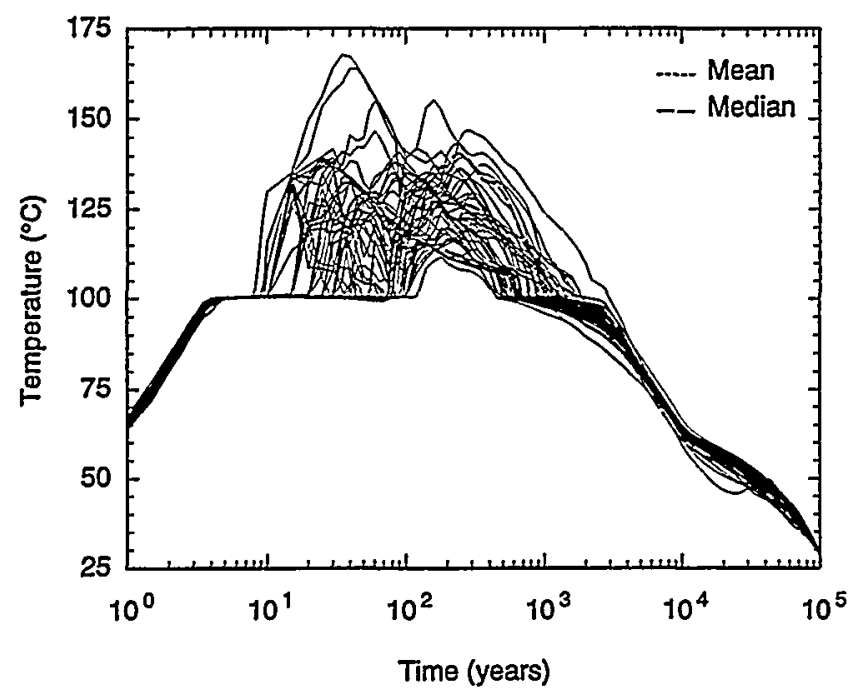

(a) Center

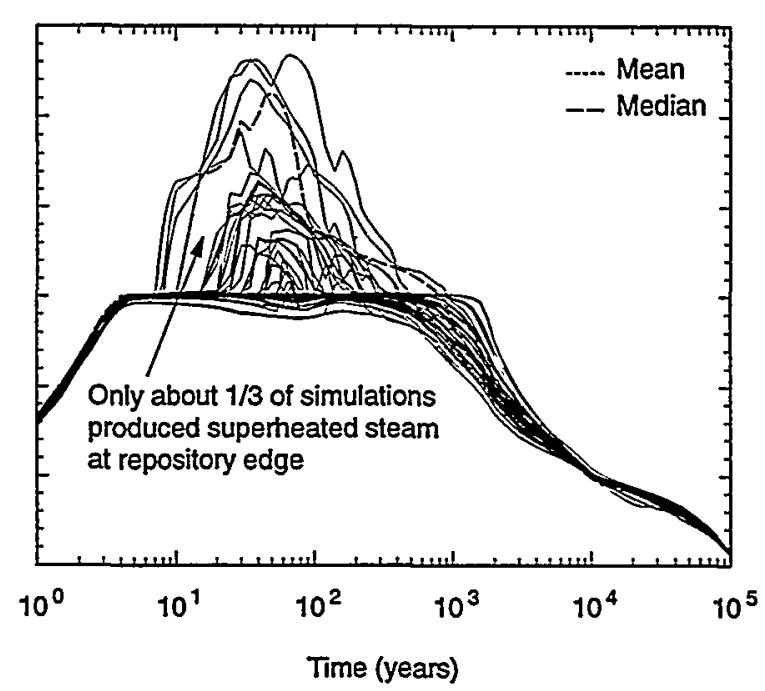

(b) Down-Dip Edge

TRI-6342-5827-1

Figure 11-22. Mass. fraction of $\mathrm{O}_{2}$ available for oxic alteration of fuel matrix at (a) center and (b) edge of potential repository over $10^{5}$ yr. Although oxygen is not available while the repository is hot, after about 1000 $y r$, sufficient oxygen diffuses (or advects) into the repository to replenish the oxygen consumed during corrosion of the containers and alteration of the waste matrices (see also Chapter 8).

Water vaporization at early times displaces the noncondensible gases $\left(\mathrm{O}_{2}\right.$ and $\left.\mathrm{N}_{2}\right)$ within the repository. The oxygen mass fraction is near zero from about 40 to $200 \mathrm{yr}$ at the repository center. Beyond $200 \mathrm{yr}$, the water vapor condenses and the oxygen diffuses back to the repository. By $1000 \mathrm{yr}$, sufficient oxygen (through diffusion and/or advection) is available to allow wet oxic corrosion of the carbon steel layer of the disposal container.

The simulation with the mean parameter values took approximately another $1000 \mathrm{yr}$ to consume the entire inventory of carbon steel (Figure 11-23; see also Figure 11-18). Diffusion and/or advection of oxygen through the tuff is sufficient to supply the oxygen consumed during corrosion and alteration of the waste. Because the diffusion of oxygen is sensitive to the porosity of the volcanic tuff, the time of corrosion can extend to $3000 \mathrm{yr}$ at low porosity values or decrease to $500 \mathrm{yr}$ at high porosity values; however, the observed variation of 500 to $3000 \mathrm{yr}$ in time is not signifcant and somewhat difficult to perceive on a linear time scale of 0 to $100,000 \mathrm{yr}$ (i.e., the consumption appears fairly instantaneous). Therefore, availability of oxygen is not a significant factor in determining the corrosion rate of materials in the repository. ${ }^{\ddagger}$ That is, there is little mathematical coupling of the source term model, CST, with the fluid flow model, BRAGFLO_T, with regard to oxygen consumption; only the coupling provided by the temperature and water saturation variables is important.

Because of the assumed sensitivity of Inconel 625 to pitting corrosion at temperatures above $80^{\circ} \mathrm{C}$, the containers readily breach, as discussed in Section 11.2 (see Figure 11-16a). An alternative to changing the material of the container is to space the waste containers such that the repository remains cooler, as in the 1994 PA (Figure 11-16c). These findings are summarized in Table 11-4.

\footnotetext{
\# However, this result does not imply that there is enough $\mathrm{O}_{2}$ to sustain a fire. A model of an individual container would be necessary to confirm the suspected inability of available $\mathrm{O}_{2}$ to sustain a fire.
} 


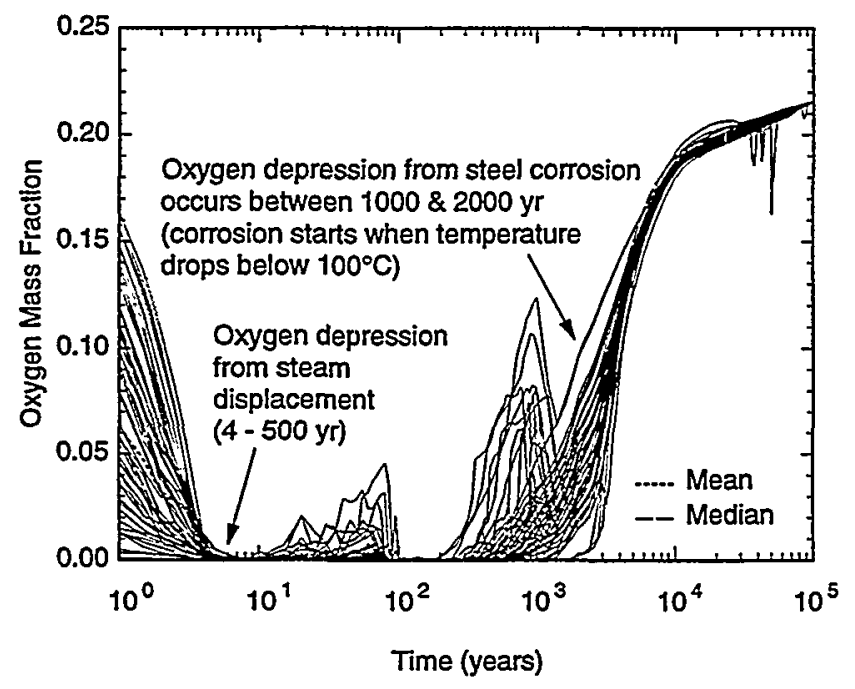

(a) Center

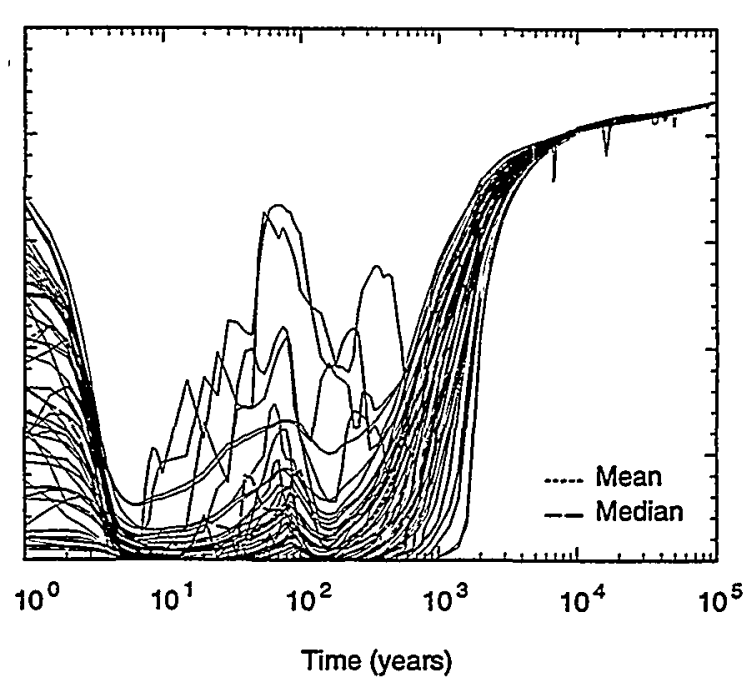

(b) Down-Dip Edge

TRI-6342-5597-5

Figure 11-23. Mass fraction of $\mathrm{O}_{2}$ available for oxic alteration of fuel matrix at (a) center and (b) edge of potential repository over 100,000 yr. Although oxygen is not available while the repository is hot, after about $1000 \mathrm{yr}$, sufficient oxygen diffuses (or advects) into the repository to replenish the oxygen consumed during corrosion of the containers and alteration of the waste matrices (see also Chapter 8).

Table 11-4. Summary of Model Results for Repository

1. The thermal loading from combining the commercial SNF with the codisposed DOE SNF/DHLW is sufficient to vaporize the water near the repository center, but after $2000 \mathrm{yr}$, the water has completely condensed to a single phase liquid (Figure 11-22a).

2. At the repository edge, some runs (about one third) produced superheated steam (Figure 11-22b), while others maintained the water in the liquid phase. Thus more liquid water is available at the repository edge for corrosion.

3. Water vaporization between 40 to $200 \mathrm{yr}$ displaces the noncondensible gases $\left(\mathrm{O}_{2}\right.$ and $\left.\mathrm{N}_{2}\right)$ within the repository (Figure 11-23).

4. Between 1000 and $2000 \mathrm{yr}$, sufficient oxygen is available through diffusion and/or advection from the surrounding tuff (Figure 11-23). 


\subsection{Findings for Geologic Barrier and Climate Change}

\subsubsection{Description}

For the potential Yucca Mountain repository, the source term model components (waste form and disposal container, discussed in previous sections) are extremely important to determining the ultimate doses; however, the geologic barrier can potentially have an influence in diminishing the peak dose.

Geologic Barrier. The stratigraphy of the tuff disposal system was idealized as a series of constant thickness hydrologic modeling units ("pancakes") with a dip of $4.6^{\circ}$ east (see Chapter 4). In the unsaturated zone, a twodimensional model was used, including two-phase flow (liquid and gas) with heat conduction, convection, and phase changes (see Chapter 8). The cross-section modeled was parallel to the $4.6^{\circ}$ east dip. The thirteen modeled layers of the unsaturated zone consisted of consecutive tuff layers with degrees of welding and porosity that were similar. The repository was assumed to be located in the unsaturated zone about $260 \mathrm{~m}$ below the surface and $380 \mathrm{~m}$ above an aquifer. The Solitario Canyon Fault was modeled as a lower permeability zone, and the Ghost Dance Fault was modeled as a higher permeability zone. Model layers were offset across the Solitario Fault but not across the Ghost Dance Fault.

The saturated zone lies under the repository and in an unconfined aquifer that is part of the larger Death Valley flow system. Regional groundwater flow below Yucca Mountain is generally to the south to southeast. The saturated zone model extended to an arbitrary $5-\mathrm{km}$ boundary. The stratigraphy in the saturated zone was simplified to nine modeling units (see Chapters 4 and 9 ). The $4.6^{\circ}$ dip east was incorporated, as well as a $1^{\circ}$ dip south.

In the saturated zone, the focus was on representing as closely as possible the observed potentiometric gradient as constrained by hydraulic head measurements taken in several wellbores. Some fault zones were invoked as barriers to match abrupt head changes in the potentiometric gradient. The Solitario Canyon, a portion of the Bow Ridge, and a zone representing the effects of the northwest-southeast trending group of faults (Drill Hole Wash and Yucca Wash Faults) were modeled as permeability barriers. The rest of the fault zones were treated as permeable zones. Model layers were not offset across the faults in the saturated zone. The saturated zone three-dimensional model included dual porosity and retardation using sampled partition coefficients $\left(\mathrm{K}_{\mathrm{D}}\right)$ (see Chapter 9).

Climate Change. The 1997 PA considered cyclical climatic change because it could enhance infiltration and thus percolation of groundwater through the unsaturated zone. Earlier performance assessments of the proposed repository at Yucca Mountain also included climatic change (although it was not modeled in a-TSPA-1997). In the $1997 \mathrm{PA}$, as in the $1994 \mathrm{PA}$, a cosinusoidal model (with a period between 40,000 and $160,000 \mathrm{yr}$ ) was used for modeling the potential change in infiltration but was not intended to predict the time when these changes occurred. Furthermore, the cosinusoidal model was assumed to model change in infiltration directly, not precipitation; hence, the model indirectly included changes from increased vegetative growth and increased evapotranspiration that result from increased precipitation. The distribution range of infiltration for dry climates was 0 to $10 \mathrm{~mm} / \mathrm{yr}$ (the maximum was increased from 1 in the $1994 \mathrm{PA}$ to 10 in the $1997 \mathrm{PA}$ ); the range for wet climates was 1 to $70 \mathrm{~mm} / \mathrm{yr}$ (the maximum was increased from 19 in the 1994 PA to 70 in the 1997 PA). The resulting average infiltration over the model grid was then varied according to a representative infiltration profile obtained from Flint et al. (draft of "Conceptual and Numerical Model of Infiltration for the Yucca Mountain Area, Nevada"). In addition to the regional variation represented by the cosinusoidal model, at one cell over the repository near the crest of Yucca Mountain, a sample factor focused infiltration between 1 and 10 times that over the rest of the repository. To compensate for focused infiltration at this point, infiltration over the remainder of the repository was slightly reduced. For modeling, the infiltration was specified using wells (Neuman boundary conditions).

\subsubsection{Parameters Sampled}

Numerous layers are modeled in the saturated and unsaturated zones, but many parameters were held constant because the emphasis of this analysis was on the waste form and container rather than the geologic barrier. In the 


\section{Selected Overall Results}

1997 PA, the bulk hydraulic conductivity, matrix hydraulic conductivity, and spacing of fractures were sampled for these unsaturated zone layers: PPw, CHnv, CHnz, and all seven modeling layers of the Topopah Spring (TSv, TSw, TSun, TSul, TSmn, TSli, TSIn). (The only difference in the Topopah Spring layers was porosity.) For all seven layers of the Topopah Spring, the vertical hydraulic conductivity was set one order of magnitude greater than the horizontal hydraulic conductivity.

The distribution coefficients for uranium on devitrified and zeolitic tuff were sampled. Also, the distribution coefficient for neptunium on vitrified tuff was sampled and for plutonium on devitrified tuff. The only geologic barrier parameter sampled in the saturated zone was dispersivity. For climate change, the minimum infiltration, maximum infiltration and cycle period were sampled, as were the infiltration concentration factor and irrigation rate (see Chapter 6).

\subsubsection{Results for Geologic Barrier}

Geologic Barrier. Even though the strata dips at $4^{\circ}$ and permeability changes between modeling layers, the water flow from infiltration is primarily vertical through the unsaturated zone. It is likely that the factor of 10 higher vertical permeability in the seven modeling units of the Topopah Spring maintains the natural tendency for vertical flow in the unsaturated zone. The increased infiltration resulted in a factor of 5 increase in the amount of water passing through the repository in the first 10,000 yr from that observed in the 1994 PA (Figure 11-24). The flow of gas is also primarily vertical. The generation of steam during the first $1000 \mathrm{yr}$ drives gas away from the repository, primarily in the vertical direction. At most times, gas flows upward toward the surface, but at $100 \mathrm{yr}$, some gas flows down below the repository and through the Ghost Dance fault, which is highly permeable. After $2000 \mathrm{yr}$, the convective gas flow diminishes as the repository cools (Figure 11-25). Hence, if breach of containers can be eliminated during the first 2000 yr when higher temperatures most influence the corrosion rates, the effects of temperature on two-phase flow will have diminished such that simple conductive heating will dominate the transport of heat away from the repository.

The majority of the highly soluble ${ }^{99} \mathrm{Tc}$ is released in the first $2000 \mathrm{yr}$ (Figure 11-26a). The resulting high concentration slug reaches the water table by 22,000 yr (Figures 11-26a and b). The travel time of the peak concentration to the 5-km boundary is another $-20,000$ yr (Figure 11-26d). Factors that affect the kinetics of the release of the ${ }^{99} \mathrm{Tc}$ (such as fraction of ${ }^{99} \mathrm{Tc}$ in the grain boundaries of the commercial SNF or alteration of fuel matrices) influence the maximum concentration reaching the water table and ultimately traveling through the saturated zone at the maximum solubility of Tc, as noted previously. The travel time of this slug is most affected by the permeability of the underlying modeling units (TS units, CHnv, and PPw). Both types of parameters were sampled and the dose results described in the next section show a wide variation in the time of the peak dose and its maximum value. Although not shown, the transport of iodine $\left({ }^{129} \mathrm{I}\right)$ is almost identical to the transport of ${ }^{99} \mathrm{Tc}$, but has a reduced concentration since the inventory of ${ }^{129} \mathrm{I}$ is less than that of ${ }^{99} \mathrm{Tc}$.

The transport of the less soluble neptunium (mean solubility $=9.6 \times 10^{-4} \mathrm{~kg} / \mathrm{m}^{3}$ ) is more gradual (Figure 11-27a). At $100,000 \mathrm{yr}$, the concentration at the water table $\left(1.0 \times 10^{-6} \mathrm{~kg} / \mathrm{m}^{3}\right)$ is $\sim 1.5$ orders of magnitude less than the solubility of ${ }^{237} \mathrm{~Np}$ (Figure 11-27b). The peak concentration along the 5-km boundary often occurred in the TSwc modeling unit, which has little retardation of ${ }^{237} \mathrm{~Np}$. However, the ${ }^{237} \mathrm{~Np}$ had to enter this unit by dispersing upward from underlying units, and so the peak concentration is located quite a distance from the repository (Figure 11-27c).

Climate Change. As discussed in the next section, the potential dose up to $30,000 \mathrm{yr}$ is dependent upon releases of ${ }^{99} \mathrm{Tc}$, while at later times the potential dose is due to ${ }^{237} \mathrm{~Np}$. Because solubility plays such an important role for ${ }^{237} \mathrm{~Np}$, its releases are dependent upon the long-term average flow rate of water through the repository. The minimum infiltration and cycle period both appeared as important parameters in the sensitivity analysis. The importance of maximum infiltration was less because the mean of the cycle period is $100,000 \mathrm{yr}$, which means that for many simulations, the infiltration model had not reached its maximum infiltration by the time the simulation concluded at 100,000 yr. The sensitivity results in the $1994 \mathrm{PA}$ were similar in that the cycle period was important in the complex PA (analysis similar to the $1997 \mathrm{PA}$ ) and all three parameters were important in some cases in the simple PA. (Of the three, however, maximum infiltration was the least important in the 1994 PA.) 


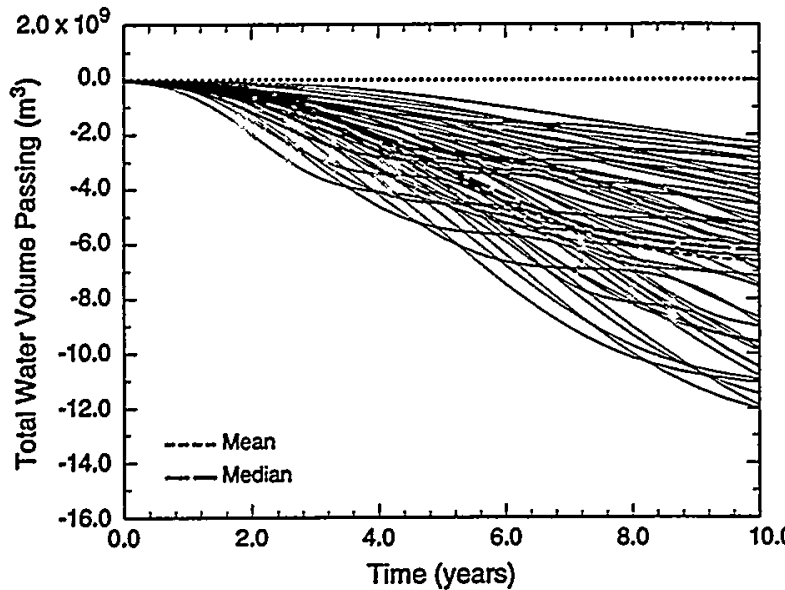

(a) Surface

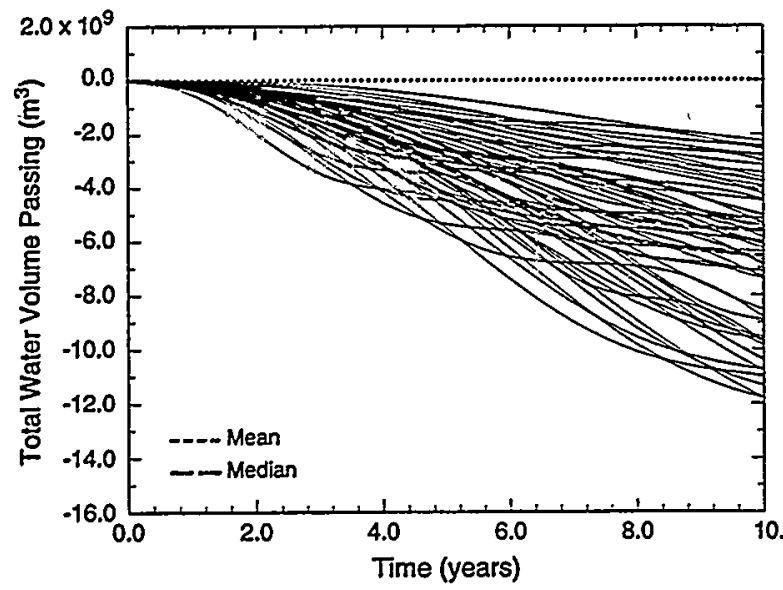

(c) Lower TSv

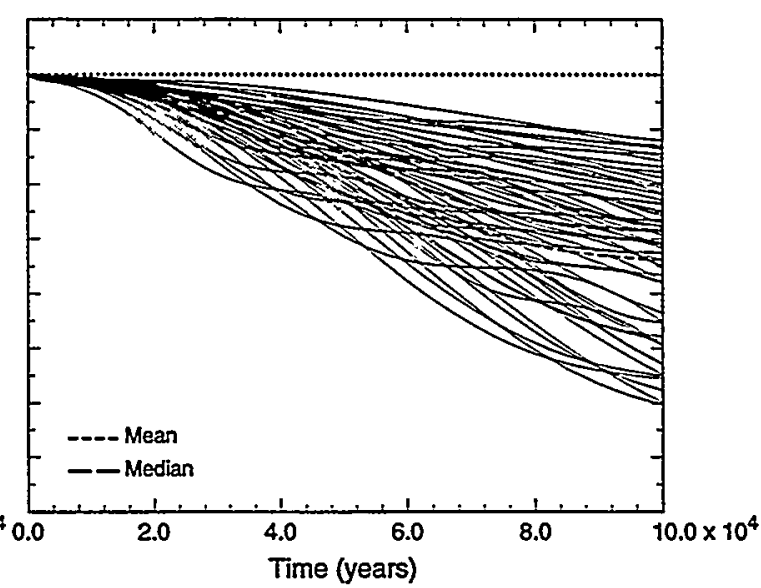

(b) Repository Top

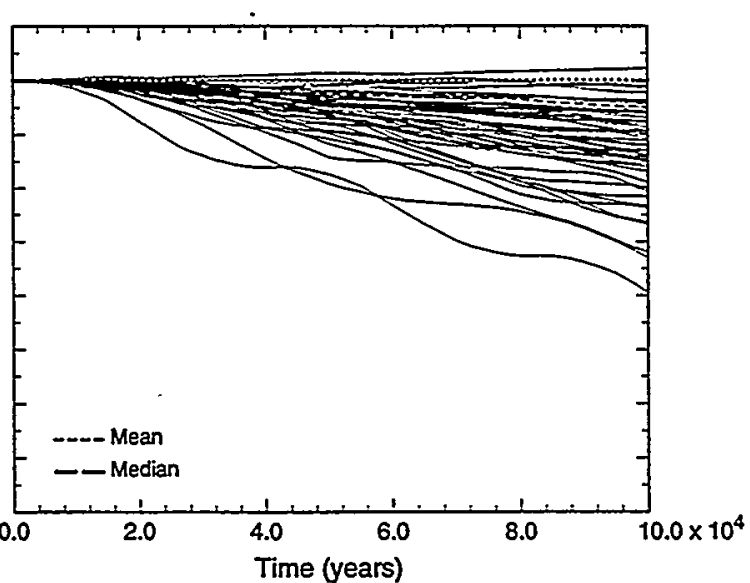

(d) Water Table

TRI-6342-5599-3

Figure 11-24. Cumulative volume of water passing vertically through various strata over the repository area in the unsaturated zone over 50,000 yr. Water moves primarily vertically except near the water table in the $\mathrm{CHnv}$ and CHnz layers where some water also flows horizontally (see transport of ${ }^{237} \mathrm{~Np}$ in Figure 11-27). 


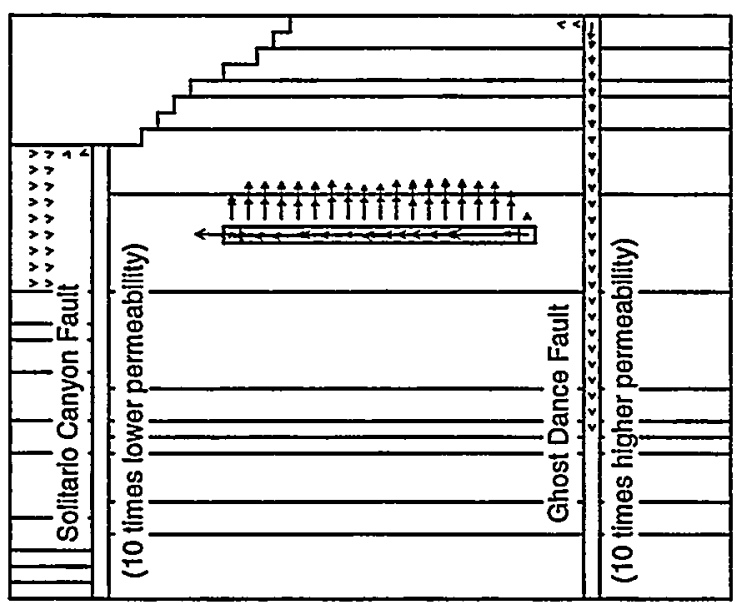

Logical Grid

(a) 10 year

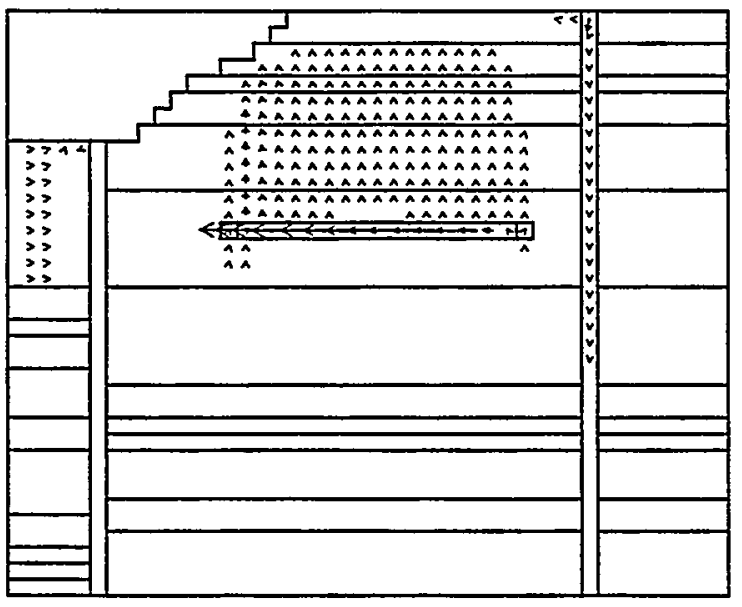

Logical Grid

(c) 500 year

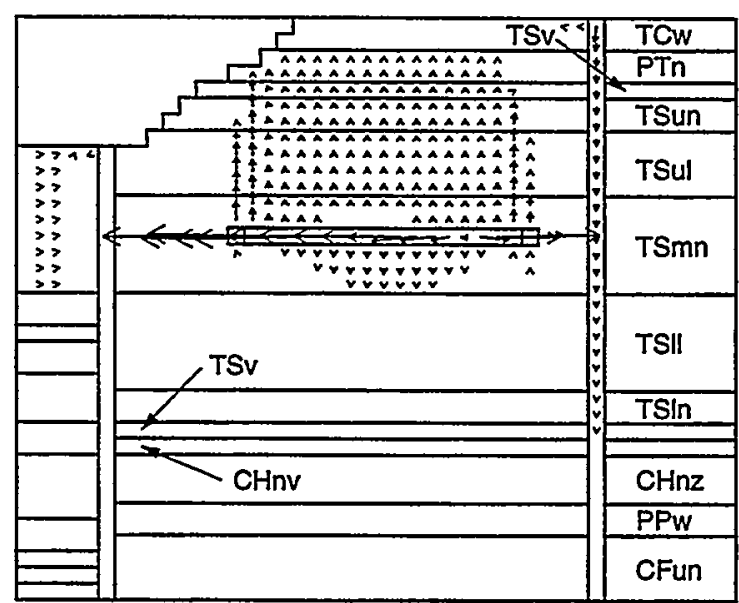

Logical Grid

(b) 100 year

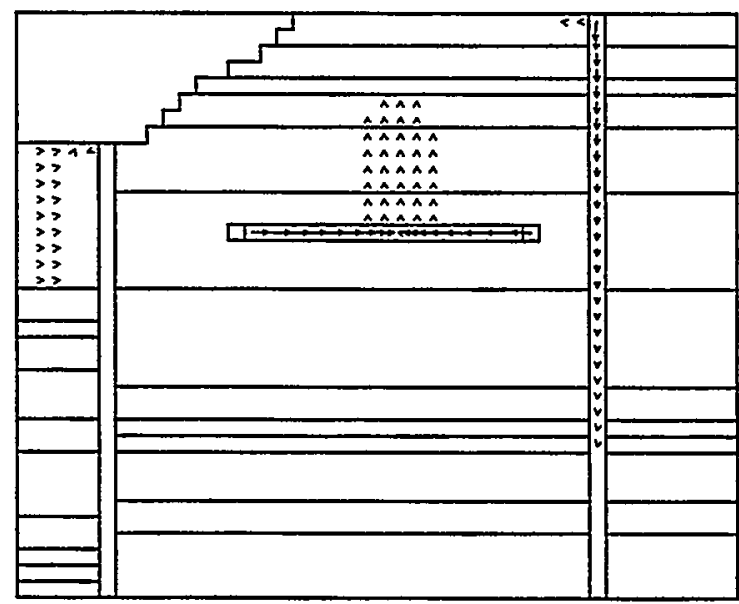

Logical Grid

(d) 2000 year

TRI-6342-5607-3

Figure 11-25. Advective gas flow field in the repository model using mean parameter values at (a) $10 \mathrm{yr}$, (b) $100 \mathrm{yr}$, (c) $500 \mathrm{yr}$, and (d) $2000 \mathrm{yr}$. The gas flow is primarily vertical because the vertical permeability is a factor of 10 larger in the seven modeling layers of the Topopah Spring than the horizontal permeability. The small volume of flow in highly permeable Ghost Dance Fault did not significantly affect the general flow field. Advective flow of gas in the strata generally stops after 2000 yr; however, diffusion of gas (primarily $\mathrm{O}_{2}$ ) continues until the carbon steel is consumed. 


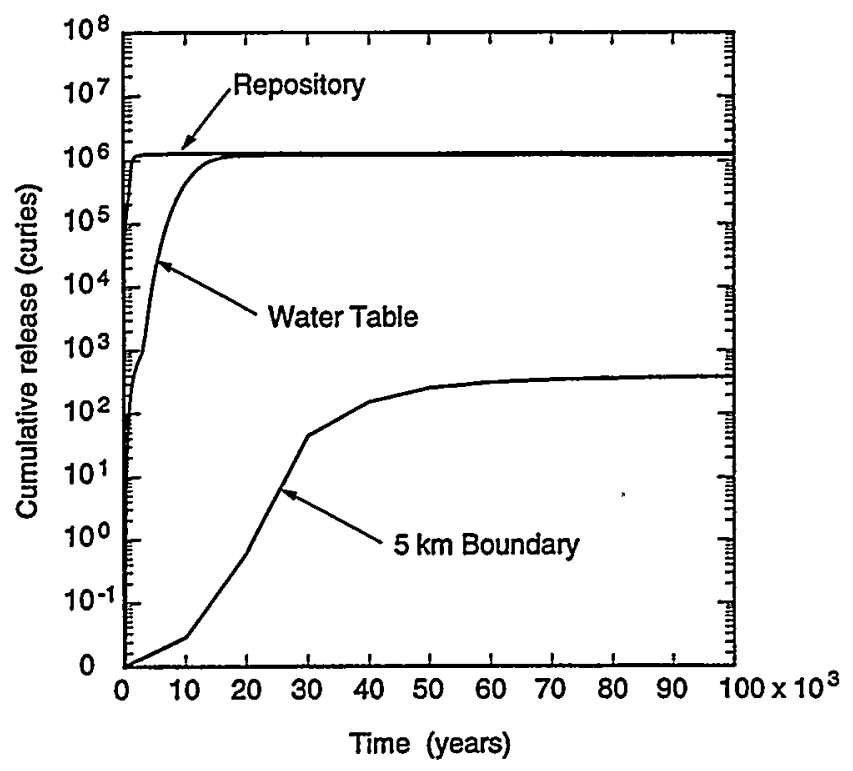

(a) Cumulative release of ${ }^{99} \mathrm{Tc}$ mean parameter values

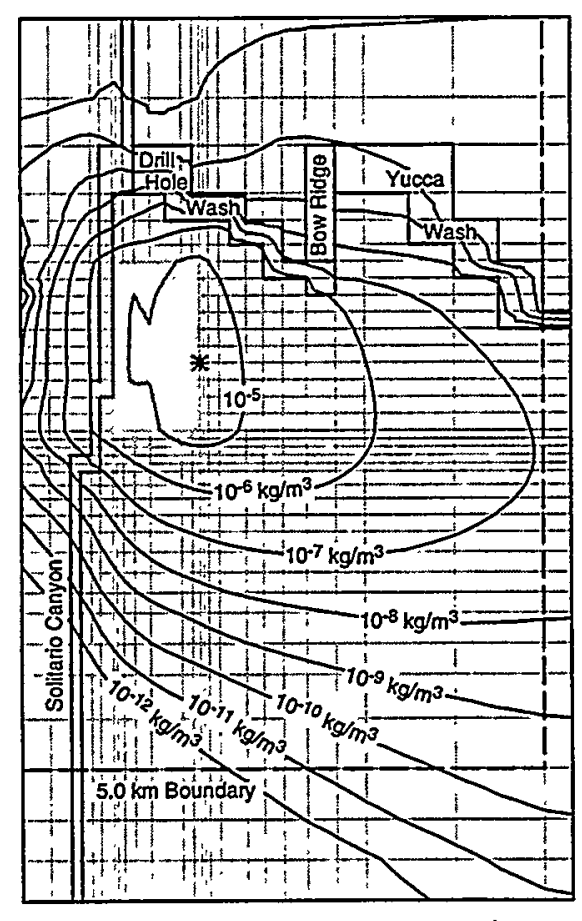

(c) 20,000 years

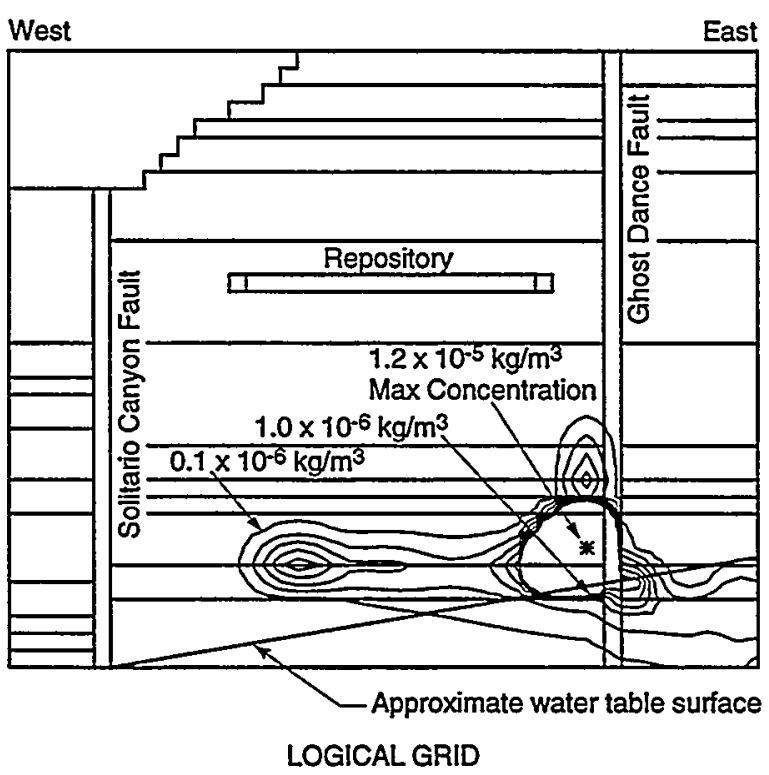

(b) ${ }^{99} \mathrm{Tc}$ at $20,000 \mathrm{yr}$

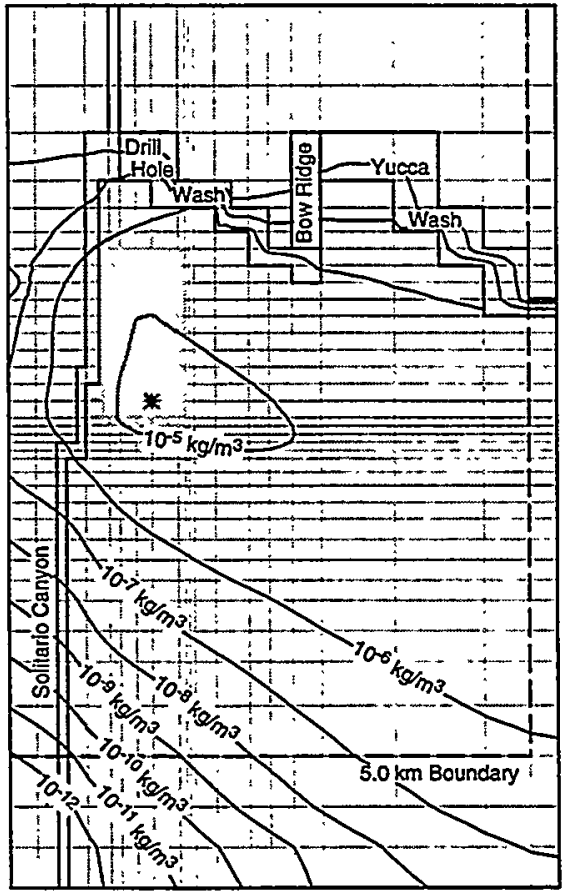

(d) 40,000 years

TRI-6342-5700-3

Figure 11-26. Releases and concentrations $\left(\mathrm{kg} / \mathrm{m}^{3}\right)$ for ${ }^{99} \mathrm{Tc}$ in deterministic run using mean parameter values. (a) Cumulative release at repository, water table, and $5-\mathrm{km}$ boundary (most of ${ }^{99} \mathrm{Tc}$ is released from repository within first $2000 \mathrm{yr}$ ), (b) concentrations in unsaturated zone at 20,000 yr (peak concentration of water table), (c) concentration in saturated zone in $P P w$ unit at 20,000 yr, and (d) concentration in saturated zone in $P P w$ unit at 40,000 yr (peak concentration along 5-km boundary). 
11. Selected Overall Results

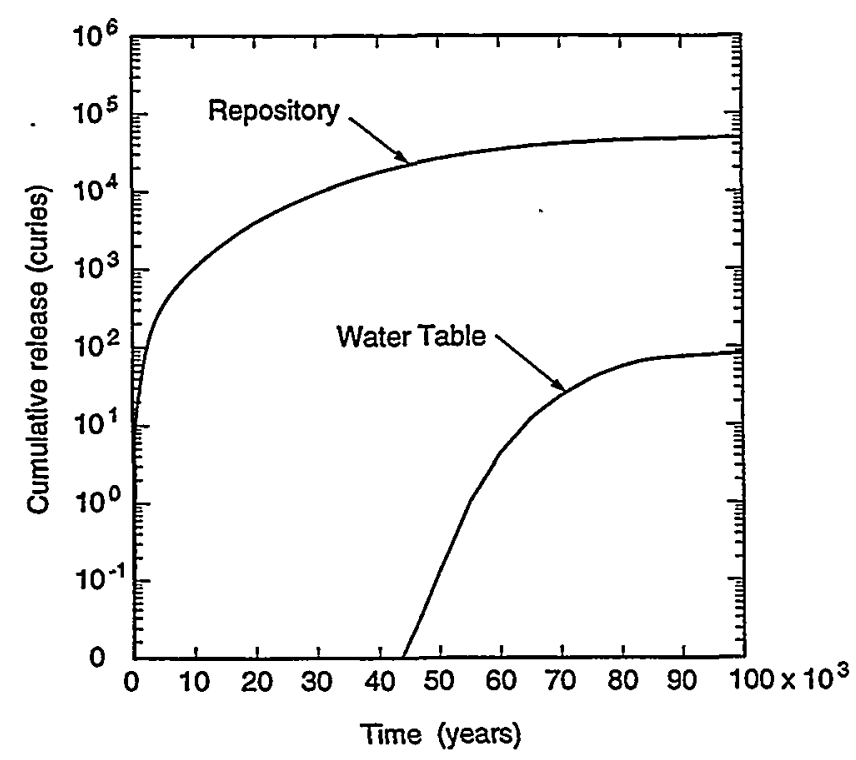

(a) Cumulative release of ${ }^{237} \mathrm{~Np}$ mean parameter values

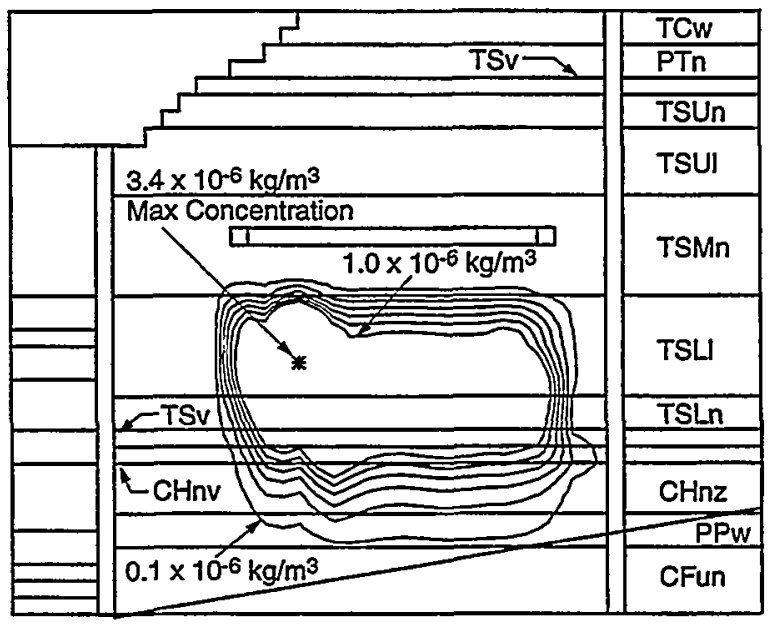

LOGIGAL GRID

(b) ${ }^{237} \mathrm{~Np}$ at $100,000 \mathrm{yr}$

TRI-6342-580t-2

Figure 11-27. Releases and concentrations $\left(\mathrm{kg} / \mathrm{m}^{3}\right)$ for ${ }^{237} \mathrm{~Np}$ in deterministic run using mean parameter values. (a) Cumulative releases at repository, water table, and 5- $\mathrm{km}$ boundary, (b) concentrations in unsaturated zone at 100,000 yr (peak concentration of water table), and (c) concentration in saturated zone at 100,000 yr (peak concentration along $5-\mathrm{km}$ boundary). 
The importance of fluid flow on time of radioisotope release is dramatic and easily demonstrated by reducing the flow through the container by an order of magnitude (flow similar to that assumed in 1994 PA). However, the total release, or at least of ${ }^{99} \mathrm{Tc}$, is not significantly influenced (Figures 11-28 and 11-29).

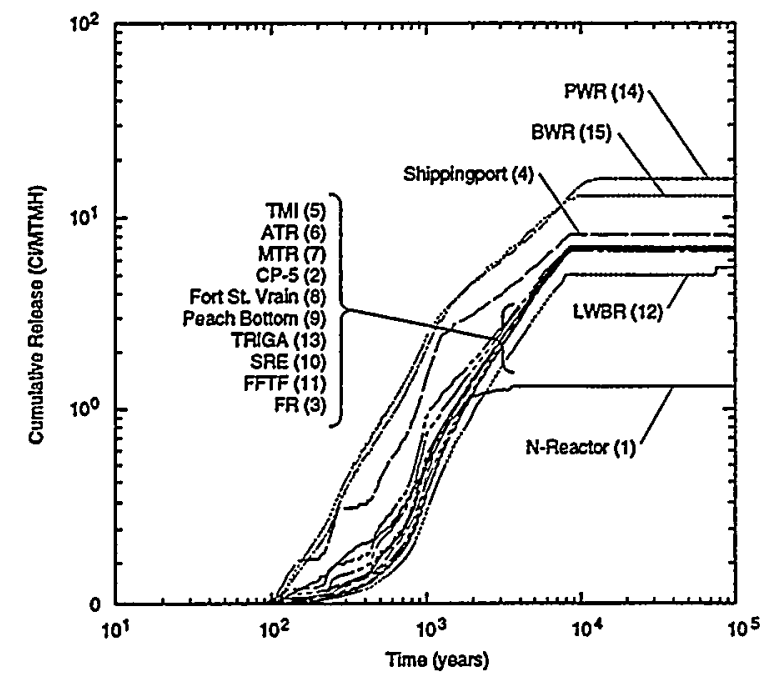

(a) Tc Release (Ci/MTHM)

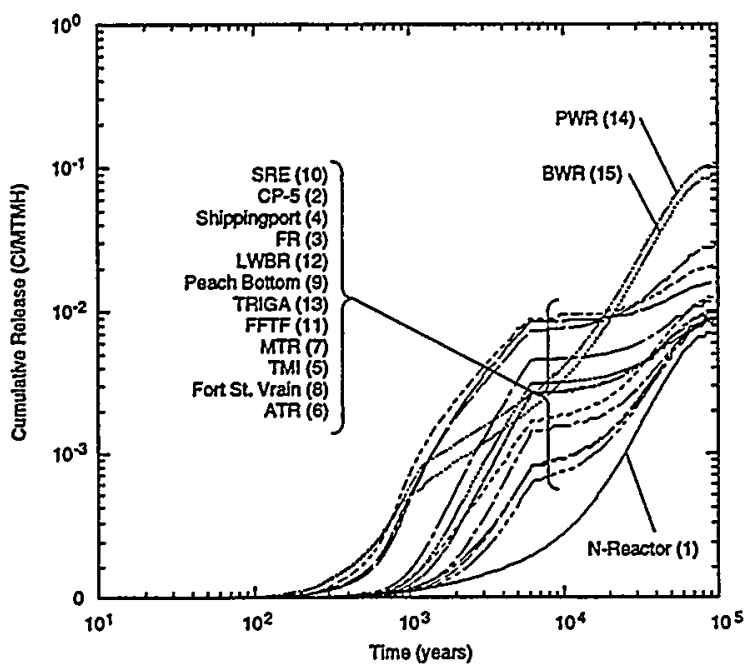

(c) Np Release (Ci/MTHM)

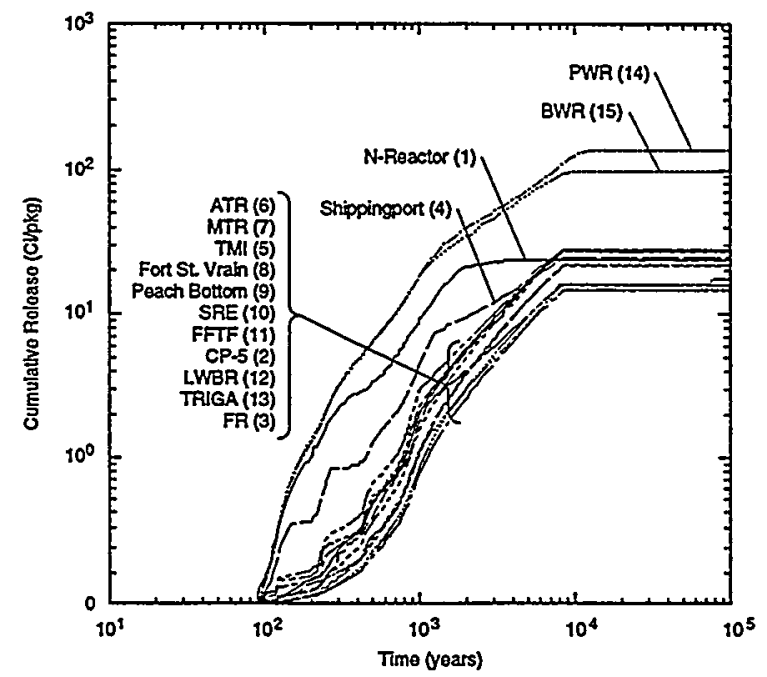

(b) Tc Release (Ci/Pkg)

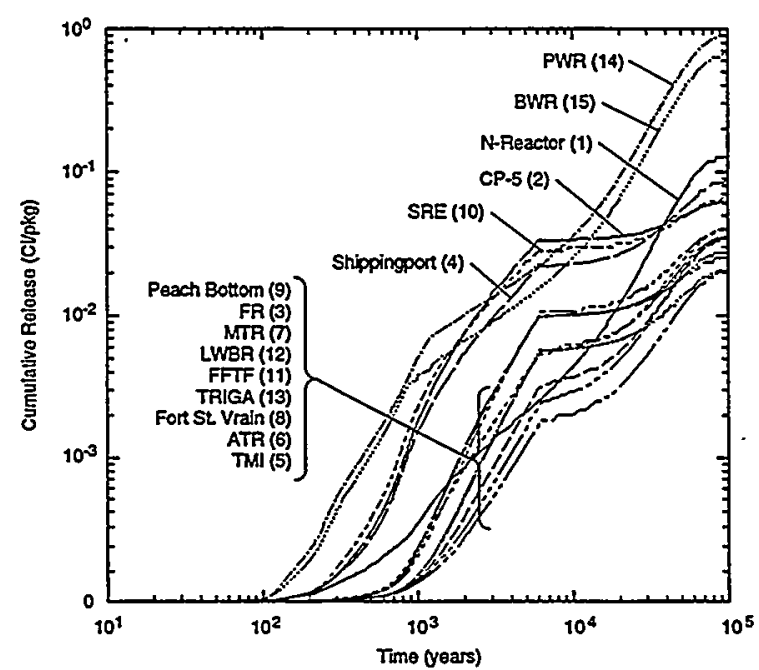

(d) Np Release (Ci/Pkg)

TRI-6342-5880-1

Figure 11-28. Release of radioisotopes are significantly delayed by just an order of magnitude reduction in water passing through the container of $(a)^{99} \mathrm{Tc}$ normalized by mass of heavy radioisotopes in inventory, (b) ${ }^{99} \mathrm{Tc}$ normalized by number of waste packages in each category, $(c)^{237} \mathrm{~Np}$ normalized by mass of heavy radioisotopes in inventory, and $(d)^{237} \mathrm{~Np}$ normalized by number of waste packages in each category. 


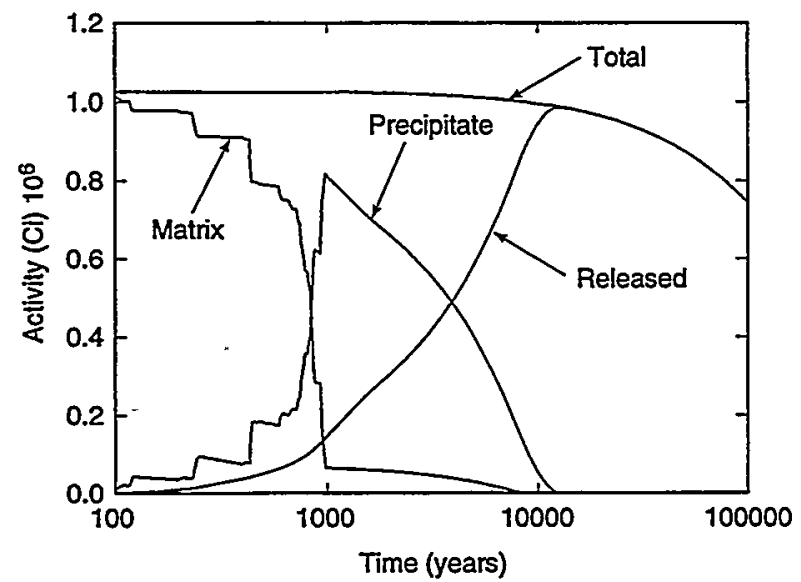

(a) ${ }^{99} \mathrm{Tc}$

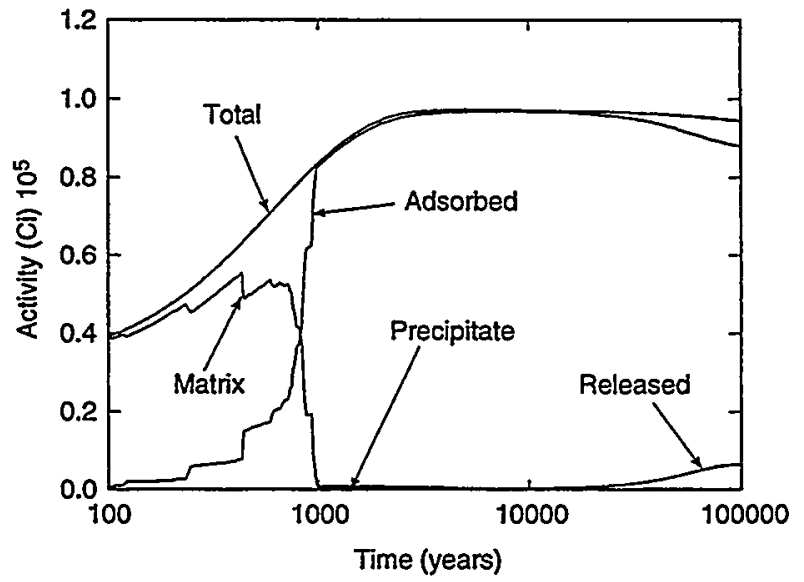

(b) ${ }^{237} \mathrm{~Np}$

TRI-6342-5879-1

Figure 11-29. The distribution of radioisotopes is significantly affected by the reduction in water passing through the container for $(a)^{99} \mathrm{Tc}$ and $(b)^{237} \mathrm{~Np}$.

These findings are summarized in Table 11-5.

Table 11-5. Summary of Model Results for Geologic Barrier and Climate Change

1. The convective gas phase flow is driven by the heating of the repository at early time and subsequent cooling at later time. As the repository cools, the gas flow weakens after $2000 \mathrm{yr}$ (Figure 11-25).

2. Gas flow (and indirectly temperature) within the unsaturated zone is highly dependent upon permeability. For the 1997 PA, the vertical permeability of all seven modeling units of the Topopah Springs was increased one order of magnitude over that used in the $1994 \mathrm{PA}$.

3. The cumulative volume of water passing vertically through various horizons, from the surface to the repository to the bottom of the Topopah Spring Formation, is similar. Only between the Topopah Spring Formation and the water table does the volume of water move horizontally as it passes through lower modeling units (see Figures 11-24, 11-26, and 11-27).

4. The amount of water passing through the repository is generaliy a factor of 10 greater in the first $10,000 \mathrm{yr}$ in the 1997 PA than in the 1994 PA because of the factor of 10 increase in surface infiltration.

5. For the highly soluble technetium $\left({ }^{99} \mathrm{Tc}\right)$, the majority is released within the first $2000 \mathrm{yr}$. The maximum concentrations of ${ }^{99} \mathrm{Tc}$ reach the vicinity of the water table at $22,000 \mathrm{yr}$ (Figure 11-26).

6. For the mean and median deterministic runs, the maximum concentration of ${ }^{99} \mathrm{Tc}$ at the $5-\mathrm{km}$ boundary in the saturated zone occurs at about 40,000 yr in the PPw modeling unit. The PPw modeling unit has the highest fracture hydraulic conductivity for the mean and median runs (Figure 11-26).

7. The transport of the less soluble neptunium $\left({ }^{237} \mathrm{~Np}\right.$ ) (mean solubility $=9.6 \times 10^{-4} \mathrm{~kg} / \mathrm{m}^{3}$ ) from the failed waste packages is more gradual (Figure 11-27a). At 100,000 yr, the concentration at the water table $\left(1.0 \times 10^{-6} \mathrm{~kg} / \mathrm{m}^{3}\right)$ is $\sim 3.0$ orders of magnitude less than the solubility of ${ }^{237} \mathrm{~Np}$ (Figure $11-27 \mathrm{~b}$ ).

8. For ${ }^{237} \mathrm{~Np}$, the maximum concentration at the $5-\mathrm{km}$ boundary often occurs in the TSwc modeling unit where there is little ${ }^{237} \mathrm{~Np}$ retardation. At $100,000 \mathrm{yr}$, the concentration is still rising at the boundary (Figure 11-27c). 


\subsection{Findings on Behavior of Overall Disposal System}

When a site applies for a license, the absolute position of the results in relation to the total system criteria of the future 40 CFR 197 regulations will be crucial. However, the purpose of this analysis is to provide guidance, not to establish the absolute position of results for purposes of compliance. Also, the use of preliminary characterization data and the lack of regulatory criteria mean that there is a large uncertainty associated with establishing these results as absolute with regard to compliance. Thus, the value of showing the absolute position here is to allow comparisons with previous and future studies.

\subsubsection{Dose Calculations}

The criteria examined are probabilistic doses received by an individual from exposure to contaminants over a 1yr period. For the dose calculations, the 1997 PA considered three cases: (1) a Ranch case, in which a rancher is exposed to radioisotopes by means of beef consumption only, (2) a Farm case, in which a member of a farming family is exposed to radioisotopes by means of food consumption, water consumption, and inhalation, and (3) a Small Community case, in which an average resident is exposed through drinking water and consumption of locally grown farm products such as vegetables, fruits, dairy, and meat products.

For all cases, dose was evaluated from peak concentrations of the transported radioisotopes- ${ }^{129} \mathrm{I}$, ${ }^{237} \mathrm{~Np}$, and ${ }^{99} \mathrm{Tc}$-at the 5-km boundary over the 100,000-yr period, i.e., as if the Rancher, Farmer, or Small Community had drilled a water well into the saturated zone at the point on the $5-\mathrm{km}$ boundary with the highest radioisotope concentrations. GENII-A used the concentrations for the transported radioisotopes from STAFF3D for the 5-km boundary location.

\subsubsection{Change of Dose With Time}

Time histories of the committed dose were generated for a 100,000-yr period for the Farm and Ranch cases, using mean and median values, as shown in Figure 11-30. A temporary local peak occurs about $40,000 \mathrm{yr}$ from ${ }^{99} \mathrm{Tc}$, but a higher dose occurs after $70,000 \mathrm{yr}$ from ${ }^{237} \mathrm{~Np}$ and continues to increase through $100,000 \mathrm{yr}$.

Similar to the Farm case, the drinking water only case has a temporary local peak dose of $250 \mathrm{mrem}$ from ${ }^{99} \mathrm{Tc}$ at about $40,000 \mathrm{yr}$ and a similar dose at $70,000 \mathrm{yr}$ from ${ }^{237} \mathrm{~Np}$ that continues to increase up to $400 \mathrm{mrem}$ at $100,000 \mathrm{yr}$. The peak dose in a-TSPA-1997 (M\&O, 1997) was $80 \mathrm{mrem}$ at $18,000 \mathrm{yr}$ from ${ }^{99} \mathrm{Tc} ;{ }^{237} \mathrm{~Np}$ did not exceed this dose in $100,000 \mathrm{yr}$ (Figure 11-31). As noted in Section 11.1, the DOE SNF and DHLW contribution to the total ${ }^{237} \mathrm{~Np}$ inventory is about $680 \mathrm{Ci}(2 \%)$. Because all cladding was assumed failed in the $1997 \mathrm{PA}$ base case, the contribution of DOE SNF and DHLW to the total inventory is also their contribution to the total doses reported here.

At $-9000 \mathrm{yr}$, in the simulation with mean parameters, radioisotopes were first detected and a dose calculated. As a measure of the nonlinearity of the modeling system, a comparison was also made between the mean of the 87 realizations and the deterministic realization using mean parameter values. In general, the consequence model is moderately nonlinear concerning the dose from ${ }^{99} \mathrm{Tc}$. A difference of a factor of 20 exists between the $4 \mathrm{rem}{ }^{99} \mathrm{Tc}$ peak at $50,000 \mathrm{yr}$ for the mean of the 87 realizations and the 200 mrem peak at $40,000 \mathrm{yr}$ for the deterministic mean realization, while a factor of 2.5 difference exists between the 1 rem from ${ }^{237} \mathrm{~Np}$ for the mean of the 87 realizations and the 400 mrem for the deterministic mean realizations at 100,000 yr. This difference in variation of ${ }^{99} \mathrm{Tc}$ and ${ }^{237} \mathrm{~Np}$ implies that uncertainty in aspects of the source term (i.e., kinetics of release) more strongly influences peak releases of ${ }^{99} \mathrm{Tc}$ than ${ }^{237} \mathrm{~Np}$. As seen in Figure 11-24 in the previous section, the variation in water flow is small in the first 10,000 to $20,000 \mathrm{yr}$ and, thus, cannot cause the variation in peak releases of ${ }^{99} \mathrm{Tc}$.

In addition, bounding calculations were performed using two approximations (discussed in TSPA-1995 [M\&O, 1995a]), i.e., the stirred tank model and the homogeneous infinite aquifer model assuming steady-state transport, to 


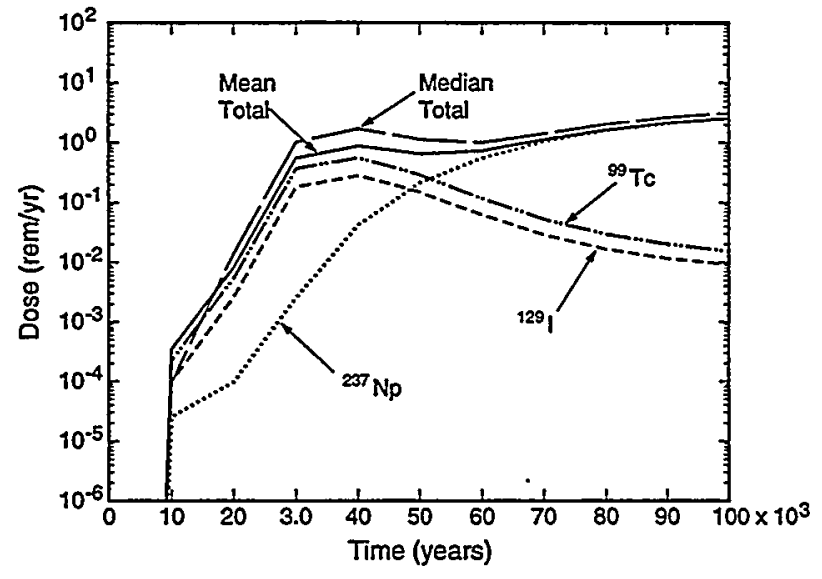

(a) Water and vegetable consumption (Farm case)

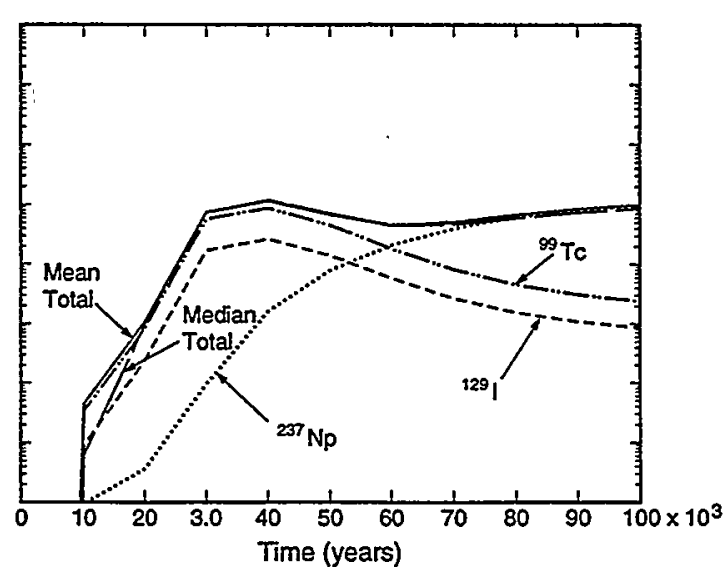

(b) Beef consumption (Ranch case)

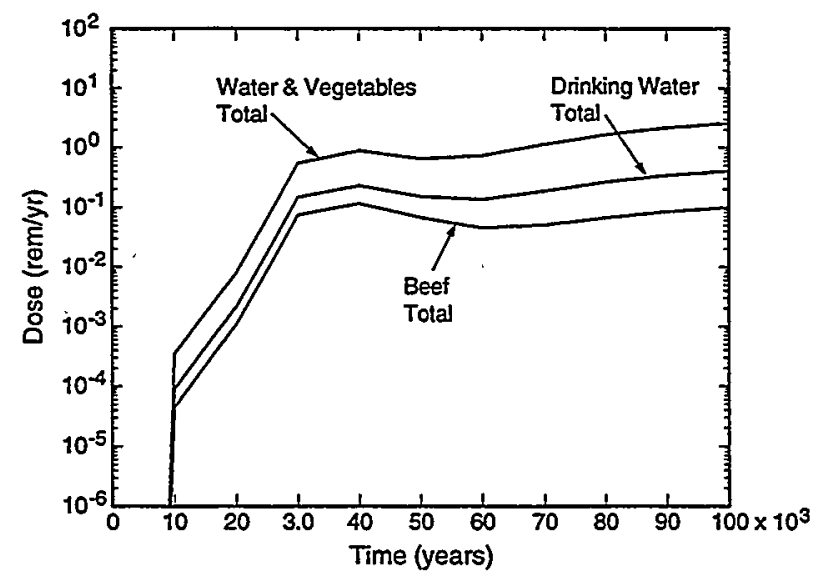

(c) Mean totals

TR1-6342-5675-0

Figure 11-30. Dose as a function of time for (a) mean and median calculations for Farm case, water and vegetable consumption; (b) mean and median calculations for Ranch case, beef consumption only, and (c) mean totals for three cases (Farm case, drinking water only case, and Ranch case). Doses from ${ }^{99}$ Tc and ${ }^{237} \mathrm{~Np}$ in the Farm case (water and vegetables) dominate the results. The doses primarily from drinking water for a critical subpopulation using the aquifer for its water supply are about seven times less than for the subsistence farmer (Farm case) but still more than the rancher consuming beef (Ranch case).

evaluate the reasonableness of the deterministic result from STAFF3D using mean parameter values. These approximations used the radioisotope concentrations in the unsaturated zone just above the water table as calculated by NUTS. With the stirred tank approximation, the peak dose was about 2 rem or about a factor of 5 higher than the corresponding peak dose of $\sim 400$ mrem at the 5-km boundary with STAFF3D (Figure 11-31). With the homogeneous infinite aquifer approximation, the peak dose was about 80 mrem or about a factor 5 lower than the corresponding peak dose of $\sim 400$ mrem. 


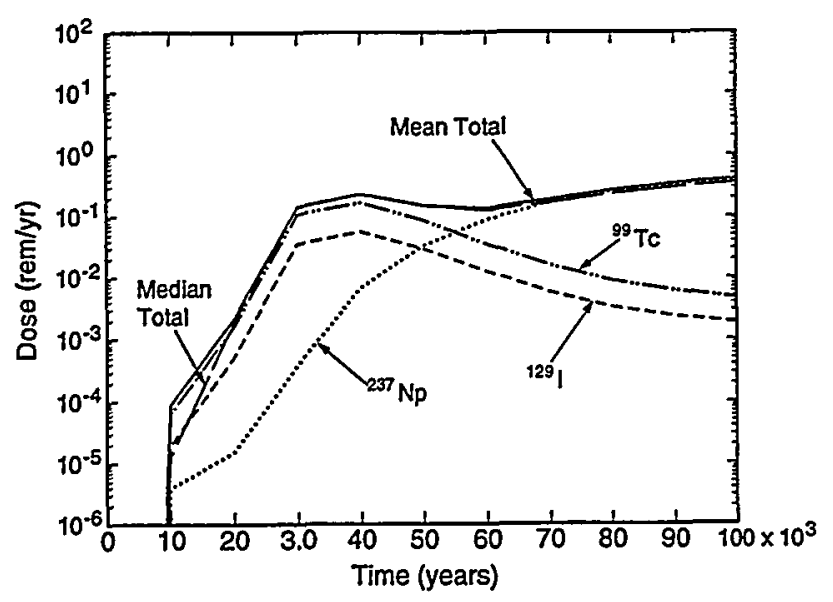

(a) Simulation with mean parameters

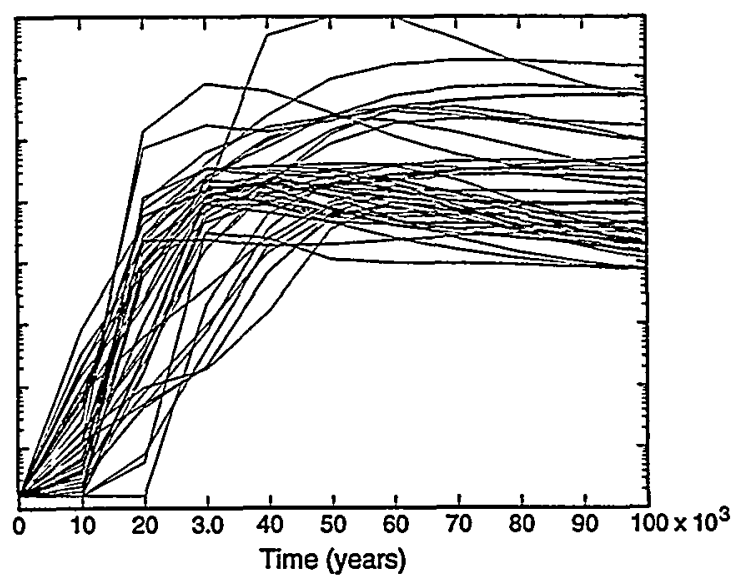

(b) 87 Realizations

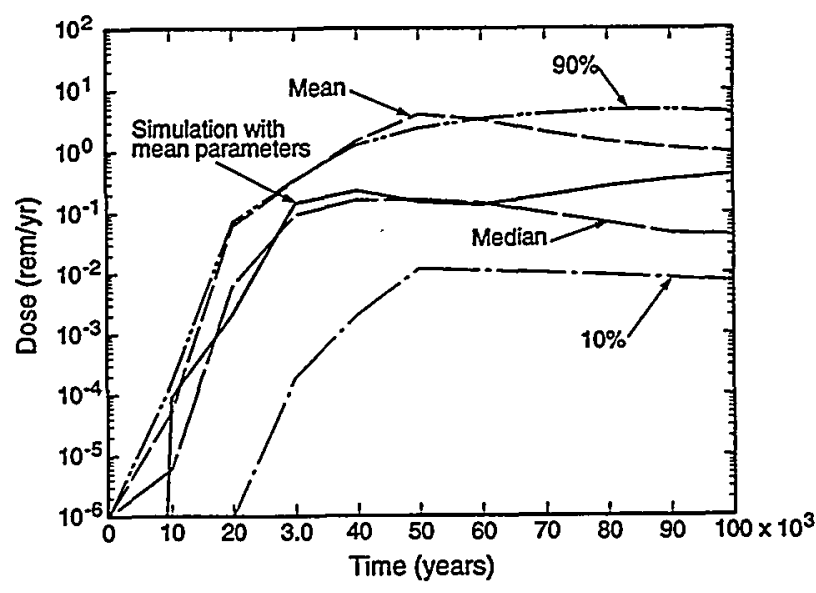

(c) Quantiles on 87 realizations

TRI-6342-5673-3

Figure 11-31. Dose at 5-km boundary from drinking water as a function of time for (a) deterministic run using mean parameter values, (b) 87 realizations in 1997 PA, and (c) quantiles on 87 realizations. The difference between the mean of the 87 realizations and the simulation with the mean parameters is greatest at early times when ${ }^{99} \mathrm{Tc}$ dominates doses, implying that uncertainty in the nonlinear aspects of the source term model is dominating the variation. At later times, uncertainty in the linear solubility limit (since two-phase flow and temperature have stabilized) appears to be dominating the variation. 
Table 11-6 summarizes these findings.

Table 11-6. Summary of Model Results for Total System Performance

1. Based on results of previous DSNF/DHLW performance assessments, the radioisotopes that contribute most to the individual dose are as follows: neptunium, technetium, and iodine ${ }^{*, \star \star}$. This result occurs because these radioisotopes are poorly retarded by the volcanic tuff geologic barrier.

2. Time histories of the committed dose for the drinking water only case, the main Farm case, and the Ranch case, using mean and median values, showed a temporary peak about $40,000 \mathrm{yr}$ from ${ }^{99} \mathrm{Tc}$. However, a higher dose occurs after $70,000 \mathrm{yr}$ from ${ }^{237} \mathrm{~Np}$ and continues to increase through $100,000 \mathrm{yr}$. Of this dose, the DOE SNF and DHLW contribute $\sim 7 \%$ and $\sim 2 \%$, respectively, i.e., DOE SNF and DHLW are $\sim 7 \%$ of ${ }^{99}$ Tc inventory and $2 \%$ of the ${ }^{237} \mathrm{~Np}$ inventory (Figure $11-30$ ).

3. Attenuation of concentration peaks is significant in the saturated zone using STAFF3D. The dose calculated at the 5-km boundary with the TSPA-1995 stirred tank model, using NUTS concentrations in the unsaturated zone just above the water table as the starting concentration, was 2 rem or about an order of magnitude higher than the corresponding peak dose of $-400 \mathrm{mrem}$ with STAFF3D (see Figure 11-31).

4. Results from the calculation of the homogeneous infinite aquifer model with steady-state transport, as described in TSPA-1995, were about 80 mrem, which is less than the STAFF3D results of $\sim 400$ mrem (Figure 11-31).

* Radioisotopes contributing to the thermal load were listed in Table 11-3.

** Uranium isotopes and, especially, its daughters including thorium, radium, and lead, become important for dose beyond $100,000 \mathrm{yr}$. 


\title{
12. Guidance to the NSNFP
}

\author{
R. P. Rechard
}

Although the DOE/EM has responsibility for the DOE-owned spent nuclear fuel and defense high-level waste, evolving regulations make it difficult at this time to set limits on the level of characterization (and possible treatment) necessary before disposal in the potential Yucca Mountain repository. Arbitrary limits can be appealing, because they encourage a straightforward course of action for DOE/EM contractors managing storage sites to prepare the DOE SNF for disposal. However, such limits not only have the potential to be extremely costly by overcompensating for the unknown, but are themselves subject to analysis in the later stages of regulatory licensing to ensure that they provide safe conditions. In addition, recent proposed amendments, along with guidance from the NAS, continue to emphasize an evaluation of the whole nuclear waste disposal system, rather than stringent regulations on each system component. In such an environment, it is even more useful to gain an understanding of the spent fuel types and their behavior in a disposal system so that the DOE/EM is well acquainted with the issues and options available as site-specific regulations on the potential Yucca Mountain repository emerge. Knowledge of the post-disposal behavior of DOE SNF can place the DOE/EM in a strong position for negotiating acceptance criteria that are prudent, cost-effective, and time-saving. Hence, the following section summarizes pertinent results described earlier as related to data needs. Section 12.1 makes a cursory evaluation of DOE SNF compared to repository acceptance criteria based on current U.S. Nuclear Regulatory Commission (NRC) requirements. Section 12.2 recommends future analysis tasks, and the final section provides general guidance on the direct disposal option.

\subsection{Guidance for Repository Data Needs Based on Performance Assessment}

A performance assessment uses a model of the geologic disposal system; hence, only data (expressed as parameters) that are used by the model can be gauged as important from the performance assessment perspective. Based on the $1997 \mathrm{PA}$, only a portion of the waste form data needed for the source-term submodel is important to calculations or decisions, as discussed in the following sections. ${ }^{*}$ Also, although a definitive evaluation of the maximum and minimum values of parameters expected to be important with regard to acceptance criteria to ensure future compliance was not within the scope of this study, significant model parameters and their importance relative to each other are identified and summarized.

\subsubsection{Inventory of Radioisotopes}

The list of radioisotopes important to repository performance is fairly short. As noted in the 1994 PA, the most important radioisotopes for which it is important to know the accurate mass or activity in the DOE SNF (and commercial SNF) are those dependent upon burnup: ${ }^{99} \mathrm{Tc},{ }^{129} \mathrm{I}$, and ${ }^{237} \mathrm{~Np}$. The $1997 \mathrm{PA}$ confirmed that ${ }^{99} \mathrm{Tc}$ was the most important isotope in determining dose in the first $10^{4} \mathrm{yr}$ and ${ }^{237} \mathrm{~Np}$ was the most important in the first $10^{5} \mathrm{yr}$, as seen in an earlier analysis. Other radioisotopes contributing to the dose for these times in the $1994 \mathrm{PA}$ were ${ }^{231} \mathrm{~Pa}$, ${ }^{129} \mathrm{I}$, and the uranium isotopes ${ }^{233} \mathrm{U},{ }^{234} \mathrm{U},{ }^{235} \mathrm{U}$, and ${ }^{236} \mathrm{U}$, with the exception of ${ }^{238} \mathrm{U}$ ). Between $10^{5}$ and $10^{6} \mathrm{yr}$, daughters of uranium-especially of ${ }^{234} \mathrm{U}$ and possibly ${ }^{238} \mathrm{U}-{ }^{226} \mathrm{Ra}$ and ${ }^{210} \mathrm{~Pb}$ contribute to dose although they are not significant in the initial inventory.

In the EPA standard, 40 CFR 191, releases were normalized by the inventory of radioisotopes; hence, if the mix of various radioisotopes were fairly constant, the absolute inventory was not significant except in determining the maximum waste allowed in the repository. If the allowable maximum dose is not normalized to the inventory in yet to be promulgated 40 CFR 197, then the accuracy of the inventory will become more important for commercial SNF.

\footnotetext{
- Source-term data other than those discussed may be needed to assure the NRC that the DOE SNF can be safely transported to the repository or moved within the repository. Furthermore, before waste is accepted into the repository, the OCRWM will need to standardize the sizes and masses of individual canisters, e.g., the DOE SNF handling containers must be easily removed from transport casks and packaged with DHLW in disposal containers. Although this other information will be a necessary part of the repository acceptance criteria, constraints other than disposal performance will determine the needed accuracy and quality of this additional information.
} 
The activity of the DOE SNF, which is largely made up of Category $1, \mathrm{~N}$-Reactor fuel, represents only $1.5 \%$ of the total initial activity of the repository. Thus its activity will likely remain within the error band of the commercial SNE. For example, only for commercial SNF is it important to accurately gauge the fraction of ${ }^{99} \mathrm{Tc}$ inventory that is released immediately when the cladding or other protective layer breaches. Therefore, when an error band of activity for each important radioisotope for the commercial SNF is known or estimated, then an allowable error for the DOE SNF can be calculated. Until the commercial error band is known, an error band of $200 \%$ for the DOE SNF is probably permissible.

Radioisotopes important to evaluating the power production from the containers, and thereby the temperature of the repository, are ${ }^{137 \mathrm{~m}} \mathrm{Ba},{ }^{90} \mathrm{Y},{ }^{238} \mathrm{Pu},{ }^{241} \mathrm{Am},{ }^{137} \mathrm{Cs},{ }^{244} \mathrm{Cm},{ }^{90} \mathrm{Sr}$, and ${ }^{240} \mathrm{Pu}$, which contribute $98.6 \%$ of the initial power.

Develop Final Spent Fuel Groups and Bounding Characteristics. Although a preliminary grouping was explored in this performance assessment for -250 types of DOE SNF, a final grouping must be established for OCRWM's license application for the potential repository at Yucca Mountain. The 13 DOE SNF categories used in the 1997 PA were based primarily on type of fuel matrix, with cladding type and condition as secondary criteria. The final grouping would benefit from information gathered in the 1997 PA. For example, linking any criticality analysis with the DOE SNF categories as modeled in this performance assessment would be difficult because the categories were not based on fissile mass. Furthermore, the results indicate that fewer categories than the 13 DOE SNF categories modeled in the $1997 \mathrm{PA}$ are necessary. Two categories (N-Reactor fuel and all other fuels) may be adequate; the use of four categories, as in the 1994 PA (N-Reactor, highly enriched, poorly clad [ATR], graphite [Fort St. Vrain], and low enriched $\left.\mathrm{UO}_{2}[\mathrm{PWR}]\right)$, is another possibility.

\subsubsection{Handling Containers for DOE SNF}

Because DOE SNF represents such a small portion of the inventory $(0.46 \%$ and $0.49 \%$ of entire inventory of ${ }^{237} \mathrm{~Np}$ and ${ }^{99} \mathrm{Tc}$, respectively), designing a robust handling container for DOE SNF is not useful. However, if other considerations demand that a more robust container be used, then DOE/EM should design a robust handling container for DHLW before designing one for DOE SNF.

\subsubsection{Solubility of Radioisotopes}

The solubility of any particular radioisotope in a specific chemical form in the repository environment will be identical for both commercial and DOE SNF; hence, this information will be developed and supplied by the OCRWM. However, the 1997 PA has confirmed that the solubility of ${ }^{99} \mathrm{Tc},{ }^{129} \mathrm{I},{ }^{237} \mathrm{~Np}, \mathrm{U}$, and Pu ought to be carefully evaluated. The results of the $1997 \mathrm{PA}$, which included higher water flow rates through the repository than previous analyses, suggest that of these radioisotopes the most important parameter by far is the solubility of ${ }^{237} \mathrm{~Np}$. For highly soluble radioisotopes such as ${ }^{99} \mathrm{Tc}$ or ${ }^{129} \mathrm{I}$, release is determined by the amount of matrix material exposed, and solubility is not a limiting factor. For ${ }^{237} \mathrm{~Np}$, however, release from any one container is controlled directly by its solubility.

\subsubsection{Corrosion Parameters}

The corrosion (or alteration) of layers and matrices can affect (a) the time needed to penetrate a layer, (b) the consumption/production rate of gas and liquid, (c) the generation of rust adsorbent, and (d) the release rate of encapsulated radioisotopes. As described more fully in Chapter 7, the corrosion rate of each material is potentially a function of the oxygen content (oxic/anoxic) of the repository and the moisture available (wet/humid/dry). The corrosion rates of the materials in the disposal container (e.g., carbon steel and Inconel 625) and the zircaloy cladding, as well as the alteration rate of the uranium dioxide matrix, will be identical for both commercial and DOE SNF. Only the stoichiometry and corrosion (or alteration) rates of materials unique to DOE SNF must be evaluated separately by DOE/EM. This material includes metallic uranium and other uranium matrices, and the aluminum, stainless steel, graphite, and 
TRISO cladding. The alteration rates used for most matrices in the 1997 PA are encompassed by the uncertainty distribution of rates for uranium dioxide. Thus, dramatic differences in release rates were not observed between commercial and DOE SNF or among different DOE SNF. Documented values from literature or experiments will be useful for evaluating the relative performance of DOE SNF and, more importantly, for substantiating the selected alteration values. Hence, to ensure the next PA calculations consider the most up-to-date information, a thorough literature search of wet/humid corrosion rates is recommended. (This study found that anoxic corrosion is not important.)

\subsubsection{Protective Ability of Layers}

In this analysis, for the two layers of the disposal container, $<1 \%$ of the Inconel 625 layer was assumed to be compromised; however, at the edge of the hot repository, the predicted protection from the two layers was minimalonly $300 \mathrm{yr}$. Conversely, the potential protection provided by the zircaloy cladding was neglected; this protection is particularly significant for commercial SNF. Currently, the YMP is reluctant to take credit for cladding protection because of the difficulty in obtaining qualified data. For example, a verifiable estimate of the percentage of intact cladding on Category 1, N-Reactor fuel, or any other SNF is expected to be difficult, and even the carefully monitored commercial SNF may not have qualified data readily available. However, the large quantities of commercial SNF combined with zircaloy's protective characteristics may encourage the YMP to run calculations for the NRC in the future, perhaps as collaborative evidence, to demonstrate zircaloy's potential protective ability, with the resulting improved performance of the repository. The NSNFP should also provide estimates with sufficiently wide uncertainty bands of the protective capability of the DOE SNF cladding.

\subsubsection{Surface Area and Mass}

Although the initial surface area and mass of protective layers of the disposal container are essential, the data will be identical for both commercial SNF and DOE SNF. Thus, only surface area and mass for cladding and matrices unique to DOE SNF are important to DOE/EM; because the mass and volume of DOE SNF is so small, rough estimates will be adequate.

\subsection{Recommended Future Analysis Tasks}

In the area of analysis, the primary recommendations are for Sandia to (1) perform a screening of features, events, and processes that are unique to DOE SNF, (2) continue to assess the importance of unique aspects of DOE SNF that cannot be screened out, (3) perform the detailed calculations from which abstractions can be produced for use by OCRWM in its TSPAs, (4) upgrade the quality assurance of the analysis and data, and (5) perform additional sensitivity analysis.

\subsubsection{Screen Features, Events, and Processes}

Over the past few years, the NSNFP has examined, through experiments and studies such as this one, a number of disposal issues concerning DOE SNF, such as combustibility and pyrophoricity. The NSNFP should now draw upon this information and formally conduct a screening of features, events, and processes. This screening would primarily entail documenting in a series of traceable records the arguments regarding reasons for screening out several issues that have been examined and are no longer required for inclusion in future performance assessments. This screening would allow the NSNFP to formally evaluate the status of the program with regard to a number of issues. Those disposal issues that cannot be screened out satisfactorily can become the focus of future studies, as described below. 


\subsubsection{Continued Assessment of Unique Aspects of DOE SNF}

The dismissal of additional disposal issues, such as criticality, requires continued improvement and evaluation of models used in the 1997 PA. (Recall that experiments for obtaining data for parameter values and for testing the fundamental premises of current models were mentioned in Section 12.1.)

Improvements to Source Term Model. A continuing evaluation and improvement of CST is recommended to ensure that the conceptual models and parameters include the latest data from YMP and other studies in the areas of hydrology and corrosion, and the solubilities, sorption and transport of radionuclides. The recommended improvements to CST include

- Replacing the fracture-drip concept in CST currently based on fracture saturation with a fracture drip concept based on fracture spacing and infiltration rate.

- Adding a module in CST for reactions in the tunnel lining invert.

- Adding the heat of the reaction from oxidation of the spent fuel matrix.

Ensuring that CST is kept current will facilitate the continued use of CST components (particularly the corrosion and fuel matrix dissolution models) and corresponding model parameters by the YMP in the Repository Integration Program (RIP) for the TSPA-VA next year.

Improvements to Unsaturated Zone Model. Recommended improvements for the unsaturated zone model include (1) more spatial variability in the strata and (2) calibration of the model with measured saturation. Also, to examine the influence of the composite porosity model, its results should be compared with results from an alternative conceptual model, dual permeability for two-phase flow.

\subsubsection{Additional Evaluations}

Continued analysis of the influence of various parameters is recommended. This analysis can serve to identify both (1) the important parameters on which the DOE/EM (through the NSNFP) should focus, (2) parameters that the OCRWM should include in their abstracted source-term models for various DOE SNF (either applied directly by OCRWM or as a cross check on any sensitivity analysis they perform), and (3) parameters that OCRWM should use in Repository Acceptance Criteria (RAC).

Link Nuclear Criticality Modeling to Geophysical Modeling. Because risk is often measured as the expected values of the consequences (i.e., probability times consequence), determining the probability and consequences of criticality requires two types of modeling. The first is geophysical modeling for probability evaluations that simulates the assembly process of fissile mass by examining the likelihood of the necessary physical, hydrologic, and geochemical conditions occurring, along with the initial conditions for the nuclear consequence calculations. The second type of modeling, nuclear criticality modeling, is needed for probability evaluations to determine the limits for critical conditions and also for consequence calculations to simulate the potential energy release.

The NSNFP performed nuclear criticality modeling in 1997. The determination of limits for critical conditions from this analysis should now be linked to the geophysical modeling of a performance assessment to further evaluate the probability of criticality. Although simulating the complete evolution of a spent fuel container and its contents after disposal is not warranted at this time, an analysis of conditions in three distinct locations should be conducted, i.e., the potential for criticality should be examined (1) when the container and spent fuel are completely degraded and the mixture is resting on the tunnel invert, (2) when the fissile material has been transported away from the tunnel and deposited along fracture walls, and (3) at a collection zone in the far field. The initial analysis should focus on criticality on the tunnel invert by examining the likelihood of separating fissile material from neutron poisons near the waste package and also the adsorption potential of the rust for fissile material and neutron poisons. 


\subsubsection{Produce Abstractions from Detailed Analyses}

The OCRWM performance assessments (i.e., TSPAs) evaluate the system based on abstractions of results drawn from detailed analyses. Currently, the OCRWM uses Sandia's formulation for the alteration and dissolution of many of the spent fuel categories. It is recommended that Sandia perform the detailed analyses of the DOE SNF/DHLW and then produce the final abstractions of these formulations for the OCRWM under Sandia's QA program. The alternative is for the OCRWM to independently verify Sandia's formulations and model parameters. The advantages of Sandia's performing the detailed analyses are that (a) Sandia has extensive understanding of the fuel and its characteristics, based on current and previous studies, (b) the appropriate detailed models are in use and proven, and (c) Sandia can apply its knowledge of the spent fuels and related issues to produce accurate and meaningful abstractions for use by the OCRWM.

\subsubsection{Upgrade Quality Assurance}

A recommended procedural improvement is to upgrade the quality assurance aspects of the analysis and data. The NSNFP can provide quality assurance for its analysis using many of the same quality assurance tools that were generated for the WIPP and approved by the DOE and EPA. Improved quality assurance of this or a future analysis will help the OCRWM benchmark and provide quality assurance of its own analysis-a very important task as the OCRWM is at the beginning of its quality assurance for the performance assessment process. In the 1997 PA, all data used in the calculations were accessed by means of a controlled database. Sources for some of the data, however, which originated in earlier project-wide studies, have not been verified. It is recommended that the data sources be gathered and verified, which would provide a consistent, quality-assured database that could be used by any team performing calculations for the YMP.

\subsection{General Guidance}

Because the mass, volume, and activity of the DOE SNF are modest in relation to the commercial SNF, future analysis of the spent fuel categories should continue to demonstrate that the unique characteristics of DOE SNF are not sufficient to adversely influence the entire disposal system. In general, the radioisotopes in the codisposal container (Categories 2 through 13) are dominated by DHLW. Therefore, direct disposal of DOE SNF will likely meet the disposal criteria if commercial SNF can meet them. (Based on results from the 1994 PA, a tuff repository containing only DOE SNF and DHLW would also likely meet disposal criteria.) Therefore, this analysis finds no difficulty with the direct disposal option. Other considerations such as transportation and storage of DOE SNF may support a need for treatment and robust packaging, but these were not examined in this study. DOE/EM should continue analysis of its spent fuel by performance assessment so that convincing arguments with regard to repository acceptance criteria can be presented to the NRC regulator. The advantage of continued analysis is that it not only informs the decision makers about significant issues but can also contribute to safe, reasonable, and cost-efficient criteria for acceptance of DOE SNF. 


\section{References}

Adriano, D.C., K.W. McLeod, and T.G. Ciravolo. 1986. "Long-Term Availability of Cm and Pu to Crop Plants," Health Physics. Vol. 50, no. 5, 647-651.

Ahola, M.P., and B. Sagar. 1993. "Modeling of Saturated Zone at Yucca Mountain, Nevada," High Level Radioactive Waste Management, Proceedings of the Fourth Annual International Conference, Las Vegas, NV, April 26-30, 1993. La Grange Park, IL: American Nuclear Society, Inc.; New York, NY: American Society of Civil Engineers. Vol. 2, 1602-1608.

Allard, B. 1982. "Solubilities of Actinides in Neutral or Basic Solutions," Actinides in Perspective, Proceedings of the 1981 Conference, Pacific Grove, CA, September 10-15, 1981. Ed. N.M. Edelstein. New York, NY: Pergamon Press. Vol. 26, 553-580.

Altman, S.J., B.W. Arnold, R.W. Barnard, G.E. Barr, C.K. Ho, S.A. McKenna, and R.R. Eaton. 1996. Flow Calculations for Yucca Mountain Groundwater Travel Time (GWTT-95). SAND96-0819. Albuquerque, NM: Sandia National Laboratories.

Amyx, J.W., D.M. Bass, Jr., and R.L. Whiting. 1960. Petroleum Reservoir Engineering: Physical Properties. New York, NY: McGraw-Hill.

Andrews, R.W., T.F. Dale, and J.A. McNeish. 1994. Total System Performance Assessment-1993: An Evaluation of the Potential Yucca Mountain Repository. B00000000-01717-2200-00099-Rev. 01. Las Vegas, NV: Civilian Radioactive Waste Management System, Management and Operating Contractor. (Available from the National Technical Information Service (NTIS), Springfield, VA, 703/487-4679, orders@ntis.fedworld.gov as DE96007840/XAB.)

Apps, J.A., C.L. Carnahan, P.C. Lichtner, M.C. Michel, D. Perry, R.J. Silva, O. Weres, and A.F. White. 1982. Status of Geochemical Problems Relating to the Burial of High-Level Radioactive Waste, 1982. NUREG/CR-3062, LBL-15103. Washington, DC: U.S. Nuclear Regulatory Commission, Office of Nuclear Material Safety and Safeguards; Berkeley, CA: Lawrence Berkeley Laboratory. (Available from the NTIS as NUREG/CR-3062.)

Asano, H., H. Wakamatsu, and M. Akashi. 1992. "Corrosion Lifetime Assessment for Candidate Materials of Geological Disposal Overpack for High-Level Nuclear Waste Canisters: Perspective of R\&D in Japan," High Level Radioactive Waste Management, Proceedings of the Third International Conference, Las Vegas, NV, April 12-16, 1992. La Grange Park, II: American Nuclear Society, Inc.; New York, NY: American Society of Civil Engineers. Vol. 2, 1658-1669.

Aziz, K., and A. Settari. 1979. Petroleum Reservoir Simulation. New York, NY: Elsevier.

Barnard, R.W., M.L. Wilson, H.A. Dockery, J.H. Gauthier, P.G. Kaplan, R.R. Eaton, F.W. Bingham, and T.H. Robey. 1992. TSPA 1991: An Initial Total-System Performance Assessment for Yucca Mountain. SAND91-2795. Albuquerque, NM: Sandia National Laboratories.

Barr, G.E., and W.B. Miller. 1987. Simple Models of the Saturated Zone at Yucca Mountain. SAND87-0112. Albuquerque, NM: Sandia National Laboratories.

Barrow, G.M. 1973. Physical Chemistry. 3rd ed. New York, NY: McGraw-Hill.

Bean, J.E., M.E. Lord, D.A. McArthur, R.J. MacKinnon, J.D. Miller, and J.D. Schreiber. 1996. "Analysis Package for the Salado Flow Calculations (Task 1) of the Performance Assessment Analysis Supporting the Compliance Certification Application (CCA)." Albuquerque, NM: Sandia National Laboratories. (Copy on file in the Sandia WIPP Central Files (SWCF), Sandia National Laboratories, Albuquerque, NM as WPO\#40514.) 
References

Bear, J. 1979. Hydraulics of Groundwater. New York, NY: McGraw-Hill.

Bear, J. 1988. Dynamics of Fluids in Porous Media. New York, NY: Dover Publications, Inc.

Bear, J., and C. Braester. 1972. "On the Flow of Tẃo Immiscible Fluids in Fractured Porous Media," Fundamentals of Transport Phenomena in Porous Media, Proceedings of the First International Symposium, Technion City, Haifa, Israel, February 23-28, 1969. Developments in Soil Science 2. New York, NY: Elsevier Publishing Company. 177-202.

Bear, J., C-F. Tsang, and G. de Marsily. 1993. Flow and Contaminant Transport in Fractured Rock. San Diego, CA: Academic Press, Inc.

Benson, L.V., J.H. Robison, R.K. Blankennagel, and A.E. Ogard. 1983. Chemical Composition of Ground Water and the Locations of Permeable Zones in the Yucca Mountain Area, Nevada. Open-File Report 83-854. Denver, CO: U.S. Geological Survey. (Available from the NTIS as DE84006458.)

Bish, D.L., F.A. Caporuscio, J.F. Copp, B.M. Crowe, J.D. Purson, J.R. Smyth, and R.G. Warren. 1981. Preliminary Stratigraphic and Petrologic Characterization of Core Samples from USW-G1, Yucca Mountain, Nevada. Eds. A.C. Waters and P.R. Carroll. LA-8840-MS. Los Alamos, NM: Los Alamos National Laboratory. (Available from the NTIS as DE82010985.)

Blankennagel, R.K., and J.E. Weir, Jr. 1973. Geohydrology of the Eastern Part of Pahute Mesa, Nevada Test Site, Nye County, Nevada. USGS Professional Paper 712-B. Washington, DC: U.S. Government Printing Office. (Available at the U.S. Geological Survey Library, 952 National Center, 12201 Sunrise Valley Drive, Reston, VA 20192, 703/648-4400, cic_lib@usgs.gov.)

Bodvarsson, G.S., T.M. Bandurraga, and Y.S. Wu, eds. 1997. The Site-Scale Unsaturated Zone Model of Yucca Mountain, Nevada, for the Viability Assessment. LBNL-40376. Berkeley, CA: Lawrence Berkeley National Laboratory; Denver, CO: U.S. Geological Survey. (Available from the Yucca Mountain Site Characterization Office, 1180 Town Center Drive, M/S 010, Las Vegas, NV 89134 as MOL.19971204.0750.)

Bonano, E.J., and W.E. Beyeler. 1985. "Transport and Capture of Colloidal Particles in Single Fractures," Scientific Basis for Nuclear Waste Management VIII, Materials Research Society Symposia Proceedings, Boston, MA, November 26-29, 1984. Eds. C.M. Jantzen, J.A. Stone, and R.C. Ewing. SAND84-0810C. Pittsburgh, PA: Materials Research Society. Vol. 44, 385-392.

Boulton, G.S., and A. Payne. 1992. Simulation of the European Ice Sheet Through the Last Glacial Cycle and Prediction of Future Glaciation. SKB Technical Report 93-14. Stockholm, Sweden: Swedish Nuclear Fuel and Waste Management Co. (Available from the NTIS as DE9416529/XAB.)

Braithwaite, J.W., and M.A. Molecke. 1980. "Nuclear Waste Canister Corrosion Studies Pertinent to Geologic Isolation," Nuclear and Chemical Waste Management. SAND79-1935J. Vol. 1, no. 1, 37-50.

Breck, D.W. 1983. "Synthetic Zeolites: Properties and Applications," Industrial Minerals and Rocks (Nonmetallics Other than Fuels). 5th ed. Ed. S.J. Lefond. New York, NY: American Institute of Mining, Metallurgical, and Petroleum Engineers, Inc. 1399-1413.

Brookins, D.G. 1978. Geochemical Constraints on Accumulation of Actinide Critical Masses from Stored Nuclear Waste in Natural Rock Repositories. ONWI-17. Columbus, OH: Office of Nuclear Waste Isolation (ONWI), Battelle Project Management Division. (Available from the NTIS as ONWI-17).

Brookins, D.G. 1981. "Primary Uranophane from the Ambrosia Lake Uranium District, Grants Mineral Belt, USA," Mineralium Deposita. Vol. 16, no. 1, 3-5.

Brookins, D.G. 1988. Eh-pH Diagrams for Geochemistry. New York, NY: Springer-Verlag. 
Brooks, R.H., and A.T. Corey. 1966. "Properties of Porous Media Affecting Fluid Flow," Journal of the Irrigation and Drainage Division, Proceedings of the American Society of Civil Engineers. Vol. 92, no. IR2, 61-88.

Broxton, D.E., R.G. Warren, R.C. Hagan, and G. Luedemann. 1986. Chemistry of Diagenetically Altered Tuffs at a Potential Nuclear Waste Repository, Yucca Mountain, Nye County, Nevada. LA-10802-MS. Los Alamos, NM: Los Alamos National Laboratory. (Available from the NTIS as DE87003802/XAB.)

Brunauer, S., P.H. Emmett, and E. Teller. 1938. "Adsorption of Gases in Multimolecular Layers," Journal of the American Chemical Society. Vol. 60, 309-319.

Brush, L.H. 1990. Test Plan for Laboratory and Modeling Studies of Repository and Radionuclide Chemistry for the Waste Isolation Pilot Plant. SAND90-0266. Albuquerque, NM: Sandia National Laboratories.

Brush, L.H. 1997. "Ranges and Probability Distribution Coefficients (Kds) for the 1997 Idaho National Engineering and Environmental Laboratory (INEEL) Performance-Assessment (PA) Calculations." Memorandum from L.H. Brush to L.J. Dotson, 7/14/97. Albuquerque, NM: Sandia National Laboratories. (On file in NSNFP Records, Sandia National Laboratories, Albuquerque, NM as NSNFP \#016).

Bruton, C.J., and H.F. Shaw. 1988. "Geochemical Simulation of Reaction Between Spent Fuel Waste Form and J-13 Water at $25^{\circ} \mathrm{C}$ and $90^{\circ} \mathrm{C}$," Scientific Basis for Nuclear Waste Management XI, Materials Research Society Symposia Proceedings, Boston, MA, November 30-December 3, 1987. Eds. M.J. Apted and R.E. Westerman. UCRL-96702. Pittsburgh, PA: Materials Research Society. Vol. 112, 485-494.

Bruton, C.J., B.E. Viani, W.L. Bourcier, K.J. Jackson, and R.D. Aines. 1990. "Geochemical Modeling: An Integrated Approach to Nuclear Waste Disposal Issues," High Level Radioactive Waste Management, Proceedings of the International Topical Meeting, Las Vegas, NV, April 8-12, 1990. UCRL-JC-102019. La Grange Park, IL: American Nuclear Society, Inc.; New York, NY: American Society of Civil Engineers. Vol. 1, 594-595. (UCRLJC-102019 is available from the NTIS as DE93009298/XAB.)

Buesch, D.C., R.W. Spengler, T.C. Moyer, and J.K. Geslin. 1996. Proposed Stratigraphic Nomenclature and Macroscopic Identification of Lithostratigraphic Units of the Paintbrush Group Exposed at Yucca Mountain, Nevada. USGS Open-File Report 94-469. Denver, CO: U.S. Geological Survey. (Available from the NTIS as DE96014541/XAB.)

Caporuscio, F.A., and D.T. Vaniman. 1985. Iron and Manganese in Oxide Minerals and in Glasses: Preliminary Consideration of Eh Buffering Potential at Yucca Mountain, Nevada. LA-10369-MS. Los Alamos, NM: Los Alamos National Laboratory. (Available from the NTIS as DE85011892/XAB.)

Carey, J.W., S.J. Chipera, D.T. Vaniman, D.L. Bish, H.S. Viswanathan, and K. Carter-Krogh. 1997. "Three-Dimensional Mineralogic Model of Yucca Mountain, Nevada. Rev. 1." Draft R1. Deliverable No.: SP344BM4. Revision 9/24/97. Los Alamos, NM: Los Alamos National Laboratory, Earth and Environmental Sciences Division. (Available from Steve J. Chipera, Los Alamos National Laboratory, Mail Stop D469, Los Alamos, NM 87545, chipera@lanl.gov.)

Carr, W.J. 1974. Summary of Tectonic and Structural Evidence for Stress Orientation at the Nevada Test Site. OpenFile Report 74-176. Denver, CO: U.S. Geological Survey.

Carr, W.J. 1988. "Volcano-Tectonic Setting of Yucca Mountain and Crater Flat, Southwestern Nevada," Geologic and Hydrologic Investigations of a Potential Nuclear Waste Disposal Site at Yucca Mountain, Southern Nevada. Eds. M.D. Carr and J.C. Yount. USGS Bulletin 1790. Washington, DC: U.S. Geological Survey. 35-49. (USGS Bulletin 1790 is available from the NTIS as DE89003048/XAB.) 
Carslaw, H.S., and J.C. Jaeger. 1959. Conduction of Heat in Solids. 2nd ed. Oxford, England: Clarendon Press; New York, NY: Oxford University Press.

Cas, R.A.F., and J.V. Wright. 1987. Volcanic Successions, Modern and Ancient: A Geological Approach to Processes, Products, and Successions. London; Boston: Allen \& Unwin.

Champ, D.R., W.F. Merritt, and J.L. Young. 1982. "Potential for the Rapid Transport of Plutonium in Groundwater as Demonstrated by Core Column Studies," Scientific Basis for Nuclear Waste Management V, Proceedings of the Materials Research Society Fifth International Symposium, Berlin, Germany, June 7-10, 1982. Ed. W. Lutze. New York, NY: North-Holland. Vol. 11, 745-754.

Clark, T.H., and C.W. Stearn. 1968. Geological Evolution of North America. 2nd ed. New York, NY: The Ronald Press Co.

Clayton, R.W., W.P. Zelinski, and C.A. Rautman. 1997. "ISM2.0: A 3D Geologic Framework and Integrated Site Model of Yucca Mountain, Rev. 00, February, 1997." B00000000-01717-5700-00004 Rev 00. Las Vegas, NV: Civilian Radioactive Waste Management System, Management \& Operating Contractor for U.S. Department of Energy, Yucca Mountain Site Characterization Project. (Available from the Yucca Mountain Site Characterization Office as MOL.19970724.0215.)

Coats, K.H. 1989. "Implicit Compositional Simulation of Single-Porosity and Dual-Porosity Reservoirs"" Proceedings of the Tenth SPE Symposium on Reservoir Simulation, Houston, TX, February 6-8, 1989. SPE 18427. Richardson, TX: Society of Petroleum Engineers. Vol. 10, 239-275. (Available from the SPE, P.O. Box 833836, Richardson, TX 75083-3836, 800-456-6863, books@spelink.spe.org.)

Coats, K.H., and B.D. Smith. 1964. "Dead-End Pore Volume and Dispersion in Porous Media," Society of Petroleum Engineers Journal. Vol. 4, no. 1, 73-84.

Coughtrey, P.J., D. Jackson, C.H. Jones, P. Kane, and M.C. Thorne. 1984. Radionuclide Distribution and Transport in Terrestrial and Aquatic Ecosystems: A Critical Review of Data. EUR 8115. Boston, MA: A.A. Balkema. Vols. 1-6.

Craig, R.W., and K.A. Johnson. 1984. Geohydrologic Data for Test Well UE-25p\#1, Yucca Mountain Area, Nye County, Nevada. Open-File Report 84-450. Denver, CO: U.S. Geological Survey. (Available from the NTIS as DE85001508/XAB.)

Cramer, J.J., and J.A.T. Smellie, eds. 1994. Final Report of the AECLSSKB Cigar Lake Analog Study. AECL-10851, COG-93-147, SKB TR 94-04. Pinawa, Manitoba, Canada: Whiteshell Laboratories. (Available from the NTIS as DE9513252/XAB.)

Cresap, D.A. 1997. "DOE SNF Sensitivity for Performance Assessment." EPS-58-97. Idaho Falls, ID: Idaho National Engineering and Environmental Laboratory. (Available from E. McBride, Document Control, National Spent Nuclear Fuel Program, emcb@inel.gov, 208/526-6837.)

Critchfield, H.J. 1983. "Climatic Classification," General Climatology. 4th ed. Englewood Cliffs, NJ: PrenticeHall. 145-161.

Crowley, K.D. 1997. "Nuclear Waste Disposal: The Technical Challenges," Physics Today. Vol. 50, no. 6, 32-39.

Czarnecki, J.B. 1985. Simulated Effects of Increased Recharge on the Ground-Water Flow System of Yucca Mountain and Vicinity, Nevada-California. Water-Resources Investigations Report 84-4344. Denver, CO: U.S. Geological Survey. (Available from the NTIS as DE85012985/XAB.) 
Czarnecki, J.B., and R.K. Waddell. 1984. Finite-Element Simulation of Ground-Water Flow in the Vicinity of Yucca Mountain, Nevada-California. Water-Resources Investigations Report 84-4349. Denver, CO: U.S. Geological Survey. (Available from the NTIS as DE85012363/XAB.)

Czarnecki, J.B., C.C. Faunt, C.W. Gable, and G.A. Zyvoloski. 1996. "On the Development of a Three-Dimensional Finite-Element Groundwater Flow Model of the Saturated Zone, Yucca Mountain, Nevada," Poster Papers, Modelcare 96, International Conference on Calibration and Reliability in Groundwater Modelling, Colorado School of Mines, Golden, CO, September 24-26, 1996. CONF-9609146-1. Washington, DC: U.S. Department of Energy, Office of Civilian Radioactive Waste Management; Springfield, VA: National Technical Information Service. 51-59. (Available from the NTIS as DE97001704/XAB.)

D'Agnese, F.A. 1994. 'Using Geoscientific Information Systems for Three-Dimensional Modeling of Regional Ground Water Flow Systems, Death Valley Region, Nevada and California." Ph.D. dissertation. Golden, CO: Colorado School of Mines, Department of Geology and Geological Engineering. (Available from Arthur Lakes Library, Colorado School of Mines, P.O. Box 4029, 1400 Illinois Street, Golden, CO 80401-0029, 303/273-3911, http://www.mines.edu/library.)

Davis, J.A., and D.B. Kent. 1990. "Surface Complexation Modeling in Aqueous Geochemistry," Mineral-Water Interface Geochemistry. Eds. M.F. Hochella, Jr. and A.F. White. Reviews in Mineralogy Vol. 23. Washington, DC: Mineralogical Society of America. 177-260.

Delany, J.M., and T.J. Wolery. 1984. Fixed-Fugacity Option for the EQ6 Geochemical Reaction Path Code. UCRL53598. Livermore, CA: Lawrence Livermore National Laboratory. (Available from the NTIS as DE85016345/ $\mathrm{XAB}$.

Doctor, P.G., A.M. Liebetrau, P.W. Eslinger, P.W. Reimus, D.M. Elwood, D.L. Strenge, D.W. Engel, J.E. Tanner, and M.D. Freshley. 1992. An Example Postclosure Risk Assessment Using the Potential Yucca Mountain Site. PNL-8081. Richland, WA: Pacific Northwest Laboratory. (Available from the NTIS as DE92014614/XAB.)

DOE (U.S. Department of Energy). 1980. Final Environmental Impact Statement, Waste Isolation Pilot Plant. DOE/ EIS-0026. Washington, DC: U.S. Department of Energy, Assistant Secretary for Defense Programs. (Available from the NTIS as DOE/EIS-0026.)

DOE (U.S. Department of Energy). 1985a. Evaluation of Commercial Repository Capacity for the Disposal of Defense High-Level Waste. DOE/DP-0020/1. Washington, DC: U.S. Department of Energy, Assistant Secretary for Defense Programs. (Available from the NTIS as DE85013230/XAB.)

DOE (U.S. Department of Energy). 1985b. An Evaluation of 'Commercial Repository Capacity for the Disposal of Defense High-Level Waste,' Response to Comments. DOE/DP-0027. Washington, DC: U.S. Department of Energy, Assistant Secretary for Defense Programs. (Available from the NTIS as DE86005423/XAB.)

DOE (U.S. Department of Energy). 1987-1988. Characteristics of Spent Fuel, High-Level Waste, and Other Radioactive Wastes Which May Require Long-Term Isolation. DOE/RW-0184. Washington, DC: U.S. Department of Energy, Office of Civilian Radioactive Waste Management. Vols. 1-8.

DOE (U.S. Department of Energy). 1988a. Draft 1988 Mission Plan Amendment. DOE/RW-0187. Washington, DC: U.S. Department of Energy, Office of Civilian Radioactive Waste Management.

DOE (U.S. Department of Energy). 1988b. Site Characterization Plan, Yucca Mountain Site, Nevada Research and Development Area, Nevada. DOE/RW-0199. Washington, DC: U.S. Department of Energy, Office of Civilian Radioactive Waste Management. Vol. 1-8. 
DOE (U.S. Department of Energy). 1988c. Internal Dose Conversion Factors for Calculation of Dose to the Public. DOE/EH-0071. Washington, DC: U.S. Department of Energy, Assistant Secretary for Environment, Safety and Health. (Available from the NTIS as DE880914297/XAB.)

DOE (U.S. Department of Energy). 1992. Characteristics of Potential Repository Wastes. DOE/RW-0184-R1. Oak Ridge, TN: Oak Ridge National Laboratory; Washington, DC: U.S. Department of Energy, Office of Civilian Radioactive Waste Management. Vols. 1-4.

DOE (U.S. Department of Energy): 1995. "Mined Geologic Disposal System License Application Annotated Outline." YMP 94-05, Revision 0. Las Vegas, NV: U.S. Department of Energy, Office of Civilian Radioactive Waste Management. (Available from the Yucca Mountain Site Characterization Office as MOL.19951018.0033, MOL.19951019.0238, and MOL.19951019.0239.)

Dudley, W.W., Jr., and J.D. Larson. 1976. Effect of Irrigation Pumping on Desert Pupfish Habitats in Ash Meadows, Nye County, Nevada. USGS Professional Paper 927. Washington, DC: U.S. Government Printing Office. (Available at the U.S. Geological Survey Library, 952 National Center, 12201 Sunrise Valley Drive, Reston, VA 20192, 703/648-4400, cic_lib@usgs.gov.)

Duffy, C.J. 1993. Preliminary Conceptual Model for Mineral Evolution in Yucca Mountain. LA-12708-MS. Los Alamos, NM: Los Alamos National Laboratory. (Available from the NTIS as DE94003899/XAB.)

Eckerman, K.F., A.B. Wolbarst, and A.C.B. Richardson. 1988. Limiting Values of Radionuclide Intake and Air Concentration and Dose Conversion Factors for Inhalation, Submersion, and Ingestion. Federal Guidance Report No. 11. EPA-520/1-88-020. Oak Ridge, TN: Oak Ridge National Laboratory; Washington, DC: U.S. Environmental Protection Agency, Office of Radiation Programs. (Available from the NTIS as DE89011065/XAB.)

Energy Policy Act. 1992. "Energy Policy Act of 1992." Public Law 102-486. (106 Stat. 2776; 42 U.S.C. 13201 et seq.)

EPA (U.S. Environmental Protection Agency). 1985. "40 CFR 191: Environmental Standards for the Management and Disposal of Spent Nuclear Fuel, High-Level and Transuranic Radioactive Wastes; Final Rule," Federal Register. Vol. 50, no. 182, 38066-38089.

EPA (U.S. Environmental Protection Agency). 1993. "40 CFR Part 191: Environmental Radiation Protection Standards for the Management and Disposal of Spent Nuclear Fuel, High-Level and Transuranic Radioactive Wastes; Final Rule," Federal Register. Vol. 58, no. 242, 66398-66416.

EPRI (Electric Power Research Institute). 1992. "Source Term in the EPRI Performance Assessment." R. Shaw, presented to Nuclear Waste Technical Review Board, Las Vegas, NV, October 15, 1992.

EPRI (Electric Power Research Institute). 1996. Yucca Mountain Total System Performance Assessment, Phase 3. EPRI TR-107191. Boulder, CO: Risk Engineering, Inc.; Palo Alto, CA: Electric Power Research Institute. (Available from EPRI, P.O. Box 10412, Palo Alto, CA, 415/855-2411.)

Ervin, E.M., R.R. Luckey, and D.J. Burkhardt. 1994. Revised Potentiometric-Surface Map for Yucca Mountain and Vicinity, Nevada. Water-Resources Investigations Report 93-4000. Denver, CO: U.S. Geological Survey. (Available from the NTIS as DE94012059/XAB.)

Fabryka-Martin, J.T., A.V. Wolfsberg, P.R. Dixon, S.S. Levy, J. Musgrave, and H.J. Turin. 1996. "Summary Report of Chlorine-36 Studies: Sampling, Analysis and Simulation of Chlorine-36 in the Exploratory Studies Facility." LA-13352-MS. Los Alamos National Laboratory Milestone Report 3783M. Los Alamos, NM: Los Alamos National Laboratory. (Milestone Report 3783M is available from the Yucca Mountain Site Characterization Office as MOL.19970211.0036.) 
Finn, P.A., J.C. Hoh, S.F. Wolf, M.T. Surchik, E.C. Buck, and J.K. Bates. In press. "Spent Fuel Reaction - The Behavior of the $\beta$-Phase over 3.1 Years," Scientific Basis for Nuclear Waste Management XX, Materials Research Society Symposium Proceedings, Boston, MA, December 2-5, 1996. Eds. W.J. Gray and I.R. Triay. Pittsburgh, PA: Materials Research Society.

Fisher, R.V., and H.U. Schmincke. 1984. Pyroclastic Rocks. Berlin; New York: Springer-Verlag.

Flint, A.L., and L.E. Flint. 1994. "Spatial Distribution of Potential Near Surface Moisture Flux at Yucca Mountain," High Level Radioactive Waste Management, Proceedings of the Fifth Annual International Conference, Las Vegas, NV, May 22-26, 1994. La Grange Park, IL: American Nuclear Society, Inc.; New York, NY: American Society of Civil Engineers. Vol. 4, 2352-2358.

Flint, A.L., L.E. Flint, and J.A. Hevesi. 1993. "The Influence of Long Term Climate Change on Net Infiltration at Yucca Mountain, Nevada," High Level Radioactive Waste Management, Proceedings of the Fourth Annual International Conference, Las Vegas, NV, April 26-30, 1993. La Grange Park, IL: American Nuclear Society, Inc.; New York, NY: American Society of Civil Engineers. Vol. 1, 152-159.

Flint, L.E., A.L. Flint, C.A. Rautman, and J.D. Istok. 1996. Physical and Hydrologic Properties of Rock Outcrop Samples at Yucca Mountain, Nevada. USGS Open-File Report 95-280. Denver, CO: U.S. Geological Survey. (Available from the NTIS as DE96014513/XAB.)

Forester, R.M., J.P. Bradbury, C. Carter, A.B. Elvidge, M.L. Hemphill, S.C. Lundstrom, S.A. Mahan, B.D. Marshall, L.A. Neymark, J.B. Paces, S.E. Sharpe, J.F. Whelan, and P.E. Wigand. 1996. "Synthesis of Quaternary Response of the Yucca Mountain Unsaturated and Saturated Zone Hydrology to Climate Change." 1996 Milestone Report 3GCA102M. Denver, CO: U.S. Geological Survey, YMP Branch. (Available from the Yucca Mountain Site Characterization Office as MOL.19970211.0026.)

Freeze, R.A., and J.A. Cherry. 1979. Groundwater. Englewood Cliffs, NJ: Prentice-Hall.

Fridrich, C.J., W.W. Dudley, Jr., and J.S. Stuckless. 1994. "Hydrogeologic Analysis of the Saturated-Zone GroundWater System, Under Yucca Mountain, Nevada," Journal of Hydrology. Vol. 154, no. 1-4, 133-168.

Frondel, C. 1956. "Part 11 Mineral Composition of Gummite," The American Mineralogist. Vol. 41, 539-568.

Galloway, D.L., E.M. Ervin, M.P. Chornak, A.C. Riggs, S.F. Diehl, J.S. Downey, A.L. Flint, L.E. Flint, S.S. Levy, G.L. Patterson, E.M. Taylor, and E.P. Weeks. 1991. "Hydrogeologic Overview and Field Trip of the Regional Ground-Water Flow System in Relation to Yucca Mountain, Nevada," Geological Excursions in Southern California and Mexico, Guidebook, 1991 Annual Meeting, Geological Society of America, San Diego, CA, October 21-24, 1991. Eds. M.J. Walawender and B.B. Hanan. San Diego, CA: San Diego State University. 474-515.

Garrels, R.M., and C.L. Christ. 1965. Solutions, Minerals and Equilibria. San Francisco, CA: Freeman, Cooper \& Co.

Godard, H.P. 1960. "The Corrosion Behavior of Aluminum in Natural Waters," The Canadian Journal of Chemical Engineering. Vol. 38, no. 1, 167-173.

Gray, W.J., and C.N. Wilson. 1995. Spent Fuel Dissolution Studies FY 1991 to 1994. PNL-10540. Richland, WA: Pacific Northwest National Laboratory. (Available from the NTIS as DE96007354/XAB.)

Gray, W.J., H.R. Leider, and S.A. Steward. 1992. "Parametric Study of LWR Spent Fuel Dissolution Kinetics," Journal of Nuclear Materials. Vol. 190, 46-52. 
Grogan, H.A. 1985. Concentration Ratios for BIOPATH: Selection of the Soil-to-Plant Concentration Ratio Database. EIR-575. Würenlingen, Switzerland: Eidgenoessisches Inst. für Reaktorforschung. (Available from the NTIS as DE86702295/XAB.)

Guzowski, R.V., F.B. Nimick, M.D. Siegel, and N.C. Finley. 1983. Repository Site Data Report for Tuff: Yucca Mountain, Nevada. SAND82-2105, NUREG/CR-2937. Albuquerque, NM: Sandia National Laboratories. (Available from the NTIS as NUREG/CR-2937.)

Haberman, J.H., and D.J. Frydrych. 1988. "Corrosion Studies of A216 Grade WCA Steel in Hydrothermal Magnesium-Containing Brines," Scientific Basis for Nuclear Waste Management XI, Materials Research Society Symposium Proceedings, Boston, MA, November 30-December 3, 1987. Eds. M.J. Apted and R.E. Westerman. Pittsburgh, PA: Materials Research Society. Vol. 112, 761-772.

Harrar, J.E., J.E. Carley, W.F. Isherwood, and E. Raber. 1990. Report of the Committee to Review the Use of J-13 Well Water in Nevada Nuclear Waste Storage Investigations. UCD-21867. Livermore, CA: Lawrence Livermore National Laboratory.

Helton, J.C., J.W. Garner, R.P. Rechard, D.K. Rudeen, and P.N. Swift. 1992. Preliminary Comparison with 40 CFR Part 191, Subpart B for the Waste Isolation Pilot Plant, December 1991. Volume 4: Uncertainty and Sensitivity Analysis Results. SAND91-0893/4. Albuquerque, NM: Sandia National Laboratories.

Hill, A.C., and G.W. Thomas. 1985. "A New Approach for Simulating Complex Fractured Reservoirs," Eighth SPE Symposium on Reservoir Simulation, Dallas, TX, February 10-13, 1985. Ed. L.C. Young. Richardson, TX: Society of Petroleum Engineers of AIME. Vol. 8, 429-440. (Available from the SPE, P.O. Box 833836, Richardson, TX 75083-3836, 800-456-6863, books@spelink.spe.org.)

Ho, C.K. 1994. "Verifying the Equivalent Continuum Model in TOUGH2." Memorandum, March 22, 1994. Albuquerque, NM: Sandia National Laboratories. (Available from the Yucca Mountain Site Characterization Office as MOL.19961114.0036.)

Holland, H.D. 1994. "Early Proterozoic Atmospheric Change," Early Life on Earth. Ed. S. Bengston. Nobel Symposium No. 84. New York, NY: Columbia University Press. 237-244.

Hsi, C.D., and D. Langmuir. 1985. "Adsorption of Uranyl onto Ferric Oxyhydroxides: Applications of the Surface Complexation Site-Binding Model," Geochimica et Cosmochimica Acta. Vol. 49, no. 9, 1931-1941.

Hudson, D.B., and A.L. Flint. 1996. "Estimation of Shallow Infiltration and Presence of Potential Pathways for Shallow Infiltration in the Yucca Mountain Area, Nevada." May 31, 1996 version. Denver, CO: U.S. Geological Survey. (Availabie from the Yucca Mountain Site Characterization Office as MOL.19961002.0067.)

Hunt, C.B. 1974. Natural Regions of the United States and Canada. San Francisco, CA: W.H. Freeman \& Co.

Huyakorn, P.S., and K. Nilkuha. 1979. "Solution of Transient Transport Equation Using an Upstream Finite Element Scheme," Applied Mathematical Modelling. Vol. 3, no. 1, 7-17.

Huyakom, P.S., and G.F. Pinder. 1983. Computational Methods in Subsurface Flow. New York, NY: Academic Press.

Huyakorn, P.S., and S.D. Thomas. 1984. "Techniques for Making Finite Elements Competitive in Modeling Flow in Variably Saturated Porous Media," Water Resources Research. Vol. 20, no. 8, 1099-1115.

Huyakorn, P.S., E.P. Springer, V. Guvanasen, and T.D. Wadsworth. 1986. "A Three-Dimensional Finite-Element Model for Simulating Water Flow in Variably Saturated Porous Media," Water Resources Research. Vol. 22, no. $13,1790-1808$. 
Huyakorn, P.S., B.G. Jones, J.C. Parker, T.D. Wadsworth, H.O. White, Jr., E.P. Springer, and H.R. Fuentes, eds. 1987. "Finite Element Simulation of Moisture Movement and Solute Transport in a Large Caisson," Modeling Study of Solute Transport in the Unsaturated Zone: Workshop Proceedings, Los Alamos, NM, June 19, 1986. NUREG/ CR-4615, LA-10730-MS. Washington, DC: U.S. Nuclear Regulatory Commission, Division of Waste Management; Los Alamos, NM: Los Alamos National Laboratory. Vol. 2, 117-173.

Huyakorn, P.S., H.O. White, Jr., and S.M. Panday. 1991. "STAFF2D Version 3.1: A Two-Dimensional Finite Element Code for Simulating Fluid Flow and Transport of Radionuclides in Fractured Porous Media with Water Table Boundary Conditions." Herndon, VA: HydroGeoLogic, Inc. (For more information, contact HydroGeoLogic, Inc., 1155 Herndon Parkway, Suite 900, Herndon, VA 20170, (703) 478-5186, http://www.hgl.com.)

Huyakorn, P.S., S. Panday, and S. Sinha. 1992. "STAFF3D-A Three Dimensional Finite Element Code for Simulating Fluid Flow and Transport of Radionuclides in Fractured Porous Media with Water Table Boundary Conditions, Version 2.0." Herndon, VA: HydroGeoLogic, Inc. (Available from the Yucca Mountain Site Characterization Office as NNA.19940427.0093.)

IAEA (International Atomic Energy Agency). 1987. "Handbook of Parameter Values for the Prediction of Radionuclide Transfer in the Terrestrial and Freshwater Environments." Draft Working Document. Vienna, Austria.

ICRP (International Commission on Radiological Protection). 1977. "Radiation Protection-Recommendations of the International Commission on Radiological Protection," Annals of the ICRP. ICRP Publication 26. Vol. 1, no. 3. New York, NY: Pergamon Press.

ICRP (International Commission on Radiological Protection). 1979. "Limits for Intakes of Radionuclides by Workers. Part 1," Annals of the ICRP. ICRP Publication 30. New York, NY: Pergamon Press.

Iman, R.L., and W.J. Conover. 1979. "The Use of the Rank Transform in Regression," Technometrics. Vol. 21, no. 4, 499-509.

Iman, R.L., and W.J. Conover. 1980. "Small Sample Sensitivity Analysis Techniques for Computer Models, With an Application to Risk Assessment," Communications in Statistics: Theory and Methods. Vol. A9, no. 17, 17491842. "Rejoinder to Comments," pp. 1863-74.

Iman, R.L., and W.J. Conover. 1982. "A Distribution-Free Approach to Inducing Rank Correlation Among Input Variables," Communications in Statistics: Simulation and Computation. Vol. 11, no. 3, 311-334.

Iman, R.L., and J.C. Helton. 1985. A Comparison of Uncertainty and Sensitivity Analysis Techniques for Computer Models. SAND84-1461, NUREG/CR-3904. Albuquerque, NM: Sandia National Laboratories.

Iman, R.L., and M.J. Shortencarier. 1984. A FORTRAN 77 Program and User's Guide for the Generation of Latin Hypercube and Random Samples for Use With Computer Models. SAND83-2365, NUREG/CR-3624. Albuquerque, NM: Sandia National Laboratories.

Iman, R.L., J.M. Davenport, E.L. Frost, and M.J. Shortencarier. 1980. Stepwise Regression with PRESS and Rank Regression (Program and User's Guide). SAND79-1472. Albuquerque, NM: Sandia National Laboratories.

Imbrie, J., and K.P. Imbrie. 1979. Ice Ages: Solving the Mystery. Short Hills, NJ: Enslow Publishers.

INEEL SNFTT (Spent Nuclear Fuel Task Team). 1997. "Technical Strategy for the Management of INEEL Spent Nuclear Fuel." INEL/EST-97-00387-Vol. 2. Idaho Falls, ID: U.S. Department of Energy; Washington, DC: U.S. Department of Energy, Office of Environmental Restoration and Waste Management. 
References

INEEL (Idaho National Engineering Laboratory). 1997. "EM/RW Repository Task Team Report: Grouping Method to Minimize Testing for Repository Emplacement of DOE SNF." Revision 0. DOE/SNF/REP-008. Idaho Falls, ID: U.S. Department of Energy, Assistant Secretary for Environmental Management.

Jackson, D. 1984. Aquatic Organism and Terrestrial Plant Data for the Biosphere Code ECOS. ANS Report 376-1. Epsom, Surrey, U.K.: Associated Nuclear Services. (Available from the NTIS as DE85781101/XAB.)

Jacobsson, A-M.M., and R.S. Rundberg. 1997. "Uranyl Sorption onto Alumina," Scientific Basis for Nuclear Waste Management XX, Materials Research Society Symposium Proceedings, Boston, MA, December 2-6, 1996. Eds. W.J. Gray and I.R. Triay. Pittsburgh, PA: Materials Research Society. Vol. 465, 797-804.

Jakob, M., and G.A. Hawkins. 1957. Elements of Heat Transfer. 3rd ed. New York, NY: John Wiley \& Sons.

Kazemi, H., L.S. Merrill, Jr., K.L. Porterfield, and P.R. Zeman. 1976. "Numerical Simulation of Water-Oil Flow in Naturally Fractured Reservoirs," Society of Petroleum Engineers Journal. Vol. 16, no. 6, 317-326.

Kent, D.B., V.S. Tripathi, N.B. Ball, J.O. Leckie, and M.D. Siegel. 1988. Surface-Complexation Modeling of Radionuclide Adsorption in Subsurface Environments. SAND86-7175, NUREG/CR-4807. Albuquerque, NM: Sandia National Laboratories.

Kerrisk, J.F. 1984. Solubility Limits on Radionuclide Dissolution at a Yucca Mountain Repository. LA-9995-MS. Los Alamos, NM: Los Alamos National Laboratory. (Available from the NTIS as DE84015084.)

Kirby, G.N. 1979. "Corrosion Performance of Carbon Steel," Chemical Engineering. Vol. 86, no. 6, $72-84$.

Kreamer, D.K., V.F. Hodge, I. Rabinowitz, K.H. Johannesson, and K.J. Stetzenbach. 1996. "Trace Element Geochemistry in Water from Selected Springs in Death Valley National Park, California," Ground Water. Vol. 34, no. 1, 95-103.

Langmuir, D. 1978. "Uranium Solution-Mineral Equilibria at Low Temperatures with Applications to Sedimentary Ore Deposits," Geochimica et Cosmochimica Acta. Vol. 42, no. 6, 547-569.

LaPlante, P.A., S.J. Maheras, and M.S. Jarzemba. 1995. "Initial Analysis of Selected Site-Specific Dose Assessment Parameters and Exposure Pathways Applicable to a Groundwater Release Scenario at Yucca Mountain." Prepared for the Nuclear Regulatory Commission. CNWRA 95-018. San Antonio, TX: Southwest Research Institute, Center for Nuclear Waste Regulatory Analyses. (For more information, contact CNWRA, P.O. Drawer 28510, San Antonio, TX 78228-0510, 512/681-4884.)

Lappin, A.R., R.G. Van Buskirk, D.O. Enniss, S.W. Butters, F.M. Prater, C.B. Muller, and J.L. Bergosh. 1982. Thermal Conductivity, Bulk Properties, and Thermal Stratigraphy of Silicic Tuffs from the Upper Portion of Hole USW-GI, Yucca Mountain, Nye County, Nevada. SAND81-1873. Albuquerque, NM: Sandia National Laboratories.

Lawson, G., and G.M. Smith. 1985. BIOS: A Model to Predict Radionuclide Transfer and Doses to Man Following Releases from Geological Repositories for Radioactive Wastes. NRPB-R169. Chilton, UK: National Radiological Protection Board. (For more information, contact the NRPB, Chilton, Didcot, Oxon OX11 ORQ England, Telephone number: 01235 822643, publications@nrpb.org.uk.)

LeCain, G.D. 1997. Air-Injection Testing in Vertical Boreholes in Welded and Nonwelded Tuff, Yucca Mountain, Nevada. Water-Resources Investigations Report 96-4262. Denver, CO: U.S. Geological Survey.

Lefond, S.J., ed. 1983. Industrial Minerals and Rocks (Nonmetallics Other than Fuels). 5th ed. New York, NY: American Institute of Mining, Metallurgical, and Petroleum Engineers, Inc. Vol. 2, 1391-1413. 
Leigh, C.D., B.M. Thompson, J.E. Campbell, D.E. Longsine, R.A. Kennedy, and B.A. Napier. 1993. User's Guide for GENII-S: A Code for Statistical and Deterministic Simulations of Radiation Doses to Humans from Radionuclides in the Environment. SAND91-0561. Albuquerque, NM: Sandia National Laboratories.

Leslie, B.W., E.C. Pearcy, and J.D. Prikryl. 1993. "Oxidative Alteration of Uraninite at the Nopal I Deposit, Mexico: Possible Contaminant Transport and Source Term Constraints for the Proposed Repository at Yucca Mountain," Scientific Basis for Nuclear Waste Management XVI, Materials Research Society Symposium Proceedings, Boston, MA, November 30-December 4, 1992. Eds. C.G. Interrante and R.T. Pabalan. Pittsburgh, PA: Materials Research Society. Vol. 294, 505-512.

Lester, D.H., G. Jansen, and H.C. Burkholder. 1975. "Migration of Radionuclide Chains Through an Adsorbing Medium," Adsorption and Ion Exchange. Eds. I. Zwiebel and N.H. Sweed. AIChE Symposium Series Vol. 71, no. 152. New York, NY: American Institute of Chemical Engineers. 202-213. (This title is also available from the NTIS as BNWL-SA-5079.)

Levy, S.S., D.S. Sweetkind, J.T. Fabryka-Martin, P.R. Dixon, J.L. Roach, L.E. Wolfsberg, D. Elmore, and P. Sharma. 1997. "Investigations of Structural Controls and Mineralogic Associations of Chlorine-36 Fast Pathways in the ESF." Milestone SP2301M4, LA-EES-1-TIP-97-004. Los Alamos, NM: Los Alamos National Laboratory. (Draft, dated 3/12/97, available from the Yucca Mountain Site Characterization Office as MOL.199701119.0044.)

Litvak, B.L. 1986. "Simulation and Characterization of Naturally Fractured Reservoirs," Reservoir Characterization Technical Conference, Dallas, TX, April 29-May 1, 1985. Eds. L.W. Lake and H.B. Carroll, Jr. Orlando, FL: Academic Press. 561-584.

Long, A., and S.W. Childs. 1993. "Rainfall and Net Infiltration Probabilities for Future Climate Conditions at Yucca Mountain," High Level Radioactive Waste Management, Proceedings of the Fourth Annual International Conference, Las Vegas, NV, April 26-30, 1993. La Grange Park, IL: American Nuclear Society, Inc.; New York, NY: American Society of Civil Engineers. Vol. 1, 112-121.

Lotts, A.L., W.D. Bond, C.W. Forsberg, R.W. Glass, F.E. Harrington, G.E. Michaels, K.J. Notz, and R.G. Wymer. 1992. Options for Treating High-Temperature Gas-Cooled Reactor Fuel for Repository Disposal. ORNL/TM12077. Oak Ridge, TN: Oak Ridge National Laboratory. (Available from the NTIS as DE92014909/XAB.)

Luckey, R.R., P. Tucci, C.C. Faunt, E.M. Ervin, W.C. Steinkampf, F.A. D'Agnese, and G.L. Patterson. 1996. Status of Understanding of the Saturated-Zone Ground-Water Flow System at.Yucca Mountain, Nevada, as of 1995. Water-Resources Investigations Report 96-4077. Denver, CO: U.S. Geological Survey. (Available from the NTIS as DE97002516/XAB.)

Luckner, L., M.Th. van Genuchten, and D.R. Nielsen. 1989. "A Consistent Set of Parametric Models for the TwoPhase Flow of Immiscible Fluids in the Subsurface," Water Resources Research. Vol. 25, no. 10, 2187-2193.

Lynch, R.W., R.L. Hunter, D.R. Anderson, F.W. Bingham, J.M. Covan, G.F. Hohnstrieter, T.O. Hunter, R.D. Klett, E.E. Ryder, T.L. Sanders, and W.D. Weart. 1991. Deep Geologic Disposal in the United States: The Waste Isolation Pilot Plant and Yucca Mountain Projects. SAND90-1656. Albuquerque, NM: Sandia National Laboratories.

M\&O (Civilian Radioactive Waste Management System, Management and Operating Contractor [CRWMS M\&O]). 1995a. "Total System Performance Assessment - 1995: An Evaluation of the Potential Yucca Mountain Repository." J.E. Atkins, J.H. Lee, S. Lingineni, S. Mishra, J.A. McNeish, D.C. Sassani, S.D. Sevougian, R.W. Andrews, and J.L. Younker. B00000000-01717-2200-00136, Rev. 01. Las Vegas, NV: TRW Environmental Safety Systems, Inc. (Available from the Yucca Mountain Site Characterization Office as MOL.19960724.0188.) 
M\&O (Civilian Radioactive Waste Management System, Management and Operating Contractor [CRWMS M\&O]). 1995b. "Controlled Design Assumption Document." O.E. Lev, R. Memory, L.J. Olguin, K.K. Bhattacharyya, H.A. Benton, S. Mishra, T.M. Williamson, and R.E. Smith. B00000000-01717-4600-00032. Rev. 01. Las Vegas, NV: TRW Environmental Safety Systems, Inc. (Available from the Yucca Mountain Site Characterization Office as MOL.19951110.0148.)

M\&O (Civilian Radioactive Waste Management System, Management and Operating Contractor [CRWMS M\&O]). 1996. "Description of Performance Allocation, Rev. 00." B00000000-01717-2200-00177-00. August 15, 1996 preliminary draft. Las Vegas, NV: TRW Environmental Safety Systems, Inc. (Available from the Yucca Mountain Site Characterization Office as MOL.19970116.0052.)

M\&O (Civilian Radioactive Waste Management System, Management and Operating Contractor [CRWMS M\&O]). 1997. "Total System Performance Assessment Sensitivity Studies of U.S. Department of Energy Spent Nuclear Fuel." A00000000-01717-5705-0017, Rev. 01. Las Vegas, NV: TRW Environmental Safety Systems, Inc.

MacDougall, H.R., L.W. Scully, and J.R. Tillerson. 1987. Site Characterization Plan Conceptual Design Report. Volume 3 Appendices A-E. SAND84-2641. Albuquerque, NM: Sandia National Laboratories.

Marsh, G.P., and K.J. Taylor. 1988. "An Assessment of Carbon Steel Containers for Radioactive Waste Disposal," Corrosion Science. Vol. 28, no. 3, 289-320.

McGuire, R.K., D.B. Bullen, N. Cook, K.J. Coppersmith, J. Kemeny, A. Long, F.J. Pearson, Jr., F. Schwartz, M. Sheridan, and R.R. Youngs. 1990. Demonstration of a Risk-Based Approach to High-Level Waste Repository Evaluation. EPRI NP-7057. Golden, CO: Risk Engineering; Palo Alto, CA: Electric Power Research Institute. (Available from the Yucca Mountain Site Characterization Office as NNA.19910813.0004)

McGuire, R.K., M.J. Apted, D.B. Bullen, S. Childs, N.G.W. Cook, K.J. Coppersmith, R.L. Keeney, J.M. Kemeny, A. Long, F.J. Pearson, Jr., B. Ross, F.W. Schwartz, M.F. Sheridan, and R.R. Youngs. 1992. Demonstration of a Risk-Based Approach to High-Level Waste Repository Evaluation: Phase 2. EPRI TR-100384. Palo Alto, CA: Electric Power Research Institute.

McKay, M.D., W.J. Conover, and R.J. Beckman. 1979. "A Comparison of Three Methods for Selecting Values of Input Variables in the Analysis of Output from a Computer Code," Technometrics. Vol. 21, no. 2, 239-245.

McKinley, P.W., M.P. Long, and L.V. Benson. 1991. Chemical Analyses of Water from Selected Wells and Springs in the Yucca Mountain Area, Nevada and Southeastern California. USGS-OFR-90-355. Denver, CO: U.S. Geological Survey.

Meijer, A. 1992. "A Strategy for the Derivation and Use of Sorption Coefficients in Performance Assessment Calculations for the Yucca Mountain Site," Proceedings of the DOE/Yucca Mountain Site Characterization Project Radionuclide Adsorption Workshop at Los Alamos National Laboratory, September 11-12, 1990. Comp. J.A. Canepa. LA-UR-91-3747; LA-12325-C. Los Alamos, NM: Los Alamos National Laboratory. 9-40. (The proceedings, LA-UR-91-3747 is available from the NTIS as DE92041241/XAB.)

Melrose, J.C. 1991. "Scaling Procedures for Capillary Pressure Data at Low Wetting-Phase Saturations," SPE Formation Evaluation. Vol. 6, no. 2, 227-232.

Miller, C.W., C.F. Baes, III, D.E. Dunning, Jr., E.L. Etnier, K.K. Kanak, D.C. Kocher, C.A. Little, L.M. McDowellBoyer, H.R. Meyer, and E.M. Rupp. 1980. Recommendations Concerning Models and Parameters Best Suited to Breeder Reactor Environmental Radiological Assessments. ORNL-5529. Oak Ridge, TN: Oak Ridge National Laboratory. (Available from the NTIS as ORNL-5529.)

Morgan, M.G., M. Henrion, and M. Small. 1990. Uncertainty: A Guide to Dealing with Uncertainty in Quantitative Risk and Policy Analysis. Cambridge, England; New York: Cambridge University Press. 
Moyer, T.C., and J.K. Geslin. 1995. Lithostratigraphy of the Calico Hills Formation and Prow Pass Tuff(Crater Flat Group) at Yucca Mountain, Nevada. USGS Open-File Report 94-460. Denver, CO: U.S. Geological Survey. (Available from the NTIS as DE95014711/XAB.)

Mualem, Y. 1976. "A New Model for Predicting the Hydraulic Conductivity of Unsaturated Porous Media," Water Resources Research. Vol. 12, no. 3, 513-522.

Napier, B.A., R.A. Peloquin, D.L. Strenge, and J.V. Ramsdell. 1988a. GENII: The Hanford Environmental Radiation Dosimetry Software System: Volume 1, Conceptual Representation. PNL-6584, Vol. 1. Richland, WA: Pacific Northwest Laboratory, Hanford Environmental Dosimetry Upgrade Project. (Available from the NTIS as DE89004462/XAB.)

Napier, B.A., R.A. Peloquin, D.L. Strenge, and J.V. Ramsdell. 1988b. GENII: The Hanford Environmental Radiation Dosimetry Software System: Volume 2, Users'Manual. PNL-6584, Vol. 2. Richland, WA: Pacific Northwest Laboratory, Hanford Environmental Dosimetry. Upgrade Project. (Available from the NTIS as DE89004445/XAB.)

Napier, B.A., R.A. Peloquin, D.L. Strenge, and J.V. Ramsdell. 1988c. GENII (Generation II): The Hanford Environmental Radiation Dosimetry Software System: Volume 3, Code Maintenance Manual. PNL-6584, Vol. 3. Richland, WA: Pacific Northwest Laboratory, Hanford Environmental Dosimetry Upgrade Project. (Available from the NTIS as DE89000482/XAB.)

NAS/NRC (National Academy of Sciences/National Research Council). 1983. A Study of the Isolation System for Geologic Disposal of Radioactive Wastes. Board on Radioactive Waste Management, Waste Isolation Systems Panel. Washington, DC: National Academy Press. (Also available from the NTIS as PB84-148584.)

NAS/NRC (National Academy of Sciences/National Research Council). 1992a. Ground Water at Yucca Mountain: How High Can It Rise? Final Report of the Panel on Coupled Hydrologic/Tectonic/Hydrothermal Systems at Yucca Mountain, Board on Radioactive Waste Management. Washington, DC: National Academy Press. (Also issued as NRC-93003273, which is available from the NTIS as DE93003273/XAB.)

NAS/NRC (National Academy of Sciences/National Research Council). 1992b. Radioactive Waste Repository Licensing: Synopsis of a Symposium. Board on Radioactive Waste Management. Washington, DC: National Academy Press. (Available from the NTIS as PB94-119419/XAB.)

NAS/NRC (National Academy of Sciences/National Research Council). 1994. Management and Disposition of Excess Weapons Plutonium. Committee on International Security and Arms Control. Washington, DC: National Academy Press. (Also available from the NTIS as PB96-203922/XAB.)

NAS/NRC (National Academy of Sciences/National Research Council). 1995. Technical Bases for Yucca Mountain Standards. Committee on Technical Bases for Yucca Mountain Standards, Board on Radioactive Waste Management. Washington, DC: National Academy Press. (Also available from the NTIS as PB96-197801/XAB.)

NCRP (National Council on Radiation Protection and Measurements). 1986. Screening Techniques for Determining Compliance with Environmental Standards, Releases of Radionuclides to the Atmosphere. NCRP Commentary No. 3. Bethesda, MD: National Council on Radiation Protection and Measurements.

Neuman, S.P., and P.A. Witherspoon. 1969. Transient Flow of Ground Water to Wells in Multiple-Aquifer Systems. Institute of Transportation and Traffic Engineering Publication No. 69-1. Berkeley, CA: Department of Civil Engineering, University of California.

Ng, Y.C., C.S. Colsher, and S.E. Thompson. 1982. Soil-to-Plant Concentration Factors for Radiological Assessments. NUREG/CR-2975, UCID-19463. Livermore, CA: Lawrence Livermore National Laboratory; Washing- 
ton, DC: U.S. Nuclear Regulatory Commission, Office of Nuclear Reactor Regulation. (Available from the NTIS as NUREG/CR-2975.)

Nimick, F.B., and J.R. Connolly. 1991. Calculation of Heat Capacities for Tuffaceous Units from the Unsaturated Zone at Yucca Mountain, Nevada. SAND88-3050. Albuquerque, NM: Sandia National Laboratories. (Available from the NTIS as DE91012366/XAB.)

Nimick, F.B., S.J. Bauer, and J.R. Tillerson. 1984. "Recommended Matrix and Rock-Mass Bulk, Mechanical and Thermal Properties for Thermomechanical Stratigraphy of Yucca Mountain, Version I." Keystone Document 6310-85-1, SLTR84-3001. Albuquerque, NM: Sandia National Laboratories. (This internal Sandia report is available from the Yucca Mountain Site Characterization Office as NNA.19891129.0284.)

Nitsche, H., R.C. Gatti, E.M. Standifer, S.C. Lee, M. Muller, T. Prussin, and R.S. Deinhammer. 1993. "Measured Solubilities and Speciation of Np, Pu and Am in Typical Groundwater (J-13) from the Yucca Mountain Region." Milestone Report 3010, LA-12562-MS. Los Alamos, NM: Los Alamos National Laboratory. (Available from the NTIS as DE94001566/XAB.)

Nitsche, H., K. Roberts, T. Prussin, A. Müller, K. Becraft, D. Keeney, S.A. Carpenter, and R.C. Gatti. 1994. Measured Solubilities and Speciations from Oversaturation Experiments of Neptunium, Plutonium, and Americium in UE-25p \#1 Well Water from the Yucca Mountain Region: Milestone Report 3329 - WBS1.2.3.4.1.3.1. LA 12563MS. Los Alamos, NM: Los Alamos National Laboratory.

NRC (Nuclear Regulatory Commission). 1977. "Calculation of Annual Doses to Man from Routine Releases of Reactor Effluents for the Purpose of Evaluating Compliance with 10 CFR Part 50, Appendix I." Regulatory Guide 1.109, Rev. 1. Washington, DC: U.S. Nuclear Regulatory Commission, Office of Standards Development. (Available from the Yucca Mountain Site Characterization Office as NNA.19870806.0032.)

NRC (Nuclear Regulatory Commission). 1981. Estimates of Internal Dose Equivalent to 22 Target Organs for Radionuclides Occurring in Routine Releases from Fuel-Cycle Facilities. Vol. III. Eds. D. Dunning, G.G. Killough, S.R. Bernard, J.C. Pleasant, and P.J. Walsh. NUREG/CR-0150, Vol. 3; ORNL/NUREG/TM-190/V3. Oak Ridge, TN: Oak Ridge National Laboratory; Washington, DC: U.S. Nuclear Regulatory Commission.

NRC (Nuclear Regulatory Commission). 1993. "Disposal of High-Level Radioactive Wastes in Geologic Repositories," Code of Federal Regulations 10, Part 60. Washington, DC: Superintendent of Documents, U.S. Government Printing Office.

NWPA. 1983. "Nuclear Waste Policy Act of 1982." Public Law 97-425, 96 Stat. 2201, 42 U.S.C. 10101 et seq.

NWPA. 1997. "Nuclear Waste Policy Act of 1997." H.R. 1270, S. 104.

NWPAA. 1987. "Nuclear Waste Policy Amendments Act of 1987 as contained in the Omnibus Budget Reconciliation Act of 1987." Public Law 100-203, 101 Stat. 1330.

Oberlander, P.L. 1979. Development of a Quasi Three-Dimensional Groundwater Model for a Portion of the Nevada Test Site. NVO-1253-14. Reno, NV: Desert Research Institute. (Available from the NTIS as NVO-1253-14.)

O'Brien, D.G. 1997. "Hydraulic Conductivity Modifications for DSNF97 Model (Parameters: BLKKGAS and HYCNDSAM)." Memorandum from D.G. O'Brien to L.J. Dotson, 6/26/97. (On file in NSNFP Records as NSNFP \#011.)

Ogard, A.E., and J.F. Kerrisk. 1984. Groundwater Chemistry Along Flow Paths Between a Proposed Repository Site and the Accessible Environment. LA-10188-MS. Los Alamos, NM: Los Alamos National Laboratory. (Available from the NTIS as DE85005598/XAB.) 
Olson, R.H. 1983. "Zeolites," Industrial Minerals and Rocks (Nonmetallics Other Than Fuels). 5th ed. Editor-inChief: S.J. Lefond. New York, NY: American Institute of Mining, Metallurgical, and Petroleum Engineers, Inc. 1391-1398.

ORNL (Oak Ridge National Laboratory). 1996. "ORIGEN2.1 Isotope Generation and Depletion Code-Matrix Exponential Method." CCC-371. Oak Ridge, TN: Oak Ridge National Laboratory, Radiation Shielding Information Center, Computer Code Collection. (For information about this document, contact the Radiation Safety Information Computational Center, Oak Ridge, TN, 423-574-6176, http://www-rsicc.ornl.gov/codelist/ccc/ccc3/ 371.)

Ortiz, T.S., R. L. Williams, F.B. Nimick, B.C. Whittet, and D.L. South. 1985. A Three-Dimensional Model of Reference Thermal/Mechanical and Hydrological Stratigraphy at Yucca Mountain, Southern Nevada. SAND84-1076. Albuquerque, NM: Sandia National Laboratories.

Panday, S., P.S. Huyakorn, R. Therrien, and R.L. Nichols. 1993. "Improved Three-Dimensional Finite-Element Techniques for Field Simulation of Variably Saturated Flow and Transport," Journal of Contaminant Hydrology. Vol. 12, no. 1-2, 3-33.

Papelis, C., and J.O. Leckie. 1988. "Adsorption of Trace Elements from Coal-fired Power Plant Effluents on Iron Oxyhydroxide: Experimental and Modeling Studies." Technical Report No. 307. Stanford, CA: Stanford University, Department of Civil Engineering.

Parsons, A.M., N.E. Olague, and D.P. Gallegos. 1991. Conceptualization of a Hypothetical High-Level Nuclear Waste Repository Site in Unsaturated, Fractured Tuff. NUREG/CR-5495, SAND89-2965. Washington, DC: U.S. Nuclear Regulatory Commission, Office of Nuclear Regulatory Research, Division of Engineering.

Peterman, Z.E., and J.S. Stuckless. 1993. "Isotopic Evidence of Complex Ground-Water Flow at Yucca Mountain, Nevada, USA," High Level Radioactive Waste Management, Proceedings of the Fourth Annual International Conference, Las Vegas, NV, April 26-30, 1993. La Grange Park, IL: American Nuclear Society, Inc.; New York: American Society of Civil Engineers. Vol. 2, 1559-1566.

Peters, R.R., and E.A. Klavetter. 1988. "A Continuum Model for Water Movement in an Unsaturated Fractured Rock Mass," Water Resources Research. Vol. 24, no. 3, 416-430.

Pourbaix, M. 1966. Atlas of Electrochemical Equilibria in Aqueous Solutions. London, UK: Pergamon Press Ltd.

Pruess, K. 1991. TOUGH2: A General-Purpose Numerical Simulator for Multiphase Fluid and Heat Flow. LBL29400. Berkeley, CA: Lawrence Berkeley Laboratory. (Available from the NTIS as DE92000755/XAB.)

Pruess, K., J.S.Y. Wang, and Y.W. Tsang. 1990. "On Thermohydrologic Conditions Near High-Level Nuclear Wastes Emplaced in Partially Saturated Fractured Tuff. 2. Effective Continuum Approximation," Water Resources Research. Vol. 26, no. 6, 1249-1261.

Rai, D., R.G. Stirckert, and G.L. McVay. 1982. "Neptunium Concentrations in Solutions Contacting Actinide-Doped Glass," Nuclear Technology. Vol. 58, 69-76.

Rautman, C.A. 1995. Preliminary Geostatistical Modeling of Thermal Conductivity for a Cross Section of Yucca Mountain, Nevada. SAND94-2283. Albuquerque, NM: Sandia National Laboratories.

Rautman, C.A., and D.A. Engstrom. 1996. Geology of the USW SD-12 Drill Hole, Yucca Mountain, Nevada. SAND96-1368. Albuquerque, NM: Sandia National Laboratories.

Rautman, C.A., and A.L. Flint. 1992. "Deterministic Geologic Processes and Stochastic Modeling," High Level Radioactive Waste Management, Proceedings of the Third International Conference, Las Vegas, NV, April 12-16, 
1992. La Grange Park, IL: American Nuclear Society, Inc.; New York, NY: American Society of Civil Engineers. Vol. 2, 1617-1624.

Rautman, C.A., and S.A. McKenna. 1997. Three-Dimensional Hydrologic and Thermal Property Models of Yucca Mountain, Nevada. SAND97-1730. Albuquerque, NM: Sandia National Laboratories.

Rechard, R.P. 1989. Review and Discussion of Code Linkage and Data Flow in Nuclear Waste Compliance Assessments. SAND87-2833. Albuquerque, NM: Sandia National Laboratories.

Rechard, R.P., ed. 1992. User's Reference Manual for CAMCON: Compliance Assessment Methodology Controller, Version 3.0. SAND90-1983. Albuquerque, NM: Sandia National Laboratories.

Rechard, R.P., ed. 1993. Initial Performance Assessment of the Disposal of Spent Nuclear Fuel and High-Level Waste Stored at Idaho National Engineering Laboratory. Volume 1: Methodology and Results. SAND93-2330/ 1. Albuquerque, NM: Sandia National Laboratories.

Rechard, R.P. 1995. An Introduction to the Mechanics of Performance Assessment Using Examples of Calculations Done for the Waste Isolation Pilot Plant Between 1990 and 1992. SAND93-1378. Albuquerque, NM: Sandia National Laboratories.

Rechard, R.P., ed. 1995. Performance Assessment of the Direct Disposal in Unsaturated Tuff of Spent Nuclear Fuel and High-Level Waste Owned by U.S. Department of Energy. SAND94-2563/1/2/3. Albuquerque, NM: Sandia National Laboratories. Vols. 1-3.

Rechard, R.P. 1998. Milestones for Disposal of Radioactive Waste at the Waste Isolation Pilot Plant (WIPP) in the United States. SAND98-0072. Albuquerque, NM: Sandia National Laboratories.

Rechard, R.P., H.J. Iuzzolino, J.S. Rath, A.P. Gilkey, R.D. McCurley, and D.K. Rudeen. 1989. User's Manual for CAMCON: Compliance Assessment Methodology Controller. SAND88-1496. Albuquerque, NM: Sandia National Laboratories.

Rechard, R.P., H. Iuzzolino, and J.S. Sandha. 1990. Data Used in Preliminary Performance Assessment of the Waste Isolation Pilot Plant (1990). SAND89-2408. Albuquerque, NM: Sandia National Laboratories.

Rechard, R.P., K.M. Trauth, J.S. Rath, R.V. Guzowski, S.C. Hora, and M.S. Tierney. 1993. "The Use of Formal and Informal Expert Judgments When Interpreting Data for Performance Assessments," Scientific Basis for Nuclear Waste Management XVI, Materials Research Society Symposium Proceedings, Boston, MA, November 30December 4, 1992. Eds. C.G. Interrante and R.T. Pabalan. SAND92-1148C. Pittsburgh, PA: Materials Research Society. Vol. 294, 943-950.

Rice, W.A. 1984. Preliminary Two-Dimensional Regional Hydrologic Model of the Nevada Test Site and Vicinity. SAND83-7466. Albuquerque, NM: Sandia National Laboratories.

Richards, L.A. 1931. “Capillary Conduction of Liquids Through Porous Mediums," Physics. Vol. 1, 318-333.

Robinson, B.A., A.V. Wolfsberg, H.S. Viswanathan, C.W. Gable, G.A. Zyvoloski, and H.J. Turin. 1996. "Modeling of Flow, Radionuclide Migration, and Environmental Isotope Distribution at Yucca Mountain." Los Alamos National Laboratory Yucca Mountain Site Characterization Project Milestone 3672. Los Alamos, NM: Los Alamos National Laboratory.

Robison, J.H. 1984. Ground-Water Level Data and Preliminary Potentiometric-Surface Maps, Yucca Mountain and Vicinity, Nye County, Nevada. USGS Water-Resources Investigations Report 84-4197. Denver, CO: U.S. Geological Survey. (Available from the NTIS as DE85001771/XAB.) 
Romney, E.M., A. Wallace, R.K. Schulz, J. Kinnear, and R.A. Wood. 1981. "Plant Uptake of ${ }^{237} \mathrm{~Np},{ }^{239} \mathrm{Pu},{ }^{240} \mathrm{Pu}$, ${ }^{241} \mathrm{Am}$, and ${ }^{244} \mathrm{Cm}$ from Soils Representing Major Food Production Areas of the United States," Soil Science. Vol. 132, no. 1, 40-59.

Ross, B., S. Amter, and N. Lu. 1992. Numerical Studies of Rock-Gas Flow in Yucca Mountain. SAND91-7034. Albuquerque, NM: Sandia National Laboratories.

Rossi, C., and J.R. Nimmo. 1994. "Modeling of Soil Water Retention from Saturation to Oven Dryness," Water Resources Research. Vol. 30, no. 3, 701-708.

Rush, F.E. 1971. Regional Ground-Water Systems in the Nevada Test Site Area, Nye, Lincoln, and Clarke Counties, Nevada. Water Resources-Reconnaissance Series Report No. 54. Carson City, NV: Nevada Department of Conservation and Natural Resources. (Available from the Yucca Mountain Site Characterization Office as NNA.19870406.0383.)

Samama, J-C. 1986. Ore Fields and Continental Weathering. New York, NY: Van Nostrand Reinhold Co.

Sanchez, L.C. 1996. "Radionuclide Half-lives and Specific Activities Obtained From ORIGEN2 Data." Memorandum from L.C. Sanchez to M. Martell, 3/28/96. Albuquerque, NM: Sandia National Laboratories. (Copy on file in the Sandia WIPP Central Files (SWCF), Sandia National Laboratories, Albuquerque, NM as WPO\#37404.)

Sass, J.H., A.H. Lachenbruch, W.W. Dudley, Jr., S.S. Priest, and R.J. Munroe. 1988. Temperature, Thermal Conductivity, and Heat Flow Near Yucca Mountain, Nevada: Some Tectonic and Hydrologic Implications. USGS OpenFile Report 87-649. Denver, CO: U.S. Geological Survey. (Available from the NTIS as DE89002697/XAB.)

Sawyer, D.A., R.J. Fleck, M.A. Lanphere, R.G. Warren, D.E. Broxton, and M.R. Hudson. 1994. "Episodic Caldera Volcanism in the Miocene Southwestern Nevada Volcanic Field: Revised Stratigraphic Framework, ${ }^{40} \mathrm{Ar} /{ }^{39} \mathrm{Ar}$ Geochronology, and Implications for Magmatism and Extension," Geological Society of America Bulletin. Vol. 106, no. 10, 1304-1318.

Scheidegger, A.E. 1960. The Physics of Flow Through Porous Media. Rev. ed. Toronto, Ontario: University of Toronto Press.

Schenker, A.R., D.C. Guerin, T.H. Robey, C.A. Rautman, and R.W. Barnard. 1995. Stochastic Hydrogeologic Units and Hydrogeologic Properties Development for Total-System Performance Assessments. SAND94-0244. Albuquerque, NM: Sandia National Laboratories.

Schoff, S.L., and J.E. Moore, comps. 1964. Chemistry and Movement of Ground Water, Nevada Test Site. Trace-Elements Investigations Report TEI-838. Denver, CO: U.S. Geological Survey. (Available at the U.S. Geological Survey Library, 952 National Center, 12201 Sunrise Valley Drive, Reston, VA 20192; 703/648-4400, cic_lib@usgs.gov.)

Scipio, L.A. 1967. Principles of Continua with Applications. New York, NY: John Wiley \& Sons.

Scott, R.B., and J. Bonk. 1984. Preliminary Geologic Map of Yucca Mountain, Nye County, Nevada, with Geologic Sections. USGS-OFR-84-494. Denver, CO: U.S. Geological Survey. (Available from the NTIS as DE85002657/XAB.)

Scott, R.B., and J.G. Rosenbaum. 1986. "Evidence of Rotation About a Vertical Axis During Extension at Yucca Mountain, Southern Nevada," EOS Transactions, American Geophysical Union. Vol. 67, no. 16, 358.

Scott, R.B., G.D. Bath, V.J. Flanigan, D.B. Hoover, J.G. Rosenbaum and R.W. Spengler. 1984. Geological and Geophysical Evidence of Structures in Northwest-Trending Washes, Yucca Mountain, Southern Nevada, and Their 
References

Possible Significance to a Nuclear Waste Repository in the Unsaturated Zone. USGS Open File Report 84-567. Denver, CO: U.S. Geological Survey. (Available from the NTIS as DE85002656/XAB.)

Sheppard, M.I., D.I. Beals, D.H. Thibault, and P. O'Conner. 1984. Soil Nuclide Distribution Coefficients and Their Statistical Distributions. AECL-8364. Pinawa, Manitoba: Atomic Energy of Canada Limited, Whiteshell Nuclear Research Establishment. (Available from the NTIS as DE85900455.)

Shinta, A.A., and H. Kazemi. 1993. "Tracer Transport in Characterization of Dual-Porosity Reservoirs," Reservoir Engineering Proceedings, SPE Annual Technical Conference and Exhibition, Houston, TX, October 3-6, 1993. SPE 26636. Richardson, TX: Society of Petroleum Engineers. 285-299. (Available from the SPE, P.O. Box 833836, Richardson, TX 75083-3836, 800-456-6863, books@spelink.spe.org.)

Simpson, J.P., and R. Schenk. 1989. "Corrosion Induced Hydrogen Evolution on High Level Waste Overpack Materials in Synthetic Groundwaters and Chloride Solutions," Scientific Basis for Nuclear Waste Management XII, Materials Research Society Symposium Proceedings, Berlin, Germany, October 10-13, 1988. Eds. W. Lutze and R.C. Ewing. Pittsburgh, PA: Materials Research Society. Vol. 127, 389-396.

Sinnock, S. 1982. Geology of the Nevada Test Site and Nearby Areas, Southern Nevada. SAND82-2207. Albuquerque, NM: Sandia National Laboratories.

Sinton, P.O. 1987. "Three-Dimensional, Steady-State, Finite Difference Model of the Ground-Water Flow System in Death Valley Ground-Water Basin, Nevada-California." Master's Thesis. Golden, CO: Colorado School of Mines, Geology and Geological Engineering Department. 145 p. (Available from Arthur Lakes Library, Colorado School of Mines, P.O. Box 4029, 1400 Illinois Street, Golden, CO 80401-0029, 303/273-3911, http:// www.mines.edu/library.)

SKB (Swedish Nuclear Fuel and Waste Management Co.) 1992. Final Disposal of Spent Nuclear Fuel. Importance of the Bedrock for Safety. SKB Technical Report 92-20. Stockholm, Sweden: Swedish Nuclear Fuel and Waste Management Co. (Copy on file in the SWCF as WPO\#47951.)

Smyth, J.R., and F.A. Caporuscio. 1981. Review of the Thermal Stability and Cation Exchange Properties of the Zeolite Minerals Clinoptilolite, Mordenite, and Analcime: Applications to Radioactive Waste Isolation in Silicic Tuff. LA-8841-MS. Los Alamos, NM: Los Alamos National Laboratory. (Available from the NTIS as DE81026686.)

Snow, D.T. 1968. "Rock Fracture Spacings, Openings, and Porosities," Journal of the Soil Mechanics and Foundations Division, Proceedings of the American Society of Civil Engineers. Vol. 94, no. SM1, 73-91.

Spaulding, W.G. 1985. Vegetation and Climates of the Last 45,000 Years in the Vicinity of the Nevada Test Site, South-Central Nevada. USGS Professional Paper 1329. Denver, CO: U.S. Geological Survey. (Available from the NTIS as DE86008699.)

Steward, S.A., and W.J. Gray. 1994. "Comparison of Uranium Dissolution Rates from Spent Fuel and Uranium Dioxide," High Level Radioactive Waste Management, Proceedings of the Fifth Annual International Conference, Las Vegas, NV, May 22-26, 1994. La Grange Park, IL: American Nuclear Society, Inc.; New York, NY: American Society of Civil Engineers. Vol. 4, 2602-2608.

Stewart, J.H. 1985. "East-Trending Dextral Faults in the Western Great Basin: An Explanation for Anomalous Trends of Pre-Cenozoic Strata and Cenozoic Faults," Tectonics. Vol. 4, no. 6, 547-564.

Stockman, C.T. 1993. "11. Gas Generation and Source Term Submodels," Initial Performance Assessment of the Disposal of Spent Nuclear Fuel and High-Level Waste Stored at Idaho National Engineering Laboratory. Volume 1: Methodology and Results. Ed. R.P. Rechard. SAND93-2330/1. Albuquerque, NM: Sandia National Laboratories. 11-1 through 11-49. 
Stockman, C.T., M.A. Martell, and J.S. Rath. 1995. "11. Parcel Corrosion and Source Term Models," Performance Assessment of the Direct Disposal in Unsaturated Tuff of Spent Nuclear Fuel and High-Level Waste Owned by U.S. Department of Energy. Volume 2: Methodology and Results. Ed. R.P. Rechard. SAND94-2563/2. Albuquerque, NM: Sandia National Laboratories. 11-1 through 11-64.

Sweetkind, D.S., and S.C. Williams-Stroud. 1996. "Characteristics of Fractures at Yucca Mountain, Nevada: Synthesis Report." Denver CO: U.S. Geological Survey for the Yucca Mountain Site Characterization Project, Las Vegas, NV. (Available from the Yucca Mountain Site Characterization Office as MOL.19961213.0181.)

Sweetkind, D.S., D.L. Barr, D.K. Polacsek, and L.O. Anna. 1997. "Integrated Fracture Data in Support of Process Models, Yucca Mountain, Nevada." USGS-YMP Branch 1997 Milestone Report SPG3M3. Administrative Report. Denver, CO: U.S. Geological Survey for the Yucca Mountain Site Characterization Project, Las Vegas, NV. (Available from the Yucca Mountain Site Characterization Office as MOL.19971017.0730.)

Swift, P.N. 1993. "Long-Term Climate Variability at the Waste Isolation Pilot Plant, Southeastern New Mexico, USA," Environmental Management. Vol. 17, no. 1, 83-97.

Szymanski, J.S. 1989. "Conceptual Considerations of the Yucca Mountain Groundwater System with Special Emphasis on the Adequacy of This System to Accommodate a High-Level Nuclear Waste Repository." Las Vegas, NV: U.S. Department of Energy, Nevada Operations Office, Yucca Mountain Project Office. (Available from the Yucca Mountain Site Characterization Office as NNA.19890831.0152.)

Tang, D.H., E.O. Frind, and E.A. Sudicky. 1981. "Contaminant Transport in Fractured Porous Media: Analytical Solution for a Single Fracture," Water Resources Research. Vol. 17, no. 3, 555-564.

Telander, M.R., and R.E. Westerman. 1993. Hydrogen Generation by Metal Corrosion in Simulated Waste Isolation Pilot Plant Environments: Progress Report for the Period November 1989 through December 1992. SAND927347. Albuquerque, NM: Sandia National Laboratories.

Telander, M.R., and R.E. Westerman. 1997. Hydrogen Generation by Metal Corrosion in Simulated Waste Isolation Pilot Plant Environments. SAND96-2538. Albuquerque, NM: Sandia National Laboratories.

Till, J.E., and H.R. Meyer, eds. 1983. Radiological Assessment: A Textbook on Environmental Dose Analysis. NUREG/CR-3332; ORNL-5968. Washington, DC: U.S. Nuclear Regulatory Commission; Oak Ridge, TN: Oak Ridge National Laboratory. (Available from the NTIS as DE84004807.)

Trauth, K.M., S.C. Hora, and R.V. Guzowski. 1993. Expert Judgment on Markers to Deter Inadvertent Human Intrusion into the Waste Isolation Pilot Plant. SAND92-1382. Albuquerque, NM: Sandia National Laboratories.

Triay, I.R., C.R. Cotter, S.M. Kraus, M.H. Huddleston, S.J. Chipera, and D.L. Bish. 1996a. Radionuclide Sorption in Yucca Mountain Tuffs with J-13 Well Water: Neptunium, Uranium, and Plutonium, Los Alamos National Laboratory Yucca Mountain Site Characterization Project Milestone 3338. LA-12956-MS. Los Alamos, NM: Los Alamos National Laboratory.

Triay, I.R., A.C. Furlano, S.C. Weaver, S.J. Chipera, and D.L. Bish. 1996b. Comparison of Neptunium Sorption Results Using Batch and Column Techniques, Los Alamos National Laboratory Yucca Mountain Site Characterization Project Milestone 3041. LA-12958-MS. Los Alamos, NM: Los Alamos National Laboratory.

Tsang, C.F., and D.C. Mangold, eds. 1984. Panel Report on Coupled Thermo-Mechanical-Hydro-Chemical Processes Associated with a Nuclear Waste Repository. LBL-18250. Berkeley, CA: Lawrence Berkeley Laboratory. (Available from the NTIS as DE85004340/XAB.) 
Udell, K.S., and J.S. Fitch. 1985. "Heat and Mass Transfer in Capillary Porous Media Considering Evaporation, Condensation and Non-condensible Gas Effects," Heat Transfer in Porous Media and Particulate Flows, Presented at the 23rd National Heat Transfer Conference, Denver, CO, August 4-7, 1985. Eds. L.S. Yao, L.C. Chow, M.M. Chen, M. Kaviany, C.E. Hickox, P. Cheng, P.G. Simpkins, and L.R. Davis. Heat Transfer Division (HTD) Vol. 46. New York, NY: American Society of Mechanical Engineers. 103-110.

Updegraff, D. 1997. "New Fracture Hydraulic Conductivities for Fault Zones." Memorandum from D. Updegraff to L. Dotson, 11/3/97. (On file in NSNFP Records as NSNFP \#036.)

van Genuchten, M. Th. 1980. "A Closed-Form Equation for Predicting the Hydraulic Conductivity of Unsaturated Soils," Soil Science Society of America Journal. Vol. 44, no. 5, 892-898.

van Genuchten, M.Th., G.F. Pinder, and W.P. Saukin. 1977. "Modeling of Leachate and Soil Interactions in an Aquifer," Management of Gas and Leachate in Landfills, Proceedings of the Third Annual Municipal Solid Waste Research Symposium, St. Louis, MO, March 14-16, 1977. Ed. S.K. Banerji. EPA-600/9-77-026. Cincinnati, OH: Municipal Environmental Research Laboratory, Office of Research and Development, U.S. Environmental Protection Agency. 95-103. (EPA-600/9-77-026 is available from the NTIS as PB-272 595/0.)

Van Konynenburg, R.A., comp. 1996. "Comments on the Draft Paper 'Underground Supercriticality from Plutonium and Other Fissile Material,' written by C.D. Bowman and F. Venneri (LANL)," Science and Global Security. Vol. 5, no. 3, 303-322.

Voilleque, P.G., G.J. Duggan, P.R. Leonard, and L.J. Coe. 1985. Environmental Radiation Doses from Difficult-toMeasure Nuclides. EPRI Report NP-3840. Rockville, MD: Science Applications International Corporation, Nuclear Environmental Services Division; Palo Alto, CA: Electric Power Research Institute. 124 pp. (Available from EPRI, P.O. Box 10412, Palo Alto, CA 94303, 415/855-2411.)

Waddell, R.K. 1982. Two-Dimensional, Steady-State Model of Ground-Water Flow, Nevada Test Site and Vicinity, Nevada-California. Water-Resources Investigations Report 82-4085, DOE/ET/44802-T5. Denver, CO: U.S. Geological Survey for U.S. Department of Energy, Washington, DC. (DOE/ET/44802-T5 is available from the NTIS as DE83009116.)

Waddell, R.K., J.H. Robison, and R.K. Blankennagel. 1984. Hydrology of Yucca Mountain and Vicinity, NevadaCalifornia: Investigative Results Through Mid-1983. Water-Resources Investigations Report 84-4267. Denver, CO: U.S. Geological Survey. (Available from the NTIS as DE85005673/XAB.)

Weast, R.C., ed. 1985. Handbook of Chemistry and Physics. 66th ed. Boca Raton, FL: CRC Press Inc.

Weeks, E.P. 1987. "Effect of Topography on Gas Flow in Unsaturated Fractured Rock, Concepts and Observations," Flow and Transport Through Unsaturated Fractured Rock. Eds. D.D. Evans and T.J. Nicholson. Geophysical Monograph 42. Washington, DC: American Geophysical Union. 165-170.

Wescott, R.G., M.P. Lee, N.A. Eisenberg, T.J. McCartin, and R.G. Baca. 1995. NRC Iterative Performance Assessment Phase 2. Development of Capabilities for Review of a Performance Assessment for a High-Level Waste Repository. NUREG-1464. San Antonio, TX: Southwest Research Institute, Center for Nuclear Waste Regulatory Analyses for Nuclear Regulatory Commission, Washington, DC. (Available from the NTIS as NUREG1464/XAB.)

White, A.F., and M.L. Peterson. 1990. "Chapter 35 The Role of Reactive-Surface-Area Characterization in Geochemical Kinetic Models," Chemical Modeling of Aqueous Systems II, American Chemical Society 196th National Meeting, Los Angeles, CA, September 25-30, 1990. Eds. D.C. Melchior and R.L. Bassett. ACS Symposium Series Vol. 416. Washington, DC: American Chemical Society. 461-475.

Wilkinson, W.D. 1962. Uranium Metallurgy. Volume II: Uranium Corrosion and Alloys. New York, NY: Interscience Publishers. 
Wilson, C.N. 1990a. Results from NNWSI Series 2 Bare Fuel Dissolution Tests. PNL-7169. Richland, WA: Pacific Northwest Laboratory.

Wilson, C.N. 1990b. Results from NNWSI Series 3 Spent Fuel Dissolution Tests. PNL-7170. Richland, WA: Pacific Northwest Laboratory.

Wilson, C.N., and C.J. Bruton. 1990. "Studies on Spent Fuel Dissolution Behavior under Yucca Mountain Repository Conditions," Nuclear Waste Management III. Ed. G.B. Mellinger. Westerville, OH: American Ceramic Society. Ceramic Transactions, Vol. 9, 423-441

Wilson, M.L. 1993. "Sensitivity Analyses for Total-System Performance Assessment," High Level Radioactive Waste Management, Proceedings of the Fourth Annual International Conference, Las Vegas, NV, April 26-30, 1993. La Grange Park, IL: American Nuclear Society, Inc.; New York, NY: American Society of Civil Engineers. Vol. 1, 14-21.

Wilson, M.L., J.H. Gauthier, R.W. Barnard, G.E. Barr, H.A. Dockery, E. Dunn, R.R. Eaton, D.C. Guerin, N. Lu, M.J. Martinez, R. Nilson, C.A. Rautman, T.H. Robey, B. Ross, E.E. Ryder, A.R. Schenker, S.A. Shannon, L.H. Skinner, W.G. Halsey, J.D. Gansemer, L.C. Lewis, A.D. Lamont, I.R. Triay, A. Meijer, and D.E. Morris. 1994. TotalSystem Performance Assessment for Yucca Mountain: SNL Second Iteration (TSPA-1993). SAND93-2675. Albuquerque, NM: Sandia National Laboratories. Vols. 1-3.

Winograd, I.J., and W. Thordarson. 1975. Hydrogeologic and Hydrochemical Framework, South-Central Great Basin, Nevada-California, with Special Reference to the Nevada Test Site. Professional Paper 712-C. Prepared for the U.S. Atomic Energy Commission. Washington, DC: U.S. Government Printing Office. (Available at the U.S. Geological Survey Library, 952 National Center, 12201 Sunrise Valley Drive, Reston, VA 20192, 703/6484400, cic_lib@usgs.gov.)

Winograd, I.J., B.J. Szabo, T.B. Coplen, and A.C. Riggs. 1988. “A 250,000-Year Climatic Record from Great Basin Vein Calcite: Implications for Milankovitch Theory," Science. Vol. 242, no. 4883, 1275-1280.

WIPP Performance Assessment Department. 1992. Preliminary Comparison with 40 CFR Part 191, Subpart B for the Waste Isolation Pilot Plant, December 1991. Volume 2: Probability and Consequence Modeling. SAND910893/2. Albuquerque, NM: Sandia National Laboratories.

WIPP Performance Assessment Department. 1997. "WIPP PA User's Manual for BRAGFLO, Version 4.10." May 12, 1997. Albuquerque, NM: Sandia National Laboratories. (Copy on file in the SWCF as WPO\#45238.)

Wolery, T.J. 1992a. EQ3/6, A Software Package for Geochemical Modeling of Aqueous Systems: Package Overview and Installation Guide (Version 7.0). UCRL-MA-110662-Pt. 1. Livermore, CA: Lawrence Livermore National Laboratory; (Available from the NTIS as DE93003359/XAB.)

Wolery, T.J. 1992b. EQ3NR, A Computer Program for Geochemical Aqueous Speciation - Solubility Calculations: Theoretical Manual, User's Guide, and Related Documentation (Version 7.0). UCRL-MA-110662-Pt. 3. Livermore, CA: Lawrence Livermore National Laboratory. (Available from the NTIS as DE93005827/XAB.)

Wolery, T.J., and S.A. Daveler. 1992. EQ6, A Computer Program for Reaction Path Modeling of Aqueous Geochemical Systems: Theoretical Manual, User's Guide, and Related Documentation (Version 7.0). UCRL-MA110662-Pt 4. Livermore, CA: Lawrence Livermore National Laboratory. (Available from the NTIS as DE93007118/XAB.)

Younker, J.L., W.B. Andrews, G.A. Fasano, C.C. Herrington, S.R. Mattson, R.C. Murray, L.B. Ballou, M.A. Revelli, A.R. Ducharme, L.E. Shepard, W.W. Dudley, D.T. Hoxie, R.J. Herbst, E.A. Patera, B.R. Judd, J.A. Docka, and L.D. Rickertsen. 1992. "Report of Early Site Suitability Evaluation of the Potential Repository Site at Yucca 
Mountain, Nevada." SAIC-91/8000. Las Vegas, NV: Technical and Management Support Services, Science Applications International Corporation. (Available from the NTIS as DE92017680/XAB.)

YMP Ref. Info. Base. 1993. "The Yucca Mountain Site Characterization Project - Reference Information Base, Version 4, Revision 7." YMP/CC-002. Las Vegas, NV: U.S. Department of Energy (NNA.930517.0092).

Zelinski, W.P., and R.W. Clayton. 1996. “A 3D Geologic Framework and Integrated Site Model of Yucca Mountain: Version ISM1.0." B00000000-01717-5700-00002, Revision 1. Las Vegas, NV: Civilian Radioactive Waste Management System M\&O Contractor. (Available from the Yucca Mountain Site Characterization Office as MOL.19970113.0085.) 


\section{Appendix A: \\ DOE Spent Nuclear Fuel Information}




\section{Appendix A}

\section{DOE Spent Nuclear Fuel Information}

\subsection{Introduction}

Allocation of repository space to DOE spent nuclear fuel (SNF) and high-level waste (HLW) glass has been identified as $10 \%$ of the 70,000 MTHM total allocated to high-level nuclear waste disposal in the repository under the Nuclear Waste Policy Act (1982) and its Amendment (1984). Within the 7,000 MTHM allocation, 1/3 of that inventory (or 2333 MTHM) was to be dedicated to DOE-owned spent nuclear fuel. The balance of the inventory (4667 MTHM equivalent) remains for defense HLW placement within the repository [Reference 1].

The existing DOE SNF inventories include approximately 2,700 MTHM of fuels considered suitable for repository disposal. A small quantity of DOE SNF will be excluded from direct disposal because they: (1) will be processing due to immediate vulnerabilities, (2) will be treated due to fuel characteristics, or (3) too few details were known to allow for assignment to a characteristic group.

Packaging fuels for criticality safety controls remains a significant issue not only due to the range of enrichments encountered with the various fuel types, but also because of the fissile material longevity beyond the design life of the repository. For purposes of packaging concepts evaluated in this performance assessment, fissile load limits. were applied to package configurations that included a co-disposal concept by mixing HLW and SNF canisters. To utilize repository standard waste package design and not generate excessive void volumes while complying with fissile load limits, a variety of SNF and HLW canister packaging configurations was used. These configurations necessarily resulted in the inclusion of much more HILW than is currently allowed under the NWPA criteria. Waste packaging for this performance assessment will examine the combination of $\sim 2,700$ MTHM SNF (367 MTHM in excess of that identified for repository disposal) and 7,977 MTHM [equivalent] HLW (3310 MTHM in excess) to promote efficient use of waste package volumes.

\subsection{Defense High-Level Waste (DHLW)/Spent Nuclear Fuel (SNF) Packages}

\subsection{DHLW Packages}

DHLW will consist of boro-silicate glasses produced at the various facilities (West Valley, Savannah River, Hanford, and the INEEL). These facilities in the past had fuel reprocessing capabilities that provided separation of fission products from the actinides. Generally, DHLW will have much lower transuranic loadings than that associated with a spent

\begin{tabular}{|l|l|l|l|}
\hline $\begin{array}{l}\text { Appendix A } \\
\text { DOE SNF Information }\end{array}$ & $\begin{array}{l}\text { Sheet } \\
1 \text { of } 75\end{array}$ & $\begin{array}{l}\text { Date: } \\
\text { January 23, 1998 }\end{array}$ & $\begin{array}{l}\text { Rev. } \\
1\end{array}$ \\
\hline
\end{tabular}


fuel package. The glass has properties prescribed by a glass standard relative to parameters deemed acceptable for disposal in a repository. All HLW glass generated across the DOEcomplex will have similar compositions.

There are four identified sources of $\mathrm{HLW}$ glasses, those being West Valley (NY), Hanford (WA), Savannah River (GA), and the Idaho National Engineering and Environmental Laboratory (ID).

West Valley waste glass originates from the only commercial fuel reprocessing facility operated in the US. The waste glass canisters from WVDP are $61 \mathrm{~cm} \mathrm{(24} \mathrm{in)} \mathrm{in} \mathrm{diameter} \mathrm{and}$ $299 \mathrm{~cm}(10 \mathrm{ft})$ long. They are constructed out of 304L SS and are approximately $0.95 \mathrm{~cm}$ $(0.375 \mathrm{in})$ thick. The projected numbers plan for 300 canisters [Reference 2]. The wastes in these packages represent fission product inventories from spent fuels irradiated in the 1950s.

Savannah River HLW glass represent the accumulated reprocessing wastes from the operation of the various production reactors at that site. These waste canisters also employ a standard $61 \mathrm{~cm}$ (24 in) diameter and a $300 \mathrm{~cm}$ (10 ft) length. Projections for canister count uses 5700 canisters [Reference 2]. These canisters also use 304L SS construction materials with $0.95 \mathrm{~cm}(0.375 \mathrm{in})$ wall thickness. Pu loadings of up to $50 \mathrm{~kg}\left(110^{\circ} \mathrm{lb}\right) \mathrm{Pu} / \mathrm{HLW}$ "log" has been proposed as a disposal scheme for $\mathrm{Pu}$ weapons-grade material [Reference 3], but modified glass inventory will not be incorporated as part of this PA.

Existing tank farm wastes at Hanford has yet to be solidified into a HIW glass form. Plans are to use existing boro-silicate glass formulations with similar properties to those used by West Valley and Savannah River. Hanford has requested a variance that would allow the use of a $61 \mathrm{~cm}$ (24 in) diameter canister but with an overall length of $\sim 450 \mathrm{~cm}(15 \mathrm{ft})$ [Reference 4]. Materials of construction and canister thicknesses are expected to parallel those used in the Savannah River glass canisters. The total number of canisters is expected to be 11,900 canisters [Reference 2].

INEEL HLW wastes have over the years been solidified as a non-corrosive, dry granular solid. However, such a form would not meet existing transportation and repository packaging requirements, so further processing of the dry solids will be required to convert this material into an acceptable HLW glass form. To maintain consistency with other sites, the HLW canister will hold to the $61 \mathrm{~cm}(24 \mathrm{in})$ diameter, $0.95 \mathrm{~cm}(0.375 \mathrm{in})$ wall thickness, and use a $450 \mathrm{~cm}$ (15 ft) length. There will be an estimated total of 550 canisters [Reference 2].

For purposes of estimating the MTHM equivalent contribution provided by each glass canister, this study used a value of 0.5 MTHM for each $300 \mathrm{~cm}$ (10 ft) canister and 0.75 MTHM for each $450 \mathrm{~cm}$ (15 ft) canister. A single set of radionuclide inventory data will be used (on a per package basis) to represent the total HLW inventory even though the actual wastes originate from different producers. The baseline inventory will represent a canister 61

\begin{tabular}{|l|l|l|l|}
\hline $\begin{array}{l}\text { Appendix A } \\
\text { DOE SNF Information }\end{array}$ & $\begin{array}{l}\text { Sheet } \\
2 \text { of } 75\end{array}$ & $\begin{array}{l}\text { Date: } \\
\text { January 23, 1998 }\end{array}$ & $\begin{array}{l}\text { Rev. } \\
1\end{array}$ \\
\hline
\end{tabular}


$\mathrm{cm}$ (24 in) in diameter and $300 \mathrm{~cm}$ (10 ft) long; a $50 \%$ increase in the radionuclide content will be used for the longer canisters.

\subsection{SNF Packages}

In the intervening years since the cessation of fuel reprocessing, DOE SNF has been grouped in various ways depending on what analysis or disposal configuration was being studied. Predominant among those groupings was fuels with common cladding characteristics (zirconium, aluminum, stainless steel), similar fuel matrix or "meat" composition (oxide, carbide, metal), and ranges of enrichments.

Fuel grouping by cladding makes sense if cladding credits prove beneficial (or detrimental) to overall fuel performance as measured by radionuclide retardation. Grouping by fuel matrix is suggested where common chemical characteristics may impact leachability/solubility issues when no credit is available for cladding retardation. Where packaging concerns must deal with the issue of criticality safety, both in operational facilities and in the repository post-closure environment, parameters such as fuel configuration, fissile loadings, container material, and/or presence of neutron absorbers must be considered.

For purposes of this performance assessment, up to 15 "categories" of fuels were to be evaluated. The 15 fuel categories are indicated on Table A.1. Evaluation of those fuels was to be completed in conjunction with, high-level waste glasses incorporated in the repository waste packages using a co-disposal concept. The categories of fuels selected for the PA modeling were selected based on the chemical composition of the fuel matrix. This basis of selection evolved from the fact that cladding is not currently "credited" for fuels identified for repository disposal. Therefore, the chemical constituency of the fuel matrix will determine the rate of release of the various radionuclides based on leachability and solubility.

The nature of the individual fuel packaging approach resulted in a combination of SNF canisters with approximate diameters of $25.4 \mathrm{~cm}, 43.2 \mathrm{~cm}$, and $61 \mathrm{~cm}$ (10 in, $17 \mathrm{in}$, and $24 \mathrm{in})$ in both $300 \mathrm{~cm}$ and $450 \mathrm{~cm}$ (10 ft and $15 \mathrm{ft}$ ) lengths. This variety of fuel canister sizes, when placed with the HLW canisters resulted in a variety of repository waste package combinations within each fuel category. Generally, fuel types (as determined by the originating reactor) within a fuel category were not mixed in common SNF canisters. This approach may create a slight increase in the SNF canister count, and hence a corresponding increase in the HLW canisters needed to meet co-disposal requirements. However, such an approach does not affect the total MTHM fuel mass.

Determination of -any fissile load limits in each SNF canister has yet to be approved for the waste repository. For purposes of this study, fissile load limits were imposed on SNF canister loadings to determine how the package count might be affected. These load limits

\begin{tabular}{|l|l|l|l|}
\hline $\begin{array}{l}\text { Appendix A } \\
\text { DOE SNF Information }\end{array}$ & $\begin{array}{l}\text { Sheet } \\
3 \text { of } 75\end{array}$ & $\begin{array}{l}\text { Date: } \\
\text { January 23, 1998 }\end{array}$ & $\begin{array}{l}\text { Rev. } \\
1\end{array}$ \\
\hline
\end{tabular}




\begin{tabular}{|c|c|c|}
\hline \multicolumn{3}{|c|}{ Table A-1: DOE Spent Nuclear Fuel Categories } \\
\hline Fuel Category & Fuel Matrix & Typical Fuel in the category \\
\hline 1 & U-Metal & $\mathrm{N}$-Reactor Fuel \\
\hline 2 & $\mathrm{U}-\mathrm{Zr}$ & Heavy Water Component Test Reactor Fuel \\
\hline 3 & U-Mo & FERMI (Enrico Fermi Reactor) Fuel \\
\hline 4 & U-Oxide Intact & $\begin{array}{l}\text { Shippingport PWR Fuel } \\
\text { Commercial PWR Fuel }\end{array}$ \\
\hline 5 & U-Oxide Failed/Declad & Three Mile Island (TMI) Fuel \\
\hline 6 & $\begin{array}{c}\text { U-Al } \\
\text { Or U-Alx }\end{array}$ & Advanced Test Reactor (ATR) Fuel \\
\hline 7 & U-Si & Foreign Research Reactor (FFR) Fuel \\
\hline 8 & $\begin{array}{l}\text { U/Th Carbide } \\
\text { Hi-Integrity }\end{array}$ & Fort Saint Vrain (FSV) Fuel \\
\hline 9 & $\begin{array}{l}\text { U/Th Carbide } \\
\text { Low-Integrity }\end{array}$ & Peach Bottom Fuel \\
\hline 10 & $\begin{array}{l}\text { U or U/Pu Carbide } \\
\text { Non-Graphite }\end{array}$ & Fast Flux Test Facility (FFTF) Carbide Fuel \\
\hline 11 & MOX & Fast Flux Test Facility (FFTF) Oxide Fuel \\
\hline 12 & U/Th Oxide & Shippingport LWBR Fuel \\
\hline 13 & $\mathrm{U}-\mathrm{Zr}-\mathrm{Hx}$ & $\begin{array}{l}\text { Training Research Isotopes- General Atomic } \\
\text { (TRIGA) Fuel }\end{array}$ \\
\hline 14 & Commercial PWR & Commercial (PWR \& BWR) Fuel \\
\hline 15 & Commercial BWR & Commercial (PWR \& BWR) Fuel \\
\hline
\end{tabular}


were adopted from a study of aluminum fuel packaging and degradation scenarios [Reference 5]. These artificial limits are not intended to be limiting values for any type or category of fuels proposed for repository disposal. The study proposed the following package loading for the DOE-owned aluminum SNF based on the fuel enrichment level:

HEU ( $(20 \%)$ should not exceed $14.4 \mathrm{~kg} \mathrm{U}-235$ equivalent LEU $(>2 \%<20 \%)$ should not exceed $43 \mathrm{~kg} \mathrm{U}-235$ equivalent VLEU ( $\leq 2 \%$ ) should not exceed $200 \mathrm{~kg} \mathrm{U}-235$ equivalent

Using this proposed fuel loading, the following package loading was developed and generally followed for this evaluation. However, exceptions to these loading recommendation do exist for a small number of packages (i.e., some package may exceed the proposed loading indicated below. As an example, a single element of the Shippingport LWBR in a disposal package will exceed the proposed limit unless the fuel is cut up in small sections for disposal. For the purpose of this evaluation, fuels like the Shippingport LWBR will be disposed intact (not cut up).).

HEU $(\geq 20 \%)$ not to exceed $14.4 \mathrm{~kg} \mathrm{U}-235$ equivalent MEU (>5\%<20\%) not exceed $43 \mathrm{~kg} \mathrm{U}-235$ equivalent LEU ( $\leq 5 \%)$ not exceed $200 \mathrm{~kg} \mathrm{U-235} \mathrm{equivalent}$

The categories or fuel groups for this PA are comprised of one or more fuel types. These types may vary in terms of physical geometry, total mass, enrichment, or burn-up. While other groupings may have segregated the fuels by cladding, the categorization of fuels for this PA resulted in analysis of fuels types by fuel matrix composition. No emphasis was placed on any further segregation by fuel cladding or enrichments within a given category. However, generally fuel types, (e.g. from two different reactors) within a given category were not "mixed" in the same SNF canister unless physical geometry, cladding, and beginning-oflife (BOL) enrichments were the same. There were no attempts to load a variety of fuels in a canister to maximize fissile loading up to a prescribed limit or to minimize void volume.

Diameter differences in the SNF canisters are not dictated by anything other than the cross-section dimensions of the fuel to be loaded, and only secondarily by the fissile loads. Canister lengths will be determined by fuel lengths, with the majority of fuels destined for loading within $300 \mathrm{~cm}$ (10 ft) long canisters. Fuel canisters $450 \mathrm{~cm}$ (15 ft) long will be reserved for those fuels requiring the length to avoid disassembly. Selectively, the longer SNF canisters could also be used to stack shorter fuels. Co-disposal options for $450 \mathrm{~cm}$ (15 ft) SNF canisters should prove substantial since OCRWM approved [Reference 4] the use of longer canisters in the HLW production facility intended for Hanford's liquid waste treatment facility.

Canister design will need to accommodate containment of the fuel load with a maximum

Appendix A

DOE SNF Information

\section{Sheet}

5 of 75
Date: January 23, 1998
Rev. 1 
pressure of 22 psia [Reference 6].

Fissile load limits on any canister are not completely inviolate. In cases where the fissile quantity in a fuel or group of fuels within a single canister approaches the limit for that canister based on BOL enrichment, the allowable fissile load may be exceeded by $20 \%$ of the established fissile limit. This variance allowance will have to be proved in a criticality safety evaluation for that specific fuel fissile loading.

\subsection{Repository Orerpacks}

Large disposal package(s) (LDPs) used for DOE EM SNF/HLW disposal will consist of one of five types of available outer containers. In all cases, the LDP will consist of an outer carbon steel layer that is $10 \mathrm{~cm}$ (3.94 in) thick, and an inner Inconel 625 layer that is $2 \mathrm{~cm}$ ( 0.78 in) thick. LDP diameters will vary depending on the configuration selected for the individual fuel canisters based on the respective fuel lengths, cross-sectional areas, and fissile content.

Design parameters may vary somewhat as repository LDP design evolves. However, the following design information was used for the packages assembled for this PA:

10-ft standard (4 HLW Canisters w/10 in SNF canister Configuration) empty package weight $\quad=16,730 \mathrm{~kg}(\sim 36,850 \mathrm{lb})$ external length $\quad=3,871 \mathrm{~mm}(\sim 152 \mathrm{in})$ internal length $\quad=3,048 \mathrm{~mm}(\sim 120 \mathrm{in})$ internal diameter $\quad=1,626 \mathrm{~mm}(\sim 64 \mathrm{in})$ external diameter $\quad=1,725 \mathrm{~mm}(\sim 68 \mathrm{in})$

15-ft standard (4 HLW Canisters w/10 in SNF canister Configuration) empty package weight $\quad=23,450 \mathrm{~kg}(\sim 51,652 \mathrm{lb})$ external length $\quad=5,247 \mathrm{~mm}(\sim 214 \mathrm{in})$ internal length $\quad=3,810 \mathrm{~mm}(\sim 150 \mathrm{in})$ internal diameter $\quad=1,626 \mathrm{~mm}(\sim 64 \mathrm{in})$ external diameter $\quad=1,725 \mathrm{~mm}(\sim 68 \mathrm{in})$

10-ft "super" (5 HLW Canisters w/17 in SNF Canister Configuration) empty package weight $\quad=22,515 \mathrm{~kg}(\sim 49,582 \mathrm{lb})$ external length $\quad=3,871 \mathrm{~mm}(\sim 152 \mathrm{in})$ internal length $\quad=3,048 \mathrm{~mm}(\sim 120 \mathrm{in})$ internal diameter $\quad=1,790 \mathrm{~mm}(\sim 70 \mathrm{in})$ 
external diameter

$=2,030 \mathrm{~mm}(\sim 80 \mathrm{in})$

15-ft "super" (5 HLW Canisters w/17 in SNF Canister Configuration)

empty package weight $\quad=31,232 \mathrm{~kg}(\sim 68,793 \mathrm{lb})$

external length

$=5,247 \mathrm{~mm}(\sim 214 \mathrm{in})$

internal length

$=3,810 \mathrm{~mm}(\sim 150 \mathrm{in})$

internal diameter

$=1,790 \mathrm{~mm}(\sim 70 \mathrm{in})$

external diameter

$=2,030 \mathrm{~mm}(\sim 80 \mathrm{in})$

Commercial (21 PWR Configuration)

interior length

external length

internal length

internal diameter

external diameter
$=31,413 \mathrm{~kg}(\sim 69,192 \mathrm{lb})$

$=5,247 \mathrm{~mm}(\sim 214 \mathrm{in})$

$=3,810 \mathrm{~mm}(\sim 150 \mathrm{in})$

$=1,790 \mathrm{~mm}(\sim 70 \mathrm{in})$

$=2,030 \mathrm{~mm}(\sim 80 \mathrm{in})$

Figures A-1 and A-2 depict the 4x1 and 5x1 arrays proposed for study in this PA. These configuration could either be in $3,871 \mathrm{~mm}(\sim 152 \mathrm{in})$ or 5,247 $\mathrm{mm}(\sim 214 \mathrm{in})$ length. 


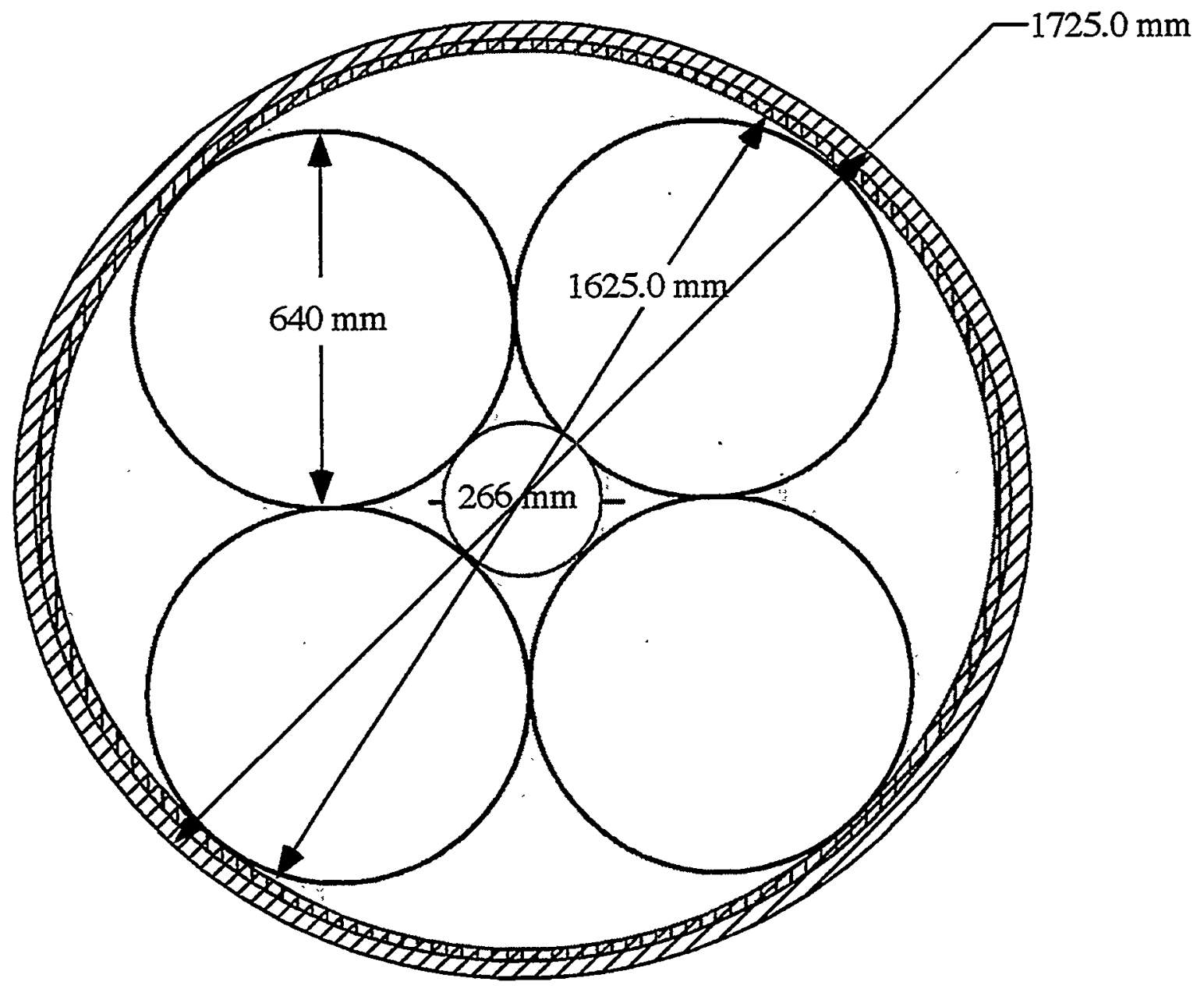

$$
\begin{gathered}
266 \mathrm{~mm} \text { OD } \\
1625 \mathrm{~mm}=63.98^{\prime \prime} \mathrm{ID} \\
266 \mathrm{~mm}=10.47^{\prime \prime} \mathrm{OD} \\
100 \mathrm{~mm} \text { CS }=4^{\prime \prime} \\
\text { III } 20 \mathrm{~mm} \text { Alloy CS }=.75^{\prime \prime}
\end{gathered}
$$

Figure A.1 Proposed 4 (HLW) x 1 (SNF) Co-disposal Package

\begin{tabular}{|l|l|l|l}
\hline $\begin{array}{l}\text { Appendix A } \\
\text { DOE SNF Information }\end{array}$ & $\begin{array}{l}\text { Sheet } \\
8 \text { of } 75\end{array}$ & $\begin{array}{l}\text { Date: } \\
\text { January 23, 1998 }\end{array}$ & $\begin{array}{c}\text { Rev. } \\
1\end{array}$ \\
\hline
\end{tabular}




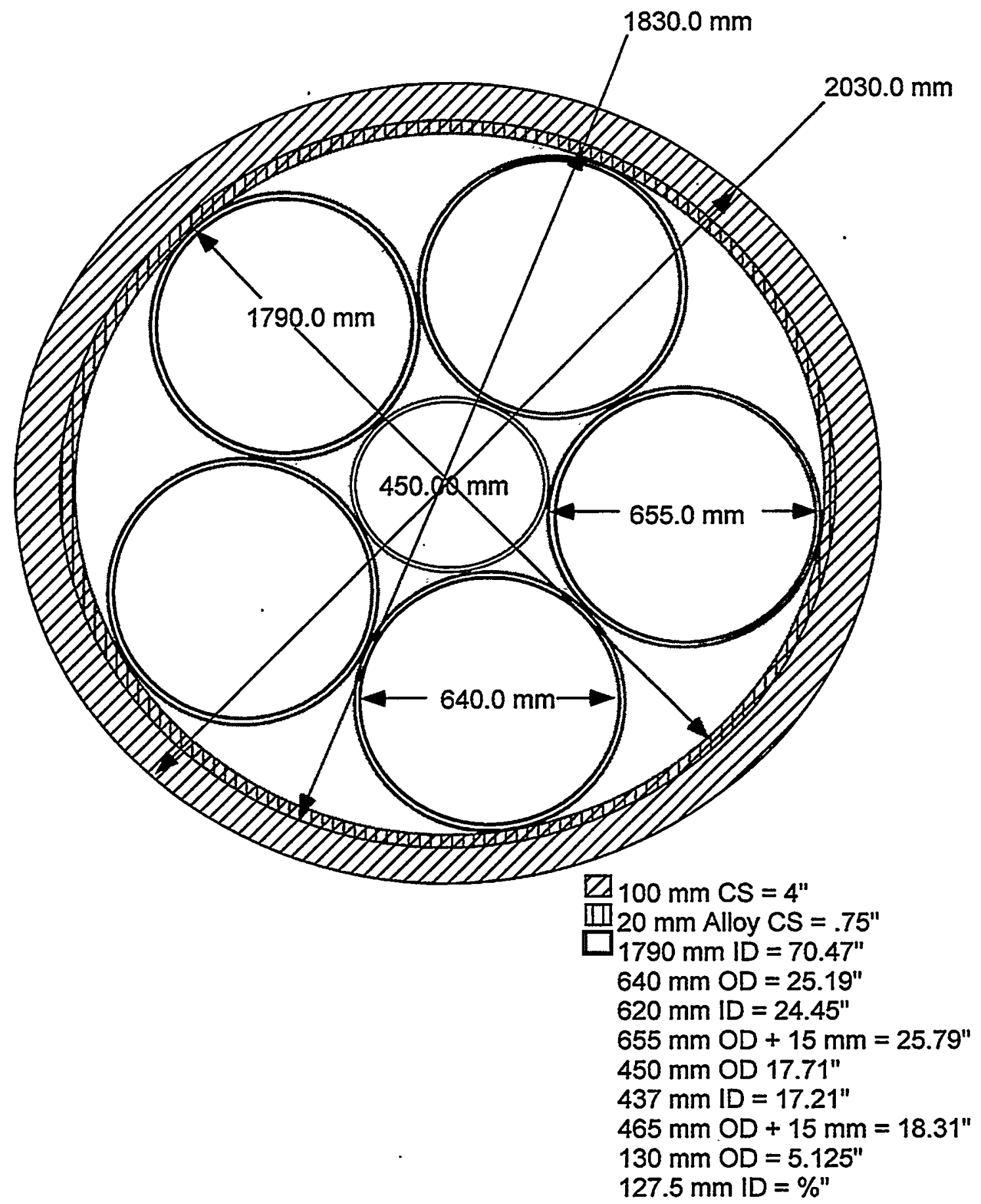

Figure A.2 Proposed 5 (HLW) x 1 (SNF) Co-disposal Package

\section{Sheet} 9 of 75
Date:

January 23, 1998
Rev.

1 


\subsection{SNF Canisters and Co-disposal Options}

The variety of individual fuels dictates both length and diameter considerations of the individual fuel canisters. The intent of canning the individual fuels is to facilitate handling the great variety of fuels'as well as the small parts and pieces.

This study will refer to $25.4 \mathrm{~cm}, 43.2 \mathrm{~cm}$, and $61 \mathrm{~cm}$ (10 in, 17 in, and 24 in) diameter canisters; standard pipe information is used to determine weights and thickness, although actual design may use modified dimensions at some later date. All canisters will be constructed of 304L SS. Typical thickness used to determine the weight will be $6.35 \mathrm{~mm}$. Table A-2 shows the canister sizes and their estimated weight based on $6.35 \mathrm{~mm}$ wall thickness.

\begin{tabular}{|c|c|c|c|}
\hline \multicolumn{4}{|c|}{ Table A-2: SNF Canister Sizes and Weight } \\
\hline $\begin{array}{l}\text { Canister Size } \\
\text { (Diameter) }\end{array}$ & Length & $\begin{array}{c}\text { Approximate Canister } \\
\text { Weight }\end{array}$ & Comments \\
\hline $\begin{array}{l}25.4 \mathrm{~cm} \\
(10 \mathrm{in})\end{array}$ & $\begin{array}{l}300 \mathrm{~cm} \\
(118 \mathrm{in})\end{array}$ & $\begin{array}{c}770 \mathrm{~kg} \\
(1,696 \mathrm{lb})\end{array}$ & $\begin{array}{l}6: 35 \mathrm{~mm} \text { wall thickness. } \\
\text { Does not include weight of } \\
\text { basket or filler materials. }\end{array}$ \\
\hline $\begin{array}{l}25.4 \mathrm{~cm} \\
(10 \mathrm{in})\end{array}$ & $\begin{array}{l}450 \mathrm{~cm} \\
(177 \mathrm{in})\end{array}$ & $\begin{array}{l}1,140 \mathrm{~kg} \\
(2,511 \mathrm{lb})\end{array}$ & $\begin{array}{l}6.35 \mathrm{~mm} \text { wall thickness. } \\
\text { Does not include weight of } \\
\text { basket or filler materials. }\end{array}$ \\
\hline $\begin{array}{l}43.2 \mathrm{~cm} \\
(17 \mathrm{in})\end{array}$ & $\begin{array}{l}300 \mathrm{~cm} \\
(118 \mathrm{in})\end{array}$ & $\begin{array}{c}1,300 \mathrm{~kg} \\
(2,863 \mathrm{lb})\end{array}$ & $\begin{array}{l}6.35 \mathrm{~mm} \text { wall thickness. } \\
\text { Does not include weight of } \\
\text { basket or filler materials. }\end{array}$ \\
\hline $\begin{array}{l}43.2 \mathrm{~cm} \\
(17 \mathrm{in})\end{array}$ & $\begin{array}{l}450 \mathrm{~cm} \\
(177 \mathrm{in})\end{array}$ & $\begin{array}{l}1,822 \mathrm{~kg} \\
(4,013 \mathrm{lb})\end{array}$ & $\begin{array}{l}6.35 \mathrm{~mm} \text { wall thickness. } \\
\text { Does not include weight of } \\
\text { basket or filler materials. }\end{array}$ \\
\hline $\begin{array}{l}61 \mathrm{~cm} \\
(24 \mathrm{in})\end{array}$ & $\begin{array}{l}300 \mathrm{~cm} \\
(118 \mathrm{in})\end{array}$ & $\begin{array}{l}1,745 \mathrm{~kg} \\
(3,844 \mathrm{lb})\end{array}$ & $\begin{array}{l}\text { Does not include weight of } \\
\text { basket or filler materials. }\end{array}$ \\
\hline $\begin{array}{l}61 \mathrm{~cm} \\
(24 \mathrm{in})\end{array}$ & $\begin{array}{l}450 \mathrm{~cm} \\
(177 \mathrm{in})\end{array}$ & $\begin{array}{l}2,442 \mathrm{~kg} \\
(5,379 \mathrm{lb})\end{array}$ & $\begin{array}{l}\text { Does not include weight of } \\
\text { basket or filler materials. }\end{array}$ \\
\hline $\begin{array}{c}64.26 \mathrm{~cm} \\
(\mathrm{MCO})\end{array}$ & $\begin{array}{l}450 \mathrm{~cm} \\
(177 \mathrm{in})\end{array}$ & $\begin{array}{l}3,685 \mathrm{~kg} \\
(8,117 \mathrm{lb})\end{array}$ & Specific to Hanford \\
\hline
\end{tabular}

\begin{tabular}{|l|l|l|l|}
\hline $\begin{array}{l}\text { Appendix A } \\
\text { DOE SNF Information }\end{array}$ & $\begin{array}{l}\text { Sheet } \\
10 \text { of } 75\end{array}$ & $\begin{array}{l}\text { Date: } \\
\text { January 23, 1998 }\end{array}$ & $\begin{array}{l}\text { Rev. } \\
1\end{array}$ \\
\hline
\end{tabular}


The following table shows the co-disposal options for the HLW and DOE SNF in the above canister sizes.

\begin{tabular}{|c|c|c|}
\hline Configuration & DOE SNF Canister Sizes & Comments \\
\hline $\begin{array}{c}5 \times 1 \\
(5 \mathrm{HLW} \times 1 \mathrm{SNF}) \\
(300 \text { or } 450 \mathrm{~cm} \text { length) }\end{array}$ & $\begin{array}{c}43.2 \mathrm{~cm} \text { diameter } \\
(17 \mathrm{in})\end{array}$ & See Figure A.2 \\
\hline $\begin{array}{c}4 \times 1 \\
(4 \mathrm{HLW} \times 1 \mathrm{SNF}) \\
(300 \text { or } 450 \mathrm{~cm} \text { length) }\end{array}$ & $\begin{array}{l}25.4 \mathrm{~cm} \text { diameter } \\
(10 \mathrm{in})\end{array}$ & See Figure A.1 \\
\hline $\begin{array}{c}3 \times 1 \\
(3 \mathrm{HLW} \times 1 \mathrm{SNF}) \\
(300 \text { or } 450 \mathrm{~cm} \text { length) }\end{array}$ & $\begin{array}{c}25.4 \mathrm{~cm}, 43.2 \mathrm{~cm}, 61 \mathrm{~cm} \\
\text { diameter } \\
(10 \mathrm{in}, 17 \mathrm{in}, 24 \mathrm{in})\end{array}$ & $\begin{array}{l}25.4 \mathrm{~cm} \text { canister for HEU. } \\
43.2 \mathrm{~cm} \text { canister for HEU \& HEU. } \\
61 \mathrm{~cm} \text { canister for LEU only. }\end{array}$ \\
\hline $\begin{array}{c}0 \times 4 \\
(0 \mathrm{HLW} \times 4 \mathrm{SNF}) \\
(300 \text { or } 450 \mathrm{~cm} \text { length })\end{array}$ & $\begin{array}{c}43.2 \mathrm{~cm}, 61 \mathrm{~cm} \text { diameter } \\
(17 \mathrm{in}, 24 \mathrm{in})\end{array}$ & LEU only \\
\hline $\begin{array}{c}4 \mathrm{MCOs} \\
(450 \mathrm{~cm} \text { length) }\end{array}$ & $64.26 \mathrm{~cm}$ diameter (MCO) & $\mathrm{N}$-reactor fuels only \\
\hline $\begin{array}{c}4 \mathrm{HLW} \\
\text { (300 or } 450 \mathrm{~cm} \text { length) }\end{array}$ & N/A & $\begin{array}{l}\text { All HLW glass canisters. No SNF } \\
\text { canisters }\end{array}$ \\
\hline
\end{tabular}

\subsection{Calculating Package Curie Loadings}

\section{DOE SNF Radionuclide Inventory}

For the DOE SNF, one or more ORIGEN-2 run of selected DOE SNF within each category was used to estimate the total radionuclide inventory for each category. The total radionuclide inventory for each category was divided by the number of packages required to dispose the entire category to come up with an average package inventory. The specific ORIGEN-2 runs used to represent each category are indicated in the Table A-4 below. As

\begin{tabular}{|l|l|l|l|}
\hline $\begin{array}{l}\text { Appendix A } \\
\text { DOE SNF Information }\end{array}$ & $\begin{array}{l}\text { Sheet } \\
11 \text { of } 75\end{array}$ & $\begin{array}{l}\text { Date: } \\
\text { January 23, 1998 }\end{array}$ & $\begin{array}{l}\text { Rev. } \\
1\end{array}$ \\
\hline
\end{tabular}


noted in the table, categories 2 , and 7 fuels are represented by ORIGEN-2 run from another category because no ORIGEN runs were available for these two categories. In the future performance assessment analysis, these inventories will be updated as the sites complete more ORIGEN runs for their fuels.

To be consistent with RW's calculation, the commercial SNF inventory were extracted form the RW 1995 TSPA report "Total System Performance Assessment - 1995: An Evaluation of the Potential Yucca Mountain Repository, November 1995".

\begin{tabular}{|c|c|c|}
\hline \multicolumn{3}{|c|}{ Table A-4 ORIGEN-2 Runs used in the Fuel Category } \\
\hline Fuel Category & ORIGEN-2 Run to Represent the category & Comment \\
\hline 1 & N-Reactor Fuel & \\
\hline 2 & Advanced Test Reactor (ATR) Fuel & $\begin{array}{l}\text { No ORIGEN run available for } \\
\text { fuels in the category. ATR was } \\
\text { used because it most represents } \\
\text { the category. }\end{array}$ \\
\hline 3 & FERMI (Enrico Fermi Reactor) Fuel & \\
\hline 4 & $\begin{array}{l}\text { Commercial PWR Fuel } \\
\text { Pathfinder Fuel } \\
\text { Power Burst Facility Fuel } \\
\text { Transient Reactor Test (TREAT) Fuel }\end{array}$ & \\
\hline 5 & $\begin{array}{l}\text { Pulstar Buffalo Fuel } \\
\text { Three Mile Island (TMI) }\end{array}$ & \\
\hline 6 & Advanced Test Reactor (ATR) Fuel & \\
\hline 7 & Advanced Test Reactor (ATR) Fuel & $\begin{array}{l}\text { No ORIGEN run available for } \\
\text { fuels in the category. ATR was } \\
\text { used because it most represents } \\
\text { the category. }\end{array}$ \\
\hline 8 & $\begin{array}{l}\text { Fort Saint Vrain Fuel } \\
\text { General Atomics-High Temperature Gas } \\
\text { Cooled Reactor (GA-HTGR) Fuei }\end{array}$ & - \\
\hline 9 & Peach Bottom Fuel & \\
\hline 10 & Fast Flux Test Facility (FFTF) Carbide Fuel & \\
\hline 11 & Fast Flux Test Facility (FFTF) Oxide Fuel & \\
\hline 12 & Shippingport LWBR Fuel & \\
\hline 13 & $\begin{array}{l}\text { Training Research Isotopes- General Atomic } \\
\text { (TRIGA) Fuel }\end{array}$ & \\
\hline 14 & Commercial (PWR \& BWR) Fuel & From RW 95 TSPA Report \\
\hline 15 & Commercial (PWR \& BWR) Fuel & From RW 95 TSPA Report \\
\hline
\end{tabular}

Appendix A DOE SNF Information
Sheet 12 of 75
Date: January 23, 1998
Rev.

1 


\section{HLW Radionuclide Inventory}

The radionuclide inventory for the HLW canister was from the RW 1995 TSPA report. According to TSPA-95, the inventory used was from the report "Characteristics of Spent Fuel, High-Level Waste, and Other Radioactive Wastes Which May Require Long-Term Isolation," DOE/RW-0184 published in 1987 [Reference 13]. Since the 1995 TSPA report radionuclide inventory was based on $300 \mathrm{~cm}$ (118 in) long, $61 \mathrm{~cm}$ (24 in) diameter standard canisters, for those SNF/HLW package combinations using $450 \mathrm{~cm}$ (177 in) $\mathrm{HLW}$ canisters, the inventory for the $450 \mathrm{~cm}$ (177 in) HLW canisters may be multiplied by 1.50 .

\section{Co-Disposal Package Radionuclide Inventory}

Individual SNF packages are assigned a representative radionuclide inventory by fuel category, based on a typical $43.2 \mathrm{~cm}$ (17 in) diameter and a $300 \mathrm{~cm}$ (118 in) long SNF package.

Example calculations:

$$
\begin{aligned}
& 0 \times 4 \text { (MCOs) }=4 * \text { Category } 1 \text { radionuclide inventory } \\
& 3 \times 1(10 \mathrm{ft})=3 * \mathrm{HLW} \text { canister }+1 \text { Category } \mathrm{x} \\
& \text { radionuclide inventory } \\
& 5 \times 1(15 \mathrm{ft})=5 *(1.5 * \text { HLW canister }) \\
& +1 \text { Category } x \text { radionuclide inventory }
\end{aligned}
$$




\subsection{DOE SNF Categories}

The following section describes the typical fuels within each of the DOE SNF categories and the various information for each of the fuel categories.

\subsection{Category 1 U-metal/zirconium}

Typical fuel: N-reactor

\section{Fuel Description}

The N-reactor fuel elements consist of two concentric tubes made of uranium metal co-extruded into Zircaloy-2 cladding. There are two basic types of fuel elements differentiated by their uranium enrichment Mark IV fuels elements a pre-irradiation enrichment of $0.947 \%$ U-235 in both tubes and an average uranium weight of $22.7 \mathrm{~kg}(50 \mathrm{lb})$. The Mark IV fuels have an outside diameter of $6.1 \mathrm{~cm}$ (2.4 in) and a length of 44, 59, 62 or $66 \mathrm{~cm}(17.4,13.2$, 24.6 , or 26.1 in). Mark IA fuel elements have a pre-irradiation enrichment of $1.25 \%$ U-235 in the outer tube and $0.947 \%$ U-235 in the inner tube. They have an average uranium weight of $16.3 \mathrm{~kg}(35.9 \mathrm{lb})$. Mark IA fuels have an outside diameter of $6.1 \mathrm{~cm}(2.1 \mathrm{in})$ and a length of 38,50 , or $53 \mathrm{~cm}(14.9,19.6$, or $20.9 \mathrm{in})$ [Reference 7].

The degraded condition of the $\mathrm{N}$-reactor fuels has created a vulnerability issue relative to their continued storage in a water environment. Breach of the fuel element cladding and long-term water storage has created an apparent uranium hydride formation. Planned remediation of these fuels currently includes drying and controlled oxidation of the hydride to an oxide for interim storage in a package labeled as a Multi-Canister Overpack (MCO) [Reference 8]. The MCO has experienced evolutionary design changes; the basic unit will contain a close packed arrangement of either Mark IV or Mark IA fuels. While the original concept of the $\mathrm{MCO}$ is not intended as a repository approved disposal package, no alternative or proposed package exists at this time. The physical size of the MCO is akin to the standard HLW glass package, and will therefore be modeled as a 4-pack within the repository overpack.

Each MCO consists of a $61 \mathrm{~cm}$ ( 24 in) outer diameter shell that is $416.6 \mathrm{~cm}$ (164 in) long. The package has a $0.95 \mathrm{~cm}(0.375$ in) wall thickness, and uses $304 \mathrm{~L}$ stainless steel construction. The approximate mass of the empty MCO is $1673.5 \mathrm{kgs}(3868 \mathrm{lb})$.

\begin{tabular}{|l|l|l|l|}
\hline $\begin{array}{l}\text { Appendix A } \\
\text { DOE SNF Information }\end{array}$ & $\begin{array}{l}\text { Sheet } \\
14 \text { of } 75\end{array}$ & Date: & January 23, 1998 \\
\hline
\end{tabular}




\begin{tabular}{|l|l|l|}
\hline Category 1 U-Metal Fuel Inventories/Information \\
\hline $\begin{array}{l}\text { Radionuclide Inventory } \\
\text { (41 isotopes) }\end{array}$ & $\begin{array}{l}\text { Refer to TSPA group listing data - Table } \\
\text { A-7 }\end{array}$ & \\
\hline Composition & $\begin{array}{l}\bullet \text { Breached fuel cladding } \\
\text { uranium metal with possible oxide } \\
\text { surface coating }\end{array}$ & \\
\hline Wet Dissolution Rate & Metal Model & \\
\hline Surface Area ( $\mathrm{m}^{2} / \mathrm{g}$ ) & $1.32 \mathrm{E}-06$ & \\
\hline Clad Failure Fraction & $100 \%$ & \\
\hline $\begin{array}{l}\text { Free Radionuclide Inventory } \\
\text { Fraction }\end{array}$ & $0.01 \%$ & \\
\hline
\end{tabular}

\section{Configuration, Mass, and Package Count}

The following table shows the disposal configuration, average package mass, repository package count, and HLW mass used to co-disposed the category 1 SNF.

\begin{tabular}{|c|c|c|c|c|c|c|}
$\# 3 \times 1$ & $\# 3 \times 1$ & $\# 4 \times 1$ & $\# 4 \times 1$ & $\# 5 \times 1$ & $\# 5 \times 1$ & $\# 0 \times 4$ \\
$10 \mathrm{ft}$ & $15 \mathrm{ft}$ & $10 \mathrm{ft}$ & $15 \mathrm{ft}$ & $10 \mathrm{ft}$ & $15 \mathrm{ft}$ & $15 \mathrm{ft}$ \\
\hline
\end{tabular}

Category 1 U metal, failed clad

- ave package mass (MT)

- repository pkg count

- HLW can count

- HLW mass (MT)

- SNF pkg count

- ave U mass $(\mathrm{kg})$

- ave. fissile mass $(\mathrm{kg})$

\begin{tabular}{rrrrrr} 
& 34.9 & 29.5 & 36.8 & & 66.41 \\
\hline & 2 & 3 & 2 & 6 & 105 \\
\hline$)$ & 6 & 12 & 8 & 30 & \\
\hline+ & 19.2 & 25.8 & 25.6 & 96.0 & \\
& 2 & 3 & 2 & 6 & 420 \\
& 951.5 & 108.5 & 252.5 & 228.8 & 18073 \\
& 11.25 & 1.05 & 2.63 & 1.65 & 60.7
\end{tabular}

Date:

January 23, 1998 
Tables A-5 and A-6 provide a summary listing of the various chemical components associated with the typical $\mathrm{N}$-reactor fuels.

Table A-5: N-Reactor Fuel Element Description

\begin{tabular}{|c|c|c|c|c|c|c|c|}
\hline & \multicolumn{4}{|c|}{ Mark IV } & \multicolumn{3}{|c|}{ Mark IA } \\
\hline Pre-irradiation enrichment of U235 & \multicolumn{4}{|c|}{$0.947 \%$ Enriched } & \multicolumn{3}{|c|}{$1.25-0.947 \%$ Enriched } \\
\hline Type-Length code ${ }^{1}$ & $\mathrm{E}$ & $\mathrm{C}$ & $\mathbf{S}$ & A & $\mathbf{M}$ & $\mathbf{F}$ & $\mathrm{T}$ \\
\hline Outer length $(\mathrm{cm})$ & $66: 3$ & 62.5 & 58.9 & 44.2 & 53.1 & 49.8 & 37.8 \\
\hline \multicolumn{8}{|l|}{ Element diameter (cm) } \\
\hline 1. Outer of outer & \multicolumn{4}{|c|}{6.15} & \multicolumn{3}{|c|}{6.1} \\
\hline 2. Inner of outer & \multicolumn{4}{|c|}{4.32} & \multicolumn{3}{|c|}{4.5} \\
\hline 3. Outer of inner & \multicolumn{4}{|c|}{3.25} & \multicolumn{3}{|c|}{3.18} \\
\hline 4. Inner of inner & \multicolumn{4}{|c|}{1.22} & \multicolumn{3}{|c|}{. 1.12} \\
\hline \multicolumn{8}{|l|}{ Cladding weight $(\mathrm{kg})$} \\
\hline I. Outer element & 1.09 & 1.04 & 0.99 & 0.79 & 0.88 & 0.83 & 0.66 \\
\hline 2. Inner element & 0.55 & 0.52 & 0.50 & 0.40 & 0.24 & 0.51 & 0.40 \\
\hline \multicolumn{8}{|l|}{ Weight of uranium in outer $(\mathrm{kg})$} \\
\hline 1. $(0.947 \% 235 U)$ & 15.96 & 15.01 & 14.15 & 10.48 & & & \\
\hline 2. $(1.25 \%$ 235U) & & & & & 11.07 & 10.39 & 7.85 \\
\hline $\begin{array}{l}\text { Weight of uranium Inner }(\mathrm{kg}) \\
0.947 \%\end{array}$ & 7.48 & 7.03 & 6.62 & 4.94 & 5.49 & 5.12 & \\
\hline $\begin{array}{l}\text { Weighted average of uranium in } \\
\text { element }(\mathrm{kg})\end{array}$ & \multicolumn{4}{|c|}{22.68} & \multicolumn{3}{|c|}{16.28} \\
\hline Ratio of Zircaloy-2 to uranium ( $\mathrm{kg} / \mathrm{MT})$ & 70.0 & 70.8 & 71.6 & 77.1 & 85.5 & 86.3 & 90.4 \\
\hline Weighted ave. $(\mathrm{kg} / \mathrm{MT})$ & \multicolumn{4}{|c|}{63.76} & \multicolumn{3}{|c|}{77.73} \\
\hline$\%$ of total elements & \multicolumn{4}{|c|}{63} & \multicolumn{3}{|c|}{37} \\
\hline$\%$ of length type of each fuel & 78 & $\begin{array}{l}10 \\
5\end{array}$ & & 7 & 87 & 10 & 3 \\
\hline Displacement Volume(l/MT uranium) & \multicolumn{4}{|c|}{66.77} & \multicolumn{3}{|c|}{66.77} \\
\hline
\end{tabular}

1. Letter code differentiates the different lengths of the Mark IV or Mark IA fuel elements, i.e. a type " $E$ " element is $66.3 \mathrm{~cm}$ long. [Hanford Irradiated Fuel Inventory Baseline]

\begin{tabular}{|l|l|l|l|}
\hline $\begin{array}{l}\text { Appendix A } \\
\text { DOE SNF Information }\end{array}$ & $\begin{array}{l}\text { Sheet } \\
16 \text { of } 75\end{array}$ & $\begin{array}{l}\text { Date: } \\
\text { January 23, 1998 }\end{array}$ & $\begin{array}{c}\text { Rev. } \\
1\end{array}$ \\
\hline
\end{tabular}


Table A-6: Chemical Composition of 105-N-Reactor Fuel Elements ${ }^{1}$

\begin{tabular}{|c|c|c|c|}
\hline Element & Uranium Alloy 601 & Zircaloy-2 & Braze Filler \\
\hline Aluminum & $700-900$ & 75 & 14 \\
\hline Beryllium & 10 & - & 4.75-5.25 wt\% \\
\hline Boron & 0.25 & 0.50 & 0.5 \\
\hline Cadmium & 0.25 & 0.50 & 0.5 \\
\hline Carbon & $365-735$ & 275 . & 500 \\
\hline Chromium & 65 & $0.05-0.15 w t \%$ & $0.05-0.15$ wt $\%$ \\
\hline Cobalt & - & 10 & 20 \\
\hline Copper & 75 & 50 & 60 \\
\hline Hafinium & & 200 & 200 \\
\hline Hydrogen & 2.00 & 25 & 50 \\
\hline Iron & $300-400$ & $0.07-0.20 w t \%$ & $0.06-0.21$ wt\% \\
\hline Lead & - & 100 & 130 \\
\hline Magnesium & 25 & 20 & 60 \\
\hline Manganese & 25 & 50 & 60 \\
\hline Molybdenum & - & 50 & 5 \\
\hline Nickel & 100 & $0.03-0.08 w t \%$ & 0.03-0.08 wt\% \\
\hline Nitrogen & 75 & 80 & 20 \\
\hline Oxygen & - & - & 230 \\
\hline Silicon & 124 & 100 & 25 \\
\hline Sodium & - & 20 & 2 \\
\hline Tin & - & $1.20-1.70$ wt\% & $1.14-1.70$ wt\% \\
\hline Titanium & - & 50 & 5 \\
\hline Tungsten & & 50 & 10 \\
\hline Uranium & Balance & 3.50 & 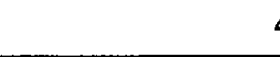 \\
\hline Vanadium & - & 50 & 5 \\
\hline Zirconium & 65 & Balance & Balanc \\
\hline
\end{tabular}

1.Concentrations given in parts per million (ppm) maximum or ppm range, unless indicated otherwise. [Ref. X]

Appendix A

DOE SNF Information
Sheet

17 of 75
Date:

January 23,1998
Rev.

1 
Figure A.3 depicts a typical N-reactor fuel element; Figures A.4a, and A.4b depict proposed layout of $\mathrm{N}$-reactor fuel packaging within an $\mathrm{MCO}$ as it was evaluated in the PA.

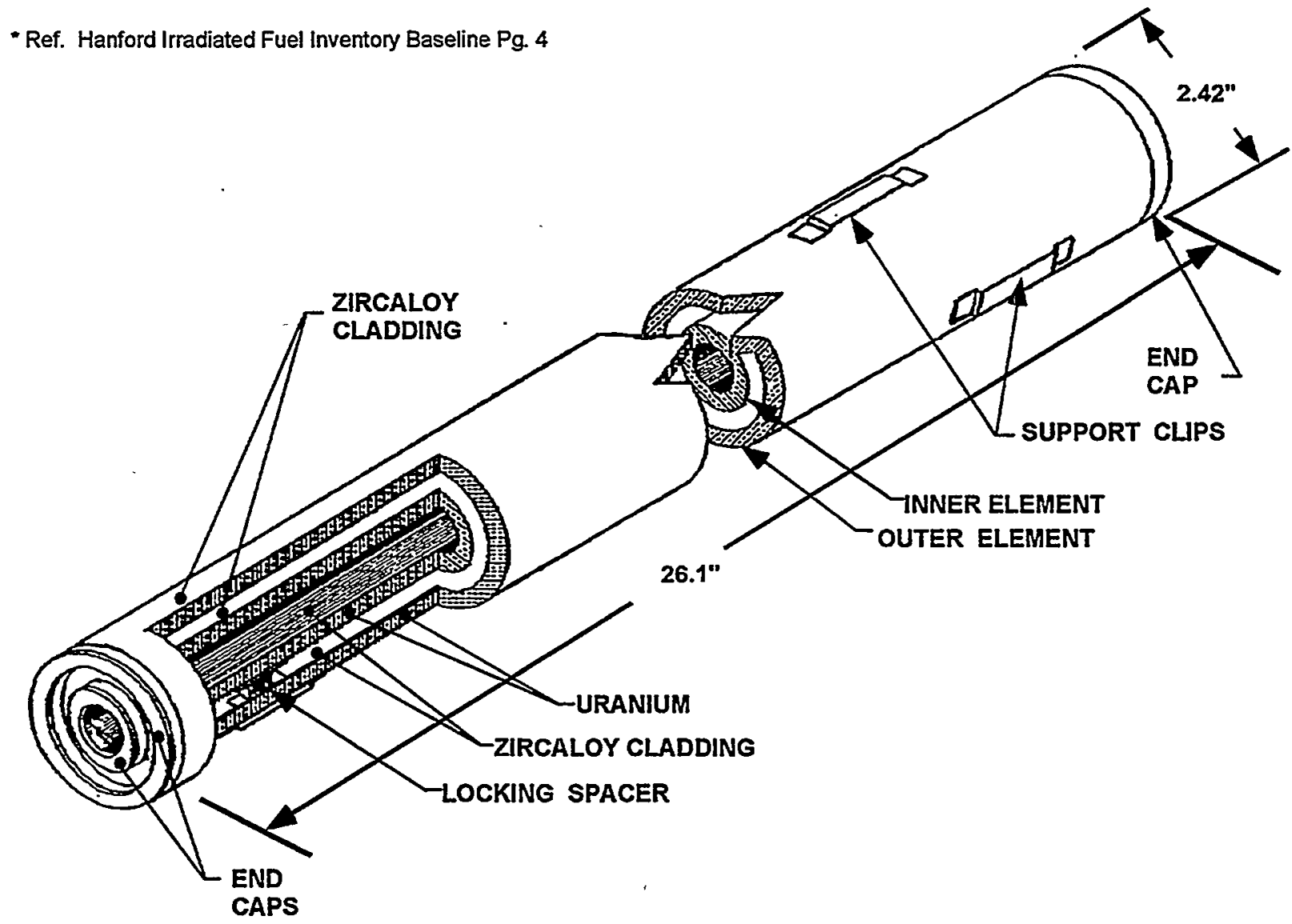

105-N REACTOR MARK IV FUEL ELEMENT ASSEMBLY

Figure A:3 N-Reactor Mark IV Fuel Element Assembly

Sheet 18 of 75
Date:

January 23, 1998
Rev. 1 


\section{Loading Arrangement for Mark IV Fuel in MCO Container.}

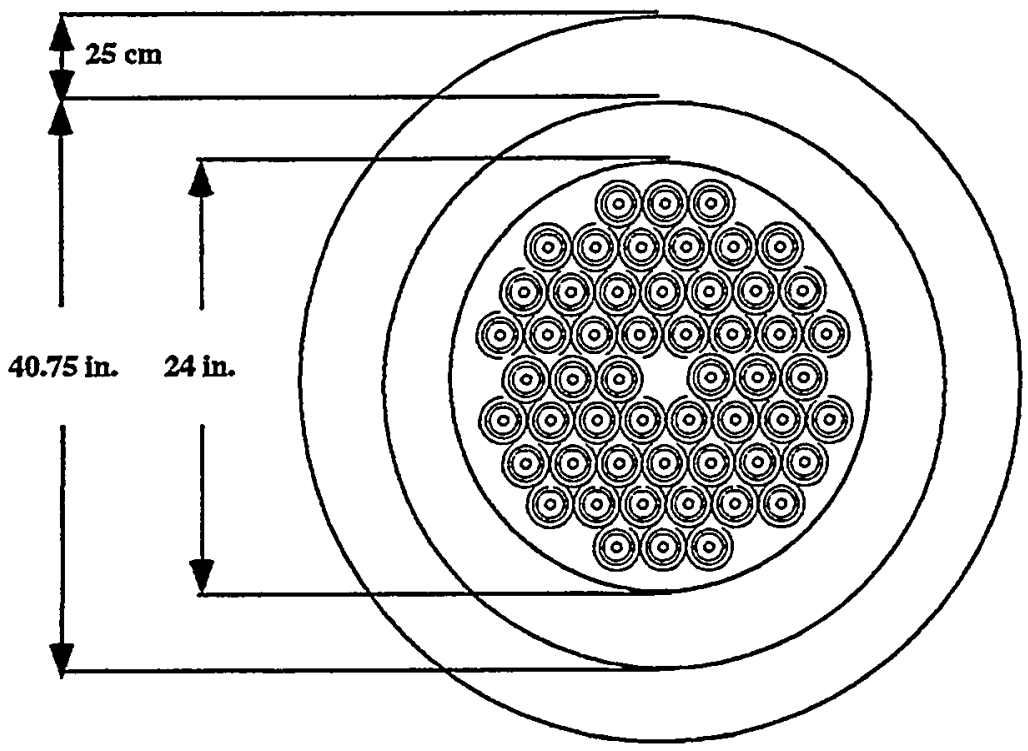

Loading Arrangement for Mark IA Fuel in MCO Container.

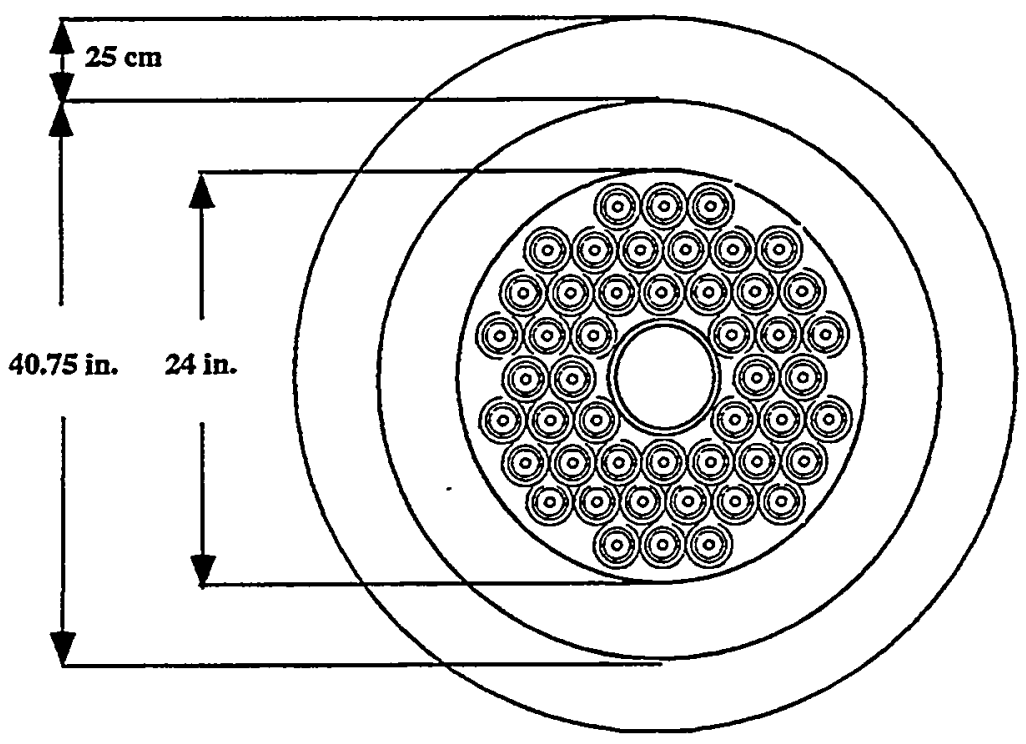

Figure A.4a (Top): Loading Arrangement for Mark IV Fuel in MCO Container Figure A.4b (Bottom): Loading Arrangement for Mark 1A Fuel in MCO Container

\begin{tabular}{|l|l}
\hline $\begin{array}{l}\text { Date: } \\
\text { January 23, 1998 }\end{array}$ & $\begin{array}{l}\text { Rev. } \\
1\end{array}$
\end{tabular}




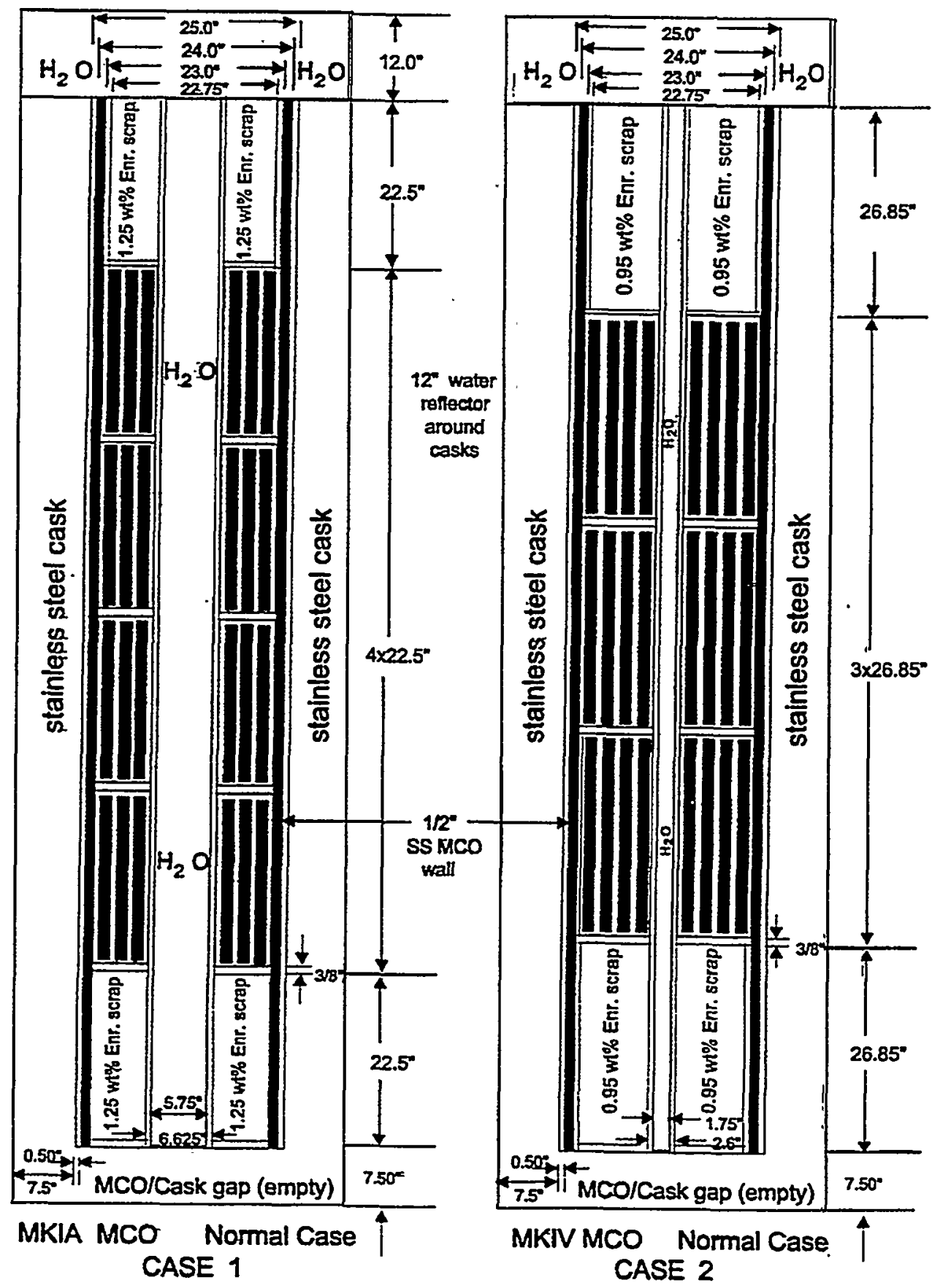

Figure A.5 MCO Axial Geometry Layout

\begin{tabular}{|l|l|l|l|}
\hline $\begin{array}{l}\text { Appendix A } \\
\text { DOE SNF Information }\end{array}$ & $\begin{array}{l}\text { Sheet } \\
20 \text { of } 75\end{array}$ & $\begin{array}{l}\text { Date: } \\
\text { January 23, 1998 }\end{array}$ & $\begin{array}{l}\text { Rev. } \\
1\end{array}$ \\
\hline
\end{tabular}




\subsection{Category 2 U-Zr/Zirconium}

Typical fuel: CP-5, HWCTR

\section{Fuel Description}

The Heavy Water Components Test Reactor (HWCTR) is a tank type, fully enriched (93\%) uranium, heavy water moderated and cooled reactor. The purpose of the reactor was to test fuel elements, materials, and components for heavy water reactors at power reactor conditions. The reactor had a nominal thermal power $61 \mathrm{MW}$. The driver fuel elements are located around the outside part of the reactor with up to 12 the test fuel elements placed in the center of the reactor.

The driver fuels are tube type design with $5.84 \mathrm{~cm}$ (2.3 in) outside diameter, $4.98 \mathrm{~cm}$ (1.96 in) inside diameter and $287 \mathrm{~cm}$ (113 in) long. The fuel meat is $0.348 \mathrm{~cm}(0.137$ in) thick consisting of $93 \%$ enriched uranium alloyed with 90.7 wt\% zirconium. Figure A.6 contains a section view of the driver element [Reference 9]. The test elements are made of natural or slightly enriched uranium metal or uranium oxide. Thus, they are not included in this category.

\begin{tabular}{|l|l|l|}
\hline Category 2 U-Zr Fuel Inventories/Information \\
\hline $\begin{array}{l}\text { Radionuclide Inventory } \\
\text { (41 isotopes) }\end{array}$ & $\begin{array}{l}\text { Refer to TSPA group listing data - Table } \\
\text { A-7 }\end{array}$ & \\
\hline Composition & $\begin{array}{l}93 \% \text { enriched uranium alloyed with } 90.7 \\
\text { wt\% zirconium }\end{array}$ & \\
\hline Wet Dissolution Rate & Metal Model & \\
\hline Surface Area (m $/ \mathrm{g})$ & $5.00 \mathrm{E}-05$ & \\
\hline Clad Failure Fraction & $10 \%$ & \\
\hline $\begin{array}{l}\text { Free Radionuclide Inventory } \\
\text { Fraction }\end{array}$ & $0.001 \%$ & \\
\hline
\end{tabular}

Appendix A DOE SNF Information

\section{Sheet} 21 of 75
Date: January 23, 1998
Rev.

1 


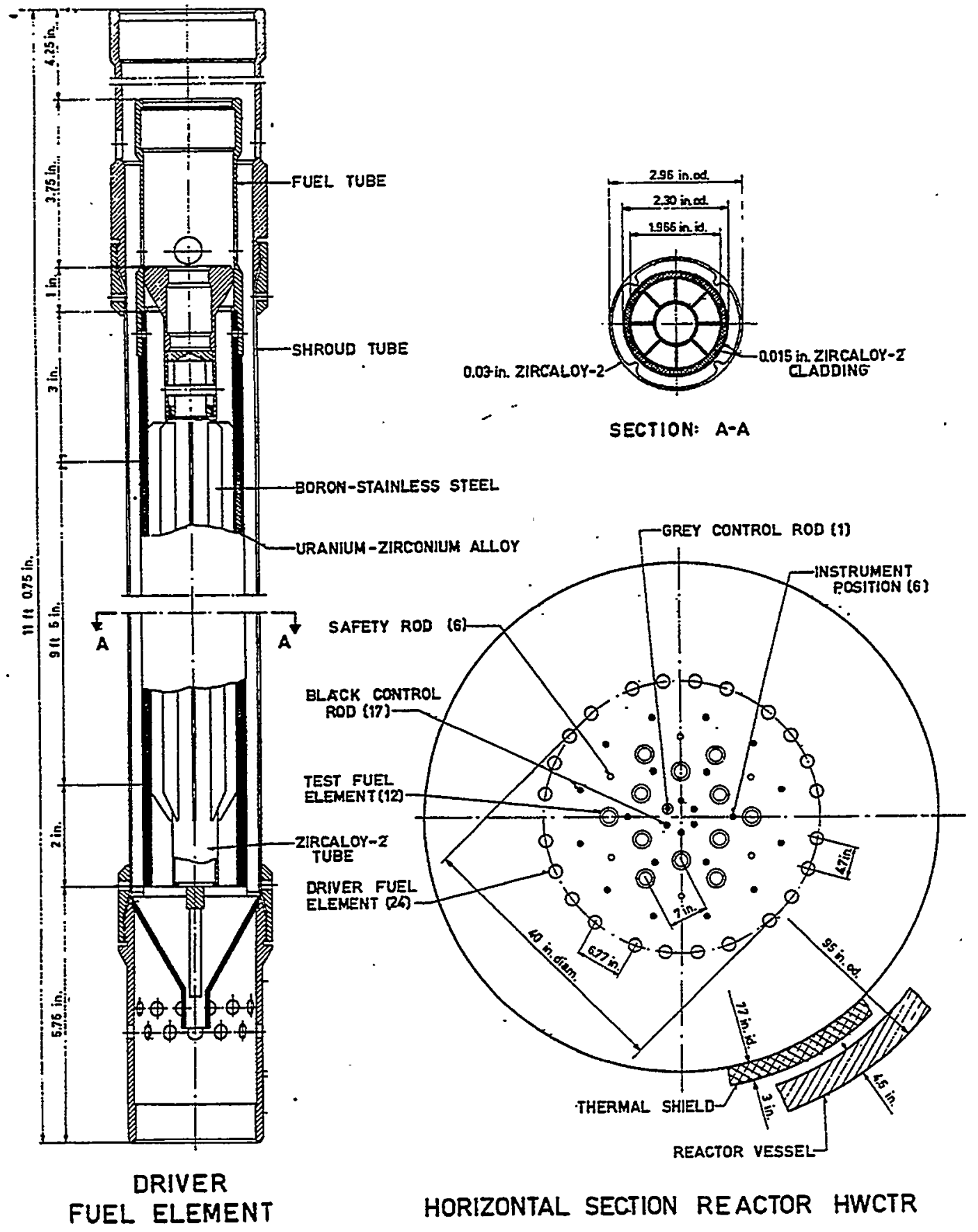

Figure A.6 Section View of the HWCTR Driver fuel and Reactor

\begin{tabular}{|l|l|l|l|}
\hline $\begin{array}{l}\text { Appendix A } \\
\text { DOE SNF Information }\end{array}$ & $\begin{array}{l}\text { Sheet } \\
22 \text { of } 75\end{array}$ & $\begin{array}{l}\text { Date: } \\
\text { January 23, 1998 }\end{array}$ & $\begin{array}{l}\text { Rev. } \\
1\end{array}$ \\
\hline
\end{tabular}




\section{Configuration, Mass, and Package Count}

The following table shows the disposal configuration, average package mass, repository package count, and HLW mass used to co-disposed the category 2 SNF.

Category 2 U-Zr, $\mathrm{Zr}$ clad

\begin{tabular}{|c|c|c|c|c|c|c|}
$\# 3 \times 1$ & $\# 3 \times 1$ & $\# 4 \times 1$ & $\# 4 \times 1$ & $\# 5 \times 1$ & $\# 5 \times 1$ & $\# 0 \times 4$ \\
$10 \mathrm{ft}$ & $15 \mathrm{ft}$ & $10 \mathrm{ft}$ & $15 \mathrm{ft}$ & $10 \mathrm{ft}$ & $15 \mathrm{ft}$ & $15 \mathrm{ft}$ \\
\hline
\end{tabular}

- ave package mass (MT)

- repository pkg count

- HLW can count

25.5

3

12

47.6

- HLW mass (MT)

38.4

- SNF pkg count

12

- ave U mass (kg)

1.6

0.44

6

30

96

- ave. fissile mass ( $\mathrm{kg})$

6

6.44

5.47 


\subsection{Category 3 U-Mo/Zirconium}

Typical fuel: Fermi

\section{Fuel Description}

Fermi was a sodium-cooled fast breeder reactor with intermediate sodium loops, sodium-to-water steam generators, and an associated steam-driven turbine-generator. The lower reactor section of the reactor vessel has a $289.56 \mathrm{~cm}$ ( 9.5 feet) outside diameter and is $245.11 \mathrm{~cm}(96.5 \mathrm{in})$ in height. Core and blanket subassemblies are housed within the lower reactor vessel and are cooled by sodium that flows from the bottom of the lower reactor through the subassemblies and up into the upper reactor vessel. Each subassembly has a nozzle attached to the bottom end for insertion into the two 2 inch support plates spaced 14 inches apart. The core and blanket of Fermi were made up of $6.72-\mathrm{cm}$ (2.646 in) square driver core and blanket subassemblies positioned to approximate a right circular cylinder approximately 80 inches in diameter and 70 inches tall. Figure A.7 shows the configuration of the core subassembly. The reactor core region was 30.5 inches in diameter and 31.2 inches tall and was completely enclosed by a thick breeder blanket that was designed to give a high breeding ratio and provide shielding.

The radial blanket fuel subassembly is made up of an inlet nozzle, a lower axial blanket, a fuel section, and an upper axial blanket. The radial blanket fuel subassemblies were made up of 25 cylindrical rods fabricated from depleted U-Mo alloy, encased in stainless steel tubes and bonded with sodium. The radial blanket subassemblies are currently stored dry in ICPP-749. The radial blanket subassembly rods contain depleted uranium and sodium and thus will be treated prior to final disposition. It isn't part of the category 3 inventory.

The Fermi driver fuel subassembly was designed with three active regions - a lower axial blanket, a fuel section, and an upper axial blanket. The lower and upper axial blanket subassemblies have been cropped off from the central core fuel section and are currently stored with the radial blanket subassemblies in ICPP-749 and will be treated prior to final disposal. A type 347 stainless steel square tube measuring 2.646 inches square with a 0.096 inch wall thickness was used as the outside structure to hold the three regions together. The fuel section contained 144 fuel pins, made up of $25.69 \%$ enriched uranium-molybdenum alloy. Four stainless steel structural support pins were inserted into the corner positions of the $12 \times 12$ array to add structural support to the fuel section and the fuel subassembly. The fuel pins were closely packed into the 2.646 inch square tube. The fuel pins were maintained on a square pitch of 0.200 inches in a cartridge made of stainless steel wires and plates.

\begin{tabular}{|l|l|l|l|}
\hline $\begin{array}{l}\text { Appendix A } \\
\text { DOE SNF Information }\end{array}$ & $\begin{array}{l}\text { Sheet } \\
24 \text { of } 75\end{array}$ & $\begin{array}{l}\text { Date: } \\
\text { January 23, 1998 }\end{array}$ & $\begin{array}{c}\text { Rev. } \\
1\end{array}$ \\
\hline
\end{tabular}




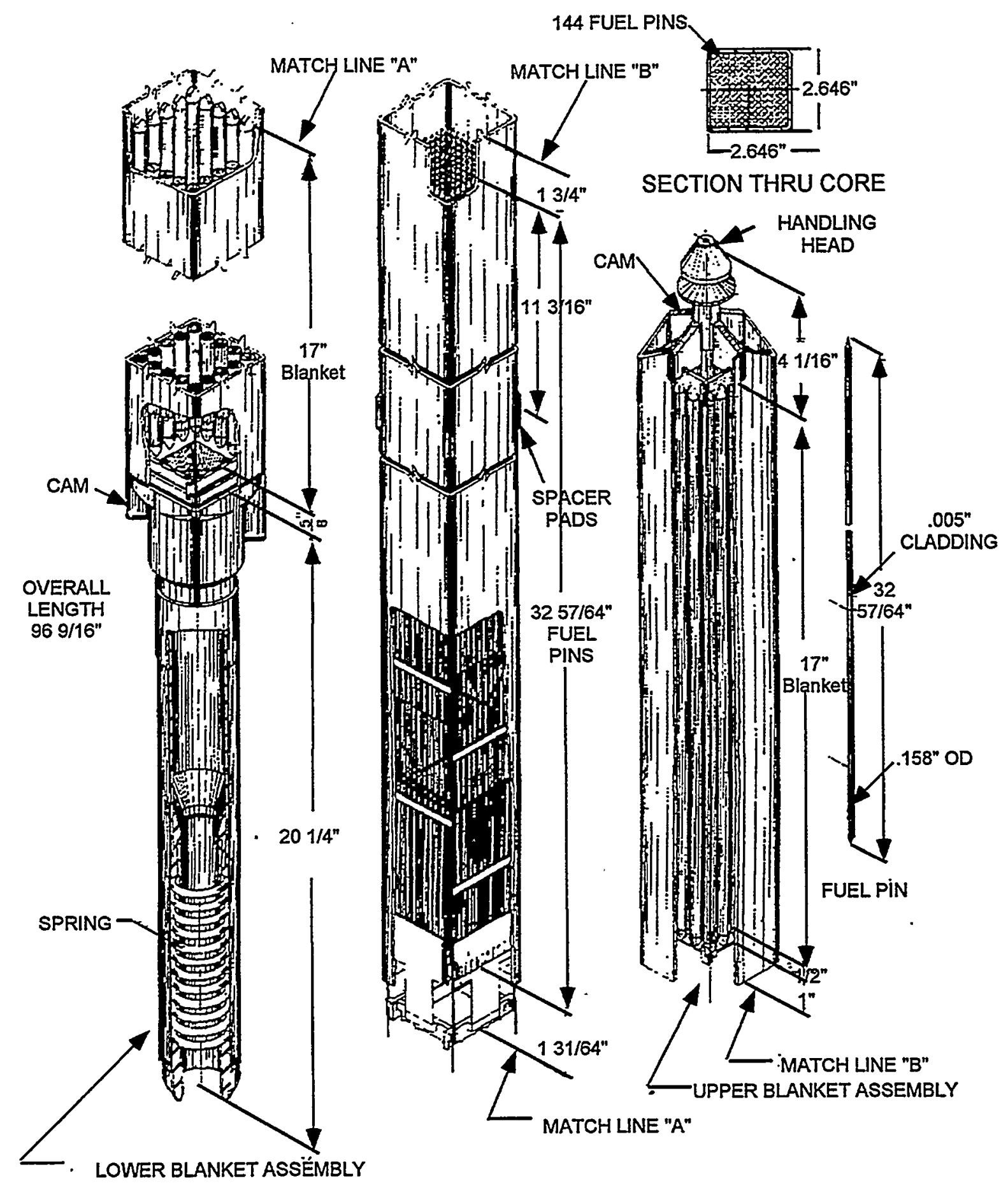

FERMI FUEL ASSEMBLY

Figure A.7 Fermi Driver Fuel Subassembly

\begin{tabular}{|l|l|l|l|}
\hline $\begin{array}{l}\text { Appendix A } \\
\text { DOE SNF Information }\end{array}$ & $\begin{array}{l}\text { Sheet } \\
25 \text { of } 75\end{array}$ & $\begin{array}{l}\text { Date: } \\
\text { January 23, 1998 }\end{array}$ & $\begin{array}{l}\text { Rev. } \\
1\end{array}$ \\
\hline
\end{tabular}


The fuel pin is made up of a solid uranium-molybdenum alloy fuel meat, 0.148 inches in diameter, metallurgically bonded to a zirconium tube. The fuel material is 90 weight percent uranium that has been enriched to a nominal 25.69 percent in U-235, and 10 weight percent molybdenum. The fuel pins were originally fabricated in lengths of 12 feet or greater and were later cut into 30.5 inch sections with the ends pointed by cold swaging. Following the sectioning, each pin was subjected to heat treatment to provide for stress relief. Next, prefabricated zirconium caps were placed on the end of each pin and secured in place by cold swaging. The total length of the fuel pins including the zirconium endcaps is 32.78 inches. A slot was made in the bottom cap of the fuel pin for anchoring purposes [Reference 10].

\begin{tabular}{|l|l|l|}
\hline Category 3 U-Mo Fuel Inventories/Information & \\
\hline $\begin{array}{l}\text { Radionuclide Inventory } \\
\text { (41 isotopes) }\end{array}$ & $\begin{array}{l}\text { Refer to TSPA group listing data - Table } \\
\text { A-7 }\end{array}$ & \\
\hline Composition & $\begin{array}{l}\text { Nominal 25.69\% in U-235, and 10 wt\% } \\
\text { Mo }\end{array}$ & \\
\hline Wet Dissolution Rate & Metal Model x 10 & \\
\hline Surface Area (m $2 / \mathrm{g})$ & $6.80 \mathrm{E}-05$ & \\
\hline Clad Failure Fraction & $10 \%$ & \\
\hline $\begin{array}{l}\text { Free Radionuclide Inventory } \\
\text { Fraction }\end{array}$ & $0.001 \%$ & \\
\hline
\end{tabular}

\section{Configuration, Mass, and Package Count}

The following table shows the disposal configuration, average package mass, repository package count, and HLW mass used to co-disposed the category 3 SNF.

Category 3 U-Mo, $\mathrm{Zr}$ clad

- ave package mass

- repository pkg count

- HLW can count

- HLW mass (MT)

- SNF pkg count

- ave U mass (kg)

- ave. fissile mass $(\mathrm{kg})$

\begin{tabular}{|c|c|c|c|c|c|c|}
$\# 3 \times 1$ & $\# 3 \times 1$ & $\# 4 \times 1$ & $\# 4 \times 1$ & $\# 5 \times 1$ & $\# 5 \times 1$ & $\# 0 \times 4$ \\
$10 \mathrm{ft}$ & $15 \mathrm{ft}$ & $10 \mathrm{ft}$ & $15 \mathrm{ft}$ & $10 \mathrm{ft}$ & $15 \mathrm{ft}$ & $15 \mathrm{ft}$ \\
\hline
\end{tabular}

\section{7}

55

220

473

55

71.45

18.2
Appendix A

DOE SNF Information

\begin{tabular}{|l|l|l}
\hline $\begin{array}{l}\text { Sheet } \\
26 \text { of } 75\end{array}$ & $\begin{array}{l}\text { Date: } \\
\text { January 23, 1998 }\end{array}$ & $\begin{array}{c}\text { Rev. } \\
1\end{array}$ \\
\hline
\end{tabular}




\subsection{Category 4 U oxide/Zirconium \& stainless steel}

Typical fuel: Shippingport (HEU), commercial (LEU), Saxton (MEU), ML-1 (HEU), PBF (MEU), FFTF-TFA (LEU)

\section{Fuel Description}

The fuels in this category generally have the characteristics found in most of the commercial fuels (PWR and BWR). For one reason or another, these fuels have ended up in the DOE SNF inventory. As an example, the commercial fuels were brought to the DOE site for examination or testing programs while some were reconfigured for the Dry rod consolidation Test (DRCT) at the INEEL. The reconfiguration involved consolidating the fuel by removing the rods and placing them into canisters that doubles number of rods in a volume equal to a standard commercial fuel assembly. Other examination or testing involved taking some of the assemblies and rods apart for post-irradiation examination. The fuel compositions, properties, and conditions are identical to the commercial fuel [Reference 11].

The Power Burst Facility (PBF) was used to test fuel materials and the driver fuel was included in the category 4 inventory. The PBF driver core fuel contains a pelletized ternary fuel (UO2-ZrO2-CaO-18.5\% enriched) surround by a Helium gas annulus, an insulator sleeve of ( $\mathrm{ZrO} 2-\mathrm{CaO})$, and cladded with $304 \mathrm{~L}$ stainless steel. This fuel is similar to commercial fuel that is made by pressing the uranium oxide into pellets. The pellets are loaded into zircaloy or stainless steel tubes [Reference 10].

Another fuels such as the Shippingport PWR Core 2 Seed 2 was also included in the category 4 inventory. The Shippingport PWR was built to demonstrate the concept of a light water, slow breeder reactor using a commercial type pressurized water reactor (PWR). This was a joint AEC/Navy project that was designed for development and demonstration purposes of this type of reactor. Bettis Atomic Power Laboratory designed the reactor. The Naval Reactors Group of the AEC directed the project, and the power was distributed by Duquesne Light Company. The Navy's NRF and ECF facilities received the fuel after it was removed from the core. The Navy played a large part in all aspects of this reactor. Shippingport was designed and built to test different core designs and explore operating variables for large-scale nuclear reactors. The reactor was of the seed and blanket type and began operation with the first core (PWR-C1) in December 1957. The seed element was a Zircaloy clad plate type fuel while the blanket fuel was in the form of pellets placed inside short ( $\left.10^{\prime \prime}\right)$ Zircaloy-2 tubes. The basic component of the seed elements was the fuel plate. A plate was formed by sandwiching an enriched ( $93 \%) \mathrm{U}-\mathrm{Zr}$ alloy strip between two Zircaloy-2 cover plates and four side strips. Figure A.8 shows the Shippingport PWR fuel subassembly [Reference 10].

\begin{tabular}{|l|l|l|l|}
\hline $\begin{array}{l}\text { Appendix A } \\
\text { DOE SNF Information }\end{array}$ & $\begin{array}{l}\text { Sheet } \\
27 \text { of } 75\end{array}$ & $\begin{array}{l}\text { Date: } \\
\text { January 23, 1998 }\end{array}$ & $\begin{array}{l}\text { Rev. } \\
1\end{array}$ \\
\hline
\end{tabular}


For purposes of this PA, these commercial or commercial like fuels will be analyzed as separate packages of SNF, but this analysis will be done using commercial fuel properties in conjunction with the PWR (Category 14) and BWR (Category 15) representing the 63,000 MTHM portion of the repository design.

\begin{tabular}{|l|l|l|}
\hline \multicolumn{3}{|l|}{ Category 4 U-Oxide Intact Fuel Inventories/Information } \\
\hline $\begin{array}{l}\text { Radionuclide Inventory } \\
\text { (41 isotopes) }\end{array}$ & $\begin{array}{l}\text { Refer to TSPA group listing data - Table } \\
\text { A-7 }\end{array}$ & \\
\hline Composition & Pressed uranium oxide pellets & \\
\hline Wet Dissolution Rate & Commercial Model & \\
\hline Surface Area (m $\left.{ }^{2} / g\right)$ & $5.179 E-05$ & \\
\hline Clad Failure Fraction & Commercial & \\
\hline $\begin{array}{l}\text { Free Radionuclide Inventory } \\
\text { Fraction }\end{array}$ & Commercial & \\
\hline
\end{tabular}

\section{Configuration, Mass, and Package Count}

The following table shows the disposal configuration, average package mass, repository package count, and HLW mass used to co-disposed the category 4 SNF.

Category $4 \mathrm{U}$ oxide, $\mathrm{Zr} / \mathrm{SST}$ clad

\begin{tabular}{|c|c|c|c|c|c|c|}
$\# 3 \times 1$ & $\# 3 \times 51$ & $\# 4 \times 1$ & $\# 4 \times 1$ & $\# 5 x 1$ & $\# 5 x 1$ & $\# 0 \times 4$ \\
$10 \mathrm{ft}$ & $15 \mathrm{ft}$ & $10 \mathrm{ft}$ & $15 \mathrm{ft}$ & $10 \mathrm{ft}$ & $15 \mathrm{ft}$ & $15 \mathrm{ft}$ \\
\hline
\end{tabular}
- ave package mass (MT)

- repository pkg count

- HLW can count

33.5

- HLW mass (MT)

- SNF pkg count

- ave U mass (kg)

- ave. fissile mass (kg)
3

9

19.35

3

$\cdot 9.64$

8.88

\section{8}

2

6

19.2

2

152.9

2.6
30.5

60

240

516

60

27.86

5.66

\section{9}

41

164

524.8

41

589.87

10.5

\section{4}

47

235

505.25

47

46.72

6.32
43.7

22

110

352

22

351.4

7.75
Appendix A

DOE SNF Information

\section{Sheet}

28 of 75
Date:

January 23, 1998
Rev.

1 


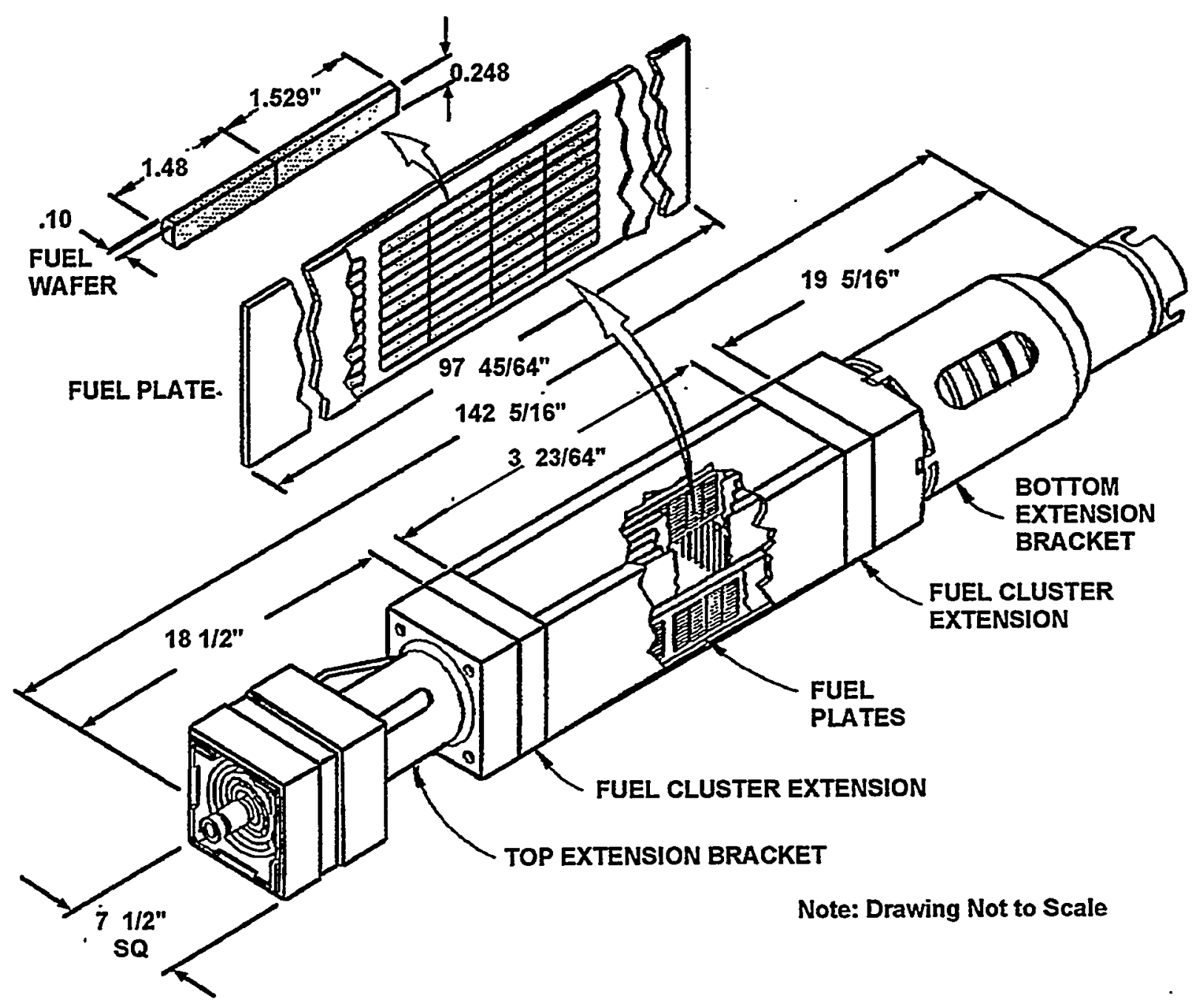

Figure A.8 Shippingport Core 2 Blanket Fuel Assembly

\begin{tabular}{|l|l|l|l|}
\hline $\begin{array}{l}\text { Appendix A } \\
\text { DOE SNF Information }\end{array}$ & $\begin{array}{l}\text { Sheet } \\
29 \text { of } 75\end{array}$ & $\begin{array}{l}\text { Date: } \\
\text { January 23, 1998 }\end{array}$ & $\begin{array}{l}\text { Rev. } \\
1\end{array}$ \\
\hline
\end{tabular}




\subsection{Category 5 U oxide/Failed clad \& Al}

Typical fuel: SM-1A, ORNL SST \& Zr (MEU), TMI-2 (LEU), HFIR, FRR, MTR

\section{Fuel Description}

The fuels in this category represent those materials that are already damaged, disrupted, or considered the least robust in terms of immediate fissile and fission product movement upon package breach. The fuels in this category have been disrupted from their original configuration for number of reasons such as operational activities, testing, accidents, or destructive examination.

The bulk of this category consists of the packaged TMI-2 debris. The fuel was a typical commercial pressurized water nuclear reactor fuel until it melted in a reactor accident. It now consists of materials with sizes ranging from fines to nearly intact assemblies. Some of which have been melted and cooled. The fuel debris was placed into three types of stainless steel canisters: filter canister that contain the fines, knockout canisters that contain gravel consistency materials, and fuel canisters that contain large pieces of melted or unaffected assemblies. The materials have been extensively characterized as part of the TMI-2 reactor analysis [Reference 11].

Primary issues related to packaging this fuel category for disposal related to: (1) packaging for criticality control and, (2) drying material to prevent gas generation. Figure A.9 shows the canister configuration for the Three Mile Island unit 2 (TMI-2).

\begin{tabular}{|l|l|l|}
\hline Category 5 U-Oxide Failed Clad Fuel Inventories/Information \\
\hline $\begin{array}{l}\text { Radionuclide Inventory } \\
\text { (41 isotopes) }\end{array}$ & $\begin{array}{l}\text { Refer to TSPA group listing data - Table } \\
\text { A-7 }\end{array}$ & \\
\hline Composition & Pressed uranium oxide pellets & \\
\hline Wet Dissolution Rate & Commercial Model & \\
\hline Surface Area $\left(\mathrm{m}^{2} / \mathrm{g}\right)$ & $5.10 \mathrm{E}-03$ & \\
\hline Clad Failure Fraction & $100 \%$ & \\
\hline $\begin{array}{l}\text { Free Radionuclide Inventory } \\
\text { Fraction }\end{array}$ & $0.01 \%$ & \\
\hline
\end{tabular}

Appendix A DOE SNF Information

\section{Sheet} 30 of 75
Date: January 23, 1998
Rev. 1 


\section{Configuration, Mass, and Package Count}

The following table shows the disposal configuration, average package mass, repository package count, and HLW mass used to co-disposed the category 5 SNF.

\begin{tabular}{|c|c|c|c|c|c|c|}
$\# 3 \times 1$ & $\# 3 \times 1$ & $\# 4 \times 1$ & $\# 4 \times 1$ & $\# 5 \times 1$ & $\# 5 \times 1$ & $\# 0 \times 4$ \\
$10 \mathrm{ft}$ & $15 \mathrm{ft}$ & $10 \mathrm{ft}$ & $15 \mathrm{ft}$ & $10 \mathrm{ft}$ & $15 \mathrm{ft}$ & $15 \mathrm{ft}$ \\
\hline
\end{tabular}

Category $5 \mathrm{U}$ oxide, mixed clad

- ave package mass (MT)

- repository pkg count

- HLW can count

- HLW mass (MT)

- SNF pkg count

- ave U mass (kg)

$\begin{array}{rrrrrr}23.96 & 35.7 & 26.3 & 35.4 & 35.6 & 47.4 \\ 382 & 19 & 11 & 27 & 26 & 44 \\ 1146 & 57 & 44 & 108 & 130 & 220 \\ 2463.9 & 182.4 & 94.6 & 345.6 & 279.5 & 704 \\ 382 & 19 & 11 & 27 & 26 & 44 \\ 6.49 & 48.79 & 22.75 & 3.15 & 13.78 & 2.86 \\ 5.47 & 9.71 & 0.65 & 2.77 & 7.87 & 1.22\end{array}$

- ave. fissile mass $(\mathrm{kg}) \quad 5.47$ 


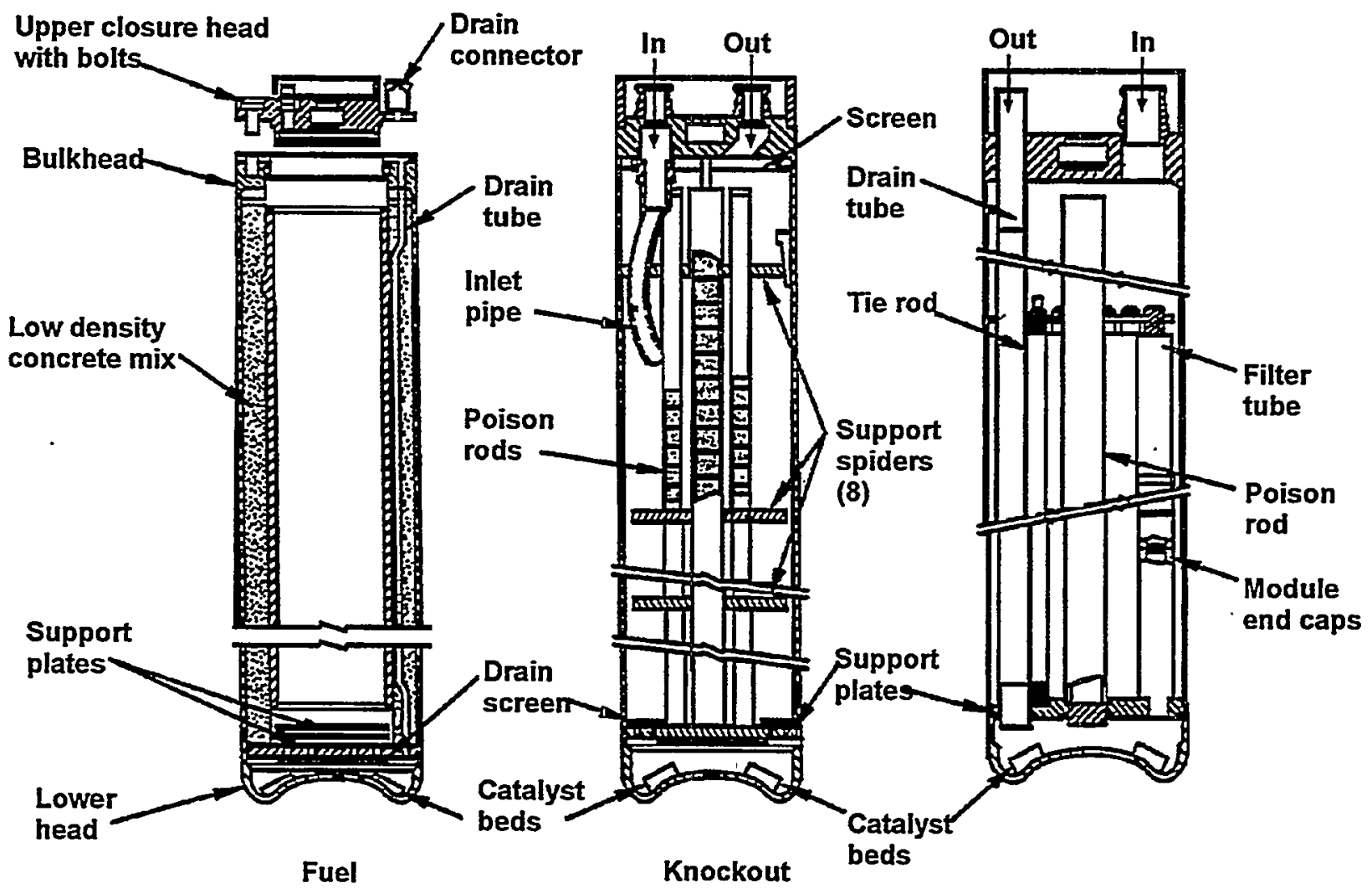

Diagram of the Three TMI-2 Canister Types

Figure A.9 TMI-2 Canister Types

\begin{tabular}{|l|l|l|l|}
\hline $\begin{array}{l}\text { Appendix A } \\
\text { DOE SNF Information }\end{array}$ & $\begin{array}{l}\text { Sheet } \\
32 \text { of } 75\end{array}$ & $\begin{array}{l}\text { Date: } \\
\text { January 23, 1998 }\end{array}$ & $\begin{array}{l}\text { Rev. } \\
1\end{array}$ \\
\hline
\end{tabular}




\subsection{Category $6 \quad \mathrm{U}-\mathrm{Al}_{x} / \mathrm{Al}$}

Typical fuel: ATR (HEU), MTR, FRR (MEU)

\section{Fuel Description}

This category includes fuels composed of a uranium-aluminum alloy. The cladding is assumed to be intact at this time, but is not considered to be a very durable material in longterm storage conditions in wet environments. The nature of the cladding suggests application of a lower allowable centerline temperature $(\sim 200 \mathrm{C})$ within a waste package.

The typical Advanced Test Reactor (ATR) a fuel element consists of 19 curved aluminum clad fuel plates swaged into two non-fueled aluminum side plates. The 19 curved (concentric) aluminum clad UAlx fuel plates form a pie shaped geometry. The fuel meat consists of UAlx, boron, and aluminum particles mixed together and pressed. into a $0.015^{\prime \prime}$ thick plate and clad with a 6061 aluminum foil (nominally 15 mils). The uranium and poison loadings are varied among the fuel plates giving a total U-235 loading of 1075 grams per fuel element [Reference 10]. Figure A.10 shows the ATR fuel configuration. below.

Other UAlx fuels are similarly constructed and generic fuel information is indicated

\begin{tabular}{|l|l|l|}
\hline Category 6 U-Al $\mathrm{Al}$ Clad Fuel Inventories/Information \\
\hline $\begin{array}{l}\text { Radionuclide Inventory } \\
\text { (41 isotopes) }\end{array}$ & $\begin{array}{l}\text { Refer to TSPA group listing data - Table } \\
\mathrm{A}-7\end{array}$ & \\
\hline Composition & UAlx dispersed in aluminum & \\
\hline Wet Dissolution Rate & Metal Model & \\
\hline Surface Area (m $\left.\mathrm{m}^{2} / \mathrm{g}\right)$ & $1.30 \mathrm{E}-03$ & \\
\hline Clad Failure Fraction & $100 \%$ & \\
\hline $\begin{array}{l}\text { Free Radionuclide Inventory } \\
\text { Fraction }\end{array}$ & $0.01 \%$ & \\
\hline
\end{tabular}

\begin{tabular}{|l|l|l|l|}
\hline $\begin{array}{l}\text { Appendix A } \\
\text { DOE SNF Information }\end{array}$ & $\begin{array}{l}\text { Sheet } \\
33 \text { of } 75\end{array}$ & $\begin{array}{l}\text { Date: } \\
\text { January 23, 1998 }\end{array}$ & $\begin{array}{l}\text { Rev. } \\
1\end{array}$ \\
\hline
\end{tabular}




\section{Configuration, Mass, and Package Count}

The following table shows the disposal configuration, average package mass, repository package count, and HLW mass used to co-disposed the category 6 SNF.

\begin{tabular}{|c|c|c|c|c|c|c|}
$\# 3 \times 1$ & $\# 3 \times 1$ & $\# 4 \times 1$ & $\# 4 \times 1$ & $\# 5 \times 1$ & $\# 5 \times 1$ & $\# 0 \times 4$ \\
$10 \mathrm{ft}$ & $15 \mathrm{ft}$ & $10 \mathrm{ft}$ & $15 \mathrm{ft}$ & $10 \mathrm{ft}$ & $15 \mathrm{ft}$ & $15 \mathrm{ft}$ \\
\hline
\end{tabular}

Category 6 U alloy, aluminum clad

- ave package mass

- repository pkg count

- HLW can count

- HLW mass (MT)

- SNF pkg count

- ave U mass (kg)

- ave. fissile mass $(\mathrm{kg})$

$\begin{array}{rr}33.7 & 47.9 \\ 231 & 519 \\ 1155 & 2595 \\ 2483.25 & 5579.25 \\ 231 & 519 \\ 8.55 & 13.38 \\ 7.09 & 8.48\end{array}$




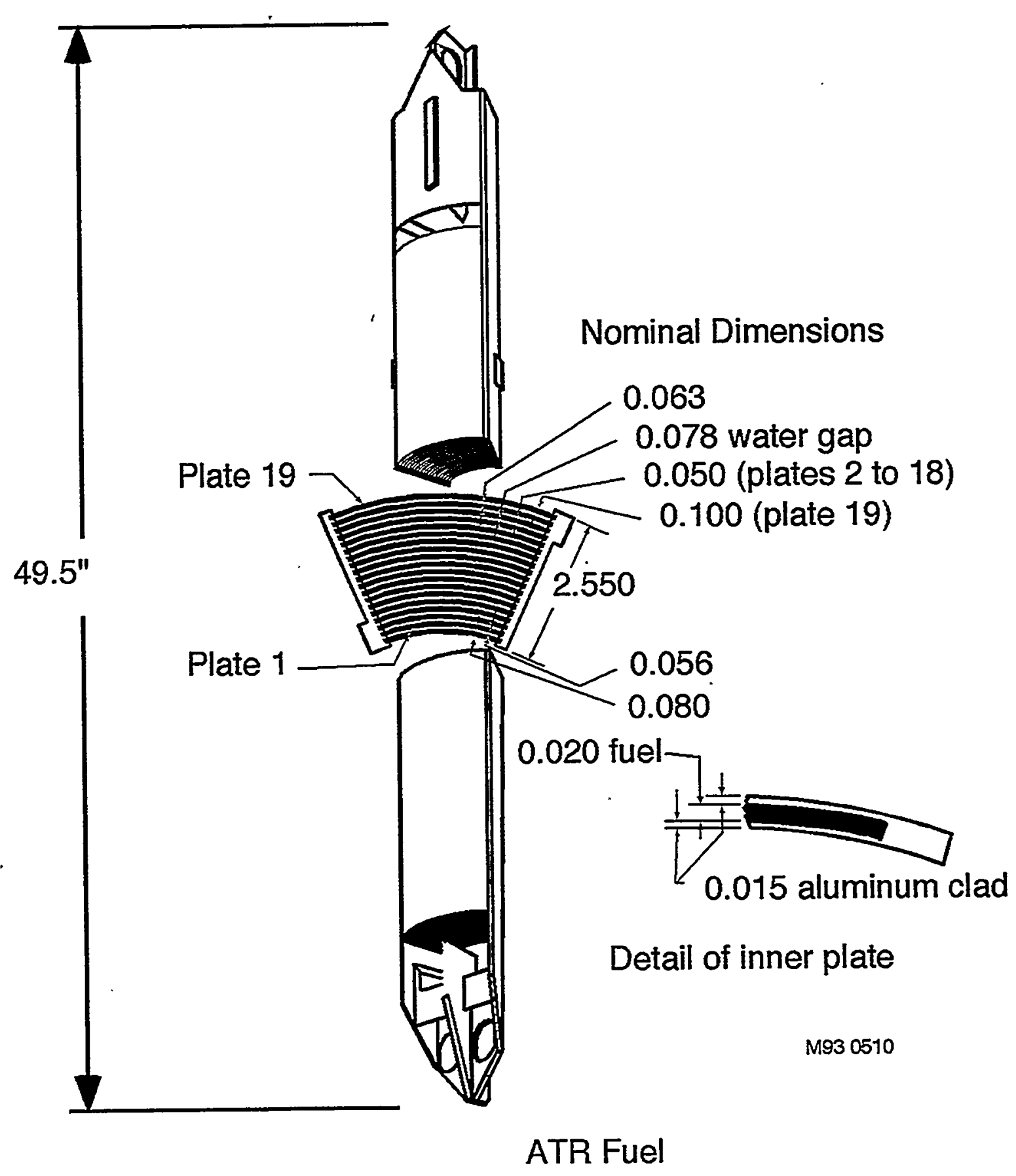

Figure A.10 ATR Fuel Element 


\subsection{Category 7 U-Si}

Typical fuel: MTR, FRR (HEU, MEU)

\section{Fuel Description}

The typical fuels in this category come from material test reactors and foreign research reactors (FRR). Most foreign research reactors will continue to operate during the next several years. Foreign reactor reactors use a number of different fuel designs. These designs can be placed into five groups: (1) plate-type design, (2) concentric tube-type design, (3) pin-type design, (4) special-type design and (5) rod-type design. The various designs are shown in the Final Environmental Impact Statement on a Proposed Nuclear Weapons Nonproliferation Policy Concerning Foreign Research Reactor spent Nuclear Fuel Appendix B, DOE/EIS0218F February 1996.

The plate type design is described here since it is used in the majority of the FRR. The thermal power of these reactors ranges from $1 \mathrm{MW}$ to $50 \mathrm{MW}$. Each fuel assembly contains between 6 to 23 plates and an initial U-235 content between 37 grams to 420 grams. The fuel matrix consists of U-Si dispersed in aluminum. Figure A.11 shows the plate type MTR element.

\section{Category 7 U-Si Fuel Inventories/Information}

\begin{tabular}{|l|l|l|}
\hline $\begin{array}{l}\text { Radionuclide Inventory } \\
\text { (41 isotopes) }\end{array}$ & $\begin{array}{l}\text { Refer to TSPA group listing data - Table } \\
\text { A-7 }\end{array}$ & \\
\hline Composition & U-Si dispersed in aluminum & \\
\hline Wet Dissolution Rate & Metal Model x 0.1 & \\
\hline Surface Area $\left(\mathrm{m}^{2} / \mathrm{g}\right)$ & $1.30 \mathrm{E}-03$ & \\
\hline Clad Failure Fraction & $100 \%$ & \\
\hline $\begin{array}{l}\text { Free Radionuclide Inventory } \\
\text { Fraction }\end{array}$ & $0.01 \%$ & \\
\hline
\end{tabular}

Date:

January 23,1998
Rev.

1 


\section{Configuration, Mass, and Package Count}

The following table shows the disposal configuration, average package mass, repository package count, and HLW mass used to co-disposed the category 7 SNF.

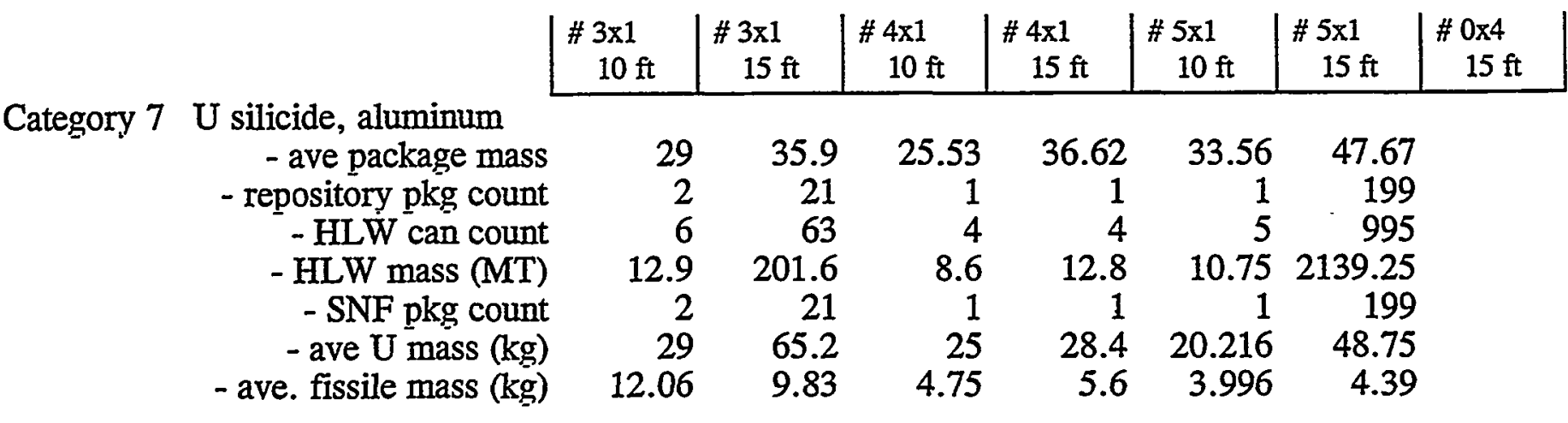




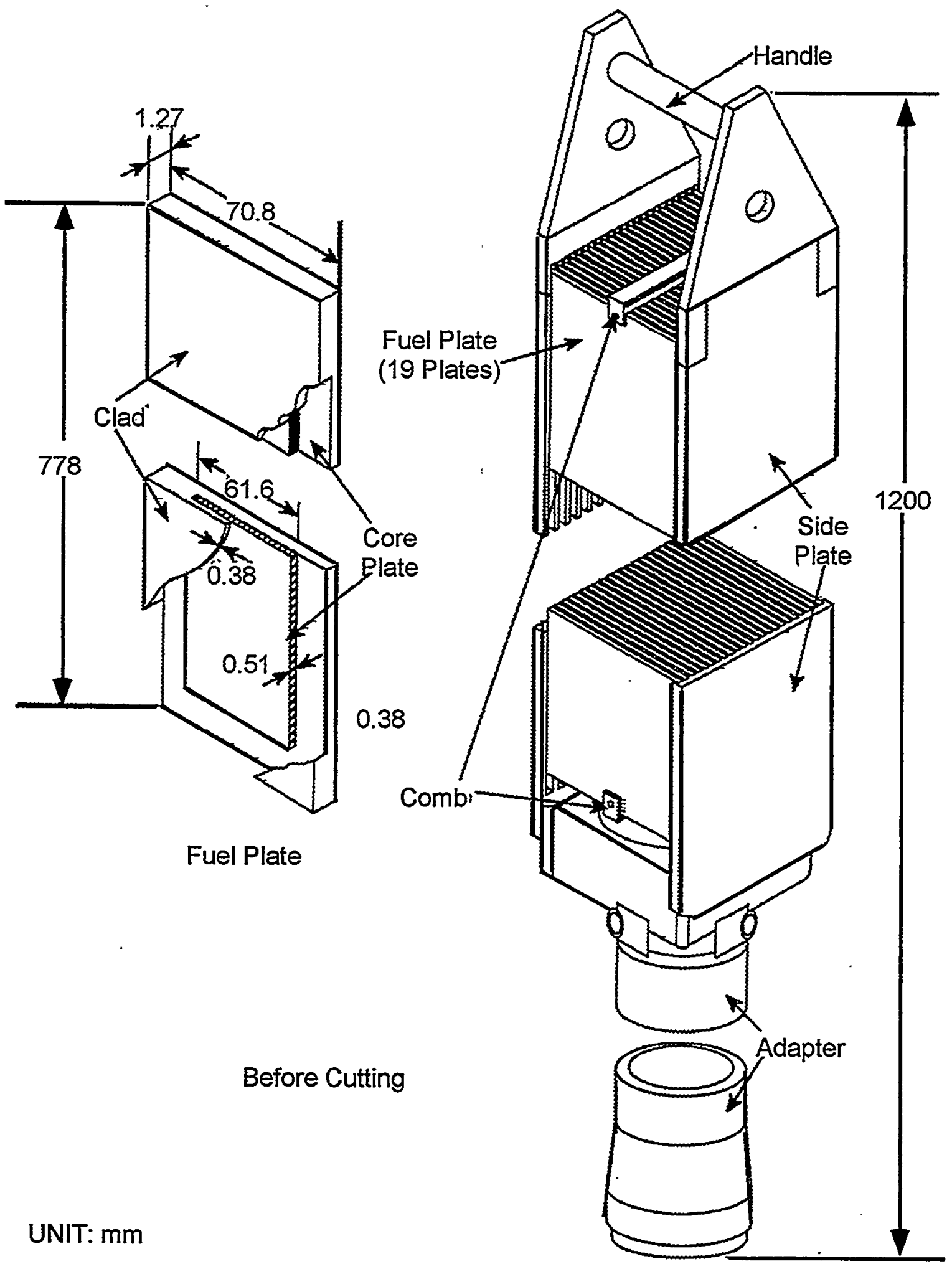

Figure A.11 Typical (Boxed-Type/Flat Plate) FRR Fuel Element

\begin{tabular}{|l|l|l|l|}
\hline Appendix A & Sheet & Date: & Rev. \\
DOE SNF Information & 38 of 75 & January 23, 1998 & 1 \\
\hline
\end{tabular}




\subsection{Category 8 U/Th carbide (hi-integrity)/graphite}

Typical fuel: Fort Saint Vrain (FSV)

\section{Fuel Description}

The FSV fuel is a graphite based fuel that was used only in the Fort Saint Vrain Reactor. An assembly is composed of a hexagonal shaped graphite block drilled with 102 coolant holes and 210 fuel holes. The fuel is made of highly enriched uranium carbide and thorium carbide spheres coated with layers of pyrolytic carbon followed by a SiC protective outer coating, which is very durable, and an outer pyrolytic coating. The fuel spheres are sintered with carbon and formed into rods, called compacts, and then stacked into the fuel holes within large hexagonal blocks of graphite. These blocks are $36 \mathrm{~cm}$ (14.172 in) across the flats, $20.6 \mathrm{~cm}$ (8.102 in) on each side, and $79.3 \mathrm{~cm}$ (31.22 in) long [Reference 11]. Figure A.12 shows the Fort Saint Vrain fuel assembly.

\begin{tabular}{|l|l|l|}
\hline Category 8 U/Th carbide (high-integrity) Fuel Inventories/Information \\
\hline $\begin{array}{l}\text { Radionuclide Inventory } \\
\text { (41 isotopes) }\end{array}$ & $\begin{array}{l}\text { Refer to TSPA group listing data - Table } \\
\text { A-7 }\end{array}$ & \\
\hline Composition & U/Th Carbide & \\
\hline Wet Dissolution Rate & Carbide Model & \\
\hline Surface Area (m²) & $3.40 \mathrm{E}-03$ & \\
\hline Clad Failure Fraction & $1 \%$ & \\
\hline $\begin{array}{l}\text { Free Radionuclide Inventory } \\
\text { Fraction }\end{array}$ & $0.001 \%$ & \\
\hline
\end{tabular}

\section{Configuration, Mass, and Package Count}

The following table shows the disposal configuration, average package mass, repository package count, and HLW mass used to co-disposed the category 8 SNF.

Category $8 \mathrm{Th} / \mathrm{U}$ carbide, graphite (FSV)

\begin{tabular}{|c|c|c|c|c|c|c|}
$\# 3 \times 1$ & $\# 3 \times 1$ & $\# 4 \times 1$ & $\# 4 \times 1$ & $\# 5 \times 1$ & $\# 5 \times 1$ & $\# 0 \times 4$ \\
$10 \mathrm{ft}$ & $15 \mathrm{ft}$ & $10 \mathrm{ft}$ & $15 \mathrm{ft}$ & $10 \mathrm{ft}$ & $15 \mathrm{ft}$ & $15 \mathrm{ft}$ \\
\hline
\end{tabular}

- ave package mass

- repository pkg count

- HLW can count

- HLW mass (MT)

- SNF pkg count

- ave U mass $(\mathrm{kg})$ 


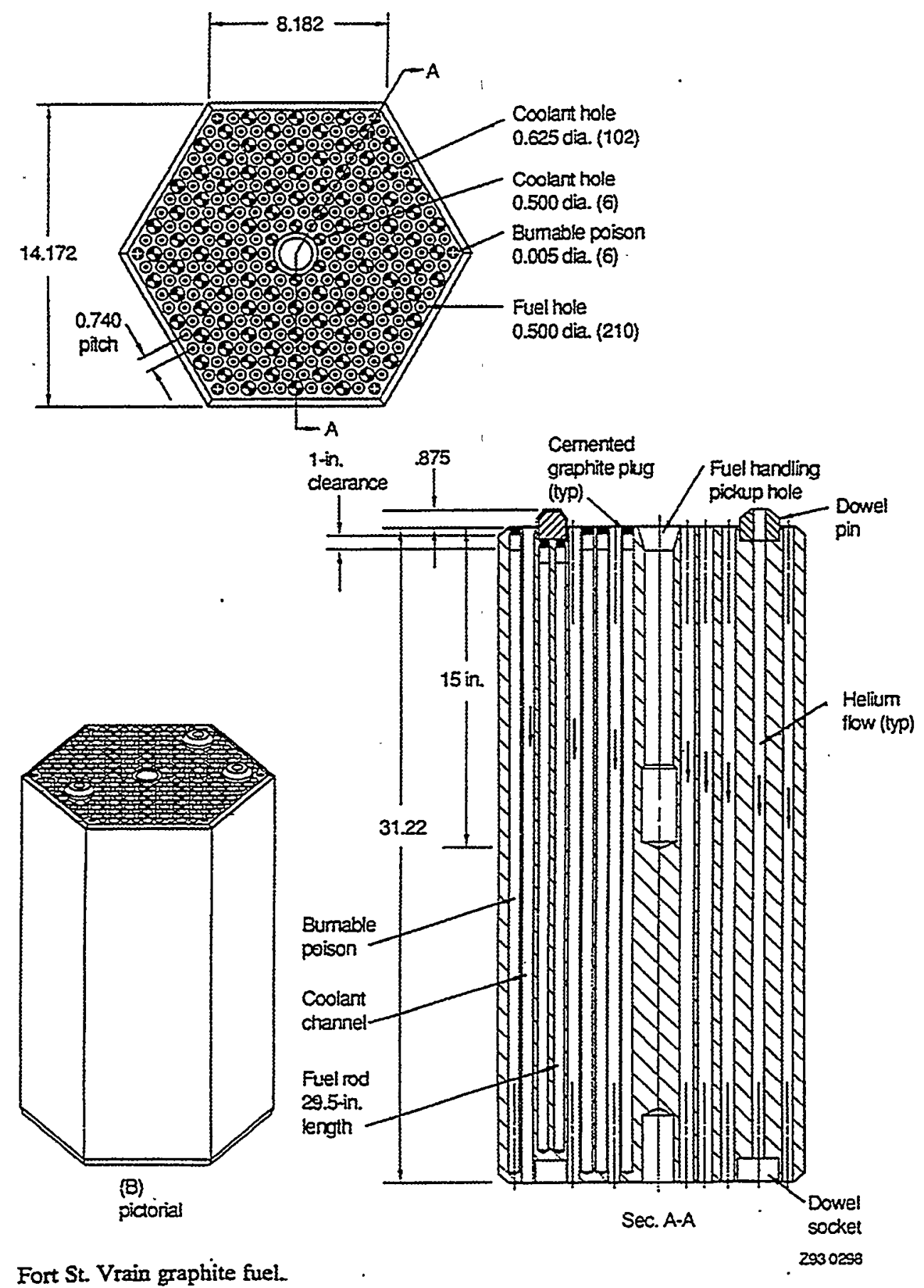

Figure A.12 Ft. St. Vrain Graphite Fuel

\begin{tabular}{|l|l|l|l|}
\hline $\begin{array}{l}\text { Appendix A } \\
\text { DOE SNF Information }\end{array}$ & $\begin{array}{l}\text { Sheet } \\
40 \text { of } 75\end{array}$ & $\begin{array}{l}\text { Date: } \\
\text { January 23, 1998 }\end{array}$ & $\begin{array}{l}\text { Rev. } \\
1\end{array}$ \\
\hline
\end{tabular}




\subsection{Category 9 U/Th carbide (low-integrity)/ graphite \\ Typical fuel: Peach Bottom}

\section{Fuel Description}

The Peachbottom (PB) Cores 1 and 2 are a graphite based fuel that is made of mixed uranium carbide and thorium carbide particles ranging from 295 to 630 microns in diameter and coated with pyrolytic carbon. However, there is no a SiC protective outer coating on the fuel particles. The particles are formed into annular compacts $7.6 \mathrm{~cm}$ (2.98 in) high with a center hole diameter of $4.45 \mathrm{~cm}(1.75 \mathrm{in})$ and an outside diameter of $6.86 \mathrm{~cm}(2.7 \mathrm{in})$. the compacts are stacked on a $76.2 \mathrm{~cm}$ (30 in) long graphite spine. Three units make up the 228.6 $\mathrm{cm}$ (90 in) long fuel section. An annular low-permeability graphite sleeve is slipped over the fuel compacts [Reference 11]. The failure rate of the particles is estimated to be considerable higher than the FSV fuel particles. Figures A.13 and A.14 show the PB fuel assembly.

\begin{tabular}{|l|l|l|}
\hline Category 9 U/Th Carbide (low-integrity) Fuel Inventories/Information \\
\hline $\begin{array}{l}\text { Radionuclide Inventory } \\
\text { (41 isotopes) }\end{array}$ & $\begin{array}{l}\text { Refer to TSPA group listing data - Table } \\
\text { A-7 }\end{array}$ & \\
\hline Composition & U/Th Carbide & \\
\hline Wet Dissolution Rate & Carbide Model & \\
\hline Surface Area $\left(\mathrm{m}^{2} / \mathrm{g}\right)$ & $5.179 \mathrm{E}-05$ & \\
\hline Clad Failure Fraction & $35 \%$ & \\
\hline $\begin{array}{l}\text { Free Radionuclide Inventory } \\
\text { Fraction }\end{array}$ & $0.0001 \%$ & \\
\hline
\end{tabular}

\section{Configuration, Mass, and Package Count}

The following table shows the disposal configuration, average package mass, repository package count, and HLW mass used to co-disposed the category 9 SNF.

Category $9 \mathrm{Th} / \mathrm{U}$ carbide, graphite (PB)

\begin{tabular}{|c|c|c|c|c|c|c|}
$\# 3 \times 1$ & $\# 3 \times 1$ & $\# 4 \times 1$ & $\# 4 \times 1$ & $\# 5 \times 1$ & $\# 5 \times 1$ & $\# 0 \times 4$ \\
$10 \mathrm{ft}$ & $15 \mathrm{ft}$ & $10 \mathrm{ft}$ & $15 \mathrm{ft}$ & $10 \mathrm{ft}$ & $15 \mathrm{ft}$ & $15 \mathrm{ft}$ \\
\hline
\end{tabular}

- ave package mass

- repository pkg count

- HLW can count

48.14

103

- HLW mass (MT)

515

- SNF pkg count

1648

- ave U mass (kg)

103

- ave. fissile mass (kg)

\begin{tabular}{|l|l|l|l|}
\hline $\begin{array}{l}\text { Appendix A } \\
\text { DOE SNF Information }\end{array}$ & $\begin{array}{l}\text { Sheet } \\
41 \text { of } 75\end{array}$ & $\begin{array}{l}\text { Date: } \\
\text { January 23, 1998 }\end{array}$ & $\begin{array}{l}\text { Rev. } \\
1\end{array}$ \\
\hline
\end{tabular}




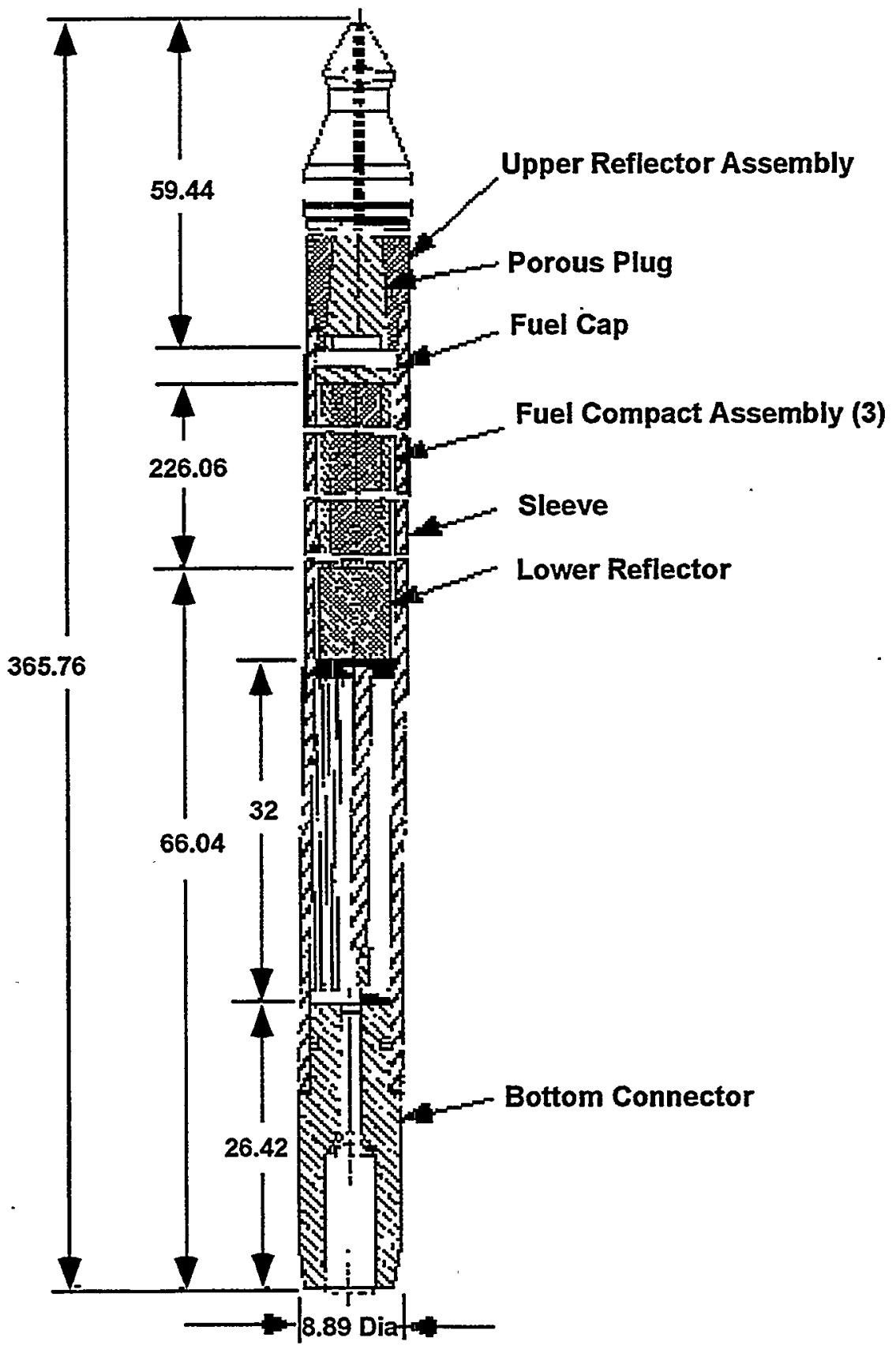

Figure A.13 Peach Bottom Unit 1, Core 1 Fuel Element (Drawing not to scale; dimensions in centimeters) 


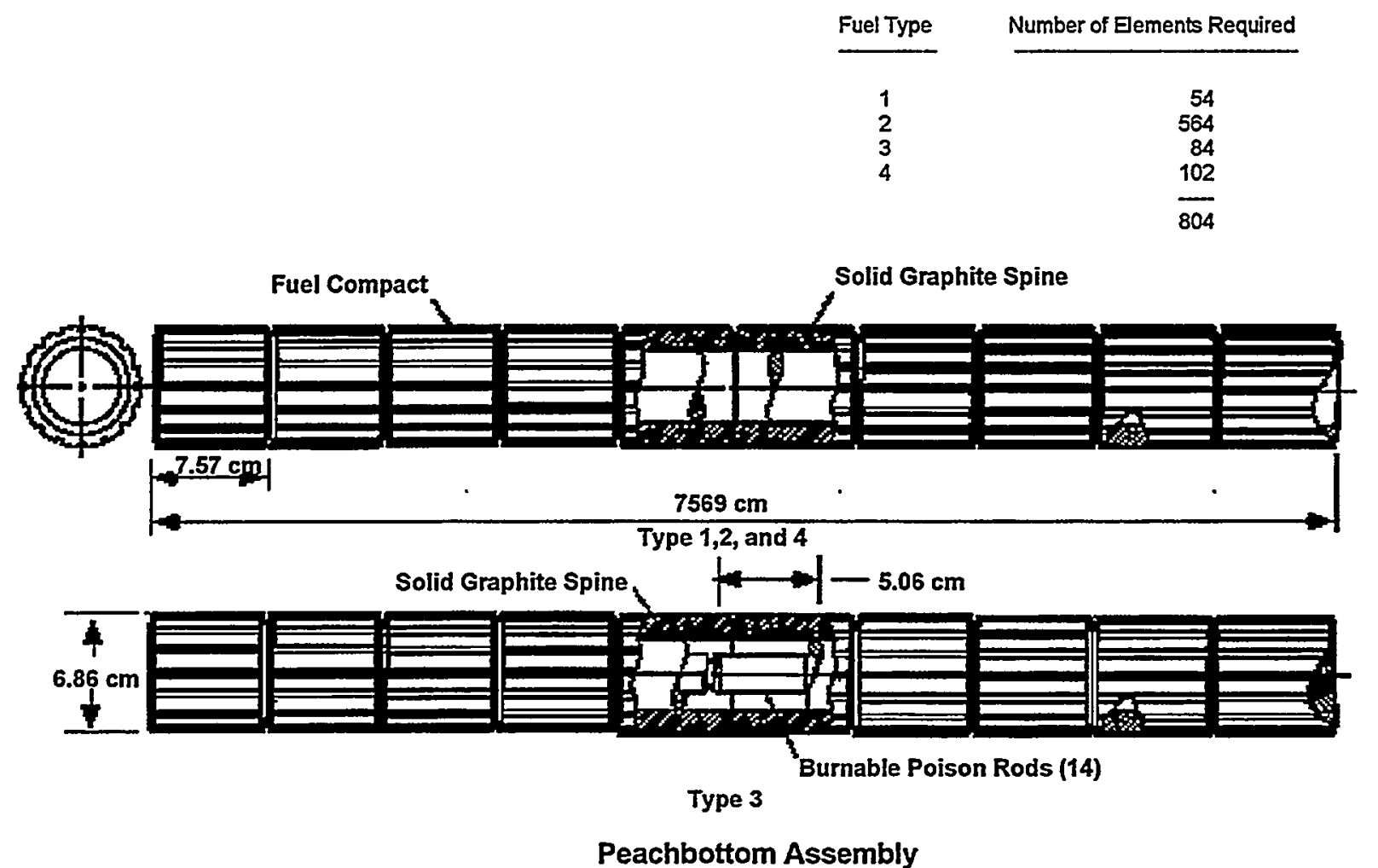

Figure A.14 Peach Bottom Unit 1, Core 1 Fuel Compacts

(Drawing not to scale)

\begin{tabular}{|l|l|c}
\hline $\begin{array}{l}\text { Sheet } \\
43 \text { of } 75\end{array}$ & $\begin{array}{l}\text { Date: } \\
\text { January 23, 1998 }\end{array}$ & Rev. \\
\hline
\end{tabular}




\subsection{Category $10 \mathrm{U} \& \mathrm{U} / \mathrm{Pu}$ carbide / non graphite}

Typical fuel: SRE (MEU FGE), FFTF Carbide (MEU FGE)

\section{Fuel Description}

Category 10 fuel are mixed carbide fuel in a non graphite matrix. A number of the fuels were test fuel assemblies (TFAs) from the Fast Flux Test Facility (FFTF). FFTF was to provide testing capability for a wide range of development needs of the United States advanced reactor program. The mission of the FFTF included irradiation and evaluation of different types of fuel assemblies and different materials for fuel assembly construction. The purposes of the TFAs varies and a few examples are indicated below. However, in general, the TFAs support the fuel or material requirements for large scale breeder reactors.

As an example, the FFTF-ACN-1 fuel in this category was tested to develop information on helium- and sodium-bonded mixed-carbide fuel pins with full length fuel columns at prototypic fluence and exposure conditions. Additionally, it tests the relative effects of $20 \%$ cold worked 316 SS and $25 \%$ cold worked D9 cladding on the carbide fuel pins. The assembly contains 18 sodium-bonded and 19 helium-bonded carbide fuel pins, enclosed in a 316 SS inner duct similar to the SRF-3. The outer region contains 90 standard driver fuel pins and is enclosed by a D9 duct [Reference 7]. The test fuel assembly's (TFAs) configuration is similar to the FFTF driver fuels shown on Figures A.15 and A.16 under category 11.

Another fuel assembly, the FFTF-AC-3 was tested in cooperative effort of the United States and Swiss governments and was part of the advanced liquid metal fast breeder reactor fuel program. The test compared performance of 66 pins containing pelletized fuel with that of 25 sphere-pac fuel pins at typical conditions of the breeder reactor. The pins are D9-clad, wire-wrapped, and were housed in a D9 duct. The fuel is mixed plutonium-uranium carbide with plutonium enrichments of $19.1 \%$ for the sphere-pac fuel and $19.7 \%$ for the pelletized fuel [Reference 7].

And the FFTF-FC-1 assembly was tested to establish performance characteristics of a full size carbide fuel assembly. The assembly contains 91 large diameter [0.94 $\mathrm{cm}(0.37 \mathrm{in})]$, D9 clad, wire wrapped, helium bonded fuel pins. The plutonium enrichment is $21.4 \%$ in uranium carbide, with $16.5 \mathrm{~cm}$ (6.5 in) top and bottom blankets [Reference 7].

\begin{tabular}{|l|l|l|l|}
\hline $\begin{array}{l}\text { Appendix A } \\
\text { DOE SNF Information }\end{array}$ & $\begin{array}{l}\text { Sheet } \\
44 \text { of } 75\end{array}$ & $\begin{array}{l}\text { Date: } \\
\text { January 23, 1998 }\end{array}$ & $\begin{array}{l}\text { Rev. } \\
1\end{array}$ \\
\hline
\end{tabular}




\begin{tabular}{|l|l|l|}
\hline Category 10 U-Si Fuel Inventories/Information & \\
\hline $\begin{array}{l}\text { Radionuclide Inventory } \\
\text { (41 isotopes) }\end{array}$ & $\begin{array}{l}\text { Refer to TSPA group listing data - Table } \\
\text { A-7 }\end{array}$ & \\
\hline Composition & U \& U/Pu Carbide & \\
\hline Wet Dissolution Rate & Carbide Model & \\
\hline Surface Area (m²) & $5.179 \mathrm{E}-05$ & \\
\hline Clad Failure Fraction & $10 \%$ & \\
\hline $\begin{array}{l}\text { Free Radionuclide Inventory } \\
\text { Fraction }\end{array}$ & $0.001 \%$ & \\
\hline
\end{tabular}

\section{Configuration, Mass, and Package Count}

The following table shows the disposal configuration, average package mass, repository package count, and HLW mass used to co-disposed the category 10 SNF.

Category Pu carbide, SST clad

- ave package mass

- repository pkg count

- HLW can count

- HLW mass (MT)

- SNF pkg count

- ave U mass $(\mathrm{kg})$

- ave. fissile mass $(\mathrm{kg})$

\begin{tabular}{|c|c|c|c|c|c|c|}
$\# 3 \times 1$ & $\# 3 \times 1$ & $\# 4 \times 1$ & $\# 4 \times 1$ & $\# 5 x \mathrm{f}$ & $\# 5 \mathrm{xl}$ & $\# 0 \mathrm{x} 4$ \\
$10 \mathrm{ft}$ & $15 \mathrm{ft}$ & $10 \mathrm{ft}$ & $15 \mathrm{ft}$ & $10 \mathrm{ft}$ & $15 \mathrm{ft}$ & $15 \mathrm{ft}$ \\
\hline
\end{tabular}

$\begin{array}{rr}25.53 & 36.65 \\ 2 & 3 \\ 8 & 12 \\ 17.2 & 25.8 \\ 2 & 3 \\ 25.14 & 31.889 \\ 2.76 & 5.641\end{array}$




\subsection{Category 11 MOX/ (Zr) (SST) (other)}

Typical fuel: GE Test ((HEU FGE), FFTF-DFA (HEU FGE, FFTF-TFA-ACO (LEU \& MEU FGE)

\section{Fuel Description}

MOX fuels are composed of a mixture of uranium and plutonium oxides within various claddings. The uranium enrichment qualifies as "low" but the plutonium content increases the effective enrichment above $15 \%$. The Fast Flux Test Facility (FFTF) driver fuel assembly (DFA) and test fuel assembly (TFA) contributed to large quantity of the fuel in this category. The standard FFTF-DFA are hexagonally shaped composed of 217 fuel pins. The assembly is $3.6 \mathrm{~m}$ (12 feet) long, $11.6 \mathrm{~cm}$ (4.575 in) wide across the hexagon flats, $13.1 \mathrm{~cm}(5.16 \mathrm{in})$ wide across the hexagon points, and weight $173 \mathrm{~kg}(381 \mathrm{lb})$. Figures A.15, and A.16 show the configuration of the standard FFTF-DFA fuel assembly [Reference 7].

\begin{tabular}{|l|l|l|}
\hline Category 11 MOX Fuel Inventories/Information \\
\hline $\begin{array}{l}\text { Radionuclide Inventory } \\
\text { (41 isotopes) }\end{array}$ & $\begin{array}{l}\text { Refer to TSPA group listing data - Table } \\
\text { A-7 }\end{array}$ & \\
\hline Composition & Mixed oxide - U oxide and Pu oxide & \\
\hline Wet Dissolution Rate & Commercial Model & \\
\hline Surface Area $\left(\mathrm{m}^{2} / \mathrm{g}\right)$ & $5.179 \mathrm{E}-05$ & \\
\hline Clad Failure Fraction & $10 \%$ & \\
\hline $\begin{array}{l}\text { Free Radionuclide Inventory } \\
\text { Fraction }\end{array}$ & $0.001 \%$ & \\
\hline
\end{tabular}

\section{Configuration, Mass, and Package Count}

The following table shows the disposal configuration, average package mass, repository package count, and HLW mass used to co-disposed the category 10 SNF.

Category $11 \mathrm{Pu} / \mathrm{U}$ oxide, $\mathrm{Zr} / \mathrm{SST}$

\begin{tabular}{|c|c|c|c|c|c|c}
$\# 3 \times 1$ & $\# 3 \times 1$ & $\# 4 \times 1$ & $\# 4 \times 1$ & $\# 5 \times 1$ & $\# 5 \times 1$ & $\# 0 x^{A}$ \\
$10 \mathrm{ft}$ & $15 \mathrm{ft}$ & $10 \mathrm{ft}$ & $15 \mathrm{ft}$ & $10 \mathrm{ft}$ & $15 \mathrm{ft}$ & 16 \\
\hline
\end{tabular}

- ave package mass

- repository pkg count

- HLW can count

- HLW mass (MT)

- SNF pkg count

- ave U mass $(\mathrm{kg})$

- ave. fissile mass $(\mathrm{kg})$

$\begin{array}{rrrr}25.6 & 36.64 & 33.8 & 48.3 \\ 16 & 327 & 7 & 2 \\ 64 & 1308 & 35 & 10 \\ 137.6 & 2812.2 & 75.25 & 32 \\ 16 & 327 & 7 & 2 \\ 9.41 & 24.15 & 48.75 & 458.86 \\ 5.04 & 7.18 & 3.67 & 8.01\end{array}$

\begin{tabular}{|l|l|l|l|}
\hline Appendix A & Sheet & Date: & Rev. \\
DOE SNF Information & 46 of 75 & January 23, 1998 & 1 \\
\hline
\end{tabular}




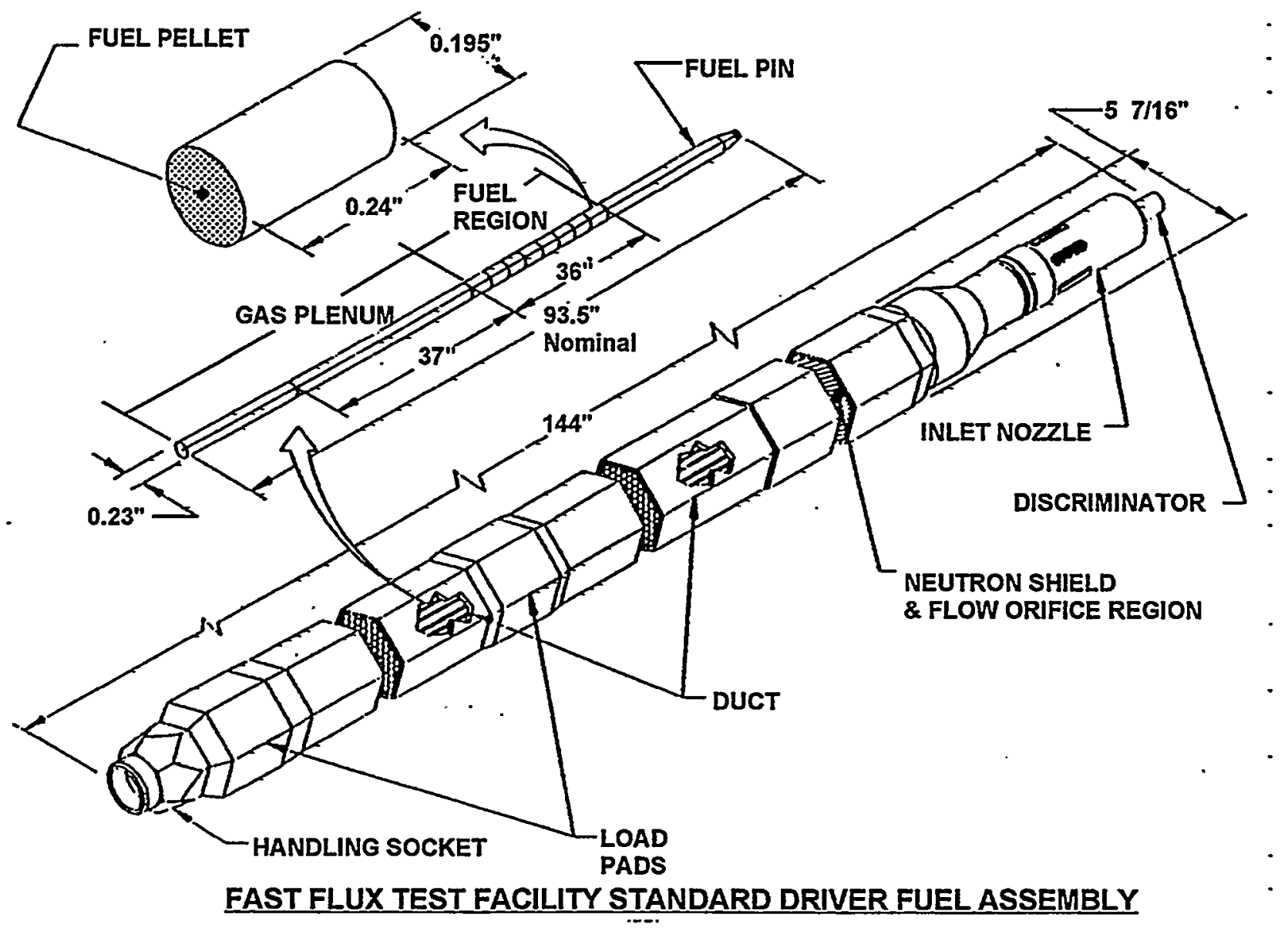

Figure A-15: FFTF Standard Driver Fuel Assembly

Appendix A

DOE SNF Information
Sheet 47 of 75
Date:

January 23, 1998
Rev.

1 


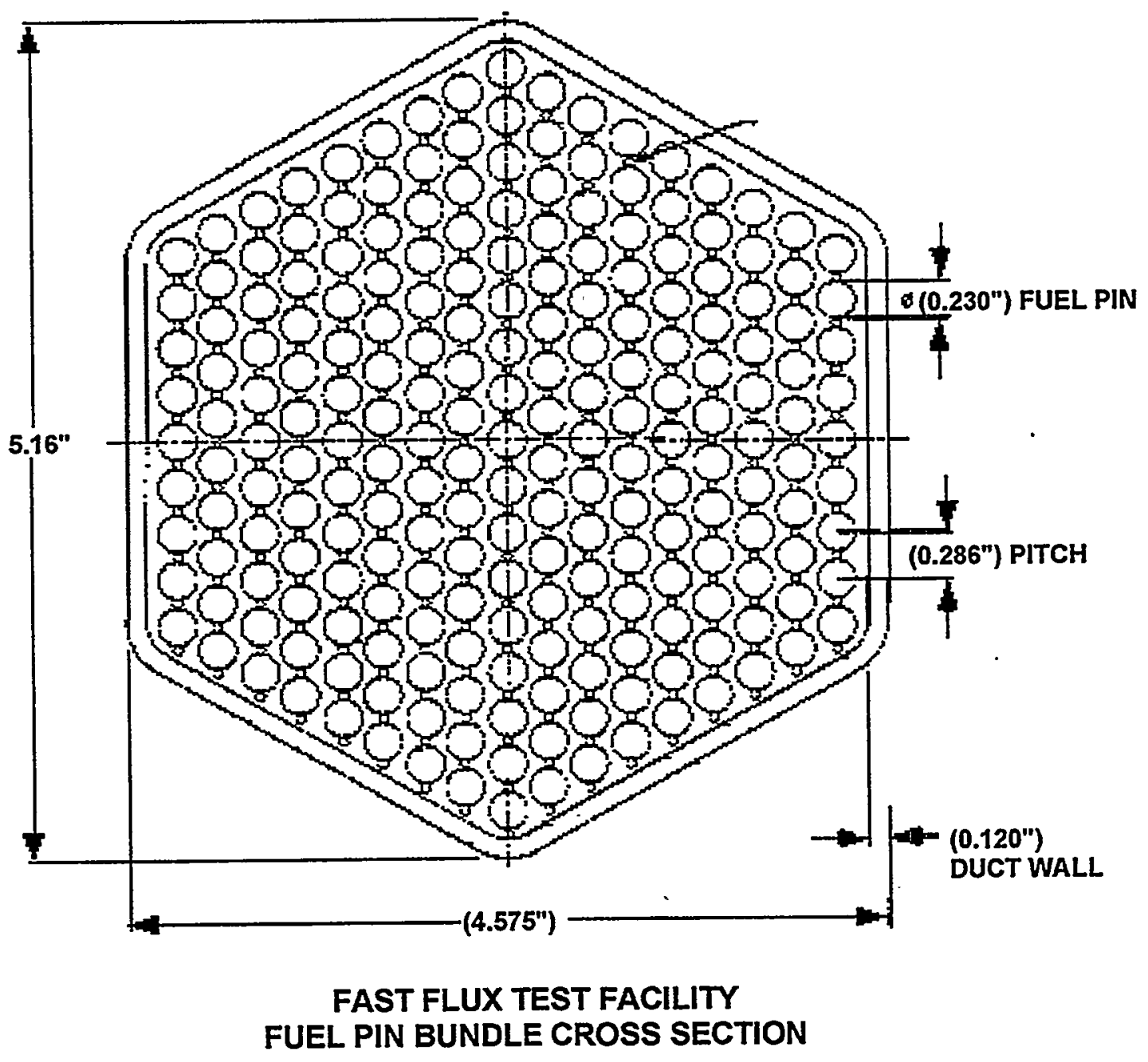

Figure A-16: FFTF Pin Bundle Cross Section 


\subsection{Category 12 U/Th oxide / (Zr) (SST)}

\section{Typical fuel: LWBR (HEU FGE), Dresden (HEU FGE)}

\section{Fuel Description}

Shippingport Light water Breeder Reactor (LWBR) fuel makes up the major inventory of the fuel in category 12. The Shippingport LWBR was used to demonstrate the production of fissile uranium 233 from thorium in a water-cooled operating reactor. The fuel was made of uranium oxide, enriched up to $98 \%$ in uranium 233 mixed with thorium oxide made into cylindrically shaped ceramic pellets. The pellets were loaded into 0.3 in diameter Zircaloy- 4 tubes whose ends are capped and seal welded. These tubes were made into assemblies. The LWBR has four different types of assemblies: 12 seed assemblies used the HEU to produce power, 12 blanket assemblies were used to capture neutrons and convert the thorium to uranium 233, and 9 type IV reflector assemblies and 6 type $V$ reflector assemblies were used to reflect neutrons back into the reactor. Figure A.17 shows the configuration of the Shippingport LWBR assembly.

\begin{tabular}{|l|l|l|}
\hline Category 12 U/Th oxide Fuel Inventories/Information & \\
\hline $\begin{array}{l}\text { Radionuclide Inventory } \\
\text { (41 isotopes) }\end{array}$ & $\begin{array}{l}\text { Refer to TSPA group listing data - Table } \\
\text { A-7 }\end{array}$ & \\
\hline Composition & U/Th Oxide & \\
\hline Wet Dissolution Rate & Ceramic Model & \\
\hline Surface Area (m²) & $1.10 \mathrm{E}-04$ & \\
\hline Clad Failure Fraction & $30 \%$ & \\
\hline $\begin{array}{l}\text { Free Radionuclide Inventory } \\
\text { Fraction }\end{array}$ & $0.00 \%$ & \\
\hline
\end{tabular}

Appendix A DOE SNF Information
Sheet 49 of 75
Date: January 23, 1998
Rev.

1 


\section{Configuration, Mass, and Package Count}

The following table shows the disposal configuration, average package mass, repository package count, and HLW mass used to co-disposed the category 12 SNF.

\begin{tabular}{|c|c|c|c|c|c|c|c}
$\# 3 \times 1$ & $\# 3 \times 1$ & $\# 4 \times 1$ & $\# 4 \times 1$ & $\# 5 \times 1$ & $\# 5 \times 1$ & $\# 0 \times 4$ \\
$10 \mathrm{ft}$ & $15 \mathrm{ft}$ & $10 \mathrm{ft}$ & $15 \mathrm{ft}$ & $10 \mathrm{ft}$ & $15 \mathrm{ft}$ & $15 \mathrm{ft}$ \\
\hline
\end{tabular}

Category $12 \mathrm{Th} / \mathrm{U}$ oxide, Zirconium

- ave package mass

- repository pkg count

- HLW can count

- HLW mass (MT)

- SNF pkg count

- ave U mass (kg)

- ave. fissile mass (kg)

33.97

69

207

445.05

69

11.99

10.85 


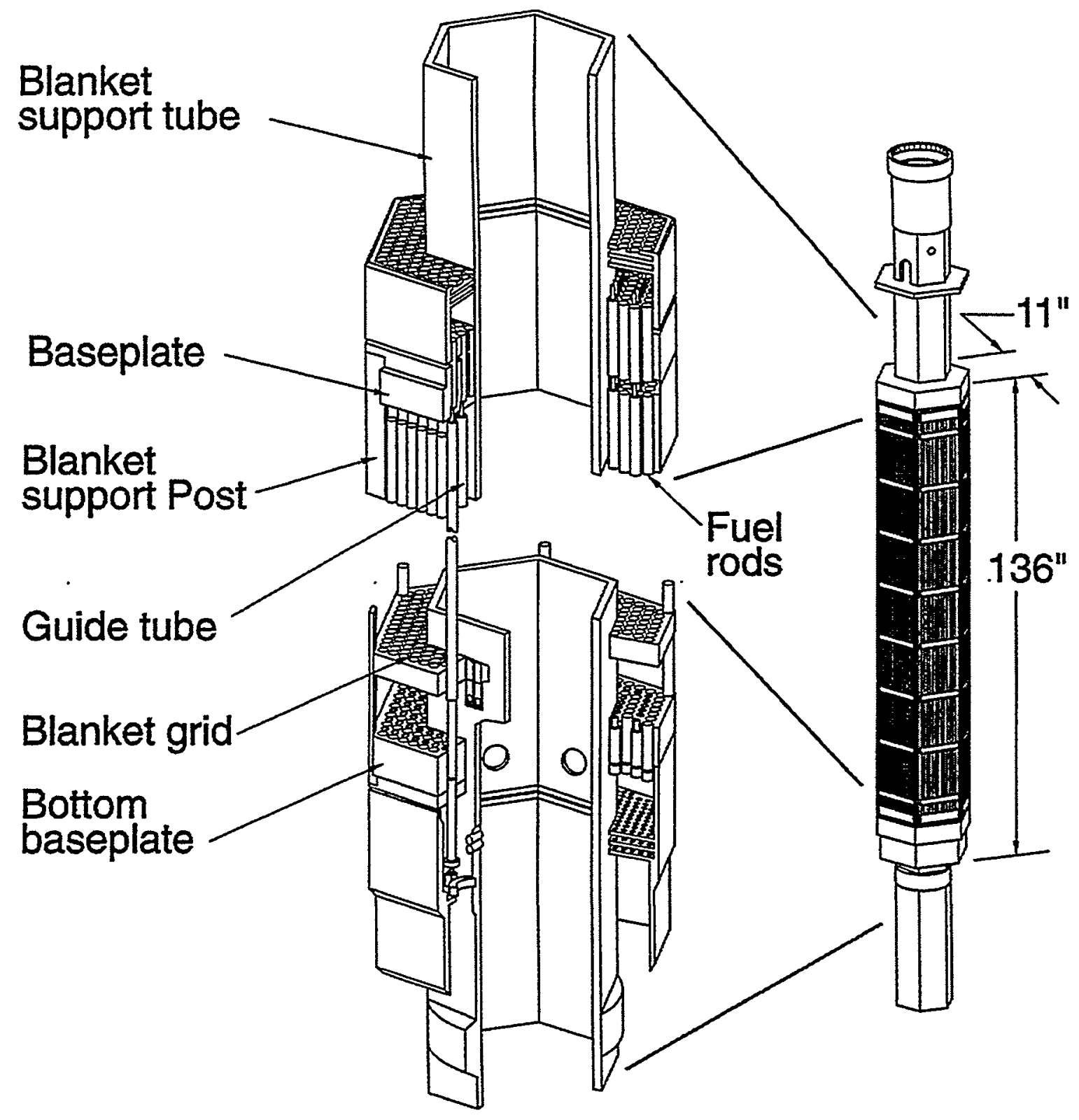

LWBR Assembly

P96 0366

Figure A.17 Shippingport LWBR Fuel Assembly

\begin{tabular}{|l|l|l|l|}
\hline $\begin{array}{l}\text { Appendix A } \\
\text { DOE SNF Information }\end{array}$ & Sheet & Date: & Rev. \\
\hline
\end{tabular}




\subsection{Category 13 U-Zr hydride / (SST) (Incaloy) (other)}

Typical fuel: TRIGA Flip (HEU), TRIGA Std. (MEU), TRIGA Alum (MEU), SNAP (HEU)

\section{Fuel Description}

Category 13 contains the fuel with the uranium/zirconium hydride matrix. Fuels from the TRIGA reactors make up the majority of the fuels in this category. The Training, Research, Isotope General Atomics (TRIGA) research reactor have been in use since 1957 throughout the United states and more than twenty countries world-wide. The TRIGA reactors are water-cooled, graphite and water reflected, pool-type research reactors which have steadystate and pulsing capabilities. There are six TRIGA reactors developed by General atomic, each having different experimental facility features to accommodate a user's specific needs.

Like all the fuels in this category, TRIGA fuel elements are made of uranium-zirconium hydride matrix which provides the reactor with its build in control and inherent safety. They are solid homogeneous all, clad with aluminum, stainless steel, or incoloy-800 and varying enrichment and weight percent of U-235 [Reference 12]. Figure A.18 shows a typical configuration of the TRIGA fuel assembly.

\begin{tabular}{|l|l|l|}
\hline Category 13 U-Zr Hydride Fuel Inventories/Information \\
\hline $\begin{array}{l}\text { Radionuclide Inventory } \\
\text { (41 isotopes) }\end{array}$ & $\begin{array}{l}\text { Refer to TSPA group listing data - Table } \\
\text { A-7 }\end{array}$ & \\
\hline Composition & U-Zr Hydride & \\
\hline Wet Dissolution Rate & Commercial Model x 0.1 & \\
\hline Surface Area (m $\left.{ }^{2} / \mathrm{g}\right)$ & $1.90 \mathrm{E}-03$ & \\
\hline Clad Failure Fraction & $0 \%$ & \\
\hline $\begin{array}{l}\text { Free Radionuclide Inventory } \\
\text { Fraction }\end{array}$ & $0.001 \%$ & \\
\hline
\end{tabular}

Appendix A

DOE SNF Information
Sheet 52 of 75
Date:

January 23,1998
Rev.

1 


\section{Configuration, Mass, and Package Count}

The following table shows the disposal configuration, average package mass, repository package count, and HLW mass used to co-disposed the category 13 SNF.

Category $13 \quad \mathrm{U}-\mathrm{Zr}$ hydride, mixed

- ave package mass

- repository pkg count

- HLW can count

- HLW mass (MT)

- SNF pkg count

- ave U mass $(\mathrm{kg})$

- ave. fissile mass $(\mathrm{kg})$

\begin{tabular}{|c|c|c|c|c|c|c|}
$\# 3 \times 1$ & $\# 3 \times 1$ & $\# 4 \times 1$ & $\# 4 \times 1$ & $\# 5 \times 1$ & $\# 5 \times 1$ & $\# 0 \times 4$ \\
$10 \mathrm{ft}$ & $15 \mathrm{ft}$ & $10 \mathrm{ft}$ & $15 \mathrm{ft}$ & $10 \mathrm{ft}$ & $15 \mathrm{ft}$ & $15 \mathrm{ft}$ \\
\hline
\end{tabular}

$25.56 \quad 48 \quad 33.77$

$\begin{array}{llr}4 & 4 & 93\end{array}$

$16 \quad 16 \quad 465$

$34.4 \quad 51.2 \quad 999.8$

$\begin{array}{lll}4 & 4 & 93\end{array}$

$8.92 \quad 25.63 \quad 20.06$

$1.75 \quad 5.06 \quad 4.67$
48.1 


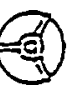

End View
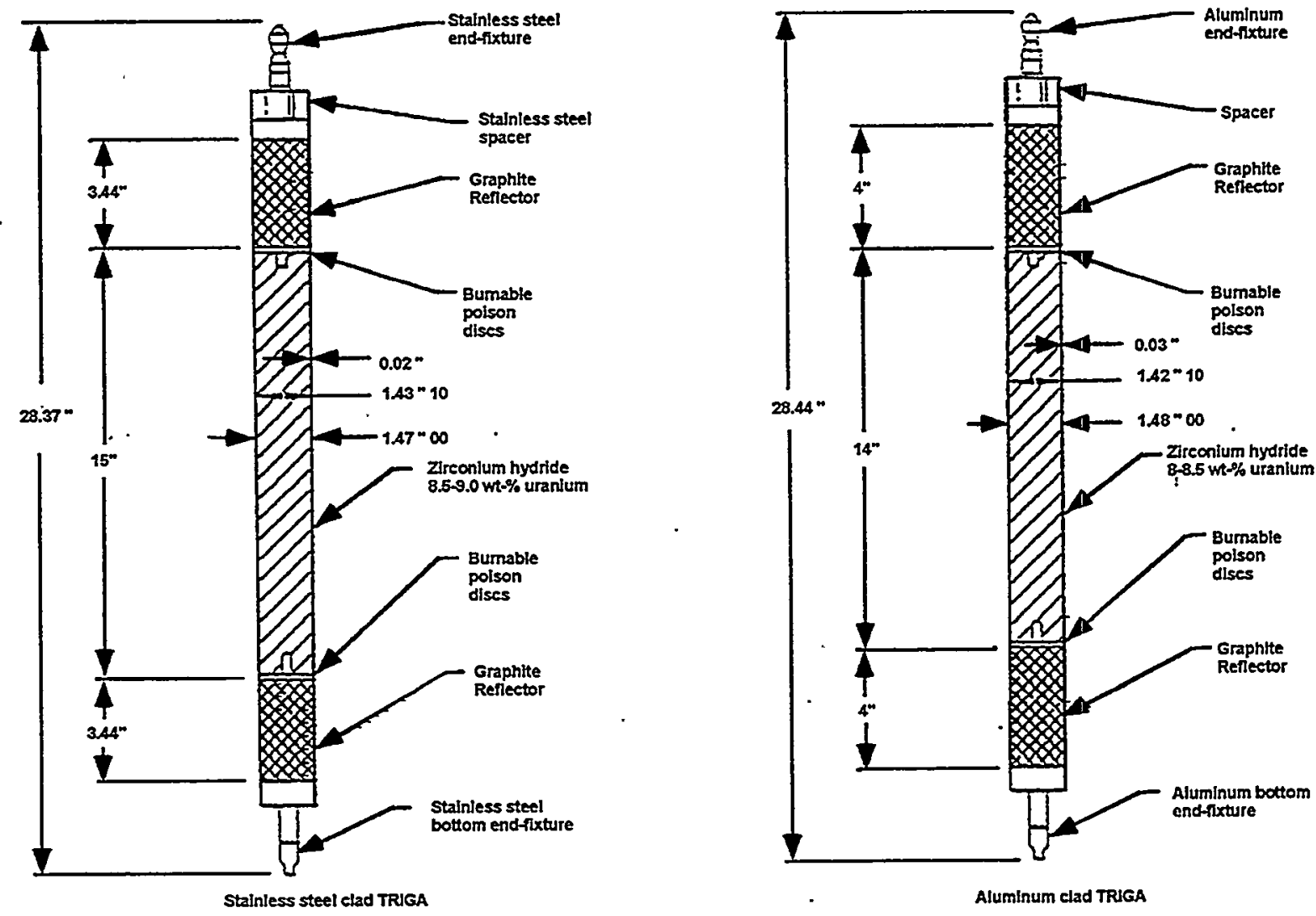

Standard TRIGA Fuel Element

Figure A.18 Standard TRIGA Fuel Element

Appendix A

DOE SNF Information
Sheet

54 of 75
Date:

January 23, 1998
Rev.

1 


\subsection{Category 14 Commercial Fuels (PWR)}

Typical fuel: Commercial Fuel

\section{Fuel Description}

For this performance assessment analysis, standard pressurized water reactor fuels from the commercial reactors are placed in category 14 . The fuels in this category represented the commercial PWR fuels that needs to be disposed of in a geologic repository. Presently, $\sim 40,785$ MTHM of the total repository capacity will be allocated to the PWR commercial SNF [Reference 13]. Since the TSPA-95 MTHM weren't tie to any particular repository Count. The following. MTHM, canister count, and MTHM/pkg values were used to represent the commercial PWR fuel. Figure A.19 and A.20 show the configurations of the PWR fuels and the proposed disposal package.

\begin{tabular}{|l|l|l|}
\hline Category 14 U-Oxide Fuel Inventories/Information \\
\hline $\begin{array}{l}\text { Radionuclide Inventory } \\
\text { (41 isotopes) }\end{array}$ & Refer to Commercial SNF data - Table A-8 & \\
\hline Composition & U-Oxide & \\
\hline Wet Dissolution Rate & Commercial Model & \\
\hline Surface Area (m $/ g)$ & $4.543 E-05$ & \\
\hline Clad Failure Fraction & Commercial SNF & \\
\hline $\begin{array}{l}\text { Free Radionuclide Inventory } \\
\text { Fraction }\end{array}$ & Commercial SNF & \\
\hline
\end{tabular}

\section{Configuration, Mass, and Package Count}

The following table shows the disposal configuration, average package mass, repository package count for category 14 SNF.

Category 14 U-Oxide, Commercial

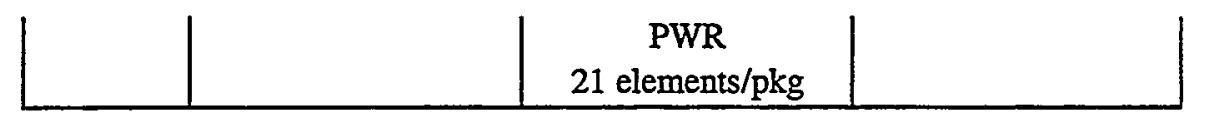

- PWR total MTHM

- ave package mass (MT)

- repository pkg count

40,400

-Approximate MTHM/pkg

-51
4,674
8.64




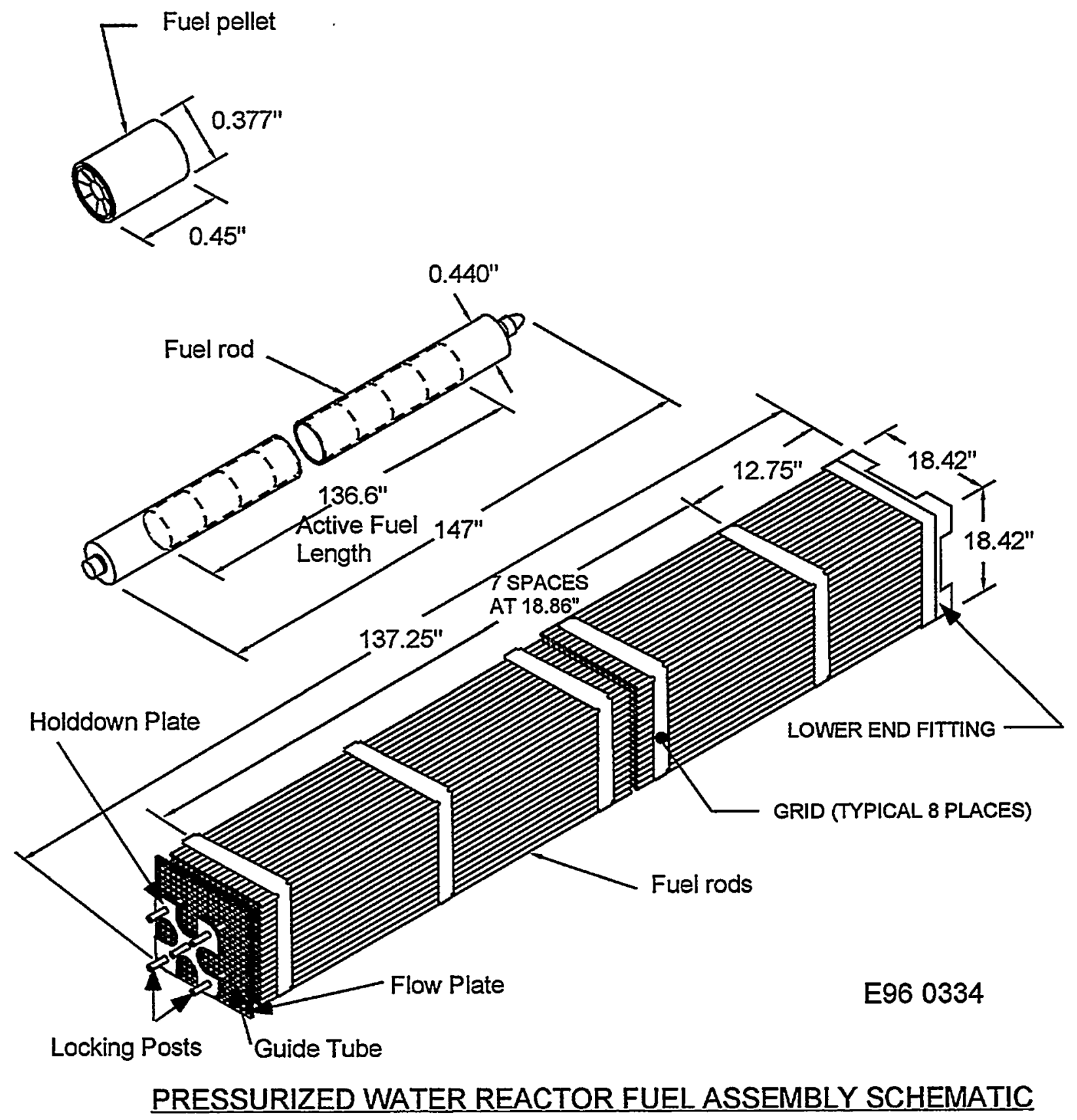

Figure A.19 Standard PWR Fuel Assembly

\begin{tabular}{|l|l|l|l|}
\hline Appendix A & Sheet & Date: & Rev. \\
DOE SNF Information & 56 of 75 & January 23, 1998 & 1 \\
\hline
\end{tabular}




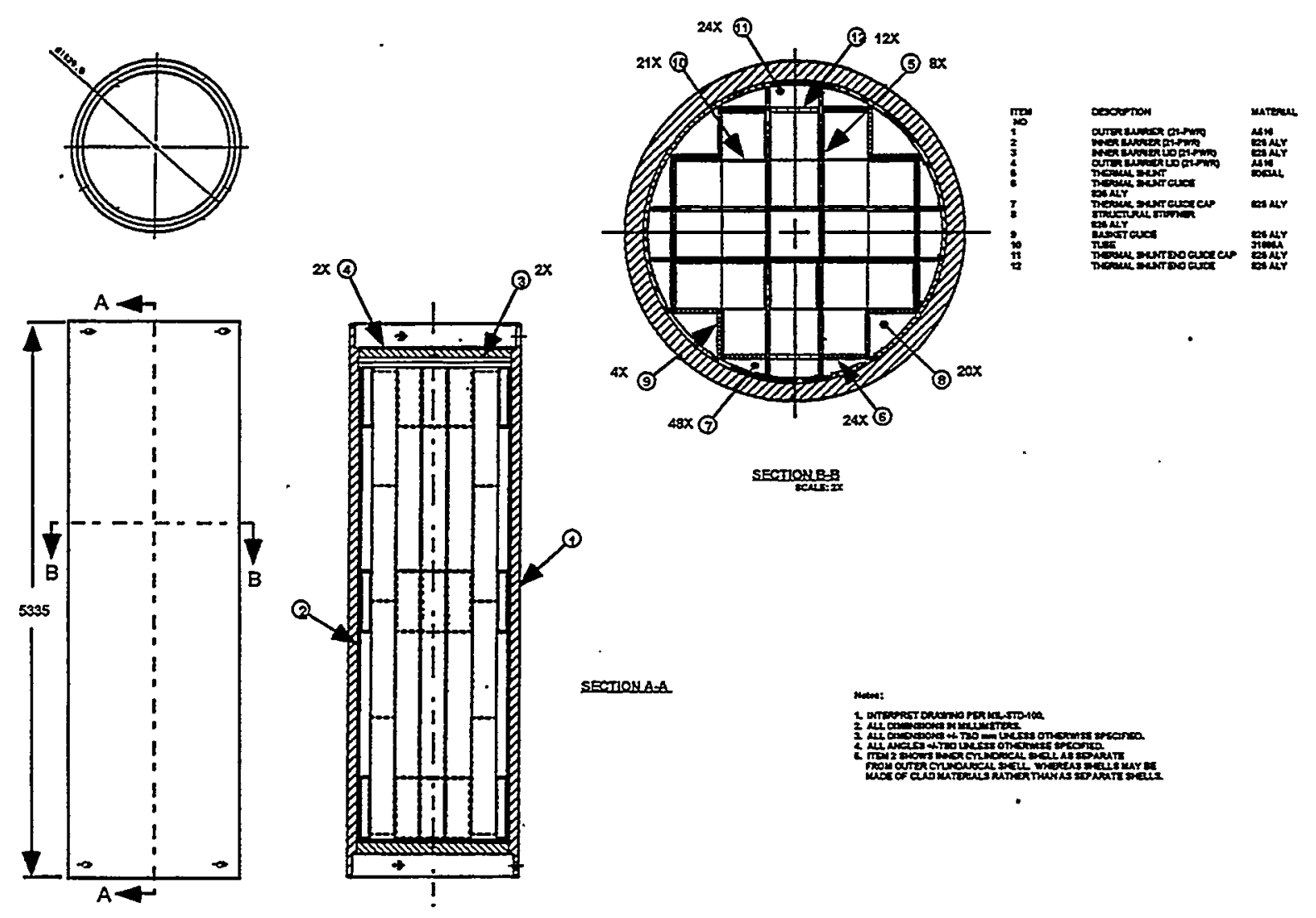

Figure A.20 Proposed PWR Loading Inside a Large Disposal Package

\begin{tabular}{|l|l|l|c}
\hline Appendix A & Sheet & Date: & Rev. \\
DOE SNF Information & 57 of 75 & January 23, 1998 & 1 \\
\hline
\end{tabular}




\subsection{Category 15 Commercial Fuels (BWR)}

Typical fuel: Commercial Fuel

\section{Fuel Description}

For this performance assessment analysis, standard boiling water reactor fuels from the commercial reactors are placed in category 15 . The fuels in this category represented the commercial BWR fuels that needs to be disposed of in a geologic repository. Presently, $\sim 22,210$ MTHM of the total repository capacity will be allocated to the BWR commercial SNF [Reference 13]. Since the TSPA-95 MTHM weren't tie to any particular repository Count. The following MTHM, canister count, and MTHM/pkg values were used to represent the commercial BWR fuel. Figure A.21 and A.22 show the configurations of the BWR fuels and their proposed disposal packages.

\begin{tabular}{|l|l|l|}
\hline Category 15 U-Oxide Fuel Inventories/Information \\
\hline $\begin{array}{l}\text { Radionuclide Inventory } \\
\text { (41 isotopes) }\end{array}$ & Refer to Commercial SNF data - Table A-8 & \\
\hline Composition & U-Oxide & \\
\hline Wet Dissolution Rate & Commercial Model & \\
\hline Surface Area (m²/g) & $4.543 E-05$ & \\
\hline Clad Failure Fraction & Commercial SNF & \\
\hline $\begin{array}{l}\text { Free Radionuclide Inventory } \\
\text { Fraction }\end{array}$ & Commercial SNF & \\
\hline
\end{tabular}

\section{Configuration, Mass, and Package Count}

The following table shows the disposal configuration, average package mass, repository package count for category 15 SNF.

Category 15 U-Oxide, Commercial

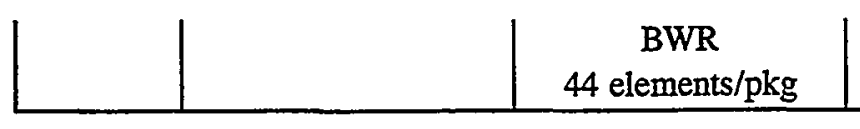
-BWR Total MTHM

- ave package mass (MT)

- repository pkg count

-Approximate MTHM/pkg

22,600

Sheet 58 of 75
Date: January 23, 1998
Rev. 1 


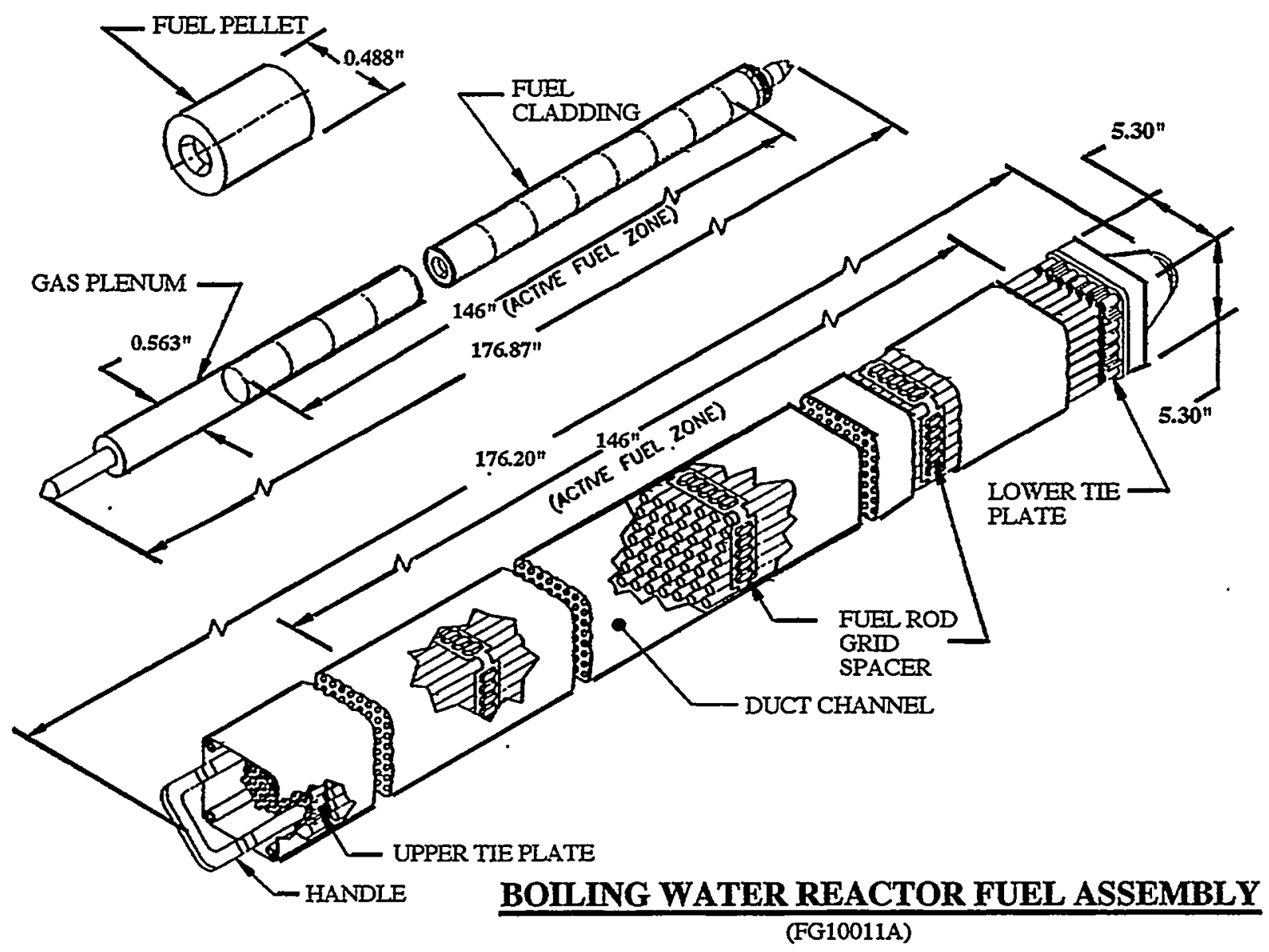

Figure A.21 Standard BWR Fuel Assembly 

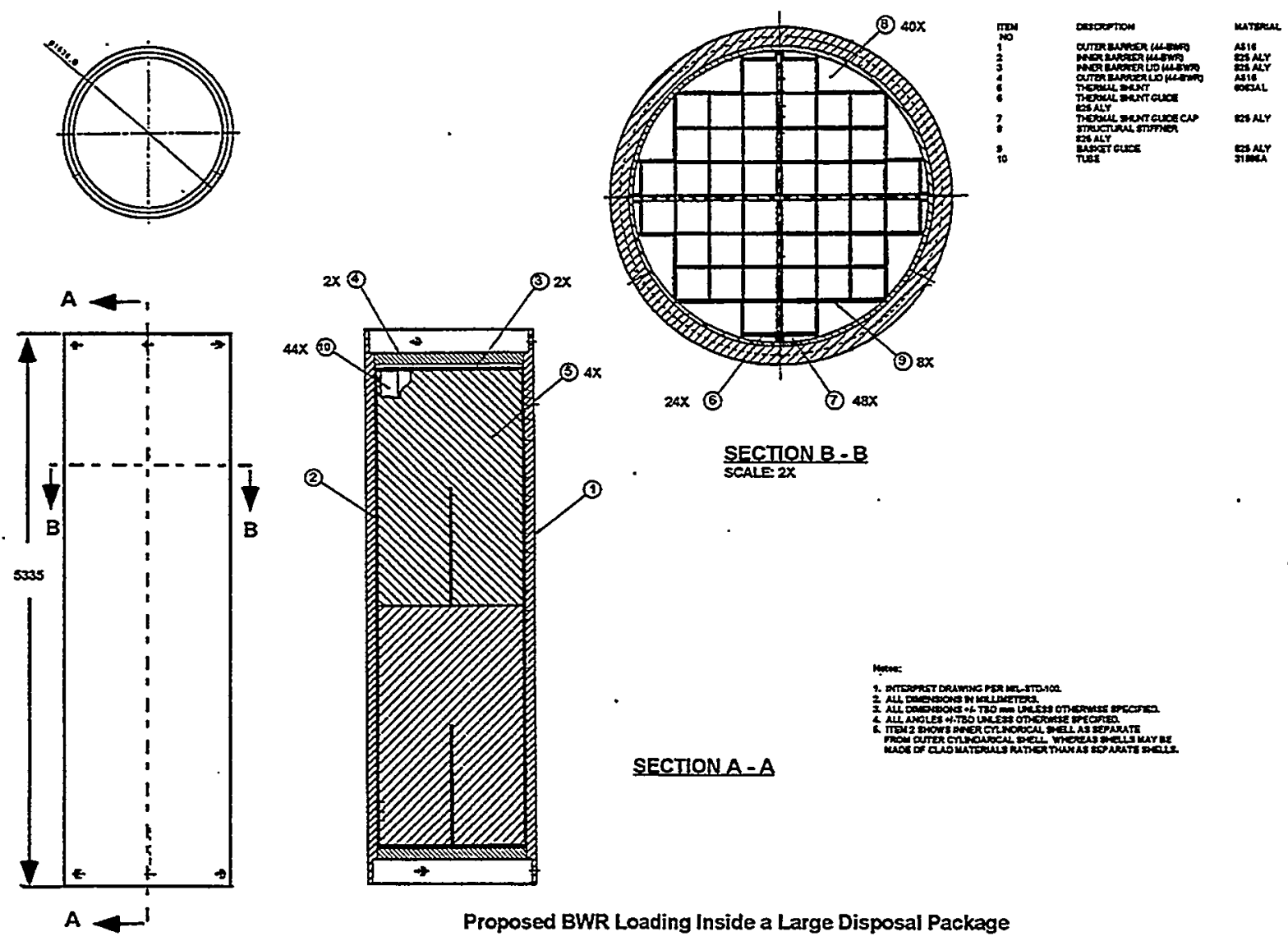

SECTION B - B

SECTIONA-A

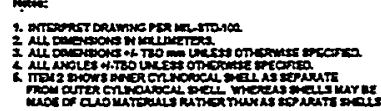

Proposed BWR Loading Inside a Large Disposal Package

Figure A.21 Proposed BWR Loading Inside a Large Disposal Package

Appendix A

DOE SNF Information

\section{Sheet} 60 of 75
Date:

January 23, 1998
Rev.

1 


\subsection{Category 16 Generic HLW}

SRS HLW product data

West Valley HLW product data

INEEL HLW product data

Hanford HLW product data

\section{Boro-silicate Glass Description}

The boro-silicate glass was developed and used in the radioactive waste because high temperature silicate melts dissolve various amounts of most other oxides and its relative resistant to "leaching" by water at temperature ranges 25-100 degree Celsius. A variety of quite different compositions had been developed to incorporate $\sim 10-30 \%$ fission products. The waste glasses are general formulated to melt at temperatures between 950 to 1150 degree Celsius with a viscosity between 5-200 poises. Regardless of these variables, the boro-silicate glass produced by the four sites will have to meet the latest U.S. Department of Energy Office of Environmental Management Waste Acceptance Product Specifications for High-Level Waste Forms (EM-WAPS).

If applicable, the glass must also meet the RW waste acceptance criteria for defense high-level waste. In the past, RW has generated a document title "Waste Acceptance Preliminary specifications for the Defense Waste Processing Facility High-Level Waste Form, $\mathrm{DOE} / \mathrm{RW}-0260$, July 1989 . It is conceivable that RW will be finalizing the acceptance criteria for the defense HLW regardless of its origin.

For the purpose of this evaluation, the HLW canister count from reference 2 will be used. The total MTHM for the total HLW inventory will be estimated using the method described in section 5.0.

\begin{tabular}{|l|l|l|}
\hline Category 16 HLW Inventories/Information & \\
\hline $\begin{array}{l}\text { Radionuclide Inventory } \\
\text { (41 isotopes) }\end{array}$ & Refer to HLW data - Table A-9 & \\
\hline Composition & Boro-silicate Glass & \\
\hline Wet Dissolution Rate & EA glass standard & $\begin{array}{l}\text { Exceeds 4,667 } \\
\text { MTHM allocation }\end{array}$ \\
\hline Total MTHM & 7,977 & \\
\hline
\end{tabular}

\begin{tabular}{|l|l|l|l|}
\hline Appendix A & Sheet & Date: & Rev. \\
DOE SNF Information & 61 of 75 & January 23, 1998 & 1 \\
\hline
\end{tabular}




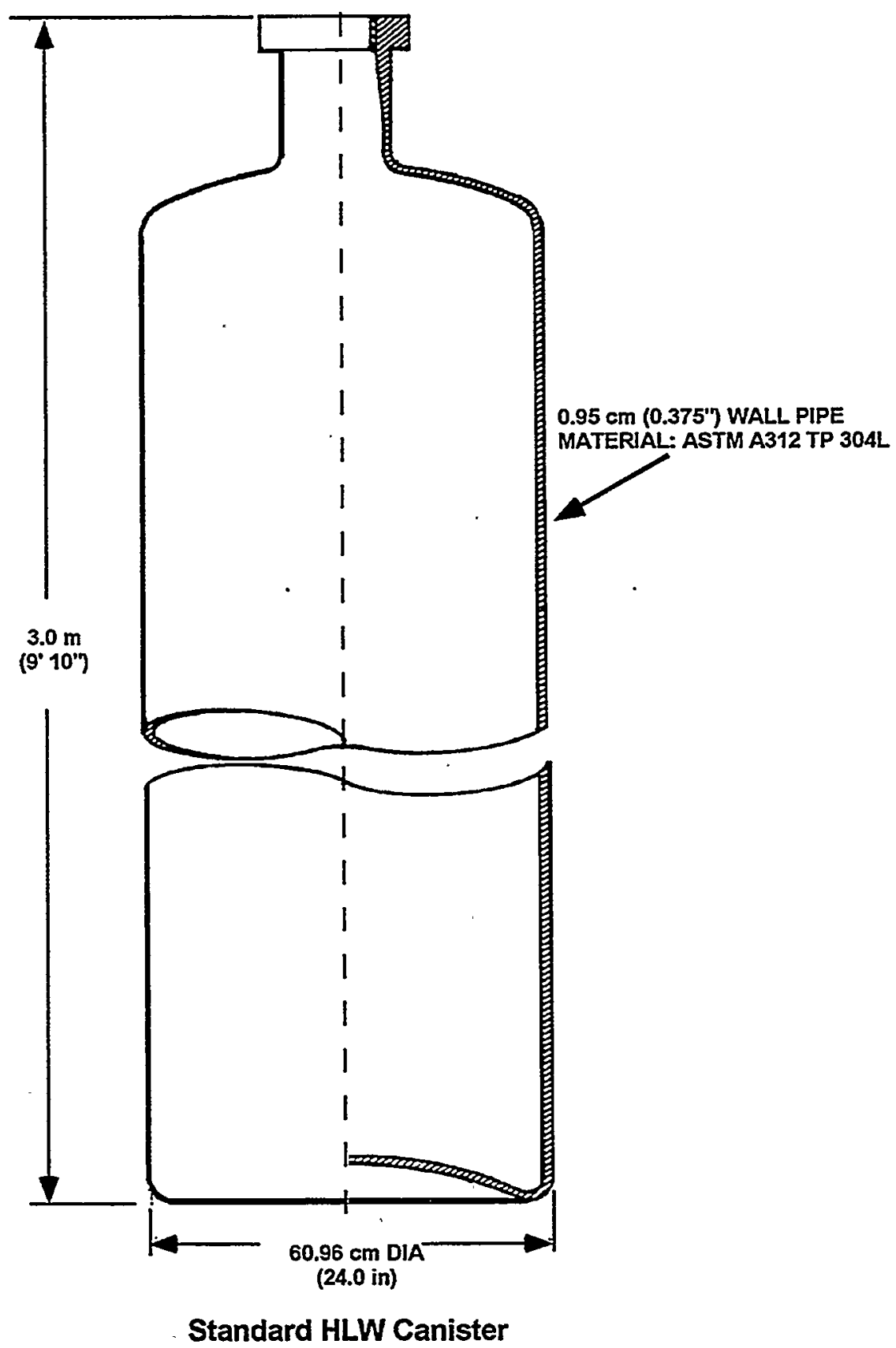

Figure A-21: Standard HLW Canister

\begin{tabular}{|l|l|l|l|}
\hline $\begin{array}{l}\text { Appendix A } \\
\text { DOE SNF Information }\end{array}$ & $\begin{array}{l}\text { Sheet } \\
62 \text { of } 75\end{array}$ & $\begin{array}{l}\text { Date: } \\
\text { January 23, 1998 }\end{array}$ & $\begin{array}{c}\text { Rev. } \\
1\end{array}$ \\
\hline
\end{tabular}




\subsection{Package Configurations}

The following section describes the proposed packages used in the performance assessment of the DOE SNF.

Individual canisters

$10 " \varnothing$ (for 4-pack HLW + 1 SNF)

Dimensions $\quad-26.6 \mathrm{~cm}$ OD, Sch $20(0.635 \mathrm{~cm}$ wall thickness); both $300 \mathrm{~cm}(770 \mathrm{~kg})$ and $450 \mathrm{~cm}(1144 \mathrm{~kg})$

Materials - can: $304 \mathrm{~L}$

- basket: borated 300 series SS

17" Ø (for 5-pack HLW + 1 SNF)

Dimensions $-45.7 \mathrm{~cm}$ OD, Such 10 ( $0.635 \mathrm{~cm}$ wall thickness); both $300 \mathrm{~cm}(1300 \mathrm{~kg})$ and $450 \mathrm{~cm}(2103 \mathrm{~kg})$

Materials - can: $304 \mathrm{~L}$

- basket: borated 300 series SS

24" Ø (for 3-pack HLW + 1 SNF)

Dimensions - $61 \mathrm{~cm} \mathrm{OD,} \mathrm{Sch} 10$ (0.635 cm wall thickness); both $300 \mathrm{~cm}(1745 \mathrm{~kg})$ and $450 \mathrm{~cm}(2442 \mathrm{~kg})$

Materials - can: $304 \mathrm{~L}$

- basket: borated 300 series SS

MCOs (specific to N-reactor fuels) (4-pack)

Dimensions - $\sim 61 \mathrm{~cm}$ OD

- length: $416.6 \mathrm{~cm}$

- weight: $3685 \mathrm{~kg}$

Materials - 304L

HLW standard canister

Dimensions - $61 \mathrm{~cm}$ OD;

both $300 \mathrm{~cm}(1745 \mathrm{~kg})$ and $450 \mathrm{~cm}(2442 \mathrm{~kg})$

Materials - can: 304L

\subsection{Criticality Issues}

Use of fissile loadings on a per SNF canister basis offer one approach to meeting one of the two contingencies needed to assure criticality safety during the operational phases of fuel handling. Criticality safety evaluations (CSEs) will be needed for all SNF canisters prior to transportation, and these CSEs ( $\mathrm{k}_{\text {eff }}<0.95$ per regulations) will need to show safety for all

\begin{tabular}{|l|l|l|l|}
\hline Appendix A & Sheet & Date: & Rev. \\
DOE SNF Information & 63 of 75 & January 23, 1998 & 1 \\
\hline
\end{tabular}


operational phases (packaging, transportation, and storage) prior to repository closure. Adoption of double contingency controls implies both monitoring and remediation capabilities in operational facilities. For geologic disposal facilities, neither monitoring nor remediation offer viable options. Furthermore, keff $=1.0$ define a criticality rather than the administrative limit of $\geq 0.95$ associated with operational facilities.

Use of other approaches to provide contingencies to assure criticality safety may allow credits for fixed neutron absorbers and/or controlled spacing of fuel assemblies if long-term performance can be demonstrated. None of the fissile limits referenced in the PA study are intended to preclude use of one or more of these options in lieu of the fissile mass limits and water exclusion bases used for this PA.

There will be a set of data needs to meet licensing requirements for waste packages intended for the repository. Some of this characterization data is expected to be fuel specific due to the variety of the fuel matrices and the resultant chemistries for a fully-flooded waste package. Parameters such as leachability, solubility, matrix dissolution rates, and surface areas are among those which can affect mobilization and transport of chemical species in a breached waste package. Very little of this type of data specific to the repository environment is actually available, Use of conservative estimates predominate the numbers used to calculate release and transport of the various chemical species

ORIGEN code data for the DOE SNF relied on ORIGEN-2 runs for one or more fuel types in each category. These runs provided a generic set of source term inventories (by category) needed to calculate both waste package thermal loads and radiation shielding. They also provide the basis for calculating the curie quantities of radionuclides available for release and transport at any point in the future.

Cladding composition and mass are available for this study on to the extent they may affect the rate of gas generation. At the present time, no cladding credits are being allowed for any of the fuels identified for repository disposal. This approach represents a bounding limit, since any allowance for retardation of radionuclide transport by the presence of intact cladding can only improve the results calculated for the waste packages.

\subsection{Packaging Combinations}

N-reactor fuels, which constitute $\sim 80 \%$ of the DOE SNF inventory, are in the process of being removed from their wet storage environment. As part of this process, they are receiving some minimal treatment to address breached and/or damaged fuels prior to dry packaging in multi-canister overpacks (MCOs). While these stainless steel packages are intended to provide only interim storage for these fuels, there are no identified packaging alternatives for geologic disposal. Therefore this PA will evaluate the $\mathrm{N}$-reactor fuel performance based on a default packaging concept, evaluating the MCOs inside a repository overpack.

\begin{tabular}{|l|l|l|l|}
\hline $\begin{array}{l}\text { Appendix A } \\
\text { DOE SNF information }\end{array}$ & $\begin{array}{l}\text { Sheet } \\
64 \text { of } 75\end{array}$ & $\begin{array}{l}\text { Date: } \\
\text { January 23, 1998 }\end{array}$ & $\begin{array}{c}\text { Rev. } \\
1\end{array}$ \\
\hline
\end{tabular}


An MCO consists of either 48 Mark 1A fuels/layer, with 6 layers/MCO, or 54 Mark IV fuels/layer with 5 layers/MCO) $7 \mathrm{~N}$-reactor elements/ canister]. ${ }^{1}$

7 elements / Mark II canister

28 elements / layer

140 elements / MCO

560 elements / repository overpack

Ft. St. Vrain fuels - stack 5-high in a $44.4 \mathrm{~cm}$ (OD) canister insert.

All other remaining (DOE-EM) fuels will be contained in:

$26.6 \mathrm{~cm}$ OD canister, or

$45.7 \mathrm{~cm}$ OD canister, or

$61 \mathrm{~cm}$ OD canister

in their appropriate length.

Internal basket designs may be incorporated into the SNF canister to facilitate packaging and/or a method of neutron absorber fixation as part of a criticality safety assurance. Such an internal basket would be similar to that being proposed for commercial fuels in the LDP.

Repository overpack

10 -cm thick carbon steel outer layer

2 -cm thick Inconel 625 


\section{Terminology}

leu $(<5 \%)$

$\operatorname{meu}(>5 \%<20 \%)$

heu $(>20 \%)$

These enrichment values were used as the division criteria for the this

performance assessment study.

MCO (multicanister overpack)

This is a Hanford design, that while intended only for interim dry storage of $\mathrm{N}$ reactor fuels, may suffice for disposal when coupled with the repository overpack in a multi-pack array

\section{MPC/DPC}

This original concept (54 OD; $15 \mathrm{ft}$ long) has encountered design holdups, but may still considered a viable package suitable for repository disposal.

repository overpack

Dual layer approach consisting of - carbon steel $(10.0 \mathrm{~cm})$, the corrosion allowance layer/ Inconel $625(2.0 \mathrm{~cm})$, the corrosion resistant layer. The repository overpack will be sized to accommodate the various package configurations described in this performance assessment report.

\section{ORIGEN codes}

Code uses to calculate radionuclide inventory needed for a variety of reasons such as performance assessment evaluation, design basis events analyses, and the Environmental Impact Statement. RW presently uses ORIGEN-S to estimate the commercial SNF radionuclide inventory. ORIGEN-II has also been used by the DOE sites to estimate radionuclide inventory.

FGE (fissile gram equivalent)

A estimate of the system reactivity contain U-233 and Pu-239 using the following equation

$=\mathrm{U}-235$ conc. $+2 *(\mathrm{U}-233$ conc. $)+2 *(\mathrm{Pu}-239$ conc. $)$

\section{LDP (Large Disposal Package)}

Replaces the terminology that previously addressed what was known as a "repository overpack" (that would accept either an MPC/DPC or $4 \mathrm{HLW}$ canisters). The design of this canister is still in a state of design flux to accommodate the proposed variant loadings to accommodate the HLW/SNF codisposal concept and the proposed Navy fuel package sizing requirements.

wet dissolution rate $\left(\mathrm{gm} / \mathrm{m}^{2} / \mathrm{yr}\right)$

\begin{tabular}{|l|l|l|l|}
\hline $\begin{array}{l}\text { Appendix A } \\
\text { DOE SNF Information }\end{array}$ & $\begin{array}{l}\text { Sheet } \\
66 \text { of } 75\end{array}$ & $\begin{array}{l}\text { Date: } \\
\text { January 23, 1998 }\end{array}$ & $\begin{array}{l}\text { Rev. } \\
1\end{array}$ \\
\hline
\end{tabular}


surface area $\left(\mathrm{m}^{2} / \mathrm{gm}\right)$

The surface area of fuel matrix material based on the degree of irradiation that will be subject to leaching. A value of $3.96 \times 10-3 \mathrm{~m} 2 / \mathrm{g}$ (Gray and Wilson, 1995) has been recommended as a reasonable minimum surface area for typical spent fuel. A maximum value of $0.1 \mathrm{~m} 2 / \mathrm{g}$ (Gray and Wilson, 1995) could be used for severely disrupted fuels such as the TMI debris.

(assignment of) clad failure fraction

Some credit to be taken for the contribution any intact cladding may provide upon initial breach of the EBS

free radionuclide inventory fraction

The fraction of radionuclides within a package available for immediate release upon initial package breach

gap radionuclide inventory fraction

Portion of the radionuclide inventory between the fuel clad and the fuel matrix itself. 
References:

1. Memorandum from Daniel Dreyfus to Jill E. Lytle dated November 9, 1995, Subject: Proposed Mix of DOE-Owned High Level Waste and Spent nuclear Fuel.

2. Integrated Data Base Report - 1994, DOE/RW-0006, Rev 11, September 1995.

3. Predecisional draft RW Report - Degraded Mode Criticality analysis of Immobilized Plutonium Waste Form in a Geologic Repository, February 1997

4. Memorandum from Ronald A. Miller to Jackson Kinzer dated April 5, 1996, Subject: Evaluation of Hanford Longer High-Level Waste Product Anister Oprion for acceptance by the Civilian Radioactive Waste management System.

5. Technical Strategy for the Treatment, Packaging, and Disposal of AluminumBased Spent Nuclear Fuel, Vol. 1, DOE Office of Spent Fuel Management, June 1996.

6. Waste Acceptance System Requirements Document, (WASRD), Rev. 02A, September 1995.

7. Hanford Irradiated Fuel Inventory Baseline, WHC-SD-CP-TI-175, February, 1993.

8. CSER 96-019, Rev. 3: Criticality Safety Evaluation Report for Spent Nuclear Fuel Processing and Storage Facilities, HNF-SD-SNF-CSER-005, February 1997.

9. Directory of Nuclear Reactors, Vol V, International Atomic Energy Agency, Vienna, 1964

10. Lockheed-Martin Unclassified Nuclear Fuel Information System (LUFIS), INEEL Spent Nuclear Fuel Program.

11. Technical Strategy for the Management of INEEL Spent Nuclear Fuel, March 1997

12. Draft Universal TRIGA Fuel Summary Report

13. Total System Performance Assessment - 1995: An Evaluation of the Potential Yucca Mountain Repository, November 1995

\begin{tabular}{|l|l|l|l|}
\hline $\begin{array}{l}\text { Appendix A } \\
\text { DOE SNF Information }\end{array}$ & $\begin{array}{l}\text { Sheet } \\
68 \text { of } 75\end{array}$ & $\begin{array}{l}\text { Date: } \\
\text { January 23, 1998 }\end{array}$ & $\begin{array}{l}\text { Rev. } \\
1\end{array}$ \\
\hline
\end{tabular}


Table A-7

\begin{tabular}{|c|c|c|c|c|c|c|}
\hline COMPLEX CUM & TSPA Category & TSPA Category & TSPA Category & TSPA Category & TSPA Category & TSPA Category \\
\hline SNF, not HLW & 1) & 1 & 2 & 2 & 3 & 3 \\
\hline & MTHM & packages & MTHM & packages & MTHM & packages \\
\hline INEEL & 34.6 & 13 & 0.04 & 9 & 3.93 & 55 \\
\hline \multicolumn{7}{|l|}{ SRS } \\
\hline Hanford & 2102.2 & 105 & & & & \\
\hline \multirow[t]{2}{*}{ Total } & 2136.8 & 118 & 0.04 & 9 & 3.93 & 55 \\
\hline & total curies & Ci/pkg & total curies & Ci/pkg & total curies & Ci/pkg] \\
\hline & & & & & & \\
\hline \multicolumn{7}{|l|}{ Isotopes } \\
\hline$\overline{\mathrm{C} 14}$ & $6.72 E+02$ & $5.70 \mathrm{E}+00$ & $6.54 \mathrm{E}-05$ & 7.27E-06 & $1.58 E+01$ & $2.87 \mathrm{E}-01$ \\
\hline$\overline{C L 36}$ & $0.00 \mathrm{E}+00$ & $0.00 E+00$ & $0.00 \mathrm{E}+00$ & $0.00 E+00$ & $4.01 \mathrm{E}-02$ & $7.30 \mathrm{E}-04$ \\
\hline NI 59 & $3.79 E+01$ & 3.21E-01 & $0.00 \mathrm{E}+00$ & $0.00 \mathrm{E}+00$ & $4.94 E+01$ & $8.97 \mathrm{E}-01$ \\
\hline NI 63 & $3.57 \mathrm{E}+03$ & $3.03 \mathrm{E}+01$ & $0.00 \mathrm{E}+00$ & $0.00 E+00$ & $1.03 E+03$ & $1.88 \mathrm{E}+01$ \\
\hline SE79 & $8.33 E+01$ & 7.06E-01 & 2.35E-01 & $2.61 E-02$ & $1.20 \mathrm{E}+01$ & 2.18E-01 \\
\hline SR90 & $4.67 \mathrm{E}+06$ & $3.96 E+04$ & $5.63 E+04$ & $6.26 \mathrm{E}+03$ & $1.07 E+04$ & $1.95 E+02$ \\
\hline ZR93 & $3.91 E+02$ & $3.31 E+00$ & $1.21 \mathrm{E}+00$ & $1.34 \mathrm{E}-01$ & $4.78 \mathrm{E}+01$ & $8.69 E-01$ \\
\hline NB93M & $3.57 \mathrm{E}+02$ & $3.03 E+00$ & $6.94 E-02$ & 7.71E-03 & $3.33 \mathrm{E}+01$ & $6.05 \mathrm{E}-01$ \\
\hline NB94 & $1.25 \mathrm{E}-04$ & $1.06 \mathrm{E}-06$ & $1.31 \mathrm{E}-05$ & $1.45 \mathrm{E}-06$ & $6.03 E+00$ & $1.10 \mathrm{E}-01$ \\
\hline TC99 & $2.80 \mathrm{E}+03$ & $2.37 E+01$ & $7.93 E+00$ & $8.81 E-01$ & $3.25 \mathrm{E}+02$ & $5.90 E+00$ \\
\hline PD107 & $1.39 E+01$ & $1.18 \mathrm{E}-01$ & 9.12E-03 & $1.01 \mathrm{E}-03$ & $9.23 E-01$ & $1.68 \mathrm{E}-02$ \\
\hline SN126 & $1.46 \mathrm{E}+02$ & $1.23 E+00$ & 2.11E-01 & $2.34 \mathrm{E}-02$ & $2.73 \mathrm{E}+01$ & 4.97E-01 \\
\hline 1129 & $6.12 E+00$ & $5.18 \mathrm{E}-02$ & $1.30 \mathrm{E}-02$ & $1.45 \mathrm{E}-03$ & $8.30 \mathrm{E}-01$ & 1.51E-02 \\
\hline CS135 & $7.43 E+01$ & $6.30 \mathrm{E}-01$ & 6.14E-02 & 6.83E-03 & $3.26 E+01$ & $5.92 \mathrm{E}-01$ \\
\hline CS137 & $6.28 \mathrm{E}+06$ & $5.32 E+04$ & $5.87 E+04$ & $6.52 E+03$ & $1.28 \mathrm{E}+04$ & $2.32 E+02$ \\
\hline SM151 & $1.36 E+05$ & $1.16 \mathrm{E}+03$ & $2.12 E+02$ & $2.36 E+01$ & $5.00 \mathrm{E}+04$ & $9.09 E+02$ \\
\hline$\overline{\mathrm{PB} 210}$ & $7.49 \mathrm{E}-08$ & $6.35 E-10$ & 6.89E-12 & $7.65 \mathrm{E}-13$ & 1.03E-06 & $1.88 \mathrm{E}-08$ \\
\hline RA226 & $2.13 E-03$ & $1.80 \mathrm{E}-05$ & $1.75 \mathrm{E}-11$ & 1.95E-12 & 4.64E-06 & 8.43E-08 \\
\hline RA228 & 1.72E-07 & $1.46 \mathrm{E}-09$ & 9.91E-13 & $1.10 \mathrm{E}-13$ & 2.95E-05 & 5.36E-07 \\
\hline AC227 & $1.01 \mathrm{E}-02$ & 8.59E-05 & $3.80 \mathrm{E}-08$ & 4.23E-09 & 5.11E-02 & $9.29 \mathrm{E}-04$ \\
\hline TH229 & 1.73E-05 & 1.46E-07 & $8.47 \mathrm{E}-10$ & 9.41E-11 & $1.70 E-05$ & $3.09 E-07$ \\
\hline TH230 & $1.90 E-03$ & $1.61 \mathrm{E}-05$ & $6.84 E-08$ & $7.60 \mathrm{E}-09$ & $8.27 \mathrm{E}-04$ & $1.50 \mathrm{E}-05$ \\
\hline TH232 & $2.25 E-07$ & $1.90 \mathrm{E}-09$ & $1.62 \mathrm{E}-11$ & $1.80 \mathrm{E}-12$ & 3.11E-05 & $5.66 \mathrm{E}-07$ \\
\hline PA231 & 2.55E-02 & $2.16 \mathrm{E}-04$ & 1.83E-06 & 2.03E-07 & $1.44 \mathrm{E}-01$ & $2.63 \mathrm{E}-03$ \\
\hline $\mathrm{U} 233$ & $1.06 E-02$ & 9.01E-05 & 7.28E-06 & $8.09 E-07$ & $7.10 E-03$ & $1.29 \mathrm{E}-04$ \\
\hline U234 & $9.02 E+02$ & $7.64 \mathrm{E}+00$ & $6.84 \mathrm{E}-03$ & 7.60E-04 & $3.55 \mathrm{E}+00$ & 6.46E-02 \\
\hline U235 & $3.45 \mathrm{E}+01$ & $2.92 E-01$ & 6.54E-02 & $7.27 E-03$ & $2.30 \mathrm{E}+02$ & $4.19 E+00$ \\
\hline 0236 & $1.31 E+02$ & $1.11 E+00$ & $2.69 E-01$ & $2.98 E-02$ & $9.00 \mathrm{E}+00$ & $1.64 \mathrm{E}-01$ \\
\hline U238 & $7.09 \mathrm{E}+02$ & $6.01 \mathrm{E}+00$ & 1.20E-03 & 1.33E-04 & $1.05 \mathrm{E}+02$ & $1.92 E+00$ \\
\hline NP237 & $7.20 \mathrm{E}+01$ & $6.10 \mathrm{E}-01$ & $1.60 \mathrm{E}-01$ & $1.78 \mathrm{E}-02$ & $2.40 \mathrm{E}+00$ & $4.36 E-02$ \\
\hline PU238 & $1.01 E+05$ & $8.56 E+02$ & $3.45 E+02$ & $3.83 E+01$ & $1.19 \mathrm{E}+02$ & $2.16 E+00$ \\
\hline PU239 & $2.30 \mathrm{E}+05$ & $1.95 \mathrm{E}+03$ & $8.57 E+00$ & $9.52 E-01$ & $1.41 E+04$ & $2.57 E+02$ \\
\hline PU240 & $1.33 E+05$ & $1.13 \mathrm{E}+03$ & $4.90 E+00$ & $5.44 E-01$ & $4.36 \mathrm{E}+01$ & $7.93 \mathrm{E}-01$ \\
\hline PU241 & $1.46 E+06$ & $1.24 E+04$ & $1.68 \mathrm{E}+03$ & $1.87 E+02$ & $4.33 E+00$ & $7.88 \mathrm{E}-02$ \\
\hline PU242 & $6.44 E+01$ & $5.46 \mathrm{E}-01$ & $7.33 E-03$ & $8.15 E-04$ & $2.73 E-07$ & $4.97 \mathrm{E}-09$ \\
\hline AM241 & $5.04 E+05$ & $4.27 \mathrm{E}+03$ & $3.08 E+00$ & $3.42 E-01$ & $3.25 E-01$ & $5.90 \mathrm{E}-03$ \\
\hline AM242M & $1.34 \mathrm{E}+01$ & $1.14 \mathrm{E}-01$ & 9.31E-03 & $1.03 E-03$ & $0.00 \mathrm{E}+00$ & $0.00 E+00$ \\
\hline AM243 & $7.60 \mathrm{E}+01$ & 6.44E-01 & $3.04 E-02$ & $3.37 E-03$ & $5.24 \mathrm{E}-09$ & $9.53 \mathrm{E}-11$ \\
\hline CM244 & $2.10 \mathrm{E}+03$ & $1.78 \mathrm{E}+01$ & $1.25 E+00$ & $1.39 \mathrm{E}-01$ & $4.88 \mathrm{E}-10$ & $8.88 \mathrm{E}-12$ \\
\hline CM245 & $9.52 \mathrm{E}-01$ & $8.06 \mathrm{E}-03$ & $6.39 \mathrm{E}-05$ & $7.10 \mathrm{E}-06$ & 1.33E-15 & 2.42E-17 \\
\hline CM246 & $1.26 \mathrm{E}-01$ & 1.07E-03 & 4.40E-06 & 4.89E-07 & $4.36 \mathrm{E}-19$ & $7.93 \mathrm{E}-21$ \\
\hline
\end{tabular}

Appendix A DOE SNF Information
Sheet 69 of 75
Date: January 23, 1998
Rev. 
Table A-7 (Continued)

\begin{tabular}{|c|c|c|c|c|c|c|}
\hline$=$ & TSPA Category & TSPA Category & TSPA Category & TSPA Category & TSPA Category & TSPA Category \\
\hline SNF, not HLW & $4 \mid$ & 4 & 5 & 5 & 6) & 6 \\
\hline & MTHM & packages & MTHM & packages & MTHM & packages \\
\hline INEEL & 79.27 & 182 & 84.11 & 167 & & \\
\hline SRS & & & 3.67 & 425 & 8.96 & 750 \\
\hline Hanford & 17.8 & 21 & 0.15 & 3 & & \\
\hline \multirow[t]{3}{*}{ Total } & 97.07| & 203 & 87.93 & 595 & 8.96 & 750 \\
\hline & & & & & & \\
\hline & total curies & Ci/pkg & total curies & Ci/pkg & total curies & Cilpkg \\
\hline & & & & & & \\
\hline \multicolumn{7}{|l|}{ Isotopes } \\
\hline$\overline{C 14}$ & $7.61 E+00$ & 3.75E-02 & $9.80 \mathrm{E}-01$ & $1.65 \mathrm{E}-03$ & $1.14 E+01$ & $1.52 \mathrm{E}-02$ \\
\hline CL36 & $0.00 \mathrm{E}+00$ & $0.00 E+00$ & $3.62 \mathrm{E}-03$ & $6.08 \mathrm{E}-06$ & $3.43 \mathrm{E}-01$ & $4.58 \mathrm{E}-04$ \\
\hline NI59 & $0.00 E+00$ & $0.00 E+00$ & $7.06 \mathrm{E}-01$ & 1.19E-03 & $6.70 \mathrm{E}+01$ & $8.93 E-02$ \\
\hline NI 63 & $3.48 \mathrm{E}+05$ & $1.72 \mathrm{E}+03$ & $8.69 \mathrm{E}+01$ & $1.46 E-01$ & $8.24 E+03$ & $1.10 \mathrm{E}+01$ \\
\hline$\overline{\text { SE79 }}$ & $3.82 E+01$ & $1.88 \mathrm{E}-01$ & $2.65 E+01$ & $4.45 E-02$ & $3.58 \mathrm{E}+01$ & $4.78 E-02$ \\
\hline SR90 & $4.81 E+06$ & $2.37 E+04$ & $7.23 \mathrm{E}+06$ & $1.22 \mathrm{E}+04$ & $8.55 \mathrm{E}+06$ & $1.14 E+04$ \\
\hline ZR93 & $1.79 E+02$ & $8.83 \mathrm{E}-01$ & $1.35 \mathrm{E}+02$ & $2.27 \mathrm{E}-01$ & $1.86 E+02$ & 2.47E-01 \\
\hline NB93M & $1.20 E+02$ & 5.93E-01 & $1.26 \mathrm{E}+01$ & $2.11 \mathrm{E}-02$ & $1.10 \mathrm{E}+01$ & $1.46 \mathrm{E}-02$ \\
\hline NB94 & $0.00 E+00$ & $0.00 \mathrm{E}+00$ & |7.74E-03 & $1.30 \mathrm{E}-05$ & $6.25 \mathrm{E}-01$ & $8.34 E-04$ \\
\hline TC99 & $1.33 E+03$ & $6.53 \mathrm{E}+00$ & $8.93 E+02$ & $1.50 \mathrm{E}+00$ & $1.21 E+03$ & $1.61 E+00$ \\
\hline PD107 & $9.72 E+00$ & 4.79E-02 & $1.38 \mathrm{E}+00$ & $2.32 E-03$ & $1.40 \mathrm{E}+00$ & $1.87 \mathrm{E}-03$ \\
\hline SN126 & $4.83 E+01$ & $2.38 \mathrm{E}-01$ & $2.62 \mathrm{E}+01$ & 4.40E-02 & $3.21 \mathrm{E}+01$ & $4.28 \mathrm{E}-02$ \\
\hline 1129 & $3.58 \mathrm{E}+00$ & $1.77 \mathrm{E}-02$ & $9.52 \mathrm{E} \div 00$ & $2.55 \mathrm{E}-03$ & $1.98 \mathrm{E}+00$ & $2.65 E-03$ \\
\hline CS135 & $3.08 E+01$ & $1.52 \mathrm{E}-01$ & $1.07 E+01$ & $1.80 \mathrm{E}-02$ & $1.29 \mathrm{E}+01$ & $1.72 \mathrm{E}-02$ \\
\hline CS137 & $6.47 E+06$ & $3.19 E+04$ & $7.73 E+06$ & $1.30 \mathrm{E}+04$ & $8.91 \mathrm{E}+06$ & $1.19 E+04$ \\
\hline SM151 & $1.10 E+05$ & $5.42 E+02$ & $3.22 E+04$ & $5.42 \mathrm{E}+01$ & $3.37 \mathrm{E}+04$ & $4.49 \mathrm{E}+01$ \\
\hline PB210 & $7.25 \mathrm{E}-06$ & $3.57 \mathrm{E}-08$ & 4.06E-06 & $6.82 \mathrm{E}-09$ & $1.07 \mathrm{E}-09$ & $1.42 \mathrm{E}-12$ \\
\hline RA226 & 3.02E-05 & $1.49 \mathrm{E}-07$ & $11.54 \mathrm{E}-05$ & $2.60 \mathrm{E}-08$ & $2.73 E-09$ & $3.64 \mathrm{E}-12$ \\
\hline RA228 & $2.62 \mathrm{E}-02$ & $1.29 E-04$ & 6.84E-03 & $1.15 \mathrm{E}-05$ & 5.49E-07 & $7.33 \mathrm{E}-10$ \\
\hline AC227 & $3.26 \mathrm{E}-02$ & $1.60 \mathrm{E}-04$ & 8.33E-03 & $1.40 \mathrm{E}-05$ & $1.28 \mathrm{E}-05$ & 1.71E-08 \\
\hline TH229 & $7.79 \mathrm{E}-02$ & $3.84 \mathrm{E}-04$ & 1.97E-02 & $3.31 \mathrm{E}-05$ & 2.72E-07 & $3.63 \mathrm{E}-10$ \\
\hline TH230 & $5.39 \mathrm{E}-03$ & 2.65E-05 & 2.27E-03 & $3.82 \mathrm{E}-06$ & $1.02 E-05$ & $1.37 \mathrm{E}-08$ \\
\hline TH232 & $2.76 \mathrm{E}-02$ & $1.36 \mathrm{E}-04$ & $7.21 \mathrm{E}-03$ & $1.21 \mathrm{E}-05$ & $1.62 \mathrm{E}-06$ & $2.16 \mathrm{E}-09$ \\
\hline PA231 & $5.71 \mathrm{E}-02$ & $2.81 \mathrm{E}-04$ & $1.48 \mathrm{E}-02$ & $2.49 \mathrm{E}-05$ & $3.82 \mathrm{E}-04$ & $5.09 \mathrm{E}-07$ \\
\hline U233 & $3.02 E \div 01$ & $1.49 E-01$ & $7.52 \mathrm{E}+00$ & $1.26 \mathrm{E}-02$ & $1.83 E-03$ & 2.44E-06 \\
\hline U234 & $2.90 \mathrm{E}+01$ & $1.43 E-01$ & $8.80 \mathrm{E}+00$ & $1.48 \mathrm{E}-02$ & $1.01 E+00$ & $1.35 \mathrm{E}-03$ \\
\hline U235 & $3.27 \mathrm{E}+00$ & $1.61 E-02$ & $1.03 E+01$ & $1.74 \mathrm{E}-02$ & $1.07 E+01$ & 1.43E-02 \\
\hline U236 & $2.73 \mathrm{E}+01$ & $1.35 \mathrm{E}-01$ & $2.93 \mathrm{E}+01$ & $4.92 \mathrm{E}-02$ & $4.06 E+01$ & $5.41 \mathrm{E}-02$ \\
\hline U238 & $2.56 \mathrm{E}+01$ & $1.26 E-01$ & $2.77 E+01$ & $4.66 \mathrm{E}-02$ & $1.14 E+00$ & $1.52 \mathrm{E}-03$ \\
\hline NP237 & $3.52 E+01$ & $1.73 E-01$ & $1.68 \mathrm{E}+01$ & 2.83E-02 & $2.34 \mathrm{E}+01$ & $3.12 \mathrm{E}-02$ \\
\hline PU238 & $3.41 \mathrm{E}+05$ & $1.68 \mathrm{E}+03$ & $3.61 E+04$ & $6.07 E+01$ & $5.02 E+04$ & $6.70 E+01$ \\
\hline PU239 & $3.76 \mathrm{E}+04$ & $1.85 \mathrm{E}+02$ & $1.09 \mathrm{E}+04$ & $1.83 \mathrm{E}+01$ & $1.93 E+03$ & $2.57 E+00$ \\
\hline PU240 & $5.61 E+04$ & $2.76 E+02$ & $4.01 E+03$ & $6.74 \mathrm{E}+00$ & $9.76 E+02$ & $1.30 E+00$ \\
\hline PU241 & $2.98 \mathrm{E}+06$ & $1.47 \mathrm{E}+04$ & $3.93 E+05$ & $6.60 \mathrm{E}+02$ & $2.60 \mathrm{E}+05$ & $3.47 E+02$ \\
\hline PU242 & $1.85 \mathrm{E}+02$ & $9.12 \mathrm{E}-01$ & $3.24 \mathrm{E}+00$ & $5.45 \mathrm{E}-03$ & $1.09 E+00$ & $1.46 \mathrm{E}-03$ \\
\hline AM241 & $3.67 E+05$ & $1.81 \mathrm{E}+03$ & $6.33 \mathrm{E}+03$ & $1.06 \mathrm{E}+01$ & $4.72 E+02$ & $6.29 \mathrm{E}-01$ \\
\hline AM242M & $5.31 \mathrm{E}+02$ & $2.62 E+00$ & $1.28 \mathrm{E}+01$ & 2.15E-02 & $1.53 \mathrm{E}+00$ & $2.04 \mathrm{E}-03$ \\
\hline AM243 & $1.68 \mathrm{E}+03$ & $8.29 E+00$ & $5.93 E+00$ & $9.97 \mathrm{E}-03$ & $4.43 \mathrm{E}+0 \mathrm{D}$ & $5.91 \mathrm{E}-03$ \\
\hline CM244 & $7.31 E+04$ & $3.60 E+02$ & $2.47 E+02$ & $4.16 \mathrm{E}-01$ & $1.82 E+02$ & 2.43E-01 \\
\hline CM245 & $2.89 E+01$ & $1.42 \mathrm{E}-01$ & $6.10 \mathrm{E}-02$ & $1.03 E-04$ & $9.29 \mathrm{E}-03$ & $1.24 \mathrm{E}-05$ \\
\hline CM246 & $4.91 E+00$ & $2.42 \mathrm{E}-02$ & 9.79E-03 & $1.65 \mathrm{E}-05$ & $6.40 E-04$ & 8.53E-07 \\
\hline
\end{tabular}

\begin{tabular}{|l|l|l|l|}
\hline $\begin{array}{l}\text { Appendix A } \\
\text { DOE SNF Information }\end{array}$ & $\begin{array}{l}\text { Sheet } \\
70 \text { of } 75\end{array}$ & $\begin{array}{l}\text { Date: } \\
\text { January 23, 1998 }\end{array}$ & $\begin{array}{l}\text { Rev. } \\
1\end{array}$ \\
\hline
\end{tabular}


Table A-7 (Continued)

\begin{tabular}{|c|c|c|c|c|c|c|}
\hline & TSPA Category & TSPA Category & TSPA Category & TSPA Category & TSPA Category & TSPA Category \\
\hline SNF, not HLW & 7 & 7 & 8 & 8 & 9 & 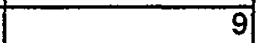 \\
\hline & MTHM & packages & MTHM & packages & MTHM & packages \\
\hline$\overline{\text { INEEL }}$ & & & 24.67 & 545 & 1.66 & 103 \\
\hline SRS & 11.4 & 225 & & & & \\
\hline \multicolumn{7}{|l|}{ Hanford } \\
\hline Total & 11.4 & 225 & 24.67 & 545 & 1.66 & 103 \\
\hline & & & & & & \\
\hline & total curies & Ci/pkg & total curies & Ci/pkg & total curies & Ci/pkg \\
\hline & & & & & & \\
\hline \multicolumn{7}{|l|}{ Isotopes } \\
\hline C14 & $4.09 E+01$ & $1.82 \mathrm{E}-01$ & $1.08 E+02$ & $1.99 \mathrm{E}-01$ & $2.24 E+00$ & $2.18 \mathrm{E}-02$ \\
\hline CL36 & $1.23 E+00$ & $5.48 \mathrm{E}-03$ & $1.36 E+00$ & 2.49E-03 & $6.35 \mathrm{E}-02$ & $6.17 E-04$ \\
\hline NI 59 & $2.41 E+02$ & $1.07 E+00$ & $8.66 \mathrm{E}+00$ & $1.59 \mathrm{E}-02$ & $8.75 \mathrm{E}-02$ & $8.49 E-04$ \\
\hline NI 63 & $2.96 \mathrm{E}+04$ & $1.32 E+02$ & $2.08 E+02$ & $3.82 \mathrm{E}-01$ & $9.02 E+00$ & $8.76 \mathrm{E}-02$ \\
\hline SE79 & $6.33 E+00$ & $2.81 \mathrm{E}-02$ & $1.48 \mathrm{E}+01$ & 2.71E-02 & $8.06 \mathrm{E}-01$ & $7.83 \mathrm{E}-03$ \\
\hline SR90 & $1.43 E+06$ & $6.37 E+03$ & $1.13 E+06$ & $2.08 E+03$ & $1.06 E+05$ & $1.03 E+03$ \\
\hline ZR93 & $3.80 \mathrm{E}+01$ & 1.69E-01 & $5.40 \mathrm{E}+02$ & $9.90 \mathrm{E}-01$ & $3.83 E+00$ & $3.72 \mathrm{E}-02$ \\
\hline NB93M & $3,36 \mathrm{E}+00$ & 1.49E-02 & $4.64 E+00$ & $8.52 \mathrm{E}-03$ & $2.77 E+00$ & $2.69 E-02$ \\
\hline NB94 & $2.24 \mathrm{E}+00$ & $9.96 \mathrm{E}-03$ & $0.00 \mathrm{E} \div 00$ & $0.00 \mathrm{E}+00$ & $2.94 \mathrm{E}-02$ & $2.86 \mathrm{E}-04$ \\
\hline TC99 & $2.14 E+02$ & 9.51E-01 & $3.72 E+02$ & $6.83 \mathrm{E}-01$ & $2.43 E+01$ & $2.36 E-01$ \\
\hline PD107 & $2.99 \mathrm{E}-01$ & 1.33E-03 & 4.38E-01 & $8.03 E-04$ & 2.85E-02 & 2.77E-04 \\
\hline SN126 & $5.89 E+00$ & 2.62E-02 & $6.96 \mathrm{E}+00$ & $1.28 \mathrm{E}-02$ & $7.45 \mathrm{E}-01$ & $7.23 E-03$ \\
\hline 1129 & $3.56 \mathrm{E}-01$ & $1.58 \mathrm{E}-03$ & $1.04 E+00$ & 1.91E-03 & 4.39E-02 & $4.26 \mathrm{E}-04$ \\
\hline CS135 & $1.44 E+01$ & $6.41 \mathrm{E}-02$ & $8.68 E+00$ & $1.59 \mathrm{E}-02$ & $1.58 E+00$ & $1.53 \mathrm{E}-02$ \\
\hline CS137 & $1.51 \mathrm{E}+06$ & $6.72 E+03$ & $1.21 \mathrm{E}+06$ & $2.21 E+03$ & $1.12 \mathrm{E}+05$ & $1.09 E+03$ \\
\hline SM151 & $1.08 \mathrm{E}+04$ & $4.81 E+01$ & $2.26 \mathrm{E}+04$ & $4.15 \mathrm{E}+01$ & $1.53 E+03$ & $1.48 \mathrm{E}+01$ \\
\hline PB210 & $2.54 \mathrm{E}-10$ & $1.13 \mathrm{E}-12$ & $2.30 \mathrm{E}-03$ & $4.22 \mathrm{E}-06$ & 1.56E-05 & $1.51 \mathrm{E}-07$ \\
\hline RA226 & $6.84 \mathrm{E}-10$ & $3.04 \mathrm{E}-12$ & 2.44E-03 & $4.48 \mathrm{E}-06$ & $5.60 \mathrm{E}-05$ & 5.44E-07 \\
\hline RA228 & 1.97E-06 & 8.77E-09 & $3.29 \mathrm{E}+00$ & 6.04E-03 & 1.43E-01 & $1.39 \mathrm{E}-03$ \\
\hline AC227 & $2.63 E-05$ & 1.17E-07 & $0.00 \mathrm{E}+00$ & $0.00 E+00$ & $1.70 \mathrm{E}-01$ & $1.65 \mathrm{E}-03$ \\
\hline TH229 & 5.37E-07 & 2.39E-09 & $1.39 \mathrm{E}+01$ & $2.56 \mathrm{E}-02$ & 4.11E-01 & $3.99 \mathrm{E}-03$ \\
\hline TH230 & 1.24E-06 & $5.51 \mathrm{E}-09$ & 9.54E-01 & $1.75 \mathrm{E}-03$ & 7.91E-03 & 7.68E-05 \\
\hline TH232 & $5.82 \mathrm{E}-06$ & $2.59 \mathrm{E}-08$ & $2.55 \mathrm{E}+00$ & $4.68 \mathrm{E}-03$ & $1.50 E-01$ & $1.46 \mathrm{E}-03$ \\
\hline PA231 & 4.22E-04 & $1.88 \mathrm{E}-06$ & $9.34 \mathrm{E}+00$ & $1.71 E-02$ & $2.89 \mathrm{E}-01$ & $2.80 \mathrm{E}-03$ \\
\hline U233 & $2.79 E-03$ & $1.24 \mathrm{E}-05$ & $3.46 \mathrm{E}+03$ & $6.35 E+00$ & $1.57 E+02$ & $1.52 E+00$ \\
\hline U234 & 7.29E-02 & $3.24 \mathrm{E}-04$ & $2.81 E+02$ & $5.16 \mathrm{E}-01$ & $2.60 E+01$ & 2.53E-01 \\
\hline U235 & $4.61 E+00$ & $2.05 \mathrm{E}-02$ & $1.08 E+00$ & $1.99 \mathrm{E}-03$ & 4.05E-01 & $3.93 \mathrm{E}-03$ \\
\hline $\mathrm{U} 236$ & $6.26 \mathrm{E}+00$ & $2.78 \mathrm{E}-02$ & $1.07 \mathrm{E}+01$ & $1.96 \mathrm{E}-02$ & $1.02 E+00$ & $9.93 \mathrm{E}-03$ \\
\hline U238 & $3.48 \mathrm{E}+00$ & $1.55 \mathrm{E}-02$ & $2.69 \mathrm{E}-02$ & $4.94 \mathrm{E}-05$ & $4.24 \mathrm{E}-03$ & $4.11 \mathrm{E}-05$ \\
\hline NP237 & 9.17E-01 & $4.08 \mathrm{E}-03$ & $8.16 \mathrm{E}+00$ & $1.50 \mathrm{E}-02$ & $4.41 \mathrm{E}-01$ & $4.28 \mathrm{E}-03$ \\
\hline PU238 & $1.27 \mathrm{E}+03$ & $5.65 \mathrm{E}+00$ & $4.08 \mathrm{E}+04$ & $7.49 E+01$ & $1.10 E+03$ & $1.07 E+01$ \\
\hline PU239 & $2.47 E+03$ & $1.10 \mathrm{E}+01$ & $1.13 E+02$ & 2.07E-01 & $2.46 E+01$ & $2.39 \mathrm{E}-01$ \\
\hline PU240 & $9.58 \mathrm{E}+02$ & $4.26 E+00$ & $1.90 \mathrm{E}+02$ & 3.49E-01 & $1.93 E+01$ & $1.87 \mathrm{E}-01$ \\
\hline PU241 & $6.14 E+04$ & $2.73 E+02$ & $0.00 E+00$ & $0.00 E+00$ & $1.80 \mathrm{E}+03$ & $1.75 \mathrm{E}+01$ \\
\hline PU242 & $1.20 \mathrm{E}-01$ & 5.33E-04 & $0.00 E \div 00$ & $0.00 E+00$ & $2.54 \mathrm{E}-02$ & $2.46 \mathrm{E}-04$ \\
\hline AM241 & $9.59 E+01$ & $4.26 \mathrm{E}-01$ & $1.87 \mathrm{E}+03$ & $3.42 E+00$ & $1.59 E+02$ & $1.54 E+00$ \\
\hline AM242M & $6.63 \mathrm{E}-01$ & $2.95 \mathrm{E}-03$ & $5.05 \mathrm{E}-01$ & $9.27 \mathrm{E}-04$ & $8.15 E-02$ & 7.91E-04 \\
\hline AM243 & $1.37 E-01$ & $6.07 \mathrm{E}-04$ & $1.33 \mathrm{E}+01$ & $2.45 \mathrm{E}-02$ & $7.90 \mathrm{E}-02$ & 7.67E-04 \\
\hline CM244 & $3.84 \mathrm{E}+00$ & $1.71 \mathrm{E}-02$ & $4.19 \mathrm{E}+02$ & $7.68 \mathrm{E}-01$ & $2.07 E+00$ & 2.01E-02 \\
\hline CM245 & $1.57 \mathrm{E}-04$ & $6.98 \mathrm{E}-07$ & $7.00 \mathrm{E}-02$ & $1.28 \mathrm{E}-04$ & $2.40 E-04$ & $2.33 \mathrm{E}-06$ \\
\hline CM246 & $1.05 \mathrm{E}-05$ & $4.67 \mathrm{E}-08$ & $3.49 \mathrm{E}-02$ & $6.40 \mathrm{E}-05$ & $7 . \overline{78 E}-06$ & $7.55 \mathrm{E}-08$ \\
\hline
\end{tabular}

Appendix A DOE SNF Information

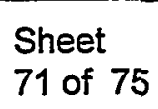

Date:

January 23, 1998
Rev.

1 
Table A-7 (Continued)

\begin{tabular}{|c|c|c|c|c|c|c|}
\hline & TSPA Category & TSPA Category & TSPA Category & TSPA Category & TSPA Category & TSPA Category \\
\hline SNF, not HLW & 10 & 10 & 11 & 11 & 12 & 12 \\
\hline & MTHM & packages & MTHM & packages & MTHM & packages \\
\hline INEEL & 0.05 & 4 & 1.55 & 25 & 49.66 & 69 \\
\hline SRS & & & & & & \\
\hline Hanford & 0.17 & 1 & 10.2 & 327 & & \\
\hline Total & 0.22 & 5 & 11.75 & 352 & 49.66 & 69 \\
\hline & & & & & & \\
\hline & total curies & Ci/pkg & total curies & Ci/pkg & total curies & Ci/pkg \\
\hline & & & & & & \\
\hline Isotopes & & & & & & \\
\hline C14 & $0.00 \mathrm{E}+00$ & $0.00 E+00$ & 2.38E-01 & 6.75E-04 & $4.74 E+01$ & 6.87E-01 \\
\hline$\overline{C L 36}$ & $0.00 \mathrm{E}+00$ & $0.00 \mathrm{E}+00$ & $4.42 \mathrm{E}-03$ & $1.26 \mathrm{E}-05$ & $1.06 \mathrm{E}+00$ & $1.53 \mathrm{E}-02$ \\
\hline N1 59 & $0.00 \mathrm{E}+00$ & $0.00 \mathrm{E}+00$ & $8.62 \mathrm{E}-01$ & $2.45 \mathrm{E}-03$ & $3.63 \mathrm{E}+00$ & $5.26 \mathrm{E}-02$ \\
\hline NI 63 & $7.36 \mathrm{E}+01$ & $1.47 \mathrm{E}+01$ & $3.66 \mathrm{E}+03$ & $1.04 E+01$ & $4.43 E+02$ & $6.41 E+00$ \\
\hline SE79 & $0.00 E+00$ & $0.00 \mathrm{E}+00$ & $5.28 \mathrm{E}-01$ & $1.50 \mathrm{E}-03$ & $1.75 E+01$ & $2.53 \mathrm{E}-01$ \\
\hline SR90 & $1.06 \mathrm{E}+04$ & $2.13 E+03$ & $5.65 \mathrm{E}+05$ & $1.61 E+03$ & $1.81 E+05$ & $2.63 E+03$ \\
\hline ZR93 & $0.00 E+00$ & $0.00 \mathrm{E}+00$ & $2.47 E+00$ & 7.03E-03 & $4.08 E+01$ & $5.91 \mathrm{E}-01$ \\
\hline NB93M & $0.00 E+00$ & $0.00 \mathrm{E}+00$ & $1.79 E+00$ & 5.09E-03 & $2.19 E+01$ & 3.17E-01 \\
\hline$\overline{\text { NB94 }}$ & $0.00 E+00$ & $0.00 \mathrm{E}+00$ & 8.03E-03 & $2.28 E-05$ & $1.10 \mathrm{E}+00$ & $1.60 \mathrm{E}-02$ \\
\hline TC99 & $0.00 E+00$ & $0.00 E+00$ & $1.84 E+01$ & $5.22 \mathrm{E}-02$ & $1.62 \mathrm{E}+02$ & $2.35 E+00$ \\
\hline PD107 & $0.00 E+00$ & $0.00 \mathrm{E}+00$ & $1.45 \mathrm{E}-01$ & $4.12 E-04$ & $1.70 \mathrm{E}-01$ & $2.47 \mathrm{E}-03$ \\
\hline SN126 & $0.00 E+00$ & $0.00 \mathrm{E}+00$ & 6.85E-01 & $1.95 \mathrm{E}-03$ & $1.96 \mathrm{E}+01$ & $2.84 \mathrm{E}-01$ \\
\hline 1129 & $0.00 \mathrm{E}+00$ & $0.00 \mathrm{E}+00$ & 5.14E-02 & $1.46 \mathrm{E}-04$ & 7.85E-01 & $1.14 \mathrm{E}-02$ \\
\hline CS135 & $0.00 E+00$ & $0.00 \mathrm{E}+00$ & $4.86 \mathrm{E}-01$ & $1.38 \mathrm{E}-03$ & $1.43 \mathrm{E}+01$ & $2.07 \mathrm{E}-01$ \\
\hline$\overline{C S 137}$ & $3.05 E+04$ & $6.11 E+03$ & $1.55 \mathrm{E}+06$ & $4.39 E+03$ & $1.76 \mathrm{E}+05$ & $2.56 E+03$ \\
\hline SM151 & $2.02 E+03$ & $4.04 \mathrm{E}+02$ & $9.90 \mathrm{E}+04$ & $2.81 \mathrm{E}+02$ & $6.43 E+03$ & $9.32 E+01$ \\
\hline PB210 & $0.00 E+00$ & $0.00 \mathrm{E}+00$ & 4.37E-08 & $1.24 \mathrm{E}-10$ & $5.57 \mathrm{E}-03$ & $8.08 \mathrm{E}-05$ \\
\hline RA226 & $3.34 \mathrm{E}-08$ & $6.69 \mathrm{E}-09$ & $\mid 1.82 \mathrm{E}-06$ & $5.18 \mathrm{E}-09$ & $3.20 \mathrm{E}-03$ & 4.63E-05 \\
\hline RA228 & $6.97 \mathrm{E}-12$ & $1.39 \mathrm{E}-12$ & 7.77E-09| & $2.21 \mathrm{E}-11$ & $5.03 E+00$ & $7.30 \mathrm{E}-02$ \\
\hline AC227 & $7.23 \mathrm{E}-09$ & $1.45 \mathrm{E}-09$ & 5.06E-07 & $1.44 \mathrm{E}-09$ & $3.05 E+01$ & $4.41 \mathrm{E}-01$ \\
\hline TH229 & $8.51 E-10$ & $1.70 \mathrm{E}-10$ & 2.67E-07 & $7.58 \mathrm{E}-10$ & $1.29 E+01$ & 1.87E-01 \\
\hline TH230 & $7.35 \mathrm{E}-06$ & 1.47E-06 & $4.00 E-04$ & 1.14E-06 & $4.88 \mathrm{E}-01$ & $7.07 \mathrm{E}-03$ \\
\hline TH232 & $1.15 \mathrm{E}-11$ & $2.31 E-12$ & $2.19 \mathrm{E}-08$ & $6.23 \mathrm{E}-11$ & $5.98 \mathrm{E}+00$ & $8.66 \mathrm{E}-02$ \\
\hline PA231 & $2.77 \mathrm{E}-08$ & $5.53 \mathrm{E}-09$ & $1.94 \mathrm{E}-05$ & $5.51 \mathrm{E}-08$ & $7.68 \mathrm{E}+01$ & $1.11 \mathrm{E}+00$ \\
\hline$\overline{U 233}$ & $1.57 \mathrm{E}-06$ & 3.14E-07 & 1.88E-04 & 5.33E-07 & $8.38 E+03$ & $1.21 E+02$ \\
\hline U234 & $5.06 E-02$ & $1.01 \mathrm{E}-02$ & $2.75 E+00$ & $7.80 E-03$ & $4.17 E+02$ & $6.04 E+00$ \\
\hline U235 & $8.44 \mathrm{E}-05$ & $1.69 \mathrm{E}-05$ & $4.36 \mathrm{E}-02$ & $1.24 E-04$ & $2.80 \mathrm{E}-02$ & $4.06 \mathrm{E}-04$ \\
\hline$\overline{U 236}$ & $1.50 \mathrm{E}-02$ & $3.01 \mathrm{E}-03$ & $1.08 \mathrm{E}+00$ & $3.06 E-03$ & $5.76 \mathrm{E}-02$ & $8.35 E-04$ \\
\hline $\mathrm{U238}$ & $1.57 E-13$ & $3.14 \mathrm{E}-14$ & $3.91 \mathrm{E}-01$ & $1.11 \mathrm{E}-03$ & 9.11E-04 & $1.32 \mathrm{E}-05$ \\
\hline NP237 & $2.70 \mathrm{E}-02$ & 5.39E-03 & $1.78 \mathrm{E}+00$ & $5.05 E-03$ & $5.03 E-02$ & $7.28 \mathrm{E}-04$ \\
\hline PU238 & $5.09 E+02$ & $1.02 E+02$ & $2.75 E+04$ & $7.82 \mathrm{E}+01$ & $1.96 \mathrm{E}+02$ & $2.84 E+00$ \\
\hline PU239 & $2.77 E+03$ & $5.53 E+02$ & $1.34 E+05$ & $3.80 \mathrm{E}+02$ & $1.27 E+01$ & $1.84 \mathrm{E}-01$ \\
\hline PU240 & $2.39 E+03$ & $4.78 \mathrm{E}+02$ & $1.16 \mathrm{E}+05$ & $3.29 \mathrm{E}+02$ & $7.24 \mathrm{E}+00$ & 1.05E-01 \\
\hline PU241 & $2.00 E+04$ & $4.00 \mathrm{E}+03$ & $1.01 E+06$ & $2.86 \mathrm{E}+03$ & $1.72 E+03$ & $2.50 \mathrm{E}+01$ \\
\hline PU242 & $6.40 \mathrm{E}-05$ & $1.28 \mathrm{E}-05$ & $2.80 \mathrm{E}+00$ & $7.95 \mathrm{E}-03$ & $1.62 \mathrm{E}-02$ & $2.35 \mathrm{E}-04$ \\
\hline AM241 & $3.48 \mathrm{E}+03$ & $6.97 E+02$ & $1.72 \mathrm{E}+05$ & $4.90 \mathrm{E}+02$ & $5.92 E+01$ & $8.57 \mathrm{E}-01$ \\
\hline AM242M & $6.05 E+00$ & $1.21 \mathrm{E}+0 \mathrm{O}$ & $3.00 \mathrm{E}+02$ & $8.51 \mathrm{E}-01$ & $6.20 \mathrm{E}-01$ & $8.99 E-03$ \\
\hline AM243 & $0.00 E+00$ & $0.00 \mathrm{E}+00$ & $2.54 \mathrm{E}+01$ & $7.22 E-02$ & $1.18 \mathrm{E}-01$ & $1.71 \mathrm{E}-03$ \\
\hline CM244 & $0.00 E+00$ & $0.00 \mathrm{E}+00$ & 1.07E+03 & $3.03 E+00$ & $1.14 \mathrm{E}+01$ & 1.65E-01 \\
\hline CM245 & $0.00 E+00$ & $0.00 E+00$ & 4.37E-01 & 1.24E-03 & $2.35 \mathrm{E}-03$ & 3.41E-05 \\
\hline CM246 & $0.00 E+00$ & $0.00 E+00$ & 7.42E-02 & $2.11 E-04$ & $1.55 \mathrm{E}-04$ & $2.25 \mathrm{E}-06$ \\
\hline
\end{tabular}

Appendix A

DOE SNF Information
Sheet 72 of 75
Date:

January 23, 1998
Rev.

1 
Table A-7 (Continued)

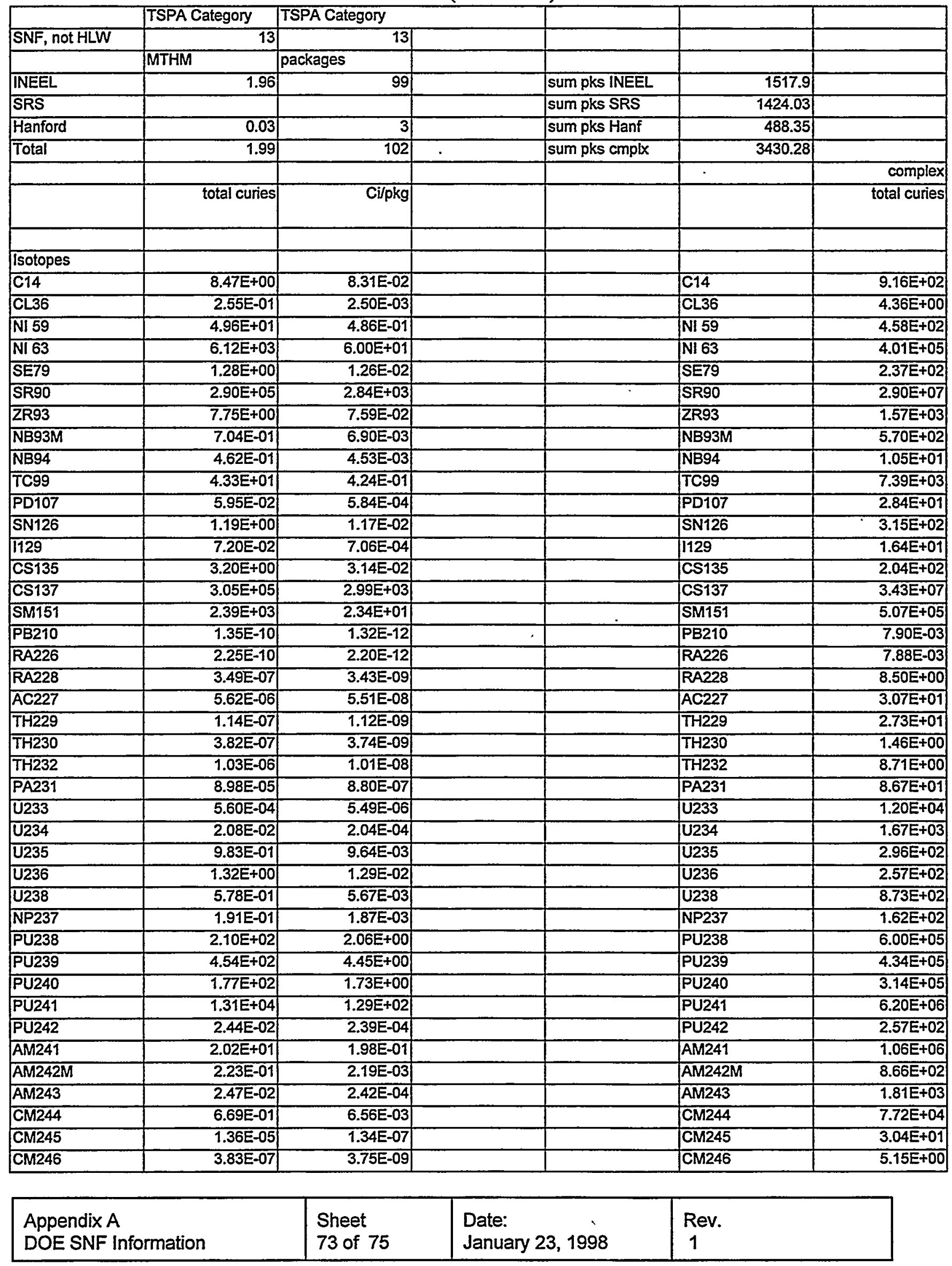


Table A-8 Commercial Spent Nuclear Fuel Inventory

From RW TSPA-1995 report

\begin{tabular}{|c|c|c|c|}
\hline Isotope & $\begin{array}{c}\text { Multi-Barrier Waste } \\
\text { Package Design - 21 PWR } \\
\text { (Ci/pkg) }{ }^{1,2}\end{array}$ & Isotope & $\begin{array}{c}\text { Multi-Barrier Waste } \\
\text { Package Design - 21 PWR } \\
\text { (Ci/pkg) }\end{array}$ \\
\hline${ }^{227} \mathrm{Ac}$ & $1.79 \mathrm{e}-4$ & ${ }^{239} \mathrm{Pu}$ & $3.56 \mathrm{e} 3$ \\
\hline${ }^{241} \mathrm{Am}$ & $3.73 \mathrm{e} 4$ & ${ }^{240} \mathrm{Pu}$ & $5.26 \mathrm{e} 3$ \\
\hline${ }^{242 m} \mathrm{Am}$ & $2.16 \mathrm{e} 2$ & ${ }^{241} \mathrm{Pu}$ & $3.39 \mathrm{e} 5$ \\
\hline${ }^{243} \mathrm{Am}$ & $2.48 \mathrm{e} 2$ & ${ }^{242} \mathrm{Pu}$ & $2.01 \mathrm{e} 1$ \\
\hline${ }^{14} \mathrm{C}$ & $1.38 \mathrm{e} 1$ & ${ }^{226} \mathrm{Ra}$ & $2.50 \mathrm{e}-5$ \\
\hline${ }^{36} \mathrm{Cl}^{3}$ & $1.11 \mathrm{e}-1$ & ${ }^{228} \mathrm{Ra}$ & $3.10 \mathrm{e}-9$ \\
\hline${ }^{244} \mathrm{Cm}$ & $1.16 \mathrm{e} 4$ & ${ }^{79} \mathrm{Se}$ & $4.41 \mathrm{e} 0$ \\
\hline${ }^{245} \mathrm{Cm}$ & $3.36 \mathrm{e} 0$ & ${ }^{151} \mathrm{Sm}$ & $3.53 \mathrm{e} 3$ \\
\hline${ }^{246} \mathrm{Cm}$ & $6.95 \mathrm{e}-1$ & ${ }^{126} \mathrm{Sn}$ & $8.50 \mathrm{e} 0$ \\
\hline${ }^{135} \mathrm{Cs}$ & $5.13 \mathrm{e} 0$ & ${ }^{90} \mathrm{Sr}$ & $3.85 \mathrm{e} 5$ \\
\hline${ }^{137} \mathrm{Cs}$ & $5.61 \mathrm{e} 5$ & ${ }^{99} \mathrm{Tc}$ & $1.40 \mathrm{e} 2$ \\
\hline${ }^{129} \mathrm{I}$ & $3.43 \mathrm{e}-1$ & ${ }^{229} \mathrm{Th}$ & $3.54 \mathrm{e}-6$ \\
\hline${ }^{93 \mathrm{~m}} \mathrm{Nb}$ & $1.82 \mathrm{e} 1$ & ${ }^{230} \mathrm{Th}$ & $3.59 \mathrm{e}-3$ \\
\hline${ }^{94} \mathrm{Nb}$ & $8.24 \mathrm{e} 0$ & ${ }^{232} \mathrm{Th}$ & $4.35 \mathrm{e}-9$ \\
\hline${ }^{59} \mathrm{Ni}$ & $2.36 \mathrm{e} 1$ & ${ }^{233} \mathrm{U}$ & $7.01 \mathrm{e}-4$ \\
\hline${ }^{63} \mathrm{Ni}$ & $3.10 \mathrm{e} 3$ & ${ }^{234} \mathrm{U}$ & $1.34 \mathrm{e} 1$ \\
\hline${ }^{237} \mathrm{~Np}$ & $4.35 \mathrm{e} 0$ & ${ }^{235} \mathrm{U}$ & $1.68 \mathrm{e}-1$ \\
\hline${ }^{231} \mathrm{~Pa}$ & $3.30 \mathrm{e} 4$ & ${ }^{236} \mathrm{U}$ & $2.72 \mathrm{e} 0$ \\
\hline${ }^{210} \mathrm{~Pb}$ & $6.75 \mathrm{e}-6$ & ${ }^{238} \mathrm{U}$ & $3.07 \mathrm{e} 0$ \\
\hline${ }^{107} \mathrm{Pd}$ & $1.26 \mathrm{e} 0$ & ${ }^{93} \mathrm{Zr}$ & $2.38 \mathrm{er}$ \\
\hline${ }^{238} \mathrm{Pu}$ & $3.05 \mathrm{e} 4$ & & \\
\hline
\end{tabular}

${ }^{1}$ Assumes 40,785 MTU PWR with a burnup of 39,651 MWd/MTU, and 22,211 MTU BWR with a burnup of $31,186 \mathrm{MWd} / \mathrm{MTU}$

2 9.74 MTHM/container, 21 PWR case

${ }^{3}$ Carbon, Chlorine, and Iodine inventory assumed to be gaseous release

${ }^{4} \mathrm{Cs}-137$ and Sr-90 inventories are from previous PA completed by DOE-EM for commercial like fuels. These inventories are not from the RW TSPA. 
Table A-9 HLW Inventory

From RW TSPA-1995 report

\begin{tabular}{|c|c|c|c|}
\hline Isotope & $\begin{array}{c}\text { DHLW Inventory } \\
(\text { Ci } / \mathrm{pkg})^{1}\end{array}$ & Isotope & $\begin{array}{c}\text { DHLW Inventory } \\
(\mathrm{Ci} / \mathrm{pkg})\end{array}$ \\
\hline${ }^{227} \mathrm{Ac}$ & $6.02 \mathrm{e}-4$ & ${ }^{239} \mathrm{Pu}$ & $4.73 \mathrm{e} 0$ \\
\hline${ }^{241} \mathrm{Am}$ & $8.65 \mathrm{e} 1$ & ${ }^{240} \mathrm{Pu}$ & $3.30 \mathrm{e} 0$ \\
\hline${ }^{242 m} \mathrm{Am}$ & $2.06 \mathrm{e}-2$ & ${ }^{241} \mathrm{Pu}$ & $1.48 \mathrm{e} 2$ \\
\hline${ }^{243} \mathrm{Am}$ & $3.67 \mathrm{e}-2$ & ${ }^{242} \mathrm{Pu}$ & $5.02 \mathrm{e}-3$ \\
\hline${ }^{14} \mathrm{C}$ & 0 & ${ }^{226} \mathrm{Ra}$ & $9.37 \mathrm{e}-8$ \\
\hline${ }^{36} \mathrm{Cl}$ & 0 & ${ }^{228} \mathrm{Ra}$ & 0 \\
\hline${ }^{244} \mathrm{Cm}$ & $1.14 \mathrm{e} 1$ & ${ }^{79} \mathrm{Se}$ & $9.18 \mathrm{e}-2$ \\
\hline${ }^{245} \mathrm{Cm}$ & $5.64 \mathrm{e}-5$ & ${ }^{151} \mathrm{Sm}$ & 0 \\
\hline${ }^{246} \mathrm{Cm}$ & $6.39 \mathrm{e}-6$ & ${ }^{126} \mathrm{Sn}$ & 0 \\
\hline${ }^{135} \mathrm{Cs}$ & $1.15 \mathrm{e}-1$ & ${ }^{90} \mathrm{Sr}$ & $9.09 \mathrm{e} 3$ \\
\hline${ }^{137} \mathrm{Cs}$ & $1.32 \mathrm{e} 4$ & ${ }^{99} \mathrm{Tc}$ & $3.30 \mathrm{e} 0$ \\
\hline${ }^{129} \mathrm{~T}$ & $1.90 \mathrm{e}-6$ & ${ }^{229} \mathrm{Th}$ & $1.51 \mathrm{e}-5$ \\
\hline${ }^{93 \mathrm{~m}} \mathrm{Nb}$ & $5.48 \mathrm{e}-1$ & ${ }^{230} \mathrm{Th}$ & $1.24 \mathrm{e}-5$ \\
\hline${ }^{94} \mathrm{Nb}$ & $3.02 \mathrm{e}-5$ & ${ }^{232} \mathrm{Th}$ & $1.05 \mathrm{e}-4$ \\
\hline${ }^{59} \mathrm{Ni}$ & $2.70 \mathrm{e}-2$ & ${ }^{233} \mathrm{U}$ & $5.84 \mathrm{e}-4$ \\
\hline${ }^{63} \mathrm{Ni}$ & 0 & ${ }^{234} \mathrm{U}$ & $5.00 \mathrm{e}-2$ \\
\hline${ }^{237} \mathrm{~Np}$ & $2.83 \mathrm{e}-2$ & ${ }^{235} \mathrm{U}$ & $7.93 \mathrm{e}-5$ \\
\hline${ }^{231} \mathrm{~Pa}$ & $9.74 \mathrm{e}-4$ & ${ }^{236} \mathrm{U}$ & $4.35 \mathrm{e}-4$ \\
\hline${ }^{210} \mathrm{~Pb}$ & $2.72 \mathrm{e}-8$ & ${ }^{238} \mathrm{U}$ & $3.78 \mathrm{e}-3$ \\
\hline${ }^{107} \mathrm{Pd}$ & 0 & ${ }^{93} \mathrm{Zr}$ & $7.01 \mathrm{e}-1$ \\
\hline${ }^{238} \mathrm{Pu}$ & $4.00 \mathrm{e} 2$ & & \\
\hline
\end{tabular}

${ }^{1}$ Assumed 4 canisters per container.

Source: DOE (1987). Same inventory as DHLW inventory in TSPA-1993.

${ }^{2}$ Cs-137 and Sr-90 inventories are from previous PA completed by DOE-EM.

These inventories are not from the RW TSPA.

Appendix A

DOE SNF Information
Sheet

75 of 75
Date:

January 23, 1998
Rev.

1 


\section{Appendix B: \\ SNF Grouping for TSPA, 2035 Data}


Appendix B: SNF Grouping for TSPA, 2035 Data 


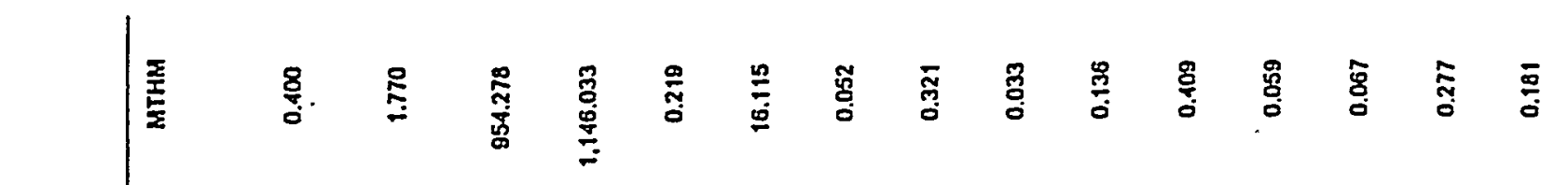

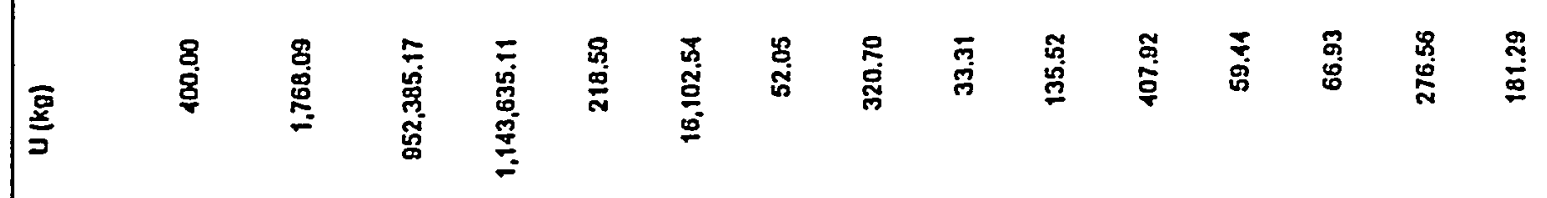

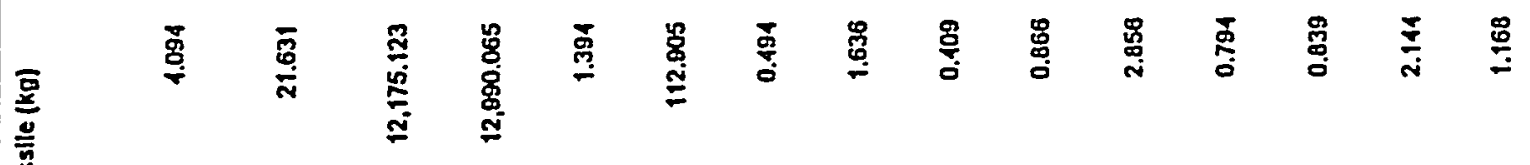

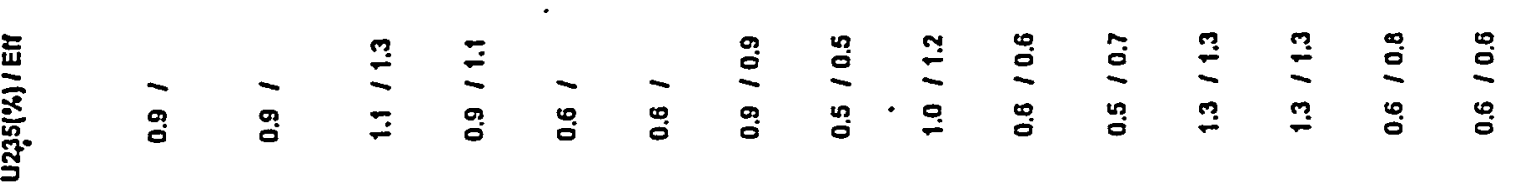

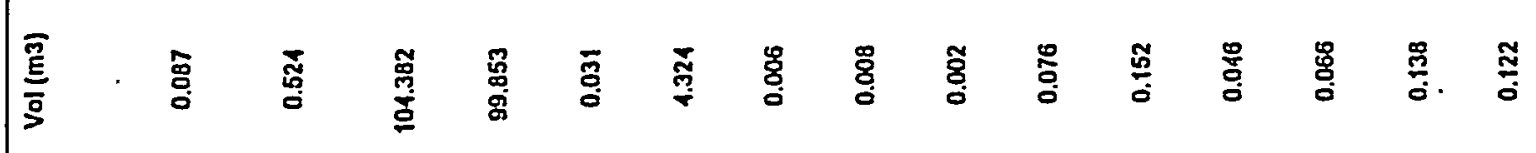

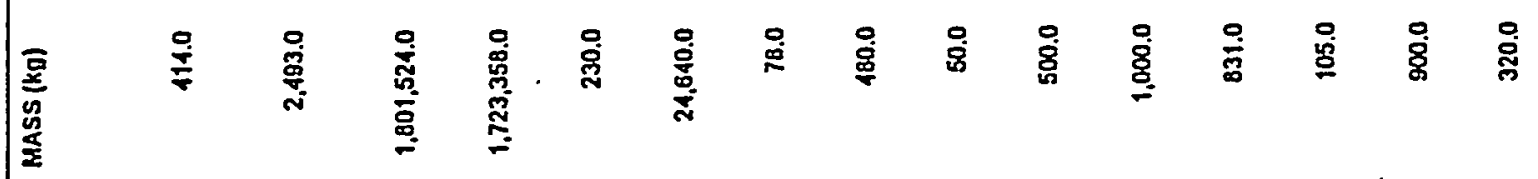

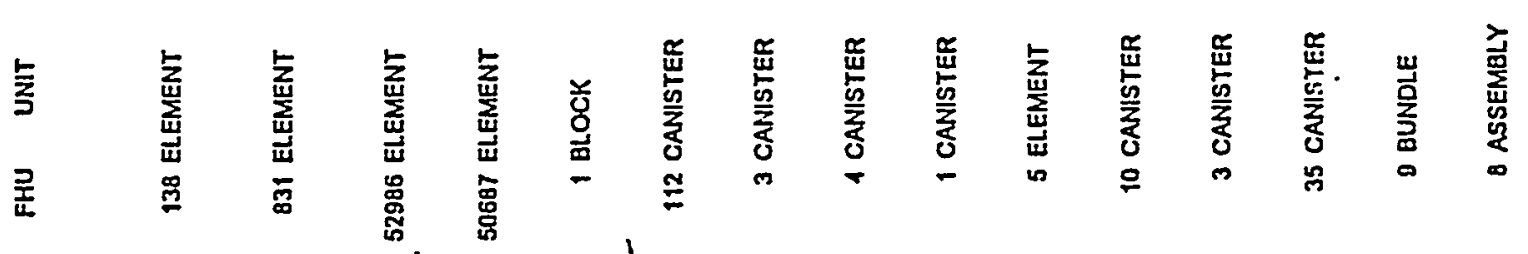

$$
\begin{aligned}
& 3 \text { I }
\end{aligned}
$$

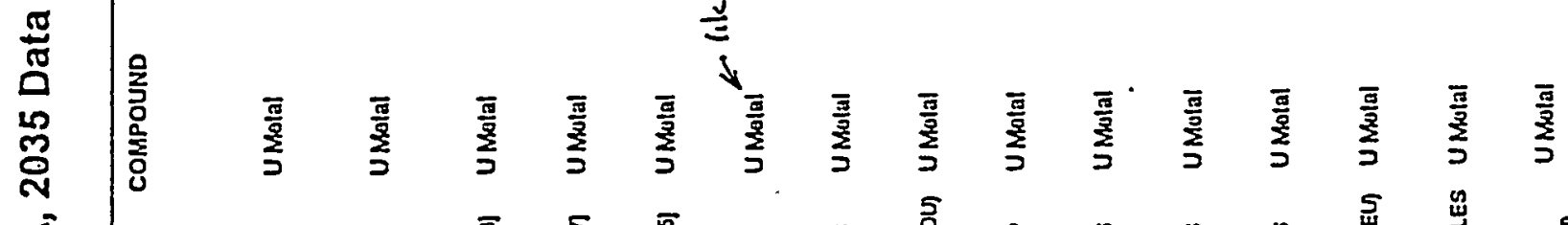

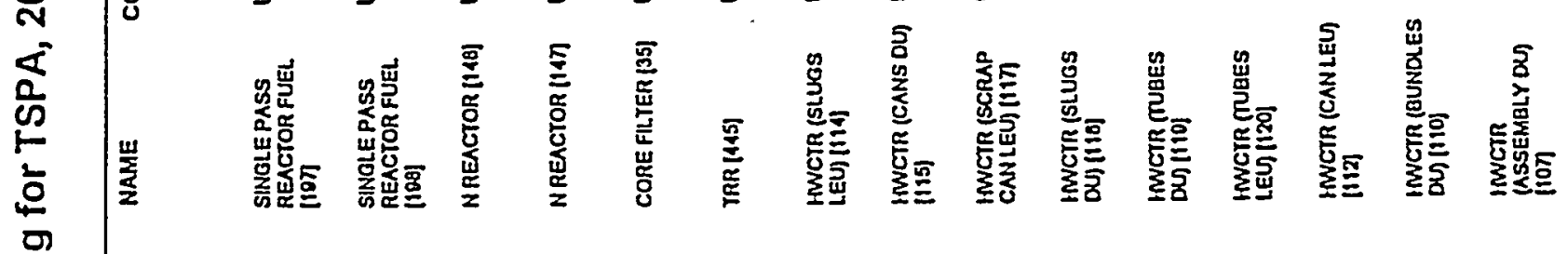

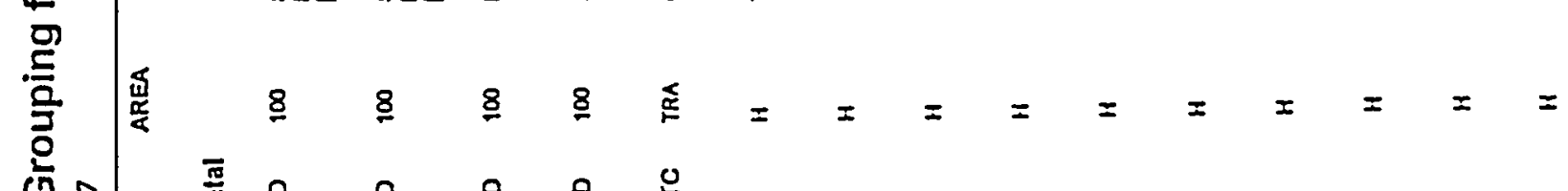

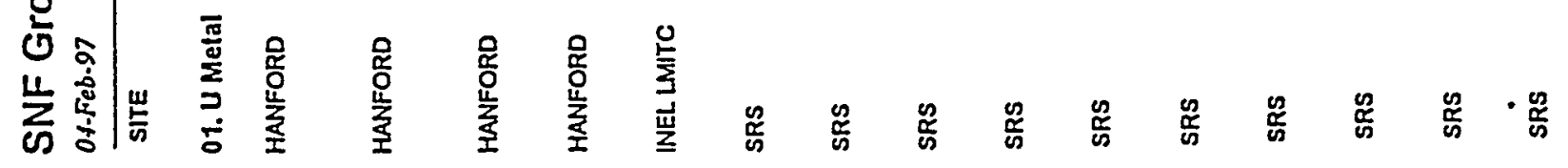


SNE Grouping for TSPA, 2035 Data

$0+-F+b-97$

TSPA CATECORY

U Mat

orus

a. U Ho

Q. 4 Oxdde inact

\section{U Oxlde Falledronetad}

OS. UAL \& UAN

07. Us:

os. Thu Carolde High Integrtiy

09. muru cartide Low Integrty

Mu carolde NonGrapht

11. MOX

12. Tur oxde

13. $12 x$

14. Sodium Bended

15. Nary

16. Not Evaluated (mise)
Sub Tout

\begin{tabular}{|c|c|c|c|c|c|}
\hline FHU & $\operatorname{Mass}(\mathrm{kg})$ & Yol $(\mathrm{m} 3)$ & $F|x=1|=\left(x_{g}\right)$ & $v\left(k_{0}\right)$ & МтиМ \\
\hline 104099 & $3,576,597.0$ & 2129 & 20.4313 & $2,132.004 .5$ & 21 \\
\hline
\end{tabular}

Sub Totat:

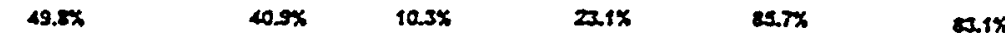

\begin{tabular}{cccccc}
$0.0 x$ & $0.0 x$ & $0.0 x$ & $0.0 x$ & 0.05 & $0.0 x$ \\
\hline 214 & 3.416 .3 & 1.2 & 999.4 & 3.930 .1 & 2.931
\end{tabular}

$\begin{array}{llllll}0.1 \% & 0.1 \times & 0.1 \% & 0.9 \times & 0.2 x & 0.2 x\end{array}$

Sub Toat

\begin{tabular}{llllll}
\hline 4023 & 187.6827 & 51.2 & 244.3 & 90,0007 & 91619
\end{tabular}

Sub Totat:

$\begin{array}{llllll}2.94 & 25 x & 25 x & 35 & 25 x\end{array}$

\begin{tabular}{llllll}
\hline soso & $395,009,9$ & 209.3 & $4,604,3$ & 86,7320 & ecsis
\end{tabular}

Sub Totat

\begin{tabular}{|c|c|c|c|c|c|}
\hline $4.3 x$ & $4.5 x$ & $28.1 \%$ & 425 & $25 x$ & $36 x$ \\
\hline & $112,007,6$ & 1273 & 5,0427 & 8,9221 & 2072 \\
\hline
\end{tabular}

Sub Toot

$\begin{array}{llllll}0.5 x & 1.35 & 6.15 & 5 x & 0.4 x & 0.35\end{array}$

\begin{tabular}{llllll}
\hline 7170 & 42336.6 & 50.8 & 1.137 .4 & 11.356 .8 & 11.356
\end{tabular}

$\begin{array}{llllll}3.45 & 0.58 & 25 x & 4.0 x & 0.5 x & 0.4 x\end{array}$

Sub Toat:

\begin{tabular}{|c|c|c|c|c|c|}
\hline 3020 & $315,046,4$ & 2126 & 994.4 & 12002 & 24.657 \\
\hline
\end{tabular}

Sub Toat

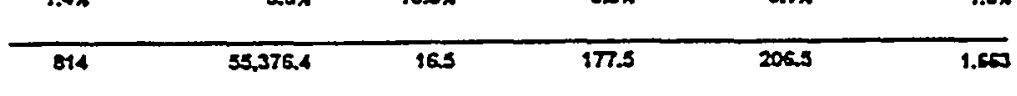

Sub Totat:

\begin{tabular}{llllll}
0.45 & $0.6 x$ & 0.85 & 0.25 & 0.05 & 0.15 \\
\hline
\end{tabular}

\begin{tabular}{llllll}
\hline 19 & 5003 & 02 & 224 & 1460 & 0.100 \\
$0.0 x$ & $0.0 \%$ & 0.05 & $0.0 \%$ & $0.0 \%$ & $0.0 \%$
\end{tabular}

Sub Touk

\begin{tabular}{llllll}
\hline 2003 & $57,509.7$ & 136.0 & 24722 & 9,6623 & 11.99
\end{tabular}

Sub Toar

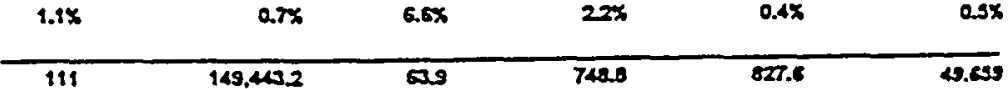

$\begin{array}{llllll}0.15 & 1.7 x & 31 \times & 0.75 & 0.05 & 1.9 x\end{array}$

Sub Tobt

\begin{tabular}{llllll}
\hline 9568 & 31.078 .6 & 0.4 & 465.4 & 1.990 .4 & 1.997
\end{tabular}

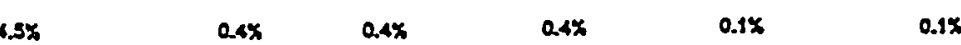

Sub Totat

\begin{tabular}{llllll}
\hline 35595 & 99,9789 & 50.2 & 2.430 .7 & 60.120 .9 & 00325
\end{tabular}

$\cdot$

Sub Totat

$16.95 \quad 24 x \quad 27 x \quad 24 x \quad \therefore 25 x$

\begin{tabular}{llllll}
\hline 6790 & $3,659,14.5$ & $\operatorname{sesh0}$ & 61.355 .4 & $05,031.4$ & 0051
\end{tabular}

sub Totat

\begin{tabular}{rrrrrr}
$32 x$ & $41.9 x$ & $425 x$ & $55.5 x$ & $25 x$ & $25 x$ \\
\hline 5213 & $46,121.3$ & 200 & 5020 & 8.344 .0 & 10.004 \\
4.45 & $0.5 x$ & 1.14 & $0.8 x$ & $0.5 x$ & $0.4 x$
\end{tabular}

Grand Total:

1. Disposition of Navy SNF will be performed by the

Navy. 2. Current plans call for treatment of sodium bonded SNF. 


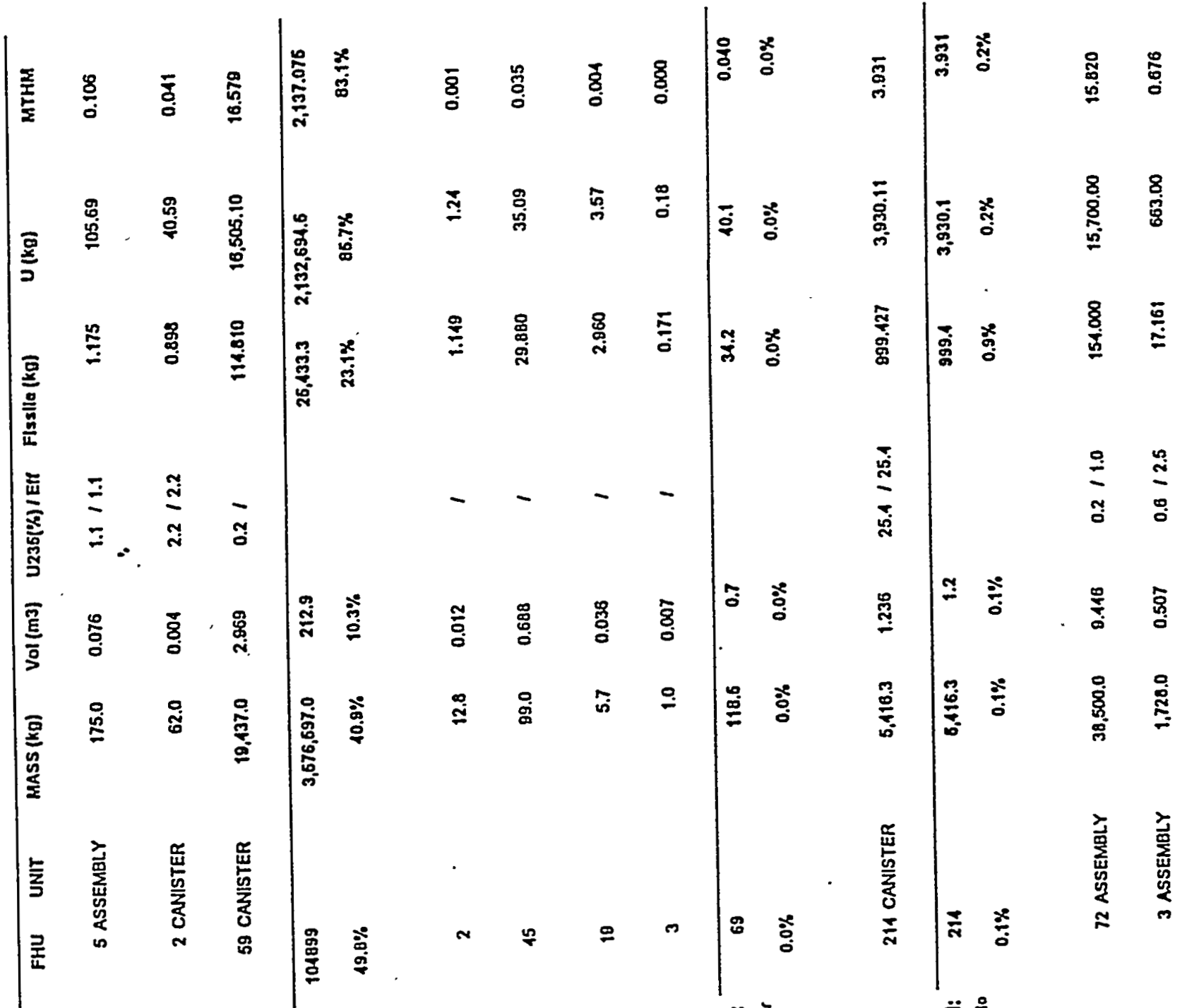

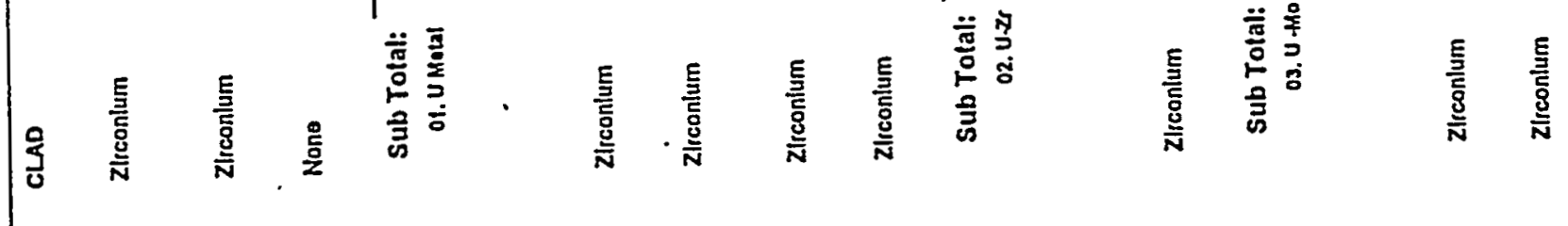

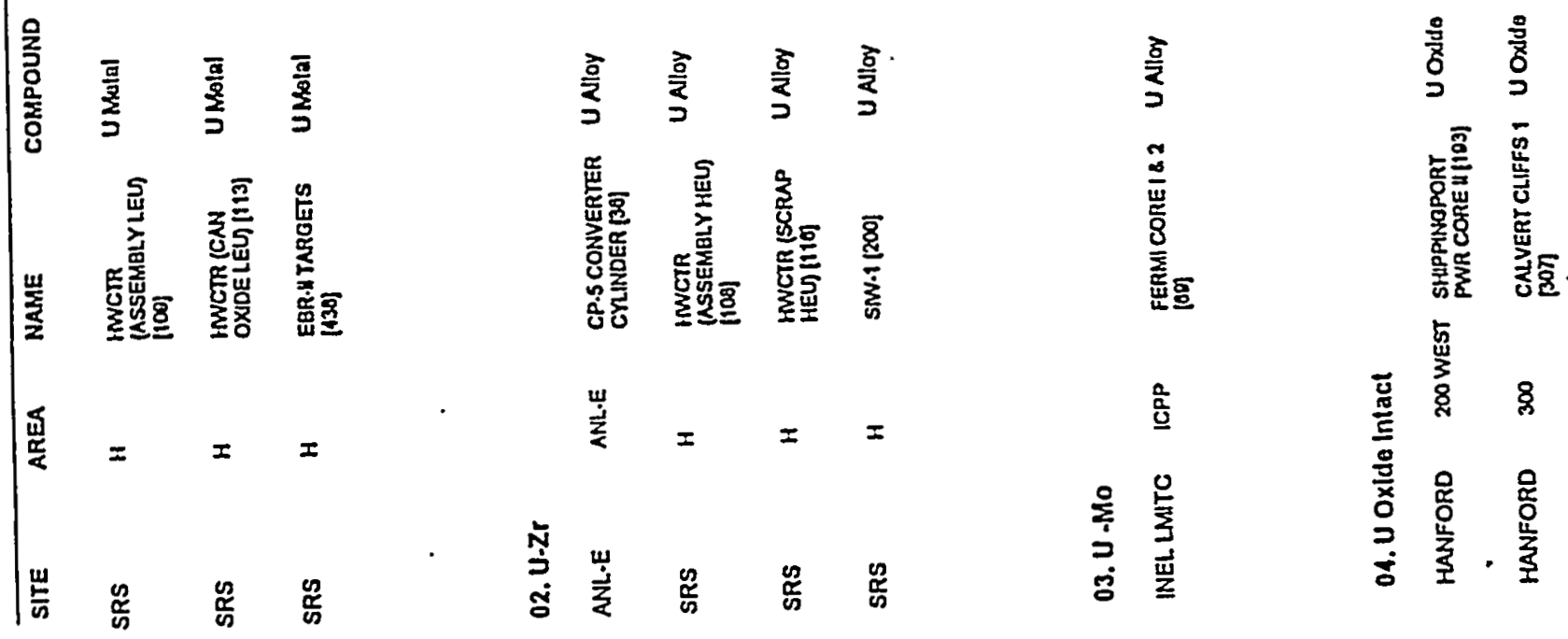




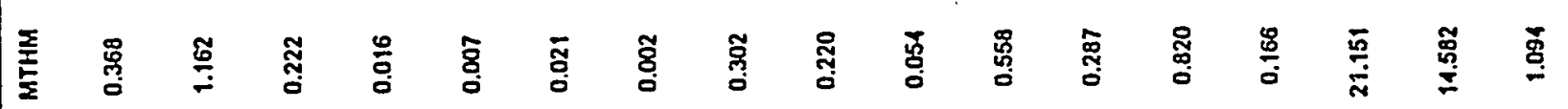

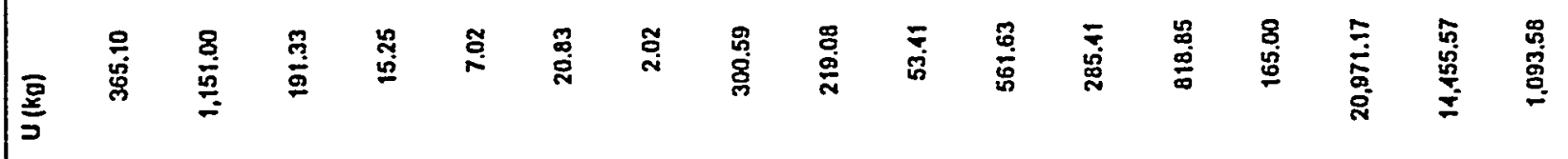

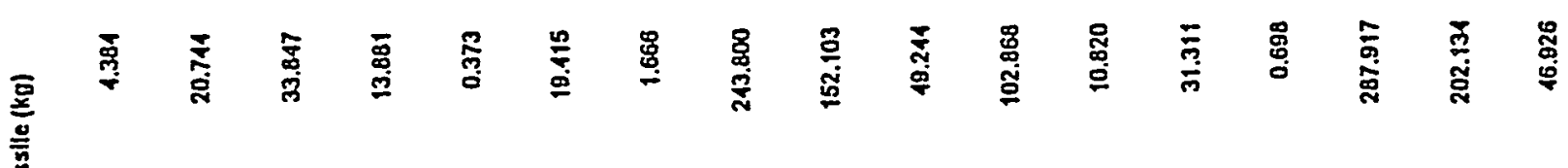

$\frac{\bar{x}}{\bar{L}}$

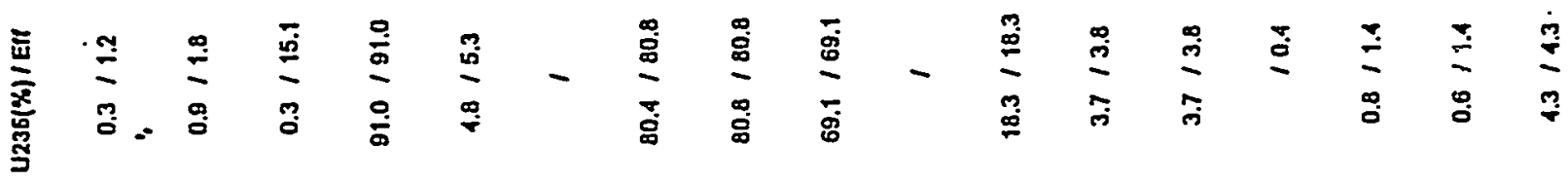

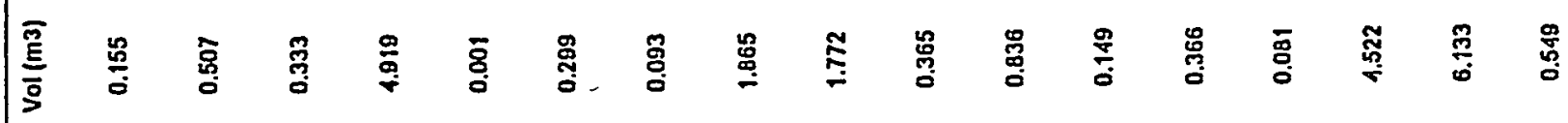

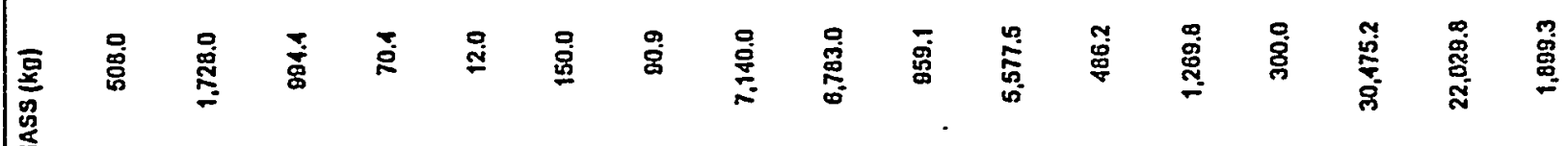

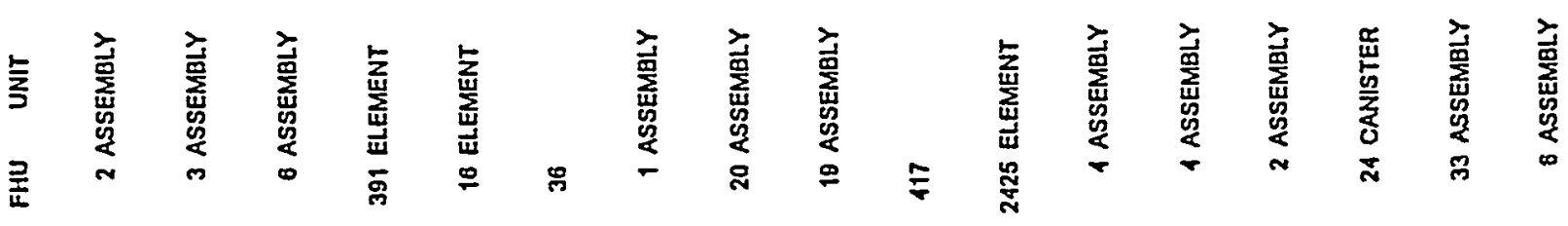

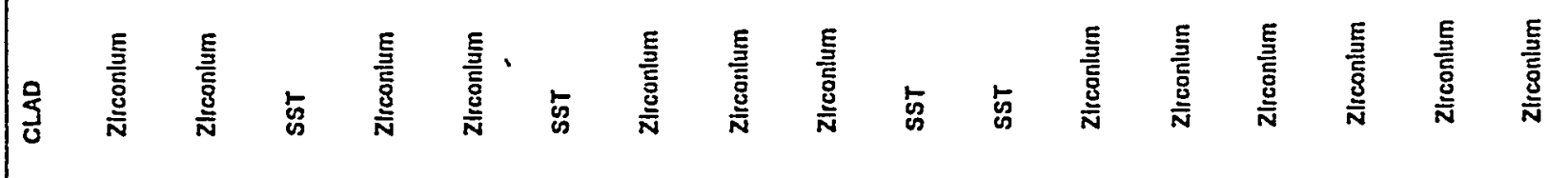

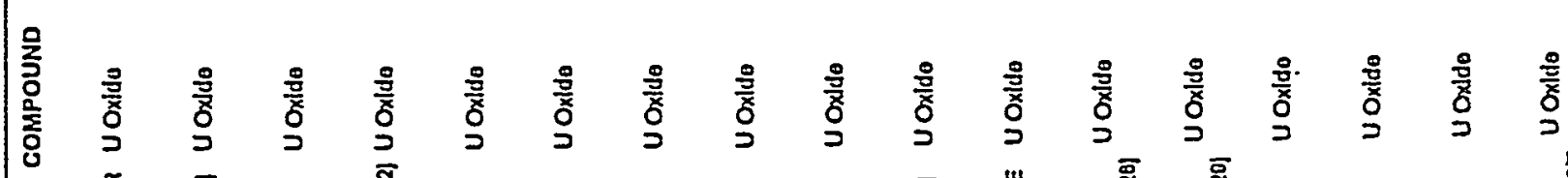

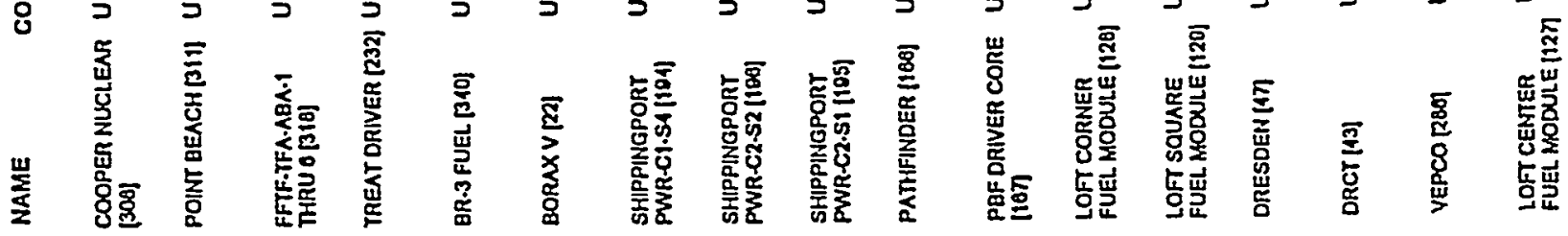

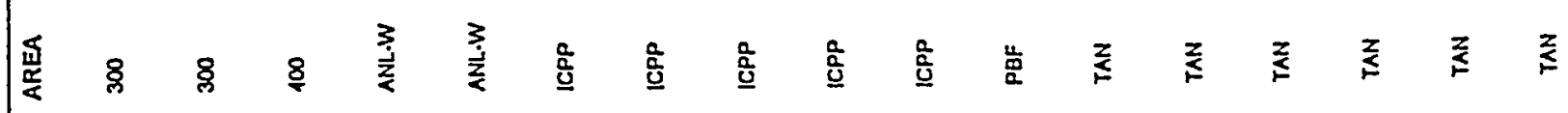

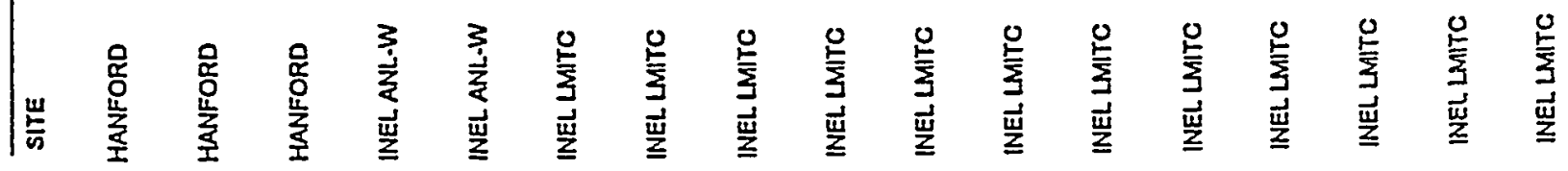




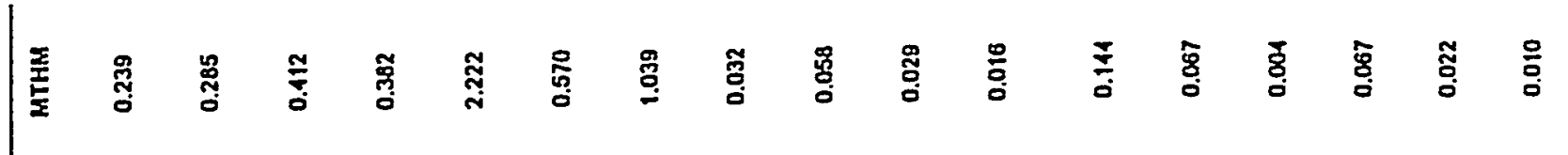

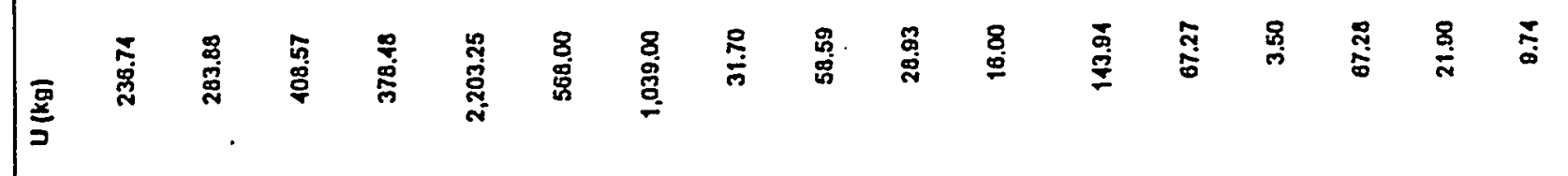

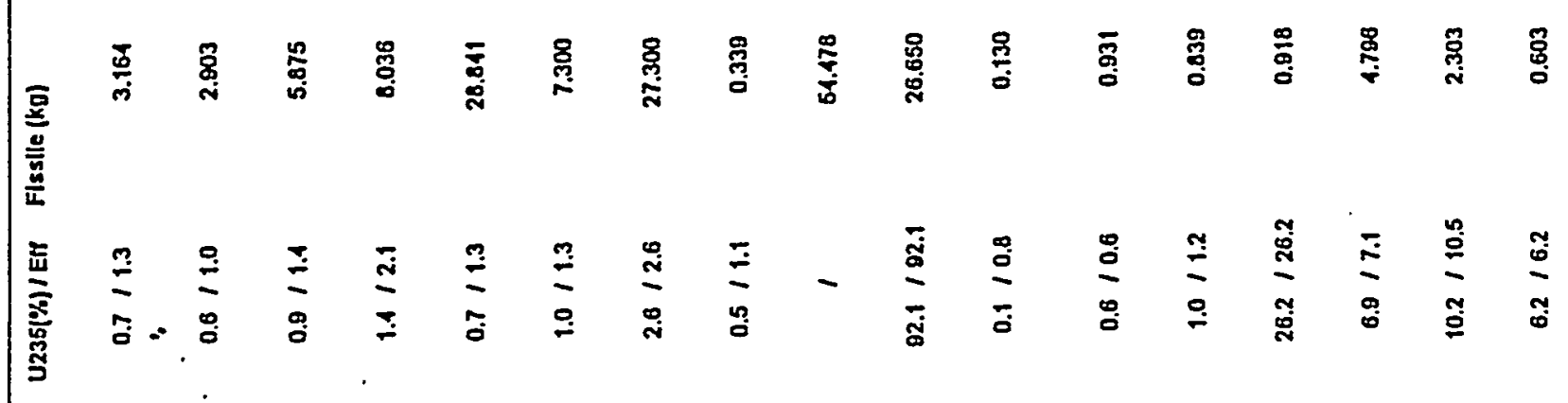

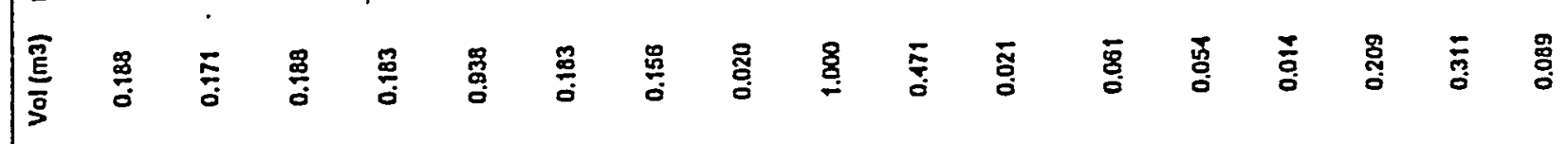

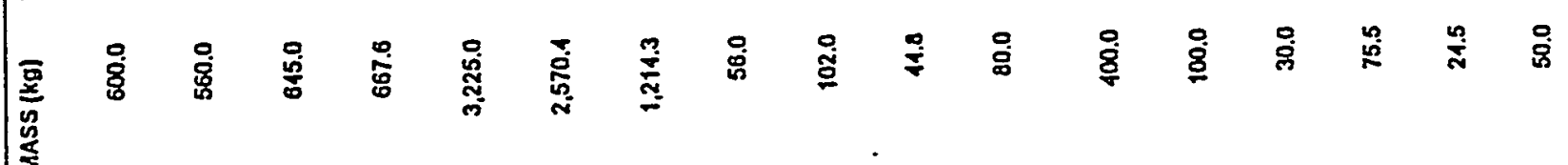
$\stackrel{n}{2}$

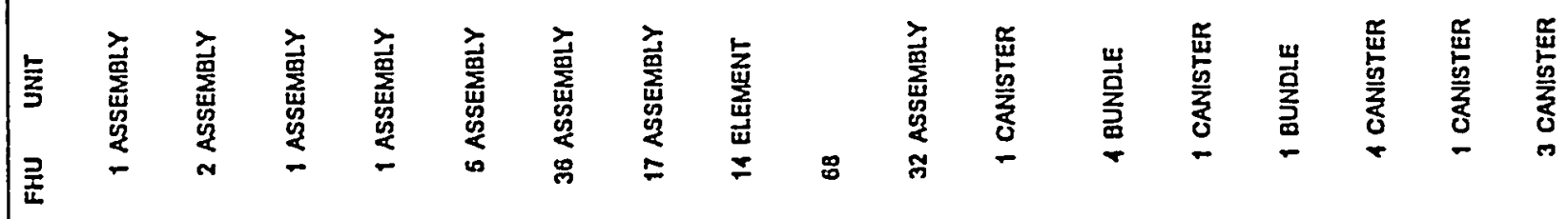

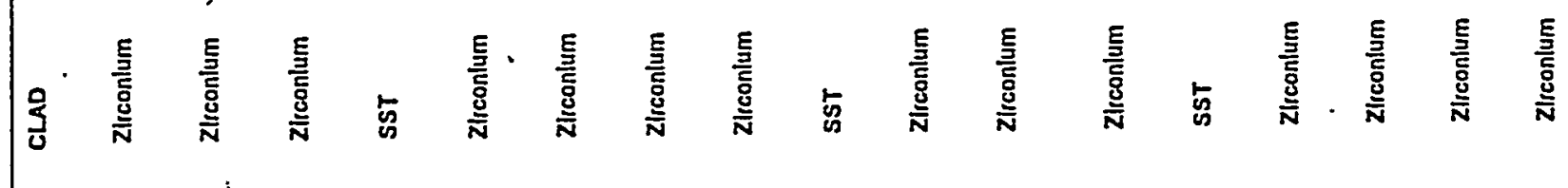

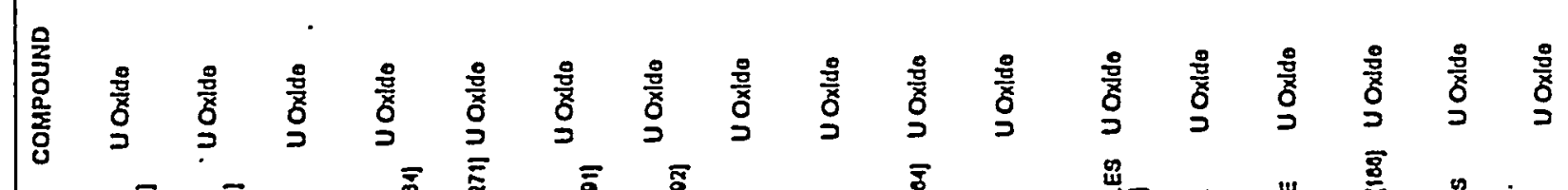

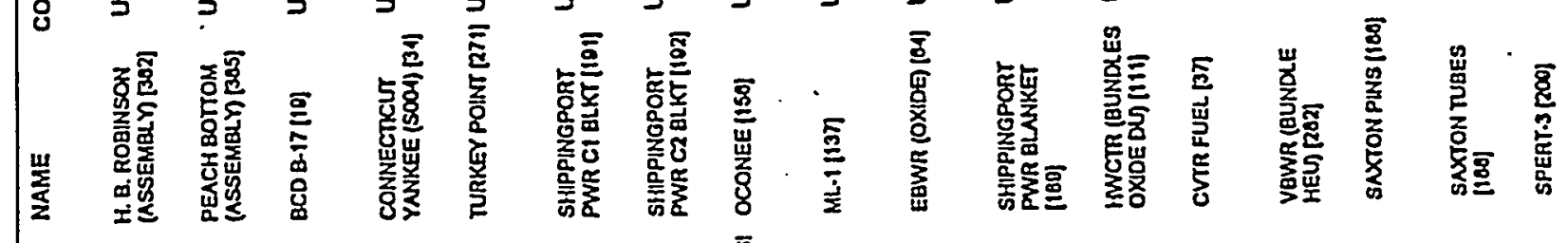

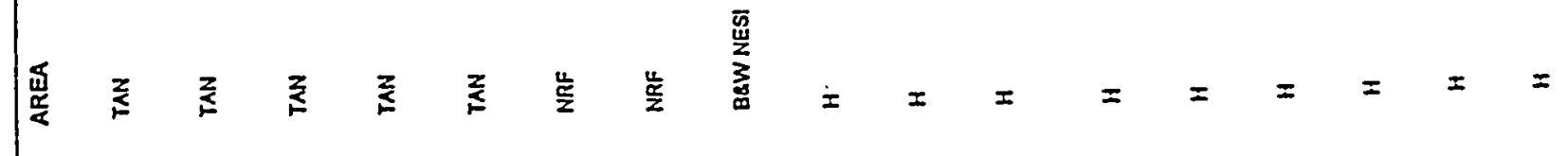

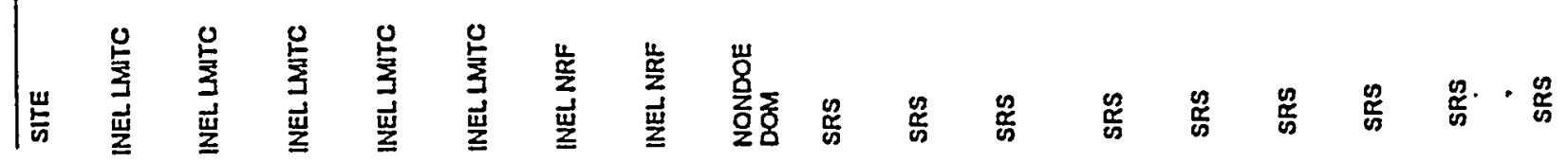




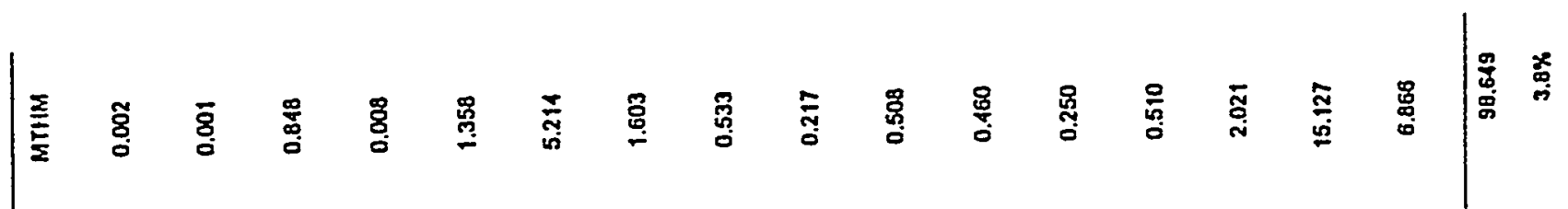

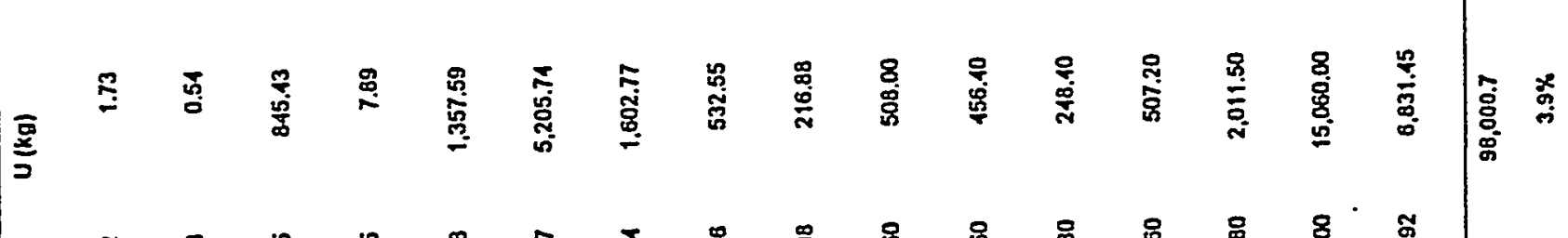

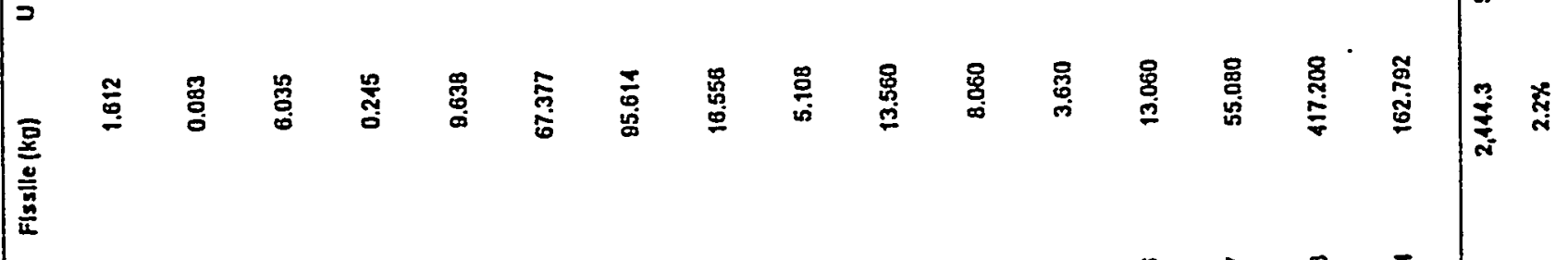

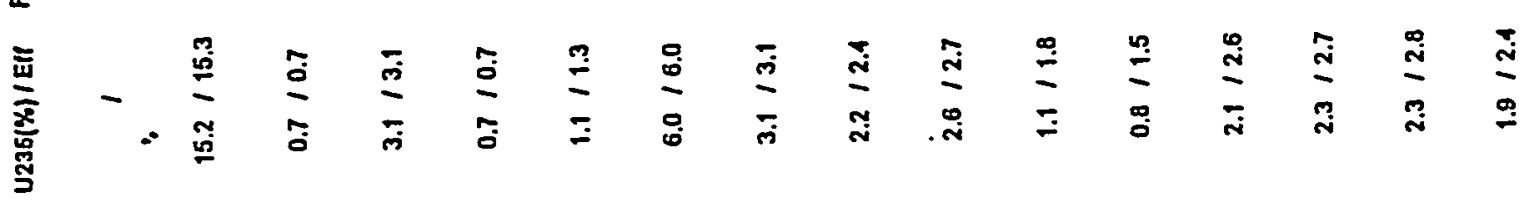

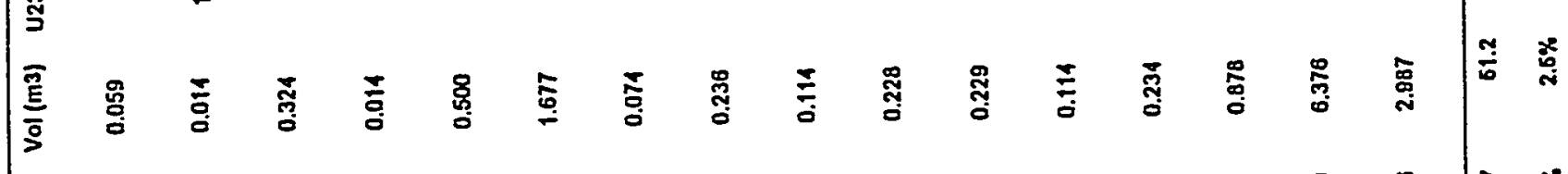

$$
\begin{aligned}
& \text { 望" }
\end{aligned}
$$

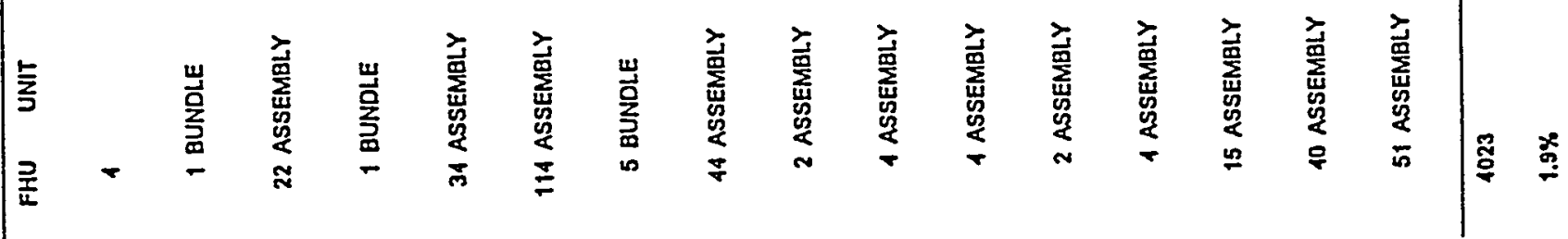

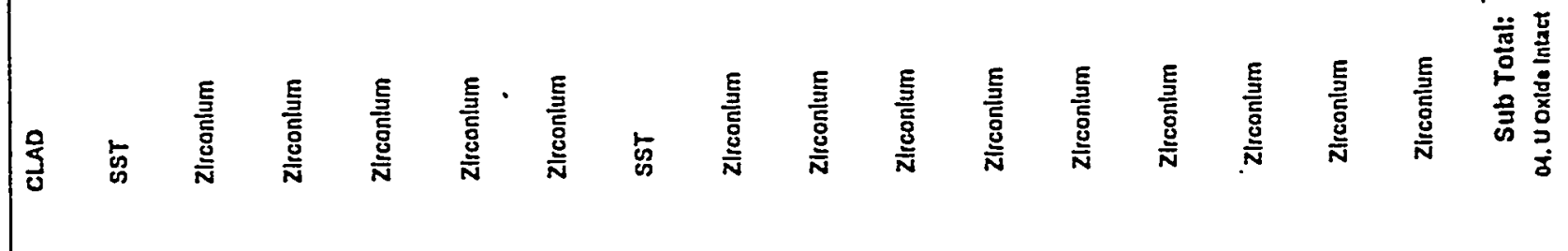

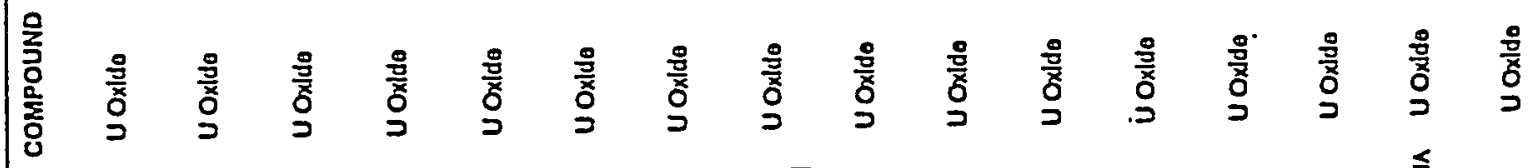

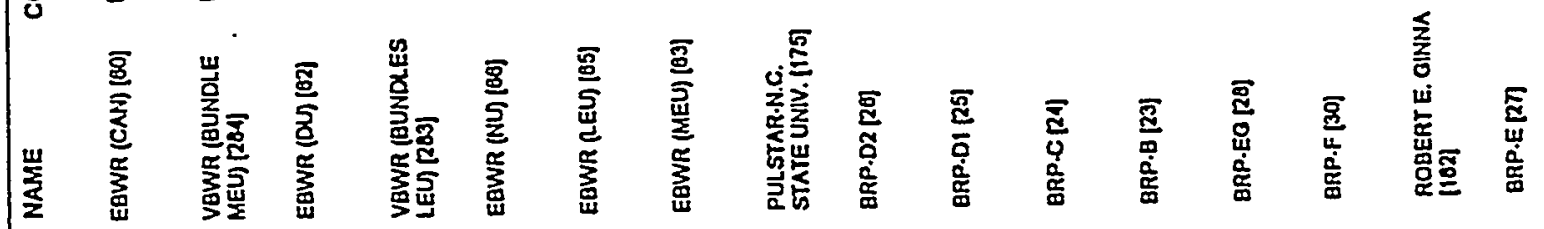

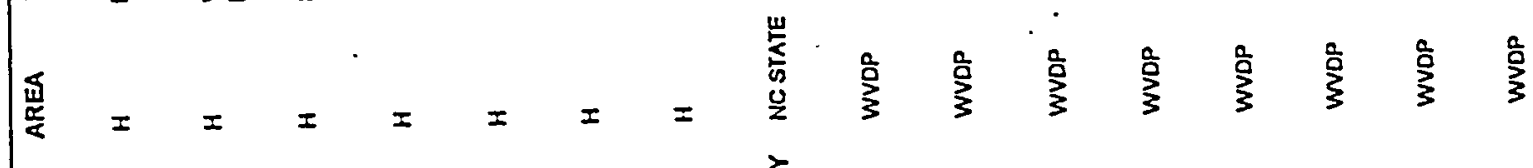

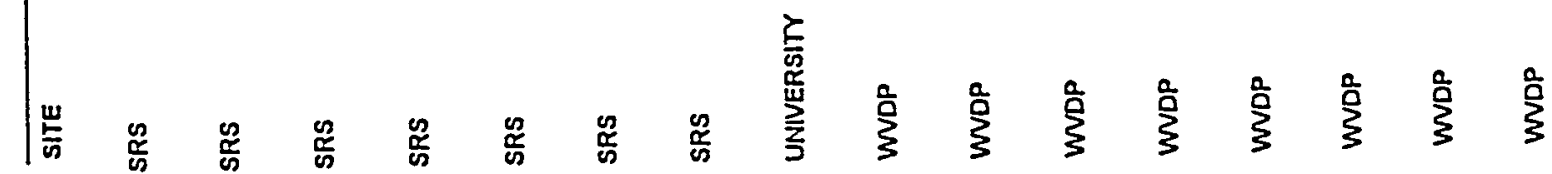




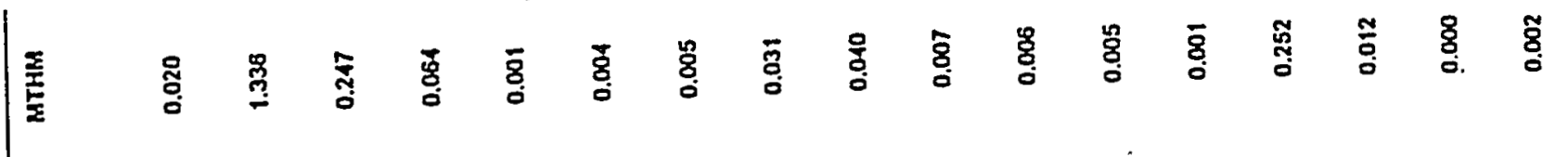

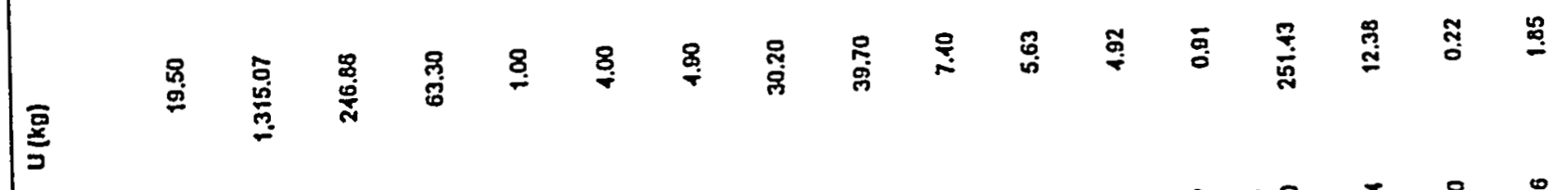

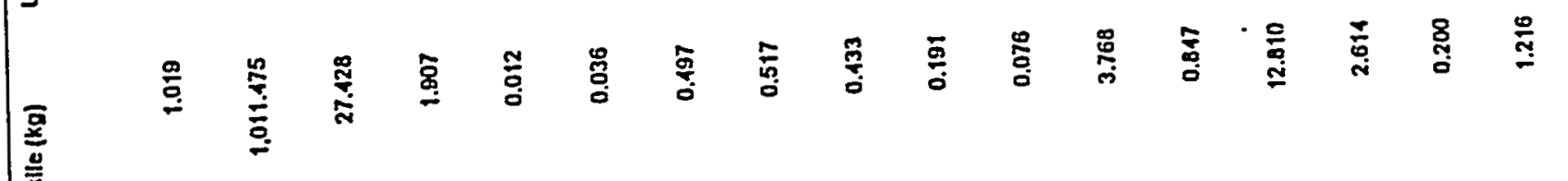

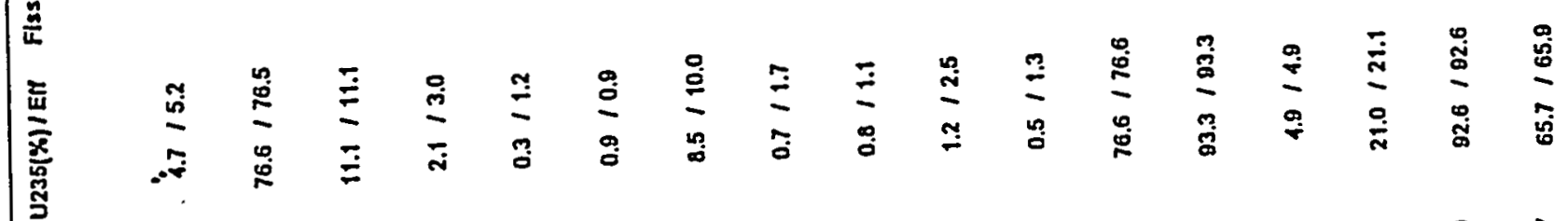

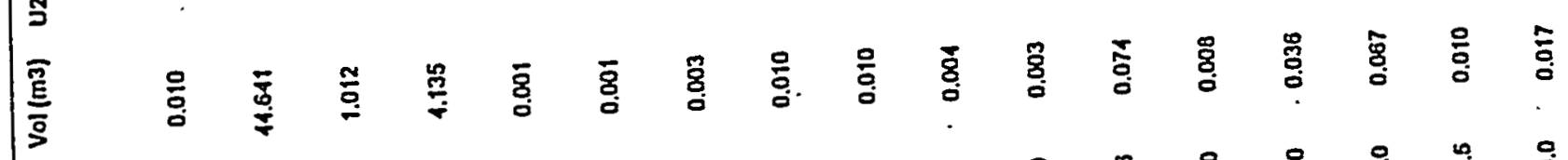

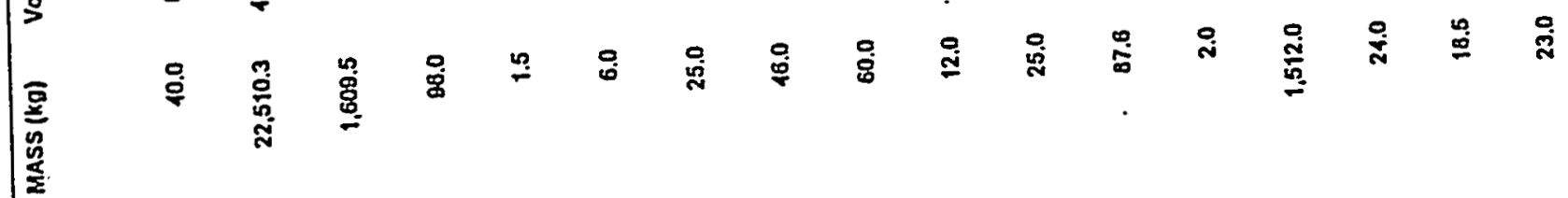

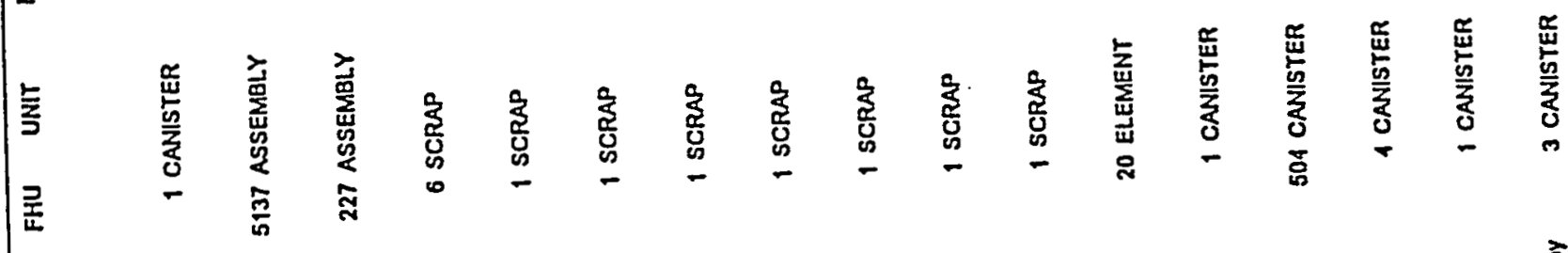

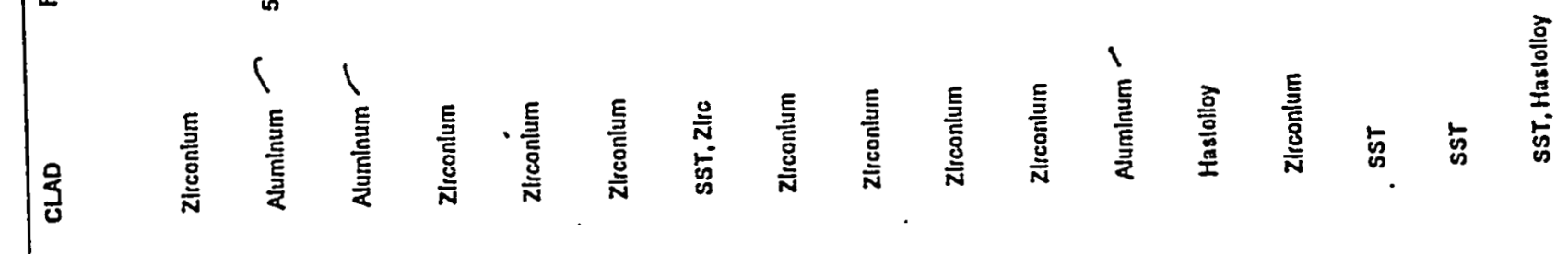

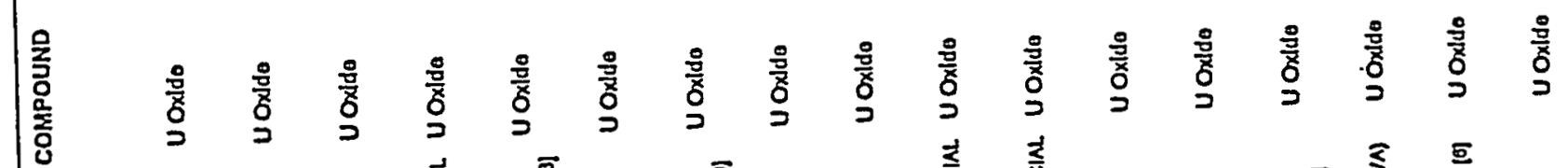

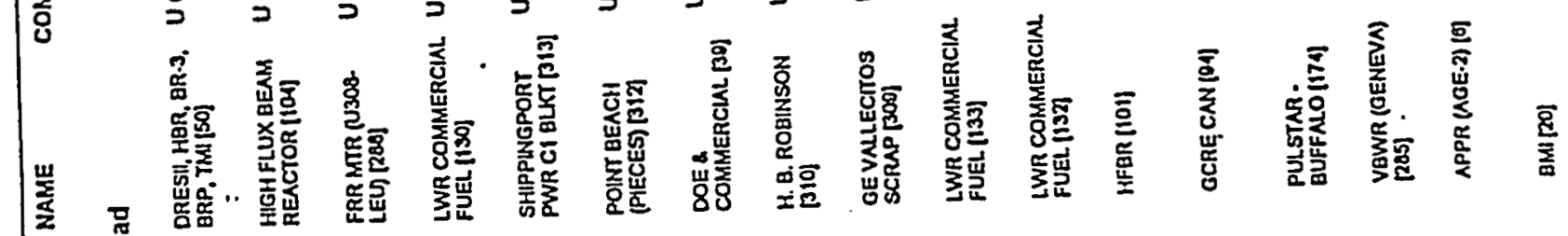

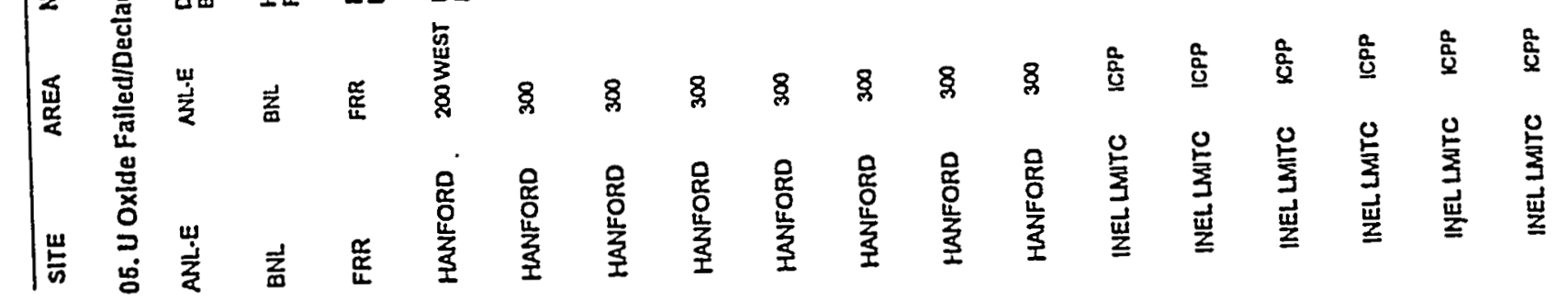


竞巻

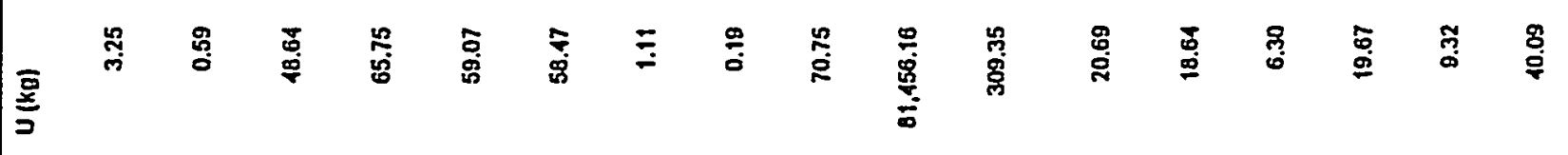

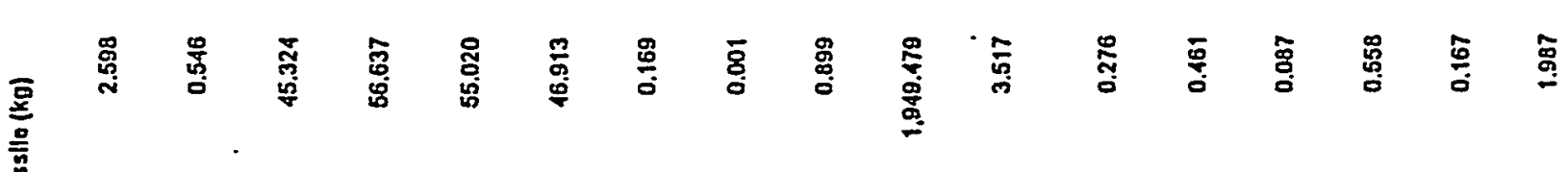

产

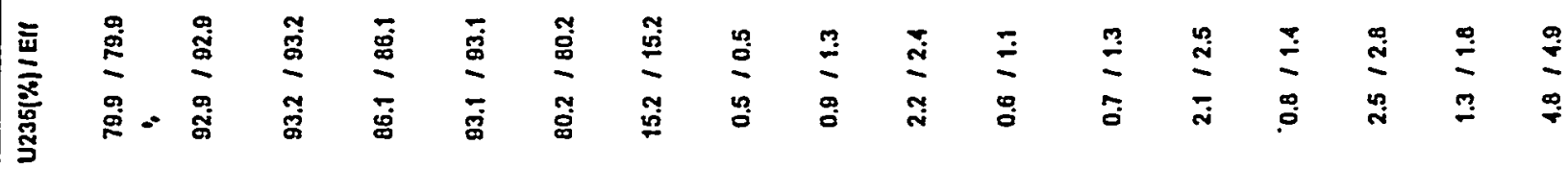

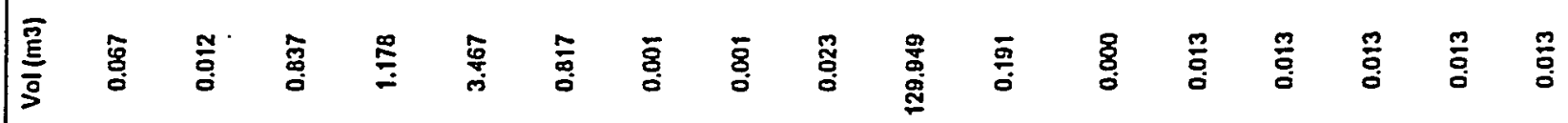

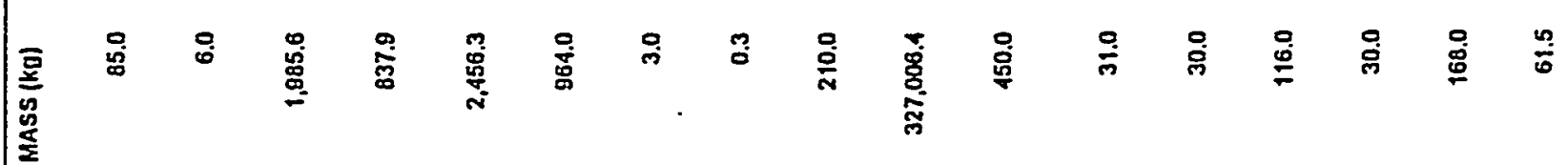

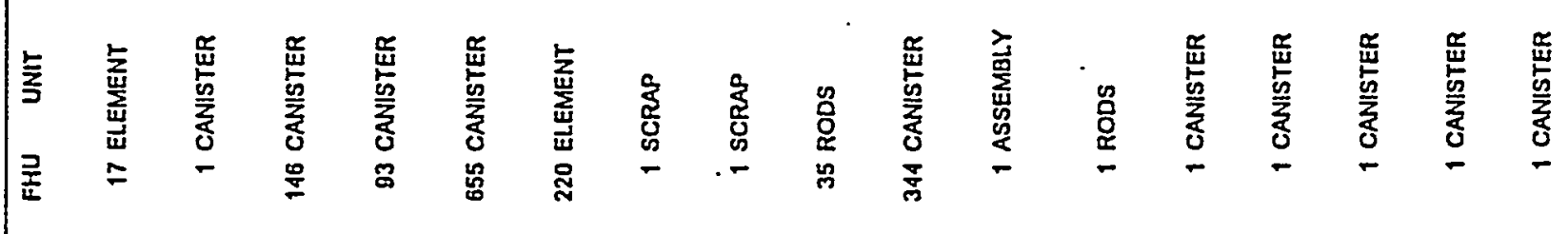

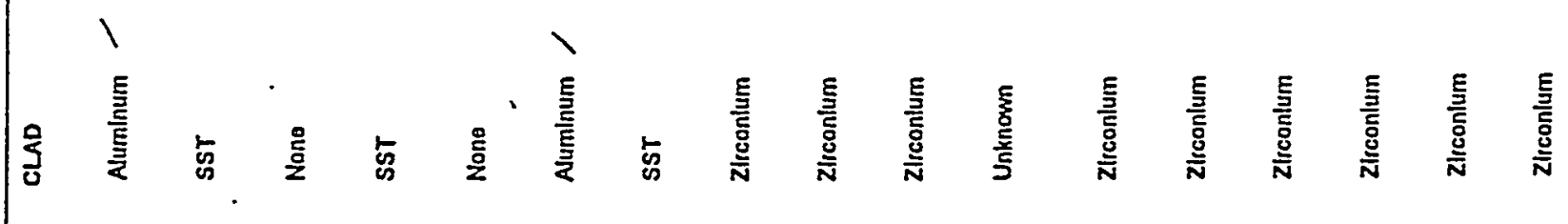

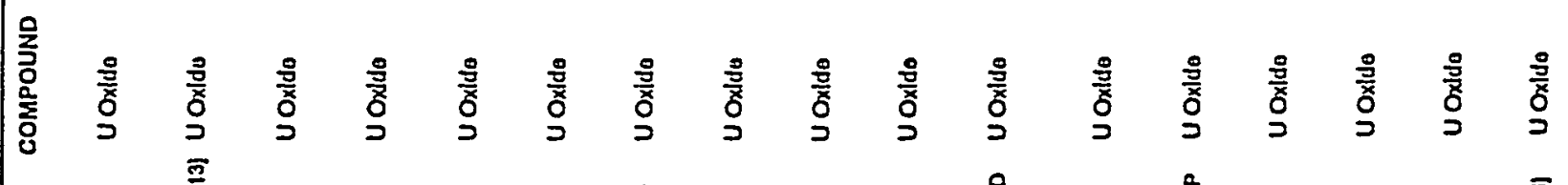

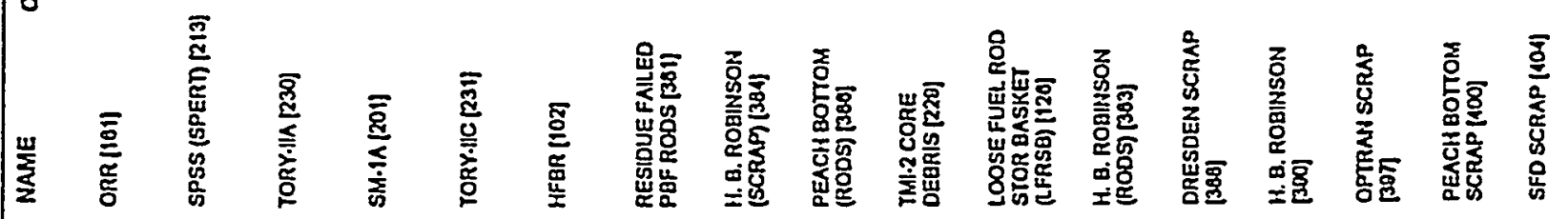

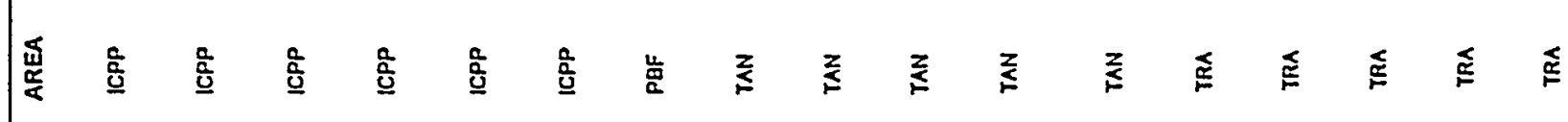

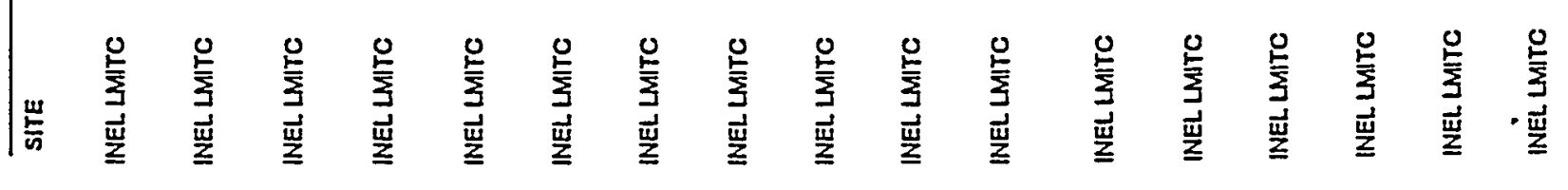




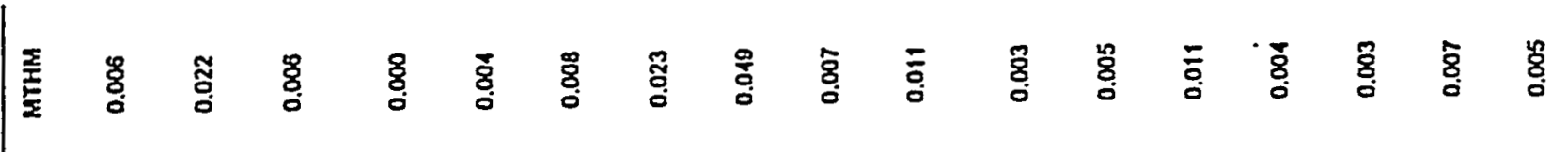

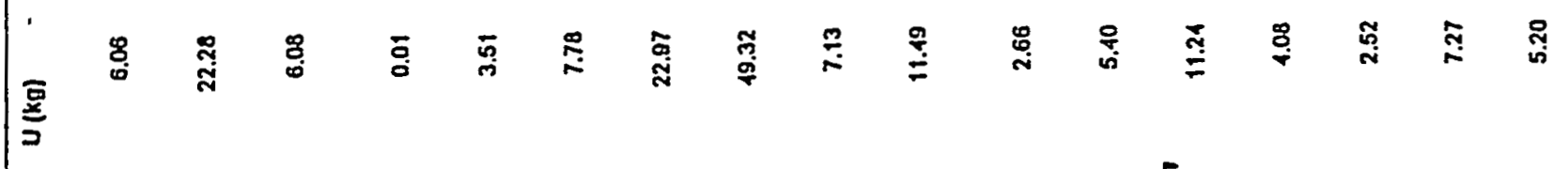

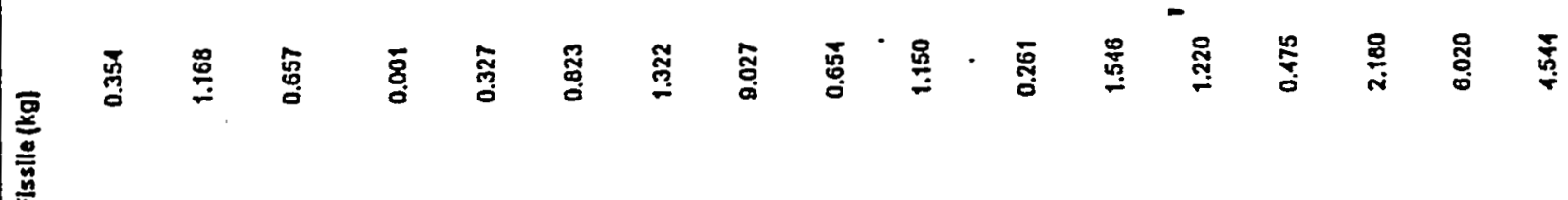

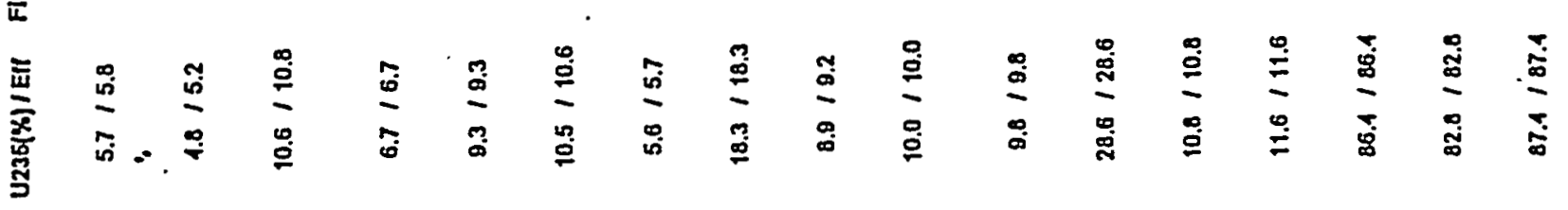

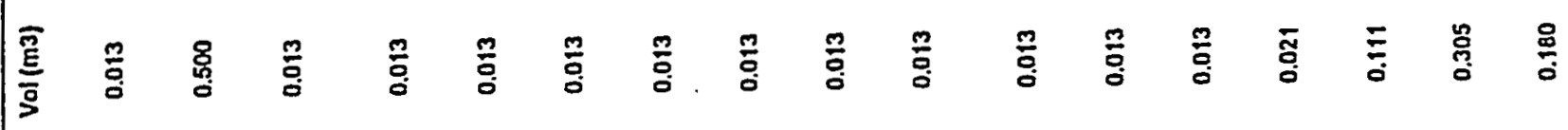

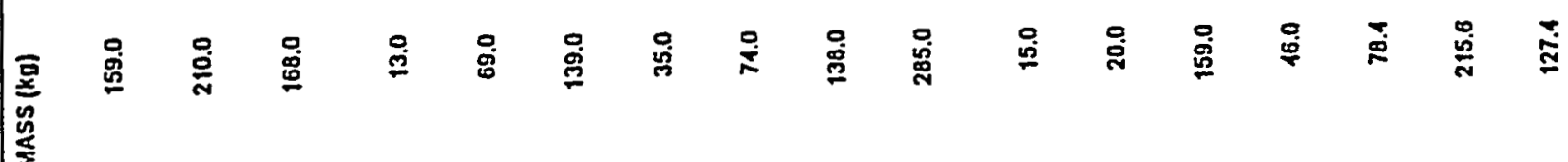

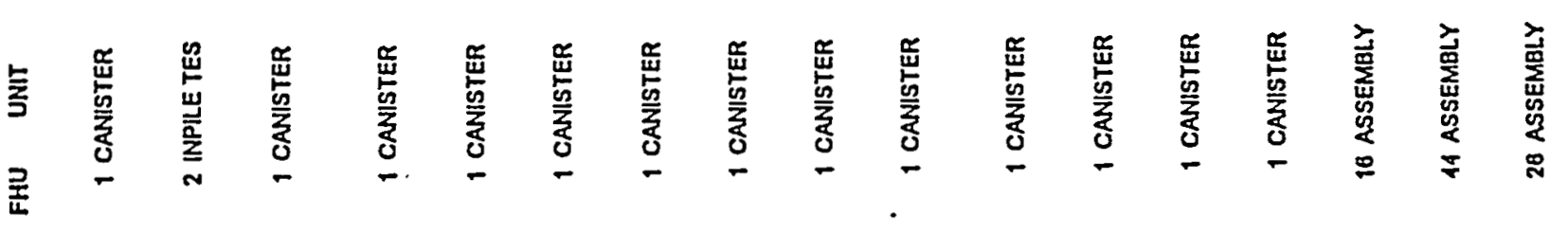

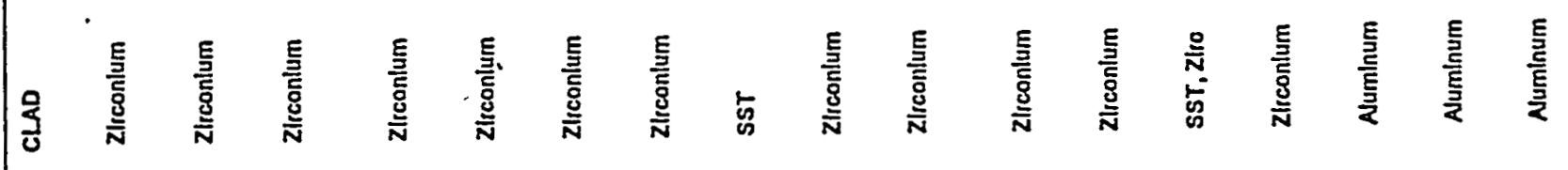

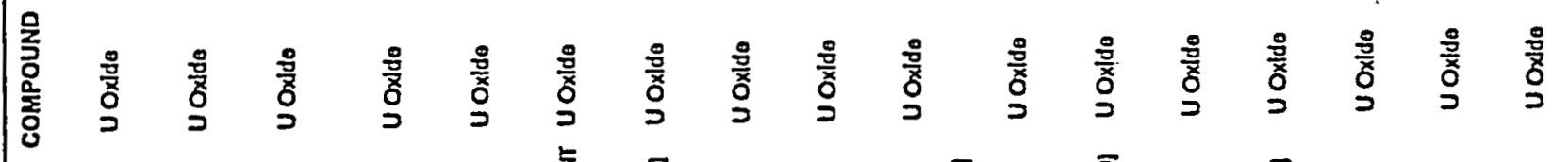

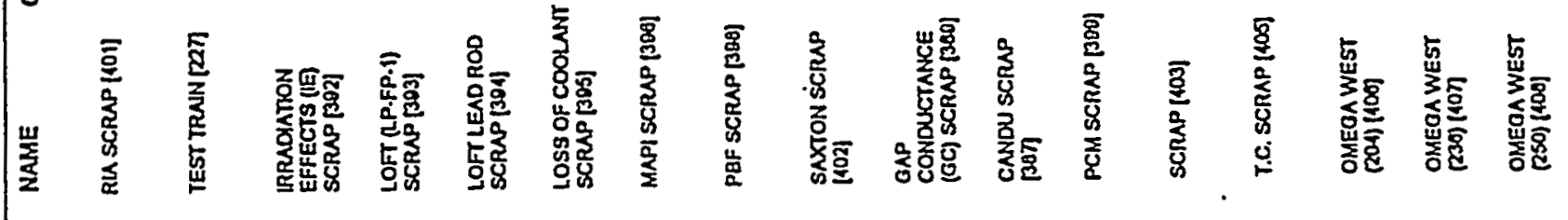

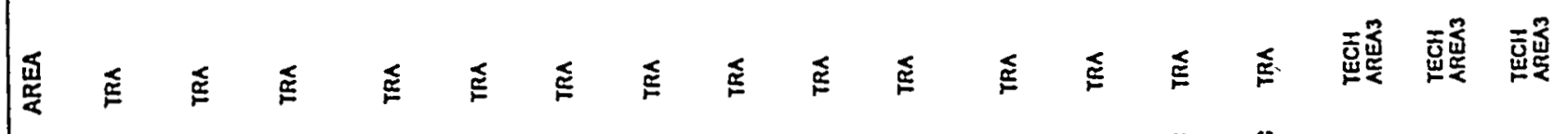

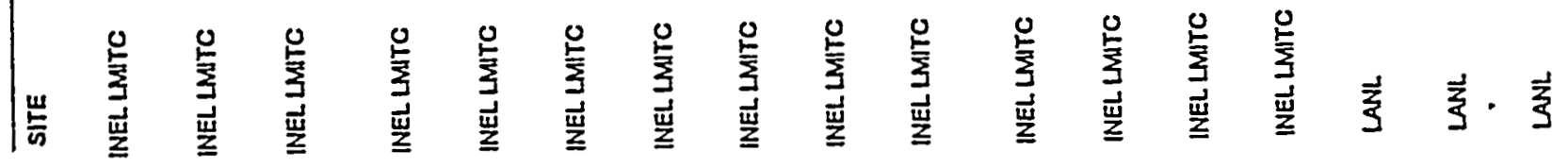




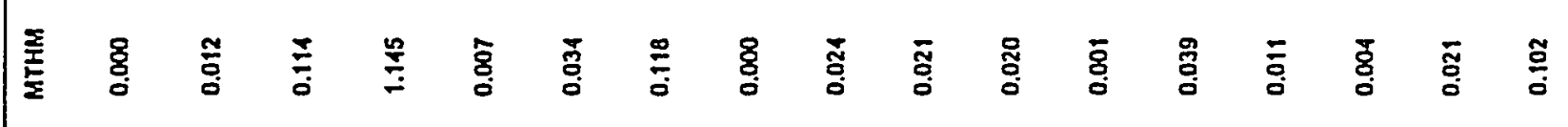

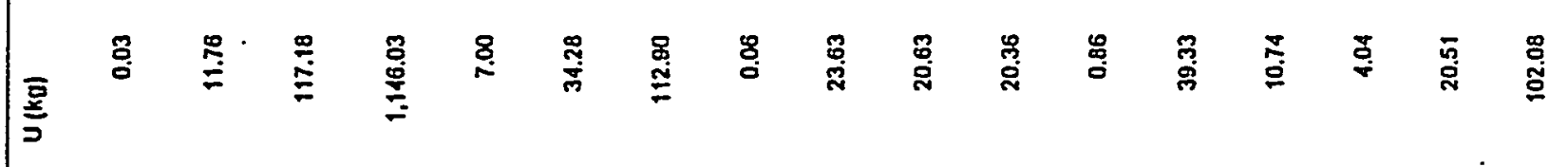

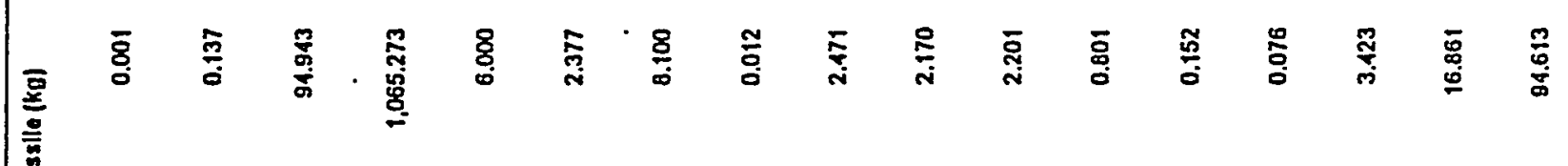

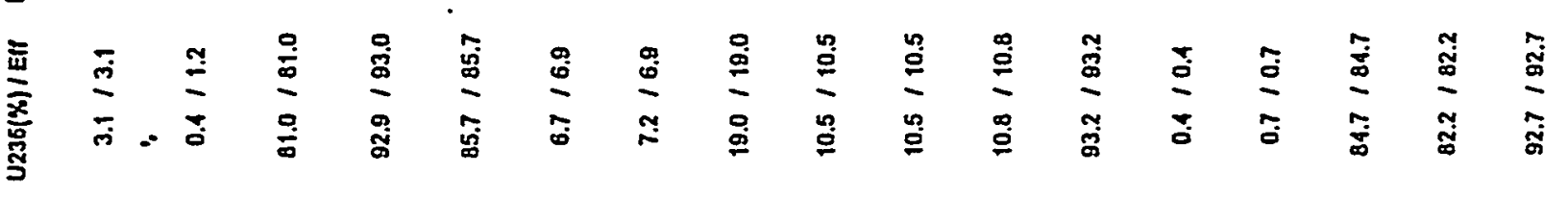

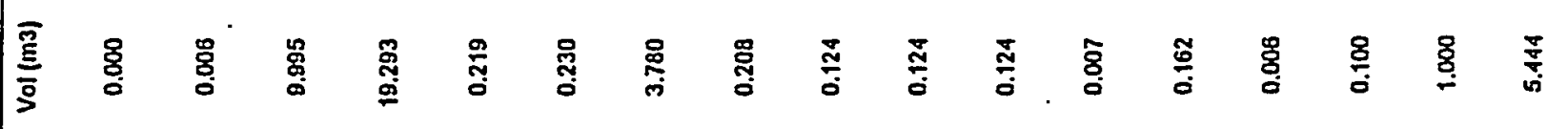

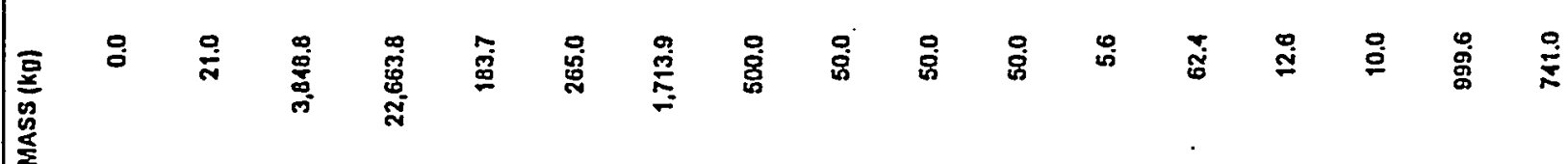

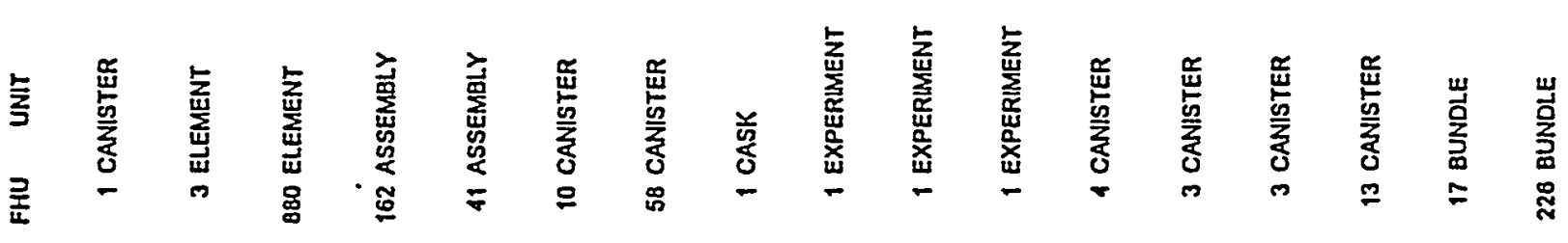

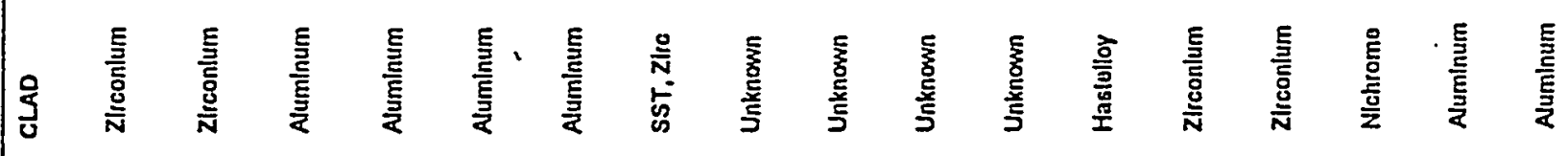

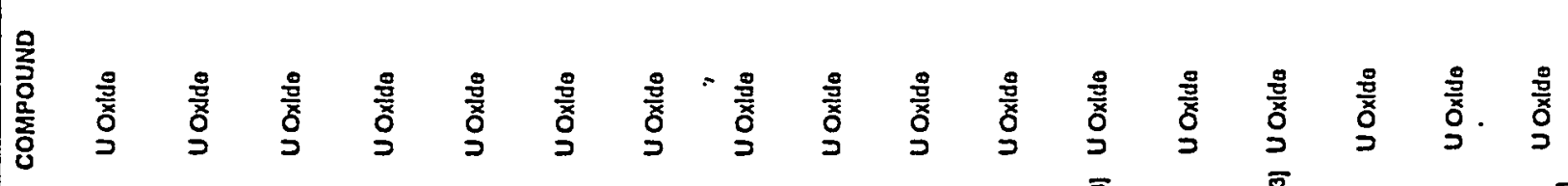

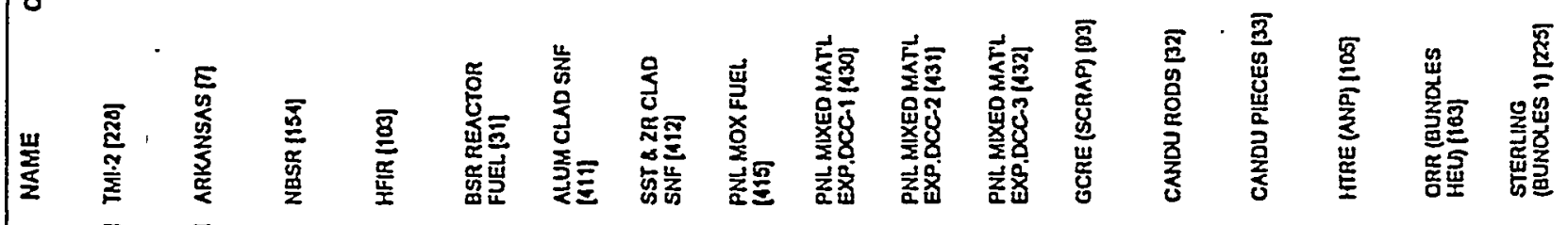

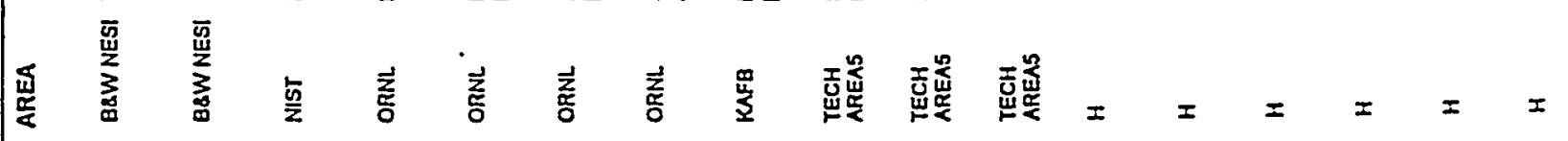

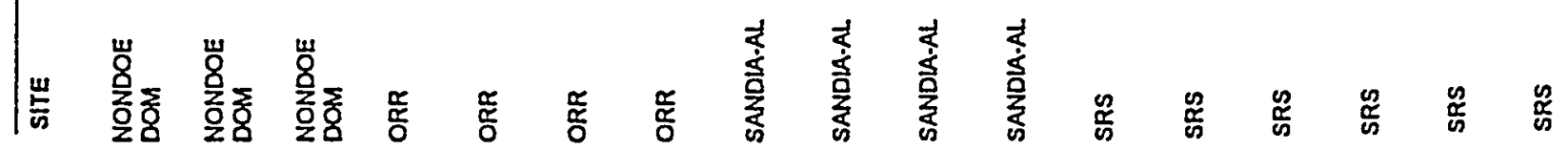




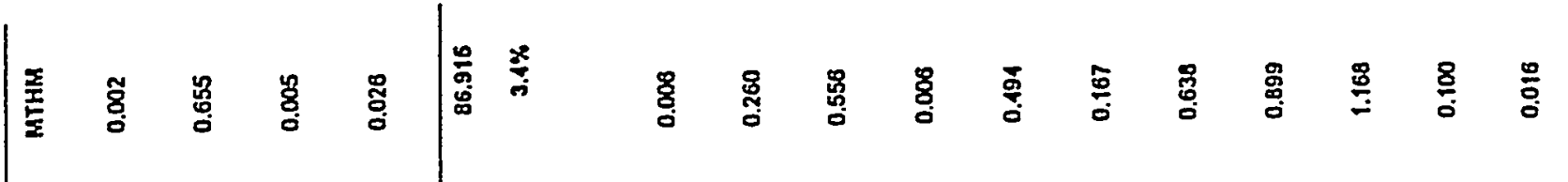

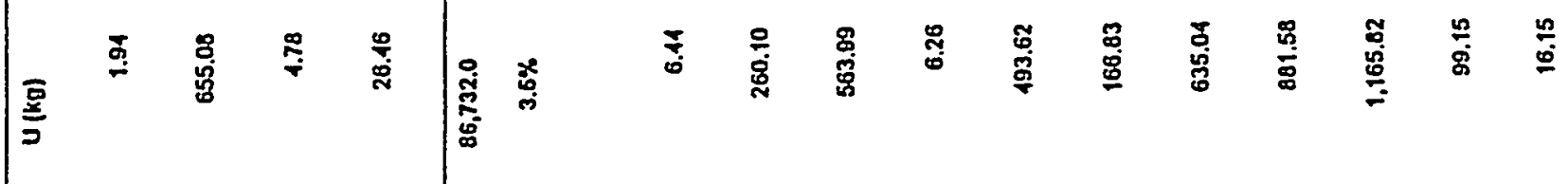

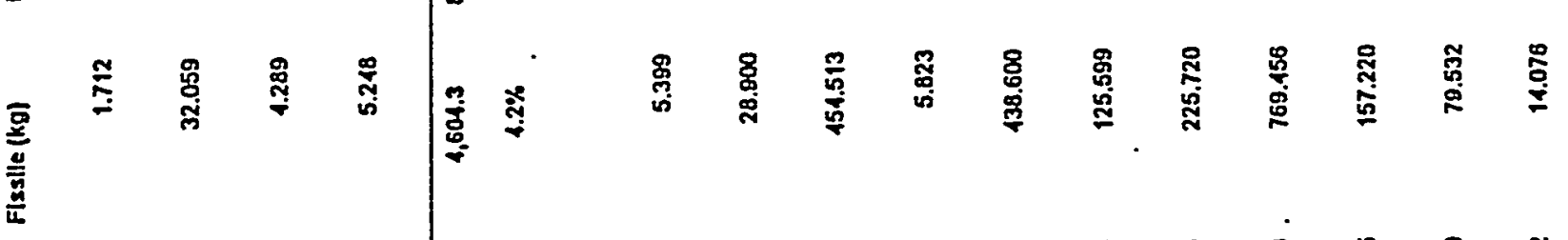

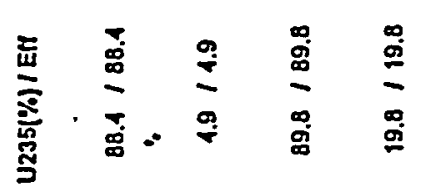

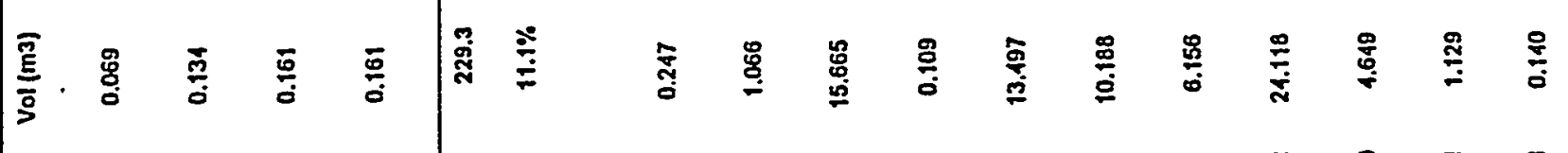

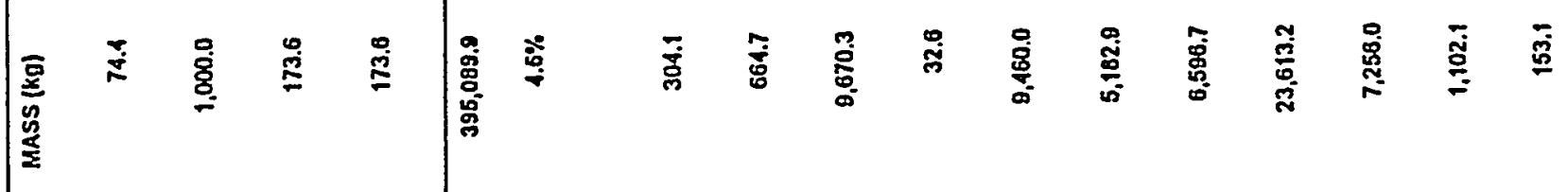

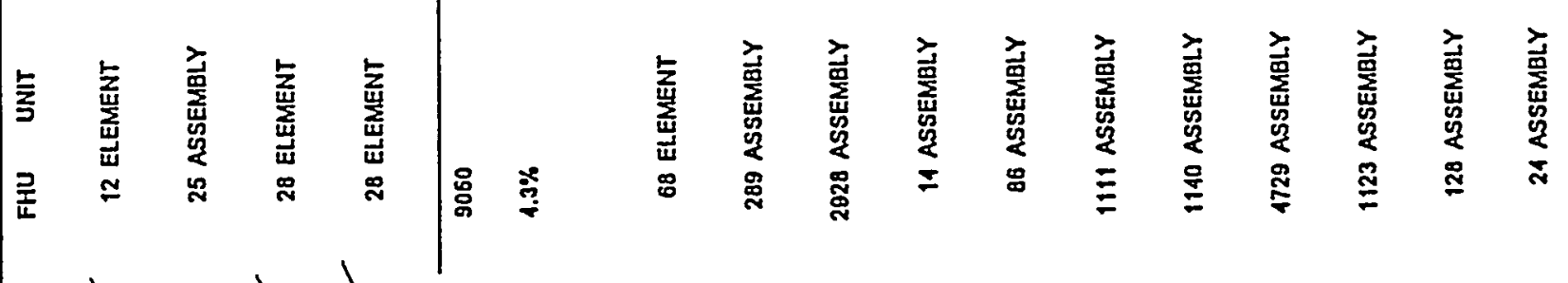

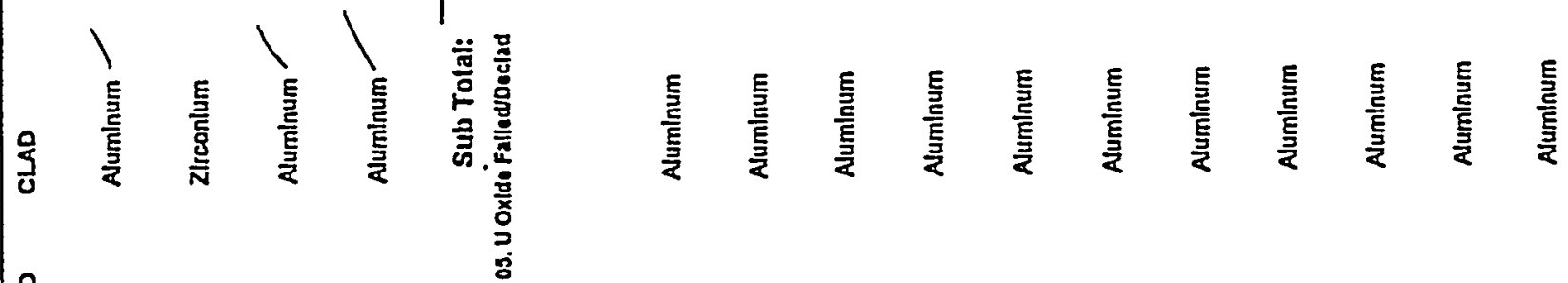

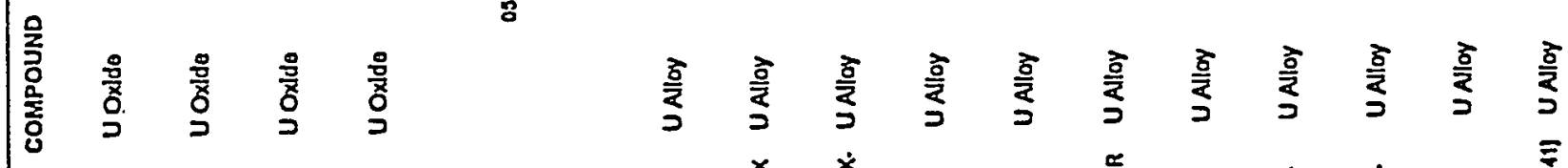

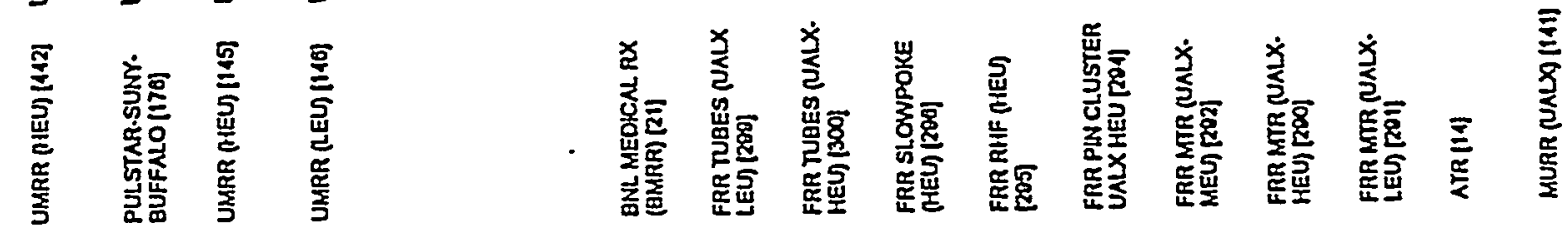

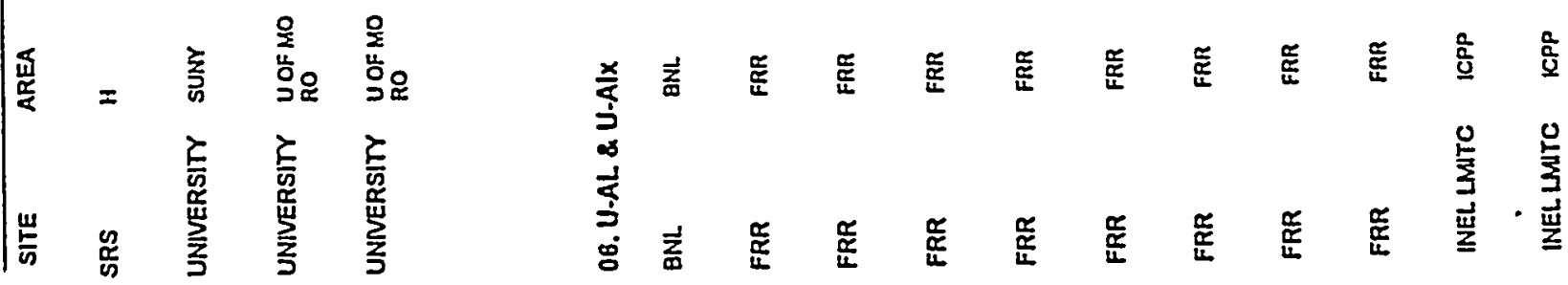




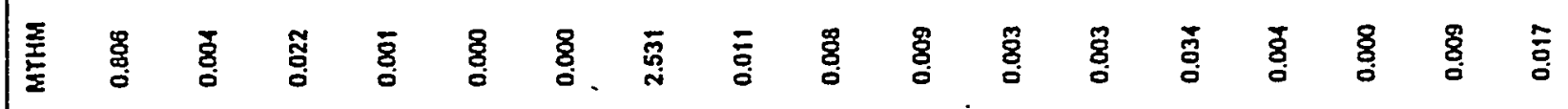

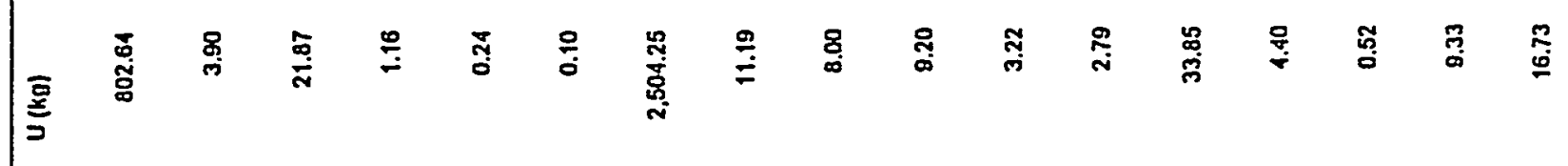

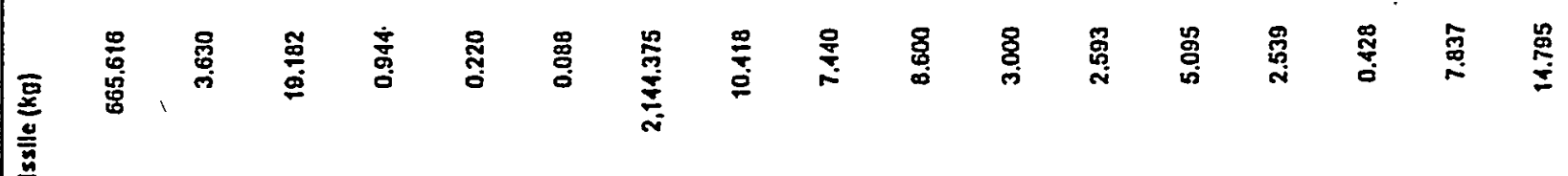

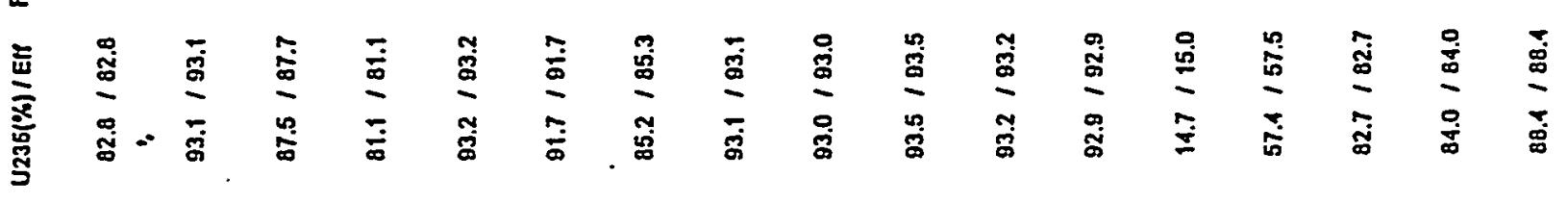

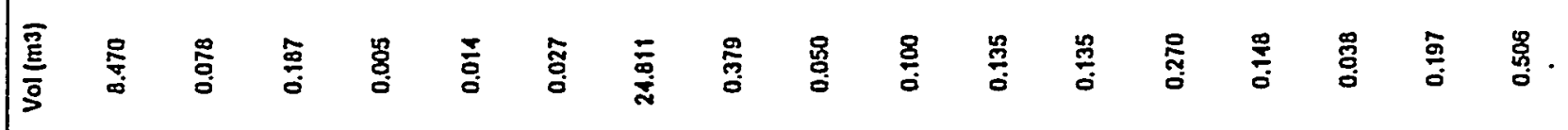

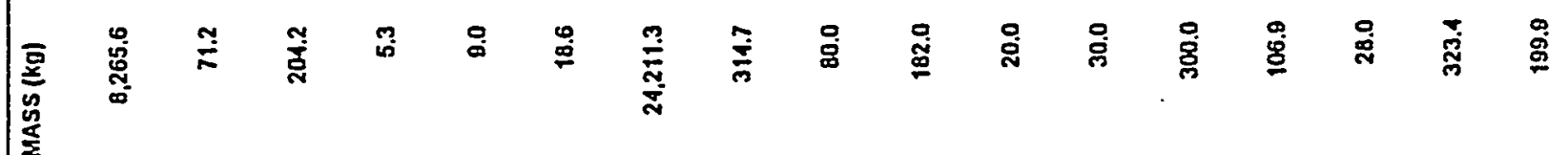

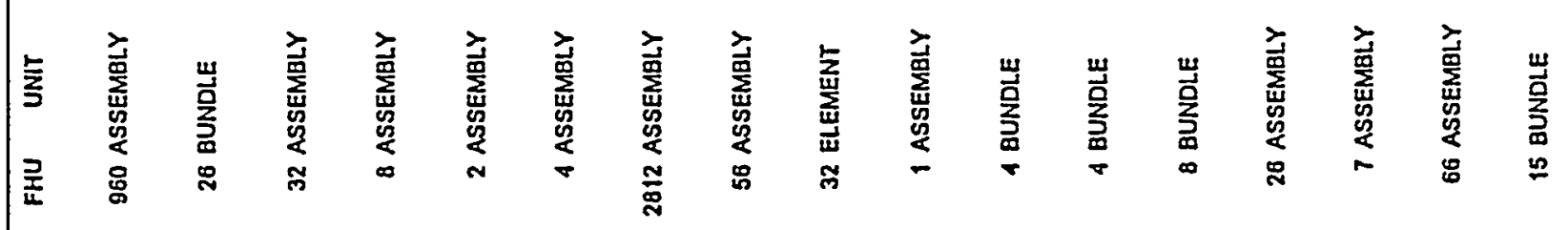

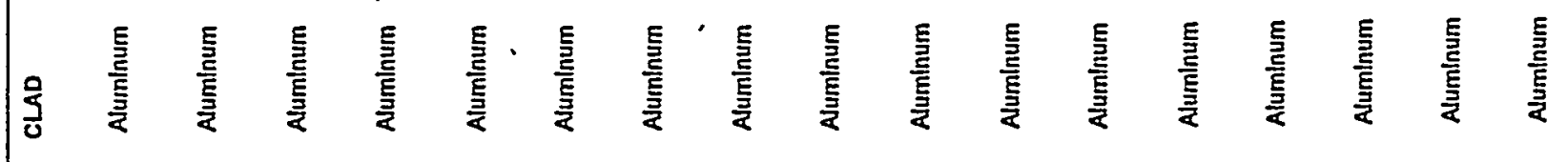

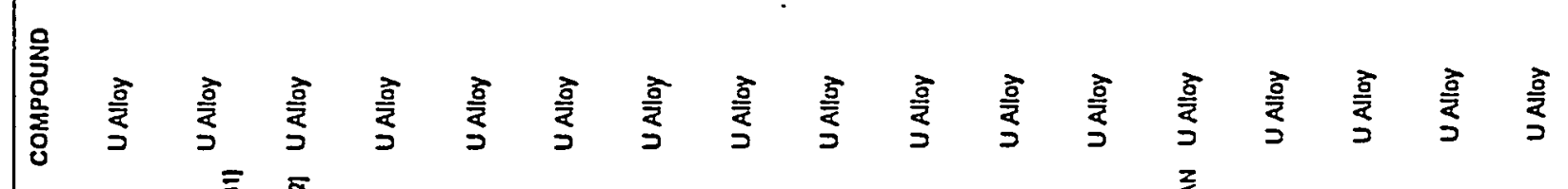

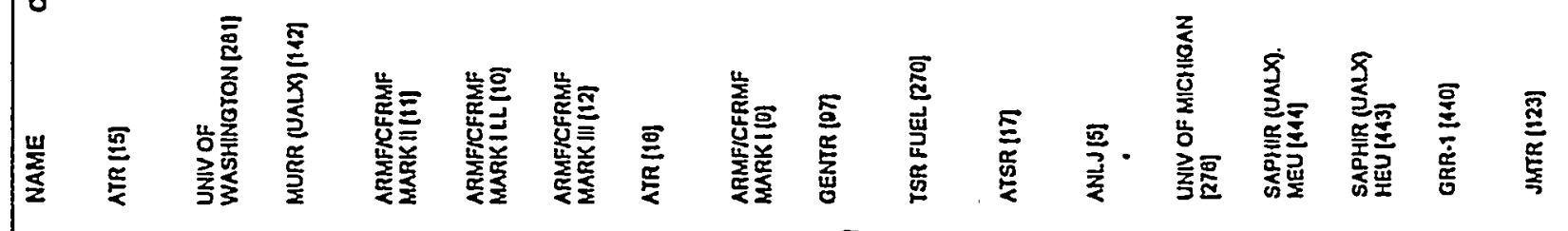

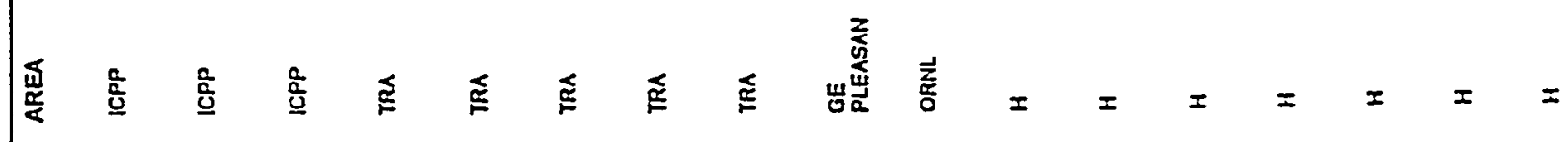

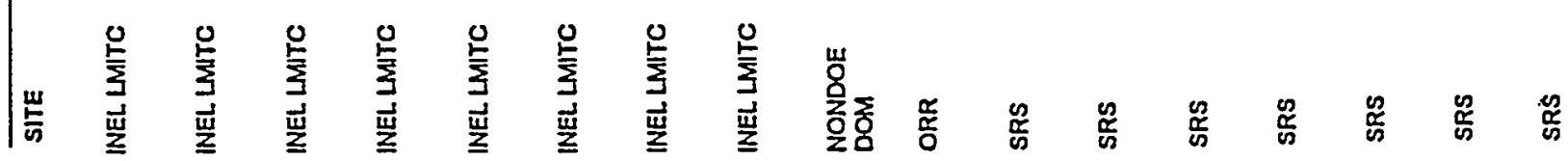


榱哭

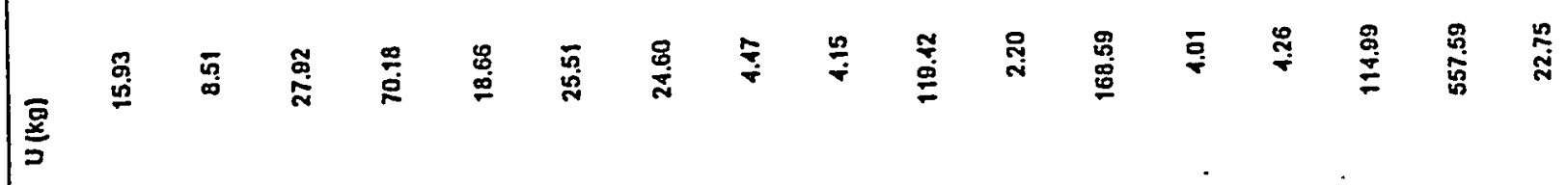

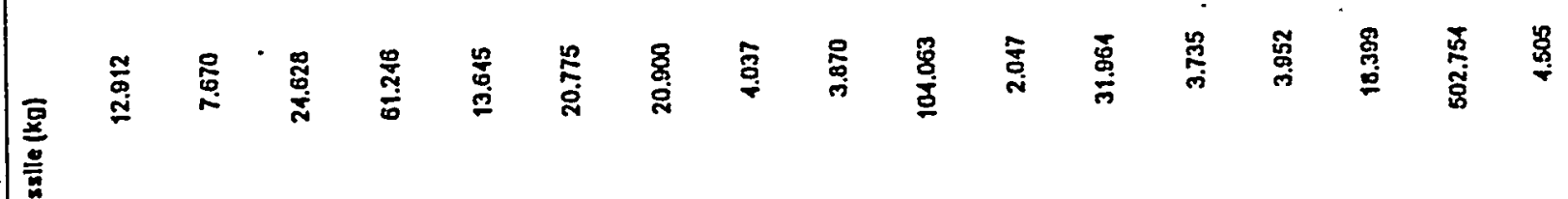

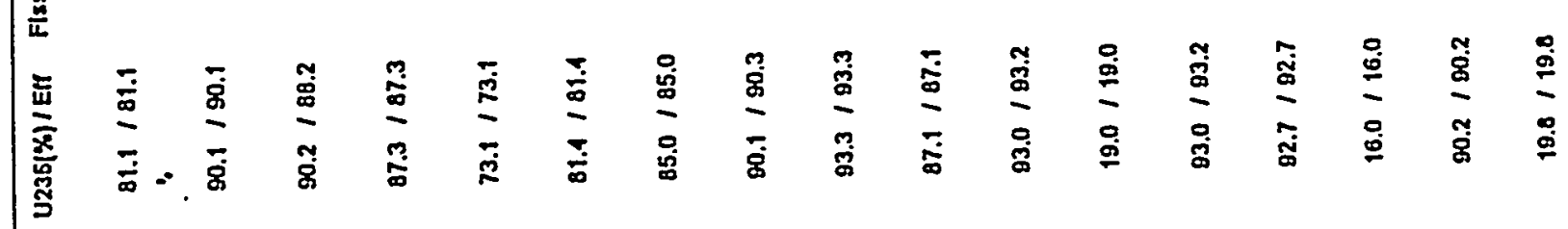

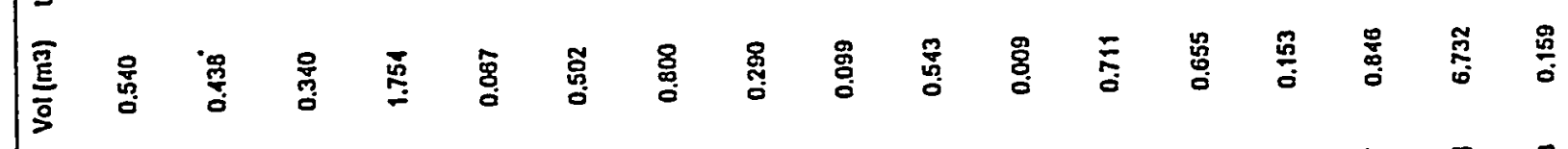

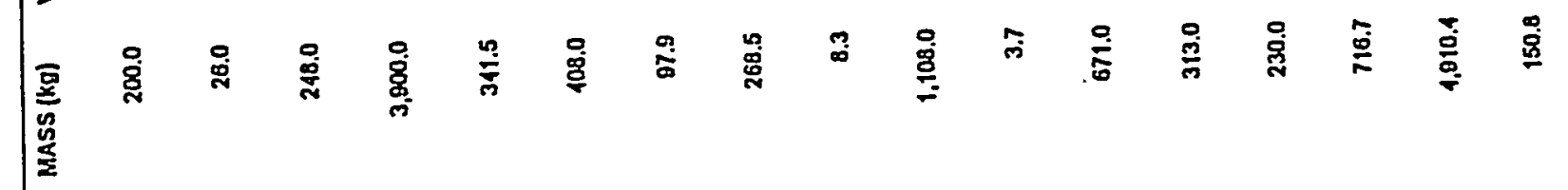

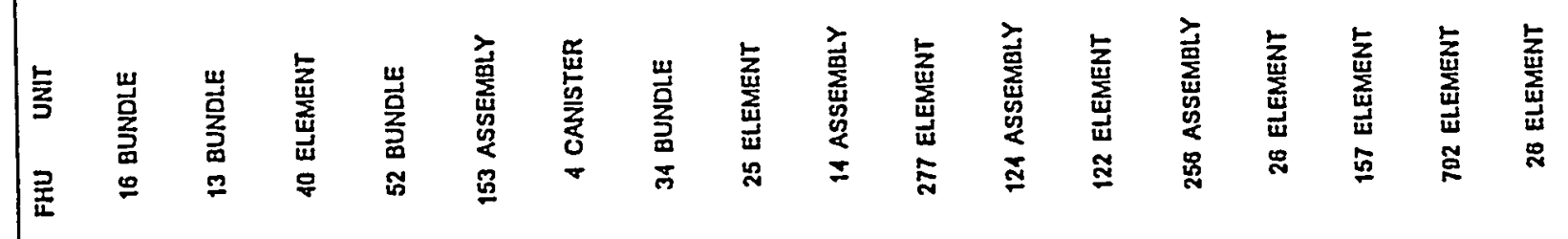
\& \|\|\|\|\|\|\|\|\|\|

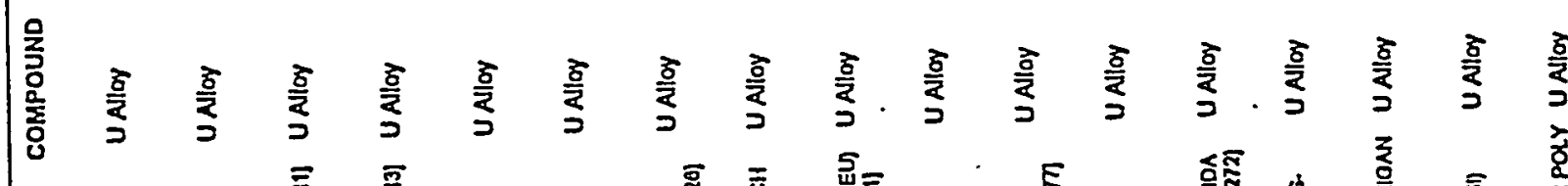

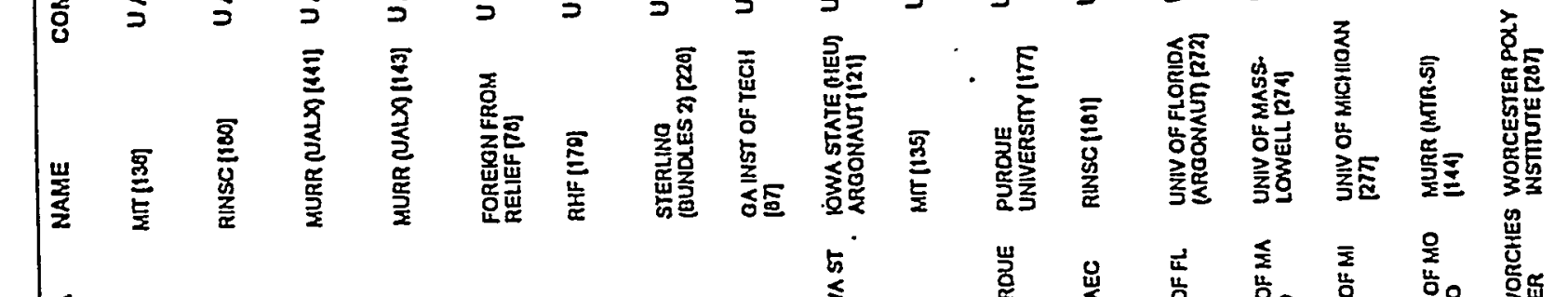

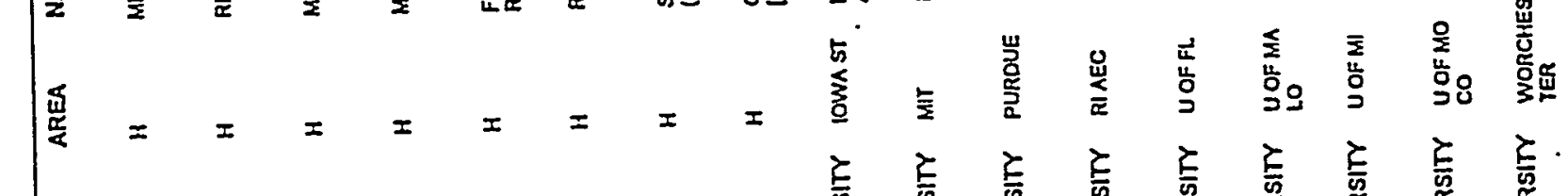

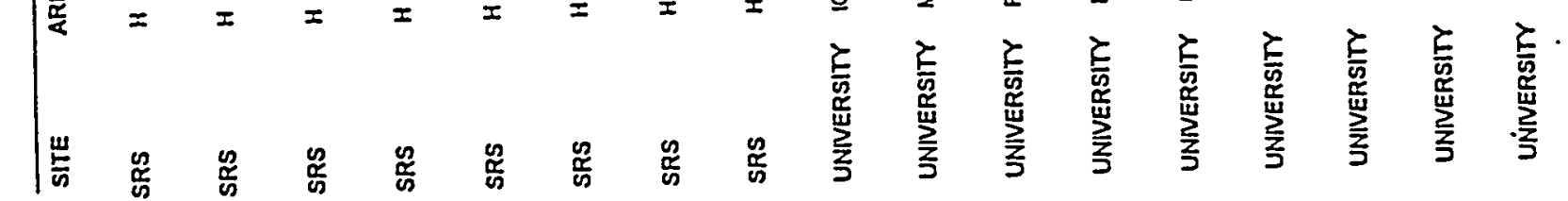




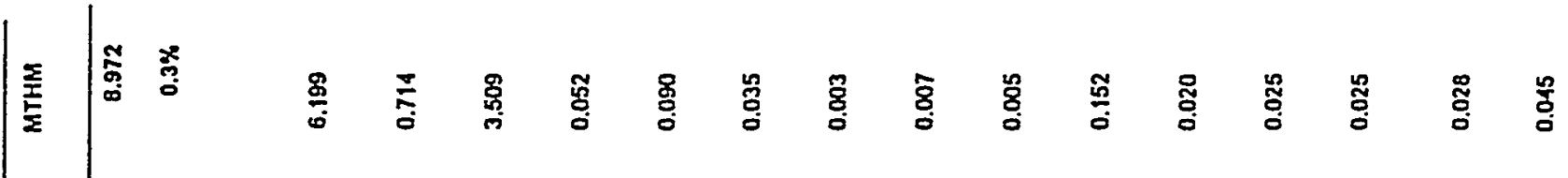

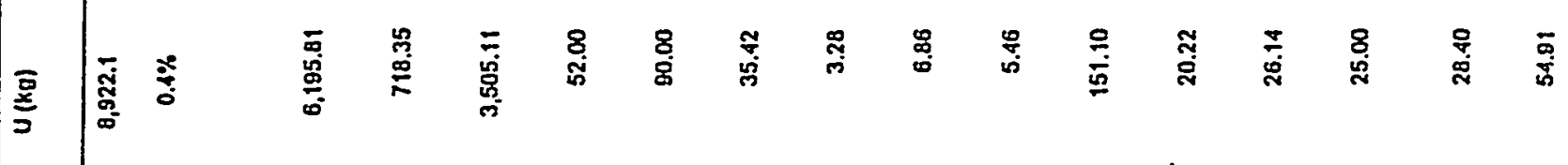

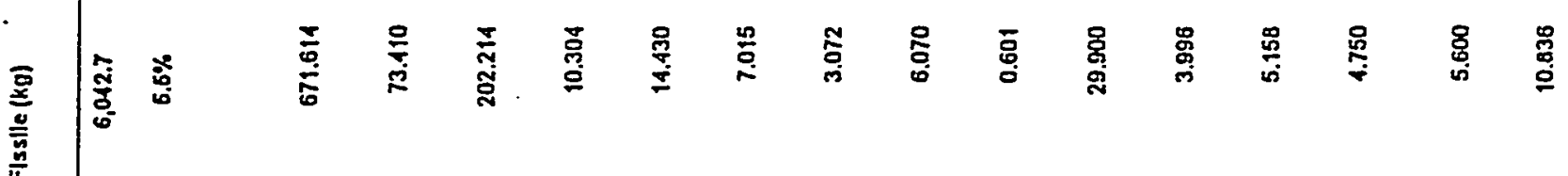

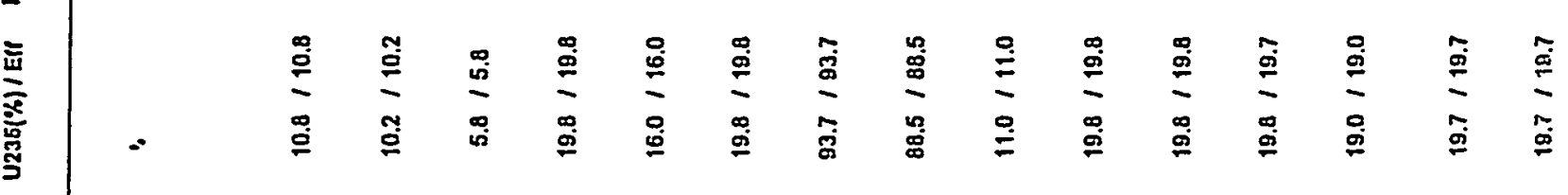

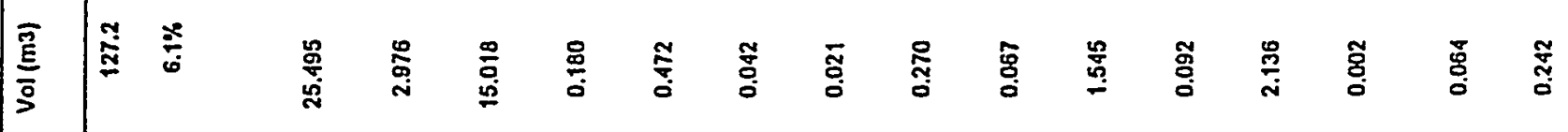

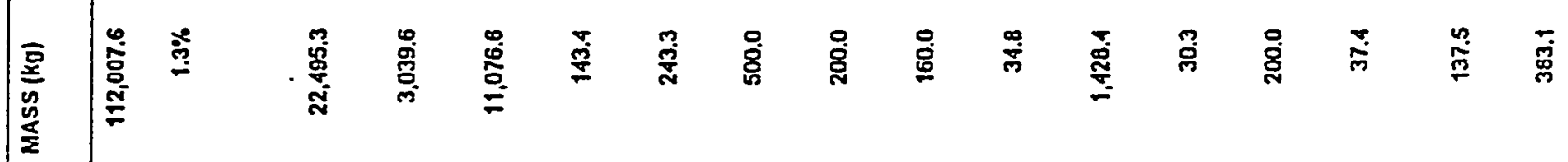

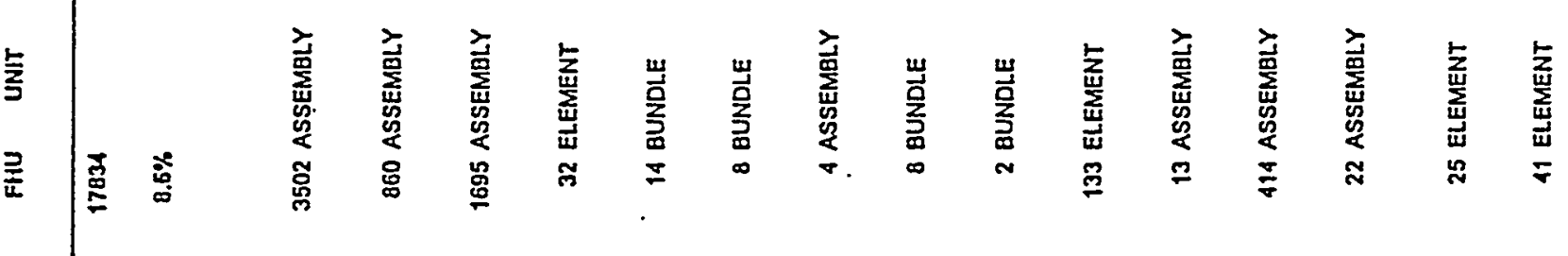

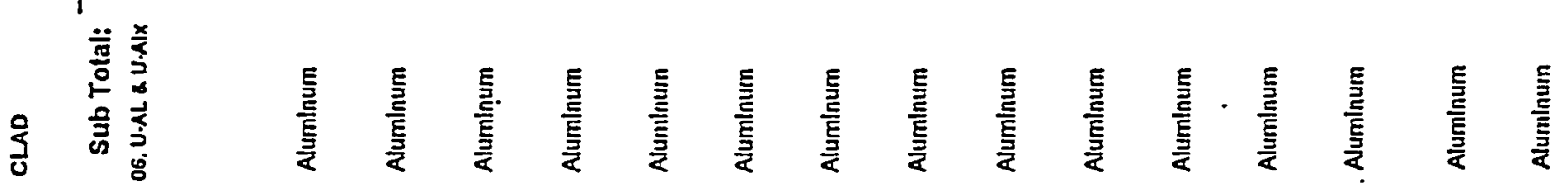

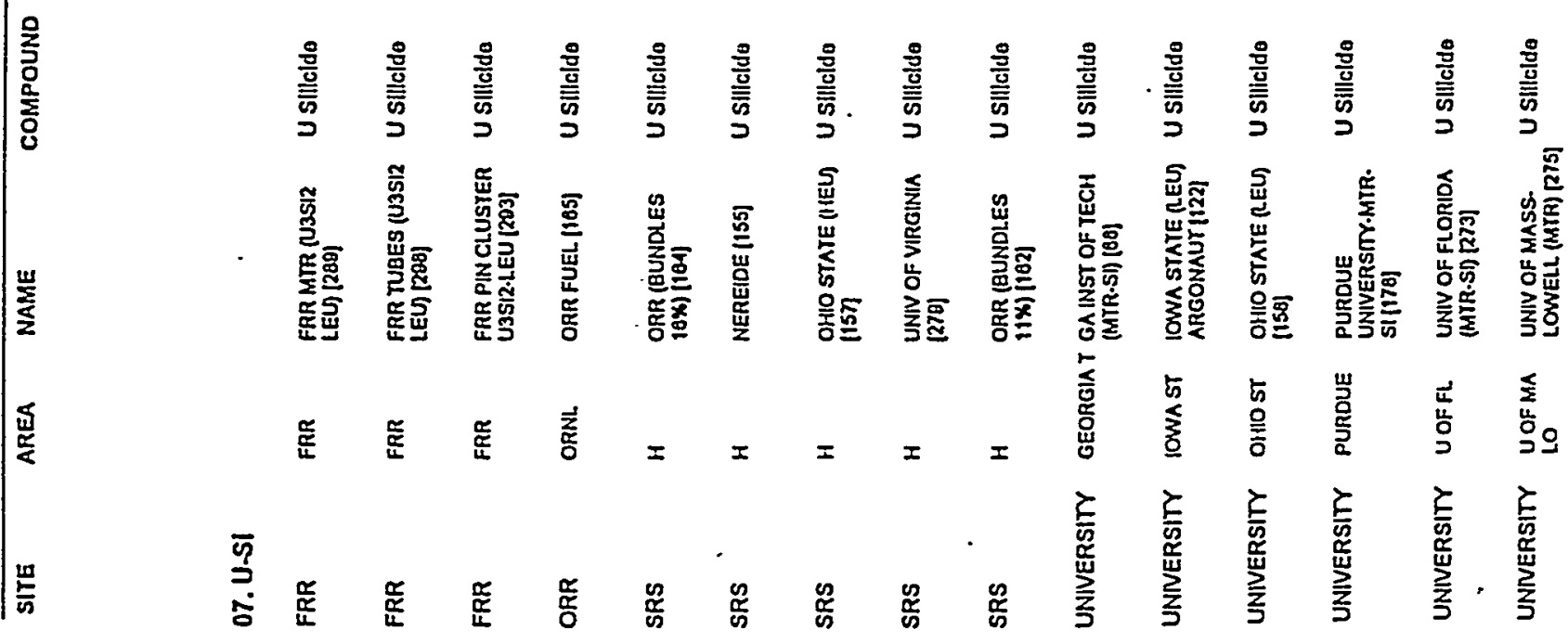




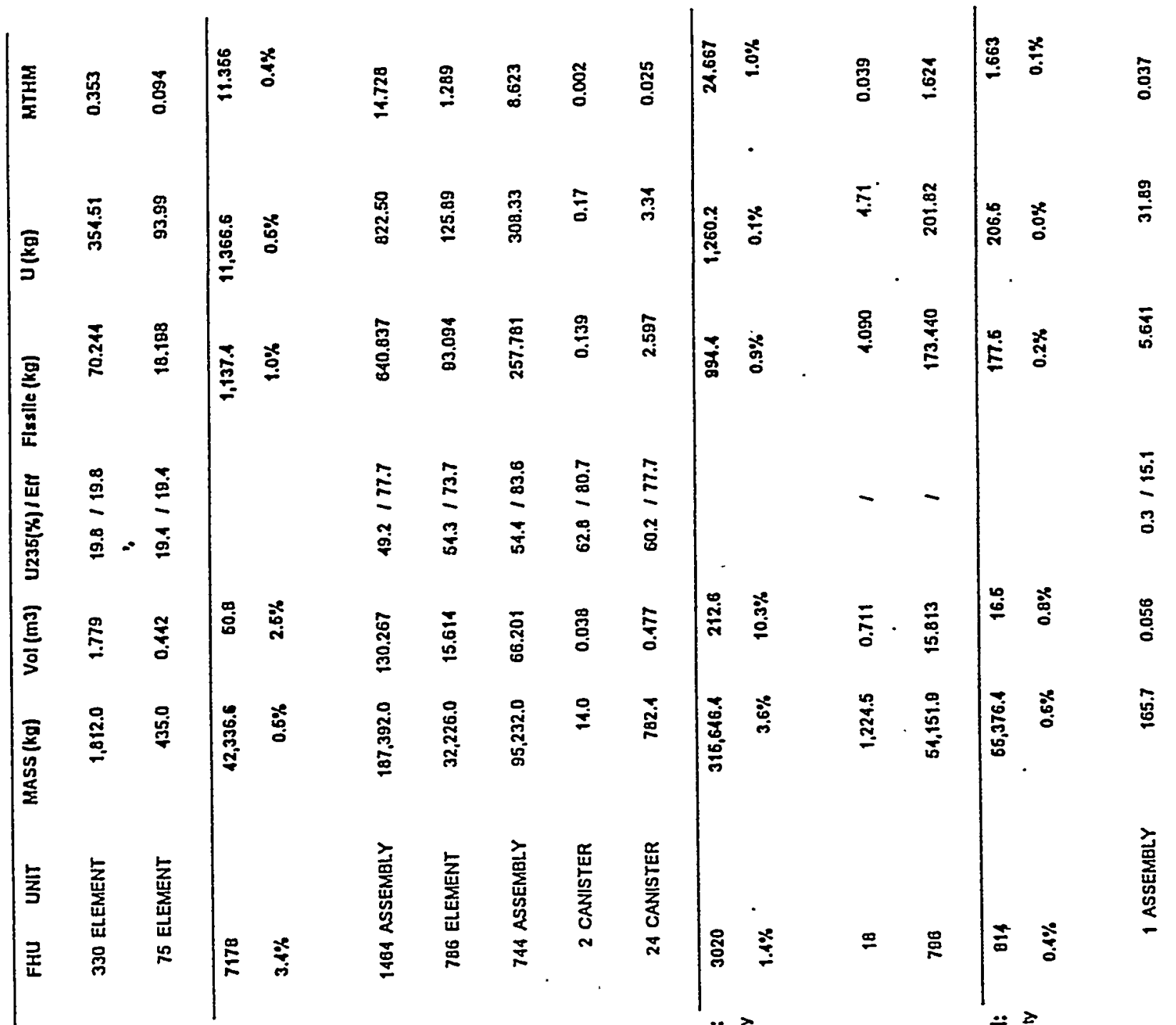

$$
\begin{aligned}
& \text { 界彭高 } \\
& \text { 豆 } \\
& \text { ร) }
\end{aligned}
$$

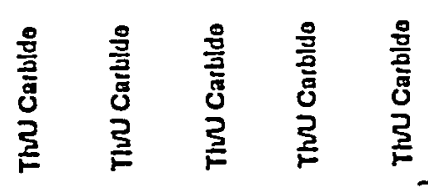

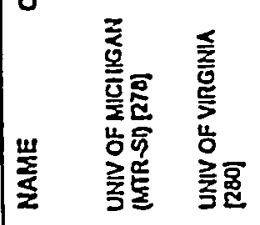

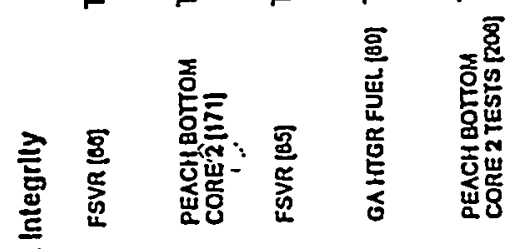

$$
\begin{aligned}
& \text { 衰 }
\end{aligned}
$$

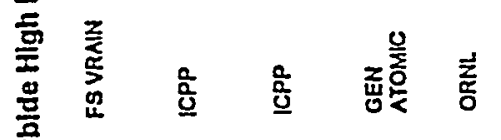

$$
\begin{aligned}
& \text { 訔喜喜 }
\end{aligned}
$$

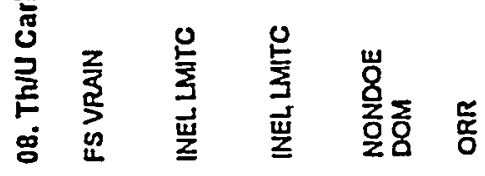

$$
\begin{aligned}
& \text { 重高高高 } \\
& \text { 滖 }
\end{aligned}
$$

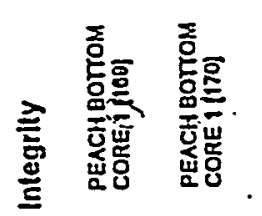

$$
\begin{aligned}
& \text { 望全高 }
\end{aligned}
$$

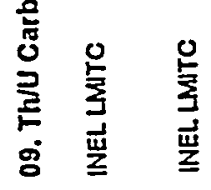

$$
\begin{aligned}
& 5 .
\end{aligned}
$$

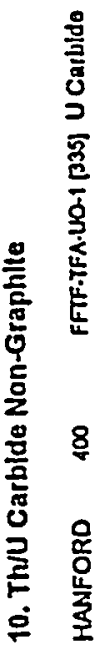




$$
\begin{aligned}
& \text { | } \\
& \text { 至 }
\end{aligned}
$$

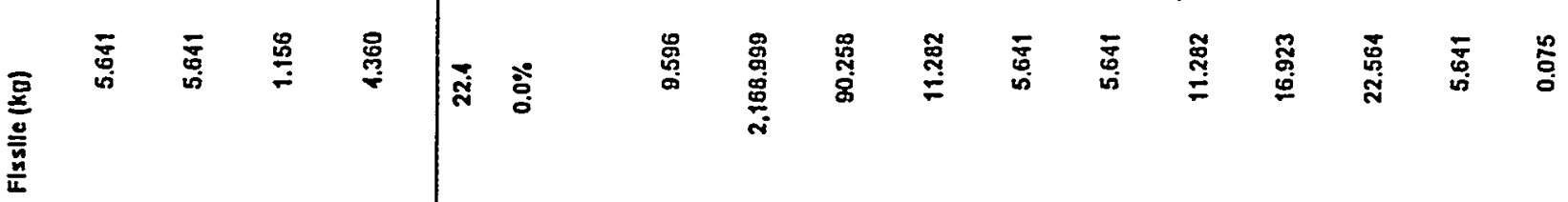

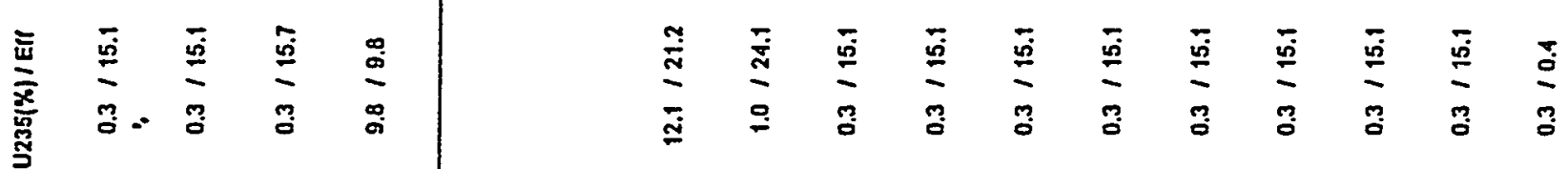

$$
\begin{aligned}
& \text { 产 }
\end{aligned}
$$

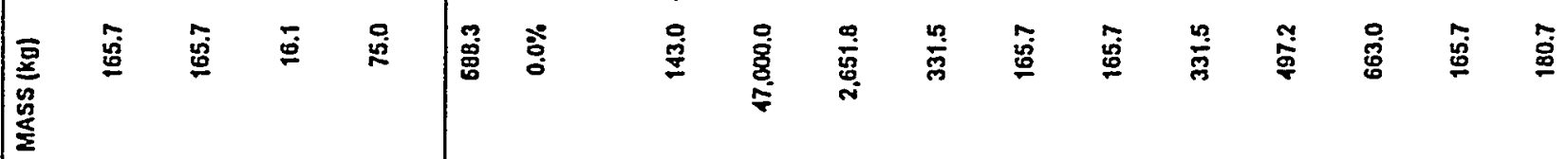

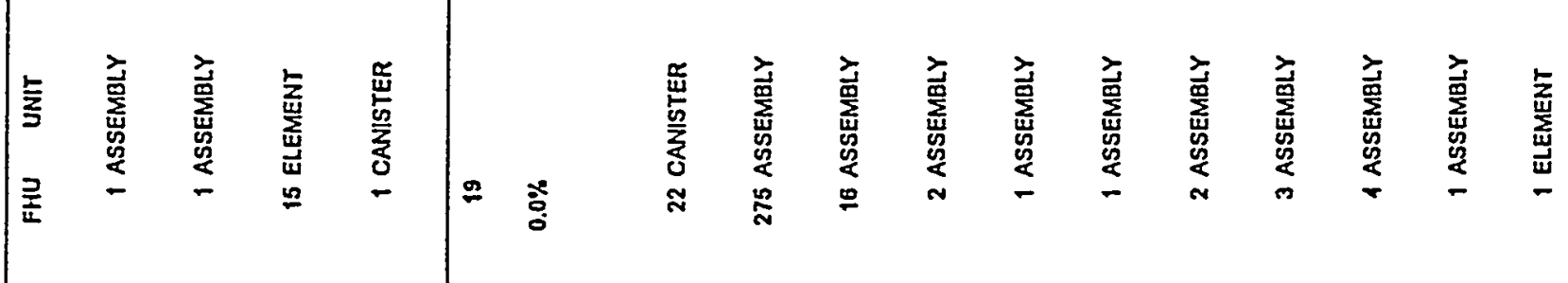

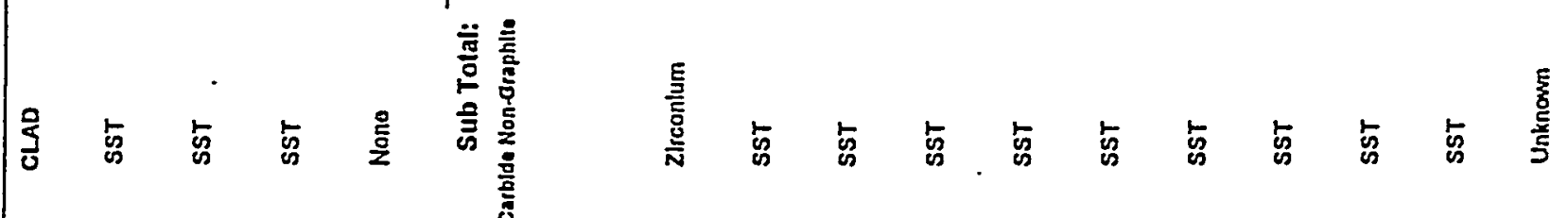

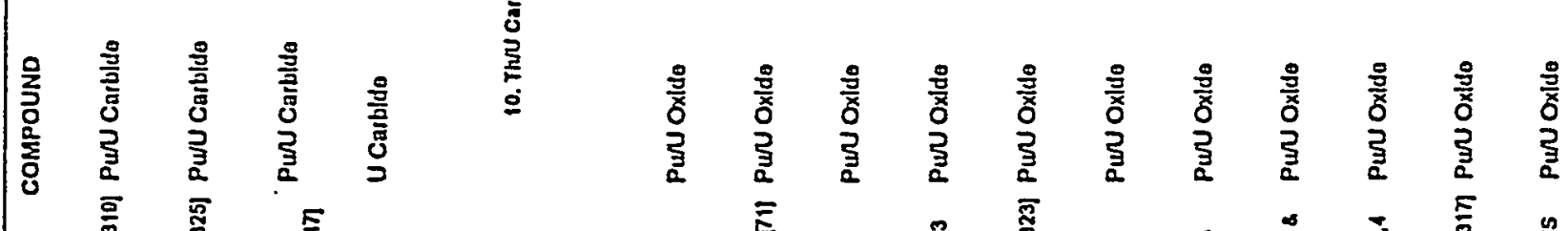

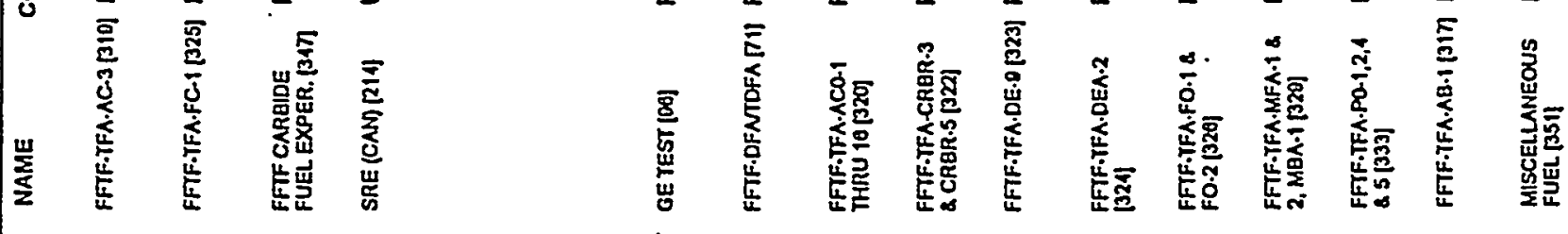

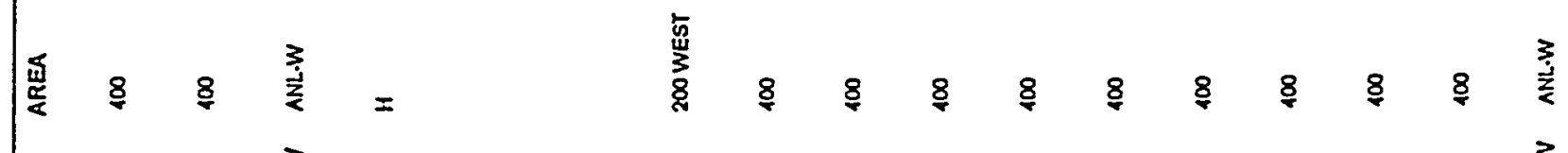

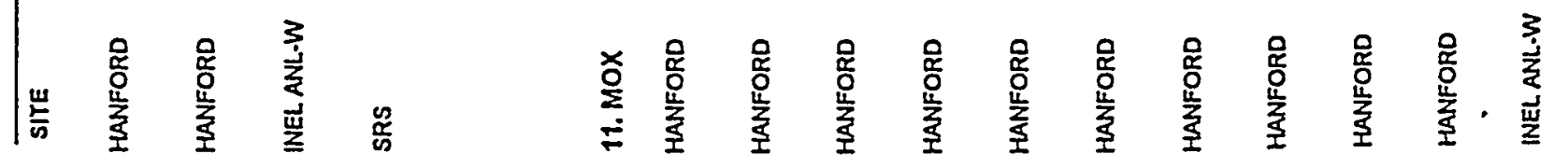




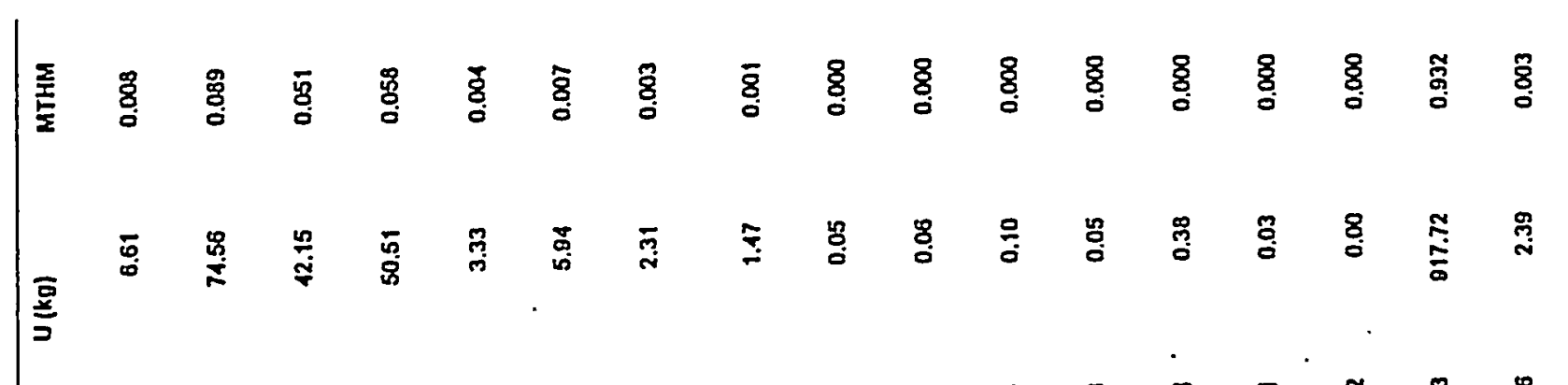

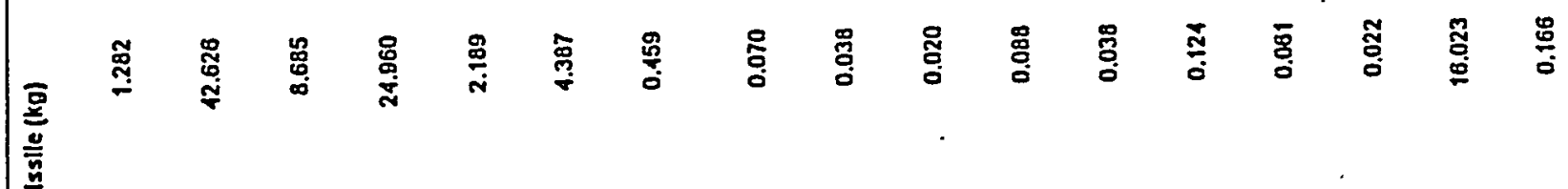

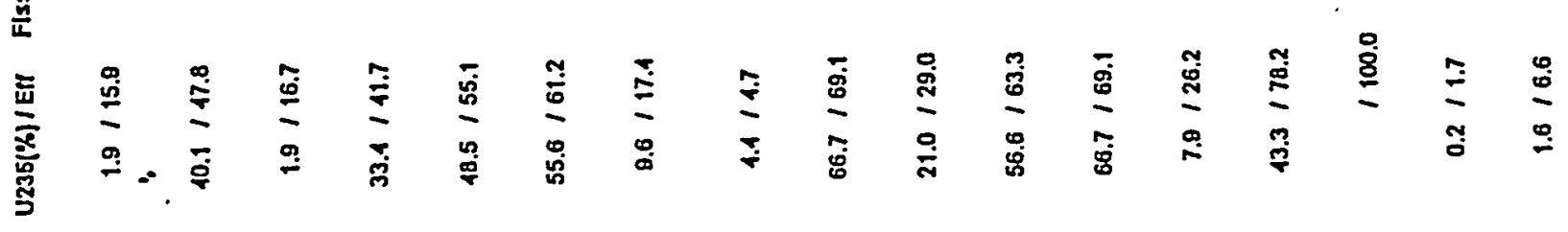

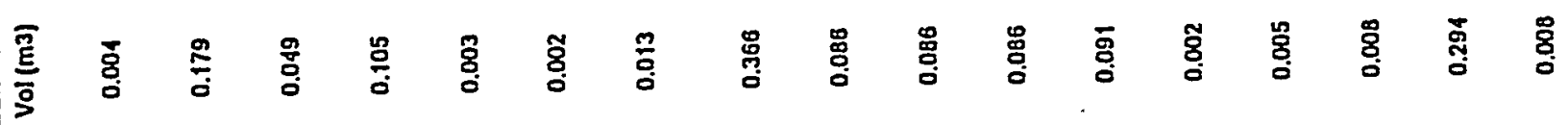

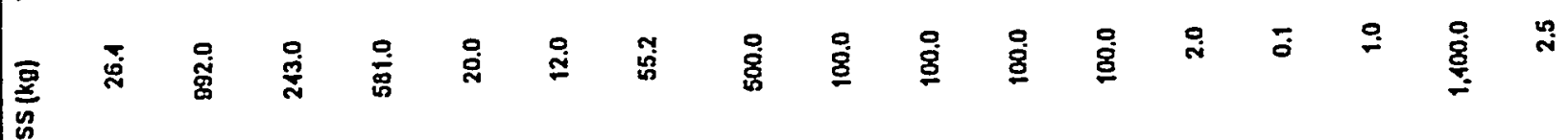
站

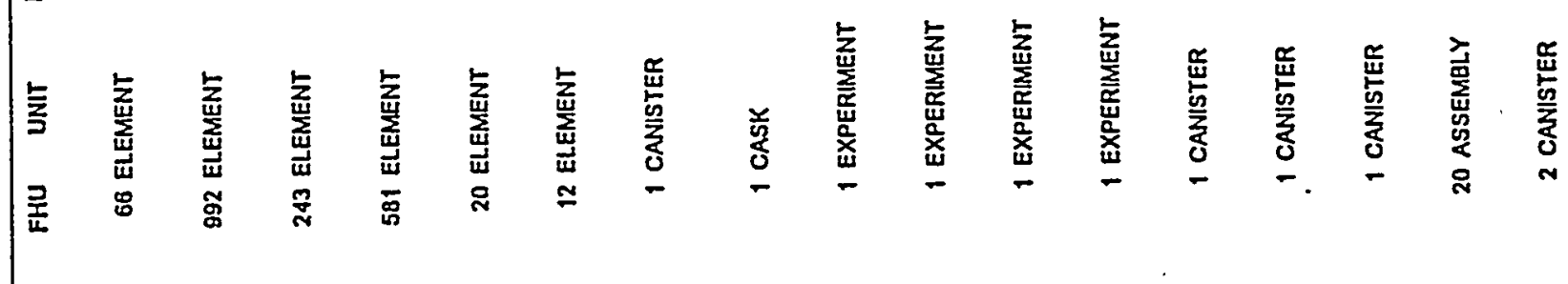

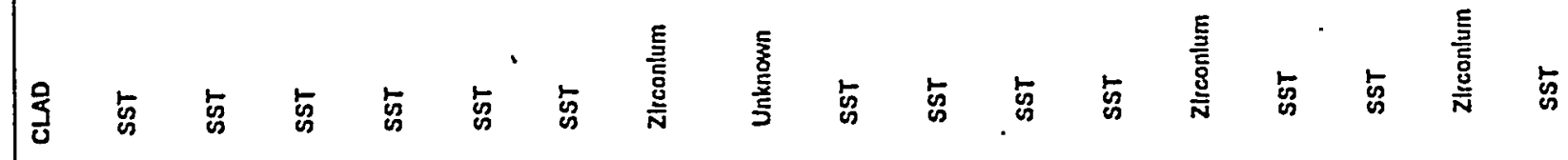

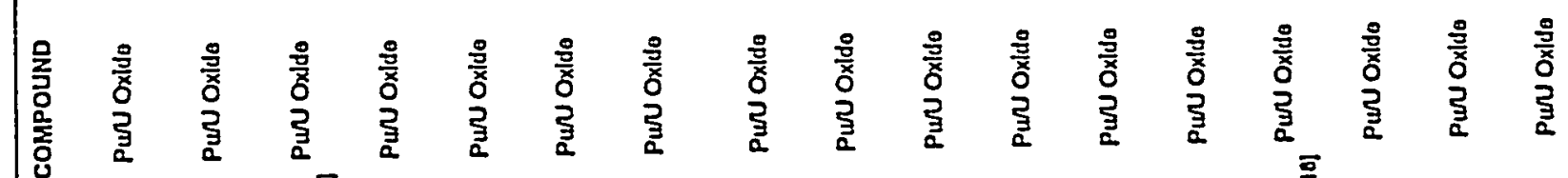

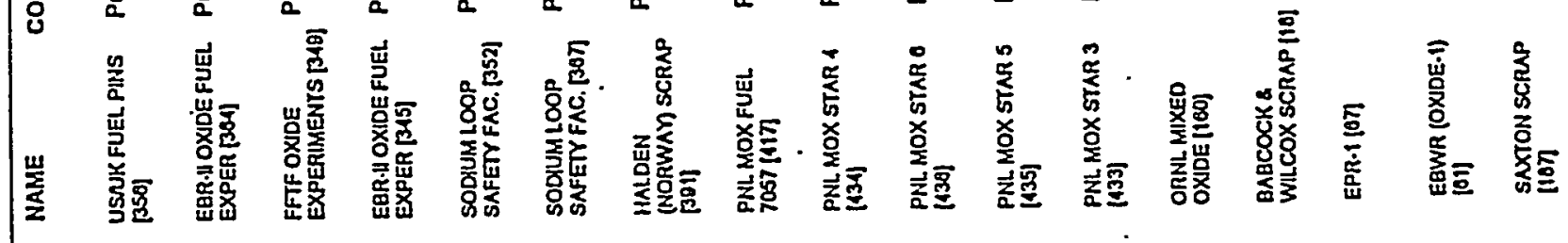

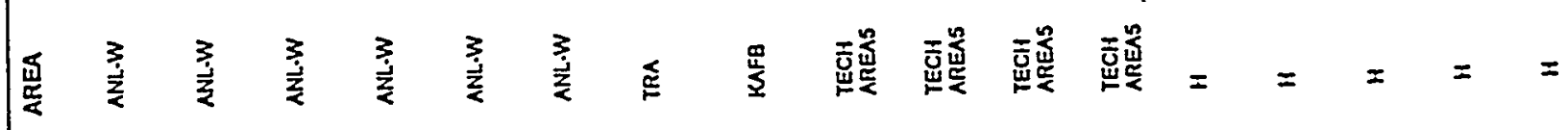

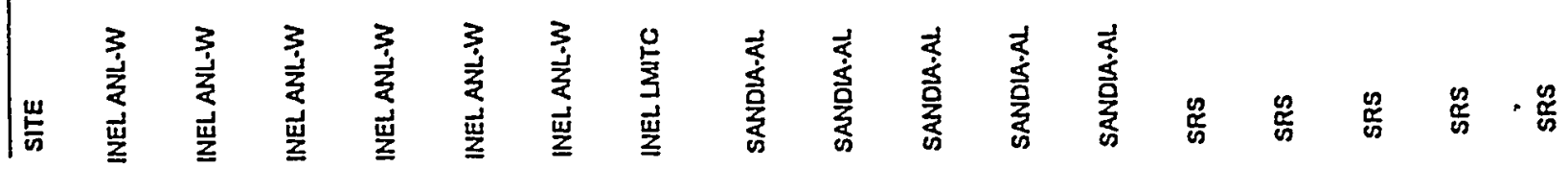




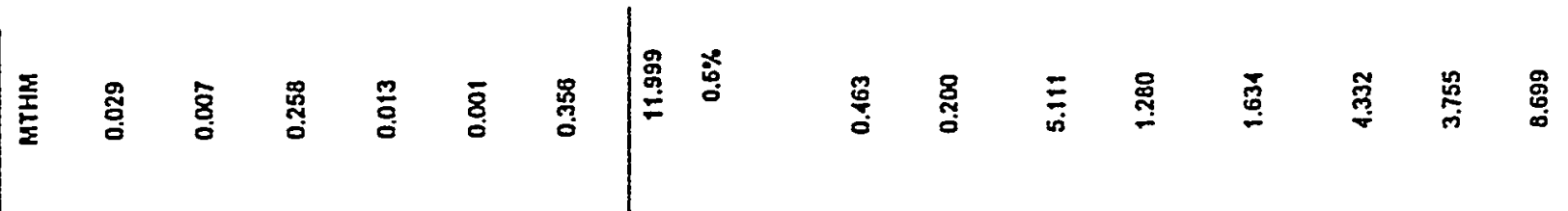

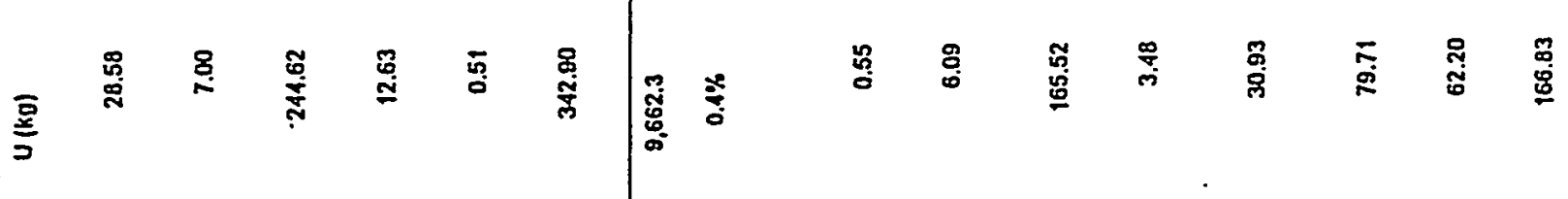

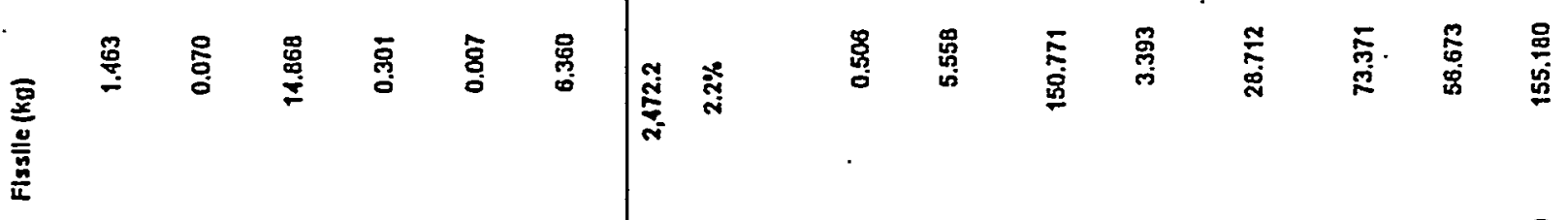

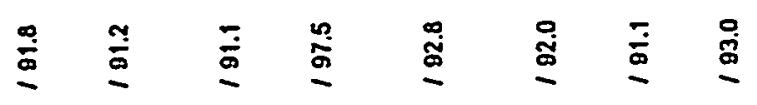

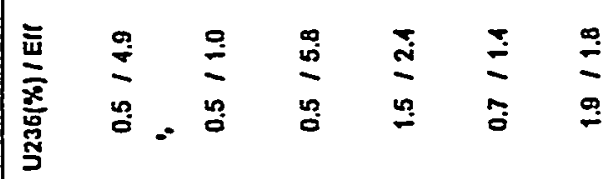

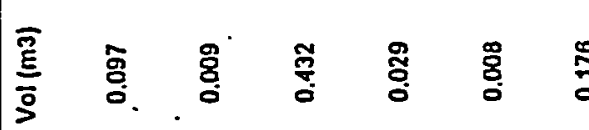

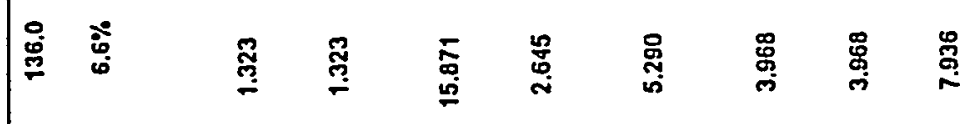

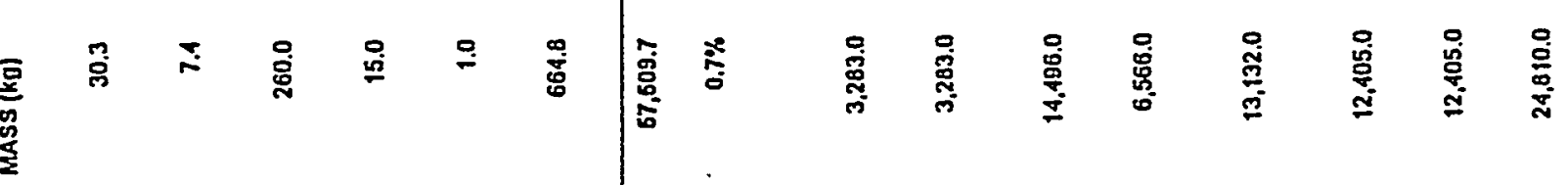

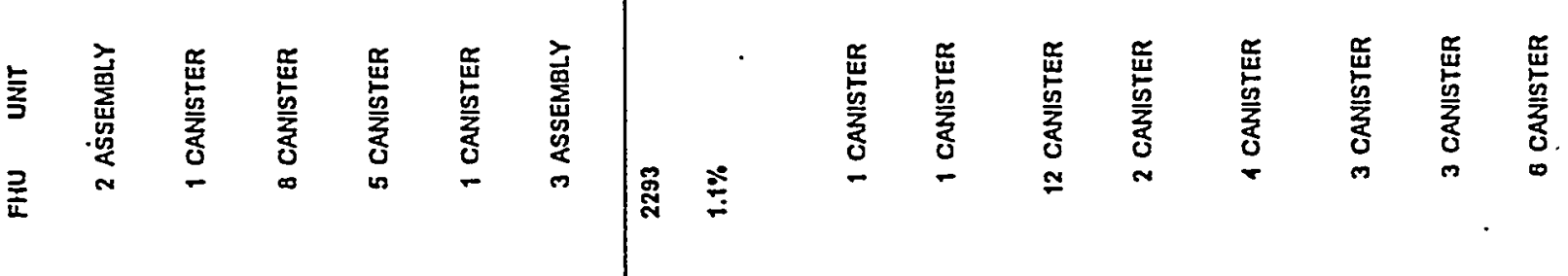

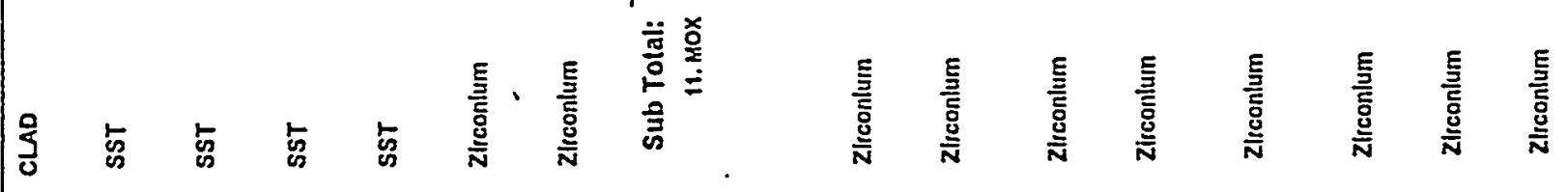

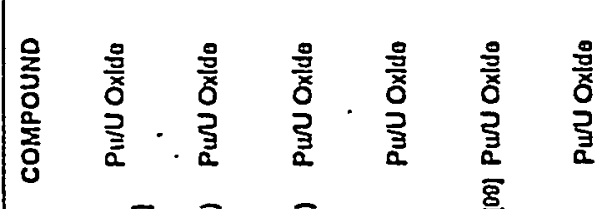

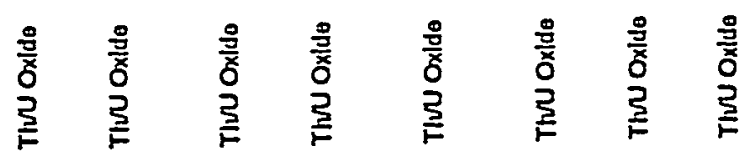

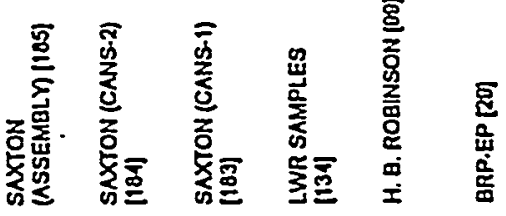

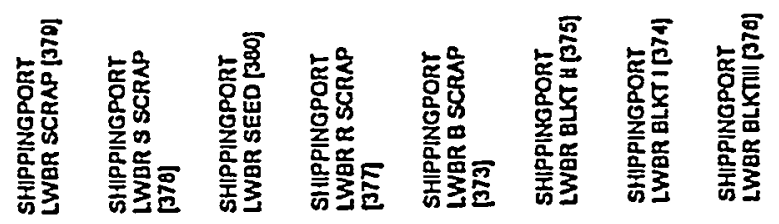

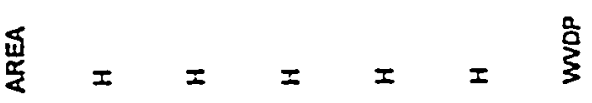

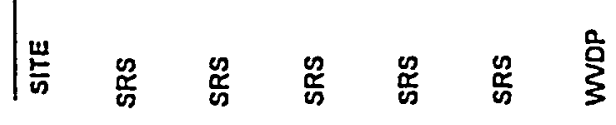

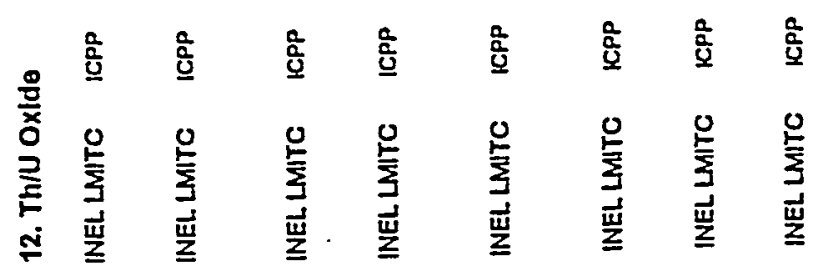




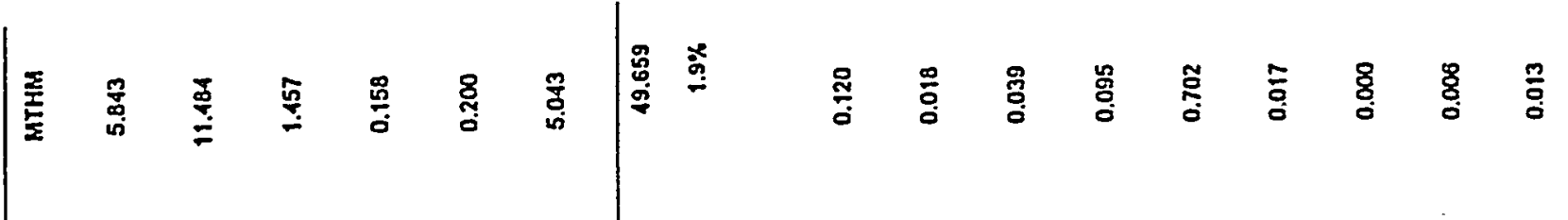

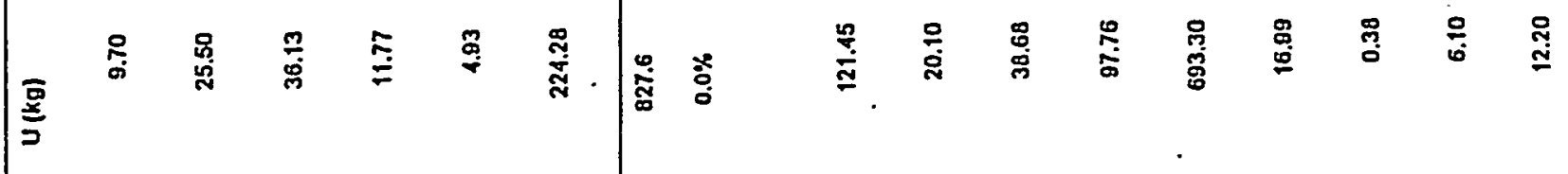

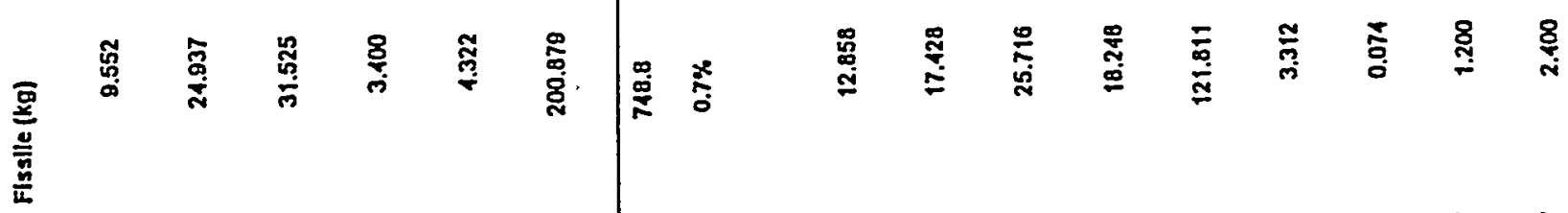

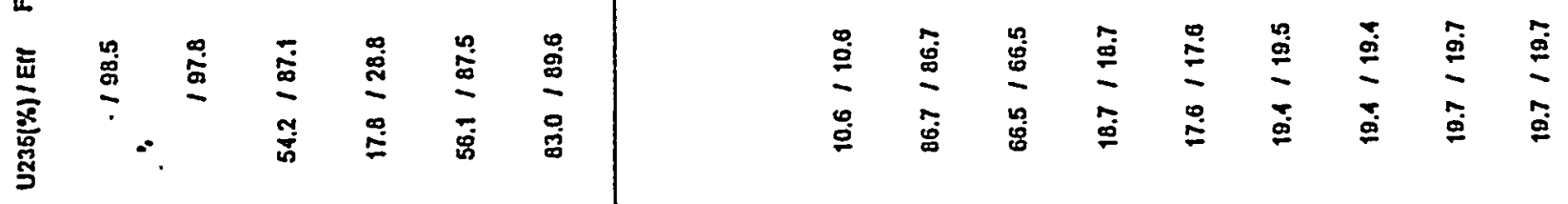

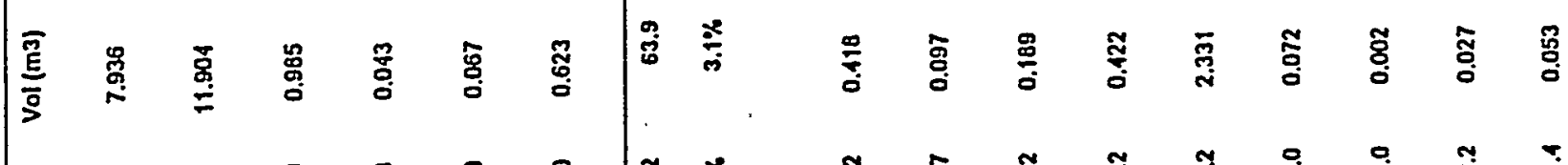

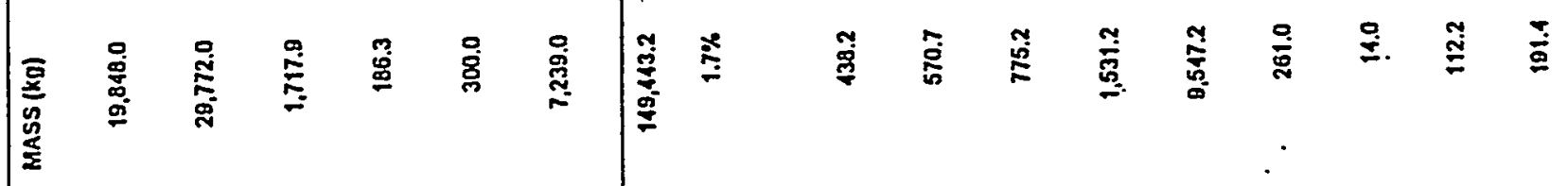

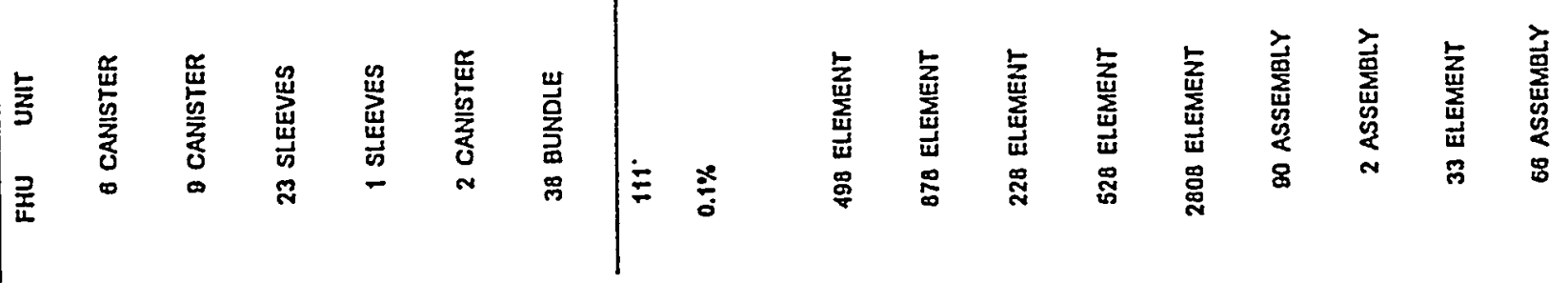

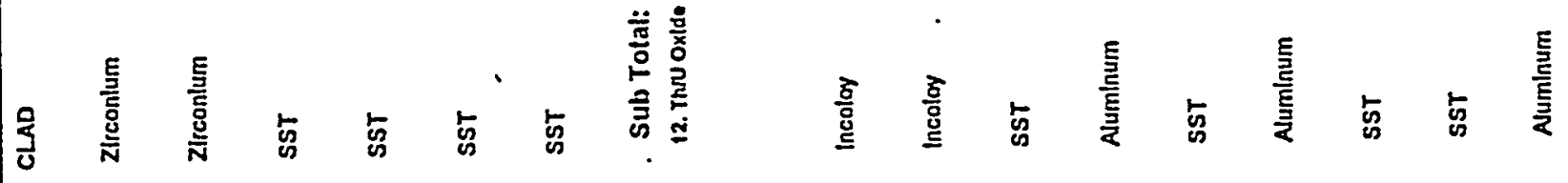

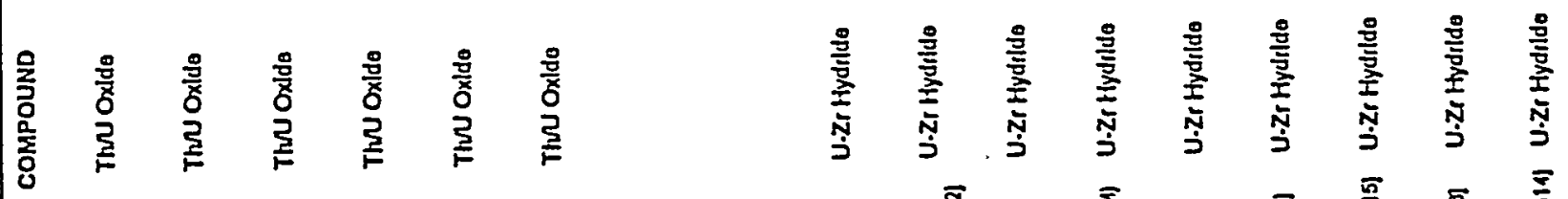

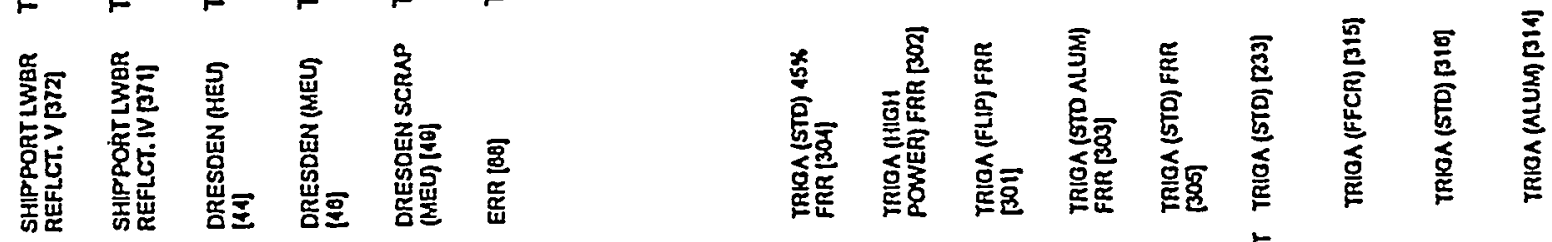

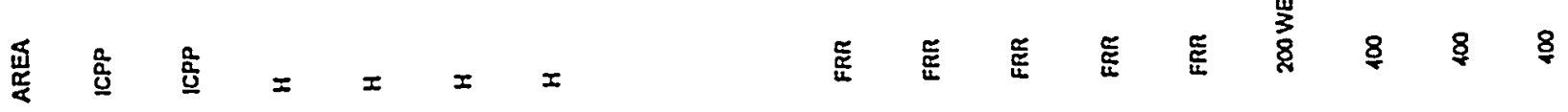

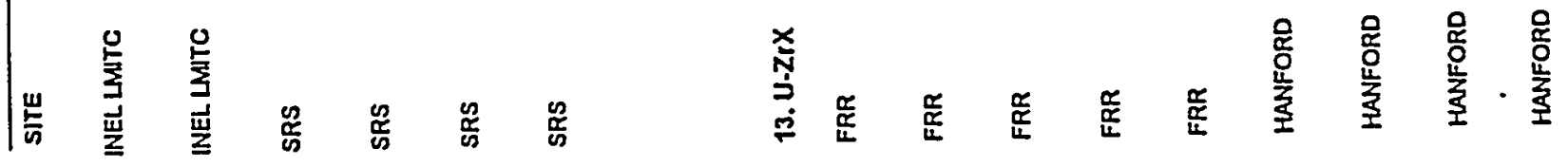


重

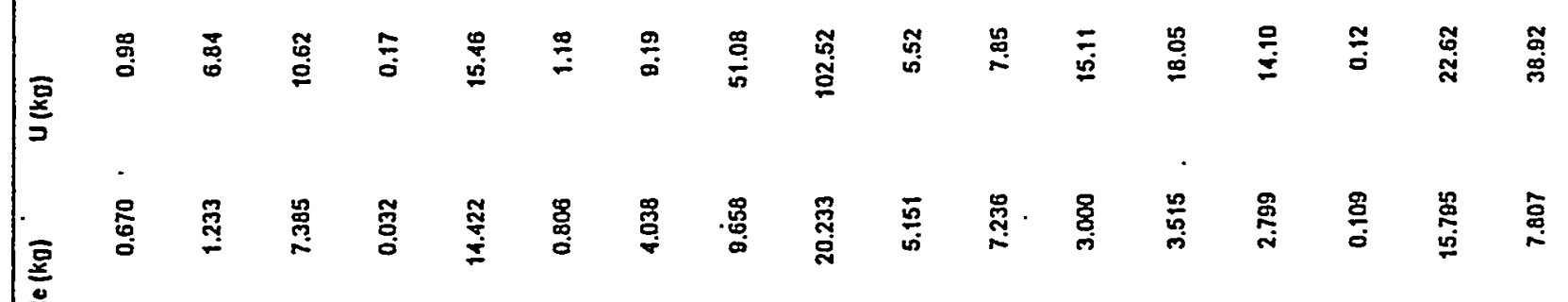

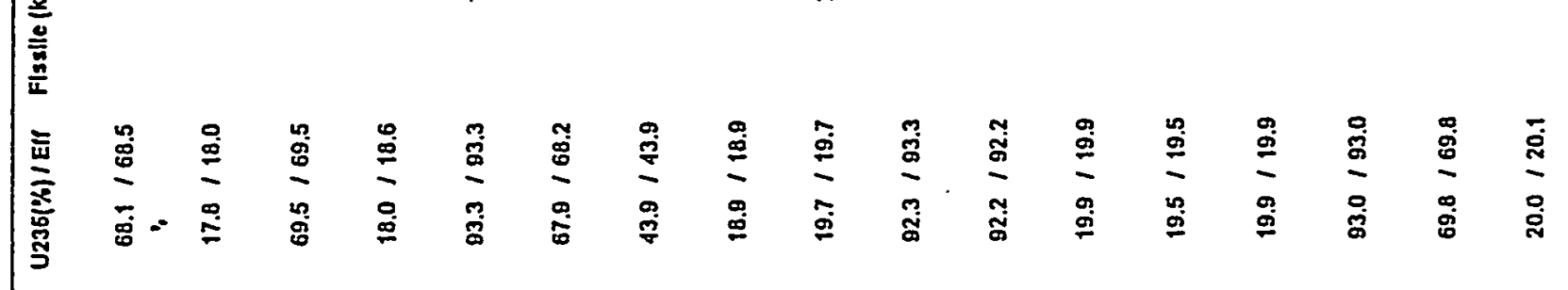

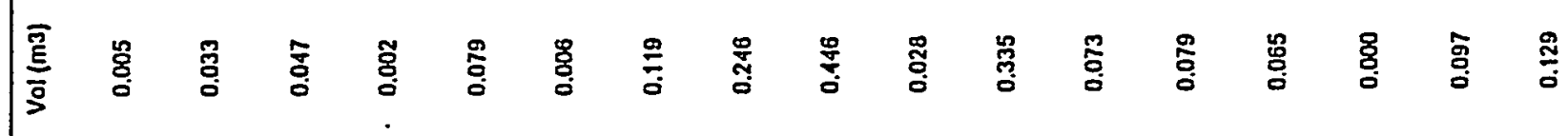

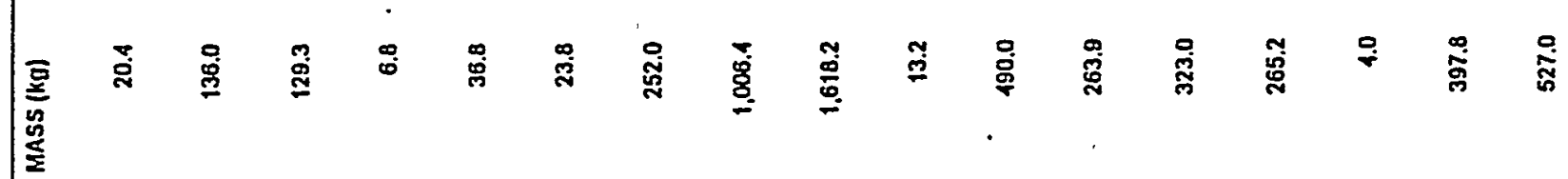

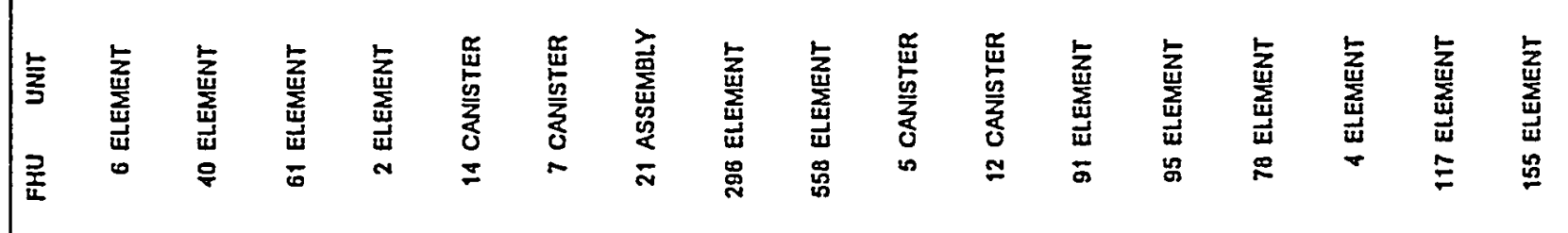

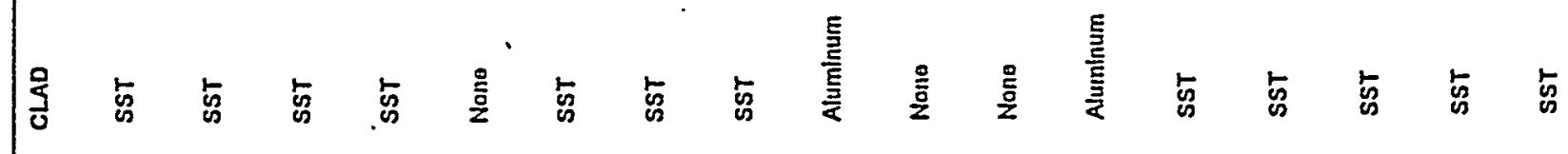

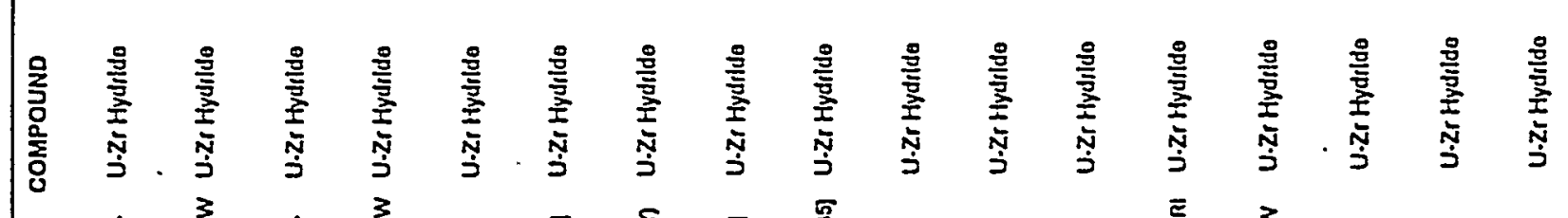

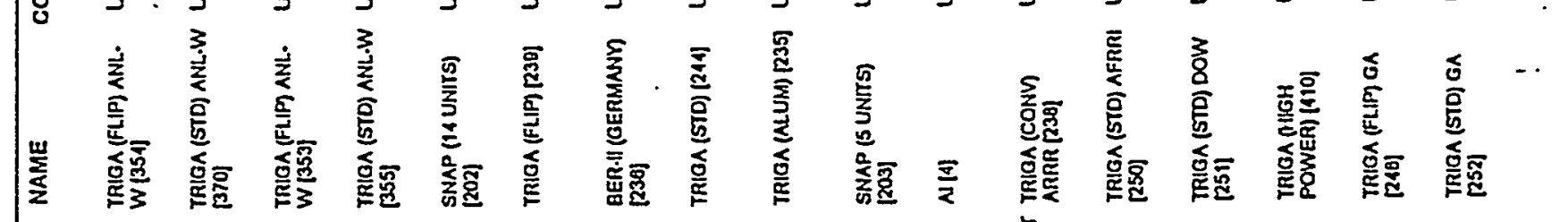

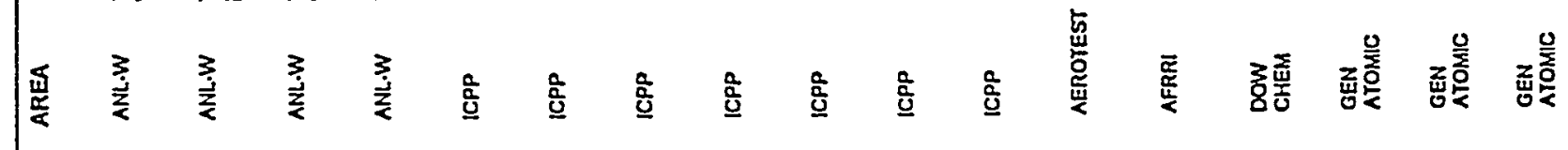

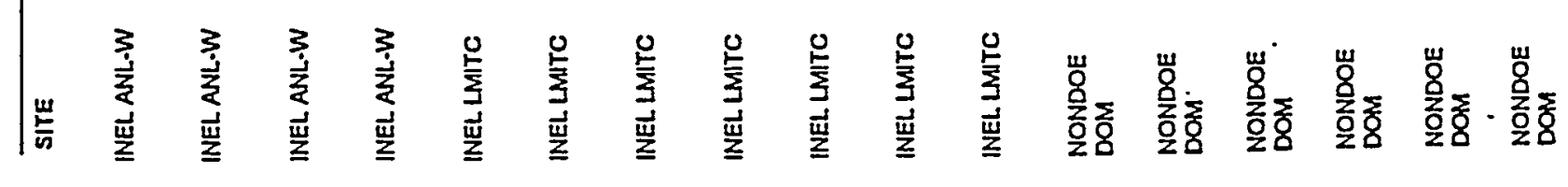




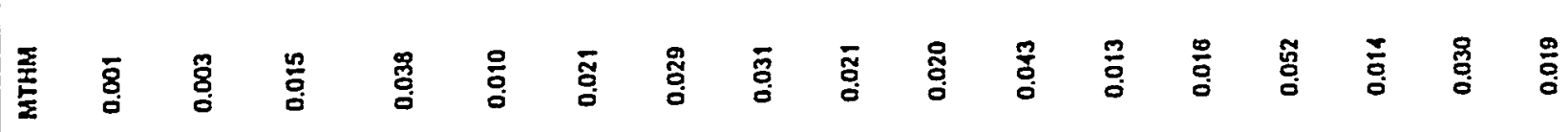

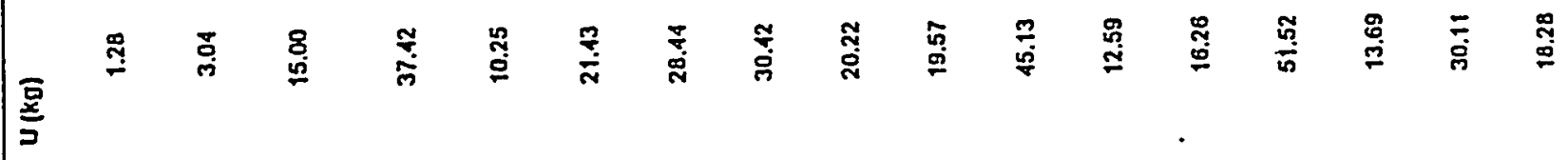

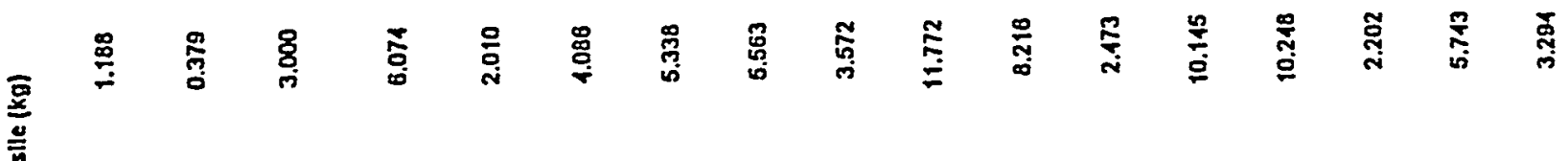

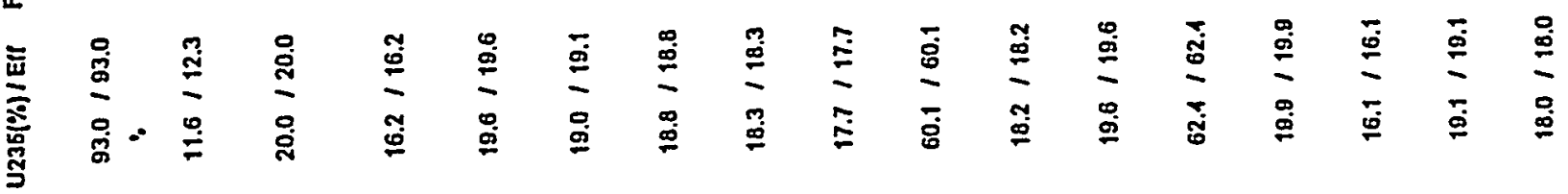

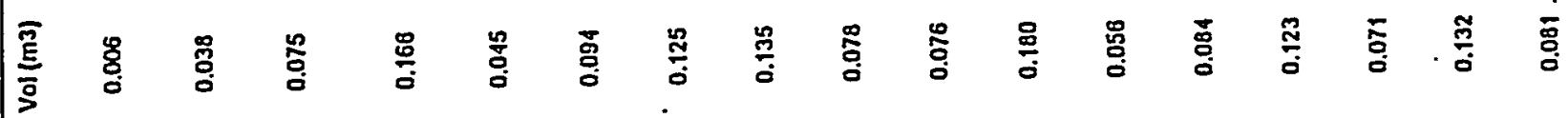

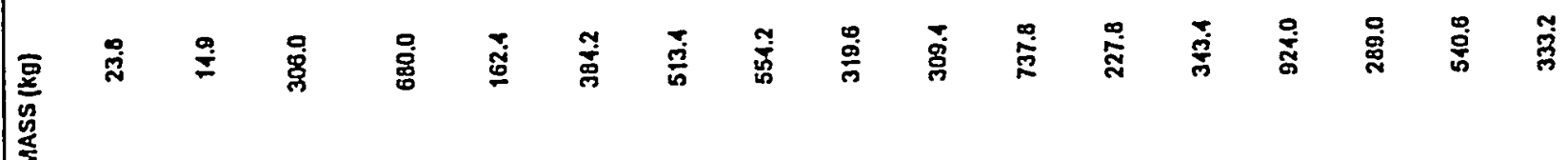

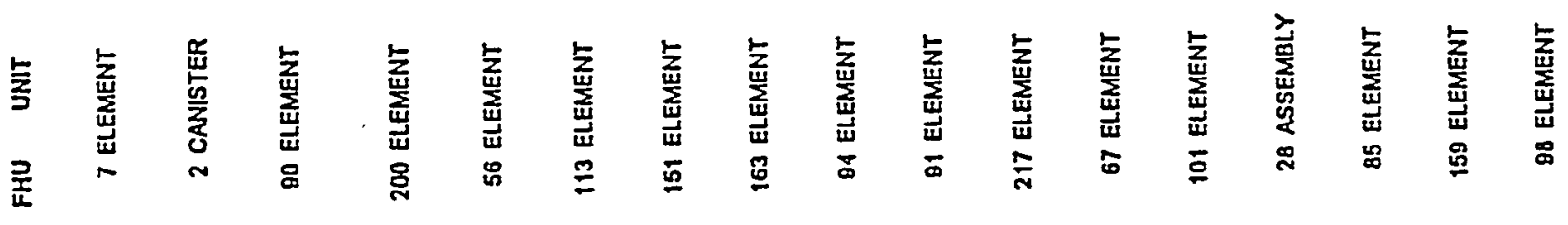

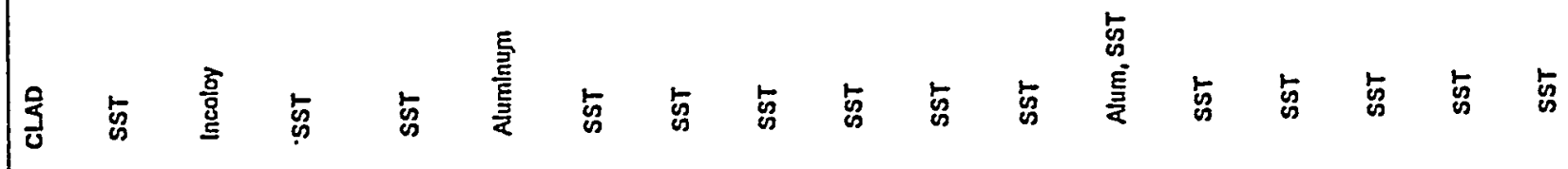

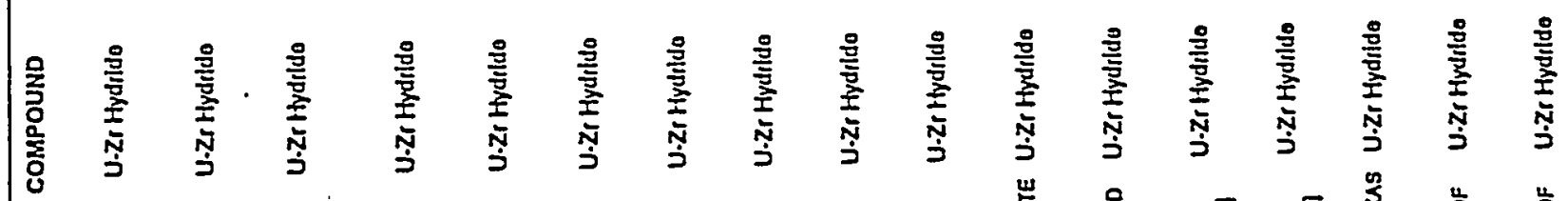

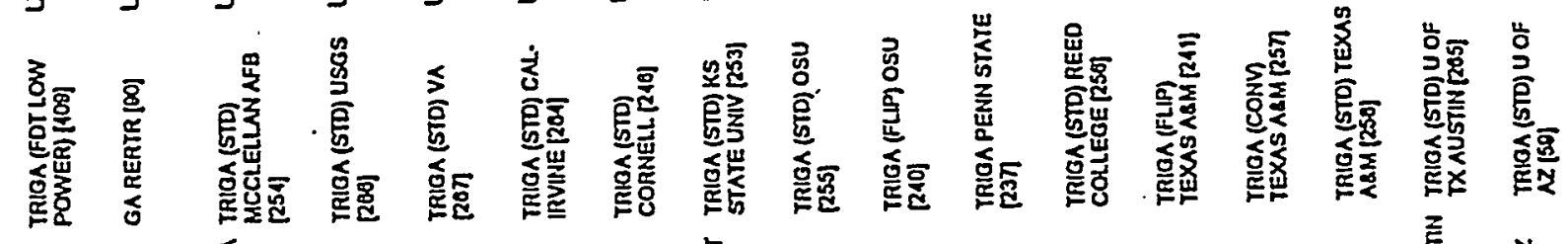

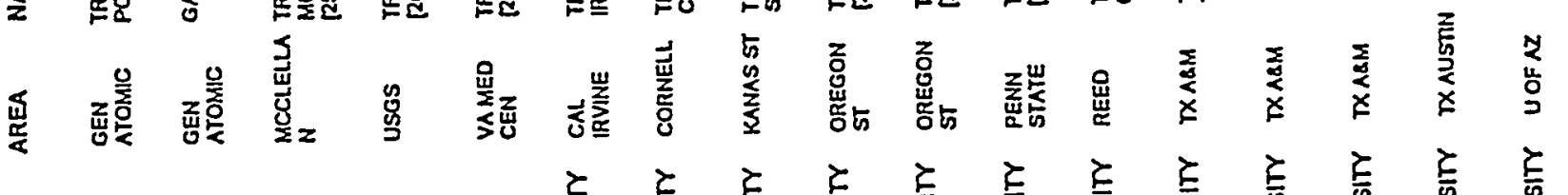

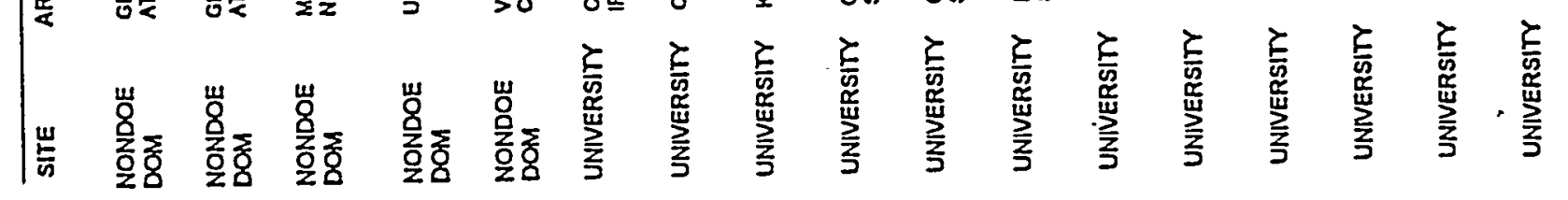




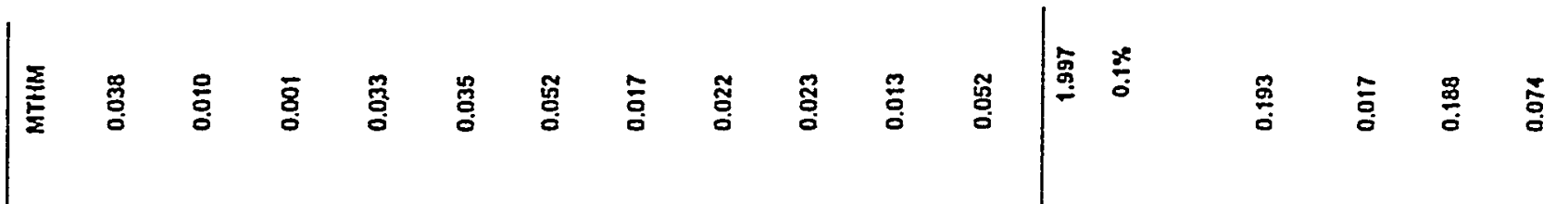

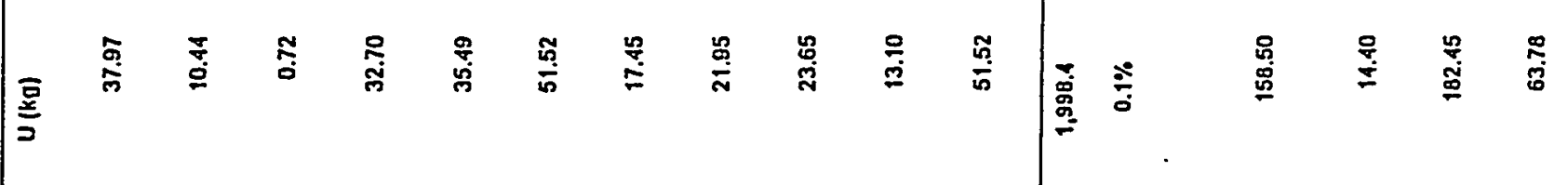

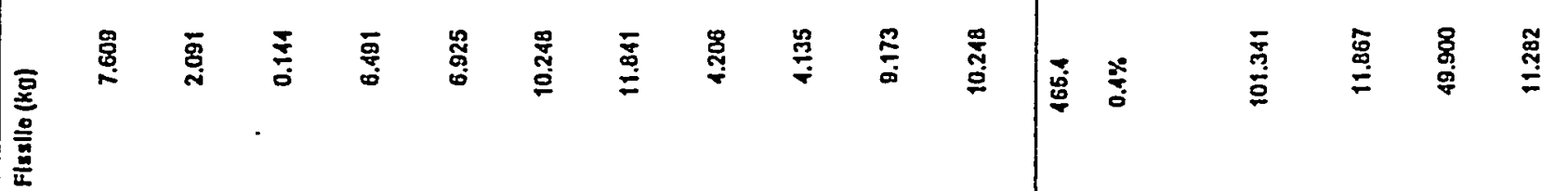

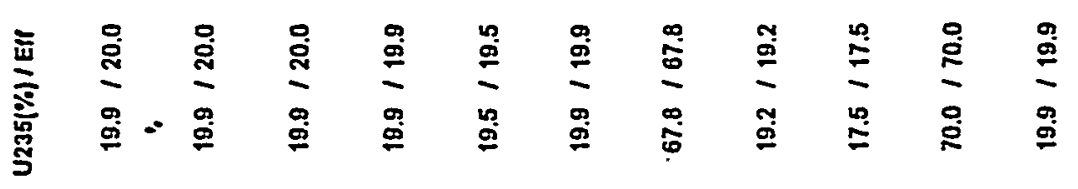

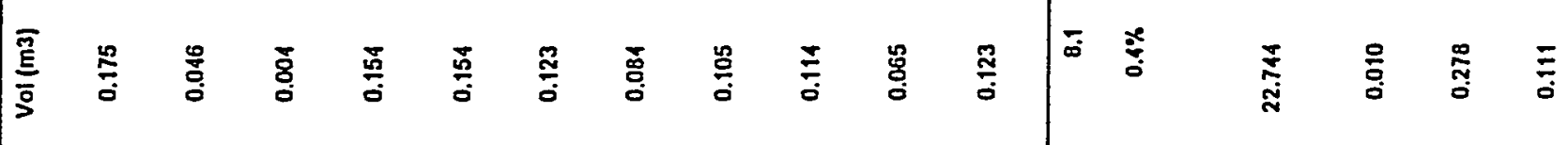

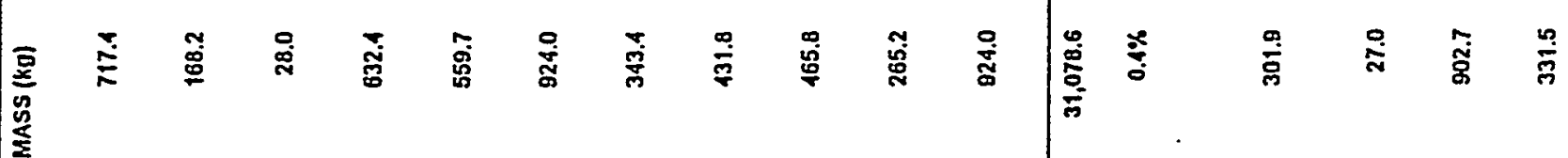

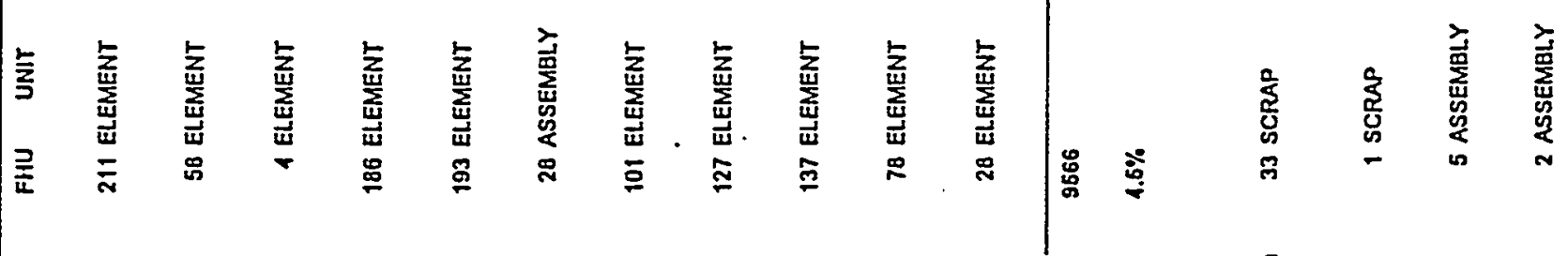

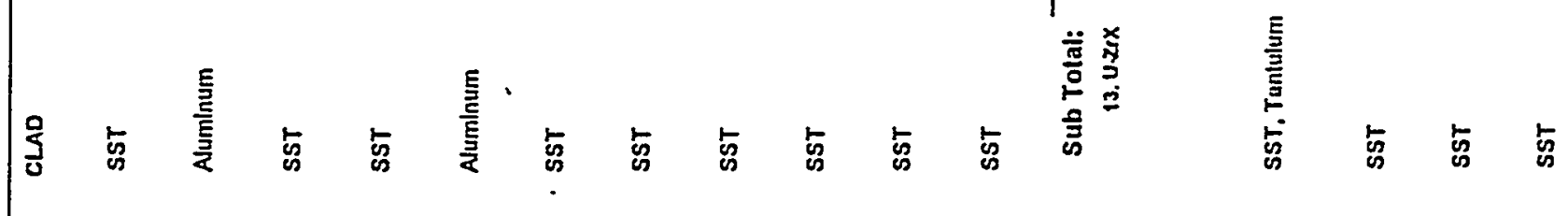

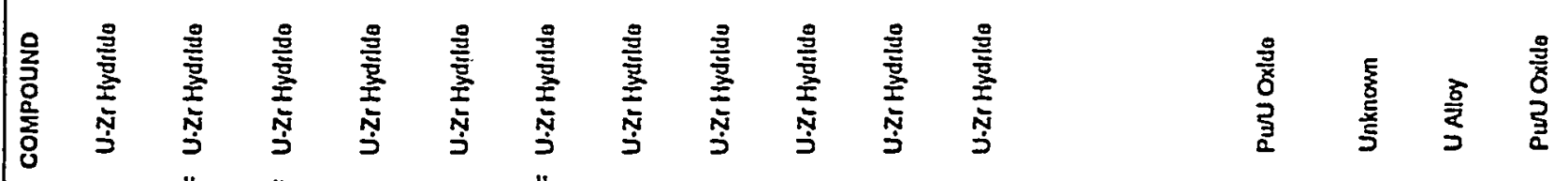

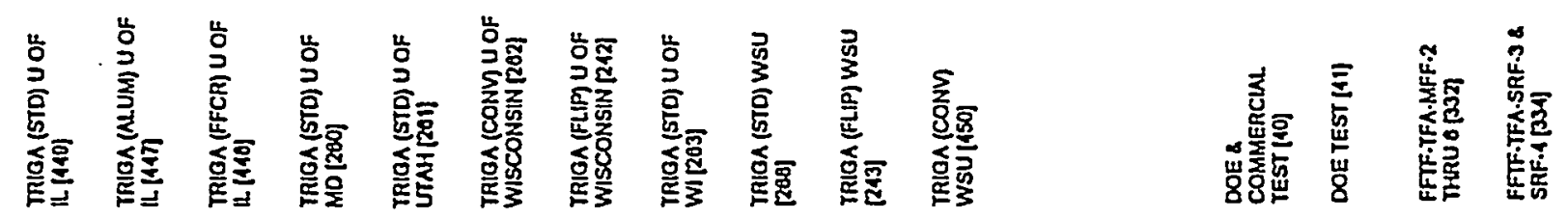

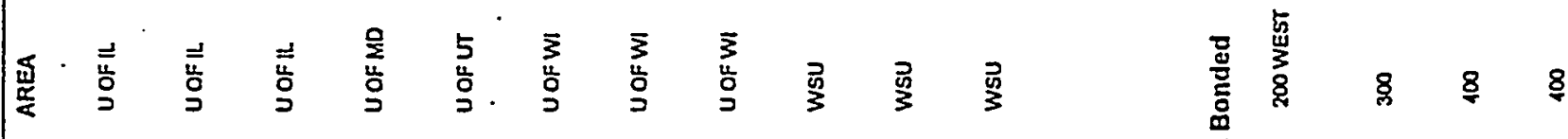

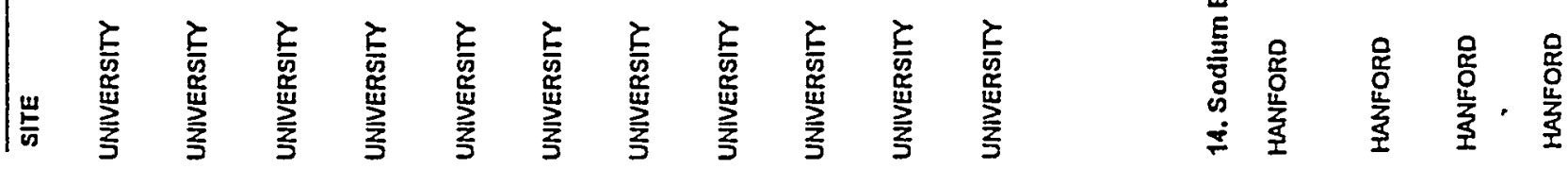




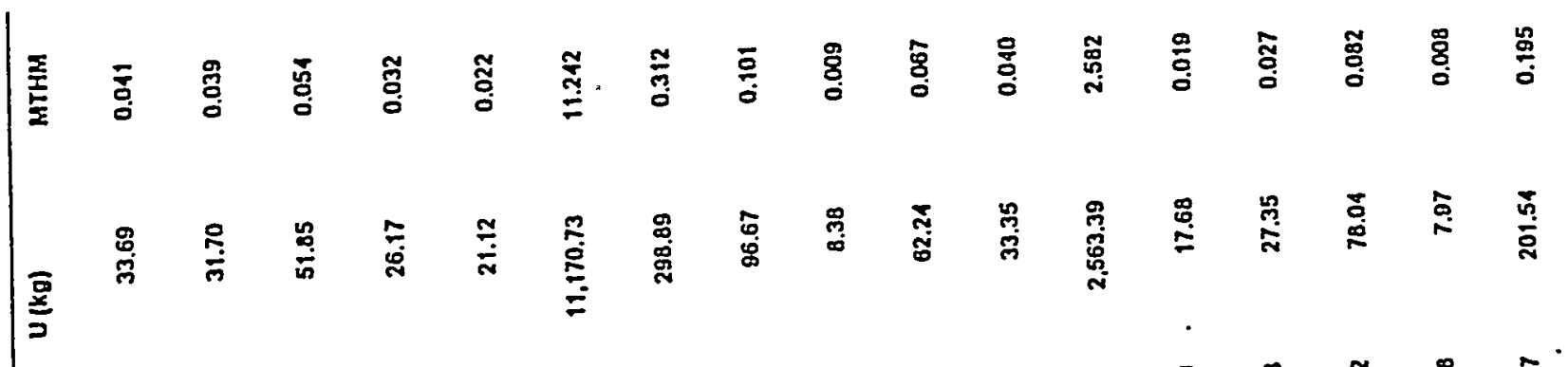

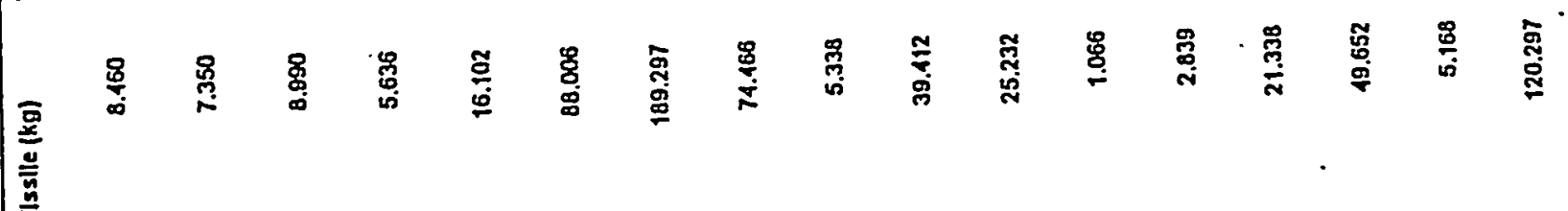

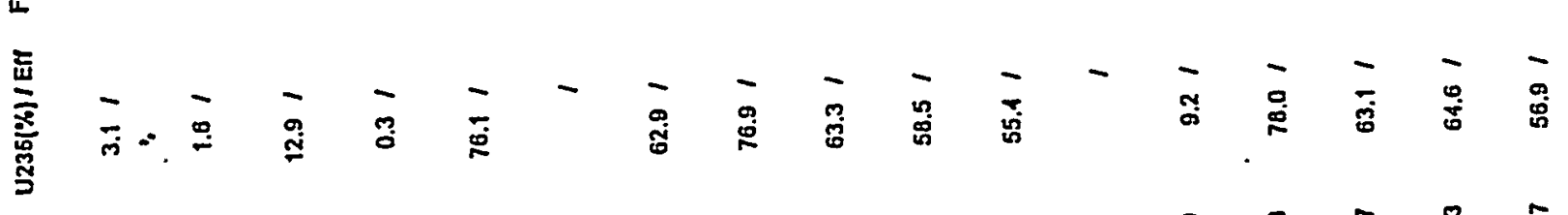

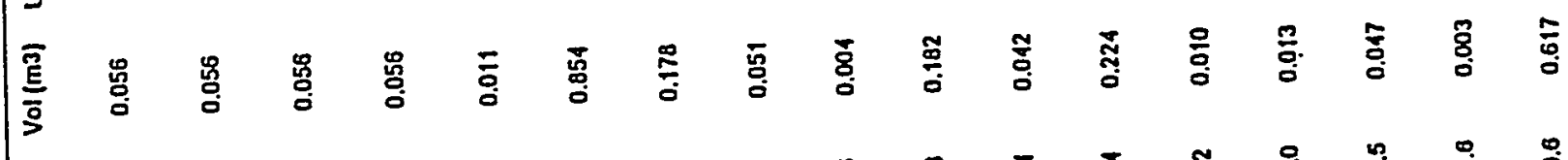

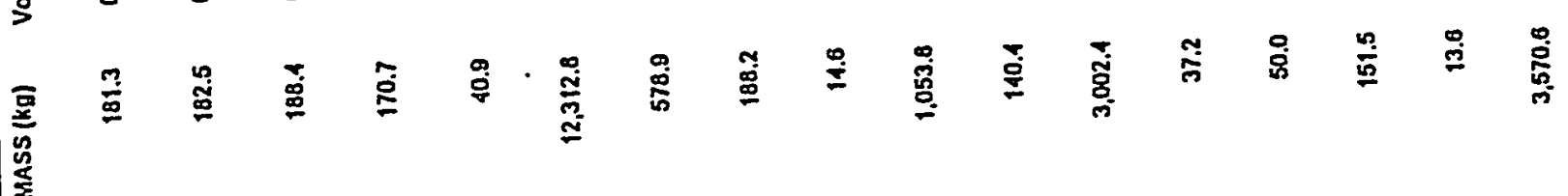

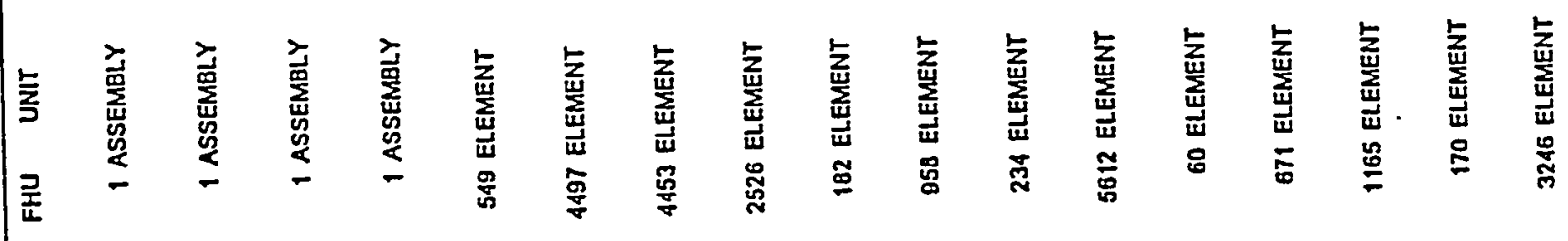

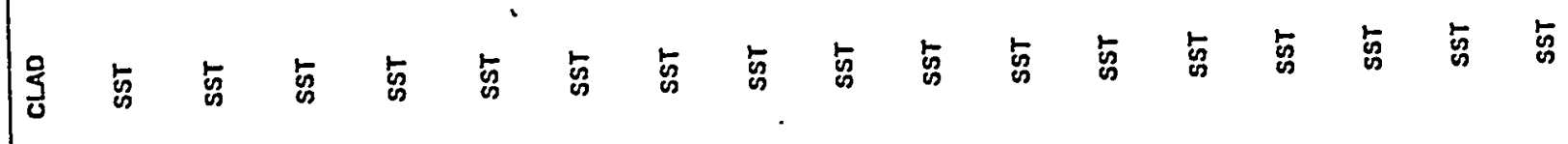

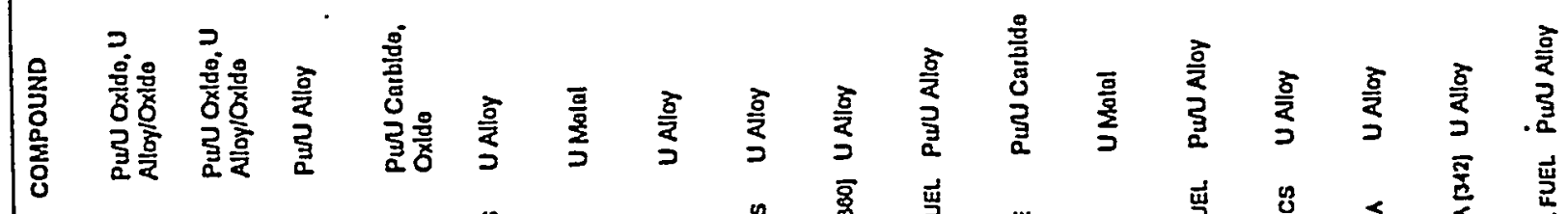

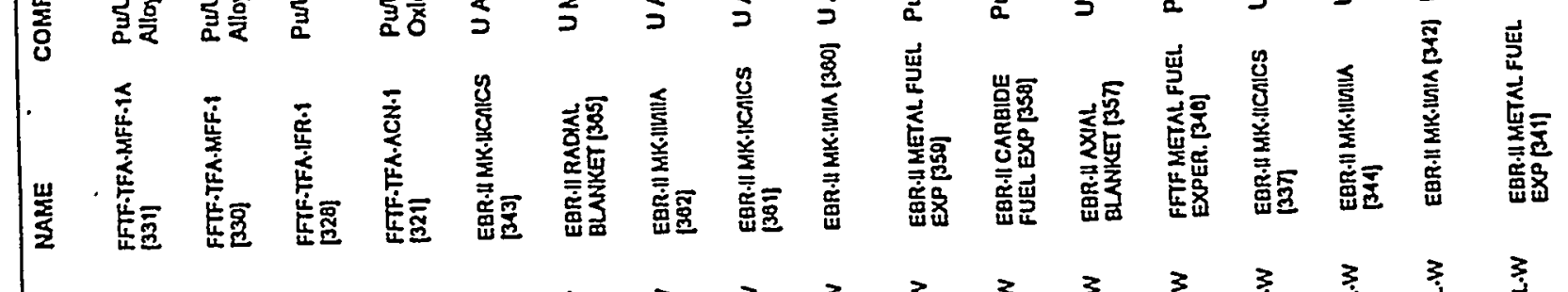
畏

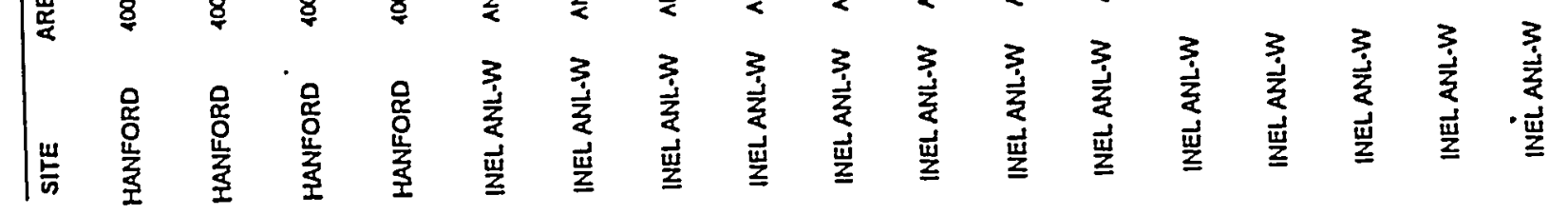




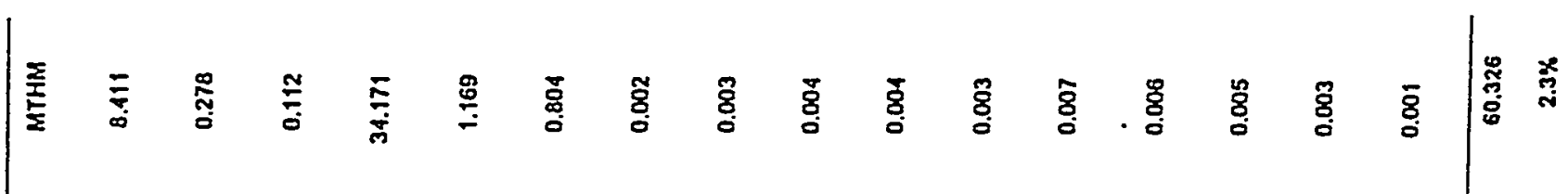

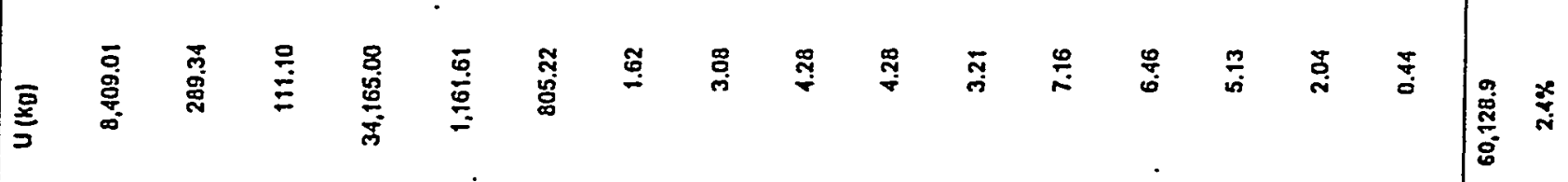

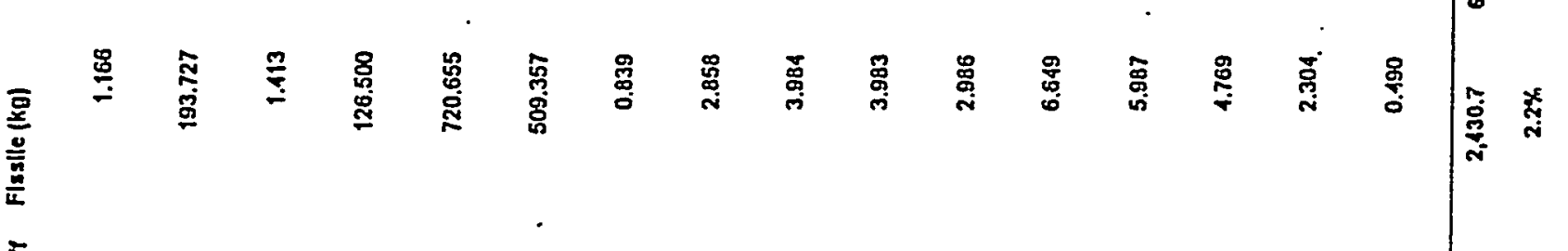

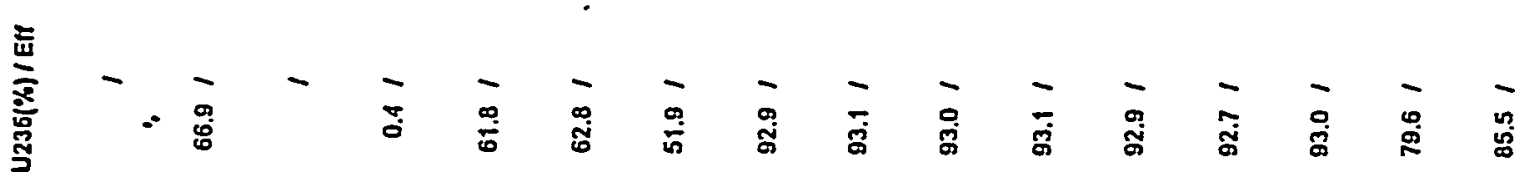

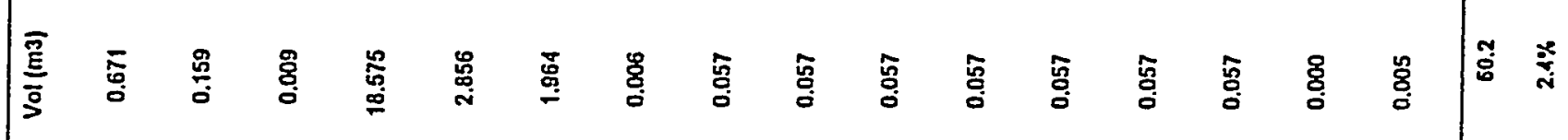

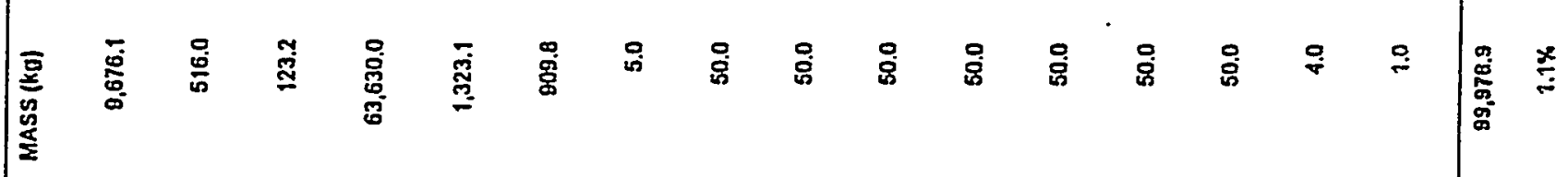

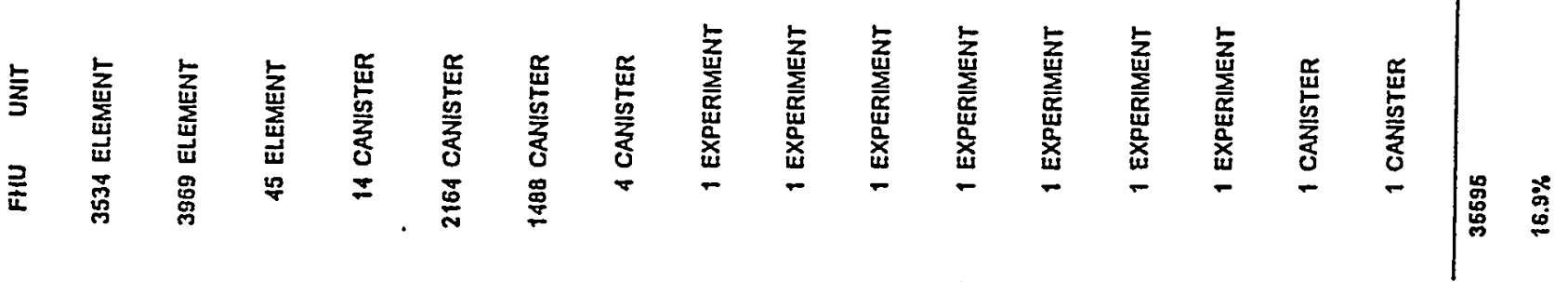

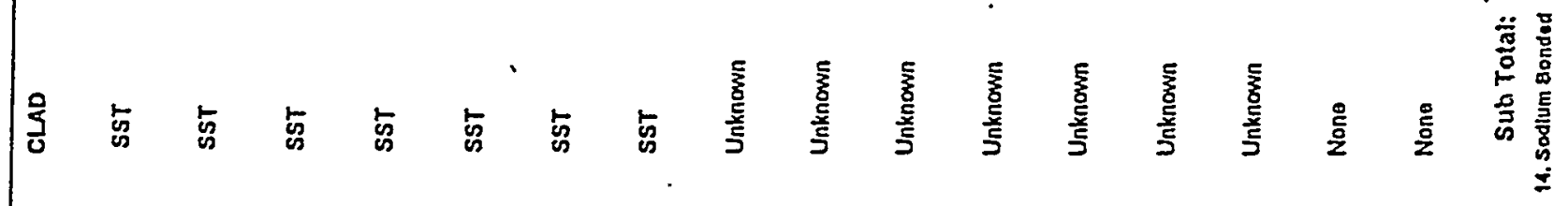

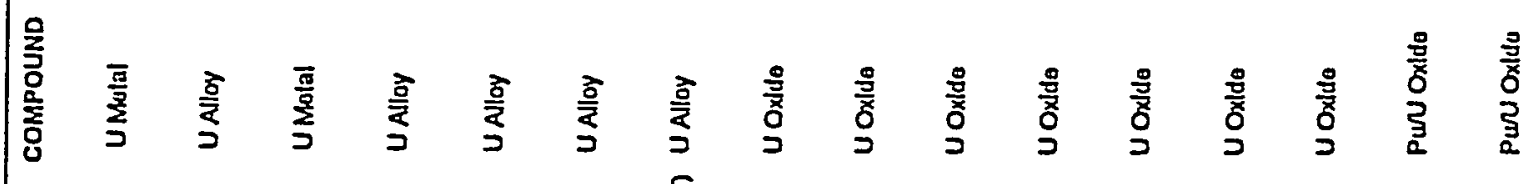

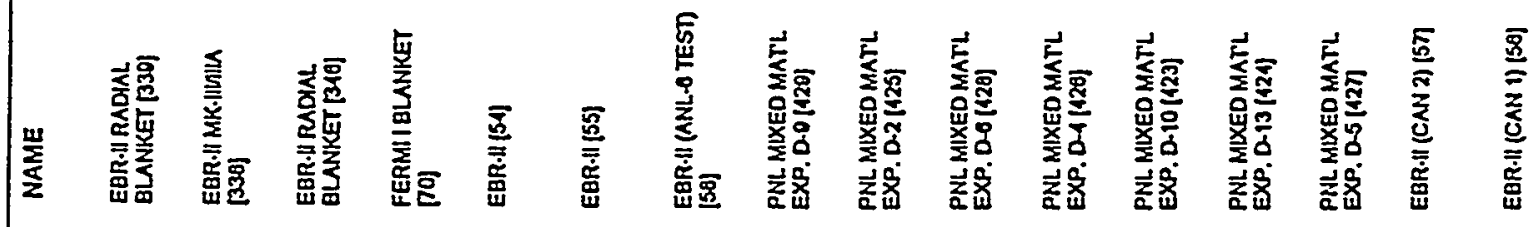

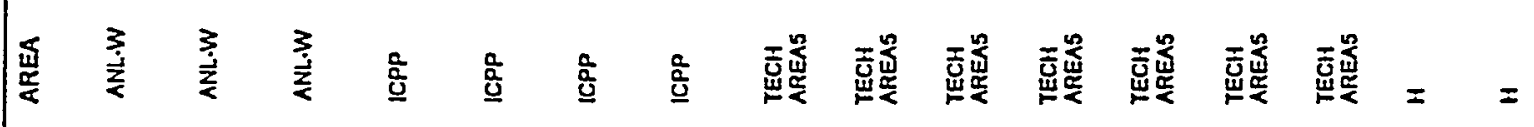

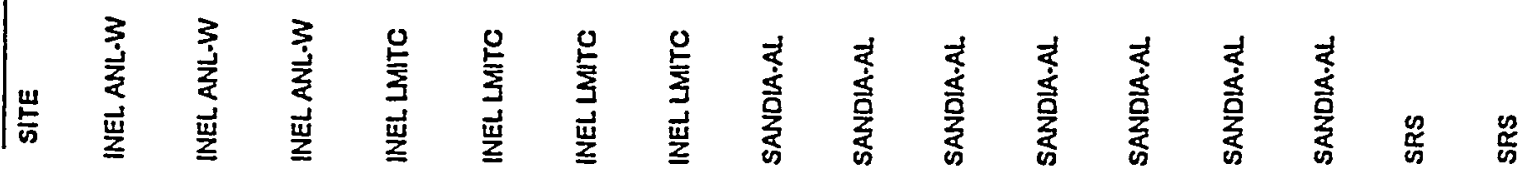




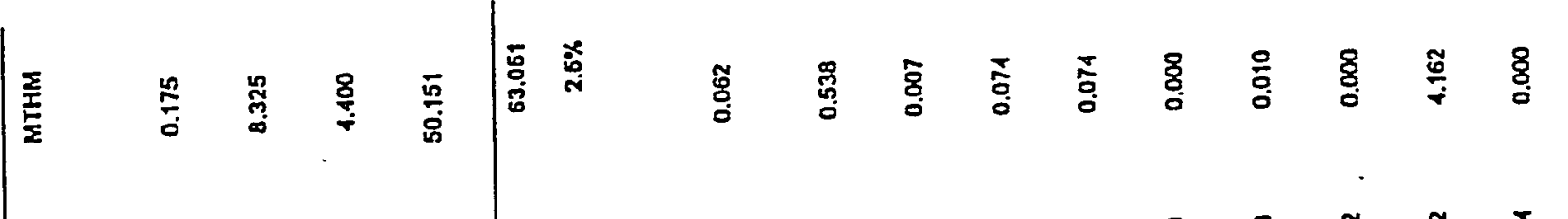

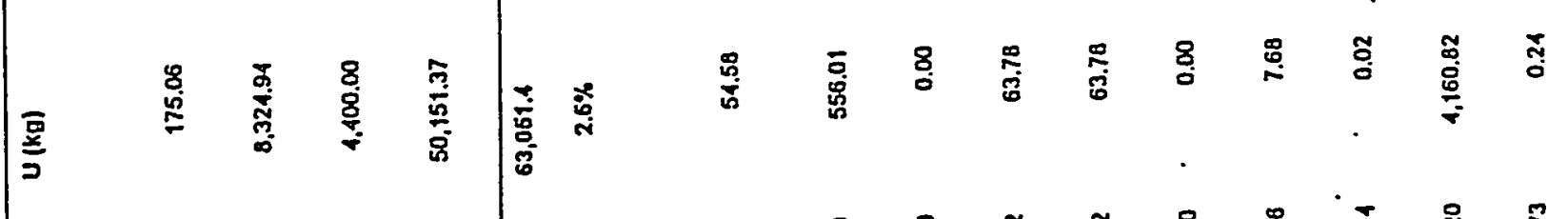

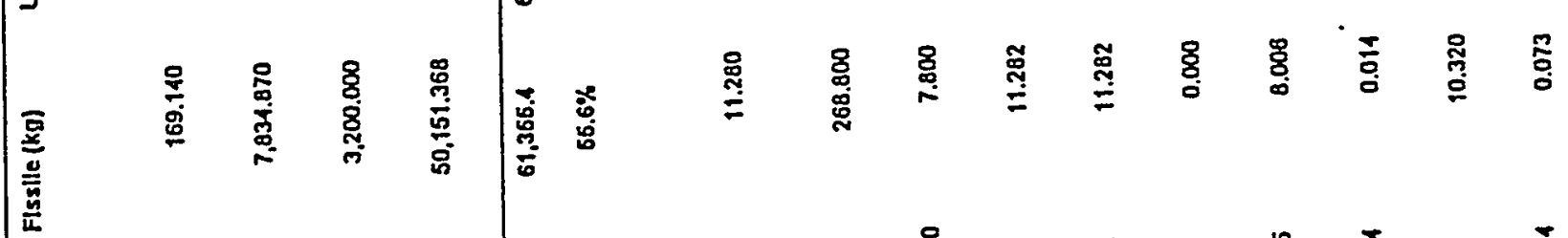

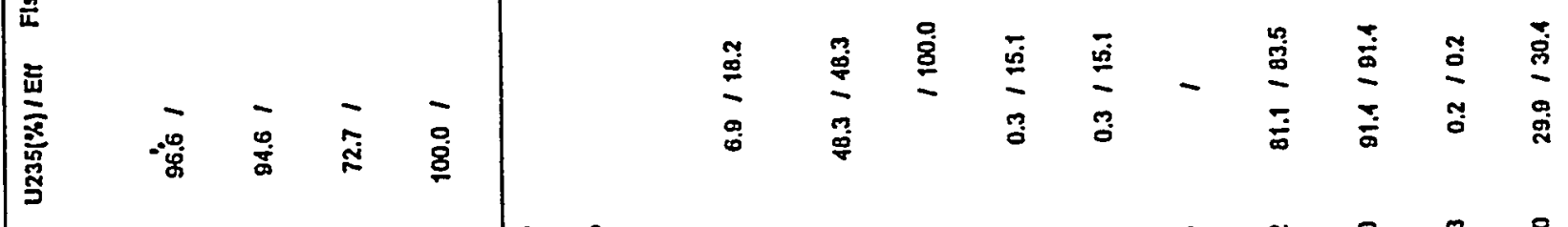

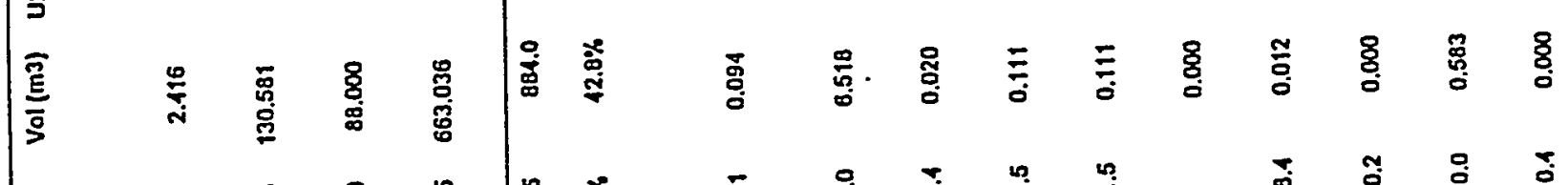

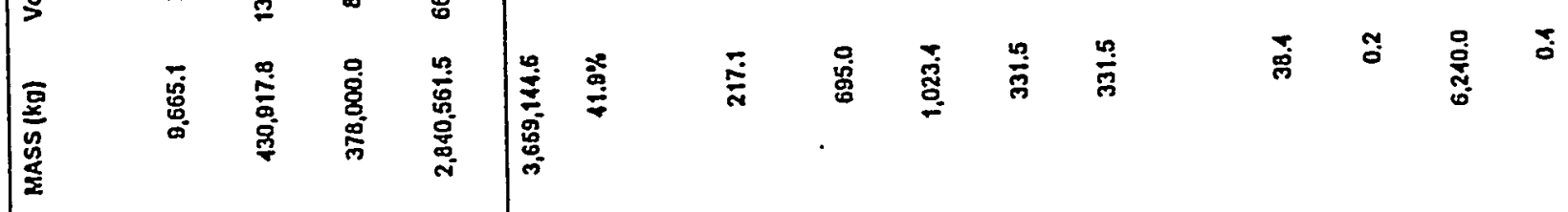

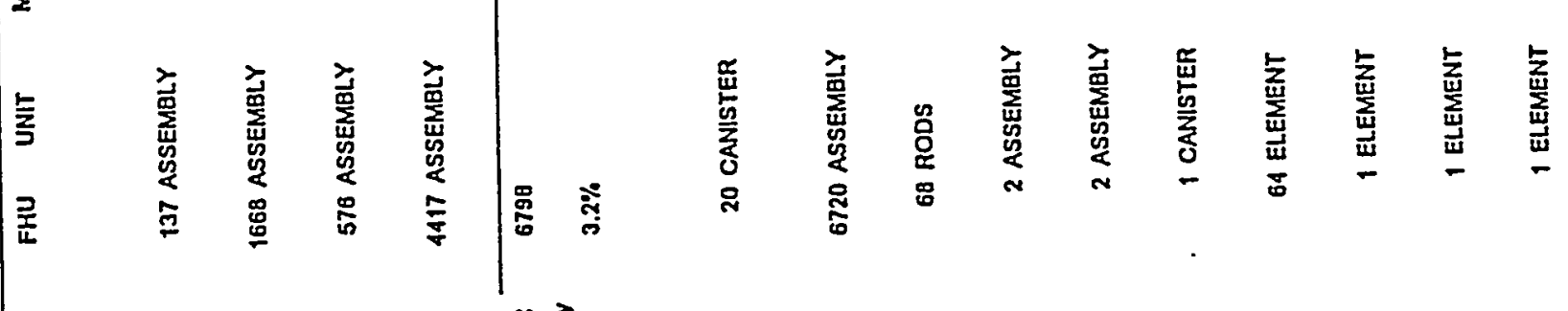

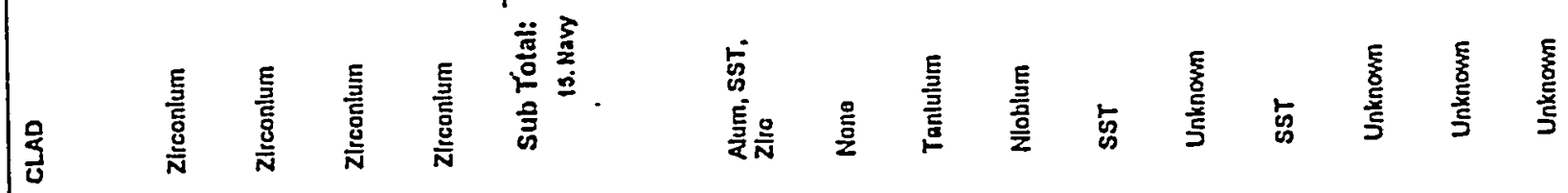

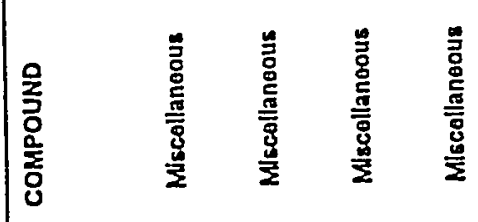

$$
\begin{aligned}
& \text { | }
\end{aligned}
$$

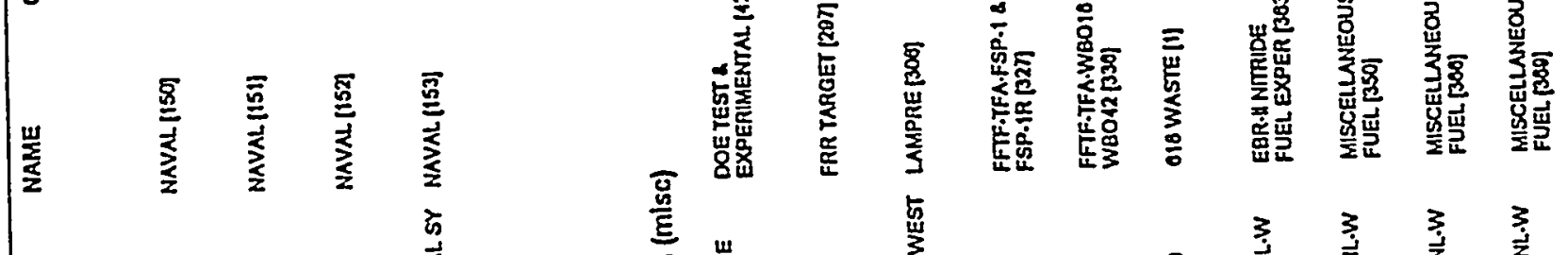

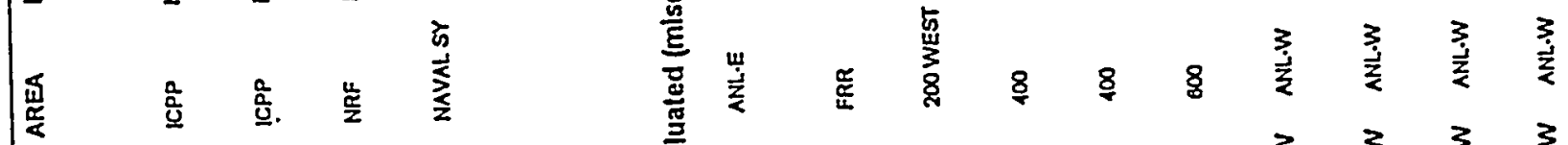

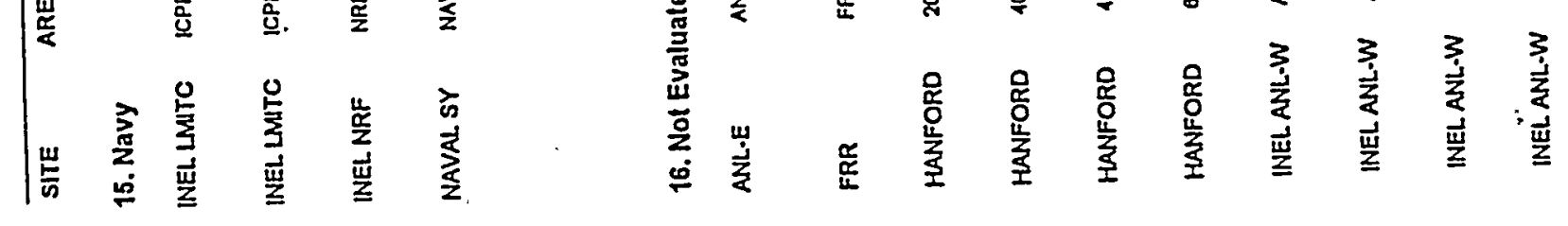




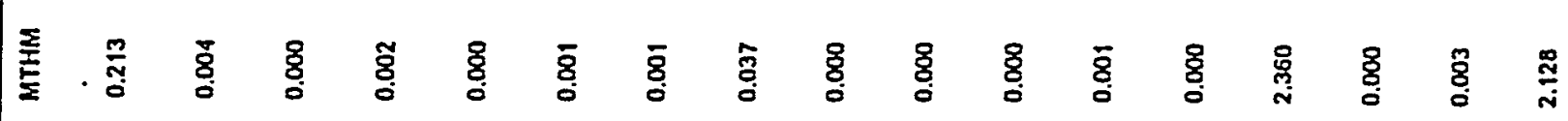

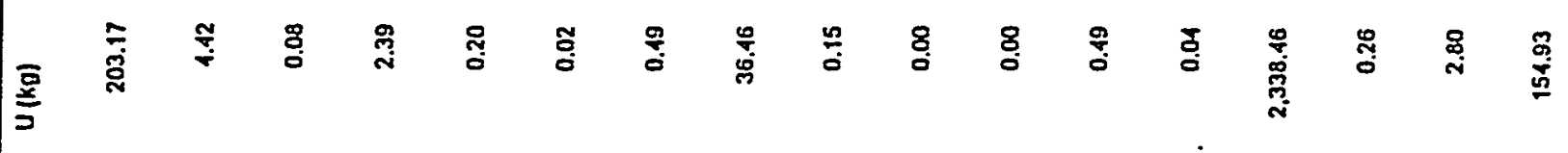

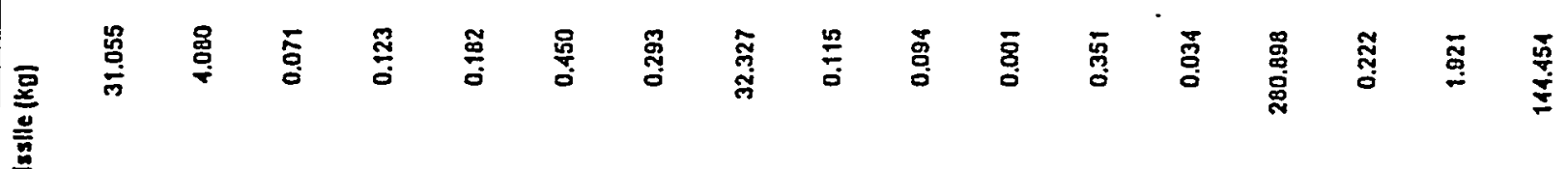

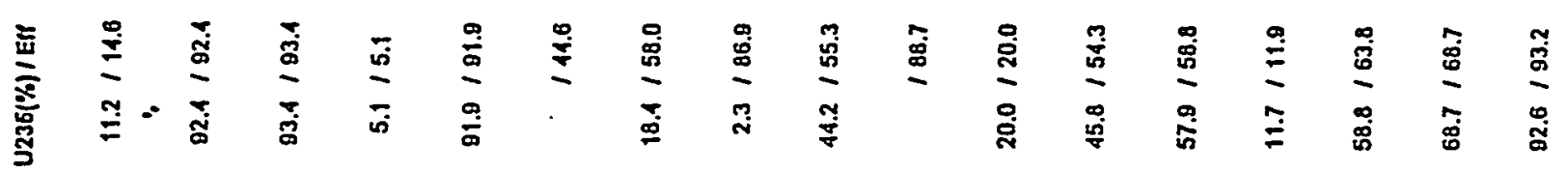

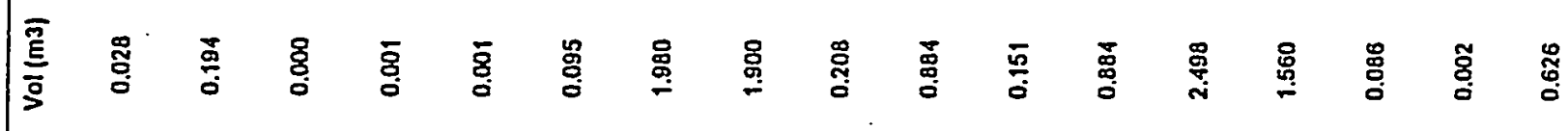

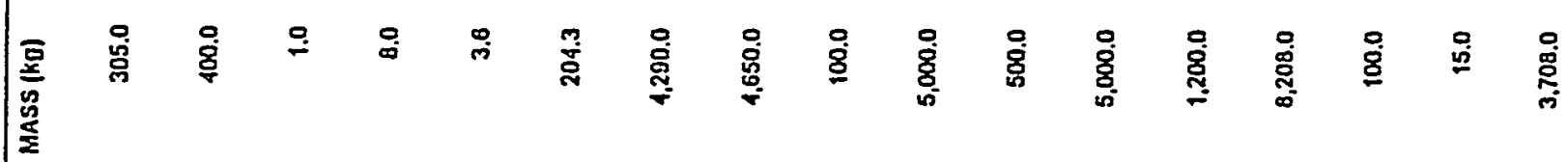

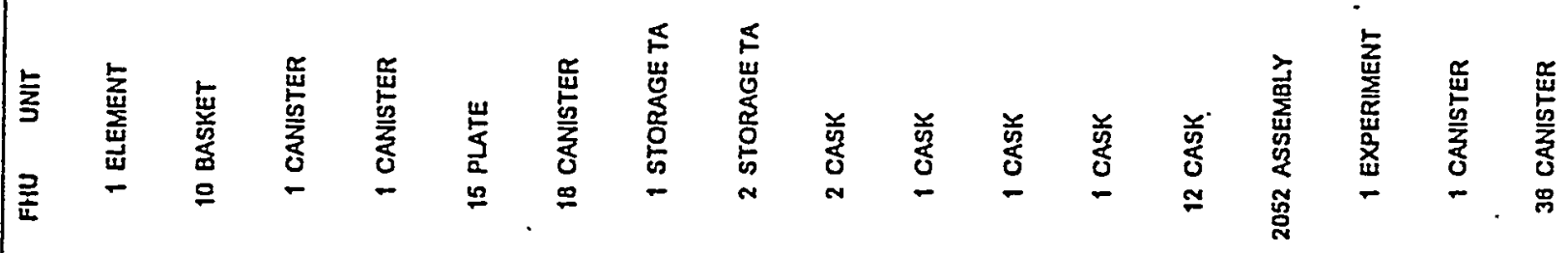

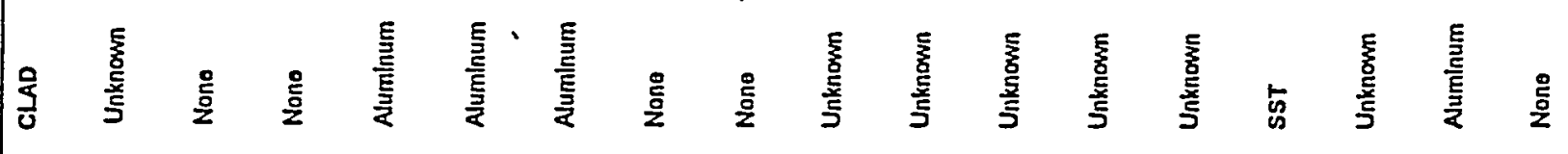

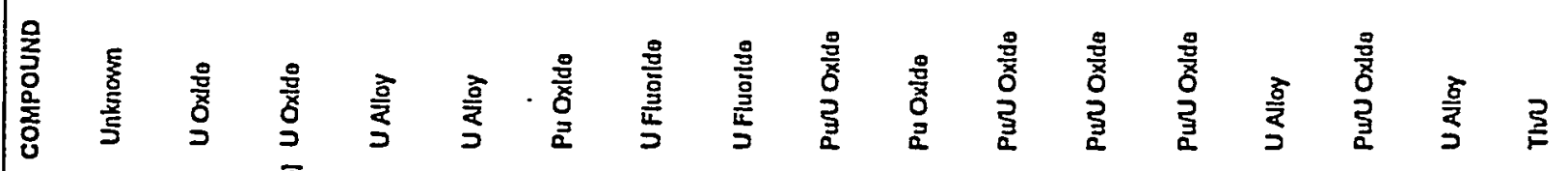

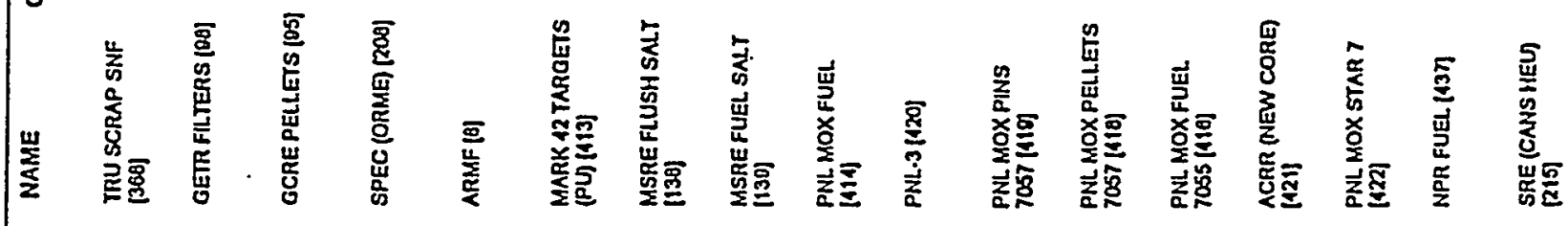

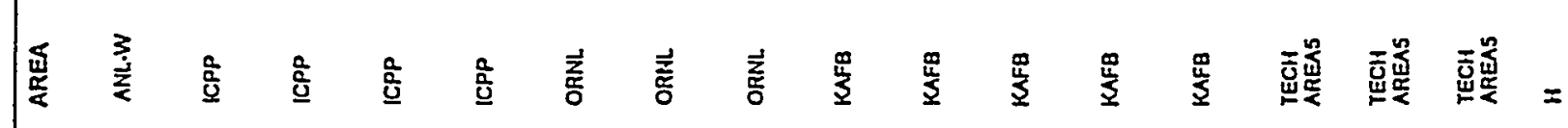

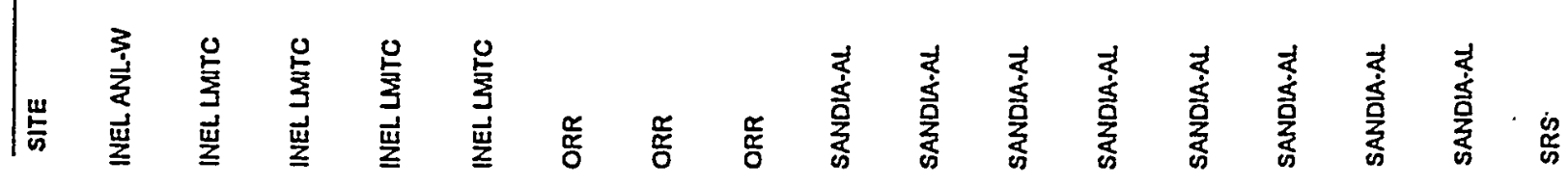




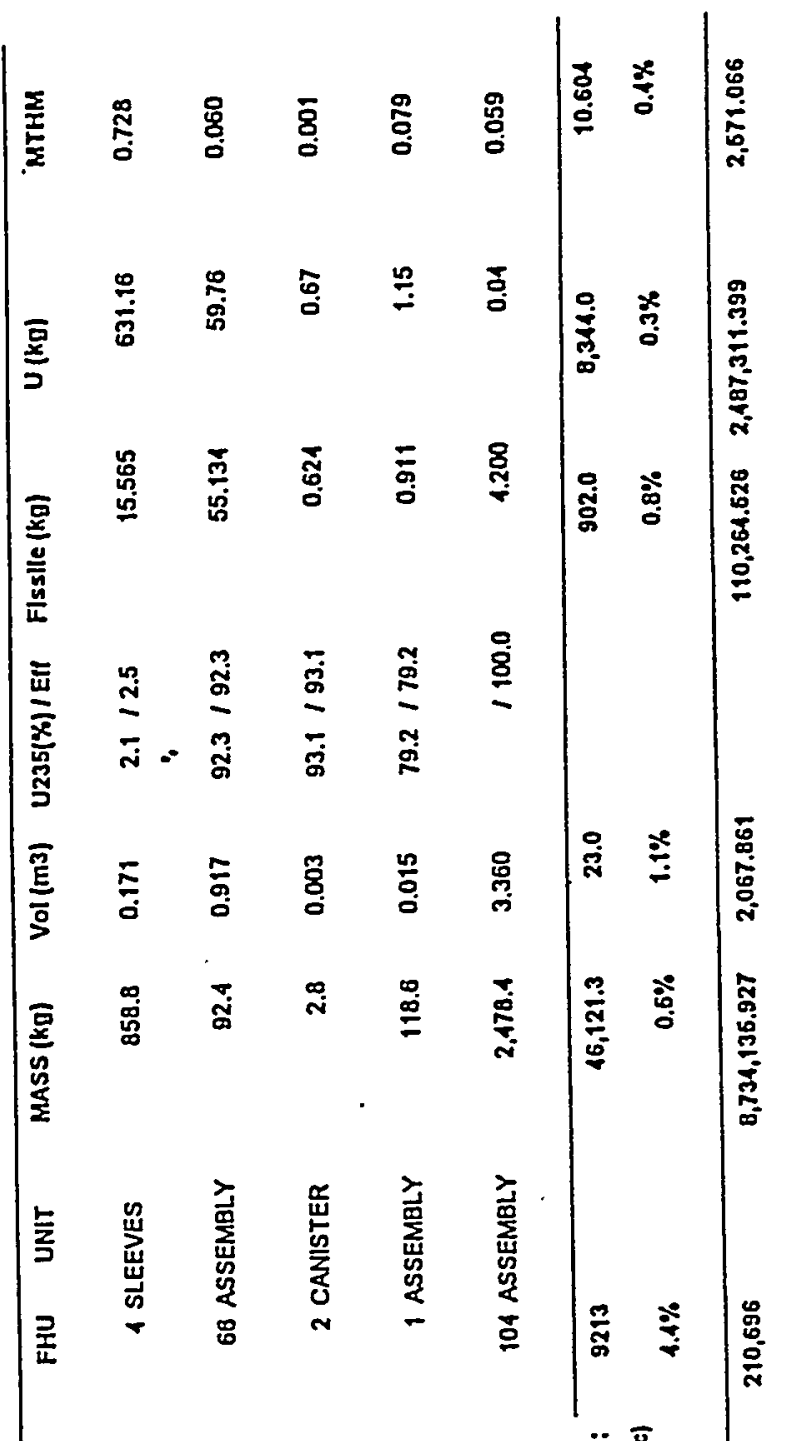

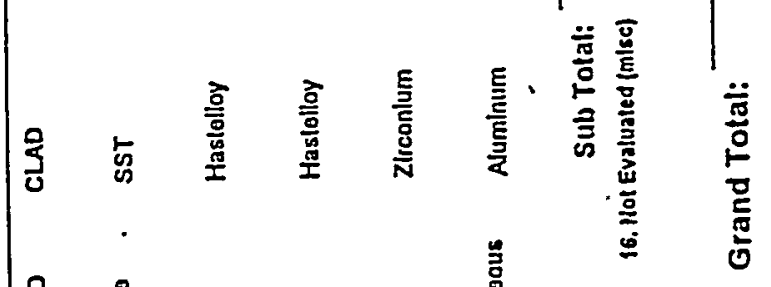

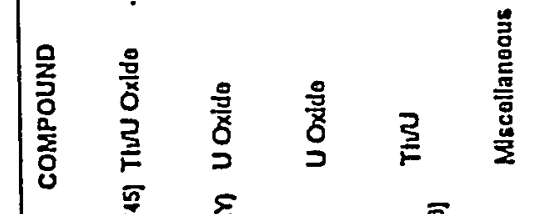

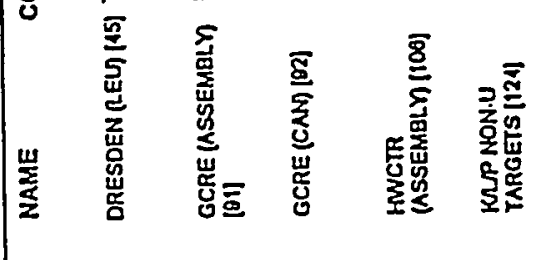

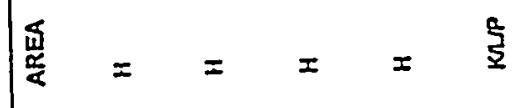

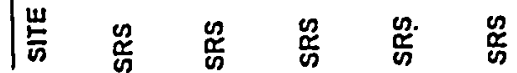




\section{Appendix C: \\ Background on 1997 Performance Assessment}


Appendix C: Background on 1997 Performance Assessment 


\section{Appendix C: Background on 1997 Performance Assessment}

\section{Background on National Spent Nuclear Fuel Program}

The Office of Environmental Management of the U.S. Department of Energy (DOE) (designated herein as DOE/ EM) is responsible for the safe disposal of the DOE-owned spent nuclear fuel (SNF) as part of the National Spent Nuclear Fuel Program (NSNFP). The NSNFP planning activity originated in 1994 when DOE directed EM-67 to "[s]afely, and efficiently manage DOE-owned SNF and SNF returned to the U.S. from foreign research reactors" and prepare it for disposal. In December 1994, EM-67 issued a "DOE-Owned Spent Nuclear Fuel Strategic Plan" reflecting the DOE mission, strategies and objectives.

Two documents, which delineate the responsibility of the NSNFP, are considered the high level requirements for this scope of work. The first is the text of Court Order (Settlement Agreement), Civil No. 91-0054-S-EJL, October 17, 1995, Section F, Paragraph 1, which stated: "The DOE shall direct the research, development and testing of treatment, shipment, and disposal technologies for all DOE spent fuel, and all such DOE activities shall be coordinated and integrated under the direction of the Manager, DOE-ID [DOE-Idaho] Operations Office." This agreement was followed by a memorandum from EM-37 Thomas P. Grumbly to the Manager of the DOE-DD Operations Office on October 26, 1995, which stated: "The INEL [now the Idaho National Engineering and Environmental Laboratory] is hereby designated as the lead site laboratory for ... coordination and integration of all non-commercial SNF activities for the Department." In response to these responsibilities, DOE-ID and its contractor, LMITCO (Lockheed Martin Idaho Technologies Company), which operates INEEL, have pursued studies on the acceptability of disposal of DOE SNF.

The overall purpose of the 1997 performance assessment (1997 PA) is to provide information and guidance to the DOE/EM with regard to the level of characterization necessary to dispose of DOE SNF in the potential Yucca Mountain repository. Although intended primarily for disposal of commercial SNF, a portion of the potential repository is reserved for DOE SNF. The Office of Civilian Radioactive Waste Management (OCRWM) of the DOE is ultimately responsible for setting repository acceptance requirements for the potential repository. The value of this study, then, is that not only can its results be used by the DOE/EM to gauge the kind of packaging or other options required in order to comply with the OCRWM's repository acceptance requirements, but the data can also be used by the DOE to develop performance-based requirements for acceptance of spent fuel.

An example can help to clarify this purpose. In comparison to commercial SNF, the percentage of DOE SNF is small: $4 \%$ of total metric tons of heavy metal (MTHM) in the potential repository, of which one fuel type, N-Reactor fuel, represents about $80 \%$. Hence, the possibility that DOE SNF characteristics will dominate the overall performance of the repository is minute. Nevertheless, at least conceptually, a small probability exists that the unique characteristics of DOE SNF could exert an unwanted influence. Without a detailed analysis to refute this possibility, a natural action would be for the repository operator, OCRWM, to establish repository acceptance criteria in the form of limits on the DOE SNF that cannot be exceeded. Once limits were established, DOE/EM would be required to demonstrate that DOE SNF meets them by taking actual measurements or establishing upper bounds through analysis of the original fuel and its current estimated condition. Particularly because many of the over 250 types of DOE SNF originate from experimental arrangements, which result in varied and nonstandard conditions compared to data on commercial SNF, this process could be time consuming and expensive. Thus, the DOE has selected a more prudent course of action by using analysis to establish performance-based acceptance criteria, a time-saving and significantly less expensive solution.

Defining performance-based repository acceptance criteria requires an accurate representation of the behavior of DOE SNF after disposal, which can demand a more detailed analysis than establishing overall repository performance, because the latter is dominated by relatively standard commercial SNF. For example, the pyrophoric nature of $\mathrm{N}$-Reactor spent fuel, because of its metallic uranium, might be expected to adversely affect conditions in the reposi- 
tory. However, $\mathrm{O}_{2}$ must be present for the pyrophyric fuel to pose a danger. Sandia has proposed that as corrosion progresses on the massive amounts of steel in the handling and disposal containers, the amount of $\mathrm{O}_{2}$ in the repository will deplete; thus, the repository environment may greatly diminish the need for concern about the metallic uranium. Consequently, this type of fuel might be accepted without restrictions, which would also eliminate the need to reprocess or otherwise condition the fuel, at additional expense. In addition, the OCRWM may benefit from this detailed analysis $\left(\mathrm{O}_{2}\right.$ transport is not currently considered in overall repository performance) because corrosion results of the analysis may be used with regard to the life of handling and disposal containers and so can aid OCRWM in decisions about further modeling refinements for licensing of the repository.

Toward this purpose, the current performance assessment (1997 PA) evaluates whether DOE SNF performs noticeably better (or worse) than commercial SNF, and identifies important parameters that influence this performance after disposal. The latter results, in particular, are intended to help define appropriate requirements for characterization with regard to DOE SNF being accepted for storage and disposal. The 1997 PA assembled data and then evaluated performance after disposal of 13 separate DOE SNF categories in containers that also include defense highlevel waste (DHLW) (i.e., the codisposal option) and 2 commercial SNF categories. A major focus of the current study is to improve the understanding of performance of spent fuel in an unsaturated tuff repository by including the most current description of the potential Yucca Mountain disposal system.

\section{Related Documents}

This performance assessment is the third phase of a study that was begun in 1993. The 1993 PA evaluated waste treatment options, ranging from no conditioning to codisposal to full reprocessing, by studying the performance of five potential waste forms. For the 1993 PA, 12,000 MTHM of waste were emplaced in two hypothetical repositories, one sited in granite (saturated) and the other in salt. In the 1994 PA, Sandia assessed one treatment option (direct disposal) for five types of spent nuclear fuel and three treatment options for calcined high-level waste in a 12,000MTHM hypothetical repository in unsaturated tuff. The performance assessments were documented in the following reports:

Rechard, R.P., ed. 1993. Initial Performance Assessment of the Disposal of Spent Nuclear Fuel and High-Level Waste Stored at Idaho National Engineering Laboratory, Vols. I and 2. SAND93-2330. Albuquerque, NM: Sandia National Laboratories

Rechard, R.P., ed. 1995. Performance Assessment of the Direct Disposal in Unsaturated Tuff of Spent Nuclear Fuel and High-Level Waste Owned by U.S. Department of Energy, Vols. 1, 2, and 3. SAND94-2563. Albuquerque, NM: Sandia National Laboratories

The major source of data for site characterization of the tuff repository was drawn from Sandia's performance assessment ${ }^{*}$ of the Yucca Mountain Project:

Wilson et al. 1994. Total System Performance Assessment for Yucca Mountain-SNL Second Iteration (TSPA1993). SAND93-2675. Albuquerque, NM: Sandia National Laboratories.

Some parameters for the 1997 PA are based on the results from an abbreviated performance assessment performed for the Yucca Mountain Project in 1996 (a-TSPA-1996):

M\&O (Civilian Radioactive Waste Management System, Management and Operating Contractor [CRWMS M\&O]). 1996, in preparation (August 15, 1996, preliminary draft). "Description of Performance Allocation." B00000000-01717-2200-00177. Rev. 00. Las Vegas, NV: U.S. Department of Energy, Yucca Mountain Site Characterization Project.

\footnotetext{
* The Performance Assessment Departments at Sandia for the Yucca Mountain Project (YMP) and the Waste Isolation Pilot Plant (WIPP) Project are both within the Nuclear Waste Management Center. Their style of calculations differ because of the stage of the projects, degree of scientific understanding of unsaturated flow, and specific requests from their respective DOE sponsors.
} 
All three of Sandia's DOE SNF/DHLW studies (1993, 1994, and 1997 PAs) used the performance assessment methodology originally developed for the study of the Waste Isolation Pilot Plant (WIPP), near Carlsbad, NM. Thus, a useful companion document that reviews the mechanics of the performance assessment process is

Rechard, R.P. 1995. An Introduction to the Mechanics of Performance Assessment Using Examples of Calculations Done for the Waste Isolation Pilot Plant Between 1990 and 1992. SAND93-1378. Albuquerque, NM: Sandia National Laboratories.

\section{Analysis Rigor}

Through joint agreement between the DOE Quality Assurance (QA) Program Manager, the INEEL NSNFP QA Program Manager, and the Sandia QA Manager, the 1997 PA is "scoping" in nature. In general, most initial performance assessments are carried out at this level because information about the disposal system under study is not completely known; analyst judgment must supplement the available information. Sandia was careful to maintain good standard scientific protocol in the three areas affecting the results: data, software, and analysis. Furthermore, rudimentary parts of the rigorous Sandia QA Program for evaluating the compliance of the WIPP by the U.S. Environmental Protection Agency (EPA) were used.

Data. Over 3,000 model parameters used in the $1997 \mathrm{PA}$ were collected in an Ingres ${ }^{\mathrm{TM}}$ relational database to ensure their traceability. Strict control of read and write access and audit trails ensure the security, integrity and traceability into and out of the database. Data were entered into the database by the Database Administrator (DBA) or designee after a written or electronic request was submitted to the DBA from the Parameter Task Leader. These requests are on file in the Nuclear Waste Management Project's Records Center for permanent archival. The model parameters have been tabulated in this report. In addition, to facilitate understanding, the report contains a discussion of the values and distribution types for important parameters (see Chapter 6 of this report).

Software. For the two-phase flow code, BRAGFLO_T, a draft user's manual was prepared that described the input, nature, and purpose of the code; the manual was filed with the NWMP Records Center. Also, some verification tests were performed. The remaining codes, some of which were modified for this analysis, had adequate user documentation consistent with the intended use of the software because either the same version or a slightly earlier version of these codes had been used in the WIPP compliance assessment. Exceptions are BRAGFLO_T, STAFF3D, CONRAD, SUM3D, and CCDFCALC (the latter code was a parent of the code used in the WIPP compliance assessment). All software codes were entered into the Software Configuration Management System (SCMS) to ensure repeatability.

Analysis. To ensure the robustness of the analysis, the simulations were run by an SCMS staff member (i.e., had no stake in the results), using only software from the SCMS, data from the database, and input files supplied by the PA analysts. This report serves as documentation of the analysis and has been reviewed by two personnel who are not working on this project directly.

QA Coordinator. A QA Coordinator was assigned by the QA Manager in Sandia's Nuclear Waste Management Center. The QA Coordinator verified that all personnel on the project were up-to-date on current training required for similar roles and responsibilities as defined for the WIPP Project.

These QA efforts have attempted to ensure that the 1997 PA represents a reasonable undertaking that includes, implements, and evaluates important phenomena, pertinent data, and the general state of knowledge regarding relevant issues. 
Appendix C: Background on 1997 Performance Assessment

\section{Description of Participants}

The project management and coordination structure is illustrated in the DOE/EM and INEEL organizational chart (Figure C-1) and the Sandia organizational chart (Figure C-2). The 1997 PA was coordinated from within Sandia's WIPP Performance Assessment (WIPP-PA) Department (6849). The technical components of the project were directed by Rob P. Rechard (Sandia) through coordination with Larry Taylor (INEEL). F.W. Bingham (Sandia) provided overall management for the project. The activity and subtask leaders offered technical guidance during the modeling development phases of the performance assessment, with the assistance of other qualified personnel from Sandia and LMITCO.

At $\mathrm{DOE} / \mathrm{D}$, Pete Dirkmaat provided project policy guidance consistent with DOE programmatic goals for the DOE NSNFP and programmatic guidance for any future applications of the 1997 PA results. The DOE NSNFP, represented by Jim Boyd, is the primary customer for the 1997 PA results, which are intended to assist the Program in its effort to attain a cost-effective strategy for the interim storage and ultimate disposal of DOE-owned spent nuclear fuel.

\section{Relationship of 1997 PA with Related Performance Assessments}

The 1997 PA (also referred to as 1997 DOE SNF/DHLW PA) can be compared not only to the 1994 PA, but also to the most recent performance assessments of the YMP (i.e., TSPA-1995 and a-TSPA-1997). The similarities and differences in techniques are summarized below.

Comparison Between 1994 and 1997 DOE SNF/DHLW PAs. The methodology for the 1997 PA is the same as that used in the previous PAs (1993 and 1994), which, in turn, were based upon the methodology originally developed for the performance assessment of the WIPP near Carlsbad, New Mexico. Differences between the performance assessments are shown in Table C-1. The major differences are that a 75,320-MTHM repository is being modeled, including 63,080 MTHM of commercial fuel; 15 spent nuclear fuel categories are modeled; and the source term/corrosion model has been enhanced, with $\mathrm{O}_{2}$ transport added. Also, the $1997 \mathrm{PA}$ expanded or changed the focus of the study in four additional ways. First, the codisposal option was used for the DOE-owned spent fuel and high-level waste. Second, dose calculations with a 100,000-yr performance period were considered. Third, the 1997 PA contains only a complex performance assessment, and does not include a simplified model for comparison as was done in 1994. For the 1997 PA, the complex model can be compared to the YMP Total-System Performance Assessment (TSPA), which uses simplified models (i.e., less interdependent). Finally, the 1997 PA models the actual Yucca Mountain site as the potential repository. The hypothetical repository modeled in the 1994 PA was small, contained only DOE-owned spent fuel and waste, and was located east of the Ghost Dance Fault; the model did not account for the effects from commercial waste.

Comparison Between 1997 DOE SNF/DHLW PA and a-TSPA-1997. The YMP has conducted several TotalSystem Performance Assessments (TSPAs), including TSPA-1991 (Barnard et al., 1991), INTERA's TSPA-1993 (Andrews et al., 1994), Sandia's TSPA-1993 (Wilson et al., 1994); and TSPA-1995 (M\&O, 1995a). As a general goal, most TSPAs were conducted to provide feedback to project participants on the significance of design and sitecharacterization data with regard to regulatory compliance. In addition, an abbreviated TSPA was performed in 1996 (M\&O, 1996; referred to here as a-TSPA-1996) to evaluate the importance of various components of the disposal system and as a parametric sensitivity study. Also, to provide a comparison between simplified and complex PAs, as was done by Sandia National Laboratories in the 1994 DOE SNF/DHLW PA, the NSNFP requested that the YMP perform another abbreviated performance assessment this year (a-TSPA-1997) with their current models, which represents the simplified PA. Table C-2 highlights differences between the 1997 DOE SNF/DHLW PA and a-TSPA-1997.

The primary sources of data for the 1997 PA and a-TSPA-1997 are similar, although for the 1997 PA, the data were taken from Sandia's TSPA-1993, and for a-TSPA-1997, the data were taken from the INTERA TSPA-1993 and TSPA-1995. Furthermore, some choices of parameters for both the 1997 PA and a-TSPA-1997 are based on the results from a-TSPA-1996. In some cases, however, where a difference in data existed, the 1997 PA attempted to use a data source consistent with a-TSPA-1997. For example, the radioisotope inventory for INTERA's TSPA-1993 uses 


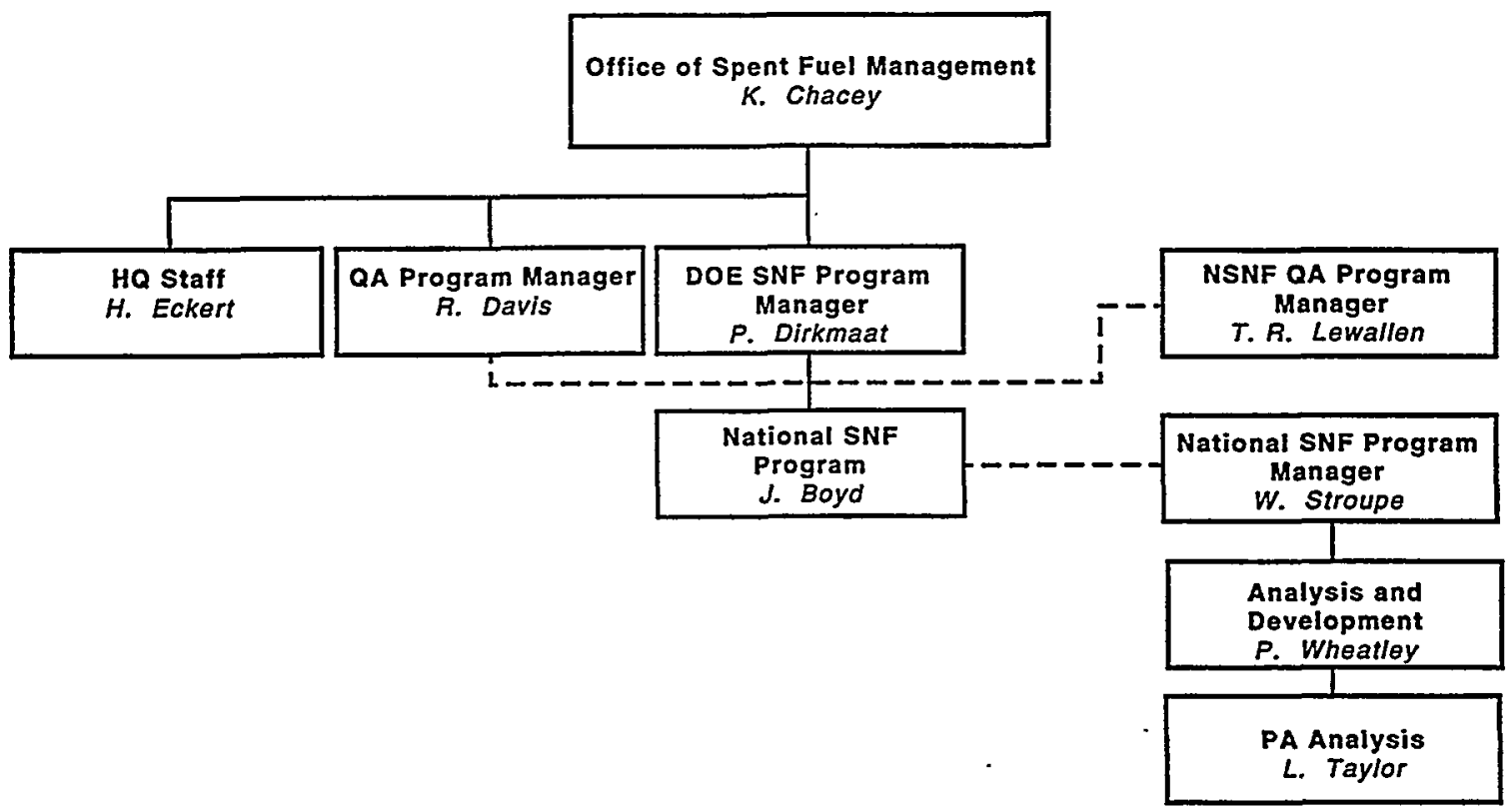

TRI-6342-5294-0

Figure C-1. Project participants from the Office of Environmental Management of the U.S. Department of Energy and Idaho National Engineering and Environmental Laboratory. 
Managers

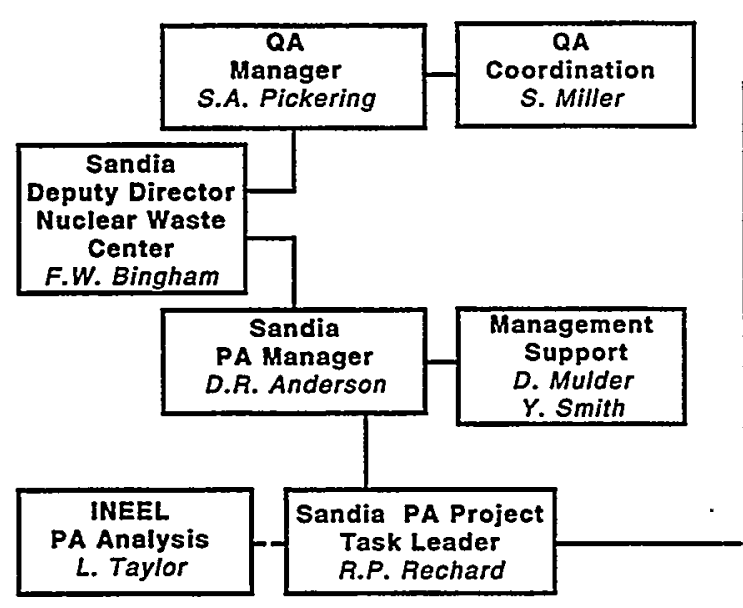
Subtask Leaders
Activities and

\section{Personnel Resources}

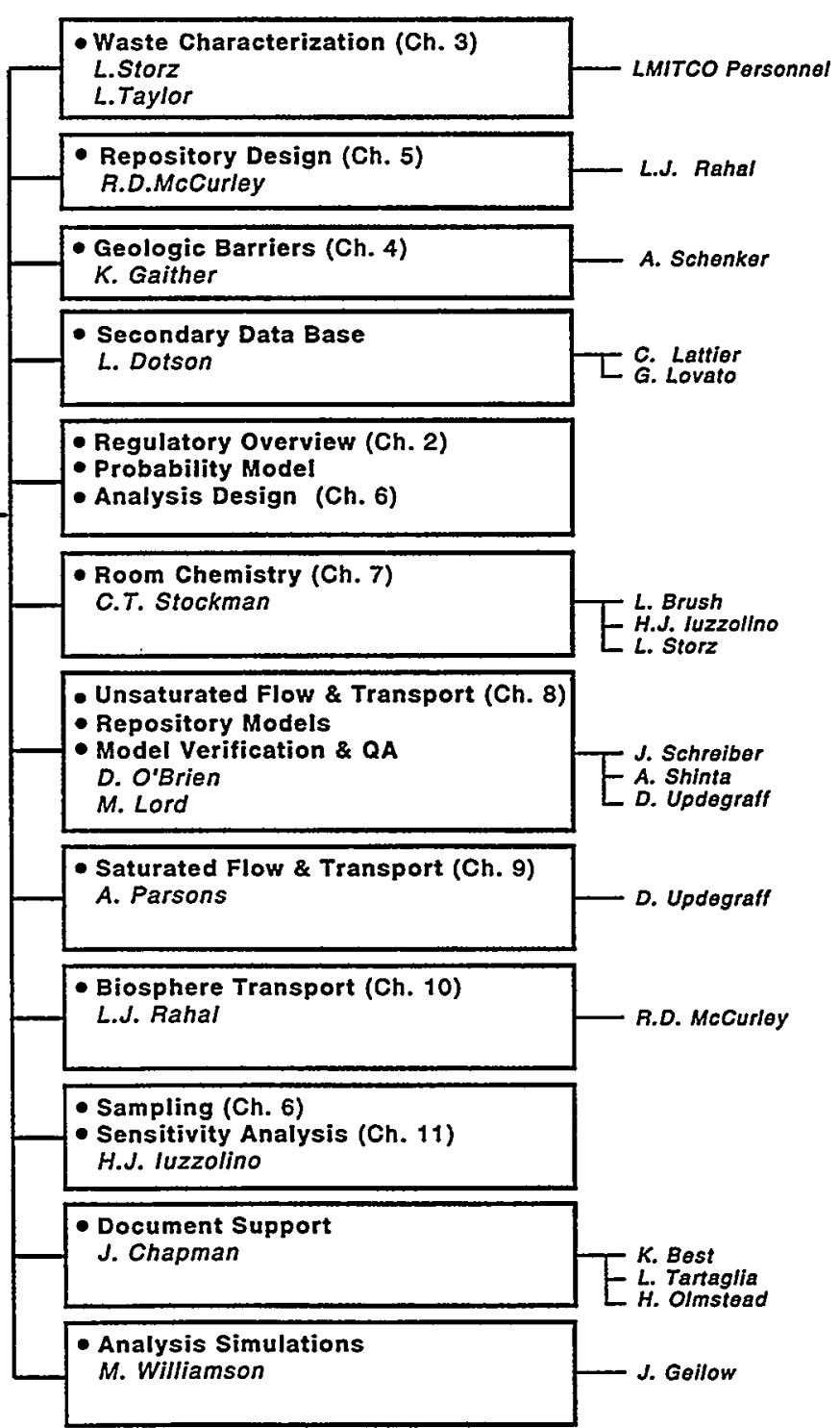

September, 1997

TRI-6342-4222-4

Figure C-2. Organizational chart for 1997 DOE SNF/DHLW performance assessment. 
Table C-1. Differences Between 1994 and 1997 DOE SNF/DHLW Performance Assessments

1994 PA

1997 PA

\section{Geologic Barrier Media}

Modeling

Engineered Barrier
Waste Package
Waste Forms

Waste Types

Waste Inventory

Containers
Tilted repository in tilted, pancake tuff strata

- No faults modeled in unsaturated zone

$\mathrm{H}_{2} \mathrm{O}$ and air transport; oxic corrosion rates
Tilted repository in tilted, pancake tuff strata

- Faults modeled in saturated and unsaturated zones

- Refinement of TS layers using new porosity data

$\mathrm{O}_{2}, \mathrm{~N}_{2}, \mathrm{H}_{2} \mathrm{O}$ transport; oxic and anoxic corrosion rates
Evaluated one waste form for DOE SNF; three forms for DHLW-glass and glass-ceramic for calcine; all waste packaged individually; solubility in silica-saturated water evaluated

INEL spent fuel (graphite, high enriched, low enriched, and Shippingport) and high-level waste (calcine); Hanford spent fuel (N-Reactor) (83\% in N-Reactor category); and HLW (glass)

- DHLW - Four separate radioisotope inventories for INEEL, Hanford, SRS, and WVDP based on 1992 DOE/RW-0184-R1 report

- DOE SNF - Radioisotope inventory of 5 categories from various sources (published and unpublished)

- Waste Cladding - Estimate made of distribution of failed cladding for each waste category

Three containers designed to meet

10 CFR 60 criteria:

a. 125-ton MPC for horizontal emplacement

1. $24.5-\mathrm{mm}$ stainless steel handling container

2. Optional $20-\mathrm{mm}$ Incoloy 825 overpack

3. 100-mm carbon steel overpack

b. LWT for vertical emplacement 9.5-mm stainless steel handling container Same thicknesses of overpacks as MPC

c. $9.5-\mathrm{mm}$ thick stainless steel handling container, overpacked for calcine and HLW with same thickness as MPC.
Evaluated disposal of DOE SNF with DHLW (codisposal option)

13 categories of DOE-owned spent fuel ( $88 \%$ in $\mathrm{N}$-Reactor, Category 1), 2 categories of commercial fuel, and DHLW (glass)

- DHLW - Used inventory from 1992 DOE/RW-0184 report.

- DOE SNF - Radioisotope inventory for 13 categories from ORIGEN2 runs made by INEEL

- Waste Cladding - For consistency with a-TSPA-1997, all cladding assumed failed

Disposal container: Large disposal package (LDP), 10-cm-thick outer carbon steel layer and 2-cm-thick inner Inconel 625 layer. (Carbon steel liner in LDP not included because information not available from INEEL.)

Handling containers:

1. Defense spent fuel, $6.35-\mathrm{mm}$ thinshelled stainless steel

2. N-Reactor fuel, 9.5-mm-thick stainless steel multi-canister overpack (MCO).

3. High-level waste,9.5-mm-thick stainless steel standard canister 
Appendix C: Background on 1997 Performance Assessment

Table C-1. Differences Between 1994 and 1997 DOE SNF/DHLW Performance Assessments (Continued)

\begin{tabular}{|c|c|c|}
\hline & 1994 PA & 1997 PA \\
\hline $\begin{array}{l}\text { Repository } \\
\text { Size }\end{array}$ & $\begin{array}{l}\text { 12,060 MTHM; MTHM of HLW deter- } \\
\text { mined by } 40 \text { CFR 191, Appendix A, } \\
\text { note 1(d) }\end{array}$ & $\begin{array}{l}75,336 \text { MTHM; MTHM of DHLW deter- } \\
\text { mined by using note } 1 \text { (d) of } \\
\text { Appendix A of } 40 \text { CFR } 191 .\end{array}$ \\
\hline $\begin{array}{l}\text { Waste Emplacement } \\
\text { Orientation }\end{array}$ & $\begin{array}{l}\text { Vertical and horizontal; distributed } \\
\text { waste packages uniformly }\end{array}$ & $\begin{array}{l}\text { Horizontal; distributed waste packages } \\
\text { uniformly }\end{array}$ \\
\hline Layout & $\begin{array}{l}\text { Hypothetical design using tunnel- } \\
\text { boring machine; } \\
\text { Horizontal - 4.3-m dia. drifts } \\
\text { Vertical - } 7.65-m \text {-dia. drifts }\end{array}$ & $\begin{array}{l}\text { YMP current design } \\
\text { 5.3-m. dia. tunnels }\end{array}$ \\
\hline \multicolumn{3}{|c|}{$\begin{array}{l}\text { Regulatory Criteria (based on } \\
\text { regulations that are currently } \\
\text { being revised) }\end{array}$} \\
\hline Total System & $\begin{array}{l}40 \text { CFR } 191 \text { 10,000-yr Containment } \\
\text { Requirements }\end{array}$ & $\begin{array}{l}\text { NAS } 1995 \text { guidance; } 40 \text { CFR } 191 \text { indi- } \\
\text { vidual dose limits; proposed NWPA } \\
\text { amendments. }\end{array}$ \\
\hline & $\begin{array}{l}\text { 10,000-yr Individual Protection } \\
\text { Requirements; dose calculated from } \\
\text { several pathways (well with sludge } \\
\text { pond, farm site, freshwater lake) }\end{array}$ & $\begin{array}{l}10,000-y r \text { Individual Protection } \\
\text { Requirements (over } 100,000-y r \\
\text { period); dose calculation for ranchers, } \\
\text { subsistence farmer, and small commu- } \\
\text { nity pathways; also dose calculations } \\
\text { with stirred tank model and homoge- } \\
\text { nous infinite aquifer model with } \\
\text { steady-state transport for comparison } \\
\text { to a-TSPA-1997. }\end{array}$ \\
\hline Components & $\begin{array}{l}10 \text { CFR } 60 \text { waste package and engi- } \\
\text { neered barrier requirements }\end{array}$ & None \\
\hline Regulatory Period & $10,000 \mathrm{yr}$ & $100,000 \mathrm{yr}$ \\
\hline
\end{tabular}

an older inventory (DOE, 1987-1988), while the Sandia TSPA-1993 and 1994 PA used a 1992 revision (DOE, 1992). The 1997 PA chose to use the older inventory (DOE, 1987-1988) for consistency with a-TSPA-1997. Note, however, that although both PAs begin with similar data, the modeling methods of a-TSPA-1997 and the 1997 PA are different, and therefore so is their use of data.

The models for a-TSPA-1997 are based primarily on INTERA's TSPA-1993 and TSPA-1995, with some modifcations made to models and repository design in anticipation of a 1998 performance assessment mandated by Congress, called the Viability Assessment, or TSPA-VA. The basic approach to code development within the YMP, however, has remained the same, i.e., developing simplified models (e.g., algebraic equations, statistical empirical equations, or lookup tables) based on a few simulations in which complex codes modeled various phenomena individually. Thus, a-TSPA-1997 assumes that many phenomena can be decoupled and expressed simply.

In contrast, the 1997 PA assumes that phenomena, especially in the unsaturated zone, cannot be decoupled (e.g., close coupling of thermal effects with the availability of water and oxygen necessary for corrosion) to evaluate penetration rates of containers. Both the simplified and complex methodologies were used in the 1994 PA, and both tech- 
niques were useful in different ways. The key to which technique is more appropriate depends on the purpose of the analysis.

Differences between the 1997 PA and a-TSPA-1997 are listed in Table C-2. Major differences are in the assumptions with regard to the source term and its coupling with information from the unsaturated zone models. For example, the 1997 PA evaluates the saturation in fractures around the container to determine corrosion rather than using a sampled value from a distribution based on calculated percolation rates from a separate analysis. However, the precise details of analysis differences are difficult, and perhaps inappropriate, to convey in a summary table. If necessary, a future report can elaborate upon this topic. 
Appendix C: Background on 1997 Performance Assessment

Table C-2. Comparison Between a-TSPA-1997 and 1997 DOE SNF/DHLW PA

\begin{tabular}{|c|c|c|}
\hline \multicolumn{2}{|c|}{$\begin{array}{c}\text { a-TSPA-1997 } \\
\text { (Simple PA) }\end{array}$} & $\begin{array}{l}1997 \text { DOE SNF/DHLW PA } \\
\text { (Complex PA) }\end{array}$ \\
\hline \multicolumn{3}{|c|}{$\begin{array}{c}\text { Engineered Barrier } \\
\text { Waste Package } \\
\text { Waste Form }\end{array}$} \\
\hline & $\begin{array}{l}\text { Source term } \\
\text { - Containers for DOE SNF assumed to fail } \\
\text { between } 600 \text { and } 10,000 \mathrm{yr} \text { (conservative); } \\
\text { containers for commercial spent fuel fail } \\
\text { over long period ( } 85 \% \text { by } 10^{6} \mathrm{yr} \text { ) } \\
\text { - Outer barrier corrosion models: humid-air } \\
\text { general and pitting, aqueous general and } \\
\text { pitting } \\
\text { - Corrosion of outer barrier controlled by rela- } \\
\text { tive humidity limit (uniform distribution) } \\
\text { humid-air corrosion below } 75 \% \text {; aqueous } \\
\text { corrosion above } 75 \% \\
\text { - Inner barrier corrosion model: assumes } \\
\text { aqueous pitting (aqueous conditions } \\
\text { assumed when pits reach inner barrier) } \\
\text { - Assumed pitting history of number of pits as } \\
\text { a function of time, giving area on waste } \\
\text { package that is available for transport of } \\
\text { mobilized radionuclides } \\
\text { - Cathodic protection of inner corrosion-resis- } \\
\text { tant barrier (Incoloy } 825 \text { ) assumed for first } \\
1000 \text { yr for } 90 \% \text { of packages but neglected } \\
\text { for most analyses. Galvanic protection con- } \\
\text { sidered. } \\
\text { - One pit completely penetrates both layers } \\
\text { - Near-field environmental conditions as- }\end{array}$ & $\begin{array}{l}\text { 1. Source term (CST) } \\
\text { - Corrosion rate of steel increased to get rapid } \\
\text { - Moilure for consistency with a-TSPA-1997. } \\
\text { in 2-phase flow code, BRAGFLO_T } \\
\text { - All alteration models use same mathematical } \\
\text { form (container \& fuel) (arrhenius rate equation) } \\
\text { - Both rate and solubility control for matrix alter- } \\
\text { - Wan } \\
\text { - Water for corrosion based on calculated satura- } \\
\text { model in BRAGFLO_T } \\
\text { - Wet and dry, anoxic and oxic corrosion } \\
\text { - Temperature, O, water saturation, and time } \\
\text { dependence of corrosion rates } \\
\text { - No cathodic protection, for consistency with a- } \\
\text { TSPA-1997 } \\
\text { - Near-field environmental conditions after } \\
\text { breach, but near field can be } \mathrm{O}_{2} \text { depleted } \\
\text { - No cladding protection } \\
\text { - } 10 \text { diffusion modeled through rubble contacting } \\
\text { container simultaneous with advecting water, if } \\
\text { present } \\
\text { - Neptunium solubility decreased two orders-of- } \\
\text { magnitude from TSPA-1993 } \\
\text { - Radionuclide sorption on corroded iron }\end{array}$ \\
\hline
\end{tabular}
sumed inside container immediately after first pit

- All radionuclides released upon package failure (no cladding protection)

- Six dissolution models; for alteration of fuel (oxide, metallic-humid oxic, metallic-wet oxic, ceramic, carbide, glass)

- Diffusive releases through pitted waste container in a-TSPA-1997 for most analyses. Advective release considered for sensitivity analyses of dissolution rate and surface area.

- Neptunium solubility decreased two ordersof-magnitude from TSPA-1993.

2. Waste intermingled

- Spent fuel (commercial low enriched): 63,000 MTHM

- DOE high-level waste (glass): 8798 MTHM*

- DOE-owned defense spent nuclear fuel: 2436 MTHM

- 83 MTU/acre

2. Waste Intermingled (same radioisotope inventory as a-TSPA-1997, but discrepancy with MTHM corrected for consistency)

- Spent fuel (commercial low enriched): 63,080 MTHM

- DOE high-level waste (glass): 9842 MTHM

- DOE-owned defense spent nuclear fuel: 2415 MTHM

- $\quad 85$ MTU/acre (85 kW/acre or $2 \mathrm{~W} / \mathrm{m}^{2}$ )

\footnotetext{
- Actual value calculated was 8798 MTHM, although it was reported as 8745 in a-TSPA-1997.
} 
Table C-2. Comparison Between a-TSPA-1997 and 1997 DOE SNF/DHLW PA (Continued)

a-TSPA-1997

(Simple PA)
1997 DOE SNF/DHLW PA

(Complex PA)

\section{Container}

3. Horizontal Emplacement

- Disposal containers:

- Large disposal package (LDP), 100-mmthick carbon steel layer and $20-\mathrm{mm}$ thick inner Inconel 625 layer

- Seven codisposal packages for DOEowned defense spent nuclear fuel possible per 13 waste categories

\section{Repository}

4. Size-74,181 MTHM

5. Orientation - N/A (one dimensional)

6. Thermal loading

- 83 MTU/acre

\section{Backfill}

7. Backfill - none except invert, with diffusion characteristics of tuff

\section{Geologic Barrier}

Unsaturated Zone

8. 6 one-dimensional flow pathways, through varying pancake stratigraphy

- Velocity fields (steady state) for fracture/ matrix abstracted from process-level models

- Percolation flux in fractures/matrix is spatially varied over repository area based on results from LBL-USGS model (avg $-6.2 \mathrm{~mm} / \mathrm{yr}$ )

- Faults not modeled

9. 1D flow with flow divided (volumetrically) between matrix and fractures

10. Particle transport

- Radionuclides transported (39); plots for top 5 dose-producing radioisotopes: Total, ${ }^{129} \mathrm{l}$, ${ }^{237} \mathrm{~Np},{ }^{99} \mathrm{Tc},{ }^{14} \mathrm{C},{ }^{229} \mathrm{Th}$

- Retardation modeled with simple, equilibrium (infinite capacity) distribution-coefficient $\left(K_{D}\right)$ model; lowest $K_{D}$ (most conservative) for each layer

- Fracture-matrix interaction simulated by Markovian process algorithm that randomly transitions particles between fracture and matrix modes

11. Gas transport - not modeled

11. Gas transport - not modeled

3. Horizontal Emplacement

- Disposal container:

- Large disposal package (LDP)

- Handling containers (nonprotective but steel mass important for $\mathrm{O}_{2}$ consumption):

- Defense spent fuel, $6.35-\mathrm{mm}$ thin-shelled stainless steel

- N-reactor fuel only, 9.5-mm thick stainless steel multi-canister overpack (MCO)

- High-level waste, 9.5-mm-thick stainless steel standard canister

- One package size used per each of 15 waste categories ( 4 package sizes modeled)

4. Size $-75,336 \mathrm{MTHM}$

5. Orientation - horizontal within tilted stratigraphy

6. Thermal loading

- $2.1 \mathrm{MTHM} / \mathrm{m}^{2}$

7. No backfill

8. Two-dimensional, tilted "paricake" stratigraphy

- Fault effects for Ghost Dance and Solitario Canyon modeled

- Refined geologic strata modeling in unsaturated zone

9. 2D composite porosity (equivalent continuum model)

- Velocity field from BRAGFLO_T used directly

- Infiltration flux spatially varied

10. Particle transport

- Radionuclides transported (3): ${ }^{129} \mathrm{I},{ }^{237} \mathrm{~Np},{ }^{99} \mathrm{Tc}$

- Sampled $K_{D}$ based on rock type (vitric, nonvitric, zeolitic)

- Transport with NUTS (dual permeability model treated as composite porosity model) 
Table C-2. Comparison Between a-TSPA-1997 and 1997 DOE SNF/DHLW PA (Continued)

a-TSPA-1997

(Simple PA)
1997 DOE SNF/DHLW PA

(Complex PA)
12. Release scenario:

- $30 \%$ packages have dripping water but water does not pass through container (radioisotopes must diffuse to edge of container); $10 \%$ dripping used for one comparison case

- For codisposal, radionuclide release from glass and fuel tracked separately

- $30 \%$ of packages have dripping water with advective flow assumed for sensitivity of dose to spent fuel dissolution rate and surface area

\section{Saturated Zone}

13. Stratigraphy

- 3D pancake with layers cut off at location of water table to make confined tilted units

- Single porous medium

- Most conservative retardation for each layer

14. Modeling

- 1-dimensional flow tubes used flux of $0.31-\mathrm{m} / \mathrm{yr}$ and porosity of 0.2 abstracted from 3D model

- Fault effects not modeled

\section{Agents Acting on Disposal System}

15. Climate - no climate cycles

\section{Biosphere Transport}

16. Dose calculations

- Maximum exposed individual located at point on accessible-environment boundary that corresponds to peak of radionuclide concentration

- 2 liter/day drinking water at $5 \mathrm{~km}$ downgradient (all water from tuff aquifer)

- Dilution method: (a) stirred tank model and (b) homogenous infinite aquifer with steadystate transport
12. Release scenario: Drips on waste package

- Percentage of packages with dripping water determined by saturation in fractures in each grid block modeling repository

- Release from each container tracked separately in source term; releases lumped together for transport in unsaturated zone

13. Stratigraphy

- 3-D pancake with water table

- Dual porosity

- Sampled ${ }^{237} \mathrm{~Np}$ retardation

14. Modeling (STAFF3D)

- 3D steady-state flow and transient transport

- Release region of radionuclides slightly below water table based on plume in unsaturated zone

- Fault effects modeled: flow barriers and separate head zones allow more accurate modeling of flow behavior

15. Climate - wet-dry cycle

- Cosinusoidal function

- Period, base peak and rate sampled

- Variable infiltration area included at surface

16. Dose calculations

- GENII-A

- Maximum exposed individual

- Exposure scenarios:

- Ranch case (beef consumption only)

- Farm case (food consumption, water consumption, inhalation)

- Small community case (water consumption and local food products)

- Maximum exposed individual located at point on accessible-environment boundary that corresponds to peak of radionuclide concentration

- 2 liter/day drinking water at $5 \mathrm{~km}$ downgradient (all water from tuff aquifer) for water consumption cases

- Comparison calculations with a-TSPA-1997

- Dilution methods: (a) stirred tank model and (b) homogenous infinite aquifer with steady-state transport 


\section{Appendix D: \\ Parameters for Waste Package Materials for Final 1997 PA}


Appendix D: Parameters for Waste Package Materials for Final 1997 PA 


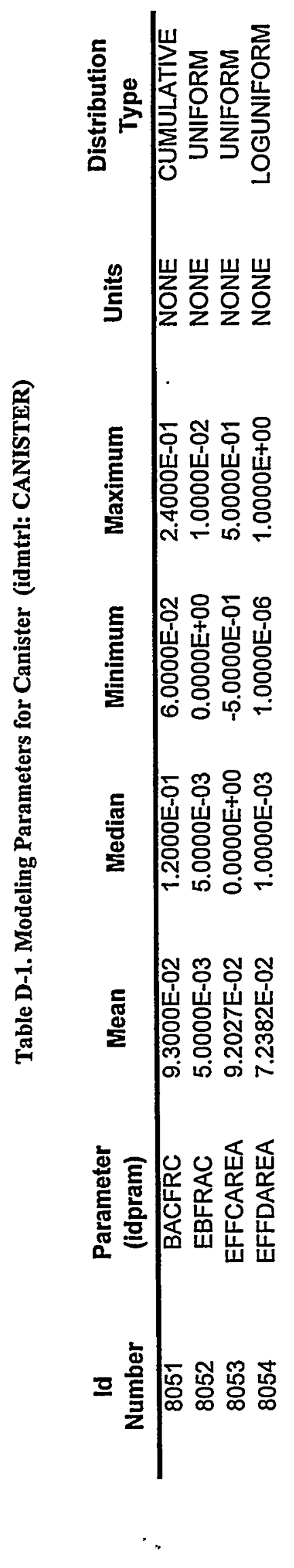




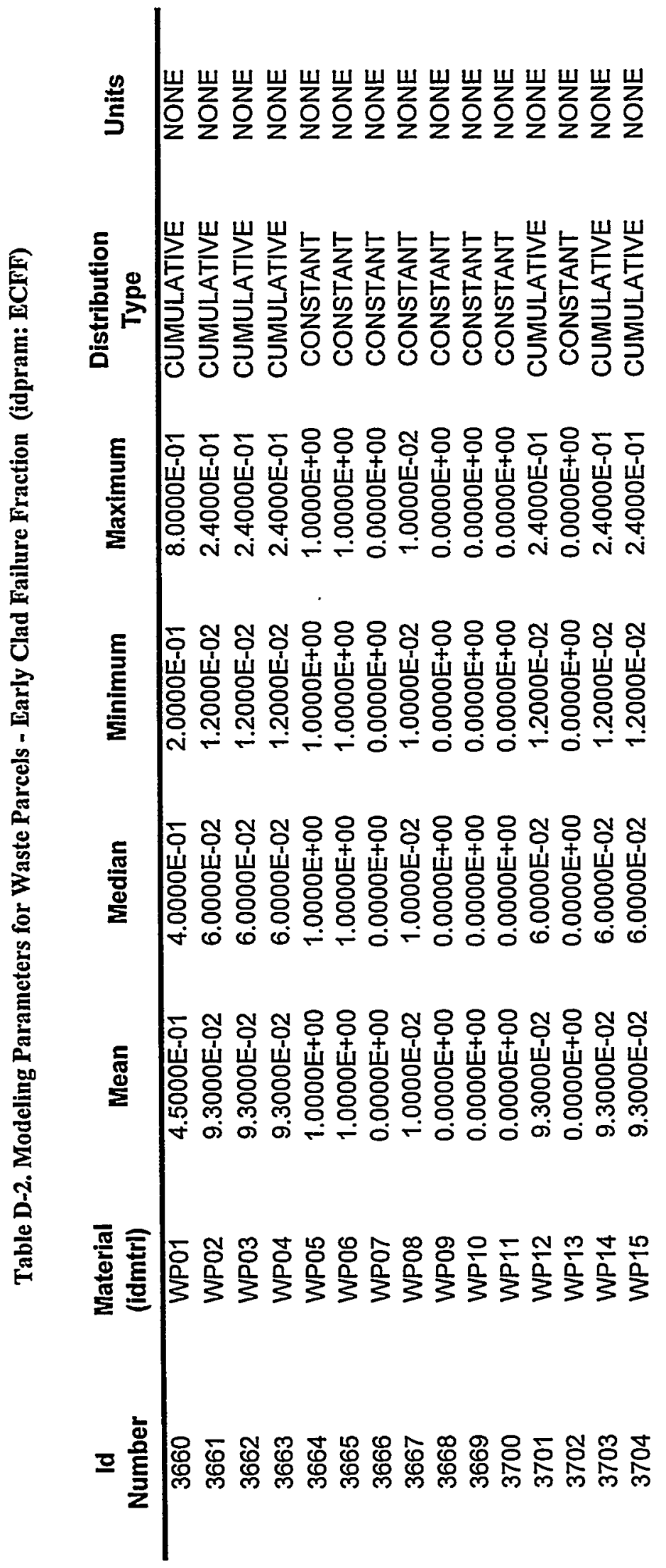




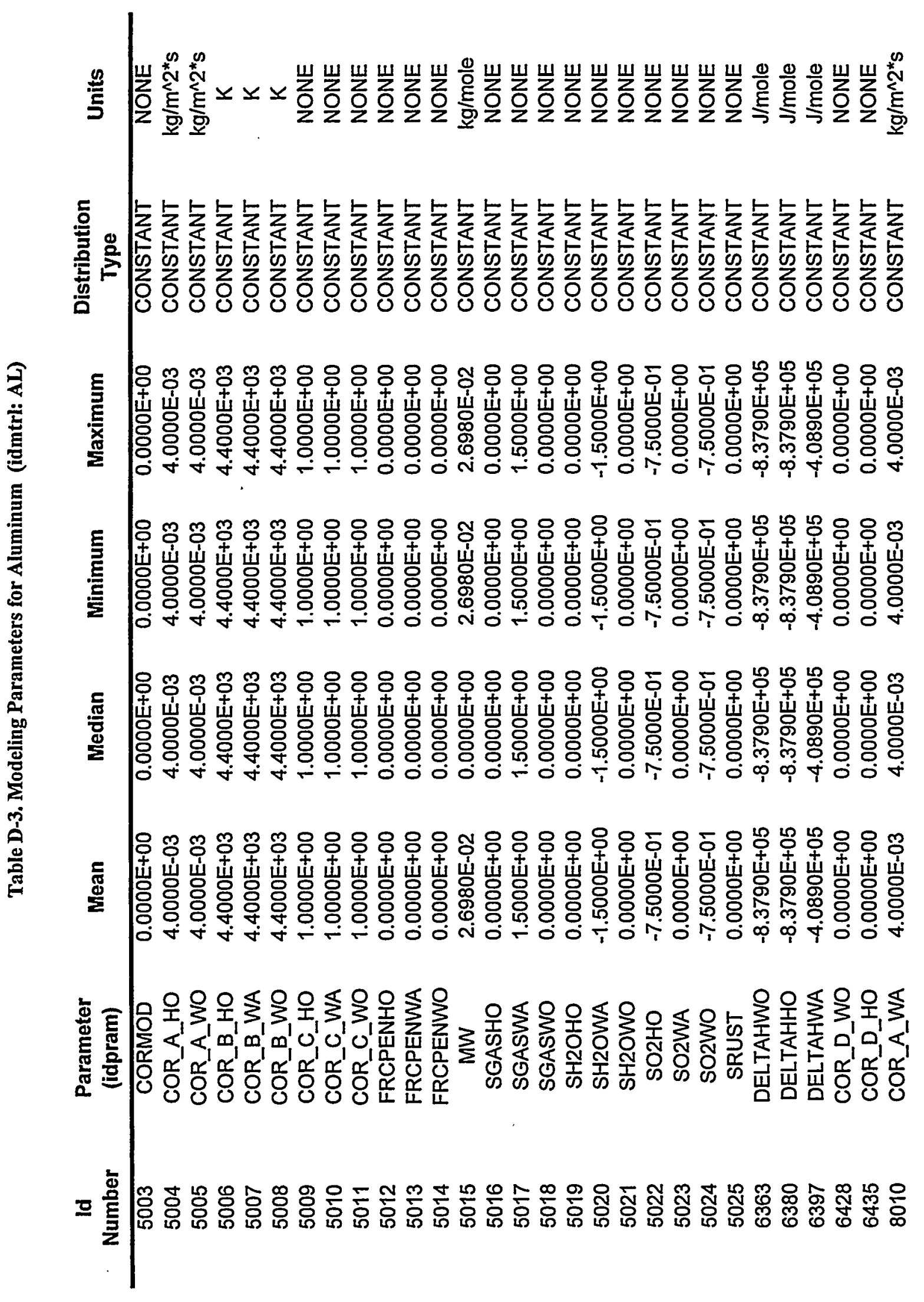




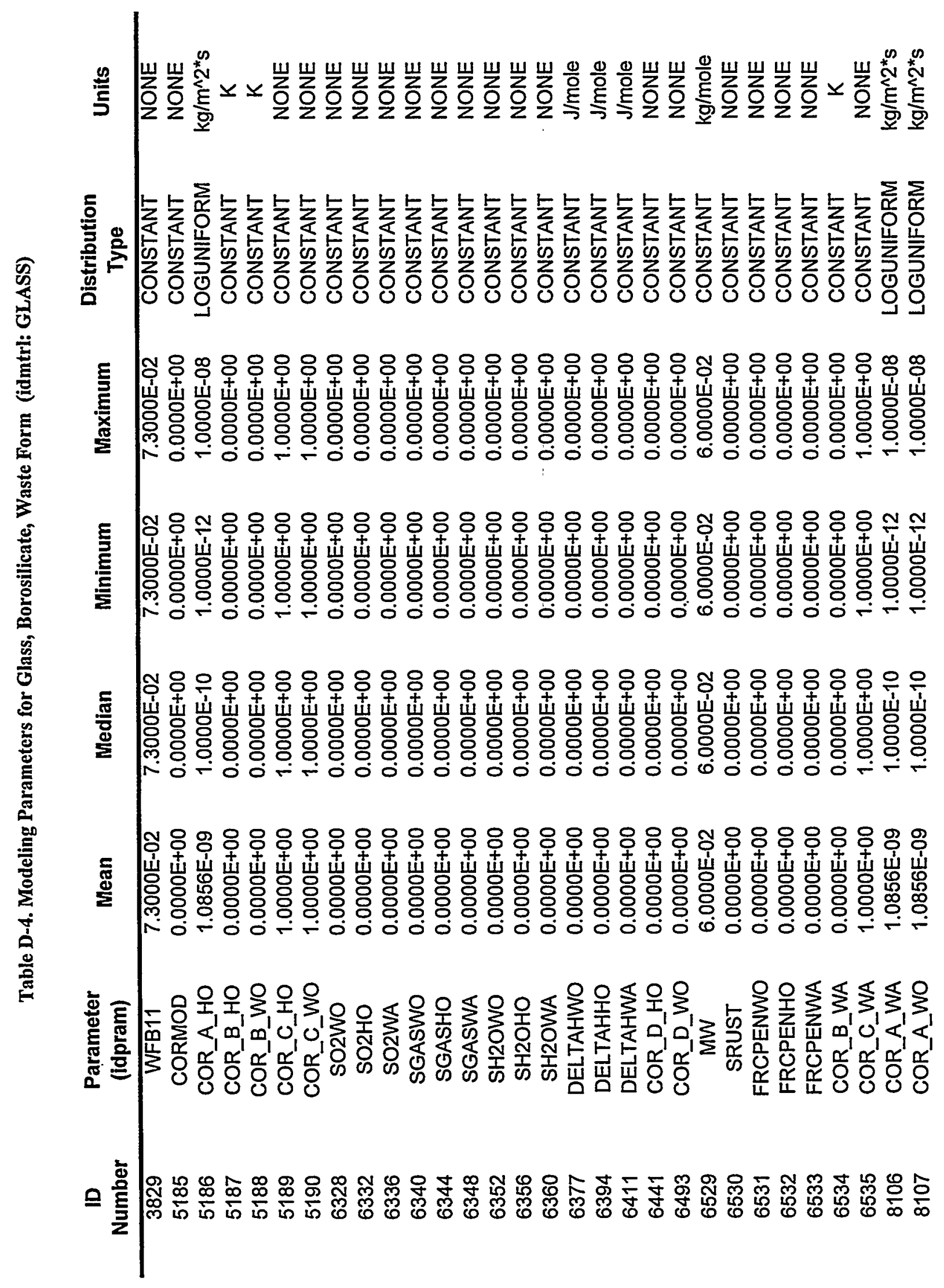




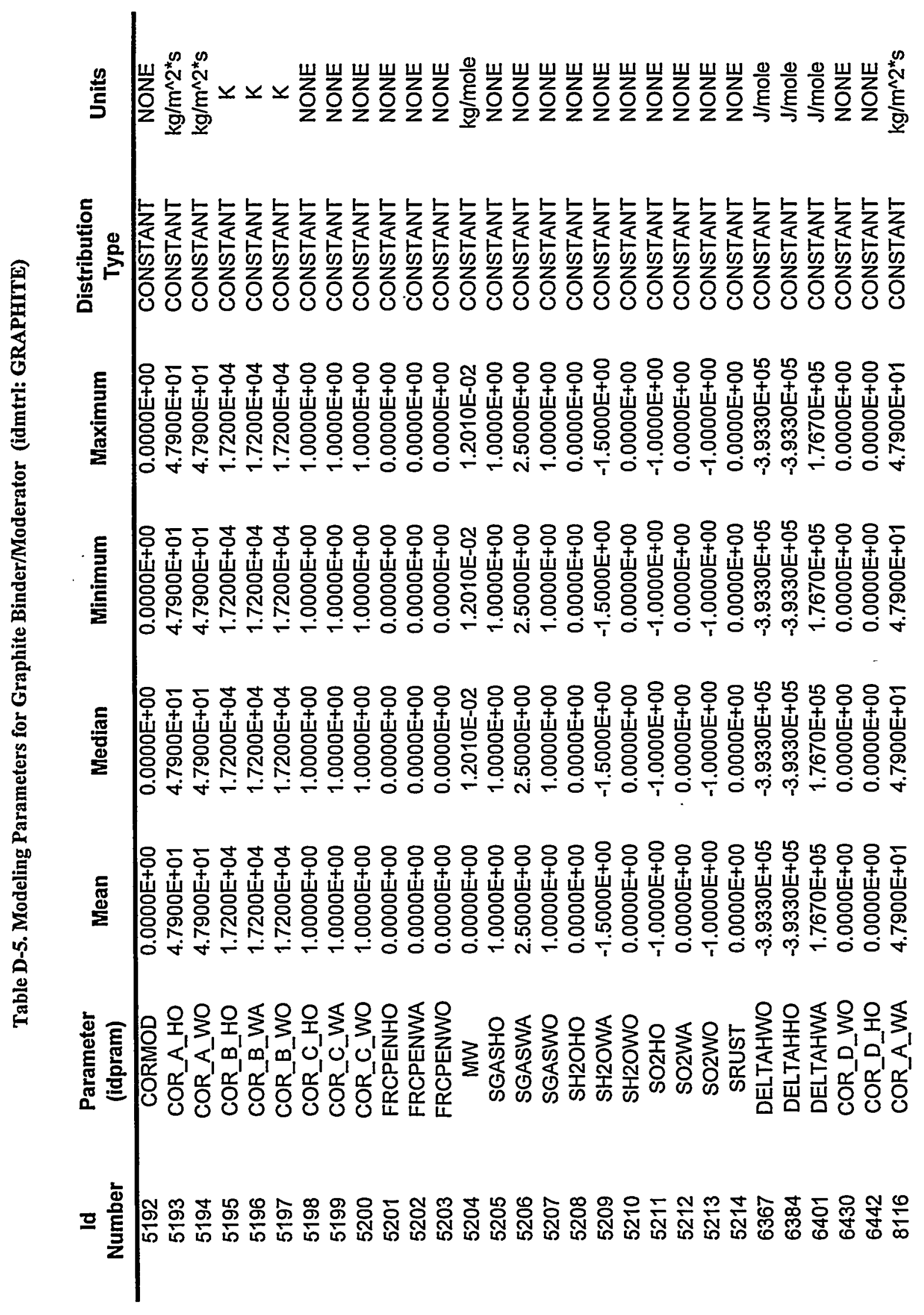




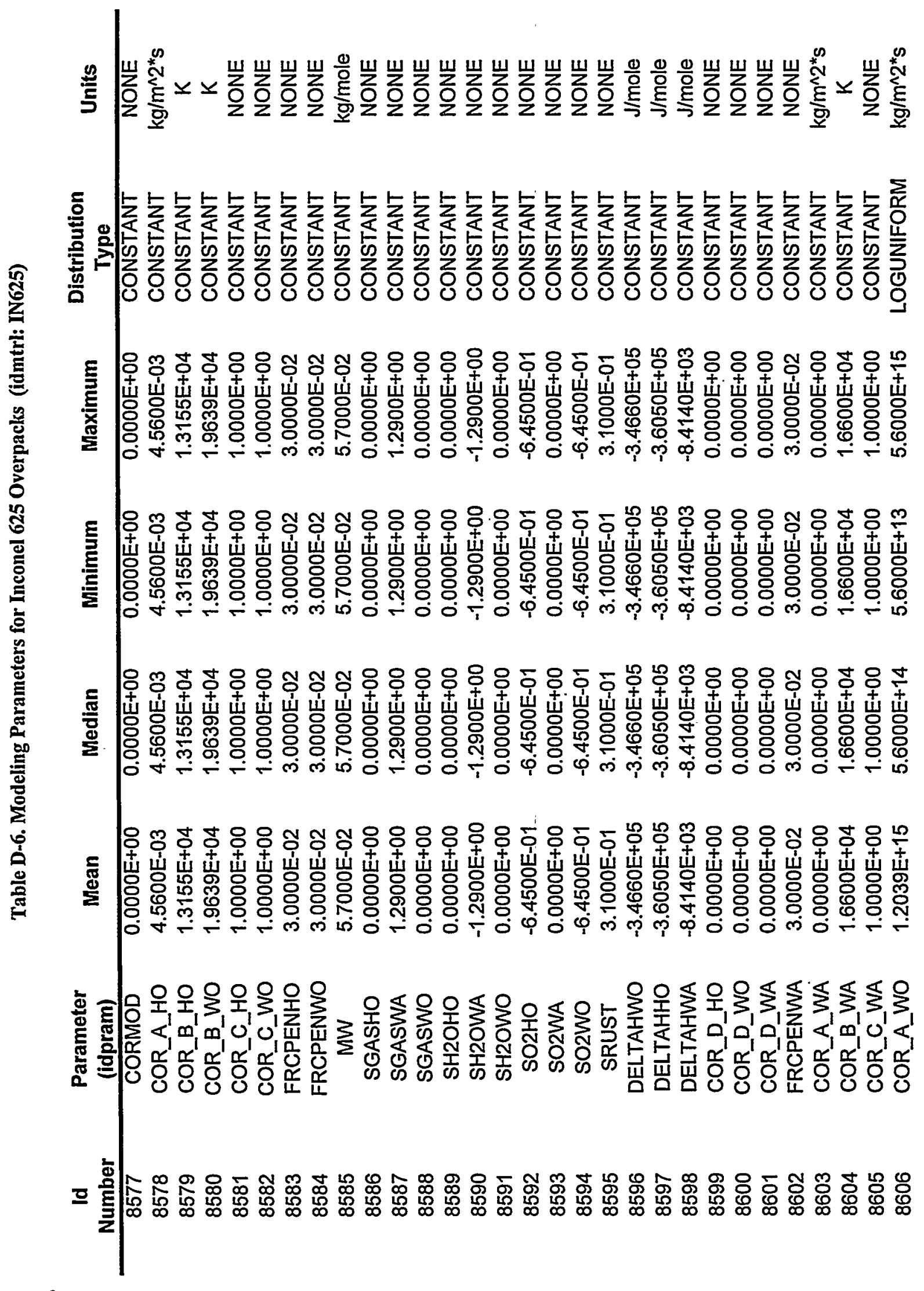




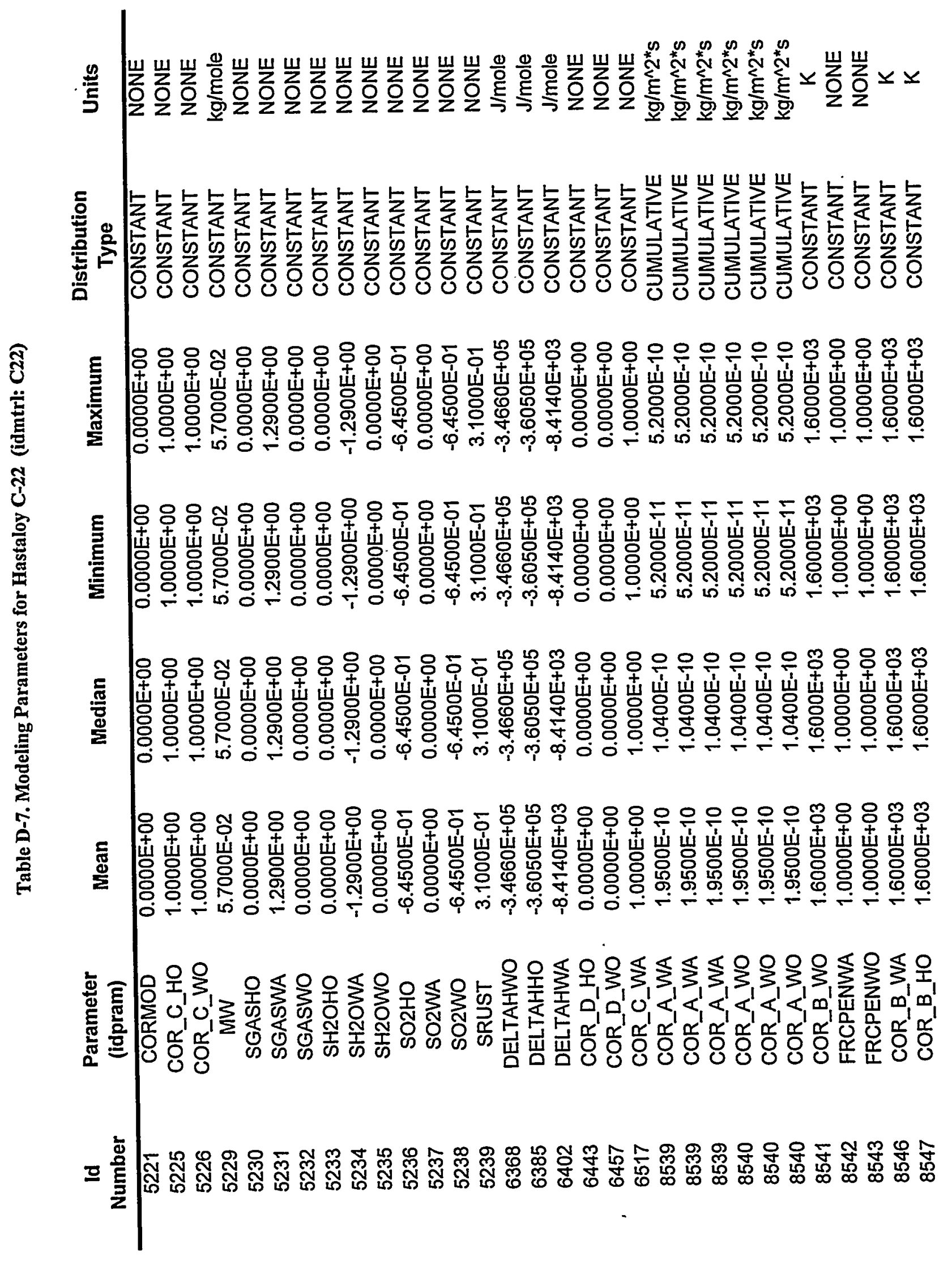




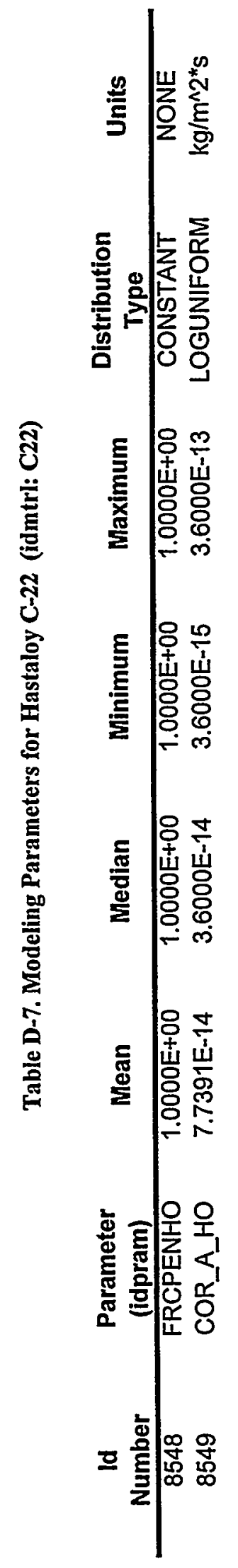

D-10 


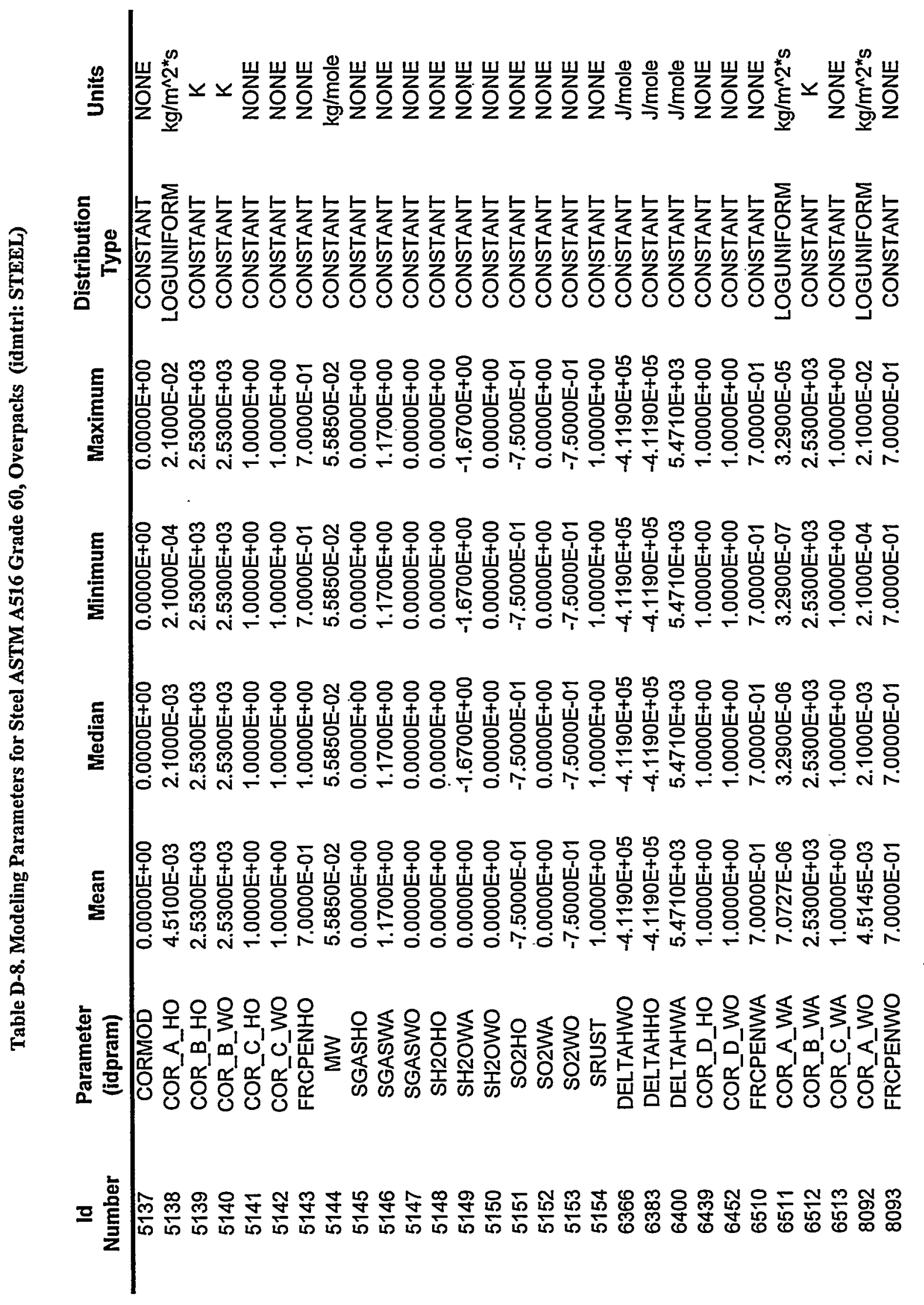




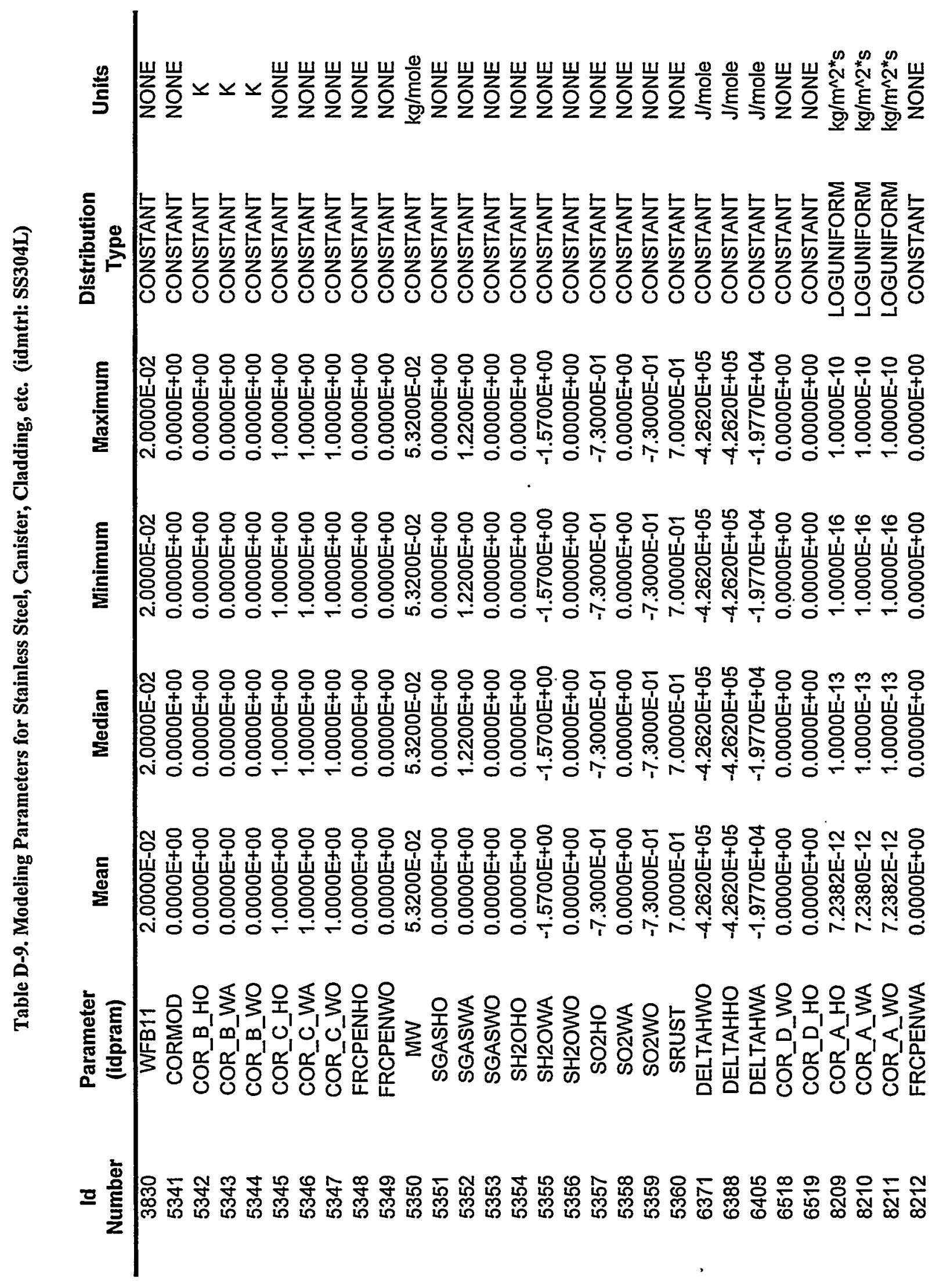




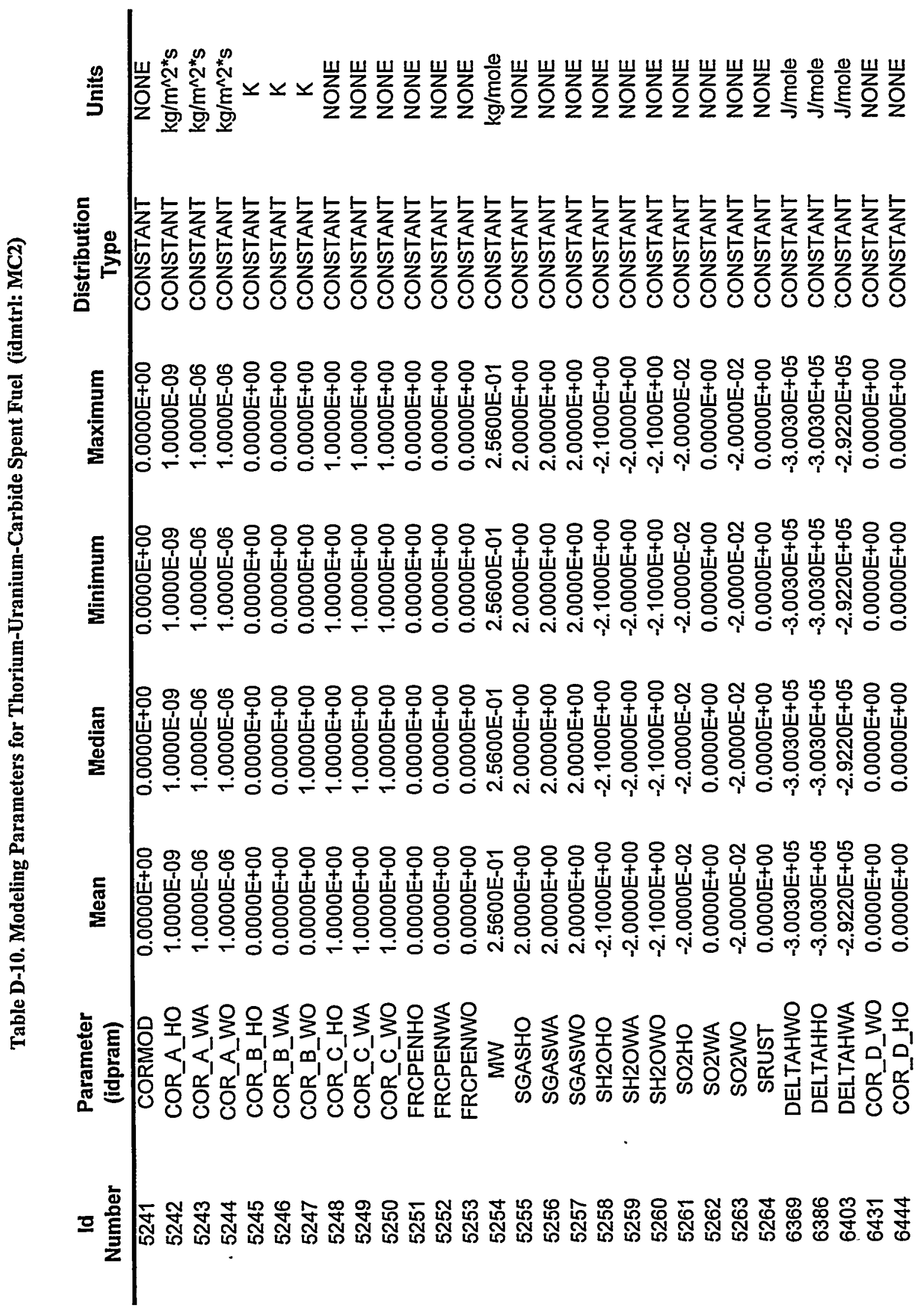

D-13 


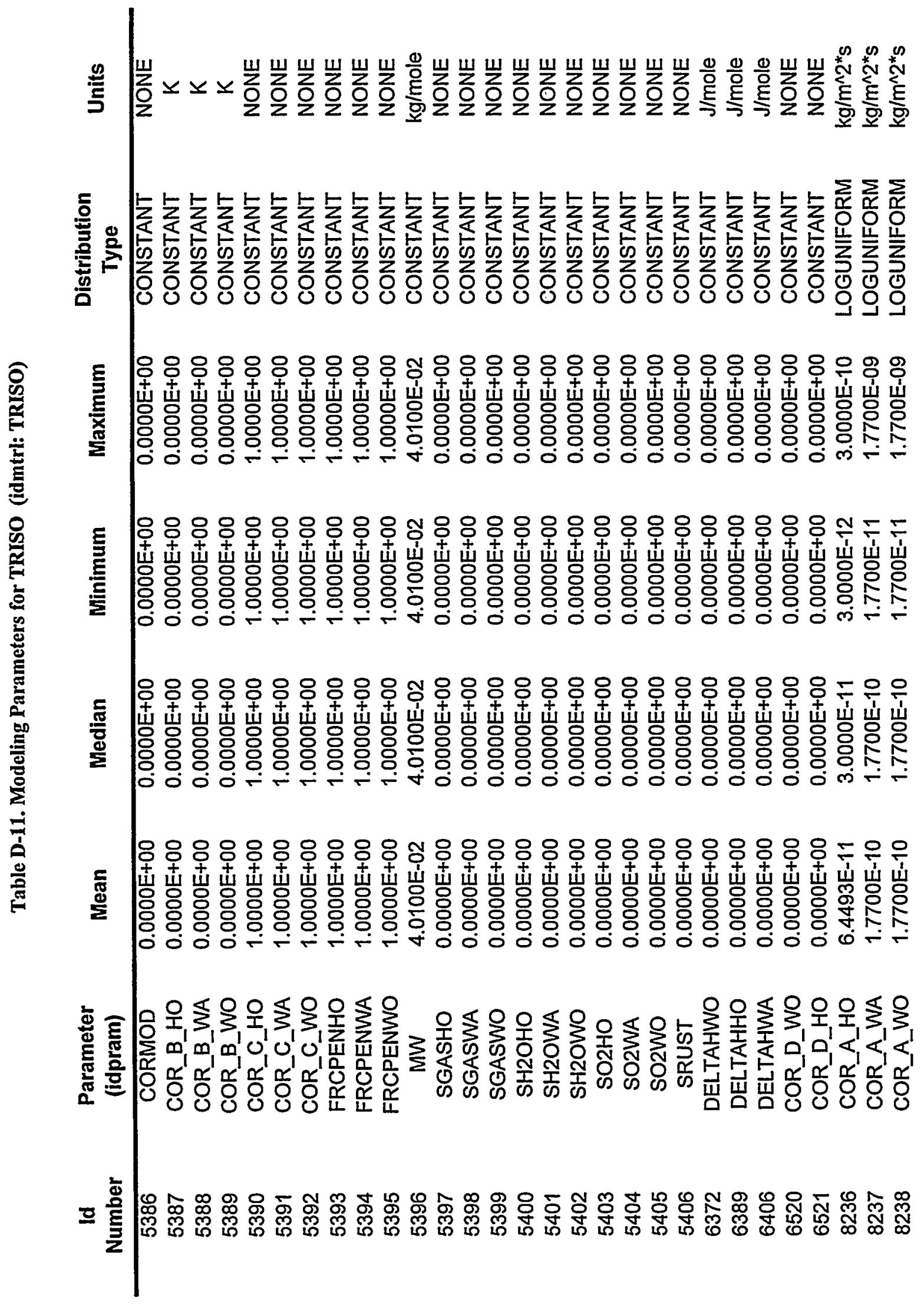




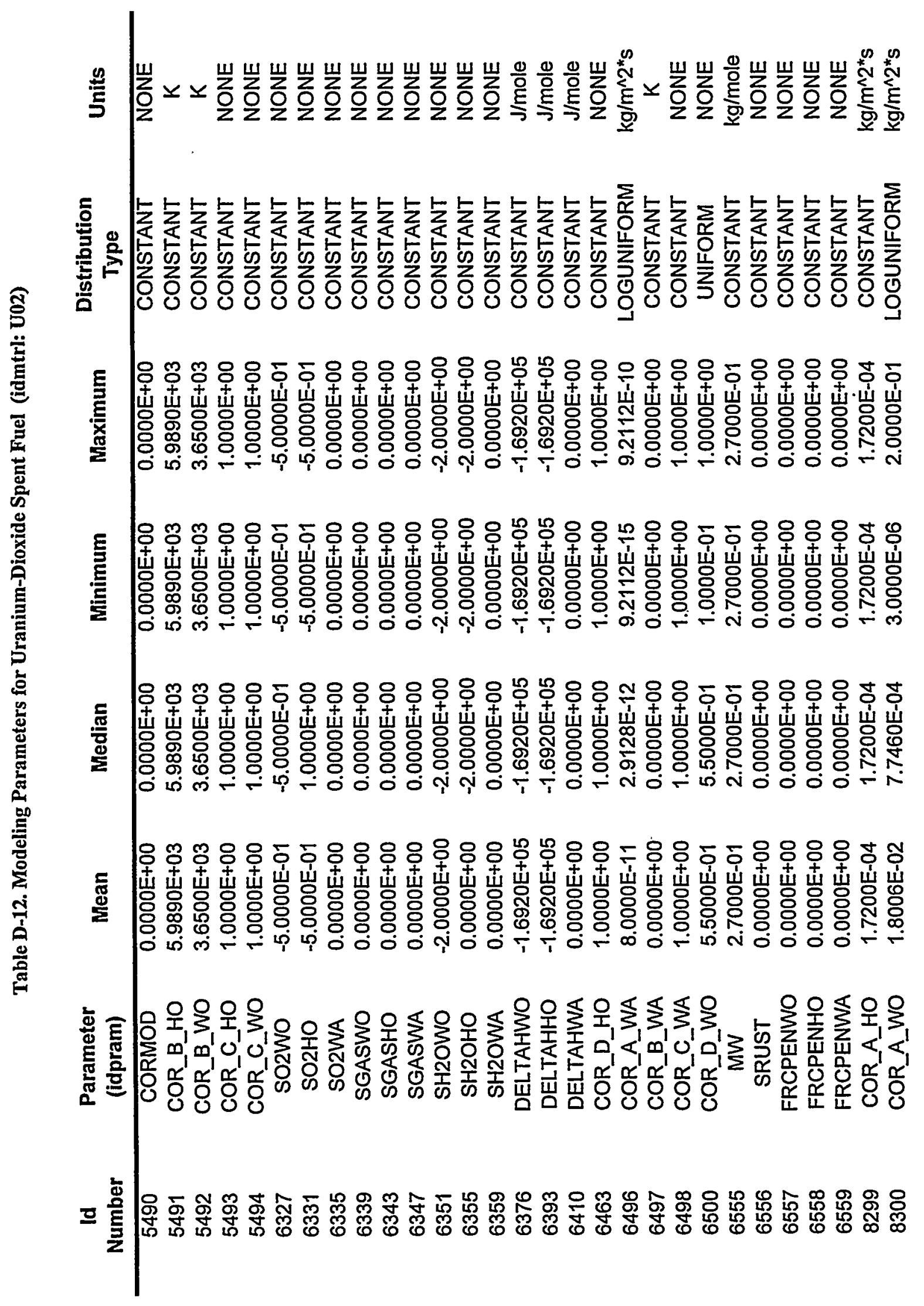




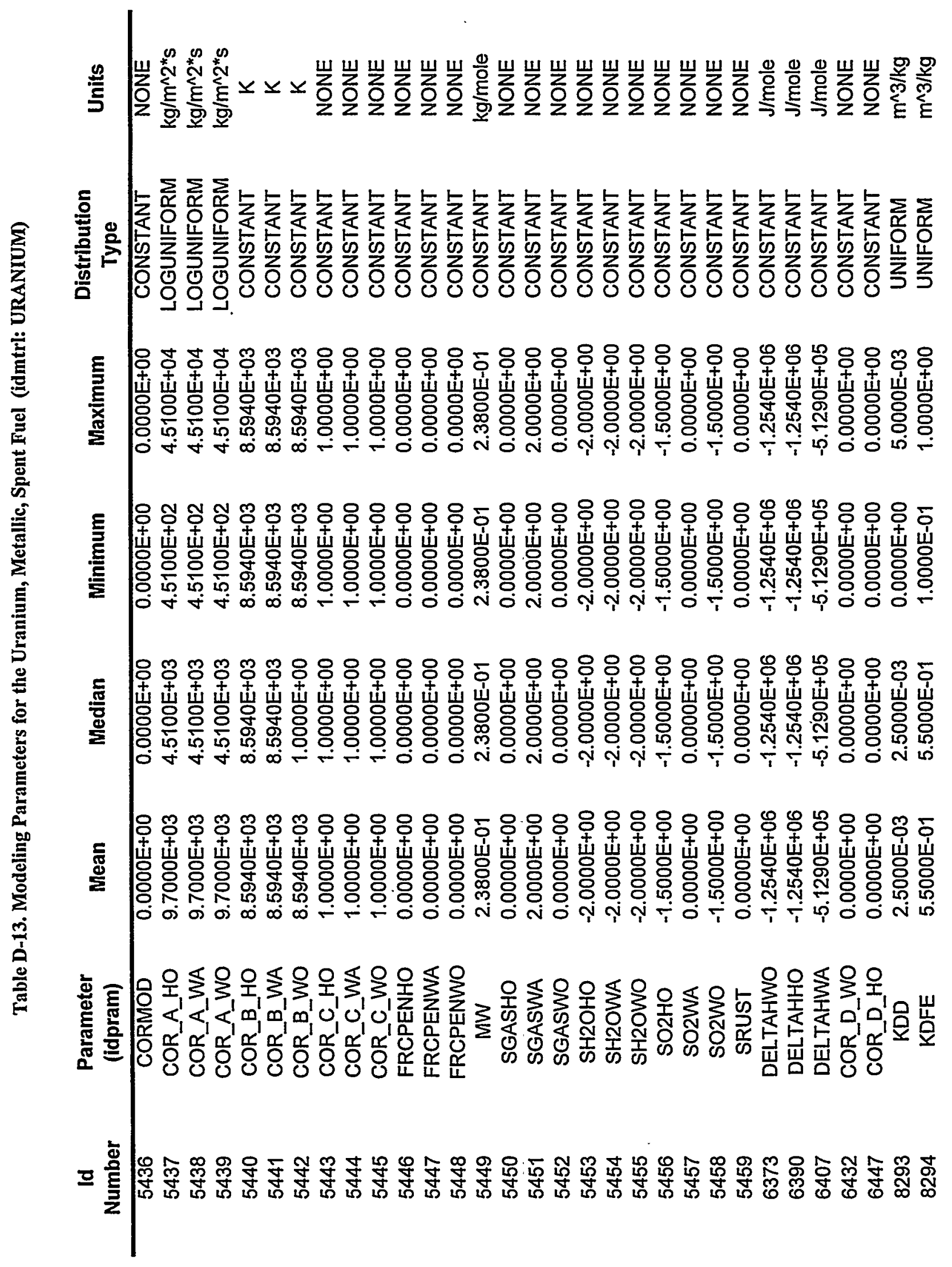




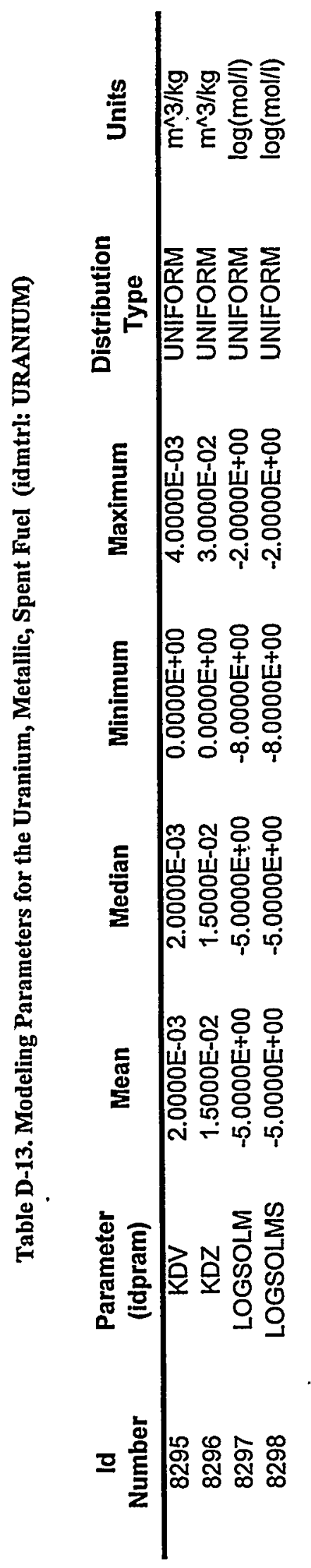




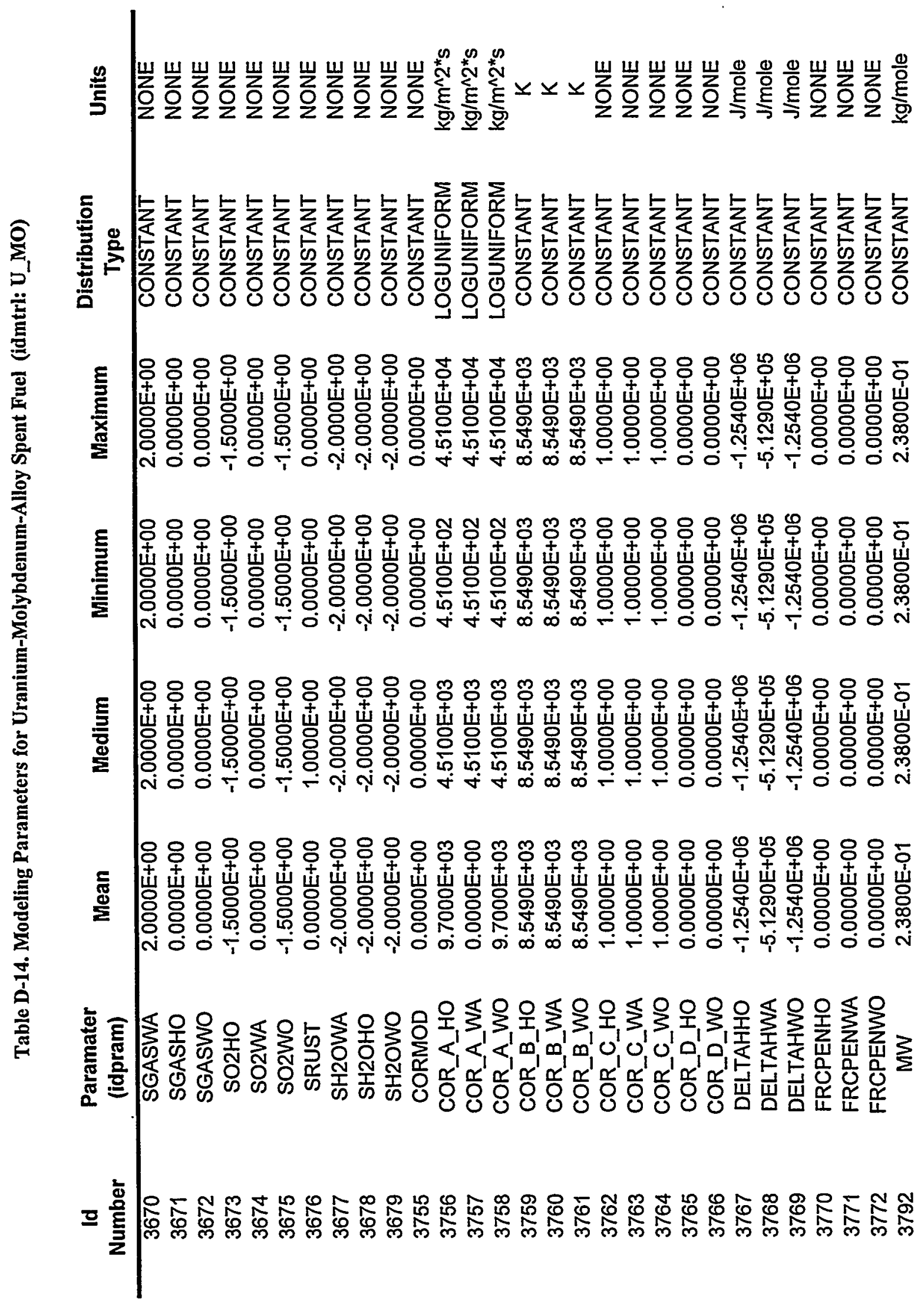




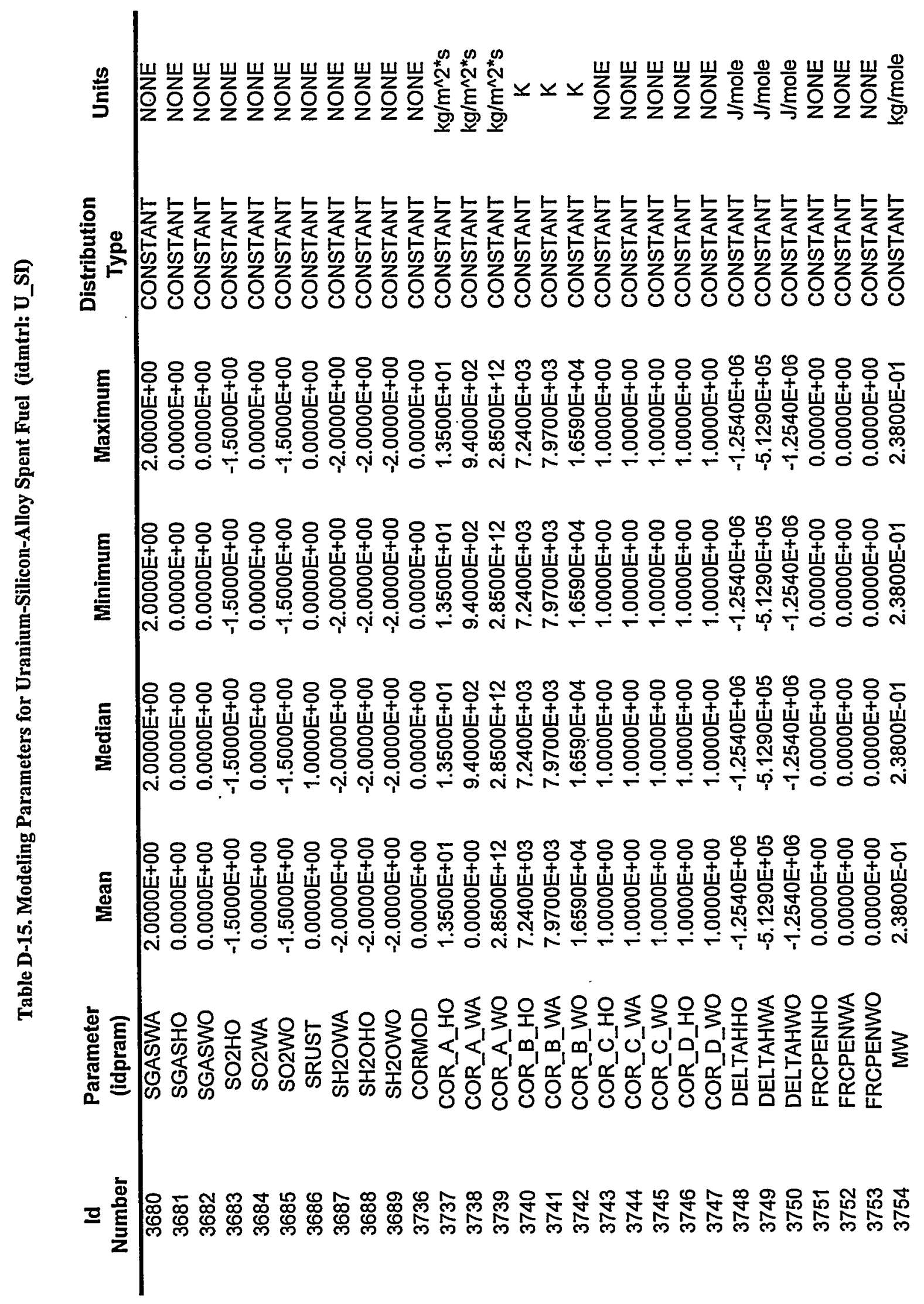




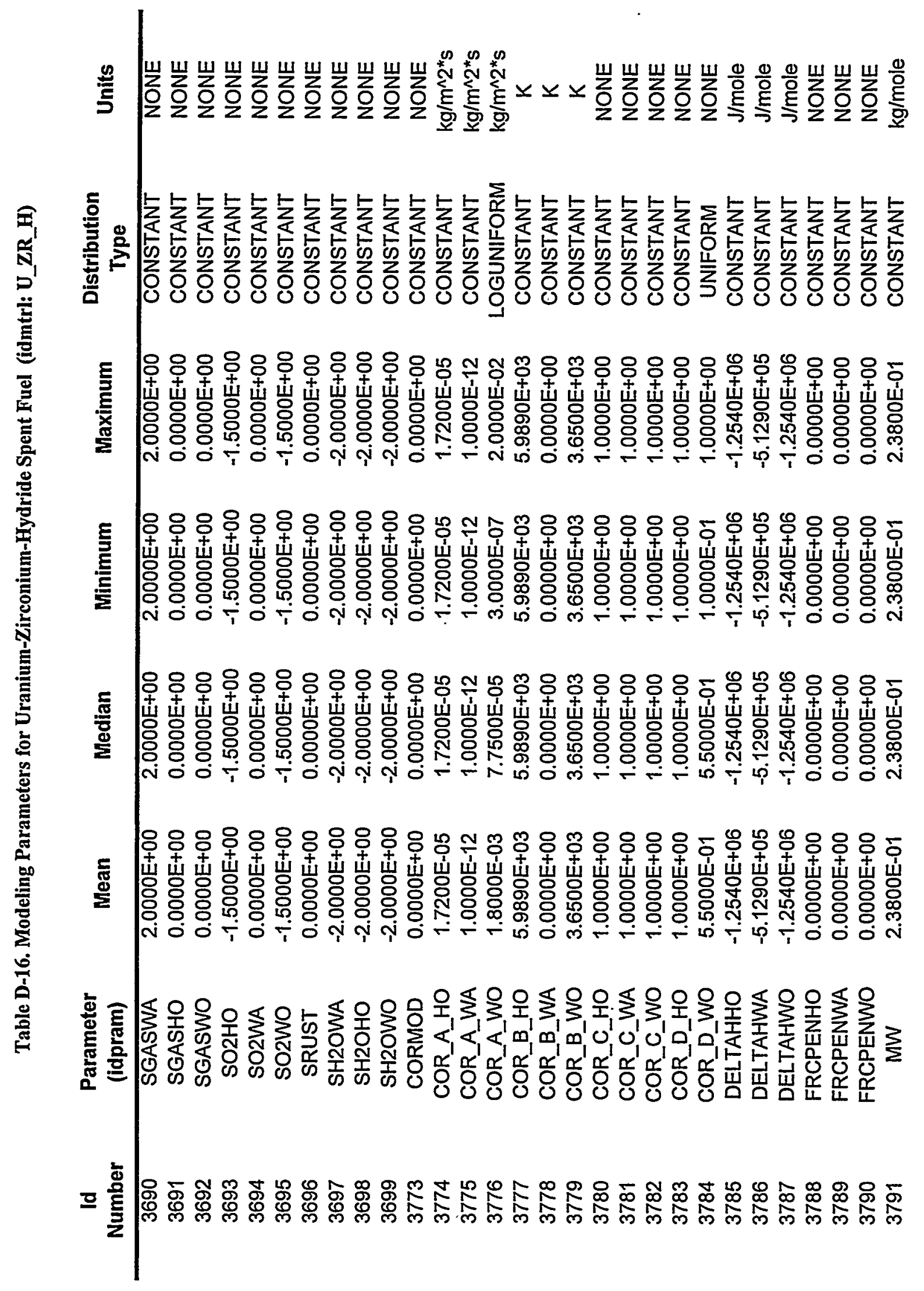




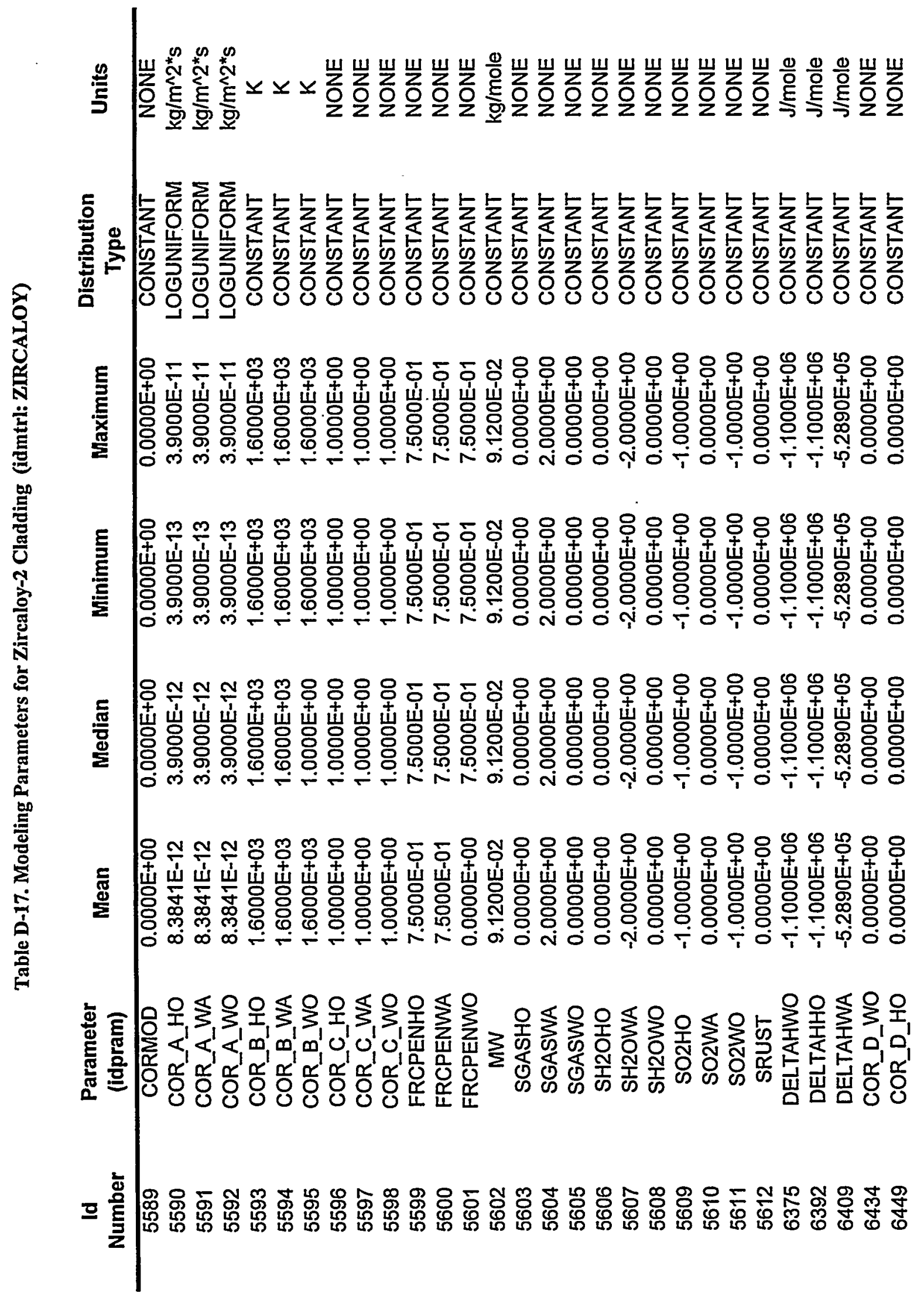

